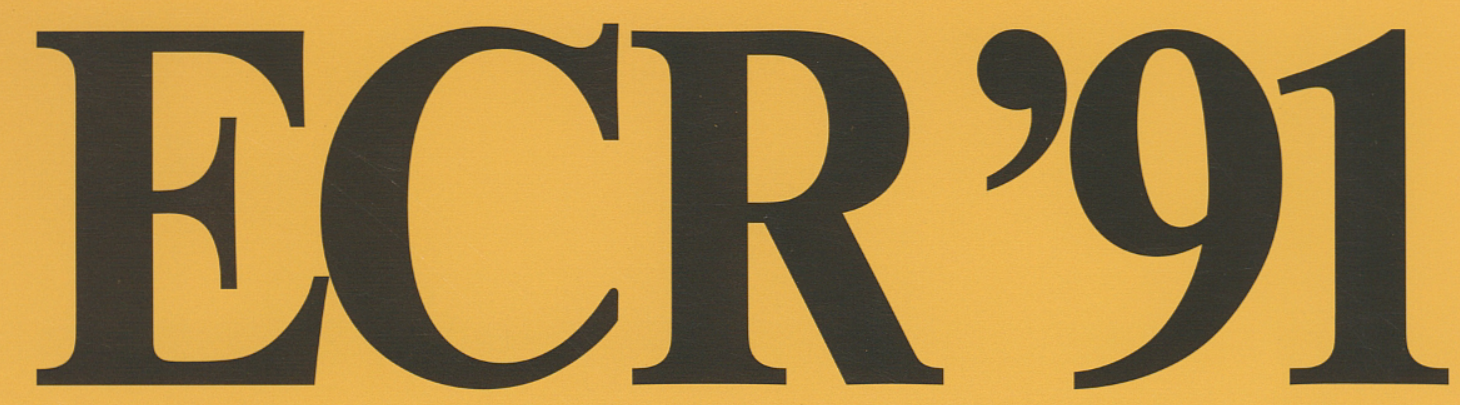

\title{
European Congress of Radiology
}

\section{Scientific Programme and Abstracts}

Supplement to

European Radiology

Vol.1

Vienna, Austria

September 15-20, 1991

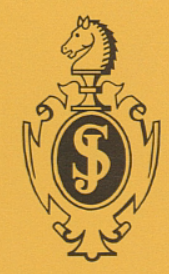

Springer International

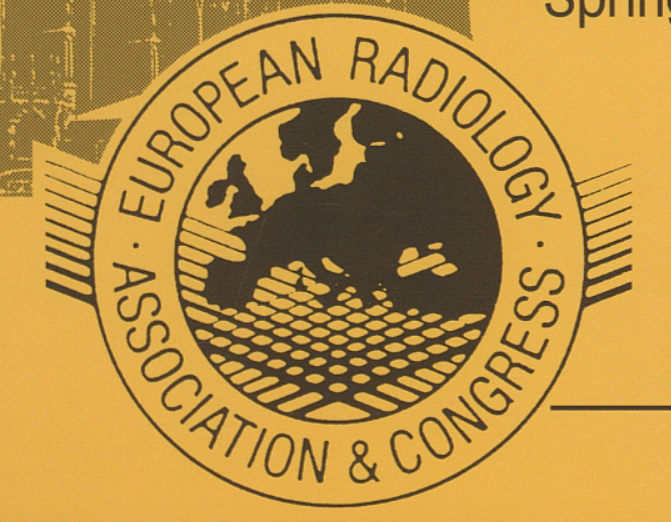




\section{Scientific Programme}

\section{Monday}

8.30-10.00 AM

\section{Room A}

\section{CQ}

\section{Basics of MR imaging \\ Stuart W. Young; Palo Alto, CA}

Since its clinical introduction in the early 1980s MRI has undergone considerable development and diffusion with new applications continually being introduced. Although there appears to be a general trend away from high field toward mid field and ultra low field scanners and the initial enthusiasm for whole body spectroscopy and multinuclear imaging has yet to come to fruition, spin echo spin warp sequences continue to be the most utilized pulsing sequences due to their ability to exploit $\mathrm{T} 1$ and $\mathrm{T} 2$ differences among the tissues and display them as gray scale images taken along any plane desired by the examiner. More recently, gradient echo and low-tip-angle methodologies have been found to be very useful for encoding flow information, detecting susceptibility phenomena, and providing very fast imaging methodologies in 3D acquisition and spoiled GRASS or turbo flash pulsing sequences (usually used in conjunction with breath holding on the part of the patient).

All parts of the anatomy from the head to the extremities are currently being evaluated by MRI and it is the modality of choice for evaluating the brain, the vertebral column and spinal chord, and the extremities. Although CT and MRI images appear at first to be comparable they are, of course, based on completely different physical characteristics of the tissue. The gray scale in CT imaging reflects the tissues ability to absorb $\mathrm{x}$-ray photons transmitted through the tissue whereas the MR signal in proton MRI reflects not only nuclear density of the protons within tissues but more importantly the biochemical nature of the tissue as reflected in the different $\mathrm{T} 1$ and $\mathrm{T} 2$ relaxation times. The use of short TR/TE sequences and long TR/TE sequences to examine various parts of the body not only provides optimal T1 and T2 tissue differences but the flow void on the short TR/TE sequences can often provide important information regarding the position and flow characteristics of blood vessels and MRI scans are often complimentary with plane radiography and CT.

\section{Basics of MR imaging - contrast agents K. J. Wolf; Berlin}

MRI offers excellent soft tissue contrast compared to other imaging modalities. Despite this outstanding advantage, research activities in potential contrast media developed promptly as early as 1980 . MRI contrast media influence imaging parameters in increasing or decreasing signal intensities in tissue having adequate concentration of this media. Paramagnetic, superparamagnetic and ferromagnetic agents influence $\mathrm{T} 1$ and $\mathrm{T} 2$ relaxation times in a specific manner.

Gd-DTPA is a typical representative of the paramagnetic-chelate group. Its biodynamic behaviour after i.v. injection is comparable to typical iodine contrast media, the biodistribution is intravascular, extracellular and interstitiell. Demonstration of local perfusion offers improved diagnostic information.

Intracellular targeting may provide advantages in term of tissue specificity, manganese and iron-compounds are tested in clinical trials. First experimental results of liposomes and monoclonal antibodies demonstrate the wide field of MR-contrast media research.

In this course typical representatives of the different groups of MR-contrast media are discussed. The actual state of clinical indications and limitations will be of special interest.

\section{Room B}

\section{BC} 101 11 Mediastinum: plain film, CT and MR correlations $\bar{M}$. Coulomb, G. Ferretti, Y. Ranchoup; Grenoble

Contribution available at the information desk.
MRI of the mediastinum is not yet as well established as CT for the evaluation of mediastinal diseases. Only in neurogenic tumors and diseases of the thoracic aorta MRI is already the method of choice instead of CT. Clinical applications of MRI in the chest have been limited by respiratory and cardiac motions interacting to produce blurring, signal loss and ghost artifacts that degrade anatomic resolution. On the other hand, CT scans show excellent contrast between the aerated lung, vascular structures and mediastinal fat. CT scans have smaller voxels and are acquired during breath holding, improving spatial resolution. The principal limitation of CT is poor inherent contrast between pathologic masses and mediastinal vascular structures, which is easily corrected by infusion of iodinated contrast media.

Technique

Cardiac gating is the most important method for reducting cardiovascular motion and flow related ghost artifacts in the mediastinum. ECG-gated images provide better visualization of mediastinal anatomy, cardiac structures and especially the great vessels. T1-weighted spin echo sequences with short TR and TE plain and after administration of paramagnetic contrast media are sufficient for the evaluation of mediastinal masses. T2-weighted spin echo sequences should not be used because of the long acquisition time, the low signal to noise ratio and many motion artifacts. For the differentiation of cystic lesions gradient echo sequences can be helpful in special cases.

CT is still the method of choice for the primary staging of bronchogenic carcinomas and malignant lymphomas. MRI, nowadays, is able to deliver the same diagnostic information as CT. The advantage of MRI is the higher contrast resolution in comparison to $\mathrm{CT}$. Using paramagnetic contrast medium MRI allows a better delineation of mediastinal lesions and a better differentiation of solid, necrotic or cystic parts of tumors. The ability to examine different planes is another advantage of MRI especially in infiltrating tumors of the chest wall or pericardium.

The differentiation of tumor tissue by measurement of the relaxation time is not possible because of overlapping. Only in cystic or fatty lesions an exact diagnosis is possible in most of the cases.

\section{Room C}

\section{Ws}

\section{4}

13 Advanced imaging of the gastrointestinal tract including MR J.Pringot; Bruxelles

The practice of GI radiology has been changed by the progresses in imaging. US and CT are not only accurate for evaluating the intramural and extraintestinal components of disease but occasionally may detect undiagnosed gastrointestinal lesions. However, they are not competing for evaluating intraluminal and mucosal disease with conventional barium examination or endoscopy which remain superior.

Dedicated US and CT studies of the GI tract require emptyness and distension of the intestinal lumen by air, water, or a positive contrast agent to avoid shortcomings due to collapsed or fluid filled intestine.

CT requires also intravenous injection of angiourographic contrast, repositioning, and thin section over the area of interest if required.

Both US and CT allow direct visualization of the GI wall thickness and surrounding structures, including the liver, CT being more global in its imaging capability and having less limitations. Endosonography is the most accurate modality available yet for the study of the upper GI and rectal wall. In particular, by demonstrating accurately the muscularis propria layer it permits accurate assessing of the depth of the mural invasion.

The indications for GI imaging include 1) diagnosing and suggesting the presence of primary disease in the GI tract, 2) evaluating the nature and extent or origin of disease in patients with known GI lesions, 3) determining the presence, location and severity of complications associated with primary GI lesions, 4) evaluating the effect of treatment on known GI lesions or of their complications, 5) diagnosing or suggesting the recurrence of disease.

Despite limitation particularly for the characterization of segmental involvement, US and CT remain the leading imaging modalities of the GI tract.

MRI may be useful in determining the extent of primary or recurrent carcinoma and in diagnosing extraintestinal complications of inflammatory disease. However, despite technological improvement and the developement of dedicated oral contrast agents, it has not been established as superior to CT. 


\section{Room E1}

\section{Re}

\section{Standard radiography of the skull}

R. Lehmann; Berlin

The basis of the necessity of a standardisation is the non-uniformity of the $\mathrm{X}$-ray-pictures of the skull in the routine. Therefore an inter- and intrainstitutional comparison of films is often impossible. The consequence is a repetition of taking of X-ray-pictures and a violation of X-ray-protection, a waste of material and of working time as well as a prolongation of the diagnostic time. Therefore a group of 10 neuroradiologists, physicists and MTRA analysed the literature and the practical and technical conditions concerning the best methods in 1973/74. The conclusion was a recommendation for the most exact and best handling methods with practical and technical instructions and criteria for the analysis of the films. The recommendations were revised after many discussions twice after some years.

These standards are compared with the guide lines of the "Bundesärztekammer" of the FRG and with the EG-guide lines.

\section{Standard radiography of the spine}

\section{J.L. Dietemann; Strasbourg}

Plain films of the spine have no or limited value in diagnosis of intradural diseases; MR imaging, myelography and myeloCT are the diagnostic procedures usually used for diagnosis of tumoral, traumatic, ischemic, inflammatory, dysraphic lesions of the spinal cord.

Plain radiographs of the lumbar spine including lateral and frontal projections (oblique views are mainly requested for demonstration of pars defects) demonstrate degenerative changes involving lower intervertebral spaces and apophyseal joints (in $95 \%$ of both sexes by 70 years of age). Isthmic spondylolisthesis is usually suspected on lateral and frontal films. In degenerative spondylolisthesis mild forward displacement ( 2 to $4 \mathrm{~mm}$ ) of L4 or L3 may be responsible for important spinal stenosis. It is difficult to evaluate the size and the shape of the spinal canal. Flexion and extension views are helpful for diagnosis of vertebral instability.

Plain radiographs of the cervical spine including lateral, frontal and oblique projections demonstrate degenerative changes usually located at C5-C6 and C6-C7 levels. Diagnosis of cervical spinal stenosis is possible by using mental transposition of the vertebral body toward the vertebral canal (Wackenheim's procedure).

Plain radiographs of the thoracic spine may often demonstrate disc herniations involving the thoracic spine; indeed more than $50 \%$ of thoracic disc herniation are calcified.

Plain radiography has an essential place in evaluation of traumatic lesions of the spine. Most tumoral lesions are demonstrated by standard films and some primary tumors will give characteristic appearance. Diagnosis of vertebral osteomyelitis becomes possible 4 weeks after the first clinical manifestations; bone scans, CT and MR may reveal earlier modifications. Paget's disease has a characteristic radiological appearance. Evaluation of spinal deformity is based on plain radiography.

\section{Room E2}

\section{RC}

\section{Imaging of soft tissue tumors K. Bohndorf; Aachen}

The application of plain radiography, ultrasonography, angiography, CT scanning and MR imaging in children and adults with soft tissue tumors will be discussed. The sensitivity and specificity of these imaging modalities in the initial diagnosis and follow-up of these tumours will be reviewed, together with their use in the localisation, characterisation and staging of disease, with special emphasis on the role of MRI. The need to distinguish recurrent disease from post-therapeutic changes will be stressed. Techniques for differentiating benign lesions from more aggressive ones will also be considered. We will also review the use of Gd-DTPA and special imaging sequences in the MR evaluation of soft tissue masses. In soft tissue tumours, plain radiography is only of value in the exclusion of bone involvement and in the demonstration of calcified structures, such as phleboliths in haemangiomas. US has a role as a screening method to confirm the clinical suspicion of a soft tissue mass, whereas angiography is only necessary in cases where the exact demonstration of the arterial supply of the tumour is of surgical interest. CT and especially MRI provide the most useful information and, although the imaging features of many masses are non-specific, characteristic appearances have been reported for certain soft tissue tumours. MRI has been shown to be most valuable in the staging of these tumours. This modality provides essential information about tumour size and extent, anatomic compartmentalisation, joint invasion and relationship to major nerves and vessels.
17 Sports injuries: imaging of tendon and ligament damages Folco Rossi; Rome

This refresher course will provide an overview of the imaging modalities currently being used to evaluate acute and chronic damages of tendons and ligaments most common in athletes active in high performance sports.

As we know, joint stability and mobility depend on a delicate interaction between different anatomical elements.

Due to the biomechanics of the joints, tendons and ligaments act as true "articular pivots", and are more and more important as regards to the functional requirements during athletic motions.

Aim of the course is therefore the presentation of common and uncommon injuries pertaining only three joints (gleno-humeral girdle, knee and subtalar joint) and three articular pivots (supraspinatus and long head of biceps tendons, cruciate and interosseus talo-calcaneal ligaments), and the evaluation and comparison of their conventional and unconventional radiological features.

\section{Imaging disorders of muscle David Wilson; Oxford}

Apart from the detection of calcification and perhaps crude assessment of fatty lesions, plain radiographs have limited value in the study of muscle disorders. Clinicians have largely depended on physical examination and biopsy. Since the introduction of ultrasound, computed tomography and magnetic resonance a plethora of data has become available. This presentation will compare and contrast these diagnostic methods illustrated by a variety of cases of muscle disease.

Ultrasound is particularly effective at demonstrating fluid containing structures and is the only means by which movement may be assessed. Although very operator dependant it is arguably the most sensitive method for imaging muscle and is probably the screening method of choice. Computed tomography lacks the soft tissue resolution of MR and is limited to certain planes. However, it remains the most precise method of detecting and analysing calcification. Magnetic resonance is a powerful but time consuming and costly tool. MR spectroscopy gives biochemical information allowing the early detection of muscular dystrophy and the study of muscle physiology.

Disease may alter muscle texture, shape, function and biochemistry. In focal disease it is important to compare the opposite side of the body. Tumours, abscess and localised haematoma may all present as mass lesions. However, there are circumstances in which a too great reliance on one method may be seriously misleading. Ischaemia and inflammation produce a different pattern of signs which are more subtile. Imaging has an important role in first confirming that the muscle is abnormal and secondly guiding biopsy. Both US and MRI allow follow up studies without the risk of ionising radiation. Tendons are commonly affected by chronic stress and inflammatory disease. Imaging may demonstrate swelling, adjacent oedema and disruption. Ultrasound may be used to show normal tendon movement confirming integrity.

\section{Room L}

\section{RC}

\section{Advanced imaging of the normal temporal bone F. Veillon; Strasbourg}

The aim of this lecture is to present the normal anatomy of the temporal bone on C.T. and M.R.I. The principal study plane is horizontal supplemented by coronal and sagittal sections. The external, middle and inner cavities of the temporal bone are presented in all three spatial planes. The ducts of the temporal bone (petromastoid canal, ducts of the bottom of the internal auditory meatus, facial canal, chorda tympani canal, tensor tympani canal, stapedius canal) and certain fissures are also studied, especially by C.T. The detailed anatomy of the ossicles, particularly the adjacent region of the footplate, is presented as well.

\section{Advanced imaging of the temporal bone: abnormal} Thomas J. Vogl; München

The temporal bone is a complex crossroad of neural, vascular and skeletal structures. Accurate imaging is crucial because clinical evaluation frequently cannot localize the lesions accurately.

Although computerized tomography (CT) was a significant imaging advance in the evaluation of the temporal bone there is a number of limitations like radiation, beam hardening artifacts and limited soft tissue resolution. In the diagnosis of tumorous lesions in the temporal bone Magnetic Resonance Imaging (MRI) has become the method of choice.

Based on experience in more than 500 patients the course will focuse on the optimized imaging technique and diagnostic criteria of temporal bone pathology. Extensively differential diagnostic criteria will be discussed and a flow chart for the diagnostic workup in these patients will be presented. New developments in threedimensional MRI techniques show an enormous 
impact on diagnostic possibilities in the temporal bone. These techniques allow the evaluation of very thin slices and the arbitrary reconstruction of outlines and surfaces.

\section{Room M}

\section{RG}

105

\section{Diagnostic imaging of the adrenal glands}

G. Marchal; Leuven

The main techniques, actually used in adrenal imaging are ultrasound, CT, MRI and nuclear medicine. The first three provide more or less accurate morphological data whereas nuclear medicine should be regarded as a technique of functional imaging.

The diagnostic capabilities of these four techniques largely depend on the type of adrenal pathology, with or without gross macroscopic change, with or without functional disorder.

Most adrenal masses originate from either cortex or medulla. The cortex which derives from the mesoderm produces the glucocorticoids, mineralocorticoids and sex steroids. The medulla, deriving from the ectoderm secretes epinephrine and nonepinephrine. Since many of the pathological adrenals produce an excess of those hormones, they frequently present with typical clinical and laboratory findings.

The role of adrenal imaging is first to detect possible adrenal pathology and to differentiate between diffuse hyperplasia, tumoral mass lesions and other secondary changes.

Up to now CT has been the method of choice to image adrenal pathology. With appropriate techniques lesions of less than $1 \mathrm{~cm}$ can be visualized. Although contrast enhancement by IV bolus technique is used to study the degree of vascularization, it does frequently not improve the differentiation between benign and malignant conditions.

More recently MRI has also be advocated. Typically TI and T2 weighted SE sequences are used for both detection and characterization. Tissue characterization is mainly based on the comparison of intensities on $\mathrm{T} 1$ and $\mathrm{T} 2$ weighted images. Comparison with the neighbouring liver as a reference is also frequently used.

Benign cortical adenomas tend to appear isointense to the liver on both $\mathrm{Tl}$ and $\mathrm{T} 2$ weighted images.

On the contrary, primary and secondary malignant lesions often display a lower intensity on $\mathrm{T} 1$ and a higher intensity on $\mathrm{T} 2$ weighted images. A careful study of the uptake and wash-out of an IV injected Gadolinium chelate can also help in the differentiation. Unfortunately there is still overlap between both groups. Therefore, clinical and laboratory data, functional imaging and biopsy remain important diagnostic tools.

\section{Diagnostic imaging and interventional procedures of the adrenals: nuclear imaging}

J.-L. Baulieu; Tours

Adrenal scintigraphy is a non invasive functional imaging procedure. At present time, the cortex and the medulla are selectively and differentially imaged using appropriate radiopharmaceuticals.

[ $\left.{ }^{131} \mathrm{I}\right]-6 \beta$-iodomethyl-19-norcholesterol (NP59) is used in aldosterone and cortisol excess. The uptake is regulated by ACTH. The normal adrenal cortex is suppressed by dexamethasone. In primary hyperaldosteronism, bilateral uptake indicates a diffuse macronodular hyperplasia. Aldosteronoma are localized with a 88 per cent accuracy. In hypercortisolism, NP59 scintigraphy differentiates a hyperplasia and an adenoma, and, in the latter case, indicates the side of the lesion.

$\left.{ }^{[131} \mathrm{I}\right]$ or $\left[{ }^{123} \mathrm{I}\right]$ meta-iodobenzylguanidine (MIBG) is a marker of the monoamine uptake function. The sensitivity and specificity for intraadrenal pheochromocytoma detection are respectively .89 and .94 . The performances in the screening of ectopic, multiple and metastatic pheochromocytoma are higher: sensitivity $=92$, specificity $=1.00$. Studies are in progress to differentiate normal from hyperplastic glands and pheochromocytoma in patients with the multiple endocrine neoplasia syndrome (MEN).

The limitations of adrenal scintigraphy are: the poor anatomic resolution, the potential pharmacological interferences (antialdosterone, labetalol), the irradiation due to ${ }^{131} \mathrm{I}$, the cost and the delay of the response ( 3 to 8 days). Therefore, nuclear medicine is not commonly used as a basic imaging procedure of adrenal glands. However it is particularly useful in the post-operative period, the MEN syndrome, the malignant pheochromocytoma, and in cases of discordant clinical biological and imaging data

\section{Guided biopsies of the adrenal glands \\ R. Oyen, G. Marchal, C. De Schepper, D. Penne, H. Rigauts, A. L. Baert; Leuven}

It is obvious that US, CT and MRI provide important anatomical information of the adrenal gland and adrenal masses. However, it remains impossible to obtain a histological or functional diagnosis from either of the imaging techniques. Adrenal masses are particularly confusing when they are associated with cancer from the lung or the breast. Moreover, normal sized and normal shaped glands do not exclude the presence of metastases. Guided biopsies are often required especially if a histological diagnosis is crucial to patient management.

Guided biopsies of the adrenals can be performed under sonographic guidance, CT-guidance, or more rarely under MRI-guidance. CT should be preferred especially for biopsy of small masses, normal sized glands, obese patients or deeply located masses.

A variety of needles is available to perform aspiration cytology, aspiration biopsy or biopsy. Several approaches are in use for the right and left adrenal gland and many devices are developed to facilitate needle guidance. The dorsal approach is preferred whenever possible. With the gantry tilting caudally, virtually all important structures can be avoided.

The most common complications are hemorrhage and pneumothorax. A major risk is the puncture of an unexpected pheochromocytoma when sudden catecholamine release can become a life-threatening situation.

The accuracy of CT-guided biopsy of adrenal masses ranges from 80 to $100 \%$, while the reported incidence of complications is low. The negative predictive value of guided biopsy of adrenal masses in oncologic patients is reported to be $75 \%$; therefore a rebiopsy can be recommended.

\section{Room N}

\section{RC}

\section{2}

\section{Endovascular imaging: current applications and methods}

\section{G. P. Feltrin, M. Chiesura C., S. Savastano, D. Miotto; Padova}

Two basic methods of endovascular imaging have been developed to complete the angiographic vascular diagnosis: angioscopy and intravascular ultrasound. Both techniques are percutaneously employed and provide good information about the endoluminal surface of target segments. Intravascular imaging is typically directed to the regions of interest outlined by diagnostic angiography and by interventional procedures for control.

Methods: The angioscopes can be coaxially inserted over a guide wire via a vascular sheath; the block of blood flow and saline flush of the artery are needed. To avoiding the possible large amount of water administration $\mathrm{CO}_{2}$ may be used to clear the lumen. The angioscopes can be employed in antegrade direction in respect to the vascular access, so that the practical use is limited to distal vessels.

The intravascular ultrasound devices are constituted as catheter varying from $5 \mathrm{~F}$ to $8 \mathrm{~F}$, introduced through an arterial sheath with or without guide wire. Two types of devices are available: mechanical and phased arrays transducers. The mechanical design gives better cross sectional images than the phased arrays design. The major advantage of phased arrays transducer is the integrated use within interventional devices avoiding the multiple catheter exchange.

Applications: The angioscopy before interventional procedure recognizes the thrombotic component of an occlusion distinguishing the hard white atheroma from new and old thrombus. After intervention it provides a good assessment of intimal surface showing flaps, dissections or very irregular and almost smooth lumen. The clinical use is limited to the medium and smaller size vessels.

The advantages of intravascular ultrasounds are: quite reliable cross-sectional diameter of the vessel, calculation of stenotic area, characterization of vessel wall, detail of intimal surface and plaque components, and correct choice of therapy. In post-procedure use the intravascular ultrasound indicates precisely the flaps or dissections related to angioplasty, atherectomy or missed by insufficient stenting. Therefore it helps to determine the end point of the procedure. The retrograde and antegrade use of ultrasound permits the application in distal and proximal vessels and all the way to the aorta.

Both techniques provide additional information not always available with angiography and are becoming necessary during interventional procedures.

\section{Current applications of angioscopy \\ $\bar{M}$. R. Rees; Leeds}

Angioscopy offers a unique method of direct endovascular imaging which enables the operator to define exactly the endoluminal surface and characteristics. Small diameter endoscopes can be inserted percutaneously into the vessel via guiding catheters or conventional balloons. Angioscopes are available that can be used in conjunction with therapeutic procedures such as angioplasty, laser angioplasty, thrombolysis and atherectomy. Current angioscopes range from $0.45-3.00 \mathrm{~mm}$ in size. Angioscopes can have working channels for guide wire insertion, balloon attachment to occlude the vessel and reduce flow and for the insertion of laser fibres and other instruments.

Angioscopy has demonstrated the effects of angioplasty on the endoluminal surface as well as determining the nature of the underlying disease enabling more accurate therapeutic choices. Angioscopy has also been useful in monitoring the endothelialisation and intimal proliferation in stent therapy. The careful documentation of endoluminal appearance may aid in the under- 
standing of the mechanisms of atheroma deposition, healing of the treated vessel and the mechanisms involved in restenosis.

\section{Room 0}

\section{RB}

26 Early diagnosis of breast cancer by mammography: macroscopical and microscopical structures

K. Prechtel; Starnberg

Early diagnosis of breast cancer rendering possible the total removal of the tumor and cure is rare. Definite delineation of carcinoma in situ (CIS) defined by limitation of tumor cells to the ductal system with intact basal membranes is not an easy task for the morphologist. CIS may be small and its borders may be clearly defined. However, it may also cover larger areas and be multifocal. In the individual case, the switch to invasive, destructive growth and metastasis cannot be predicted.

At present, mammography is the best diagnostic means for the detection of early breast cancer lesions targeted at visualization of microcalcifications. Palpation may only detect larger areas of tumor growth. According to the WHO classification, there are two basic types of noninfiltrating breast cancer: ductal carcinoma in situ (DCIS) of medium or large cell type and lobular carcinoma in situ (LCIS), mostly of small cell type differing in their biological behaviour. Histologically $70 \%$ of DCIS exhibit microcalcifications; however, only a part of these present radiographically. LCIS with its more favorable biological behaviour is calcified more less frequently and less intensively and may therefore escape radiological diagnosis more often. At present, noninvasive and small invasive carcinoma (pTla) with no lymphnode involvement associated with a very favorable prognosis is rare. In daily routine diagnosis, $95 \%$ of breast cancers are invasive and have spread more or less. The mos frequent type, ductal carcinoma (NOS), comprising $70 \%$ of cases, may be detected radiographically before reaching palpable size by its calcifications (calcium phosphate) in its intraductal as well as its invasive parts. As a diagnostic target, microcalcification may gain importance with increasing tumor size. The other histological types i.e. lobular carcinoma and special types display less pronounced calcifications and may escape mammographic diagnosis. Patterns of calcification may be similar in breast cancer and benign breast lesions. Therefore the plea for carcinoma-specific calcification patterns appears questionable to the histomorphologist.

\section{Identification of malignant lesions by microcalcifications Eric J. Roebuck; Nottingham}

To detect the calcification which suggests the presence of malignancy, it is first necessary to demonstrate them on the mammogram. Assume a perfect radiographic technique and a mammographic apparatus with an appropriately small focal spot and adequate compression. What then is necessary is a high resolution film/screen combination with high contrast characteristics. The film processing must be carefully controlled to maximize resolution and contrast and must not have a high "base plus fog" level which will make the calcifications look grey.

The perceived resolution and contrast depends also on viewing conditions. To this end a film with a high "D max" will appear better than one with identical characteristics, but with a grey background. Similarly, the viewing screen around the mammogram must be darkened and ambient light in the viewing room must be minimised.

In the search for calcification one should always use a magnifying glass.

Having discovered calcifications we reach the first decision point. There are three possible options:

1. The calcifications are sufficiently suspicious of malignancy that tissue diagnosis is mandatory.

2. They are sufficiently characteristic of a benign process that they can be ignored and the patient reassured.

In both these instances further diagnostic imaging is unnecessary and wasteful. 3. If the calcifications cannot be classified into one of the first two categories with confidence, then further imaging assessment is indicated.

The prime tool for imaging assessment of calcification is radiographic magnification, with or without a localised compression device.

There are only three possible outcomes to assessment.

1. Tissue diagnosis mandatory

2. Reassure and discharge.

3. Keep under surveillance.

The role of fine needle aspiration cytology (FNAC) must be considered here. 1. In all cases classified on imaging grounds as "tissue diagnosis mandatory" FNAC should be performed in an attempt to obtain a pre-operative diagnosis of cancer, so that definitive treatment decisions can be made by the surgeon and patient. In this group any FNAC result other than "malignant" should be ignored and the patient scheduled for open excision biopsy.

2. In patients assessed and then thought to be benign on imaging, a case can be made for FNAC to be performed, providing that all results other than "malignant" or "suspicious of malignancy" are ignored for management purposes

3. Those patients with equivocal imaging assessments should have FNAC performed, if necessary with a repeated FNAC 4-6 weeks after the first.

4. A "malignant" or "suspicious of malignancy" result means surgery - either definitive or for diagnosis

5. A good sample of benign epithelial cells might in some circumstances give sufficient confidence to discharge the woman, but in the case of calcification the possibility that such cells might have been obtained from tissue just above or below a duct containing malignant cells is so high and I would prefer to ignore the result. These patients, together with those having inadequate or equivocal FNAC results should be kept under surveillance. An interval les than one year before re-imaging is valueless in these circumstances, although a case can be made for interim clinical and BSE examinations.

Finally, to consider those characteristics which make a diagnosis of malignancy or benignity more likely. It must be stressed that absolute differentiation is very commonly impossible; one should just total those observed feature suggesting malignancy and those suggesting benignity, and then make judgement knowing what the outcome of that opinion means in management terms.

The features to be considered are:

1. Particle size

Large or small, variable or uniform.

2. Particle shape

Linear (casting), irregular (granular), variable or uniform

3. Particle orientation

In rows or clustered.

4. Particle distribution

Scattered or localised.

5. Shape of cluster

Rounded, oval or not.

6. The presence of any associated soft tissue mass or parenchymal deformity. The diagnosis is more likely to be malignant if the calcified particles are small, variable in size or shape, arranged in rows or clusters and if the clusters are any other shape but round or oval.

\section{Mammographic interpretation of circumscribed lesions} J. L. Lamarque, J. Pujol, M. J. Rodière; Montpellier

\section{Histological basis of the mammographic image}

Anatomico-histological correlation studies have permitted researchers to determine the ultrastructural foundations of the mammographic image.

The constituent elements which can be found in the radiographic image are the conjonctive tissue, more or less fibrosed, water, fat and calcium.

The mammographic sign is the diagnostic element to which careful attention should be paid; it can be fibrous, hydric, calcic or fatty. It is not and never will be epithelial for reasons of technical and histological perceptibility.

It can be made up of an association of diverse fundamental elements in one of the following configurations:

- either systematized or circumscribed: this is the pathological focus whether it is calcic, fibrous or another type

- or non systematized or diffuse: the sign is spread out in a more or less uniform manner over the entire mammary area.

Table I presents the various diagnostic types encountered.

II. Suspect infraclinical signs

The infraclinical sign represents an important part of the mammography, as no palpable element comes to the aid of the diagnosis.

The detection of an abnormal infraclinical structure thus poses the problem of the surgical indication of exeresis and histological control. Two complementary elements help in the decision:

- clinical, in its anamnestic part: interrogation, notion of biological or familial risk, etc...

- directed cytology in the case of a clustered sign or of a zone in the case of a non clustered sign.

The threefold CRC ensures the reliability of the diagnosis and leads to the decision of exeresis or simple surveillance. 


\begin{tabular}{|c|c|c|}
\hline & Clustered & non clustered \\
\hline Fibrous signs & $\begin{array}{l}\text { Localized dystrophy } \\
\text { nodule (S) } \\
\text { stellate image }(\mathrm{S})\end{array}$ & $\begin{array}{l}\text { Generalized dystrophy } \\
\text { micronodular } \\
\text { macronodular } \\
\text { miked }\end{array}$ \\
\hline Hydric signs & $\begin{array}{l}\text { cyst }(\mathrm{S}) \\
\text { localized dystrophies }\end{array}$ & $\begin{array}{l}\text { Conjonctivo edematous } \\
\text { Breast I } \\
\text { Conjonctivo edematous } \\
\text { Breast II } \\
\text { Conjonctivo edematous } \\
\text { Breast III } \\
\text { (overal blurring of } \\
\text { matrices): Liquid like }\end{array}$ \\
\hline Calcic signs & $\begin{array}{l}\text { Benign calcifications } \\
\text { suspect } \\
\text { macro-calcifications } \\
\text { zone (S) }\end{array}$ & $\begin{array}{l}\text { Diffuse } \\
\text { microcalcifications }\end{array}$ \\
\hline
\end{tabular}

\section{Room R}

\section{RC}

In 1991 the American Cancer Society anticipates that there will be 43,300 new cases of primary malignant tumors involving the head and neck. The management of these tumors represent complex and difficult approaches in order to maximize the potential for local control, the maximum in terms of survival and the minimum in terms of complication.

The majority of tumors of the head and neck present as locally advanced carcinomas and it is this group of malignant tumors that have served as a primary area for investigation of new and innovative treatment programs in management. The various clinical trials that have been pursued involve studies using radiation therapy alone, studies using hypoxic cell radiation sensitizing agents, and studies using radiation therapy along with cytotoxic chemotherapy with or without surgical management.

In large measure these clinical trials have been restricted to patients who have local and regionally advanced head and neck cancers with no evidences of distant metastatic disease with the selection criteria for patients being precisely defined.

The goal of the refresher course presentation will be to present those trials where significant advances have been made in the management of head and neck cancer. These involve the use of radiation therapy alone with altered fractionation techniques including hyperfractionation, accelerated fractionation and concomitant boost radiation therapy. On this background of these trials using altered fractionation, newer concepts in management will be presented.

The clinical trials related to hypoxic cell sensitizers will present the data from the use of Misonidazole and standard radiation therapy, Misonidazole and non-standard radiation therapy as well as the data beginning to emerge using SR-2508 (Etanidazole).

Clinical trials relative to radiation therapy with cytotoxic drugs will review the use of sequential chemotherapy in radiation therapy, simultaneous chemotherapy and radiation therapy, sequential chemotherapy, radiation therapy with surgery, interdigitated chemotherapy and radiation therapy as well as how these findings can be integrated into future research efforts in head and neck cancer.

Unfortunately, the 1990's began without compelling evidence that any alternative is better than conventionally delivered radiation therapy with or without surgery although many promising leads are beginning to appear. It is obvious that tumors arising in the head and neck region will continue to serve as a major area for innovative new concepts in cancer treatment. 
High resolved (multislice and single slice) films of the thorax and of the abdomen demonstrate the three principle aspects of the method: 1. High signal-high resolution images with absence of susceptibility artifacts. 2 . Freezing of motion even in the heart and great vessels. 3. Delineation of organs and vessels with continuous contrast due to $\mathrm{T} 1, \mathrm{~T} 2$, flow and contrast agent perfusion. Additionally principal experiments are provided, that show the influence of further TE-reduction on the quality of gradient echo imaging. In conclusion the fast high resolved snapshot FLASH MR images more and more provide the quality of spin echo images, however, about 20 times faster. Resulting clinical applications are discussed.

\section{SS \\ 11.01 MR Ultrafast sequences - new techniques}

\section{$10.30 \mathrm{AM}$}

30 Quantitative high-speed MR imaging using snapshot FLASH MRI A. Haase, D. Matthaei, D. Henrich, R. Deichmann; Würzburg

Purpose: The purpose of this study is the investigation of snapshot-FLASH MRI techniques for quantitative measurements of MR relaxation times (T1, $\mathrm{T} 2, \mathrm{~T} 2 *$ ), flow and diffusion parameters, and MR spectroscopic data.

Methods: We use a high-speed gradient echo technique, with a repetition time of less than $4 \mathrm{msec}$ and a gradient echo time of less than $2 \mathrm{msec}$. Usually $128 \times 128$ and $256 \times 256$ image matrices are used. The techniques are applied in a $4.7 \mathrm{~T}$ experimental and a $2 \mathrm{~T}$ whole body Bruker magnet.

Results: We obtain quantitative MR parameters in animal and human studies, as long as the repetition times can be short (of the order of $3 \mathrm{msec}$ ) and the flip angle of the excitation pulses are less than 10 degree. Experiments are possible and can be quantified even using high spatial resolution.

Conclusions: These techniques will improve the diagnostic value of MRI in abdominal and cardiac studies.

\section{$10.40 \mathrm{AM}$}

31 Clinical valence of a T1- and T2-weighted ultra fast gradientecho-technique (UGE) within seconds

W. Dewes, U. Hau, P. Benz, A. Seeliger, B. Junk, B. Kramann, E. Oberhausen, U. Piepgras; Homburg/Saar

We report about the clinical valence of an self developed T1- and T2-weighted ultra fast gradient-echo-technique (UGE).

The sequences are implemented on an 1,0 T MR-Tomograph with a field strength of $6 \mathrm{mT} / \mathrm{m}$. We used the sequences additionally to the normal SE- and GE-sequences in 60 patients. With SE-, GE- and UGE-sequences anatomical structures, pathological changes and artefacts were compared.

Possibilities of application in routine MR-imaging are shown.

\section{$10.50 \mathrm{AM}$}

\section{Ultrafast MR imaging of the liver using TurboFLASH and segmented k-space acquisition}

B. Wallner, W. Weidenmaier, R. R. Edelman, UIm

Purpose: We developed a technique, which allows high resolution T1- or T2-weighted imaging of the whole liver within a breathhold intervall.

Methods: We employed a turboFLASH sequence (TR/TE/Flipangle $6.5 / 3 / 10$ ) that covers $\mathrm{k}$-space in four segments. T1-weighting was achieved by using a 180 -degree prepulse, T2-weighting with a 90-180-90 prepulse. We examined 44 patients with focal liver lesions and compared the images and the contrast with standard spinecho (SE) sequences.

Results: Lesion-liver contrast of the fast sequences was superior to $\mathrm{T} 1$-weighted and similar to T2-weighted SE sequences with a long echo time of $120 \mathrm{~ms}$. Fat and liver signal could be nulled with the segmented turboFLASH method at inversion delays of 10 and $300 \mathrm{~ms}$, respectively; this was not possible with standard sequences. The images were uniformly free of respiratory artefacts, whereas the SE images were degraded by motion in some patients. Conclusions: The segmented turboFLASH method is feasible to obtain breathhold images with arbitrary image contrast. It may prove useful for the detection and characterization of hepatic lesions and shortens imaging time compared with standard sequences.

\section{$11.00 \mathrm{AM}$}

\section{Delineation of moving organs and dynamic contrast on high resolved snapshot FLASH MR images}

D. Matthaei, A. Haase, D. Henrich, D. Ratzel, E. Duehmke; Göttingen

With fast MR imaging it becomes possible to freeze organ motion and to demonstrate physiologic processes realtime. With acceleration of the snapshot FLASH MR sequence first realtime images could be demonstrated of the cardiac cycle and of relaxation processes with a method other than Echo Planar. Most fast MR imaging approaches had principal but not practical success due to low signal to noise and low in plane resolution. Here we present results of experiments, that show the use of the snapshot FLASH sequence with high inplane resolution. Thus in less than $1 \mathrm{sec}$ a $256 \times 256$ matrix can be measured with whole body snapshot FLASH MR imaging.

11.10 AM

34 PHASE-MAPPING-technique for the evaluation of aortic valve stenosis by MR

G. Engels, E. Müller, K. Reynen, N. Wilke, K. Bachmann; Erlangen

Purpose: New MR-techniques for quantitative blood flow registration as PHASE-MAPPING (a two-dimensional space-resolved technique with a time-averaged measurement of blood flow) now are available for the diagnostics of valvular heart disease. First results in grading aortic valve stenosis by blood flow measurements in the ascending aorta by PHASEMAPPING on a WHOLE Body MR Unit (Magnetom, 1.5 T., Siemens) are shown in comparison to continous wave Ultrasound-Doppler.

Methods: The PHASE-MAPPING-technique is used in a plane perpendicular to the blood flow through the ascending aorta in a defined distance from the aortic valve. 15 patients were examined, 7 of whom suffered from an aortic valve stenosis. The shape of blood profile as well as graduation of aortic valve stenosis are validated by Ultrasound Doppler.

Results: We find high concordance in comparing the results of MR-and Doppler-technique with respect to the evaluation of aortic valve stenosis. Good reliability and practicability of the demonstrated MR-method are shown.

Conclusions: With respect to our results by PHASE-MAPPING the development of an alternative and competing MR-method for the evaluation of valvular heart disease and shunt diagnostics seems possible.

\subsection{AM}

\section{Time-optimized T2-weighted MR imaging}

Peter Schubeus, Bernhard Sander, Wolfgang Schörner, Uwe Tosch, Michael Deimling, Roland Felix; Berlin

Purpose: Aim of the present study was to evaluate, whether lowering the flip angle in proton density- and T2-weighted double-spin echo sequences allows for shortening TR and imaging time without relevant changes of image quality. Methods: 10 patients with cerebral white matter lesions were investigated with an 1.5 T Magnetom (Siemens) using a conventional double-spin echo sequence $(\mathrm{TR}=2500 \mathrm{~ms}, \mathrm{TE}=15$ and $70 \mathrm{~ms})$ and time-optimized double-spin echo sequences $(\mathrm{TR}=1900 \mathrm{~ms}, \mathrm{TE}=15$ and $70 \mathrm{~ms})$ at flip angles of $90^{\circ}, 80^{\circ}, 70^{\circ}$, $60^{\circ}$, and $50^{\circ}$.

Results: Lowering the flip angle resulted in less $\mathrm{T} 1$-contrast and a relative increase of $\mathrm{T} 2$-contrast. At a flip angle of $70^{\circ}$ contrast-to-noise values between lesions and brain (CNR 22,4 vs. 21,1) as well as image artifacts of the time-optimized sequence were similar to the conventional spin echo sequence, however, imaging time was shortened about $25 \%$.

Conclusions: Lowering the flip angle in double-spin echo sequences allows for shortening TR and imaging time without relevant degradation of image quality.

\subsection{AM}

36 Texture analysis of magnetic resonance images: a method to improve tissue characterization

F. Di Salle, M. Cesarelli, A. Pepino, R. Elefante, M. Bracale; Napoli

The multiparametric correlation of MR signal intensity with tissue molecular composition provide a reliable tool for the identification of normal and pathologic tissue components.

Even if Magnetic Resonance provides an high efficacy in discriminating tissues it is not very uncommon to experience situations in which this technique does not produce a complete identification of lesions, and the radiologist can need further information to obtain a definite differential diagnosis.

An image analysis technique could provide useful information about tissue structure by means of a texture analysis and a signal intensity pattern recognition. This information can be used to generate artificial images in which the pixel intensity is based on regional texture considerations. The authors present their method for texture analysis and its application on a first group of normal and pathologic images.

The advantages of this technique of image processing, consisting in a further amplification of MR information on tissue structure, are discussed in comparison with traditional pre- and post-contrast MR images. 


\section{$11.35 \mathrm{AM}$}

37 Contrast optimization in flash sequences for the investigation of bone tumors

R. Erlemann, U. Stöber, C. Drews, P. E. Peters; Münster

Purpose: With phantom studies and theoretical estimations including correction of flip angle distribution over the slice profile, contrast in FLASH sequences was optimized for a replacement of spin echo sequences for staging of bone tumors.

Methods: Contrast between probes simulating bone tumors and muscle or bone marrow achieved with FLASH sequences $\left(\beta: 10^{\circ}-90^{\circ}\right.$, TR: $40-320 \mathrm{~ms}$, TE: $10-20 \mathrm{~ms}$ ) was measured and theoretically calculated.

Results: Contrast was closer correlated with the flip angle than with TR or TE. For delineation of tumor from muscle only a FLASH sequence with a large flip angle following intravenous administration of Gd-DTPA can be recommended, if a tumor shows a clear Gd-DTPA uptake. With all FLASH sequences analyzed, no sufficient contrast between "tumor" without Gd-DTPA uptake and muscle was obtained. Maximal contrast between "tumor" and bone marrow was achieved with small flip angles; and an additional peak was noted with large flip angles and short TR. Experimentally measured $\mathrm{T} 2 *$-dependent contrast were nearly identical to theoretically calculated contrast without correction of flip angle distribution. For calculation of T1-dependent contrast correction of flip angle distribution over the slice profile was of high value.

Conclusions: FLASH sequences can replace T1-weighted but not T2-weighted $\mathrm{SE}$ sequences for staging of bone tumors.

\section{$11.45 \mathrm{AM}$}

\section{Proton NMR relaxation times and biomechanics of human vascular wall: an experimental study at 4 and $60 \mathrm{MHz}$}

Ph. Vinee, B. Meurer, A. Constantinesco; Freiburg

Purpose: Understanding the influence of viscoelastic characteristics of human vascular wall on its relaxation times would possibly allow noninvasive characterization of its stress state by MRI.

Methods: In vitro, we measured 2 viscoelastic parameters, water content, monoexponential $\mathrm{T} 1$ and $\mathrm{T} 2$ at $4 \mathrm{MHz}$ of 41 human vascular wall samples at 5 localizations: arterial, venous, pulmonary. We performed lysis of collagen and elastin for 5 aortas, measuring viscoelasticity, biexponential T1, T2 and Van Vleck's second moment (characteristic for the rigidity of a polymer) at $60 \mathrm{MHz}$. Results: A MANOVA test showed significant difference $(\mathrm{p}<.001)$ within the 5 vascular localizations for tissue water content, viscoelastic and relaxation parameters at $4 \mathrm{MHz}$. A multiple regression analysis demonstrated that $\mathrm{Tl}$ and $\mathrm{T} 2$ are in significant correlation $(\mathrm{p}<.01)$ with both viscoelastic parameters and water content (with preponderance of a viscoelastic parameter over water content for T2). At $60 \mathrm{MHz}$ there was a significant correlation $(\mathrm{p}<.05)$ between $\mathrm{Tl}$ and a viscoelastic parameter as well as between the NMR second moment of a compartment that showed to be collagen and the slope at high strain of the strain-stress curve of the tissue. We also quantified by NMR the amount of collagen and collagen bound water in the tissue characterized by $\mathrm{T} 2$ $\approx 15 \mu \mathrm{s}$ and contributing by 6 to $9 \%$ to the total FID signal amplitude. Conclusions: Viscoelastic characteristics of human vascular tissue appear to be co-responsible for differences of relaxation times $\mathrm{T} 1$ and $\mathrm{T} 2$ between vascular localizations and between patients. Viscoelastic parameters contribute to NMR tissue characterization as they are as significantly correlated with $\mathrm{T} 1$ and $\mathrm{T} 2$ values than water content. Further development could lead in direction of noninvasive measuring of the stress status of tissues like vascular wall or myocard.

\section{$11.55 \mathrm{AM}$}

39 MR imaging in patients with traumatic and inflammatory diseases of knee and ankle: comparison between $2 \mathrm{~T}$ and $0.23 \mathrm{~T}$

A. Mundinger, M. Ioannidou, A. Beck, E. Dinkel, G. Sigmund; Freiburg

Fifty-six patients with traumatic and 117 patients with inflammatory lesions of the knee and ankle were examined at $2 \mathrm{~T}$ (69 examinations) or $0.23 \mathrm{~T}$ (104 examinations) MR system. The quality of all images was assessed by the consensus of three radiologists on a scale of diagnostic $(3+, 2+)$ and non-diagnostic $(1+)$ image quality. More than 90 percent of images in both systems were diagnostic. However, high field imaging provided better image quality compared to low field imaging due to a higher proportion of $3+$ quality images at $2 \mathrm{~T}(2 \mathrm{~T}: 56 / 69=81 \%$ versus $0.23 \mathrm{~T}: 51 / 104=49 \%$; $\mathrm{p}<0.001)$

\section{Room B}

\subsection{Brain, Tumors}

\section{Economics of modern neuroradiology (Keynote Lecture)}

\section{J.M. Caillé; Bordeaux}

The best economic policy in health care necessarity implies quality in other words value for money.

Along these lines, we shall have to examine the position of radiology in medicine and more particularly, the position of neuroradiology in radiology and in the neurological sciences.

Radiology is a discipline whose application supposes the acquirement of specific knowledge in both medicine and technology, the utilization of diagnostic and therapeutic tools.

The utilization of such tools obviously entails technical competence but most importantly specific clinical competence.

Specialization in modern radiology - in so far as it is considered as a discipline - must be achieved not according to the different techniques but according to the different medical disciplines. Tomorrow's radiologist will be a specialist in organs not a specialist in techniques and the neuroradiologist will be one of them.

The annual incidence for disease and injury of the nervous system is $2.5 \%$ (2.500 per 100.000 population), the prevalence is $9.5 \%$. Most often in neuroradiology, signs and symptoms reflect only the localization of the lesion but are unable to assign a precise etiological diagnosis. Neuroradiology which provides precise localization and data about the nature of the lesion leads to the exact diagnosis of the lesion. At present neurology only provides first crude screening. Neurological patients are sorted out and possible diagnostic hypotheses may be put forward. In most cases such diagnostic hypotheses are invalidated by imaging.

Modern neuroradiology can and must carry out the first clinical screening. A precise etiological diagnosis must be proposed without the neurologist's mediation but this is possible only if the neuroradiologist's competence in the four following areas: technical and clinical knowledge, research, teaching, is available.

If neuroradiology sets up a sensible political line in the forthcoming years, good value for money will be provided.

All these points will be presented and discussed.

\subsection{AM}

\section{Preoperative embolization of intracranial meningiomas: angio- graphic and surgical correlations}

G. E. Klein, J. Lammer, F. Waltner, K. A. Hausegger, F. Flückiger, M. Mokry; Graz

Purpose: Preoperative embolization of intracranial meningiomas should facilitate their total resection. The aim of this study was to evaluate the efficacy of embolization in tumors with external CAS only and those tumors with external and internal CAS.

Methods: In 90 patients a preoperative embolization was performed. After CT and/or MR examinations embolization was performed in the same session as the diagnostic angiography. We routinely used 3 French Microcatheters with steerable guidewires for superselective angiography and embolization. As embolic agent, polyvinyl alcohol foam (PVA) particles were used.

Results: Marked hemorrhage during surgery occured only in one of 47 patients with only external CAS. In five of 43 patients with internal and external CAS significant intraoperative bleeding occured, 4 of them had predominantly internal CAS.

Conclusions: The use of Microcatheter in combination with DSA enables an effective and safe embolization.

The preoperative embolization appeared to be very useful in all patients especially in large tumors with predominant external CAS and in skullbase meningiomas.

\subsection{AM}

\section{$42{ }^{1} \mathrm{H}$ NMR spectroscopic imaging of human brain tumors}

$\bar{W}$. Heindel, P. Luyten, J. Bunke, H. Kugel, J. den Hollander, G. Friedmann Köln

Purpose: In this preliminary study we evaluated the diagnostic value o spatially resolved metabolic information provided by proton NMR spectros. copic imaging (SI) which supplements the morphological information from "conventional" MRI.

Methods and Materials: Using a clinical $1.5 \mathrm{~T}$ whole body MR system localized 'H MR SI was performed in 28 patients suffering from gliomas. The spatial distributions of choline-containing compounds (Cho), creatine $(\mathrm{Cr})$ $\mathrm{N}$-acetyl aspartate (NAA) and lactate ( $\mathrm{Lac}$ ) were displayed as spectroscopic images and correlated with "conventional" MRI. 
Results: The metabolite maps showed pronounced heterogeneity across the tumorous regions: NAA was decreased in tumors to a different extent. Cho has been found increased 2 to 3 times in the solid part of gliomas compared to normal brain. Lac was often increased, but the distribution of high Lac differed from the distribution of high Cho. One tumor cyst as well as postsurgical defect within a recurrent tumor also showed high lactate, but no Cho.

Conclusions: ${ }^{1} \mathrm{H}$ MR SI adequately displays the heterogeneity of tumor metabolism and by that way helps to identify the metabolically most active parts of the tumors.

11.05 AM

\section{Localized ${ }^{1} \mathrm{H}$ NMR spectroscopy in 75 patients with a human brain tumor: clinical evaluation}

Ph. Demaerel, K. Johannik, P. Van Hecke, G. Wilms, G. Marchal, Ch. Plets, M. Lammens, J. Goffin, A. L. Baert; Leuven

Purpose: To evaluate the sensitivity and specificity of ${ }^{1} \mathrm{H}$ spectroscopy in brain tumors.

Methods: Seventy-five patients with a primary or secondary tumor were examined with ${ }^{1} \mathrm{H}$ single volume spectroscopy at $1.5 \mathrm{~T}$, using the STEAM method.

A histological diagnosis was available in seventy cases. The tumor was localized on a gradient-echo sequence and a volume of interest was chosen centrally in the tumor.

Results: Distinct abnormalities were seen in all tumor spectra as compared to the normal brain spectra. For those patients in which a normal brain spectrum could not be acquired, the spectral peak intensities were compared to those obtained in a group of 15 healthy volunteers.

A decrease in $\mathrm{N}$-acetylaspartate and/or Phosphocreatine/creatine peak intensities was seen in most tumor spectra as well as changes in the Choline peak. An increased Lactic acid peak was more often observed in malignant tumors.

No absolute tumor specific characteristics could be found. A strongly increased Choline peak was a constant finding in meningiomas and oligodendrogliomas.

Conclusions: ${ }^{1} \mathrm{H}$ spectroscopy is for the moment of limited clinical usefulness and further extensive research is needed. Right now it is obvious that the technique will contribute to a better insight of brain metabolism.

\subsection{AM}

44 Prominent meningeal enhancement adjacent to non meningiomatous malignant lesions on Gd-enhanced MR images

G. Wilms, M. Lammens, G. Marchal, Ph. Demaerel, F. Van Calenbergh, J. Goffin, C. Plets and A. L. Baert; Leuven

Prominent meningeal enhancement was noted in 10 out of $61(16 \%)$ superficial malignant intracranial tumors, studied with Gd-enhanced MRI during a 2 year period. It concerned 6 glioblastomas, 3 metastases and 1 case of dural metastasis.

Seven cases underwent surgery. In 4 cases, there was extensive leptomeningeal invasion in the center of the lesion. In two of these lesions there was firm attachment of the center of the tumor to the dura, but without dural invasion despite extensive external carotid artery supply to the tumor in one case. In two cases the overlying dura was normal, without leptomeningeal tumoral invasion. In the case of dural metastasis, huge nodular lesions were present a the inner aspect of the dura. In none of the cases the prominent dura enhancement adjacent to the tumor corresponded to tumoral invasion nor extension to the dura.

Prominent meningeal enhancement on Gd-enhanced MRI appears to be much less frequent in malignant tumor than in meningioma. As in meningioma it seems not to point to tumoral invasion but to reactive changes of the dura.

\subsection{AM}

\section{CNS-Lymphomas in AIDS-patients: spectrum of morphological appearances}

J. Berkefeld, C. Lang, H. Hacker, W. Schlote, U. Madlehner; Frankfurt

In our experience with the evaluation of CNS-disease in 250 AIDS-patients the radiological diagnosis of primary CNS-lymphoma was surprisingly difficult. Only $45 \%$ of the cases were diagnosed correctly from the first CT- or MRI-examination. Therefore the CT- and MRI-morphology of 32 CNS-lymphomas was reviewed after death and compared with the results of neuropathological studies.

We found a large spectrum of morphological appearances mimicing nearly every kind of space-occupying process in the CNS. Solid or necrotic circumscript tumors $(65 \%)$ could be distinguished from diffuse or disseminated spreading lymphomas $(35 \%)$. In the latter group the radiological aspect was difficult to differentiate from encephalitic pictures. The histological examination confirmed the hypothesis of different types of tumor-growth and -spreading.

In conclusion the variety of CT- and MRI-morphology of CNS-lymphomas in
AIDS-patients is greater than reported before and can mimic many other tumors and inflammatory processes of the brain.

\subsection{AM}

46 MRI aspect of cerebral tumors appearing unenhanced and hypo- or isodense on CT (Report of 40 cases). Multicentric study Bruxelles/Strasbourg (Belgium-France)

D. Baleriaux, C. Matos, C. Grand, D. Krause, J. L. Drape, J. L. Dietemann, J. Tongio; Bruxelles

Purpose: To evaluate MRI capabilities in the study of those tumors appearing as iso- or hypodense on CT, or unenhanced after iodinated contrast injection: 40 cases are reviewed.

Methods and Materials: MRI studies included T1, T2 and Gadolinium-enhanced imaging. MRI and CT results were compared.

Results: Tl results were comparable to CT; tumors appeared as irregular, hypointense areas with ill-defined limits. On T2, all tumors presented as high signal intensity lesions with well defined boundaries. Edema was often mild or absent.

Intratumoral cystic formations were more frequently detected on MRI than on CT.

Gadolinium enhancement was obtained in 8 cases: this finding illustrates the higher sensitivity of MRI Gadolinium studies compared to iodinated CT.

Tumor location mainly involved the frontotemporal region. On histology, low or intermediate grade oligodendrogliomas or astrocytomas were found. In five cases high-grade tumors were found: these were more posteriorly located (parieto-occipital region), were surrounded by edema and showed strong enhancement.

Conclusions: MRI is the modality of choice for the preoperative work-up of tumors appearing hypodense on CT, and which are classically of low grade. The five high-grade tumors encountered in this group had a particular location (parieto-occipital) and degree of contrast enhancement with Gadolinium.

\section{$11.45 \mathrm{AM}$}

\section{MRI Follow-up of low- and intermediate-grade brain tumors:} multicentric study

D. J. Krause, J. L. Drape, J. L. Dietemann, J. Tongio, D. Baleriaux, C. Grand, C. Matos; Strasbourg/Bruxelles

Purpose-Materials: The aim of this ongoing study is to show the value of MRI in the follow-up of 30 patients with brain tumors; these low- or intermediategrade tumors had MRI features which correlated well with the histological diagnoses (January 1987-December 1990). All tumors were either astrocytomas or oligodendrogliomas mainly located in the frontotemporal region. Therapeutic decisions can be discussed:

- functional location of the tumor in association with poor clinical symptoms. - no clear-cut understanding of the spontaneous evolution concerning low grade tumors.

Results: MRI findings for 20 operated patients were as follows:

- Good sensitivity of T2 sequences, which differentiated between tumors and postoperative sequelae.

- Fourteen patients have a satisfactory postoperative evolution; follow-up MRI examinations (over 18-24 months) were unchanged.

- In 6 patients, MRI showed recurrences at the margins of the operative site (4 cases before 18 months and 2 cases at 4 years).

MRI findings for 10 unoperated patients showed:

- a distinct evolution of the tumor in 7 cases (increased tumoral volume and a mass effect) after 24 months.

- unchanged appearance on short-term follow-up (less than 15 months) in 3 cases.

Conclusions: The authors stress the value of MRI in the follow-up of low- and intermediate-grade tumors, especially in comparison with CT. They discuss a timetable for follow-up examinations. In the follow-up of these patients, gadolinium is essential for detecting foci of malignant transformation.

\subsection{AM}

48 Comparative analysis of residual or recurrent brain tumors with positron emission tomography (FDG-PET) and MRI/CT Hong W. Chin, David Lefkowitz, Ronald L. Eisenberg; Shreveport, LA, Omaha, NE

Purpose: We report the comparative analysis of follow-up FDG-PET and $\mathrm{MRI} / \mathrm{CT}$ studies in primary and recurrent brain tumor patients who were treated with surgery, external beam radiotherapy (EBR) and/or stereotactic interstitial brain implantation (SIBI).

Methods: Post-therapy PET studies were obtained and correlated with corresponding MRI/CT to examine the diagnostic value of FDG-PET in assessing residual tumor, recurrence, and tumor response as well as radiation necrosis Six patients received surgery, EBR, and SIBI; and four patients had surgery and $\mathrm{EBR}$ 
Results: While MRI/CT findings of mixed enhancement in the original tumor site made it difficult to differentiate actual tumor from residual changes of previous therapy, FDG-PET was able to verify biologically active tumors. PET identified four residual tumors, one recurrence, and one radiation necrosis. All six primary tumors showed complete responses to implantation therapy, however one recurred in another site.

Conclusions: FDG-PET appears useful in the evaluation of metabolically active brain tumors, and is the only reliable imaging modality in post-therapy assessment of tumor control, progression, and recurrence.

\section{Room C}

\section{SS}

\subsection{CT General - MRI General - Comparison CT - MRI} $10.30 \mathrm{AM}$

\section{Additional procedures to improve accuracy of abdominal CT} J.M. Friedrich, P. Schnarkowski, E. Merkle, K. A. Schumacher; UIm

Intestinal contrast is of great importance for diagnosing abdominal diseases in CT. We studied the influence of various contrasting modalities in opacifying the gastrointestinal tract.

Dynamic abdominal CT was performed in 273 patients. 6 homogeneous population groups differed in one single determinant: concentration of contrast medium, application of an activating gastrointestinal motility agent, addition of hydroxymethylcellulose, timing of contrast medium intake, dietary preparation.

Significant improvement of abdominal CT imaging was achieved by application of metoclopramide ( $\mathrm{p} 0.01$ ) and a good dietary preparation (p 0.01). Fastening of abdominal transit increased the homogeneity of the contrast medium and allowed the imaging of more distally located bowel loops. In these patients contrast medium intake has been to be prolonged until the abdominal CT had been started to compensate the rapid gastric passage by metoclopramide. Hydroxymethylcellulose increased the distension of the small bowel loops and the diagnostic accuracy of the peritoneal and retroperitoneal space (p 0.01 ).

Adequate intestinal contrast significantly improves the image quality in abdominal CT. The concentration of contrast medium is of less importance than decreasing the transit time by pharmacological activation or dietary preparation.

\section{$10.40 \mathrm{AM}$}

50 Clinical evaluation of a new gastrointestinal contrast agent for $\overline{a b}$ dominal and pelvic MR imaging

C. Caron, J. J. Railhac, M. Kasbarian, P. J. Valette, C. Roy; Angers

Purpose: AMI 121 is an oral superparamagnetic iron oxide MR contrast media. The aim of this study is to assess safety and efficacy during abdominal and pelvic examinations.

Methods and Materials: A multicentre trial was carried out in 100 patients with pancreatic, lymphatic or pelvic pathology. MRI examination (T1 and T2 sequences) was performed before and after oral administration of 500 to $900 \mathrm{ml}$ of AMI 121 ( 87 to $158 \mathrm{mg}$ of iron). Clinical and biological safety were evaluated during 2 days. Efficacy criteria were improvement in gastrointestinal tract visualisation, in abdominal organs delineation and in diagnostic quality of MR examination.

Results: With AMI 121 visualisation of stomach, duodenum and small bowe was improved as well as delineation of pancreas, lymph nodes and pelvis in both $\mathrm{T} 1$ and T2 sequences. No artefacts due to concentration of AMI 121 in the bowel were visualized. Safety was good. Digestive effects were reported for some patients. They were considered as mild. Some cases of variation of iron and ferritin were observed 24 hours post injection without any clinical effect. Conclusions: AMI 121 is safe and useful as an oral contrast agent in abdominal and pelvic pathologies.

\section{$10.50 \mathrm{AM}$}

\section{Manganese-DPDP as a new T1-contrast agent. MR-imaging of the upper abdomen}

G. Branding, B. Hamm, P. Teller, A. Bock, K.-J. Wolf; Berlin

Purpose: To evaluate the signal enhancing effect of the new hepatobiliary contrast-agent Manganese-DPDP (Mn-DPDP) on liver, spleen, pancreas, kidney and stomach.

Methods and Materials: 20 patients were examined prior to and half an hour after intravenous application of $10 \mu \mathrm{mol} \mathrm{Mn-DPDP} / \mathrm{kg}$ at $1.5 \mathrm{~T}$. Tl-weighted $\mathrm{SE}(\mathrm{TR}=500 / \mathrm{TE}=15)$ and $\mathrm{GRE}(\mathrm{TR}=160 / \mathrm{TE}=5 / \alpha=80)$ images of the upper abdomen were obtained in each patient. Signal enhancement of liver, spleen, renal cortex and medulla, pancreas and stomach wall was measured. Results: Signal enhancement by Mn-DPDP was more pronounced in the GRE than in the SE sequences. Increase of signal intensity (measured in the GRE sequences) was highest in the liver: $82,3 \%( \pm 15 \%)$, followed by the spleen: $18,8 \%( \pm 11,8)$, pancreas: $38,8 \%( \pm 7,1 \%)$, renal cortex: $81,7 \%( \pm 5,1 \%)$, renal medulla: $32,8 \%( \pm 8,9 \%)$ and stomach wall: $64,5 \%( \pm 12,1)$. A remarkably improved differentiation of renal cortex and medulla was observed.

Conclusions: Due to its hepatobiliary and partial renal excretion Mn-DPDP leads to a strong enhancement in liver tissue and renal cortex. However previously unexpected contrast enhancement was found in the spleen, pancreas and stomach mucosa. The underlying reasons for this enhancement are yet unknown.

$11.00 \mathrm{AM}$

52 MRI staging of esophageal cancer with a new oral paramagnetic preparation

P. Pavone, G. Cardone, G. Albertini P., R. Occhiato, R. Crisci, F. Coloni, R. Passariello; L'Aquila

Purpose: To evaluate the results obtainable with the use of a specific contrast agent preparation for esophageal opacification in cancer patients.

Methods: 8 patients with histologically proven esophageal cancer were included in the study.

MRI examinations were performed with a 0.5 Tesla superconductive equipment. Axial, sagittal and coronal T1 weighted spin echo images were acquired with non gated technique.

The contrast agent preparation was obtained by emulsifying two different commercially available agents. A specific esophageal contrast agent for CT was used, presenting high viscosity (TAC Esofago, distributed by Bracco I.C. in Italy, produced by EZ.E.M. in U.S.A.). As paramagnetic contrast agent we used Gd-DTPA (Magnevist, Schering, Germany).

Results: In all cases a strong signal intensity of the lumen of the esophagus was achieved, both above and at the level of the stricture. This allowed detailed evaluation of the intramural extent of the lesion, showing 3 cases of concentric and 5 cases of eccentric neoplastic growth. In the sagittal images a complete evaluation was performed, with unique display of the tumor and lumen opacified. Periesophageal invasion was also documented.

Conclusions: This technique could be proposed for routine evaluation of esophageal cancer with MRI.

\subsection{AM}

\section{Immunoscintigraphy in the follow-up of colorectal cancer - A comparison with $\mathrm{CT}$ and MRI \\ G. Leinsinger, H. Denecke, H. Schmidt, C. M. Kirsch; München}

In the follow-up of patients (pts) with colorectal cancer early detection of tumor relapse is essential to improve the prognosis by surgical treatment. The aim of the present study was to validate immunoscintigraphy in detecting local recurrencies or metastases of colorectal cancer. The study includes $155 \mathrm{pts}$. Immunoscintigraphy was performed using a cocktail of 131-I labelled monoclonal antibodies to CEA and CA 19-9 (2 mg F(ab') $\left.)_{2} ; 70-140 \mathrm{MBq}\right) .3$ and 5 days after injection SPECT-images were acquired using a rotating gamma camera equipped with a half inch crystal. All findings were compared with the results of CT. In addition, MRI with Gd-DTPA enhancement was performed in a subgroup of pts $(n=12)$ with suspected local recurrency of rectal cancer. All results were comfirmed by subsequent surgery and histology. Local recurrency was confirmed in 53 pts. Sensitivity of immunoscintigraphy in visualizing local recurrency was $81 \%$, whereas CT was negativ or questionable in $29 \mathrm{pts}(55 \%)$. MRI confirmed the results of immunoscintigraphy in $6 / 12 \mathrm{pts}$, but contributed additional information in $4 / 12$ pts. Sensitivity of immunoscintigraphy in detecting liver metastases was $87 \%$ (CT: $71 \%$ ), lymph node metastases $79 \%$ (CT: $50 \%$ ) and peritoneal carcinosis $60 \%$ (CT: $7 \%$ ). Specificity of immunoscintigraphy in detecting local recurrencies was $82 \%$, liver metastases $95 \%$, lymph node metastases $96 \%$ and peritoneal carcinosis $98 \%$. These results were similar to the specificity found by CT. Therefore, immunoscintigraphy using SPECT improves the early detection of local recurrencies and metastases of colorectal cancer, especially in those cases with non-conclusive results of CT. In some cases MRI using Gd-DTPA can contribute additional information in visualizing local recurrencies of rectal cancer.

\section{$11.20 \mathrm{AM}$}

54 Detection of focal hepatic masses: comparison between MR imaging and CT during arterial portography (CTAP)

Luigia Rota, G. Carlo Barazzoni, Roberto Dore, Giuseppe Di Giulio, Andrea Villa, Antonio Nicolato; Pavia

Our purpose was to compare the sensitivity of CTAP and MR imaging in the preoperative evaluation of patients with neoplastic involvement of the liver. Methods: 28 patients with known primary or secondary hepatic malignancies were examined over a 15 -months period for possible hepatic resection. All 28 patients had a visceral angiogram to determine the position of the catheter in 
the SMA or in the splenic artery and subsequently CTAP; the MR imaging was performed on superconducting whole-body imager operating at $1.5 \mathrm{~T}$. The patients underwent both $\mathrm{T} 1$ - and $\mathrm{T} 2$-weighted pulse sequences. We evaluated number, size and location of each focal mass.

Results: Both in surgical and non-surgical patients (16 and 12) the detection rate of CTAP was higher than MR SE T2 sequences ( 84 vs $70 \%$ ); MR SE T1 sequences were generally poorer of informations. In all true-positive lesions the diagnosis was definite with CTAP and MR imaging; CTAP detected 5 small lesions (less than $2 \mathrm{~cm}$ ) not found on MR; two false-positive on CTAP were due to cavernous hemangiomas and one small angioma on MR was a metastasis at surgery.

Conclusions: Accurate preoperative imaging evaluation of the liver is essential. In our study CTAP was more sensitive in the detection of hepatic lesions than MR imaging, mainly if small. Therefore MR imaging improves the rate and confidence of focal hepatic lesion detection.

\subsection{AM}

\section{Hepatocellular carcinomas with excessive copper accumulation:} $\overline{\mathrm{CT}}$ and MR findings

Kiyohide Kitagawa, Osamu Matui, Masumi Kadoya, Makoto Hirano, Shinji Masuda, Yasuni Nakanuma; Takaoka

Purpose: To evaluate the correlation between the excessive copper accumulation in hepatocellular carcinomas (HCCs) and their findings of CT and MR. Materials and methods: Histologically proven $58 \mathrm{HCCs}$ were retrospectively studied with orcein stain. Findings of $\mathrm{CT}$ and MR were analyzed according to two groups; three orcein-positive HCCs and 55 orcein-negative HCCs.

Results: Three orcein-positive HCCs were demonstrated as diffuse high density on non-enhanced CT. Calcification, hemorrhage and surrounding fatty infiltration were not found in these cases. Electron X-ray microanalysis elucidated the characteristic peak of $\mathrm{Cu} \mathrm{K} \alpha(8.040 \mathrm{KeV})$ in one case and its copper content was $400 \mu \mathrm{g} / \mathrm{g}$ dry weight. But our estimated increase of the attenuation value was only $0.5 \mathrm{HU}$. Other orcein-negative $\mathrm{HCCs}$ were dipicted as low density on non-enhanced CT. We could not find any difference of MR findings between these two groups.

Conclusions: Orcein-positive materials, which were equivalent to copperbinding protein, might be attributed to the high attenuation value of HCC on non-enhanced CT. Copper ions coordinated to copper-binding protein do not influence on MR imagings, because copper accumulated in HCC is suggested to be diamagnetic (univalent).

\section{$11.40 \mathrm{AM}$}

\section{Comparison of MR, US and CT in the detection and staging of pancreatic tumors}

H. Rigauts, G. Marchal, A. L. Baert; Leuven

Purpose: Twenty patients with histological proven pancreatic neoplasm were examined with MR, US and CT. The value of these three methods in the detection and staging was compared.

Results: For detection, ultrasound and MR scored equally well but both scored better than CT. For the staging, MR scored better than CT and CT scored better than ultrasound

In MRI the best results for both tumor detection and staging were obtained with the regular T1W SE sequence. T2W SE sequences were of almost no help. Fast breathholded TIW imaging (RASE, FLASH) with or without Gadolinium enhancement also did not improve the overall results.

The better results of MR in comparison with CT are explored by the higher inherent image contrast, particularly in the presence of pancreatic lipomatosis. Methods: MRI at 1.0 T included SE T1 and T2W images, breathholded T1W RASE and FLASH sequences, some with additional Gd enhancement. CT included plain and bolus enhanced dynamic scanning.

US was performed on state of the art equipment (Acuson, ATL).

$11.50 \mathrm{AM}$

\section{MR Imaging of pancreatic disease with oral magnetic particles: comparison with $\mathrm{CT}$}

M. Zalcman, B. Lalmand, T. F. Jacobsen, J. Struyven; Brussels

Purpose: To evaluate the effect of a new oral negative contrast in MR studies of the pancreas by comparison with computed tomography.

Methods: MR studies of 24 patients with documented pancreatic disease (acute pancreatitis, chronic pancreatitis, pancreatic cancer) who were each given $0.5 \mathrm{I}$ of CMP (a crystalline magnetic iron oxide supported on a carrier matrix of monodisperse polymer particles) at a concentration of $0.5 \mathrm{gr} / \mathrm{l}$. T1- and $\mathrm{T} 2$-weighted spin echo sequences were applied at 1.5 Tesla. These studies were compared with CT examinations performed within one week of the MR examination. All MR and CT scans were reviewed and evaluated by three observers.

Results: No significant adverse effects occurred. No contrast related artifacts were recorded. Good distribution of the contrast was observed in the stomach in $80 \%$ of cases, in the duodenum in $84 \%$ and in the jejunum in $48 \%$. Pancreas delineation was evaluated as sufficient in $92 \%$ of cases.

MR information was equal to CT in $48 \%$ of cases, superior to CT in $19 \%$ and inferior to CT in $33 \%$. This loss of information was due in nearly all cases to lack of visualisation of pancreatic calcifications in chronic pancreatitis and cannot be improved by contrast medium.

Conclusions: Excellent contrast enhancement was obtained in the proximal part of the digestive tract with CMP. Although the value of MR imaging of the pancreas remains controversial, we feel this contrast medium will be of great importance in the improvement of the diagnostic value of MRI in the digestive field.

\section{Room E1}

\section{SS} $10.30 \mathrm{AM}$

\subsection{Vascular imaging and PTA}

58 Digital peripheral angiography with stepping and subtraction: comparison with other techniques

Thomas Hilbertz, U. Fink, R. Beck, U. Eberwein, H. Berger; München

Purpose: The aim of this study was the evaluation of digital peripheral angiography with stepping and subtraction (DPSA) in comparison to other imaging modalities.

Methods: In a prospective, randomized study including 60 patients new DPSA was compared to digital peripheral angiography without subtraction (DPA), DSA with stationary series and conventional angiography. Besides image quality and diagnostic reliability we analysed the duration of examination, the amount of contrast medium and X-ray exposure.

Results: Using DPA and DPSA complementary stationary series were necessary in 1 out of 20 patients (conventional angiography: 6 out of 20, DSA: 1 out of 20). The reason for this is the opportunity to watch contrast flow during the examination on the monitor to trigger table stepping by a hand switch. Examination time and the amount of contrast medium could thus be reduced by about $15 \%-20 \%$. Image quality was excellent in all patients, who underwent DSA and DPSA. Both digital subtraction techniques supplied superior image quality especially in the lower limb in comparison with conventional angiography and DPA. X-ray exposure was similar for conventional angiography, DPA and DPSA. DSA with stationary series revealed a three times increased dose.

Summary: Our results indicate that DPSA might become the method of choice in peripheral angiography.

\section{$10.40 \mathrm{AM}$}

59 Intravascular ultrasound as an adjunct to angiography during percutaneous interventional procedures

D. Vorwerk, R. W. Günther, J. M. Neuerburg, D. Andreopoulos, P. Keulers; Aachen

Intravascular ultrasound (IVUS) was used as an adjunct to angiography for additional information during interventional procedures pre and post treatment in 42 patients.

IVUS was performed using a $20 \mathrm{MHz}$ mechanical rotating single-element transducer covered by a $6 \mathrm{~F}$ side-saddle catheter for arterial and a $9 \mathrm{~F}$ catheter for venous studies. Arterial lesions $(n=36)$ were located in the aorta $(n=3)$, pelvic $(n=25)$ and femoral arteries $(n=8)$, venous application was performed in 6 patients. Interventions included stent placement, thrombectomy, angioplasty, atherectomy or caval filters.

IVUS offered complementary information to angiography in two-third of cases thus being of therapeutic relevance in one third. Severity of dissections or stenoses was more accurately depicted by IVUS; length of a diseased area, vessel diameter and exact anatomic position of important structures (orifices, branching arteries) were more exactly identified by IVUS.

Intravascular ultrasound is easily applicable and offers additional information which is important to decide on the success of percutaneous treatment, choice of additional intervention and exact placement of filters and stents.

\section{$10.50 \mathrm{AM}$}

\section{Intra-arterial Doppler ultrasound in the prediction of the early outcome of percutaneous peripheral angioplasty}

G. G. Hartnell, R. Parry, E. Berry, J. Wilcox, M. Horrocks, P. Murphy; Bristol

Following percutaneous angioplasty (PTA) the risk of early reocclusion is difficult to predict using angiography which assesses adequate flow poorly. We have applied intra-arterial Doppler ultrasound to the immediate assessment of the results of PTA to see if it is possible to use this to predict patients at risk from early reocclusion.

A $20 \mathrm{MHz}$ pulsed Doppler catheter (Millar Instruments) with a $3 \mathrm{~F}$ tip is used in conjunction with a dedicated Doppler spectrum analyser (DopStation, 
SciMed). Experimental work using a constant flow rate phantom have confirmed a consistent relationship between measured and actual flow velocities.

In vivo measurements have been made in 14 patients having PTA for femoropopliteal lesions (11) or femoral distal graft stenosis (3) and various methods of Doppler spectral analysis (flow velocities, resistance and pulsatility indices, return of normal triphasic waveform) correlated with early results following PTA

Early results indicate that intra-arterial Doppler is a useful method for assessing the haemodynamic result of PTA and may be useful for identifying patients at high risk of reocclusion who would benefit from further PTA or other intervention to prevent reocclusion.

\section{$11.00 \mathrm{AM}$}

61 Percutaneous transluminal angioplasty in the treatment of critical lower limb ischaemia

P. Matsi, H. Manninen, M. Suhonen; Kuopio

Purpose: To evaluate the utility of percutaneous transluminal angioplasty (PTA) in the treatment of critical lower limb ischaemia.

Patients and methods: During a one-year period PTA was performed on 32 patients (mean age 69 years, range 34-84 years) with critical limb ischaemia. 10 patients suffered from rest pain (Fontaine IIIb) and 22 patients had ulcus or gangrene (Fontaine IV). 52 arteries were dilated and 13 occlusions were recanalized with conventional PTA techniques. One third of the dilatations $(16 / 52)$ and recanalizations $(4 / 13)$ were infrapopliteal.

Results: The primary success rate of the dilatations was $96 \%(50 / 52)$ and of the recanalizations $85 \%(11 / 13)$. A successful venous bypass operation was subsequently performed on one of the patients with unsuccessful recanalization. After a follow-up period varying from 1 to 12 months, $80 \%(8 / 10)$ of the Fontaine IIIb group patients and $45 \%(10 / 22)$ of the Fontaine IV group patients have avoided major amputation. The overall death rate during follow-up is thus far $15 \%(5 / 32)$.

Conclusions: PTA is a feasible approach to the treatment of critical lower limb ischaemia in patients with atherosclerotic changes too extensive for vascular reconstructive operations. As a less invasive and inexpensive procedure, PTA is also a good alternative to vascular surgery as a primary treatment, especially in older patients in poor physical condition.

\subsection{AM}

\section{Percutaneous transluminal angioplasty (PTA) of crural arteries} F. Flueckiger, J. Lammer, E. Pilger, G. E. Klein, K. Hausegger, F. Waltner, M. Aschauer; Graz

Purpose: We describe our technique and results of PTA of the arteries below the knee in patients with clinical stages Fontaine IIb-IV.

Methods: We dilated 125 crural arteries (stenosis or short occlusion) in 88 patients. In 67 patients crural PTA was done in combination with a dilatation in the femoropopliteal region. Stenoses were passed by a steerable guide wire, dilatation was performed by a $5 \mathrm{~F}$ balloon catheter ( 2.5 to $4 \mathrm{~mm}$ ). Spasms were treated by nitroglycerine, nifedipine or prostaglandine E1. Heparin-5000 U during, $1000 \mathrm{U} / \mathrm{h}$ for $24 \mathrm{~h}$ after PTA. Long-term therapy-anticoagulants or acetylsalicylic acid/dipyridamole. Before and after PTA as well as in follow up (3-38 months) the Fontaine stage, the pulses and the ankle-brachial-index $(\mathrm{ABI})$ were evaluated.

Results: We achieved a primary success rate of $89 \% .81 \%$ of patients showed improvement (ABI, pulse, relieve of pain) after PTA and $68 \%$ long-term improvement. 2 patients showed clinical deterioration after PTA. Complications: spasm $n=3$, local thrombosis $n=2$, intimal flap with significant stenosis $\mathrm{n}=3$, peripheral emboly $\mathrm{n}=2$, dissection $\mathrm{n}=1$. Thromboses and emboli were treated by fibrinolysis or aspiration.

Conclusions: PTA of crural arteries is a safe procedure with a low complication rate and satisfactory long-term results. PTA of crural arteries is necessary to improve a reduced outflow after a recanalization procedure in the femoropopliteal axis. Fontaine stage IIb should be accepted as an indication for PTA of crural arteries.

\subsection{AM}

\section{Intra-operative angioplasty in the treatment of multisegmental} peripheral vascular disease

A. M. Cook, J. F. Dyet, W. C. Hartley, A. A. Nicholson, J. M. D. Galloway, A. R. Wilkinson; Hull

Purpose: A series of fourty three cases of intra-operative angioplasty is presented, and this form of treatment in patients with symptomatic multisegmental peripheral vascular disease is discussed.

Methods: The operative procedures were femoro-femoral cross over grafts in seven cases, aortofemoral grafts in two cases, femoro-popliteal grafts in six cases and proximal femoral segment endarterectomies and/or profundaplasties in twenty eight cases. Angioplasty for a significant inflow iliac stenosis or outflow femoral stenosis from the surgical procedure was performed synchronously with the surgery.

Results: The overall results show that of the fourteen patients with limb threat, eleven limbs were salvaged with three of these now asymptomatic and eight claudicants, with a mean follow-up of thirty four months. In the claudicant group of twenty nine patients with a mean follow-up of twenty months, sixteen are asymptomatic, five significantly improved, one unchanged and seven required further procedures for progressive disease at a mean time of eighteen months.

Conclusions: The results demonstrate that intra-operative angioplasty is able to effectively treat significant disease beyond the operative site and that this combined approach to multisegmental disease can obviate the need for more extensive surgical procedures in these high risk patients.

\section{$11.30 \mathrm{AM}$}

64 Color-coded Doppler-sonography: an ideal method for follow-up after anatomical and extra-anatomical By-passes of the lower extremities

V. Metz, W. Dock, K. Eibenberger, F. Grabenwöger; Wien

Purpose: The aim of the study was to determine the accuracy of different clinical and imaging methods for follow-up of anatomical and extraanatomical bypasses.

Methods: 20 femoropopliteal bypasses and 29 extra-anatomical ( 9 axillofemoral, 20 femorofemoral) bypasses were examined with color-coded Doppler sonography and pulsed Doppler sonography. Angiography was used as gold standard. Additionally the extra-anatomical bypasses were examined clinically: palpation of the bypasspulsations, Fontaine staging and Doppler indices.

Results: Both sonographic methods provided a correct diagnosis in all cases. The outcome of palpation of bypasspulsations was also correct in all cases of axillofemoral bypasses, but false in 3 cases of femorofemoral bypasses. Fontaine staging and the Doppler indices were incorrect concerning the evaluation of patency of bypassgrafts in 3 cases each. 2 seromas, 2 bypassstenoses and 1 bypassaneurysm were additional findings with the sonographic methods. These findings could not be recognized with angiography and clinical methods.

Conclusions: Sonography turned out to be the ideal method for follow-up. Palpation, Doppler index and the Fontaine method were of less value. Angiography should be performed only if further vascular reconstruction is planned.

\subsection{AM}

\section{Qualitative and quantitative findings of color Doppler before and after PTA of hemodialysis fistulas}

P. Landwehr, A. Tschammler, Th. Krahe, K. Lackner; Würzburg

Purpose: To define the clinical value of color Doppler flow imaging (CDFI) of hemodialysis fistulas in selecting patients for shunt angioplasty and in performing follow-up studies after PTA.

Methods: CDFI (QAD 1, 7.5 MHz) before and after PTA of 34 insufficient hemodialysis shunts. Qualitative analysis: localization and characterization of stenoses, decision about the PTA approach (retrograde, antegrade, additional arterial puncture). Quantitative analysis: degree of the stenoses, integral shunt flow volume.

Results: In 33/34 cases DSA during PTA confirmed the findings of CDFI. After PTA, patency of the dilated or stented segments, residual stenoses as well as complications (aneurysm, hematoma) could be detected reliably by CDFI. Shunt flow volume before PTA was $\bar{x}=188.1 \mathrm{ml} / \mathrm{min}$ (SD 122.7). PTA was clinically successful in 27 patients. In this group flow volume increased to $\overline{\mathrm{x}}=511.9 \mathrm{ml} / \mathrm{min}(\mathrm{SD} 237.4)$ after PTA $(\mathrm{p}<0.005)$. In the remaining cases no significant improvement of the shunt function could be observed (shunt flow $\overline{\mathrm{x}}=205.7 \mathrm{ml} \pm 167.9 \mathrm{ml} / \mathrm{min}$ after PTA).

Conclusions: Color Doppler is of high clinical value in hemodialysis shunt insufficiency to localize obstructions and to decide about technical possibility of shunt PTA. Complications of PTA, residual and recurrent stenoses can easily be identified non-invasively. Flow volume measurements allow quantification of the success of PTA.

\section{$11.50 \mathrm{AM}$}

66 Combined laser- and balloon angioplasty of venous stenotic lesions in patients with hemodialysis fistulas

E. Gmelin, M. Zwaan, H. Kagel, G. Oehlert, H.-D. Weiss; Lübeck

Venous stenoses or occlusions of hemodialysis fistulas at the forearm are mainly caused by subintimal fibrosis. In these cases complete dilatation also with high pressure balloons ( $10 \mathrm{~atm}$ or more) may be impossible. In fourteen patients balloon angioplasty was combined with laser ablation, two cases with occlusions (4-15 cm in length), twelve cases with recurrent (previous dilatation) stenoses $(1-4 \mathrm{~cm})$. In six patients laser angioplasty was performed initially, in eight patients after unsuccessful balloon dilatation. 
Lasersystem: Candela MDL-2000 pulsed dye laser, pulse repetition frequency $10 \mathrm{~Hz}$, - energy 80-100 mJ - duration 1 microsec., wavelength $504 \mathrm{~nm}$. Number of pulses 200-1000/procedure. Laser-ring-catheter: 7-9 F multifibre (12-19 single fibres) introduced with guide wire.

Balloon angioplasty: $5-\mathrm{F}$-catheter, balloon diameter 5-6 mm, pressure $10 \mathrm{~atm}$. Results: In all cases complete dilatation could be achieved without any residual stenosis.

Complications: Two occlusions several hours after intervention, in one case caused by intimal flap, in the other by thrombosis (patient hat $15 \mathrm{~cm}$ long thrombus before angioplasty). In 12 patients vessel remained patent up to the present moment (four months).

Conclusions: Laser ablation of venous stenotic lesions due to subintimal fibrosis is very helpful as an additional means to balloon angioplasty in patients with insufficient hemodialysis fistulas.

\section{$11.55 \mathrm{AM}$}

\section{Recanalization of arterial occlusions using a specially designed steering catheter}

M. Suhonen, H. Manninen, P. Matsi; Kuopio

Guide wires alone and straight or general purpose angiography catheters have poor steerability when used for the recanalization of atherosclerotic occlusions of lower extremity arteries. To overcome this problem, a special catheter was designed to improve the steering of guide wires during recanalization. The catheter has a $5 \mathrm{~F}$ diameter and a lumen for 0.035 guide wire. A metallic mesh layer within the wall provides good torque control. Its $1.5 \mathrm{~cm}$ long softened tip has a $30^{\circ}$ angle for steering and for finding the right direction for the guide wire. Especially when used with hydrophile "glide"-wires, it gives superb control for probing through the occluded segment. It can also be used to steer guidewires to different side branches eg. the infrapopliteal arteries. We used this catheter in 48 patients with 3 iliac artery occlusions and 45 femoropopliteal occlusions. The mean occlusion length was $6 \mathrm{~cm}(2-15 \mathrm{~cm})$. The primary technical success rate was $88 \%(42 / 48)$ and primary clinical success rate $79 \%$ $(38 / 49)$. The reasons for technical failures were heavy calcification in three (3) cases and subintimal passage in three (3) cases. In conclusion this type of catheter is very helpful in routine recanalization and has improved our primary technical success rate.

\section{$11.58 \mathrm{AM}$}

68 Effect of hydrophilic guidewires on the recanalisation of occluded femoro-popliteal arteries. A randomised controlled trial

G. G. Hartnell, R. Parry, A. Jones, R. N. Baird, P. Murphy; Bristol

36 patients with lifestyle limiting symptoms due to femoro-popliteal occlusions were randomised to have recanalisation initially attempted with conventiona (Group 1-19 cases) or hydrophilic guidewires (Group 2-17 cases). In group 1 recanalisation rate was $100 \%$, hydrophilic wires were required in 8 . In group 2 recanalisation rate was $94 \%$, conventional wires were required in 5 . The length of occlusion was greater in group 2 which also required longer screening times and more contrast. Overall success rate was $97 \%$

The use of hydrophilic guidewires is associated with a very high success rate for recanalising occluded femoro-popliteal arteries.

\section{Room E2}

\section{SS}

\subsection{Soft Tissues}

\subsection{AM}

\section{Superior tibio-fibular ganglia - a spectrum of imaging abnormalities} with clinical correlation

V. Cassar-Pullicino, D. Grier, I. W. McCall; Oswestry

The clinical and radiological features of twenty patients with superior tibiofibular ganglia are presented. The unusual spectrum of clinical presentation is correlated with the radiological appearances and the optimum mode of imaging.

The imaging methods encompass plain film radiography, superior tibio-fibular joint arthrography, Computed Tomography, Ultrasound, and Magnetic Resonance Imaging.

The results show that diagnostic and therapeutic difficulties arise from the tendency to spread, often by a peduncle into the neighbouring tissues. The swellings migrate from the joint and may be subcutaneous, subperiosteal, intramuscular, or may involve the common peroneal nerve by compression or infiltration. In the absence of a swelling, the exclusion of such a lesion by the imaging methods described, is essential in the clinical setting of tibial compartment syndrome, as well as spinal nerve root compression.

The radiological characteristics are reproducible with frequent demonstration of asymptomatic contralateral disease. Pre-operative delineation of the presence, extent and communications are essential requirements to reduce the significant recurrence rate. The etiological mechanism of spread and therapeutic implications based on these radiological features are discussed.

10.40 AM

70 Achille's tendon healing: 12 month follow-up using magnetic resonance imaging

C. F. Pope, E. Dillon, P. Jokl, K. Lynch, D. Brown, P. Anson, F. Avery, W. Heinz; Portland, ME

Purpose: Symptomatic Achille's tendon abnormalities (rupture, tendonitis, tendinosis) were evaluated using MRI during the healing phase after either surgical or conservative treatment to identify findings that would indicate healing or would allow complications such as infections or incomplete repair to be recognised.

Methods: A total of 24 patients have been studied. 18/24 had surgery (16 tendon rupture) and $6 / 24$ were managed conservatively (I rupture). Pretreatment MRI studies were obtained in $11 / 24$, at 3 months in $9 / 24$, at 6 months in $9 / 24$ and 12 months in 12/24. Axial and sagittal spin-echo (TR2000, TE20, $80 \mathrm{msec}$ ) and gradient-echo (TR800, TE16 msec, Flip 30 degrees) images were correlated with clinical results.

Results: All tendons had intra-tendinous fluid spaces at 3 and 6 months after surgical repair of tendon rupture. One developed a peritendinous abscess. This was demarcated by the peri-abscess granulation tissue. One tendon showed a region of persistent thinning with rerupture at 6 months. All tendons showed progressive signal decrease at the healing site during follow-up. By 12 months, uniform low signal intensity indicative of dense collagen fiber was seen in all tendons. Significant tendon thickening was present in all surgically treated cases even at 12 months.

Conclusions: MRI is useful to assess Achille's tendon healing. Post-operative studies show persistent intra-tendinous fluid collections which can complicate interpretation in patients in whom infection is suspected. Although tendon remodelling occurs, persistent tendon thickening is observed even at 12 months following injury.

10.50 AM

\section{The role of MRI in the diagnosis of muscle diseases}

W. A. Kaiser, B. C. G. Schalke, M. Reiser; Bonn

Purpose: The potentials of MRI in diagnosis and monitoring of therapy of muscle diseases have been evaluated.

Patients and methods: Since March 1984, 320 patients suffering from various muscle diseases including progressive muscular dystrophies (70 pats.), myotonic dystrophy (20 pats.), neural muscular atrophy (20 pats.), myosities (24 pats.), sarcoidosis ( 9 pats.), metabolic or histological defects (43 pats.), spinal muscular atrophy (13 pats.), tumors (18 pats.), trauma (18 pats.), stenosis or occlusion of vessels ( 32 pats.) have been examined using superconductive magnets (Siemens Magnetom 0.35-1.5T).

Results: According to 4 morphological criteria (symmetric, asymmetric, homogeneous, focal), characteristic MR-patterns of muscle diseases of the upper limb could be described:

Pattern I: acute myositis, neural muscular atrophy, alcoholic myopathy, diabetic myopathy, cortisone myopathy, ischemia/vessel stenosis or occlusion, mitochondrial myopathy, uremic myopathy.

Pattern II: chronic DMD, chronic myositis, status after bilateral poliomyelitis. Pattern III: limb girdle dystrophy, chronic myositis, centronuclear myopathy, familiar hypokaliemic paralysis.

Pattern IV: status after unilateral poliomyelitis, trauma, tumor, hematoma etc Conclusions: MRI is highly valuable for the diagnostic strategy of muscle diseases, e.g. diagnosis and differential diagnosis, planning of muscle biopsy and operation, control and monitoring of therapy (e.g. cortisone).

\section{$11.00 \mathrm{AM}$}

72 The role of sonography and color Doppler in the musculoskeletal pathology

L. Bacarini, A. Rabassini; Treviso

Purpose: The role of sonography to detect some "critical regions" (as shoulder, elbow, hip, knee and ankle) is discussed.

Methods: Both children and adults were tested by high frequency $(5,7.5 \mathrm{MHz})$; static and dynamic tests were always performed. Color Doppler investigation were made in all the soft tumors and tumor-like conditions.

Results: Shoulder: rotator cuff tear and related pathology (i.e. tendon biceps) is discussed. Elbow: some inflammatory and degenerative periarticular diseases (epicondilitis, bursitis, arthritis). Hip: screening and follow-up of the neonatal hip (according to Graf); presence of joint fluid in arthritis. The contribute in rotational deformities is very poor. Knee: patellar tendons and articular cartilage pathology, effusions and popliteal cysts were evaluated. Ankle: Achilles tendon pathology was tested specially in the traumatic accidents. Soft tissues tumors: benign and malign tumors were examined by sonography and Doppler. 
Conclusions: Our results suggest that sonography is the first, noninvasive imaging method in the musculoskeletal pathology detection. Sonography should be performed as test of choice in children (hip dysplasia, hip and knee effusions) and in traumatic conditions. Color Doppler improve diagnostic accuracy in the assessment of neoplastic masses.

\subsection{AM}

\section{Evaluation of soft tissue masses by magnetic resonance imaging} J.M. Hawnaur, J.P. R. Jenkins, I. Isherwood; Manchester

Purpose: The aim of this study was to review our experience of MRI in soft tissue masses, with particular reference to the role of Gadolinium-DTPA as a means of increasing diagnostic accuracy.

Methods: MRI findings were reviewed in 142 patients $(61 \mathrm{M}, 81 \mathrm{~F}$, aged 5 months to 84 years) presenting with a palpable soft tissue mass. MRI was performed at $0.26 \mathrm{~T}$, using various receiver coils according to the size and position of the lesion. $\mathrm{T} 1$ - and $\mathrm{T} 2$-weighted scans were obtained in all patients, supplemented in 68 by $\mathrm{Tl}$-weighted scans after $0.1 \mathrm{mmol} / \mathrm{kg}$ body weight Gadolinium-DTPA (Gd-DTPA), (Magnevist, Schering-AG) intravenously.

Results: Hemangiomas, lipomas, and most desmoid tumours were characterised on T1- and T2-weighted scans alone, the use of Gd-DTPA being of little additional diagnostic value. Gd-DTPA was helpful in differentiating benign solid tumours e.g. neurofibromas and myxomas from cystic masses including ganglion and synovial cysts. All primary soft tissue sarcomas enhanced markedly on T1-weighted scans after Gd-DTPA, demonstrating tumour inhomogeneity and necrosis better than on pre-Gd-DTPA scans.

Conclusions: Many benign soft tissue lesions can be characterised on conventional T1- and T2-weighted MR sequences alone. Gd-DTPA is most useful in the assessment of soft tissue sarcomas.

\section{$11.20 \mathrm{AM}$}

\section{Morton neuroma: US and MRI evaluation}

P. L. Paesano, M. Venturini, G. Dal Conte, P. Rivoltini, A. DelMaschio; Milan

Purpose: Morton neuroma is a focal mass of perineural fibrosis involving the plantar digital nerves of the foot. Both high resolution ultrasound (US) and MRI have been used in the diagnosis of this neuroma. We compared the diagnostic accuracy of this imaging modalities in the evaluation of Morton neuroma.

Methods and Materials: 30 patients clinically suspected of Morton neuroma where included in this study. Surgery confirmed the diagnosis in 27 patients. All the patients where preoperatively studied by high resolution US $(7.5 \mathrm{MHz}$ linear probe) and 10 patients by MRI (sagittal and coronal TIW $5 \mathrm{~mm}$ sections).

Results: US visualized 25 out of 27 neuromas (92.6\%). Two small neuromas were not visualized by US due to the small size of the lesions ( 3 and $3.5 \mathrm{~mm}$ ). In 3 patients US correctly identified other diseases responsable for the (pain/bursitis or capsulitis) MRI only visualized 2 out of 10 neuromas $(20 \%)$. Conclusions: In our experience high resolution US is an accurate diagnostic tool in the evaluation of Morton neuroma. On the contrary in this study the sensibility of MRI was very small as we visualized only two neuromas out of ten patients. This can be due to poor spatial resolution of our technique $(5 \mathrm{~mm}$ slice thickness).

\section{$11.30 \mathrm{AM}$}

\section{O. Bottinelli, A. Bozzini, R. Anguissola, E. Genovese, \\ I. Fulle,}

B. Guglielmoni, F. Calliada, M. Bergonzi, R. Campani; Pavia

The purpose of the research is to evidence the hand complex structure and, in particular, to point tendons with their inflammatory pathology; furthermore it is possible to study eventual subcutaneous expansive processes and other kinds of pathology, such as, for example, the carpal tunnel syndrome.

High frequency probe $(7.5 \mathrm{MHz})$ with interposition of spacing material are used; there are no standard scansion, but both the palmar and the dorsal face are examined with all the possible scansions.

130 patients have been examined, clinical symptomatology was represented by pain, localized or diffused tumefaction, paresthesias and motor deficit in the movements of flexion and extension. The examination has shown:

-70 cases of pathologic conditions of tendons (tendosynovitis, peritendinitis, tendineous cysts).

-25 cases of granuloma due to a foreign body.

-5 cases of Dupuytren disease.

In the remaining cases, the examination has given negative result.

To sum up we can say that hand sonography has shown to be useful for the study of the main part of tendinous and subcutaneous pathology whose diagnosis was mostly clinical, as these structures can't be displayed by using traditional radiology.

\section{$11.40 \mathrm{AM}$}

\section{Carpal Tunnel Syndrome: Diagnosis with MR Imaging} B. Hamm, A. Kleindienst, F. Boegner, S. Seyfert, K.-J. Wolf; Berlin

Purpose: To evaluate the normal anatomy of the carpal tunnel by MRI and to establish diagnostic criteria for carpal tunnel syndrome (CTS).

Methods: 21 normal volunteers and 97 patients with CTS were examined (1.5 T, pulse sequences: SE 500/15 and SE 2280/15-90). The measurements presented in the results section were done at three levels: 1. distal radius, 2. pisiform bone (point of entry into the carpal tunnel), 3 . hamate bone (narrowest segment of carpal tunnel). 58 CTS patients underwent surgery.

Results: The median nerve could be delimited in all volunteers and patients. The following criteria for CTS were established after comparing the 97 patients with 21 normal subjects: a) swelling of the nerve at the level of the pisiform bone ( $\mathrm{p} \leq 0.005) ; b)$ increase in signal intensity of the nerve in the T2-weighted image $(p<0.05) ; c)$ palmar bowing of the flexor retinaculum $(p<0.001)$. In addition, early and intermediate stage of CTS on the one hand and advanced stage of CTS on the other hand could be differentiated on the basis of the signal intensity of the median nerve and increased swelling on the level of the distal radius.

Conclusions: Typical signs of CTS can be demonstrated by MRI. Additional lesions in the carpal tunnel, the primary cause of median nerve compression, are detectable by MRI. Fibrosis of the median nerve as a sign of advanced CTS can be differentiated from edema.

\section{$11.50 \mathrm{AM}$}

\section{Quantification of muscle composition in the elderly using magnetic} resonance imaging and spectroscopy

Sharmila Majumdar, Sarah J.Nelson, Daniel B. Vigneron, Alexander R. Margulis; San Francisco, CA

Aging causes a decline in physical capacity and is accompanied by a reduction in muscle size, alteration of the number of fast twitch muscle fibres and increased fatty infiltration into the muscle. We are applying Magnetic Resonance Imaging and Spectroscopy techniques to quantify these changes in an elderly population (aged 60-70) and comparing the results with similar data obtained from a younger population $(25-35)$. Ten individuals are being considered in each group. The parameters being studied are the differences in $\mathrm{T} 1$ and $\mathrm{T} 2$ relaxation times, the relative fat/water content of the muscle and the distribution of P-31 metabolites such as inorganic phosphate, phosphocreatine, phosphodiesters and ATP. We have observed an increase in the extent of fatty infiltration in muscle as a function of age, as well as differences in the spatial distribution of the fat within the muscle. To quantify these differences, histograms of the relaxation time distributions were extracted from regions of the anterior tibialis muscle, the gastrognemius muscle and sub-cutaneous fat. For the younger individuals, the histogram of muscle $\mathrm{T} 2$ values was narrow and separated from the sub-cutaneous fat; for the older population, fatty infiltration caused the range of muscle T2's to be much broader and to overlap with the fat values. Initial results with P-31 spectroscopic imaging also indicate that there are differences in the resting distribution of metabolites between the two populations. We are in the process of correlating the differences in MR parameters with muscle strength to see whether they can be used to infer loss of muscle function.

\section{$11.55 \mathrm{AM}$}

\section{Ultrasound tomography of soft tissues and venous vessels in secondary edema of the upper limbs} A. F. Tsyb, I. Kh. Mukhamedzhanov; Obninsk

The paper is concerned with ultrasound investigation that permits obtaining objective data on changes in the thickness, structure, borders and density of soft tissues in upper limb lymphedema. Moderate thickening of the skin and subcutaneous fat with slight consolidation of the latter was noted in patients with mild edema. The thickness of the group of muscles at the shoulder level was decreased in each $3 \mathrm{rd}$ patient. Thickening of the skin and subcutaneous fat more than 1.5 -fold was noted in edema of average severity. In severe edema the skin and subcutaneous tissue got thicker 2-2.5 times and acquired a dense regular spotty pattern. Echogeneity of the skin was reduced, and it often became merged with the subcutaneous fat. Cavities filled in with edematous fluid were found in the zone of maximum edema. Ultrasound phlebography made it possible to determine the state of major veins in upper limb edema and to abandon roentgenocontrast investigation of veins in a majority of cases. 


\section{Room L}

SS

\subsection{Temporal bone and orbit}

\section{$10.30 \mathrm{AM}$}

79 HR-CT of the temporal bone with multidimensional image reconstructions

F. Astinet, U. Keske, P. Uhrmeister, M. Langer, C. Zwicker, R. Felix; Berlin

Patients and methods: HR-CTs ( $1 \mathrm{~mm}$ slice thickness, $2 \mathrm{sec}$ cycle time) were performed with a unit having a $1024 \times 1024$ pixel display matrix in 100 patients with illness of the petrosa.

Results: The evaluation of the 2D secondary reconstruction showed that the use of the thinnest possible, continuous slices $(1 \mathrm{~mm})$ usually resulted in an image quality sufficient for diagnosis in 2D-CT. This is additionally combined with a substantial reduction in patient stress (no problems with positioning, no further radiation exposure) and a considerable saving in time. A 3D display of the base of the skull was performed in cases of extensive bony destruction to permit a clear visualisation of complex bony structures. Disadvantages of the 3D-CT include the deficient display of soft-tissue structures and the inferior spatial resolution as well. Furthermore there is no possibility of producing detailed internal views, i.e. of the ossicular chain, or cotour smoothing by means of a filtering algorithm. Threedimensional differentiation of a soft-tissue tumor or finer bone arrosions is still impossible.

Conclusions: It was demonstrated that the high spatial resolution with the resultant possibility of differentiating even fine anatomical structures offers extended diagnostic possibilities for clarifying illnesses of the auditory and vestibular apparatus. The primary or secondary reconstructed $2 \mathrm{D}-\mathrm{CT}$ slices continue to be the basis of diagnosis.

\section{$10.40 \mathrm{AM}$}

\section{Normal variations of the temporal bone: high-resolution CT appear- ances}

Noriaki Tomura, Kenji Hirata, Masaaki Shindo, Toshio Kato; Akita

Purpose: To study the CT appearances of various normal variations of the temporal bone, which are important as preoperative informations.

Methods: The subjects were 182 cases of which high-resolution CT were performed. Prospective TARGET reconstructions with continuous axial 1.5-mm-thick sections parallel to the Reid's base line were carried out. We investigated the incidences of 6 kinds of normal variations; 1 . incomplete bony covering of high-placed jugular bulb, 2. severe asymmetry of the jugular foramen, 3. anteriorly located sigmoid sinus, 4 . deep sinus tympani, 5. patulous internal auditory canal, 6 . large cochlear aqueduct. These were defined by literature or our criteria.

Results: The incidences of those variations were as follows: $1.3 .8 \%, 2.0 .8 \%, 3$. $3.3 \%, 4.7 .0 \%, 5.3 .3 \%$ and $6.9 .5 \%$.

Conclusions: Recognition of normal variations of the temporal bone was important at operation for the inner and middle ear, and the posterior fossa. This study indicated that high-resolution CT provided an important preoperative information of the temporal bone.

\section{$10.50 \mathrm{AM}$}

\section{Auditory canal atresia: a comparison between the findings at $\mathrm{CT}$ and at operation}

Hannibal Sökjer, Lars Ekvall; Linköping

Methods: The CT-findings were reviewed in all patients with atresia operated on during 1985-1990. Coronal CT was always done. No patient was younger than 5 years at $\mathrm{CT}$, thus avoiding sedation.

Results: CT-findings influencing the operative approach were: the pneumatisation of the temporal bone, the patency of the oval window and the location of the facial nerve.

In most patients middle ear reconstruction was done, while fenestration was done when reconstruction was not possible for anatomical reasons, e.g. malposition of the facial nerve. Fenestration did not lead to improved hearing unless the middle ear air reached at least one of the windows.

The facial nerve was reliably shown at CT. Its relationship to the oval window can only be judged in coronal projection.

Even severe narrowing of the oval window allowed reconstruction which was not possible when the window was definitly closed or when the facial nerve covered it.

Conclusions: CT should be done in coronal projection. Care should be taken to outline the facial nerve. In patients with severe underdevelopment of the middle ear any operation is meaningless. The appearances of the ossicles is of little importance.
$11.00 \mathrm{AM}$

82 Evaluation of temporal bone malformation in children with microtia on the basis of CT-scan and MR-image

A. Kukwa, J.Walecki, E. Grabska, K. Kizler, M. Pietniczka-Zaleska; Warszawa

Indications for surgical reconstruction of the external acustic meatus and middle ear in children with microtia require very accurate evaluation of the inner ear and middle ear structures. The presence of middle ear cleft and ossicles with the antrum and presence of the air cells in the mastoid process confirm that there constructive surgery may be successful. For this purpose CT-scan and MR-image of temporal bone in 30 children with microtia and external ear malformation was made. The children who entered the study were either not examined previously or with earlier made external acustic meatus reconstruction, but without full reconstruction of the middle ear structures due to severe malformation of the tympanic cavity. Reconstruction of MAE was made for better utilization of hearing aid. However due to substantial malformation of the middle ear, decision of ossicular chain reconstruction was delayed. Further CT-scan and/or MR-image will enable us to follow the progress of the ariation process in the middle ear cleft. CT-scan of the pyramide provides better localisation of the facial nerve canal and its route in and out of the tympanic cavity. Furthermore with the use of this method it is possible to establish the direction of the axis of the pyramides, the semi-circular canals plain and the cochlea, which provides better way of evaluation and prognosis for the subsequent management.

\subsection{AM}

83 Is C.T. scanner reliable for diagnosing the presence or absence of cholesteatoma (with reference to 210 operated cases)? F. Veillon, D. Charneau, P. Baur, M. Bintner, P. Bloch; Strasbourg

Object: The object of this study is to evaluate C.T. scanner performance in the positive diagnosis of cholesteatoma or non cholesteatomous hyperplasia.

Methods: 210 patients, referred for chronic otitis media, were studied by CT scan in the axial and coronal planes. We confronted the CT results with the surgical findings.

Results: The diagnostis of cholesteatoma rests on the association of an amputation of the scutum $(116 / 164$ cases $=71 \%)$, the presence of ball shaped soft tissue in the lateral epitympanic recess, the severity of ossicular destruction, and the appearance of the antrum. In non cholesteatomous hyperplasia, there is hardly ever any amputation of the scutum $(1 \mathrm{case} / 46=2 \%)$, soft tissue may or may not be present, with air-bubble spotting (24/46 cases $=$ $63 \%)$

Global diagnostic sensitivity of C.T. is $85 \%$, specificity is $93 \%$, positive predictive value is 0,98 and negative predictive value is 0,63 .

Conclusions: C.T. is diagnostic of cholesteatoma in $85 \%$ of cases. The remaining undiagnosed $15 \%$ should benefit from MRI with I.V. Gadolinium injection.

\subsection{AM}

84 Malignant external otitis: comparison of MRI and CT R. Brüning, J.Gülden, M. Naegele, A. Lienemann, B. Wöll, V. Kunze, M. Reiser; Bonn

Purpose: Malignant external otitis is a severe bacterial infection of the temporal bone and the surrounding soft tissues. The purpose of this study is to compare the potential of MRI to CT for diagnostic workup of malignant external otitis.

Methods: CT and MRI are performed at 14 patients with suspected otitis. MRI scans are obtained at a 1.5 Tesla superconducting unit (Philips), using a standard headcoil. Gd-DTPA is administered at the regular dose $(0.1 \mathrm{mmol} / \mathrm{kg})$. During and after injection additional to the routine sequences a set of very fast sequences are obtained in order to record the dynamic enhancement in tissue.

Results: High resolution CT proves to be superior for depiction of destruction of the cortical rim.

MRI is capable of multiplanar imaging without moving the patient. Contrast-enhanced MRI is highly sensitive for delineation of inflammation in the temporal bone.

Repeating sequences of short acquisition time, MRI becomes a dynamic study. The uptake-pattern after injection gives additional information regarding the vascularity of the process. Thus, MRI may become a sufficient tool for grading the acuteness of inflammation, and the response to therapy. 


\subsection{AM}

\section{Retinoblastoma: evaluation with $\mathrm{CT}$ and MR imaging}

$\bar{R}$. G. H. Tan, M. J. Hendriks, K. E. W. P. Tan, L. M. Ramos; Utrecht

To evaluate the effectiveness of CT and MR imaging in diagnosing and staging of retinoblastoma, the authors studied 8 patients in whom retinoblastoma was clinically suspected.

MR imaging was performed with a 0.5 Tesla MR imager. CT images, obtained with a 350 -Tomoscan, were made for comparison with the MR image. Surgical correlation was available.

Five out of eight patients were surgically proven retinoblastoma, three out of eight appeared to be Coats' disease.

MRI was not as specific as CT in diagnosing retinoblastoma due to its lack of sensitivity in detecting calcification.

MRI and CT were equally effective in staging the disease in all patients.

Due to its greater sensitivity in differentiating subretinal fluid from tumourmass, MRI proved very accurate in differentiating retinoblastoma from Coats' disease. In three cases we correctly diagnosed Coats' disease on the bases of MR findings alone.

MRI and CT are equally effective and complementary in confirming the diagnosis and determining the prognosis and work-up.

\section{$11.40 \mathrm{AM}$}

86 A comparative study of ultrasonography (US) and computed tomography (CT) in orbital diseases

Miklós Barta, Péter Rácz, Tamás Puskás, Ida Szépe, Judit Ferentzi, Györgyi Munkácsi; Szombathely

Purpose: How can be used a non-ophthalmological ultrasound instrument in diagnostics of orbital diseases?

Methods: US examinations were carried out with Picker LSC 7000 real-time instrument by a $5 \mathrm{MHz}$ linear transducer applying a Kitecko jel-cushion. The orbit was swept by US through closed eyelids. The ultrasonographical findings were verified by CT (Somatom DRH-2 Siemens) examinations, as well as by clinical course and histological findings of the disease.

Results: 140 US and CT examinations respectively were evaluated. The intraocular lesions - in size $\geq 2 \mathrm{~mm}$ - proved exactly visible by US from the dorsal surface of the lens to the back wall of the globe. The sensivity of US had got the same extent to the extraocular, intraconal orbital processes.

Conclusions: The ultrasound technique described was practically suitable to clarify the clinical problems being in connection with intraocular pathological processes. In case of some kind of extraocular tumors and endocrine orbitopathies the use of CT is not necessary. On the other hand CT examination is indispensable if the disease is extraconally located or it destroys the orbital wall.

\section{$11.50 \mathrm{AM}$}

\section{Orbital varix-diagnosis by color-coded Doppler sonography and Computed tomography}

S. Trattnig, M. Eilenberger, H. Schurawitzki, P. Hübsch, B. Schwaighofer; Vienna

Purpose: Determination whether color-coded Doppler sonography provides additional information in the diagnosis of orbital varices.

Methods and Materials: Three patients (27 a, m; $29 \mathrm{a}, \mathrm{f} ; 38 \mathrm{a}, \mathrm{f})$ who have been suffering from intermittent unilateral proptosis for years were examined. This symptom is caused by straining, coughing, positional increase of venous pressure in the head and neck region and Valsalva manoeuver. Examinations were performed by computed tomography (axial and coronal $2-4 \mathrm{~mm}$ sections, with additional injection of contrast media and zooming of the orbital region) and color-coded Doppler sonography (Acuson $128 \mathrm{~T}^{\mathrm{m}}$ ) with multiplanar sections in different positions and dynamic evaluation during Valsalva-manoeuver.

Results: In our patients computed tomography revealed a space-occupying orbital mass of soft-tissue density with moderate or marked enhancement following intravenous injection of contrast media. A specific diagnosis was impossible. By color-coded Doppler sonography the lesion became colorcoded in positions increasing the venous pressure. During Valsalva-manoeuver filling of this vascular space with flow toward the transducer, demonstrated in red and on relaxation flow away from the transducer depicted in blue with decrease of the lesion could be demonstrated.

Conclusions: Color-coded Doppler sonography with real-time imaging of flow and the possibility of dynamic evaluation and examination in different positions permits a definitive diagnosis of this vascular disorder without the need of confirmatory invasive techniques, such as orbital venography.

\section{Room M}

Ss

\subsection{Kidney imaging}

$10.30 \mathrm{AM}$

\section{Kidney imaging - introduction (Keynote Lecture)} Sven Dorph; Herlev

Since the early days of radiology, imaging of the kidneys has been one of the major challenges in medicine.

Kidney imaging has undergone a tremendous development during the past 40 years. Until then only conventional X-ray technique was available and the contrast media, that gradually became at hand, had so serious and frequent side effects that their use was limited to imperative cases. The introduction of the 3-iodinated water-soluble contrast media in the 50's suddenly changed the use of contrast media to a relatively safe procedure, which became even safer by the introduction of the nonionics. Along with the improvements on conventional X-ray techniques the past 20 years technical development has brought a large number of new imaging techniques using X-rays and other energy sources that have made it possible not only to present the kidneys in every macroscopic outer and inner detail but also to provide important information on their functional state.

This paper, introducing the Kidney Imaging Scientific Session, will give a short survey of the strengths and weaknesses of old and new imaging techniques for the study of the kidney in the context of various typical and clinically important diagnostic problems.

This includes: X-ray scout films, intravenous urography, direct pyelography, renal angiography, renoscintigraphy, renal ultrasonography, renal computed tomography and magnetic resonance imaging.

Examples of radical changes in diagnostic strategy caused by the introduction of newer modalities will be illustrated, as well as important residual indications for older, but meritious examinations such as urography and renal angiography.

It should be remembered, however, that most diagnoses are made using the joint contribution of 2 or more diagnostic modalities.

\section{$10.45 \mathrm{AM}$}

89 Renal angiomyolipoma: comparison of CT and MRI for detection and characterization

M. F. Bellin, S. Attias, F. Richard, E. Auberton, C. Radier, J. Grellet; Paris

Purpose: To compare the diagnostic usefulness of CT and MRI in the detection and characterization of renal angiomyolipoma.

Methods and Materials: Pre-operative CT and MRI studies were retrospectively reviewed in 12 patients with surgically proved angiomyolipoma of the kidney. SE T1 and T2-weighted images were performed in all cases; fat saturation images were available in 2 cases.

Results: There were multiple angiomyolipomas in 4 cases of tuberous sclerosis. The size of the tumors ranged from $1.5 \mathrm{~cm}$ to $13 \mathrm{~cm}$. CT revealed negative densities in 11 cases. MRI identified the presence of fat in 11 cases. One fibrous angiomyolipoma was missed by CT and MRI. A hemorrhagic component was present in 3 cases; it was depicted with MRI and CT in 2 cases. Fat presaturation was effective to distinguish fat from hemorrhage. MR multiplanar imaging allowed a clear delineation of the masses.

Conclusions: Spin-Echo MRI enables detection and characterization of renal angiomyolipoma equivalent to that of CT. Fat saturation is useful to separate the fatty and hemorrhagic components of the tumor.

\section{$10.55 \mathrm{AM}$}

$\underline{90} \mathrm{CT}$ and US findings of renal angiomyolipomas in the tuberous sclerosis

M. Y. Sadıkŏglu, M. Parlak, B. Adapınar, E. Tuncel; Bursa

Tuberous sclerosis (TS) is an inherited neurocutaneous disorder in which the characteristic renal lesion is the angiomyolipoma, present in $40 \%$ to $80 \%$ of patients with this disorder. In this study, kidneys of 14 patients with TS were evaluated by US and CT. Ultrasonographic findings were correlated with CT findings. In the eight of patients (57\%), multiple renal angiomyolipomas bilaterally were detected. The size of the lesions ranged from very small to large. CT is the best diagnostic method for evaluation of angiomyolipomas because it identifies fatty tissue. US provides a useful screening and follow-up method without radiation. 
91 Comparison of ultrasonography, computed tomography and magnetic resonance imaging of renal carcinoma extension into the renal vein and inferior vena cava

C. Roy, A. Meyer, G. Spittler, D. Jacqmin, P. Bourjat; Strasbourg

We compared these 3 methods with 12 patients. In 11 cases, ultrasonography made the diagnosis. With CT the diagnosis was made in 10 cases but the site of the upper pole of the thrombus was seen in only 6 cases. On MRI, diagnosis and localization was made in the 12 cases; but in one the thrombus wasn't well seen because of unadherent to the wall. Magnetic resonance imaging seems us slightly, more sensitive than ultrasonography.

11.08 AM

92 Venous extension of renal adenocarcinoma - effectiveness of gradient recalled echo MR imaging

M. F. Bellin, C. Radier, F. Richard, P.M. Lugagne, E. Auberton, J. C. Bousquet, C. Chatelain, J. Grellet; Paris

Purpose: To assess the ability of GRASS sequences (a limited flip angle MR technique) to detect venous extension of renal cell carcinoma.

Methods and Materials: 35 patients with proved renal adenocarcinoma were studied using MRI at $1.5 \mathrm{~T}$. GRASS sequences $(\mathrm{TR}=33 \mathrm{~ms}, \mathrm{TE}=13 \mathrm{~ms}$, flip angle $=30^{\circ}$ ), $\mathrm{T} 1$ - and $\mathrm{T} 2$-weighted spin-echo sequences were performed. MRI findings were reviewed and compared with $\mathrm{CT}$ and histologic findings in all patients.

Results: GRASS images depicted tumor thrombus in the main renal vein in $8 / 35$ patients and in the inferior vena cava in $4 / 35$ patients. There was one false negative finding for renal vein thrombus (sensitivity $=89 \%$, specificity $=$ $100 \%$ ). Patency of the inferior vena cava was correctly assessed in all cases. The upper pole of the tumor thrombus was clearly delineated in all cases.

Conclusions: GRASS technique is fast, highly sensitive to flow and does not require any contrast material injection to visualize renal vessels.

GRASS technique is an accurate imaging modality to detect venous extension of renal adenocarcinoma.

\section{$11.18 \mathrm{AM}$}

\section{Imaging of renal sarcomas}

L. Dalla-Palma, R.S. Pozzi-Mucelli, P. Pavlica, C. Zuiani; Trieste

Purpose: To evaluate the imaging findings of rare malignant tumors of the kidney.

Methods: 12 patients with renal sarcomas, including 5 leiomyosarcomas, 4 liposarcomas, 1 fibrosarcoma, 1 histiocytic fibrous sarcoma, 1 emangiopericytoma, and 22 patients with sarcomatoid renal cell carcinoma were retrospectively evaluated. Patients were examined using intravenous urography, ultrasonography, computed tomography and angiography.

Results: The most significant imaging findings for each modality and type of tumor are presented and discussed. CT and angiography are the best modalities to show the findings suggestive of renal sarcomas. These included: a) capsular, sinusal location of the mass; b) fatty density within the mass (suggestive of liposarcoma); c) hypovascularity with capsular vessels in angiography. The capsular location and vascularity, which are the most significant findings, are related to the origin from the connective tissue of the renal capsule of these masses.

Conclusions: Imaging modalities can show some characteristic features of renal sarcomas. However, the findings cannot be considered specific and therefore the prospective is still difficult basing on present imaging modalities.

\section{$11.28 \mathrm{AM}$}

\section{Accuracy of diagnostic imaging in nephroblastoma before preoper- ative chemotherapy}

K. Rieden, A. Weirich, J. Tröger, W. Semmler, R. Ludwig; Heidelberg

Preoperative chemotherapy for patients between 6 months and 16 years is a main part of the therapeutic management of Wilms' tumour according to the international study SIOP 9 GPO. Before treatment the diagnosis is made only by diagnostic imaging without biopsy. Therefore we wanted to verify the accuracy of diagnostic imaging.

From January 1989 to November 1990156 patients with known histology have been registered in Heidelberg - the study centre for the German group. $67 \%$ of these patients have been treated with preoperative chemotherapy, initially diagnosed by IVP, US, CT and MRI without preceeding biopsy. $92,4 \%$ of these preoperatively treated patients had a Wilms' tumour or one of its variants. 5 patients had a different malignant tumour. 3 cases, i.e. $2,3 \%$ of the preoperatively treated, or $1,9 \%$ of all registered patients, had benign tumours of the kidney. In consequence of atypical diagnostic findings some patients were excluded from the trial before preoperative chemotherapy. They showed signs of extrarenal tumours or benign lesions of the kidney like neuroblastoma, renal bleeding, angiomyolipoma and reflux nephropathia. The morphology of Wilms' tumours revealed a characteristic inhomogeneity increasing after injection of contrast medium. Intratumoural bleeding and cystic areas occurred frequently. Calcifications were extremely rare. The pretherapeutic diagnostic imaging had a sufficient predictive value to start preoperative chemotherapy without diagnostic biopsy. A difficulty for diagnostic imaging remains to differentiate the very rare benign cystic nephroma from malignant nephroblastoma.

\subsection{AM}

95 Nuclear magnetic resonance tomography in staging of renal tumors $\bar{W}$. Fiegler, M. Laniado, W. Kornmesser, C. Claussen; Berlin

NMR was performed in 110 patients, and the results were compared with surgical findings and computed tomographic studies. Renal tumors were staged using the system proposed by Robsen, and invasion of the renal pelvis by the tumor was also considered. The accuracy of NMR in staging of renal tumors was $79 \% .6$ tumors were overstaged, while 10 tumors were understaged. Sensitivity was $75 \%$ and specificity $100 \%$ in demonstration of thrombus within the renal vein or the inferior vena cava. In evaluation of infiltration of the renal capsule NMR attained a sensitivity of $91.3 \%$ and specificity of $86 \%$. Tumors of the renal pelvis were not demonstrable as filling defects in magnetic resonance images, but infiltration of renal parenchyma by these tumors was demonstrated by NMR. Both computed tomography and NMR imaging failed to demonstrate invasion of small renal veins by the tumor in 3 patients. NMR demonstrate excellent tumor-invasion of blood vessels, but the segment of the renal vein near the hilum is poorly delineated. NMR requires oral administration of a contrast medium for differentiation between lymph nodes and the gut.

\section{$11.43 \mathrm{AM}$}

\section{Computed tomographic analysis of renal calculi}

J.A. Sanchez-Corral, F. J. Burgos, S. Avila, J. C. Saez, E. Garcia; Madrid

The knowledge of stone composition is an important factor in stone fragmentation.

One hundred and twenty calculi of known composition have been analyzed in vitro by C. T. Diameter (D), area (A), mean global density (MGD), central density (CD), peripheric density (PD), maximum density (MXD) and minimum density (MND) have been measured. Standarddeviation (S) of MGD, CD, and PD have been also determined as lithiasic homogeneity index. With MGD is possible to distinguish calculi of uric acid (AU) and phosphate ammonicum-magnesicum (FAM) from a group of calculi composed by oxalate monohidrate $(\mathrm{OXMH})$, oxalate dihydrate $(\mathrm{OXDH})$, carboapatite $(\mathrm{PC})$ and oxalate-phosphate calcium.

Differentiation between these 4 compositions is possible with the help of $C D$, MND, S CD, and S MGD parameters (p inferior to $0.01 ; 95 \%$ of security), and CT morphological pattern.

\section{$11.48 \mathrm{AM}$}

97 The sensitivity and specificity of single dose Enalapril scintigraphy in the evaluation of renovascular hypertension

Ö. Kapucu, T. Arinsoy, T. Aras, B. Erbas, E. Varoğlu, F. Balkancı, C. Turgan, G. Erbengi, S. Cağlar, C. Bekdik; Ankara

The aim of this study was to compare the results of renal scintigraphy with administration of Tc-99m-DTPA (before and after $20 \mathrm{mg}$ of oral enalapril) with the results of the digital substraction angiography to evaluate the sensitivity and specificity of renal scintigraphy in the diagnosis of renovascular hypertension. The results of 15 consecutive patients $(6$ Female, 9 Male, mean age: $35.9 \pm 7.8$ ) are in the Table.

Left ${ }^{\text {Basaline }}$ Right $\quad$ Left $\quad$ Right

\begin{tabular}{lrlrrrrr}
\hline $\mathrm{GFR}$ & $50.16 \pm 23.6$ & $52.6 \pm 22.9$ & $51.99 \pm 27.8$ & $50.77 \pm 30.5 \mathrm{ml} / \mathrm{min}$ \\
$\mathrm{T}_{\max }$ & $4.83 \pm$ & \pm .1 & $5.57 \pm 2.9$ & $5.00 \pm 4.6$ & $5.56 \pm 3.6 \mathrm{Min}$. \\
$\mathrm{T} 1 / 2$ & $8.28 \pm 3.5$ & $9.06 \pm 3.6$ & $8.74 \pm 4.4$ & $8.19 \pm 3.5 \mathrm{Min}$.
\end{tabular}

The sensitivity of renal scintigraphy in renovascular hypertension was $75 \%$, the specificity of our test was $80 \%$. Renal scintigraphy study failed to detect renal artery stenosis in patients with atrophic kidney and severe parenchymal diseases. Renal scintigraphy with and without enalapril should be used as a screening test before digital subtraction angiography. 


\section{$11.53 \mathrm{AM}$}

98 Renal scintigraphic Captopril test in multiple renal arteries F. Barbano; E. Perrone, S. Modoni, G.Petracca Ciavarella, F. Florio, S. Petronelli, G. Valle, V. Frusciante; San Giovanni Rotondo

The aim of this study is to evaluate the role of deconvolution analysis and MTT (mean transit time) parametric imaging in the processing of renal sequential angioscintigraphy in order to detect the presence and to assess the physiological meaning of multiple arteries. The transfer function has been obtained using Diffy's matrix algorithm; however the initial height $(\mathrm{IH})$ has been calculated according to Rutland method.

In the last two years 234 patients have been submitted to renal scintigraphic captopril test (RSCT) in our department. $25 / 234$ pts have shown high values of MTT calculated from a curve of the whole renal parenchima and segmental abnormalities of MTT image: $6.0 \pm 1.2 \mathrm{~min}(\mathrm{~m} \pm \mathrm{sd}$ ). In 15 controls MTT was $4.0 \pm 0.8 \mathrm{~min} ;$ a statistically significant difference was found comparing controls with our 25 pts by student $T$ test for unpaired data $(p<0.0005)$. In all cases intravenous or intraarterious DSA has confirmed the exsistence of multiple renal arteries. In $22 / 25$ pts DSA showed significant renal artery stenosis in one or more of multiple renal arteries according to standard arteriographic parameters. However in 3 pts renal artery that supply the district affected in MTT imaging was quite normal.

We conclude that RSCT associated to deconvolution analysis and to MTT image is a sensitive tool to detect segmental functional abnormalities and that a hemodynamically significant stenosis may occur also without radiologic classical signs.

\section{Room N}

\section{Ss} 10.30 AM

\section{Mediastinal masses in lymphoma: posttherapeutic aspects (Keynote Lecture) \\ Jacques Frija; Paris}

The post-treatment masses are a crucial therapeutic problem during the course of lymphomas. We have observed a post treatment residual mass of more than $2 \mathrm{~cm}$ in diameter in $13.3 \%$ of Hodgkin's diseases and $14.7 \%$ of non Hodgkin lymphomas. Generally these masses are detected either by a chest X-ray when they are large or a CT examination when they are smaller. The chief question is: are these masses malignant or not? Neither standard X-ray nor CT can respond to the question. MR is very useful because it can sometimes identify a fibrotic mass. We have studied 57 post-treatment residual masses with MR. Of these 57 masses, 34 had an intermediate signal with T1- and T2-weighted sequences: 33 out $34(97 \%)$ were fibrotic. Twenty-three masses had an intermediate signal on $\mathrm{T} 1$-weighted sequences and an hypersignal on T2-weighted sequences: 9 were malignant and 14 were not malignant. We used 44 times an injection of Gadolinium and in 14 cases we observed a fixation of Gadolinium: 7 masses were malignant and 7 were not malignant. We studied Gallium scintigraphy of 18 masses ( 16 in the mediastinum and 2 in the abdomen). Of these 18 masses, 15 did not fix Gallium and were not malignant, 2 fixed Gallium and were malignant and 1 had a very small and diffuse fixation and was not malignant. We conclude of this work that there is a high probability $(97 \%)$ of a post-treatment mass to be non-malignant when it has an intermediate signal on T1-and T2-weighted sequences and when it did not fix Gallium. Gadolinium is not useful. The other situations are ambiguous but when a mass has an hypersignal on T2-weighted sequence and it fix Gallium it is highly suspect to be malignant.

\section{$10.45 \mathrm{AM}$}

100 MRI with Gadolinium of post-therapeutic residual masses during lymphomas

J. Frija, C. Bris, C. Yana, F. Cyna-Gorse, P. Ollier, Y. Miaux, J. Tredaniel, P. Brice, J. D. Rain, M. Laval-Jeantet; Paris

Aim: To test MRI with and without intravenous Dota-Gadolinium (Gd), and Gallium scintigraphy (Ga) for the determination of malignant aspect (MA) or not (NMA) of post-therapeutic residual masses (PTRM) during lymphomas. Methods: Prospective study about 57 PTRM of 44 patients in complete remission and 13 patients with relapse. All patients had T1-weighted (T1SE) and T2-weighted spin-echo (T2SE) sequences. 44 patients had a third T1SE after Gd. The signal of the PTRM was compared to the normal muscles signal. 17 patients had Ga. Histological proof was obtained for six patients. The median survey was 18 months for 33 patients with Hodgkin disease and 44 months with non-Hodgkin lymphoma.

Results: When PTRM was isointense to the muscles during T1SE and T2SE it was NMA in 33 out of 34 cases $(97 \%)$. When PTRM was isointense to the muscles during T1SE and hyperintense during T2SE it was MA 9/23 (39\%) and
NMA 14/23 (61\%). After Gd, the PTRM was isointense in 30 out of 44 cases: $28 / 30$ cases were NMA. After Gd the PTRM was hyperintense in 14 out of 44 cases: in $7 / 14$ it was either MA or NMA. Ga was negative in $15 / 17$ cases when the PTRM were NMA, and 2/17 cases when the PTRM were MA.

Conclusions: When a PTRM is isointense during T1SE and T2SE it is NMA in $97 \%$ of the cases. When PTRM is hyperintense in T2SE MRI cannot be conclusive. Gd does not alter the results. Ga seems to be very precise.

\section{$10.55 \mathrm{AM}$}

\section{High resolution CT (HRCT) for detecting lung tissue damage after radiotherapy \\ B. Allgayer, O. Wittmann, A. Heuck, K. Mühlbauer; München}

Purpose: To compare the value of high-resolution CT (HRCT) slices $(1 \mathrm{~mm})$ with conventional CT slices $(10 \mathrm{~mm})$ in patients with breast cancer after radiotherapy,

Methods and Materials: 50 patients with breast cancer were investigated with HRCT and conventional CT before, 6 weeks, 4 months and 6 months after radiotherapy.

Results: Pathologic findings were found with HRCT in $76 \%$ of pat. and with conventional CT in $64 \%$ of pat. after 6 weeks. After 4 months and 6 months no difference between HRCT and conventional CT was found. HRCT offers additional information on discrete pulmonal reactions and pulmonal features of pneumonitis.

Conclusions: HRCT is more sensitive than conventional CT to assess early radiation induced pneumonitis.

\section{$11.05 \mathrm{AM}$}

102 Postmediastinoscopy changes in chest CT F. Saéz, J.M. Peña, E. Astigarraga, E. Canalis, O. Rodríguez, B. Múgica; Bilbao

Chest CT pre- and postmediastinoscopy was performed in 6 uncomplicated patients, to evaluate if normal postmediastinoscopy changes could simulate mediastinitis or another iatrogenic pathology.

Postmediastinoscopy chest CT was practiced one day and five days after the procedure.

CT was evaluated for the presence of fluid collections, abnormal air and replacement of the mediastinal fat by soft tissue density.

Only small air bubbles but no fluid collections were observed, as well as some replacement of the normal mediastinal fat.

We conclude that normal postmediastinoscopy CT changes have to be considered when evaluating patients with suspected iatrogenic mediastinitis.

\section{$11.08 \mathrm{AM}$}

\section{CT findings in lung transplantation}

P. Loubeyre, D. Revel, J. F. Mornex, T. Kikano, M. Amiel, F. Pinet; Lyon

This study was designed to define the CT findings during rejection, infection and miscellaneous complications (lymphoma, recurrence of the primary lung disease).

Methods: 135 CT examinations serially performed in 15 patients have been studied by two observers. The presence of various findings was assessed: pleural effusion, alveolar opacities, consolidations, micromodules, ground glass aspect, bronchiolectasis and correlated with the pathological analysis of transbronchial lung biopsy specimen $(n=41)$ and the clinical follow-up with and without acute lung rejection treatment.

Results: Alveolar opacities and consolidation in the posterior part of the lower lobes were observed in almost all the cases during first two weeks after surgery. The persistance of abnormalities in the upper lobes and the recurrence of abnormalities within lung parenchyma usually suggested acute as chronic rejection and/or infection. Although, ground glass appearance was always observed during the early stage of acute rejection $(n=4)$, nodular opacities and bronchiolectasis was mostly encountered during chronic lung rejection $(n=5)$. Conclusions: From this series of patient, CT appears as an aid to the diagnosis of various complications in lung transplantation. However, the definite differential diagnosis between rejection and infection seems not to be possible using $\mathrm{CT}$ alone.

\section{$11.18 \mathrm{AM}$}

\section{Interest of ultra fast CT (UFCT) for pulmonary follow up in heart} lung transplantation (HLT)

A. Hernigou, M. Marotel, J. P. Couetil, R. Guillemain, M. H. Bihet, C. Amrein, M. C. Plainfosse; Paris

Purpose: Results of UFCT were compared with those of respiratory function tests and transbronchial biopsy (TBB) in the pulmonary follow up of HLT. Methods and Materials:- Ten HLT patients were analysed through a follow up period of $1-12$ months and all lesions confirmed by TBB or positive response 
to therapy.

- 43 high resolution examinations by UFCT were performed (slice thickness $3 \mathrm{~mm}$ - exposure time/slice: $300 \mathrm{msec}$ ).

Results: - Acute rejection occured 12 times with reticular micronodular interstitial infiltration and consolidations. TBB was negative in three and respiratory functions tests were unreliable during the first month after HLT.

- Obliterative bronchiolitis occured in 2 cases with patchy ground glass alveolitis, emphysema and bronchectasia. This pattern appeared while respiratory function tests impaired but TBB changes were delayed.

- Evolutive bronchectasia appeared in 6 other patients and intercurren complications occured in three patients (infection, pulmonary dissection, and a nodular lymphoma).

Conclusions:- UFCT reliably contributes to the follow up of HLT. Better than chest radiographs UFCT shows signs and extension of rejection and guides biopsies.

- It allows repetitive and comparative examinations even with impaired pulmonary function or ventilatory assistance when respiratory function tests are unfeasible.

- It studies the whole lung better than TBB.

\subsection{AM}

\section{Contribution of the radiologic examination in spontaneous rupture of esophagus}

B. Bui, G. Schmutz, G. Massard, J.M. Wihlm, M. Y. Jeung, D. Regent; Sherbrooke/CDN

Purpose: To determine the contribution of the radiological imaging in diagnosis of spontaneous rupture of esophagus.

Methods: We retrospectively reviewed 17 cases of Boerhaave's syndrome seen over a 10 years period (May 1980-July 1990). Each patient underwent chest radiograph and esophagograms. A CT scan was performed in 6 cases.

Results: Chest radiographies were positive in 15 cases $(88.2 \%)$ with pneumomediastinum ( 7 cases), subcutaneous emphysema ( 3 cases), pleural effusion (11 cases) and hydropneumothorax (3 cases). Chest radiographies were considered as normal in 2 cases. Esophagograms were positive in 13 cases $(76 \%)$. In 4 cases, extravasation of contrast from esophagus was not visualized but esophagus presented large ulcerations. In one case, no significant abnormalities of the esophagus were seen. CT scans were always positive: pleural effusion ( 2 cases), hydropneumothorax (4 cases), subcutaneous emphysema (1 case) and pneumomediastinum ( 3 cases). Clinical presentation was atypical in 7 cases $(41 \%)$ and radiological examinations were helpful to affirm the diagnosis: Chest radiography (3 cases), esophagogram (2 cases) and CT scans (2 cases).

Conclusions: The diagnosis of Boerhaave's syndrom is mainly clinical but radiologic examinations may be helpful in atypical clinical presentations.

\subsection{AM}

106 The value of supine chest radiography in the diagnosis and quantification of pleural effusions. Comparison with sonography

K. Eibenberger, W. Dock, V. Metz, F. Grabenwöger; Wien

Purpose: The purpose of this prospective study was to verify of the value of the supine chest radiograph with regard to the recognition and quantification of pleural effusions.

Methods: The findings of supine chest radiographs were compared to those of chest sonography in a total of 320 pleural cavities. A moderate or large effusion was diagnosed on supine chest radiographs if one of the following radiographic signs was present: increased homogeneous density of the hemithorax, loss of the hemidiaphragm silhouette, meniscus sign, or apical capping. A small effusion was diagnosed if blunting of the costophrenic angle was the only radiographic sign present. Sonographically a moderate to large pleural effusion was diagnosed if the distance between the parietal and visceral pleura was more than $20 \mathrm{~mm}$; a small effusion if the maximal distance was between 5 and $20 \mathrm{~mm}$.

Results: The correct identification and quantification of a pleural effusion was possible by supine chest radiograph alone in only $55 \%$ of cases (176/320). In $20 \%$ supine chest X-ray was falsely negative and in $15 \%$ falsely positive. In $10 \%$ an existing effusion was in fact recognized on chest X-ray; however, the amount of effusion was falsely estimated.

Conclusions: Supine chest radiography is only a moderately sensitive and specific method for detecting pleural effusions, and often fails to estimate the amount of fluid.

\subsection{AM}

\section{$107 \mathrm{CT}$ and MR evaluation of benign pleural fibroma}

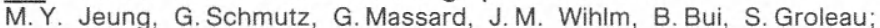
Sherbrooke/CDN

Purpose: To determine the contribution of CT and MR imaging in the diagnosis and evaluation of benign pleural fibroma
Methods: During 5 years (November 1985-October 1990) we performed CT scans and MR imaging in 8 patients ( 4 women and 4 men, age range: 49-73 years - mean 51) with proven pathologic benign pleural fibroma. In all patients MRI were obtained with a superconducting magnet operating at $0.50 \mathrm{~T}$ using the spin echo technique (T1- weighted and T2-weighted sequences).

Results: The size of the tumor exceeds at least one dimension $3 \mathrm{~cm}$. (size range $3-13 \mathrm{~cm}$, mean $7 \mathrm{~cm}$ ). On CT scans tumors appeared uniform, well defined with low or iso densities. After IV contrast material no enhancement was noticed. On MR images, tumors had a heterogenous appearance. The main tumor was of low or iso intensity on T1-weighted images and of high intensity on T2-weighted images with central cleftlike areas of low intensity.

Conclusions: MR imaging was equal to CT scans in delineating the extension of the tumor and in evaluation the connections with mediastinal structures However, MR imaging was superior to CT scans in characterizing the internal architecture and in determining the connections with pleural and with diaphragm.

\section{Room 0}

\section{SS}

\section{$10.30 \mathrm{AM}$}

\subsection{Mammography and sonography}

108 Characterization of clustered microcalcifications in mammograms with a new image analysis system: results in 241 patients

R. Gilles, F. Bouvet-Lefevre, M. Attal, H. Benali, G. Contesso, J. Masselot, E. Kahn; Villejuif

Introduction: Breast cancers without clinical manifestation are most often associated with isolated clustered microcalcifications in mammograms. Unfortunately this highly sensitive sign has a poor specificity. We developed an image analysing system to characterize benign isolated clustered microcalcifications in the breast to avoid unnecessary biopsies.

Methods and Materials: All the women who underwent a biopsy for isolated clustered microcalcifications at the Institut Gustave Roussy from January 1987 to March 1990 were reviewed. We recorded clinical, histological data and two pre-operative mammograms. We checked presence of microcalcifications within the biopsies. Pre-operative mammograms were digitized (matrix $512 \times 512,256$ grey level). We developed an image analysing system on a SUN $3 / 160 \mathrm{c}$ computer to quantify the number, the density (number per unit area), the optical density of microcalcifications, their morphology (compactness) and spatial distribution (moments of inertia, mean distance, HU moments). Statistical analysis was performed using a classification and regression tree on the whole set of women and cross-validation quantified the corresponding error rate.

Results: 241 women (163 benign, 78 malignant) were included in this retrospective study. Seven groups contained only benign cluster ( 70 patients). The corresponding error rate was $12 \%$

Conclusions: Characterization of benign microcalcification is possible. However the error rate doesn't permit routina use by now.

\section{$10.40 \mathrm{AM}$}

\section{Measurement of the diameter of malignant breast tumors} W. Gordenne, J. M. Lemaire; Liège

Purpose: The appreciation of the T of the TNM classification is up to now a clinical one - It would be more precise to adopt the X-rays or the ultrasound measurement.

Methods: One hundred cases of breast cancers are measured by clinical appreciation and are compared to mammographic and ultrasound diameters. Each measure is compared to the anatomical great diameter of the tumor.

Results: When tumors are of a nodular type, the discrepancy between the different measurements is not very important. Is is not the same when scirrhous tumors are concerned. Generally speaking, the clinical diameter of the tumor is more important than the mammographic and moreover the echographic one. Conclusions: Nowadays, when TNM classification is concerned, it would be more precise to adopt the tumor measurement by an imaging technique.

\section{$10.50 \mathrm{AM}$}

\section{Rare breast cancer}

\section{J.Sabaté, A. Lazo, A. Jiménez, A. Lozano: Sevilla}

The majority of the work about breast is based on study of standard cancer, without specifying the different histopathologic subtypes. For this reason we propose to study the radiological manifestations with the object of seeing if there is a clear correlation between the mammographic aspect and the anatomopathologic form.

We revised 1.000 cases of breast cancer, with anatomopathologic correlation, in the Oncologic Hospital of Sevilla (Spain), between 1981 and 1987.

We found 4 carcinomas ductals in situ, 18 lobulars infiltrating, 3 lobulars in 
situ, 21 medulars, 15 comedocercinomas, 11 colloids, 10 papillars, 8 intracystics, 3 apocrins, 2 carcinomas with cells of a ring seal, 1 mixture, 1 adenoid cystic and 1 indeterminite.

\section{$11.00 \mathrm{AM}$}

\section{Radiology of the male breast}

W. Buchberger, T. Penz, A. Furtschegger, A. Oberhauser; Innsbruck

a) Mammographic evaluation of the male breast can be difficult because of the variety of mammographic patterns in gynecomastia and its similarity with benign masses, inflammatory disease and carcinoma. The purpose of this study is to describe the mammographic and sonographic appearance of various conditions in male breast enlargement and to evaluate sonography as an additional diagnostic tool.

b) Mammograms and sonograms of 62 men with breast enlargement were reviewed. In 20 cases of gynecomastia a correlation of mammographic and sonographic patterns and pathohistologic features was undertaken.

c) Gynecomastias of up to 6 month's duration were histologically of the florid type and showed a nodular or nodular/dendritic mammographic pattern; sonography usually demonstrated a focal hypoechoic area. Gynecomastias of longer duration representing the fibrous type showed mammographically a dendritic or triangular pattern and sonographically hyperreflexive breast parenchym. Typical signs of malignancy were demonstrable in all 3 carcinomas, but also in 2 cases of mastitis.

d) Mammography and sonography are able to differentiate reliably true gynecomastia from pseudogynecomastia. Mammographic and sonographic patterns of gynecomastia are dependant on its histological type and duration. Gynecomastia and especially inflammatory lesions may not always be readily differentiated from carcinoma. Sonography can be helpful by demonstrating hyperreflexive glandular tissue, whereas carcinomas are usually hypoechoic.

\subsection{AM}

112 Rare sonographic finding: intraductal solitary papilloma (I.S.P.) of the breast. Report of twelve cases

A. Cilotti, P. Bagnolesi, R. Lencioni, D. Caramella, C. Bartolozzi; Pisa

Purpose: define the US characteristics of the rare I.S.P. and discuss the differential diagnosis with similar focal lesions of the breast.

Methods: Twelve I.S.P. were found among 31 cases of sero-haematic discharging breasts, all of them submitted to US and cytology. Four patients underwent mammography, 3 fine needle biopsy, 1 ductography. Surgery was performed in all patients.

Results: Three types of lesions were observed: a) solid hypoechoic nodule with peripheral anechoic areas ( 7 cases); b) periareolar overdistended duct, filled with dense material ( 1 case); c) papillary mass appreciable within a cystic cavity, corresponding to papillary cystoadenoma (4 cases). In 8 cases where cytology resulted positive, surgery was performed. In 4 patients with negative result, mammography, ductography and FNB allowed the proper diagnosis to be reached.

Conclusions: Sonography combined with the cytological samples proves itself able to gain the right diagnosis in 8 out of 12 patients $(66 \%)$. This is noteworthy considering the low reliability of mammography in young patients and the scarce tolerance of ductography in general. The 3 above-mentioned sonographic types seem to circumscribe the differential diagnosis and, consequently, the following diagnostic route.

\section{$11.20 \mathrm{AM}$}

\section{Mammographic and sonographic appearance of cystosarcoma phylloides}

Ph. Hendrickx; Hannover

Cystosarcoma phylloides is characterized by frequent local recurrences, by questionable dignity and by the often large extent. This very rare tumour is mentioned in the literature mostly as a case report.

During the years 1974-1989 a group of 15 cases could be collected. Starting from the different pathological and anatomical features, the typical mammographic and sonographic criteria are described.

The outline of the tumour is smooth and often lobulated. The solid component has echo levels lower than surrounding breast tissue and mostly is homogeneous. However, cystic parts are possible. Endotumoural calcifications are very rare. In all cases a zone of dorsal acustic enhancement was found.

All possible differential diagnoses are demonstrated.

In very large breast tumours with slow and intermittent episodic growth the tentative diagnosis Cystosarcoma phylloides should more frequently be considered.
11.30 AM

114 Benign mammographic and sonographic changes following breast conserving therapy of mammary carcinoma

R. Kindinger, J. Teubner, P. Diezler, M. Georgi; Tübingen, Mannheim

Mammography and sonography were evaluated for their contribution to the follow-up of typical benign patterns after breast conserving therapy. These patterns and their development were described and measured out by a detailed catalogue. Since July 198561 patients with breast carcinoma and following breast conserving therapy were examined regularly and included into this study. 2928 sonographic documentations made during standardized real-time sonography and 784 mammograms were evaluated. The mean observation time was 44 months.

Typical mammographic patterns are: mammary and areolar skinthickening, increase of mammary transparence, increase of interstitial alterations in subcutaneous fat tissue and in the glandular body. Typical sonographic patterns are: mammary skinthickening (during radiation therapy in the opposite breast too), increase of echogenity in fatty tissue, decrease of echogenity in glandular tissue, worsened demarkation of the glandular body and the submammarian structures.

Cooper's ligaments seemed to be not as typically alterated as the other findings. All alterations are postoperatively visible, but get enforced by the following radiation therapy. Within 12 months these alterations have their maxima and up to 3.5 years they diminish or disappear totally.

In conclusion mammography and sonography are supplementary methods characterizing peri- and postherapeutical alterations.

\section{$11.40 \mathrm{AM}$}

115 Is sonography of axillary lymph nodes a completion of mammographic examination of the breast?

Th. Heuchemer, J. M. Friedrich, S. Vogeser; UIm

In our department we practice approximately 2200 mammographic examinations every year. In a prospective study we have tried to find out if there is more information about axillary lymph nodes when sonography is used as a additional part of the routine examination of the breast.

In 69 patients with breast cancer detected with mammography from $9 / 89$ until $12 / 90$ we got more information about axillary lymph nodes by sonography of the axilla than by palpation. Using high-resolution sonography of axillary lymph nodes we found a sensitivity of $76 \%$ and a specificity of $93 \%$ for detecting axillary lymph node metastases in patients with breast cancer, compared with $69 \%$ sensitivity and $60 \%$ specificity for palpation. As a further result, palpable enlarged lymph nodes are not always visible in sonography if they are altered by chronic inflammation or by fibrosis.

We consider axillary lymph node sonography to be a useful completion of the routine triple diagnosis of the breast, if breast cancer is suspected or discovered or if enlarged axillary lymph nodes are palpable.

\section{$11.50 \mathrm{AM}$}

116 Neovascularisation in breast carcinoma - assessment by color Doppler flow mapping

Stefan Delorme, Hans-W. Anton, Michael V. Knopp, Hans-Ulrich Kauczor, Ingeborg Junkermann, Dietrich v. Fournier, Gerhardt van Kaick; Heidelberg

Purpose: To evaluate the use of a semiquantitative method to assess the degree of vascularity by color Doppler flow mapping.

Methods: 30 patients with clinically and radiologically highly suspicious breast nodules were examined by B-mode ultrasonography and color flow mapping. Objective analysis using the number of nutritive vessels, flow velocities and pulsatility indices was performed as well as subjective analysis regarding degree and reticularity of intratumoral vascular formations.

Results: All nodules were histologically proven to be invasive carcinomas. Nutritive vessels were seen in $85 \%$. In $70 \%$, intratumoral vessels could be demonstrated, ranging from isolated but reproducible color speckles over contiguous or branching small vessels to higher grade reticular formations. The degree of vascularity correlated with the tumor size, although huge nodules with poor vascularity as well as small tumors with multiple vessels were observed.

Conclusions: With respect to vascularity, mamma carcinomas seem to represent a heterogenous group. Color Doppler flow mapping introduces a method for a semiquantitative assessment of the degree of neovascular formations and might thereby allow identification of patients at high risk for distant metastases. 
117 Indices of conspicuity in mammographic diagnosis

E. James Potchen, Norman Sadowsky, Arlene E. Sierra; East Lansing, MI

The occurrence of breast cancer found on a subsequent screening mammogram provides an opportunity to assess the performance of observers in breast cancer detection. Some breast cancers are clearly missed on the initial mammogram, whereas others are obscured by overlying structural detail of normal breast tissue. The distinction between an observer error and a masked lesion is important in ascertaining responsibility for "missing the lesion". We have used the conspicuity factors described by Kundel and Revesz (AJR, June 1976) to access chest $x$-ray interpretation and applied them to the detection of early non palpable breast cancer. This provides us with the opportunity to distinguish those cancers that were missed by the error in observation from those which are not detected because of the nature of the disease in the woman's breast. Breast density, background complexity, signal to noise, and image mottle are relevant in assigning responsibility for the detection of non palpable breast cancer. These studies have major implications in evaluating physician performance, quality assurance, and medical legal liability.

\section{Room R}

SS

\subsection{Importance of diagnostic imaging in radiotherapy I} $10.30 \mathrm{AM}$

\section{Importance of diagnostic imaging in head and neck tumors (Keynote Lecture)}

H. Frommhold; Freiburg

The definition of the target volume for head and neck tumors of F. Gauwerky got a new dimension with new imaging methods: With the introduction of ultrasonography and computerised CT scanners and and Magnetic Resonance Tomography (MRT), with the capability of threedimensional visualisation of the tumor together with the surrounding areas of possible tumor spread new techniques were available in diagnostic imaging.

$60 \%$ of all patients with tumors of the head and neck and the maxillo-facial region can be cured today. But, radical surgical intervention does in many cases not go along with the aim of good cosmetic and functional results. Apart from small tumors (as for example isolated carcinomas of the larynx [vocal cord, T1 NO MO] and the early tumors of the nasopharynx) radiotherapy is not the method of choice (Sack, 1990) since structures with tumor invasion and areas of risk are located extremely close for head and neck tumors. For certain tumors after thorough evaluation of advantage versus risk, radiotherapy will be used primarily and/or together with surgery (Hünig, Wey 1989). The capability of ionising radiation can only be completely exhausted, when the target volume is defined three-dimensionally. The calculated dose distribution of a computerised treatment planning system should be checked using dose-volume-histograms.

For imaging in the head and neck area apart from x-ray films and conventional tomographic $\mathrm{x}$-ray films (as equivalent to simulation and direct field control), high resolution computed tomography (HRCT) and MRT are clinically well established diagnostic methods which lead directly to a threedimensional irradiation planning. Although both methods have often been used supplementing each other, only recently their diagnostic values and the corresponding therapeutic consequences have been compared (van Waes et al. 1991, Knauf and Thiel 1991). These publications show the increasing importance of the MRT for tumor diagnosis, for the treatment and the follow-up of tumors of the head and neck and the maxillo-facial region (approx. $90 \%$ correct results of MRT - with the exception of central nervous system, vertebral column, intervertebral spaces and ligaments).

It might be expected that MRT will take the place of the computed tomography since besides topographic localisation of the tumor, differentiation of benign and malignant tissue will be possible (high resolution MRI, spectroscopy with strong magnetic fields). Only if the base of the skull is involved (e.g. tumor invasion through the foramina or osseous arrosion) both methods should be applied.

Own results together with the established diagnosis and the resulting radiooncological therapy decisions (HPRT, afterloading, IORT, radiochemotherapy, hyperthermia, etc.) will be presented and discussed.

\subsection{AM}

\section{Quantitative imaging for radiotherapy in thyroid disease (Keynote} Lecture)

V. Ralph McCready, Mary O'Connell, Maggie Flower, Clive Harmer; Sutton, Surrey

The thyroid gland is still the only organ where systemic radiotherapy can be achieved with a high degree of success. It is the ideal mode of therapy requiring only a drink and sometimes isolation for radiation protection reasons.
However in both benign and malignant disease of the thyroid gland there are unexplained failures in treatment. We have therefore investigated in detail the dosimetry of and response to radioiodine therapy in both groups of patients to improve the prescription of radioactivity.

Benign Disease: There is a recognised long-term risk of hypothyroidism in patients with Grave's disease. The risk increases with higher radiation doses. Until recently our policy was to give a low activity of I-131 (70 MBq) every six months until euthyroidism was achieved. Using Postiron Emission Tomography (PET) (with I-124) the functioning volume was assessed and the radiation dose given by the fixed activity of $\mathrm{I}-131$ was calculated. There was a wide variation in the radiation dose received by the thyroid gland. The results demonstrated that the errors in radiation dose measurement depend more upon the accuracy of volume measurement than any other factor. Correlation with clinical progress indicate that the dose to give the highest probability of euthyroidism is 40 Gray. By using thyroid imaging to ensure that the radioiodine is distributed uniformly in the gland and to measure the radiation given by the test activity the radioactivity required to give 40 Gray can be calculated.

Malignant Disease: Studies using PET and I-124 demonstrate a wide variation in dose to metastases and primary tumour. Up to 384 Gray has been required to completely ablate normal residual thyroid tissue. Successful ablation of soft tissue metastases has been achieved with a dose of 150 Gray while bone metastases which are associated with a poor prognosis show measured doses of the order of 20 Gray.

Conclusions: Quantitative imaging in thyroid disease is essential to calculate the radiation dose delivered to both benign and malignant disease. PET (using I-124) has been proved to provide the most accurate method of measuring functioning volumes. Accurate prescriptions of I-131 to give specific doses known to be effective to thyroid tissue should help increase successful therapy. In benign disease and avoid unnecessary radiation morbidity in differentiated carcinoma of the thyroid where the actual radiation dose to primary or secondary tumours is low and further treatment is unlikely to benefit the patient.

\section{$11.00 \mathrm{AM}$}

120 Importance of diagnostic imaging in radiotherapy: chest tumors (Keynote Lecture)

Richard Pötter; Münster

Diagnostic imaging technology for thoracic oncology has evolved during the last decade adding CT and to some extent MRI to conventional chest radiography. Guidelines for step-wise imaging based on the potential benefit for the different clinical applications are necessary. Thoracic radiooncology as primary or adjuvant treatment represents the clinical setting for which thoracic imaging is evaluated. As there is no direct tumor access thoracic radiotherapy always relies on imaging methods. The basic radiooncological demands for imaging after diagnosis are the following: before radiotherapy: reliable staging for decision making on palliative vs. curative treatment, precise and reproducible delineation of tumor, target, and organs of risk; after radiotherapy: detection of recurrence and pathological changes in organs of risk (both referred to radiation dose distribution for the assessment of dose-effect relationships). Positioning and breathing have to be adjusted in a reproducible manner to the conditions of radiotherapeutic management. Recommended imaging techniques are for tumor and lymph node bearing areas: in lung cancer chest X-ray and CT with little additional information from MRI and conventional tomography; in esophageal cancer barium esophagogram and CT with additional information from MRI and endosonography (questionable); in malignant lymphoma chest X-ray and CT or MRI and mediastinal sonography for follow-up; in pleural neoplasms and pulmonary metastatic disease chest X-ray and CT; in thoracic sarcoma chest X-ray and MRI (CT).

A suggested approach for radiooncology-guided imaging for the different thoracic tumors will be presented.

Along these guidelines statements will be made from the point of view of thoracic radiooncology related to the impact of diagnostic imaging in chest tumors on the indication and performance of radiotherapy and on the follow-up period after radiotherapy.

\subsection{5 - 11.30 AM: Discussion}

\section{$11.30 \mathrm{AM}$}

121 Late bone marrow changes in Hodgkin's disease patients. A characterization with proton chemical shift imaging

B. Dietl, H. U. Kauczor, G. Brix, W. Semmler, G. van Kaick, M. Wannenmacher; Heidelberg

Purpose: To measure by means of quantitative chemical shift imaging (CSI) the late therapy induced changes in bone marrow of Hodgkin's disease (HD) patients.

Methods and Materials: 15 HD patients treated with radiotherapy (RT) alone and radiochemotherapy (RCT), age at treatment between 11 and 48 years, 
posttreatment interval between 15 and 124 months were studied with a $1.5 \mathrm{~T}$ MR-imager. For the fat-water separation in-phase and opposed-phase (SE $1200 / 22$ ) images were generated according to the Dixon method, with a modified postprocessing.

Results: Long term fatty replacement of the irradiated bone marrow only (up to 124 months). Visualization of the radiation fields as areas of high signal intensity in the T1-weighted images. Marked increase of fat signal in the CSI without a quantitative difference in the RT vs. RCT patients group, the lowest applicated dose being $30 \mathrm{~Gy}$. No age dependence.

Conclusions: Fatty replacement of the bone marrow is a long term effect in HD patients induced by irradiation not by chemotherapy, probably due to an obliteration of the microvasculature.

\subsection{AM}

122 May a new TNM be proposed in staging of breast cancer $(\mathrm{BC})$ ? The role of internal mammary lymphoscintigraphy (IML)

D. Casara, D. Rubello, G. Saladini, F. Calzavara; Padua

To current time, TNM evaluation of lymphatic invasion in BC is based exclusively on axillary node involvement (ANI). The present study was undertaken to demonstrate the importance of internal mammary node involvement in TNM of BC. During a $7 \mathrm{yrs}$ period, 526 female patients (pts) with $\mathrm{BC}$ were studied. Age ranged $24-75$ yrs, mean 49 . All pts were treated by surgery and radiotherapy, 174 also by hormone/chemotherapy. On the basis of classical TNM, 105 pts were classified as stage I, 247 stage II, 147 stage III, 27 stage IV. IML resulted pathological in $24 \%$ of cases and in $12 \%$ of pts with histological negative ANI. Moreover, in 258 pts IML-results were correlated to the results of bone scans yearly performed during a 5 yrs follow-up. In IML positive pts, bone $\mathrm{mts}$ resulted more frequent than in IML negative pts, $58 \%$ vs $42 \%$, p $<.01$. Regarding to survival a significant difference in IML positive vs IML negative pts was observed, $60 \%$ vs $85 \%, \mathrm{p}<.01$. In conclusion, our data strictly suggest that IML in pts with $B C$ represents a sensitive indicator of internal mammary lymph node status. Moreover, long-term results in our series regarding the appearance of bone mts during the follow-up and survival rate, indicate the important prognostic value of IML for these pts. IML is recommended to be performed in all pts with $\mathrm{BC}$ to better define the lymphatic involvement for TNM staging and thus for a better choice of the therapeutic approach.

\section{$11.50 \mathrm{AM}$}

\section{High dose short fractionation whole brain irradiation: follow up} with MR

K. Pfändner, A. Atzinger, N. Obletter, P. Held, S. Braitinger; Passau

Patients with cerebral metastases usually have only a very reduced survival. From a palliative point of view a short treatment time is desirable. We compare conventional fractionation and high single doses with respect to remission and side effects.

High dose short fractionation yields a better remission of the metastases but produces more serious white matter changes, specially large zones of high signal in T2. The correlation with clinical signs is in discussion. Brain necrosis seems not to occur in the first year after irradiation. In conclusion we believe that the short treatments schedules can be used when life expectance is not longer than one year.

\section{Room S}

\section{SS 04.05 Hepatic interventional radiology}

\section{$10.30 \mathrm{AM}$}

\section{Experimental study of segmental ablation of the liver by trans-} portal Ethanol injection

Toshio Kaminou, Kenji Nakamura, Noriaki Usuki, Keiji Takada, Sumio Takashima, Yasuto Onoyama; Osaka

Purpose: Segmental ablation of the liver by transportal ethanol injection was developed to treat hepatic tumor, and named Selective Segmental Sclerotherapy (SSS). We evaluated the therapeutic effect of SSS experimentally in rats.

Methods and Materials: Forty-three male Wistar rats were treated by selective portal catheterization. Ethanol was injected $0.16-1.24 \mathrm{ml} / \mathrm{kg}$ into a portal branch with the catheter in the wedged position. The ethanol in blood from the inferior vena cava assayed. Portography was done before and after the injection. The rats were killed and examined pathologically.

Results: In all rats, segmental hepatic necrosis and occlusion of the injected portal branch occurred. The blood levels of ethanol remained below toxic levels. Tissue damage was not found in the control lobes of the liver or elsewhere.

Conclusions: SSS may be a safe and effective therapy for hepatic tumor.
$10.40 \mathrm{AM}$

125 Papaverin-HCL, a new concept of spasticolysis in the intraarterial transcatheteral chemoembolization of the liver

Dieter Liermann, C. Hottenrott, M. Lorenz, J. Kollath; Frankfurt/Main

Purpose: Since 1986 in the Univ. of Frankfurt following a special protocol of treatment of tumors of the liver about 300 embolizations had been done. Histologically we found Met. of CRC in $47,5 \%$ and in $32,7 \%$ LCC. In difference to other authors we positionate the catheter in a more central vesselsegment and embolize after application of norepinephrine the multilocular tumors of the liver. In about $10 \%$, there appears a severe spasticity of the vessels caused by catheter manipulation or a high dosis of norepinephrine which prevents from embolization therapy. The use of Papaverin-HCL allows to continue the procedure after spasticolysis.

Methods: We use $50 \mathrm{mg}$ Papaverin-HCL in case of spasticity intraarterial transcatheterial up to the spasticolysis of the vessels, but without disturbing the effect of norepinephrine in the distribution of the bloodflew from the healthy vessels to the tumortissue as decided as therapy concept. Patients mentioned only a hot feeling in the stomach but no pain.

Results: In all cases we used Papaverin-HCL spasticolysis was sufficient and vasoconstriction in the healthy tissue after norepinephrine correct thus chemoembolization was possible.

Conclusions: The use of Papaverin-HCL seems to be a good spasticolyticum in the $10 \%$ of spasticity after catheter manipulation without disturbing the norepinephrine effect afterwards.

\section{$10.45 \mathrm{AM}$}

\section{Segmental hepatic embolization for hepatocellular carcinoma} Shinichi Hori, Etsuo Inoue, Yoshifumi Narumi, Makoto Fujita, Chikazumi Kuroda; Osaka

Purpose: We have attempted to solve problems of hepatic embolization such as pain and impairment of normal hepatic tissue by inserting a catheter to the relevant hepatic segmental artery.

Methods and Materials: Segmental embolization of the hepatic artery was carried out in 29 patients with hepatocellular carcinoma which was located within a single segment. In all patients, where hepatic cirrhosis was accompanied, total hepatic arterial embolization seemed risky. Total hepatic embolization was carried out in 32 patients with similar hepatocellular carcinoma. Embolization was done with a gelatin sponge, Lipiodol and antineoplastic agents.

Results: A patient would certainly feel less pain after the segmental embolization than after total liver embolization. Segmental embolization achieves a higher necrotic effect of the main tumor compared with proximal embolization. There is no significant improvement of the necrotic effect of the surrounding lesions. Survival rate was $83 \%$ in one year and $76 \%$ in two years. Conclusions: The same therapeutic result as total liver embolization can be expected inspite of small volume of embolization. The segmental embolization of hepatic tumors gives a patient less pain and less impairment of hepatic function.

\section{$10.55 \mathrm{AM}$}

\section{Segmental arterioportal chemoembolization for hepatocellular} carcinoma

Hiromichi Oi, Masaki Matsushita, Hideya Yamazaki, Jun Okamura; Osaka

Segmental arterioportal chemoembolization was performed in 34 patients with hepatocellular carcinoma. Administration of anticancer drugs from the hepatic artery into the portal vein through the arterioportal communication, permits chemotherapy against the extracapsular infiltration of the tumor nourished by the portal vein blood flow. Emulsion of $10-80 \mathrm{mg}$ (mean $38 \mathrm{mg}$ ) of doxorubicin and $2-20 \mathrm{ml}$ (mean $11 \mathrm{ml}$ ) of iodized oil was infused from a segmental artery of the liver until the emulsion overflowed into the segmental portal branches around the tumor. Additional gelatine sponge was given to all cases except five. Seven cases underwent hepatectomy and these specimens revealed necrosis of both the main tumor and extracapsular infiltration, and also local necrosis of normal liver parenchyma around the tumor. The one-year cumulative survival rate for the other 27 cases was $75.8 \%$, and three-years was $42.1 \%$.

The local necrosis and reactive inflammatory changes were complications of this therapy in normal parenchyma of the targeted segments, but it did not affect other residual liver parenchyma and was effective method for the treatment to the localized hepatocellular carcinoma. 


\section{$11.00 \mathrm{AM}$}

128 Usefulness of segmental Lp-TAE (hepatic segmental chemoembolization using Lipiodol mixed with anticancer agent) for hepatoma Hideo Uchida, Naoki Matsuo, Kiyoshi Nishimine, Yukihiro Nishimura, Hiroshi Sakaguchi, Tetsuya Yoshioka, Noritada Nagano, Hajime Ohishi; Nara

Purpose: To further improve the therapeutic effect of transcatheter arterial embolization of hepatocellular carcinoma, we developed "Segmental Lp-TAE" which is transcatheter hepatic segmental or subsegmental arterial chemoembolization using Lipiodol mixed with an anticancer agent followed by the injection of gelatine sponge particles introduced into the tumor-bearing hepatic segment as the target area and evaluated the usefulness of this procedure.

Methods: Segmental Lp-TAE was carried out in 72 patients with hepatocellular carcinoma, 11 patients who underwent hepatectomy and the anti-tumor effect and influence on non-tumor areas executed by Segmental Lp-TAE were studied histologically. The effectiveness of Segmental Lp-TAE was evaluated by follow-up CT patterns, histopathological results and survival of unresectable cases.

Results: Complete necrosis of the tumor was attained in 9 of 11 cases. The cumulative survival rates were approximately $90 \%$ at 1 year and $65 \%$ at both 2 and 3 years which compared favorably with all patients undergoing conventionial Lp-TAE.

Conclusions: It was concluded that this technique does not adversely affect normal tissues, and does reinforce the effect of TAE.

\subsection{AM}

\section{Perfusion of liver malignancies under influence of Norepinephrine} U. Lörcher, D. Liermann, J. Peters; Frankfurt/Main

Purpose: The effect of norepinephrine on the perfusion of liver malignancies was studied in order to optimize selective chemoembolization.

Methods: Before chemoembolization we performed a dynamic CT-study of the liver of 28 patients. First we injected into the celiac artery via catheter $12 \mathrm{ml}$ of contrast medium $2 \mathrm{ml} / \mathrm{sec}$. Then $0.2 \mathrm{mg}$ of norepinephrine was given immediately followed by injection of $6 \mathrm{ml}$ contrast medium $(1 \mathrm{ml} / \mathrm{sec})$. Scantime was $12 \mathrm{sec}$ in both cases. From this scan 23 images were reconstructed, one every $0.5 \mathrm{sec}$. Thus we showed as well as the arterial as well as the portal phase of perfusion. 24 hours after embolization CT of the liver was performed in order to compare the distribution of lipiodol with the perfusion found by angio-CT. Results: After administration of norepinephrine we found a dramatic reduction of the perfusion of liver, spleen and stomach in 20 patients whereas the contrast enhancement of the tumor increased. In 5 patients we found perfusion of the tumor only after norepinephrine. In 8 patients there was no difference before and after norepinephrine. The same pattern of distribution was found after embolization.

Conclusions: In 3 of 4 cases the perfusion of liver tumors can selectively be increased by the administration of norepinephrine. Thus norepinephrine improves embolization of liver tumors.

\subsection{AM}

130 Hepatocellular carcinoma - combined treatment by hepatic chemoembolisation and systemic chemotherapy (HCE)

A. Gebauer, M. Baur, R. Walter, Ch. Dittrich, H. Schurawitzki; P. Ferenci, R. Mallek, A. Fritsch, A. Gangl, D. Tscholakoff; Vienna

Purpose of the prospective study was to evaluate the feasability and efficacy of repeatable combined hepatic artery chemoembolisation and systemic chemotherapy (HCE) in nonresectable hepatocellular carcinoma (HCC) in comparison to natural course of the disease (no treatment).

Methods and Materials: Out of 33 pts with histological proven HCC 14 pts entered the control group due to contraindications against HCE. In 19 pts HCE was tried by superselective angiographic application of cysplatin $(20 \mathrm{mg} / \mathrm{m} 2)$-lipiodol emulgation followed by occlusion of the hepatic artery with resorbable gelfoam powder. On the day 2 cisplatin $(60 \mathrm{mg} / \mathrm{m} 2)$ was given intravenously, additionally interferon alpha2c (7 MIE s.c.), was given on days 2, 5, 9 and 12 after embolisation. Follow-up studies included dynamic CT, US and alpha fetoprotein (AFP) level in both groups.

Results: In 4 pts CHE could not be performed due to hepatic artery stenoses $(n=2)$, intrahepatic AV-shunt $(n=1)$ or arterial spasm $(n=1)$. Six pts had 3 or more cycles of therapy and showed according to objective remission criterias (WHO) partial remission $(n=1)$, no change $(n=4)$, progressive disease $(n=1)$ 9 pts had $<3$ cycles of therapy, 2 of them are still treated. In 4 of them AFP level decreased by $50 \%$. Complications possibly related to $\mathrm{HCE}$ included abdominal pain $(n=10)$, GI-tract ulceration $(n=3)$, portal vein thrombosis $(\mathrm{n}=2)$ and Mallory-Weiss-syndrome $(\mathrm{n}=1) .4$ months survival in the controlgroup was $0 \%$ (med. 2 months), $30 \%$ for HCE (med. 5 months). In conclusion HCE seems to be a feasible and efficacious therapy in patients with nonoperable HCC.
11.30 AM

131 Results of repeated chemoembolization of hepatocellular carcinoma

R. Wickenhöfer, I. Hasan, H. Schüller, M. Reiser, J. Hartlapp; Bonn

a) Chemoembolization (ChE) is a very effective procedure in the therapy of hepatocellular carcinoma (HCC). It is the only procedure, that can increase survival significantly.

b) Staging was made by the TNM-System. In inoperable tumors primary ChE was performed with Epirubicin A $\left(50 \mathrm{mg} / \mathrm{m}^{2}\right)$, Cis-Platin $\left(30 \mathrm{mg} / \mathrm{m}^{2}\right)$ and Lipiodol $(10-12 \mathrm{ml})$. Patients underwent monthly outpatient examinations, where also blood concentration of the tumor markers AFP and CEA were analyzed. Every three months a computed tomography (CT) was made. In relation to the findings of the $\mathrm{CT}$ and the values of the tumor markers a re-embolization was performed.

c) Since 198812 patients with HCC in clinical stage IVa had been treated in accordance with the above mentioned scheme. Therapy was easily tolerated. 2 patients had to be treated four times, 4 patients three times. For the next 4 patients a sufficient reduction of tumor size could be achieved with 2 embolizations; in the other 2 patients a single embolization was necessary. Under this therapeutic regime tumor progression could be stopped in every case. Tumor markers returned to normal.

d) Survival of untreated patients with $\mathrm{HCC}$ and of patients, who had received systemic chemotherapy, lies between 1.4 and 3.5 months. With repeated ChE a highly significant increase of survival can be achieved.

\section{$11.40 \mathrm{AM}$}

132 Management of splenohepatic steal after liver transplantation R. Langer, M. Langer, A. Scholz, P. Neuhaus, R. Felix; Berlin

Purpose: The prospective study was performed to consider the therapeutic modalities for patients with splenohepatic steal after orthotopic liver transplantation (OLT).

Methods: 23 posttransplant i.a. DSAs of the celiac axis were performed after OLT. A splenohepatic steal could be demonstrated in 7 patients, 3 times a transcatheter embolisation of the splenic artery was performed.

Results: Out of 120 OLT during September 1988 and January 19917 patients developed a posttransplant hypersplenism and an elevation of the transaminases. Posttransplant i.a. DSA revealed a splenohepatic steal syndrome with an early and high perfusion of the splenic artery and a delayed and slow perfusion of the hepatic artery. In 3 patients an embolisation with coils of the splenic artery was performed which resulted in a partial or total occlusion of the splenic artery and a normal hepatic perfusion. Because of the immunosuppression both patients with a total occlusion of the splenic artery developed splenic abscesses so that splenectomy became necessary. 3 other patients were treated by splenectomy. All laboratory findings normalized after radiologic interventions or operation.

Conclusions: Splenohepatic steal is a rare complication after OLT, resulting mostly in a hypersplenism. Because of the immunosuppression the treatment should be splenectomy.

\section{$11.45 \mathrm{AM}$}

\section{Percutaneous aspiration and Ethanol sclerotherapy of sympto-} matic hepatic cysts

A. Leinonen, T. Siniluoto, M. Päivänsalo, A. Karttunen, M. I. Kairaluoma, I. Suramo; Oulu

This study was undertaken to elucidate the usefulness of percutaneous aspiration and ethanol sclerotherapy in symptomatic congenital hepatic cysts.

Patients and methods: Between November 1985 and August 1990 all patients with symptomatic congenital hepatic cysts in Oulu University Central Hospital were prospectively subjected to sonography-guided percutaneous aspiration and temporary injection of $99 \%$ ethanol into the cyst. Eleven patients had a solitary cyst and eight a polycystic liver disease. 49 cysts were treated. The aspiration volume of the cysts ranged from 95 to $8100 \mathrm{ml}$, mean $950 \pm 2000 \mathrm{ml}$. Small cysts were treated twice with alcohol injection, the large ones three times at the same sitting. The volume of alcohol was related to the size of the cyst $(25$ to $100 \mathrm{ml}$ per injection). Blood alcohol content was measured after completion of the procedure. Routine follow-up scans and laboratory tests were requested next day and 1, 2, 6, 12, 18 and 24 months after the treatment. All patients were followed up for at least one year.

Results: Small solitary cysts usually disappeared totally. In cases of large cysts a small residual cyst without symptoms was found many times. A cure was achieved with one alcohol sclerotherapy. The size of many cysts increased temporarily during the first month after the procedure but then decreased continuously. No recurrences were found during follow-up and all patients were doing well. The mean blood alcohol content after sclerotherapy was $0.43 \pm 0.38 \%$. Only minor side effects (transient pain and temperature elevation) occurred, but no changes in laboratory tests were seen. 
134 Percutaneous debridement of complex pyogenic liver abscesses: technique and results

L. B. Morettin; Galveston, TX

Purpose: The presentation intends to demonstrate and illustrate the author's approach and technique in the treatment of complex liver abscesses that persisted or recurred following percutaneous catheter drainage.

Methods and Materials: Six patients with failed surgical (2), or percutaneous catheter drainage (4), were treated utilizing an instrument designed specifically for the purpose. Contrast enhanced CT scans of the liver were obtained on all patients. Four patients were chronically ill but stable. Two patients were septic, hypotensive and considered life threatened. All abscesses were pyogenic. Two due to E. coli and four mixed flora (E. coli, Klebsiella and gram-positive cocci).

Results: In all cases CT revealed multiloculated abscesses containing large central debris and a halo of compromised parenchyma with diminished perfusion. Debridement was successful in all 6 cases resulting in complete healing, (4-12 days). Details of procedure and postoperative management will be presented. Two cases of infected cystic cholangiomas were referred for treatment; CT findings will be described which permitted differentiation from primary abscesses, resulting in exclusion from the study.

Conclusions: Percutaneous debridement of pyogenic liver abscesses and diseased peripheral parenchyma is a useful technique, with promising results in chronic unresolved liver abscesses and may be life-saving in critically ill patients. 


\section{Room A}

\section{HL}

\section{Holzknecht-Lecture}

Radiological management of vascular anomalies of the lung J. Rémy; Lille

Vascular anomalies of the lung may be classified into 7 main topics:

1. Pulmonary arterial,

2. Pulmonary venous,

3. Pulmonary arterio-venous,

4. Systemic arterial,

5. Systemic venous,

6. Systemic arteriovenous,

7. Lymphatic.

Each of them may also be sub-classified into six subchapters including, for most of them: anomalies of origin - size - route - drainage - number - connections.

Radiological management of these malformations is well undertaken when embryologic data are integrated. For example, unilateral "absence" of a pulmonary artery is very often a misnomer. CT of the hypoplastic lung, in demonstrating normal intra-pulmonary angioarchitecture brings the proof of a well organized primitive pulmonary plexus. "Absence" should be replaced by "occult" pulmonary artery with and without intra-pericardial segment. This distinction may prove helpful in the surgical management. When the occult artery and the aortic arch are on opposite sides, a diverticulum of the innominate artery is often found indicating fetal systemic blood supply to the occult artery with obliteration soon after birth. This obliteration is well explained by ductal tissular composition of the distal part of the sixth aortic arch. There is also a well understandable association between development of the truncoconus, right and left pulmonary arteries and the lateralisation of the aortic arch.

Each vessel's anomaly of the lung must always be investigated on embyrologic, hemodynamic (pre- and post-natal) and morphologic data. 


\title{
Room A
}

\section{SS 11.08 Neuro-Flow, Head}

\author{
$2.00 \mathrm{PM}$
}

\section{Magnetic resonance imaging of cerebral blood flow by Gadolinium-DTPA bolus-tracking \\ J.C. Böck, B. Sander, J. Hierholzer, J. Haustein, W. Schörner, R. Felix; Berlin}

Purpose: The purpose was to study normal perfusion patterns in volunteers and perfusion abnormalities in patients with cerebral pathology.

Methods: In all patients an HM-PAO-SPECT was performed for comparison ( $555 \mathrm{MBq} 99 \mathrm{mTc}-\mathrm{HM}-\mathrm{PAO}$, data acquisition by rotating gamma-camera in a $64 \times 64$ matrix, axial slice thickness $=7 \mathrm{~mm}$ ). We studied 12 normal volunteers, 15 patients with cerebral ischemic disease and 12 patients with brain tumors ( $1.5 \mathrm{~T}$, single-slice FLASH, TR $=25 \mathrm{~ms}, \mathrm{TE}=20 \mathrm{~ms}$, flip angle $=10^{\circ}$, $230 \mathrm{~mm}$ field of view, $64 \times 128$ matrix, $8 \mathrm{~mm}$ slice thickness, 2-D Fourier reconstruction, 30 sequential images during 80 seconds). At the beginning of the data acquisition gadolinium-DTPA $(0.1 \mathrm{mmol} / \mathrm{kg}$ body weight, Magnevist, Schering AG, Berlin, Germany) was injected manually during 6-7 seconds into an antecubital vein.

Results: Reversible symmetric cortical signal intensity reduction of $19 \pm 7 \%$ was seen in normals. Areas of decreased or absent signal intensity reduction corresponded to areas identified as hypoperfused on SPECT. Increased signal intensity reduction corresponded to hyperperfusion on SPECT.

Conclusions: We conclude that Gd-DTPA-based MR-perfusion studies demonstrate normal and abnormal perfusion patterns and that these results correlate with the reference technique (SPECT).

\subsection{PM}

\section{MRI of multiple occult arteriovenous malformations of the brain and spine}

G. Dooms, P. Mathurin, G. Cornélis; Brussels

Purpose: A retrospective study was performed to assess the incidence of multiple occult arteriovenous malformations of the brain and the value of various imaging sequences for detecting that particular disorder.

Methods: The MR examinations were performed with a superconducting magnet (Philips Gyroscan S15) operating at 1.5 Tesla. Various imaging sequences were performed: $\mathrm{T} 2$-weighted $(\mathrm{TR}=2.18 \mathrm{sec}$ and $\mathrm{TE}=50$ and $100 \mathrm{msec})$, T1-weighted $(\mathrm{TR}=0.415 \mathrm{sec}$ and $\mathrm{TE}=20 \mathrm{msec})$ and $\mathrm{FFE}$ $\mathrm{T} 2$-weighted $\left(\mathrm{TR}=0.5 \mathrm{sec}, \mathrm{TE}=20\right.$ and $35 \mathrm{msec}$ and flip angle $\left.20^{\circ}\right)$ images. Results: Among the 164 patients with occult AVM's reviewed for that study, 14 $(8,5 \%)$ presented with multiple occult AVM's, distributed everywhere within the cerebral and cerebellar hemispheres and the brainstem. In two patients, multiple intracerebral occult AVM's were associated with one location within the thoracic spinal cord. When studying the family members of the 14 patients with multiple occult AVM's, two familial cases were found (two members of the same family had occult AVM's). The best imaging sequences for detecting the exact number of lesions and to characterize them as occult AVM's was the FFE T2-weighted sequence in all patients.

Conclusions: In conclusion, multiple occult AVM's are not a rare disease. When present, there may be a need for searching for other locations within the spinal cord and for presence of the same pathology in the often asymptomatic family members.

\section{$2.20 \mathrm{PM}$}

\section{Comparison MR-angio digital angiography in the epiaortic vessels pathology}

A. Carriero, L. Salute, A. Toppetti, A. Iezzi, L. Bonomo; Chieti

The atheromatous lesion and other neck arterial vessels pathologies are the most frequent cause of cerebrovascular disease.

In order of time magnetic resonance angiography (MR angio) is the last technique that can allow to identify the neck vessels morphology.

The authors assessed the ability of a magnetic resonance angiography technique to provide accurate, detailed images of neck arteries in comparison to DSA.

40 patients with cerebrovascular disease were studied comparing DSA to MR angio. MR imaging was performed with a $1.5 \mathrm{~T}$ system, dedicated coil and gradient-echo sequences.

Refocusing sequences for the flow were acquired on coronal and sagittal planes by following parameters: TR of $40 \mathrm{~ms}$, TE of $10 \mathrm{~ms}$, flip angle $25^{\circ}$, acquisition volumes of $80-85 \mathrm{~mm}$ with 64 partitions.

Digital angiography represented totally 101 vascular lesions: 27 slight $(>30 \%)$ stenosis, 19 moderate ( $31-70 \%)$ stenosis, 12 heavy ( $71-99 \%)$ stenosis, 7 occlusions, 14 coiling, 7 kinking and 15 hypoplasia of vertebral artery.

In slight stenosis MR angio underestimated this lesion in 11 of 27 patients $(40,7 \%)$. In moderate and severe stenosis MR angio tends to overestimate the lesions: in the moderate MR angio overestimated the $31,5 \%$, in the severe the $16,6 \%$.

In the hypoplasia of vertebral artery MR angio overestimated $16,6 \%$ of the cases.

In coiling and kinking MR angio has the same results as DSA.

\subsection{PM}

\section{MRI-angiography in the evaluation of intracranial aneurysms}

C. Louail, F. Blanc, H. Loiseau, J. M. Franconi, W. Kawiecki, D. Gense de Beaufort, J. M. Caillé; Bordeaux

The authors evaluated the contributions and limitations of angiography by magnetic resonance in the diagnosis, the pretreatment evaluation and the surveillance of intracranial aneurysms.

Methods: Ten intracranial aneurysms were explored with conventional angiography and MRI-angiography. The examinations were performed with a Magnetom SP Siemens 1.5 Tesla, using 3D FISP acquisition with short TR, as well as conventional T1-and T2-weighted spin-echo sequences. The analysis of the results comprised the spin-echoes, the $3 \mathrm{D}$ sections and the reconstructions by the MIP method.

Results: All the aneurysms were visualized with this technique but a good analysis of the neck was not possible because of insufficient spatial definition. In two cases of partially thrombosed aneurysms, the MRI-angiography provided a better definition of the relations with adjacent vessels than did conventional angiography, thus giving important informations for the evaluation before surgery.

Conclusions: The place of MRI-angiography in relation to conventional angiography has yet to be established and will need further assessment. MRI-angiography appears to be interesting in the evaluation of unoperated intracranial aneurysms because it is not traumatic. It also appears particularly interesting in the preoperatory evaluation of partially thrombosed aneurysms, defining the relations of the aneurysm to the adjacent vessels.

2.40 PM

140 Assessment of cerebral perfusion with dynamic contrast-enhanced MR-imaging

B. Wallner, W. Weidenmaier, R. R. Edelman; Ulm

Purpose: We used a T2*-weighted gradient-echo sequence to study regional brain perfusion in patients with tumors, stroke and demyelinating disease. Methods: Regional cerebral perfusion was assessed with contrast-enhanced MR imaging. After bolus administration of Gd-DTPA sequential gradient-echo images (FLASH, 35/10/25 for TR/TE/Flipangle) were obtained with an acquisition time of $2.5 \mathrm{sec}$ per image. The method was used in 7 patients with brain tumors, 15 patients with multiple sclerosis and 9 patients with stroke.

Results: In the patients with tumors, the nidus showed increased perfusion, whereas the edema was hypoperfused. Perfusion abnormalities showed a larger extension than the edema on T2-weighted images. In the patients with acute stroke, perfusion abnormalities were seen earlier than on conventional MR and in patients with chronic stroke perfusion abnormalities were more extensive than on routine MR. In patients with multiple sclerosis hypoperfusion of the white matter relatively to gray matter was seen.

Conclusions: Dynamic contrast-enhanced MR imaging provides information about cerebral perfusion not available with conventional MR. The technique can be included in routine imaging protocols and lengthens examination time only by a few minutes.

\subsection{PM}

141 Magnetic resonance imaging of normal and pathologic $\overline{\mathrm{CSF}}$-motion using retrospective gating

Bernhard Sander, Uwe Tosch, Peter Schubeus, Wolfgang Schörner, Roland Felix; Berlin

Purpose: Aim of the present study was to visualize the pulsatile motion of cerebrospinal fluid (CSF) and to differentiate between normal and pathologic patterns of CSF-motion.

Methods: Five normal volunteers and 10 patients with enlarged ventricles were examined with an $1.5 \mathrm{~T}$ Magnetom (Siemens AG, Erlangen) using a steady state free precession sequence (PSIF) and retrospective gating (TR $=20 \mathrm{msec}$, $\mathrm{TE}=31 \mathrm{msec}$, flip angle $80^{\circ}$, acquisition time $4 \mathrm{~min}$., field of view $230 \mathrm{~mm}$, $256 \times 256$ matrix). During the measurement with the fast T2-weighted PSIF-sequence the electrocardiogram of the patient was recorded. After the measurement the acquired magnetic resonance datas were retrospectively 
sorted according to the cardiac cycle. So we obtained 16 sagittal images of the CSF representing 16 phases of CSF-motion during one averaged cardiac cycle. Results: Normal volunteers showed pulsatile decrease of signal intensity near the foramina of Monroi, in the aquaeduct and in the spinal canal. Compared to the normal volunteers the patients showed more decreased signal intensities of CSF and phase shifts.

Conclusions: Retrospective gating allows for visualization of CSF-motion and seems to be helpful in the diagnosis of hydrocephalus.

3.00 PM

142 Magnetic resonance imaging of cavernous sinus subarachnoid diverticulum

G. Dooms, P. Mathurin, G. Cornélis; Brussels

Purpose: A retrospective study was performed to assess the MR appearance of cavernous sinus subarachnoid diverticulum in patients presenting with nerve palsies related to a clinical cavernous sinus syndrome.

Methods: Four patients (one male and three females) (age range: $24-72$ years) were included in the study. MRI findings were correlated with computed tomography and metrizamide CT cisternography findings in all patients. The MR examinations were performed with a superconducting magnet Philips Gyroscan S15 operating at 1.5 Tesla. T1 $(\mathrm{TR}=415 \mathrm{msec}$ and TE $=20 \mathrm{msec})$ and $\mathrm{T} 2(\mathrm{TR}=2100 \mathrm{msec} ; \mathrm{TE}=50$ and $100 \mathrm{msec})$ weighted images were performed in every patient. T1-weighted images were also performed following IV. injection of gadolinium-DTPA ( 0.1 millimole per kilo body weight).

Results: In all four patients presenting with six cavernous sinus lesions, MRI studies demonstrated enlargement of the cavernous sinus. The lesion within the cavernous sinus appeared multiloculated in one patient; its signal intensity follows that of the CSF in the optochiasmatic cistern on both T1- and T2-weighted images. There was no contrast uptake of the tumor after contrast injection. Therefore, differential diagnosis with other more common intracavernous lesions (including meningiomas, neuromas and aneurysms) was very easy to perform.

Conclusions: In conclusion, MRI is an exquisite noninvasive modality for identifying this relatively rare entity affecting the cavernous sinus and for performing the differential diagnosis between various pathological processes affecting the cavernous sinus.

\subsection{PM}

143 Proton decoupled NOE enhanced phosphorus chemical shift imaging of the human brain

H. Kolem, M. Schneider, K. Wicklow, T. Miyazaki, R. Sauter; Erlangen

Purpose: Low signal-to-noise ratio (SNR) is one of the problems of localized in vivo phosphorus brain spectroscopy. Therefore we developed a technique using the Nuclear Overhauser Effect (NOE) and decoupling effects. Thereby this technique increases SNR by $50 \%$ and improves the spectral resolution. Methods: A 1.5 Tesla whole body MR system for imaging and spectroscopy (Siemens Magnetom) was used. In order to achieve the NOE enhancement and decoupling we used a second RF transmitter working on the proton frequency. For localization we used the CSI technique. This results in 64 simultaneously acquired spectra showing also the spatial distribution of metabolites.

Results: Initially a SNR increase of $50 \%$ and an improvement of spectral resolution compared to measurements without the second RF transmitter was confirmed in volunteer measurements. Then we applied the technique to patients with brain tumors showing the spatial distribution of metabolites. Fifteen minutes of measurement time gave sufficient SNR to determine the $\mathrm{pH}$ value and the relative intensities of PME, PDE, PCr.

Conclusions: As this technique is easy to apply (no specific adjustment required) and as it gives data sets with spatial and spectral resolution in only fifteen minutes measurement time it is a valuable improvement of in vivo brain phosphorus spectroscopy.

\subsection{PM}

144 MR-angiography of the carotides: a comparison of two MR-techniques

W. Weidenmaier, B. Wallner, K. A. Schumacher, J. M. Friedrich; UIm

For 28 patients with angiographic and sonographic verified stenoses of the extracranial carotid arteries we compared the "bright-blood" and "black blood" MR-angiography.

Flow-compensated gradient echo sequences were used to get "bright-blood" images.

Spin echo sequences with presaturation of the moving spins were supplied to create black blood images.

The obtained two- and three-dimensional acquired images were used to reconstruct projection angiograms $(\mathrm{MIP}=$ maximal/minimal intensity projection). We found an overestimation of the stenosis using the bright blood technique. Black blood images confirmed more precisely with the angiographic results. An underestimation was not observed.
3.23 PM

145 MR-imaging of cerebral flow dynamics using MR-angiography and selective presaturation

B. Wallner, W. Weidenmaier, J. Vogel; UIm

Purpose: A technique to delineate vascular territories and to show the presence and directionality of flow with MR-angiography is demonstrated.

Methods: Flow dynamics were studied using a 2D-gradient-echo sequence with flow-compensation (FLASH, 40/10/30 for TR/TE/Flipangle). Selective presaturation was applied consecutively to the left and right carotids, ophthalmic and basilar arteries. We studied 25 patients with high-grade stenosis of carotid arteries, 12 patients with carotid occlusion, 5 patients with subclavian-steal syndrome and 3 patients with arteriovenous malformations. The results were compared with Doppler-ultrasound and conventional angiography.

Results: Selective MR angiography demonstrated collateral blood flow in all of the cases and correlated well with Doppler and angiography. Supply of the posterior cerebral arteries from the carotids was demonstrated in two patients, which was not seen by Doppler ultrasound. The flow dynamics in the patients with subclavian steal could also be shown and correlated with angiography. In the patients with AVMs, the source of blood supply could be demonstrated by MR.

Conclusions: Selective MR angiography reliably demonstrates cerebral flow dynamics and gives information about vascular territories, an information not provided by other MR imaging modalities. The technique is rapid and lengthens the study time for a routine MR examination by only a few minutes.

\subsection{PM}

146 Computerized tomography (CT) and Magnetic resonance imaging (MRI) assessment of the brain state in malignant arterial hypertension V.Dmitriev, G.Pogrebnaya, Yu. Belenkov, G. Arabidze, D. Geleznov; Moscow

The aim of investigation was to determine character of brain pathologic changes on MRI and CT dates in patients (pts) with MAH, complicated by hypertonic encephalopathy.

The investigation was performed on 35 pts with MAH, on Bruker BMT-1100 tomograph (Germany), $\mathrm{SE}$-sequence, $\mathrm{TR}=2000 \mathrm{msec}, \mathrm{TE}=34 \mathrm{msec}$, and on CT-tomograph CMS "Quad I" (GB).

CT and MRI have discovered equally assymetric dilatation of ventricular system: lateral ventricular bodies weight was $2,37 \pm 0,08 \mathrm{sm}(\mathrm{p}<0,005)$. Regions of low density $(24,1 \pm 3,5 \mathrm{ehH})$ in white matter were determined in 18 pts $(50 \%)$ on CT-dates. Simultaneously regions of high signal intensity $(\mathrm{T} 2=125,7+3,7 \mathrm{msec})$ in white matter were discovered with MRI in all $(n=35)$ pts, and were estimated as lacunar infarcts. Periventricular edema was discovered only with MRI, characterised with $\mathrm{T} 2=135,7 \pm 2,5 \mathrm{msec}$ ( $\mathrm{p}<$ $0,001, \mathrm{n}=35$ )

MRI and CT have the same sensitivity in the ventricular brain system estimation, simultaneously MRI possesses higher sensitivity in lacunar infarcts and periventricular edema diagnosis.

\section{Room B}

\section{SS} 2.00 PM

\section{Spiral volumetric CT (SVCT) of the mediastinum \\ Philip Costello, Christian Ecker, Dirk Blume; Boston, MA}

Purpose: To assess the effectiveness of contrast enhanced volume scans of the mediastinum with a 12 second breathhold.

Methods: A CT scanner (Siemens Somatom Plus) with a modified transport system, continuously rotating allowing $12,1 \mathrm{sec}$. scans through a $12 \mathrm{~cm}$ volume of the thorax; reconstruction software corrects for the helical scanning geometry. A $12 \mathrm{sec}$ scan was performed during a single breathhold from the aortic arch to the diaphragm at a $10 \mathrm{~mm} / \mathrm{sec}$ table advance. $50 \mathrm{cc}$ of Renografin 60 were infused via an antecubital vein, at $3 \mathrm{cc} / \mathrm{sec}$ for $16 \mathrm{sec}$. The final images were reconstructed $4 \mathrm{~mm}$ apart. 16 patients with lung carcinoma and 4 with lymphoma were assessed.

Results: All studies were of excellent quality with mediastinal and hilar pathology clearly defined. Nodes and blood vessels were easily distinguished. With a single breathhold, there were no misregistration artifacts and smooth transition from one slice level to the next. Reconstructed and 3-D images of high quality were possible. With only $50 \mathrm{cc}$ of contrast, there was optimal contrast utilization and since total scan time was 12 seconds, patient tolerance and cooperation were excellent.

Conclusions: SVCT allows for high quality mediastinal CT with only small volumes $(50 \mathrm{cc})$ of contrast material and a short scan time. For staging malignant disease, the technique is fast and well tolerated. 


\section{$2.10 \mathrm{PM}$}

\section{Optimization of technique in CT of lung disease} U. Lörcher, J. Peters, M. Gossmann; Frankfurt/Main

Purpose: So far no optimized technique for $\mathrm{CT}$ studies of diffuse pulmonary disease has been described. Until now only $10 \mathrm{~mm}$ scans (standard algorithm) have been compared with $1-2 \mathrm{~mm}$ scans (high resolution algorithm).

Methods: 100 patients with suspected interstitial lung disease have been studied with different techniques. We compared 10,5 and $1 \mathrm{~mm}$ scans with normal and $\mathrm{HR}$ algorithm with each other. The images were presented 5 radiologists with different window settings (from $500-2500 \mathrm{HU}$ for window width and window center between -200 and $-800 \mathrm{HU}$ ) in order to find optimal images. Results: Visualisation of linear structures (septal and fibrotic) is significantly better in $5 \mathrm{~mm}$ scans than with $10 \mathrm{~mm}$ technique and does not show any loss of information in small round lesions. $5 \mathrm{~mm}$ scans are definitely better in visualisation of nodules and their differentiation from vessels than $1 \mathrm{~mm}$ scans and there is no important reduction of quality as far as linear structures are concerned. Window width of $2000 \mathrm{HU}$ and a center between -350 and $-500 \mathrm{HU}$ yield an optimal visualisation of the combination of air containing (emphysema) and solid (tumor, mediastinum) structures. This window also allows the visualisation of the pleural interface.

Conclusions: CT examination of diffuse lung disease should be performed with $5 \mathrm{~mm}$ scans using HR algorithm. In general window settings of 2000/-350 to $-500 \mathrm{HU}$ are optimal.

\subsection{PM}

\section{Slide selection for high resolution computed tomography (HRCT): a sampling approach}

Claudia Henschke, Graham Kalten; New York, NY

HRCT of diffuse diseases is useful to characterize the abnormalities, to measure the overall disease severity, to detect the differential distribution throughout the lung (apex to base, central to peripheral) and to assess the change in these findings over time. Since contiguous HRCT sections throughout the lung are not practical in terms of radiation exposure, time, cost nor necessary for the assessment of any given disease process, the relevant question is how to optimumly select HRCT sections and how to standardize the methodology of the slice selection to enhance the comparability between institutions, particularly for the rare diseases. The lung is defined as the total population of secondary pulmonary lobules. It is assumed that any disease process will allow either counts or measurements, $X$, to be made on any given slice. Various optimum sampling schemes are developed for diseases which have a a) uniform distribution of the abnormality throughout the lung, b) a linear change from apex to base, c) a non-linear change from apex to base Different criteria for the slice selection are also presented and compared. Possible selection criteria are: the minimum number of slices to achieve a specified precision of the estimate of the true population count or measurement, $\mathrm{X}$; minimum radiation dose for a given level of precision; the minimum number of slices to assess differential distribution of the abnormality from the apex to the lung; minimum number of slices to adequately assess a change in the abnormality over time to initiate or change a given intervention and now the sample size is dependent on the risks and benefits of any given intervention.

\section{$2.30 \mathrm{PM}$}

\section{0 is the quality of routine chest computed tomography improved by} changing to a high resolution algorithm?

M. E. Roddie, S. J. White, J. E. Jackson, A. Adam; London

Purpose: To assess the effect of changing to a high-resolution reconstruction algorithm on image and diagnostic quality of standard chest computed tomography and to compare this with fine-section, high-resolution CT.

Methods: Forty patients with a variety of pulmonary diseases underwent chest CT using a Somatom Plus scanner (Siemens) and had $10 \mathrm{~mm}$ and $2 \mathrm{~mm}$ sections performed at 4 representative levels. Standard and high-resolution reconstructions were compared for parenchymal detail, noise and diagnostic quality by two radiologists blinded to the reconstruction and clinical data. The high-resolution $10 \mathrm{~mm}$ and $2 \mathrm{~mm}$ sections were similarly evaluated.

Results: The lung parenchymal detail was significantly better with the $10 \mathrm{~mm}$ high-resolution algorithm than with the standard algorithm and the mediastinal images were of equal diagnostic quality. There was, however, no increased diagnostic yield from the high-resolution $10 \mathrm{~mm}$ sections. The $2 \mathrm{~mm}$ high-resolution reconstructions not only showed superior lung parenchymal detail compared with the $10 \mathrm{~mm}$ sections but were of significantly higher diagnostic quality for lung pathology although moderate to severe degradation of mediastinal image quality occurred.

Conclusions: The high-resolution reconstruction algorithm should replace the standard algorithm for routine chest CT although thin sections will still be necessary to fully evaluate any abnormality detected.
2.40 PM

151 Comparison of different CT-techniques in the evaluation of diffuse interstitial lung diseases

H.-J. Triebel, G. Kanzow, H. Magnussen, E. Buecheler; Hamburg

Purpose: To compare standard $8 \mathrm{~mm}$-scans with $\mathrm{HR} 8 \mathrm{~mm}$-scans and $\mathrm{HR}$ $2 \mathrm{~mm}$-scans in the evaluation of diffuse interstitial lung diseases.

Methods and Materials: 42 patients with histologically proven interstitial lung diseases underwent CT. The scanning routine initially consisted of standard $8 \mathrm{~mm}$-scans at $8 \mathrm{~mm}$ or $16 \mathrm{~mm}$ intervals, three of them being reconstructed to a HR-algorithm. Later HR $8 \mathrm{~mm}$-scans were obtained directly with three of them being reconstructed to a standard algorithm. In all patients HR $2 \mathrm{~mm}$-scans were obtained additionally at the levels of the aortic arc, the tracheal carina and the right hemidiaphragm.

Results: Although HR $8 \mathrm{~mm}$-scans did not yield additional details, parenchymal alterations were more precisely displayed than the standard 8 mm-scans. HR $2 \mathrm{~mm}$-scans were superior to both, standard $8 \mathrm{~mm}$ - and HR $8 \mathrm{~mm}$-scans in evaluating small lesions (e.g. small cysts) and combined patterns (e.g. reticular densities within ground-glass opacities). These scans allow for a direct visualization of the pulmonary fissures and thereby a delineation of the pulmonary lobar anatomy.

Conclusions: We recommend HR $8 \mathrm{~mm}$-scans when investigating diffuse interstitial lung diseases. HR $2 \mathrm{~mm}$-scans should be obtained to depict certain lesions more clearly. HR $2 \mathrm{~mm}$-scans are essential when a "road map" for planning a broncho-alveolar lavage is required.

\section{$2.50 \mathrm{PM}$}

152 CT lung density measurements correlated with pulmonary function tests in patients with early emphysema and COPD J. A. Verschakelen, A. Heremans, L. Van Fraeyenhoven, M. Demedts, A. L. Baert; Leuven

Purpose: To evaluate the potential role of CT lung density (LD) measurements in the diagnosis of emphysema and COPD.

Methods: Fourty five patients entered the study: group $A(n=13)$ had functional characteristics of emphysema, group B $(n=10)$ had pulmonary function tests suggestive for COPD without emphysema and group $C(n=22)$ had no signs of COPD or emphysema. LD was measured at TLC and RV using 2 methods (a sector and whole lung field method) and correlated with lung function tests (LFT).

Results: There was a significant correlation between both methods for LD measurement $(p<0.001)$. Significant correlations were found between LD and FEVI, FEV1/VC, FRC, RV, Raw (all \%pred.) (p < 0.001) and TLC and RV/TLC (\%pred.) $(\mathrm{p}<0.05)$. LD at FRC correlated better with PFT than LD at TLC. Group A differed significantly from groups B and C in DLCO ( $<<$ $0.001)$. Groups $A$ and $B$ did not differ significantly from each other in FEVI/VC or LD. However groups A and B differed significantly from group C in FEV1/VC, RV and LD ( $\mathrm{p}<0.003)$.

Conclusions: CT lung density can be reliably measured but gives a better reflection of hyperinflation, which can be present in COPD even in the absence of emphysema, than of the destructive enlargement of distal airspaces, which is typical of emphysema only.

\section{$3.00 \mathrm{PM}$}

153 Densitometric evaluation of high resolution CT-images in healthy and diseased lungs

Rainer Rienmüller, Jürgen Behr, Willi Kalender, Thomas Beinert, Irene Altmann, Manfred Merin; München

Purpose: To describe normal and diseased lungs quantitatively by determining the frequency destribution of CT density values (CTDV) of HRCT-images. Methods: 57 patients divided with respect to their vital capacity (VC) and specific resistance (SR) in those with normal, restrictive, obstructive and restrictive \& obstructive lung function and 6 healthy controls with normal VC and SR were studied using HRCT at $50 \%$ of actual measured VC determined and maintained spirometrically during each exposure. The lung parenchyma was isolated and a quantitative analysis of CT density histograms performed. Results: 4 CT density intervals A (with CTDV $-900 \mathrm{HU}), \mathrm{B}(-899 \ldots-800 \mathrm{HU}$ ), $\mathrm{C}(-799 \ldots-700 \mathrm{HU})$ and $\mathrm{D}(-699 \mathrm{HU})$ were identified in which the calculation of the frequency (relative percents) of CTDV enabled a discrimination not only between patients with normal and pathologic ( $\mathrm{p} 0,01)$ lung function but also between healthy controls and diseased individuals $(p 0,05)$, both with normal $\mathrm{VC}$ and $\mathrm{SR}$.

Conclusions: The determination of the frequency of CTDV in the established CT density intervals from spirometrically standardized HRCT-images provides objective quantitative data reflecting changes of pulmonary structure corresponding to lung function impairment. 


\subsection{PM}

154 Evaluation, rate and treatment of complications after thoracic percutaneous CT guided needle biopsy

Roberto Dore, Giuseppe Di Giulio, Regina Bellantuono, Enrico Di Maggio, Paolo Cremaschi, Patrizio Vitulo; Pavia

Purpose: We have analyzed the complications caused by percutaneous needle biopsy (NB) of chest lesions with the aim to perform these procedures in patients not admitted to hospital.

Methods: We have considered a series of $1020 \mathrm{CT}$ guided NB performed from 1983 to 1990145 for mediastinal and 875 for lung or pleural lesions. Complications were evaluated by CT or clinically.

Results: Complications burdened 359 NB (35\%); they were more rare in mediastinal NB $(13,2 \%)$ than in lung or pleural NB $(24,8 \%)$. Only $23,7 \%$ of all complications were clinically symptomatic. Complications were: 187 pneumothorax (PN) (18,3\% of all NB), 17 of these were treated with needle aspiration $(1,6 \%), 14$ with chest tube $(1,3 \%) ; 165$ pulmonary bleeding $(\mathrm{PB})(16,1 \%), 43$ haemoptysis $(4,2 \%)$. Risk of PN enhanced to 1.9 and 3.4 when superficial are compared with deep lesions; patients upon and patients under 60 years of age had same risk.

Conclusions: CT guide has reduced the risk of the heaviest complications. The high rate of $\mathrm{PB}$ discovered by $\mathrm{CT}$ calls attention to the haematogenous spread of NB tumors. With adequate management of minor complications it is possible to perform NB of chest lesions in the majority of not admitted patients.

\section{$3.20 \mathrm{PM}$}

155 Chiba needle aspiration biopsy of thoracic lesions guided by CT S. Bešlić, F. Dalagija, A. Lovrincěvić, I. Lincender, M. Ibralić; Sarajevo

Percutaneous transthoracic aspiration biopsy (PTAB) is an interventional radiologic procedure with the aim of histologic confirmation or elimination of malignant nature of the lesion.

The development of digital techniques (introduction of CT and US) and small gauge needles increased the interest for this method. CT scanner enables the accurate definition of the athry point of puncture, guide, precise visualisation of the needle and safe analysis of complications.

The type of the puncture needle is very important for the exact diagnosis. Out of 163 Chiba needle punctures $84,7 \%$ of cases had cytologically and pathohistologically representative samples.

The most frequent complication was pneumothorax in $9,1 \%$.

The presented paper shows the evaluation of the method and improvement of Chiba needle, relating to the results of other needle types of various authors. Our results are satisfying with small number of complications.

\subsection{PM}

\section{Fine-needle aspiration biopsy of mediastinal lesions}

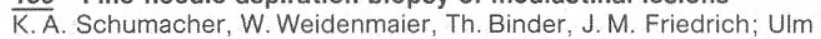

Mediastinal masses can exhibit characteristic radiographic findings in CT and MR. However, to assess the diagnosis invasive procedures have to be employed. Therefore, the diagnostic value of CT-guided fine-needle aspiration biopsy (FNAB) performed with lesions located in all mediastinal areas was examined in 92 patients in which less invasive procedures had not achieved reliable results. Dependent upon the site of the lesion punctures were performed with or without traversion of the lung. Diagnosis obtained with the aid of FNAB were confirmed by pathology and clinical follow-up. The technique yielded a high percentage of correct classification $(>90 \%)$ in cases of malignant tumors as well as in lesions characterized as benign.

\subsection{PM}

\section{A comparative study of CT and transesophageal echography in} seven cases of mediastinal and paramediastinal masses

J. Paez Moreno, J. Vicente Rueda, J. M. Franco Zapata, J. Garcia GarciaRevillo, M. Ramos Gomez; Cordoba

We had studied, during two months (January and February '90), seven patients with mediastinal and paramediastinal masses. The masses were discovered by simple radiography of thorax. Afterwards the patients were studied with transesophageal echography completed with CT. Finally the results of both procedures were compared one to each other.

All patients were males, between 47 and 71 years old. The pathological findings of the masses showed four epidermoid carcinomas, an oat cell carcinoma, an adenocarcinoma and one neurinoma.

We describe the procedure that we follow with the transesophageal echography and the relationships between the echographical and CT images. Finally the morphological features obtained by echography were evaluated in order to investigate the malignancy of processes.

\section{Room C}

\section{SS}

\subsection{General I}

2.00 PM

158 Cineradiography after total laryngectomy

Heribert Müller-Miny, David W. Eisele, Bronwyn Jones; Münster

Purpose: To determine the pharyngeal findings after total laryngectomy compared with clinical data, operation reports, histological findings, tumour staging, radiation therapy and follow up data.

Methods and Materials: We reviewed the cineradioesophagrams of 51 patients with a total number of 68 cineradioesophagrams after total laryngectomy. In 16 patients cineradiography were performed to rule out a fistula, 28 patients were examined for dysphagia and 7 for a suspected recurrence of the tumour. Results: $52 \%$ of all patients had a pseudodiverticulum, and $47 \%$ a narrowing of the upper surgical line. We could not find a relationship to the shape of the sature line of the neopharynx. There was a coincidence in the development of fistulae $(10 \%)$ with large tumours and bilateral neck dissection. Only the minority of this patients were radiated preoperatively. All patients with pouches of the lower surgical line $(6 \%)$ had a history of postoperative fistulae. We found a high accuracy of cineradiography in the determination of recurrences $(6 \%)$ without any false positive or negative result.

Conclusions: Cineradiography after laryngectomy can determine the reason for dysphagia and local recurrence with great confidence.

\subsection{PM}

159 Radiological management of post-surgical strictures of the upper gastro-intestinal tract Alan Grundy, Cynthia Sampson; London

Stricture formation following surgical procedures in the upper gastro-intestinal tract is a common problem. Radiologically guided balloon dilatation is now an accepted method of treatment.

The radiological management of 36 consecutive patients is presented.

20 female and 6 male patients, age range 18-80 years (mean 58 yrs) were referred for radiological management.

21 patients had surgery for malignant disease: 13 for gastric carcinoma, 2 for gastric lymphoma, 3 for esophageal carcinoma, 2 for laryngeal carcinoma and one patient for pancreatic carcinoma. 15 patients had surgery for non-malignant disease: 5 had surgery for morbid obesity, 5 for achalasia, 3 fundoplications for hiatus hernia and 2 gastro-enterostomies for duodenal ulceration.

The procedure was performed under intravenous sedation in all patients. Catheter placement was achieved under radiological guidance in 28 patients and under combined radiological and endoscopic guidance in 8.

There was one technical failure due to inability to negotiate a strictured anastomosis. No complications relating to the procedure occurred.

Symptomatic relief was achieved in all patients who had a successful dilatation. Symptom free intervals ranged from 5 days to 2 years.

Radiological guided dilatation is a safe and effective means of managing post-surgical strictures.

\subsection{PM}

160 Endosonography and CT scan for the prognosis of esophageal squamous cell carcinoma

L. Henry, R. Sanzari, G. Genin, P. Fouque, J.C. Souquet, P. J. Valette, R. Lambert; Lyon

The goal of this study was to compare endosonography (ENS) and computed tomography $(\mathrm{CT})$ in determining the prognosis of patients presenting esophageal squamous cell carcinoma treated by non-surgical procedures. From pathological correlations with resected specimen, it has already been demonstrated that ENS allowed a more precise staging of esophageal squamous cell carcinoma than CT scan.

Methods: ENS and CT scan were performed in 88 patients before treatment and were interpreted independently. Patients were staged according to the 1987 TNM classification. They were treated in the same unit with a multimodal non surgical protocol (laser, radiotherapy and chemotherapy). Actual survival curves were compared by the logrank test.

Results: For the parietal invasion, patient staged T1 at ENS had a significantly better survival than T2 patients (at 36 months: $62 \%$ and $28 \%$ respectively). CT scan was unable to separate these two groups of patients. Prognosis of T3 and T4 patients at ENS was similar at 18 months (about 20\%), while CT scan demonstrated a larger difference ( $42 \%$ vs $18 \%$ respectively). Although patients staged $\mathrm{N}+$ had a significantly poorer prognosis than N0 patients both at CT scan and ENS, ENS depicted more $\mathrm{N}+$ patients.

Conclusions: Although ENS and CT scan are efficient for the overall prognosis value of oesophageal squamous cell carcinoma, this study demon- 
strates ENS superior to CT scan for small tumors, and CT scan more adapted than ENS for larger tumors.

\subsection{PM}

\section{Radiology of the esophagus in HIV-infected patients}

$\bar{T}$. E. Mayer, V. Jacobi, H. Fuchs; Frankfurt/Main

We reviewed 300 barium esophagrams in double and mono contrast technic done out of 2000 HIV positiv patients in Frankfurt.

Frequency of opportunistic infections and sensitivity of the radiographies in correlation to stadium, clinical symptoms, follow up after treatment and endoscopic findings are reported.

Plaques as specific signs of candida-esophagitis and often large superficial ulcers in the distal esophagus in CMV were found.

Because of the noninvasive and unpainful barium studies, they are recommended if there are no typical clinical signs of candida-esophagitis or therapy is not effective.

\section{$2.33 \mathrm{PM}$}

\section{Radiology of the stomach and small intestines in HIV-infected patients}

T. E. Mayer, V. Jacobi, H. Fuchs; Frankfurt/Main

200 barium double contrast radiograms of stomach and duodenum followed by 70 common intestine passages and 25 double contrast enemas of HIV-positiv patients are reviewed.

13 lymphomas, kaposi sarcoma and other tumors were found as mass lesions with and without ulcerations, 2 lymphomas showed lung filiae. Infections and other benign lesions are demonstrated and correlated to clinical and histological results.

Because of the little clinical symptoms of gastrointestinal tumor and the relatively high frequency of these lesions in HIV patients esophagograms often should extended to a full examination of the upper gastrointestinal tract, always added by a common follow through passage.

\subsection{PM}

163 Bacterial ileocaecitis: a "new" disease

J. B. C. M. Puylaert, J. L. Seelen; The Hague

Purpose: To determine the incidence and sonographic features of bacterial infection of the ileocaecal area in patients with clinically suspected appendicitis.

Methods and Materials: Retrospective study of US examinations in 786 conse cutive patients with suspected appendicitis.

Results: In $91(11.6 \%)$ US revealed a characteristic image of enlarged mesenteric lymph nodes and mural thickening of the terminal ileum and caecum, while no inflamed appendix could be visualized. In 64 of these a bacterial infection was confirmed: Yersinia enterocolitica in 28, Campylobacter jejuni in 24, Salmonella enteritidis in 11, Yersinia pseudotuberculosis in 1. In the other 27 , bacteriological tests were either negative (17) or not done (10). Only 34 of 91 had diarrhea. Six of the 91 patients underwent surgery, in all of them the appendix removed was normal. The other 85 patients recovered with conservative treatment. In 37 of these 85 a planned appendictomy was cancelled because of the sonographic findings.

Conclusions: Bacterial enteritis limited to the ileocaecal area (bacterial ileocaecitis) seems to be responsible for an appreciable number of superfluous appendicectomies. It has characteristic sonographic features enabling ready distinction from appendicitis and thus preventing an unnecessary operation.

\section{$2.41 \mathrm{PM}$}

\section{Low-dose C.T. of the abdomen in follow-up studies of oncologic patients}

Alfonso Frigerio, Vincenzo Marra, Gianluca Macchia, M. Teresa Taricco; Torino

Oncologic patients can undergo frequent C.T. examinations for follow-up purposes. In some instances, young patients with lymphomas or testicular cancers are checked bi-annually by C.T. of the abdomen, resulting in considerable radiation doses and an increased risk of developing a second malignancy.

No standard C.T. technique is defined for the follow-up study of the abdomen, yet examinations are generally performed with use of 10 -mm-thick, contiguous sections at $120 \mathrm{kVp}$ and $\mathrm{mAs}$ in the range 200-390 per section, or more.

We developed a technique employing non-contiguous sections at $120 \mathrm{kVp}$ and with low mAs parameters (in the range 120-260).

In 10 patients, radiation dose to thyroid gland was measured by T.L.D.s and the visualization of anathomic details was evaluated by blind reading of lowversus high-dose images, performed by two experienced radiologists.
Results show the potential for obtaining clinically useful images with low-dose C.T. of the abdomen.

\subsection{PM}

\section{US follow-up of transjugular liver biopsy}

E. Coderc, Y. Ajavon, D. Meseure, N. Sellier, F. Mal, P. Callard; Bondy

The transjugular liver biopsy is a safe technique used in patients presenting with abdominal dropsy or coagulation diseases.

To prove the accuracy of a such approach a sonographic follow-up has been performed in 50 patients one hour after biopsy.

In cases of US abnormality or secondary clinical diseases (pain) the patients have been controlled the next day.

The most patients present with a small hyperechoic area, near the right hepatic vein; the location with the capsule is well seen with high frequency transducers Larger hyperechoic area $(2-3 \mathrm{~cm})$ are always resolutive on the second follow-up US study.

\section{$2.47 \mathrm{PM}$}

\section{CT-Guided fine needle biopsy in recurrent rectal carcinoma

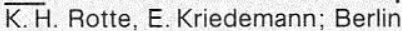

CT is a reliable method in detection of recurrent rectal carcinoma. Before decisions about an appropriate treatment can be done, a cytological or histological proof is required. CT guided fine needle biopsy was performed in 45 patients with suspicious presacral masses after abdomino-perineal rectum resection. Histological and/or cytological diagnoses were proved in $82 \%$ of all biopsies. These findings were corresponding with the clinical and final diagnoses. The advantages and limitations of CT guided fine needle biopsy in recurrent rectal carcinoma are discussed.

\subsection{PM}

167 Hepatic arterial ruptures complicating liver transplantation H. Caillet, P. Cauquil, M. Cauquil, Y. Ajavon, A. Elmaleh, J.P. Verdier, D. Castaing, H. Bismuth, J.P. Tessier; Villejuif

Hepatic artery and hepatic pseudoaneurysms ruptures are rare but serious complications of hepatic transplantation.

Since 1984, 640 liver transplantations were performed on Paul Brousse hospital for varying pathologies. After surgery, seven big arterial bleeding occured $(0,9 \%)$; of these seven cases, three were caused by hepatic artery rupture and four by pseudoaneurysm rupture. Five of the seven patients died. In all cases, liver transplantation was difficult.

All vascular injury but one were caused by sepsis, and occured after a undetermined cause septicemic syndrome. The last vascular injury was caused by percutaneous biopsy.

Six of the seven patients had imaging studies. Sonography ( 3 cases) found sub hepatic hypoechoic collection in two and failed in one. CT ( 4 cases) found sub hepatic collection in three cases and failed in one. Angiography (3 cases) depicted a pseudoaneurysm in all three cases.

At the present time, our behaviour is to perform systematic angiography in patients with undetermined cause septicemic syndrome to research a pseudoaneurysm. And if angiography is normal, to puncture systematically any sub hepatic collection to know bacteriological condition of this collection.

\section{$3.00 \mathrm{PM}$}

168 Radiologic interventions after extended liver resection and transplantation

R. Langer, A. Scholz, M. Langer, F. Astinet, F. J. Ferstl; Berlin

Purpose: The study was performed to evaluate, if re-operation can be avoided by radiologic interventions in patients with complications after hepatic resection and liver transplantation (OLT).

Methods and Materials: In 110 patients after OLT and 95 patients after hepatic resection 45 radiologic interventions were performed in 36 patients under US-, $\mathrm{CT}$-, and/or fluoroscopic guidance.

Results: The indications for radiologic interventions were temperatures of unknown origin or abdominal encapsulated fluid collection. 6 punctures were performed for bacteriologic examinations, the remaining interventions were done therapeutically, including 3 embolisations of the splenic artery in cases with splenohepatic steal after OLT, and 34 drainages of hematomas, abscesses, or bile leakages after extended left hepatic resection. All drainages were successful so that re-operations became unnecessary. After embolisation of the splenic artery 2 splenic abscesses occured so that splenectomy was performed. Conclusions: Percutaneous punctures and drainages are the modalities of choice in cases with postoperative fluid collection after OLT or hepatic resection. By these procedures re-operation can be prevented. In patients with splenohepatic steal embolisation of the splenic artery should be avoided because of the patients' extended immunosuppression. 
169 Liver metastasis detection: comparative sensitivities of US, CT and MR imaging for preoperative evaluation

Karl Wernecke, Ernst Rummeny, Georg Bongartz, Pierre Vassallo, Dietmar Kivelitz, Werner Wiesmann, Peter E. Peters, Berthold Reers, Maximilian Reiser, Wilfried Pircher; Münster

Purpose: To evaluate the sensitivity of US, CT and MR in the detection of hepatic metastases, a prospective study was conducted in 75 consecutive patients with gastrointestinal tumors admitted for surgical therapy.

Methods: Sonography was performed by experienced radiologists with convex transducers of 3.5 and $5.0 \mathrm{MHz}$. Three non-invasive CT techniques were used: unenhanced CT scans, the "incremental bolus dynamic scan technique" and delayed scanning 4 to 6 hours after bolus injection of $60 \mathrm{~g}$ of iodine. MR-images $(1.5 \mathrm{~T})$ were acquired as presaturated T1- and T2-weighted spin echo sequences. Each examination was performed blindly and the results were compared using surgical examination and intraoperative high resolution sonography of the liver as the gold standard.

Results: $65(68.4 \%)$ of 95 lesions were detected by CT, 60 lesions $(63.2 \%)$ by MR and 50 lesions $(52.6 \%)$ were detected by sonography. While lesions measuring from 1 to $2 \mathrm{~cm}$ were demonstrated almost equally well by CT and MR $(74.4 \%$ and $76.9 \%)$ the detection rate of smaller lesions $(<1.0 \mathrm{~cm})$ decreased more drastically with MR (31.4\%) than with CT $(48.6 \%)$. Sonography showed a sensitivity of only $20.0 \%$ with the smaller lesions.

Conclusions: At present, for the precise assessment of number, size, and location of focal hepatic lesions, CT has the highest overall sensitivity $(68.4 \%)$ of all cross-sectional imaging techniques. On combining the information from all imaging techniques, the overall sensitivity of $76.8 \%$ still remains unsatisfactorily low, as CT and MR have a size threshold of about $1.0 \mathrm{~cm}$ and are relatively unreliable for the detection of smaller lesions.

\section{$3.20 \mathrm{PM}$}

170 Close monitoring of liver metastases with MRI and US in patients with solid tumours

A. Giovagnoni, G. Argalia, P. Ercolani, A. Piga, R. Cellerino, P. Perotti, G. M. Giuseppetti; Torrette di Ancona

Purpose: The purpose of this study was to evaluate the diagnostic value of MRI and US in monitoring liver metastases from solid tumours in patients on chemotherapy.

Methods: 20 patients with liver metastases were evaluated with liver MRI and US before treatment and monthly thereafter (a total of $80 \mathrm{MRI}$ studies and 80 US were blindly evaluated). MRI investigations were performed using a superconductive 1.0 T (Magnetom) tomograph. Axial T1 $(500 / 17)$ and T2 (2000/19-90) pulse sequences were used. US examination was performed using an Ansaldo AU 590, convex array $3.5 \mathrm{MHz}$. Findings were correlated with clinical parameters.

Results: 1. Major discordances between MRI and US were found in detection and structure evaluation of the metastatic lesions, in particular following treatment.

2. Particularly with MRI, reduction of existing lesions was observed in responding patients early during treatment, with no effect of subsequent courses.

Conclusions: Despite the utility and cost-effectiveness of US in diagnosis and monitoring liver metastases, its role should be considered controversial; modifications induced, on the lesion and/or liver parenchyma, by treatment, may induce a lower contrast between lesion and liver, with subsequent poor performance of the technique. Both techniques, when employed in close monitoring of liver lesions show the maximum effect of treatment to be obtained with the first courses.

\section{Room E1}

\section{SS}

\subsection{New Techniques in PTA}

\section{$2.00 \mathrm{PM}$}

171 Peripheral vessels: angioplasty, atherectomy and stenting (Keynote Lecture)

E. Zeitler; Nürnberg

The peripheral arterial occlusive disease (PAOD) is essentially due to the risk factors cigarette smoking, diabetes, age, hypertension and hypercholesterolemia. Most patients with PAOD Fontaine classification IIa to IV can be treated with one of the multiple interventional radiological techniques (IR). With the modifications of Grüntzig's balloon catheter - in combination with modern types of guidewires - more than $80 \%$ of the PAOD-patients can be treated successfully. Localized excentric stenoses can be treated with atherectomy devices. Total occlusions with short history can be treated with local thrombolysis (urokinase, rt-plasminogen) in combination with PTA.
Occlusions with longer history can be recanalized in up to $80 \%$ with special rotating devices (ROTAX, BARD; RAT-PAT or others), and with laserassisted angioplasty. In case of good peripheral run-off vessels, the femorocrural bypass may be a strong alternative for patients with gangrene.

But in patients with bad peripheral run-off, all possibilities of modern angioplasty have to be exhausted before leg amputation. In patients with dissection, calcified stenoses or arterial collaps in the iliac and proximal femoral arteries, the application of an intraarterial stent (Palmaz, Strecker, Wallstent or others) can be an excellent alternative to vascular surgery.

Angioplasty is also indicated before and after vascular surgery to improve the in- and outflow condition. In addition to the transfemoral route of angioplasty from the ipsi- and contralateral side, the transpopliteal retrograde technique becomes more and more important in cases with scars in the groin or long distance occlusions. Primary success rates of balloon angioplasty today vary between 70 and $96 \%$, the 5 year patency rate between 60 and $85 \%$, depending on the type of the lesion. Also the three year patency after stent application in the proximal high flow arteries is excellent.

Close interdisciplinary cooperation is necessary. Indications, results, criticism and special recommendations will be part of the lecture.

\subsection{PM}

172 Long-term patency rate of iliac and femoral arterial occlusive disease treated with flexible Tantalum stents and a new anticoagulation therapy

E.P. Strecker, D. Liermann, B. Hagen, H.R. D. Wolf, B. Schneider; Karlsruhe

Methods: Clinical studies with expandable stents have proved that primary PTA results can be optimized, and long-term results appear for iliac arteries promising, for femoral arteries there are laking and controversial informations demanding new therapeutic concepts.

Methods: So far over 200 patients with stenosis and occlusions of iliac and femoral popliteal arteries where treated with flexible tantalum stents following primarily insufficient PTA results. Because of the known tendency of stent restenosis in femoral arteries 40 patients received for three weeks two types of new molecular heparinoids to decrease the proliferation of the smooth muscle cells causing intimal hyperplasia.

Results: There was no recurrent disease in iliac artery lesions, the longest observation time being 45 months. This was confirmed by clinical examinations with evaluation of the ancle-arm Doppler indexes and angiograms. In femoral lesions restenosis were found in 25 percent beginning 4 months after implantation. Those lesions responded well to repeat angioplasty, although second and third recurrencies were observed. Better results were obtained with the three weeks application of heparinoids.

Conclusions: The developed vascular stent therapy reveals good patency rates for stenosed and occluded iliac arteries. Recurrent disease is relatively high in stented femoral arteries, the developed new anticoagulation therapy promises better patency rates in this anatomical area.

\section{$2.25 \mathrm{PM}$}

\section{Long-term patency of stented arteriovenous shunts}

D. Vorwerk, R.W. Günther, K. Bohndorf, D. Kistler, U. Gladziwa, H. G. Sieberth; Aachen

Self-expandable endoprostheses were used as an alternative percutaneous approach after failed or complicated balloon angioplasty of arteriovenous shunts.

18 av fistulae were treated. 16 lesions were situated in the draining brachial $(\mathrm{n}=15)$ or central $(\mathrm{n}=1)$ veins of hemodialysis fistulae (9 PTFE shunts, 7 Brescia-Cimino shunts). In 2 patients, venous stenoses (1 common iliac, 1 common femoral vein) after surgical av fistulization for iliac thrombosis were stented. Indications for stenting were an insufficient result of balloon angioplasty in 7 patients or complications after treatment in 11 patients.

One acute thrombosis leading to permanent shunt occlusion occurred. Within an average follow-up period of 13.6 months, mean interval of repeated intervention within the shunt was 5 months; shunt patency was $28 \%$ at 24 -month follow-up. Due to reintervention, chance for shunt survival, however, was $79 \%$ at 2-year follow-up.

Stents have potentials to improve the acute results of failing percutaneous shunt management and allow prolongation of shunt survival in those cases. Restenosis by hyperplasia, however, cannot be prevented by stent placement.

\section{$2.35 \mathrm{PM}$}

174 Renal artery stenting with the Wallstent ${ }^{R}$ endoprosthesis G. Wilms, P.Peene, A. L. Baert, A. Nevelsteen, R. Suy, R. Verhaeghe, J. Vermylen, R. Fagard; Leuven

Renal artery stenting with the Wallstent ${ }^{\mathrm{R}}$ endoprosthesis was performed in 13 renal arteries of 14 patients. A total of 18 stents were placed. Indication concerned restenosis after dilatation in 9 patients, insufficient result after 
dilatation in 5 cases and obstructing intimal flap postdilatation in 1 patient. 7 lesions were postostial, 8 were ostial and postostial. Main technical problems concerned protrusion of the stent within the aorta, that was noted in 8 patients In two patients, malpositioning of the stent, due to poorly predictable shortening, required placement of a second stent. Complications concerned a case of massive cholesterolembolisation and a case of unexplained transient hematuria, proteinuria and deterioration of renal function. Of 7 stented renal arteries that underwent repeat angiography, 1 was occluded requiring thrombolysis and dilatation. Another showed relapse of stenosis due to shortening of the stent, and required redilatation and later on restenting. This stent occluded after 1 month. In this patient and in 3 other cases angiography disclosed tissue build-up within the stent, but without significant stenotic effect after follow up of $6.7 \pm 3.4$ months. Late follow up on blood pressure and stent patency will be presented.

\section{$2.45 \mathrm{PM}$}

\section{Transluminal angioplasty and endovascular prosthesis (Strecker)} in the treatment of renal artery stenosis

Aytekin Besim, Ferhun Balkancl, Ayșenur Cila, Cetin Turgan, Turgay Arinsoy; Ankara

Purpose: We have evaluated the efficiency of the tantalum stent (Strecker) as a supplementary to PTA in the treatment of renal artery stenosis.

Methods and Materials: 12 stents were placed in the renal arteries of 6 cases of fibromuscular dysplasia (FMD), 5 cases of atherosclerosis and one case of Takayashu arteritis. Involvement of left renal artery was in 3 cases and right renal artery in 9 cases. Indications were immediate failure of PTA or restenosis in early follow up. Direct implantation of the stent was done in 5 cases of FMD immediately after PTA because of high recurrence rate in this group.

Results: Stent placement was technically possible and successful in all cases. Follow up control was available from 8 months to one and a half year. No stent migration, thrombosis or restenosis was observed.

Conclusions: The Strecker flexible balloon expandible tantalum stent is safe and successfull in the treatment of stenosis after the failure of PTA. Because of high recurrence rate in long term follow up after PTA in FMD patients: direct implantation of the stent seems to be a promising approach in the treatment of this group with renal artery stenosis.

\section{$2.55 \mathrm{PM}$}

\section{A new therapy of intimahyperplasia after stent-implantation in peripheral vessels}

Dieter Liermann, H.D. Böttcher, J. Kollath, V.Scherkamp, B. Schopohl, K. Breddin; Frankfurt/Main

Purpose: Unfortunately intimahyperplasia after stent-implantation in peripheral vessels will appear after 6 months or later, causing a restenosis and a reocclusion of the vessel sometimes. All wellknown methods like laser, atherectomy or Re-PTA are not able to prevent the stented area from a restenosis sufficiently. Using an afterloading technique in the stented area, there seems to be an interesting way to prevent restenosis caused by intimahyperplasia.

Methods: Since 1987 Tantalum balloon-expandable stents are implanted after recanalization and PTA of different vessel-localisations. In some cases especially in the adductorial channel restenosis by intimahyperplasia occurs 6 months after stent-implantation. Laser and Re-PTA suffers also only for an period up to the next intimahyperplasia. In such a situation, after recanalisation of the occlusion by PTA or Laser we use an endovascular afterloading therapy with a single dosis of 12 Gy after positioning catheter in the right position under fluoroscopy with a 197 Iridium-HDR afterloading method. Results: The follow up of the patients treated with these methods showed up to now (longer than 6 months) no rezidives of intimahyperplasia.

Conclusions: If long-therm results will be as positive, a routinously afterloading therapy in peripheral vessels should be discussed.

\section{$3.00 \mathrm{PM}$}

\section{A new "Over the wire" pullback atherectomy catheter-PAC}

$\bar{R}$.I. White Jr., D. F. Denny Jr., T. A. Fischell, R. E. Fischell, D. Markowitz, M. Yoselevitz, J. Pollak; New Haven, CT

Purpose: A new percutaneous atherectomy catheter (PAC), introduced over a guidewire, has been designed for retrograde removal of atheroma from the superficial femoral, popliteal and trifurcation arteries.

Methods: The design of the catheter included a conical cutter and collecting chamber. Introduced through a percutaneous sheath over an 0.018 inch guidewire, catheters varying in diameter from $2.0-3.5 \mathrm{~mm}$, sequentially shave and collect atheroma. Rotation of the cutter at 2000 RPM is provided by a battery operator rotator. PAC was tested in 10 severely diseased cadaver superficial femoral arteries (SFAS) mounted in a perfusion chamber and in 3 in situ cadaver SFAS via arterial cutdown.

Results: In the 10 mounted and perfused SFAS, stenosis was reduced $97 \pm 2 \%$ to $22 \pm 4 \%$. In the 3 in situ SFAS, PAC atherectomy resulted in a reduction of stenosis from $92 \pm 4 \%$ to $18 \pm 7 \%$. Large particle emboli were not observed. Pathology of PAC atherectomy resembled surgical endarterectomy. Excellent luminal restoration was achieved with and without balloon angioplasty in the first 7 patients who have undergone PAC atherectomy for SFA occlusive disease since May 1990. An additional 3 patients comprising the Phase 1 trial will be treated over the spring and summer.

Conclusions: The current PAC design provides simplified atheroma removal of diffuse and focal occlusive disease of the femoropopliteal artery.

\subsection{PM}

178 Late results of "Simpson-Atherectomy" in peripheral arteries H. Lugmayr, M. Deutsch, O. Pachinger, W. Pauer; Wels

Beetween March 1988 and November 198994 patients with peripheral vascular desease were treated with the Simpson-Atherectomy catheter.

During this period atherectomy was done in all patients, so far morphology allowed the intervention. Patency rate in a non selected material was $68,5 \%$ after 12 months, $56,4 \%$ after 18 months and $42,3 \%$ after 24 months.

It therefore equals balloon dilatation. Simpson-Atherectomy therefore is not recommanded generally.

Good morphological and late results are found in solitary excentric stenoses and in significant residual stenoses after balloondilatation. These indications therefore are now recommended as ideal.

\section{$3.20 \mathrm{PM}$}

\section{Initial results of a randomised trial of Simpson atherectomy versus} balloon angioplasty of femoral-popliteal stenosis

A. V. Tielbeek, G. H. M. Landman, J. Buth; Eindhoven

Patients with different types of stenosis in the femoral-popliteal traject were randomised to treatment using the Simpson atherectomy catheter or conventional balloon angioplasty. Combination of these methods was not performed in this group of patients. Informed consent was obtained in all patients. From January 1990 to April 1991, Simpson atherectomy and balloon angioplasty was performed in 40 patients. In 30 patients, atherectomy or balloon angioplasty was done percutaneously and in 10 , it was performed after surgical exposure of the femoral artery in the operating room.

Results: Atherectomy in the angiographic room was technically unsuccessful in only one patient because of obesitas. No significant dissection, distal embolisation or bleeding were observed in the 40 patients.

Histologic pattern of removed atherosclerotic material, angiographic and clinic short-term results will be reported in detail.

\subsection{PM}

180 Early results with Rotation-Aspiration-Thromboembolectomy catheter after Starck (RAT)

H. Lugmayr, M. Deutsch, O. Pachinger, W. Pauer; Wels

The common therapeutic approach for recent thromboses and embolisms was up to now local lysis. RAT offers two alternatives: First aspiration and second removal by a spiral which is drilled into the plug like a corkscrew. Using this method we successfully treated 11 embolisms of the A. poplitea and 3 extensive thromboses of the A. fem. sup. between September und December 1990.

In the case of embolisms we were able to remove the embolic material in all cases. In case of thrombosis the underlying arterial stenoses could always be treated during the same session.

RAT therefore can be described as a precious addition to interventional therapy of peripheral vessels, which allows either reduction in the dosis of lytic agents or complete renunciation of local lysis in many cases.

Compared to open operation emboli can be removed completely using minimal invasive therapy.

We cannot offer any findings concerning late results up to now.

\section{Room E2}

\section{SS}

$2.00 \mathrm{PM}$

\subsection{Chest}

\section{Imaging immunodeficiency in children (Keynote Lecture) Jack O. Haller; Brooklyn, NY}

The normal functions of the immune system entail a complicated interplay of lymphocytes, phagocytes and various effector and mediator systems. Immune deficiencies refer either to defects in function or frank absence of these features. The common denominator to all diseases of the immune system is infection. The essential criterion for diagnosing immune deficiency is the perception that the child is not capable of handling infections normally. This 
clinical suspicion demands an understanding by the radiologist of the body's range of normal responses to different organisms and a familiarity with the ways in which immune deficiencies may present. Pattern recognition is vital to diagnosis. There are two main types of immune deficiency diseases, primary and secondary. It may be quite evident, for example, that the child has a condition, disease or treatment known to be associated with immune deficiency such as prematurity, infancy, malnutrition, malignant disease, cytotoxic or corticosteroid therapy. Acquired infection such as AIDS may render the child immune deficient as well. These are considered secondary immune deficiency. Alternatively the child may simply present with an infection of a type, severity, pattern or course that implies opportunism. The organism has taken advantage of a defect in host response prompting a search for an underlying congenital immune deficiency.

This talk will highlight the key imaging features common to both primary and secondary immune deficiencies with special emphasis on AIDS and the contribution of older and newer modalities to its diagnosis.

\subsection{PM}

\section{Persistent pneumonia in childhood}

M.-H. Smet, K. De Boeck, G. Marchal, A. Lerut, J. Verschakelen; Leuven

Purpose: In children with persistent pneumonia the incidence and nature of underlying disease were investigated. The value of a follow-up chest X-ray 17 to 21 days after acute pneumonia was evaluated.

Methods: We prospectively evaluated 298 children with acute pneumonia and treated accordingly, but whose follow-up chest X-ray still showed a marked consolidation 17 to 21 days later.

Results: 50 patients presented with persistent pneumonia. 5 children were referred because of persistent pneumonia but in fact had the coincidence of fever, respiratory tract infection and a consolidation on chest X-ray mimicking a pneumonia. In 45 patients a true pulmonary problem was present. An underlying disorder was found in 38 of 45 children: congenital lung malformation (8), congenital cardiovascular anomaly (8), bronchiectasis (6), immunodeficiency (5), gastroesophageal reflux (5), foreign body aspiration (3), cystic fibrosis (2) and immotile cilia syndrome (1).

Physical examination was still positive 17 to 21 days after the acute onset of pneumonia in 37 children.

Conclusions: Persistent pneumonia is not an unusual problem in childhood and is suspicious of underlying disorder. Routine repeat chest X-ray in children with acute pneumonia may not be necessary unless there is clinical evidence of persistent respiratory difficulty or failure to thrive.

\subsection{PM}

\section{Primary tumours of lungs and pleura in children Sorna Thurairajasingam; Salford}

The purpose of this study was to observe the incidence and prognosis of primary tumours of lungs and pleura in children.

A survey was made of all children suffering from malignant disease of chest admitted to Royal Manchester Children's Hospital over the last 15 years.

Plain films, computed axial Tomography, Bronchoscopy and Biopsy were employed in the diagnosis. There were 4 cases of primary tumours of lungs and one of pleura as follows:

LUNGS

Pulmonary blastoma 1

Carcinoma of lung 2

Fibrosarcoma 1

PLEURA

Rhabdomyosarcoma 1

All tumours had invaded the mediastinum at presentation.

Except the fibrosarcoma, the rest produced distant metastases. Despite treatment, all proved fatal.

Primary tumours of lungs and pleura are rare in children. They carry a bad prognosis.

\section{$2.35 \mathrm{PM}$}

\section{Congenital leukaemia}

Sorna Thurairajasingam; Salford

A baby girl born by caesarian section at 41 weeks presented with abdominal masses after 5 weeks. Ultrasound Scan showed the masses to be due to massive hepatosplenomegaly. Her white cell count was extremely high. Bone Marrow examination revealed null cell type of acute lymphoblastic leukaemia. Fits occurred soon after. Chest X-rays showed bilateral lung infiltrates. Despite treatment, she developed respiratory and renal failure and died. All clinical features and diagnosis before 3 months of life lead to the conclusion that this was congenital leukaemia with grave prognosis because of the very high white cell count and central nervous system involvement.

\subsection{PM}

185 MRI in the follow-up of children after angioplasty of coarctation of the aorta (CoA)

Ch. Ehrenheim, W. Burchert, H. Kaemmerer, W. Wilken, I. Luhmer, H. C. Kallfelz, H. Hundeshagen; Hannover

Aortic aneurysm and restenosis are the most severe postinterventional complications after angioplasty of $\mathrm{CoA}$ and require regular follow-up. Besides the routine examinations, 20 children ( 15 boys, 5 girls) underwent noninvasive MR imaging within 3 months to $51 / 2$ years after dilalation.

4 children showed pathological changes of the ascending aorta, 2 of them had a moderate ectasy, two had a severe dilatation of $\geq 5 \mathrm{~cm}$ in diameter. The aortic arch was normal in 15 cases, 5 children showed small diameters. The origin of the left subclavian artery was displaced distally in 7 patients. In all cases mild to moderate changes of the wall or the lumen were observed at the site of CoA. The descending aorta was moderate spindly dilated in 12 children. In three cases a circumscribed aneurysm was found.

The comparison with clinical examinations showed, that MRI can be applied preferably in the follow-up of patients after angioplasty of CoA, especially in children.

\section{$2.41 \mathrm{PM}$}

186 Contribution of MRI in the evaluation of anomalies of the aortic arch complex in infants

B. Kastler, A. Livolsi, Y. Lebras, P. Germain, R. M. Allal, J. L. Dietemann, D. Willard, A. Wackenheim; Strasbourg

Purpose: Evaluate the utility of MRI in identifying Aortic Arch Anomalies (AAA) in a very low aged pediatric population.

Methods: 15 infants aged 4 to 91 days underwent MR imaging on a $0.5 \mathrm{~T} \mathrm{GE}$ MR Max imager with ECG gated single echo SE sequences. 10 patients had symptoms and 5 findings of vascular ring (VR) on barium oesophagography. 3 patients had additional complex cardiac anomalies (CHD) - all were also examined by 2 D Echo.

Results: (confirmed by surgery: 7 and autopsy: 1).

In 3 patients MRI excluded AAA. In 8 patients it precisely confirmed the type of AAA (double aortic arch, aberrant left subclavian artery, circumflex retrooesophageal Ao Arch). In one patient MRI just confirmed on Ao A interruption and in the 3 patients with complex CHD MRI complemented $2 \mathrm{E}$ results (subdiaphragmatic TAPVD, left aberrant subclavian artery, compression of left main bronchus by PDA and left pulmonary artery), 2 DE was diagnostic in none of the VR.

Conclusions: MRI is safe and efficient in the diagnostic management of AAA in infants.

\section{$2.51 \mathrm{PM}$}

\section{Dilatative cardiomyopathy versus myocarditis in pediatric} patients: assessment by means of MRI

P. Di Renzi, M. G. Gagliardi, E. Di Cesare, M. Bevilacqua, R. Passariello; L'Aquila, Rome

Purpose: To obtain a non invasive diagnosis of myocarditis in pediatric patients with dilated heart. When diagnosis of myocarditis by means of endocardial biopsy is made, further evaluation needs on or more endocardial biopsies.

Methods: 19 pediatric patients ( 5 controls, 7 with dilatative myocardiopathy or pregress myocarditis and 7 with active myocarditis) underwent MR investigation by means of a $0.2 \mathrm{~T}$ permanent magnet with spin-echo sequences in axial planes. T1 weighted sequences and Proton Density and T2 weighted sequences were obtained in the same planes. Myocardial/skeletal muscle region of interest ratio was calculated for $\mathrm{T} 1, \mathrm{PD}$ and $\mathrm{T} 2$ weighted sequences. Mean percentual differences between $\mathrm{T} 1$ and $\mathrm{PD}$ and $\mathrm{T} 2$ sequences were also obtained. Results were blindly compared to endocardial biopsy.

Results: $t$ test for unpaired data (controls $+\mathrm{II}^{\circ}$ group versus myocarditis) shows the following results:

- SE T1 Weighted sequences

- SE PD sequences $(T E=90 \mathrm{~ms})$

$\mathrm{p}=$ not significant

$-\delta \% \mathrm{~T} 1-\mathrm{TE}=90$

$\mathrm{p}<0.005$

$-\delta \mathrm{T} 1-\mathrm{TE}=110$

$\mathrm{p}<0.01$

$\mathrm{p}<0.001$

Conclusions: Our study demonstrates that it is possible to obtain a non invasive diagnosis of myocarditis and perhaps avoid or reduce endocardial biopsies. 


\subsection{PM}

188 Pulmonary $\mathrm{x}$-ray changes after therapeutic use of Surfactant in the respiratory distress syndrome (RDS) of the newborn

C. Müller-Leisse, U. Bick, J. Tröger, J. Meyer zum Wendischoff, G. Jorch; Aachen, Münster, Heidelberg

Purpose: To determine typical pulmonary $\mathrm{x}$-ray changes after therapeutic use of surfactant and analyze their relationship to the clinical response

Methods: As part of a multicenter surfactant rescue study, the chest $\mathrm{x}$-rays of 239 preterm and term infants were analyzed (gestational age $30.0+3.2$ weeks). To study the influence of surfactant administration on radiographic appearance, 138 patients with the clinical and radiological diagnosis of typical RDS were selected, in whom adequate chest $\mathrm{x}$-rays before and within 48 hours after treatment were available.

Results: In 52 of 138 patients a symmetrical improvement of pulmonary aeration was observed after surfactant administration, corresponding to a significant lower posttreatment oxygen requirement in $85 \%$ of these patients. Asymmetric clearance especially on the right side was common (58 of 138). In most cases, these focal air disturbances disappeared within two to five days. 17 patients showed no change or deterioration of ventilation, in the remaining 11 patients a determination was not possible because of other findings (e.g. pneumothorax or interstitial emphysema).

Conclusions: Radiographic improvement of pulmonary aeration after surfactant treatment correlates well with amelioration of the clinical situation. Transient focal air disturbances after surfactant administration are common, however usually without clinical significance.

\subsection{PM}

189 The relationship of fluid restriction during the first month of life to the occurrence and severity of bronchopulmonary dysplasia in low birth weight infants. A one-year radiographic follow-up

O. K. T. Tammela, F. P. Lanning, M. E. Koivisto; Oulu

One hundred consecutive low birth weight (LBW) infants $(<1751 \mathrm{~g})$ were randomized into a study group having a restricted fluid intake until four weeks of age and a control group following the fluid regimen conventionally used in the hospital. Chest X-rays were examined on admission, at the ages of 3 days, 7 days, two weeks and four weeks and at two-monthly visits to the outpatient clinic up to one year of age or until the chest examinations were normal. The severity of hyaline membrane disease (HMD) and typical radiologic abnormalities of bronchopulmonary dysplasia (BPD) were assessed.

Twelve of the patients succumbed, one in the study group and eleven in the control group. The study group seemed experience less severe HMD than the controls. $54 \%$ of the former and $32 \%$ of the latter were alive and had no radiographic signs of BPD at four weeks of age $(p<0.05)$. The difference between the groups in the cumulative number of normal chest X-ray examinations during the follow-up was even more significant. The percentage of normal $\mathrm{X}$-rays at one year of age was $92 \%$ in the study group and $72 \%$ in the control group $(\mathrm{p}<0.01)$.

These results suggest that fluid restriction for the first four weeks of life can lower the incidence of radiologic abnormalities typical of BPD obtained during the first year of life in LBW infants. Pulmonary edema seems to be a significant etiologic factor causing HMD to develop into chronic lung disease.

\subsection{PM}

\section{Tracheal stenosis: assessment by MRI and endoscopic investiga- tions}

M. Stiskal, A. Neuhold, B. Schwaighofer, W. Geissler, L. Wicke; Wien

Purpose: MRI was performed to determine the length and width of tracheal stenosis in children. These results were compared to endoscopic findings.

Methods and Materials: 20 children were investigated with a 0,5 T superconductive unit using a SE $3 \mathrm{D}$ overcontiguous slice technique. $\mathrm{T} 1$ weighted images (TR: 550/30) were obtained in axial, sagittal and coronal planes, T2 weighted images (TR: $1500 \mathrm{~ms}$, TE: $50 / 100 \mathrm{~ms}$ ) only in axial planes. Sections of $4 \mathrm{~mm}$ slice thickness and a matrix of $256 \times 256$ were used. The FOV was $160 \mathrm{~mm}$. Results: Our results demonstrate that the sensitivity and specificity of MRI and endoscopy were equal in the evaluation of long distance tracheal stenosis. Short distance stenosis, especially in the subglottic space, were better demonstrated with endoscopy than with MRI because of the limited spatial resolution of MRI. MRI was superior to endoscopy in the evaluation of the parapharyngeal structures and the tracheostoma region.

Conclusions: MRI is a noninvasive technique and can replace endoscopy in follow up studies of the tracheal stenosis in children. Only in children with short stenosis in the subglottic space additional endoscopy should be performed.

\section{Room L}

\subsection{Spine}

\section{Nuclear imaging of the spine (Keynote Lecture)}

\section{Fischer; Kassel}

\section{Bone scintigraphy}

One of the most useful tools of nuclear medicine is skeletal imaging. The number of bone scintigraphies has increased since 1971 when $99 \mathrm{~m}$ Tc labelled phosphonates were introduced for this procedure. Nuclear medicine imaging with "bone seeking" radiopharmaceuticals is highly sensitive but less specific. The localization of these agents in normal bone is desirable. The relative bone uptake depends on vascularity (local blood flow), bone formation and ionic exchange. Increased tracer uptake is not in itself diagnostic for malignancy, nor does a positive bone image differentiate between malignant and benign tumors. All areas of increased osteogenic activity secondary to tumors, trauma or other diseases may be visualized. Development of new radiopharmaceutical agents and improvement of gamma cameras provide clear visualization of the skeleton. The major indication for radionuclide imaging of the spine is the localization of primary bone tumors or the search for metastases in patients under treatment for malignant tumors. The second major indication is the evaluation of the spine as a source of pain without apparent radiological signs. In some cases (f.e. osteoid osteoma) spine imaging may be helpful for localization of remnant tumor tissue immediately after surgery. For differentiation of various bone lesions quantitative analysis of bone scans, SPECT or the use of other radiopharmaceuticals are needed. In this field imaging of neoplastic and inflammatory lesions of the spine with $67 \mathrm{Ga}$ or radiolabelled monoclonal antibodies plays a role.

Nuclear imaging of the spine is the most sensitive investigation for the detection of bone pathology. Because of the non-specificity additional radiographs are needed in many patients.

II. Bone mass measurement

Osteoporosis is the most common disease of the skeleton. It is characterized by an increased bone mass loss. In the U.S. about 1.2 million fractures because of osteoporosis are observed each year, half of them in the spine. Measurement of bone mineral content (BMC) of the lumbar spine may help for calculating fracture risk and to monitore patients under antiosteoporotic treatment. During the last 2 decades various methods were developed with different precision, reliability and radiation exposure. After using $153 \mathrm{Gd}$ as radionuclide source for dual-photon absorptiometry (DPA) DXA using an X-ray tube is superior for measuring BMC because of high photon flux $(1: 500)$, better reproducibility and shorter scan time. With DXA lateral scanning of lumbar spine sensitivity of this procedure is similar to QCT.

\subsection{PM}

192 Differential features in the diagnostic imaging of vertebral collapse

M. G. Bonetti, T. Scarabino, A. Casillo, G. M. Giannatempo, M. Cammisa; Rome

146 consecutive pts. with 547 collapsed vertebrae underwent projective radiography (PR: conventional and/or digital technique) and MRI. Out of the serie, 70 pts. also underwent CT and 30 bone scan. Morphologic and SI patterns of collapsed vertebrae were analized in order to predict the nature of the lesion, either benign (B) or malignant (M). The following parameters were considered: vertebral endplates appearance (focal concave, B 10\%, M 1\%; diffuse concave, B $24 \%$, M 16\%; focal angled, B and M $11 \%$; diffused angled, B $11 \%$, M $26 \%$ ), neural arch (B 3\%, M 16\%), paravertebral soft tissue (B 6\%, M $29 \%$ ), spinal canal (B 11\%, M 20\%) and disc (B 24\%, M $8 \%$ ) involvements. MRI signal showed 4 patterns: low SI in T1-WI and high SI in T2-WI (B 2\%, M 49\%), low $\mathrm{SI}$ in all sequences (B 7\%, M 25\%), isointense signal in all sequences (B $50 \%, \mathrm{M}$ $21 \%$ ), high SI in all sequences (B $41 \%, \mathrm{M} 0 \%$ ). Recently STIR sequence was implemented and used in 15 more pts., proving to be very sensitive to pathological vertebral bone and disc changes and very useful in shortening examination time. The following diagnostic protocol for vertebral collapse is proposed: 1 . PR; 2. MRI; eventually 3 . CT, on selected pathological levels. Bone scan is useful only in finding out bone metastases other than spine one.

\subsection{PM}

\section{Real time multi planar CT and SPECT scanning in investigation of spondylolysis}

N. Raby, S. Mathews; London

Twenty nine patients with chronic low back pain and spondylolysis were investigated with real time multiplanar CT (MPR) and nuclear medicine SPECT scans. The results were compared with clinical evaluation and the traditional method of external lumbar immobilisation in a plaster jacket (POP test) to determine whether scanning could predict those patients who would benefit 
from a spinal fusion.

MPR was particularly useful in identifying disc abnormalities especially in allowing evaluation of encroachment of nerve roots within exit foramina. Two patients with significant disc disease and one with facet joint abnormalities were identified which resulted in planned fusion operations being superceded by more appropriate treatment. Seventeen patients were managed conservatively. All had normal SPECT scans. Of 12 patients considered for surgery on the basis of positive POP tests 6 with positive SPECT scans were rendered symptom free. Three had persistence of symptoms following spinal fusion, all of these had negative SPECT scans.

We conclude that the combination of MPR and SPECT will identify a treatable cause of pain in patients with spondylolysis in $40 \%$ of cases. SPECT scanning is more accurate than the traditional method of identifying these patients who will benefit from spinal fusion and can prevent inappropriate fusion operations from being performed.

\subsection{PM}

194 The importance of radiologically measured lumbosacral angle in cases with discopathy

Mehmet K. Kagan, Ismail Yavuz; Istanbul

We have tried to establish a relation between discopathy at lumbar region and lumbosacral angle. The importance of lumbosacral angle has been argued for long years.

In our study, we have measured lumbosacral angle by FERFUSON method from the lumbosacral lateral radiography. We have seen that the lumbosacral angle is narrower in the cases with discopathy then in the normal cases.

In every patient having lumbalgy complaint, we think that it is useful to measure lumbosacral angle for the early diagnosis of discopathy since it is a simple, easy, practical and economical method.

\subsection{PM}

195 Functional atlanto-occipital (C0-C1) block in flexion-extension radiographs of cervical spine

H. Manninen, K.-A. Lindgren, L. Niemitukia, M. Hakola; Kuopio

Purpose: To evaluate motion of upper cervical spine, especially atlanto-occipital joint in flexion extension radiographs.

Methods and Materials: Lateral radiographs taken during maximal passive flexion and extension of the cervical spine of 100 consecutive patients with cephalalgia and neck pain were retrospectively analyzed. Posterior interspace between the occiput and posterior arch of $\mathrm{Cl}(\mathrm{CO}-\mathrm{Cl}$ distance) as well as between posterior elements of $\mathrm{C} 1$ and $\mathrm{C} 2$ ( $\mathrm{C} 1-2$ distance) in flexion and extension films were measured. Native image findings were registered, too. Results: Mean increase of $\mathrm{C} 0-1$ distance from extension to flexion was $4.4 \pm 3.7 \mathrm{~mm}$ ( $1 \mathrm{SD}$ ). In 16 cases the $\mathrm{C} 0-1$ distance remained unchanged, or paradoxically decreased during flexion, a situation which can be called a functional $\mathrm{C} 0-\mathrm{C} 1$ block. In these cases $\mathrm{C} 1-2$ interspace compensatorily increased during flexion. No statistically significant correlations (khii-square test) were found between $\mathrm{C} 0-\mathrm{Cl}$ motion and the native film findings (discus degeneration, spondyl- and facetarthrosis, scoliosis, straightened lordotic curve) or radiological hypo- or hypermobility of the lower cervical spine. Clinically these patients suffered mainly from cephalgia and showed limited and often painful extension of the neck in physical examination.

Conclusions: Functional $\mathrm{C} 0-\mathrm{Cl}$ is a relatively frequent, independent finding in flexion extension radiographs and seems to associate with cephalgia and painful extension of the cervical spine.

\section{$2.55 \mathrm{PM}$}

\section{Intervertebral disks MRI evaluation in athletes}

C. Bartolozzi, D. Caramella, V.Zampa, E. Tinacci, F. Balducci; Pisa, Florence

Purpose: Our aim was to demonstrate by Magnetic Resonance Imaging (MRI) the intervertebral disk damage caused by volley-ball sport activity, particularly in relation to different training procedures.

Methods: We selected a clinically asymptomatic population of 45 professional volley-ball players ( 21 males, 23 females), with ages ranging from 17 to 26 , who have been active in agonistic activity for a period ranging between 3 and 11 years. Twenty-five evenly matched swimmers were selected as control group. All the cases were studied with a $0.5 \mathrm{~T}$ MR scanner, using SE pulse sequences (long TR, long TE) and GRE pulse sequences (large flip angle, short TE). Disk degeneration was diagnosed on the basis of decreased signal in SE sequences; disk bulging and herniation were diagnosed on the basis of the morphological findings on two planes.

Results: In the group of volley-ball players we found 3 cases of simple disk degeneration, 3 cases of bulging, 6 cases of bulging and degeneration, 7 cases of hernia and degeneration (overall positive rate: $42 \%$ ). In the control group the positive rate was $20 \%$. We found a significant increase of positive cases when considering athletes with less or more than 7 years of activity $(37 \%$ and
$46 \%$ respectively); moreover the correlation with the type of training showed that when the reviewed training procedures were defined "uncorrect" (on the basis of the calculated strain on the column) the positive rate was $59 \%$, when they were considered "correct" the positive rate sharply fell to $22 \%$.

Conclusions: Our data show that volley-ball players are more likely to develop disk damage compared with other athletes, depending on the different degree of stress on the column. MRI seems the ideal method to diagnose and follow-up such modifications.

\subsection{PM}

\section{Segmental atrophy of the paraspinal muscles}

$\overline{\mathrm{Paul}} \mathrm{E}$. Andersen; Odense

Fatigue and lumbar muscular discomfort are frequent symptoms in the history of patients submitted to computed tomography of the lumbar spine. Analysis of over 1000 lumbar C.T.s has revealed a very large number of cases of segmental atrophy of the paraspinal muscles. The most frequent cause is laminectomy which in about $50 \%$ creates an atrophy at the level of operation and one or two vertebral segments above and/or below the resected lamina. The atrophy is visible a few months after the operation and does not regress. Severe atherosclerosis of the aorta, especially when located to the posterior wall of the vessel, is another cause of atrophy, this type usually being more pronounced than the post-operative atrophy. Aortic aneurysms may also be followed by often extensive atrophy.

More seldom an infection of neurologic tissue such as poliomyelitis is the cause of atrophy.

Typical cases demonstrate the various forms and extent of atrophy.

\subsection{PM}

198 Radiation osteoporosis, an estimation with single energy QCT Kinji Nishiyama, Kouji Kitatani, Tokurou Higashihara; Kinki, Kansai

Purpose: It was well known that irradiation to bone introduced osteoporotic change, however, quantitative analysis of radiation osteoporosis had not been carried out. In this report, radiation osteoporosis was analysed with single energy quantitative CT method.

Methods and Materials: Seven patients of uterine cervical cancer were treated with prophylactic para-aortic irradiation of $45 \mathrm{~Gy}$. These cases were designated as radiation group. Nine patients of uterine cervix cancer who were not irradiated to the para-aortic region at the same period constituted control group. Change of bone marrow content (BMC) of the 3rd lumber trabecular bone of these patients were evaluated with single energy quantitative CT Standard phantom employed was $\mathrm{CaCO}_{3}$ phantom (Tyugai Co. LTD).

Results: Mean pre-treatment BMC of radiation and control group were 150 and $128 \mathrm{mg} \mathrm{CaCO}_{3} \mathrm{eq} / \mathrm{cm}^{3}$, respectively. The difference between two groups was not significant. Mean BMC of the radiation and control group 3 months after irradiation were 94 and 122, respectively. Change of BMC was only -6 in the control group, whereas, -57 reduction was seen in the radiation group. Difference of reduction between both groups were statistically significant $(\mathrm{p}=.01)$. It was concluded that irradiation significantly reduced BMC in 3 months.

\section{Room M}

\section{SS} $2.00 \mathrm{PM}$

05.06. Prostate gland and gynaecologic neoplasms

\section{Prostate gland: visualization of internal anatomy with MRI} U. Keske, P. Uhrmeister, R. Langer, M. Langer, H. Kohl, R. Felix; Berlin

Purpose: The value of magnetic resonance imaging in visualizing internal prostatic anatomy was evaluated.

Methods and Materials: 66 patients without known genitourinary disease aged 22-70 years, were examined. A 1.5 T magnet (Magnetom, Siemens) with a whole body coil with T1- $(500 / 15)$ and double-echo SE-sequences (protonweighted and $\mathrm{T} 2,2500 / 15 / 90)$ was used

Results: On T1-weighted and proton-weighted images, the prostate appears homogenous without zonal discrimination. These sequences allow good visualization of the surrounding structures. T2-weighted MRI enables delineation of the peripheral zone as hyperintense to the central zone in every subject. The transitional zone presents hyperintense to both. The urethra may be seen as hyperintense within the central zone. The prostatic capsule appears almost isointense to the levator ani muscle. It can be differentiated from the latter by a thin, hyperintense layer which represents the periprostatic plexus. With increasing age, the prostate - especially the central zone - enlarges and the central zone develops a more irregular contour. Adenoma was clearly seen in 5 patients.

Conclusions: MRI visualizes the complex architecture and age-related changes of the prostate gland. For imaging of the internal prostatic structures, 
T2-weighted images should be preferred, whereas delineation of the surrounding structures is best in T1-weighted images.

\subsection{PM}

\section{Sonography in the diagnosis and the treatment of prostate abscesses}

R. Oyen, L. Baert, A. L. Baert; Leuven

Abscesses of the prostate are an infrequent complication of acute prostatitis. E Coli is most often found in patients with functional or organic urethral obstruction, while Staphylococcus Aureus is found in patients with a septic focus elsewhere.

10 cases of abscesses of the prostate were reviewed. A hyporeflective cystlike lesion could be recognized in the peripheral zone or on the midline behind the urethra at the level of the colliculus seminalis. A thick wall was not always present (4), while in some lesions liquid layering was seen (3).

The lesions were drained transperineally under sonographic guidance and antibiotics were instilled. All patients recovered well and became symptom free. Abscedation should be thought of in patients with acute prostatitis or subacute prostatitis especially when a cystic lesion is localized in the peripheral zone.

Abscedation of a pre-existing cyst was seen in 3 Müllerian duct cysts. In 3 cases MRI and I CT were performed, showing a hyperintense lesion on the T2-weighted images and a hypodense lesion on the contrast enhanced series respectively.

\subsection{PM}

\section{The significance of a hypoechoic area in the central and peripheral zone of the prostate: A study of 227 cases}

R. Oyen, L. Baert, G. Marchal, A. L. Baert; Leuven

Purpose: To determine the positive predictive value of rectal ultrasound of the prostate for a hyporeflective lesion of the prostate.

Methods and Materials: 227 patients with a hyporeflective lesion in the central zone or in the peripheral zone on transrectal ultrasound (TRUS) of the prostate had a transrectal biopsy under sonographic guidance using a $18 \mathrm{G}$ needle with the Biopty-gun.

Results: 114 hyporeflective lesions were adenocarcinoma, while 113 were benign. The majority of the latter were normal. In 22 biopsies acute or chronic prostatitis was seen. In 2 biopsies obvious glandular atrophy was described while in 3 others fibrosis was seen. Digital rectal examination (DRE) was abnormal in 96 cases of adenocarcinoma. In the benign group DRE was suspect in 43 cases, in 61 cases the DRE was normal, in 9 DRE was abnormal but not malignant. In the overall population of patients with a clinical palpable hyporeflective nodule, $69.0 \%$ were malignant. A hyporeflective non-palpable lesion was malignant in $22.7 \%$ in this series. The positive predictive value (PPV) of TRUS is $50.2 \%$. The PPV for DRE was $69.0 \%$, the negative predictive value (NPV) of DRE $79.5 \%$. The PPV of TRUS for the last 50 hyporeflective lesions was $58 \%$, the PPV for DRE $72.2 \%$, the NPV $78 \% .10 \%$ of the hyporeflective tumors were not palpable.

Conclusions: The value of TRUS for hyporeflective lesions in the peripheral and central zones of the prostate can considerably be increased with DRE. Even then there still remain discrepancies which should be biopsied.

\section{$2.30 \mathrm{PM}$}

\section{First results using Gd-DTPA in MRI of prostatic cancer} Anne Sparenberg, Bernd Hamm, Dorothea Gutschow, Peter Hammerer K.-J. Wolf; Berlin

Purpose: The zonal anatomy of the prostate is well delineated in MRI. Cancer can be detected as a focal hypointense lesion in the peripheral zone. It has not yet been investigated whether MR diagnosis of this type of tumor is markedly improved by using Gd-DTPA.

Methods and Materials: In a prospective study, 40 patients with prostatic cancer were examined by MRI (1.5 tesla, Siemens; T1- and T2-weighted SE-sequences in axial and sagittal plane). All patients received i.v. injections of Gd-DTPA $(0.1 \mathrm{mmol} / \mathrm{kg})$. They also underwent radical prostatectomy.

Conclusions: 1. An inhomogenous signal enhancement in prostate cancer was observed after using Gd-DTPA.

2. Delineation of the prostatic capsule was improved in small lesions.

3. After contrast agent application, infiltration of the neighboring structures was easier to detect.

4. The overall accuracy increased from $85 \%$ (precontrast) to $91 \%$ (postcontrast).

\section{$2.33 \mathrm{PM}$}

203 Prostatic carcinoma: a comparison between MRI and TRUS Anne Sparenberg, Bernd Hamm, Peter Hammerer, Johanna Burmester, K.-J. Wolf; Berlin

Purpose: Newly developed surgical methods of radical prostatectomy with the aim of preserving the periprostatic neuroplexus are making new demands of imaging procedures. Beside the diagnosis of lymphatic invasion, they are expected to diagnose local tumor extension as accurately as possible.

Methods and Materials: Seventy patients with bioptically confirmed prostatic carcinoma were examined with MRI (1.5 Tesla, Magnetom, Siemens) and TRUS (7.0 MHz, Sectorscanner, Bruel + Kjaer) during a prospective study. All patients underwent radical prostatectomy including lymphadenectomy. MRI was performed in T1- (SE 500/15) and T2- (SE 2500/90) sequences in multiplanar layers. TRUS was done in axial and sagittal planes.

Results: 1 . Tumors were more easily detected by MRI ( $89 \%)$ than by TRUS $(80 \%)$.

2. The delineation of seminal vesicle infiltration was better in MRI $(85 \%)$ than in TRUS $(71 \%)$.

3. Both methods did not allow exact determination of the tumor volume.

4. Infiltration of organs and bone metastases were not completely shown by TRUS, and lymph-node staging was limited.

Conclusions: 1. Staging is best performed using MRI.

2. Screening and biopsy are best performed with TRUS.

\section{$2.43 \mathrm{PM}$}

204 Transrectal ultrasonography of prostatic cancer treated by external beam therapy

A. Scherrer, H. Piollet, P. Vincent, F. Reboul; Avignon

Purpose: To assess the ultrasonic aspect of irradiated prostatic gland and the impact of transrectal ultrasonography (TRUS) on the follow up of patients. Methods: 42 patients were examined using a $5 \mathrm{MgHz}$ biplane transrectal probe just before a routine follow up consultation (mean 3 years after treatment). The examination was done by the same radiologist without any information about the stage and location of the initial tumor or PSA level. All charts were reviewed one year after the examination to validate conclusions of the consultation.

Results: It appears that there is a great difference between clinical examination and TRUS findings. 14 patients had hypoechoic focal zones. In 8 cases the hypoechoic zone was contralateral to the primary tumoral site. 3 recurrences were clinically present, 1 with a normal TRUS and 1 with contralateral echographic signs.

Conclusions: Irradiated prostates are difficult to explore by TRUS because heterogeneity and calcifications. TRUS may have clinical utility in case of high PSA level and normal digital examination or bone scintigraphy. We do not advocate routine TRUS of irradiated prostate.

\section{$2.53 \mathrm{PM}$}

205 Accuracy of CT and FNAB of localized prostate carcinoma and bladder carcinoma

R. Oyen, F. Ameye, H. Van Poppel, L. Baert, A. L. Baert; Leuven

Purpose: To study the sensitivity, specificity and accuracy of CT and FNB in the staging of lymph nodes in localized prostate carcinoma and in bladder carcinoma.

Methods: 1. A retrospective study of 73 patients with a T2 prostate carcinoma; a routine CT of the pelvis considered to be N0, a radical prostatectomy and pelvic lymphadenectomy was performed.

2. Fine needle aspiration biopsy (FNAB) of enlarged regional and juxtaregional lymphnodes $(>1 \mathrm{~cm})$ in 20 patients with bladder carcinoma and 18 patients with prostate carcinoma.

Results: 1 . Sensitivity of CT was $73 \%$, specificity $100 \%$ and accuracy $94 \%$. Out of 73 patients 4 false negatives were seen, 3 with micrometastases in a normal sized lymphnode, and 1 with massive metastases not enlarged nodes.

2 . In the group of 38 patients where a transperitoneal or a paraspinal retroperitoneal FNAB was performed, 30 biopsies turned out to be positive, 7 were true negative and 1 false negative, leading to a sensitivity of $96.7 \%$, a specificity of $100 \%$ and an accuracy of $97 \%$.

Conclusions: CT combined with FNAB of suspected nodes is a highly reliable method for staging of localized prostate carcinoma and bladder carcinoma.

\subsection{PM}

\section{Contrast-enhanced MR imaging of cervical carcinoma} Siegfried Thurnher, Maeve McPhillips, Borut Marincek; Zürich

Purpose: To assess the potential of contrast-enhanced MR imaging in the evaluation of cervical carcinoma.

Methods and Materials: In 31 patients with histologically proven cervical 
carcinoma spin-echo-MR studies were performed before and after administration of Gd-DOTA $(0.1 \mathrm{mmol} / \mathrm{kg})$. Contrast-enhanced T1-weighted MR images were compared to unenhanced T2-weighted MR images in the transaxial plane.

Results: Clinical staging was correct in 19 out of 31 patients (accuracy $61.2 \%$ ). Overall accuracy of MR staging was $83.8 \%$. In 2 patients with small stage I a carcinoma, tumors were neither detected by means of unenhanced nor enhanced MR imaging. In 8 studies, T2-weighted MR images were superior to contrast-enhanced MR images owing to better definition of tumor margins. However, image quality was decreased due to peristaltic artifacts in 10 patients After Gd-DOTA, marked enhancement of the masses and metastases was noted. Improved evaluation of tumor infiltration to the parametrium or adjacent organs was achieved by contrast-enhanced MR imaging in 10 patients.

Conclusions: Contrast-enhanced MR imaging is a valuable method for evaluation cervical carcinoma, and may be used when unenhanced T2-weighted MR images are suboptimal or equivocal. Improved image quality attainable in the pelvis may provide additional essential information in tumor staging.

\subsection{PM}

\section{Cancer related urological complications in gynaecologic oncology patients}

Kornélia Szluha, Mózes Péter; Debrecen

1. Retrospective analysis of the urological complications in the last 5 year in our clinic treated 3455 gynaecologic oncology patients.

2. In 492 patients screened out by isotope nephrography, conventional urography was performed. An increasing number of patients were subjected to ultrasonographic examination of the urinary tract, as primary screening. Only in the last 2 years could we detect with CT urological complications.

3. In 279 patients the pathologic finding developed due to the malignant tumour and/or the treatment procedures. By 150 patients could we detect cancer related urographic findings. Out of them in 19 patients ultrasonographically guided nephrostomy was performed.

4. The conclusion of the authors is that the role of ultrasonography in screening has a growing importance but in some cases only the urography can show us the place of ureter complications. Even in early stages of gynaecologic malignancy several forms of early and late urological complications have to be taken in consideration. Complex modern imaging and laboratory examinations are necessary not only in the stage of diagnosis and treatment, but also at scheduled post-treatment follow-up examinations.

\subsection{PM}

208 Evaluation of local prostate cancer: a comparison between transrectal ultrasonography and histopathology

M. Norberg, M. Häggman, T. Andersson, C. Busch, A. Magnusson; Uppsala

Purpose: The aim of this study was to investigate the ability of the transrectal ultrasound (TRUS) to detect, stage and determine the size of localized prostate cancer.

Methods: Thirty-seven patients with localized prostate cancer, selected for radical prostatectomy, were examined with TRUS prior to surgery. Hypoechoic areas were interpreted as tumors. Bulging adjacent to a hypoechoic area was interpreted as extra capsular extension (ECE). Thirty-four patients underwent radical prostatectomy while three patients with positive lymph nodes were offered other treatment. Following formalin fixation the specimen was whole organ mounted and the size of the tumor was measured and evaluated for grade and ECE.

Results: Tumor was demonstrated histologically in all 34 specimens. Of the 26 tumors detected by TRUS, the size was underestimated in 20. Twenty-six tumors also had microscopic ECE, only 2 of which were detected by TRUS. Conclusions: A normal TRUS examination does not exclude the presence of a tumor. In this study TRUS underestimated the size of the tumor, was not reliable in detecting ECE and was not useful in preoperative staging.

\section{Room N}

SS

\subsection{Brain, Varia}

\section{$2.00 \mathrm{PM}$}

209 Biomagnetic multichannel measurements - A new tool for functional diagnosis (Keynote Lecture)

R. Helle, H. Reichenberger, S. Schneider, K. Abraham-Fuchs, G. Röhrlein, J. Uebler; Erlangen

Measurements of electric surface potentials of the human body are routinely used in clinical diagnosis to investigate the function of organs. The electric sources in the body that create these electric potentials simultaneously produce magnetic fields, that can also be measured outside the body. Compared to electric surface potentials magnetic fields are much less influenced by tissue surrounding the source. They therefore render possible the localization even of deep lying sources. Biomagnetic multichannel systems enable simultaneous acquisition of the magnetic field distribution at a sufficient number of points. Total measurement time is reduced to a couple of minutes which is feasible for clinical examinations. The universal biomagnetic system KRENIKON ${ }^{R}$ has been developed for investigations of brain and heart. 37 sensor coils are arranged in a flat array of $19 \mathrm{~cm}$ diameter. Superconducting quantum interference devices (SQUIDs) act as sensitive amplifiers for the tiny biomagnetic fields. A shielded room reduces external interference. Depending on signal to noise ratio superficial electric sources can be localized with a spatial accuracy of better than $0.5 \mathrm{~cm}$ and sources $7 \mathrm{~cm}$ deep with an accuracy better than $2 \mathrm{~cm}$, temporal resolution is typically $1 \mathrm{~ms}$. Diagnostic information is significantly enhanced by projecting the biomagnetic localizations into diagnostic images yielded e.g. from MRI. Applications demonstrate the localization and propagation of epileptic activity at the brain, and of ectopic activity at the heart. Clinical trials are now under way to investigate the full clinical potential of biomagnetism.

\subsection{PM}

210 Brain magnetic resonance imaging in patients with hepatic encephalopathy and portosystemic shunt

Noriharu Yanagimachi, Tsuneya Watabe; Kanagawa

While the involvement of globus pallidus in hepatic encephalopathy (HE) and portosystemic shunt (PS) has been well documented pathologically, there were a few neuroradiological reports addressing unexplained pallidal brightness on T1-weighted spin-echo MR images in these conditions. To clarify the relationship between pathological alterations and MR signal patterns, we analyzed MR images of 18 patients with $\mathrm{HE}$ and PS on $1.0 \mathrm{~T}$ and $1.5 \mathrm{~T}$ MR units, combined with $\mathrm{T} 1$ and $\mathrm{T} 2$ measurements of arbitrary areas of the brain including the pallidal regions in normal and abnormal subjects. All of 18 patients revealed the peculiar brightness distributed in the pallidonigral regions on T1-weighted images without apparent abnomality on T2-weighted images. The $\mathrm{T} 1$ and $\mathrm{T} 2$ measurements in 14 patients showed expected $\mathrm{T} 1$ shortening with minimal $\mathrm{T} 2$ shortening in the bright regions. Of interest was unexpected $\mathrm{T} 1$ shortening even in the cerebral white matter, where the pathological changes by HE and PS were known to be minimal. Though the actual mechanism of these alterations of signal intensity and $\mathrm{T} 1$ value requires further investigation, brain MR findings in HE and PS suggests a condition of focal and diffuse pathological processes in the brain, probably pertinent to a systemic metabolic change.

\subsection{PM}

211 Assessment and neurologic correlation of vascular indentations on the brain stem

G. Reuther, S. Müller, G. Fahrendorf, G. Bongartz; Münster

Purpose: To record and to assess the significance of contour indentations of the brain stem by adjacent vessels.

Methods and Materials: 1000 consecutive MR exams of the brain were scrutinized for infratentoriell neurovascular relationships. Patients with indentations of the brain stem next to vessels were evaluated for deficiencies of cranial nerves and long ascending/descending systems by evoked potentials unless an underlying neurologic disorder was present.

Results: The total incidence of neurovascular contour alterations was $8.3 \%$ increasing with age. 3 different types in cross-sectional morphology could be identified. $2 / 3$ were due to contact of the left vertebral artery to the upper medulla. No correlation with neurologic deficiencies could be found.

Conclusions: Indentations of the brain stem due to adjacent vessels are a not too rare finding especially in the elderly, but even strong indentations are unlikely to cause neurologic deficiencies.

\subsection{PM}

212 MR assessment of neurological form of Wilson's disease N. Bešenski, T. Babić, P. Jurković; Zagreb

Twenty-two patients ( 9 women and 13 men) aged from 7-35 $( \pm 22,05)$ with clinical and biochemical findings of Wilson's disease are analyzed. In our study were included only patients with cerebral involvement in Wilson's disease.

The duration of the disease since diagnosis was approximately 3,5 years. The most frequent symptoms were dysarthria (18/22), tremor $(17 / 22)$, rigidity $(13 / 22)$ and cerebellar ataxia $(12 / 22)$. Ten patients underwent MRI examinations (our unit employs a permanent magnet operating at $0,2 \mathrm{~T}$ ). All patients also underwent CT examinations. In $3 / 10$ cases MRI showed no abnormalities. Subcortical atrophy with widening of the ventricle system was found in $3 / 10$ cases.

The lesions in nucleus lentiformis in $2 / 10$ patients, thalamus $(1 / 10)$ and nucleus caudatus $(1 / 10)$ were best demonstrated on $T 2$ weighted sequences as 
hyperintense areas.

Unilaterally abnormalities did not exist in our group of patients. Generally there was no correlation between MR findings and clinical neurological symptoms. CT scans showed hypodense lesions in caput nuclei caudati in $5 / 22$ patients and nucleus lentiformis in $8 / 22$ patients. The other $9 / 22$ patients had cortical atrophy on CT scans. The abnormalities established with CT scans were unspecific. In our experience MRI is very sensitive method for visualization of cerebral abnormalities in Wilson's disease, especially when combined with other neurological symptoms and signs.

\subsection{PM}

213 Neurofibromatosis: MR appearance of deep cerebral abnormalities

S. Magnaldi, R. M. White, R. N. Bryan; Trieste

Purpose: to explain the nature and significance of some deeply located areas of altered signal intensity, using Magnetic Resonance Imaging in patients with Neurofibromatosis (NF).

Methods: a retrospective evaluation of the clinical records and the cranial MR examinations of 24 patients ( 21 with neurofibromatosis type 1 and 3 with neurofibromatosis type 2 , mean age, 16.2 years, range, 2 to 42 ) and a prospective study of 4 hereditary NF-1 families with an affected parent and child were carried out.

Results: the MR examinations showed the presence of foci of prolonged T2 relaxation, primarily in the globi pallidi, thalami and the dentate nuclei of the cerebellum, in 22 of 29 patients, most of which younger than 15. Some of the foci in the globi pallidi exhibited increased signal intensity on T1-weighted images as well. NF-2 patients had periventricular foci of prolonged T2 relaxation, but did not have dentate and basal ganglia lesions. In $10 \mathrm{NF}-1$ patients sequential MR studies were available as well: in these subjects, 5 lesions decreased in size, 3 were unchanged and 2 lesions increased in size. In the prospective study, the parents never had the lesions, whereas all the children did.

Conclusions: the deeply located areas of abnormal signal intensity affect only NF-1 patients and are transient, because these lesions are present only in young subjects and in the children of NF families.

\subsection{PM}

\section{Stereotaxic localisation by MRI}

J.Rousseau, D. Gibon, P. Clarysse, S. Blond, N. Bradai, X. Marchandise; Lille

Purpose: MRI is known as the best imaging procedure in 3-D localisation for stereotaxic neurosurgery. But several peculiar difficulties require specific adaptation. Our work set a new method to localise targets in stereotaxic frame. Methods: The MRI studies are performed using a 0.5 T GE MR-Max imager. Four boxes $\left(0.15 \mathrm{ml} \mathrm{CuSO}_{4}\right.$ solution $)$ are inserted into the plastic intracranian holders of Talairach's frame before the examination. Thirty $7 \mathrm{~mm}$-thick sagittal slices spread over the whole head and provide sagittal localisation of landmarks, of tumour and of AC-PC line. Twenty axial slices, parallel to AC-PC line, provide visualisation of the whole brain. The procedure lasts about 10 minutes. Images data are removed to a Personal Computer. Specific software has been developed: recognition and barycentric coordinates determination of the four landmarks; determination of the reference stereotaxic trihedron; displaying of the proportional grid of Talairach.

Results: Quality control checked millimeter accuracy of the origin of the trihedron. Systematic geometrical errors have been carefully evaluated. An original procedure and corrective algorithms have been implemented. Checks on in vivo studies have been designed to detect geometrical distortion or patient's movements.

Conclusions: Our three-dimensional localisation method appears to be quite precise for clinical use. Millimeter accuracy is achieved in the three dimensions. Duration of MRI examination is not a restricting factor, mainly since the setting of the patient is easily done. Required equipment is simple and cheap. The only restricting features are the setting of intracranial Talairach's holders prior to MRI, and a careful quality control of the imager.

\subsection{PM}

\section{Improved safety and accuracy of stereotactic biopsy by a calcu- lated display of the planned probe track on axial CT slices}

\section{Van Roost, L. Solymosi; Bonn}

Purpose: Stereotactic systems are designed to hit point-like intracranial targets. Their routine performance does not comprise a display of the intended transcerebral and translesional probe path. Implementing the latter can improve accuracy, compared to standard serial biopsy.

Methods: We use the Riechert stereotactic frame, the settings of which are expressed as polar coordinates. Here, the probe track can be mathematically described as a vector. We modified the computation program for the conversion of cartesian into polar coordinates, so that a more manifold use of the vector becomes possible. By allowing for a metric variation of the vector's magnitude, any point on the probe track is definable in terms of cartesian coordinates and thus can be traced on CT scans during the biopsy planning stage.

Results: We employed this technique in 53 stereotactic procedures. In 42 out of 49 serving for biopsy, a clear-cut diagnosis was obtained. Furthermore, displaying a planned probe track on axial CT images provided a better resolution with less slices than a probe simulation on a CT stack reconstruction. Conclusions: The imaging detail obviously helps in selecting representative sites for biopsy along the whole probe track, whereas dangerous sites for biopsy as e.g. vessel-bearing structures can be avoided.

\subsection{PM}

216 Isolated neurofibroma and schwannoma of the sciatic nerve: ultrasound and MRI features

S. A. Sintzoff Jr., W. O. Bank, P. A. Gevenois, J. Noterman, J. FlamentDurand, J. Struyven; Brussels

A 53 year old women was studied with ultrasound for suspicion of a compressive mass of the popliteal region.

Ultrasound demonstrated two masses on the external branch of the sciatic nerve. The masses were hypoechoic with distal sound enhancement. MRI was performed and demonstrated both masses. Their signal intensity was that of the normal nerve on T1 weighted images and brighter on T2 weighted images. Intense gadolinium uptake was observed in one of the two lesions.

Surgery and histopathologic examination were performed. One mass had the histological features of a neurofibroma and the other had those of a schwannoma. The latter corresponded to the lesion which exhibited the gadolinium enhancement.

Thorough physical and neurological examinations, MRI studies of the central nervous system and skeletal radiographs failed to demonstrate any additional lesions.

Final diagnosis was isolated neurofibroma and schwannoma of the sciatic nerve.

\subsection{PM}

$217{ }^{123} \mathrm{~J}$-lomazenil-SPECT: Benzodiazepin-receptor distribution in relation to medication

H. Steinert, S. Schlegel, K. Hahn; Mainz

${ }^{123} \mathrm{~J}$-Iomazenil provides the opportunity to measure benzodiazepine-receptors by single-photon-emission-tomography (SPECT) in vivo. 90 minutes after injection of ${ }^{123} \mathrm{~J}$-Iomazenil the average count rate in various regions of interest were measured and the ratios between pons and different brain areas were calculated.

Preliminary analyses revealed a $40 \%-70 \%$ decrease of receptor bindings in frontal, temporal and occipital regions after long term medication with lorazepam, flunitrazepam and clonazepam.

\section{$3.21 \mathrm{PM}$}

218 Atypical band heterotopia - case report

M. Gallucci, A. Bozzao, O. Migliori, A. Spendiani, A. Cifani, R. Passariello; L'Aquila

Band heterotopia is a rare migration disorder described since 1989 by J. Barkovich. In band heterotopia layers of ectopic gray matter are present in the subcortical white matter following the circonvolutions. These layers are symmetrically evident in both emispheres. In all the cases this abnormality is associated with severe psychomotor. In our case a strongly asymmetric band heterotopia was shown by RM in a normally developed young woman suffering from seizure. This observation suggests that a milder form of band heterotopia may exist thus changing the prognostic value in this abnormality. Pathogenetic considerations may eventually be deducted.

\section{$3.24 \mathrm{PM}$}

219 The use of miniaturized guide wire guided microcatheters in extreme low-diameter cerebral vessels

B. Richling, G. Bavinzski; Wien

The evaluation of guide wire guided flow independend microcatheters with progressive softness makes it possible to reach peripheral vascular territories by the endovascular route. The limits of an endovascular approach were set rather by the diameter of the catheter tip in relation to the lumen of the vessel than by navigational problems. Further miniaturization of these guide wire guided catheters (TRACKER 10) enables endovascular access to smallest cerebral vessels not reachable by conventional techniques. This is possible due to decreased outer diameter of guide wire, catheter tip and -shaft, the mechanical stability of the system remaining almost unchanged.

Examples of accesses to pediatric cerebellar arteries or branches originating 
from the carotid syphon demonstrate the utility of this system. Finally special problems and risks caused by the use of a very thin microcatheter and guide wire in cerebral vessels are pointed out.

\section{Room 0}

\section{SS}

$2.00 \mathrm{PM}$

\section{Digital radiography (Keynote Lecture)}

Peter Aspelin; Huddinge

The last decades have seen an explosive development of diagnostic radiology, with the advent of a number of new modalities. This development is parallel to the technical development as a whole, especially the rapid progress in computer science. Not only are several of the new modalities, like CT, MRI, and PET, dependent on advanced computer technology, but during the last years we have also seen a computer based change of the traditional, conventional radiography: digital radiography.

Digital radiography is defined as a common name for all methods, imaging plate system or any other, that use computers instead of traditional photographic films for collecting, processing, displaying and storing radiological information.

The potential advantages are manyfold:

1. Data processing with possibilities for enhanced information from one and the same exposure.

2. Broad exposure range, with possibilities for lowering the exposure dose, and avoiding repeat exposures.

3. Development of the filmless radiologic department, in which all radiologic information is primarily handled by computers, and displayed on monitors. The potential disadvantages are mainly the limited spatial resolution and the huge amount of data that may be difficult to handle conveniently with the technology of today. The possibilities and limitations using digital radiography are still only known in part - much research is still to be done. This concerns not only the direct technical questions, nor the diagnostic advantages and limitations inherent in the method. It also concerns the working situation for the radiologist and the relation between radiologists and clinicians, when all radiologic information is quickly and easily available, not only at the department of radiology, but at the same time anywhere else.

In the following papers, several of these aspects will be discussed and answers will be given, but $\mathrm{I}$ am sure that we also will face new challenging questions.

\section{$2.15 \mathrm{PM}$}

221 Nonlinear subtraction for correction of beam hardening effects in dual-energy storage phosphor radiography

M. Prokop, A. Meschede, A. Memis, C. Schaefer, M. Galanski; Hannover

Purpose: Beam hardening effects in dual energy (DE) subtracted images result in incomplete removal of overlying bone and soft tissue structures. We present simple nonlinear subtraction techniques for beam hardening correction of DE images obtained by standard storage phosphor radiography equipment.

Methods and Materials: Storage phosphor DE images were obtained with a single shot technique with $\mathrm{K}$-edge pre-filtering and a sandwich cassette. Processing of $1 \mathrm{~K} \times 1 \mathrm{~K} \times 12$ bit images was performed on the standard postprocessing console (Digiscan 901, Siemens) and the host computer (Microvax II, DEC). Nonlinear subtraction coefficients were estimated by the absorption in the first storage phosphor screen. Optimum curves for nonlinear subtraction of images were determined experimentally.

Results: Nonlinear subtraction could be optimized interactively and allowed for almost complete separation of bone and soft tissue structures on the subtracted dual energy images. Improvements over linear subtraction were most marked on bone images of the chest.

Conclusions: Nonlinear subtraction is a suitable method for beam hardening correction in DE radiography resulting in an adequate separation of bone and soft tissue structures.

\subsection{PM}

\section{Computer stimulation of dose reduction in digital storage phosphor radiography}

D. Urbach, W. Döhring, R. Urbach; Hannover

Purpose: We present a new computer program which simulates dose reduction by calculating, generating and superposing the image noise occuring in exposures with lower doses.

Methods and Materials: The simulation software was programmed in Fortran on a DEC MicroVax II which is linked to a storage phosphor radiography system (Digiscan 901). The program includes system dependent parameters and noise distribution statistics to calculate the increase of image noise. To compare the computer simulated dose reduction with real dose reduced image we used

1. series of homogeneous phantoms (three different materials) and

2. anthropomorphic phantoms with five different exposures and

3. hand radiograms of volunteers with two different exposures $(40 \mathrm{kVp}, 64 \mathrm{mAs}$ and $16 \mathrm{mAs}(\mathrm{n}=15), 64 \mathrm{mAs}$ and $32 \mathrm{mAs}(\mathrm{n}=15))$.

Results: Any digital radiogram can be taken to generate the equivalent of a series with varying doses for detection studies. Statistical parameter like standard deviation and noise distribution statistics and the optical impression could be imitated by the program.

Conclusions: For the first time it is possible to determine the admissible dose reduction for the detectibility of a specific finding by any single radiogram of a patient; serial films with different exposures are not necessary.

\subsection{PM}

\section{Influence of different noise sources of a radiographic system on the image quality}

W. Müller, H. Eschenbacher; Erlangen

a) Digital radiographic systems based on the image intensifier television chain exist for many years and the properties of their single components (e.g. the image intensifier or the television pick up tube) are principally known.

However to optimize a radiographic system it is not sufficient to have a basic understanding of single components but it is necessary to study the mutual interactions of all components and the external influences (e.g. the scatter radiation) on the system.

b) One possibility to analyse such complex system is to demonstrate the effects of quantum noise, structure noise, electrical noise and the influence of the total MTF on the image. This method allows us to "accompany" the image through the imaging system and to spot critical system components.

The method we developed is a combination of computer simulations and physical experiments and can be summarized as follows:

- Experimental determination of the distribution of the scatter radiation behind an irradiated phantom.

- Calculation of the total radiation behind the phantom.

- Experimental determination of structure noise, electrical noise and total MTF.

- Calculation of noisy images by taking into account the different frequence spectra and studying the influence of each noise source on low contrast details.

\subsection{PM}

\section{Noise suppression techniques for dual-energy storage phosphor radiography}

M. Prokop, H. Shin, C. Schaefer, M. Galanski; Hannover

Purpose: Dual energy (DE) subtracted radiographs suffer from a poor signalto-noise ratio. To obtain images of diagnostic quality, often a marked dose increase is necessary. We present simple and advanced algorithms for noise suppression in DE radiography that can be implemented on standard storage phosphor equipment and allow for iso-dose DE imaging.

Methods and Materials: DE images were obtained with standard storage phosphor equipment (Digiscan 901, Siemens). We used a single shot technique with $\mathrm{K}$-edge prefiltering and a sandwich cassette for simultaneous acquisition of high and low energy images. Image processing algorithms for noise suppression were implemented for a $1 \mathrm{~K} \times 1 \mathrm{~K} \times 12$ bit matrix on the standard postprocessing console and on the host computer (Microvax II, DEC). Algorithms are based on the negative correlation of image noise in bone and soft tissue images.

Results: Simple noise suppression algorithms that could be performed on the postprocessing console (processing time $<20 \mathrm{~s}$ ) reduced noise levels to those in unsubtracted images but reintroduced soft tissue and bone contours. On the host computer, advanced prototype algorithms could be developed that markedly reduced these contour artifacts.

Conclusions: Digital noise suppression allows for high quality dual energy imaging, even with conventional exposure dose.

\section{PM}

225 Computer simulation of radiographic patterns in digital storage phosphor radiography

D. Urbach, W. Döhring, R. Urbach; Hannover

Purpose: A new software (DIPHA, digital phantom) for simulation of structures relevant in diagnostic and experimental digital radiography will be presented.

Methods and Materials: The software was programmed in Fortran on a DEC MicroVax II. The host computer of the image processing unit Digiscan 901 . The digital radiograms were obtained by a storage phosphor radiography system (Fuji 901). DIPHA can be transfered to other digital systems.

Results: DIPHA enables to generate

1. geometric patterns like different edge function, drill hole phantoms, lead grid phantoms or test images 
2. anthropomorphic patterns like pulmonary nodules, emphysematic bullae, interstitial lines, osteolytic and osteoplastic bone lesions or fracture lines. These details can be blurred by a selectable degree and superimposed to other digital radiograms.

Conclusions: DIPHA proved to be an important aid in

1. the development and conduction of clinical studies

2. analyses and standardization of image processing software

3. quality assurance of hard- and software components. Digital phantoms generated by DIPHA are superior to conventional phantoms when the influence of the data acquisition system is undesired and exact defined patterns are demanded.

\subsection{PM}

\section{Can digital radiography already replace the traditional techniques in abdominal X-ray studies?}

B. Krug, K. F. R. Neufang, G. Friedmann, P. Siemens, Th. Küpper; Köln

Purpose: The value of digital radiography in abdominal X-ray examinations was evaluated by comparing digital luminescent radiography (DLR) with conventional screen-film systems (CFS), and digital image intensifier radiography (DIR) with photofluorography (PF), respectively.

Methods: In the first group (DLR/CFS) 71 matched conventional and digital plain films, 140 excretory urographies, 22 lymphographies, 9 cholezystographies, 16 phlebographies and 156 contrast examinations of the GI-tract were obtained by CFS speed class 200 and DLR (PCR-SP, Philips). In each patient a single digital exposure was carried out additionally to one of the conventional radiographs of an examination. In the second group (DIR/PF) 92 conventional and digital contrast examinations of the upper GI-tract were compared following the same schedule (Sircam 103 and Polytron 1000, Siemens). In each digital technique two differently postprocessed images were obtained from one exposure. Digital and conventional images were evaluated in a blinded fashion by 4 radiologists using a questionaire.

Results: DLR and DIR proved to be diagnostically equivalent to the conventional techniques. High spatial frequency enhancement (DLR) and displays in positive contrast (DIR) did not provide further diagnostical information. Conclusions: Digital radiography (DLR and DIR) has the potential to replace analogue techniques in abdominal radiography.

\subsection{PM} 227 Interstitial lung disease: a ROC study comparing digital storage
phosphor chest radiographs and conventional filmscreen chest radiographs

W. Dölken, A. E. Horwitz, Th. Krahe, P. Landwehr, K. Lackner; Würzburg

Digital storage phosphor chest radiographs and filmscreen chest radiographs from 60 patients have been taken using identical radiation dose. 29 patients fulfilled the criteria postulated to include them into the group of patients with interstitial lung disease. 31 patients without pathologic interstitial lung alterations made up the "normal" group. Postprocessing of the digital images has been performed using the method of unsharp masking with constant parameters for every patient. Images with high frequency and with medium frequency enhancement have been used.

An ROC analysis with 10 observers showed no statistical significant difference $(\alpha=0,05)$ between the two compared technologies. A correlation analysis of the profusion of the pathologic opacities according to the ILO/UC classification showed a significant positive correlation $(p<0,001)$ for the digital and the conventional images with a correlation coefficient of $r_{S}=0,853$.

\section{Room R}

SS

16.02. Importance of diagnostic imaging in radiotherapy II $2.00 \mathrm{PM}$

\section{Diagnostic imaging in gastrointestinal tumors (Keynote Lecture) W.Wenz; Freiburg}

In case of imaging gastrointestinal tumors, the importance of conventional imaging methods changed. Endoscopy is the method of choice now. Nevertheless, the tumorous invasion of the wall as well as regional or distant metastases cannot be detected. The lecture refers to indications for double contrast demonstration of the GI-tract (such as early carcinomas, intramural tumors, and tumors of the small intestine). Concrete examples show the advantages of computer assisted tomography, and first possibilities of MRI.

\subsection{PM}

229 The value of diagnostic imaging in the treatment of CNS tumors (Keynote Lecture)

Ch. B. Ostertag; Freiburg

Computed tomography imaging modalities such as X-ray-CT and MR-CT have produced an unprecedented euphoria among neuroradiologists. MR providing not only a superior contrast discrimination also has, though yet to be developed, a potential to probe body chemistry in vivo. However, the usefulness as a clinical instrument for therapeutic purposes (e.g. neurosurgery, brain tumor radiotherapy) is still fragmentary. The principle role of computed tomography is not tissue characterization analysis, i.e. suggesting a histologic diagnosis. For the time being the principal role is morphologic coordinate determination, i.e. computed tomography is conceived primarily as an instrument to better understand an individual patient's pathologic brain anatomy in all its different aspects. The key problem in neurosurgery as well as in radiotherapy of CNS tumors concerns volume definition and selection, i.e. the definition of a lesion within an individual brain: the discrimination of contours and borders of a lesion, the composition of a lesion (solid-cysticcalcified-mixed), the relative position of major vessels (displaced/incorporated/neoformation), the relative position of neighboring of CSF spaces (compressed-enlarged-distorted sequestrated) are of utmost importance for therapeutic decisions. It is uncertain at this point whether tissue characterization can replace the histological diagnosis. At present the legal environment makes a histological (biopsy) diagnosis (versus a radiographic diagnosis) mandatory. Tissue characterization as a substitute for a histological diagnosis is a longterm research objective. The midterm research objective, however, is an improvement of working conditions in terms of improved accuracy, quality, efficiency, availability and cost effectiveness for therapeutic manoeuvers.

\subsection{PM}

230 The contribution of nuclear medicine in the pre- and posttreatment evaluation of cancerous disease (Keynote Lecture) J. Frühling; Brussels

As a technique from the general point of view, nuclear medicine using physiological tracers and routinely digitalized data, evaluated and treated by computers, presents some major advantages, such as: good reproducability of the results, relatively high sensitivity, low exposure to irradiation and acceptable social costs. The morphological data, obtained by nuclear imaging techniques should be considered as the expression of functional structures. Moreover, the same tracers are often used in a specific diagnostic and therapeutic aim on a given organ.

Adapted to the natural history of the cancerous disease, nuclear diagnostic studies (imaging, metabolic and dynamic ones) deal with primary tumor visualization, locoregional evaluation, the diagnosis of metastatic localizations, - correct staging being the basis of a successful treatment - with control of therapeutic efficacity, follow up and the evaluation of complications.

For in vivo primary tumor visualization among the well-known and numerous tumor markers only those which are metabolically specific (131-I, 75-Se-cholesterol, 131-I- or 123-I-MIBG) play a decisive role in tumor localization and identification. As far as the other products are concerned, the most contributive is still $67-\mathrm{Ga}$. The classical organscintigraphy adds its useful diagnostic contributions, either by positive findings (hot zones), or by negative contrast (cold zone). The study of local perfusion can demonstrate, using computer analysis, by the observation of elevated values during the initial phase, in comparison with adequate reference regions, the existence of a malignant lesion. During the last 10 years, the introduction of radioimmunoscintigraphy constitutes the most significatif progress presented by the oncological nuclear medicine at least as far as the physiopathological concept is concerned. Nevertheless, due to technical problems the place of radioimmonoscintigraphy in the daily clinical practice is still limited.

When staging and follow-up are concerned bone scintigraphy is the first and most sensitive examination if metastases are to be demonstrated or excluded, the morphological bone study being, together with the cardiac investigations the most commonly requested examination in a nuclear medicine department. We should still emphasize the importance of lymphoscintigraphic investigations in the evaluation of nodal attempt or relapse.

In order to realize a control of therapeutic efficacity if performed under reproductible technical conditions isotopic measurements of the size, uptake and perfusion of primary and secondary tumor lesions allow the evaluation of the response of the tumor to anticancer treatment. Size measurement is often limited by resolution problems, but measurement of modifications in tumoruptake and perfusion, constitute a promoting field, especially when PET studies are concerned.

Complications in case of cancer can be due to the evolution and extension of the underlying disease and/or to the side effects of treatment. Thus, thromboembolism, infection, hematologic complications, cardio- and nephrotoxicity are the most important clinical consequences, where isotopic methods, often the simplest and least invasive diagnostic tests among those available, are routinely used in the clinical management of the patients. 


\section{Assessment of PET for therapy monitoring of unresectable} thoracic tumors

Michael V. Knopp, Ludwig G. Strauss, Uwe Haberkorn, Antonia Dimitrakopoulou, Helge Bischoff, Franz Oberdorfer, Christian Manegold, Wolfgang Maier-Borst, Gerhard van Kaick; Heidelberg

Purpose: To assess the potential benefit and use of positron emission tomography (PET) with F-18-deoxyglucose (FDG) for improved therapy management of patients with unresectable thoracic tumors.

Methods and Materials: 45 patients with unresectable tumors were enrolled in a monitoring study. All patients were imaged prior to therapy using a whole-body PET scanner. Each patient additionally was studied by plain film and CT. Only patients with confirmed histology by mediastinoscopy or bronchoscopy were included. 40 patients received chemotherapy whereas 5 received primary radiation therapy. More than 25 follow-up-studies have been already performed.

Results: All malignant lesions showed high FDG-uptake and were clearly visualised by PET. The change in FDG accumulation after the second cycle of chemotherapy or the midpoint of radiation therapy proved to be a reliable predictor of the therapy outcome determined at final staging after therapy. The conventional evaluation only correctly predicted $70 \%$ of the responders and $40 \%$ of the non-responders.

Conclusions: Positron emission tomography with FDG enables a reliable and timely evaluation of response to an on-going therapeutic protocol. It is clearly superior to morphologic evaluation of response and provides an opportunity for improved therapy management if alternative therapeutic strategies are available.

\subsection{PM}

\section{Changes in regional cerebral blood flow (rCBF) in HMPAO-SPECT during and after brain irradiation - preliminary results of a prospective study}

W.-H. Dingler, Z. Lapukins, D. Beil, B. Kober, H.-K. Deininger; Darmstadt

In a prospective study of tumor patients, the $\mathrm{rCBF}$ is investigated with 99m-Tc-HMPAO-SPECT and TCT under standardized conditions. We studied patients receiving prophylactic brain irradiation, patients with brain metastases or brain tumors and head and neck cancer patients, receiving irradiation of the base of the skull. The aim of the study is to compare the rCBF before, during and every three months after radiation therapy. As of Dec. 20th, 1990, we have follow up studies of 38 patients. During radiation we saw increased $\mathrm{rCBF}$ of the hemispheres in $23 / 38$ patients. Even in patients with small cerebral irradiation volumes these changes were seen.

12 patients have been followed up for three months or more. $9 / 12$ patients show a cerebral hyperperfusion during radiation. 4/9 show a normalization of the $\mathrm{rCBF}$ after one month, 2 patients after two months and 2 other patients after three months. 1 patient had no normalization after three months. 3 patients show a reduced cerebral perfusion, 2 of them were normalized after one month. The other was still pathologic after four months. Basically the reduced cerebral perfusion correlated to clinical neurologic deterioration. We will present a renewed analysis of this study as of Aug. 15th, 1991.

\subsection{PM}

233 Follow up of patients with ovarian cancer: computed tomography, radioimmunoscintigraphy and second look with immunohistochemistry in comparison

G. Barzen, W. Richter, C. Zwicker, W. Friedmann, H., Eichstädt, R. Felix; Berlin

The follow up of patients suffering from ovarian cancer with noninvasive methods is still unsatisfactory. In this prospective study the value of Radioimmunoscintigraphy (RIS) with iodine 131 labelled OC 125 F(ab)2 antibodyfragments was proven in comparison to Computed Tomography (CT) and Second Look (S.L.). The results were compared with immunohistological findings and $\mathrm{Ca} 125$ serum levels. Planar scintigraphies were performed up to 7 days post injection. Tumor/Nontumor tissue ratios $(\mathrm{T} / \mathrm{N})$ were calculated and CA 125 serum levels estimated. In 12 patients immunohistological examination of the resected tissue was performed with the identical antibody. CT, S.L. and RIS were performed within 18 days.

Results: 31 of 44 patients had a local recurrency and or peritoneal carcinosis within the pelvis. Sensitivity was $70 \%$ and specificity $63 \%(\mathrm{~T} / \mathrm{N}>1,3)$, and $49 \%$ and $91 \%(\mathrm{~T} / \mathrm{N}>1,6)$ for RIS and $52 \%$ for CT. 22 of 44 patients had peritoneal carcinosis in the abdominal cavum. Sensitivity for RIS was 57\% versus $32 \%$ for CT. 5 of 44 patients had liver metastasis. RIS detected 2 and CT 5 cases.

Conclusions: Radioimmunoscintigraphy is the suitable method for detection

\section{Room S}

SS

\subsection{Biliary imaging - Biliary Interventional Radiology} $2.00 \mathrm{PM}$

234 The radionuclide investigation of the GI tract (Keynote Lecture) Otmar Schober, Klaus F. Gratz; Münster, Hannover

Nuclear medicine non-invasive diagnostic procedures partly compete, partly correlate, and are partly specific and superior to morphologically characterized imaging modalities. It is the aim of the summary to compare radionuclide imaging and absorption tests with other imaging modalities and clinical tests in terms of sensitivity and specificity, advantages and problems with the yield of a rational diagnostic approach for the indications of the radionuclide investigations of the gastrointestinal (GI) tract in clinical routine work.

- Scintigraphic evaluation of oesophageal transit, gastroesophageal reflux, and gastric emptying.

- Hepatobiliary imaging

- Differential diagnosis of liver tumors.

- Hepatic scintigraphy for evaluation of liver grafts.

- Gastrointestinal bleeding sites.

- Detection of infection using specific, radiolabelled cells, and unspecific tracers.

- Tumor imaging of the GI tract using and radiolabelled monoclonal antibodies.

- Resorption tests, e.g. Schilling test, Se-75HCAT test.

The radionuclide investigations of the GI tract has become considerably more complicated during the last years and have to be considered as different instruments in the beneficial concert of diagnostic procedures for the patient.

\section{$2.15 \mathrm{PM}$}

235 Imaging of polypoid endoluminal growing bile duct tumors E. Ponette, G. Biebau, J.Gelin, G. Marchal, A. L. Baert, W. Van Steenbergen, R. Kerremans, K. Geboes; Leuven

We reviewed the radiological documents and protocols of 196 cases of bile duct tumors examined over a period of 12 years: 20 of them (10.2\%) presented with a polypoid endoluminal growth. The aim of this study was to provide a better knowledge about the radiological features of this less frequent kind of tumor.

The mean age of these 20 patients was 63.6 years and the sex distribution was equal between males and females.

The correct lesion-diagnosis was provided in $100 \%$ by as well E.R.C.P., P.T.C., U.S. as C.T., and in $42 \%$ by arteriography. Correct diagnosis of tumor was made by E.R.C.P. in $86 \%$, by P.T.C. in $86 \%$, by U.S. in $65 \%$, by C.T. in $64 \%$ and by arteriography in $25 \%$. A correct tumoral diagnosis of $100 \%$ could be reached by combination of several examination techniques.

The mean tumoral length was $3.5 \mathrm{~cm}$ (extreme values: 0.5 and $10 \mathrm{~cm}$ ). Distribution of these 20 tumors involved as well the several segments of the common bile duct, as the right and left hepatic ducts.

The anatomopathological diagnosis was: cholangiocarcinoma ( 7 cases), adenocarcinoma of Vater's ampulla (4), villous adenoma of Vater's ampulla (2), cystadenoma (1), cystadenocarcinoma (1), hepatocellular carcinoma (1), apudoma (1) and metastases (3).

2.25 PM

\section{Adenomas and adenomatosis of the bile ducts}

V. Vilgrain, Y. Menu, P. Ponsot, G. Amouyal, B. Gayet, J. Belghiti, H. Nahum Clichy

Purpose: to describe ultrasonography (US), endoscopic sonography, and computed tomography (CT) of adenomas and adenomatosis of the bile ducts. Methods and Materials: five patients with cholestasis or obstructive jaundice were studied. All cases had US, CT, and cholangiography. One patient had endoscopic sonography of the bile ducts. All patients were operated on, and had surgical and histopathological correlation.

Results: in all cases US and CT showed bile obstruction with dilated intrahepatic bile ducts. In four patients a bile duct mass on the common bile duct was discovered. This mass appeared hyperechoic without posterior shadowing on US, and hyperdense on CT. On cholangiography the tumor made an intraluminal defect. In one patient, the common bile duct was enlarged on US and CT. No mass was seen. The bile appeared heterogeneous with echogenic foci on US and on endoscopic sonography. The intraluminal defect on cholangiography corresponded to mucus secretion.

Histological examination revealed in all cases adenomatous proliferation of the bile duct. Malignant transformation was present in four patients. Surgery 
discovered intrahepatic adenomatous lesions in two patients.

Conclusions: US, endoscopic sonography, and CT may play an important role for the diagnosis of adenomas or adenomatosis of the bile duct. Although these tumors are uncommon, malignant transformation occur frequently, and surgical treatment is required.

\section{$2.35 \mathrm{PM}$}

\section{The radiological spectrum of primary sclerosing cholangitis: a new classification}

Charles B. L. M. Majoie, Jacques W. A. J. Reeders, Joost B. Sanders, Peter L. M. Jansen; Amsterdam

Purpose: To evaluate a radiographic classification of endoscopic retrograde cholangiopancreatography (ERCP) findings in patients with primary sclerosing cholangitis (PSC).

Methods: The cholangiograms of 40 patients with PSC collected over the past 10 years were retrospectively reviewed.

The ERCP findings were classified into 3 types of intra- and 4 types of extrahepatic features based on the extent of bile duct narrowing, the severity of narrowing, contour abnormalities and prestenotic dilatation.

Results: In 35 cases ( $88 \%$ ) both intra- and extrahepatic ducts were involved. In 2 cases only the intrahepatic and in 3 cases only the extrahepatic ducts were involved. Multiple strictures with saccular dilatations and decreased arborization was the most common type of intrahepatic duct involvement ( 22 cases, $55 \%$ ). In the case of extrahepatic duct involvement an irregular margin of the duct with distinct diverticulum-like outpouchings and narrowing was most often seen ( 16 cases, $40 \%$ ). These intra- and extrahepatic abnormalities were usually seen in combination.

Conclusions: We conclude the classification of ERCP findings into 3 types of intra- and 4 types of extrahepatic features as useful in classifying patients with PSC because the different types of duct involvement have different diagnostic and therapeutic implications.

\section{$2.45 \mathrm{PM}$}

\section{The aetiology of obstructive jaundice based on patterns of biliary tree dilatation}

J. M. E. Kirk, A. Moody, A. Grundy, A. E. A. Joseph; London

Purpose: To assess the relationship in cases of obstructive jaundice between aetiology and the ratio of intrahepatic to extrahepatic duct dilatation.

Methods: The ratio of the diameter of intrahepatic to extrahepatic ducts (e/i ratio) was measured at percutaneous transhepatic cholangiography (PTC) in 31 patients with obstructive jaundice and in 7 controls. Ultrasound examination of a further group of 18 patients was performed and each ascribed to one of three groups, 1 . disparate dilatation where the common bile duct was markedly dilated with minimal or no dilatation of the intrahepatic ducts, 2 . proportionate dilatation where both intrahepatic and extrahepatic ducts were proportionately dilated, 3 . reverse disparity where the intrahepatic ducts were dilated disproportionately compared to the extrahepatic ducts.

Results: There was a highly significant difference $(\mathrm{p}<0.001)$ at PTC between the $\mathrm{e} / \mathrm{i}$ ratio for patients with common bile duct calculi and those with carcinoma of the pancreas. Ultrasound scans were then interpreted in the light of these findings. All three patients with disparate dilatation and eight patients with proportionate dilatation on ultrasound had common bile duct calculi subsequently confirmed. Two patients with proportionate dilatation had benign strictures. Four patients with reverse disparity had carcinoma of the pancreas and the fifth had a large mass in the head of the pancreas which was either chronic pancreatitis or a carcinoma.

Conclusions: We conclude that disparate or proportionate dilatation is more likely to be associated with a calculus or benign obstruction and reverse disparity with malignant obstruction.

\subsection{PM}

\section{CT of intrahepatic lithiasis}

S. Debernardi, M. Giudice, S. Kienle, E. Tola, D. Regge, G. Gandini; Torino

Since April 198365 patients with intrahepatic lithiasis have been treated transhepatically in our Department. CT scans were performed in 20 patients most of which with known massive lithiasis in order to assess the exact location and to predict stone composition.

In a small group of patients with cholangitis bile duct stones were unknown previous to $\mathrm{CT}$.

At first 8800 and then $9800 \mathrm{CT} / \mathrm{T}$ General Electric scanners were employed. Exams were performed with overlapping sections of $10 \mathrm{~mm}$ thickness, both without and with i.v. injection of contrast material.

CT was able to visualize stones in 16 of 20 patients $(80 \%)$; in 4 patients assessment of stone distribution was more accurate than with PTC. In 8 patients with aereobilia, CT was by far superior to US in evaluating the exent of lithiasis.

Furthermore in 2 patients CT visualized segmentary dilatation of peripheral duct and indirectly predict lithiasis.

Concluding we feel patients with massive lithiasis should be systematically submitted to CT as this exam has proven useful and at times superior to PTC and US in the evaluation of the distribution of biliary stones. Furthermore CT may give useful indication on the composition of stones especially if contact dissolution is to considered.

\section{$3.05 \mathrm{PM}$}

240 Retained intrahepatic stones; percutaneous removal with a preshaped angulated catheter in $\mathbf{1 7 0}$ patients

Byung Ihn Choi, Joo Koo Han, Man Chung Han; Seoul

Purpose: To establish the usefulness of fluoroscopically guided percutaneous radiologic interventional procedures with a preshaped angulated catheter for the removal of multiple intrahepatic biliary stones.

Methods: One hundred and seventy patients underwent percutaneous stone removal utilizing a preshaped angulated catheter and a Dormia basket under fluoroscopy with a combination of techniques including irrigation-suction, crushing of large stones, balloon dilatation of strictures and extracorporeal shock wave lithotripsy. The procedure was performed through a mature T-tube tract (168 patients) and a mature transhepatic tube tract ( 2 patients). Stones were exclusively intrahepatic in 125 patients, whereas 45 patients also had stones in the common bile duct.

Results: In 84 (49\%) of 170 patients, the stones were completely removed and in $36(22 \%)$ patients most of the stones were removed. Overall success rate was $71 \%$. Angulation deformity of the biliary tree and multiple biliary stricture were the factor most often responsible for failure. No significant complications were observed.

Conclusions: Fluoroscopically guided percutaneous interventional procedures with a preshaped angulated catheter is an useful complementary procedure to surgery for patients with retained intrahepatic stones. The major benefits of individually fitted angulated catheter are its safety and easy access to small peripheral bile ducts.

\subsection{PM}

\section{Non-surgical ablation of the gallbladder}

C. C. Coleman, J. A. Vennes, I. P. Posalaky, K. Amplatz; Minneapolis, MN

Fifteen canines underwent a midline laparotomy for subsequent gallbladder ablation. The cystic duct was temporarily clamped during the ablation. Temperature probes were placed in the liver parenchyma adjacent to the gallbladder, on the gallbladder surface and in the lumen of the gallbladder. A 7-Fr catheter with multiple sideholes was placed into the gallbladder. Hot contrast medium was introduced as an ablative agent. The mean temperature in the gallbladder lumen was $80^{\circ} \mathrm{C}$, in the adjacent liver $43.5^{\circ} \mathrm{C}$, and on the gallbladder surface $45.8^{\circ} \mathrm{C}$. Following, the cystic duct was unclamped, temperature probes and catheter were removed and the laparotomy closed in standard fashion. Two animals served as controls, using room temperature contrast medium.

In the hot contrast group, one animal was sacrificed at 2 weeks, one at 4 weeks, one at 8 weeks, one at 12 weeks and one at 6 months. Eight animals were sacrificed at one year. One animal was a surgical failure, and in one animal, the gallbladder ruptured, necessitating sacrifice at 17 days.

There was complete obliteration of the gallbladder in all animals. In the control group, the gallbladders were normal at 12 and 13 weeks. In the one-year follow-up group in one animal, the neck of the gallbladder remained intact. The remainder of the gallbladder was a fibrous strand. In three animals, there was a small $1-2 \mathrm{~cm}$ cystic area in the fundus of the gallbladder with a monolayer of cuboidal epithelium.

\subsection{PM}

\section{Mesenteric and retroperitoneal fibrosis as an unusual presen-} tation of a cholangiocarcinoma

J. C. Vilanova, R. Bassaganyas, X. Serres, J. J. Teruel, J. Barcelo, D. del Hoyo; Girona

A 74 year old woman was admitted to our hospital with indisposition, loss of weight and abdominal bloating. The abdominal ultrasound exam showed bilateral obstructive uropathy and extrahepatic obstructive jaundice with ascites. The Computed Tomography showed that the mesentery and the retroperitoneal area were heterogeneous and of soft tissue masses enclosing them. The retroperitoneal extension throughout the pelvis was bringing out a bilateral obstructive uropathy.

The autopsy revealed a huge desmoplastic fibrosis which occupied practically the entire abdominal cavity. Their findings were secondary a common bile duct carcinoma.

An exhaustive review of the medical literature corroborates the unusual presentation of this case. 
243 Malignant obstructive jaundice: decompression by external biliogastric fistula

K. A. Schumacher, J. M. Friedrich, W. Weidenmaier, E. Stange; UIm

In rare cases of malignant bile duct obstruction in which internal biliary drainage cannot be established by implantation of a stent, the externally drained bile can be recycled by connecting the transhepatic catheter to a percutaneous gastrostomy. In 3 patients submitted to this procedure the decrease in serum bilirubin and alkaline phosphatase was comparable with that observed with conventional methods, cholangitis occuring in one case could be controlled by antibiotics. There were no symptoms of biliary gastritis during observation periods of up to 5 months. The biliogastric fistula may help to avoid electrolyte imbalance and/or malabsorption resulting from excessive loss of bile in cases of external drainage. 


\section{Room A}

CC.

\section{1}

244 Brain MR morphology and functional anatomy: the role of diffusion weighted imaging

Graeme M. Bydder, Joseph V. Hajnal; London, Wembley

Diffusion weighted imaging provides a new and important approach to imaging the anatomy of the brain. It provides a unique method for demonstrating white matter tracts as a function of their fibre direction and is more sensitive than conventional techniques in demonstrating myelination. It also provides unique information about the movement of water in health and disease.

The technique employs pulsed gradient spin echo sequences and depends on the fact that water diffusion across myelinated fibres is restricted by the presence of myelin whereas diffusion along the fibres relatively unrestricted. If sentitizing gradients are applied parallel to fibres there is a net loss of signal but if they are applied perpendicular to fibres there is relatively little loss of signal. Tracts can be demonstrated in the corpus callosum, pons and brain stem. In disease, diffusion is often increased but in some circumstances water may be trapped within damaged myelin fibres resulting in a reduction of apparent diffusion coefficient and a high signal.

The implications for anatomy of the brain of diffusion weighted sequences are as great as those of the high contrast between gray and white matter shown with MR ten years previously using $\mathrm{Tl}$ weighted sequences.

\section{Pathomorphology: tumors vs. infections} William G. Bradley; Long Beach, CA

In the brain, the clinical history is probably the best means to discriminate intracranial masses into neoplastic or infectious/inflammatory categories. Nonhemorrhagic neoplasms tend to present insidiously rather than abruptly and infections tend to be associated with fever, known systemic infection or AIDS and elevation of the erythrocyte sedimentation rate. Inflammatory diseases such as tumefactive multiple sclerosis may present abruptly like tumors, however, they are usually associated with additional periventricular white matter disease which should provide a clue to the diagnosis.

When faced with an intracranial mass without the benefit of clinical history, there are several morphologic features which may aid in their distinction. If the infection has progressed from a cerebritis to a frank abscess, there should be a relatively smooth enhancing rim following administration of Gadolinium-DTPA and the liquefied center should be bright on T2-weighted images. While neoplasms may be centrally hecrotic, the peripheral enhancement tends to be less regular than that seen in the capsule of an abscess. Prior to liquefaction, enhancing cerebritis may be indistinguishable from the enhancing portion of the tumor, however, the cerebritis is generally associated with a greater amount of vasogenic edema and mass effect, however, this is not a certain sign.

In certain instances, infection can be distinguished from tumor on the basis of location within the brain. Infections arising from adjacent sinusitis, otitis or mastoiditis, should be easily diagnosed by contiguity to the previously infected extracranial space. Unfortunately, blood-borne infection (e.g., from subacute bacterial endocarditis or sepsis) may not be distinguishable from blood-borne metastatic disease as both tend to go to the grey white junction and engender a relatively large amount of vasogenic edema. If clinical history does not provide the necessary information to distinguish these two entities, then biopsy may be necessary.

\section{Room B}

\section{BE}

\section{1}

246 Analysis of the pulmonary hilum: Plain film and CT Peter Armstrong; London

The analysis of the pulmonary hilum requires a detailed understanding of normal anatomy. The major components of the normal hilar shadows are the blood vessels: normally, neither lymph nodes nor bronchial walls contribute significantly to the bulk of the hila. The presentation will, therefore, review the anatomy of the hilar blood vessels, emphasizing where they may be confused with masses when seen end-on or in cross section, and will also discuss those portions of the hila that are devoid of major blood vessels, since in these "bare areas", even very small masses may be confidently diagnosed.

\section{Analysis of the hilum: MR}

\section{B. Mayr; Munich}

MR has been shown to be a useful modality in the evaluation of hilar abnormalities. For a sufficient visualization of hilar lesions an appropriate imaging technique is necessary.

This requires multiplanar imaging, often ECG-gating, T1 weighted images, partially sequences with long repetition times. Knowledge of the normal anatomy in the transverse, coronal and sagittal planes is the basis of a sufficient diagnosis. In most cases MR will be used in the detection and evaluation of hilar masses or evaluation of an equivocal abnormal hilum. Additionally hilar lymphadenopathy can be assessed in different diseases. MR provides information quite similar to sufficiently performed contrast $\mathrm{CT}$ in the evaluation of hilar diseases. MR should be used, if diagnostic problems cannot be solved by CT

\section{Room C}

\section{RC}

248 Role of radiology of the colon as compared with endoscopy E. Ponette, A. Van Steen, P. Aerts, A. L. Baert; Leuven

Detection of small mucosal lesions is not a monopoly of endoscopy: the barium enema also reveals small ulcera and small nodules. On the other hand both techniques make diagnostic mistakes even in the mucosal pathology, so they have already for this bare reason a complementary function.

The barium enema has advantages in comparison with endoscopy, in the first place for the demonstration of a low-degree, but diagnostical important, stenosis, for the determination of the length of a high-degree stenosis, for the demonstration of lesions proximal to a stenosis and for the demonstration of fistulae. Further the barium enema is more sensitive in the detection of submucosal lesions of the colon and extrinsic processes which displace the colon. Moreover the barium enema allows to make up a balance - useful for therapy and follow-up - of the distribution of the lesions in diffuse colon diseases, to perform almost always a complete investigation of the colon as far as the caecal bottom and also to examine patients with acute abdominal pain syndromes. Finally the barium enema is mostly better tolerated, shows less complications, diminishes the number of required coloscopies and increases the coloscopic efficiency.

As a result the barium enema precedes an eventual endoscopy, except when it concerns acute hemorrhage and in those cases where clinically an ano-rectal problem is suspected.

\section{Room E1}

\section{RC}

\section{Mechanical devices in peripheral vessel angioplasty} F. Mahler, J. Triller, D. Do; Bern

In addition to the balloon-catheter for PTA a number of new methods have been developed in recent years. We report on four mechanical devices for recanalization of the vessels of which we have made our own experience. The fast rotating Kensey-catheter has not been proven superior to balloon PTA in our hands neither to remove organized or fresh thrombotic occlusive matter. A slowly rotating catheter (Rotacs Vallbracht) facilitates primary recanalization of long occlusions prior to balloon dilatation in femoral and iliac vessels, however not without failures. Atherectomy by the Simpson-catheter is advocated most for femoral stenoses that are also suited best for balloon PTA alone. It provides samples for histological examination but is indicated only in special situations. The TEC-system combines the principles of pulverization by a rotating knife and aspiratioin to remove occlusive material. In our experience it produces a relatively small lumen in proportion to the puncture hole and does not work without additional balloon PTA. Of the numerous mechanical devices under development clinical long term results are not yet available. In our opinion for all of these newer technologies both their relative effectiveness and the extent to which they may compare or complement balloon PTA remain to be determined.

\section{Peripheral vessel angioplasty: mechanical devices Dominique Desbrosses; Strasbourg}

Mechanical devices used in peripheral vessel angioplasty can be divided in: - atherectomy devices: "Simpson", T.E.C., "Bard System"...

- rotating devices: Kensey catheter, Rotablator, Rotacs.

- angiosonoplasty devices.

The results published with rotating devices are distributed as follows:

Rotacs: primary successes: $73 \%$, mid-term patency: $48 \%$ widely patent, $16 \%$ restenosis, $36 \%$ reocclusion.

Kensey catheter: primary successes: $76 \%$, mid-term patency: $70 \%$. 
Rotablator: (in stenoses): primary successes: $94 \%$, mid-term patency: $79 \%$. The best indications, for each device, can be established as follows:

Rotacs: Occlusions less than $10 \mathrm{~cm}$ of length, with proximal stump, without a large collateral vessel orginating proximal to the occlusion.

Kensey: Occlusions less than $11 \mathrm{~cm}$ of length, not healively calcified and with a good run-off.

Rotablator: Long ang calcified stenoses, especially in distal vessels.

Angiosonoplasty devices are still in an experimental stage but initial results are encouraging.

We have to keep in mind, that mechanical devices are more expensive and take more time than balloon agioplasty. So they must have better primary success rate and long-term patency rate than balloon angioplasty.

For all these devices we need, now, prospective and randomized studies to define their place in comparison with balloon angioplasty and surgery.

\section{Percutaneous Simpson atherectomy}

\section{Manuel Maynar Moliner; Las Palmas}

Technological advances and acceptance of percutaneous techniques had led to the development and testing of several new devices for the treatment of peripheral atherosclerotic disease. The Simpson atherectomy catheter is one of these new devices that permits percutaneous removal of atheromatous material, resulting in a smooth vessel wall.

The Simpson atherectomy device is a catheter with a distal metal capsule that cuts and extracts the obstructive atherosclerotic material. The capsule has a window in one side and a balloon positioned opposite the window to force the capsule against the atheromatous material. A cutting device within the capsule rotates at $2000 \mathrm{rpm}$. A floppy guidewire is fixed to the distal end of the capsule to aid in catheter placement.

Its use has not been recommended for the treatment of total occlusions. Nevertheless on the assumption that the atherectomy should provide better long term clinical results than angioplasty, because it removes the obstructing material, we have used the atherectomy device in patients with complete total occlusions.

We have also found the atherocath useful in the management of postangioplasty intimal flaps.

Ninety five atherectomies with the Simpson Athereocath were performed in 89 patients over three year follow-up. Eighty-eight males and 7 females, age range 35-84, mean of 61,08. Sixty-eight stenoses and 27 total occlusions. The segmental distribution was as follows: 37 in the iliac artery, 46 in the superficial femoral artery, 12 in the popliteal artery area. Ankle-brachial index (ABI) before atherectomy was 0,53 . All patients were evaluated before, immediately after, and in the follow-up visits by physical examination and ABIs. Digital subtraction angioigraphy (DSA) was used in the follow-up of $25 \%$ of the patients.

The average increase in $\mathrm{ABI}$ was 0.87 . This $\mathrm{ABI}$ value has decreased to 0.80 at 36 months and in those patients that remain asymptomatic. Associated procedures included angioplasty in 57 cases and thrombolysis in 2 cases.

At 36 months follow-up $78,94 \%$ of the treated vessels remain patent, $17,89 \%$ reoccluded, and 3,15\% were lost to follow-up. Eighty-two percent of the stenosis and $70,37 \%$ of total occlusions were patent.

In conclusion we think the atherectomy catheter has been proved useful not only in the treatment of arterial stenosis but also in the management of total occlusion and it could be a good alternative to surgery in the treatment of intimal flaps.

\section{Room E2}

\section{RC}

\section{6}

252 Spine related disorders: Intervertebral discs

W.P. Butt; Leeds

Abstract available at the information desk.

\section{Spinal stenosis}

\section{J. Haller, D. Wimberger; Vienna}

Spinal stenosis has been defined as a condition with reduced size of the spinal canal or its lateral recesses, causing compression of the thecal sac and its contents. Regardless of its congenital or acquired origin, spinal stenosis occurs most commonly in the lumbal or cervical regions.

Plain film examination is still the basic method and has to precede other imaging modalities. Plain film information enables correct performance and interpretation of myelography, CT and MRI examinations. Furthermore displacement of vertebral bodies and movements of articular facets can be evaluated with functional examinations. CT using a wide window is the method of choice to visualize osseous (mostly degenerative) spinal stenosis. The relationship of these changes to the thecal sac can be seen best with computer assisted myelography (CAM). Plain myelography depicts the configuration of the intradural spaces, stenosing changes can only be deduced indirectly. MRI provides information equally to CAM, but is non invasive, and reveals soft tissue alterations better than CT. Thus MRI may replace CAM, at least in the lumbar region. To our experience, in about $10 \%$ of the cases with cervical spinal stenosis, CAM becomes necessary after MRI in order to get more osseous information. Contrast media supported MRI is most sensitive in spinal stenosis caused by soft tissue changes. Failed back surgery syndrome, for instance, which affects about $25 \%$ of the patients after disc surgery, has become a main MRI indication. In $80-90 \%$ of these cases a clear distinction between scar and disc tissue can be done. Furthermore, inflammatory alterations and their therapeutic response can be monitored and blastomas causing spinal stenosis can be diagnosed and delineated best with MRI.

In conclusion, today examination of spinal stenosis is a domain of CT and MRI, supported by plain film informations. Invasive myelography is loosing its importance.

\section{Room L}

\section{RC}

254 Modern plain film analysis of pre- and postoperative congenital heart disease

Kenneth E. Fellows; Philadelphia, PA

In spite of new technology such as Doppler echocardiography and cardiac MRI, and despite surgical emphasis on complete repair of defects in infancy, plain films remain important to the evaluation of children with congenital heart disease. The overall heart size and the characteristics of pulmonary blood flow remain essentials not available from other diagnostic studies. Moreover, clues to anatomic abnormalities which may influence catheterization and surgical repair are present in the mediastinal configuration (i.e., azygous continuation of the inferior vena cava), the cardiac shape (i.e., juxtaposition of the atrial appendages), and abnormalities in the bony thorax (i.e., rib notching from collaterals in tetralogy of Fallot).

Deep hypothermic cardiac arrest and other developments allow the neonatal surgical correction of simple and complex types of congenital heart disease. Examples are complete repair of tetralogy of Fallot, arterial switching for d-transposition, and the Fontan procedure (direct caval-pulmonary artery anastomosis) for hearts with only one ventricle (i.e., tricuspid atresia and the hypoplastic left heart syndrome). Additionally, some cadiovascular anomalies are now better treated by catheter techniques rather than surgery, examples being patent ductus arteriosus, recurrent coarctation, valvular and peripheral pulmonic stenosis, and (potentially) multiple and residual muscular ventricular septal defects.

Not only is there important preoperative information on plain films for each of these congenital heart diseases, but the postoperative films are essential to the discovery and documentation of complications. Familiarity with these diseases, their surgical corrections, and the postoperative configuration of the heart is necessary to detect complications which arise, and to recognize the normal post-surgical morphology which will characterize the films throughout adolescence and adulthood.

\section{Room M}

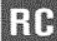

\section{The right place of prostatic ultrasonography} L. Lemaitre, E. Mazeman; Lille

The role of ultrasound prostatic cancer has generated a great deal of controversy with regard to its early detection and improved staging.

After initial enthusiasm for this new technique, further data now leads us to reassess its place in the diagnosis of prostatic cancer. As technology and our experience in this field continue to evolve, recommendations may now be made concerning the indications for ultrasonography.

1. Transrectal US is a complement to digital examination, increasing diagnostic suspicion of cancer when it reveals a palpable lesion in the peripheral zone as being hypoechoic. US guided biopsy may enable more tissue sampling of the hypoechoic lesion. There is no reliable specific appearance on sonograms that allows accurate differentiation between benign and malignant areas. Thus a hypoechoic lesion is an indication for biopsy.

2. Transrectal US may demonstrate non-palpable prostate cancer in patients with positive results after laboratory tests. The prostatic specific antigen (P.S.A.) is considered as a sensitive marker for prostate cancer but elevated serum levels are also observed in patients with acute prostatitis and benign prostatic hypertrophy.

3. US guided systematic biopsy of the contralateral lobe is useful. There is no specific ultrasonic pattern and 19 to $32 \%$ of the prostatic cancers are quite uniformly isoechoic.

4. No properly controlled study supports use of transrectal US as a primary screening exploration for the detection of curable prostate cancer.

5. The tumor volume evaluated by US is one of the most reliable predictive factors of cancer progression. 
6. Transrectal US may reveal capsular penetration and seminal vesicle invasion in patients with clinically localized cancer but it obviously is unable to detect a microscopic invasion.

In the preoperative assessment of $\mathrm{BPH}$, US is a useful means of evaluating $\mathrm{BPH}$ volume, post voiding residual urine and other consequences of outflow obstruction.

Finally transrectal US may provide us with instructive information in patients suffering from prostatitis or other diseases of the seminal tract.

\section{$256 \mathrm{MRI}$ of the prostate}

H. M. Pollack, Philadelphia, PA

This part of the course will deal with the techniques and applications of prostatic MRI. Techniques for conventional body coil MRI will be discussed first, followed by a review of the anatomy of the prostate as seen by MRI. A clear description of the internal architecture of the gland including its various zones, will be presented with special attention to the effects of changing pulse sequences and varying anatomic planes on the appearance of intra- and extraprostatic structures. Subsequently, attention will be directed to endorectal surface coil imaging with emphasis on techniques, as well as differences in the appearance of the prostate gland between surface coil and body coil imaging. The improved resolution of structures such as the neurovascular bundles made possible with endorectal surface coil MRI will be illustrated.

Examples of the usefulness of the surface coil technique in staging carcinoma of the prostate will be emphasized and data will be given comparing its staging accuracy with other modalities.

The value of surface coil imaging in certain benign conditions of the prostate and its adnexae, such as seminal vesicle and utricular disorders will also be discussed and illustrated.

\section{Room N}

RE

\section{7}

\section{IA-DSA in neuroradiology}

H. Zeumer; Hamburg

Since the introduction of computerized tomography the indication for cerebral angiography has changed significantly. Cerebral angiography is not a screening method anymore. Angiological information as for the morphology of arteriosclerotic lesions, the demonstration of collateral pathways in cerebral vascular occlusive disease and the anatomical analysis of cerebral vascular malformations have become more important in the last years. Due to the development of digital subtraction angiography the method can now be performed much faster under online visual control using lower concentrated contrast media. These capabilities not only improve the technical quality of the method but reduce the inherent risk of this invasive technique. Concomittantly with and enhanced by DSA transvascular therapeutical techniques have been developed and improved in the last years.

\section{PET studies in cerebral vascular disease}

\section{W.-D. Heiss; Köln}

Studies of various physiologic variables in early stages of ischemic stroke by PET of O-15 and F-18 labelled tracers have improved our understanding of pathophysiologic mechanisms leading to ischemic cell damage. Especially uncoupling phenomena among regional perfusion and metabolism indicate viable tissue in demand of blood supply (e.g., increased oxygen extraction fraction as a sign of viable tissue suffering from misery perfusion) or pathologically altered energy metabolism which may be detrimental for cell survival (e.g., glucose uptake far beyond oxygen supply as a sign of anaerobic glycolysis with excessive production of lactic acid). In addition, flow and metabolic images in stroke cases usually demonstrate diaschisis or remote metabolic depression as an indicator of the effect of a focal lesion on whole brain function and rehabilitative capacity.

Since large scale controlled clinical trials as the only means to prove the efficacy of treatment are extremely costly and time consuming there exists a need to select drugs and therapeutic strategies with a potential for effectiveness for such studies. The various aspects of flow and metabolic changes observed by PET in early stroke can serve as a study paradigm to indicate therapeutic efficacy. This correlation of effects on pathologically altered variables as demonstrated by PET and the clinical efficacy in multicenter studies is evident from studies with the $\mathrm{Ca}^{++}$channel blocker nimodipine, which was shown to improve misery perfusion in penumbra zones, decreased glucose metabolism in deactivated regions, and clinical outcome after ischemic stroke. As also indicated from other trials in progress PET studies in early stroke can be utilized to close the gap between experimental testing and clinical application and may help in defining treatment protocols for multicenter studies.

\section{Room 0}

WS

259 Clinical relevance of MR-spectroscopy Ole Henriksen; Copenhagen

Non-invasive measurements of tissue metabolism by MR-spectroscopy are a powerful tool in biomedical research. In principle such measurements are of great clinical relevance. So far, a really breakthrough in the sense of appellation of MR-spectroscopy in clinical routine in a broader sense remains to be seen. Among the reasons are technical difficulties, which have to be dealt with, including development of methods for volume selection which give reproducible results. Another basic problem is the inherent low sensitivity of MR, which constrains the spacial resolution within a measurement time tolerable for the patients. Finally, a lot of research is needed to establish relevant clinical diagnostic tests.

Recent results indicate that proton spectroscopy may be relevant for clinical investigations of the brain, bone marrow, liver, and skeletal muscle. Phosphorous spectroscopy is used for clinical evaluation of skeletal muscle metabolism during exercise. Another topic is brain asphyxia in newborn infants.

Conclusions: Spectroscopy methods for clinical research carried out on human beings are now available. The research activities are growing and the results obtained indicate that MR-spectroscopy surely will be of clinical relevance in the future.

\section{Room R}

\section{SS}

\subsection{Importance of diagnostic imaging in Radiotherapy III}

\subsection{PM}

\section{Primary CNS lymphoma: assessment of treatment strategies} R. Wurm, H. Pape, F. Düster, N. Zamboglou, G. Schmitt; Düsseldorf

The objective of this retrospective study was to analyse current treatment strategies of primary CNS lymphoma at our department. From 1980-1989 26 patients ( 14 female, 12 male) with primary lymphomas of the CNS were treated by radiotherapy. The age incidence ranged from 18 to 78 years, with a mean age of 62 years. 14 were high grade, 5 low grade and 7 unclassifiable lymphoma. 23 primary CNS lymphoma were localised supra- and 3 cases infratentorially. 22 patients had whole brain radiotherapy up to 30-50 Gy, which was combined in 2 cases with MTX intrathecally. In 4 patients a boost was added to the entire tumour up to $60 \mathrm{~Gy}$ and in 4 cases craniospinal irradiation was performed. During radiotherapy clinical symptoms improved in $80 \%$. Control CT and MRI scans revealed an initial response rate of $85 \%$. Median survival was 14 months and the median time to recurrence 12 months. 16 patients developed a local recurrence and 6 patients a recurrence outside the primary lesion. In 4 cases additionally CSF seeding was found at the time of recurrence. Although the data as heterogeneous as those reported in the literature, they support the temporary efficacy of radiotherapy for primary CNS lymphoma. But a clear guidance of treatment can not be obtained. Under such circumstances, a multidisciplinary approach integrating surgery, radiotherapy and chemotherapy as well is one we should aim for.

\subsection{PM}

\section{Radiosurgery of cerebral arterio-venous malformations with a} dynamic stereotactic single high dose irradiation

R. Engenhart, B. Wowra, B. Kimmig, K. H. Höver, R. von Kummer, M. Wannenmacher; Heidelberg

Since 198490 patients with inoperable cerebral arterio-venous malformations or residual malformations after embolization or surgery were treated in Heidelberg. A commercial $15 \mathrm{MeV}$ linear accelerator with special collimators was used for dynamic stereotactic irradiation technique delivering a sharp dose gradient at the edge of the target volume. The AVM field size treated ranged from 10 to $54 \mathrm{~mm}$ (mean $29 \mathrm{~mm}$ ). The location, size and venous drainage was assessed according to the Spetzler grading system: grade III 22 pat.; grade IV and $V 30$ pat.; definitive inoperable 30 pat.s. Operation was performed in 11 pat. and endovascular embolization in 6 pat. in order to reduce the AVM size. The prescribed doses at the isocenter varied from 10 to $36 \mathrm{~Gy}$ and were given as a single fraction in all pat.s. To date 30 pat.s were followed by repeat angiographic studies at two years post treatment. We found a significant dose effect relationship: pat.s who received less than $12 \mathrm{~Gy}$ at the edge of the pathological vessels showed no change. Dose effect studies indicate that superior results occur in AVM's treated with margin doses between 18 and $25 \mathrm{~Gy}$. A complete obliteration was found in $15 / 30$ pat.s $(50 \%)$ a partial obliteration in $6 / 30$ pat.s $(30 \%)$. Side effects occured in $13 / 90$ pat.s (14\%). 10 pat.s developed reversible, 3 developed permanent neurologic deficits within the latency of 6 to 12 
months. Rebleeding was seen in 7 pat.s after irradiation.

This results are preliminary in respect to the 30 pat.s. However, the technique is as effective as proton-beam therapy, at least.

\subsection{PM}

\section{MR-assisted brachytherapy on esophageal carcinoma}

H. Aydin, J. Bachmann, H.v. Lieven, M. Sens; Giessen

Magnetic Resonance Imaging enables an exact coronar and sagittal topography of oesophageal cancers. In our Institute patients with oesophageal cancers become a pretherapeutic CT and MR-Scan. The inoperable cases become a Teletherapy up to $50,0 \mathrm{~Gy}$, delivered in five weeks with a low-dose Cis-Platin or 5-FU-Mitomycin C Radiosensitizing on days 1 to 5 . We apply then a boost of 2- up to $4 \times 4$ GyHD Iridium HDR Afterloading Therapy with the oscillating Buchler-System. Before the application of Afterloading-Boost, the patient is examined on MR with the Afterloading Catheter in oesophagus. The catheter is filled with diluted gadolinium to determine its position, moreover the both margins of the therapy-length are marked with thin metallic wires which can also be seen on MR-Film. The sagittal and coronar MR-Picture is enlarged to $1 / 1$ real size and overexposed with isodose-curve of Afterloading-therapy plan. If necessary, correcture of the therapy-length or dosis distribution can be undertaken. The combination of MR-Scan with the placed and marked AL-Catheter and isodose-chart allows an optimum placement of the catheter and dose calculation. The dose on critical regions such as spinal cord can be determined easily and exactly. The therapeutical response is promising.

\subsection{0-5.30 PM: Round Table Discussion with Keynote Speakers}

\section{Room $S$}

\section{WS}

\section{3}

263 Planning the radiology department for the year 2000: introductory remarks

Antonio Chiesa; Brescia

Since the advent of Computed Tomography and Ultrasounds, the Radiology Department has undergone the most impressive modifications among all hospital departments. In the last 15 years, technical and clinical needs transformed Radiology into Diagnostic Imaging. The consequences of this change are not yet exhausted: MRI, Computed Radiography, and PACS are few examples of areas which are still on a fast growing development.

Therefore it is very difficult to identify and propose a Department of Radiology (or Diagnostic Imaging?) which could fit the needs of the beginning of the next millenium. Nine years before this beginning, some trends are easily identified, many others can be foreseen, few others may hypothetically be proposed. The Authors involved in this Workshop will try to define and to present these trends.

As a first introductory remark it is important to draw the attention on the many differences which characterize the situation of existing Radiology Departments: the updating of these Departments will be much more frequent and important than the building and the equipping of the new ones.

A survey based on 1989 data was carried out in 10 countries of Europe, and analyzed 120 hospitals. The surveyed hospitals were 46 teaching, 72 general and 2 specialistic hospitals. The grouping according to the size showed this distribution: 16 small size (below 400 beds), 38 medium size ( $400-800$ beds), and 66 large hospitals (more than 800 beds). The data collected presented many differences from one site to the other and meant that the projects on planning, on building or re-building, and on technology acquisition should be different according to the hospital size and to the local situation.

A second remark points out some important general criteria which should be taken into account for any project on planning. The following points are to be considered:

- the changing spectrum of patients (aged patients are continuously increasing) and of diseases (AIDS, more vascular and neurological disorders, more grafts and more reconstructive surgery). This evolution will demand a corresponding modification of radiological facilities;

- the large amount of information which can be acquired from digital sources (e.g. flow and diffusion measurements, spectroscopic signals, volume and motion analysis), and which is not based on images. The coexistence of images and of different parameters has to largely modify the information flow into the Department;

- the shifting of invasive examinations (angiography, interventional procedures) from $x$-ray based activities to non-x-ray activities (endoscopic ultrasounds, laser procedures). The consequence could be the disappearance from the Radiology Department of any interventional work;

- the management of images, as foreseen by PACS and IMAC implementation;

- the upgrading and the maintenance of equipment;

- the economic constraints which push towards a more difficult funding for Radiological Departments in an era of reduction of health care resources;
- the need for a change of medical habits. Due to this change the radiologists have to keep the guidance responsability for the most suitable examination to be performed for a given suspect of disease.

Many of the above mentioned criteria are not really dealing with the planning of Radiology Departments. Nevertheless they greatly influence the final result, and their importance cannot be underestimated.

\section{Building or extending a radiology department. The planning phase Eric J.Roebuck; Nottingham}

A series of well defined steps are essential to successful planning.

Step 1 Establish a core project team.

Step 2 Get to know the background.

This entails: defining the parameters available - space finding, political constraints etc. defining the needs in terms of work volume and specialised requirements (Angiography, MRI etc). This needs to be done with future developments in mind - population growth and movements - technical advances etc.

Existing and planned facilities available in the locality should be assessed - do these also need modification (e.g. should one centralise an MRI service).

Step 3 Decide on this basic provision in terms of the number and type of Radiological Rooms. From this will flow the size of supporting facilities administrative, clerical, waiting space, staff accommodation etc.

Step 4 Get to know the architect - well, he is the key to a successful design.

Step 5 Define several options which could fulfill the basic provision objectives.

Step 6 Option appraisal.

Develop a short list of options by matching them against the background, and judge if they will fully fulfill the defined needs. The benifits and costs of each option should be assessed, including capital, estimated revenue costs and the probable time scale, with any phasing proposals.

Step 7 The "core" project team should be enlarged to include all interested parties.

- Administrator/Manager

- Architect

- Quantity Surveyor

- Mechanical Engineer

- Electrical Engineer

- Radiologist

- Radiographer

- Radiological Engineer

- Radiation Protection Advisor

The full project team should define the future departmental policy, and the functional relationships of facilities to be provided. The work flow pattern should also be defined, and a commissioning plan developed.

Step 8 The layout of Radiological Rooms and associated areas can now be designed using ergonomic models to ensure efficient and comfortable working practices.

265 Impact of the changing workload and technical developments on the department planning

O. Pohlenz, E. Heidtmann; Hamburg

A. The work load of an imaging department of a 1100 beds hospital has been repeatedly analysed since 1978 with the following results:

1. makked and steady growth of ultrasound and CT

2. slow but steady increase of invasive/interventional procedures

3 . decrease (disappearance) in number of some types of conventional radiography

4. slow but steady emergence of MRI

B. Our results are compared with those from 30 other imaging departments in 6 European countries.

C. For future planning these results lead to the following consequences:

1. Equipment and other type of hardware

a) only few but more multipurpose conventional general duty equipment, but still some dedicated units (i.e. for mammography, angiography, pediatrics, urology)

b) increase in number of units for ultrasound, CT, and MR

c) increase in number of small, more decentralized imagers with easy production of hard-copies

d) more computers, electronics and network.

2. Image (-production, -processing, -archiving)

a) Replacement of conventional film by computers and associated electronics with

b) corresponding data handling (i.e. PACS, RIS, Teleradiology etc.)

c) HOWEVER: parallel handling of "PCR-technology" along with conventional (old) techniques and films (i.e. screens and monitors, films and electronic images) for a longer period of time (years!)

3. Room planning

a) decentralized work stations with centralized administration by linkage to a central data net

b) rooms (for roentgen, ultrasound, CT, MR, intervention) with adequate larger size for

- more personnel in interdisciplinary action 
- more space around the patient

- computer hardware

- air-conditioning

- image analysis, post-processing, presentation.

c) rooms designed for organisation, administration, and (interdisciplinary) communication employing old and new techniques.

\section{The department of diagnostic imaging towards 2000 A.D.} Holger Pettersson; Lund

To foresee the future of diagnostic imaging is not only challenging and in many aspects impossible. It is also demanding and necessary, because such forecasts are more than intellectual games - what we think and discuss today will to some extent change and shape the future.

Based on the development we know today, and the trends we can see concerning changes in the spectra of patients, the handling of medical information, and the approach to treatment, some suggestions may be given.

1. The equipment will naturally differ with the economic circumstances and the size of the department, but virtually all large regional or university departments will be well equipped with ultrasound, scintigraphy, CT, MRI and probably PET. The conventional radiography of today will be digital, be it a development of the imaging plate system we see today, or some other approach. Also the small radiologic departments in the private or county setting, will need all the modern modalities, including MR.

2. Storage and communication of the medical information will be digitized. This will mean planning and construction of networks within the department of radiology, and in the hospital between the department of radiology and the wards, operating rooms, intensive care units, etc. For communication between hospitals the telecommunication systems will be used. Data compression techniques, based on new approaches, have developed, making the information easy available and communicable, also from large archives.

3. Interventional radiology has developed considerably. It will be performed by specialists, who are recruited both from radiologists and clinicians. From the patient's point of view it is totally unimportant if the doctor who treats him in the interventional room calls himself radiologist, surgeon, or internist - all the patient wants and needs is a skilled specialist. If this specialist is a radiologist who has learned clinical skills, or a clinician who has learned radiology, is less important. Both radiologists and clinicians of today have to consider ths carefully.

4. Concerning organization, the large departments will be organized according to organ areas, and the different organ specialists - surgeons, internists, pathologists, radiologists, etc. - will work close together towards the same goal, both in the research and the daily routine: the benefit of the patient. It means that the border between the different specialities within the same organ area partly have dissolved. But still the highly specialized radiologist has basic knowledge of diagnostic imaging as a whole, and he/she shares the heavy and expensive equipment with his/her fellow radiologists in one and the same department of radiology. In smaller departments, the radiologist must be more all-round, with updated basic knowledge in radiology as a whole, even if he is specialized in few organ areas.

5. Working conditions, relations between radiologists and between radiologists and clinicians, will gradually change because of the changes described above. In planning the future department, it is extremely important that we realize that the extensive technical progress in radiology will change the discipline of radiology thoroughly and the demands on the radiologist correspondingly. In the future, the radiologist will be concerned not only with morphologic changes, but also with functional changes, like flow and diffusion, volume and motion. This will be based on images, but also on other types of signals, for instance spectroscopy. It is important that we realize this today. Then we can plan the future department to cover the demands of tomorrow, and we can prepare ourselves and our colleagues towards our common goal, the best benefit of our patients.

Closing remarks

Antonio Chiesa; Brescia 



\section{Room A}

\section{BC 311}

The examination of the paediatric brain and spinal cord by magnetic resonance imaging and by this the diagnosis of their abnormalities is not merely an extrapolation of the adult experience, as children are not "small adults". The special knowledge of and experience in the child's central nervous system anatomy and its development, its variations, and pathologies is essential, as is the special application of MRI techniques in children.

An essential outline will be presented encompassing sedation, special techniques, anatomical variations, and principles of diagnosis often unique or at least peculiar to paediatric pathology. Special attention will be paid to a practical diagnostic approach towards congenital abnormalities; neoplasms of the brain and spinal cord and vertebral abnormalities, and in particular their appropriate and necessary clinical presentations.

\section{MR imaging of the head and neck}

\section{Thomas J. Vogl; München}

\section{Magnetic Resonance Imaging (MRI)}

is evolving into an effective tool with which to image the head and neck, its strength based on excellent soft tissue contrast, spatial resolution and multiplanar imaging capability. The increasing experience with the paramagnetic contrast medium Gd-DTPA in the head and neck suggests that there will be variable utility depending on the site under investigation and the questions being asked. At the moment there are two different settings for the use of Gd-DTPA. In some of these Gadolinium is definitely useful, in others it might be useful depending on the individual observer.

The skull base and petrous bone is a complex crossroad of endocrine, neural, vascular and skeletal structures. Accurate imaging is crucial because clinical evaluation frequently cannot localize the lesions accurately.

Although computerized tomography (CT) was a significant imaging advancement in the evaluation of the skull base, there are a number of limitations like radiation, contrast enhancement, beam hardening artifacts and limited soft tissue resolution. So Magnetic Resonance Imaging (MRI) has become the modality of choice in the assessment of most tumors of the skull base and adjoining structures.

In the face and nasopharynx MRI has shown some relative deficiencies due to the fact that both the air and the bone produce no signal (black) on all MR pulse sequences because of their inadequate number of mobile protons. However, new scientific data have proven that MRI has a major impact in the diagnosis, treatment planning and follow-up evaluation of neoplastic diseases in the head and neck.

Clinical examination with endoscopy remains the best method for assessing the mucosa in the oropharynx and oral cavity. Although tissue diagnosis from imaging modalities alone is not possible CT and MRI are important for localization of potential biopsy sites. The most frequent reason for referal to MRI is to assess deep soft tissue extent of the primary lesion in order to support treatment planning.

The goal of salivary gland imaging is threefold:

1. Diagnosis of intraglandular vs. extraglandular lesions, 2. differentiation of benign from malignant lesions and 3 . demonstration of the facial nerve in relation to a tumor. In this area MRI is able to provide the needed information without the associated risks of CT.

Both high-resolution CT and MR imaging exquisitely display normal and pathological anatomy of the neck. However, accurate assessment of the neck requires a thorough knowledge of both the complex anatomy and scope of pathologic entities although CT and MR imaging may have relatively poor specificity. In the detection of metastatic nodal disease there are several features in imaging studies which are highly indicative of metastatic disease including central nodal necrosis peripheral nodal enhancement.

New developments in 3D-techniques have shown an enormous impact on diagnostic possibilities in the head and neck. These techniques allow the evaluation of very thin slices and the arbitrary reconstruction of outlines and surfaces. Based on MRI data sets all 3D-techniques are characterized by lower signal-to-noise ratio compared to conventional sequences and time-intensive postprocessing.

Many clinical spectroscopic studies employing in vivo 31-Phosphor and 1 Proton-spectroscopy suggest the clinical utility for the studies of metabolic and ischemic disease affecting metabolism. Abnormalities in 31 Phosporspectroscopy, observed in neoplasms, are similar to those seen in other pathologic states. So it seems likely that spectroscopy can serve only as an adjunct to other diagnostic tools in these cases.

On contrary Proton $(1 \mathrm{H}) \mathrm{MR}$-spectroscopy may be useful for characterizing a

variety of lesions of the head and neck and skull base. Future developments will show whether spectroscopy will eventually become routine thereby limiting the need for tumor biopsies. Additional research and blind clinical tests will be necessary in the future. So Head and Neck radiology continues to become a further exciting subspeciality within radiology and neuroradiology.

\section{Room B}

CE

\section{Diagnosis and primary tumor characteristics (T-Factor)} J.P. Senac; Montpellier

\section{Staging for nodal and distant metastases (N-M-factors), clinical stage, treatment options and restaging}

Peter Vock; Bern

A shift in the surgical approach to non-small cell lung cancer (NSCLC), a modified TNM-staging system, the technical development in non-invasive imaging methods, new knowledge about normal lymph node size and anatomical location, and changing results in radiologic-pathologic correlation analysis all contribute to the fact that the preoperative assessment of nodal metastases from lung cancer is a complex subject and has caused controversial discussions. Based on the topography of hilar and mediastinal lymph nodes, the pathways of lymphatic drainage from the lung to the mediastinum and the current TNM-staging categories, the role of CT and MRI is analyzed with regard to methodology, criteria of pathology and histologic verification. The reasons for the wide ranges of reported specificity and sensitivity are analyzed. Based on this recent knowledge and the treatment options, a pragmatic approach to nodal staging is suggested.

The brain, liver, adrenal glands, bone and bone marrow, and the kidneys are the preferred locations of distant metastases from lung cancer of all histologic types; large-cell carcinoma has some predilection for the gastrointestinal tract. Largely used imaging methods to search for brain metastases are CT or MRI, for liver metastases CT, ultrasound or MRI, for adrenal metastases CT or MRI and for bone metastases nuclear bone scans. The need for brain and bone screening generally depends on the cell type and the presence of a clinical suspicion.

In NSCLC, surgery is the treatment of choice in all cases with clinical stages I and II and, increasingly, IIIa whereas stages IIIb and IV are treated by chemotherapy and/or radiotherapy.

In the follow-up after treatment, complications of treatment, the multicentricity of lung cancer and infections due to the suppression of the immune system have to be differentiated from local or distant recurrences of the primary tumour.

\section{Room C}

\section{WS}

271 Technique and interpretation of the double contrast barium enema

C. I. Bartram; London

Diagnostic accuracy depends on the quality of examination technique and interpretation. There is an inter-relation between preparation and suspensions, magnesium based purgatives creating a "gel effect" to improve coating. Carbon dioxide is recommended as its rapid reabsorption reduces discomfort afterwards. An i.v. smooth muscle relaxant should be used routinely and hyoscine butylbromide is a more effective than glucagon in the colon. An integrated technique using a disposable barium and carbon dioxide filled bag will be described, with details of how to fill the bowel, film sequences, and dealing with common problems

Inherent diagnostic limitations are lesions that do not alter the fine mucosal coating $(<0.2 \mathrm{~mm}$ thick), such as angiodysplasia and microscopic colitis. Relative limitations are polyps $<5 \mathrm{~mm}$ which are difficult to distinguish from other ring shadows - notably residue. Polyp detection is reduced in diverticular disease. Compression helps show polyps as filling defects in barium pools. The double contrast en face texture of mucosa is normally smooth. The innominate groove and lymphoid hyperplasia patterns are normal variants. The coating viewed tangentially creates mucosal "lines". Normal lines due to haustra etc. must be distinguished from abnormal lines formed by menisci at tumour edges. Inflammation creates a granular or ulcerated surface, and alters smooth muscle tone, causing blunting or loss of haustration, narrowing and shortening of the bowel, and widening of the presacral space. Both mucosal and structural changes are reversible. The instant enema is a limited double contrast study without bowel preparation designed to assess patients with known colitis, and provide a map of the colitis. The relationship between the double contrast barium enema and colonoscopy in polyp detection and colitis will be discussed. 


\section{Room E1}

\section{R6 302}

272 Local arterial thrombolytic therapy: current practice and methods E. Starck; Kasse

Basic handling, dosage and laboratory monitoring of the different pharmaceutic thrombolytic agents, streptocinase, urocinase and plasminogen activator will be discussed. The way of local application is very important for the reopening rate. Long term lysis increases bleeding complications significantly. Mechanically accelerated lysis solves the most important problems either with pulsed spray application, pre balloon-dilatation or with rotating devices (RAT) increasing the surface for the local lytic agent. Removal of bad or unlysable components by aspiration increases the success rate significantly. Combinations of several angioplastic means serves best for acceptable lyses times with improved successrates and low complications.

\section{Local arterial thrombolytic therapy: current practice and methods $\overline{R . F}$. Dondelinger; Luxembourg}

Local arterial thrombolytic infusion has evolved as an established therapeutic modality of vascular occlusion in conjunction with percutaneous thrombus fragmentation and aspiration. Local thrombolytic infusion can be applied alone or combined with percutaneous transluminal angioplasty, percutaneous vascular stenting or surgery. Among indications, occlusions of the arteries of the lower limbs and upper limbs, of the abdominal aorta, and the superior mesenteric artery will be detailed in various clinical conditions. Streptocinase, Urocinase and recombinant tissue plasminogen activator are in current clinical use; the different mechanisms of these drugs will be discussed. Recommendations on technique of local infusion, haematological and radiological monitoring of the patient during treatment will be given. Dose regimen of these different drugs and results obtained will be presented with a particular emphasis put on cause of failure and complications and their prevention.

\section{Room E2}

\section{RC}

274 Imaging of joints - current practice: knee

Maximilian F. Reiser; Bonn

MRI has become a highly important tool for the evaluation of various disorders of the musculoskeletal system. Injuries of the knee joint are very frequent. In this course the technique of MRI examination, normal anatomy and variants as well as the pathological findings and the diagnostic value in various disorders will be addressed.

T1-and T2-weighted spin-echo sequences are advocated by most authors as the adequate imaging technique. Meniscus and cartilage lesions, however, are advantageously visualized using gradient-echo techniques. Multiplaner MR anatomy and various anatomic variants will be discussed in order to enable the differentiation of normal from pathological findings. MRI has gained high accuracy for the detection and grading of meniscal lesions. High signal intensity foci extending to the articular surface are indicative of meniscal tears, whereas high signal intensity confined to the meniscus substance are found in degenerative alterations. Normal ligaments and tendons exhibit low signal intensity, independent of the imaging parameters selected. Focal or diffuse signal increase is observed in lesions of the cruciate and collateral ligaments and of the patellar tendon. The degree of injury of ligament can be accurately assessed using MRI.

Controversy exists concerning imaging techniques and diagnostic utility of MRI for the visualization of normal and pathological joint cartilage. The benefits and drawbacks of various pulse sequences and of intraarticular administration of Gd-DTPA will be discussed.

MRI proved to be superior to other imaging modalities for the detection of aseptic necroses and osteochondritis dissecans. This holds also true in occult fractures of bony elements adjacent to the knee joint.

Inflammatory disorders, e.g. septic arthritis and rheumatoid arthritis, are reliably detected using MRI. Dynamic Gd-DTPA enhanced scans allow for clear depiction of synovial proliferations within the knee joint.

The choice of the diagnostic procedure and the significance of MRI for the work-up of knee joint pathology will be discussed. In conclusion, MRI can even know be recommended as the imaging modality of choice for the evaluation of internal derangements of the knee joint and it should be performed prior to arthroscopy.

\section{Imaging of joints - current practice: the shoulder} Bryan J.Preston; Nottingham

Plain films in conjunction with the history and clinical examination usually lead to an appropriate imaging strategy and clinical management. Two films at right angles are mandatory for assessing injuries at the gleno-humeral joint and upper humerus. It may be difficult to obtain a good lateral film and special projections have been devised to overcome this. Posterior dislocations of the humerus are easily missed if only an antero-posterior film is obtained and this is especially so if there is an associated fracture. Notches in the humeral head may be associated with recurrent dislocation at the gleno-humeral joint. The labral detachments which occur in recurrent dislocations are best demonstrated by double contrast CT arthrography although MRI is beginning to play an increasing role in detection of the abnormalities. Subluxations, dislocations and fractures at and around the acromioclavicular joint may involve angled up, weight bearing and axial views.

In patients with subacute and chronic pain in the absence of significant trauma it is helpful to consider the region as consisting of three compartments, namely the gleno-humeral joint, the subacromial space and the acromio-clavicular joint. Specific conditions tend to involve a particular compartment and they will be discussed. Polyarthritis such as rheumatoid arthritis tend to involve all compartments of both shoulders. Psoriatic arthropathy will involve both shoulders asymmetrically.

Double contrast arthrography until recently has been the method of diagnosing tears of the rotator cuff and capsulitis. MRI is now considered to be the modality of choice. Sonography has also been employed to detect tears of the rotator cuff but the results seem to be very variable.

Bone and soft tissue tumours are usually investigated in the conventional manner employing plain films, scintigraphy, CT and MRI.

\section{Imaging of the wrist}

Jean Garcia; Geneva

Injuries of the wrist are very common; clinical and plain film diagnosis is generally correct. Subtle fractures, particularly those involving the hook of hamate and the triquetrum, require conventional tomography or CT. CT is also of value in the evaluation of wrist instability, fracture healing (scaphoïdnon-union) and post-surgical follow-up.

Arthrography is very helpful in case of persistent wrist pain after trauma. Use of fluoroscopy during injection may demonstrate the ligament disruption before contrast medium excess. Digital arthrography combines the advantages of fluoroscopy and videotaping, visualizing the flow of contrast medium Three-compartment injection should be performed for an accurate diagnosis. The major findings are disruption of the scapho-lunate, lunate-triquetral ligaments, complete or partial tear of the triangular fibro-cartilage complex and capsular rupture or adhesive capsulitis.

MR imaging is the most advanced technique for soft-tissue evaluation, using high-field-strength $(1,5 \mathrm{~T})$, surface coils (FOV $8-10 \mathrm{~cm}), 3 \mathrm{~mm}$ slices. The imaging planes (transaxial, coronal, sagittal) are adapted for the specific problem. Cortical bone, bone marrow, hyaline cartilage, tendons, ligaments, muscles and neurovascular bundles are well differentiated because of their appearance on T1 and T2 images. Clinical indications for MR are known carpal tunnel syndrome (sinovitis of the flexor tendons or space-occupying lesion), triangular fibro-cartilage lesions (degenerative and post-trauma), sinovitis, arthritis (early stages), ischemic necrosis of the scaphoïd and Kienbock's disease (MR is the most sensitive technique), and tumor (MR is not specific but offers a very good delineation).

\section{Room L}

\section{RC}

277 Imaging of the salivary glands

\section{Peter M. Som; New York, NY}

This course will emphasize some of the exciting recent advances in the application of Magnetic Resonance Imaging (MRI) to the evaluation of salivary gland lesions. Specific attention will be given to diseases that involve the parotid glands because of the associated complications that can occur to the facial nerve.

Initially, the inflammatory and nontumorous diseases of the major salivary glands will be examined with a discussion of the pathophysiology of these diseases. The limited use of plain films and sialography will be discussed and the application of computed tomography (CT) and MRI will also be explored. Next, tumors of the major salivary glands will be evaluated and the pros and cons of the use of CT and MRI will be discussed. Extension of tumors from the retromandibular or deep portion of the parotid gland into the parapharyngeal space will be shown and a method for distinguishing a parotid origin from an extraparotid origin of a parapharyngeal space lesion will be examined. The imaging appearance of primary extraparotid parapharyngeal space salivary gland lesions that arise in salivary rest tissue will be shown.

The critical points to evaluate concerning malignant tumors will be emphasized so that the radiologist gives the clinician the optimal information necessary to determine the best treatment philosophy.

Lastly, minor salivary gland neoplasms will be explored and their imaging appearances and how they may serve to distinguish these tumors from squamous cell carcinomas will be discussed. 


\section{Room M}

\section{WS}

278 Doppler ultrasound and MRI of the transplanted kidney

J.F. Moreau, O. Helenon, E. Attlan, M. Souissi, J. Chabriais, N. Lebo; Paris

Duplex and color doppler (DCD) has become the screening test enabling to suppress many indications of invasive arteriography. In early post-operative anuria, it enables to diagnose accurately: 1 . the acute thrombosis of the renal artery (absence of flow, mute image of the vascular tree, visualization of the arterial stump); 2. the acute thrombosis of the renal vein (at the arterial leval: peaked systolic frequency shifts and plateau-like frequency shifts during diastole; mute image of the venous tree).

Provided the PRF is as low as possible, DCD is contributive in the detection of segmental or lobar arteries lesions inducing large infarcted areas (focal lack of vascular signal in hypo- or anechoic cortical areas). Because DCD is operatordependent and apparatus-dependent and not sensitive enough, the interlobular and nephronic vascularization cannot be evaluated accurately at present, when a nephropathy decreases the renal blood flow. This explains why DCD is not diagnostic in the differential between acute rejection, acute tubular necrosis and cyclosporin nephropathy, except when a massive cortical necrosis argues toward rejection; on the other hand, DCD is prognostic when the renal blood flow is increasing or not under treatment.

In the later stages, DCD provides diagnostic data on renal artery stenosis (high-frequency shifts at the site of the stenosis with post-stenotic turbulent flow) and can detect post-biopsy false aneurysms and arterio-venous fistulae. Because US, IVU and/or CT solve most of the urological complications, MRI is rarely indicated in obstructive uropathies or fluid collections. The major interest raises from the possibility to study morphologically and functionally all the renal parenchyma and its vascularization, with an emphasis on the poles which are not easily studied by DCD. Using Gd-DOTA after a non-contrast preliminary study in $\mathrm{T} 1$ - and $\mathrm{T} 2$-weighted sequences, dynamic sequences demonstrate, in normal kidneys, an early cortical enhancement followed by a rapid parenchymal signal homogenization, then a pelvi-calyceal system enhancement on delayed images. Renal infarction and cortical necrosis are well demonstrated by MRI: infarcted areas are not visible in T1-weighted SE sequences but T2-weighted SE sequences exhibit a slight increase of signal intensity when the necrotic area is ischemic, or a pale or heterogeneous signal when hemorrhagic. There is no enhancement on them after Gd-DOTA injection.

Whether by US or by MRI, no histological diagnosis can be made. Using Factorial Analysis Dynamic Structures software with contrast-enhanced CT or $\mathrm{MR}$, it is possible to study separately all the components of the renal vascularization from the tubular pathway, and more precisely the medullary component. This provides useful etiological and prognostic data.

\section{Radiological management of renal transplants: Nuclear medicine} Henrik S. Thomsen; Herlev

The most frequent and important complications of renal transplantation, vasomotor nephropathy, acute rejection, and cyclosporin A nephropathy, are diffuse processes. Their functional and perfusional consequences are usually more prominent than their structural effects. For this reason radionuclide studies being able to quantify perfusion and function have assumed a dominating role in the control of renal grafts. None of the radionuclide agents is specific enough to demonstrate a particular cause of renal impairment with certainty. In general serial quantitative radionuclide studies are needed in documenting improvement or deterioration in perfusion and/or parenchymal function. The fact that there are several different methods to follow the kidney suggests that there is not one ideal, but the most commonly adopted procedures are perfusion studies (so-called angioscintigraphy) and renography. The perfusion study gives a histogram of count rates (first passage curve) over the graft that depends on the vascular transit of the tracer and the total vascular volume in the kidney. The blood flow can be assessed both qualitatively and quantitatively. The graft renogram mirrors the effective plasma flow and tubular uptake and excretion if either ${ }^{131} \mathrm{I}$ hippuran, ${ }^{123} \mathrm{I}$ hippuran or ${ }^{99 m} \mathrm{Tc}$ $\mathrm{MAG}_{3}$ are used. The method of evaluating the graft is adapted from that used for "normal" kidneys. These radionuclide investigations have the advantages of being non-invasive and can be repeated frequently. This is important in a situation where clinical condition may change rapidly, e.g. acute graft rejection is not an all-or-none process but a crescendo attack by the body.

The more specific radionuclide methods using e.g. ${ }^{125} \mathrm{I}$ fibrinogen, ${ }^{99 \mathrm{~m}} \mathrm{Tc}$ sulphur colloid, ${ }^{67} \mathrm{Ga}$ citrat, and labelled patellets, lymphocytes and leukocytes have not gained wide acceptance due to a high frequency of false negative and false positive results.

\section{Room N}

RC

\section{7}

280 Imaging of cerebrovascular pathology A. Thron; Aachen

Imaging modalities in cerebrovascular diseases may be divided in 1 . non-invasive and 2 . invasive techniques.

1. CT is still the most important non-invasive examination in acute hemispheric stroke, giving a clear and quick distinction between an ischemic and a hemorrhagic event.

MRI gives additional information in infarction of the brainstem and may give essential information in suspected cerebral sinus thrombosis. However, in order to avoid misinterpretation, profound knowledge of flow effects and of the time dependant alteration in the signal intensity pattern of an intraluminal thrombus is needed. The main advantage of MRI lies in its superiority in demonstrating blood in subacute and chronic stages, as for example in cavernous angiomas. Furthermore, MRI may contribute to an early non-invasive diagnosis of the source of an intracranial hemorrhage (aneurysm, AVM, neoplasm) or of the cause of vessel occlusion as in vertebral and carotid artery dissection.

The clinical value of MR-angiography has still to be determined.

SPECT and PET-examinations mainly serve scientific purposes.

2. Digital subtraction angiography, performed as an intraarterial and selective procedure, remains the method of choice in the evaluation of arterial and venous vessel pathology. This includes demonstration of site and type of vessel occlusion in thrombembolic disease, arterial wall dissection and angiitis as well as evaluation of small and large aneurysms, AV-malformations, venous angiomas and thrombosis of cerebral sinus and veins.

Imaging of cerebrovascular pathology should be interpreted with regard to a pathogenetically orientated classification of brain infarction.

Typical but not pathognomonic lesions may be demonstrated if brain infarction is due to

- cerebral microangiopathy, causing lacunar infarctions and/or diffuse vascular demyelinisation or

- cerebral macroangiopathy, causing territorial infarcts in thrombembolic events and terminal zone infarctions in hemodynamically relevant stenoses.

\section{Peritumoral brain oedema}

P. Huber; Bern

The low density area around a tumour visualized on a CT scan is an expression of the extent and degree of the oedema caused by the fluid leakage from the tumour vessels which propagates into the surrounding brain tissue. Peritumoral oedema profiles were estimated by measuring the CT-numbers of consecutive tissue blocks of $3.0-3.6 \mathrm{~mm}$. The distance of oedema spreading is determined by the amount of fluid accumulation in the white matter immediately bordering the tumour. Pressure gradients and bulk flow are involved in the development of peritumoral oedema. Neither the size of the tumour nor the histology has a close relationship to the amount of pertumoral oedema. The localisation of an oedema producing tumour seems to be of some importance.

\section{Room 0}

\section{RG}

282 Supplementary investigations in diagnosis of breast diseases: needle aspiration cytology

N. Bjurstam; Göteborg

\section{Abstract available at the information desk.}

\section{Ultrasound of the breast} R. Ch. Otto; Baden

The challenge of applying sonography to the identification of breast lesions has been approached at several occasions. Semeiology described by various authors permits evaluation of different nodular formations which are palpable or visible on mammograms. Cysts may be differentiated from solid nodules with a high degree of certainty. The adequate management of cysts becomes feasible (puncture and pneumocystography) and solid nodules may be punctured under permanent visual control. Technique and examples will be shown and discussed. There are special ultrasound phenomena indicative for benignity and malignancy. Irregular borders, spicules and polymorphous structure, especialy attenuation of posterior echos with acoustic shadowing are most suspicious for cancer. Fibroadenomas instead, show a clear outline and evenly distributed posterior accentuation. The results of sonography indicate the importance of this imaging technique, though ultrasound examination on its own usually remains insufficient to evaluate breast disease or to screen for breast cancer. It may be helpful, however, for more precise interpretation of special mammographic findings. During the last years sonography became an 
essential supplement for breast examination due to its easy and inexpansive application.

\section{MRI of the breast}

Sylvia H. Heywang-Köbrunner; München

Introduction: Based on initial reports much hope was raised concerning a tissue characterization by MRI in breast diagnostics. This has not been fulfilled by plain MRI yet, but interesting and diagnostically important information has been gained by contrast-enhanced (c.e.) MRI using Gd-DTPA.

Methods: The special technical prerequisites for breast examination are presented. They concern choice of breast coil, necessary resolution, slice thickness, elimination of artifacts. For plain-MRI no generally valid recommendation concerning choice of pulse sequence yet exists. For c.e. MRI $3 \mathrm{D}$-gradient echo fast imaging sequences are presently best suited. The use of water-selective imaging will be discussed.

Results: Plain MRI has turned out more difficult than expected and has remained a matter of research. Therefore only a short overview will be given. Experiences with c.e. MRI worldwide comprise about 1500 cases (including over 700 cases examined at our institution). So far all malignancies enhanced significantly, whereas many benign changes don't enhance noticeably, some enhance variably.

Conclusions: Due to its excellent sensitivity and negative predictive value c.e. MRI has proven very valuable as an additional method for improved distinction between irregularly-shaped dysplasia or scarring and malignancy and for diagnostic problems in mammographically dense breasts.

\section{Room R}

\section{RE}

316

285 The role of radiation therapy in cancers of the prostate, urinary bladder, and testicle

Gerald E. Hanks; Philadelphia, PA

Long term results of radiation therapy in prostate cancer will be shown that accurately define the role of radiation therapy as compared to radical prostatectomy. This will include comparing the available 15 year data in patients of unknown lymph node status and the available 5 and 10 year data on patients with surgically negative lymph nodes. The roles of PSA, I-125 implant and post prostatectomy radiation will also be discussed and preliminary results demonstrating reduced morbidity with conformal 3D planned treatment of early prostate cancer will be shown.

The results of RTOG trials in bladder conserving studies of urinary bladder cancer will be summarized that emphasize the importance of including radiation in the multimodal treatment program.

The treatment of seminoma with radiation will be shown through fifteen year results that emphasize the success of treatment and the need for careful radiation technique and dose selection. 
The authors discuss the contribution of the 3 dimensional sequence in comparison with classical T1-weighted sequences. They analyse the contribution of this technique to the diagnosis of extra-axial lesions, its anatomical definition, its interest in the assessment of the extension of the lesions, and, finally, the total duration of the examination.

Results: Three dimensional imaging is an interesting technique because:

\section{Room A}

\section{SS}

11.04 Head and neck - bone-marrow

\section{$10.30 \mathrm{AM}$}

286 MR angiography of the head and neck region: technique and correlation with conventional angiography

Th. Vogl, J. Balzer, J. Stemmler, L. Ziegler, H. Schedel, J. Lissner; Munich

Purpose: Findings of different MR angiographic (MRA) techniques in the head and neck were compared with the results of high resolution digital subtraction angiography (DSA).

Methods and Materials: MRA was performed in 80 patients at 1.5 Tesla (Magnetom) with either a receiving and transmitting head coil or a receiving neck coil. Basic technique was the "Time Of Flight" MRA, employing FISP 3D or FLASH 2D sequences. Selective MRA was obtained by using an additional saturation pulse. MRA images were postprocessed with a ray tracing method. So far 30 patients with various lesions in the head and neck were studied with both MRA and DSA.

Results: Compared with DSA a similar delineation of vascular anatomy and arterio-venous malformations especially with extra- and intracranial vessels was possible. $56 \%$ of the patients underwent arterial MRA with manifestation of malformations such as stenosis, aneurysms or vessel displacement. Venous MRA was performed in $34 \%$ cases, $10 \%$ underwent arterial and venous MRA. Due to the construction of the neck coil and the limited FOV MRA revealed disadvantages for the evaluation of supraaortic vessels.

Conclusions: MRA proved to be a powerful tool in the preoperative visualization of vascular anatomy and arterio-venous malformations. Pitfalls are changes in flow-velocity and direction with possible overestimation of stenosis grade. As MRA can be easily added to a MRI examination and is a non-invasive technique it might replace conventional angiography in certain cases.

\section{$10.40 \mathrm{AM}$}

\section{MRI of the salivary mass lesions using stir sequences}

M. J. Ereño, A. Ferrero, J.C. Ruiz, J. Negueruela, J.C. Martin-Urreta, J. Reyzabal; Bilbao

Purpose: To evaluate the usefulness of MRI of the salivary mass lesions using STIR sequences versus T1-W, PD, and T2-W studies.

Methods and Materials: 15 examinations on patients with suspected salivary mass lesions were studied using a $0.5 \mathrm{~T}$ superconducting magnet and shortinversion-recovery pulse sequences. In all cases, additionally, T1, PD and T2-weighted images were performed using different multislice acquisitions and compared with the STIR sequences. The cases were confirmed by surgery and histology.

Results: (Preliminary results.)

MRI has identified 15 lesions using STIR sequences at the moment.

The masses were identified in all cases ( $100 \%$ sensitivity), even small ones. Lesions histologically diagnosed as pleomorphic adenoma were predominantly homogeneous as well as smoothly marginated in STIR images. Other salivary tumors were often heterogeneous.

On Tl-weighted image, the lesions showed low signal intensity and high signal intensity on T2-weighted image in most of cases, with any intermedial signal in malignancies.

By Short-TI-IR (STIR) image all the lesions were shown more definetely in high signal intensity than by $\mathrm{T} 2-\mathrm{W}$ image.

Conclusions: We consider MRI using STIR is to be useful in the evaluation of salivary gland tumors.

\section{$10.45 \mathrm{AM}$}

\section{Contribution of 3 dimensional imaging to the examination of skull base tumors}

C. Louail, M. Raynaud, D. Gense de Beaufort, J.F. Gréselle, J. M. Caillé; Bordeaux

The purpose of the present study was to evaluate a new technique, 3 dimensional imaging. This technique can perform reconstructions in every plane of space thanks to volumetric acquisition.

Methods: Examinations were performed on 30 patients with skull base tumors, using a Magnetom Siemens 1.5 Tesla. In every case, the 3 dimensional sequence after injection of Gadolinium was compared with a spin-echo Tl-weighted reference sequence (TR: 500; TE: 15), performed in at least two different planes and after injection of Gadolinium.

- it is as sensible as spin echo sequences in the positive diagnosis of extra-axial tumors when used together with the injection of Gadolinium.

- the assessment of the extension of the lesions is more precise than with spin-echo sequences because $3 \mathrm{D}$ imaging can obtain thin sections (about $1 \mathrm{~mm}$ ) in every plane of space.

- it represents a considerable gain of time, which makes $3 \mathrm{D}$ imaging very valuable in comparison with spin-echo sequences.

Conclusions: Three dimensional imaging with injection of Gadolinium appears to be a very promising technique in the exploration of skull base tumors, allowing a precise, reliable and fast diagnosis.

\section{$10.55 \mathrm{AM}$}

289 Quantitative long TR gradient recalled chemical shift imaging of bone marrow: Correlation with histology

Manfred Seiderer, Hubert Wagner, Axel Staebler, Josef Lissner; Munich

Purpose: Gradient recalled (GR) long TR imaging with opposed magnetization for fat and water protons results in low signal intensity (SI) of red bone marrow and high signal intensity of pathology.

Methods: Measurements were carried out on a $1.0 \mathrm{~T}$ imager (Magnetom, Siemens AG) with TR $=500 \mathrm{~ms}$ and $\alpha=90^{\circ}$. For quantitative assessment of SI 10 autopsy specimen of normal and diseased femur and vertebral bodies, which were compared with histology, were imaged. TE was varied from 11 to $22 \mathrm{~ms}$ in steps of $1 \mathrm{~ms}$.

Results: SI of intact red marrow in opposed images was below $35 \%$ of SI for in phase images. The fat content of these specimen ranged from $40-60 \%$. SI of tumors with a fat content $\leq 5 \%$ did not show a decline of SI, resulting in a contrast factor of 3 or more compared to intact red marrow. Tumors with a fat content identical to intact red marrow $(40-60 \%$ ) (AML) showed a signal decrease identical to red marrow and could not be separated with opposed gradient recalled imaging.

Conclusions: Opposed GR images introduce a new parameter to MRI which is primarily based on the macroscopic fat-to-water ratio within a voxel. SI is very sensitive to variations of this ratio, especially in cases like red bone marrow where the physiologic range of fat and water bound protons results in very low SI.

\subsection{AM}

290 MR-examination of the bone marrow in children with diseases of the haemapoetic system and special regard to posttherapeutic changes

A. Seeliger, U. Ballhof, N. Graf, B. Junk, P.Benz, U. Hau, W. Dewes, B. Kramann; Homburg/Saar

In 41 children with different diseases of the bone marrow MR-examinations of both tibiae and knees were performed. 21 children got one or more controls. $\mathrm{T} 1$ - and T2-weighted spin-echo-sequences and T2-weighted gradientecho-sequences were applicated.

Before therapy a diffuse infiltration of the bone marrow was detectable with a loss of signal intensity in $\mathrm{T} 1$-weighted images and an increase in T2-weighting. Normalising of MR-findings was later than clinical remission. In relapses there were hypointense spots in $\mathrm{T} 1$-weighting and different behaviour in T2-weighting.

MR-imaging of bone marrow seems to be useful for the staging of diseases of the haematopoetic system. Especially T2-weighted sequences promise the discrimination between posttherapeutic spots, infiltrations, necrosis and fibrosis.

\section{$11.15 \mathrm{AM}$}

291 Magnetic resonance imaging of bone marrow in multiple myeloma Gunnar Aström, Kristina Carlson, Rickard Nyman, Håkan Ahlström, Christer Sundström; Uppsala

Purpose: To assess the value of MRI in multiple myeloma.

Methods: 59 patients with multiple myeloma were studied by MRI $(0.5 \mathrm{~T})$ and conventional radiography. Sternum, thoracic- and lumbar spine, sacrum, pelvis and proximal parts of the femurs were examined with $\mathrm{Tl}$-weighted $(500 / 30)$ sequences with and without Gd-DTPA-enhancement, phase-contrast $(1500 / 37)$ and T2-weighted sequences. The signal intensity in the bone marrow were subjectively assessed and compared to histopathology and clinical investigations.

Results: Almost all focal lesions in plain radiographs corresponded to areas of low signal intensity in the Tl-weighted images and a high signal intensity in the phase-contrast images. The phase-contrast images demonstrated more and 
better demarcated focal changes than did the T2-weighted images. The variation of signal intensity in bone marrow corresponded well to the cellularity ( $\%$ haematopoetic cells of a given bone marrow section area). Repeated MRI showed progress/regress in the lesions. After administration of Gd-DTPA it might be possible to distinguish between active- and non-active lesions.

Conclusions: MRI was superior to plain radiographs in the evaluation of the extent of multiple myeloma, and was also valuable in the treatment follow-up.

11.25 AM

292 Effect of radiation therapy on thoracic and lumbar bone marrow: evaluation with MR imaging

D. F. Yankelevitz, C. I. Henschke, P. H. Knapp, L. Nisce, Y. Yi, P. Cahill; New York, NY

Bone marrow suppression is often the limiting factor in the toxicity of chemotherapy or radiation therapy. To determine if MR imaging will provide a means of quantitating bone marrow changes, we have undertaken a serial prospective study of 12 patients with lung cancer and lymphoma, and quantitated the changes in signal intensity of vertebral bodies in response to therapeutic irradiation. Quantitative and qualitative assessment of T1-weighted sagittal images (0.6 Tesla, TR-750/TE-33) before radiotherapy, during and after therapy showed increased signal intensity in the irradiated spine. These changes appeared as early as 2 weeks after beginning irradiation, continued to increase until a maximum value was attained, and then persisted during the followup period of 2 years. Patients with lymphoma demonstrated a significantly higher $(\mathrm{p}<.04)$ pretreatment to maximum posttreatment signal intensity ratio while their pretreatment values were significantly lower ( $p$ $<.01$ ). The latter may be due to the lower proportion of pretreatment yellow marrow in the patients with lymphoma who are significantly younger ( $33 \mathrm{vs} 62$ years), while the former may be related to their higher proportion of available hematopoetic marrow to undergo fatty replacement, or to the irradiation of both the thoracic and lumbar spine. The persistance of elevated signal intensity for as long as 2 years suggests an endpoint in the process of marrow conversion, but not reversal in the form of regeneration or reconversion. Quantitative MR evaluation of bone marrow may be of considerable clinical value as a non invasive means of monitoring the toxicity of radiotherapy or chemotherapy.

\section{$11.35 \mathrm{AM}$}

\section{Improvement in the staging of small cell lung cancer by MRI of bone marrow \\ G. Layer, K. Jarosch, H. Bischoff, C. Manegold, H.-U. Kauczor, W. Semmler,} G. v. Kaick; Heidelberg

Purpose: To evaluate the diagnostic contribution of bone marrow MRI in the initial staging of small cell lung cancer (SCLC).

Methods: 29 patients with histologically verified SCLC were examined for possible bone marrow infiltration using a 1.5 Tesla whole body scanner (Magnetom SP, Siemens, Erlangen). Contiguous, coronal slices (thickness: $5 \mathrm{~mm}$, FOV: $50 \mathrm{~cm}$, matrix size: $256 \times 256$ ) were obtained from the lumbar spinal column down to the proximal femurs utilizing a T1-weighted SE sequence $(600 / 15,2$ acquisitions). All patients simultaneously underwent routine clinical staging procedures (radionuclide bone scan, iliac crest biopsy, abdominal US, additional CT of brain and abdomen in suspicious cases). Results: According to the conventional clinical staging protocol 13 patients were in a limited stage of SCLC, 16 in an extensive stage. 6 patients with limited disease and 11 patients with extensive disease were found to have bone marrow hypointensities according to MRI. All lesions clearly detected in bone scan could be confirmed. MRI demonstrated additional bone marrow lesions in 6 patients who had been previously found negative both in bone scintigraphy as well as in iliac crest biopsy.

Conclusions: Bone marrow is a common site of metastasis in SCLC. Radionuclide bone scan detects only metastases destroying marrow-free bone; iliac crest biopsy has limited sensitivity. Hence, bone marrow MRI should be an integral part of the primary staging protocol according to our results.

\section{$11.45 \mathrm{AM}$}

294 Localized in-vivo ${ }^{1} \mathrm{H}$ spectroscopy and chemical shift imaging of the bone marrow in leukemic patients

H. Bongers, F. Schick, M. Skalej, W. I. Jung, H. Einsele; Tübingen

Purpose: To determine the role of 'H MRS and chemical shift imaging of the central bone marrow in follow up studies of leukemic patients.

Methods: Localized in-vivo ' $\mathrm{H}$ spectroscopy was performed in 6 healthy volunteers and in 18 leukemic patients (AML, ALL, CML). In 12 patients the study was repeated up to 3 times during and after chemotherapy. We used a double spin echo localisation method implemented at a 1.5 Tesla whole body unit. A surface coil was placed beneath the lumbar spine and used in receive mode. A cubic voxel of $(13 \mathrm{~mm})^{3}$ was chosen in a mid-lumal vertebra. For relax- ometric data spectra with varying TE $(50-400 \mathrm{msec})$ at a TR of $2 \mathrm{sec}$ and a varying TI $(50-1800 \mathrm{msec})$ at a fixed TR of $3 \mathrm{sec}$ were recorded. T1- and T2-times were calculated for the spectral fat- and water-components. Additionally fat/water images (SENEX) were recorded in a representative mid-sagittal slice.

Results: Patients with acute leukemia showed a severe reduction or complete loss of the fat signal. The initially shortened $\mathrm{Tl}$ of the water signal increased during therapy. In nonresponders neither the fat content nor relaxometric data changed. The spectral fat content was inversely correlated with the cellularity in iliac crest biopsies, that were available in all patients. The amout of the fat signal in the fat images correlated well with our spectral findings. In cases of inhomogeneous bone marrow infiltration fat/water images showed the infiltration pattern with high contrast.

Conclusions: Our findings suggest that in-vivo ' $\mathrm{H}$ spectroscopy of the lumbar spine and fat/water images allow to discriminate responders and nonresponders during chemotherapy in leukemic patients and might replace some iliac crest biopsies in the future.

\section{$11.55 \mathrm{AM}$}

\section{MRI of the spine in patients with leukaemia} M. Sens, G. Bachmann, G. Pralle, W. Rau; Giessen

We investigated the spines of patients in different stages of leukaemia with routine radiography, MRI and CT. The examinations were done to see whether there was a involvement of the vertebrae or not. We performed MRI with fat-suppressed-technique, $\mathrm{T} 2$-weighted sequences and $\mathrm{T} 1$-weighted sequences before and after administration of Gd-DTPA. The examinations were performed before and after therapy. CT was done to obtain bone-densitometry and we looked for the change in bone-density in the course of therapy. The diagnosis was verified with a bone marrow biopsy. One aim of the study was to compare the accuracy of the methods concerning the bone marrow involvement in leukaemia. Further, we wanted to see if it is possible to find criteria for a positive response to treatment in order to manage therapy properly and efficient.

\section{Room B}

\section{Ss} $10.30 \mathrm{AM}$

\subsection{Cancer of the lung}

296 Endobronchial tumours in patients with bronchogenic carcinoma: assessment by computed tomography and bronchoscopy A. Tschammler, M. Höhmann, W. Riedel, K. Lackner; Würzburg

The correlation of computed tomography (CT) and bronchoscopy in the assessment of bronchial wall abnormalities and of endobronchial tumours in patients with bronchogenic carcinoma was evaluated.

Methods: CT scans and bronchoscopic findings of 48 patients showing hilar masses and of 12 patients with normal hila (control group) were reviewed. Criteria for endobronchial tumours at CT were endobronchial masses or an irregularity of the bronchial wall. Narrowing of bronchi with regular bronchial wall wasn't judged as a sign of endobronchial tumour. To localize bronchial abnormalities the central bronchial tree was divided in 10 segments.

Results: 35 patients $(=73 \%$ ) had abnormalities of the bronchial wall (average $1.6(1-4)$ segments). Bronchoscopy and CT agreed in $96 \%$ of the examinations in detecting bronchial abnormalities, but the number of abnormal segments differed in $54 \%$ of the examinations. Bronchoscopy detected endobronchial masses in 27 patients (average $1.6(1-4)$ segments). The assessment of endobronchial tumours by CT and bronchoscopy matched in $83 \%$, but only in 14 patients $(=52 \%)$ in terms of numbers of infiltrated segments. There were no false results in the control group.

Conclusions: CT findings of bronchial abnormalities and endobronchial tumours correlated closely with bronchoscopic findings, but CT misjudged frequently the extent of bronchial abnormality.

\section{$10.40 \mathrm{AM}$}

\section{Reliability of CT in predicting the resectability of lung cancer} S. Lähde, P. Rainio, M. Päivänsalo, I. Suramo; Oulu

In order to assess the reliability of thoracic CT in predicting the resectability of lung cancer a prospective study was performed on 96 consecutive patients who underwent a thoractomy.

The tumours were staged at CT according to the international staging system for lung cancer, and assessed to be resectable, potentially resectable (stage III a), or non-resectable.

The percentages of radical resections in the groups of resectable and potentially resectable were $86.6 \%$ and $63 \%$, respectively. The need for lobectomy vs. pulmectomy was correctly predicted in $81.3 \%$ of the patients with radical resection. One patient with a tumour predicted non-resectable underwent 
thoracotomy and the CT prediction proved correct. The insufficiency of CT to define lymph node metastases and infiltrative tumour growth was considered a marked disadvantage of the method.

\section{$10.50 \mathrm{AM}$}

\section{Reliability of staging $\mathrm{CT}$ in predicting the survival of patients with lung cancer}

S. Lähde, P. Rainio, M. Päivänsalo, P. Kärkölä, I. Suramo; Oulu

The purpose of this investigation was to assess the predictive value of a preoperative staging $\mathrm{CT}$ on the survival of the patients with lung cancer. In 1985-88 207 patients underwent CT of mediastinum, upper abdomen and head. Out of them 157 were operated on. Among them 71 tumours were assessed at CT to be of stage I, 30 of stage III a-b and 45 were classified as indeterminate stage due to an inconclusive finding in the respect of mediastinal tumour involvement. Survival of the patients with stage I tumour was $91 \%$ after one year, $85 \%$ after two years, and $80 \%$ after three years. The respective percentages for patients with IIIa or indeterminate tumours were $65 \%, 57 \%$ and $53 \%$, and for the non-operated patients with stage IV tumour $28 \%, 21 \%$ and $21 \%$. The initial results indicate that the preoperative staging CT is valuable in predicting the long term benefit of the surgical cure in lung cancer.

\section{$11.00 \mathrm{AM}$}

\section{Clinical effectiveness of CT in preoperative staging of non-small cell lung cancer}

A. Olazabal Zudaire; R. Rosell Costa; Badalona

Purpose: To determine the clinical effectiveness of $\mathrm{CT}$ for preoperative staging of non-small cell lung carcinoma (NSCLC). Methods and Materials: During a of non-small cell lung carcinoma (NSCLC).

Methods and Materials: During a five years period, 160 patients with clinically resectable NSCLC were examined prospectively by CT scan. Patients with surgical contraindications, low performance status and/or metastases were excluded. CT scans were obtained with a Somaton DR2 Siemens. Contiguous slices ( $10 \mathrm{~mm}$ interval, $8 \mathrm{~mm}$ thickness) from lung apex to adrenals glands were taken. AJCC staging system was used. Definitive staging was achieved by thoracotomy and histopathological evaluation of resected specimens.

Results: The sensitivity (S) for detection of N1 disease was $29.6 \%$. For N2 disease, the $\mathrm{S}$ was $88.8 \%$ and specifity (So) $92.6 \%$, with a positive and negative predictive values of 78 and $96.6 \%$. The S for involvement of thoracic wall was $70 \%$ and $\mathrm{Sp} 81 \%$. For detection of mediastinal involvement, the $\mathrm{S}$ and $\mathrm{Sp}$ was 73 and $41.6 \%$.

Conclusions: Overall accuracy for T parameter was $82.5 \%$. Accuracy for $\mathrm{N}$ parameter was $79.4 \%$. CT is no effective to detect N1 disease, association between tumoral size larger than $3 \mathrm{~cm}$ and mediastinal lymph node metastases is demonstrated $(\mathrm{p}=0.001)$. Optimum cut-off point for mediastinal lymph node size is $15 \mathrm{~mm}$ (ROC curve). Performance of CT in evaluation of involvement of thoracic wall and mediastinal structures is low. No additional gain is supplied by CT in T1NO tumors compared with plain chest radiograph.

\section{$11.10 \mathrm{AM}$}

300 Preoperative T-staging of bronchogenic carcinomas: comparison of MR imaging and $\mathrm{CT}$

B. Mayr, M. Lehnhart, L. Sunder-Plassmann, M. Seiderer, W. Permanetter; Munich

Purpose: In this study MR imaging and CT were compared in those patients, in whom the chest $\mathrm{x}$-ray did not allow a sufficient evaluation of the local tumor spread.

Methods: 73 consecutive patients with a bronchogenic carcinoma were examined by $C T$ and MR imaging $(1,0 \mathrm{~T})$. All diagnoses were confirmed by operation and histopathological examinations (stage T1: 6 patients, stage T2: 35 patients, stage $\mathrm{T} 3: 21$ patients, stage $\mathrm{T} 4: 11$ patients).

Results: T-staging was correct in $77 \%$ of patients by both modalities. In the detection or exclusion of malignant bronchial lesions CT was slightly more correct than MR, whereas MR was more correct than CT in the diagnosis of an infiltration of the pericardium and the heart. Both $\mathrm{CT}$ and MR were equal in the evaluation of an infiltration of large vessels.

Conclusions: In the preoperative T-staging of bronchogenic carcinomas MR and $\mathrm{CT}$ provide nearly similar information. Because CT is less expensive and more accessible, it should be used for routine examinations.

\subsection{AM}

301 Gd-DTPA enhanced MRI - dynamic study in lung cancer patients Shuji Adachi, Michio Kono, Masahiko Kusumoto, Eiichiro Itouji, Eiro Sakai, Koji Tanaka; Kobe

Purpose: Evaluation of dynamic study of Gd-DTPA enhanced MR imaging (Gd-MRI) in differentiation between hilar lung cancer and the peripheral lung disease, and in differentiation between the residual or recurrent tumor and radiation pneumonitis.

Methods and Materials: We performed Gd-MRI in 82 patients with lung cancer and the diagnoses were confirmed by operation or autopsy. MRI was taken by $\mathrm{T} 1$-weighted spin echo sequence with $0.5 \mathrm{~T}$ or $1.5 \mathrm{~T}$ machine before and after i.v. administration of Gd-DTPA and sequential scans were performed $3,10,20,30,(60,90,150)$ minutes after injection.

Results: In all cases, the tumors were gradually enhanced after Gd-DTPA injection, and the enhancement of the tumor continued for more than 30 minutes. However necrotic area within the tumor was not enhanced. Peripheral lung diseases were rapidly enhanced and washed out in the early phase. Therefore there was some difference between the tumor and peripheral lung diseases. Pleural effusion was not enhanced immediately after Gd-DTPA injection but gradually enhanced 150 minutes later.

Conclusions: Dynamic study of Gd-MRI can distinguish the tumors from peripheral lung diseases and radiation pneumonitis. Therefore it is considered to offer more information concerning tumor extent, inner structure, and pleural effusion.

\subsection{AM}

\section{MR imaging of direct mediastinal invasion in bronchogenic} carcinoma

M. Cerioni, L. Carotti, E. Ravasi, L. Amoroso, R. Lanza; Ancona

Purpose: to assess the role of MR imaging compared with CT in the evaluation of the relationship of the primary lesion to the mediastinal structures in patients with central bronchogenic carcinoma.

Methods: MR imaging and CT were performed in 37 patients who subsequently underwent surgery, biopsy, or follow-up after radio-chemotherapy. MR imaging was performed with $1.5 \mathrm{~T}$ superconducting magnet, and cardiac gating was used for acquisition. Chest imaging was performed with use of a spin echo technique.

Results: MRI demonstrated 14 masses without involvment of the mediastinum and 16 with invasion. In this group MR findings demonstrated focal invasion of mediastinal structures in 10 cases and considered unresectable 6 cases. MRI sensivity and specificity were $77 \%$ and $85 \%$ respectively.

Conclusions: MRI appears to offer advantages relative to CT to demonstrate neoplastic involvement of the vessels and other mediastinal structures and focal invasion of mediastinal fatty tissue. Coronal and sagittal plane images were an advantage of MRI in detecting mediastinal involvement of bronchogenic carcinoma.

\section{$11.40 \mathrm{AM}$}

\section{Recurrent nerve palsy due to bronchogenic carcinoma: evaluation} of mediastinal causes

Stefan Delorme, Michael V. Knopp, Hans-Ulrich Kauczor, Uwe Haberkorn, Hans-G. Bauer, Gerhard van Kaick; Heidelberg

Purpose: To determine typical CT findings in the mediastinum of patients with recurrent nerve palsy due to lung cancer.

Methods: We retrospectively reviewed the CT findings of 20 patients with laryngoscopically confirmed vocal chord palsy at the time of diagnosis of histologically confirmed bronchial carcinoma. Image analysis included size, extent and localization of tumor as well as metastatic lymphadenopathy.

Results: In most of the cases the primary tumor was located in the left upper lobe and showed extensive metastatic disease to the anterior mediastinum or to the aortopulmonary window. The bulk of tumor widely exceeded the common findings at the time of diagnosis. The aortic arch was contacted in all cases, more frequently by lymph node metastases than by the primary tumor itself. Conclusions: Recurrent nerve palsy in patients with lung cancer indicates an extensive progression of the disease. The infiltration at the aortic arch is more commonly due to metastatic adenopathy than the primary tumor.

\section{$11.50 \mathrm{AM}$}

\section{Stents in the tracheobronchial system - first results}

Dieter Liermann, U. Lörcher, K. Rauber, D. Kronnenberger, E. P. Strecker, J. Meier-Sydow, J. Kollath; Frankfurt/Main

Purpose: Especially malignant stenosis in the tracheobronchial system is an unsatisfying situation for surgical treatment as well as for lasertherapy because of the early recurrency of the stenosis. Stent-implantation into the tracheobronchial system seems to be a helpful method in such a situation.

Methods: After animal experiences with self-expandable and balloon-expandable stents in Frankfurt and Gießen and clinical experiences with more than 100 patients in vascular or biliary system since 1987, we started with the stentapplication with stents of a minimal diameter of $14 \mathrm{~mm}$ (expanded) over wire under endoscopical and fluoroscopic control.

Results: Because of the high flexibility insertion of the Strecker Tantalum Stent causes no problems. Because of the low resistance of the stent, deformation with a relatively narrowing of the stent in some parts take place. Nevertheless 
the narrowing causes insufficient respiration.

Conclusions: The respiratory insufficiency of all patients before treatment allows them only surviving staying in a clinical bed. After stent all patients leave the hospital without respiratory insufficiency. The above described method seems to be senseful and helpful to increase life-quality for the patients.

\section{$11.55 \mathrm{AM}$}

305 Clinical experiences with endotracheal Gianturco-Z-Stents Klaus Rauber, Bernd Weimar, Martina Hofmann, Wigbert S. Rau; Giessen

We perorally inserted endotracheal or endobronchial Gianturco-Z-Stents in 14 patients on 15 occasions for the relief of severe dyspnea due to tracheobronchial-stenoses. In three cases this was done under bronchoscopic control in all others under fluoroscopy with local anasthesia only.

Three patients had benign, all others malignant tracheal or bronchial stenoses All patients experienced immediate relief of their severe dyspnea that had led to the stent-implantation. Patients survival ranged from 10 days to meanwhile 16 months. There was only one patient with severe complications: In a patient with tracheomalacia the initially chosen stent was too small and dislocated so that it had to be extracted endoscopically. The second placed stent in the same patient showed a strutfracture 3.5 months after insertion. The loose fragments were extracted, the patient got a silicone-rubber-stent for treatment of his tracheomalacia.

According to our experiences Gianturco-Z-Stents are a valuable tool in the treatment of otherwise untreatable severe malignant tracheobronchial stenoses.

\section{Room C}

\section{SS}

\section{$04.09 \mathrm{CT}$ of the Gl tract}

$10.30 \mathrm{AM}$

\section{The use of intra-venous contrast in the staging of gastro-intestinal pathology}

H. Rigauts, G. Marchal, A. L. Baert; Leuven

Purpose: The subject of the study was to evaluate if systematic i.v. contrast enhancement of the intestinal wall can improve the C.T. diagnosis of gastrointestinal pathology.

Methods: The studied population consists of 350 patients with known or clinical suspicion of inflammatory or tumoral pathology of the stomach, duodenum or colon. Patient preparation includes distension of the interested bowel segment with tap water and the consecutive scanning during a fast i.v. infusion of a contrast medium.

Images are evaluated for lesion detection, differentiation between tumoral and inflammatory pathology and for transmural extension. The C.T. findings are correlated with surgery, endoscopy, pathology and follow up under medical treatment.

Results and conclusion: Despite the use of additional i.v. contrast, differentiation between tumoral and inflammatory pathology can not be solved and local tumoral staging is not improved compared to published studies using a positive intraluminal contrast without additional i.v. contrast enhancement

\section{$10.40 \mathrm{AM}$}

$307 \mathrm{CT}$ in the preoperative assessment of locally advanced carcinoma of the cardia

M. C. Freund, W. Jaschke, M. Georgi; Mannheim

Introduction: The therapy of carcinoma of the cardia includes not only radical curative but also palliative tumor resection. In addition less invasive procedures can be applied like the palliative endoscopic pertubation (PEP) combined with possible laser ablation or intraluminal dilatation. In order to avoid exploratory laparotomy of inoperable patients with diaphragmatic involvement preoperative staging was performed utilizing computed tomography (GE 8800 \& 9800).

Methods and Materials: 52 patients with histologically proven adenocarcinoma of the cardia were studied. The CT criteria utilized to judge diaphragmatic involvement was the obliteration of the peridiaphragmatic rat planes and deformity of the diaphragmatic contour. Lymph nodes with a diameter of more than $1.5 \mathrm{~cm}$ were considered metastatic.

Results: Sensitivity and specificity of CT for diaphragmatic involvement was $80 \%$ and for lymph node involvement $60 \%$

Conclusions: In our experience CT scanning has a poor diagnostic accuracy in judging diaphragmatic and especially lymph node involvement in adenocarcinom of the cardia and does not preclude exploratory laparotomy.

\section{$10.50 \mathrm{AM}$}

\section{$308 \mathrm{CT}$ follow-up of transgastric endoscopic drainage of perigastric} pseudocysts

P. Braude, D. Van Gansbeke, B. Lalmand, M. Aissa, M. Cremer, J. Struyven; Brussels

Purpose: Endoscopic gastro-cystostomy progressively becomes an attractive alternative to the surgical enteric derivation of perigastric pseudocysts subsequent to pancreatitis. The aim of this study is to determine what is to be expected from the CT evolution of perigastric fluid collections after transgastric endoscopic drainage.

Methods: We reviewed the $\mathrm{CT}$ examinations performed before and after (short and long term follow-up) the endoscopic procedure in 24 patients. Perigastric pseudocysts ranged from small and well marginated fluid collection to large and partially septated retroperitoneal collection, extended to the left and/or right prerenal spaces; all these collections presented a close contact with the gastric wall.

Results: The CT features have been correlated with the clinical evolution and the technical endoscopic aspects (site and size of the gastrostomy; presence of transgastric device). Long term follow-up showed complete resolution of the fluid collections. Two patients presented early complications which required percutaneous drainage ( 1 case) or surgery ( 1 case).

Conclusions: CT is the most appropriate procedure for the follow-up of transgastric endoscopic drainage of perigastric pseudocysts.

\section{$11.00 \mathrm{AM}$}

\section{CT in primary tumours of the small bowel}

J. C. Vilanova, J. Pont, R. Bassaganyas, X. Serres, J. Ferrer, M. D. Figueras; Girona

Primary tumours of the small bowel are frequently and typically nonspecific in their clinical presentation consequently, it is often difficult to detect them by traditional radiograpic studies.

We present a retrospective study of 44 primary small bowel tumours evaluated by Computed tomography (C.T.). They were eighteen adenocarcinomas, nine lymphomas, four leiomyomas, four leiomyosarcomas, three lipomas, three adenomas, and three carcinoid tumours. The lymphoma tended to be large and annular; the adenocarcinoma tended to be shorter and ulcerated; the leiomyosarcomas tended to be bulky and heterogeneous and lipomas had a homogeneous low attenuation of fat. All the tumours were histologically verified, except for the lipoma. The common use of C.T. as a method to evaluate patients with vague abdominal symptoms is likely to afford the initial opportunity to detect and characterize a tumour of the small bowel. Certain patterns of C.T. findings may enable the radiologist to diagnose or at least it can strongly suggest individual tumour types.

\subsection{AM}

310 Segmental omental infarction masquerading as appendicitis: incidence, US and CT findings

J. B. C. M. Puylaert, J. L. Seelen; The Hague

The purpose of this study is to add a new entity to the list of conditions that clinically may mimic appendicitis, but have typical US and CT features enabling rapid differentiation.

Patients and methods: From a group of 440 patients with clinically suspected appendicitis, in five cases US and CT findings were compatible with the diagnosis of segmental omental infarction. We studied the clinical, sonographic and $\mathrm{CT}$ findings in these 5 patients.

Results: US revealed an immobile, ovoid, circumscript, hyperechoic, non-compressible mass of intraabdominal fat at the spot of maximum tenderness. The mass adhered to the peritoneum. CT found a corresponding area of high density fat with an inhomogeneous, streaky pattern. No associated abnormality of bowel or appendix was demonstrated. None of these 5 patients were operated. In all 5 both clinical symptoms and US and CT abnormalities gradually resolved.

Conclusions: Although no histological proof was available, the US and CT findings in all 5 were alike and typical, and corresponded to the entity called "segmental omental infarction" in the radiological and the surgical literature. In all 5 the consistent US and CT findings at least allowed to exclude appendicitis, thus preventing an unnecessary operation. The relatively high incidence of $5 / 440(1.1 \%)$ suggests actual underestimation.

\section{$11.15 \mathrm{AM}$}

311 Improved accuracy of $\mathrm{CT}$ in rectal cancer staging using water enema

G. Angelelli, L. Macarini, L. Lupo, F. Martino, V. Memeo; Bari

Recently we have proposed water enema during $\mathrm{CT}$ in the staging of rectal cancer, in order to improve the image of the rectal wall and the surrounding 
tissues or organs. The efficacy of the method was tested comparing two groups of patients suffering from rectal cancer. One (57 patients) was staged using water enema for parietal evaluation, the other (64 patients) using dense contrasts (iodate solutions or baritate suspensions). The accuracy of both series and diagnostic gain with confidence limit $95 \%$ were calculated. The histological modified Duke's classification has been employed. Total accuracy of CT using water enema as regards stage classification was $84.2 \%$, using dense contrasts $62.5 \%$ with a diagnostic gain of $21.7 \pm 16(+37.7,+5.7)$. The accuracy on wall invasion detection was: $94.7 \%$ vs $60.9 \%$ with an improvement of $33.8 \pm 16(+49.8,+17)$; as regards lymphonodes involvement $80.7 \%$ vs $70.31 \%$ with a difference of $10.4 \pm 17(+27.4,-6.6)$ and as regards extension to other organs $96.5 \%$ vs $92.21 \%$ with a difference of $4.3 \pm 12(+16.3,-8)$.

Although the $95 \%$ estimation of diagnostic improvement ranges between a large interval, our results show that water enema during CT is a reliable for staging rectal cancer.

\section{$11.25 \mathrm{AM}$}

312 Computed tomography and endosonography in the preoperative staging of rectal carcinoma

K.-H. Rotte, L. Kluhs, H. Kleinau, E. Kriedemann; Berlin

Imaging techniques can provide the surgeons with necessary information about tumor extension, which are needed for the planning of the surgical approach.

In a study of 65 patients we compared the preoperative findings of computed tomography (CT) and endorectal sonography (ES) with the surgical specimen. In T-staging ES showed an accuracy of $86 \%$ and CT of $76 \%$. A precise staging of lymph node metastases was possible neither by ES nor by CT. Advantages and limitations of both methods are discussed.

Conclusions: Endosonography, combined with rectoscopy is favoured as the first imaging procedure. CT is recommended in endosonographic doubtful findings and in patients with tight stenoses.

\section{$11.35 \mathrm{AM}$}

\section{Contribution of $\mathrm{CT}$ examination in bowel obstruction}

G. Schmutz, R. K. Chhem, D. Bergeron, F. R. Plante, J. M. Burel, D. Regent Sherbrooke, Québec

Purpose: To determine the contribution of CT in the diagnosis and the etiology of bowel obstruction.

Methods: During two years (September 1988-October 1990) we evaluated 67 patients with a clinical diagnosis of bowel obstruction. Diagnosis was established by surgery ( 31 cases), barium radiography ( 22 cases) and clinical follow-up (14 cases). Eleven patients proved to have no bowel obstruction etiologies included tumoral lesions (17 cases), hernias and peritoneal adhesions (19 cases), Crohn's disease ( 7 cases) and miscellaneous lesions (14 cases).

Results: Fifty-five patients were identified as obstructed with two false positives. No signs of obstruction were obtained in twelve patients with two false negative. Thus the sensitivity of CT for detecting obstruction was 94.0 percent, the specificity 81.0 percent and accuracy achieved 92.0 percent. The etiology of obstruction was correctly identified by CT in 36 cases ( 67 percent). All patients with tumoral lesion and with Crohn's disease were correctly identified. Peritoneal adhesions were frequently difficult to identify.

Conclusions: CT is highly accurate to diagnose bowel obstruction and to establish the etiology of the obstruction, especially in neoplastic conditions.

\section{Room E1}

\section{SS}

\section{$10.30 \mathrm{AM}$}

\section{Long-term follow-up after local thrombolysis in the treatment of peripheral arterial occlusion}

U. Blum, A. Beck, G. Grosser, W. Heiss, A. Frank; Freiburg

Peripheral arterial occlusions are invariably caused by a blood clot from either in situ-thrombosis or embolism.

Intraarterial infusion has become an acceptable approach of recent peripheral arterial occlusion (including recent occlusion of bypass grafts).

A retrospective study was undertaken for evaluating the impact of local thrombolysis on the treatment of peripheral arterial occlusion. The study population consisted of 354 patients treated between $1984-1990$ by streptokinase $(n=55)$, urokinase $(n=223)$ and rt-PA $(n=76)$. All had angiographically documented thrombotic or embolic occlusion.

A total of $60 \%(n=212)$ were successfully treated primarily. The follow-up studies included angiography, oscillography or conventional duplex US. After 12 and respectively 24 months the cumulative patency rate was still $69 \%$.

The results indicate that regardless of the thrombolytic agent the rate of recanalization is similar and there is no significant reduction of the bleeding rate when using rt-PA as thrombolytic agent.

$10.40 \mathrm{AM}$

315 Effect of local thrombolysis on the clinical outcome in acute renal artery occlusion

U. Blum, P. Billmann, A. Beck, F. Nitzsche, D. Kramer; Freiburg

Acute renal artery occlusion is a rarely recognized clinical entity. The clinical symptoms are protean and an accurate diagnosis often is not established until irreparable renal injury has occurred. There is considerable controversy regarding precise guidelines for the treatment of renal artery embolic disease. We administered local thrombolysis in 11 patients with angiographically documented complete renal artery occlusion. All patients were treated successfully with streptokinase $(n=1)$ and urokinase $(n=10)$.

The follow-up studies (performed between 24 and 48 months after treatment) included the following techniques: laboratory studies, renal scan, enhanced CT and MRI. Although the treatment was initially successful these studies demonstrated considerable loss of functioning parenchyma comparable to isotopic studies performed prior to treatment.

In contrary to the current literature we advocate systemic heparinization as the therapy of choice when the "warm" ischemic tolerance of the kidney which is in the order of 90 minutes is exceeded.

\section{$10.50 \mathrm{AM}$}

316 Percutaneous treatment of iliac artery occlusions - fibrinolysis, PTA, stents

K. A. Hausegger, J. Lammer, E. Pilger, G. E. Klein, F. Flückiger, F. Waltner, M. Lafer, M. Aschauer; Graz

Purpose: To evaluate the safety and efficacy of percutaneous recanalization therapy with special regard to the benefit of vascular stents.

Methods: The study consists of 2 groups of patients. Group I $(n=17)$ : treated with systemic or local fibrinolysis (FL) and PTA alone (follow-up: 12-69 months). Group II $(\mathrm{n}=25)$ : treated with FL, PTA and stent (follow-up: 2-20 months).

Results: The initial recanalization rate was $83 \%(35 / 42)$. Systemic FL caused dissolution of the appositional thrombus in $3 / 14$ patients (21\%), local in $13 / 22$ cases $(59 \%)$. In group I FL and PTA revealed a satisfactory initial and longterm result in $4 / 17$ patients $(23 \%) .10 / 17$ patients $(59 \%)$ showed unsatisfactory angiography results after FL and PTA. However, 2 of those showed good clinical results. 4 patients had reocclusion within 1 year, 2 showed no clinical improvement. 2 were lost of follow-up.

In group II FL and PTA alone was successful in $5 / 25$ patients $(20 \%) .15$ patients got stents $(60 \%)$. One early stent thrombosis was successfully treated by local FL. In the control intervall all stents are patent. Complications: 3 hematomas, 2 peripheral emboli.

Conclusions: Recanalization of iliac artery occlusion after local FL is an efficacious and save procedure. However, in only about $20 \%$ FL and PTA alone will show good initial results. Thus at least $60 \%$ of the patients need endovascular stents to achieve a good anatomical vascular restoration.

\section{$11.00 \mathrm{AM}$}

\section{Renal transplant artery stenosis in children}

Anje M. Spijkerboer, Willem P. Th. M. Mali, Raymond A. M. G. Donckerwolcke; Utrecht

Six children with transplant renal artery stenosis causing severe and uncontrollable hypertension were treated with Percutaneous Transluminal Angioplasty (PTA). Little has been written about PTA of transplant renal arteries in children. Retrospectively, we examined the effect of PTA on hypertension. Seven successful angioplasties were performed in these six children, and the follow-up period at the end of the study varied between 3 and 66 months. The stenosis was situated at the site of the anastomosis in 4 children and was distal to the anastomosis in 2.

PTA resulted in improvement of hypertension in all patients.

Cure of hypertension without the need of medication was not achieved. Major complications or graft loss did not occur.

Our findings show that PTA of transplant renal artery stenosis in children is an acceptable first choice of treatment.

\subsection{AM}

\section{PTA in dialysis access fistula stenosis: bad results?}

W. Richter, M. Riedl, E. Dünser, F. Meisl, H. Kern, H. Umek; Vienna

39 PTA have been performed at 29 patients with dialysis access fistula stenosis. In 2 cases a dilatation could not be achieved, in 1 case the stenosis could not be 
passed by the catheter and therefore the intervention could be carried out in $36 / 39$ cases $(95 \%)$.

There were 14 Cimino shunts, 1 Omniflow, 7 Goretex prostesis and 7 homologous vein grafts.

The degree of the stenosis was: $33 / 39(84,6 \%)$ above $70 \%$ and $6 / 39(15,4 \%)$ between $40 \%$ and $70 \%$. Most stenosis were within the venous branch of the fistula.

Out of the 36 performed PTA a dilatation of the stenosis to less than $50 \%$ of its original diameter or to a degree of less than $30 \%$ was considered to be successful and could be achieved in 29 PTA ( 21 patients). $2 / 21$ patients could not have been followed up, in 3/27 PTA the fistula became occluded within the first week after. Of the remaining 24 successful interventions the patency rate after $3,6,12$ and 24 months was $60 \%, 45 \%, 26 \%$ and $13 \%$.

The causes for these-compared to other studies - relatively poor results will be analysed, among others an early angiographic control for early redilatation will be a matter of discussion.

\subsection{AM}

\section{PTA of crural arteries}

\section{Oertl, W. Horvath, Ch. Luft, D. Haidinger; Linz}

Purpose: Reevaluation of the indications for PTA of crural arteries under the aspect of modern equipment.

Methods: From 1986 to 1990 we dilated 170 crural arteries in 125 patients mainly with $5 \mathrm{~F}$-balloon catheters with balloon diameters from 3 to $5 \mathrm{~mm}$. These were inserted over steerable 0.020 guide wires with floppy tips. PTA of crural arteries alone was performed in 35 cases. Interventions in combination with PTA in the femoropopliteal region were done in 69 cases, with laser angioplasty in the same region in 15, with local lysis of recent thromboses in 30 and with surgery in 21 .

Results: Primary success was achieved in 166 cases (primary success rate $97,6 \%$ ). Complications included one rupture of a fibular artery and one intimal flap causing occlusion (both treated by the vascular surgeon). In one case a hematoma at the puncture site was evacuated. Long time results were calculated by the life table analysis and showed a cumulative patency rate of $79,8 \%$ after 1 year, $75,3 \%$ after 2 years and $64,6 \%$ after 3 years.

Discussion: With improvement of catheters, guide wires and contrast media PTA of crural arteries has become a safe procedure. Therefore not only stage III and IV disease may be treated but also stage II b.

\subsection{AM}

320 Profundus venous thrombosis of lower limbs: phlebography matching on outcomes of two therapeutic modalities

R. Crespi Porro, N. Campi, S. Fontana, F. di Giusto; Tradate/Varese

Purpose: The purpose of the present paper is the comparison between two different kinds of thrombolytic therapy in patients suffering from venous thrombosis.

Methods: In a set of ten patients carrying profundus venous thrombosis of the lower limbs, come to our observation in the last year (1990), we have separated two groups according to the therapeutic protocol to which they were included at random: first group: local thrombolitic therapy with continuous infusion of urokinase lasting for 72-96 hours; second group: general thrombolitic therapy with intravenous injection of heparin and Sintrom for 10-12 days.

In the patients of the first group, phlebographic controls in the distance have shown profundus circulus recanalization from the fifth day since the beginning of the management, lasting in time, even if with large remains, which have never conditioned the patency or the function of the profundus circulus which was previously occluded. In the patients of the second group, phlebograpic controls in the distance, usually starting from the twentieth day from the beginning of the therapy, have shown a persistent obliteration of the profundus circulus and the appearance of large collateral circles through superficial veins. Conclusions: In general, the patients of both groups have shown an improvement of the clinical picture, more precocious and evident in patients of the first group.

\section{$11.40 \mathrm{AM}$}

321 Deep venous thrombosis: color-doppler-flow-imaging (CDI) in the control of lysis efficacy

R. Wildling, A. Lederer, H. Steiner, E. Deu, M. Stiskal; Graz

Purpose: To evaluate the accuracy of color-Doppler-imaging in comparison with contrast venography in the control examinations being performed in the course of lysis therapy.

Methods and Materials: 69 sonographic and phlebographic studies of 22 patients $(7 / 3,12 / 3,3 / 4-12$ women, 10 men, age $32-67,22$ legs - left/right $12 / 10$ ) could be compared. The sonographic examinations, including the $\mathrm{v}$. iliaca externa, v. femoralis, v. poplitea and the calf veins were carried out with an ACUSON 128 (ACUSON; montain view, calif.) equipped with a $5 \mathrm{MHz}$ and $7 \mathrm{MHz}$ imaging probe and $3.5 \mathrm{MHz}$ pulsed Doppler. For documentation a video-recorder and a polaroid camera was available. Lysis agents were rtPA, urocinase and streptocinase.

Results: In 14 cases thrombosis affected the whole leg, in 6 cases it came up to the middle of the thigh and twice until to the knee.

7 patients could be treated successfully, a partial recanalisation was achieved in 5 patients, 10 patients showed no significant change.

Comparing the two methods of examination we had 58 corresponding sonographic and phlebographic results and one false-positiv sonographic.

Conclusions: CDI is able to replace phlebography in the control of lysis efficacy in the femoro-popliteal segment and has a high accuracy in the calf.

\subsection{AM}

322 Local intraarterial lysis of occluded bypasses in lower extremities A. Scholz, R. Langer, M. Langer, R. Felix; Berlin

Purpose: In a prospective study 24 patients with occluded bypasses of the lower extremities were treated by intraarterial fibrinolysis.

Methods and Materials: transcatheter fibrinolysis was performed after acute occlusion. The thrombolytic agent used in this study was urokinase.

Results: In $83 \%$ of the patients an almost complete thrombolysis could be achieved in the first 2 hours after application of 300000-500000 U urokinase. The therapy was then prosecuted over 12 hours (10000-20000 U/h). In 2 patients catheterisation of the proximal anastomosis of the bypass was not possible. In 2 other patients intraarterial lysis was not successful because of a bad peripheral run off. Major complications were not registered.

Conclusions: In our opinion intraarterial fibrinolysis with urokinase is the method of choice after acute lower extremity bypass occlusion.

\section{$11.53 \mathrm{AM}$}

323 Out-patient thrombo-atherolysis following transluminal angioplasty

L. Horváth, A. Kiss, I. Battyány, M. Romhányi, K. Hazafi; Pécs

Platelet aggregation inhibitors and anticoagulants have been used as post-angioplasty medication so far. Now enzyme stimulants were tried in this program to prevent reocclusion following the Dotter's procedure. Surprisingly additionally to the well proved prevention of reocclusion even the remaining deposited clots and atheromata could be reduced or even removed by stimulated enzyme activity. First two groups of patients were treated paralel (100-100 cases) with dicumarol or sodium pentosane polysulphate. Later all together 203 patients were accumulated in the latter group. Anticoagulants have long been regarded as the most successful means to prevent reocclusion following transluminal angioplasty. In this study enzyme stimulants (pentosane polysulphate) resulted in an even higher patency rate than dicumarol. A 5-year accumulated patency rate will be presented. It is demonstrated that thrombolysis and atherolysis can be performed by longterm oral medication too.

\section{Room E2}

\section{SS}

\subsection{Knee}

\section{$10.30 \mathrm{AM}$}

324 MRI of laser induced meniscal lesions: Evaluation of dependence on surrounding medium and lesion size

M. Naegele, M. Leunig, W. Lumper, F. Gamarra, A. E. Goetz; Bonn

Purpose: Purpose of the study was to determine whether MRI is able to demonstrate small laser induced meniscal lesions and how the surrounding medium affects their detectability.

Methods: Nine bovine menisci were studied with 12 laser induced lesions per meniscus. Two different lasers were used (ER-YAG, HO-YAG). MRI was performed with an experimental Helmholtz surface coil; Sequence: Threedimensional FLASH, flip angle 50 degree, 1 -mm sections, 2 data acquisitions. Each meniscus was examined in room air, in $.9 \% \mathrm{NaCl}$, and in Gd-DTPA solution without a change in position. All laser induced lesions were histologically prepared, and all lesion sizes measured with a digital imaging system. MRI examinations were evaluated blindly by two radiologists (without knowledge of laser system, lesion numbers and surrounding medium).

Results: Detectability rate of lesions greater than $1 \mathrm{~mm}$ : Room air: $60-80 \%$; saline: 30-60\%; Gd-DTPA: 90-100\%. Lesion smaller than $1 \mathrm{~mm}$ : Room air: 30-60\%; saline: 20-40\%; Gd-DTPA: 40-60\%.

Conclusions: Gd-DTPA improves the detection rate of meniscal lesions greater than $1 \mathrm{~mm}$ in comparison with room air and saline as the surrounding medium. Meniscal lesion smaller than $1 \mathrm{~mm}$ has a low detection rate with all three mediums. Gd-DTPA as an arthrographic agent might assist clinical workup of meniscal lesions. 
325 Stability of osteochondral fragments of the femoral condyle: MR imaging with pathohistologic correlation in an animal model G. Adam, M. Bühne, C. Nolte-Ernsting, A. Prescher, K. Bruchmüller, W. Küpper, K. Bohndorf, R. W. Günther; Aachen

The stability of surgically induced osteochondral fragments of the femoral condyle was examined by MR imaging using plain and contrast enhanced T1and plain T2-weighted spin-echo sequences in an animal model in 9 dogs. Animals were followed up and sacrificed between the 34 th and 196 th day after surgery. MR images were compared to the pathohistologic findings.

We found two loose and seven stable fragments on autopsy. A well defined line of high signal intensity between the fragment and the epiphysis showed high enhancement after contrast medium application in loose fragments. Histology displayed a vascularized granulation tissue at the interface. In animals with stable fragments a similar, but irregular defined interface line was observed on plain sequences too, but no enhancement was noted after contrast medium application. Histology showed intact bone trabecula at the interface within the complete repaired fracture.

Contrast enhanced MR imaging allowed an exact delineation of the interface line of unstable osteochondral fragments in this animal model and their differentiation from a similar line observed in stable fragments. However, the interface line of stable fragments could not be explained by histology and reflects probably differences of proton binding or distribution in the osteoid of healing osteochondral fragments.

\section{$10.50 \mathrm{AM}$}

\section{Changes in the MRI appearance of the ACL graft during the initial postoperative 12 months}

A. Smith, C. F. Pope, P. Jokl, K. Lynch; New Haven, Portland

Purpose: Patients with Anterior Cruciate Ligament (ACL) reconstructions not infrequently experience injuries in the first 12 months following surgery. This study was performed to establish if graft abnormalities could be identified and whether MRI could play a role in monitoring postoperative rehabilitation.

Methods: 14 patients, age range 15-28 years were scanned postoperatively at 1.5 months $(14 / 14), 6$ months $(13 / 14)$ and 12 months $(13 / 14)$ using a 1.5 Tesla MRI scanner. Axial, sagittal and coronal images (TR 1500 \& 2000, TE $20,80 \mathrm{msec} ; 5$ \& $3 \mathrm{~mm}$ thickness) were examined for overall graft appearance (signal intensity, thickness, joint fluid). Standard clinical stability tests were obtained at similar intervals.

Methods: All grafts were identified as intact at 12 months. At 1.5 months all grafts had signal intensity approaching normal low signal collagen tissue. Increased signal occurred in grafts by 6 months $(13 / 14)$ while at 12 months only $4 / 14$ showed a signal reduction that might correspond to an increase in the amount of normal tendon fibres.

Instrumented ligament laxity tests varied significantly between the 1.5 and 12 month assessment compared to the 6 month evaluation.

Conclusions: Contrary to previous reports, the ACL graft can be assessed on routine post-op MRI scans. Graft signal intensity was most like normal tendon early after graft surgery while little change in the ACL graft occurred between 6 and 12 month scans.

\subsection{AM}

327 Gd-DTPA-enhanced MRI in hemophiliac arthropathy of the knee joint

V. Kunze, M. Nägele, H. Eickhoff, B. Wöll, M. Reiser; Bonn

Purpose: The study was performed to determine the diagnostic value of contrast-enhanced MRI of the hemophiliac knee joint arthropathy.

Methods: 24 male patients, $7-33$ years

MRI-Sequences: 1. Spin-Echo T1-w 500/30 ms (pre and post i.v. Gd-DTPA); 2. SE T2-w $(1600 / 20 / 120) ; 3$. Dynamic FFE-Sequence $\left(50 / 12 \mathrm{~ms} / 70^{\circ}, 8\right.$ consecutive slices with a time interval of $23 \mathrm{~s}$ ); Contrast medium. Magnevist ${ }^{R}$ (Gd-DTPA), $0.1 \mathrm{mmol} / \mathrm{kg}$ bw; Technique: surface coil, Philips Gyroscan 1,5 $\mathrm{T}$;

Results: T1-w sequence + Gd-DTPA: Synovial proliferations could be differentiated from joint effusion, ligaments and menisci. Thinning of hyaline cartilage could be verified.

Dynamic T1-w FFE + Gd-DTPA: The perfusion of the synovial proliferations could be assessed reliably. Susceptibility artifacts correlated with hemosiderin depositions from previous hemorrhages.

Summary: Contrast enhanced MRI studies provide important information on perfusion of synovial proliferations and may be useful for precise evaluation of joint damage in hemophiliac arthropathy.
328 Cartilagineous lesions of the knee: evaluation with MR and arthroscopic comparison

E. Cerofolini, E. Gallo, A. Burani, A. Vaccari, S. Boschi, A. Montorsi, M. Marchetti; Modena

In order to evaluate the accuracy of MR in the detection and grading of articular cartilage lesions of the knee, 35 young adults (mean age 35 ) with clinical signs of chondral pathology and a negative standard X-ray film were studied both with MR and arthroscopy; results were compared.

A 1.5 $\mathrm{T}$ magnet and a cylindrical receive-transmit coil were used; both spin-echo and gradient-echo sequences were performed. For the grading of the lesions the arthroscopic classification of Noyes was employed. MR findings included: loss of cartilage thickness, modification of signal intensity and alterations of subchondral bone. Arthroscopy detected 26 lesions in 20 patients while MR recognized 21 lesions in 15 patients both examinations were negative. All of the lesions missed by MR were grade 1 (softening).

In 15 out of 21 lesions the grading also agreed. In 4 lesions grade $3 \mathrm{~B}$, confirmed at arthroscopy, MR clearly showed erosion of subchondral bone that were not visible in the X-ray film, even retrospectively. MR appears to be a very accurate and specific imaging modality to ascertain cartilagineous lesions of the knee (no false positive result); if grade 1 lesions are not considered, its negative predictive value is also very high allowing to correctly select patients who need an arthroscopy examination for appropriate surgical treatment.

\section{$11.20 \mathrm{AM}$}

329 The effects of quadriceps contraction on the biomechanics of the knee in extension

B. Gülek, Z. Ziylan, S. Seber; Eskișehir

In this study we aimed to investigate the effects of contraction of the quadriceps muscle on the biomechanics of the knee. 60 healthy knees were studied and axial tomographic sections were obtained before and after quadriceps contraction in the extended knees. Patellar tilt angles, femoral trochlear depths, femoral trochlear angles and congruence angles were measured on slices passing through the thickest portion of the patella. In addition, patellar elevation and lateralization during quadriceps contraction were measured. It was seen that contraction of the quadriceps muscle in the extended knee pulled the patella upwards and pushed it laterally. Also, the patellar tilt angles and femoral trochlear angles were increased after contraction of the quadriceps. A decrease in femoral trochlear depths was also noted. Finally, the congruence angles were increased during quadriceps contraction. We came to the conclusion that contraction of the quadriceps muscle tended to dislocate the patellas laterally and upwards, making alterations in the biomechanics of the knees studied.

\subsection{AM}

330 The MRI appearance of the healing patella tendon: 12 month follow-up

A. Smith, C. F. Pope; P. Jokl, K. Lynch; New Haven, Portland

Purpose: Healing tendon has a similar appearance to tendonitis or tendinosis. This study evaluated the healing patella tendon following patella tendon graft excision to assess the sequential changes that occur on MR images post-operatively.

Methods: 14 patients ( 7 females, 7 males), mean age 21.1 years (range $15-28$ ) were scanned preop, and postop at $1.5,6$ and 12 months using a 1.5 Tesla MRI scanner, a dedicated extremity coil and spin echo pulse sequences. Axial, sagittal and coronal images (TR 1500 \& 2000, TE $20,80 \mathrm{msec} ; 5$ \& $3 \mathrm{~mm}$ thickness) were examined for overall tendon appearance, the sagittal and axial dimensions $(\mathrm{mm})$ and the degree of patella/femoral cartilage overlap (patella baja). Quadriceps and hamstring muscle group strength was assessed at similar time points.

Results: The tendon shows size changes between preop and postop scans that are significantly different at all time points (ANOVA) $\mathrm{P}=0.0001$. The degree of patella/femoral overlap increases for postop 1.5 and 6 months $(P=0.005)$ but at 12 months was not significantly different from preoperative measurements. Muscle groups approach similar strengths at 12 months.

Conclusions: Although no persistent tendon thickening occurs unlike that seen following rupture, abnormalities exist in the patella tendon even 12 months following graft excision. These may mimic an infected or partially torn tendon. 
$11.40 \mathrm{AM}$

331 The evaluation of patellar localization during different degrees of flexion, extension, and extension with quadriceps contraction in 60 healthy knees by means of computerized tomography

B. Gülek, Z. Ziylan, S. Seber; Eskișehir

In this study, we aimed to investigate if the patella was centrally or laterally located during $90^{\circ}-60^{\circ}-30^{\circ}$ of flexion, extension, and extension with quadriceps contraction in 60 healthy knees. Despite the claims made by Albee in 1915, and Helfet in 1963 and 1974, that the patella was centrally located in extension and tends to lateralize during flexion, Schutzer et al in 1984 showed by means of computerized tomography, that the patella starts to be central after $10^{\circ}$ of flexion but is not centrally located during extension. We used the same method used by Schutzer et al, and measured the congruence angles and patellar tilt angles in 60 healthy knees. Conventional axial roentgenograms were obtained in $30^{\circ}-60^{\circ}-90^{\circ}$ of flexion, but during extension and extension with quadriceps contraction, computerized tomography was utilized to obtain axial slices. All knees were found to be centrally located during the degrees of flexion, but lateralization occured during extension. Quadriceps contraction during extension increased the lateralization of the patellas.

11.50 AM

\section{The accuracy of MRI in the traumatic knee at 1.5 Tesla. Correlation} with arthroscopy

Ernesto Gómez-Catalán, Vicente Lozano, Octavio Uriol, Maria Muñoz, José Nazar, Carlos Barranco; Madrid

We discuss the impact of MRI for the evaluation of traumatic knee. 150 knee examinations were retrospectively analyzed in order to identify normal variants and detect types of injuries. Using a spin-echo imaging in the sagittal and coronal planes, with a combination of $\mathrm{T} 1$ and $\mathrm{T} 2$ weighted, and, axial imaging trying depict the patello-femoral joint best. For tears of the medial and lateral meniscus the accuracy of MRI were $98 \%$ and $88 \%$, respectively. When the anterior cruciate ligament was well visualized, rate were $95 \%$, and for the posterior was $97 \%$. Other injuries were too evaluated. In summary, MRI is the technique of choice.

\section{$11.53 \mathrm{AM}$}

\section{Transverse geniculate ligament of the knee: appearance on plain films}

S. A. Sintzoff jr., P.A. Gevenois, C. Matos, I. Gillard, Y. Andrianne, J. Struyven; Brussels

Purpose: We wish to report the appearance of a normal anatomic structure, the transverse geniculate ligament of the knee, which we have seen in 4 patients on lateral plain films of the knee. To our knowledge it has only been described on MRI examinations.

Methods and results: Our 4 patients had symptoms attributed to meniscal tears diagnosed by MRI and confirmed by arthroscopy. On lateral plain films of these patients, the transverse geniculate ligament could be seen as a small rounded opacity surrounded by fat density, situated anterior to the knee joint. MRI examinations were available for all these patients and demonstrated a thick, normal intensity transverse ligament. In all cases, this ligament was separated from the capsule by fatty tissue. This feature made the ligament evident on lateral plain films.

Conclusions: The transverse geniculate ligament of the knee is a normal anatomic structure that should be recognized when seen on lateral plain films of the knee.

\subsection{AM}

334 Pigmented villonodular synovitis of the knee joint: CT and MRI diagnosis

A. Barile, E. Fascetti, C. Masciocchi, R. Passariello; L'Aquila

In 13 patients, CT detected a nodular mass (density 60-90 H.U.) at the level of the knee joint. MRI like CT detected the lesion, its extension, and the relationship with the other anatomical structures. MRI differentiated the lesion into two groups: the first group (10 cases) with low signal intensity on T1- and T2-weighted images, and the other group ( 3 cases) with high signal intensity on T2-weighted images. Surgery and histological examination defined PVNS in the first group, and hyperplastic reactive synovitis in the second. In conclusion MRI is better than CT in differentiating PVNS from other synovial diseases.

\section{Room L}

\section{SS}

08.02 Salivary glands and neck

\section{$10.30 \mathrm{AM}$}

\section{Head and neck sonography (Keynote Lecture) N. Gritzmann; Salzburg}

High resolution transducers ( $\geq 5 \mathrm{MHz}$ ) are used to examine the orbit, sinustract, salivary glands, tongue and floor of the mouth and the cervical soft tissues.

In the orbit, sonography can be used to delineate tumors and foreign bodies and to measure the orbital muscles. In the sinustract cystic and solid pathologies can be differentiated. Sonography is used to delineate T1 and T2 tumors of the tongue and floor of the mouth.

Most of the tumors of the salivary glands can be localized and delineated by sonography. US is used to evaluate inflammations and concrements. In most conditions, sonography can be used to delineate cervical tumors. Sonography proved to be highly sensitive in cervical lymph node staging. Sonography should be used for follow up of tumor-patients in the ENT region, because subclinical recurrencies can be detected.

The relationship of the tumor and the great cervical vessels can be assessed. The lecture will demonstrate the clinical value of sonography in the most common clinical conditions.

\section{$10.45 \mathrm{AM}$}

336 Ultrasound in salivary gland diseases - Comparison with alternative imaging methods

E. Rinast, E. Gmelin; Lübeck

a) Salivary glands are easily accessible for ultrasound examinations because of their shallow position. Alternative imaging methods use ionizing radiation (CT), are additionally invasive (sialography) or not readily available (MR). b) In 41 patients with salivary gland diseases "small-parts" sonography, high resolution computed tomography, conventional and digital subtracted sialography were performed and the results compared with operative findings or clinical follow up, respectively.

c) In the case of salivary gland masses, the sensitivity of ultrasonography, CT and sialography was $100 \%, 82 \%$ and $71 \%$, respectively, while in the case of sialadenitis the respective figures were $54 \%, 69 \%$ and $85 \%$. Image quality of digital subtraction sialography was superior or equal to that of conventional sialography in $96 \%$.

d) In all cases of salivary gland diseases ultrasound should be the first imaging procedure. If a tumor is not confirmed, digital subtraction sialography should be employed to visualize inflammatory changes, while in most cases of salivary gland masses no further imaging will be necessary.

\section{$10.55 \mathrm{AM}$}

\section{Sonography of the salivary tumors}

M. J. Eren̈o, J. C. Ruiz, J. Negueruela, J. C. Martin, I. Azkuna, B. Canteli; Bilbao

Purpose: To study the efficacy of sonography for the diagnosis of salivary tumors.

Methods and Materials: 78 patients with parotid and/or submandibular masses, later confirmed by surgery or biopsy were studied. The age of the patients ranged from $2 \mathrm{~m}$ to 78 years. 29 were female and 37 were male. High frequency transducers ( 5 and $7.5 \mathrm{MHz}$ ) were used. The salivary glands on both sides were examined in transverse and longitudinal sections.

Results: The results of this study were: 35 mixed Tumors, 11 Adenolymphomas, 7 Epidermoid Carcinomas, 8 lipomas, 3 lymphomas, 1 Monomorphic Adenoma, 1 Cylindroma, 1 Lymphangioma, 1 Hemangioma, 1 Metastases and 9 Adenopathies.

The sonographic criteria evaluated were: number, location, size, echo-structure and tumoral borders.

In our study the sensitivity of sonography in detection of salivary gland tumors was $100 \%$. The accuracy was lower than the sensitivity.

There were different sonographic features for benign and malignant lesions but not a specific correlation with histology.

Conclusions: In our experience sonography was a valuable primary evaluation of salivary gland tumors, but was impossible to define the final histology.

\section{$11.05 \mathrm{AM}$}

338 MR evaluation of salivary gland lesions with STIR and Gd-DTPA R. Chaudhuri, J. Crossman, J. B. Bingham, M. Gleeson; London

Purpose: Most patients with salivary gland disease may be accurately assessed by clinical examination. Relatively few need detailed imaging to stage their disease and plan surgical treatment. The purpose of this study was to determine 
the most efficient MR sequences for assessment of salivary gland lesions. Methods: Thirteen patients with presumed parotid masses were imaged using a 1.5 T Philips Gyroscan, with a standard head coil. T1-weighted spin-echo and STIR sequences were used, in multiple planes, in all cases, and six subjects also had Gd-DTPA enhanced scans.

Results: T1-weighted spin-echo images were most useful for visualising anatomical structures and identifying the course of the facial nerve. Most lesions were visible with T1-weighting but were more conspicuous on STIR images, because of suppression of surrounding fat and increase in water content of pathological tissues. Use of Gd-DTPA added no further information as most lesions enhanced only slightly. STIR allowed clear resolution of lesions down to $6 \mathrm{~mm}$ diameter, which could, in retrospect, be identified on T1-weighted images, but with difficulty. These small lesions were surgically confirmed. Axial and coronal planes were found more useful than the sagittal plane.

Conclusions: A combination of Tl-weighted spin-echo and STIR sequences displays anatomy and pathology with accuracy in both extensive and subtle disease.

\section{$11.15 \mathrm{AM}$}

\section{Detecting and diagnosing parotid tumors with MRI. 146 patients} studied

Nicole J. M. Freling, Eduard L. Mooyaart, Cees J.P. Thijn, Albert Vermey, Arend K. Panders; Groningen

We studied 146 patients, clinically presenting with a parotid mass. Using a combination of T1 and T2 weighted pulse sequences on a $1.5 \mathrm{~T}$ system (Philips Gyroscan) we were able to predict the correct localisation, intrinsic or extrinsic, deep or superficial, in $99 \%$

MRI predicted the correct nature of a mass in $83 \%$. Histopathologic confirmation was obtained in 139 of 146 patients. MR correctly predicted a benign nature of a mass in $90 \%$. A malignant lesion was correctly identified in $76 \%$. Pleomorphic adenomas have a low signal intensity on $\mathrm{T} 1$ and a high signal intensity on T2 weighted images. Their inhomogeneous aspect, mainly noted on T2 weighted images, may be due to different proportions of cells, chondroid, mucoid and collagen within these tumors. Small malignant tumors resembled benign lesions.

MRI is a highly sophisticated imaging modality for the detection of recurrent pleomorphic adenomas. Sensitivity in this series is $95 \%$, specificity $99 \%$, PPV $95 \%$ and NPV $99 \%$.

MRI is a useful adjunct to clinical judgement, not only to confirm the presence and determine the extent of a tumor, but also in predicting the nature of a parotid mass lesion

\section{$11.25 \mathrm{AM}$}

340 Plain and Gd-DTPA enhanced MR imaging in Sjögren's-syndrome of the parotid gland: New developments

Th. Vogl, S. Dresel, M. Späth, H. Schedel, J. Lissner; Munich

Purpose: New results of MR imaging in Sjögren's disease may offer new possibilities in diagnostic imaging.

Methods and Materials: Twenty-eight patients suffering from immunohistological and serological ensured Sjögren's-syndrome were examined in transverse and coronal orientation, using plain and Gd-DTPA enhanced Tl-and T2-weighted images.

Results: In acute stages plain T2-weighted images showed a swollen gland with prolonged T2-relaxation times and high signal intensity in these images. In chronic stages, a gland of normal size and also prolonged T2-relaxation times could be seen. Additionally, in all cases there was a non-homogeneous internal pattern with a characteristic speckled, honeycomb-like appearance, especially in T2-weighted images. After administration of Gd-DTPA no additional information was extracted. Our experience rendered possible to develop 4 stages to diagnose Sjögren's-syndrome in differentiation to inflammatory changes (no characteristic changes - nodular and swollen gland).

Conclusions: In a preliminary study the typical appearance of Sjögren's-syndrome allows to differentiate this disease from inflammatory changes as well as from tumors using plain $\mathrm{T} 1$ - and $\mathrm{T} 2$-weighted sequences. Administration of Gd-DTPA does not lead to advantageous diagnostic results.

\section{$11.35 \mathrm{AM}$}

\section{Use of the ESWL in the therapy of the sialolithiasis}

Dieter Liermann, J.Peters, W. Kater, J. Kollath, K. Bitter; Frankfurt/Main

Purpose: The surgical therapy of sialolithiasis especially in the parotic gland was always complicated by the risk of damage of the nerv. facialis and necessarily accompanied with a clinical stay. Our purpose was to desintigrate the stones by ESWL in an ambulant session without risk of damage of the parotid gland.

Methods: An exact supervision and documentation before, during and after the ESWL by sonography and sialography is absolutely necessary. We used an
Modullith $^{\mathrm{R}}$ SL 10 Lithotripter with in line ultrasound-scanner over waterbolster with $1500 \mathrm{Imp}$. and a frequency of $2 \mathrm{~Hz}$ by $16-18 \mathrm{KV}$. For documen tation we use a $10 \mathrm{MHz}$ ultrasound sonde and a sialography with a Siemens Digitron DSA.

Results: Sialolithiasis was treated in 26 patients in an non-inflammatory stadium with 33 stones .8 out of 9 stones in the parotid gland and 11 out of 24 stones in the submandibular gland had been desintigrated totally by ESWL technique.

Conclusions: Exact supervised ESWL of sialolithiasis is an helpful and senseful therapy with less risk for the patient, ambulant treatment and a good success rate.

\section{$1.45 \mathrm{AM}$}

342 Evaluation of acute and chronic sialadenitis by ultrasound (US) S. A. A. Qaiyumi, H. Milbradt, M. Prokop, K. H. Hessling, R. Schmelzeisen, M. Galanski; Hannover

In a prospective study of 32 patients, we examined the diagnostic value of US in acute and chronic sialadenitis.

Methods and Materials: We used Sonoline AC/SL US-machines (Siemens) and 7.5 and $5 \mathrm{MHz}$ transducers. 25 patients with chronic sialadenitis and 7 patients with acute sialadenitis could be examined. Sonographic findings were correlated to histological results and clinical course.

Results: Correct sonographic identification was possible in 23 out of 25 patients with chronic sialadenitis. US imaging showed slight volume enlargement of salivary glands with inhomogeneous hypoechoic echotexture and often small cystoid areas due to ductal ectasis. US of 2 patients was normal though histologic evaluation demonstrated slight chronic sialadenitic changes. 7 patients with acute sialadenitis could be identified correctly, and their course under antibiotic therapy could be controlled sonographically. US of acute sialadenitis showed an evident swelling of salivary glands with inhomogeneous or homogeneous transformation of echotexture. Additional information about abscesses and calculi could be obtained. In summary US demonstrated high accuracy in the diagnostic evaluation of acute and chronic sialadenitis.

\section{$11.50 \mathrm{AM}$}

\section{Ultrasound: Long to short axis quotient versus $\mathrm{CT}$ to discriminate} metastatic cervical lymph nodes

H. J. Steinkamp, N. Hosten, C. Zwicker, K. Neumann, R. Felix; Berlin

AIM: Ultrasound (=US) and CT were used preoperatively for lymph node staging in patients with head and neck malignancies. Generally, specificity of US and CT is considered low. Size of lymph nodes does not correlate well with metastatic involvement. In our study the ratio of long and short diameter of suspicious lymph nodes was calculated to enhance specificity of US.

Methods: 138 patients with head and neck cancer were preoperatively examined with $\mathrm{CT}$ and ultrasound. CT criteria for metastatic investigation of lymph nodes were lymph node size $(>15 \mathrm{~mm})$ and structural characteristics (inhomogeneity, central hypodensity with rim enhancement). Ultrasound criterium for metastatic investigation of the lymph nodes was a long to short axis quotient smaller than 2 . Results were than compared with histologic findings.

Results: Sensitivity of CT $(93 \%)$ and ultrasound $(93 \%)$ was equal. US specificity of ultrasound $(95 \%)$ was clearly higher than CT $(66 \%)$. False positive findings: US 5\%; CT 34\% false negative findings: US 7\%; CT 7\%. Accuracy: US $94 \%$; CT $79 \%$

Conclusions: 1. CT criteria of metastatic investigation yield a high sensitivity and low specificity. In $34 \%$ reactive enlarged lymph nodes and metastatic lymph nodes can't be differentiated 2. Ultrasound: The ratio of long to short axis yield a high sensitivity and high specificity to discriminate reactively enlarged lymph nodes from metastatic lymph nodes. 3. Ultrasound ratio of long to short axis compared to CT yields a clearly higher specificity and accuracy for discriminated of metastatic lymph nodes from reactively enlarged lymph nodes.

\section{$11.55 \mathrm{AM}$}

344 What is the importance of three dimensional ultrasonography (3D US) in the diagnosis of thyroid carcinomas?

Remide Arkun, Erkan Sevinç, Şebnem Örgüç, Ayten Süngüoǧlu, Hadi Özer; Bornova-Izmir

Ultrasound (US) has become an important assessment in the evaluation of thyroid disease. When performed with high-frequency transducers, US is highly sensitive in enabling the detection of nodular lesions but it is not specific for distinction of malignant from benign nodules that have been found.

Twenty-two cases who had solitary thyroid nodules were examined in two steps. First, not only nodular lesions but also all thyroid gland was examined by small parts scanner which had a high-frequency transducer. And then only nodular lesion was examined by $3 \mathrm{D}$ US and obtained three different images on axial, coronal and sagittal planes. Fifteen of these patients underwent 
operation. Pathologic, conventional and 3D US findings were then correlated. In 3D US images, especially on sagittal plane displayed better the interior structure and border of lesion than conventional US.

Although US is not highly specific for distinction malignant from benign nodules, the new diagnostic modality 3D US seems to obtain more valuable information about the nature of lesions.

\section{Room M}

\section{SS}

\subsection{Doppler in Urology}

$10.30 \mathrm{AM}$

\section{Duplex Doppler of the renal tract (Keynote Lecture)} P.A. Dubbins; Plymouth

The kidney is a highly vascular organ receiving approximately $20 \%$ of the cardiac output. The blood supply to the kidney has a major role not only in the physiology and pathology of the kidney itself but also plays a role in the pathogenesis of, for example, hypertension. That pathology of the kidney could be reflected in changes in the blood supply and venous drainage was first documented on ultrasound by the recognition of enlarged renal veins in certain vascular carcinomata. This reflected the significant arterio-venous shunting that may occur in certain renal tumours. However, the crude demonstration of enlarged or dilated vessels of supply and drainage provides little information about the physiology and pathology of blood flow to the kidneys.

The advent of duplex systems where imaging ultrasound is allied to a Doppler flow meter has made possible the study, in detail, of blood flow to the kidney in health and disease. It is possible to document increased volume flow in response to certain physiological stimuli such as fluid and protein loads as well as identifying renal shutdown in cardiovascular shock. Furthermore, it is now possible to demonstrate changes not only in volume flow but also in the characteristic pattern of flow in systole and diastole that occur in response to different diseases. In addition, characteristic velocity changes in the main renal artery can be identified in renal artery stenosis and in abnormalities of the renal vein such as renal vein thrombosis.

Early optimism about the specificity of certain simple mathematical ratios based on systolic and diastolic flow velocities have not been supported by subsequent research work. However, the application of simple indices to the solution of complex pathological problems involving vessels of supply and drainage as well as the renal parenchyma are at best crude. More sophisticated analysis of the flow pattern to the kidneys in different conditions may allow the further characterisation of disease processes. Already, however, it is possible to identify alterations in blood flow as well as to document perfusion patterns with colour flow Doppler thus adding a functional element to the traditional role of ultrasound imaging of the renal tract.

\subsection{AM}

\section{Results of Dopplersonography in renal artery stenosis and status post PTA}

E. Gutierrez, E. Dünser, W. Richter, F. Meisl, H. Umek; Wien

The aim of this study was to evaluate dopplersonographic parameters (maximal systolic velocity (MSV), enddiastolic velocity (EV), resistance index $(\mathrm{RI})$ ) in comparison to angiographic findings in renal artery stenosis.

80 angiographically verified normal kidney arteries showed a distribution of normal maximal systolic velocities (MSV) between 60 and $140 \mathrm{~cm} / \mathrm{sec}$. with an accumulation between 80 to $100 \mathrm{~cm} / \mathrm{sec}$.

The average enddiastolic maximal velocity was found between 25 to $35 \%$ of MSV.

70 angiographically verified stenoses of renal arteries showed an elevation of MSV between 110 to more than $400 \mathrm{~cm} / \mathrm{sec}$. There was no statistically proofed correlation between the angiographic degree of stenosis and elevation of MSV; but provided that a renal artery can be visualized sonographically in a sufficient way the dopplersonographic accuracy of the diagnosis of a renal artery stenosis shows a sensitivity of 90 to $93 \%$ and a specificity of 95 to $100 \%$ (with an upper limit of normal MSV between 140 to $160 \mathrm{~cm} / \mathrm{sec}$.)

In 15 patients with renal artery stenosis and subsequent PTA the follow up by dopplersonography showed equivalent results compared to angiography (follow up up to 24 monthes).

Conclusions: Additionally to angiography Dopplersonography is a reliable non-invasive method for diagnosing a renal artery stenosis and for the follow up after PTA.
$10.55 \mathrm{AM}$

347 Characterization of the vascularisation of renal tumors by color Doppler US

A. Denys, O. Hélénon, E. Atlan, Y. Chrétien, B. Dufour, J. F. Moreau; Paris

Purpose: The aim of this prospective study is to evaluate the contribution of color doppler imaging for the detection of neovascularisation and for the characterization of renal tumors.

Methods and Materials: We explored 24 tumors: 19 carcinomas including 3 cystics and 5 benign tumors.

Results: The results were correlated to angiographic ( $23 / 24$ cases) and pathologic datas (23/24 cases). The neovascularisation is found in each vascularised tumor ( 0 false negative). Pulsed doppler seems to be more sensitive than color doppler for the hypo-vascularised tumors. Spectral analysis found 3 different kinds of curves, but cannot differentiate malignant from benign tumors. Conclusions: Color doppler imaging seem to be an accurate technique for the detection of neovascularisation but not specific in the characterization of renal tumors.

\subsection{AM}

\section{Color-Doppler imaging of renal cell carcinoma: a prospective study M. F. Bellin, G. Ittah, F. Richard, C. Radier, C. Chatelain, J. Grellet; Paris}

Purpose: To analyze the color-Doppler features of renal cell carcinoma (RCC), and to assess the effectiveness of color-Doppler in the pre-operative staging of RCC

Methods and Materials: 35 patients with surgically proved RCC were examined on an Acuson 128 unit. The color flow pattern within and around the tumor was studied; a spectral analysis of the tumor vessels, of the ipsilateral renal artery and vein was made using pulsed-Doppler. The results of colorDoppler concerning the tumor staging were correlated with CT, MRI (Signa GE $1.5 \mathrm{~T}$ ) and pathology in all cases.

Results: Color-Doppler depicted intra-tumoral vessels in $34 / 35$ cases, located at the periphery of the tumor in $27 / 35$ cases and in the center of the tumor in 19/35 cases. Pulsed-Doppler identified three types of flow-pattern: high systolic velocity $(>0.25 \mathrm{~m} / \mathrm{s})$, with large systolic-diastolic gradient, low systolic velocity $(<0.25 \mathrm{~m} / \mathrm{s})$, continuous flow with low velocity. ColorDoppler clearly depicted patency of the main renal vein in $26 / 35$ cases; it correctly assessed venous extension in 7 cases. There were 2 false negative findings.

Conclusions: Color-Doppler provides information on blood flow that supplements the information gained by routine sonography.

\section{$11.15 \mathrm{AM}$}

349 Kidney transplant renal artery stenosis: Evaluation with continuous Doppler

G. Alvarez, A. Gomez, B. Asenjo, M. Gonzalez, M. Cabello, M. A. Frutos Malaga

Purpose: We present our experience in the detection of renal artery stenosis in 20 patients. All patients had arterial hypertension and in five there was also a progressive deterioration of the renal function with an increase in serum creatinine.

Methods: The transplanted kidney was studied with a Duplex-Doppler Diasonic DRF-300 with pulsed Doppler transducers of $3.5 \mathrm{MHz}$ and the renal artery with continuous Doppler transducers of $3 \mathrm{MHz}$

Results: The intrarenal curves presented in all cases a pattern of low resistance and in three cases there was a damping of systolic-diastolic signal secondary to a renal artery stenosis greater than $90 \%$. Stenosis was assumed when the Doppler frequency was higher than $7.5 \mathrm{KHz}$ between the transplanted kidney (low resistance pattern) and the iliac artery (high resistance pattern).

Conclusions: All twenty patients fulfilled a requirement for a Doppler frequency higher than $7.5 \mathrm{KHz}$, and in all these angiography was performed confirming the presence of arterial stenosis. There was a correlation between the Doppler frequency obtained and the degree of stenosis.

\subsection{AM}

\section{Doppler ultrasound examination of renal allografts - resistive index versus pulsatility index}

P. Hübsch, S. Trattnig, F. M. Kainberger, A. Globits, O. Traindl; Vienna

AIM: For the analysis of the Doppler waveform in the examination of renal allografts, the resistive indices (RI) and the pulsatility index (PI) are reported to be of value. Since there is no comparative study in the literature, we have assessed both parameters to determine their diagnostic value in the evaluation of rejection.

Methods: 34 patients were examined 1-6 hours before a percutaneous needle biopsy of the graft was done. The results of RI and PI measurements were 
compared with each other, and with histology.

Results: 3 groups of patients could be identified on the basis of the threshold values recommended in the literature $(\mathrm{RI}=90 \%, \mathrm{PI}=150 \%): 1$. normal RI (mean $63.5 \%$ ) and PI (mean $117.4 \%$ ), 2. normal or borderline RI (mean $80.8 \%$ ) and elevated PI (mean 195.6\%), 3. elevated RI (mean 99.5\%) and PI (mean $352.8 \%$ ). No significant correlation with histology was found.

Conclusions: The PI seems to be more sensitive than the RI for the detection of a pathologic blood flow pattern in renal allografts. Especially when the RI is borderline, the PI should be calculated additionally. Nevertheless, needle biopsy is indispensable to determine the cause of graft dysfunction.

\subsection{AM}

351 Gd-DOTA MR imaging and color Doppler US characteristics of renal transplant infarction

O. Hélénon, E. Attlan, Ch. Legendre, J. M. Correas, M. Souissi, J. Chabriais, H. Kreis, J. F. Moreau; Paris

Purpose: To demonstrate Gd-DOTA MRI and color Doppler US characteristics of renal transplant infarction.

Methods and Materials: we examined 22 transplanted kidneys with areas of infarction $(n=11)$, cortical necrosis $(n=9)$ and complete renal infarction with artery thrombosis $(n=1)$ or acute vein thrombosis $(n=1)$. MR study consists of T1-weighted and T2-weighted images and fast dynamic MRI using gradient echo sequences ( 9 to 12 slices) during a breath hold, followed by T1-weighted spin echo sequence 9 to $15 \mathrm{~min}$. after Gd-DOTA injection. 19 patients were also explored with color Doppler US. The diagnosis was confirmed by needle biopsy ( 10 cases) or transplant nephrectomy ( 12 cases) with macroscopic correlation.

Results: Color Doppler US provides early detection of arterial or venous thrombosis and areas of infarction in renal parenchyma, but is less specific than Gd-DOTA enhanced MRI and insufficient for the precise delimitation of infarction. Gd-DOTA enhanced MRI provides a very precise delimitation of infarcted areas, particularly in cases of small defects of perfusion or cortical necrosis.

Conclusions: Color Doppler US permits an early detection of infarction in most cases and is useful to diagnose vascular thrombosis. Gd-DOTA MRI provides an accurate and noninvasive evaluation of perfusion defects.

\subsection{AM}

352 Follow up - study of renal transplants by duplex Doppler-and gray scale-ultrasound examination

O. Jansen, P. M. Rob, V. Schmidtke, N. Marienhoff, E. Rinast, H.-D. Weiss; Lübeck

In the early postoperative period there are four main nonvascular complications which can cause renal transplant dysfunction - interstitial and vascular rejection, acute tubular necrosis and Cyclosporin nephrotoxicity. Single duplex ultrasound examination can not discriminate between these four deseases because of lack of specificity.

Over a period of 18 months we sampled standardized duplex indices (resistance index, pulsatility index) and gray scale parameter (parenchyma/ pyelon-index) of 388 follow up examinations in 77 patients in the early posttransplantation phase. The retrospective analysis of these datas showed significant differences in the behaviour of duplex indices and gray scale parameters during the courses of the different diseases. We designed a diagnostic pattern which includes cut off values of duplex and real time parameters to distinguish interstitial rejection, vascular rejection, acute tubular necrosis and Cyclosporin nephrotoxicity. This diagnostic pattern showed a retrospective sensitivity of $84 \%$ and specificity of $81 \%$ in our patients.

To our opinion a periodical examination by duplex and gray scale sonography every three days in the immediate posttransplantation phase is essential to make this diagnostic pattern a valid instrument in the monitoring of renal transplants.

\subsection{AM}

\section{Intrarectal Doppler studies of arterial flow during endo-urethral hyperthermia: preliminary results}

R. Oyen, F. Ameye, L. Baert, G. Marchal, A. L. Baert; Leuven

Purpose: Cellular necrosis and vascular thrombosis are described to be the effect of hyperthermia in the heated area of the prostate. The purpose of this study was to determine if changes in arterial blood flow could be detected in patients prone to endo-urethral hyperthermia for benign prostatic hyperplasia. Methods and Materials: A Transesophageal Endoscopic $5 \mathrm{MHz}$ phased array transducer (ATL, Ultramark 9) was used intrarectally. The hyperthermia was performed with the BSD 300 equipment. Blood vessels were detected with colour doppler and subsequently measured.

Results: In 9 patients, intrarectal doppler studies were done prior to, during and after the hyperthermia. No statistical significant differences in resistive index could be measured nor in the peri-urethral area, nor in the peripheral zone $(p>0.05)$. For comparison endorectal doppler studies were done in 2 patients with BPH, 2 with prostatic carcinoma and 1 with acute prostatitis. In the latter, the inflammatory area was clearly hypervascular, with statistical significant differences in the resistive index between the pathologic area and the normal prostate $(p<0.01)$. No specific changes were seen in the patients with the prostatic carcinoma.

Conclusions: Further studies have to be done to find out if there are objective changes in intraprostatic blood flow during hyperthermia or specific changes in prostatic disease.

\section{Room N}

\section{SS} $10.30 \mathrm{AM}$

\subsection{Dementia, Vascular 1}

\section{Brain imaging in dementia (Keynote Lecture)}

\section{U. Buell; Aachen}

Reflecting neuropathology, cortical (Alzheimer (DAT), Pick), subcortical (Huntington, Parkinson, Wilson) or mixed-type dementia (D) (multiinfarct (MID), Jakob-Creutzfeld) may be separated from white matter D (AIDS, Binswanger (DBT), NP-Hydrocephalus, ED). Methods, aimed at imaging function (PET, SPECT) give insight into regional cerebral blood flow (rCBF) or blood volume (rCBV) and into metabolic rates ( $\mathrm{rCMR})$. CT and MRI, displaying morphology, evaluate degrees of atrophy, periventricular lucency and pattern of angiopathical involvement (macro-, micro-). Combined evaluation provides access to an in-vivo classification of D.

Atrophy is a main but unspecific finding in nearly all types of D. Widened sulci and fissures are potentially filled with enlarged veins (MRI) and increased BV (SPECT). Functional changes correlate with the severity of D. In DAT, subsequently to the clinical onset, CBF and CMR are reduced condordantly, symmetrically or not, mainly in frontal and/or parietal cortex. In severe DBT, $\mathrm{CBF}$ and $\mathrm{CMR}$ are below normal in both, white and grey matter, preceeding the demential presentation. In AIDS, rCBF may be focally reduced in the grey matter. CMR is below normal in the caudate nucleus in Huntington very early. MID was found to be reflected by lacunes or macroangiopathy or a combination thereof. Exploring patients suspected to have D by means of such procedures may be helpful in the differential diagnosis of $\mathrm{D}$ versus the symptom complex of depression, where no rCBF reduction occurs in non-organic depressive patients. On the other hand, $\mathrm{rCBF}$ pattern of low flow may be typical in advanced cases of the various types of D only. Atrophy, however, adds to such findings simply by having reduced the brain volume concerned and thus $\mathrm{CBF}$ and $\mathrm{CMR}$.

\section{$10.45 \mathrm{AM}$}

355 Acetazolamid induced changes of cortical $\mathrm{rCBF}$ in patients with cerebrovascular disease - a HMPAO-SPECT study

S. Asenbaum, I. Podreka, B. Schuster, T. Czech, B. Reinprecht; Vienna

a) The purpose of the study was, to demonstrate changes in $\mathrm{rCBF}$, by calculating the cerebral HMPAO uptake, at patients with cerebro-vascular disease and to determine the vascular reactivity by provocation with acetayolamid (Diamox).

b) 19 patients (mean age: 63 a, 16 male and 3 female) have been examined two times with Tc-99m HMPAO-SPECT, whereas at the second time one week later $1 \mathrm{~g}$ acetazolamid i.v. was injected in advance. All patients suffered at least once an ischemic cerebro-vascular incident (4 transient ischemic attacks, 4 RIND's and 11 minor strokes) and most of them showed an occlusion (in 5 patients) or a severe stenosis (in 11 patients) of the internal carotid artery on the corresponding side. - The global and hemispheric HMPAO uptake, calculated as $\%$ of injected dosis per $100 \mathrm{ml}$ of brain tissue were calculated. To determine the cortical HMAPO uptake, 17 regions of interest (ROI)/hemisphere were drawn on 4 adjacent transversal slices ( $21.9 \mathrm{~mm}$ thick).

c) The global as well as both hemispheric HMPAO uptakes increased significantly after Diamox (at about $28 \%$, respectively $25 \%$ on the symptomatic and $30 \%$ on the non-affected side). But the difference of the HMPAO uptake between the two hemispheres was more pronounced in the second investigation. - The regional evaluation of the uptake showed 1. a significant increase over all ROI's after Diamox, but on a lower level on the affected side, 2. a significant uptake-difference in corresponding ROI's latero-frontal, central, inferior parietal and in the thalamus, and with Diamox although mesiofrontal, superior parietal, occipital and in the basal ganglia, 3. a significant increase in asymmetry of both hemispheres under Diamox in the fronto-mesial, central, inferior parietal and superior occipital region.

d) Beneath the existing difference in HMPAO uptake of the hemispheres, even the affected side showed a significant increase of the uptake after Diamox. But the asymmetry between the hemispheres increased in special cortical regions, which could be regarded as an indication for an operation. 
356 Tc-99m-HMPAO-SPECT in patients with reversible cerebrovascular disease (CVD): comparison of the sensitivity without and with acetazolamid

W.-H. Dingler, D. Beil, H.-K. Deininger; Darmstadt

SPECT imaging of the brain was performed in 66 patients with reversible CVD utilizing Tc-99m-HMPAO; 35 patients were studied after intravenous administration of $1 \mathrm{~g}$ Acetazolamid, 31 patients got none. Sensitivity of these two methods were compared differently in reversible hemispheric (TIA) and vertebrobasilar insufficiency (VBI). Cerebral infarction was excluded by TCT. Sensitivity in TIA without provocation was $55 \%$, in VBI $36 \%$. Acetazolamid improved sensitivity to $90 \%$ and $64 \%$. In reference to total CVD sensitivity increased from $48 \%$ to $80 \%$. 11 patients who were investigated with both methods within a few days, showed an increase of sensitivity from $45 \%$ to $81 \%$. 3 patients with amaurosis fugax and severe stenosis of the internal carotid artery were normal with Acetazolamid. However, in 8 patients with asymptomatic severe carotid stenosis the ipsilateral hemispheric blood flow was reduced by the provocation test.

Conclusions: SPECT imaging with Tc-99m-HMPAO shows in patients with reversible CVD in provocation with Acetazolamid a distinct higher sensitivity.

\section{$11.05 \mathrm{AM}$}

\section{Dopamine D2 receptor imaging with I-123-IBZM-SPECT in patients with Parkinsonian syndromes}

K. Tatsch, J. Schwarz, W. H. Oertel, C.-M. Kirsch; Munich

This study investigates the diagnostic potential of dopamine D2 receptor imaging with I-123-IBZM (3-iodo-6-methoxybenzamide)-SPECT for discriminating idiopathic Parkinsons's disease (IPD) from parkinsonian-like syndromes (PS).

Six normal controls and 31 untreated patients with IPD $(n=13)$ and PS $(n=18)$ were studied. The diagnosis was established clinically and by positive/negative response to dopaminergic drugs. SPECT was performed 2 hours after application of $185 \mathrm{MBq}$ I-123-IBZM. With regions of interest basal ganglia/frontal cortex (BG/FC) ratios were calculated (normal controls: $1.37 \pm 0.05$ SD). All patients with IPD (13) had normal binding of IBZM in the striatum $(\mathrm{BG} / \mathrm{FC}: 1.34 \pm 0.05) .17 / 18$ patients with $\mathrm{PS}$ showed significantly $(\mathrm{p}<0.001)$ reduced striatal uptake of IBZM (BG/FC: $1.16 \pm 0.07)$. In one PS-patient SPECT was normal, though no improvement on L-Dopa was observed. In 4/18 IBZM binding was reduced unilaterally with clinical symptoms contralaterally in 3. The other cases presented symmetrical findings. IBZM-SPECT has a sensitivity of $100 \%$ and specificity of $94 \%$ for differentiating IPD from PS. IBZM-SPECT may well discriminate between IPD and PS in untreated patients with parkinsonian syndromes. Detectable changes in striatal D2 receptor binding provide an early, quick and objective diagnosis which has no side effects and is independent of subjective interpretations of the clinical response to dopaminergic therapy.

\section{$11.15 \mathrm{AM}$}

358 SPECT of the D2 dopamine receptors with $1-123$ iodobenzamide J.-L. Baulieu, D. Guilloteau, M.-J. Ribeiro Barras, S. Chalon, S. Markabi, B. Gaymard, A. Autret, J.-C. Besnard; Tours

a) The aim of this study was to develop SPECT of the D2 dopamine receptors of the human brain using I-123 iodobenzamide ( ${ }^{123}$ IBZM).

b) ${ }^{123} \mathrm{IBZM}(100 \mathrm{MBq})$ was injected into 14 subjects: 3 control, 4 patients with untreated Parkinson's disease, 1 patient with untreated Alzheimer's disease, and 6 patients treated with dopaminergic agonists (L-dopa, bromocriptine) or antagonist (pipamperone). One hour after injection, anterior, posterior and lateral views of the head were obtained; brain SPECT was performed using a single head rotating gamma camera in $64 \times 40$ seconds steps of acquisition, and a filtered backprojection algorithm (Sophy Camera - Sopha Medical). The ratio of the mean activity in the striatum to the cerebellum $(\mathrm{s} / \mathrm{c})$ was calculated. c) The mean brain uptake was 3 per cent of the injected dose. The distribution of the activity on the brain slices was described according to three types of patterns: type I: predominant uptake in the basal ganglia observed in the control subjects and in patients with Parkinson's disease; type II: cortical activity without visualization of the basal ganglia observed in 5 of the 6 treated patients; type III: uptake in the basal ganglia associated with a cortical activity observed in the patient with Alzheimer's disease. The s/c ratio was increased in untreated Parkinson patients $($ mean $=1.9)$ and decreased in the Alzheimer patient $(1.3)$, compared to the normal subjects (mean $=1.7$ ).

d) These results confirm the faisability, the specificity and the potential interest of the D2 dopamine receptors SPECT using ${ }^{123}$ IBZM, for the investigation of neurological and psychiatrical disorders.
359 Acetazolamide stimulation test for the assessment of cerebrovascular reserve capacity by transcranial Doppler and ${ }^{99 m}$ TC-HMPAOSPECT

K. Rosenkranz, R. Langer, J. Hierholzer, W. Hepp, J. Palenker, R. Felix; Berlin

Purpose: The vasodilatating effect of acetazolamide on cerebral arterioles was used to study the cerebrovascular reserve capacity (CVRC) in patients with cerebrovascular disease.

Patients and methods: 20 patients with symptoms of cerebral ischemia and angiographically confirmed unilateral stenoses and occlusions of the extracranial internal carotid artery (ICA) and 20 controls were studied by a $2 \mathrm{MHz}$ transcranial Doppler. Time-mean velocity $\left(\mathrm{V}_{\text {mean }}\right)$ and pulsatility index $(\mathrm{PI})$ in the middle cerebral artery (MCA) were measured before and $10 \mathrm{~min}$. after intravenous application of $1 \mathrm{~g}$ acetazolamide. In addition, the patients

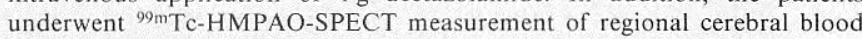
flow $(\mathrm{rCBF})$ at rest and after stimulation with $1 \mathrm{~g}$ acetazolamide.

Results: In 10 patients with ICA-stenoses $>80 \%$ and -occlusiuons, $\mathrm{V}_{\text {mean }}$ increase and PI-decrease in the postobstructive MCA as well as the increase of the ipsilateral $\mathrm{rCBF}$ were reduced in comparison with the contralateral side. The remaining 10 patients showed a normal $\mathrm{V}_{\text {mean }}$-increase and PI-decrease as well as a normal increase of $\mathrm{rCBF}$ after acetazolamide injection without significant side differences.

Conclusions: The acetazolamide stimulation test with combined measurement of cerebral blood flow velocity and $\mathrm{rCBF}$ in cerebrovascular disease can be helpful to identify patients with hemodynamic ischemia.

\section{$11.35 \mathrm{AM}$}

\section{Color-coded Doppler sonography of vertebral arteries} S. Trattnig, P. Hübsch, B.Schwaighofer, D. Pölzleitner, F. Kainberger Vienna

Purpose: To determine the visualization of the extracranial portions of the vertebral arteries and to describe the appearance of different vertebral artery disease with color-coded Doppler sonography.

Methods and Materials: We studied 42 patients without and 86 patients with a history of vertebrobasilar disease by using a color-coded Doppler sonography unit equipped with 7,5 and 5-MHz linear-array transducers (Acuson $128 \mathrm{~T}^{\mathrm{m}}$ ). Additionally, nine patients with subclavian and vertebral artery reimplantation to the common carotid artery were examined. All pathologic findings were confirmed at angiography.

Results: The origin of the vertebral artery was visualized in $87,2 \%$ of cases on the right and $70,9 \%$ on the left side. The intertransverse portion of the artery was visualized in $95 \%$ on the right and $97 \%$ on the left side. The atlas loop could be visualized in $88,4 \%$ on the right and $84,9 \%$ on the left side. Pathologic findings were hypoplasia (4/86), stenosis (19/86), dissection $(2 / 86)$, occlusion $(7 / 86)$, kinking (12/86), and the subclavian steal syndrome (3/86). A typical color pattern was demonstrated in hypoplastic arteries, high-grade concentric stenoses at the origin, and dissections in the atlas loop, as well as in patients with subclavian steal syndrome.

Conclusions: Color-coded Doppler sonography seems to be a promising, noninvasive imaging technique for the evaluation of vertebral artery disease.

\section{$11.45 \mathrm{AM}$}

\section{Early C.T. signs of cerebral ischemia}

X.Serres, J.C. Vilanova, J.Teruel, I. Lladó, J. Barceló, R. Bassaganyas, A. Dávalos; Girona

A group of 116 patients were included in a clinical-biochemical-radiological protocol of an ischemic stroke. All of them had 3 C.T.-scan examinations, the first one was made as early as possible, and always before the 8th hour of the onset of symptoms, and the others at 7 th and 60 th day of evolution.

We retrospectively reviewed the new signs of recent infarction (lentiform sign and an insular cortex hypodensity) and we added to the already known classical findings (hypodensity of the lesion, vascular hyperdensity and mass effect). During the review, we realized that the rest of the grey matter is shown as effective as the lentiform nucleus or insular ribbon in the early hours of infarction. As a matter of fact, caudate nucleus, thalamus and the rest of cortical gyri presented a blurring when ischemia affected them.

The rate of effectiveness is from $52 \%$ of the caudate nucleus to $75 \%$ of the lentiform nucleus as disorders of the grey matter. In hemispherics infarctions, $25 \%$ of the sylvian arteries were hyperdense, and between $30 \%$ and $40 \%$ appeared with mass effect and hypodensity superior to 5 H.U. To sum up, $82 \%$ of the examinations had one or various of the signs mentioned above which allows a very early diagnosis in the majority of cases. 
11.55 AM

362 The study of brain perfusion with $99 \mathrm{mTC}-\mathrm{L}, \mathrm{L}-\mathrm{ECD}$ in patients with cerebral disorders

Rongfu Wang; Suresnes

In recent year, brain perfusion imaging has been greatly achieved, but $99 \mathrm{mTc}-\mathrm{L}$, L-ethyl cysteinate dimer (ECD) is still not in clinical routine use in Europe. This study presented the clinical evaluation for the study of brain perfusion with $99 \mathrm{mTc}$-ECD in 15 clinically normal subjets and 35 patients with various cerebral diseases ( 6 with stroke, 14 with transient ischemia and 15 with neurological disorder). At the same time, brain imaging in 5 normal subjets and 10 patients with $99 \mathrm{mTc}$-HMPAO and ECD was performed. Our studies demonstrate favourable characteristics of ECD as a potential cerebral blood flow markes because of its readily available from a freege-dried vial and easily prepared with radiochemical purity of $>94 \%$ and labelled yield of $>97.5 \% .35$ patients showed significantly abnormal hypoperfusion in various degree. 8 of 10 patients with $99 \mathrm{mTc}$-HMPAO indicated abnormal, but 2 were disputable. This suggests that $99 \mathrm{mTc}$-ECD has a better quality of imaging with enhanced contrast owing to its faster clearance from the blood and prolonged brain retention. We conclude that $99 \mathrm{mTc}-\mathrm{L}, \mathrm{L}-\mathrm{ECD}$ is a suitable agent for brain perfusion imaging and comparable to HMPAO. However, the exact trapping mechanisms and their relationship with potential clinical applications still remain to be investigated.

\section{Room 0}

\section{SS} $10.30 \mathrm{AM}$

03.01 Stereotactic localization and fine-needle-biopsy

363 Mammography - methodology and screening (Keynote Lecture) Audrey K. Tucker; London

Dramatic advances have occurred in breast imaging with improvement of films, processing and apparatus.

Lesions of 2.0 or $3.0 \mathrm{mms}$ can now be demonstrated by $\mathrm{x}$-rays and the significance of these lesions must be assessed. No screening programme should be undertaken without assessment facilities.

Ultrasound is an invaluable tool for the differentiation of solid from cystic lesions and also to demonstrate masses in the very dense breast. It can be used for the localisation of impalpable but ultrasonically visible suspicious lesions. $\mathrm{X}$-ray mammography is at present the only accepted modality for population screening. Factors influencing screening programmes include compliance, age of onset and frequency of screening, detection rates and accuracy of interpretation.

Compliance is greatest with direct invitation, particularly in urban districts. Current evidence for reduction of mortality supports screening for women in the over- 50 group but is contraversial in the 40-50 decade. Optimum frequency of screening is also under discussion. Trials are progressing in the UK to investigate one or two views, frequency and optimum age at onset of screening. Significant reduction in the benign-to-malignant ratios can be achieved by using pre-operative stereotactic aspiration and cytology.

For newer modalities digital radiography is being developed and MRI spectroscopy holds interest for the future though MRI is not practical for routine imaging at present.

A team with experience, expertise and good training in mammography, ultrasound and cytology is vital for the success of any screening or diagnostic programme.

\section{$10.45 \mathrm{AM}$}

\section{Stereotactical localisation for cytological puncture and histo- logical biopsy at small lesions in mammography}

M. Bauer, R. Schulz-Wendtland, F. Kommoss, K. Prinz, F. Richard; Freiburg

Since November 1987 we use at the Department of Gynaecological Radiology (University of Freiburg) at $N=199$ patients a stereotactical equipment compatible to the mammography system for cytological puncture, histological biopsy and preoperative marking. After localisation of the lesion by computerized help, the puncture, biopsy, preoperative marking is done manual. The physical exactness is $\pm 1 \mathrm{~mm}$, clinical the maximum variation after 81 preoperative markings $\pm 5 \mathrm{~mm}$. Until $11 / 90$ we carried out 118 stereotactical punctures for cytological diagnosis - a conformity of cytological and histological diagnosis was found in $82 \%$. The advantages and disadvantages of the method will be discussed in view of their possibilities in breast diagnostic.
$10.55 \mathrm{AM}$

365 Mammography-guided stereotactic fine-needle aspiration cytology of clinically occult breast lesions: comparative study with surgical biopsy results

T. Franquet, C. de Miguel, R. Cozcolluela; Pamplona

Purpose: To assess the accuracy of fine-needle aspiration citology (FNAC) in providing pathologic diagnosis of clinically occult mammographic lesions by prospectively performing mammography-guided FNAC in 100 women and to determine whether a benign cytologic diagnosis can be sufficiently specific to characterize the mammographic abnormality.

Methods and Materials: One hundred consecutive FNAC were performed on clinically occult breast lesions. Based on high mammographic/cytologic index of suspicion for malignancy, 40 of 100 patients underwent open biopsy. Cytologic and histologic findings were compared with mammographic findings.

Results: Cytologic aspirate was adequate in $81 \%$ of cases. There were 16 cancers (two masses, thirteen calcifications, and one stellate lesion). Cytology provided specific diagnoses in $66 \%$ of cases. In $15 \%$ of cases cytology was reported as benign but without a specific diagnosis. A protocol for further diagnostic and therapeutic decisions, was developed to follow-up those discordant cytologic and mammographic findings. Of the 40 breast biopsy specimens, 24 were benign and 16 were malignant. Comparing cytologic and histologic diagnosis, FNAC had a sensitivity of 0.85 and specificity of 1.00 versus 0.87 sensitivity and 0.85 specificity for mammography.

Conclusions: Stereotactic FNAC can provide specific diagnosis of benign and malignant breast disease but cytology must always be correlated with mammography to avoid false-negative results. Our results also suggest that this technique may reduce the number of breast biopsies for benign lesions.

11.05 AM

366 Stereotactic breast punctures in patients with non-palpable lesions

Jutta Peters, Ulrich Lörcher; Frankfurt/Main

Purpose: Purpose of the study presented here is to present the results of our 18 months experience with stereotactic breast puncture of non-palpable lesions, especially microcalcifications.

Methods: During the last 18 months 95 stereotactic breast punctures were performed in patients with non-palpable lesions that were difficult to evaluate radiologically. The system we used for the punctures was the Philips Mammodiagnost UC with Cytoguide. Technical aspects of the system and precision are discussed.

Results: Of the 95 patients punctured 30 had two controls after 6 months respectively, 30 had one control puncture and 35 did not have a control puncture yet or had malignant cytology to begin with and were operated on. So far in the non-malignant cases no follow-up showed malignancy and the ones that showed malignant cytology in the first place proved to be malignant after biopsy.

Conclusions: The Mammodiagnost UC combined with Cytoguide is a very accurate and reliable system that helps to clarify the dignity of non-palpable breast lesions.

\subsection{AM}

367 Perspective and applicative methodologies of stereotactic biopsy in mammographic context

C. Gilardoni et al.; Mandello Lario

The stereotactic proceedings allow to reach, with high precision, a lesion or an anatomic structure, previously identified, after having defined the three dimensional co-ordinates in a settled reference system.

Now the stereotactic units, used to hit the centre of a non palpable suspectlesion utilize two kinds of devices:

- DEDICATED: planned and used exclusively for stereotactic.

- NON-DEDICATED: supplied to complete a mammographic equipment. Both devices are based on the same geometric principle.

The target of this communication is to analyse the physical characteristics of a stereotactic unit and to verify the difference between the DEDICATED and NON-DEDICATED equipments.

In this lecture we will show some important results about this argument.

\subsection{AM}

368 Relevance of bioptic mammary sampling with stereotaxy E. Reginato, H. Almolla, A. Varcasia, F. D'Agostino; S. Marco Argentano

The early diagnosis of infraclinical lesions reduces death rate in mammary carcinoma.

Methods: Mammography with Mammogil (Gilardoni) and stereotaxy with Biopsygil (Gilardoni). 
Methods and Materials: 586 mammographies, 48 cytological examinations on 42 patients: The mammographic examination has shown: 10 benign lesions, 6 malignant lesions, 22 uncertain lesions and 4 microcalcific foci.

Results: Localization was $100 \%$, insufficient sampling in 8 cases $(20 \%)$. In 34 cases: 5 were non invasive ductal carcinomas, 3 invasive ductal carcinomas and 2 lobular carcinomas.

Conclusions: Stereotaxy shows sensitivity and specificity close to $100 \%$. Adequate procedure reduces unnecessary biopsies. In radiologically benign lesions, unsuspected carcinomas can be diagnosed.

\section{$11.28 \mathrm{AM}$}

369 Non palpable breast lesions: use of preoperative marking in 974 cases

G. Wolf, P. Fitzal; Vienna

Over a period of 14 years we have carried out 41.000 mammographies.

During these examinations we found 974 occult changes that had not appeared clinically and needed further checks. The changes were the reason for aspiration biopsy and the use of preoperative markings, 306 of which were carried out with conventional localisation instruments and several different localisation helps. In December ' 86 we were supplied with a stereotactic instrument. Since then we have been using it for 665 preoperative markings and punctions, 52 of which we had to carry out conventionally. The number of preoperative markings is a lot higher than the numbers of aspiration biopsy. Recently the number of punctions has increased due to the introduction of the stereotactic instrument. The operation statistics have been changed in the past years. While in the first 4 years - 1976 to 1980 - the number of preoperative localisations was 8,25 per year, in the next years the number of interventional measures increased.

In the last years the $\mathrm{B} / \mathrm{M}$ index levelled off the $2: 1$.

In our research series we could localize 34 preinvasive carcinomas successfully.

\subsection{AM}

\section{The radiological examination of breasts after conservative surgery of breast cancer}

P. Göblyös; Budapest

The radiological examination of the operated breast is more difficult, than that of the not operated ones, and is especially difficult after the conservative surgeries, recently becoming more frequent. The postoperative irradiation increases the difficulties, because it causes alterations, too.

Our experiences are as follows:

The operative influence of breast causes lasting alterations in the breasts in two-thirds of cases. These signs can mimic the indirect, and even the direct signs of malignant tumours.

These signs can appear after the conservative operation in most different combination. The most important task is a possible separation of a new cancer from the skar-phenomena.

In our University until now there have been made about 60 such operations among these the postoperative radiological examinations raised suspicion of a malignant recidive three times, there were 2 really malignant among them.

The Liquid-Cristal-Thermography is a very useful supplementary method for mammography, but neither method could give any direct differential-diagnostic sign.

Author verifies the above said by showing typical radiograms.

Conclusions: The accuracy of diagnostics is increased by close team-work between surgeons and radiologists.

\subsection{AM}

371 The Mama Program - a national breast cancer screening strategy Gisela Gästrin; Helsinki

Mammographic examination is the most sensitive method in the early diagnosis of breast cancer. Different screening strategies have been developed to use this technique in different populations.

Direct screening with mammographic examination of large populations is expensive. Consequently, studies have been established to evaluate the effectiveness of strategies, that combine mammographic examination with either breast physical examination (BPE) or breast self-examination (BSE).

The comprehensive, continuous Mama Program combines initial teaching of the BSE-technique and a surveillance-system of the BSE-habit with a special strategy for self-referral and mammographic examination of women with self-discovered changes of the breasts. The program was developed in Finland and the Mama-study was established in 1973 in co-operation with women's organizations and the Finnish Radiological Society.

The effectiveness of the screening program is evaluated in terms of compliance, incidence, tumor size and stage, survival and mortality from breast cancer in a 13 years follow-up, being the first time published in 1991 . Implementation of the program in Finland as a national breast cancer screening program required limited cost.
Initiatives in different countries can be supported on the basis of experiences from Finland. Material for national programs can be made available.

\section{$11.58 \mathrm{AM}$}

\section{Mammography screening in Finland}

Martti Pamilo, Peter B. Dean, Osmo Räsänen; Helsinki

A nationwide mammography screening program started in Finland in 1987. Screening covers women aged $50-59$ years. The screening started with steadily increasing number of birth-year cohorts so that all cohorts are screened until 1991. Women aged 60 or more can also be included in the screening program. The Health Centers are responsible for the arrangement of the screening and the Central Hospitals are responsible for the quality control. The health centers can do the screening themselves or they can buy the service. There are two major screening organizations based on private practice: The Cancer Society of Finland and a health testing organization owned by several municipalities. Personal invitation letters are sent and two-view mammography technique is used with two year intervals. Interpretation of the mammography screening films is based on double reading of all the films. The radiologists interpreting the screening films are also responsible for all the further studies needed including additional views, coned down views, magnified views, pneumocystography, galactography, ultrasonography and fine needle aspiration. If it is impossible to exclude malignancy with these methods the women are referred for surgery in central hospital. The Finnish Cancer registry is responsible for the evaluation of the screening.

In 198764471,198887035 and 1989129651 women were invited to be screened. The attendance rate was $89 \%$ every year. In $19874.8 \%, 19884.5 \%$ and 1989 $3.3 \%$ of those screened were recalled for further studies and respectively $1.15 \%$, $0.83 \%$ and $0.65 \%$ were referred for surgery. Histology revealed breast cancer in $0.38 \%, 0.36 \%$ and $0.34 \%$ of those screened. The positive predictive value (cancers per operation) was 33\% in $1987,44 \%$ in 1988 and $53 \%$ in 1989 . Most of the screening detected breast cancers have been local, TIS + Stage I tumours, $62.2 \%$ in $1987,64.2 \%$ in 1988 and $63.6 \%$ in 1989 . The number of screening detected breast cancers is 2.5 times the normal breast cancer incidence in this age group.

The results of the fourth screening year will be also presented.

\section{Room R}

\section{SS} $10.30 \mathrm{AM}$

\subsection{Treatment planning in radiotherapy I}

\section{The development of modern treatment planning (Keynote Lecture)} Peter J. D. K. Dawes; Newcastle upon Tyne

The essential nature of ionising radiation beams is to give a greater dose near the surface of the body than at depth. Strategies were rapidly developed to use more than one beam to redress the balance in favour of a high tumour dose. In combination with this, knowledge of tumour spread has greatly improved since 1950. Since 1975 imaging techniques have refined the localisation of tumours. Many imaging techniques are now used in Radiotherapy Departments suitably modified for use on radiotherapy simulators. The challenge of the last decade of the twentieth century is to integrate the newest techniques into departments and use the information with planning systems to improve treatments.

Recording information dynamically, usually by digital methods will allow much more information to be recorded and perhaps analysed later in the light of new developments. Here the challenge is to decide how to organise treatment planning so the appropriate techniques are used, recorded and later analysed to improve results.

\section{$10.50 \mathrm{AM}$}

374 Clinical and organizational aspects of introducing a 3-D dose planning system in the department (Keynote Lecture)

Torgil R. Möller, Inger-Lena Lamm; Lund

Depending on the existing level of sophistication at the department as regards treatment planning, the introduction of a fully 3-D TPS influences departmental activities in various ways.

Much more complete topographical information is needed, i.e. more CT-scans have to be obtained in order to fully utilize the volumetric concept. Almost all working time for active treatment planning by radiotherapists and dosimetrists will be spent in front of the graphics display defining tumour volume, clinical target volume, and planning target volume, as well as the arrangement of beams and the design of blocks, compensators etc. Thus, depending on the number of treatment plans carried out, a sufficient number of work stations must be available.

This change in way of working also puts demands on the treatment planning system itself. The man-machine interface is critical, but has to be a compromise between the demand of being simple to learn and easy to use, which usually also means a restriction of functions, versus flexibility which usually follows 
complexity and a wide range of applications. For the interactive part of treatment planning, including optimization, speed is essential, while for the final dose plan accuracy is important. The system must comply with existing treatment techniques, and it should be possible to taylor e.g. the hardcopy output to the demands of the department.

It is obvious, that a full 3-D plan is not necessary for all patients in the department, but should be applied in specific situations, such as design of new treatment techniques, quality control of existing techniques, and individual dose plans for very complex treatments.

\subsection{AM}

\section{Clinical impact of treatment planning on the daily practice of radiotherapy (Keynote Lecture)}

J.P. Gérard, L. Sentenac, P. Romestaing; Lyon

- Decision to irradiate: The physician defines the aim of treatment (curative or palliative), the volume to be irradiated and the dose. This is the medical prescription.

- Treatment planning: It is a technical interactive process to achieve the prescription with the rational use of available facilities of the department.

- Clinical impact: The relevant end points are: local control, side effect, survival and cure. It is important to assess the role of the different steps of the treatment planning: positioning and immobilisation devices imaging system, beam positioning and shaping, simulator, dosimetric calculation and display (computer, software), control films. When and how these facilities must be used? How sophisticated should they be?

- It is recommended: 1 . to use a common language for prescription, recording and reporting of all the relevant informations (ICRU report). 2. to have a regular quality control program of the different facilities used for treatment planning. 3. to work in close collaboration between Physicians, Physicists, Technicians. All the different parts of the chain are of equal importance. - During treatment daily reproducibility of the irradiation is essential. In vivo dosimetry and on line imaging can be useful to detect a random or systematic error.

\section{$11.30 \mathrm{AM}$}

\section{Postoperative radiotherapy after pneumectomy for lung cancer.} Impact of modern treatment planning

P. Phlips, P. Rocmans, P. Vanderhoeft, P. Van Houtte; Brussels

In our prior randomized trial, postoperative radiation was associated with poor survival after pneumectomy, the technique used was advocated to explain this difference. So, we reviewed our experience over the last years to see the impact of modern treatment facilities.

From 1970 till 1985, 490 consecutive patients were seen in the department; after a surgical resection 126 had a pneumectomy and a complete resection for a T1 T2 NO tumor ( $54 \mathrm{pts}$ ) or a T3 N1 N2 (72 pts). Among the 54 pts with NO tumors, postop radiation was delivered with a Co60 unit (60 Gy in 6 weeks). The 5 year survival rate was $5 \%$ compared to $22 \%$ after surgery alone. In contrast, 19 pts with T3 N1 N2 tumors were treated with CT scan for dosimetry, treatment planning and linear accelerator. A dose of 56 Gy was delivered. The 5 year survival rate reached $22 \%$ compared to only $10 \%$ for a series of patients treated earlier with the Co60 technique described for the NO tumors. This benefit was achieved by reducing the toxicity without decreasing the local control.

So, adequate techniques allow to deliver safely high doses to the mediastinum even after a pneumectomy for lung cancers.

\section{$11.40 \mathrm{AM}$}

\section{Treatment results of the multi-leaf conformation therapy}

Atsuro Terahara, Keiichi Nakagawa, Katsuyuki Karasawa, Yukimasa Aoki, Atsuo Akanuma, Yuzo Onogi, Nobuharu Muta, Kouichi Sakata, Kenji Hasezawa, Yasuhito Sasaki; Tokyo

In 1983, a newly developed linear accelerator treatment unit which is equipped with a set of multi-leaf collimators was installed in our department. Since then up to now, 550 cases has been treated with this unit.

Purpose: Aim of this study is to evaluate clinical availability of the multi-leaf conformation therapy.

Methods and Materials: This study includes multiport irregular field radiotherapy with multi-leaf collimators as well as rotational multi-leaf conformation therapy. The numbers of the cases according to primary sites are as follows: 85 brain tumors, 126 head and neck tumors, 74 lung and mediastinum tumors, 50 bone and soft tissue tumors, etc. The analysis of the results was performed with Kaplan Meier method.

Results: 5 years survival rate of grade III astrocytomas and glioblastomas treated with this method is $45.6 \%, 20.0 \%$ respectively. 27 months survival rate of the lung tumors is $12.7 \%$.

Conclusions: We can deliver the more doses by sparing the adjacent normal tissue with this method than non-conformation therapy with such as paralle opposite beam. The multi-leaf conformation therapy is considered to be a useful method to improve the treatment results of the radiation therapy.

11.50-12.00 AM Discussion

\section{Room S}

\section{SS 12.01 Computer science in radiology - Expert systems and computer-aided diagnosis}

378 Expert systems image analysis and computer-aided diagnosis (Keynote Lecture)

Heber Mac Mahon, Kunio Doi, Maryellen L. Giger, Kenneth Hoffmann, Shigehiko Katsuragawa, Shigeru Sanada; Chicago, IL

Digital radiography provides several practical advantages over conventional systems, such as the ability to retrieve, transmit and duplicate images electronically, without loss of quality. The diagnostic advantages of digital imaging, however, are less certain, except in a few specific applications. Radiologists, who are primarily concerned with making diagnoses accurately and efficiently, have not had a strong incentive to convert to digital radiography systems.

The implementation of computer-aided diagnosis by systems may alter this perception. Until recently, the term computer-aided diagnosis referred to techniques which used relatively simple computer algorithms to assist radiologists or clinicians in decision making, by providing recommended courses of action or differential diagnoses, based on data supplied by the physician. A new generation of computer-aided diagnostic schemes are being developed which involve direct analysis of image data, in order to detect and measure abnormal findings in the radiograph. These include techniques which analyze chest radiographs to detect nodules, interstitial disease, pneumothoraces and cardiac abnormalities; mammograms for masses and microcalcifications; and angiograms for stenoses. In addition to detecting the abnormal findings, these programs can provide additional quantitative information such as the type and intensity of interstitial lung disease, or the extent of a vascular stenosis and its effect on blood flow.

After detection and measurement, additional techniques such as neural network programs have the potential to offer prioritized differential diagnoses based on the nature of the detected findings and the clinical history.

A logical culmination of these various approaches would be a multi-facetted computer based scheme that would be applied to all digital images prior to their final interpretation. The powerful combination of the computer's potential to detect and measure pathology, with the broad experience and clinical insight of the radiologist, promises to offer a decisive advantage over conventional radiography. Thus, computer-aided diagnosis may serve as a critical catalyst for the widespread implementation of digital radiography.

\section{$10.45 \mathrm{AM}$}

379 Neures: computer assisted diagnosis of CT-images with analytic and neuronal methods

G. Zöllner, A. Wackenheim, J. F. Rapp, L. Steib, J. L. Dietemann; Strasbourg

AIM: create a framework for computer assisted diagnosis of 2D-CAT-scan images of the spine.

Methods: combining a module for image analysis, based on analytical techniques, a neuronal network module and a rule-based expert program. After automated pre-segmentation of images with an unsupervised-learning clustering algorithm, the following image classes are created: bone, vascular, muscles, canal and fat. Axial images are selected for further analysis with respect to their anatomic position by a neuronal network trained with a supervised learning procedure. Further analysis is guided by an expert system which, dependent upon the anatomical localisation of the slices and the results obtained by specific analysis, choses the steps to execute. For diagnoses, where defined parameter sets exist, an analytic parameter extraction method is used (example: spinal stenosis with canal diameter or surface: identification of the vertebral canal, measures of the diameters or the surface). The parameters coming from the image analysis module are evaluated by an expert system. For diagnosis where shape recognition plays an important role, shape recognition is done by analytical methods or a neuronal network, trained with pathological images.

Results: Recognition of anatomic structures: $90 \%$. Spinal stenosis found corresponding to the radiologists definition.

Conclusions: Within a user-friendly environment based on XWINDOWS a framework for computer assisted diagnosis of the spine has been developped, which allows (without user interaction) to identify anatomic structures and spinal stenosis. 
$10.55 \mathrm{AM}$

380 Applications of neural networks to diagnostic radiology V. Cavallo, F. Giovagnorio, D. Messineo; Roma

Neural network try to simulate on a computer the biological neural system, according to the connectionistic theory.

They are made up of nodes (neurons) and connections (synapses).

The first ones are the fundamental units, usually organized on three layers: input, hidden and output layers. The connections have inhibitory and excitatory capabilities, because of different weights given to them during the training.

The proposed system is made with a shell from California Scientific Software, called BrainMaker Professional, which was employed to build a diagnostic helper system for hepatic cirrhosis with sonographic and laboratory data.

The net is organized on three layers, an external one with 21 inputs, an hidden and an output external one.

The preliminary phase consisted in training the system with typical features of healthy and cirrhotic subjects, utilizing 136 cases divided in 68 cirrhotics and 68 normals.

The adjustment and validation of system needed the employment of randomized group of cases; the accuracy of final diagnosis has been checked out.

The interest aroused from the results will cause a development of the network based on the analysis of a winder group of cases and the introduction of differential diagnosis possibily.

$11.05 \mathrm{AM}$

381 An expert system for microcomputer-aided diagnosis of the prostatic transrectal ultrasonotomography

C. Oton, M. Pastor, J. Fernandez-Ramos, L. F. Oton, J. Hernandez-Armas; Tenerife

Expert systems and other artificial intelligence techniques have been for a long time, a field reserved for big computers. The revolutionary changes in Personal Computers capabilities in the last years have opened this new doorway and their use is now quickly extending to many medical specialities.

To make an expert system actually useful, we think it should not try to replace the human expert but to collaborate with him, discussing the cases like another specialist in a clinical session. The expert system has the inconvenience of the lack of "common sense" but the advantage of an unbeatable memory.

We have made a quantitative analysis of the signs of 430 transrectal ultrasonotomographies with proved diagnosis, to prepare a base of objective data to teach an expert system the probability of each diagnosis with different sonographic signs.

We present a tested Investigation Prototype of an expert system to aid the radiologist in the diagnosis of the transrectal ultrasonography of the prostate that can work on a 80386 PC under a LISP-based expert system shell named "GOLDWORKS II". Our program includes advice and bibliography in connection with the particular case and explanations of the reasons why he has reached this conclusions.

\subsection{AM}

382 User-friendly electronic film library for $\mathrm{CT}$, MRI, and ultrasound David Gur, Walter F. Good, Catherine M. Burzik, Paul J. Scanlon, Glenn S. Maitz; Pittsburgh, PA

A fast, easy-to-use, manufacturer-nonspecific archival display and filming system was assembled and tested in the clinical environment. The system allows CT, MRI, or ultrasound operators to film (laser print - Kodak Ektascan) procedures as they would under normal conditions and at the same speed $(\sim 0.7 \mathrm{sec} /$ image $)$. In this system, optical character recognition software decodes patient identification information and the type of examination and builds a directory for the archival database in a manner which is transparent to the operator (no dual entry required). Images are stored on an optical disk jukebox (Kodak ODL6800) and can be retrieved to soft display for review and/or reprinting. The system can be easily attached to multiple devices (currently three) that are manufactured by the same or different vendors. Preliminary clinical evaluations ( $>500$ cases) clearly indicate that such a concept can successfully replace and/or serve as a backup to conventional film libraries. Technologist and physician responses to the system have been favorable.
SS

12.01 Computer Science in Radiology - PACS I

$11.20 \mathrm{AM}$ 383 A second generation PACS concept (Keynote Lecture)
M. Osteaux; Brussels

The first generation PACS realisation. Why they failed to meet the clinical requirements

The first generation PACS implementations, as developed in the last decade, were mostly technologically driven. Most proved technically quite successful, but it is however generally accepted that they failed to demonstrate a real utility in the clinical environment and were not cost effective. This failure is partially due to: the lack of maturity of the technical tools (storage, network, digital radiology), the absence of recognized standards and some misconceptions in the systems themselves.

The New Generation-Medically driven-PACS concept

A synthesis between the knowledge acquired from the first implementations, the progress of the available technologies, and the cooperative work undertaken by the partners of our research consortium or innovative general concept of the future of PACS can be presented as an integrated but decentralized structure, where small or medium size units are specialized to suit the requirements of specific clusters of users in the hospital.

Characteristics: medically driven to suit the needs of the different cluster of users. Namely: an adaptive user interface, options for modular architecture (specialized small systems, integrated as a logical whole with a distributed network); "open" architecture, multivendor environment, making use of recognized standards and a maximum use of digital interfaces; architecture allowing progressive and phased approach; upgradability; distributed data base and/or multiple local temporary store with prefetching preloading capability; integration with radiological and/or hospital information system inclusive provision for prefetching and preloading strategies; knowledge based networking allowing an intelligent distribution of the image within a distributed network.

\subsection{AM}

\section{The Vienna SMZO PACS project}

Walter Hruby, Hans Mosser, Alfred Mandl, Michael Urban, Helmut Hradil; Vienna

Purpose: This paper presents the implications of the decision to implement a totally digital radiology department of a new built 1400-bed teaching hospital, opening in March 1992 in Vienna, Austria. There will also be outlined the concept of implementing a total PACS integrated into a radiology- and hospital image and information management system with the inherent goal of replacing the current film based methods.

Methods and Materials: System overview:

+ FDDI backbone (100 Mbits/second)

+ decentralized archiving

+8 modules for image acquisition and/or display

+ Direct connection of archives and workstations with FDDI backbone

+ standardized components: ACR-NEMA standards/SPI-specification

+ Multifunctional workstations for primary diagnosis with interactive manipulation possibilities

+ Numerous remote viewing stations on PC-basis

Conclusions: In planning the radiology department of a brand new hospital the rapid technological advances have to be considered. Since almost all diagnostic modalities will be digital, including digital radiography and -fluoroscopy, the operational structure and design of a total PACS was planned in detail by a team of computer engineers and radiologists. The participation of the radiologist - the final user of this system - as a prerequisite of a successful performance is emphasized in this paper.

\section{$11.45 \mathrm{AM}$}

\section{The PACS project at the University of Florence}

Davide Caramella, Raffaella De Dominicis; Pisa

The PACS related research started at the University of Florence in 1988, prompted also by the participation of our University to two European Projects focused on digital image transmission (RACE - TELEMED and AIM PACS-IMACS).

Furthermore our cooperative effort in building an image database, within the framework of the TELEMED Project, allowed us to approach the issues of image capture, image file format, database architecture and query languages. From these experiences the actual PACS Project has now come to the implementation phase: the main feature of our installation is a backbone able to support the IEEE 802.6 DQDB protocol. This high performance protocol is going to be adopted on the Florence Wide Area Network (WAN), to which the University is connected, and will be able to handle the heavy traffic due to the diffusion of diagnostic images among many users in the metropolitan area. 


\section{PACS Trieste project: phase 2}

L. Dalla Palma, F. Stacul, P. Giribona, W. Ukovich, P. Cortivo, J. Moda; Trieste

Purpose: The goal of the project is the implementation of a large area metropolitan PACS connecting the four main Public Hospitals of Trieste and some Remote Health Centers to a central archive.

Methods and Materials: Since September 1988 a PACS (CommView by AT\&T and Philips) has been operating in the Radiology Department of the University Hospital, Trieste. In the first phase of the project the system was evaluated as far as operational, technical, clinical and economic aspects are concerned. The system offered definite advantages (quicker and more efficient retrieval of old examinations, reduction of archive size and of devoted personnel, more efficient communication within the Radiology Department), but several relevant limits were faced as well. In the second phase of the project the effects of the connection via optical fiber with a system located in another hospital 6 $\mathrm{km}$ away were assessed.

Results: A central archive collected images coming from the two hospitals and allowed a direct access to images from each Radiology Department. Data of a qualitative and quantitative analysis are available, including evaluation of frequency of retrievals, retrieval times, archive size, required resources and impact on patient management and teaching. 


\section{Room A}

\section{HL}

The Other Dimension

387 Adaptation deficiency of human reason $\overline{R u p e r t ~ R i e d l ; ~ V i e n n a ~}$

On the basis of the evolutionary theory of knowledge developed by Konrad Lorenz, biologists today view evolution as a knowledge-acquiring process. From this perspective there emerges an epistemological dilemma stemming from our insight into the origin of the preconditions of human reason. The aprioris of our reason prove to be genetically acquired adaptation, the a posteriori adaptation products of our species.

Genetic adaptation, however, has been overrun by cultural development. As a result, those inborn decision aids which evolved by selection to cope with the vastly simpler life problems of fish or apes no longer prove equal to the complexity of today's problems. This, in fact, has taken place within our industrialized, sociocapitalistic, "success-oriented society".

These adaptation deficiencies are the subject of my speech. They affect both our senses and our mechanisms of knowledge.

With regard to our senses, it is evident that we perceive a one-dimensional time and a three-dimension space, although we have known since Einstein that we live in a four-dimensional space-time continuum. The simple solution conveyed by our senses is sufficient for the medium-sized cosmos in which we live. It is only on the very large and very small scales that our expectations lose their validity. This example also shows that we are able to overcome the deficiencies of our adaptation through experiment and experience. Although we cannot change our senses, we can alter our insight.

This problem does not unduly affect us, because it involves different dimensions. However the following approaches are dimensionless and thus also affect us here on earth.

First, there is the way in which we believe we approach truth. We act as though confirmation of a prognosis makes the occurrence of the subsequent prognosis more probable. Such a process is logically unfounded.

Second, there is the way in which we appraise quantities and qualities. We act as though we can ignore the nonidentical in the identical but add the imagined identical. In conjunction with our definitional form of logic, the result is that we are unable to understand the origin of new qualities. We do not even notice that quantitative changes alone must give rise to new qualities.

Third, there is the way in which we approach causality. We assume that identical things must necessarily have the same cause. However, in doing so, we apply a simplification in which we assume the existence of linear relationships as well as beginnings and ends of cause-effect chains, neither of which exists in this world.

Fourth, we experience those subsystems or subactions to be purposeful which contribute to the realization or preservation of higher-level systems or actions, but only to the extent that we believe we see ourselves with our personal conditions and concerns reflected in them. Our comprehension does not take into account purposes that go beyond these bounds. It is also for this reason that we fail to understand our inescapable function in the biosphere.

In my speech I hope to uncover these deficiencies, explain their original successes and show how we have reached a state where we can and must overcome these deficiencies through experience, if only to preserve our species. 
Conclusions: The use of these additions to the basic inflow angiography methods clearly enhance contrast resolution of MR angiograms in up till now "difficult" anatomical areas.

References: 1. Groen J. P., de Graaf R. G., van Dijk P., Abstracts SMRM 1988, p906 2. de Graaf R. G., Groen J. P., Abstracts SMRM 1990, p499.

\section{Room A}

\section{SS}

\subsection{Flow, angio}

$2.00 \mathrm{PM}$

388 Optimized phase-contrast MR-angiography: Implementation and clinical results

Richard Hausmann, Gerhard Laub; Erlangen

Purpose: Two different techniques are presently used for magnetic resonance angiography (MRA): time-of-flight and phase-contrast techniques. Both have their advantages and drawbacks. Despite its advantages like complete background suppression and variable flow sensitivity, phase contrast angiography suffers from very long acquisition times. A new and faster acquisition scheme for phase-contrast MR angiograms was developed and tested on volunteers as well as selected patients.

Methods: The new sequence scheme combines one flow-compensated and three-flow-encoded acquisitions. This leads to four measurements per slice instead of 6 in the standard phase contrast method. Taking the complex difference of each flow-encoded data set from the flow-compensated set cancels stationary tissue signal. The flow-compensated part of the data set can be viewed as a usual time-of-flight angiogram. Optimization was performed on a standard 1.5 T MAGNETOM with volunteers and selected patients.

Results: We applied the method to the intracranial vasculature using different flow sensitivity ranges from $20 \mathrm{~cm} / \mathrm{sec}$ up to $100 \mathrm{~cm} / \mathrm{sec}$. Both $2 \mathrm{D}$ and $3 \mathrm{D}$ images show good vessel delineation and complete background suppression. Typical scan time for a full 3D data set with 64 partitions and $1 \mathrm{~mm}$ inplane resolution is approximately $20 \mathrm{~min}$.

Conclusions: This method respresents a promising approach towards making phase-contrast angiography more clinically useful by decreasing its scan time by $33 \%$.

\subsection{PM}

389 Magnetic resonance (MR) angiography and blood flow measurements in patients with peripheral arterial occlusive disease before and after percutaneous transluminal angioplasty (PTA)

Manola Koch, S. E. Maier, C. von Weymarn, A. Bollinger, G. K. von Schulthess; Berlin, Zürich

Purpose: MR angiography together with quantitative blood flow measurements provides both morphologic and functional information about the arterial system. Measurements performed before and after PTA of arterial lesions allow a quantification of the therapeutic results.

Methods: So far 23 patients (aged between 45 and 80 years) with peripheral arterial disease stage II-III of the femoral and iliac arteries have been examined before and after PTA. Multiple slice inflow angiography with three dimensional reconstruction of the arterial tree was performed on a Philips S 15 1.5 $\mathrm{T}$ scanner. The velocity dependent phase shift in images of an ECG-triggered multiple phase acquisition in planes orthogonal to the vessel axis was used to determine the temporal flow rate proximal and distal to the stenoses.

Results: The angiograms prove a successful therapy in 11 cases, an improvement for 9 patients and an indifferent state in 3 cases. Normalization of the mean flow rate and the pulsatility of the blood flow is visualized in temporal flow rate curves.

Conclusions: MR angiography is a new non invasive method to study the morphology in diseased vessels. Quantification of blood velocity and flow rate at different instants of the heart cycle opens a new possibility to quantify the severity of vascular lesions and the efficiency of their treatment.

\subsection{PM}

\section{Improvements in MR inflow angiography}

P. van Dijk, R. G. de Graaf, J.P. Groen, J. Dam, M. S. Silver; Best

Purpose: Flow pulsatility and vessel or body motion lead to loss of contrast and definition in arteries in some parts of the body, imaged with $2 \mathrm{D}$ or $3 \mathrm{D}$ inflow MR angiography (1). Also unsaturated fat may obscure some vessels when using a maximum intensity projection algorithm. The purpose of this study was to devise methods to overcome these problems.

Methods and results: Reduction of pulsatility and motion artifacts was obtained by synchronizing data acquisition to ECG with a special gating strategy (2), while FastScan (subsecond imaging) was used to make breathhold scans in the abdominal region. In combination with fat suppression techniques angiograms of high quality were produced with surface coils on both $0,5 \mathrm{~T}$ and

\subsection{PM}

3913 dimensional phase sensitive MR angiography with $0.3 \mathrm{~T}$ vertical field MRI

Shigeru Watanabe, Hiroshi Nishimura and Shinji Kawasaki; Chiba

Purpose: Our main purpose is to develop 3 dimensional MR angiography for better visualization of blood vessels with $0.3 \mathrm{~T}$ vertical field MRI system, and to evaluate the detectability of small aneurysm, arteriovenous malformation and stenosis of arteries.

Methods: We have developed 3 dimensional phase sensitive MR angiography obtained by sequence subtraction of dephasing gradient echo from rephasing (velocity-compensated) one. After reconstruction of phase-corrected complex subtraction, MR angiograms were obtained with a ray tracing of the new modified maximum intensity projection at various viewing angles. These images are displayed on CRT in cyclic mode to depict the front-rear relation of vessels for recognition.

Results: With a repetition time (TR) of $40-100 \mathrm{msec}$, a TE of $15 \mathrm{msec}$, the two volumetric images may be acquired. The slab thickness for 33 partitions varied from $32 \mathrm{~mm}$ to $128 \mathrm{~mm}$, resulting in an effective section thickness of $1.0 \mathrm{~mm}$ to $4.0 \mathrm{~mm}$. There was improved delineation of mid cerebral artery branches and anterior cerebral arteries at low flip angle.

Conclusions: Even if we have used 0.3 T MRI system, our phase sensitive MR angiography provides an excellent method to visualize intracranial vessels. The new modified MIP can improve the front-rear recognition of vessels in 3 dimensional display without any illusion.

\section{$2.40 \mathrm{PM}$}

\section{MR-angiography of flow disturbances in peripheral arteries} B. Krug, H. Kugel, G. Friedmann, J. Bunke, P. van Dijk, W. Groß-Fengels, R. Schmidt; Köln

Purpose: Poststenotic flow phenomena were studied experimentally (a) and clinically (b) on a 1.5 Tesla MR-imager (Gyroscan S 15, Philips), in order to investigate information contained in signal loss along flow direction.

Methods: a) Concentric stenoses in Tygon tubes (range $28 \%$ to $96 \%$ ) were examined with different flow rates between 0 to $20 \mathrm{~cm}$ per second. SE-(TR $500 \mathrm{~ms}$, TE $27 \mathrm{~ms}$ ) and GRE-sequences (TR $60 \mathrm{~ms}$, TE $27 \mathrm{~ms}, \mathrm{FA} 15^{\circ}$ ) were used, modulus and phase images being reconstructed from each data set. The length of the poststenotic flow void was determined to analyse the influence of stenosis grade and flow rate.

b) Patients with flow disturbances of the lower limb proven by conventional angiography were further investigated by MR-angiography (MRA) using 2D-inflow and phase contrast techniques.

Results: a) The length of the poststenotic flow void increased with the extent of the stenosis and the flow rate. The correlation of modulus and phase images allowed the differentiation of turbulent and nonturbulant stenotic flow.

b) Clinically significant stenoses were accurately diagnosed by MRA, while stenoses less than $50 \%$ were occasionally missed. Phase contrast techniques allowed the interpretation of some artifacts observed in time-of-flight MRA. Conclusions: MRA is an useful, non-invasive method for evaluating peripheral arterial disease.

\section{$2.50 \mathrm{PM}$}

\section{Real-time interactive color flow MRI}

S. J. Riederer, R. L. Ehman, R. C. Wright, A. E. Holsinger, R. C. Grimm, H. W. Korin, D. G. Brown; Rochester, MN

Purpose: The purpose of this work was to investigate the feasibility of performing vascular magnetic resonance (MR) imaging using real-time techniques. Magnitude information was to be displayed in gray scale and directionality and velocity of flow superimposed in color.

Methods: Flow compensated (FC) and flow encoded (FE) images were continuously acquired using ultra-short TR $(\sim 11 \mathrm{msec})$ data acquisition. Both images were immediately reconstructed using a MR fluoroscopy apparatus. Each magnitude FC image was displayed in gray scale and the phase difference between FC and FE images converted into a color overlay. The cycle time for one complete image was about $3 \mathrm{sec}$. The pulse sequence was modified so that many scan parameters were alterable in real time.

Results: Preliminary results in phantoms demonstrate the ability of the technique to image flow at physiological rates and to interactively adjust slice position, slice orientation, and directionality of flow encoding. In vivo results demonstrate the ability to image vasculature in the head, neck, thorax, and abdomen. 
Conclusions: Initial results are promising and we conclude that real-time interactive vascular imaging with MR is feasible.

\subsection{PM}

394 Analysis of the abdominal aortic flow using MR phase-shift velocity mapping

Makoto Amanuma, Makoto Hasegawa, Raad H. Mohiaddin, Atsuko Heshiki, Donald B. Longmore; Saitama

Purpose: To characterize blood flow and its regional distribution in the abdominal aorta using magnetic resonance phase mapping technique.

Methods: Ten normal subjects were examined with a 0.5-T superconducting system. Field Even Echo Rephasing (FEER) technique with and without velocity encoding was used to process transverse cine velocity maps at three different levels of the descending aorta. Aortic flow at three levels were measured and flow in the celiac trunk, superior mesenteric artery, and renal arteries were also calculated. Aortic dimention, distensibility, and flow pattern were analyzed.

Results: Total blood flow in the abdominal aorta above the celiac trunk and below the renal arteries were $4094 \pm 1600$ and $1602 \pm 549 \mathrm{ml} / \mathrm{min}$, respectively. The net aortic flow above the celiac trunk was persistently forward during the entire cardiac cycle while there was considerable back flow (13\% of total instantaneous flow) in the aorta below the renal arteries during early diastole. While there was a $30 \%$ reduction in cross sectional area between these two levels, wall distensibility shows no statistically significant difference.

Conclusions: MRI is a suitable technique for qualitative and quantitative assessment of blood flow in the abdominal aorta and its main branches.

\subsection{PM}

\section{MR angiography of renal arteries: optimization of a 3D sequence} in normal volunteers and patients

P. Loubeyre, P. Garcia, D. Revel, C. Porcher, M. Amiel; Lyon

The goal of this study was to optimize that different parameters of a 3D fast sequence designed for MR angiography of renal artery.

Methods: 10 normal volunteers and 5 patients suspected of renal artery stenosis were studied on a $1.5 \mathrm{~T}$ magneton (SIEMENS) using a 3D FISP sequence focused on their renal arteries. This sequence was based on the time of flight phenomenon and images reconstructed with the maximum intensity projection mode.

Different parameters of the sequence: TR, flip angle, partition number and sampling volume, field of view and average number have been varied one by one in the same subject. The imaging data were judged by two independant observers in order to evaluate the image quality using a scale technique.

Results: After a consensus between the 2 observers, the more adapted sequence should include a $30^{\circ}$ pulse angle, with a TR of 30 to $40 \mathrm{msec}, 2$ averages, using a FOV of $250 \mathrm{mn}$ for a $40 \mathrm{mn}$ imaging volume with 32 partitions for a 8 min acquisition time.

In 5 patients, the MR data were correlated with renal artery DSA with IV injection of $\mathrm{CM}$.

Conclusions: The origin and the first centimeters of both renal artery were precisely evaluated using this non-invasive imaging modality, allowing to propose it as a screening method for the assessment of hypertension.

\subsection{PM}

\section{Comparison of MR-angiography and DSA in the evaluation of vascular diseases of the hand}

D. Hahn, L. Ziegler, Th. Vogl, H. Schedel, G. Küffer, F. Spengel; Munich

A prospective study including 30 patients with different vascular diseases of the hand was performed. The aim of the study was to evaluate if the MR-angiography is able to replace the intraartertial DSA.

Different MR-angiography-(MRA)techniques for demonstrating arterial anatomy and pathology of the hand were compared with a high resolution digital subtraction angiography (DSA). Vascular imaging was performed with a 1.5 T Magnetom using flow related and flow enhanced techniques. Processing was done by multiple intensity projection (MIP). DSA was performed by a transbrachial approach with a $1024 \times 1024$ matrix.

The most suited MRA sequence was a FISP 3D gradient echo sequence with a flip angle of $20^{\circ}$ and flow related acquisition sensitive to slow flow. Venous presaturation was not needed.

MRA allowed a comparable imaging of pathological changings in comparison to DSA in over $90 \%$ of the cases. MRA as a non-invasive technique can replace DSA in many cases. Especially in patients with thrombangitis MRA seems to be the method of choice.

\section{Room B}

SS $2.00 \mathrm{PM}$

\subsection{Infectious disease of the lung}

397 The role of conventional chest radiology in the management of Pneumocystis carinii Pneumonia in AIDS-related patients

B. Gómez Ansón, J. Albillos Merino, J. Ferrando Alvarez-C., R. Rubio Garcia, J. Manrique Chico; Madrid

Purpose: Our aim was that of analyzing the role of conventional chest radiology in diagnosis, evolution prior and after treatment, and prognosis in AIDS-patients with PCP.

Methods: Clinical records and chest films of 25 AIDS-patients with PCP were reviewed. Data were stored and statistically analyzed by means of the Sigma software and a PC AT-286.

Results: Average time between clinical and radiological manifestations was 3 to 6 weeks. 24 patients $(96 \%)$ showed radiological manifestations at admittance. Evolution prior to treatment was as follows: $5(27,8 \%)$ towards parenchymal consolidation; $4(22,2 \%)$ improved; $9(50 \%)$ showed no changes. After treatment, $13(59,1 \%)$ resolved; $1(4,5 \%)$ evoluted towards fibrosis; $2(9 \%)$ developed ADRS; and $6(24 \%)$ did not change. $5(20 \%)$ patients, who died, did not show any specific radiological features.

Conclusions: 1. Radiology is not useful for early diagnosis.

2. Radiology is useful for assessing diagnosis, having a great sensibility (96\%), but little specificity.

3. Radiology may serve as an evolution control prior and after treatment, having a good correlation with clinical changes.

4. No radiological features can be used as prognostic factors.

\subsection{PM}

398 HR-CT of interstitial pneumonias in immunocompromised patients P. Uhrmeister, F. Astinet, M. Langer, C. Zwicker, N. Schönfeldt, R. Felix; Berlin

Purpose: This study was performed to evaluate the higher spatial resolution of HR-CT in diagnosis of interstitial pneumonia by patients being immunocompromised.

Methods and Materials: 31 Patients with known immunodeficit were examined by HR-CT. The thickness of the slices, performed in five predefined locations, was $1 \mathrm{~mm}$ with two seconds cycle time. The scans were compared with corresponding standard-CT and chest radiographies.

Results: In comparison the HR-CT was the most sensitive method in diagnosis of interstitial pneumonia with a sensitivity of $92,5 \%$ respectively $77 \%$ of standard-CT and $69 \%$ of chest radiography. Furthermore pleural changes were recognized best by HR-CT. In analogy to pathological studies it was possible to visualize the mode of extension of interstitial pneumonias from the bronchovascular bundle to the alveolar walls and the interstitial tissue in the secondary pulmonary lobe by HR-CT.

Conclusions: HR-CT improves the diagnosis accuracy of interstitial pneumonias by immunocompromised patients. The high spatial resolution enables to describe the mode of extension in analogy to pathological studies.

\subsection{PM}

\section{CT findings in tuberculosis of the lung}

U. Lörcher, J.Peters, R. Buhl; Frankfurt/Main

Purpose: Tuberculosis should be considered in differential diagnosis of parenchymal lung disease if CT is performed.

Methods: 42 patients with proven tuberculosis were examined by HRCT. One half of the patients were immundepressed by malignancies, chemotherapy, steroids or HIV infection.

Results: 40 patients showed a typical miliar distribution of small nodules or nodular infiltrates along the broncho-vascular bundle. We found cavities in 21 patients, segmental or lobular infiltrates in 10 , a diagonal profusion in 5 , pleural effusion in 4 and pericardial effusion in 3 cases. Mediastinal lymph nodes were found in all patients.

Conclusions: HRCT shows characteristic signs in pulmonary tuberculosis which make differential diagnosis easier.

\subsection{PM}

400 Pulmonary tuberculosis: radiological differences between

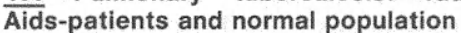

M. Torres, M. Macias, P.Valdes, J.Sanchez, J. M. Antunez, F. Serrano; Malaga

Purpose: Since the first cases of Human Immunodeficiency Virus infection were described at the beginning of the last decade, not only have the frequency and clinical and radiological patterns of pulmonary opportunistic infections 
changed, but also those produced by common agents such as Mycobacterium Tuberculosis. The aim of this work, was to compare the thoracic patterns of TBC in the general population and HIV (+) patients, as well as determine the value of radiology in the identification of these.

Methods: We studied a series of 291 patients with TBC, 241 HIV (-) and 50 HIV $(+)(65 \%$ intravenous drug abusers), in whom chest $x$-ray was performed on admission and evolutionary controls. $42 \%$ of the HIV $(+)$ cases were discovered as a result of these initial tests and $8 \%$ had no known risk factor. Results: The most common radiological patterns were, in HIV (-) bronchogenic disemination $49 \%$, pleural effusion $23 \%$, nodules $14 \%$, adenopathies $6 \%$, micronodular $3 \%$ and others $25.7 \%$. In HIV (+) were micronodular $52 \%$, adenopathies $13 \%$, pleural effusion $11 \%$ and others $24 \%$.

Conclusions: These results lead us to believe that the micronodular pattern with no other known causes of immunosuppression obliges us to determine HIV markers, whether or not there are associated risk factors, since, according to the literature, this pattern reflects the poor immunological response in HIV positive patients.

\subsection{PM}

\section{Respiratory complications of varicella in children}

Jose M. Llerena, Marcos Herrera, Vicente Garcia-Medina, Juan D. Berna; Minneapolis, MN

Purpose: To evaluate the radiographic findings of respiratory complications of varicella among immunocompetent and immunodeficient children.

Methods: We retrospectively reviewed the charts and chest X-rays of 26 children hospitalized because of respiratory complications of varicella. Nineteen $(73 \%)$ patients were healthy at the time of acquiring varicella and the remaining seven $(17 \%)$ had an underlying immunodeficiency (malignancyimmunosuppressive therapy in 3 cases and moderate-severe desnutrition in 4). Results: There was no significant difference in the mean age (2.5 years) of immunocompetent and immunodeficient children. Chest X-rays showed a multifocal bronchopneumonic pattern in $18(69.2 \%)$ children, lobar pneumonia in $5(19.2 \%)$, interstitial pneumonitis in $2(7.7 \%)$ and diffuse miliary nodules in $1(7.7 \%)$. The interstitial and micronodular patterns were only seen among immunodeficient patients. Positive cultures were found in $17(65.4 \%)$ cases, with predominance of Gram positive bacteria. Fifteen $(57.7 \%)$ of the patients died; mortality among immunodeficient children $(85.7 \%)$ was higher than that seen among immunocompetent patients $(52.6 \%)$.

Conclusions: There was predominance of bacterial infections and bronchopneumonic of lobar pneumonic patterns among children with respiratory complications of varicella. Interstitial and micronodular patterns, usually seen in viral infection, were uncommon and occurred only among immunodeficient patients. Respiratory complications of varicella were highly fatal mainly among immunodeficient patients.

\subsection{PM}

\section{Diagnosis of PCP, chest radiography versus computed tomography scan}

V. Jacobi, I. Harth, R. Mulert-Ernst, J. Berkefeld, Th. Meyer, Th. Labuda, H. Fuchs, S. Staszewski; Frankfurt/Main

From 1982 to 1990 in the university hospital of Frankfurt PCP was found in 247 $(42 \%)$ out of 547 HIV-infected patients as first manifestation of AIDS. Chest radiography is of considerable importance in the diagnosis of $\mathrm{PCP}$, the most common first manifestation of AIDS.

The first visible radiological signs of PCP are relatively elevated hemidiaphragms. The basic examination of an HIV-infected patient therefore should include chest radiography for later comparisons. Also in the early state of PCP there is found a so-called ground glass pattern. In patients who have not been treated thickened septal lines are seen.

The typical ground glass pattern is bilateral and symmetric, predominantly seen in the middle and lower zone, peripheral zones are left out.

Often a computed tomograph scan is necessary for the diagnosis of PCP whereby a similar pattern is seen, i.e. a bilateral ground glass pattern leaving out segments and subsegments. By this other infections imitating a PCP can be excluded.

\section{$2.53 \mathrm{PM}$}

403 Pneumococcal infections in HIV-infected patients, different radiological findings
V. Jacobi, I. Harth
R. Mulert-Ernst,
H. Fuchs,
G. Just-Nübling,

S. Staszewski, S. Rehmet; Frankfurt/Main

From January to December 1990280 HIV-infected patients with pulmonary diseases were studied. Diagnostic criteria included a chest radiography and bacteriological cultures from induced sputum. Additional computed tomography scans of the chest were conducted in 100 patients. In $10 \%$ of all cases PNEUMOCOCCI could be cultured from the induced sputum.

Radiographic findings were different. A typical lobular pneumonia could only be found in 1 patient.

$25 \%$ of the radiographic findings showed a typical, PCP-like pattern. In computed tomography scans of these patients a nodular interstitial pattern was found as diagnosed in case of atypical PCP. Further studies should be conducted to find out whether this pattern is caused by PNEUMOCYSTS CARINII or by PNEUMOCOCCI

\subsection{PM}

404 Role of CT in the diagnosis and evolution of pulmonary mycosis in the immunocompromised patient

Elisabetta Tola, Sonia Biselli, Marisa Giudice, M. Cristina Martina; Daniele Regge, Giovanni Gandini; Torino

Diagnosis and evolution of fungal infection is of extreme importance in the immunocompromised host, especially in patients with acute leukemia, with pharmacologically induced granulocytopenia and in patients submitted to renal transplantation with $\mathrm{T}$ lymphocite depletion. In both cases treatment must be interrupted until complete regression of infection.

We review our series of 11 patients with biopsy proven pulmonary mycosis. All patients have performed at least 3 high resolution CT examinations in order to evaluate regression and eventually complete healing of the lesions before continuing therapy.

\subsection{PM}

\section{Pulmonary tuberculosis in the developing countries} Giuliano Bacheca; Viterbo

The author worked as radiologist in the italian Health Cooperation's Programme in the North Uganda. He points out that TB is still one of the major health problems of this country because of its high incidence, morbidity and mortality. The growing spread of AIDS also terribly worsened the present situation. The firststep of the anti-TB campaign in all the tropical countries is the improvement of the low living conditions together with BCG vaccination. Moreover the diagnostic goal is the finding the TB open forms by both bacteriological examination and the chest X-ray. The treatment follows by 3 -drugs chemotherapy. The radiological features are described, emphasizing the high frequence of the florid forms due to a less resistance. TB frequently produces a picture of bilateral bronchopneumonia or segmental pneumonia SUBSPECIALTY with patchy consolidation and cavitation associated with enlarged lymphnodes.

\subsection{PM}

\section{The use of thoracic needle biopsy in the diagnosis of pulmonary infections}

D. J. Conces, R. L. Schuen, D. D. Davidson; Indianapolis, IN

To evaluate the usefulness of thoracic needle biopsy in the diagnosis of pulmonary infections, 733 biopsies were reviewed. Biopsies were performed using a team approach which included both a radiologist and a cytopathologist.

The primary differential diagnosis prior to biopsy was cancer in 641 patients and infection in 92 patients. Of those with suspected infections, 71 were immunocompromised. In patients with suspected infection a specific organism was identified in 47 cases. A specific benign noninfectious diagnosis was made in 8 cases, including pulmonary infarct (3), lipoid pneumonia (2), hematoma (1), hamartoma (1), and radiation change (1). Malignant disease was identified in 5 patients; lymphoma (3), bronchoalveolar carcinoma (1), and chronic lymphocytic leukemia (1). Biopsies in 26 patients provided nonspecific results. Biopsy material in 6 patients was so scant as to be deemed inadequate.

Pneumothorax was the most common complication of the procedure occurring in 14 patients. These were observed in 10 patients with 4 patients requiring placement of a small caliber chest tube. Even though many of the patients were thrombocytopenic, hemoptysis occurred in only 5 patients. In all cases it was self limited.

We conclude that needle biopsy is a useful technique in the evaluation of patients with suspected pneumonia. It is especially useful in the immunocompromised patient in whom the identification of a specific organism is critical.

\subsection{PM}

407 Imaging-guided percutaneous catheter drainage of thoracic fluid collections

M. Castrucci, R. Mellone, G. B. Minio Paluello, A. DelMaschio; Milan

Purpose: pleural and pulmonary collections can be treated in selected cases with imaging-guided percutaneous drainage (PTCD) using small catheters as an alternative to conventional chest-tube or surgery.

Methods: 32 patients underwent PTCD for lung abscesses (5), pleural effusion (5) or empyema (8), pneumothorax (8), hemothorax (3), post-surgical pio/idro-pneumothorax (3). At the beginning, PTCD were used after failure of 
thoracostomy-tube ( 5 cases). The PTCD was performed by CT-guidance, in not extended lesions; US-guidance was preferred for pleural effusions. 8 to 14 Fr. catheters were used, inserted mostly with Seldinger technique. The Trocar technique was performed in pneumothorax.

Results: PTCD was curative in 28/32 patients; in 4/32 patients, we obtained resolution of symptoms without cure (mesotelioma (1), bullous emphysema (1), large bronchopleural fistula in neoplasm (1), leakage of main bronchus post-pneumonectomy (1)). In our series there were no major complications and no conventional chest-tube, after PTCD, was required.

Conclusions: the percutaneous thoracic drainage imaging-guided procedures were effective in thoracic fluid collections. The use of small diameter (8-14 Fr.) drainage catheters, with Seldinger technique, is therefore proposed as the first choice for treatment of thoracic collections, representing a less invasive procedure compared with conventional chest-tube placement.

\section{Room C \\ SS \\ $04.03 \mathrm{CT}$ of the liver}

\section{$2.00 \mathrm{PM}$}

\section{Spiral-CT and 3D-imaging of liver masses}

J.Gmeinwieser, A. Wunderlich, M. Strotzer, W. Bautz, P. Gerhardt; Munich

Purpose: The aim of the study was, to develop a method for semiautomatic 3D-reconstruction of liver masses, normal parenchyma and intrahepatic portal and venous vessels.

Methods and Materials: In 15 cases of primary and secondary tumors the liver was scanned with a spiral-CT mode (Somatom Plus/Siemens) during a single breath-hold while a contrast agent was injected intravenously using a bolustechnique. For semiautomatic 3D-reconstruction we modified the software of the MIPRON-system (KONTRON/Eching). Different anatomical structures were represented in different colors.

Results: Spiral scanning provided an excellent basis for 3D-imaging of the liver. The volume data files had no gaps. The short scanning time allowed a constant maximum enhancement of the intra- and extrahepatic vessels. After initial segmentation the interesting structures could be reconstructed automatically without major problems.

Results: 3D-reconstruction allowed an easy perception of number, size and site of liver masses and of their relation to surrounding veins and portal vessels. This resulted in a better and faster understanding of the segmental location of these tumors and thus in an easier pre-op planning of liver resections.

\subsection{PM}

\section{Monitoring of regional chemotherapy for metastatic liver disease by CTA and CTP}

M. Galanski, S. Reichelt, E. Schmoll, A. Chavan, M. Prokop, C. Schaefer, R. Raab, H. J. Schmoll; Hannover

Purpose: In patients undergoing regional chemotherapy for metastatic liver disease, the vascular supply of metastases (arterial or portal venous) and the pattern of liver perfusion via the port systems have to be known. The purpose of this study was to determine the relative value of $\mathrm{CT}, \mathrm{CT}$-arteriography (CTA) and CT-portography (CTP) in answering these questions.

Methods and Materials: In 25 patients CTA and CTP followed by post-contrast CT were obtained before and every 3 months after beginning of regional chemotherapy. Contrast agent was injected either via a port system (arterial or portal venous) or via a transfemoral catheter. CTA was performed by flow-controlled perfusion and by pressure-controlled injection.

Results: CTP with immediate and delayed scanning was found to be the most accurate method to determine size and number of lesions. Both, CTA and CTP are mandatory to evaluate the vascular supply of each metastasis and to estimate the perfusion capability of each port system. CTA best demonstrated changes in flow distribution and offers the possibility of intervention by injection of microspheres to reduce hyperperfused areas.

Conclusions: Both, CTA and CTP are required for adequate therapy control in regional therapy of liver metastases.

\subsection{PM}

\section{Intraarterial angio-CT before chemoembolization}

\section{U. Lörcher, D. Liermann, J. Peters; Frankfurt/Main}

Purpose: Perfusion of liver malignancies was studied in order to plan chemoembolization.

Methods: The liver of 63 patients ( 18 hepatocellular carcinoma, 7 cholangiocarcinoma, 23 metastatic colon carcinoma, 7 metastatic carcinoma, 8 other metastases) was investigated by dynamic intraarterial angio-CT before chemoembolization. Perfusion characteristics were compared with the distribution of contrast medium after embolization.

Results: We found 2 types of perfusion in angio-CT: 1. peripheral hypervascu- larity in the arterial phase of metastases of colo-rectal carcinoma and HCC and peripheral plus irregular internal hypervascularity in primary HCC. 2 . Hypovascularity in cholangiocarcinoma an metastatic carcinoma of the breast. Contrast distribution after embolization showed comparable results.

Conclusions: Thus angio-CT is able to predict the possible value of chemoembolization in that patients with hypovascularized lesions don't profit from embolization.

\subsection{PM}

411 3D-fusion of CT and DSA-sequences for exact correlation between liver segments and metastases

N. Rilinger, L. Köhler, A. Hewett, H. Niemann, P. Jensch; Oldenburg

An indispensible precondition to curative liver surgery is the exact topographic assignment of metastases to liver segments. It was the aim of this study to develop a computer based frontend for the $3 \mathrm{D}$ presentation of combined CT and DSA examinations.

Methods: Parallel algorithms and neural networks serve in reconstructing $3 \mathrm{D}$-models of liver veins out of bi-plane angiographic images. CT was used to extract and reconstruct the liver surface and the metastases.

Results and conclusion: The resulting model is visualized by using calculated transparency and artificial illuminations and allows the exact topographic assignment of metastases to liver segments.

\subsection{PM}

412 Lipiodol CT in patients with malignant liver tumours - is it worth the trouble?

\section{A. Adam, P. Dawson, L. Banks; London}

In the last few years there has been a vogue, emanating initially from Korea and Japan, for the use of Lipiodol embolization of the hepatic artery in an attempt to enchance the yield of CT of the liver in patients with hepatic malignancy. Specific patterns of uptake have been described for different kinds of liver lesion and great claims have been made for its usefulness.

We present our data on 21 patients with a variety of primary and metastatic liver tumours examined in this manner in whom all definitive diagnoses were confirmed histologically either by percutaneous needle biopsy or at subsequent surgery. In only one patient did the procedure reveal a previously undetected lesion and in no case did it affect clinical management. Interpretation was difficult in a number of cases and a false positive result was seen at the site of the liver biopsy. Another patient with a malignant phaeochromocytoma exhibited a pattern "characteristic" of haemangioma. Another patient developed a calculous cholecystitis as a complication.

We concluded that Lipiodol enhanced CT is not risk-free and does not usually affect the management of patients with hepatic malignancy.

\section{$2.43 \mathrm{PM}$}

413 Liver metastases from colorectal cancers: detection and localization with $\mathrm{CT}$ portography

Ph. Soyer, D. Vavasseur, A. Roche, F. Breittmayer, M. Gad, M. Sapoval, M. Levesque; Colombes

Purpose: To evaluate the accuracy of CT portography (CTP) in detecting and locating hepatic metastases to the Couinaud liver segments.

Methods and Materials: Twenty-three patients were prospectively studied before surgery by ultrasound (US), bolus CT and CTP. Sixty-one metastases were surgically and pathologically confirmed.

Results: CTP had a total detection rate (sensitivity) of $92 \%$. CTP was falsely positive in 2 cases and misdiagnosed the presence of metastases in 5 cases. CTP permitted to localize accurately 48 metastases to specific segments and plannify preoperatively the surgical procedure.

Conclusions: This study demonstrates that CTP is the most reliable imaging technique for detecting hepatic metastases and determining preoperatively their segmental location.

\section{$2.53 \mathrm{PM}$}

\section{Quantitative dynamic $\mathrm{CT}$ of malignant liver tumors} C. Zwicker, M. Langer, R. Langer, K. Rosenkranz; Berlin

Purpose: To evaluate and quantify the perfusion pattern of hepatocellular carcinomas, cholangio-/cholecystocarcinomas and metastases in subsecond dynamic CT.

Methods and Materials: 71 dynamic CT studies without and with table incrementation were performed prospectively using a Somatom Plus and a computerized contrast injection. Time density curves for aorta, liver and tumor were achieved and the values of the quotients $\mathrm{T}=$ time to peak-tumor: time to peak-liver and $\mathrm{D}=$ maximal enhancement-tumor: maximal enhancement-liver were compared.

Results: 17 of 20 hepatocellular carcinomas were hypervascularized in the 
arterial phase of perfusion and revealed a mean $T=0,48$. The density elevation of these tumors was reduced to $1 / 2$ the time of the surrounding liver with a slightly elevated maximum of $\mathrm{D}=1,14.11$ of 13 cholangio-/ cholecystocarcinomas were hypovascularized with an average $\mathrm{T}=2,8$ and $\mathrm{D}=0,74.38$ patients had hypovascular metastases with a mean $\mathrm{T}=2,3$ and $\mathrm{D}=0,55.2$ patients revealed hypervascular metastatic lesions with a $\mathrm{T}=0,24$ and $\mathrm{a} \mathrm{D}=$ 1,04 .

Conclusions: Quantitative dynamic CT can differentiate hypervascularized hepatocellular carcinomas and metastases from hypovascular metastatic lesions and cholangio-/cholecystocarcinomas.

\subsection{PM}

\section{Perfusion characteristics of hepatic cavernous hemangioma using intravenous CT-angiography (IVCTA)}

J. Gaa, M. Schütz, H. K. Deininger, S. Saini; Darmstadt, Boston

Purpose: The $\mathrm{CT}$ diagnosis of hepatic cavernous hemangioma $(\mathrm{HCH})$ is based on the analysis of dynamic and delayed patterns of lesion contrast enhancement. Using a fast CT scanner (Somatom Plus) in conjunction with a low dose, high rate intravenous contrast injection technique (IVCTA) we examined the early perfusion profile of $\mathrm{HCH}$.

Methods and Materials: Thirty patients with 35 proven $\mathrm{HCH}$ were studied with IVCTA. In this technique 15 scans are performed in the first $30 \mathrm{sec}$ after bolus $(6 \mathrm{ml} / \mathrm{sec})$ injection of $50 \mathrm{ml}$ iodinated contrast medium. Thenafter $2 \mathrm{scans} / \mathrm{min}$ are obtained up to $30 \mathrm{~min}$.

Results: Three new criteria were utilized to make a specific diagnosis of $\mathrm{HCH}$ : 1. detection of an intense mural nodular enhancement in the arterial phase, 2. well-defined nodular area(s), 3. centripedally oriented enlargement of the nodular region(s). These criteria were seen in $31(89 \%)$ of 35 hemangiomas. In contrast the "classic" Freeny-Marks criteria were present in only $24(69 \%)$ of 35 hemangiomas.

Conclusions: Our results show that these new criteria at IVCTA with a fast CT scanner may provide greater specificity in the diagnosis of $\mathrm{HCH}$.

\subsection{PM}

416 The role of $\mathrm{CT}$ in patient management after liver transplantation M. Galanski, J. Drewes, G. Gubernatis; Hannover

Purpose: Goal of the study was to examine the value of CT in diagnosis and differentiation of complications following liver transplantation

Methods and Materials: CT studies of 43 liver transplant patients were analysed with respect to the clinical question and were correlated with clinica and histological data and with the clinical outcome. Main indications for liver transplantation were end-stage liver disease and primary carcinoma.

Results: Frequent indications for CT examinations were unspecific deterioration of patient condition or liver function tests. Infections of unknown origin, tumor recurrence, or vascular complications were less frequent. The most common abnormalities on CT were patchy parenchymal inhomogeneities, geograpic low density lesions, and periportal low attenuation areas. Hypodense parenchymal areas were found to be suggestive of ischemic reactions. Periportal low-attenuation areas alone, without any other abnormalities, were fairly common, nonspecific and not indicative of transplant rejection in particular. However, periportal hypodensities combined with diffuse liver swelling were nearly always associated with severe transplant dysfunctioning neccessitating re-transplantation.

Conclusions: CT examinations for complications of liver transplantation are helpful only in the diagnosis of vascular complications and for the differentiation of focal space occupying lesions and therefore should be restricted to those indications.

\subsection{PM}

\section{CT-Assessment of liver regeneration after partial-hepatectomy: a prospective diagnostic study}

R. Maas, M. S. Beese, G. Krupski, U. Meyer-Panwitt; Hamburg

Introduction: The increasing frequency of liver-surgery during the last years faces the diagnostic radiologist more often with a pre- and postoperative liver-CT. There was interest in 2 items:

1. early postoperative imaging of the liver

2. whether and how liver regeneration does occur in man.

Methods and Materials: high resolution fast scanner (Siemens Somatom Plus), matrix $512 \times 512$, volumetry by means of contour tracing and sumation of areas. CT-protocol: pre-surgery, and postsurgery after 10 days, 3, 6 and 12 months. Volume determination of resected specimen.

Twenty patients ( 16 malignant, 4 benign tumors) with more than one year follow-up.

Results: 1 . Accuracy of volume determination by CT of phantoms $=3 \%$ 2. Although considering the resected parenchyma, there is a transient early increase of liver volume (hyperaemia?, edema?, narcotic drugs?)

3. Transient early decrease of liver density.
4. No clear correlation between amount of resection and process of liver regeneration in man (multiple regression: $\mathrm{r}=0.10, \mathrm{t}=0.29, \mathrm{~s}=0.77$ )

5. Significant correlation between remaining parenchyma and regeneration (multiple regression: $\mathrm{r}=0.85, \mathrm{t}=4,26, \mathrm{~s}=0.002$ )

6. No tendency of complete regeneration within one year.

\section{$3.28 \mathrm{PM}$}

\section{Hilar hepatic nodule: a pitfall in CT-portography} C. Pérez, J. Llauger, E. Castañer, A. Zidan, A. Giménez; Barcelona

CT during arterial portography (CTAP) is becoming the most sensitive method of hepatic enhancement to detect focal lesions.

Our experience with CTAP has led us to detect a hepatic pseudolesion, radiologically indistinguishable from a tumor. This pseudometastases is characteristically located in subcapsular areas of the left hepatic lobe, near the portal vein. In our 3 cases, normal hepatic parenchyma was surgically demonstrated in this zone.

We describe this pseudolesion, non previously reported; causes that may explain its appearance are discussed. Typical radiological findings allow their recognition and, in patients with other metastatic nodules potentially operables, surgery must not be obviated.

\section{Room E1}

\section{SS}

\subsection{Interventional procedures in the peripheral} vasculature

\section{$2.00 \mathrm{PM}$}

419 Mechanical revascularization of occluded iliac arteries using self-expandable endoprostheses

D. Vorwerk, R. W. Günther; Aachen

Mechanical revascularization combining careful balloon dilatation and self-expanding endoprostheses was used as an alternative approach to percutaneous treatment of iliac occlusions.

Traversal by catheter was achieved in 61 out of 83 patients presenting with iliac occlusions. Subsequent stent placement was performed in all 61 patients. The occluded segments were situated in the common iliac $(n=37)$, external iliac $(\mathrm{n}=16)$ or the common and external iliac artery $(\mathrm{n}=8)$ with a mean length of 5.5 (range $1-22) \mathrm{cm}$. Mean Doppler index was 0.55 . Stage II was found in 54 , stage III/IV in 7 patients.

Vascular restoration was achieved in all 61 patients. Reocclusion within 2 months occurred in 3 patients $(4.9 \%)$. One acute embolization occurred necessitating surgical intervention. Mean follow-up period was 17 months, restenosis occurred in 3 patients, Life-table analysis revealed a long-term patency of $93 \%$ at 6 and of $83 \%$ at 24 -month follow-up.

Combination of underdilatation and stent placement offers a high technical success in recanalizing iliac occlusions. Rate of embolization is relatively low compared to balloon dilatation alone.

\subsection{PM}

\section{Experience with various stents in the treatment of iliac obstruc- tions}

Georg Küffer, Th. Pfluger, W. Kellner, F. Spengel; Munich

Purpose: To present our experiences with the various stent concepts so as to delimit possible ranges of indications for the individual stents.

Methods and Materials: In 18 months 36 stents were implanted in 26 common iliac and in 5 external iliac arteries of 28 patients. These included 26 Palmaz stents, 8 Strecker stents and 2 Wallstents. Bilateral stents were given to three patients. Two of them were aortic bifurcation supports. The indications were $4 \times$ a recanalized common iliac artery occlusion, $4 \times$ an unsuccessful PTA of restenoses PTA/AE, and $16 \times$ an insufficient PTA result of eccentric/amorphous stenoses. In 4 patients a stent was implanted for primarily morphological reasons, regardless of the PTA result.

Results: All stent operations were primarily successful. The mean pressure gradient after regular PTA was reduced from $11.9 \mathrm{~mm} \mathrm{Hg}(\mathrm{sd} 8,6)$ to $1.6 \mathrm{mmHg}$ (sd 2,1). Complications included two patients with local haemorrhage and one patient with embolism in the popliteal artery. In the average follow-up period of 11,5 months 18 patients have been symptom-free.

Conclusions: According to our experience the Palmaz stent is particularly suitable for hard eccentric plaques on the aortic bifurcation. The two flexible stents (Strecker/Wallstent) are more suitable for bended vessels with soft lesions. 
421 Is there a surgical contraindication against stents and is it possible to rescue a misplaced stent?

D. Liermann, M.Zegelman, J. Kollath, P. Satter, E.P. Strecker; Frankfurt/Main

Especially surgical colleagues resist often against stent implantation because of the fear to loose any surgical vessel treatment except Bypass after stent implantation. We 've now placed more than 100 endovascular stents since 1987 in iliac or peripheral stents. In two series we tried to show, that an endovascular stent Strecker-tantalum stent - is to rescue after misplacement without difficulties and that a surgical treatment after stent implantation like the TEA is possible without problems.

Methods: In the first serial we tried to rescue a placed stent over a 8-F-introducer sheat (Terumo) and a special moveable barb set. To tear back the stent into the introducer sheat and to put it out of the vessel. In the second serial we tried a TEA of the stented area, where the ringstripper cut a typical cylinder of the atherosclerotic vessel wall overpassing the stent.

Results: As well the rescue of the placed stents through the introducer sheat as well as the TEA in a stented region succeded in all cases without any problems and without damage of the vessel wall.

Conclusions: It is very easy to rescue a misplaced stent without risk of incomplete rescue or vessel wall damage. A stent is no contraindicative against a TEA later on.

\subsection{PM}

\section{Control of life threatening hemorrhage resulting from fractures using embolization with Ethibloc and GAW-coils}

W. Jaschke, H. P. Busch, E. Wetzel, M. Georgi; Mannheim

Goal: Fractures may be accompanied by massive hemorrhage. We report our experience with transcatheteral embolization for control of bleeding.

Methods: Between February 1988 and September 199011 patients with bleeding resulting from fractures caused by blunt trauma were embolized (pelvis: 9 patients; skull: 1 ; femur: 1 ). After selective catheterization Ethibloc was injected in fractions of $0.1 \mathrm{ml}$. The effect of embolization was monitored by fluoroscopy. GAW-coils were used to protect arterial branches which did not sustain bleeding. In a few cases, GAW-coils were used to prevent loss of Ethibloc emboli through the arterial stump. The effect of embolization was evaluated by the amount of blood transfusions required after the procedure. Results: In all 11 patients bleeding was successfully controlled by embolization. However, two patients died of cardiac failure within 24 hours, another 3 patients died of acute adult respiratory distress within 4 weeks. The other 6 patients survived. No complications were observed. We conclude that embolization is a safe and effective mean for control of traumatic bleeding. However, despite effective control of hemorrhage a high percentage of patients die due to reasons associated with hemorrhagic shock.

\section{$2.40 \mathrm{PM}$}

423 Complete arterial obstructions of the lower limbs: the place of percutaneous atherectomy

P. Maquin, H. Rousseau, M. Levade, E. Bloom, B. Escude, F. Joffre, J. J. Railhac; Toulouse

Purpose: Evaluation of the Simpson catheter in the treatment of complete arterial obstructions.

Methods: Seventeen patients ( 12 men, 5 women, 51 to 83 years old), presenting with iliac $(\mathrm{n}=1)$ and femoro-popliteal $(\mathrm{n}=16)$ symptomatic occlusions (mean length: $23 \mathrm{~mm}$ ), were treated by atherectomy. A previous recanalization with balloon angioplasty was necessary in 4 patients, along with a complemental dilatation in 14 cases.

Evaluation included repeated clinical and doppler examinations, angiography (6 months after or in case of clinical worsening).

Results: - Initial results: disappearance of the claudication was noted in all patients with a mean 0,4 increase in the ankle-arm-index. A residual stenosis lower than $30 \%$ was noted in 16 cases (the severity of the residual stenosis was lower when the catheter used was a 9 french, and when the lesion was short and non calcified. Seven mild dissections were noted. One distal embolization occurred without sequelae.

- Mid-term results: clinical and doppler improvement remained stable in 15 patients with a maximum follow-up of 32 months (mean: 17,3 months); one restenosis at four months; one death, non related to the arterial disease, at 6 months.

Conclusions: atherectomy along with a conventional angioplasty seems to be a safe and effective treatment of short femoro-popliteal obstructions.
424 Results of secondary Simpson-atherectomy (S-AE) in fem.pop. obstructions after unsuccessful balloon angioplasty Georg Küffer, Th. Pfluger, L. Ziegler, J. Sinzinger; Munich

Purpose: To present our experience with the Simpson Atherocath in treatment of residual stenoses or occlusions after failed PTA or other angioplasty technique.

Methods: In 30 months we conducted a secondary S-AE in fem.pop. obstructions in 22 of 82 patients. In 4 patients it was necessary to correct plaque complications after regular PTA of complex stenoses. In 14 patients with fem.pop. occlusions, plaque dissections by the atherectomy had to be eliminated since otherwise a reocclusion was imminent. Three times the operation was preceded by a recanalization with an excimer laser. The mean occlusion length was $4.9 \mathrm{~cm}$.

Results: The operations were all technically successful. Themean plaque weight was $142 \mathrm{mg}$ (35-491 mg). In 21 of 22 patients there was a clinical success postatherectomy.

Conclusions: Secondary S-AE is currently the most effective ablative angioplastic technique in fem.pop. vessels. Its complementary use with other techniques increases the rate of success of the angioplasty. The use of narrowlumen fem.pop. vessel stents can thereby clearly reduced.

\section{$3.00 \mathrm{PM}$}

425 Cell biological and morphological studies on Simpson atherectomy specimens of primary lesions and restenoses

W. Krings, W. Schlumberger, M. Thie, H. Robenek, F. J. Roth, P. E. Peters: Münster

During the early stages of atherogenesis, smooth muscle cells (SMC) migrate from the media to the intima of the arterial wall, where they proliferate and synthesize large amounts of extracellular material. Most of our present knowledge concerning the regulation of these processes is derived from studies on cultured cells. Atherectomy with a Simpson catheter enables a detailed characterization of percutaneously removed human atheromatous plaque material.

Our cell biological investigations are aimed at a comparative characterization of excised material from primary lesions and restenoses to yield further insight into the mechanisms of plaque formation. We used flow-cytometric analyses and cell type-specific antibodies to obtain quantitative data on the amount and the types of cells that proliferate and data on their phase of cell cycle. Furthermore, with immunhistological labelling methods of selected extracellular matrix proteins we would like to contribute to a better understanding of the structure and composition of the extracellular matrix and their interaction with cells in atherosclerotic plaques. In addition, localization of cells containing elevated levels of mRNA for matrix proteins was achieved by in situ hybridization with cDNA probes.

First results obtained with the mentioned methods on 10 specimens will be presented.

\subsection{PM}

426 Radiofrequency thermoprobe (PTA associated), in recanalization of femoro-popliteal arteries: 12 months follow-up results in 16 cases M. Castrucci, M. Venturini, R. Varagona, R. Chiesa, G. Melissano, P. Spiegel, A. DelMaschio; Milan

Purpose: Hot-tip Laser assisted angioplasty has been successfully used for treatment of superficial femoral and popliteal arteries occlusions. The purpose of this clinical experimentation was to assess the value of the Radiofrequency (RF) Thermoprobe (Thermoplaster-AIS), which is a low cost technology compared with laser based systems.

Methods: 16 SFA/popliteal occlusive lesions (15 pts) were treated by RF-Thermoprobe and PTA. Pre-intervention clinical and angiographic criteria of selection were: 1 . length $0-10 \mathrm{~cm} ; 2$. impossible recanalization by angiographic guide-wire; 3 . not heavy calcified plaques; 4 . fair run-off; 5 . Fontaine stages IIA, IIB and III. Post-treatment Ankle/Brachial indices were checked immediately post the intervention and monthly for 12 months, and compared with pre-treatment $\mathrm{A} / \mathrm{B}$ data.

Results: good results in recanalization was obtained in $15 / 16$ RF-Thermoprobe/PTA procedures. No recanalization in one patient due to heavy resistant localized plaque. $1 / 15$ patients had a low increase of $A / B$ index and low improvement of claudication. $1 / 15$ patients reoccluded after 13 months.

Conclusions: our preliminary study demonstrated good results and therapeutic effects of the RF-Thermoprobe recanalization technique in selected obstructive lesions. The Thermoplaster-AIS presents the following advantages: 1. low cost energy and device; 2. safety (system battery $12 \mathrm{~V}$ operating); 3 . handleness (portable device); 4. application of the probe on angiographic guide-wire. 


\section{Additional interventions supporting laser angioplasty of peripheral vessels}

W. Horvath, M. Oertl, Ch. Luft, D. Haidinger; Linz

Purpose: Laser angioplasty for recanalization of peripheral arteries has become a frequently used method. The purpose of this study is to show that numerous additional interventional methods are necessary to achieve satisfactory results.

Methods: From 1987 to 1990 we treated 166 peripheral arteries with laser assisted balloon dilatation using a neodyme-YAG-laser. Complications (embolism and early reocclusions) were treated by aspiration and local lysis with urokinase in 12 cases. To improve long term results balloon dilatation of stenoses of the inflow area was performed in 35 cases, of the outflow area in 26 cases. In 8 patients PTA of recurrent stenoses in the recanalized segment was necessary, and also 4 of 8 reocclusions were treated by PTLA.

Results: The high primary success rate of $90.9 \%$ for laser angioplasty is partly attributed to the above described additional interventions. For example all 8 cases of peripheral embolism were successfully treated by aspiration or aspiration combined with local lysis. Also the 4 early reocclusions could be reopened by these methods.

Discussion: The above results show the importance of additional interventions for primary success, but it is also very likely that long term results are improved by treating in- and outflow problems.

\section{Room E2}

\section{SS}

\section{$2.00 \mathrm{PM}$}

428 Decreased acromiohumeral distance: relationship with rotator cuff pathology

F. Sáez, J. Negueruela, J. C. Martín, E. Elizagaray, L. Larrea, J. M. Peña; Bilbao

Purpose: To prove the diagnosis of rotator cuff tear when the acromiohumeral distance is $<6 \mathrm{~mm}$ (measured by plain film), as well as to evaluate the extent of the rupture and the presence of associated lesions.

Methods and Materials: In a series of 187 patients, we have found 21 cases of acromiohumeral distance $<6 \mathrm{~mm}$. The average age was 70 years. All of them have been studied by means of plain films, ultrasound and CT-arthrography and 51 of them were also examined with $0.5-\mathrm{T}$ MR imaging.

Results: Every case showed large supraspinatus tears. In 7 cases the infraspinatus was also involved. In 10 other shoulders the subscapularis, infraspinatus and supraspinatus were involved. 1 case included rupture of the teres minor. 14 cases showed bilateral cuff tears. All cases presented severe atrophy of several muscles of the cuff. Half the patients had prominent deltoid atrophy. Cuff tear arthropathy was observed in 4 cases. Pathology of the biceps tendon (tendinitis, rupture, luxation) was demonstrated in 17 cases. Degenerative changes were seen in 17 cases. 6 cases of adhesive capsulitis and 1 case of distrophic calcification were encountered.

Conclusions: When the plain film shows an acromiohumeral distance $<6 \mathrm{~mm}$, no other exploration is necessary to prove large rotator cuff tear with marked muscle atrophy. Surgery would only be indicated to avoid intractable pain, because function is not likely to be recovered.

\subsection{PM}

\section{Shoulder sonography: evaluation of impingement syndrome} Pekka Farin, Heikki Jaroma; Kuopio

1. The purpose was to evaluate the appropriate technique for sonographic evaluation of the impingement syndrome and to discuss the reliability of sonographic findings.

2. During scanning a dynamic method with lateral and anterior elevation of the arm was used and at the same time attention was paid to the bursal system, especially to the gradual distention of the bursa.

3. In 102 of 381 patients studied, surgical diagnosis was available for correlation. Among this group there were seven false-negative and three falsepositive sonographic findings. A comparison of sonographic with surgical findings demonstrated a sensitivity of $81 \%$ and a specificity of $95 \%$ in stages I-III, and a sensitivity of $71 \%$ and a specificity of $96 \%$ in early stages I and II. 4. Although, there are many pitfalls in shoulder sonography, the results of dynamic examination with fluoroscopic radiography provide valuable information in patients with suspected early-stage impingement syndrome. This method seems to have its own place in assessing early-stage impingement syndrome, but it cannot, unlike MR imaging, to demonstrate the causes for this disability.
430 Shoulder sonography: different role in the acute and in the chronic patient

P. Bagnolesi, A. Cilotti, R. Lencioni, D. Caramella, C. Bartolozzi; Pisa

Purpose: define the clinical conditions which benefit or not by sonography and ascertain the reliability of the different signs in the acute and in the chronic patients.

Methods: thirty-six patients ( 16 with history of acute trauma, 20 with chronic history) underwent sonography. Arthrography was performed in 6 acute patients (demonstrating thinning of the cuff) and in 12 chronic ones ( 2 presenting nonvisualisation of the cuff, 10 diffuse thinning of it). Surgical control was done in 14 acute and in 10 chronic patients.

Results: cuff tears were found in all 14 acute patients and no sonographic false was found in them. A tear was present in 16 chronic patients, presenting as nonvisualisation of the cuff ( 9 cases) and as diffuse thinning ( 7 cases). Cuff thinning resulted true positive in 7 patients, false positive in 4 .

Conclusions: the signs referred demonstrate different meaning whether they occur in the acute or in the chronic patient. In the former, focal discontinuity, nonvisualisation of the cuff and diffuse thinning of it, hold same importance, indicating a lesion and can be regarded with confidence.

In the chronic patient focal discontinuity is rarely observable. While nonvisualisation of the cuff indicates a tear, its thinning may or may be not due to it.

\subsection{PM}

431 Postoperative computed tomographic examination of the shoulder

G. Helweg, D. zur Nedden, A. Oberhauser, R. Knapp, H. Resch; Innsbruck

Purpose: Modern surgical treatment of atraumatic and traumatic recurrent shoulder dislocations aimes the elemination of recurrence causing factors by over 150 different surgical procedures reported in the literature. The choosen operation method depends for cases of anterior and posterior instability. To prove the stabilizing parameters and the success of the operation CT is accurate und reliable

Methods and Materials: Between 1984-1990 312 patients unterwent a shoulder operation. Out of that number 65 unterwent CT postoperatively. 41 of them after receiving a wedge shape bone graft at the glenoid rim.

Results: CT under standardized examination technique; in our study fokused stone those who revealed bone graft reconstruction of glenoid rim, demonstrate in all cases no resorption of the bone graft and an excellent joint cartilage with new formations of cartilagineous rim cartilage in four cases.

Conclusions: Postoperative CT under standardized examination conditions reveales even without contrast excellent results in observation of the operation success and should be carried out especially in cases of bonegraft implants.

\section{$2.40 \mathrm{PM}$}

432 MRI of the labral capsular complex: normal morphologic patterns and variations

Christian H. Neumann, Stephen S.Petersen, Arlon H. Jahnke; San Francisco, CA

Purpose: This study was undertaken to describe the normal appearance and variations of the labral capsular complex on MRI to serve as a reference for the interpretation of clinical cases.

Methods and Materials: 52 asymptomatic shoulders were imaged by $1.5 \mathrm{~T}$ MR using a 5 inch receiver coil pair. The anterior and posterior glenoid labrum and capsular insertions were critically examined on $4 \mathrm{~mm}$ axial $\mathrm{T} 1$ and $\mathrm{T} 2$ weighted and MPGR images.

Results: Marked variability in the normal morphology of the anterior labrum was confirmed. The most common pattern was triangular $(50 \%)$, followed by rounded $(20 \%)$, cleaved $(14 \%)$, notched $(8 \%)$, and flat $(7 \%)$. The posterior labrum exhibited a much more consistent pattern and was triangular in most patients $(73 \%)$ and labral absence was not uncommon $(8 \%)$. Focal increased signal within the labrum was noted 13 times. Capsular insertion tended to be types one and two anteriorly ( $97 \%)$ and type one posteriorly (100\%). The labral capsular appearance matched the other shoulder in only half the cases. No Hill-Sachs lesions or degenerative changes were noted.

Conclusions: Variability and characteristic MRI patterns of the normal labral complex were described in this study. Awareness about these normal patterns is believed essential for interpretation of clinical cases at a diagnostic quality similar or better than other imaging methods. 
$433 \mathrm{MRI}$ and CT-arthrography of the labral capsular complex in shoulder instability: A prospective study of $\mathbf{4 5}$ patients with surgical and arthroscopic proof

Christian H. Neumann, Stephen S. Petersen, Arlon H. Jahnke, Lynne S. Steinbach, Frank W. Morgan; San Francisco, CA

Purpose: This study was undertaken to assess the diagnostic value of MRI in patients with suspected shoulder instability and provide a comparison with CT-Arthrography.

Methods: 45 consecutive patients with shoulder instability and symptoms of undetermined etiology were imaged by $1.5 \mathrm{~T}$ MRI and CT arthrography prior to arthroscopy or surgery. Interpretation was done by three radiologists blinded to the clinical and surgical diagnosis. The anterior and posterior labrum and capsule and humeral head were critically assessed. Sensitivity, specificity, accuracy, predictive values and ROC curves were calculated including a statistical analysis. Surgical and arthroscopic proof was available in all patients.

Results: Sensitivity, specificity and accuracy of MRI in the anterior and posterior labrum was $85 \%, 90 \%$ and $88 \%$ comparing favorably with CT-Arthrography $66 \%, 90 \%$ and $88 \%$. Hill-Sachs lesions were better seen by MRI. Both modalities were unsatisfactory evaluating capsular pathology. The results were statistically significant.

Conclusions: MRI provides accurate assessment of the anterior and posterior labrum and humeral head when performed with state of the art equipment. It may become the preferred diagnostic imaging procedure in patients with suspected instability not at least because of its non-invasiveness and more comprehensive assessment of the shoulder than CT-Arthrography.

\section{$3.00 \mathrm{PM}$}

434 Shoulder MR: imaging: improvements of routine examination using fast gradient recalled and fat saturated sequences

Martin Vahlensieck, Sharmila Majumdar, Mariela Resendes, Philipp Lang, Harry K. Genant; San Francisco, CA

Purpose: Common MRI protocols of the shoulder joint include T1 and T2 weighted spin echo sequences. The purpose of this study was to shorten imaging time and to improve contrast resolution of routine examinations, particularly the differentiation between peribursal fat and fluid.

Methods: We compared different SE sequences (T1, Density-weighted, T2, with and without fat saturation) with different gradient recalled sequences (GRE), (T1, Density-w., T2/T1, T2* with and without fat saturation. TR's: 70, $100,200,500$; TE's: $12,15,20,30,35,60 ; \Theta: 5,30,40,90)$ in 7 volunteers on a 1.5 T System (GE Signa, shoulder coil, FOV: $16 \mathrm{~cm}$, slice thickness/spacing: $4 / 1.5 \mathrm{~mm}, 8$ slices in the coronal oblique plane, Matrix $128 \times 256,2 \mathrm{NEX}$ ). Results: High S/N was observed on a T2/T1-w. GRE sequence and its corresponding fat-saturated sequence. T1-w. GRE showed a satisfying S/N and few susceptibility artifacts. A Density-w. GRE demonstrated similar muscle and fat signal. Fat saturated GRE sequences with short TR, short TE and intermediate flip angle suppressed fat signal completely and highlighted appearance of tissues with high water content - such as the hyaline cartilage.

Conclusions: A short TR, short TE and intermediate flip angle sequence could be of value in routine protocols of the shoulder. Preliminary observations indicate that our fat saturated GRE technique assists in distinguishing fat from fluid, and extensive clinical investigations to prove its capabilities are currently being conducted.

\subsection{PM}

\section{Athletes acute dislocation of the shoulder; staging of intra- and extraarticular lesions using MRI}

C. Masciocchi, A. Barile, E. Fascetti, R. Passariello; L'Aquila

The purpose of this report is to illustrate our preliminary experience with magnetic resonance imaging (MRI) in the evaluation of athletes with acute anterior dislocation of the shoulder in order to demonstrate its potential utility in clinical practice. Twenty-nine patients with acute anterior dislocation of the shoulder were evaluated with MRI using a 0.5 superconductive system (Esaote Biomedica MR 5000) and a 0.2 permanent system (Esatom PM 4000). In all cases a "dedicated" surface coil was used. Transverse and two oblique planes, perpendicular and parallel to the glenoid surface, were used. In all patients comparison between the surgical findings and the results of MRI was possible. All scans were considered of diagnostic quality. Twenty-two patients had an anterior labrum tears and in six of these patients an associated Hill-Sachs lesion and in one case an associated Bankart lesion were demonstrated. In seven cases MRI showed complicated lesions. Six patients had an anterior labrum tear with a rotator cuff lesion and one patient had an anterior labrum tear with a long head of biceps lesion. Surgical confirmation of these findings was available in all patients. In conclusion the result of this preliminary experience demonstrates that MRI has considerable potential in the evaluation of acute anterior dislocation of the shoulder in the athletes.
436 Recurrent dislocation of the shoulder joint: MR versus radiologic imaging Uwe Tosch, B. Sander, D. Büscher, M. Kretschmar, R. Felix; Berlin

Purpose: The diagnostic value of various imaging techniques in recurrent shoulder dislocation were correlated.

Methods: Fifteen patients were preoperatively investigated at a Magnetom SP (1.5 Tesla) with an oval surface coil $(25 \times 15 \mathrm{~cm})$. In all cases axial and sagittal T1 (SE $500 / 15 \mathrm{~ms}$ ) and T2 (SE 2500/15-90 ms) weighted images were performed. MR was correlated with conventional radiographs and axial CT examinations.

Results: Apart from Hill-Sachs defects, which were found to a various extent in all patients, MR helped to diagnose rotator cuff lesions in 13 of 15 patients. Additionally defects of the anterior glenoid labrum and an elongation of the capsule of the shoulder joint were found in 12 cases. These findings were not seen in CT and proved true by surgery.

Results: MR imaging proved to be a useful non invasive technique in diagnosing shoulder joint disease and seems to be superior to CT imaging.

\section{Room L}

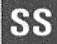

\subsection{PM}

\section{Nuclear medicine procedures in children}

I. Gordon; London

Abstract available at the information desk

\subsection{PM}

438 Acute renal vein thrombosis in children; early detection with duplex Doppler ultrasound and color flow imaging

S. Trattnig, K. Frenzel, M. Eilenberger, A. Khoss, W. Ponhold, B. Schwaighofer; Vienna

Purpose: To describe the morphology and findings of duplex Doppler ultrasound and color-coded Doppler sonography (CCDS) of native kidneys with clinical suspected renal vein thrombosis in order to establish an early diagnosis.

Patients and methods: Two children were included in the study: One neonate, who was transferred to hospital because of hypoglycemia and convulsions. The laboratory findings showed transient hematuria and thrombocytpenia. An abdominal mass was palpable on the right. An-11-year-old boy developed a swollen leg on the right side after autotransplantation of his right kidney to the right iliac fossa. Autotransplantation was necessary because of persistency of renal hypertension due to a long, high-grade stenosis of the right renal artery. Examinations were performed with a duplex Doppler (Ultramark 4, ATL) and color-coded Doppler sonography unit (Acuson $128 \mathrm{~T}^{\mathrm{m}}$ ).

Results: In both children duplex Doppler ultrasound demonstrated en enlarged kidney with loss of cortico-medullary delineation. The spectral tracing showed peaked systolic frequency shifts and retrograde plateaulike frequency shifts during diastole in larger renal vessels. No venous signal could be recorded. CCDS demonstrated a reverberating oscillation of blood flow within the main renal artery and proximal branches without color-coding of peripheral vessels. In addition, CCDS was helpful in the detection of partial vena cava inferior thrombosis in the newborn and iliac vein thrombosis in the patient with autotransplantation.

Conclusions: Dynamic evaluation by duplex Doppler sonography and CCDS may be considered as characteristic of acute renal vein thrombosis and seem to permit definitive diagnosis in the early stage without any need of confirmatory invasive procedures such as renal angiography or venography.

\subsection{PM}

439 RARE-MR-urography in the diagnosis of upper urinary tract abnormalities in children

G. Sigmund, B. Stoever, L. B. Zimmerhackl, A. Frankenschmidt, E. Nitzsche, J. Hennig; Freiburg

Purpose: RARE-MR-urography (Rapid Acquisition with Relaxation Enhancement) is a fast MR imaging technique that selectively depicts fluid by heavy T2-weighting, but without contrast medium application. In the present study the method was evaluated in children with upper urinary tract abnormalities by comparing its results with those of conventional methods.

Patients and methods: From 9/1989 to 11/1990, RARE-MR urograms were prospectively evaluated to diagnose upper urinary tract abnormalities in 55 children. The method is performed in several planes and combined with a coronal, T1-weighted spin-echo sequence. 
Results: Forty out of 42 dilated renal pelvises and 21 out of 24 dilated ureters were identified, only mildly dilated ones were missed. The kidneys without excretory function on excretory urography (EU) were clearly depicted by RARE-MR-urography. No differentiation could be made between vesicoureteral reflux and non-refluxive dilatation. All 19 kidneys with hydronephrosis resulting from pelviureteric obstruction and all eight renal duplications with a dilated segment were identified.

Conclusions: RARE-MR-urography is a new tool for diagnosing urinary tract abnormalities in children without ionizing radiation, contrast media, or general anesthesia. A dilated urinary tract can be shown in one image displaying the entire urinary system, similar to EU. Despite some limitations, it might - in certain cases - replace EU.

\section{$2.35 \mathrm{PM}$}

440 What's about the contralateral kidney in unilateral multicysticdysplastic kidney?

K. Schneider, J. Wisser, T. Schramm, M. Kellner, H.-G. Dietz; Munich

Multicystic-dysplastic kidney disease (MCKD) is the most frequently prenatally diagnosed dysplasia of the kidneys. Prognosis depends mainly on the state of the contralateral kidney, because MCKD usually has no function. There is reported in the literature that growth of the contralateral kidney starts only after birth. From 1982 to 1990 we examined 36 newborns with prenatally detected MCKD within 10 days after birth. The contralateral kidney looked normal (size and morphology) in 27. Obstructive hydronephrosis (ureteropelvic-junction obstruction, ureterovesical junction obstruction), duplication anomalies or hypoplasia were present in 6 patients; bilateral MCKD were seen in 3 .

Five of the remaining 27 kidneys were considerably enlarged by the first sonographic examination, but were otherwise normal. This suggests that in some patients with MCKD hypertrophy of the contralateral kidney even starts before birth.

2.45 PM

441 Ovarian cysts in the fetus and neonate - changes in sonographical pattern in the follow-up and management

C. Müller-Leisse, U. Bick, K. Paulussen, J.Tröger, Z. Zachariou; Aachen, Münster, Heidelberg

Purpose: To evaluate the changes of sonographic patterns of neonatal ovarian cysts perinatally and to discuss their management.

Methods and Materials: In a multicenter trial clinical, surgical and sonographic data of 44 neonatal ovarian cysts, 39 detected prenatally, 5 postnatally, were reviewed concerning histology, course and management.

Results: Of the 39 prenatally detected cysts 32 were purely cystic, 3 echogenic or had a mixed pattern, in 4 no data were available. In 19 of the 32 the cystic appearance changed perinatally from purely cystic to a mixed pattern being independant on the size of the cyst. 25 of the 44 cysts were treated surgically, histologic data are available. 19 were controlled sonographically: in 12 cysts there was complete resolution within 1 year postpartum the size of these cysts varied between 5 and $60 \mathrm{ml} .2$ infants with cysts presented as emergency cases, both cysts had a volume greater than $100 \mathrm{ml}$.

Conclusions: The change of ultrasound pattern from purely cystic to a mixed pattern perinatally is typical for hemorrhage and should not be indicative of surgical management.

Conservative management is indicated unless the cyst is space-occupying, there is no regression, the sonographic pattern is atypical or in case of emergency.

\section{$2.55 \mathrm{PM}$}

442 Posterior urethral polyps in infants and children: ultrasonographic and radiodiagnostic imaging

C. Gervas, J. Gutierrez, L. De Pablos, I. Pastor; Madrid

Posterior urethral polyps are a rare cause of bladder outlet obstruction in boys, with only 70 cases reported in the literature up to 1990 .

We present five cases of children between the age of four months and three years who developed repeated urinary infections and/or difficult micturition, and recurrent hematuria in one case.

A voiding cystourethrogram revealed an oval filling defect at the bladder neck in two cases and posterior urethra in the other three, along with a thickened and trabeculated bladder and important reflux in three cases. Ultrasonography demonstrated a small mobile solid polypoid mass emerging from the bladder neck in two cases.

Excretory urogram was normal in two cases, hydronephrosis noted in three. Diagnosis was confirmed by cystoscopy. All polyps were resected with a total remission of symptoms.

Pathology showed in all cases benign fibrous-epithelial polyp.

\section{$2.58 \mathrm{PM}$}

443 The "acute scrotum" in Henoch-Schönlein Purpura: ultrasonographic findings and literature review

G. Pozo, M. Miralles, V. Lozano, J. Boned; Madrid

Although in Henoch-Schönlein Purpura (HSP) every organ system may be virtually affected, there is only one previous reference in the radiologic literature with description of US alterations in male external genitalia involvement. Very uncommonly it may be the first symptom of HSP, and the presentation as "acute scrotum" may be confused with a testicular torsion.

We report two cases of acute scrotal involvement in two patients with HSP. In one of them the involvement was bilateral in the course of other general symptoms. In the other was unilateral and isolated, making difficulties in differentiating from testicular torsion. The US appearance with tests minimally affected or normal, helps in the diagnosis.

\subsection{PM}

\section{Acute scrotum in childhood: ultrasound diagnosis} B. Hamm, F. Fobbe; Berlin

Purpose: Assessment of the diagnostic value of gray-scale ultrasound (US) and color-coded duplex sonography for acute scrotum in childhood.

Methods: Ninety-four children (ages: 1 day to 16 years) underwent high-resolution ultrasound ( 5 and $7.5 \mathrm{MHz}$ ) following the clinical diagnosis of acute scrotum. Forty-one children were additionally examined by color-coded duplex sonography. Thirty-six children were submitted to surgery following ultrasound examination.

Results: The most frequent diagnoses were testicular torsion $(23 \times)$, hydatid torsion $(19 \times)$, epididymitis $(14 \times)$ and scrotal trauma $(8 \times)$. US proved a useful supplementary procedure to clinical examination in the diagnosis of acute scrotum. The tentative clinical diagnosis could be confirmed in $43 \%$ of the cases, and in $22 \%$ of cases therapeutically relevant additional information was obtained by US. However, in 16 cases it was impossible to distinguish between testicular torsion and inflammation by conventional US. In comparison, this differentiation of considerable therapeutic relevance was possible in $96 \%$ of cases using color-coded duplex sonography.

Conclusions: Ultrasound improves the diagnosis of acute scrotum, reducing surgical exploration to the therapeutically indispensable minimum. The great accuracy of color-coded duplex sonography in the diagnosis of suspected testicular torsion deserves special mention in this context.

\subsection{PM}

\section{US in the evaluation of the testes in adrenogenital syndrome}

P. L. Paesano, A. Vanzulli, F. Braggion, G. Chiumello, A. DelMaschio; Milan

Purpose: Testicular masses in males with the adrenogenital syndrome (AGS) have been recognized since 1901. The cells of origin of these "tumors" are probably adrenal cortical rest or pluripotential cells derived from the adrenogenital ridge. They respond to elevated ACTH levels. The frequency of these testicular "tumors" in patients with AGS is unknown; no Ultrasound (US) patterns of these lesions are reported. Purpose of the study is the identification of these lesions in patients with ASG by scrotal US.

Methods: Twenty boys (age 10-16 yrs) affected by AGS were evaluated with scrotal US (7.5 MHz, linear probe). On clinical examination of the scrotum 19 patients were negative; 1 patient had some irregularities on testicular surface. Results: We found 4 patients $(4 / 20=20 \%)$ with testicular lesions; two brothers affected by 11-Hydroxylase deficiency and two unrelated children. At US examination bilateral, focal, sometime confluent hypoechogenic lesions were found, with diameters ranging from 5 to $20 \mathrm{~mm}$. It is to remark that ACTH levels and testicular masses were not reduced by administration of large doses of dexamethasone in two patients.

Conclusions: The incidence of testicular lesions we have found in patients with AGS suggest a more widespread use of US screening in these patients.

\subsection{PM}

\section{Radionuclide diagnosis of retroperitoneal tumors in children} B.F. Sinyuta, V. V. Shishkina; Kiew

The study was designed to evaluate the results of radionuclide treatment administered to 60 children with retroperitoneal tumors and tumor-like lesions using $99 \mathrm{~m}$ Tc-Pyrphotech, (Technephore), $113 \mathrm{~m}$ In-Indiphore. Within the first 30 minutes a dynamic scintigraphy of retroperitoneum was performed, including renal scintigraphy. Within 1 and 3 hours following administration of these drugs we carried out static retroperitoneal and skeletal scintigraphy. The method revealed metastizing into bones, urinary dynamic disorders, as well as changes in anatomic and topographic renal status and function, enabling visualization of neuroblastomas and Wilms tumors, the diagnostic efficacy reaching about $90 \%$, as well as renal development abnormalities associated with tumors. Evaluation and comparison of changes in anatomic and 
topographic status of kidneys on scintigrams obtained within first minutes and changes in drug distribution in tumor sites within 1 and 3 hours enabled determination of a number of scintigraphic features. The latter are helpful in the differential diagnosis of Wilms tumors, neuroblastoma, malignant renal lymphomas, renal development abnormalities associated with tumors, including observation of findings of excretory urography, ultrasonography and CT scanning, which appeared difficult to interpret.

\section{Room M}

\section{SS 05.05 Bladder}

$2.00 \mathrm{PM}$

\section{Imaging of the reconstructed bladder (Keynote Lecture) \\ H.M. Saxton; London}

For some 40 years the commonest bladder "substitute" in severe bladder disorders has been an ileal conduit emptying via a stoma into a drainage bag. Recently attempts have been made to achieve urine storage with no external stoma and with preservation of the urethra or the provision of continent access for catheterisation.

The material described consists of 111 cases of cystoplasty in two forms: 1. Augmentation cystoplasty ${ }^{(44)}$ : In severe chronic cystitis, bladder instability or neuropathic bladder. The aim is to increase bladder capacity and reduce over contractility by interrupting muscle fibres. With sphincter incompetence or overaction, an artificial urinary sphincter (AUS) is used.

The bladder is opened transversely and a segment of ileum or caccum introduced, the ureters implant normally.

2. Replacement cystoplasty(67): For patients with no appreciable bladder remnant eg following operation for malignant disease. The inverted caccum is attached to the bladder base or urethra. An artificial sphincter may be needed if sphincter function is impaired. The ureters are implanted in the upper part, in the caecum itself.

In both types of operation voiding is by sphincter relaxation and abdominal straining.

Imaging

The material presented is the assessment of the functional results of operation using video urodynamics (VUD). The particular features noted were the capacity of the "bladder"; its compliance; the presence or absence of spontaneous contractions, usually colonic, and whether these resulted in leakage; the effectiveness or otherwise of the sphincter mechanism and the adequacy of voiding.

Augmentation cystoplasty

Of 44 patients $21(47.7 \%)$ had a completely satisfactory result with no leakage and with adequate voiding or drainage via a catheter. Among 27 without AUS leakage occurred in 9 due to sphincter weakness $(33.3 \%)$ as against $7(26 \%)$ with colonic contractions. Of 17 with artificial sphincters only $2(11.7 \%)$ showed stress leakage and 6 contractile leakage.

Replacement cystoplasty

34 out of $67(50.7 \% \mathrm{p})$ had effective storage and emptying. Among 23 with AUS $6(26 \%)$ showed contractile leakage: 4 showed sphincter weakness. In 44 without AUS $16(36.3 \%)$ showed pressure leakage: 8 were associated with a functionally ineffective cystoplasty with a capacity of less than $100 \mathrm{ml}$. There were $7(16 \%)$ with sphincter weakness.

Conclusions

High pressures in the substituted or augmented bladder due to colonic contractions do not cause leakage if the sphincter mechanism is adequate. VUD has also shown functional inadequacy of the replaced bladder in a small group, mainly those without sphincters. Substitution and augmentation procedures promise improved functional results for patients whose bladders are severely diseased and VUD is helpful in assessment and in indicating appropriate management.

\subsection{PM}

\section{Radiological studies before bladder reconstruction}

J. Hughes, C. Evans, T.P. Stephenson; Cardiff

Over 200 patients have been treated by bladder reconstruction in our centre in the past 10 years. Radiological studies are essential prior to reconstruction especially in the neuropath, before undiversion and in complex cases. IVU and isotope studies (DTPA or MAG 3) will determine total and relative renal function and more importantly, ureteric size, motility and degree of obstruction, if any.

Videocystography will determine the size and configuration of the bladder on which augmentation or substitution will depend, augmentation being preferable if the bladder is larger than $150 \mathrm{cc}$ and not trabeculated. Visualisation of the bladder neck, sphincter and the leak pressure will determine if an artificial urinary sphincter is necessary.

In patients for undiversion the loopogram will determine whether part of the loop can be used for the reconstruction and reimplantation. Urethrography is necessary to determine whether reconstruction is feasible when the patient has had a cystectomy.

Radiological studies will thus usually, but not always determine the precise nature of the reconstructive technique to be used.

\section{$2.25 \mathrm{PM}$}

\section{C.T. staging of urinary bladder tumours O.P. Sharma; Bombay}

Staging of carcinoma of urinary bladder is very important from view point of choices of therapy and also has prognostic implications. A system for staging of urinary bladder tumours was introduced by Jewett and Strong in 1946 and later modified by Marshall. However, most accepted classification is that, adopted by International Union Against Cancer (Wallace et al.).

Computed tomography is a noninvasive diagnostic modality which precisely delineates the disease in the bladder, involvement of adjacent structures, nodal status and distant metastases to liver etc. and renal function.

In this presentation effort will be made in 132 cases evaluated by C.T. Stage accuracy in surgically confirmed cases. C.T. scan has ability to show whether tumour is less than stage $\mathrm{B} 2$ or more, on which depends the treatment modality. Stage B2 or lesser lesions are treated by local excision, stage C lesions by radical cystectomy and or radiation, while stage D lesions are unsuitable for surgical resection.

We were able to achieve $100 \%$ accuracy in less than B2 lesions, while in stage C lesions $75 \%$ and in stage D $61 \%$ accuracy. Our nodal accuracy being $73 \%$.

Thus with C.T. staging, modality of treatment can be predetermined and unnecessary surgery be avoided.

\section{$2.35 \mathrm{PM}$}

\section{The role of MRI in the staging of urinary bladder carcinoma} Jelle O. Barentsz, Sjef H. J. Ruijs, Gerd Rosenbusch, Frans M. J. Debruyne, Herbert F. M. Karthaus, Theo W. J. Witkamp; Nijmegen

Purpose: Evaluation of MRI in relationship to clinical staging method, CT, and histopathologic findings in 60 patients with urinary bladder carcinoma. Methods: The staging results of CT, MRI, and the clinical staging method are compared to histologic findings in 60 patients ( 38 had cystectomy, 22 had deep TUR including a follow-up of $1-3$ years).

Results: In T staging MRI proved to be more accurate than CT ( $85 \%$ and $58 \%$ respectively). Only differentiation between stage T3A and T3B was equal for both methods. The clinical staging method was far less accurate from stage T2 on (accuracy $33 \%$ ), but proved to be useful in superficial tumors (stage T2 or less).

In assessing the $\mathrm{N}$ stage, MRI turned out to be the best imaging method. A 95\% accuracy was found for MRI versus $82 \%$ and $85 \%$ for CT and lymphography respectively.

Also distant metastases (M staging) can be found by MRI (in 9 out of 10 patients).

Conclusions: It can be concluded, that MRI and clinical staging are supplementary. In superficial tumors (stage T2 or less) the clinical staging is more accurate, whereas MRI is superior to all other methods in more infiltrative tumors (stage T2 or higher).

\subsection{PM}

\section{Staging bladder neoplasms with magnetic resonance imaging} Luis Mazaira, Vicente Lozano, Inmaculada Ayala, Manoli Rebolledo; Madrid

Authors report a retrospective study with MR imaging in the determination of local disease extent (staging), of primary bladder neoplasms. This paper describes our initial experience with a total of 20 patients, using a $1.5 \mathrm{~T}$. superconducting Magnetom Siemens. We have used a double echo technique ( $\mathrm{TR}=1300 \mu \mathrm{sec}, \mathrm{TE}=20-90 \mu \mathrm{sec})$, trying decrease imaging time. The results were compared and verified with findings at biopsy, surgical exploration, or follow-up.

In conclusion, we have obtained a high "accuracy and specificity" for evaluation of deep muscle invasion, perivesical extension, and adjacent visceral infiltration. Problem areas include detection of pelvic lymph node metastases.

\subsection{PM}

452 MRI of urinary bladder carcinoma: tumorstaging and Gadolinium contrast enhanced imaging

E. Doringer, H. Joos, R. Forstner, H. J. Schmoller; Salzburg

Purpose: Is a better preoperative tumorstaging possible using Gd-DTPA contrast enhanced MRI?

Methods: Before onset of therapy 33 patients were examined with $\mathrm{T} 1 \mathrm{w}$ images before and after administration of $0,1 \mathrm{mmol} / \mathrm{kg} \mathrm{Gd}$-DTPA as well as T2 w images in at least 2 planes. Results were compared with the final pathological classification after TUER and bimanual palpation. The signal intensity ratio of 
tumor tissue/fat and tumor/muscle were calculated on T1 $w$ images and after Gd-DTPA and examined for their statistical significance.

Results: The increase in signal intensity of the tumors after administration of Gd-DTPA was statistically significant (Wilcoxon test $p<0,01$ ). Staging: The stages Tis-T2 were summarized. Correct staging was successful in the precontrast diagnosis $(\mathrm{T} 1, \mathrm{~T} 2)$ in $8 / 15$ cases $(60 \%)$, after Gd-DTPA in $11 / 15(73 \%)$. T3a: no benefit from Gd-enhanced images. MRI was $2 \times$ correct, 2 over-, 1 understaged. T3b $(n=8), T 4(n=7)$ : These stages were correctly recognized in all cases, including one case where the infiltration of the rectum was seen only after Gd-DTPA. In relation to all stages the Gd contrast enhanced MRI showed correct staging in 28 of 33 tumors $(84,8 \%)$, the precontrast MRI (T1, T2) in 25 of 33 tumors $(75,7 \%)$

Conclusions: Because of the relatively short acquisition time of $\mathrm{T} 1 \mathrm{w}$ images and the specific tumor enhancement, administration of Gd-DTPA proved to be of help in the staging of urinary bladder carcinoma.

\section{$2.58 \mathrm{PM}$}

453 Possibilities of the diagnostic of the urothel-carcinoma - a comparative study with $\mathrm{CT}$, MR and radio-immuno-scintigraphy P. Benz, E. Becht, A. Seeliger, B. Junk, H. Hadidi, U. Hau, W. Dewes, E. Oberhausen; Hamburg

The aim was to point out the different diagnostic abilities of modern imaging methods as CT, MR and radio-immuno-scintigraphy (RIS).

35 patients with histological proven urothelian carcinoma and different grading and staging were examined with all three methods and the results operatively verificated.

All three methods showed a sensitivity of more than $90 \%$ and different specificities between 70 and $95 \%$.

An optimated diagnostic proceeding in patients with urothelian carcinomas is formulated concerning proof of the primary tumor and existance of metastasis. Practicability and diagnostic valence of the methods are discussed.

\section{$3.08 \mathrm{PM}$}

454 Pulse sequence optimization in 100 patients with urinary bladder carcinoma at $1.5 \mathrm{~T}$

Jelle O. Barentsz, Sjef H.J. Ruijs, Leon J.T.O. van Erning, Chris J.G. Bakker; Nijmegen

Purpose: To determine the optimal pulse sequence(s) for staging urinary bladder cancer (UBC).

Methods: In 14 patients with UBC T1 and T2 values of perivesical fat, bladder wall, urine, and tumor were estimated. On the basis of these values contrast matrices were calculated, from which the sequences with optimal contrast between these structures could be derived. Additionally, the changes in contrast between these structures were illustrated by synthetic imaging. To confirm the value of the found pulse sequences in staging of UBC, they were applied for 100 patients with UBC.

Results: Optimal staging of $\mathrm{UBC}$ requires at least 2 pulse sequences:

1. A T1 weighted pulse sequence ( $\mathrm{SE} / 750 / 15)$ is optimal to determine tumor invasion in perivesical fat, and to recognise enlarged lymphnodes and bone marrow infiltration.

2. A T2 weighted pulse sequence (SE/2100/30,100) is optimal to determine tumor invasion in bladder wall, prostate, seminal vesicles or uterus, and to differentiate between tumor and fibrosis.

The STIR sequence $(\mathrm{IR} / 2000 / 175 / 60)$ is superior to the SE/2100/30,100 sequence to recognise bone marrow metastases.

Conclusions: Optimal pulse sequences for staging UBC are SE/750/15 and $\mathrm{SE} / 2100 / 30,100$. In bone marrow metastases the IR/2000/175/60 sequence has advantages over the $\mathrm{SE} / 2100 / 30,100$ sequence.

\subsection{PM}

\section{Gd-DTPA in MR diagnosis of urinary bladder carcinomas} Anne Sparenberg. Bernd Hamm, Peter Hammerer, Monika Samberger, K.-J. Wolf; Berlin

Purpose: It is known that MRI is superior to CT in the diagnosis of bladder carcinoma, especially due to the visualization of intramural tumor growth. Whether the application of Gd-DTPA markedly improves MR diagnostics has not yet been clarified.

Methods and Materials: In a prospective study 110 patients with urinary bladder carcinoma were examined by MRI (1.5 tesla, Siemens). SE-sequences (T1- and T2-weighted) in axial and sagittal planes were used for all patients. Seventy patients additionally received i.v. injections of Gd-DTPA $(0.1 \mathrm{mmol} / \mathrm{kg}) .80$ patients underwent TUR, in 230 patients a cystectomy was performed.

Results: 1. Contrast application considerably improved accuracy in tumors staged Tis and $\mathrm{Tl}$ - from $33 \%$ (precontrast) to $71 \%$ (postcontrast). 2. The tumor infiltration of neighboring organs (T4) was better demarcated using Gd-DTPA. 3. The diagnosis of $\mathrm{T} 2$ to T3b tumor stages was not improved by GD-DTPA. 4 .
Total accuracy in the bladder carcinoma group increased from $69 \%$ (precontrast) to $83 \%$ (postcontrast).

Conclusions: 1. Performance of T1- and T2-weighted spin-echo sequences. 2. Intravenous application of Gd-DTPA is indicated if infiltration of the bladder wall or neighboring organs has not been clarified.

\section{Room N}

\section{SS}

\subsection{PM}

\section{Imaging in diseases principally affecting white matter (Keynote Lecture)}

Brian E. Kendall; London

Irregular periventricular and discrete foci of altered signal (increased $\mathrm{T} 1$ and T2), mainly in white matter, visible in the great majority of clinically definite MS correspond to plaques on histology. A significant relationship has been confirmed between total plaque volume and neuropsychological but not physical disability, which is influenced by spinal cord and posterior fossa lesions.

Similar changes occur in -

1. c. $70 \%$ of adults with optic neuritis: $40 \%$ of these develop clinically definite MS within 1 year. Optic neuritis with normal brain on initial MRI did not develop evidence of MS within a year. STIR shows plaques in $84 \%$ of symptomatic and $20 \%$ of asymptomatic nerves: lesions longer than $1.0 \mathrm{~cm}$ or in the optic canal have poor visual prognosis.

2. c. $75 \%$ with clinically isolated brainstem symptoms, $36 \%$ with acute and $61 \%$ with chronic cord lesions, again with a higher rate of progression to overt MS. In acute plaques damage to the blood-brain barrier allows leakage of GdDTPA over a period of 2-8/52: T1 and T2 increase as water protons replace focal myelin loss. Loss of water in subacute lesions may reduce visibility on MRI. Gliosis follows and watery cytoplasm within fibrillary astrocytic processes increase $\mathrm{T} 1$ and $\mathrm{T} 2$, less than in acute lesion.

Images simulating MS occur with

1. non-inflammatory small vessel disease - relative differences include grey matter involvement: subependymal, brainstem, corpus callosum and $U$ fibre involvement and cerebellar and brainstem atrophy are more frequent in MS and plaques are more often visible on T1W images.

2. vasculitis - often causes cortical involvement and smooth periventricular changes. Bechet's disease favours the brainstem and may cause mass effect simulating glioma.

3. allergic encephalomyelitis - a monophasic disease, with lesions occurring during a short time-period: new foci after $6 / 12$ suggest MS. In exanthemata single, sometimes large, or diffuse small lesions occur and usually regress but resolution may be incomplete. SSPE is generally evident clinically before small white matter lesions appear on MRI.

4. neurosarcoidosis - meningeal and multiple cortical involvement may distinguish. Neurobrucellosis may rarely cause demyelination simulating MS. Demyelination may simulate

1. dysmyelination. Inherited enzyme defects and leucodystrophies of unknown aetiology characteristically but not invariably cause symmetrical lesions.

2. large isolated focal lesions, including infarcts, neoplasms and acute haemorrhagic leucoencephalitis. Large plaques with mass effect mainly involve white matter and are rarely solitary: irregular ventricular narrowing suggests neoplasm or granuloma. Central pontine myelinolysis even if atypically located generally complicates sodium depletion. Progressive multifocal leucoencephalopathy follows immunosuppression: white matter is predominantly but not exclusively involved.

3. Progressive cerebellar degenerations, in which periventricular signal change may occasionally accompany the symmetrical atrophy.

\section{$2.15 \mathrm{PM}$}

\section{White matter lesions in psychiatric patients: a retrospective MR study}

T. Becker, E. Teichmann, E. Hofmann, M. Warmuth-Metz, M. Nadjmi; Würzburg

a) Aim: To establish prevalence, distribution and age dependence of white matter lesions (WML) in psychiatric disorders.

b) T2-weighted MRI scans were retrospectively reviewed for hyperintense focal WML in 100 psychiatric patients. Diagnosis was determined according to ICD-9 and DSM-III R.

c) Mean age was 48 years, mean WML number was 12. Correlation of WML number with age was positive $(r=0.3)$. There was a close correlation with periventricular hyperintensity, not with ratings of Virchow-Robin spaces. Both hemispheres were involved in a comparative manner, with a strong frontal and parietal lobe predilection. WML number in patients with affective disorders did not differ from demented subjects.

d) There were no gross differences when the present sample was compared with 
non-psychiatric controls. WML do not discriminate demented subjects from patients with affective disorders.

\subsection{PM}

458 Unspecific white matter lesions: prevalence, number and distribution in cranial MRI

E. Hofmann, M. Sedaghat-Kerdar, T. Becker, M. Warmuth-Metz, M. Nadjmi; Würzburg

a) Aim: To establish data about prevalence, number and topography of unspecific white matter lesions as seen on MRI.

b) T2-weighted MRI scans of 83 patients were retrospectively reviewed for hyperintense focal white matter changes. Patients with known inflammatory CNS disease were excluded.

c) There was an approximately linear increase of prevalence and number of lesions with patient age. Prevalence ranged from $18 \%$ in the second decade to over $90 \%$ in those over 70 ys. There was a close correlation with the degree of concomitant periventricular hyperintensity. However, rating of VirchowRobin spaces did not correlate with the number of white matter lesions. Both hemispheres were involved nearly equally with a minimal right side preponderance. Lesions showed a strong dilection for frontal and parietal lobes. d) The present study provides statistical and topographic data for assessment of white matter lesions which are a common finding in daily MRI routine.

\section{$2.35 \mathrm{PM}$}

\section{CT- and MRI long term follow-up in the differential diagnosis of AIDS related brain diseases}

\section{A. Schwartz, M. Mull, G. Arendt, A. Aulich; Düsseldorf}

Purpose: Patients with CNS-manifestations in acquired immunodeficiency syndrome were examined by long-term follow-up CT- and MRI scanning to evaluate the early beginning of opportunistic brain infection and/or encephalopathy.

Methods: During the past five years 84 patients with focal neurological symptoms and/or signs of demantia out of 380 HIV infected individuals were followed up by CT and MRI-scans.

Results: $22(26 \%)$ patients had normal scans, $34(40,1 \%)$ clinically showing slight focal symptoms had contrast enhancing nodular structures in CT and MRI scans as characteristic findings of opportunistic brain infections disappearing in the follow-up scans under adequate therapy. Radiological criteria of underlying encephalopathy (11 patients) could be detected only by follow-up examinations. In 17 (20\%) patients further pathological findings could be confirmed: cortical atrophy $(n=9)$, hydrocephalus $(n=4)$, infarct $(n=2)$ and astrocytoma $(\mathrm{n}=1)$.

Conclusions: Short term follow-up scans only have a value in measuring time course and therapy effectivity of opportunistic brain infections often localized in the basal ganglia. In contrast, encephalopathy only could be proved by consequently undertaken long term follow-up scans. Further studies must prove, if radiological methods will be able to reveal signs of HIV related brain diseases not only in clinically symptomatic but in asymptomatic individuals giving further informations on the time course of these diseases.

\section{$2.45 \mathrm{PM}$}

\section{MR imaging of herpes simplex encephalitis}

Ph. Demaerel, G. Wilms, W. Robberecht, K. Johannik, P. Van Hecke, H. Carton; Leuven

The Magnetic Resonance (MR) findings in 8 patients with herpes simplex meningo-encephalitis were reviewed. A total of 14 examinations were analyzed. The most important finding was the high signal intensity area in the temporal lobe(s) with its typical configuration known from CT. Meningeal enhancement after Gd-DTPA administration was clearly visualized in 4 patients.

The hemorrhagic changes are much better seen on MRI. When adequate motion control can be achieved, MRI becomes the examination of choice in the diagnosis and follow-up of Herpes simplex encephalitis. Also localized ${ }^{1} \mathrm{H}$ NMR spectroscopy proved promising in the evaluation of neuronal loss in Herpes simplex encephalitis.

\section{$2.55 \mathrm{PM}$}

\section{Intracranial tuberculoma, neuroradiologic findings}

N. Bargalló, J. Berenguer, C. Nicolau, X. Tomás, G. Garcia, J. M. Mercader; Barcelona

Purpose: Appearance by computed tomography (CT) and magnetic resonance (MR) of intracranial tuberculomas are described.

Methods and Materials: We present seven patients, six males and one female (age range 19-80 mean 42), with intracranial tuberculomas studied over the past four years. Three patients had AIDS, among the four non-AIDS patients, one had undergone hepatic transplantation. All cases were studied with CT and five underwent MR. Diagnosis of intracranial tuberculoma was histologically determined in four patients and in the remaining patients was made by good response to specific therapy.

\begin{tabular}{llllll}
\hline Results & $\begin{array}{l}\text { Number } \\
\text { of lesion }\end{array}$ & location & $\begin{array}{l}\text { meningeal stage } \\
\text { enhance- } \\
\text { ment }\end{array}$ & $\begin{array}{l}\text { hydro- } \\
\text { evolution } \\
\text { cephalus }\end{array}$ \\
\hline 1. CT, MR & single & cerebellum & no & incipient & yes \\
2. CT, MR & single & cerebellum & no & mature & yes \\
3. CT & single & occipital 1. & no & - & no \\
4. CT, MR & single & brain stem. no & mature & no \\
5. CT & single & parietal 1. no & - & no \\
6. CT, MR & single & protuberance no & abscess & no \\
7. CT, MR & multiple & $\begin{array}{l}\text { cerbellum, no } \\
\text { incipient }\end{array}$ & no \\
& & frontal 1. & & \\
\hline
\end{tabular}

Conclusions: 1-CT scans normally show iso or hyperdense lesions with hypodense surrounding edema and ring or nodular enhancement. 2-MR studies are more sensitive and discriminative than CT scans, particularly on T2-weighted sequences, to assess the evolution stage of the lesions, showing several features depending on grade of maturity. 3-No significant differences were observed between the AIDS and non AIDS patients, although no definitive conclusions may be drawn due to the small number of patients studied.

\subsection{PM}

462 Non-contrast MR cisternography and digital subtraction cisternography in CSF fistula

A. K. Wakhloo, M. Schumacher, V. van Velthoven; Freiburg

Exact preoperative localization of dural defect in CSF rhinorrhea is essential for successful surgery.

Heavily T2 weighted MR images (modification of the rapid acquisition with relaxation enhancement imaging), digital subtraction cisternography (DSC) in real time technique ( 3 pictures/s) and subsequently coronal CT cisternography (CTC) was carried out in 16 patients with traumatic, postoperative and spontaneous CSF rhinorrhea and 1 patient with CSF otorrhea. MRI performed in 5 cases, depicted the CSF fistula in 3 patients. DSC revealed the exact site of leakage in $9 / \mathrm{II}$ cases with active CSF leakage. DSC performed in non-drop period ( 6 cases) could delineate the area of dural defect in one case only after artificial increase of CSF pressure. CT cisternography confirmed the findings except in the latter case. Surgery was carried out successfully in 9 cases and confirmed the predicted site of dural defect.

It is suggested that heavily T2 weighted MR images are helpful in detecting the CSF fistula in severe CSF thinorrhea. DSC can be recommended in addition to CT cisternography especially when artificial increase of CSF pressure is required in non-drop period.

\subsection{PM}

463 Progressive diffuse leukoencephalopathy (PDL) in AIDS, a distinct lesion pattern detectable by CT and MRI

H. Henkes, J. Artigas, R. Jochens, W. Schörner, G. Huber, T.Scherb, U. Piepgras; Homburg/Saar

We report upon a distinct pattern of white matter lesions in AIDS that by means of CT and MRI can definitely be differentiated from other types of CNS involvement in the disease. The clinical appearance is that of a profound psycho-organic syndrome.

Patients and methods: The present study is based on the clinical and imaging data of 350 MRI examinations, carried out in 220 patients with central nervous system manifestations of the HIV infection. All studies have been performed using a 3rd generation CT scanner and a $0.5 \mathrm{~T}$ MRI unit. The imaging protocol was in accordance with clinical routine procedures, including contrast enhanced studies in both imaging modalities and MR imaging with T1- and T2-weighted sequences.

Results: In 7 AIDS patients, presenting with severe dementia, we observed bilateral-symmetric, confluent white matter lesions, located in the frontal and parietal lobe. The affected brain regions were hypodense on CT and hyperintense on T2-WI in MRI, without mass effect or contrast enhancement. CT failed in the detection of brain stem involvement (i.e. Wallerian degeneration) that was clearly seen on MRI. Pathologically, we observed diffuse loss of myelin, subcortical invasion of macrophages, vacuoles in the white matter and multinucleated giant cells.

Conclusions: For the bilateral-symmetric, frontally accentuated leukoencephalopathy in AIDS patients, detected by CT or MRI, we propose the acronym "PDL". It can be defined by a clear cut CT and MRI appearance as well as by distinct neuropathologic findings. Most probably, this pattern represents a rare, severe variant of HIV leukoencephalopathy. 
Room 0

\section{SS}

15.05 New developments in US, various

\subsection{PM}

\section{New technical developments in ultrasound (Keynote Lecture)} P.N.T. Wells; Bristol

Ultrasonic Doppler studies of blood flow are giving new insight into vascular disease and tissue characterisation, particularly in the detection of malignancy. Improved spatial and contrast resolution resulting from the use of wideband transducers has led to more reliable detection of, for example, liver metastases. Speckle reduction may provide additional advantages. Intracavitary and intravascular probes are being developed. Techniques for resolution improvement based on phase aberrration correction and system optimisation are promising. Sensitivity and the vector problem limit the usefulness of colour flow mapping but time domain processing may provide some solutions. Contrast agents that can cross the lungs are available and novel contrast enhancement schemes are being investigated. There is great interest in three-dimensional data acquisition and image display; it is now recognised that ultrasonic scanners will have to be integrated with image management and communications systems.

\subsection{PM}

465 Conditions for an in-vivo-like phantom for evaluating duplex and colour-coded Doppler devices

Ph. Hendrickx, F. Brassel, U. Roth, H. Wagner; Hannover

Quantitative measurement of blood flow gets an increasing value in vascular pathology. Especially for duplex and colour-coded Doppler devices it is of first importance to check under realistic conditions, how far the indicated blood velocities agree.

Therefore, an experimental circulatory phantom was constructed, taking into account following conditions:

1. The design consists of a piston-diaphragm metering pump with an adjustable piston volume and frequency of stroke.

2. Silicone tubings with different elasticity and diameters comparable with cadaver arteries.

3. Blood or an ultrasonic blood-like solution as circulating fluid.

4. Surrounding soft tissues of the artery near the Doppler probe.

5. The transducer should be manually guided.

Thus, comparable to physiological frequency spectral displays, different types of wave forms (e.g. monophasic, triphasic) were initiated to analyse the reliability of duplex and colour-coded Doppler devices for practical use.

\section{$2.25 \mathrm{PM}$}

\section{Continuous wave, pulsed wave and velocity mapping ultrasound Doppler techniques: properties and limitations}

Dietmar Hiller, Hans Kaarmann; Erlangen

Introduction and refinement of ultrasound Doppler techniques has resulted in the possibility of qualitative and quantitative non-invasive measurement of flow velocities in the body, and specifically, the haemodynamic properties of the heart, large vessels and vascularized tissue. For optimal use, as well as proper interpretation of results and for comparison of ultrasound to other invasive and non-invasive methods, it is important to consider the physical and technical concepts of each of the ultrasound Doppler modalities.

The different Doppler techniques, like continuous wave, pulsed wave and velocity mapping (color flow imaging) are reviewed, their principles and technical implementations are discussed and their properties and limitations, in particular relating to sensitivity and accuracy, are critically evaluated.

\section{$2.35 \mathrm{PM}$} 467. A new phantom for quality assurance of color-coded US flow
equipments

R. Novario, A. Goddi, A. Crespi, L. Conte, L. Bianchi, C. Bianchi, C. Mordacchini; Varese

Colour-doppler US imaging is now in routine clinical use for the evaluation of blood flow in vessels. We propose a special phantom for a QA program of colour-coded flow equipments. It is composed of an empty container into which various inserts can be applied permitting the reproduction of two opposed parallel flows of a synthetic blood. The container is then filled with a tissue equivalent solution. Known flows were made to run using a pump, a potentiometer and a flowmeter. It is possible to vary the liquid flow, the caliber of the simulated blood vessels, their inter-axial distance and their depth below the surface of the phantom. Results concerning measurements on two commercial devices are presented and their different characteristics are pointed out.
2.45 PM

468 The performance characteristics of a new film/screen system J.Beutel, B. A. Apple, D. J. Mickewich, R. Shaw; Wilmington, DE

Models are able to predict how the image quality of film screen systems varies as a function of systems design parameters. In particular, these models demonstrate that significant image quality improvements are available if printthrough were eliminated. An obstacle to practical implementation of this advantage has been the absorption behavior of silver halide films. Recent developments indicate that there may be a solution to this problem. We describe the image quality characteristics of an experimental film/screen system which approaches model predictions.

\section{$2.55 \mathrm{PM}$}

469 The imaging performance of high-strip-density radiographic grids - A physical and clinical evaluation H. Aichinger, F. Staudt, W. Müller; Erlangen

Purpose: The employment of antiscatter radiographic grids in combination with a moving Bucky tray is the most widely used method for reducing the amount of scattered radiation and for improving contrast of radiographic images. Modern grid technology makes the manufacturing of high-stripdensity radiographic grids possible, so that movement of the grids during exposure to radiation is not necessary. Additional advantages include reduction of geometric unsharpness, as well as the potential for decreased patient dose through improvement of the primary transmission and the elimination of decentering.

Methods and Materials: Conventional $8 / 40$ and 12/40 grids were examined and compared with a new stationary $17 / 70$ grid with regard to their grid performance parameters. With the help of computer simulations, as well as phantom and patient exposures, the resulting image quality was investigated. Results: The new high-strip-density grid $17 / 70$ provides contrast improvement factors and Bucky factors comparable to or slightly better than those obtained with a conventional $12 / 40$ grid. On the condition that paper is used as interspace material the primary transmission can be improved, whereby a slightly increased scatter transmission can be tolerated.

Conclusions: High-strip-density grids can be used in place of conventional grids without compromising image quality. As a result of the improved primary transmission and the elimination of decentering, the patient dose can be reduced. These advantages are of great importance, especially in digital radiography.

\subsection{PM}

470 First results of a prototype device for universal realtime stereo X-ray fluoroscopy with digital image processing for interventional procedures

G. Hahm, T.Peters, D. Tampieri, J.Weigl, H. Sklebitz, H.-D. Hofmockel, H. Horbaschek, R. Ethier; Erlangen

Purpose: Increased three dimensional visualization for interventional procedures, utilizing a new imaging system which provides real time three dimensional fluoroscopic images.

Methods: A prototype stereo X-ray TV and cine system has been adapted for the needs of the practical evaluation in clinical environment. This system operates with commonly available components in reference to the imaging chain and does not require specialized equipment regarding the $\mathrm{X}$-ray image intensifier, TV camera, ghost image suppressions and digital image processing. Results: Present day technological advances in image pick-up, image processing and display technology enable a decisive step towards a flexible clinically useful X-ray stereo fluoroscopic system. This system has potentially promising applications in all types of interventional radiology, such as angio exams in neurology, cardiology etc. First clinical results from this prototype system at the Montreal Neurological Institute of McGill University will be demonstrated and discussed.

Conclusions: Stereo fluoroscopy offers great potential advantages for all interventional applications due to better visualization, increased working speed, reduced radiation and results in safer procedures.

\subsection{PM}

\section{Sandwich dosemeter for quality assurance in diagnostic radiology} H. Lenzen, M. Fiebich, N. Meier; Münster

During the last years quality control grew up to a well established procedure in the diagnostic radiology. An important aim was to distinguish errors in the developing process and in the X-ray equipment respectively. Unfortunately this desired separation can be only incompletely obtained using the actual version of DIN 6868. The developing process can be checked tolerably exact whereas the test results of the X-ray equipments are also depending on the choosen charge of films, the film cassette and the developing process. Such a 
dependence results from the use of films as a measuring instrument for output dose and energy of X-rays. Moreover the actual method of dose measurement (input dose) is rather insensitive to $\mathrm{mAs}$ variations using free exposure technique. With a sandwich dosemeter (semiconductors before and behind the absorber) input and output dose can be measured simultaneously. The dose D2 (behind the absorber) is highly sensitive to $\mathrm{mAs}$ variations and the quotient D1/D2 to variations of energy. A prototype of the described sandwich dosemeter was developed and investigated in the clinical routine. As benefits can be presented a clear separation of developing process and X-ray equipment, a raised sensitivity to dose and $\mathrm{kV}$ variations and a simplified measurement saving up to $75 \%$ films.

\section{Room R}

\section{SS}

\subsection{Treatment planning in radiotherapy II}

$2.00 \mathrm{PM}$

\section{External beam treatment planning - can we deliver what we plan? (Keynote Lecture)}

R. M. Harrison; Newcastle upon Tyne

Many sources of uncertainty exist in the delivery of the prescribed dose to the specified target volume in external beam radiotherapy. Their magnitudes are compared with recommendations for the overall accuracy of dose delivery $( \pm 3.5-5.0 \%)$. Five dose intercomparisons are reviewed, including a recent UK survey undertaken by the Institute of Physical Sciences in Medicine (IPSM). For phantom doses, standard deviations in the ratio of stated to delivered doses range from $1-3.5 \%$, with larger uncertainties when irradiation of volumes within anthropomorphic phantoms is considered. In addition, uncertainties will arise from geometrical discrepancies; the role of portal films in their assessment is also considered. Imaging during radiotherapy will be increasingly important in the development of complex conformal treatments, where the shape of the target volume may be irregular. Specific recommendations for future work include:

(I) the development of on-line portal imaging so that patient movement and positional errors can be examined both during and between treatment fractions.

(II) regular quantitative comparisons of portal images with simulator images, though the development of digital imaging. This work might exploit the advances in digital transducer design such as photostimulable luminescence and image intensifier-TV technology currently employed in diagnostic imaging systems.

(III) thorough evaluation of complex radiotherapy treatment planning system algorithms.

(IV) continued refinement and standardisation of dosimetry protocols.

\subsection{PM}

473 Sectional images as a valuable aid in brachytherapy planning Experiences in gynecologic radiotherapy-patients with magnetic resonance, computed tomography and real time ultrasound imaging (Keynote Lecture)

B. F. Schmidt, P. Hirnle, Th. W. Kaulich, M. Skalej, M. Bamberg; Stuttgart

In 29 patients (pts) with cervical or endometrial cancer magnetic resonance images (MRI) of the pelvis were taken in three dimensions. In all pts a non artifact giving plastic HDR-afterloading-brachytherapy dummy-applicator was inserted in intrauterine brachytherapy position just before imaging. By this means valid information became available concerning the topographic relationships between the individual tumor size and shape, the organs at radiation risk, and the applicator. Superimposition of different brachytherapy isodose patterns allowed election of the best fitting one. The MRI-based planning procedure resulted in an individual optimization of the brachytherapy isodose-distribution in $48 \%$. In about $18 \%$ considerable technical mistakes in brachytherapy could be avoided. In 10 of these pts pelvic CT-scanning and percutaneous ultrasound were done also for brachytherapy-planning purpose. Comparison of the three methods showed the significant advantages of MRI, but showed also some practical advantages of ultrasound.

474 CT and MRI for radiation therapy planning and therapeutic monitoring in CNS-tumors

S. Sehlen, D. Matthaei, M. Busch, K. Dieckmann, E. Dühmke; Göttingen

CT in CNS-tumors for treatment planning is regarded the primary imaging modality. The role of NMR remains to be defined due mostly to the multitude of MR sequences and their varying informations. Here an investigation of about 40 patients is presented with both methods (CT and NMR) to compare their treatment planning capabilities. Standard CT and MRI were compared by different clinicians for their capability to localize the optimal target volume in order, to minimize radiation damage to normal tissue. Different MR sequences were additionally used for first descriptive evaluations.

The patients were affected by the following diseases: 2 lymphoma, 2 germinomas, 4 oligodendrogliomas, 5 glioblastomas, 5 astrocytomas, 9 metastases, 1 craniopharyngioma, 1 ganglioglioma, 2 medulloblastoma, 1 aesthesioneuroblastoma, 1 glioma of the optic nerve, 1 glomus tumor, 2 pituitary adenoma, 2 eosinophilic granulomas, 1 ependymoma, 1 rhabdoid tumor.

MRI was found to have superior information in the recognition of the relationship edema/tumor, in the demonstration of multiple site disease, and in the regions prone to CT-artifacts. CT proved to be diagnostically useful for planning of radiation therapy of tumors with anatomical relationship to bone structures. Additionally experience with experimental MR sequences is presented, that promises MR advantages for treatment planning concerning fast arbitrary contrast, high resolution (3D in a couple of seconds) and vascular informations.

\section{$2.50 \mathrm{PM}$}

475 Multiaxial overcenterable conformation radiotherapy for retroperitoneal lymph node metastases

T. Takahashi, K. Machida, N. Honda, T. Mamiya, T. Takishima, T. Kamano, S. Tamaki; Saitama

Geometrically, radiation treatment planning of retroperitoneal lymph nodes is complex as the target volume looks like a curved cylinder of varying crosssection with two wings in pelvic region. In order to deliver a homogeneous tumoricidal radiation dose to an accurately localized target volume in retroperitoneal and pelvic area and not to irradiate tissue outside that volume, computer controlled multiaxial overcenterable conformation radiotherapy is proposed.

The system includes overrunning multileaf collimator with independently movable multiple pairs of jaws to define unsymmetric irregular fields. The developed components of treatment planning units consist of 3D-treatment modelling, -dose calculation and -display capabilities, with volume dose histogram.

Also using multiaxial conformal rotation technique, we have applied this method to the radiotherapy of involved retroperitoneal and pelvic lymph nodes in various tumors of pelvic organs so that it has become possible to conform radiation more nearly to the target volume of irregular shape and non axial extention and thereby to spare adjacent normal tissue.

This approach to local control may prove clinically effective and provide a viable alternative to other forms of radiation.

\subsection{PM}

476 Digital reconstructed radiograph (DRR), a helpful instrument for patient positioning in radiation treatment planning

J. Romahn, J. Doll, G. Gademann, W. Schlegel, G. van Kaick, M. Wannenmacher; Heidelberg

The improvement of modern imaging methods (CT, MRT) and their combination with sophisticated $3 \mathrm{D}$ treatment planning systems allows a better definition of tumor and target volumes and this makes a more tumor related distribution of dose possible, by use of irregular field configurations and of more complicated irradiation techniques.

On the premises of identical patient position during planning procedures and therapy it is difficult to find exactly the computer calculated position of central beam axises.

DRR allows a reconstruction of plane film radiographs on the basis of the same set of CT data used for the computer planning of radiation treatment. It observes the projection rules of deverging beams and is capable of image reconstruction under any wanted angle or any focus film and focus object distance. Furtheron it is possible to display the tumor outline and the irregular field shape within the DRR.

This combination of a "beams eye view projetion" of the tumor with anatomic landmarks (bone structures) allows a better precision of patient positioning in treatment simulation. By digital overlay of DRR and simulator radiographs any incorrect position of the patient is easy to detect.

\subsection{PM}

477 Abdominal MR tomography and MR angiography in radiotherapy treatment planning

M. Müller-Schimpfle, G. Layer, A. Köster, B. Kimmig, G. Brix, L. Schad, W. Semmler, G. van Kaick; Heidelberg

The aim of our study was to achieve individual field definition in subdiaphragmatic radiotherapy by visualization of target volume using MR tomography (MRT) and MR angiography (MRA).

Images were obtained using a 1.5 Tesla Siemens Magnetom SP. Five frontal 
slices parallel to $\mathrm{L} 1$ were acquired by rapid acquisition spin-echo technique within a 14-second breathholding period (SE 150/10; NEX $=1$, matrix size $128 \times 256$ ). Additionally, frontal MRA-acquisition in breathhold technique was performed using a sequential FLASH-2D sequence (FLASH-2D $30 / 10 / 30^{\circ}, \mathrm{NEX}=1$, matrix size $256 \times 256$ ). For reconstruction of the MR-angiogram we used a maximum-intensity-projection (MIP) algorithm. The data sets of optimal MR tomogram and MR angiogram were matched and superimposed by a computer program. The MRT/MRA-superposition was geometrically projected onto simulation film in correct magnification. Spatial distortion due to magnetic field inhomogeneities was particularly considered by phantom measurements. The resulting data were analyzed by a $2 \mathrm{D}$ polynomial using computer software. A distortion of less than $4 \mathrm{~mm}$ within a radius of $14 \mathrm{~cm}$ was evaluated.

The presented method showed excellent applicability in cases of malignant lymphoma. Using the MRT/MRA-superposition demarcation of vascular architecture from parenchymatous organs was achieved. By projection of the MR-superposition onto simulation film accurate and convenient definition of the individual irradiation field was performed.

\subsection{PM}

478 Use of imaging techniques in the treatment planning and evaluation of transperineal implants

B. Tamler, B. James, D. Goffinet, D. S. Kapp, M. Bagshaw; Boston, MA

Purpose: Transperineal implants are often done open or blindly with or without a template guide. The adequacy of implant treatment and the incidence of morbidity are dependant on accurate trochar placement. An imaging protocol has been developed to aid in the planning of transperineal implants for brachytherapy or hyperthermia. The protocol involves obtaining a preoperative MRI for determining anatomic measurements, using 3D-graphics to aid in implant design, and post-operatively obtaining a CT scan for implant position verification and for final loading or hyperthermia planning. Three implant cases are reviewed and the impact of this protocol evaluated.

Methods: All patients underwent standard sagittal and axial MRI imaging of the pelvis on a $1.5 \mathrm{~T}$ GE Signa system coil. The tumor volume, perineal-tumor distance and angulation, tumor length and minimum pubis-rectum distance were measured. Axial scans were digitized using customized software and displayed on a Silicon Graphics workstation. A post-implant CT scan was obtained with a GE 9800 HighLite system and was likewise digitized and displayed in $3 \mathrm{D}$

Results: MRI measurements allowed for accurate preoperative determination of the optimum number and length of implanted trochars. The 3D graphical display allowed for easy visualization and planning of possible implant tracts, number of trochars needed to cover the target volume, potential implant difficulties and template angulation. The post-implant CT confirmed trochar position. 3D representation of the CT made for easy assessment of coverage of implant and relationship of implant to sensitive structures. Differential loading decisions were thus easily made.

Conclusions: The described protocol aids in implant planning and design, enhancing the delivery of treatment and evaluation of transperineal implants.

\subsection{5-3.30 PM Discussion}

\section{Room S}

\section{SS}

\section{SS 12.02 Computer Science in Radiology - PACS II}

\subsection{PM}

\section{PACS - Experiences and future developments}

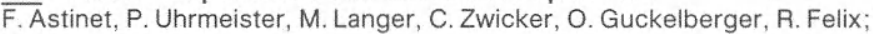
Berlin

\section{Current Status:}

Within the Berlin-Communication-Project (BERKOM) PAC-Systems were installed in the two locations of the Radiological Department of the R. Virchow University Clinic at Charlottenburg and Wedding, about $10 \mathrm{~km}$ apart. In all 5 CTs and 2 MRI-units are connected to the local PACS by an ETHERNET based LAN. Communication and Image Transfer between both PAC-Systems works via an ISDN-B broad band network (140 Mbit/s). The actual effective data transfer rate is limited to $200 \mathrm{Kbit} / \mathrm{s}$ due to the use of ETHERNET bridges ( $2 \mathrm{Mbit} / \mathrm{s}$ ) instead of high capacity BERKOM-gateways and internal "organisational" problems in the PACS computer software. Experiences:

- excellent image quality without loss in information during data transfer - evaluation and reporting time on PACS is nearly 2 times longer than for conventional hard copy analysis

- monitor reporting at the PACS-unit improves the subjective diagnostic accuracy due to possibilities of image manipulation and postprocessing Future developments:
- reduction of access time to the archive and acceleration and simplification of the operating system

- monitor synchronisation and command processing for digital conferences - application of PACS as central evaluation and reporting station in a digital radiological department by realisation of a simultaneous background data transfer during examination from the imaging modality and integration of a Hospital Information System (HIS).

\section{$2.10 \mathrm{PM}$}

\section{Impact of PACS on radiologists' daily work}

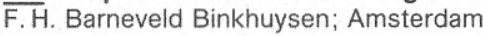

Although in Western countries there is hardly any radiologist using PACS in daily routine, it is clear that in future image information will be digital. Nowadays the role of PACS in radiology is being increasingly evaluated. This presentation will summarize and discuss the radiological experiences of different PACS sites so far.

Experiences with PACS have generated a lot of unsolved questions such as: - What is the impact of PACS on the reading of radiological examinations? - What is the impact of PACS on the relationship between radiologists and referring physicians?

- After years of centralization, will radiology again decentralize by the possibilities of PACS?

- Who is responsible for the radiological information?

- What has to be done by radiologists to keep control of radiology?

\subsection{PM}

\section{Open PACS by standards. Distributed image data-base and image} analysis in a XWINDOWS environment

\section{G. Zöllner, A. Wackenheim, J. F. Rapp, L. Steib, B. Kastler; Strasbourg}

AIM: Create an open image data-base and image analysis system in a network of workstations.

Methods: use of standards for communication (TCP/IP), data base query language (SQL), network (ETHERNET) and computer system (UNIX). Data-base- (ORACLE) and image analysis software (VISILOG) have been transformed into servers which allow different workstations of the network to query the image-base and to use the procedures of image-analysis locally. The distributed structure of the data-base (ORACLE) permits the user to access data distributed over the network without knowledge of their location. Images and their description are integrated into the data-base. This assures the advantages of data base management with respect to data protection and data loss. The interfaces have been written in C. Queries and image analysis are integrated into a XWINDOWS based user interface.

Results: The system allows distributed image display and analysis in a net of UNIX and XWINDOWS workstations. Workstations of different manufacturers can be used within the net (SUN and IBM). Duration of the consultation and display $(256 \times 256$ images $)$ in a moderately charged local network: $1-2 \mathrm{sec}$. Conclusions: an open PACS- and image analysis system can be created using standards UNIX, SQL, TCP/IP and XWINDOWS. This guarantees speed, easy portability, low cost and stability.

\subsection{PM}

482 "PACS to the future": a modular approach to PACS development Osman Ratib, Yves Ligier, Matthieu Funk, Gerhard Trayser, Christian Girard, René Perrier, Denis Hochstrasser, Jean Raoul Scherrer; Geneva

Purpose: To achieve a progressive phased implementation of a hospital-wide PACS based on an open architecture with multivendor equipment.

Methods: The Geneva PACS is a Hospital-wide Image Management System for radiological and non-radiological images. It is part of one of the widest hospital information systems (HIS) in Switzerland (Diogene system). It is based on a multi-vendor open architecture and a set of widely available industry standards, namely: Unix, TCP-IP and SQL-based distributed database (INGRES). A distributed architecture with servers of two types is used: the Archive Servers connected to the sources of images and equipped with large optical disk libraries (Juke Boxes) and Display Servers distributed over the hospital. A standard image storage format (PAPYRUS) was developed based on the ACR-NEMA standard. To provide a more uniform user interface on a variety of workstations a common platform for image display and manipulation called OSIRIS is developed based on $\mathrm{X}$-11-OSF/Motif windowing system. This platform is designed to be portable to a variety of computers and is written in the object oriented language $\mathrm{C}++$ to be easily expandable and adaptable to different needs.

Results: The current configuration comprises a first cluster of an Archive Server connected to 2 CT scanners, an MRI scanner and an ultrasound unit. A Display Server supports a high resolution workstation with two $2 \mathrm{~K} \times 2 \mathrm{~K}$ displays and several workstations with single $1 \mathrm{~K} \times 1 \mathrm{~K}$ display. A concurrent project is underway for the implementation of multimodality workstations in intensive care units. A second PACS cluster for emergency room is under 
development. Future plans include the integration of images from digital angiography and cardiology, Nuclear Medicine and images from non radiological sources such as protein electrophoresis, autoradiography and histological images.

\section{$2.40 \mathrm{PM}$}

483 PAPYRUS: a generic image file format for digital medical images Yves Ligier, Osman Ratib, Matthieu Funk, Christian Girard, Gerhard Trayser; Geneva

Purpose: To define a unique file format for the storage of any kind of medical images as well as the related clinical information to be used in multimodality image archiving and communication systems.

Methods: As part of a hospital-integrated PACS at the University Hospital of Geneva, a standard file format (PAPYRUS) was developed based on the ACR-NEMA specifications on image communication. The PAPYRUS format can be referred to as an "encapsulated" format for ACR-NEMA messages. One of the main features is the notion of folder. It provides a way to deal with sets of images. Related images can be distributed in separate files or directly in the same folder file.

Results: Display and image manipulation programs are being developed based on the PAPYRUS image file format as part of the Geneva PACS project. This format has been also discussed and adopted by 11 countries of the European TELEMED project (teleradiology and remote expert consultation in Europe) as well as by some US partners such as UCLA.

Conclusions: A message format (ACR-NEMA) was available for the communication of images, but nothing appropriate was available for the storage and archiving of images. The PAPYRUS file format, based on the ACR-NEMA format, allows to fill this lack. It allows to transfer and exchange images between different PACS systems independently from the hardware or the operating systems. It also doesn't require any special file management or communication protocols. Images can be exchanged over standard communications protocols such as FTP or even telephone lines.

\section{$2.50 \mathrm{PM}$}

\section{Connection of an hypermedia program to a PACS system: early results and goals}

Paolo Pavone, Giovanni de Gasperis, Carlo Catalano, Antonio Agnifili, Luca Marsili, Giampiero Cardone, Roberto Passariello; L'Aquila

Purpose: To verify the utility of connecting to PACS an hypermedia program, allowing nonsequential, nonlinear, branched access to information stored in a number of media, including text, static images, animated sequences and sound. Methods: A Macintosh computer (Apple computer, Cupertino, U.S.A.) was connected with Ethernet link to our PACS system. Image format was converted to TIFF Macintosh compatible format for image display and manipulation. Radiological images entered the computer directly or through a CCD digitizer. A "Supercard" hypermedia software was used, with 256 gray levels.

Results: The easy programming of the hypermedia software allowed preparation of teaching programs with little image degradation. However, the display console of the Macintosh computer was not employed for routine diagnostic purposes, mostly for the presence of other sophisticated viewing console in the PACS structure.

Conclusions: The connection of the hypermedia software to the PACS system is considered mostly valid for the preparation of teaching programs.

\section{$3.00 \mathrm{PM}$}

\section{Evaluation of PACS versus hard copy for image analysis \\ M. Langer, F. Astinet, R. Langer, C. Zwicker, O. Guggelberger, K. Rosenkranz, R. Felix; Berlin}

Purpose: Comparison between PACS and conventional hard copies with regard to diagnostic accuracy, time effects, and feasibility.

Methods: In a prospective study the acceptance, the time requirements, and the diagnostic accuracy of film reading with PACS and hard copies were evaluated by analyzing the subjective opinions of different radiologists. In a clinical study 300 CT-examinations were compared (PACS versus hard copy) for diagnostic accuracy and time requirements.

Results: It could be demonstrated that time requirements for conventional film analysis (including search for the hard copies and fixing them at the light box) is equal to image analysis with PACS. A relevant problem for PACS image analysis at our institution is the lack of a long term digital film archive. This makes a simultaneous reading of old hard copies in comparison to actual examinations on a PACS screen necessary. This is time consuming and leads to reduced acceptance of PACS by radiologists. The subjective diagnostic accuracy was judged better on PACS, due to the possibility of windowing and centering.

Conclusions: Evaluation on a PACS is at least equal to hard copy reading regarding time requirements and judged superior for diagnostic accuracy of actual CT and MRI studies.

\section{$3.10 \mathrm{PM}$}

486 ISDN-PACS for multimedia communication applications in radiology and nuclear medicine departments

N. Rilinger, H. Niemann, A. Hewitt, J.Schwanke, L. Köhler, P. Jensch; Oldenburg

It is our aim to use the advantages of the ISDN-technology for a medical end-user by supporting him with integrated image/voice/data information. Methods: An image/voice/data management and communication system using ISDN-technology has been designed.

Results: The system allows 1 . to acquire multiple image modalities from several hospital departments, 2. to complement visual results with voice and other data, and 3 . to distribute integrated multimedia information to medical end-users such as surgeons and therapists. The flexibility and the acceptance of ISDN-PACS for multimedia communication will be addressed by reviewing several departmental cases.

\subsection{PM}

\section{A new task in radiology: the information and communication} coordinator

Hans Mosser, Michael Urban, Walter Hruby; Vienna

Purpose: Goal of this paper is to present a new type of task for the radiologic community: The radiology information and communication coordinator (RICC). The need for such a coordinative and integrative function in the rapid spreading digital environment in radiology departments is evident, if the radiologists are to be more vital involved in PACS and related issues, which affect so intensively their working structure and organization, comparable to the wide spread of office automation in the eighties.

Methods and Materials: In planning a large scale PACS for the SMZO hospital in Vienna, Austria, the necessity of a RICC within the PACS planning team was recognized. A radiologist out of this team was appointed to perform this task. The specific radiologic experience of such a person may be an important contribution in the various planning stages with regard to the radiologic needs and requirements.

Results: In the planning phase, after vendor selection, the RICC already could achieve major changes and modifications of the initial concept, without additional costs, by verbalizing and emphasizing the necessity of various radiologist's requirements.

Conclusions: The rapid advances in information and computer sciences in radiology are obvious through the growing number of PACS installations in radiology departments throughout the world. Yet problems concerning this development become evident in the poor user acceptance of these systems. This reflects a gap between radiologists (as the end users) and system developers. To overcome this problem a radiologist of the department should function as a RICC, being the key link between radiologists and system engineers.

This paper outlines in detail duties and responsibilities as well as qualificationprerequisites and specifications for a RICC, and examines its impact on departmental structure.

\subsection{PM}

\section{Image communication in ambulant medicine}

\section{H. M. Klein, W. Schneider, J. Nawrath; Aachen}

In the development of a concept for the use of digital communication media for private practice, an inquiry was carried out concerning the usual transfer of images and reports.

A total of 139 general practitioners were questioned as to the amount and acquisition method of medical images required in their practice. Nearly all used diagnostic images and most were equipped with imaging facilities. Sonographic and X-ray systems were most widespread, but there was usually little direct communication concerning diagnostic images or reports between doctors.

Our inquiry has shown a large demand for digital image processing and communication systems and suggests a sufficiently high acceptance rate.

\section{$3.26 \mathrm{PM}$}

\section{PACS: requirements on teleradiology systems}

E. List-Hellwig, O. Rienhoff, K. J. Klose; Marburg

The teleradiology project of Marburg University is presented: The transmission of CT-Scans between radiology departments of two hospitals $(3,5 \mathrm{~km}$ apart) and image transmission inside the main hospital complex. The hardand software basis of the project is a Siemens PACS connecting the two hospitals with a glassfibre ethernet network and linking different types of workstations and video screens into the PACS network.

The evaluation of literature and of recent teleradiology projects as well as our own experience shows: besides technical aspects the introduction of teleradi- 


\subsection{PM}

490 Initial experience in the networking of $1024^{2} \times 8$ bit fluoroscopic images into a functioning workstation

D. M. Hynes, J. A. Rolands, E. W. Edmunds, A. J. Porter; Toronto, Ont

Purpose: The purpose of this presentation is to investigate the technical and economic feasibility of networking ultrasound, nuclear medicine, and fluoroscopy in a busy, clinical environment.

Methods: The authors have been developing a local PACS network which receives digital images from three ultrasound units (different manufacturers) and two nuclear medicine units. All images are immediately transferred to a file server $\left(10,000\right.$ images at $512^{2} \times 8$ Bit) from which two workstations $(640$ MB disk - 1500 images) can call up images for diagnosis and archiving. This system is currently being interfaced with the departmental DecRAD R.I.S.

Results: Current works in progress describes technical problems and their solution, when $1024^{2} \times 8$ Bit general fluoroscopic images are entered into the system from four different digital fluoroscopic rooms. This experience will be supported by clinical material. Potential economic benefits will be reviewed. Conclusions: The system described above is in daily clinical use, and preliminary assessment suggests it to be economically viable and clinically realistic.

3.34 PM

\section{Clinical evaluation of a mini-PACS}

A. Memis, M. Prokop, C. Schaefer, A. Meschede, M. Galanski; Hannover

Purpose: In our institution, a mini-PACS (video-link DS-1000, $1 \mathrm{~K} \times 1 \mathrm{~K}$ monitor) is installed between the radiology department and a mixed oncologic/cardiac ward (distance: ca. $200 \mathrm{~m}$ ). Purpose of this study was to evaluate the diagnostic efficiency of such a mini-PACS, its potential and limitations, and to examine whether it is clinically acceptable.

Methods: The study group consisted mainly of oncologic and cardiac patients that often require fast access to radiographic films for decisions about immediate treatment for pulmonary infections or cardiac failure. Over a period of three months, clinically required p.a. and lateral chest radiographs of 117 patients were obtained in digital storage phosphor technique $(0.2 \mathrm{~mm}$ pixel size). The digital data were processed to approximate conventional image characteristics and transferred by video link to a monitor display $(1 \mathrm{~K} \times 1 \mathrm{~K})$ on the ward. Images were available a few minutes after exposure. The four clinicians on the ward were asked to answer a standardized questionnaire each time they used the monitor.

Results: Basis for the following results were all 88 completed questionnaires: Within one hour, $76 \%$ of the radiographs were reviewed; within four hours, additional $22 \%$. For $52 \%$ of the patients, diagnostic information of the radiographs had therapeutic consequences on the same day, for $10 \%$ within one hour. By subjective impression, $90 \%$ of the monitor-displayed images were considered sufficient. Additional hardcopies were required in $5 \%$ of cases.

Conclusions: Dependent on the spectrum of disease, a radiographic communication system that provides immediate access to the radiographs can considerably shorten time between diagnostic procedures and therapeutic consequences. 


\section{Room A}

\section{GE}

\section{1}

492 MR of cranial nerves and basal cisterns

J.Vignaud, C. Pharaboz, G. Cosnard; Paris

Detailed anatomy of the cisternal part of the cranial nerves is demonstrated. This study is performed with a signa G.E M.R UNIT (1.5 T), using various sequences. Precise imaging of the nerves, in longitudinal and cross sections is performed thanks to reformated images from the data of thin (1-2 mn) and joining axial sections.

Precise relationship of the nerves with the surrounding vessels is emphazised. A listing of the pathology is given for all the nerves, and some examples are shown.

\section{$493 \mathrm{MRI}$ of the orbit}

G. Wilms, G. Marchal, Ph. Demaerel, A. L. Baert, G. Dralands; Leuven

Contribution available at the information desk.

\section{Neuroradiological evaluation of sellar and parasellar lesions G. Scotti; Milano}

The Sella Turcica is an anatomo-topographical entity with great functional and clinical relevance: it contains the pituitary gland and it is surrounded bilaterally by the cavernous sinuses containing cranial nerves and carotid siphon while superior to it lie the optic chiasm and the hypothalamus.

Clinical presentation of lesions in this area include neuroendocrine disfunction, visual disturbances, diplopia, trigeminal neuralgia.

The best radiological depiction of anatomy in this region is provided by Magnetic Resonance; plain films and particularly politomography have lost all their indications and should not be used any more. Computed tomography, though highly informative, is seriously challenged and may be abandoned in a near future.

An appropriate MR evaluation of the sella required thin slices ( $2 \mathrm{~mm}$ or less) obtained at least in two orthogonal imaging planes, T1 weighted. T2 w images are frequently required particularly for para- and suprasellar lesions as well as the use of the paramagnetic contrast agent Gd-DTPA.

The type and number of diseases that affect this area is very extensive, from pituitary adenomas to craniopharyngiomas, meningiomas, aneurysms, gliomas of the optic chiasm and hypothalamus, infections, histiocytosis, congenital anomalies. MR allows clear recognition of the lesions, definition of their size, topography, relationship with surrounding structures, intrinsic characteristics. Differential diagnosis may be very accurate. Preoperative or pretreatment angiography or biopsy is seldom needed.

\section{Room B}

\section{CE}

495 The impact of C.T. and U.S. in the diagnoses and management of pleural disease

C. D. R. Flower; Cambridge

Until the advent of these imaging modalities radiologic diagnosis of pleural disease depended on chest radiography. This has severe limitations, particularly with regard to the demonstration of complicated pleural fluid collections and pleural tumours.

Ultrasound and computed tomography are complementary techniques which have advanced our understanding of pleural abnormalities. They enable more accurate diagnosis than simple chest radiography and are invaluable in the implementation of interventional procedures for the drainage of fluid and air collections, and the biopsy of pleural and chest wall masses.

It is likely that magnetic resonance imaging will prove as helpful but as yet its role in the investigation and management of pleural problems is not fully determined.

\section{Diaphragm: plain film, US, CT and MR}

J.A. Verschakelen; Leuven

Radiology of the diaphragm is often difficult. In part because many diaphragmatic abnormalities are obscure clinically, but mainly because there is no imaging technique that can clearly and entirely visualize the diaphragm. Nevertheless the radiologic evaluation of the diaphragm is important in many clinical situations. Because diaphragmatic abnormalities detected on conven- tional chest radiographs are often non specific, other additional imaging techniques such as fluoroscopy, ultrasound, computer tomography and magnetic resonance imaging are often necessary. This paper will discuss the normal anatomy and physiology of the diaphragm and will give an overview of the role of the different imaging modalities in the diagnosis of hernia, traumatic rupture, tumors, eventration and dysfunction of the diaphragm.

\section{Room C}

\section{RC}

\section{4}

497, 498 CT and MRI of focal and diffuse liver disease

Y.M. Menu and D. G. Mathieu; Paris-Creteil

During the past decade techniques for imaging the liver have proliferated dramatically. Developments in radionuclide imaging, ultrasonography, digital angiography, computed tomography and magnetic resonance imaging (MRI) have provided unprecedented possibilities for studying liver tumors.

Benign tumors of the liver include essentially hemangioma, focal nodular hyperplasia (FNH), and hepatocellular adenoma (HA). Hemangioma are relatively common, but $\mathrm{FNH}$ and $\mathrm{HA}$ are rarer tumors. Whereas it is well known that $\mathrm{HA}$ is related to oral contraceptive $(\mathrm{OC})$ intake, the responsibility of OCs in the occurrence of either hepatic hemangioma or FNH has not been established. The purpose of this course is to correlate the macroscopic appearances of these benign liver tumors (FNH and HA) with their features obtained by MR imaging and Dynamic CT and to compare the results of both imaging modalities. We will emphasize the different roles and applications of contrast agents particularly with gradient echo imaging and ultrafast techniques.

The malignant hepatic tumors include essentially metastases and hepatocellular carcinomas.

For the detection of liver metastases, particularly in the preoperative setting, the roles of sonography, dynamic CT, delayed CT and CT during arterial portography are discussed. The use of paramagnetic and superparamagnetic contrast agents on MR are emphasized.

For the evaluation of hepatocellular carcinoma, sonography and angiography with injection of Lipiodol and delayed Lipiodol CT are recommended. The role of MRI to distinguish this tumor from regenerative nodules is discussed. Finally, fatty infiltration can raise a difficult dilemma with other liver lesions, particularly in case of focal nodular lesions. The role of percutaneous fine needle aspiration and guided biopsy are also discussed.

\section{Room E1}

\section{RC}

499 Laser angioplasty: technique and clinical results

J. Lammer; Graz

Basic principals

The purpose of percutaneous transluminal laser angioplasty (PTLA) is to ablate obstructing thrombi and atheromatous plaques by means of tissue vaporization. This can be achieved with continuous wave (cw) or long pulsed lasers due to the photothermal effect alone, as well as by short pulsed lasers (nanosecond range) due to the photothermal and photoacoustic effect.

Currently various lasers are under experimental and clinical investigation for PTLA: Excimer-laser (308 nm, pulsed), Argon-laser $(488 \mathrm{~nm}, 514 \mathrm{~nm}, \mathrm{cw})$, Dye-laser (504 nm, pulsed), Nd: YAG-laser (1064 nm, cw, pulsed), HolmiumYAG-laser ( $2100 \mathrm{~nm}$, pulsed). The laser light is guided through 300-600 micron glass fibers in combination with transparent contact probes (sapphire probe, SLT; spectraprobe, Trimedyne), or through multifiber catheters.

PTLA of peripheral arteries

Technique

PTLA can be done after insertion of a catheter introducer sheath under local anesthesia. The tip of the laser catheter has to be in contact with the obstructing plaque. After successful recanalization additional balloon angioplasty (PTA) of residual stenoses is required. Heparinization with 5000 IU during and $1000 / \mathrm{h}$ for 48 hours after the procedure is recommended.

Results: PTLA was carried out successfully in iliac and femoro-popliteal artery occlusions. In femoro-popliteal artery occlusions with a mean length of $8-10 \mathrm{~cm}$ the initial recanalization rates were $70-85 \%$. In comparison to conventional PTA, PTLA resulted in higher recanalization rates in long occlusions of $8 \mathrm{~cm}$ or more. Perforations were observed in less than $5 \%$. The 6 months patency rate was $74-80 \%$, the 3 years patency rate $52-63 \%$, depending upon outflow and clinical stage.

Conclusions: PTLA is technically still in its infancy. Early clinical trials revealed recanalization rates and long-term patency rates similar to those of PTA. However, the objective of PTLA to improve long-term patency rates is not achieved yet. 
500 Laser angioplasty

D. C. Cumberland; Sheffield

Abstract available at the information desk.

\section{Room E2}

\section{WS}

\section{6}

\section{Intervertebral spinal procedures - discography}

I.W. Call; Oswestry

The investigation of the intervertebral disc by the injection of contrast medium has been practiced for more than 40 years. The advent of CT and now MR which are non-invasive and permit the diagnosis of herniation of the nucleus pulposus and degeneration of the disc have significantly reduced the need for discography, although CT discography has been shown to be the most accurate method of diagnosis of disc herniation. Unfortunately degeneration of discs and annular tears shown on MR are also present in asymptomatic subjects, and there are no specific features on MR that identify the painful disc in non specific back ache and leg pain. Discography with pain provocation remains the only method and has an accuracy in this group of patients of $82 \%$ with a positive predictive value of $89 \%$. Pain has been shown to be rare in asymptomatic subjects, and a recent study reports a false positive rate of $0 \%$, but false negative studies have been reported in patients with pain who subsequently respond to spinal fusion. Complications are few but infection of the disc space occurs in $0.4 \%$ of patients despite careful aseptic techniques.

\section{Percutaneous biopsy}

\section{D.J. Stoker; London}

Disagreement still exists over the various methods of biopsy. However the inaccessibility of the vertebral body makes the use of a guided biopsy technique, performed by the radiologist, no longer controversial as open biopsy can only offer a destructive approach. The prevalence of metastatic carcinoma in the spine and the frequency of infective lesions centred on the disc spaces, makes the technique one that should be practised by radiologists in the majority of district general hospitals and not confined to tumour centres. The importance of the radiologist as a clinical colleague in the management of such problems in the spine, with preliminary advice on imaging procedures prior to biopsy is emphasized. In this presentation, the approach and choice of biopsy technique will be discussed, together with the importance of a close understanding of the problems that may be encountered by the histopathologist.

\section{Facet joint arthrography}

A. Chevrot, C. Vallee, F. L'Huillier, B. Rousselin, O. Richard, D. Godefroy: Paris

Lumbar posterior arthrography becomes easy when using a new approach, puncturing the inferior articular recess.

On a prone patient, the entry point is located under the hip of the inferior articular process of the superior vertebra. Injection of a small amount of contrast medium opacities the articular space which is ring shaped with a superior bulging (superior recess in the foramen) and an inferior bulging (subcutaneous inferior recess).

The articular filling is painless. The borders of the space are smooth. The opacification is homogeneous.

Pathological cases include painful filling, irregular borders with sometimes diverticula or cysts, horizontal or vertical leakage towards on other articular cavity can be seen.

Intraarticular injection of local anesthesic $(1 \mathrm{ml})$ can lead to a disparition of the pain, that confirms the pathological nature of the joint. Intraarticular injection of long delayed action corticoids can be performed helping to recover during several months.

\section{Automated percutaneous lumbar discectomy (percutaneous nucleotomy)}

H. Weigand; Wiesbaden

The percutaneous nucleotomy or the Onik-procedure is described as a new interventional percutaneous radiological technique for lumbar disc removal. The own results in 350 patients since October 1987 are perfectly correlated with other surgical and microsurgical techniques with and without endoscopic control. The percutaneous nucleotomy seems to be very valuable in every level of the lumbar spine.

Precise indications and technique under fluoroscopic control and documentation may ensure 70 to $80 \%$ good and excellent results. In comparison with other interventional treatments the percutaneous nucleotomy is safe, relatively simple and without major complications. In the near future the percutaneous nucleotomy will have a certain place in the treatment program of radicular back pain.

\section{Room L}

RC

\section{Non accidental injury}

Helen Carty; Liverpool

NAI is a problem of greater or less degree in all societies, though far commoner in the first world. The apparent increase in incidence may simply reflect an increased awareness of the problems or may be a true reflection of stresses and strains of modern society. The term NAI is unfortunate as it implies deliberate abuse, but in fairness to the perpetrators of the injuries, terrible though these are, the act is seldom premeditated. The injuries seen by radiologists are usually the result of temporary loss of self control, although this can be repetitive. The original term used by John Caffey to describe the problem was "The parent child stress syndrome", which more truly reflects the clinical situation. Nonetheless, all radiologists should be familiar with the radiological manifestations, so that a child may be protected by timely intervention.

The radiological manifestation may be divided into three groups, the skeletal lesions, brain injury, and visceral and soft tissue injury.

The role of the radiologist in the diagnosis of NAI will be discussed with illustrative cases.

Skeletal injury will be discussed under the headings of those with a high specificity for NAI, and those that are common but less specific. The mechanism of the cause of the fractures and the long term orthopedic implications will be addressed.

Brain injury can be devastating. Brain injury occurs by one of two mechanisms, direct trauma and shaking. Both will be illustrated and followed by a brief discussion of prognosis.

Visceral and soft tissue injury is much rarer and is protean in manifestation. Abdominal and chest injuries occur through violent beating and punching: Pharyngeal tears through penetrating injury and rectal and vaginal tears through sexual abuse.

Having made or suggested a diagnosis of child abuse, there are ethical and legal implications for the radiologist. These will be addressed.

\section{Room M}

\section{RC 405}

\section{Imaging of the bladder and rectum. II. US} Bruno D. Fornage; Houston, TX

This course reviews the current techniques of indications for, and results with ultrasound (US) examination of the bladder and the rectum, with emphasis placed on endosonographic techniques.

Bladder. The techniques of US examination of the bladder include the transabdominal, transurethral, transvaginal and transrectal approaches. The norma US anatomy is presented. Trànsabdominal scans allow assessment of many of the nontumoral pathologic conditions affecting the bladder, including calculi, hematomas, severe cystitis, ureteroceles, trabeculation, diverticuli, and changes secondary to neuromuscular disorders. Tumors of the bladder wall can usually be visualized on transabdominal scans, but staging is not reliable because of the suboptimal visualization of the detrusor on these scans. Perivesical abnormalities can also be appreciated transabdominally. Transurethral scanning is usually performed in conjunction with cystoscopy; staging of bladder tumors is the major indication for transurethral US and will be discussed in detail. Transvaginal US can be used to evaluate cystoceles, urethroceles, and stress incontinence. In the male, transrectal voiding US allows assessment of bladder neck dysfunctions and abnormalities of the prostatic urethra.

Rectum. The various transducers available for endorectal US (EUS) examin ation of the rectum are reviewed with their specific advantages and drawbacks EUS is capable of distinguishing the various layers of the normal rectal wall, and EUS staging of rectal carcinoma is primarily based on the relation of the tumor to the hypoechoic muscularis propria layer. Perirectal lymph nodes can also be demonstrated, but EUS may fail to differentiate between benign and metastatic lymph nodes. EUS has proved to be more accurate than palpation and CT and is becoming the technique of choice for preoperative local staging of rectal carcinoma. EUS also contributes significantly to the early detection of local recurrences following anterior resection of rectal carcinoma. The role of EUS in inflammatory conditions of the rectum is also discussed.

\section{Imaging of the bladder: computed tomography and magnetic resonance} Janet E. Husband; Sutton

Computed Tomography $(\mathrm{CT})$ and Magnetic Resonance Imaging (MRI) are both powerful tools for evaluating the pelvis. During the last decade CT has made a major impact on staging patients with bladder cancer with accuracy rates ranging from $60-90 \%$, and MRI now challenges the role of CT in this and 
several other areas of body imaging. The major limitations of CT in staging bladder cancer are (i) the inability to stage early tumours confined to the bladder wall, (ii) the inability to diagnose early perivesical tumour spread and the difficulty in detecting early organ invasion. Furthermore, patients who have been previously treated with radiotherapy are difficult to assess because fibrosis of the bladder wall cannot be distinguished from tumour.

MRI has advantages over CT which may provide more accurate staging in certain patients. Thus with MRI the bladder wall can be assessed on T2 weighted images providing information regarding the stage of early tumours confined to the bladder wall. The multiplanar capability of MRI also facilitates the assessment of extravesical spread in some patients. Organ invasion may be demonstrated with MRI more readily than with CT. However, MRI, like CT, has important limitations. These include the length of the examination time, the presence of chemical shift artefacts and the inferior spatial resolution of MRI compared with CT. Several series have now been published comparing the accuracy of CT with MRI for staging primary bladder cancer. These results suggest that MRI has a small but significant advantage over CT, although the range of accuracy figures is similar for both techniques.

The assessment of lymph node status is an integral part of tumour staging. Both CT and MRI may detect enlarged pelvic lymph nodes greater than $1.0-1.5 \mathrm{~cm}$ in diameter. Although little comparative work has been published, early results suggest that CT and MRI are similar in their ability to detect lymph node metastases from bladder cancer.

\section{Room N}

\section{RQ}

407

\section{Trauma of skull and brain}

\section{P. Huber; Berne}

In cases of acute head injuries, CT is the examination which provides all informations needed for the emergency treatment of the patients. Intra- and extracerebral hematomas are easily depicted as well as contusions, brain swelling and fractures of the skull. Of importance are follow-up studies in patients deteriorating hours or days after the trauma. Of special importance is the evaluation of the size of hemorrhagic contusions in patients with coagulopathies.

\section{Brain trauma: medicolegal aspects}

\section{J. C. Froment, L. Daligand; Lyon}

Brain traumatisms are frequent (about 300000 vehicular accidents each year in France).

Medical aspects include all relationships between law and imaging.

X-Ray examinations (CT-scan, Angio) play a very important role for the diagnosis of intracranial injuries. It appears now that skull X-Rays are not routinely indicated in patients with head trauma. On the contrary, CT-examinations of the brain have to be performed early to detect surgical lesions, especially extradural hematomas.

The radiologist may have to establish the proof of the traumatism (in child abuse for instance) or to diagnose preexisting (ischemic, tumoral) lesions and their link of causality with the trauma.

An other aspect is to make sure of brain death before organ transplants: bilateral carotid arteriography is the most efficient way to prove absence of brain circulation.

Expertal imaging contributes to the evaluation of sequelae for disability assessment and damage repair. Examinations required for the expertise have to be non invasive (CT-scan without contrast, MRI). T1-, T2-, T2*-weighted MRI is very contributive to the detection of intracranial abnormalities (hemorrhagic lesions, diffuse axonal injury, high-signal changes in the white matter..). But the estimation of brain damages is mostly based on clinical symptoms and furthermore there is not always a total convergence between morphological aspects and functional symptoms.

Of course, medicolegal aspects of brain traumatisms are subject to the legislations of each country, which will evolve towards harmonization in 1992.

\section{Room 0}

\section{WS 415}

510 MR imaging of diffusion and perfusion

D. Le Bihan; Bethesda, MD

The effects of molecular diffusion on the NMR signal have been described long ago and powerful diffusion measurement techniques using NMR have been extensively used in physics and chemistry. The recent application of such principles to clinical MR imaging represents a spectacular and somewhat unpredicted development with tremendous clinical potential. Diffusion MRI is the only method which can investigate non-invasively molecular displace- ments in the micrometer range, which is the size of many biological structures. Water mobility has been found to differ between tissues (solid tumors versus cysts, recurrent tumor versus radiation damage or edema) and physiological states (hyperacute brain ischemia). Diffusion has also been found to be anisotropic in brain white matter, which allows the myelin fiber orientation to be determined and mapped using a color table. The sensitivity of diffusion to temperature offers the possibility of real-time non-invasive temperature imaging, of great interest in monitoring clinical hyperthermia or laser procedures. Diffusion thus appears as a new and promising source of contrast in MR images.

Perfusion imaging can be achieved without using contrast agents from extensions of diffusion imaging, considering the random orientation of capillaries in most tissues. Another promising approach is based on the dynamic imaging of the passage of a bolus of contrast agent, such as Gd-DTPA, through the capillary bed. Preliminary results have been obtained with both techniques, essentially in the brain. With the speed of Echo-Planar Imaging, diffusion and perfusion images can now be obtained also in mobile tissues, such as eyes or kidneys. Perfusion MR imaging is an important field of research, given the high spatial and temporal resolution and the safety of the technique. However, further work is required before these new methods can be competitively and reliably used clinically.

511 Evaluation of tissue perfusion. Possibilities and limits of sonography

Michel M. J. Dauzat; Nîmes

Many sonographic techniques are currently and widely used for the clinical assessment of the vascular function: continuous wave and pulsed wave Doppler, duplex Doppler, color coded Doppler imaging, and color velocity imaging. Besides, some systems are dedicated to volumetric blood flow measurement (attenuation compensation, transit time measurement...), while some are designed to intra-operative flow measurement, and others are elaborated for the measurement of vessel wall compliance.

On the other hand, several methods have been proposed for the evaluation of tissue perfusion using these different techniques:

- volumetric blood flow measurement,

- indirect, semi-quantitative evaluation of distal impedance,

- direct or indirect assessment of the velocity profile,

- arterial wall morphological and mechanical evaluation.

In the clinical routine, the assessment of the blood velocity profile by means of real time spectral analysis of Doppler signals ensures the diagnosis and semi-quantitative evaluation of arterial stenoses. Various indices calculated from systolic and diastolic blood velocities are related to distal circulatory impedance, thus allowing to evaluate the functionial consequences of arterial obstructions. B-mode sonography is very conclusive for the detection and characterization of atheromatous plaques in arteries, and of thrombus in peripheral veins.

Nevertheless, although these relatively simple, mostly qualitative, methods have been proven to be clinically efficient, many technical and practical problems remain partly unsolved as regards to quantitative assessment of vascular function using ultrasound. The noninvasive evaluation of the arterial wall compliance, using B-mode and/or Doppler sonography, with or without measurement of the pulse wave velocity, offers valuable data for epidemiological studies, but the large number of proposed methods contrasting with the relatively small number of users suggests that there is no consensus in this field. There is a great interest in volumetric blood flow measurement using ultrasound, especially for physiological research, pharmacological studies, and intensive care. The main causes of error, for such methods, are related to the diameter and cross sectional area measurement, the Doppler angle of incidence, and the spatial averaging of blood flow velocities. Different technical and methodological approaches have been proposed to overcome these difficulties. None has been fully accepted so far.

However, as technological progress is rapid and tremendous, adequate solutions can be expected for the near future. Therefore, the sonographic methods, being relatively inexpensive, currently available, and noninvasive (i.e. preserving the normal physiological conditions) will probably remain very attractive for the assessment of vessel function and tissue perfusion.

\section{Room R}

\section{SS} 4.00 PM

$$
\text { 16.05 Treatment planning in Radiotherapy II }
$$

Round Table discussion with Keynote speakers 


\section{Room S}

\section{Features and economics of PACS}

C.F. C. Greinacher; Erlangen

Talking about PACS there is still a need to define what is meant by this term "PACS" (or IMAC, or Image Information System for Radiology, or ...). Two definitions, independent from each other, are used as an introduction into the workshop:

a) PACS, a certain configuration of diagnostic imaging equipment, communication networks, image storage devices, image workstations and hard and soft copy devices.

b) PACS, a computerised system, meeting the expectations as perceived by radiologists, radiographers and administrators concerning the primary benefits of the electronic technology.

Based on the latter definition, expected key issues and their technical feasibility are discussed: Improved image archiving, fast image communication, reduced film consumption, reduced personnel, improved image manipulation and reduced film losses.

Examples of system configurations for typical applications are shown. The economics of PACS are discussed under the assumption, that PACS - in general - will not provide additional revenue for the hospital. Cost justification of PACS depends on two issues: Cost savings as well as improvements provided by the system for the patients and/or for the users which hospitals are willing to pay for.

\section{Communication systems in radiology}

\section{Heinz U. Lemke; Berlin}

With digital imaging, multi-media MR structures, computer assisted methods for information management, and modern digital communication technology, new types of communication channels are being realised. They augment the traditional communication paths in health care or allow for new methods of medical interaction. Various health care zones and associated users may be part of a distributed information management and communication system. Medical imaging workstations become part of different types of communication infrastructures commonly referred to as Picture Archiving and Communication Systems (PACS) and Telemedicine (TM).

Several concepts and realisations of picture archiving and communication systems and teleradiology systems demonstrate this point. There exist open and closed systems, single-vendor and multi-vendor systems, star, bus, and ring topologies, as well as a multitude of different types of terminals which may start from simple, up to highly sophisticated workstations and optical archiving systems.

Communication in radiology is essentially multi-media. Many projects in the USA and Europe are concerned with this type of information transfer not only in PACS but also in telemedicine applications. Teleradiology facilities are being developed in these countries using smallband and broadband ISDN or similar network infrastructures.

Specific application tools provided with these networks can enable health care professionals to engage in the following activities:

1. consultation for diagnostic and therapeutic planning,

2. case presentation to one or more members of a medical group,

3. method presentation in the on- or off-line working mode,

4. introductory and advanced teaching,

5. data bank access, and

6. expert (centre) referral.

Some critical aspects of these developments such as standards and security will be reviewed.

\section{Image processing and data compression}

\section{A. Todd-Pokropek; London}

A Picture Archiving and Communication System (PACS) can be considered as providing an electronic lightbox. However, potentially great value can be derived from such systems using them to provide additional functions to those of a basic display. Image processing tools now available enable the user to perform not just the conventional contrast enhancement and display manipulation familiar from CT and MRI, but to perform segmentation and quantification, generate functional images and enable the use of expert system based advisory system to assist classification and interpretation. Display of data in 3-D and the registration and superimposition of features of images from different modalities is also potentially valuable. The transmission and archiving of large quantities of image data suggests that data compression is also required, and, if legally acceptable, of a non-reversible type. Data compression ratios of the order of 20:1 can be achieved with no apparent loss of clinical image quality. Data compression can also be considered as providing a compact description of an image and therefore as part of image processing. Additional work concerning standards for data formats, data compression, and the human computer interface is urgently required. 


\section{Room A}

\section{GE}

\section{MR of the chest: mediastinum and lung} Jacques Frija; Paris

During this course we shall emphasize the technical aspects of MR-study of the chest in order to reduce the time of the examination. Then it will be showed the normal results and the limitations of this technique. We will emphasize the now deceptive but promising results of the study of the lungs. Then we shall show the aspects of the invasion of the mediastinum by tumors, and the unique MR capability to identify the structure, specially fibrotic post-treatment masses, of mediastinal tumors.

\section{MRI of the heart and great vessels}

\section{Gustav K. von Schulthess; Zurich}

MRI of the heart and chest is still the most challenging of all MR examinations. A bewildering array of techniques have been made available over the past five years. ECG-triggering is mandatory and the major choice on current imagers is between SE and GRE sequences, although Echoplanar Imaging (EPI) undergoes trials at several institutions. Conventional and oblique imaging as well as schemes to obtain the same slice at various points in the cardiac cycle are used. Which technique is used depends largely on the type of information desired.

Vascular morphological abnormalities such as in patients with congenital heart disease require SE imaging usually in at least two imaging planes and reconstruction of phase images in addition to magnitude images. T2-weighted images are often not required. In the case of mass lesions, $\mathrm{T} 1$ - and T2-weighted images are mandatory and, since in the heart long echo times strongly degrade image quality, Gadolinium enhanced images are often very helpful. GRE sequences are used for functional studies of the heart. However, most such examinations are still experimental in nature. In the future they will be mainly used for the quantitative determination of flow and motion of the heart and great vessels

Current indications for MRI of the heart and great vessels are the investigation of complex congenital heart disease, cardiac and paracardiac masses and degenerative diseases of the aorta. The inclusion of GRE-based MR function studies are rapidly gaining acceptance as their use becomes easier, faster and quantitative. EPI imaging of the cardiovascular system promises real time images of the heart, perfusion studies and MR guided vascular interventions.

\section{Room B}

\section{EC 501}

517 Ventilation-perfusion scintigraphy in pulmonary embolism: current status

B. D. Bok; Clichy

Contribution available at the information desk.

518 Pulmonary embolism: angiography, CT and MR

D. Musset; Clamart

Pulmonary embolism is a frequent, serious disease for which an absence of diagnosis may have severe consequences. The aim of the course is to compare present days methods of diagnosing PE, exclusive of those designed to identified lower limb thrombosis.

Pulmonary angiography remains the reference examination but its place in the diagnosis of thromboembolic disease remains open. The main questions in this field we have to discuss are the following: What is the best site for injection? What is the best type of equipment? What are the best X-ray incidences? What results may be expected and what complications? What precautions are needed to reduced such complications? and finally how does it compare with the other methods for the diagnosis of pulmonary embolism? Other techniques such as $\mathrm{CT}$ and MR can play only a secondary role even though in certain cases they may have originally diagnosed the disease. The indications for angioscopy and intravascular US have not yet been completely defined but they may be useful, especially in cases of chronic thromboembolic disease.

519 Pulmonary embolism: an integrated approach to the diagnosis D. J. Delany; Southampton

Pulmonary embolism is a very common disease in patients who are, or have recently been hospitalised. Anticoagulation treatment is considered to be

efficacious but carries a significant morbidity risk. Therefore, the diagnosis of pulmonary embolism should be made as accurately as modern medicine permits. Clinical diagnosis is notoriously inaccurate because of the many processes other than pulmonary embolism that cause acute onset of chest pain, breathlessness and hypoxia. It is suggested that the diagnostic sequence should begin with as good a plain film examination of the chest as is permitted by the patient's condition. Interpretation of this should be carried out by a specialist chest radiologist, along with a careful review of any previous radiographs that are available, or the radiological record. The plain films may thus lead to an understanding of the symptomatology, without the need to invoke a diagnosis of acute pulmonary embolism. Failing this, further imaging techniques, such as ultrasound or CT may lead to other diagnoses that have caused the same symptoms being investigated.

If no other diagnosis can be substantiated, then a ventilation/perfusion lung scan should be performed. There are very few exceptions to this requirement. Patients who are extremely ill or unstable should probably be submitted for early pulmonary angiography, so that thrombolytic therapy can be applied if appropriate. It is often suggested that patients with plain film abnormalities are not appropriate subjects for scanning, but there is frequently valuable information to be obtained from the regions of the lung that are normal to plain $\mathrm{x}$-ray. Further than this, the perfusion scan is extremely helpful in guiding the angiographic search from embolism. It seems self evident that lung scanning should be carried out as soon as possible following the suspicion of a pulmonary embolism, to increase the chances of imaging the physiologic disturbance that caused the event. The scanning should be carried out with the best available technology, with multiple views of the perfusion agent and appropriately matched ventilation images.

The lung scan should be interpreted by a nuclear medicine specialist and then a final clinico-radiological diagnosis rendered collaboratively by the chest radologist and the nuclear medicine specialist. If at this stage, a concensus high or low probability decision is reached, then that should be the determination With any other outcome, the patient should be referred for angiography which should rapidly be performed, preferably by the thoracic radiologist or an angiographer, who has had specialty training in right heart catheterisation. Only in this way can a radiology department be said to be providing an adequate answer to the clinical question - Does this patient have the disease pulmonary embolism?

\section{Room C}

\section{RP}

\section{4}

520,521 Diagnostic and therapeutic management of abdominal contusions

R. F. Dondelinger and

J. C. Kurdziel; Luxembourg

Computed tomography $(\mathrm{CT})$ has been proven effective in the diagnostic work-up of polytraumatized patients who have sustained blunt injury to abdominal parenchymas (liver, spleen, kidney, pancreas), to the retroperitoneal space, the pelvis and bone structures. Thoracic CT, which is extremely valuable in polytrauma victims, will not be discussed here. CT examination, comprising the pelvis, the abdomen and the thorax is performed following initial resuscitation in all polytraumatized patients who are kept in an haemodynamically stable condition. Results from a series of 226 consecutive patients confirm diagnostic accuracy of $\mathrm{CT}$ and the low rate of false negative findings; diagnostic errors will be discussed. The actual trend in management of solid organ contusions is conservatism. Pros and cons, indications and results of conservative treatment of hepatic and splenic trauma are detailed. Visceral abdominal angiography is indicated when a bleeding source has been evidenced or is strongly suspected on CT or when haemorrhage persists after surgery. Principles, technique, pros and cons of haemostatic visceral embolization are discussed. Subsequent CT examinations are indicated during hospitalization to evidence unresolved haematomas, abscesses and other fluid collections, many of them being amenable to percutaneous treatment. An integrated radiological management of blunt abdominal contusions is best achieved by a combination of CT and angiography. A dedicated trauma radiologist must be available; vicinity of the CT room and the angiography suite is necessary and these rooms must also be located close to the emergency room.

\section{Room E1}

\section{RG 507}

522 Diagnosis in cerebrovascular malformation M. Nadjmi; Würzburg

In diseases of the CNS great progress has been made during the last years not only in neuroradiological diagnosis but also in endovascular therapy. Advanced methods of choice are:

Intraarterial digital subtraction angiography 
3D-computer tomography

MR-angiography

By use of microcatheters each desired part of the cerebral vasculature can be reached up to a lumen of $2 \mathrm{~mm}$.

The second part of the presentation (b) deals with diverse embolisation agents. The risk of diagnostic angiography has been minimized by intraarterial digital angiography with a shortened examination time and by application of non-ionic contrast media. Intraarterial DSA is not restricted to imaging of intraarterial aneurysms and arteriovenous angiomas by injection of contras medium into the main cerebral vessels, but also yields a hemodynamic analysis of the vascular malformation by placement of the microcatheter within the malformation itself. The anatomical and hemodynamic information obtained is important both for planning of interventional neuroradiological therapy and in choice of operative procedures.

\section{$\underline{523}$ Interventional neuroradiology in aneurysms and arteriovenous malformations of the brain}

L. Picard, S. Bracard, R. Anxionnat, D. Roy, T. Boulanger, A. Per, J. Torrens; Nancy

Endovascular treatment of intracranial vascular malformations is now widely accepted as an important part of the multidisciplinary approach in the management of these lesions. In brain AVM's, many parameters must be considered in order to choose the best protocol of embolization : angioarchitecture - topography in relation to functional areas - hemodynamic characteristics, etc... The embolization itself is realized after hyperselective catheterism of the nidus with a progressive suppleness mircrocatheter. For preoperative embolization, any kind of embolic material may be used. However, permanent occlusion can only be achieved with N-Butyl polymer. The best results are obtained with small AVM's and direct AV Fistulas. In giant AVM's, partial embolization may alleviate the symptomatology when headaches, seizures, of neurological deficits secondary to a steal syndrome are of a main concern. Therapeutic association with surgery and radiosurgery are used more and more.

Endovascular treatment of aneurysms may be obtained either by direct endosaccular occlusion (with balloons or micro-coils) or by occlusion of the parent vessels. This depends on the size of the aneurysm, its topography and the capacity to enter the aneurysmal neck with the microcatheter. A double lumen microcatheter can solve the deadspace problem raised by the need to fill the balloon with a polymerizing substance. With this catheter, very small aneurysms can be treated. Indications, results and complications are discussed according to the aneurismal sites.

\section{Room E2}

\section{WS}

\section{6}

524 Staging of skeletal tumors - initial staging: local aggressiveness and diagnosis

Holger Pettersson; Lund

Modern therapy of musculoskeletal tumors has changed the demands on the preoperative radiologic work-up. Given the combination of limb saving surgery and aggressive oncologic treatment, the management of patients with musculoskeletal tumors today should be monitored at tumor centers, by a group representing at least oncology, orthopedic tumor surgery, pathology, cytology, radiology.

In the preoperative evaluation, the radiologist has to evaluate three important parameters: the local aggressiveness of the tumor, the diagnosis, and the loca extent. In this first paper we will discuss local aggressiveness and diagnosis. 1. Local aggressiveness

The local aggressiveness may be determined mainly for skeletal tumors, using conventional radiography, while little may be said about the soft tissue tumors from a radiologic point of view. Evaluation of local aggressiveness of skeletal tumors takes into account bone destruction, bone reaction and periosteal reaction. Using the terms designed by Lodwick, a three-graded scale may be made up, dividing the tumors into latent, active and aggressive tumors. It should be noted that this primarily is an evaluation of aggressiveness and not of malignancy, even if the malignant tumors most often are found in the aggressive group. Scintigraphy may add information as to the aggressiveness of skeletal tumors, while the other modalities are of limited value.

Concerning soft tissue tumors, little can be said on the aggressiveness from a radiological point of view. All deep soft tissue tumors (situated below the deep fascia) should be regarded as aggressive and malignant, till otherwise proved. 2. Diagnosis

The diagnosis of musculoskeletal tumors may be difficult, and in the work up of the radiologic diagnosis, several parameters should be taken into account: A. Local aggressiveness of the tumor

B. Singularity or multiplicity of the tumor

C. Localization in the body and in the bone

D. Specific radiologic patterns

E. Site and shape of the tumor
F. Age of the patient and pertinent clinical information

For bone tumors, the conventional radiography is important, not only for evaluation of aggressiveness, but also for assessment of localization, and specific radiologic patterns. Such patterns may be seen also with CT and MRI. Scintigraphy is used for evaluation of singularity or multiplicity, while site and shape is best seen using CT or MR.

For soft tissue tumors, MRI is the most profitable modality for diagnostic evaluation as a whole.

A meticulous analysis of all the parameters will then provide the basis for the radiologic diagnosis. However, it should be born in mind that the final diagnosis always is histopathologic.

\section{Primary bone tumors: local evaluation}

\section{Vanel; Villejuif}

Conservative limb salvage surgery is more and more used for malignant bone tumors. Local recurrences are almost always fatal. So the evaluation of local extension before surgery must be perfect.

MRI is by far the best technique for local evaluation because of the high contrast and the choice of the axis of the slices without moving the patient. The extension into the marrow defining the level of resection, skip lesions, the involvement of the epiphyseal plate and the joint, the soft tissues, the vessels are very accurately evaluated. The only limitations are very small lesions, and a possible overevaluation of the tumor especially on T2 weighted images. If MR is not avalaible, CT can be used.

Staging of skeletal tumors: evaluation and follow up of treatment A. M. Davies; Birmingham

If, after radiological detection, staging and biopsy a skeletal tumor is shown to be a benign lesion definitive treatment can be undertaken immediately - be it excision, currettage, or just re-assurance and observation. Locally aggressive lesions, with a high propensity to local recurrence (e.g. giant cell tumor), will require long term six monthly radiographic follow up. Bone scintigraphy should be interpreted with caution as increased activity at the surgical site may persist for several years. The majority of bone sarcomas, chondrosarcoma excepted, will undergo an intensive chemotherapy regime prior to surgery. In this period all three readily available imaging modalities, plain radiography, bone scintigraphy and CT as well as MRI, where available, may be used to assess tumor response to chemotherapy. The relative merits of each technique and correlation with the resected pathological specimen in this context are discussed.

Following surgery imaging is employed to monitor for local recurrence and evidence of metastatic spread. Sarcomas tend to metastasise to the lungs and all cases are routinely followed up with CT examinations of the chest. The problems of differentiating viable from non viable tumor tissue on chest CT is discussed. With the advent of chemotherapy a higher percentage of patients are developing metastases to bone without intervening visceral involvement, for which bone scintigraphy is valuable.

Complications of endoprosthetic replacement, chemotherapy and radiotherapy are discussed and the importance of close clinical-radiological correlation stressed.

\section{Room L}

\section{WS}

\section{8}

527 CT and MR imaging of the pharynx and the floor of the mouth normal anatomy and benign lesions

Sven G. Larsson; Uppsala

New imaging techniques like computed tomography and magnetic resonance imaging are able to demonstrate the complex anatomy of the pharynx and the floor of the mouth.

Normal anatomy

The base of the tongue part of the anterior boundary of the oropharynx is separated from the oral tongue by the circumvallate papillae. The tongue itself consists of both extrinsic and intrinsic musculature. The tongue is attached to the hyoid bone, mandible and the styloid process of the skull base by the extrinsic muscles. The three extrinsic muscles are the genioglossus muscle and the interdigitating hyoglossus and styloglossus muscles. The bulk of the tongue is made up of four intrinsic muscle groups.

The supporting structure and diaphragm of the floor of the mouth is the mylohyoid muscle. The anterior bellies of the digastric muscles run superficial and the geniohyoid muscles run deep to the mylohyoid muscle. The subman dibular salivary gland wraps around the posterior free margin of the mylohyoid muscle thereby forming a deep and a superficial portion. The duct after leaving the deep portion of the gland runs in the gutter between the mylohyoid and the hyoglossus and genioglossus muscles.

Benign lesions

Congenital muscle anomalies as well as anomalies in the decent of the thyroid gland can be encountered in the base of the tongue and the floor of the mouth. 
Also conditions associated with motor disturbances of the muscles of this part of the body can be analyzed. The tongue plays a central role in the sleep apnea syndrome and CT can therefore be used to study the size of the tongue and its relationship to the soft palate and the oropharyngeal airway. The palatine tonsils rest between the anterior and posterior faucial pillars. Benign calcifications, so called "tonsillar stones", can be found quite frequently. Benign disease of the tonsils are primarily inflammatory which may lead to abscess formation.

\section{CT and MR imaging of the pharynx and floor of the mouth: malignant lesions and tumor staging} Martin Lenz; Munich

Primary CT criteria for the presence of orofacial tumors are destruction of anatomical landmarks (muscle, bone), infiltrative and asymmetrical growth and the obliteration of fat tissue compartiments. Enhancement after iv-contrast or tumor necrosis are direct tumor signs but not seen in all cases and thus only secondary criteria. Computed tomography has an overall sensitivity of $82 \%$ in the T-staging of orofacial tumors which is reduced to $54 \%$ in the oroal cavity by metallic artefacts while the detection of small and superficially spreading tumors is impaired by their lack of enhancement after iv-contrast.

Magnetic resonance imaging on the other hand offer excellent tissue contrast that allows to depict even small tumors. MR sensitivity is significantly improved if Gadolinium-DTPA, especially in combination with fast gradient echo sequences, is used for iv-contrast. Additional advantages of MR imaging are the availability of any desirable imaging plane, the better demarcation of blood vessels and the absence of bone and metal artifacts that impair CT diagnostics in the oral cavity. In $78 \%$ of cases MR imaging is superior to CT in differentiating tumor from surrounding tissues.

\section{Room M}

\section{RC}

\section{Diagnostic imaging of the renal infections (acute)}

\section{Dalla-Palma; Trieste}

Taking into account the pathology, and simplifying the terminology very often misused in the literature, the acute renal infections can be classified in bacterial nephritis, diffuse and focal, abscess and pyonephrosis. The most common agents are: escherichia coli, proteus mirabilis, klebsiella pneumoniae and staphilococcus aureus.

The clinical pattern is very characteristic for diagnosing the urinary infection but it is insufficient for evaluating the kind of pathological involvement of the kidneys and the extrarenal extension of the lesion.

Ultrasonography (US) and Computed Tomography (CT) are very useful to recognize and to stage the lesions for the best therapeutical decision, medical or surgical

Urography has a low sensitivity and specificity. U.S. has a good sensitivity for renal lesions but not for the extrarenal extension.

$\mathrm{CT}$ has an extremely high sensitivity and diagnostic accuracy.

The imaging modalities are also indicated for the follow-up during medical therapy.

\section{Diagnostic imaging of the renal infections (chronic) \\ F. Stacul: Trieste}

Earlier detection and appropriate treatment of the disease and of the predisposing factors are decreasing the frequence of chronic renal infections. We shall focus on the following pathological entities:

Chronic recurring pyelonephritis, related to stones or obstruction of the urinary pathway. The contribution of the radiologist concerns the detection of predisposing factors and of the degree of parenchymal involvement.

Xanthogranulomatous pyelonephritis and renal malacoplakia. These granulomatous inflammatory diseases are quite rare and symptoms are often poor. The plain film of the abdomen and urography can be of some help in xanthogranulomatous pyelonephritis. CT features may be characteristic in this disease and can allow a preoperative detection and an evaluation of the extension that otherwise is usually quite difficult.

Renal tuberculosis. The radiologist has a central role in the diagnosis and follow-up of this disease. Tubercolosis can involve the cortical tissue only or, more often, the collecting system. In such a case, three ways of evolution can be recognized: spread in the excretory pathway, development of cavities which do not communicate with the collecting system, recurrent "poussées". Urography with nephrotomography allows to detect peculiar findings in the renal parenchyma and particularly in the excretory pathway. CT may give more information on the distruction of renal parenchyma. Ultrasonography can be a valuable tool in the follow-up of the cavities which do not communicate with the collecting system.

Other less common chronic infections include renal cholesteatoma, fungal infections and AIDS - associated nephropathy. However the radiologist must be confident with the possible radiological findings because of the increasing frequency of the latters.

\section{Room N}

\section{RC}

\section{Embolization: techniques and applications} A. Roche; Villejuif

Abstract available at the information desk.

\section{Embolization: techniques and applications Anne P. Hemingway; Sheffield}

Percutaneous transvascular embolization, i.e. the deliberate occlusion of blood vessels, is employed in a wide variety of clinical situations including haemorrhage, vascular malformations, tumours, hypersplenism, priapism, ablation of organ function, varices and varicoceles.

A meticulous angiographic technique under aseptic conditions is essential. A wide range of embolic materials is available ranging from particulate emboli such as Ivalon, to liquids such as hypertonic dextrose and alcohol, and including balloons and steel coils.

Awareness of and avoidance of potential complications, which may be immediate or delayed, and either systemic or focal and related to the angiographic procedure or the embolization itself, is critically important.

The workshop will discuss in detail indications for embolization, patient selection, informed consent, vascular technique, choice of embolic agent and avoidance of complications. Illustrative examples of haemorrhage, AVM's and tumours will be shown.

\section{Room 0}

RG 503

533, 534 Quality assurance in mammography

V. Barth and

M. Friedrich; Esslingen, Berlin

Quality assurance in mammography must be divided into a technical as well as an educational and medical section.

The scope of technical quality assurance is the control of the expert use of dedicated mammographic equipment including the appropriate image receptors by well-trained technical personell. An overview of the state of the art of mammographic equipment and image receptors will be given. The problems of film processing, dark room conditions and the mammographic application technique will be discussed. Problems of mammographic application technique as e.g. the correct positioning of the breast and a satisfactory penetration of all parts of the parenchyma will be highlighted.

An individual training program is necessary in order to motivate and convince radiographers of the special nature and requirements of the mammographic procedure and to achieve constant and high-level mammographic quality. The technical and organizational prerequisites for the implementation of a nationwide mammographic breast screening program will be defined. An evaluation of the respective results of various terminated and ongoing screening programs will be given.

\section{Room R}

\section{RC}

535 Recent advances in the treatment of lung cancer Hans-Peter Heilmann; Hamburg

Lung cancer is not a uniform disease, but a variety of tumors with different behavior, complex treatment protocols and normally unfavorable prognosis. Advances, however, has been achieved in different aspects. CT-Scanning and MRI, e.g., give better information on the extent of the disease and the development of pulmonary changes during treatment. Lung function studies have been improved by quantitative lung scans in order to correlate lung function to therapeutic strategy.

In prognosis and treatment, is has become evident that the old scheme "smallcell" and "non-small-cell" cancers is not sufficient. About $25 \%$ of all cancers have a mixed cellularity, even combining small-cell with non-small-cell tumors. On the other hand, in the group of non-small-cell cancers the behavior of adenocarcinomas, e.g., differ totally from squamous cell carcinomas, showing similarities to small-cell tumors. Therapeutic strategies have been proposed with respect to those differences.

In non-small-cell lung cancer (NSCLC), surgery is still the most effective treatment modality. The majority of NSCLC, however, can not be treated by 
surgical procedures. Patients in good condition (Karnofky-Index $\geq 70 \%$ ) with no distant metastases can be referred to high dose radiotherapy with curative intent. 5-year-survival is about $7 \%$ for the whole group, in early cases $11-20 \%$. Treatment protocols with altered fractionation (e.g. CHART) give interesting early results, but long term effects are not known.

Adjuvant irradiation has been a controversy for long time. Today, it is obvious that early cases or even patients with small hilar lymph nodes don't need adjuvant irradation when the postoperative status is $\mathrm{R} 0$. On the other hand, adjuvant postoperative irradiation improves results in T3-4-cases and especially in N2-3 tumors. 5-year-survival is about $20 \%$.

Chemotherapy is still the main treatment in small-cell lung cancer (SCLC), but drugs and strategies have changed. The role of radiotherapy in the management of SCLC is established, but there are controversies on time of irradiation, dose and prophylactic cranial irradiation. 5-year-survival in limited disease-case is about 7\%-11\%.

In palliation, better understanding of the development of the disease gives possibilities to tailor treatment to the individual in order to get maximum effect without overstraining the patient. 


\section{Room A}

\section{SS 11.11 Heart, Chest}

\section{$10.30 \mathrm{AM}$}

\section{Evaluation of hypertrophic cardiomyopathy by MRI}

E. Di Cesare, R. Occhiato, P. Di Renzi, R. Passariello; L'Aquila

10 patients, affected by hypertrophic cardiomyopathy, 4 females and 6 males, in age from 33 to 55 (mean 40.2), and 5 male patients, with secondary myocardic hypertrophy with age range from 36 to 59 (mean 47.2) underwent Magnetic Resonance Imaging examination with gated Spin-Echo T1 and T2 weighted sequences for a morphological evaluation and signal analysis. Gradient refocusing sequences were employed for functional evaluation. The same patients were submitted to mono- and bidimensional echocardiographic examination (HP sonos 1000). After comparison between MRI and Echo data, we found a correlation coefficient of 0.62 for apex thickness, $\mathrm{R}=0.85$ for intraventricular septum, $R=0.84$ for thickness of the posterior wall, 0.76 for the lateral wall, and 0.79 for the area at papillary level. In addition, signal intensity analysis was performed in 5 patients affected by secondary myocardic hypertrophy and in other 5 patients with hypertrophic cardiomyopathy. The analysis was performed at the level of the septum and the values were normalized with the signal intensity of the fat. In secondary hypertrophy we found lower values as compared to the ones of primary hypertrophy.

\section{$10.40 \mathrm{AM}$}

\section{In-vitro behaviour of mechanical heart valves in a $1.5 \mathrm{~T}$ supercon-} ducting magnet

H. Frank, P. Buxbaum, S. Globits, L. Huber, H. Mayr, D. Glogar, H. Imhof; Vienna

We examined seven commonly used mechanical heart valves in a $1.5 \mathrm{~T}$ magnetic field, concerning deflection and change in temperature during gradient echo sequences. The valves were mounted on a special suspension and deflection was measured at the entrance of the coil. Change in temperature in air as well as waterbath was measured using a thermo element. Three measurements were done for each valve and than averaged.

Results: The Björk Shiley valve showed the greatest deflection with $1.8 \mathrm{~mm}$ at the coil entrance, according to $0.1 \mathrm{~g}$ static deflection. All other valve types showed lower values. During acquisition gradient echo sequences no deflection or change in temperature was detected. Concerning the temperature at the valve ring, we found an increase in 3 valve types, most prominent in the Medtronic valve with $+0.6 \mathrm{C}$.

In conclusion using a $1.5 \mathrm{~T}$ Magnet most commonly used valve types show little deflection and increase in temperature without clinical significance.

Thus, implantation of a heart valve prosthesis is no contraindication for MR-examination in a $1.5 \mathrm{~T}$ magnet.

\section{$10.50 \mathrm{AM}$}

538 Cardiac cine magnetic resonance imaging at low intensity field P. Di Renzi, E. Di Cesare, C. Simonetti, R. Occhiato, C. Catalano, L. Marsili, F. Caramia, P. Pavone, R. Passariello; L'Aquila

Purpose: To assess the feasibility of 0.2 permanent unit in the evaluation of morphological and functional cardiac parameters.

Methods: 42 subjects ( 5 with valvular involvement, 17 well trained athletes, 10 volunteers, 4 postoperative controls for Ascending Aortic Aneurysms, 5 dilatative cardiomyopathy, 1 hypertrophic cardiomyopathy) underwent Cardiac Cine Magnetic Resonance (CCMR) investigation with a 0.2 Permanent Magnet (Esaote MP 4000) by Gradient Refocusing sequences $\left(\mathrm{TR}=50 / \mathrm{TE}=23 / \mathrm{FA} 45^{\circ}\right)$ for their different cardiac problems or voluntary study to assess right heart chambers.

Results: Morphological parameters (areas, volumes and masses) showed a high correlation with 2-D echocardiography ( $\mathrm{r}$ range $0.72-0.90$ ) as well as functional data in case of valvular or prosthetic insufficiency. Very high quality images of the right atrium and right ventricle were obtained. Overall assessment of performances was made by two expert radiologists. Results were evaluated as competitive with those of own personal radiologic experience and with those reported in the literature.

Conclusions: CCMR imaging at low intensity field allows to obtain good quality images comparable to those obtained by means of medium $(0.5 \mathrm{~T})$ and high $(1.5 \mathrm{~T})$ intensity field units.

539 MRI and echocardiography in the assessment of pericardial involvement by mediastinal lymphomas

J.D. Tesoro-Tess, S. Biasi, L. Balzarini, E. Ceglia, R. Petrillo, Y. Reyner, R. Musumeci; Milano

1. Pericardial involvement is a relatively frequent event both in Hodgkin's disease (HD) and non-Hodgkin lymphoma (NHL), mostly in presence of bulky disease. Since MRI seems to have increased possibilities in comparison with CT utilizing multi-planar and multi-echo sequences, a chest MRI combined with echocardiography was performed in 26 consecutive previously untreated patients with mediastinal HD and NHL.

2. MRI was performed with a $1.5 \mathrm{~T}$ superconductive magnet. Transverse and coronal ECG-gated $8 \mathrm{~mm}$ thick mainly T1-weighted images were acquired (TR = R-R., TE/17). US examination was made according to standard planes (long and short axis combined with apical four chambers view) using both a 3.5 and $5.4 \mathrm{MHz}$ probes.

3. In $8 / 26(30.1 \%)$ patients adenopathies extended beyond the cardiac contours in contiguity with the pericardium. US correctly identified a pericardial effusion, present in $7 / 8(87.5 \%)$, whereas MRI only in $5(62.5 \%)$. On the contrary the panoramic view offered by MRI permitted to better define the relationships between adenopathies and parietal pericardium as well as extrapericardial diffusion.

4. In conclusion MRI should be preferred to CT in the staging of chest HD and NHL but followed by echocardiography if pericardial effusion is suspected or critical mediastinal involvement is depicted.

\section{$11.10 \mathrm{AM}$}

540 Evaluation of tumor invasion to heart and great vessels by cine MR imaging

Masayuki Mori, Kiyoshi Murata, Masashi Takahashi, Tsutomu Sakamoto, Mitsuru Komizu, Hirofumi Kato, Rikushi Morita; Shiga

Purpose: To evaluate the usefulness of cine MR imaging for the diagnosis of invasion of lung cancer and mediastinal tumors to the heart and great vessels. Methods and Materials: 21 patients including 6 with lung cancer, 5 with esophageal cancer, and 10 with mediastinal tumors, which were confirmed by operation, were scanned using a 1.5 T MR imaging system (Signa, GE). Following the conventional cardiac-gated spin echo imaging, cine MR imaging was performed with field echo sequence through the levels at which the tumor attached to the heart and great vessels. These 21 examinations were interpreted independently by three experienced radiologists about tumor invasion to the heart and great vessels on conventional MR imaging and cine MR imaging, separately. The diagnostic sensitivity and specificity of each imaging was calculated, comparing MR findings with surgical findings.

Results: Cine MR imaging showed the sensitivity of $100 \%$ and specificity of $89 \%$, while the sensitivity and specificity of conventional MR imaging was $70 \%$ and $53 \%$, respectively.

Conclusions: Cine MR imaging may provide additional information about tumor invasion to the heart and great vessels in patients who showed equivocal findings by conventional static imaging modalities.

\section{$11.15 \mathrm{AM}$}

541 Tumor invasion of the pleura and chest wall: detection with contrast enhanced MRI

R. Bittner, W. Schörner, H. Hieckel, R. Loddenkemper, A. Grassot, D. Kaiser, R. Felix; Berlin

Purpose: To evaluate criteria of Gd-DTPA enhanced MRI in the detection of malignant pleura and chest wall invasion.

Subject and methods: 25 patients with different pleura related masses were investigated. MR images were generated with $0.5 \mathrm{~T}$ or $1.5 \mathrm{~T}$ superconductive systems. All patients were examined with ECG-gated T1- and T2-weighted (TI-WI/T2-WI) SE sequences. The T1-WI were repeated after IV application of Gd-DTPA $(0.1 \mathrm{mmol} / \mathrm{kg} \mathrm{BW})$. Slice thickness was $4-8 \mathrm{~mm}$ in transverse and coronal plane. Surgery confirmed malignant invasion of chest wall and/or pleura in 22 of the 25 patients.

Results: Two different features were evaluated for tumor detection with Gd-DTPA enhanced MRI. The first represents an enhancing lesion within the chest wall muscles. The second feature stands for enhancing pleural structures. Those findings were confirmed by lesions of increased SI within the chest wall and/or the pleura in the T2-WI. In all 18 patients with chest wall lesions surgery revealed tumor invasion. The pleural feature was found in 19 patients, in 16/19 malignancy was confirmed. In 3 patients we had false positive results due to non-malignant pleural inflammation. As an additional finding, pleural thickening was detected in all 16 patients with malignant pleural disease, pleural inflammation showed no thickening.

Conclusions: Characteristic features of malignant chest wall invasion in Gd-DTPA enhanced MRI are contrast enhancing lesions within the chest wall. Enhancing pleural structures alone appeared not to be specific for malignant 
invasion, pleural inflammation may have similar MR appearance. In those patients pleural thickening was indicative for malignant disease.

\subsection{AM}

542 Comparison of utility of CT and MR for preoperative diagnosis of N factor of primary lung cancer - cases where diagnosis by CT and MR are different -

Soichi Akata, Dai Kakizaki, Kouji Kawana, Kimihiko Abe, Saburo Amino: Tokyo

1. Purpose: Correct diagnosis of $\mathbf{N}$ factor in primary lung cancer is quite important to select the method of the treatment. In the present study, we compared the abilities of preoperative CT and MR to diagnose $\mathrm{N}$ factor in primary lung cancer.

2. Methods: 80 cases of primary lung cancer were studied. The equipments we used were Toshiba TCT-900S and superconductive MRT-50A with 0.5 Tesla. As positive for metastasis, we defined more than $10 \mathrm{~mm}$ of long and short diameters of each.

3. Results: MR was useful for hilar lymphnodes due to the absence of signals for blood vessels, although it had poor ability to detect calcification and exhibited poor spatial resolution. Sensitivity, specificity and accuracy of CT were $81.8 \%, 80.3 \%$ and $80.5 \%$, respectively, while those of MR were $77.9 \%$, $83.2 \%$ and $82.2 \%$, respectively, indicating almost the same level of diagnosis. 4. Conclusions: Considering the result in the lymphnode cases $(33 / 544)$ in which diagnosis by CT and MR were different from each other, it can be said that at least either of CT and MR can give a correct diagnosis in most of the cases. Thus, the rate of correct diagnosis can be increased by concomitant use of both methods.

\subsection{AM}

543 Bronchogenic carcinoma: value of MRI in " $T$ " (TNM) parameter assessment

S. Baldelli, P. Ercolani, E. Bichi Secchi, S. Gasparini, E. De Nigris, A. Giovagnoni; Ancona

Purpose: The purpose of this work is to verify the diagnostic value of MRI in "T" parameter assessment (TNM classification) of lung cancer in comparison with CT findings and surgical-pathological data.

Methods: 104 patients ( $88 \mathrm{M}, 16 \mathrm{~F}$ ) with radiographic, CT, bronchoscopic and cytologic diagnosis of lung cancer underwent MRI examination. In $70 \%$ of cases the lesions were peripherically localized. A superconductive $1.0 \mathrm{~T}$ (Magnetom) tomograph unit was used. Multiplanar, T1 and T2 weighted images were obtained. Two observers evaluated independently MRI and CT findings in order to check lesion's parameters for "T" stage assessment (AJCC - TNM classification schema). A surgical, pathological proving or follow-up were obtained in all cases.

Results: The results show that in "T1" stage (12 patients) CT was more accurate $(100 \%)$ than MRI ( $85 \%)$. In "T2" stage (22 patients) MRI understaged 3 lesions $(13 \%)$ while CT misinterpreted 8 lesions $(30 \%)$. In cases with thoracic wall infiltration (14 patients) "T3" stage the diagnostic accuracy was $85 \%$ for MRI and $60 \%$ for CT. In "T4" stage (52 patients) MRI made an easy evaluation of great mediastinal vessels infiltration than $\mathrm{CT}$.

Conclusions: CT remains fundamental for bronchogenic carcinoma staging; however in cases with suspicion of chest wall (T3) or vessels (T4), infiltration MRI investigation is recommended.

\section{$11.45 \mathrm{AM}$}

\section{MR angiography in the evaluation of vena cava inferior and venous} vessels of the pelvis

L. Ziegler, Th. Vogl, H. Schedel, G. Küffer, M. Seiderer, D. Hahn; München

Purpose: Different MR angiography techniques (MRA) for demonstrating venous anatomy and pathology were compared with venography.

Methods: Vascular imaging at 1.5 T Siemens Magnetom was performed with sequential 2D GREW (Flash) sequence (TR/TE $=32 / 8, \alpha=60^{\circ}$, matrix $=256 \times 256)$ and presaturation pulses were applied to suppress selectively signal from inflowing spins. 3 probands and 5 patients were examined and compared with venograms.

Results: Compared with venography a similiar delineation of occlusion was achieved and additional demonstration of bypassing veins was possible. Conclusions: MRA is an accurate, noninvasive mean of detecting occlusions of vena cava inferior and pelvic veins
$11.48 \mathrm{AM}$

545 Pitfalls in magnetic resonance imaging of adenoid cystic carcinoma of the trachea

Ronald L. Embry, Dean J. Shanley; Honolulu, Hawaii

Adenoid cystic carcinoma (cylindroma) of the trachea is an uncommon malignancy which can be treated with positive results by surgery and radiation therapy. Pretherapeutic assessment is important in determining the extent of local tumor invasion and the involvement of mediastinal structures, especially if surgery is proposed as part of therapy. A case of adenoid cystic carcinoma of the trachea, evaluated best by multi-planar MRI in multiple sequences is discussed, together with the pitfalls of chemical shift artifact in the distal trachea. The possibility of artifacts affecting interpretation of images can be minimized by avoiding the use of gradient recall echo sequences and variable bandwidth protocols.

\subsection{AM}

\section{Popliteal artery entrapment demonstrated by MRI}

Ernesto Di Cesare, Paolo Di Renzi, Giuseppe Morettini, Roberto Passariello; L'Aquila

Entrapment of the popliteal arteries is a congenital disorder resulting from an abnormal relationship between the arteries and muscles.

Two patients with suspected popliteal entrapment were studied by Magnetic Resonance Imaging. The examinations were performed by a superconductive magnet operating at $0.5 \mathrm{~T}$ using gradient echo sequences with rephasing (TR $100 \mathrm{~ms}$, TE $20 \mathrm{~ms}$ ), with $7 \mathrm{~mm}$ slice thickness and $20 \mathrm{~cm}$ of view field. We examined the leg at rest and during active plantar flection (against resistance). Both patients were submitted to angiography and results were compared. MRI showed to be useful in demonstrating the relationship between muscle and popliteal arteries. After active plantar flection we observed signal loss in both patients related to stenosis of the arteries as showed at angiography. In order to compare stress images we studied also four volunteers by means of MRI and did not any signal alterations in the popliteal arteries.

\section{Room B}

\section{SS 01.01 Digital radiography}

10.30 AM

\section{Optimized digital imaging for routine chest radiography} U. Neitzel, I. Maack; Hamburg

Separation of detection and display in digital radiography allows the optimal fitting of the detector for high sensitivity and large dynamic range whereas the conspicuity of details can be enhanced by image processing. Chest radiography is the most demanding application in this respect because of the large variations in density between lung and mediastinum. This requires wide latitude and high detail contrast simultaneously. We have developed a procedure of image processing and display which can be described as 'digital beam equalisation'. It consists of a locally adaptive dynamic range compression and detail enhancement. Application of the procedure to chest images gives results which look like optimal conventional images in the lung fields and displays also full information in mediastinal and retrocardiac regions. We feel that this new image display method presents all the information in one which is usually displayed in two images of one exposure. The paper includes remarks on conditions for the detector to achieve full benefit of this processing method.

\subsection{AM}

\section{Dual-energy chest radiography with storage phosphors}

M. Prokop, C. Schaefer, A. Memis, J. W. Oestmann, M. Galanski; Hannover

Purpose: The complex composition of soft-tissue, air and calcified structures in a chest radiograph frequently aggravates the detection and classification of lesions. Dual energy (DE) subtraction has the potential to decrease the effects of obscuring rib structures and aid in the differential diagnosis of calcified from non-calcified structures. However, image quality is often seriously compromised by the high noise level.

Methods and Materials: DE images were obtained with standard storage phosphor equipment (Digiscan 901, Siemens). We used a single shot technique with $\mathrm{K}$-edge pre-filtering and a sandwich cassette for simultaneous acquisition of high and low energy images. Image processing algorithms for noise suppression were implemented for a $1 \mathrm{~K} \times 1 \mathrm{~K} \times 12$ bit matrix on the standard postprocessing console and on the host computer (Microvax II, DEC).

Results: DE subtraction improves the differentiation of calcified from non-calcified structures and in the detection of lesions that are obscured by overlying ribs. With application of noise suppression algorithms some skeletal 
contours are reintroduced into the image, however, the significantly improved signal to noise ratio allows for the detection of even small structures. Non-linear weighting factors that correct for beam hardening effects considerably improve the imaging of the spine and the lateral rib contours in the subtracted bone images.

Conclusions: DE subtraction decreases the obscuring effects of overlying skeletal structures in chest radiography. Application of noise suppression algorithms and non-linear weighting factors significantly improves the quality of the subtracted images.

\section{$10.50 \mathrm{AM}$}

\section{Digital storage phosphor imaging of interstitial lung disease:} impact of post-processing

C. M. Schaefer, R. Greene, M. Prokop, J.W. Oestmann, M. Galanski; Hannover

Purpose: The ability to resolve the fine linear structures of interstitial lung disease is one measure of the limiting performance characteristics of an imaging system.

Methods and Materials: We compared conventional film radiography (FR) and six algorithms of isodose storage phosphor digital radiography (SR) $(0.2 \mathrm{~mm} \times 10$ bit pixel matrix $)$ in the detection of interstitial lung abnormality documented by computed tomography ( 40 patients with abnormality and 25 normal control subjects). Performance was evaluated by an analysis of variance of average receiver operating characteristics area $\left(A_{2} \pm\right.$ standard error) of 2730 observations by six readers.

Results: The moderately and the more markedly high frequency edge enhanced algorithms of SR (SRh and SRhi) were equivalent to FR. The defaul mode of SR (SRd), the low and medium frequency edge enhanced algorithms of SR (SRm and SRI), and the grey scale reversed mode (SRg) were inferior to $\mathrm{FR}:(\mathrm{FR}=.88 \pm .01, \mathrm{SRhi}=.88 \pm .01, \mathrm{SRh}=.86 \pm .01, \mathrm{SRd}=.84 \pm .01$, $\mathrm{SRg}=.83 \pm .02, \mathrm{SRI}=.80 \pm .03, \mathrm{SRm}=.78 \pm .03, \mathrm{p}<.05)$.

Conclusions: We conclude that high frequency edge enhanced algorithms of $\mathrm{SR}$ can perform as well as FR in the detection of interstitial disease.

\section{$11.00 \mathrm{AM}$}

\section{Postprocessing of digital chest radiographs: which is the best} compromise?

M. Prokop, C.Schaefer, J.W. Oestmann, S. Reichelt, M. Galanski; Hannover

Purpose: We compared the performance of four classes of unsharp mask filtering algorithms for storage phosphor chest radiographs $(1744 \times 2144 \times 10$ bit matrix $)$ to that of iso-dose conventional radiographs $(\mathrm{FR})$ in the detection of different types of lung pathology.

Methods and Materials: Three types of subtle lesions (nodules, fine lines and micronodular opacities), chosen to represent various spatial and contras requirements were superimposed on chest radiographs of 10 healthy volunteers. Unsharp mask filtering was performed with "small" (kernel length = $\left.1.44 \mathrm{~mm}, \mathrm{RS}_{\mathrm{s}}\right)$, "medium" ( $5 \mathrm{~mm}, \mathrm{SR}_{\mathrm{m}}$, current industry default), "large" $\left(25 \mathrm{~mm}, \mathrm{SR}_{1}\right)$ and "ultra-large" $\left(70 \mathrm{~mm}, \mathrm{SU}_{\mathrm{ul}}\right)$ filter kernels. A total of 10.800 observations by eight radiologists were evaluated according to ROC analysis. Significance of differences was tested by analysis of variance $(p<0.05)$. Results: In the detection of nodules, $F R, \mathrm{SR}_{\mathrm{ul}}$ and $\mathrm{SR}_{\mathrm{l}}$ were superior to $\mathrm{SR}_{\mathrm{m}}$ and $\mathrm{SR}_{\mathrm{s}}$. In the detection of micronodular opacities, $\mathrm{FR}$ was superior to all $\mathrm{SR}$ algorithms; $\mathrm{SR}_{\mathrm{ul}}$ and $\mathrm{SR}_{\mathrm{l}}$ were better than $\mathrm{SR}_{\mathrm{m}}$. In the detection of lines, $\mathrm{FR}$ was only superior to $S R_{u l}$; all other $S R$ versions were equivalent to $F R$.

Conclusions: Edge enhancement with large kernels $\left(\mathrm{SR}_{1}\right)$ seems to be the most appropriate standard postprocessing algorithm to simultaneously match linear, nodular and micronodular structures.

\subsection{AM}

\section{Dual-energy subtraction imaging with digital luminescent radio- graphy in thoracic diagnostics}

R.-D. Müller, M. Voss, V. Heer, E. Löhr; Essen, Erlangen

A digital dual-energy subtraction is applicated in order to improve the demonstration of calcified and noncalcified pulmonary nodules.

Methods: A copper filter was placed between two imaging plates in one cassette to separate the effective energy of the $x$-ray spectrum between the $x$-rays absorbed in both imaging plates. The first plate showed a "bone-emphasized" radiograph, the second a "soft-tissue" image. The subtracted image was made from two original images multiplied by special weighting factors using a Digiscan System (Siemens AG.). Image post-processing of the digital data enables the elimination of either skeleton or soft tissue structures. We examined the influence of filter thickness, tube voltage and $\mathrm{mAs}$-product on image quality and signal-to-noise ratio.

Results: Advantages are demonstrable in detection of calcified and noncalcified pulmonary nodules simulated in an anthropomorphic thorax phantom as well as in patients with pulmonary metastasis.
Conclusions: The one-shot dual energy subtraction improves the recognition of pulmonary nodules and skeleton lesions. We have to enlarge energy separation, one of the most important factors determining the image quality for dual-energy subtraction. We also have to optimize image processing in order to reduce signal-to-noise ratio and radiation exposure.

\section{$11.20 \mathrm{AM}$}

\section{Comparison of AMBER and conventional film in CT-documented} chest disease

Petra A. M. Bun, Leo J. Schultze Kool, Piet van der Merwe, Henri Lemmers, Jo Hermans; Leiden

Purpose: To compare conventional and AMBER radiographs with CT scans as a standard.

Methods: A total of 150 patients with a suspected or known chest abnormality underwent chest CT. In each case, posteroanterior and lateral radiographs of the chest with both conventional radiography and AMBER were obtained on the same day as the CT scan. Four radiologists read the conventional and AMBER films. For both systems, the same film screen combination was used (TMG-Lanex-regular). The lesions were divided into three groups: pulmonary, mediastinal, and pleural. The readers were asked to indicate the presence of an abnormality according to a five-level scale of confidence. The data were analyzed with receiver operating characteristic methods.

Results: Tests of performance indicated that AMBER is at least as effective as conventional radiography in the overall detection of all three types of abnormalities. There were fewer interpretation errors with AMBER. Pulmonary opacities in the cardiac and diaphragmatic regions and mediastinal lesions especially are detected more easily with AMBER.

Conclusions: AMBER radiographs are superior to conventional radiographs in the detection of lesions in the retrocardiac, subdiaphragmatic, and mediastinal regions.

\section{$11.30 \mathrm{AM}$}

553 Photostimulable phosphors computed radiography: experience with portable chest films

J. A. Verschakelen, F. Dhaenens, G. Marchal, A. L. Baert; Leuven

Purpose: Clinical evaluation of portable chest films obtained with a prototype digital phosphor plate scanner (resolution $2048 \times 2496 \times 12$ bit, Agfa).

Methods: A digital phosphor plate scanner was used to produce bedside chest films from 1100 patients. The images were interpreted by 4 radiologists. In 300 patients, the influence of dose and image processing was studied in a double blind study. In 700 patients, starting from 1 exposure, images were sensitometrically postprocessed on 2 films. One to visualize the mediastinum and the other to see the lungs. Diagnostic quality and ability to identify catheters was examined. In 400 patients phosphor images were compared with conventional portable film-screen systems.

Results: Best results were obtained with a dose equal to $80-100 \%$ of the dose used with film-screen systems. Images optimized to visualize the lungs were significantly $(p<0.001)$ preferred for interpretation of the lungs, to images optimized to visualize the mediastinum. The reverse was true $(p<0.001)$ when visualization of the mediastinum on both images was compared. Detection of catheters was significantly $(p<0.001)$ better on mediastinal than on lung images. The phosphor images were in $75 \%$ of the cases preferred to conventional film screen systems.

Conclusions: Phosphor images were preferred above conventional film screen combinations, were scored as average to good for image quality and diagnostic value and showed less exposure errors. Only small dose reduction was possible.

\section{$11.40 \mathrm{AM}$}

554 Performances of fluoroscopic and digitalized fluoroscopic images in detection of pulmonary nodules and diffuse infiltrative diseases (DID) in comparison with conventional chest radiographs

\section{J.F. Biquet, A. Albert; Liège}

Purpose: Since 1987 we have been performing chest X-ray of hospitalized patients with a Large Field Image Intensifier (LFII) (SIEMENS ${ }^{\circ}$ ). An original adaptation of our installation allowed to obtain simultaneously a photofluorogram (FI) and a $1024^{2}$ digital image (DI) with a single exposure. The aim of this paper is to evaluate the performances of F1 and DI in the detection of nodules and DID in comparison with the conventional full size technique (FS). Methods: By using F1, DI and FS, 6 observers were asked to detect 39 one centimeter nodules disposed on the back side of an embedded human chest $\left(\mathrm{AGFA}^{\circ}\right)$. In another experiment, 6 observers were asked to detect 22 patients affected with DID out of a group of 40 . The reference method in this study was high resolution C.T.

Results: No statistical difference was found between F1 and FS in the detection of nodules and DID. On the other hand, DI were statistically less accurate in the diagnosis of these two diseases.

Conclusions: These two experiments are concordant with our three years 
clinical practice. The good spatial resolution of F1 allows this method to detect subtle pathologic images. It is not the case of DI whose spatial resolution and signal/noise ratio are perfectible. However, DI shortens the time of chest X-ray and is useful to the diagnosis by immediate control of the image as soon as it is displayed in the TV monitor: Equivocal images can be checked under fluoroscopic control immediately.

\section{$11.50 \mathrm{AM}$}

\section{New techniques in chest imaging - a comparison of five imaging} methods

H. P. Busch, M. C. Freund, K. J. Lehmann, M. Georgi, K. Richter; Mannheim

In this study new digital and analogue techniques for chest imaging were compared with conventional film-screen radiography. The spatial resolution, the entrance dose and the detection of pulmonary nodules (FROC-study) were studied with Slot(AMBER)-technique, digital image intensifier radiography, storage phospor radiography, $100 \mathrm{~mm}$ technique and film screen radiography. Eight radiologists of two different institutions (Mannheim, Berlin-Buch) graded the image quality of 50 patients. The results demonstrated a lower image quality of $100 \mathrm{~mm}$ technique and digital image intensifier radiography compared with film-screen radiography. AMBER-technique and storage phospor radiography were superior to film-screen radiography. The AMBERtechnique shows best results in mediastinum as well as in the retrocardial and retrodiaphragmal space.

\section{Room C}

\section{SS 04.02 General II}

$10.30 \mathrm{AM}$

\section{Uncommon neoplasms of the gastrointestinal tract (Keynote Lecture)}

Daniel J. Nolan; Oxford

Benign neoplasms such as leiomyoma are uncommon in the stomach but when present they have characteristic appearances. Metastases may cause unusual changes in the stomach. The duodenum is an uncommon site for primary neoplasms but the duodenum is the most common site in the small intestine for primary carcinoma. Malignant neoplasms may spread directly to involve the duodenum from the pancreas, colon, kidney, gallbladder and bile ducts.

Primary carcinomas may involve the jejunum but are extremely rare in the ileum whereas the ileum is the site of most small intestinal carcinoid tumours and lymphomas. Carcinoma of the caecum may spread via the lymphatics to the terminal ileum and the small intestine may be invaded by neoplasms from other abdominal organs. Blood-borne metastases may be seen in any part of the small intestine.

The colon may occasionally be the site of a primary lymphoma. Mesenteric spread of gastric and pancreatic carcinoma to the colon is uncommon but the appearances are characteristic.

\section{$10.45 \mathrm{AM}$}

557 Standardized, fractionated, oral double contrast examination of the small bowel using Metoclopramid and Ceruletid

W. Weidenmaier, J. M. Friedrich, K. A. Schumacher, G. Bargon; Ulm

Purpose: An oral standardized small bowel examination with documentation mostly independent of investigator experience, reduction of examination time and $\mathrm{x}$-ray dose, improvement of visualisation and evaluation of motility. Methods: Oral application of $400 \mathrm{ml}$ Barium-Tylose Suspension, followed by injection of $10 \mathrm{mg}$ Metoclopramid to homogenize and stimulate the transport out of the stomach. Motivation of the patient to drink 1000-1400 ml Tylose suspension when the stomach is emptied. Following a double contrast of the jejunum and of the proximal ileum, Ceruletid was injected iv. to stimulate the peristaltics. Observation and documentation ( $\mathrm{x}$-ray-Film, Video). The value of these drugs has been assessed in a prospective study in 326 patients with suspected Crohn's disease.

Results: Improved visualisation (terminal Ileum) from 80 to $100 \%$, increase of the diagnostic validity from 65 to $96 \%$, reduction of examination time from 90 to 67 minutes, shortening of fluoroscopy time from 11 to $7.2 \mathrm{~min}$ with an according dose reduction.

Conclusions: The intravenous use of Metroclopramid and Ceruletid reduces the time required for fractionated double contrast examination of small bowel. It also improves visualisation of individual sections of the small bowel, particularly the terminal ileum. The Video documentation permits the evaluation of small bowel motility.
$10.55 \mathrm{AM}$

$558{ }^{99 m}$ Tc-HMPAO labelled leucocytes in the assessment of inflammatory bowel activity - comparison with endoscopy and histology T. Lantto, K. Järvi, I. Krekelä, E. Lantto, M. Vorne; Hollola

10 patients (pts) with proven inflammatory bowel disease (IBD) were imaged at $2 \mathrm{~min}, 0,5 \mathrm{~h}, 2 \mathrm{~h}$ and $4 \mathrm{~h}$ with ${ }^{99 \mathrm{~m}} \mathrm{Tc}-\mathrm{HMPAO}$ labelled leucocytes. The activity of IBD was scored visually (score $0-3$ ) from the endoscopically and histologically confirmed segments $(n=28)$ without knowing the result of these examinations. The scintigraphic results were compared with the activity grading of endoscopy and histology (scores $0-3$ ).

In 20/28 segments the visual and histological score were equal; in 6 segments the visual was 0 (normal), but histological score was 1 (mild) and in 2 segments the visual score was 2 (moderate), but histological score was 1 because of the shifted activity from the more proximal intense inflammation. In moderate and severe histological changes the scores were equal. The endoscopy and scintigraphy agreed in 16/28 segments; the endoscopy showed higher score in 9 and lower in 3 segments. The scoring of histology and endoscopy agreed in $17 / 28$ segments; the endoscopy underestimated inflammatory activity in 5 segments and overestimated in 6 segments. $2 \mathrm{~h}$ images had the highest accuracy with histology.

${ }^{99 \mathrm{~m}} \mathrm{Tc}-\mathrm{HMPAO}$ labelled leucocytes agreed better with the histological changes than endoscopy and the correlation was complete in moderate and severe changes of IBD.

\section{$11.00 \mathrm{AM}$}

\section{Imaging of idiopathic chronic ulcerative enteritis} J. Andreu, L. Casas, P. Vicente de Vera, R. Puy, J. Capellades, R. Salvador; Barcelona

Idiopathic Chronic Ulcerative Enteritis is a rare entity characterized by the association of ulcers of the small bowel with a malabsorption syndrome. Clinical, radiological and histological correlations in 2 cases are presented. Barium studies and CT findings were important in the diagnosis. Differential diagnosis and the relationship of this process with celiac disease and intestinal lymphoma are discussed.

\section{$11.03 \mathrm{AM}$}

560 Retrospective study of 255 patients with ischemic colitis: radiology in comparison with endoscopy

J.W. A. J. Reeders, Ad. C. Wevers, G. Rosenbusch, G. N. J. Tytgat, A. J. Bakker; Amsterdam

Purpose: To compare different radiological and endoscopical manifestations of ischemic colitis in the various stages.

Methods and Materials: Of 255 patients with various stages of ischemic colitis, barium enema examinations were performed in $33 \%$ of those studied radiologically and colonoscopy was performed in $26 \%$ of those studied endoscopically.

Results: Thumbprinting (submucosal edema/hemorrhage) and/or transverse ridging were more frequently seen $(69 \%$ and $59 \%$, respectively, of patients studied radiologically and endoscopically) in the early stages (1-7 days) of colonic ischemia, but we also occasionally found late manifestations (after 14 days) of thumbprinting ( $22 \%$ and $27 \%$, respectively)

Ulceration (ragged sawtoothed irregularity or spiculation of the bowel wall) (both $44 \%$ ) was present at the end of the first week, with a peak frequency in the 4 th to 6 th week ( $50 \%$ and $68 \%$, respectively), after which the frequency of occurence decreased.

Stricturing and/or tubular narrowing ( $67 \%$ and $32 \%$, respectively) were found in the late stages (after 4 weeks) of colonic ischemia, representing the fina status of the bowel wall, accompanied by progressive fibrosis and mucosal reorganization. Sacculations (pseudodiverticula of the antimesenteric colonic wall) occurred in a late stage of colonic ischemia, after 1-3 months ( $72 \%$ and $9 \%$, respectively).

Conclusions: Early ischemic manifestations (thumbprinting, transverse ridging, ulceration) and late ischemic manifestations (stricturing and/or tubular narrowing, sacculation) corresponded with radiological and endoscopic findings.

\section{$11.13 \mathrm{AM}$}

561 The "equivocal" lesion in the follow-up of colorectal cancer in computed tomography: the need for radioimmunoscintigraphy G. Barzen, R. Richter, C. Zwicker, W. Böse-Landgraf, R. Raakow, R. Felix; Berlin

The aim of this prospective study was to investigate if Radioimmunoscintigraphy (RIS) is able to demonstrate local recurrency of colorectal cancers in patients with equivocal or negative CT scans. We examined 53 patients $6-52$ months after primary operation of colorectal cancer with "equivocal" (scare/ 
tumor/inflammation) ( $\mathrm{n}=26$, group A) or "conclusive positive/negative" computed tomography results $(n=27$, group B). CT of the abdomen in $8-10 \mathrm{~mm}$ contiguous sections was performed after application of oral and intravenous contrast media. RIS was done with $99 \mathrm{~m}-\mathrm{TC}$ labelled monoclonal-CEA-antibodies (BW 431/26) in SPECT and planar technique 3-6 and $22-31 \mathrm{~h}$ p.i. Results were proven by operation $(\mathrm{n}=40)$ and followup/biopsy $(\mathrm{n}=13)$.

Results: Group A: sensitivity for recurrency was (if there had to be a "clear cut decision" for CT) (CT/RIS): $53 \% / 77 \%$ and $69 \% / 77 \%$ for specificity. Group $\mathrm{B}$ (in case of conclusive CT results): (CT/RIS) 78\%/88\% and $94 \% / 83 \%$ for sensitivity and specificity. In total 27 metastatic lesions were detected (liver $\mathrm{n}=18$, lung $\mathrm{n}=9$ ). By RIS only 5 of 18 liverlesions demonstrated an antibodyaccumulation ("hot lesion") whereas as 10 showed an equivocal "cold lesion". Lung metastasis was detected by RIS in 1 of 9 cases.

Conclusions: RIS is able to demonstrate recurrency in case of false negative or equivocal CT results, but there are also false negative results in RIS for local recurrency and especially in case of liver and lung metastasis.

11.23 AM

562 A comparative study using CT and immunoscintigraphy (IS) in the detection of metastases and recurrences in patients with colorectal carcinoma

N. Rilinger, D. L. Munz, H. Niemann, H. J. Halbfaß, H. J. Illiger; Oldenburg, Göttingen

It was the aim of this study to elucidate the diagnostic value of $\mathrm{CT}$ and immunoscintigraphy in the detection of recurrent colorectal carcinoma.

Methods: We studied 41 patients (pts) aged between 39 and 80.39 pts suffered from colorectal carcinoma, 2 from other tumours. All CT-scans were performed using a Siemens Somatom DRH-System. In 26 pts CT examination was carried out after i.v. application of contrast material, in 15 pts with suspected liver metastases CT arteriography was performed. IS was performed using Tc 99m labelled intact anti-CEA-monoclonal antibody (MAb). In $26 \mathrm{pts}$ the MAb was administered i.v., in 15 pts i.a. Planar scanning up to 30 hours p.i. was performed and supplemented in special cases by ECT technique. All findings were confirmed if possible by surgery.

Results: The following lesions were detected: 1 . liver metastases IS 39/CT 62; 2. lymphnode metastases IS $3 / \mathrm{CT} 1 ; 3$. lung metastases IS $0 / \mathrm{CT} 1 ; 4$. local recurrences IS $8 / \mathrm{CT} 8$. All findings were confirmed by surgery, except the liver metastases where only in $20 \%$ partial hepatectomy was possible.

Conclusions: CT has a higher diagnostic value in the detection of liver metastases than IS. However, in cases of extrahepatic tumour deposits IS can be the decisive method in handling the colorectal carcinoma.

\section{$11.33 \mathrm{AM}$}

\section{Evaluation pouchography after proctocolectomy and ileal-j-pouch} anal anastomosis

R. L. Vernacchia, S. Papa, S. Brusori, G. Poggioli, E. Ciccarese, G. Gavelli; Bologna

Since 1985 a dynamic radiological study has been employed regularly, in association with anal manometry and endoscopy, to assess pelvic ileal reservoir function after restorative proctocolectomy.

This series is composed of 107 patients ( 95 ulcerative colitis and 12 familial polyposis) submitted to two-three staged procedure of proctocolectomy Brooke ileostomy - ileal-J-Pouch anastomosis, and closure of the diverting ileostomy.

The evaluation pouchography is performed before transit restoration, to determine the function and morphology of the pouch, and detect complications.

A hydrosoluble contrast medium delivered by a Foley catheter positioned through the diverting ileostomy into the loop afferent to the pouch, evaluate transit time, healing of the pouch and ileo-anal suture lines, pouch morphology, compliance, maximum tolerable volume, threshold capacity and the presence of inflammatory or ischemic phenomena.

The dynamic pouchography assesses evacuation rate, emptying modality, modification of the ileo-anal and pouch-anal suture line.

The data show good correlation between radiological continence, manometry, endoscopy and patient's ability to retain and evacuate spontaneously.

The future goal is to set up a reliable radiological method of assessing pouch morphology and function, using manometry in selected cases.

\section{$11.38 \mathrm{AM}$}

564 Diagnostic and therapeutic impact of defecography in Descensus Perinei Syndrome (DPS)

A. Oberhauser, K. Wicke, R. Knapp, W. Buchberger, F. Conrad, D. zur Nedden; Innsbruck

a) Between 1988 and 1990 we performed 114 investigations on 91 patients with clinical symptoms of DPS to evaluate defecation problems and to control operative results.

b) Equipment: Philips Urodiagnost 1 with a specially designed absorption filter.

Documentation: a.p. and lateral plain filmes before and after defecation, $100 \mathrm{~mm}$ spot films $(2 / \mathrm{sec}$.) during defecation.

c) In 86 patients DPS could be verified. Additional findings were mucosal prolaps, intussusception, rectocele or dysfunction of the pelvic floor muscles which were demonstrable in $74 \%$. All patients, who underwent surgical therapy, were controlled postoperatively. In $70 \%$ of this group defecography documented satisfying results.

d) Defecography is a simple method for diagnosis and therapy planning in DPS. In addition it provides information about primary and secondary changes such as mucosal prolaps, invagination or muscle dysfunctions of the pelvic floor. It is the method of choice for documentation of postoperative results.

\section{$11.48 \mathrm{AM}$}

\section{$565 \mathrm{X}$-ray examination in the intestinal localization of lymphomas} F. Pomerri, P. C. Muzzio, S. Curtolo, S. Romani; Padua

The purpose of this study is to confirm the value of $x$-ray examination in the diagnosis of submucosal rising pathologies like lymphomas of the gut. In spite of the important improvements of radiological techniques recently obtained, the risks of radiological examination are negligible in comparison with endoscopy ones (perforation, bacterial and viral infections). 24 patients, having gastrointestinal lymphoma, underwent a double contrast radiological examination with specific method for every intestinal tract, with histological confirmation of the diagnosis. We found 1 esophageal localization, 12 gastric, 9 duodenal and low intestine, 4 colon-rectal; 2 patients with multiple localizations Lymphomas of the gut have 4 different macroscopical manifestations: stenosis, proliferation, infiltration, excavation; these different appearances are not correlated with a different histolocical type. It is possible to make a right diagnosis in $19 / 24$ patients $(79.16 \%)$; a patient with a gastric initial state lymphoma had no radiological diagnosis; in the other cases radiological approach allowed, at least, the diagnosis of pathological alteration. Because of its reliability and very low rate of complication, $x$-ray examination must be considered the first time of a diagnostic process, in the diagnosis of lymphomas too.

\section{$11.58 \mathrm{AM}$}

566 Peritoneography in the pre-operative diagnosis of inguinal hernia V. Garcia-Medina, J. D. Berna, E. Capitan, J. Llerena, J. Guirao, J. GarciaMedina, M. Madrigal, J. L. Genoves; Murcia

Purpose: When unilateral inguinal hernias are diagnosed by physical examination a contralateral hernia may be present but not palpable. We prospectively evaluated the role of peritoneography in the preoperative diagnosis of unsuspected contralateral inguinal hernias among patients with clinical diagnosis of unilateral hernias.

Methods and Materials: 64 patients with clinically detected unilateral inguinal hernia (55 right, 9 left) underwent preoperative peritoneography using $40 \mathrm{cc}$ of non-ionic contrast injected into the peritoneum in the left lower quadrant without complication. After rolling the patient and increasing the intraabdominal pressure, an inguinal hernia was diagnosed when contrast material extended into a hernial sac in the inguinal region. Surgical confirmation was obtained in all cases.

Results: Peritoneography confirmed unilateral inguinal hernia in all 64 patients with positive physical examination. 20/64 (31.2\%) patients had contralateral hernias diagnosed by peritoneography all confirmed at surgery. A higher percentage of contralateral hernia was found among patients with palpable left inguinal hernia $(6 / 9: 67 \%)$ than among patients with palpable right hernia (14/55: N 25\%).

\section{Room E1}

\section{SS}

\section{$10.30 \mathrm{AM}$}

\subsection{Vascular imaging}

567 Visual and computer-based estimation of atherosclerosis in femoral arteriograms - Radiological methods in the probucol quantitative regression swedish trial (PQRST)

L. Bergstrand, U. Erikson, S. Nilsson, G. Stenport; Uppsala

Purpose: In an ongoing trial we do aorto-femoral overview arteriographies which we score visually and correlate to several computer-based atherosclerosis-related endpoints, based on data from one of the femoral arteries.

Methods: Prerandomization material comprising 301 patients with hypercholesterolemia, 39 of which had symptoms of peripheral vascular disease (PVD). Aorto-femoral overview arteriography was performed and each artery was 
scored visually $0-4$ where $0=$ no lesion and $4=$ total occlusion. Arteriography of a $20 \mathrm{~cm}$ segment of the superficial femoral artery (SFA) was digitized and by computerized image processing several parameters were calculated. Results: The visual score ranged between 0 and 2.47 (mean value $0.63, \mathrm{SD}$ 0.42 ). The score was higher in the PVD (mean value 1.29, SD 0.37) than in the non-PVD group (mean value $0.52, \mathrm{SD} 0.33$ ). The lumen volume ranged between 1287 and 13864 (mean value 5972, SD 2007). The range of edge roughness was $0.52-2.36$. The correlation between visual score and lumen volume was -0.39 (Spearmean -0.32 ) and between visual score and edge roughness $\mathrm{r}=0.64(0.48)$.

Conclusions: Lumen volume and edge roughness in a $20 \mathrm{~cm}$ of the SFA both correlated significantly with visual scoring of aorto-femoral arteriograms. The correlation with roughness was higher.

\subsection{AM}

568 Influence of interpolated soft tissues on measurements with (colour-coded) duplex devices

Ph. Hendrickx, U. Roth, F. Brassel, H. Wagner; Hannover

Using a self-constructed, practically orientated phantom for evaluating Doppler Duplex equipment, the influence of superimposed slices of various soft-tissues on flow quantification, on the spectral waves and on colour-coded visualisation of the arteries was examined.

Various thick muscle-, fascia- and fatlayers were interpolated between the artery and the Doppler probe. Comparative measurement of peak systolic and peak diastolic velocity, enddiastolic velocity, mean velocity, vesseldiameter, bloodflow and "pulsatility index" were performed by conventional duplex (Diasonics DRF 400) and a colour-coded doppler devices (Angiodynograph). The results of these examinations show a clear underestimation of all velocities and flows. The angiodynographic measured mean velocity e.g. sunk about $10 \% / \mathrm{cm}$ using muscle tissue, $35 \% / \mathrm{cm}$ using fat and $15 \% /$ fascia. For the duplex device the underrating halved. The resulting changes of spectral waveform, "pulsatility index" and coded colours are demonstrated.

The study shows the reason why (colour-coded) duplex examinations of deep vessels (e.g. femoral artery in the adductor canal) frequently are problematic.

\section{$10.50 \mathrm{AM}$}

569 Detection of deep venous thrombosis (DVT) by high-resolution realtime ultrasonography (US): evaluation of the vein diameter as diagnostic criterion

J. Fernández Sánchez, W. Bücklein, E. Klotz; Augsburg

Purpose: The ability of US for the diagnosis of lower-extremity DVT has been proved in several studies. It is considered that the acutely thrombosed vein is distended. However, it has not been reported a quantitative study about the diameter of thrombosed veins. The purpose of this prospective study was to evaluate the vein size as US criterion for the diagnosis of fresh DVT.

Methods and Materials: During a 24-month period 280 limbs of 262 patients with suspected acute lower-extremity DVT were examined by US with a 7.5 $\mathrm{MHz}$ transducer. Venography and duplex Doppler studies were performed in 169 and 218 cases, respectively. The explorations were analyzed for the presence of DVT, localization of the thrombus and size of thrombosed vein. In 45 subjects ( 90 extremities) without venous disease (normal collective) the diameter of deep veins was measured. Comparisons of the vein size between thrombosed and normal vein were performed using Wilcoxon test. Statistical significance was defined as $\mathrm{p} \leq 0.05$.

Results: DVT was diagnosed in $34 \%$ of all femoral and popliteal veins (US accuracy: $99 \%$ ), with following distribution: 79 thrombi in the common femoral vein (CFV), 99 in the superficial femoral vein (SFV) and 90 in popliteal vein $(\mathrm{PV})$. There was a significant difference between the mean diameter of thrombosed veins (CFV: 1.31, SFV: $1.16, \mathrm{PV}: 0.90 \mathrm{~cm}$ ) and those of normal veins (CFV: 0.80 , SFV: 0.62 , PV: $0.40 \mathrm{~cm}$ ).

Conclusions: We conclude that the vein size is an accurate, simple, reproducible, reliable US criterion for the diagnosis of a fresh DVT.

\subsection{AM}

\section{Sonographic methods in the diagnostics of early and late compli-} cations after reparative operations in arterial occlusions

Ludomir Stefańczyk, Andrzej Papiewski, Sławomir Jedrzejczyk, Tomasz Pertyński; Łódź

The authors present the great value of sonographic methods in the diagnostics of the complications after reparative operations in the arteries. Siemens Sonoline SL-2 duplex doppler and Toshiba Sonolayer SSA 270-A colour doppler were used.

In this study 280 examinations in 88 patients with intraoperative verification. The study shows the great accuracy $(90 \%)$ of the method in the evaluation of graft potency and pseudoaneurysms. The case of rare complications, like aneurysmal degeneration of venous graft are presented. The difficulties in the evaluating of abdominal complications (heamatoma and abscess) were also shown, especially during the first few days after operation.

Sonographic methods enable to perform sufficient evaluation of occlusion and stenosis of prosthetic arterial grafts and pseudoaneurysms in the anastomosis also aneurysmal degeneration of venous grafts.

In the diagnostics of haematomas and retroperitoneal abscesses CT is recommended in some cases as a complementary method.

\subsection{AM}

571 Lower extremity arterial pathology: diagnosis with conventional and colour Duplex-Doppler prior to angiography

G. Alvarez, B. Asenjo, A. Gomez, J. J. Muñoz, M. Torres, A. Escaño; Malaga

Purpose: We present a series of 35 patients in whom the lower extremities were studied by ultrasound Duplex-Doppler ( 20 conventional and 15 colour) and who later underwent arteriography of the same region. They all presented at least one risk factor for arteriosclerosis and a history of intermittent claudication and three had already had percutaneous transluminal angioplasty (PTA).

Methods: The study began in the common femoral artery and lead to the superficial femoral and popliteal arteries as far as the distal part, using high frequency transducers $(7.5 \mathrm{MHz})$. The distal trunks were checked when selective localized pathology was suspected in the region.

Results: The sensibility was $80 \%$ for haemodynamically significant stenosis and attained almost $90 \%$ for obstructions.

Conclusions: Duplex-Doppler is an excellent method for mapping the lower extremity arteries in patients with a high risk of arteriosclerosis or as a long term follow up in patients undergoing peripheral vascular surgery or PTA.

\section{$11.20 \mathrm{AM}$}

\section{Color Doppler US of femoral artery iatrogenic complications}

Elisabeth Attlan, Mourad Souissi, Olivier Hélénon, Alban Denys, Jean-François Moreau; Paris

Purpose: To assess the value of Color Doppler US in the detection and follow-up of femoral artery punctures complications.

Methods and Materials: 27 studies have been performed from November 89 to November 90 with a 3.75 or $5 \mathrm{MHz}$ transducer, following punctures of femoral artery for angioplasty or coronarography in patients under anticoagulant therapy.

Results: In all cases, Color Doppler US permitted to differentiate the three main complications. 7 pseudo aneurysms were characterized by a swilling flow into the false aneurysm. 4 arterio-venous fistulas showed a turbulent flow on Color Doppler images with an extravascular signal relative to tissue vibrations. Specific pulsed Doppler findings were also demonstrated in the artery and draining vein. 7 hematomas showed a hypoechogenic mass without any Doppler signal.

Conclusions: Color Doppler US appears to be an accurate and non invasive method for the detection and the assessment of iatrogenic femoral artery complications.

\subsection{AM}

\section{MR angiography of lower limb arteries as follow-up after PTA} B. M. Cramer, E. Schlegel, M. Boos, G. Laub; Wuppertal, Erlangen

Purpose: Comparison of conventional and MR-Angiography regarding stenoses and occlusions.

Methods: 160 procedures of either modality were performed on arteriosclerotic patients prior and post PTA. We used 3D-Fisp-sequences with re-phased and de-phased subtracted images on a Siemens 1.5 T-Magnetom. Results: There was excellent correlation of conventional and MR-Angiography. Length of occlusions, degree of stenosis and number of collateral vessels were visualized in all situations except one: Short and high grade stenoses may be visualized as occlusions because of the limited local resolution. All intimal tears were depicted by MR-Angiography.

Conclusions: MR-Angiography proved clinically useful as regional, non-invasive follow-up after PTA and may vary after vascular surgery as well. Lack of spacial resolution was very well compensated by the high flow sensitivity of the method.

\subsection{AM}

574 Duplex and color Doppler sonography of dialysis arteriovenous fistulae

M. Maynar, R. Reyes, J. M. Pulido-Duque, E. Gorriz, C. Garcia-Morales, A. Cruz; Las Palmas

Thirty-one Doppler color ultrasound (DCU) studies were performed in 30 patients with Cimino-Brescia hemodialysis shunts. The average speed and flow in the brachial artery and in the arterialized vein were determined for each patient. A morphological study was made of the whole vascular circuit deter- 
mining the level of the arteriovenous anastomoses and its characteristics. Nineteen patients had a fistulogram performed by puncture of the brachial artery. DCU was compared with the fistulogram, getting a good match in 18 patients. We found stenosis in 7 cases, thrombosis in 1 case, venous aneurysms in 14 cases, and radial steal syndrome in 5 cases. Mean flow in the proximal artery was $661.1 \mathrm{ml} / \mathrm{min}$; mean flow in the vein was $458 \mathrm{ml} / \mathrm{min}$.

$\mathrm{DCU}$ is an accurate noninvasive means of evaluating arteriovenous dialysis fistulae before and after treatment as well as on follow-up.

\section{$11.50 \mathrm{AM}$}

\section{Advantages of ultra-sound guided approach of jugular and femoral axis}

P. Maquin, B. Coustets, N. Railhac, J. Giron, H. Rousseau, J. J. Railhac; Toulouse

\section{Ultra-sound guidance was used in 45 patients for:}

- the insertion through the internal jugular vein of: a definitive vena cava filter in 23 patients or a temporary vena cava filter along with fibrinolytic therapy in 15 patients,

- the insertion of a central venous catheter for acute collapse after failure of conventional catheterization ( 2 cases),

- antegrade puncture of the femoral common artery in 5 obese patients (for peripheral atherectomy with the Simpson catheter).

All punctures were performed under local anaesthesia and guided by a 7,5-MHz transducer.

Catheterization was achieved in all cases with no complications. It is mostly interesting to avoid damage to nearby vessels when a fibrinolytic therapy is planned.

\section{$11.53 \mathrm{AM}$}

\section{3D-reconstruction of intravascular ultrasound images}

H.M. Klein, R.W. Günther, M. Verlande, J. Kelch, D. Vorwerk, W. Schneider, M. Hamm; Aachen

A system for 3D-imaging of intravascular ultrasound sequences was developed. Imaging was performed using a $20 \mathrm{MHz}$ intravascular ultrasound system. A specially designed motor-assembly controlled the withdrawal of the ultrasound catheter withdrawn in equidistant steps or at a constant retraction speed. Vessel curvature was documented by two rectangularly positioned angiograms. A true $3 \mathrm{D}$-model of the vessel was created by rearranging the ultrasound images according to the angiographic shape. 3D-rendering was realized using a voxel-based image processing system, which is applicable to all spatially consistent cross-sectional imaging modalities. Accuracy of the system was tested in models and post-mortem arteries. We found a sufficiently high accuracy of the vessel reconstructions. The system was tested in ten patients during angioplastic therapy of arteriosclerotic occlusions.

$3 \mathrm{D}$-intravascular ultrasound provides additional information for interventional procedures. However, some steps of the reconstruction process like the selection of relevant structures are time-consuming - automatization of these parts represent work-in-progress.

\section{Room E2}

\section{SS}

\subsection{Tumours I}

$10.30 \mathrm{AM}$

\section{The radiologist's role in management of bone tumours (Keynote Lecture)}

D. J. Stoker; London

The aim of treatment of primary tumours of bone is for the institution of effective early therapy with where appropriate, the preservation of the affected limb. These primary malignancies are rare and such treatment is best carried out following referral to a centre dedicated to the specialty, of which there are a number in Europe.

The clinical radiologist, like the surgeon, the oncologist and the pathologist, is a full and important member of the team. He is involved from the time of the initial presentation and throughout the treatment and follow-up periods.

The symbiotic relationship between radiologist and histopathologist is important and both should know the others strengths and weaknesses so that mistakes are avoided.

Important considerations in radiological management relate to assessing response to oncological and surgical treatment, measurement of prosthetic replacements and the control of complications.
$10.45 \mathrm{AM}$

578 Magnetic resonance imaging of the aneurysmal bone cyst Ph. Vinee, G. Sigmund, J. C. Dosch, C. P. Adler; Freiburg

Purpose: Only a few authors dealed with MRI of the Aneurysmal Bone Cyst $(A B C)$, reporting small series. We contribute to further description of the ABC's MRI characteristics.

Methods: We have seen 6 patients ( 12 to 25 years old) with $\mathrm{ABC}$ (the diagnosis being confirmed by surgical excision). MRI at magnetic field of 0.23 or $0.5 \mathrm{~T}$ was performed as well as plain radiography ( 2 cases also had CT, 3 had an angiographic examination). Only spin-echo sequences were used, T1, T2 and "rho"-weighted. 2 cases had additional RARE-hydrography sequences (with very strong $\mathrm{T} 2$-weighting).

Results: We found the MR image of $\mathrm{ABC}$ to be very variable in morphology and signal. Fluid-fluid levels are seen in only 4 patients and are anyway not pathognomonic for $\mathrm{ABC}$. A low-signal rim was found in all cases in at least one sequence.

We describe 3 categories of MRI signal for $\mathrm{ABC}$ depending on the content of the cyst (correlated with its age):

- low-signal type: beginning $\mathrm{ABC}$ containing no blood but mainly fibrotic material,

- intermediate type: $\mathrm{ABC}$ with blood-filled cavities but no blood degradation products,

- inhomogeneous type: corresponding to the classically described $\mathrm{ABC}$ with blood and sedimental blood degradation products.

Conclusions: MRI allows more than morphology and gives information about the content and age of the $\mathrm{ABC}$. We propose a 3-class classification of $\mathrm{ABC}$ in MRI, correlating with histology.

\section{$10.55 \mathrm{AM}$}

579 Differentiation of giant cell tumors from aneurysmal bone cysts R. Erlemann, P. Wuisman, G. Edel, G. Reuther, P. E. Peters; Münster

Purpose: In long bones, epiphyseal extension of aneurysmal bone cysts (ABC) and no epiphyseal involvement of giant cell tumors (GCT) are known. Diagnostic criterions were analysed, which improve the differentiation between $A B C$ and GCT.

Methods: Radiographic morphology and patient's age of $72 \mathrm{ABC}$ and $47 \mathrm{GCT}$ located in humerus, femur or tibia were analysed.

Results: 51 lesions extended into the epiphysis ( $42 \mathrm{GCT}, 9 \mathrm{ABC}$; PPV of $\mathrm{GCT}=82 \%$ ). In patients under $17 \mathrm{y}, \mathrm{ABC}$ were exclusively found, while in older patients 42 of 49 lesions were GCT (PPV $=86 \%$ ). 35 lesions showed no epiphyseal involvement ( $30 \mathrm{ABC}, 5 \mathrm{GCT}$; PPV of $\mathrm{ABC}=86 \%$ ). In patients under $20 \mathrm{y}, \mathrm{ABC}$ were exclusively found $(\mathrm{PPV}=100 \%)$, while in older patients only 4 of the 9 lesions were $\mathrm{ABC}(\mathrm{PPV}=44 \%)$. All lesions located within the shaft were $A B C$. Changes related to LODWICK IA were found more often in $A B C$ (6) than in GCT (2), while LODWICK IC was more often observed in GCT (16) than in ABC (7); and LODWICK II was exclusively noted in GCT (5). LODWICK IB was found in both tumors with similar frequencies.

Conclusions: Besides tumor localization, considering patient's age improves the differentiation of $A B C$ from GCT. With LODWICK IA ABC and with LODWICK II GCT should be considered; a location within the shaft is diagnostic for an $A B C$.

\section{$11.05 \mathrm{AM}$}

\section{MRi of eosinophilic granuloma: a report of 10 cases} A. De Schepper, H. De Gryse, F. Ramon; Edegem

Eosinophilic granuloma (EG) is a relatively uncommon disease (less than $1 \%$ of all tumorlike conditions) and a great mimicker on conventional radiology and computed tomography.

In order to assess the value of Magnetic Resonance Imaging (MRI) in the diagnosis of EG, we reviewed the records of 10 patients with histologically proven EG examined over a period of three years. MRI was performed on a 0.5 $\mathrm{T}$ unit. T1-weighted and T2-weighted spin-echo images were obtained. In 4 patients, Tl-weighted spin-echo images were carried out after intravenous injection of Gadolinium-DTPA.

For all lesions signal intensity ( $\mathrm{SI}$ ) on all pulsing sequences, before and after contrast administration, was compared to the SI of muscle and fat. Besides SI, we evaluated size and shape, delineation and homogeneity and looked for the presence of periosteal reaction, sclerotic borders, button sequestra and accompanying soft tissue masses. Finally we compared SI and morphology of all lesions with the age of the patients, the appearance on conventional radiography and the histology, in order to find a correlation between the MR findings and the maturity of EG.

On T1-weighted images nearly all lesions were iso- to hyperintense compared to the SI of muscle. On T2-weighted images they were mostly hyperintense compared to the SI of fat. All lesions showed a definite enhancement after contrast administration. Accompanying soft tissue masses and changes of SI in the adjacent bone marrow were better depicted on MRI. A button sequestrum 
was present in two cases and the integrity of adjacent vertebral discs reinforced the diagnosis of EG in a cervical vertebra plana in two cases. The lobular structure of the lesions was demonstrated in 8 cases.

\section{$11.15 \mathrm{AM}$}

581 Imaging techniques in the osteoid osteoma: differential diagnosis A. Zerbi, N. Trenti, L. Balzarini, F. De Marchi, S. Zappala', I. Bosi; Milano

The new imaging techniques available such as scintigraphy, CT and MR, made the radiological diagnosis of the osteoid osteoma more and more accurate. Osteoid osteoma is a disease histologically well known. 64 osteoid osteoma, 104 chronic osteitis, 4 eosinophilic granuloma and 6 Brodie's abscess have been treated in our institute since 1965 .

Authors present the clinical and imaging differential diagnosis between those diseases compared to the histological evaluation.

The differential diagnosis between osteoid osteoma, eosinophilic granuloma, chronic osteitis and Brodie's abscess is now reliable in most cases if in addition to the traditional X-ray techniques, scintigraphy, CT and MR are available.

\subsection{AM}

\section{MR imaging of tumors and pseudotumors of the soft tissues of the hand}

Mourad Souissi, Noelle Stempfle, Olivier Helenon, Fabienne Thibault, Joel Chabriais, Jean-Francois Moreau; Paris

Purpose: To locate and to assess the extent of masses of the hand preoperatively.

Methods and Materials: 25 examinations were performed in 14 patients presenting with at least one mass of the hand. The protocols were similar $(0.5 \mathrm{~T}$ magnet, surface coil, T1-weighted $(\mathrm{TR}=500 \mathrm{~ms}, \mathrm{TE}=26 \mathrm{~ms})$ and T2-weighted ( $\mathrm{TR}=1800 \mathrm{~ms}, \mathrm{TE}=60 / 120 \mathrm{~ms}) \mathrm{SE}$ sequences$)$.

Results: Out of 6 patients examined primarily, there were 6 benign tumors: 2 lipomas, 1 synovial cyst, 2 congenital muscular abnormalities, 1 chondroma). Out of the 8 patients previously operated on, MR detected accurately recurrences in 7 cases ( 2 angiomas, 1 schwannoma, 1 benign histiocytofibroma, 1 histiocytofibrosarcoma, 1 extensive fibrosis), and no recurrence in 1 case (histiocytofibrosarcoma grade 1)

Conclusions: MRI provides interesting contributions in 1. the preoperative staging of a tumor; 2 . the delination of the mass and the identification of the anatomical structure from which it has developed (muscle, tendon, synovial sheath...); 3. sometimes the tissular characterization (lipoma, cyst...); 4. the post-operative follow-up, specially when the clinical examination is difficult.

\section{$11.35 \mathrm{AM}$}

\section{Ultrasound in the evaluation of subcutaneous lipoma}

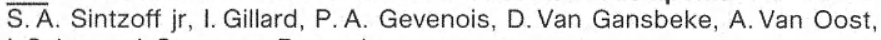
I. Salmon, J.Struyven; Brussels

Purpose: The purpose of the study is to evaluate the performance of ultrasound in the evaluation of suspected subcutaneous lipoma.

Methods: 21 patients were prospectively evaluated with an Acuson 128 computed tomography unit equipped with a $5 \mathrm{MHz}$ transducer. The following parameters were studied: the size, the margins, the echogenicity towards the surrounding fatty tissue and the presence of distal sound enhancement or acoustic shadowing. MRI was performed in 4 patients with large masses with ill-defined margins. Surgery and pathological examination were performed in all patients.

Results: The histological diagnosis included 1 epidermoid cyst, 1 liposarcoma and 19 benign lipomas. The patients have been classified in 3 different groups on the basis of sonographic features. The first group includes the 4 patients who underwent MRI: at ultrasound, all had large heterogeneous tumor with posterior acoustic shadowing or ill-defined margins. MRI demonstrated typical lipoma in 3 cases as proven by the pathological examination. The last case did not exhibit a lipoma pattern on MRI and corresponded to the liposarcoma. The second group included 3 patients with hyperechoic small subcutaneous tumors. All were confirmed to be lipomas. The last group included 14 patients with hypoechoic heterogeneous well-defined masses. 6 masses had distal acoustic enhancement, 5 were proven lipomas and one an epidermoid cyst that was indistinguishable from the other masses on the basis of sonographic criteria. The other 8 masses exhibited no acoustic enhancement and were all proven to be lipomas.

Conclusions: Ultrasound is a useful method in the evaluation of small subcutaneous lipomas. For large masses, especially when the margins are ill-defined, ultrasound cannot make the differential diagnosis with other soft tissue tumors and MRI may be helpful.

\subsection{AM}

584 The role of OPT in Gardner syndrome

G. M. Danesini, S. Bergonzi, A. Laffranchi, P. Sala, G. Viganotti, A. Guzzon; Milano

Gardner syndrome is a familial adenomatous polyposis coli (FPC) associated to extraintestinal lesions like osteomas of the skull and jaws, dental abnormalities, epidermal cysts, soft tumors (desmoid included), pigmented ocular fundus lesions. The purposes of this study are to analyse by orthopantomography dental abnormalities and jaws osteomas that frequently occur in FPC and to assess whether panoramic dental films may be useful on routine examination of FPC in the relatives. Osteomas are bone lesions, as radiodensities, either circumscribed or in clusters or wide-spread, often clinically occult, in the mandible and/or maxilla. Dental abnormalities include supernumerary teeth, malformed or malpositioned teeth, abnormal root formation, congenitally missing teeth, fused roots and impactions. In the National Cancer Institute of Milan, we have reviewed $89 \mathrm{x}$-ray films in FPC patients from 1984 to $1990 ; 50$ patients had osteomas and/or dental abnormalities of the jaws.

Panoramic dental radiograph may identify early in life these diseases which often go before the serious intestinal lesions and high risk pedigrees.

\section{Room L}

\section{SS} 10.30 AM

\section{MRI of the larynx (Keynote Lecture)}

J.D. Piekarski, F. Héran; Paris

MR imaging of the larynx play a significant role in exploring deep structures of the larynx. Compared to CT, MRI has many potential superiority: multiplanar capability, superior soft tissue definition. Technical considerations are important for MR imaging of the larynx depending of the type and strength of the magnet. Movements artifacts should be reduced by using shortest acquisition time as possible. Fat planes are easily visualized and are important landmarks. Because of a similar signal beetwen fat and enhanced lesion, Gadolinium should be used with caution. The possibility to perform direct sagittal and frontal planes explains easily the interesting role of MRI, in detecting pre-epiglottic, paraglottic and subglottic extension in laryngeal carcinoma. The role of MRI in the detection of cartilage invasion is more controversal.

In the field of post-treatment survey MRI should play also a major role in the future, especially with fast scanning technique.

The place and the role of MRI of the larynx as compared to CT will be discussed, especially in the field of laryngeal cancer.

\section{$10.45 \mathrm{AM}$}

586 In vitro study of larynx carcinoma: comparison of histologic and NMR imaging results

M.Spanoghe, C. Kloeck, P. van de Heyning, A.van der Linden, E.van Marck, D. Lanens, F. van de Vyver, C. van Laer; Wilrijk

Purpose: The purpose of this study was to evaluate the spread of larynx carcinoma, in order to substitute the peroperative microscopic oriented histological sectioning (MOHS) technique.

Methods: Axial NMR images of fresh and fixed larynx specimens were obtained after laryngectomy of patients with larynx carcinoma, (iv. inj. Gd-DOTA) on a $1.9 \mathrm{~T}, 30 \mathrm{~cm}$ bore Bruker Biospec spectrometer with an Aldermann Grant RF coil $(8 \mathrm{~cm})$. Spin-echo sequence: T1 (3D, contrast: $\mathrm{TR} / \mathrm{TE}=320 / 8$, without contrast: $\mathrm{TR} / \mathrm{TE}=320 / 40$ ), $\mathrm{T} 2$ (multi-slice, TR/TE: 3000/80); in-plane resolution: $0.3 \mathrm{~mm}, 1.7 \mathrm{~mm}$ thick, FOV $=7 \mathrm{~cm}$, matrix $256^{2}$. The images were compared with axial whole-organ $10 \mu \mathrm{m}$ histologic slices.

Results: An excellent correlation between histology and T2 weighted NMR images was observed while T1 weighted images with and without contrast were less helpful in delineating the tumor.

Conclusions: It is concluded, that T2 weighted images enable delineation of the tumor including detection of cartilage destruction and detection of tumor outside the thyroid cartilage. This appears essential for the further course of the surgical intervention and for the final outcome.

$10.55 \mathrm{AM}$

587 MR imaging with gadolinium-DTPA in carcinomas of tongue, oroand hypopharynx

R. Einspieler, G. Ranner, F. Ebner; Graz

Objectives: In a prospective study the value of i.v. Gd-DTPA in addition to non-contrast imaging in evaluating $\mathrm{T}$ - and $\mathrm{N}$-stage of carcinomas of the upper 
digestive tract was analyzed.

Methods: A superconductive magnet (Gyroscan S15, Philips) was used. Tl-weighted images with and without contrast medium were obtained, a dosage of $0,2 \mathrm{mmol}$ per $\mathrm{kg}$ Gd-DTPA was administered.

Results: 24 patients with a primary diagnosis of squamous cell carcinoma of tongue, oro- and hypopharynx were examined. Histopathological classification was performed in all tumors. Marked contrast enhancement produced by Gd-DTPA was observed in all carcinomas and in normal pharyngeal mucosa. The clinical T-stage of the tumors in comparison to surgical evaluation was diagnosed correctly with MRI in 9 patients, as opposed to the clinical stage in $7 / 11$. The surgical $N$-stage showed a correlation with MRI in $8 / 11$ patients, was overstaged in 1 and understaged in 2 patients.

Conclusions: In tumors stage TI and TII we found similar results in comparison to clinical examination including ultrasound; in tumors stage TIII and TIV the true tumor extension was better assessed by MRI particularly in tumors adjacent to the hypopharynx.

\subsection{AM}

588 MR imaging of patients with head and neck tumors presenting cranial nerve palsies

Annick Delvalle, Robert Sigal, François Janot; Villejuif

Purpose: Sixteen patients presenting with head and neck malignant tumors extending to intracranial nerves were retrospectively studied (nasopharyn $\mathrm{x}-4$; parotid-2; ethmoid-7; larynx-2; spino-cellular carcinoma of the skin-1). The aims of the study were: 1. to establish correlations between imaging and clinical data, 2. to assess the value of the different MR imaging sequences and 3. to evaluate the specific problem of perineural extension.

Methods: MR exams were performed on a $1.5 \mathrm{~T}$ system, with multidimensional T1- and T2-weighted sequences; Gadolinium was routinely injected. CT was done in all cases.

Results: The patients presented with a total of 49 cranial nerve palsies. MR findings were positive in 44 cases (CT: 31 ). In 4 patients, only MR made the diagnosis. Gadolinium Tl-weighted sequences were the most sensitive, but optimal diagnosis stems from a comparison with $\mathrm{T} 1$ and $\mathrm{T} 2$ pre Gadolinium sequences. Extension to bony structures were made with MR in 9 cases (CT: 10 ); to the brain in 3 cases (CT: 0 ), to the cavernous sinus in 10 cases (CT: 5 ), to the nerves in 7 cases (CT: 2 ).

Conclusions: MR imaging should be the first exam in patients with head and neck tumors presenting with nerve palsy. It allows for assessment of critical areas (cavernous sinus, orbital apex, meninges, brain) and thus guides therapeutic planning: i.e. complete or partial surgery and/or radiotherapy.

\subsection{AM}

589 A functional imaging approach to speech: first results of MR-SNAPSHOT-FLASH-imaging of the articulators

B. Wein, W. Angerstein, M. Drobnitzky, S. Klajman; Aachen

Purpose: MRI-SNAPSHOT-FLASH-technique was used to image the tongue and other articulators in functional positions during speech and to study coordinations and misfunctions of the articulators.

Methods and Materials: The investigations were performed using a $1.5 \mathrm{~T}$ clinical MR-imager with circular polarized head coil or Helmholtz neck coil with a $30-\mathrm{cm}$ field of view. Sagittal tomograms were recorded in Tl-weighted SNAPSHOT-FLASH sequences (TI: $900 \mathrm{~ms}$. TR: $7.5 \mathrm{~ms}$. TE: $4 \mathrm{~ms} .128 \times 256$ matrix) in the midsagittal median plane of the floor of mouth. 20 healthy subjects and 10 patients with peripheral and central articulatory dysfunctions were examined. To get representative functional positions all subjects were asked to utter long vowels, nasals and gutturals for at least $2 \mathrm{~s}$.

Results: All obtained MR-images were functionally meaningful and allowed effective analysis of form and positioning of lips, tongue, hard palate, soft palate and epiglottis. Pathologic form and positioning of the tongue and the soft palate can be effectively discriminated from normal.

Conclusions: MR-SNAPSHOT-FLASH imaging is a fast and convenient new approach to demonstrate and document functional forms and positions of all articulators during articulation. This noninvasive method is indicated for all patients with central or peripheral articulatory dysfunctions.

\section{$11.25 \mathrm{AM}$}

590 Evaluation geometry of the cranio-facial skeleton using CT-scan and MR image with special computer programme

A. Kukwa, J.Walecki, R. Staroński, M. Pietniczka-Zalẹska, M. Tulibacki, I. Rejniak; Warszawa

Recurrent episodes of the upper airway obstruction during sleep may become a consequence of anatomical abnormalities causing narrowing of it in the oropharyngeal region. For this an evaluation of the orofacial skeleton is the most important step in the diagnosis - Obstructive Sleep Apnea Syndrome (OSAS). For this reason all disharmonies in geometry are noticed and calculated by selectively prepared set: Pc-computer, CCD-camera, Videograber and soft-ware. On the basis of lateral view of CT-scan of the head and neck area the cranio-facial measurements are automatically taken. Fifty-eight (anthropometric) variabilities defining soft tissue and bony landmarks of the cranium and upper airway from patients suspected of the OSAS are compared with corresponding measurements obtained from controls. These results of it indicate that there are a number of differences between OSA patients and control group. The most striking are the enlarged tongue and soft palate which provides the basis for surgical treatment using Uvulopalato-pharyngoplasty (UPPP). OSA patients exhibit a retroposition of the maxilla and hyoid bone with significantly longer hard palate. This latter places the already elongated soft palate closer to the posterior pharyngeal wall, narrowing as a result of it the passage between naso- and oropharyngeal airways. In OSA patients the hyoid bone also more inferiorly located compared to the control. All of these are the best tool for diagnosis and indications for surgery as well as consecutive evaluation of the upper airway passage after treatment.

\section{$11.35 \mathrm{AM}$}

591 The subcricoidal impression of the pharynx - a differential-diagnostic problem in the differentiation from early cancer Chr. Hannig, H. Daschner, A. Wuttge-Hannig; Munich

a) In the anterior wall of the pharynx during bolus-passage two impressions are to be observed for a short period of 0.2 seconds: A permanent impression is caused by the cricoid-cartilage, a second one, the so-called subcricoidal impression demonstrates a great variability in its configuration. The latter is caused by a compression of the venous-plexus and pharyngeal mucosa during pharynx-contraction and it's often not to be differentiated from a neoplasm of the pharyngeal front-wall.

b) By means of High-Speed-Cineradiography on $35 \mathrm{~mm}$-film and a frame-rate of 50-100 frames/sec. a differentiation between permanent and inconstant impression is possible with high precision because of the high temporal and spacial resolution of this method.

c) In $93 \%$ of our 1298 patients an inconstant subcricoidal impression was to be diagnosed. In 47 cases a malignant tumor was suspected by means of bariumswallow, CT or MRI. This finding could be confirmed in 23 cases, in 24 cases cineradiography revealed benign alterations like venous structures.

d) The diagnostic work-up of early cancer in the retrocricoidal region usually includes CT, MRI and endoscopy. High-Speed-Cineradiography can help to avoid false positive findings.

\subsection{AM}

592 Videofluorographic evaluation of postoperative oral and pharyngeal dysfunction

A. Steudel, E. K. Walther, M. Reiser, F. Christ, M. Deroover; Bonn

Purpose: To determine the frequency and type of oropharyngeal dysfunction that occur after cancer resection and reconstructive procedures.

Methods and Materials: Videofluorographic deglutition examinations of 56 patients were reviewed after pharyngeal tumor resection. 48 patients complained of dysphagia, 22 had clinical signs of prandial aspiration.

Results: Oral-stage impairment ( $27 \%$ of cases) included loss of bolus control, prolonged bolus delivery, and delay in swallowing initiation. Pharyngeal-stage abnormalities $(41 \%)$ included severe motility dysfunction, incomplete bolus ejection, and defective laryngeal closure. Minor liquid aspiration was detected in 22 individuals, and major aspiration was found in nine.

Conclusions: Videofluoroscopy plays an important role in postoperative investigation and management of deglutition rehabilitation.

\section{$11.55 \mathrm{AM}$}

593 Sonography of the thyroid gland in schoolchildren after Chernobyl accident in 4 years

G. A. Subovsky, O. B. Tararuchina; Moscow

Ultrasound scanning of the thyroid gland have been conducted in schoolchildren living in conditions of iodine endemiae. The 1st group of 900 schoolchildren with dose radiation of thyroid gland $400 \mathrm{mZv}$. The 2 nd group of 600 schoolchildren with dose $200 \mathrm{mZv}$. No one of the children showed clinical signs of disease of thyroid gland, but hyperplasia revealed by palpation. All studies have been conducted by the same investigator, using the same apparatus SAL-38B TOSHIBA, equipped the transducer at $7,5 \mathrm{MHz}$ and water-bag kit with high resolution. The results have been compared with group of 550 schoolchildren without radiation. All kinds of discovered pathological structure changes of the thyroid gland can be divided into following 4 groups: 1 . nodes and cysts 2 . thyroiditis 3 . hypoplasia 4 . hyperplasia. $3,9 \%$ of structure changes were revealed in control group, and $10 \%$ in 1 and 2 groups. Children, having changes in gland, have been admitted to the Children Hospital for full-scale clinical-laboratory examination. Sonography is the best method of mass prophylactic examination of children in endemic region. 


\section{Room M}

\section{SS}

\subsection{Kidney imaging}

$10.30 \mathrm{AM}$

594 The radionuclide investigation of the urinary tract (Keynote Lecture)

Keith E. Britton; London

The exclusion of obstructive nephropathy, the effect of an increased resistance to outflow, is the first evaluation of the urinary tract: by total and relative renal function; by measurement of the parenchymal transit time index and the pelvic transit time; and by the response to frusemide qualitatively and qantitatively relating response to uptake through calculation of the output efficiency. The second evaluation is ureteric peristalsis and the recognition that the ureteric response to increased resistance to outflow is an increase in frequency and reduction in amplitude of contraction. Ureteric spasm does not occur. The basal pressure rises smoothly and is interpreted at cerebral level as colic. Disordered peristalsis may be evaluated by setting serial regions of interest during the Tc-99m MAG3 study and analysing the space/time relationships. Vesicoureteric reflux and Yo Yo duplex uretero ureteric reflux may also be analysed by regional renal activity time curves. Direct radionuclide micturating cystography is an alternative. By these means the pathophysiology of the ureters, reflux and obstructive nephropathy are available to the nephro-urologist.

\section{$10.45 \mathrm{AM}$}

\section{Dual-energy imaging in genitourinary radiography with storage phosphors}

C. Schaefer, M. Prokop, A. Meschede, E. Schlick, M. Galanski; Hannover

Purpose: The detection of pathology in genitourinary radiography is frequently compromised by overlying faeces or bowel gas shadows. Dual energy (DE) subtraction might decrease the detracting effects of overlying structures by separately displaying soft tissues and calcific or contrast-opacified structures.

Methods and Materials: DE images were obtained with standard storage phosphor equipment (Digiscan 901, Siemens). We used a single shot technique with $\mathrm{K}$-edge prefiltering and a sandwich cassette for simultaneous acquisition of high and low energy images. Image processing algorithms for noise suppression were implemented for a $1 \mathrm{~K} \times 1 \mathrm{~K} \times 12$ bit matrix on the standard postprocessing console. Abdominal plain film radiographs for detection of urinary calculi and excretory urograms for detection of filling defects were presented in a subtracted and in the ordinary mode.

Results: Dual energy subtraction significantly improves lesion detection in those cases where pathology is obscured by overlying faeces or bowel gas shadows. Without interfering anatomic noise it proved not to be advantageous. The application of noise suppression algorithms was obligatory to achieve an adequate signal to noise ratio for the diagnosis of small lesions.

Conclusions: Dual energy subtraction increases performance by reducing the impact of bowel gas. Noise suppression algorithms are obligatory.

\section{$10.55 \mathrm{AM}$}

\section{Quality of urography and renal clearance of contrast media} Henrik S. Thomsen, Aage Vestergaard; Herlev

One hundred patients with normal serum creatinine concentration had an intravenous urography with either diatrizoate or iopamidol after randomization. A pilot study including 11 healthy volunteers showed a good correlation $(r=0.97)$ between single sample 51-Cr-EDTA and contrast medium clearance. Employing Renalyzer PRX 90, the plasma concentration of iodine (contrast medium) was determined on blood samples drawn approx. 3 and $4 \mathrm{~h}$ from one sample obtained $4 \mathrm{~h}$ post contrast and from multiple samples (2 drawn $3 \mathrm{~h}$ and 2 drawn $4 \mathrm{~h}$ post contrast). The urograms were assessed blindly as regards nephrographic and pyelographic opacification as well as the overall diagnostic quality was assessed. The clearance was found to vary between 42 and $125 \mathrm{ml} \times \min ^{-1} \times 1.73 \mathrm{~m}^{-2}$ at both determinations. The $3 \mathrm{~h}$ single sample value correlated well $\left(\mathrm{Cl}_{3+4}=\mathrm{g} \quad\left(\mathrm{Cl}_{3}\right) \pm 2.1\right.$ at isotonic regression; $\mathrm{Cl}_{3+4}=-8+1.0 \mathrm{Cl}_{3} \pm 2.1$ at linear regression) with the multiple sample value. No systematical connection of practical significance was found between the clearance and the assessment of the urograms. Considerable variation was found in the clearance among not only the poor but also the good urograms. No demonstrable differences of clinical significance were found between the two media. It is concluded that it is not possible to assess clearance from the appearance of the nephrogram and pyelogram or from the overall diagnostic quality in routine urographies regardless of whether ionic high-osmolar or non-ionic low-osmolar contrast media are employed in patients with normal serum creatinine levels. The glomerular filtration rate can easily be determined by measuring the contrast medium concentration in a plasma sample obtained $3 \mathrm{~h}$ post contrast.
$11.05 \mathrm{AM}$

597 Value of the partial body time-activity-curve for the determination of renal function

U. Keske, A. Debrand-Passard, W. Richter, M. Cordes, R. Felix; Berlin

Purpose: The diagnostic utility of a partial body time-activity-curve (PBTAC) taken during renal sequential scintigraphy for the documentation of renal function was evaluated.

Patients and methods: Data of 257 patients that underwent renal scintigraphy with $80 \mathrm{MBq}$ Tc-99m-MAG 3 were reviewed. A PBTAC was established by a region of interest above both kidneys.

Results: Measurements of the percental decrease of the PBTAC at different times $\left(t_{1}\right.$ and $t_{2}$ ) revealed an exponential relation with the Tauxeclearance. Correlation improves with increasing difference between $t_{1}$ and $t_{2}$ and is best for PA $2 \mathrm{~min} / 25 \mathrm{~min}$ :

clearance $=10,89 \times \exp (0,0484 \times \mathrm{PA})$

$(\mathrm{r}=0.88$, standard deviation $=58.8 \mathrm{ml} / \mathrm{min})$.

Relationship to the two-compartment-clearance was also exponential $(r=$ $0.80, \mathrm{n}=16$ ). The measurements proved to be reproducible with $\mathrm{r}=0.97$ $( \pm 10.3 \%, \mathrm{n}=30)$.

Conclusions: In contrast to clearance calculations by plasma samples, this procedure can easily be performed at the end of each examination to estimate renal function. However, it should not be implemented as the only method to calculate renal clearance in clinical routine.

\section{$11.15 \mathrm{AM}$}

598 CT planimetry of renal parenchyma: effectiveness in the evaluation of separate renal function

M. F. Bellin, P. Gobin, F. Richard, M. O. Bitker, P. Conort, C. Chatelain, J. Grellet; Paris

Purpose: The aim of this work is to compare the effectiveness of CT renal planimetry versus scintigraphy and clearance of creatinine in the evaluation of separate renal function in patients with urologic disease.

Methods and Materials: 35 patients were included in this prospective study. The results of CT $(n=35)$ were compared to those of renal scintigraphy $(n=$ $23)$ and separate clearance of creatinine $(n=12)$ using a nephrostomy catheter. The volume of renal parenchyma was measured for each kidney on contiguous, $1 \mathrm{~cm}$ thick slices, performed after injection of iodinated contrast medium

Results: The linear correlation factor was excellent $(r=0,939)$ between the planimetric data and the reference methods. The maximum difference we observed was $15 \%$. The correlation factor was not significantly modified when patients with pyelonephritis or renal failure were excluded.

Conclusions: CT renal planimetry is an accurate, semi-quantitative method in the evaluation of separate renal function.

\section{$11.25 \mathrm{AM}$}

599 Microangiographic changes in diabetic nephropathy in rats and humans

J. Merikanto, M. Päivänsalo, S.-O. Hietala, E. Hägg, F. Lithner; Oulu

The aim of the study was to compare microvascular changes of kidneys in diabetic and control rats and diabetic autopsied patients. Half of the patients had additionally hypertension or other renal diseases.

Microangiographic and histopathologic examinations of kidneys were performed in 19 autopsied human diabetics, 7 control rats and 15 experimental diabetic rats at time periods varying from 3 to 9 months after diabetes had been induced.

Depending on the duration of diabetic disease various preglomerular and glomerular vascular changes were seen. Diabetic rats especially those with the longest diabetic duration, demonstrated fewer glomeruli, more aglomerular vessels in the outer region of the cortex, diminished contrast filling of the glomeruli, more abnormalities of peritubular capillaries, vasae rectae, arteries and arterioles as compared to nondiabetic rats. Several microangiographic similarities were seen in diabetic rats and human diabetics. However, certain differences were detected depending on additional renal diseases in human diabetics.

\section{$11.35 \mathrm{AM}$}

600 Acute segmental alterations of renal function after ESWL: evaluation of morphologic and functional changes with dynamic MR studies G. P. Krestin, R. Fischbach, G. K. v. Schulthess, K. F. R. Neufang; Zürich, Köln

Purpose: To evaluate morphologic and functional alterations of the kidneys after ESWL using fast dynamic contrast-enhanced MR examinations. Methods and Materials: Dynamic MR studies were performed by repeated imaging in the coronal plane after administration of Gadolinium-DTPA 
$(0.1 \mathrm{mmol} / \mathrm{kg})$ before, and $24-48$ hours after ESWL for renal calculi in 25 patients. Temporal resolution was $10 \mathrm{~s} /$ image.

Results: Before ESWL 21 patients had normally functioning kidneys, characterized by a marked signal intensity decrease in renal medulla $30-40 \mathrm{~s}$ after onset of cortical perfusion. This is due to the concentration of Gd-DTPA in the collecting ducts and the consecutive T2-shortening. After ESWL a regional loss of the concentration ability in the medulla as sign of a segmental functiona damage could be seen in 7 out of 21 cases. Morphologic alterations (parenchymal hemorrhage, perirenal hematoma, hemorrhage in cortical cysts and hydronephrosis) were detected in 12 cases. The severity of the visible alterations correlated with the energy and number of the shock waves.

Conclusions: Dynamic contrast-enhanced MR imaging is a valuable method for the assessment of morphologic as well as functional changes after ESWL. Acute functional alterations could be localized and visualized as segmental damages of glomerular and tubular function.

$11.45 \mathrm{AM}$

601 Outpatient intraarterial DSA to rule out renovascular hypertension in kidney transplant recipients

S. H. Duda, C. M. Erley, P. Huppert, J.-P. Wakat, M. Sökler, T. Risler, C. D. Claussen; Tübingen

The purpose of this prospective trial was to determine the feasibility and safety of digital subtraction angiography with intraarterial catheterization in renal allograft recipients on an out-patient basis.

All hypertensive pts. $(n=18)$ underwent ia DSA (1024-matrix) with 4F catheters after noninvasive imaging procedures. There was a 4 hour rest in the angiography ward post examination. Serum creatinine was assessed before and 3 days after DSA (total load of contrast medium: $24 \mathrm{ml}$ LOCM for 2 series: $300 \mathrm{mgI} / \mathrm{ml}$ ).

We identified 4 pts. with renal transplant artery stenosis. There were no bleeding complications. The rise of serum creatinine was not significant and no impairment of graft function occurred.

In conclusion ia DSA of renal grafts may be performed with good image quality and without and increased risk of procedure related complications even in out-patients.

\section{Room N}

\section{SS} $10.30 \mathrm{AM}$

\subsection{Pediatric neuroradiology}

602 Perinatal brain damage: clinical and advanced imaging (Keynote Lecture)

Olof Flodmark; Stockholm

Routine clinical imaging of the neonatal brain is important as an adjunct to the clinical evaluation of the neonate's central nervous system. Imaging of neonatal brain damage has a primary clinical interest to the neonatologist who in the short term needs to know if intracranial hemorrhage is present. In assessing long term prognosis, it is important to evaluate the extent and type of hypoxic/ischemic damage to the brain. Despite shortcomings, neurosonography is the primary imaging tool in most neonatal centers. A majority of the clinical imaging challenges can be solved using this technique despite ultrasonography being operator dependent, with the quality of the study is intimately related to the skill and experience of the sonologist as well as his/her knowledge of the anatomy and pathology of the neonatal brain. With awareness of the limitations of neurosonography in mind, the radiologist can select and utilize other imaging modalities, primarily CT scanning, to properly solve the diagnostic problem presented to him.

Perinatal brain damage is one of the major reasons for permanent mental and motor handicap in children today. Ongoing research to define these pathophysiological events with the goal to prevent or limit perinatal brain damage is extremely important. Advanced neonatal life support and monitoring of vital signs have significantly increased our knowledge about these mechanisms. However, in vivo imaging of the neonate's brain is an equally important part of this scientific effort. Application of cranial Magnetic Resonance Imaging (MRI) and Positron Emission Tomography (PET) in the study of the destressed neonate has already significantly increased our understanding of these events. However, the complicated logistics of performing an MRI or PET scan will for still some time prevent any of these methods from becoming a routine clinical imaging tool.

\section{$10.45 \mathrm{AM}$}

603 Morphological and functional aspects in neuronal migration anomalies

Manfred Hermes, Gisela Huber, Thomas Scherb; Homburg/Saar

a) Evaluation of imaging techniques in anomalies of neuronal migration. b) 5 patients $(6 \mathrm{mo}-35 \mathrm{y})$. MRI including IR sequences: CCT; Tc-HMPAOSPECT.

c) Neuronal migration anomalies are very heterogeneous, and CT and MRI present an excellent view of the various parenchymous changes regarding their degree and location. They are frequently associated with vascular malformations in the Sylvian fissure. These can be shown, but the non-invasive imaging techniques do not permit to analyse arterial or venous structures. In such cases the likewise non-invasive HMPAO-SPECT provides additional functional information about the regional cerebral blood perfusion at the site of the anomalies.

d) Besides the documentation of the macroscopical anatomy of parenchymous changes in CT or better with multiplanar tomography in MRI it is recommended to perform a HMPAO-SPECT study. From this functional study may be expected therapeutically relevant aspects especially regarding the leading clinical symptoms: the seizures.

\section{$10.55 \mathrm{AM}$}

604 Diagnosis of cerebral malformations by MRI and real-time sonography

D. Wimberger, G. Zoder, G. Bernert, H. Imhof; Vienna

Purpose: To examine diagnostic facilities of MRI compared with Real Time (RT) Scan findings, concerning cerebral malformations.

Methods and Materials: We examined 33 infants and young children in whom a cerebral malformation had been diagnosed or assumed by RT sonography. MRI was done using a $1.5 \mathrm{~T}$ superconducting system $\mathrm{T} 1$ and $\mathrm{T} 2 \mathrm{w}$. images were performed in 3 planes. For sonography we used a mechanical sector scanner working at 5 or $7.5 \mathrm{MHz}$.

Results: RT scan diagnoses were: Dandy Walker Complex $(n=11)$ agenesis of corpus callosum $(n=5)$, holoprosencphaly $(n=2)$, septal agenesis $(n=10)$, combined malformations $(n=10)$, not classifiable $(n=2)$. In classifiable cases RT and MRI diagnoses correlated. In 2 cases which could not be classified sonographically, a definite MRI diagnosis was possible. Complementary MRI informations concerning disturbances of myelinisation, grey/white matter differentiation and associated malformations could be won in 17 cases and changed the therapeutical management or prognostic predictions $9 \times$. In 1 case sonographical information was superior to MRI.

Conclusions: MRI seems to be a useful diagnostic tool in infants and young children with cerebral malformations. RT sonography will remain the imaging method of choice for screening purposes.

\section{$11.05 \mathrm{AM}$}

605 Variations of the posterior pituitary gland in children: Assessment by $1.5 \mathrm{~T}$ MR imaging

M. Schütz, M. Koschut, J. Gaa, V. Fiedler, H. K. Deininger; Darmstadt

Purpose: On T1-weighted images the posterior aspect of the normal sellar content usually displays high intensity signal (HIS) corresponding to the posterior lobe of the pituitary gland. We describe location, size and shape of the HIS in children.

Methods and Materials: Pituitary glands of 250 children (aged from 3 days to 14 years) were studied by $1.5 \mathrm{~T}$ magnetic resonance (MR) imaging. T1-weighted images (repetition time $=250-600 \mathrm{~ms}$, echo time $=15 \mathrm{~ms}$ ) were obtained in the sagittal plane using $2-5 \mathrm{~mm}$ slice thickness. Patients with intrasellar or parasellar lesions and/or with history or symptoms of pituitary gland diseases were excluded.

Results: In $92 \%$ of all children the HIS was seen intrasellar ( $91 \%$ directly posterior, $3 \%$ superior-posterior of the anterior pituitary gland and $6 \%$ on the sellar floor). In 6 cases ( $2.4 \%$ ) we found the HIS ectopic in the superior portion of the infundibulum or in the hypothalamic region. In the sagittal plane the size of the HIS is between $1 \times 1$ and $4 \times 2 \mathrm{~mm}$, the shape is in $38 \%$ oval, in $37 \%$ round, in $17 \%$ concave-convex and in $8 \%$ like a thin line. We saw no influence of sex and age.

Conclusions: In children the posterior pituitary gland is usually seen as-a bright signal in numerous variations. The posterior pituitary ectopia seems to be a normal variant and should not be mistaken for a hypothalamic lesion.

\section{$11.15 \mathrm{AM}$}

\section{A proposal for $\mathrm{CT}$ staging of retinoblastoma} Mohamed Banna; Riyadh

Retinoblastoma is the most common and most malignant ocular tumor in children. Despite the important role of CT in the diagnosis and management of 
this tumor, there has been no apparent attempt to use it in the staging of the disease. The CT scans of 100 children with retinoblastomas were reviewed and categorised into four stages.

Stage I: Tumor, confined within the globe.

IA: Single or multiple tumor nodules occupying less than $25 \%$ of the globe.

IB: Single or multiple tumor nodules occupying $25-50 \%$ of the globe.

IC: Single or multiple tumor nodules occupying more than $50 \%$ of the globe.

Stage II: Tumors with limited extension into the optic nerve and/or the sclera.

Stage III: Tumors with orbital involvement.

Stage IV: Tumors with CNS and/or other system involvement.

The results of the CT analysis seem to correlate with the expected outcome of the disease.

11.25 AM

607 Magnetic resonance imaging in pediatric neuromuscular disorders

Gin-Chung Liu, Yuh-Jyh Jong, Chi-Hsiun Chiang; Kaohsiung

Purpose: Differentiation of different types of pediatric neuromuscular disorders by Magnetic Resonance imaging (MRI).

Methods: Totally 38 cases [Duchenne's muscular dystrophy (DMD) 15, spinal muscular atrophy (SMA) 10, dermatomyositis/polymyositis (DM/PM) 4, congenital myopathy (CM) 2, arthrogryposis multiplex congenita (AMC) 2, myotonic dystrophy (MD) 1, lipid storage myopathy (LSM) 1, mitochondrial myopathy (MM) 1, Pompe's disease 1 and myasthenia gravis (MG) 1] were investigated with a $0.5 \mathrm{~T}$ superconductive scanner. $\mathrm{T} 1$ - and $\mathrm{T} 2$-weighted spin echo sequences in axial and coronal projections of calf and thigh muscles were obtained. For SMA and AMC, evaluation of cervical cord with same techniques were also performed.

Results: MRI clearly demonstrated the abnormality of subcutaneous fat and muscular bundles with its advantages of multiplanar capability and excellent tissue contrast. The findings of MRI are : a) DMD: preserved muscular groups of gracilis $(15 / 15)$, sartorius $(12 / 15)$, semitendinous $(9 / 15)$. b) SMA: increased subcutaneous fat in all subtypes with ragged islands of muscular tissue within a large mass of panniculus adiposus in intermediate type. c) acute stage of DM/PM: high T2-signal intensity. d) CM: partly preserved semimembranous and biceps muscle. e) LSD: increased subcutaneous fat. f) AMC: severe fatty degeneration. g) Pompe's disease: preserved muscle with mild degeneration. $h$ ) MD and MG: normal.

Conclusions: MRI provides a complementary study and guidance of muscular biopsy for differential diagnosis of neuromuscular disease and can serve as a tool for follow up study of disease progression or treatment response.

\section{$11.35 \mathrm{AM}$} 608 Pediatric CNS focal white matter abnormalities on MRI: clinical

F. Ebner, G. Ranner, M. Millner; Graz

Objective: The objectives of the recent study were to evaluate the prevalence and clinical significance of focal white matter abnormalities (WMA) in neuropediatric patients.

Methods: 1.5 Tesla superconductive magnet (Gyroscan S15, Philips). IR and SE pulse sequences were employed. MR findings were correlated with the patients history, clinical presentation and laboratory findings.

Subject and methods: Within a time period of 3 years 873 consecutive children (age 0-18 years) were referred to MR of the CNS to evaluate various neurologic deficits.

Results: In infancy (age $0-2$ years) $2 / 81$ patients $(2,5 \%)$ exhibited focal brain WMA abnormalities. In childhood between 3 and 9 years $8 / 179$ children had focal WMA $(4,5 \%)$. The most frequent etiology in this group was encephalitis $(n=4)$ : measles, herpes, Hemophilus, Candida albicans. In group III (age 10-14 years) $17 / 162$ children $(10,5 \%)$ had focal WMA, 6 of them were proved clinically to suffer from multiple sclerosis. All of them had clinical definite or laboratory supported definite MS. In 5 children the presence of WMA was associated with clinically long standing partial or generalized epilepsy. In adolescence from 15 to 18 years $26 / 202$ patients demonstrated brain WMA on $\operatorname{MR}(12,9 \%)$.

Conclusions: CNS focal WMA in a neuropediatric population were frequently associated with epileptic seizure disorders. There was an unexpected high frequency of MS in the age group 13 to 18 years. Frequent causes of WMA in pediatric patients were CNS infections, trauma, chemotherapeutic agents and presumed hypoxia-ischemia states.
11.45 AM

\section{$609 \mathrm{MRI}$ in adrenomyeloneuropathy}

W. Köhler, A. Bergin, S. Naidu, H.W. Moser, A. Moser, D. H. Edwin, A. J. Kumar; Berlin, Baltimore, MD

Adrenomyeloneuropathy (AMN) is a phenotypical variant of X-linked childhood adrenoleukodystrophy leading to adrenal dysfunction and central and peripheral nerve system disturbances in young male adults and approximately $15 \%$ of the heterozygote females.

Basis for the disorder is a peroxisomal enzymatic defect with a consecutive accumulation of saturated very long chain fatty acids in body fluids and tissues, mainly the adrenal gland and cerebral white matter, inducing a so far unknown, probably immunological, mechanism that finally leads to demyelination.

Our aim is to show CNS-involvement by brain and spinal cord MRI in 101 patients ( 76 males 25 , females) actually participating a therapeutic clinical trial.

Cerebral involvement can be demonstrated by brain MRI in $47 \%$ of the males, but only in $16 \%$ of the females. All patients with a normal brain MRI are ether asymptomatic or show exclusively clinical signs that may implicate a spinal cord involvement, a suggestion which is supported by abnormal SSEP's in all cases and additional spinal cord MRI's showing, partly severe, thoracic cord atrophy in 25 of 27 cases.

40 patients show brain MRI abnormalities in following areas:

Cerebral white matter $77,5 \%$ (frontal $27,5 \%$, temporal $42,5 \%$, parietooccipital $65 \%$ ); Corpus callosum $42,5 \%$; Optic system $75 \%$; Auditory system $55 \%$; Corticospinal system $80 \%$; Sinothalamic system 70\%; Cerebellum 22,5\% and cerebral atrophy in $30 \%$. Clinical correlations will be given.

Our results give a complete overview of MRI changes in AMN providing implications for pathogenetic aspects and diagnostic strategies.

\subsection{AM} 610 Evaluation of pediatric brain tumors with prohance

S. N. Nadel, L. Gray, J. Debatin, H. Friedman; Durham, NC

As part of a Phase III clinical trial, 22 children with known or suspected primary brain tumors underwent Magnetic Resonance Imaging (MRI) before and after intravenous injection of $0.1 \mathrm{mmol} / \mathrm{kg}$ Gadoteridol, ProHance (Squibb Diagnostics, Princeton, NJ), a new nonionic gadolinium chelate. Ages ranged from 5 months to 17 years with 10 males and 12 females evaluated. Tumors imaged: ependymoma-4; glioma-5; astrocytoma-5; oligodendroglioma-3; medulloblastoma-3; pineal tumor-2; and neuroblastoma-1. Laboratory analyses included complete blood count, chemistry, electrolytes, hepatic function and iron studies both before and 24 hours after contrast agent administration. All imaging was performed on a GE Signa 1.5 T system (GE, Milwaukee, WI). Comparison study obtained within 6 weeks of this study: enhanced MRI - 14 and enhanced CT -8 .

No statistically significant changes related to contrast administration were identified after the administration of ProHance. In this select group of patients, the ProHance-enhanced study provided more diagnostic information in 17 of the 22 cases. There was improvement in lesion detection, number of lesions identified, improved visualization and disease classification.

This initial trial suggests that ProHance is a safe and efficacious agent for the pediatric neurooncology patient.

\section{$11.55 \mathrm{AM}$}

\section{MRI and clinical findings in myotonic dystrophy (MD)}

\section{G. Bachmann, M. Damian, S. Trittmacher; Gießen}

Purpose: We wanted to correlate clinical and MRI findings in patients with MD.

Methods: 40 patients with MD underwent MRI of the brain, facial skull and the muscles of the lower limbs. We used T1 and T2 weighted sequences.

Results: MRI shows the dystrophic changes in the skeletal muscles due to MD in early cases, when neurologic examination is still negative. The degree of changes in MRI correlates with the results of neurophysiologic examinations like EMG. MRI of the brain shows diffuse atrophic changes and a characteristic focal increase of signal intensity in the white matter. These changes in the brain correlate with the intellectual impairment of the patients demonstrated in clinical performance tests.

Conclusions: MRI is the ideal method to show the spectrum of morphologic changes in brain and skeletal muscles in patients with MD. 


\section{Room 0}

\section{Ss}

\section{$03.03 \mathrm{MRI}$ and digital radiography}

\section{$10.30 \mathrm{AM}$}

612 Use of contrast enhanced MRI of the breast in diagnostic problems

S. H. Heywang-Köbrunner, R. Beck, T. Hilbertz, K. Diergarten, W. Permanetter; München

Purpose: Differential diagnosis of discrete and uncharacteristic changes may cause significant problems, when detection of carcinomas at earlier and preinvasive stages is attempted. Sensible use of all diagnostic possibilities is necessary to avoid too many excess biopsies of benign changes.

Methods: Because of the excellent results of contrast enhanced MRI in about 300 biopsy-proven cases, contrast enhanced MRI has more and more been applied as a supplementary method in diagnostic problems. Meanwhile the value of MRI in such cases has been checked in over 200 patients, who have all been biopsied or followed since more than 2 years.

Results: In these preselected cases supplementary MRI was able to improve sensitivity (from $74 \%$ to $100 \%$ ) and specificity (from $32 \%$ to $53 \%$ ). It was helpful in the distinction of irregularly shaped or asymmetric tissue (dysplasia, scarring vs. carcinoma) and in equivocal findings within dense tissue (palpable abnormality, search of primary tumor). It is not recommended for distinction between inflammation and malignancy or for differentiation of microcalcifications.

Conclusions: MRI helps improve diagnostic accuracy in problem cases without increase of the number of recommended biopsies.

\subsection{AM}

\section{Contrast-enhanced MRI in breast masses}

Junichi Hachiya, Tsuneaki Seki, Toshiaki Nitatori, Tateo Korenaga, Yoshiro Furuya; Tokyo

Purpose: Evaluation of accuracy of contrast-enhanced MRI in breast masses with emphasis on comparison to mammography and ultrasonography.

Methods: A total of 51 cases including carcinoma $(n=36)$, malignant phyllodes tumor $(n=1)$, fibroadenoma $(n=7)$ and cyst $(n=7)$ was examined by $0.5 \mathrm{~T}$ superconductive MRI unit. Intravenous administration of $0.1 \mathrm{mmol} / \mathrm{kg}$ of GD-DTPA was given in all cases and dynamic study was performed in 40 cases using rapid gradient-echo pulse sequence.

Results: Mass lesions were correctly detected by MRI in all but 2 cases of malignant tumors, and in all cases of fibroadenoma and cysts. Differential diagnosis of masses based on MR image characteristics was correct in $75 \%$ of malignant tumors, $86 \%$ of fibroadenoma and $100 \%$ of cysts. These results were favorably compared to those of mammography and ultrasonography.

The most important features of MR images that aided differential diagnosis were: 1 . significant difference $(p<0.05)$ of dynamic profile of carcinoma and fibroadenoma, and 2. presence of peripheral ring contrast enhancement in $30 \%$ of carcinomas reflecting increased capillary neovascularization and central necrosis. Diagnosis of mammography and ultrasonography was not in agreement each other in 6 cases in detection and in 10 cases in differential diagnosis. All of these cases were correctly diagnosed by MRI.

Conclusions: MRI can be a reliable method in diagnosis of breast masses. It can afford important additional informations in problematic cases in which mammography and ultrasonography are not in agreement.

\section{$10.50 \mathrm{AM}$}

614 MRI with Gd-DTPA in the evaluation of recurrent breast carcinoma P.Ercolani, G. M. Giuseppetti, E. De Nigris, S. Baldassarre, A. Segoni, A. Giovagnoni; Ancona

Purpose: We consider the role of contrast enhanced MRI in the clinical situation of suspected recurrent breast carcinoma.

Methods: Twenty-seven patients $(47 \mathrm{y}$.) with suspected recurrent breast carcinoma were studied. Seven previously were treated by mastectomy and twenty by lumpectomy combined with radiotherapy. Recurrence was suspected following X-ray mammography or clinically by the presence of a palpable mass or nipple discharge. MR studies were performed on $1.0 \mathrm{~T}$ MAGNETOM. T1 (500/17) and T2 (2000/90) weighted spin-echo images were obtained and gradient-echo images (FLASH 2D) $\left(.04 / 10 ; \mathrm{FL}=70^{\circ}\right)$ were performed before and following the intravenous injection. Dynamic studies were performed in all cases of a distinct mass was demonstrated. Gradient-echo images were obtained at nineteen seconds intervals for eight minutes.

Results: The dynamic enhancement profiles of recurrent tumor, fibrous masses and hematomas were obtained. There were six recurrent tumors and all showed marked enhancement. The dynamic profiles of tumors showed an enhancement rapidly within the first two minutes following Gd-DTPA and then retain enhancement for at last five minutes.
Conclusions: MRI in the clinical situation of suspected recurrent breast carcinoma can differentiate recurrent tumor from post-treatment fibrosis and the other post-treatment effects.

\section{$11.00 \mathrm{AM}$}

\section{Pitfalls in MR-examinations of the breast}

\section{W. A. Kaiser; Bonn}

Purpose: Magnetic Resonance was considered as being not very informative in the diagnosis of small breast carcinomas, mainly due to the great variety of examination techniques used. In the past 6 years, the measurement techniques have been optimized.

Methods: Since March 1984 a total of more than 550 MR-breast examinations have been performed (Siemens Magnetom, 0.35-1.5 T). In 372 measurements, a "dynamic" MR-technique was applied, i.e. imaging in short time intervals before and after the injection of $0.1 \mathrm{mmol} / \mathrm{kg} \mathrm{Gd}$-DTPA. In order to assess the efficacy of MRI, clinically and/or mammographically difficult cases were examined in the "dynamic" study; 223 cases had a histopathological correlation, the others had a follow-up study of up to 5 years.

Results: According to the diagnosis of carcinoma, MRI had a sensitivity of $98.4 \%$, a specificity of $98.1 \%$, a positive predictive value of $91.3 \%$, a negative predictive value of $99.7 \%$ and an accuracy of $98.1 \%$. The optimized measurement conditions as well as the multiple possibilities of error are described, e.g.: patient positioning, function and quality of the breast coil, dosage and injection technique of contrast medium, time intervals of images after the injection, measurement sequences, phase encoding direction, adjustment of the transmitter and receiver, image interpretation.

Conclusions: Using an optimized dynamic technique, MRI is able to proof or exclude a breast carcinoma with a high reliability.

\subsection{AM}

\section{MR-guided needle localisation of breast lesions}

R. Beck, S. H. Heywang-Köbrunner, Th. Hilbertz, K. Diergarten, W. Permanetter; Munich

For those cases, where nonpalpable suspicious lesions in the breast were detected by MRI alone, an exact preoperative marking became necessary. Therefore an MR-guided needle localisation technique has been developed at our institution. In principle our MR-localisation technique is similar to the usual CT-localisation technique. First the complete breast is imaged before and after Gd-DTPA with the usual 3D-Flash pulse sequence. After needle insertion and injection of marking solution (charcoal) into the suspicious area, the complete breast is imaged once more to demonstrate the exact location of the marking solution. The charcoal remains within the tissue until removed by the surgeon.

So far altogether 31 lesions have been marked by this technique in 27 patients. All lesions could be identified by the surgeon and the distance between lesions and marking solution was generally below $1 \mathrm{~cm}$.

This localisation procedure allows the necessary identification of non-palpable lesions, which are detected with MR-imaging, by the surgeon and by the pathologist.

It is absolutely necessary because of the well-known variable shape of the breast.

\section{$11.20 \mathrm{AM}$}

617 Breast imaging: digital radiography with stimulable phosphor plates

D. Jimenez, E. Fraile, I. Fernandez, F. Aguado, D. Montero, C. Alvarez, J. Martin; Madrid

1. The purpose of our work is to evaluate the CR system in the study of the breast.

2. To accomplish that, three well-trained mammography radiologists have studied the images obtained from our first four hundred patients. The images have been read both in hard copy and high resolution monitor. According to the pathological findings we classified the images in four categories: definitively negatives, probably negatives, probably positives and definitively positives. All the positive cases have been checked by puncture, guided biopsy or surgery. With the pathological findings, we studied the radiological signs of both benign and malignant lesions.

3. We have appreciated very much the post-processing facility of digital radiography, mainly because: improves image quality, affords greater flexibility in images display, field always consistent contrast on images, avoid incorrect exposures repetitions. We have also achieved a reduction of $20 \%$ of dosis radiation without decrease the diagnostic quality, which is particulary important on follow up studies.

4. Diagnostic possibilities of this digital technique are at least similar to the conventional one, even though both of them achieve a high rate of diagnosis accuracy. Archiving images and their retrieval and reevaluation is really useful and practical. 
mobilization or protection of normal tissues adjacent to the treatment area and 618 Comparison of digital mammography in dual image output format and conventional mammography

Roland J.Scheck, Mareike Kessler; Munich

Purpose: High contrast resolution, an essential requirement in mammography, can hardly be achieved by a single digital image. Therefore we investigated a technique with two complementary hardcopy images different in window width.

Methods: In 134 cases both conventional and digital mammograms were obtained at identical exposure factors. Hardcopies of the digital mammograms were made in single ( 50 cases) or double ( 84 cases) image output format. By manipulating the window width a complementary pair of a low resp. high contrast image was printed. Conventional and digital images were evaluated according to overall image quality, contrast resolution, spatial resolution, detectability of masses and microcalcifications.

Results: Digital mammograms in the dual image output format were judged equal or even slightly better than conventional ones for images quality and contrast resolution. However, spatial resolution of microcalcifications was superior on conventional radiographs. It was difficult to achieve good contrast resolution and depict the whole density range of the breast including the skin as well on a single digital image.

Conclusions: Mammograms obtained by digital luminescence radiography (two processed images of different window width) are judged at least equal to conventional mammograms in the detection of masses and groups of calcifications. For the morphologic analysis of microcalcifications a higher resolution computed radiography system will be required.

\subsection{AM}

\section{Application of digital radiography and PACS system in a senology department}

S. Corcione, B. Bagni, G. Vita; Ferrara

The use of digital radiography has improved significant advantages by virtue of its superior image quality, sensitivity, image-processing capacity and exposure dose reduction.

The electronic components of the PACS System enable a more efficient utilization of medical images and data. These advantages take a particular value in a Senology department according to the increasing number of required examinations for prevention. In particular, PACS System allows a more efficient diagnosis by giving an output of the necessary images when they are required so as to control the rapidly rising medical costs.

The authors report their experience in the elaboration of digital mammographic images and in their departmental transmission with the Philips PCR-PACS System.

\subsection{AM}

620 A charge-coupled device based, quasi real-time imaging system for pre-surgical needle localization and fine needle aspiration biopsy procedures in mammography

Hans Roehrig, Tim B. Hunter, Emre Toker; Tucson, AZ

A Charge-Coupled Device (CCD) based electronic imaging system is proposed to overcome limitations of conventional film/screen mammography systems at no additional risk or discomfort to the patient. The presentation summarizes the design, implementation and evaluation of a prototype system incorporating the latest advances in X-ray intensifying phosphor screen, fiber optic reducer, and CCD technologies. System design is beased on an X-ray intensifying screen fiber optically coupled to a high resolution, cooled, low-noise, scientific CCD detector. The performance of the prototype system is compared to theoretical predictions and to conventional film/screen detector. Patient images acquired on the prototype system are presented. Initial clinical study results indicate that the CCD-based system can provide "film-quality" images within seconds of X-ray exposure.

\section{Room R}

\section{Ss}

\subsection{Intraoperative external radiotherapy and new modalities of interstitial radiotherapy I}

10.30 AM

621 IORT clinical results in tumours of multiple sites (Keynote Lecture)

Felipe A. Calvo; Pamplona

Intraoperative radiotherapy (IORT) is a developmental technique for loco-regional intensification of cancer treatment. IORT permits an excellent radiation dose distribution in a surgically selected target volume, and the

not involved by tumor. During the last decade a significant number of studies have reported the initial clinical and experimental results stablishing the feasibility, tolerance and local tumor control rates that might be expected from the integration of IORT in the treatment management of human cancer. Single institution IORT trials have identified as dose-limiting tissues the peripheral nerves, duodenum and ureters, and the strong relation of local tumor control promotion with the excellence of tumor resection. Multi-institutional IORT efforts have demonstrated the interest and feasibility to develop trials in a Cooperative Group setting, and phase III trials have been activated.

From the clinical experience generated at the University Clinic of Navarra the data shown in the table has been selected in order to summarize a 5 years period of active protocols using IORT as a loco-regional intensification cancer treatment modalities.

\begin{tabular}{llll}
\hline Tumor site & \#PTS & LC $(\%)$ & AS $(\%)$ \\
\hline Head \& neck & 21 & 43 & 32 \\
Lung & 60 & 71 & 19 \\
Gastric & 40 & 78 & 62 \\
Pancreas & 25 & 72 & 5 \\
Colo-rectal & 49 & 80 & 33 \\
Bladder & 22 & 96 & 62 \\
Gynecologic & 27 & 63 & 25 \\
Sarcomas & 19 & 95 & 71 \\
Osteosarcoma & 23 & 96 & 89 \\
Ewing & 20 & 95 & 63 \\
\hline
\end{tabular}

PTS: patients; LC: local control; AS: actuarial survival

These clinical results have to mature in the future, but indicate that IORT is a valuable technique to incorporate in Institutions particularly devoted to cancer treatment.

\subsection{AM}

\section{IORT clinical trials in the RTOG (Keynote Lecture)}

Gerald E. Hanks; Philadelphia, PA

The RTOG has completed 6 phase II trials of IORT that were begun in 1985. The sites were pancreas cancer, gastric cancer, rectal cancer, cancer of the bile duct, cervix cancer and retroperitoneal sarcomas. Two hundred and fifty patients have been entered with 125 receiving IORT.

The results from phase II trials in gastric, rectal and pancreas cancer are encouraging and a phase III trial has begun in 1990 in rectal cancer, and additional phase III trials will begin in 1991 in pancreas cancer and 1992 in gastric cancer.

Results from the phase II trials will be presented along with a summary of physics quality assurance procedures.

\subsection{AM}

623 IORT in gastrointestinal and pancreatic cancer (Keynote Lecture) Normann Willich; Munich

IORT as an useful adjunct in the treatment of gastric cancer can improve the survival rates after curative resection, as it has been demonstrated by Japanese trials. Besides of some reports on similar experience from pilot studies there are only a few randomized trials evaluating the subject. A Munich randomized trial was activated in Jan. 1990 comparing radical resection with N2-lymphadenectomy \pm IORT (28-35 Gy depending on residual mass). Until March 199135 patients were entered on the trial. So far no adverse effects were seen in the IORT treated patients. A similar multiinstitutional study will be opened in the next future by EORTC.

Rectal cancer has been treated by IORT only in a small extent in Europe. Foreign studies indicate a reduced local recurrence rate after curative resection. For treatment of recurrence IORT is effective with respect to palliative effects like pain control.

There is a large experience in IORT for pancreatic cancer. Due to the characteristics of the disease there is only a slight or no prolongation of survival in unresectable cases, despite the local tumour control is about $75 \%$. The mainly advantageous effects are impressive pain alleviation in about $85 \%$ of the patients as well as a stabilization of the body weight and the Karnofsky index. IORT for resectable cases may slightly improve the survival rates.

Despite of only a few reports on complications of IORT, there were some cases of gastro-intestinal bleedings after IORT and high-dose external beam irradiation in our own experience.

In the future the definite place of IORT in the treatment of gastro-intestinal and pancreatic cancers should be defined in controlled trials.

11.20-11.30 AM: Discussion 
11.30 AM

624 IORT in head and neck surgery

A. Nilles, P. Stoll, H. Frommhold; Freiburg

IORT offers new aspects in the treatment of head and neck tumors. It is possible to apply a high dose to the surgically revealed site before and/or after tumor resection is done. By using a linear accelerator providing electrons with energies ranging from $6-21 \mathrm{MeV}$ and by attaching individualized cones areas areas within a depth of about $6 \mathrm{~cm}$ and a size between 4 and $12 \mathrm{~cm}$ can be treated by IORT.

Head and neck tumors are especially qualified, for the required surgical techniques reveal the area of interest completely. Critical structures such as bones, nerves, vessels, salivary glands and the covering tissue can be spared by accurately placed cones in a sufficient size and by using the appropriate energy. Damage of myocutaneous flaps or free vascularized grafts used to close the defect probably can be avoided by reduction of the postoperative external beam therapy.

Within a coordinated team approach patients with squamous cell carcinomas of the floor of the mouth were treated with IORT. The indications for treatment were 1 . aggressive primary cancer, 2 . recurrent cancer after complete external beam radiation, 3 . close margins. Treatment results will be presented.

No major complications have been observed so far (12-16 months observation).

\section{$11.40 \mathrm{AM}$}

\section{Intraoperative radiotherapy (IORT) in miscellaneous tumors of the upper abdomen}

Felipe A. Calvo, Manuel Santos, Oscar Abuchaibe, Javier A. Cienfuegos, Fernando Pardo, José M. Berian; Pamplona

1. Purpose: IORT, a treatment modality developed to intensify loco-regional therapy in patients undergoing cancer surgery, has been explored in miscellaneous tumors located in the upper abdomen.

2. Methods: The loco-regional treatment protocol consisted in maximal surgical tumor resection, IORT boost to the tumor, residual disease or tumor bed area, using moderate single radiation doses with electron beams ( 10 to 20 Gy, 6 to $20 \mathrm{MeV}$ ). External irradiation was omitted in patients with recurrences to previous radiotherapy, and in those of pediatric age.

3. Results: 30 patients were treated in a 5 year period (1984-1989): 18 male and 12 female. Mean age was 48 years (range 3 to 79 years). Disease was recurrent to previous therapy in 10 cases. Tumor locations were: kidney (18), adrenal gland (4), lymph nodes (5), bile duct (1), stomach (1) and duodenum (1). At the time of this analysis the patient follow-up ranges from 4 to $53+$ months (median 10+ months). Actuarial survival rate for the entire group is projected $30 \%$ at 5 years. Actuarial rate of freedom from local relapse is projected $77 \%$. Treatment tolerance was acceptable

4. Conclusions: Selected indications of IORT in miscellaneous tumors of the upper abdomen might induce high local tumor control rates. IORT is a valuable technique to be considered systematically for patients undergoing cancer surgery.

\section{$11.50 \mathrm{AM}$}

626 Intraoperative radiotherapy (IORT) of brain tumors: technical notes, MRI findings and preliminary results

Ch. Rübe, N. Willich, H. Fritsch, B. Fink, Z. Kiszel; Munich

Since $1 / 90$ we treated 13 malignant cerebral tumors (10 malignant gliomas, 2 primary cerebral melanomas, 1 metastasis) with IORT. After maximal tumor removal the patients were irradiated with single doses between 20 and $25 \mathrm{~Gy}$ with a round shaped electron tubus (diameter $5-7 \mathrm{~cm}$ ). The electron energy ranged from 12 to $18 \mathrm{MeV}$.

As an early result there were no major complications on terms of neurological symptoms due to irradiation nor wound infection. Postoperative CT- and MRI-studies were performed routinely within 48 hours and 2-15 days after the irradiation to demonstrate tumor rest or tissue alteration due to IORT. MRI up to 15 days after IORT demonstrated a well demarcated contrast enhancing area on T1-weighted images corresponding to the irradiation field, an effect not seen on CT and in non irradiated patients.

Follow-up studies in regard to this phenomenon and first clinical results are presented.
Room S

SS 10.30 AM

627 Strategic value of numbers in planning of radiologic departments (Keynote Lecture)

Carl B. A. J. Puylaert; Utrecht

The most important factor now in planning is the expected workload, and the question of centralisation (as surface and patient pro room are well-known). In the past three decades conventional radiology conquered the resistance against its expansion by rising and collecting worldwide figures to persuade authorities. In many countries, especially in the lesser developed, the figures of ISPRAD still help to establish radiology.

(Int. Symp. Planning Radiological Dept., 1972 Otaniemi, 1976 Philadelphia, 1980 Amsterdam, 1984 Puerto Rico, 1988 Florence, 1992 Bergen).

The aggressive countercurrents to CT were predicted in the 4 year meetings on the Impact of Modern Techniques by Margulis real think-tanks.

And even if the heat of that fight may be over, we can deplore the loss of diagnoses, therapies and lives.

Centralized conventional radiology had two powerful supports: Cost + Radiohygiene. CT still has both, MRI only one, US neither.

The real reason (and possibility) to keep the radiology in our hands (centralized, intact) is in the end only quality. That is by knowledge of the radiologist, and objectivity (without foreknowledge and assured after-affirmation). Quality is the only reason to wager the fight - not chauvinism or self-interest.

In the latter days many disturbant facts appear, also in our literature, disputes over "Turf" battle (Evens; Rogers + Linton; Levin and Matteucci), "Self referral" (Hillman a.o., N.E.M.J.; Hielman), "Greater control by Academic Departments", "Efficiency of expensive apparatus in one hand", "Competence", "Intervention versus scalpel", can only be settled by figures. Limitation by radiation scare too. Finally, if we want to prove our quality also than figures are necessary. To begin with Ultrasound, where a real battle is going on and loss of quality is not to be feared (Isprad V Florence).

The old law of Descartes - that Science is only Science if it can be proven by figures - keeps valid.

In the end - now iconographic apparatus can be used by technically untrained doctors, it could easily become apparent that our real speciality is anatomy, and pathologic anatomy, visualized with our radiologic (iconographic) apparatus (Ruys, in print) (to be proven by perception tests in figures!).

The author has held an enquiry and will show some results, but a main fact arises foremost, and that is that much too few figures are known and collected, and that all Radiological Societies should start nationally the collection thereof and internationally the study. I would suggest that the Standing Committee of the ISPRAD should put into this circuit as the only group with experience before its next Congress in 1992 in Bergen (Dr. Göthlin).

One fact in nearly all answers to the enquiry is, by the way, that the consumption of X-ray examinations, steady for many years, has risen in the last year(s) $5-10 \%$.

\subsection{AM}

\section{Quality assurance: dose to the patient and image quality (Keynote Lecture)}

J. J. Broerse, J. Zoetelief; Leiden, Rijswijk

Quality assurance in X-ray medical diagnosis is defined as an organized effort by the staff operating a facility, to ensure that the diagnostic images are of sufficiently high quality so that they consistently provide adequate diagnostic information at the lowest possible cost and with the least possible exposure of the patient to radiation. Especially for large scale screening programmes such as mammography carried out in asymptomatic women, analysis of the risk-benefit relation, results in a recommended value below $4 \mathrm{mGy}$ for the average absorbed dose in the glandular tissue.

Investigations of absorbed doses and image quality in various $\mathrm{X}$-ray examinations performed in European clinics revealed appreciable dose variations by more than tenfold at equal image quality. These variations could be attributed to the use of out-of-date X-ray equipment or to non-optimal radiological techniques. On an international scale a number of procedures have been recommended to reduce the collective equivalent dose in diagnostic X-ray examinations without loss of diagnostic information. The following methods could contribute to an appreciable dose reduction: 1 . definition of strict referral criteria; 2 . use of patient compression when appropriate; 3 . beam collimation; 4. increase of filtration; 5 . use of rare earth screens; 6 . change from chest fluoroscopy to radiography whenever possible; and 7. optimal operation of film processing. It has been estimated that such procedures would reduce the total annual collective equivalent dose from medical X-ray examinations in the United Kingdom from a level of 16000 manSv to 8500 manSv which would prevent between 100 and 250 radiation induced cancer fatalities.

Within the framework of a coordinated CEC programme, quality criteria have 
been formulated for diagnostic radiographic images for a restricted number of examinations: chest, skull, lumbar spine, pelvis, urinary tract and breast. For some of these examinations details will be presented on the diagnostic requirements, the criteria for good imaging performance and examples of good radiographic techniques.

\section{$11.00 \mathrm{AM}$}

629 Strategies for patient dose measurement in diagnostic radiology R. M. Harrison, K. Faulkner, C. L. Chapple; Newcastle upon Tyne

Purpose: The ALARA principle implies that formal strategies should exist for the measurement of patient dose in diagnostic radiology. These would also enable the exploration of dose-image quality relationships. This paper outlines a practical strategy for dose assessment.

Methods: Examinations such as IVU, barium meal, barium enema and lumbar spine contribute significantly to the collective dose to the UK population. For each examination, techniques for dose assessment are described using TLD and/or dose-area product meters. In addition, a novel method of measuring doses on-line using a microcomputer interfaced to the X-ray generator and dose-area product meter is described.

Results: The recommended strategies offer an efficient way to monitor regularly those examinations whose doses contribute significantly to population dose. Comparisons of the on-line system with phantom dosimetry using TLD show good agreement with the traditional methods.

Conclusions: Patient doses in selected examinations should be regularly assessed. TLD and dose-area product measurements should be made at least annually for a randomly selected group of at least 20 patients for each examination. This information may also be acquired using an automated system of dose measurement and acquisition.

\subsection{AM}

\section{Population dose assessment on radiodiagnostic in Portugal} $\bar{R}$. Serro, J. V. Carreiro, J. Galvăo, R. Reis; Sacavém

A survey of radiodiagnostic installations was carried out in Portugal covering 75 permises considering public hospitals, local and regional public health centers in a total of $175 \mathrm{X}$-ray tubes surveyed by using the new NEXT methodology covering permises data, tube and operator data and projection data. Average values of voltage, current time product, HVL, ratio of beam area to film area and source to film distance for twelve most frequent projections are reported as well as the skin entrance exposure and the doses to some organs. Considering the total film consumption in 1986, the number of examinations per inhabitant and the mean number of films per examinations, it was estimated as $600 \mu \mathrm{Gy}$ the annual whole body dose "per caput". It was also estimated as $230 \mu \mathrm{Gy}$ the genetic significant dose considering the mean value of the relative frequency of examinations below the mean age of child bearing.

11.20 AM

\section{The dose to the sella turcica in the radiography of the teeth of both arches}

A. Pastremoli; Bologna

Purpose: In the radiography of the teeth of both arches, the sella turcica and then the pituitary gland are radiated. Aim of this research is the experimental evaluation of the exposure dose in connection with the radiologic method used. Methods: A phantom made by an adult man skull of average size with holes filled with water was radiated with the same radiologic techniques and parameters used for the livings. The evaluation of the radiations dose to the sella turcica was made by thermoluminescent dosimeters (Ca F2 : TLD-200) placed inside it.

Results: It turned out that the exposure dose to the sella turcica was the following: $73,19 \mu \mathrm{Gy}$ using a long cone dental X-ray unit (14 exposures); $109,54 \mu \mathrm{Gy}$ with an orthopantomograph (OPT) for film-screen examination (1 exposure with $14 \mathrm{~mA}$ ) and $58,40 \mu \mathrm{Gy}$ for photostimulable plate-digital images acquiring system (PCR Philips) examination (1 exposure with $6 \mathrm{~mA}$ ).

Conclusions: The digital OPT allows a radiography of the teeth of both arches with the lowest dose to the sella turcica. The radiation dose reduction is only $7 \%$, even if the attenuation of the number of photons primary beam is more than $50 \%$ of the traditional OPT. Experimental examination of this result are now in progress in order to consider its dependence on geometric factors for dosimetry (rotational beam).

\subsection{AM}

632 Globus pharyngis versus heartburn - indicators for reflux disease? Anita Wuttge-Hannig; Munich

Aim of the study is to find out which of the both symptoms, globus pharyngis or heartburn, is more frequently seen in our patients suffering from a gastroesophageal reflux.
Until now we found 182 patients with a gastro-esophageal reflux by means of video-fluoroscopy. 112 of them presented a globus pharyngis sensation, but only 87 suffered from heartburn. A special interest was focussed on the function of the upper esophageal sphincter, pharyngeal function, the esophageal peristalsis and the esophago-gastric junction. The esophago-pharyngeal interactions turned out to be an important factor. Globus sensation was found to be mainly due to a dysfunction of the upper esophageal sphincter and this dysfunction was caused by an underlying esophageal disease.

Therefore a consequent antireflux medication was able to abolish as well globus pharyngis as heartburn. Our observations confirm the thesis that globus sensation is more common in reflux disease than heartburn. Theese findings will be proofed in a larger patient-collective.

11.30 AM

633 A multicenter evaluation of guidelines for use of standard projections in common radiological examinations

I. Matthews, D. Yates; Cardiff

Patients undergoing diagnostic X-ray examinations are investigated using a variety of projections for the same examination type. This occurs despite evidence which indicates that a certain choice of projections is acceptable for radiological diagnostic purposes and therefore, additional projections are undesirable.

An observational study of radiological practice in 30 hospitals was carried out between 1983-86 and led to the formulation of guidelines of choice of projections for standard examinations. A multicenter study, conducted under the auspices of the Royal College of Radiologists, was commenced in 1989 with the aim of evaluating the implementation of these guidelines. The main objective was to effect a consequent reduction in variation in patient dose by implementing changes in clinical practice in X-ray departments.

High levels of compliance exceeding $80 \%$ are achievable in all non-contrast examinations although considerable differences in compliance exist between hospitals for certain examination types. Factors responsible for non-compliance have been identified, and practical recommendations are made to ensure a high level of compliance and consequently optimise clinical practice.

The effective implementation of these guidelines into clinical practice offers the opportunity to enhance quality standards in radiodiagnostic care. 


\section{Room A}

\section{HL}

Roentgen-Lecture

634 Imaging of the lymphatic system

P.E. Peters; Münster

Lymphangiography is the only direct radiologic approach to the visualization of the lymphatic system. However, there are many obstacles to this method: no consistent opacification of mediastinal lymph nodes, no opacification of mesenteric lymph nodes, limited accuracy even in areas accessible to the oily contrast medium and, last but not least, the time consuming and invasive cannulation of the lymphatic vessels.

The sum of these obstacles led to numerous attempts to replace lymphangiography with non-invasive or less-invasive imaging modalities. The list of such alternative imaging methods is long:

- indirect lymphangiography

- lymphscintigraphy

- ultrasound

- computed tomography

- magnetic resonance imaging

- and many other specific techniques such as immunoscintigraphy of lymph node metastases of specific tumors, gallium scintigraphy etc.

The current role of cross-sectional imaging in the staging of Hodgkin's disease (HD), Non-Hodgkin's lymphoma (NHL) and in the evaluation of lymph node metastasis of the most common malignant tumors will be reviewed.

In the "outlook" part of the lecture new results of ultrasound studies will be presented which allow the analysis of the internal structure of normal and deseased superficial lymph nodes. In addition, the most fascinating potential application of paramagnetic or superparamagnetic contrast media in Magnetic Resonance Imaging as well as recent developments in immuno-scintigraphy will be discussed. 
techniques. MR with Gadolinium enhancement is more accurate in differentiating tumoral extension from fibrous scars induced by previous pelvic surgery.

Conclusions: In our experience at Institut Curie where only stage 1 and $2 b$ cervix carcinoma undergo surgery after Curietherapy, MR imaging is superior

Room A

SS

\subsection{Abdomen I - Pelvic organs I}

2.00 PM

635 A new, objective classification of cervical carcinoma on the basis of MRI morphometry

G. Ranner, F. Ebner, H. H. Hofmann, J. Haas; Graz

Objective: The present study was designed to determine the exactitude of in vivo MRI measurements of tumor volume, which is the single most important prognostic factor in carcinomas of the uterine cervix.

Methods: Tumor volumes from serial giant sections of the specimens obtained at radical hysterectomy were compared with tumor volumes measured in vivo by MRI. A 1.5 Tesla magnet was used (Gyroscan S12, Philips). Tumor was delineated on long TR, long TE spin echo pulse sequences, ROI were defined and volume data accumulated.

Results: 67 cases of cervical carcinomas stage I to IIb had undergone radical hysterectomy, tumor morphometry on the specimens and preoperative in vivo MRI. There was a highly significant correlation of histomorphometric volumes and in vivo MRI volumes $\left(\mathrm{r}^{2} \cdot 0,983\right)$. In evaluation of tumor extension to the parametria MRI accuracy was $74 \%$ (FIGO $60 \%$ ) in urinary bladder invasion MRI was correct in $96 \%$, in rectum infiltration in $96 \%$, infiltration of the fornix vaginae $87 \%$ and in evaluation of lymph node involvement MRI was correct in $64 \% .29,7 \%$ of all patients had positive lymph nodes (macro- and micrometastases).

Conclusions: MRI may provide an objective basis for precise classification of cervical cancer and for objective comparison of surgery and radiotherapy. The volumes obtained by MRI correlated well $\left(\mathrm{r}^{2} .0,983\right)$ with dose obtained by histomorphometric analysis of the surgical specimens.

\subsection{PM}

\section{Advanced cervical carcinoma treated with preoperative chemotherapy: MR imaging findings}

S. Sironi, A. Vanzulli, G. L. Taccagni, A. DelMaschio; Milan

Purpose: The purpose of this study was to assess the capability of MR imaging to determine tumor regression in patients with advanced cervical carcinoma treated with preoperative chemotherapy.

Methods and Materials: Nineteen patients with cervical carcinoma (stage IIB or higher) treated with a combination of cisplatin, vinblastine and bleomycin were considered. Each patient underwent MR imaging before and after chemotherapy. Postchemotherapy MR images were obtained 1-4 days prior to surgery. All patients underwent radical hysterectomy providing specimens for pathologic correlation. T1 weighted (SE 350/25) and T2 weighted (SE 2000/40-90) images in sagittal and axial planes were obtained at 0.5 T.

Results: In assessing the extent of tumor after chemotherapy MR imaging findings closely approximated the pathologic findings in the hysterectomy specimens, with correct estimates in 15 of 19 cases. The extent of disease was underestimated in 3 patients; in these three cases microscopic examination showed residual parametrial invasion that was not identified on MR images. In one patient the extent of tumor after chemotherapy was overestimated with MR imaging.

Conclusions: The results indicate that in patient with advanced cervical carcinoma MR imaging can provide a reliable means of determining tumor regression after preoperative chemotherapy.

\subsection{PM}

\section{Uterine cervical carcinoma $=$ value of MR and CT imaging}

Thun Nguyen-Tan, Thu-Ha Dao, Liliane Ollivier; Paris

Purpose: To demonstrate value of MR imaging in staging and lymph nodes metastases detection in carcinoma of the cervix compared to CT imaging.

Methods and Materials: 68 consecutive untreated cervical carcinoma (stage 1, 2, 3 FIGO) underwent CT and MR examinations performed within 10 days. CT images were obtained on the CGR-CE 10000 scanner after IV iodine contrast media and MR imaging was performed with a $0.5 \mathrm{~T}$. superconducting magnet GE-CGR MR-Max and Gadolinium enhancement was available in 45 examinations. The prospective results of both techniques are correlated to the clinical and pathologic findings.

Results: Due to its superior tissue characterization and its multiplane section ability, MR imaging is superior to CT in tumor delineation. Endocervical, vaginal, bladder tumoral extension are well demonstrated with MR. Parametrial involvement and lymph nodes metastases are equally detected by both detection.

\subsection{PM}

638 Monitoring response of cervical carcinoma to radiotherapy by magnetic resonance imaging

J. M. Hawnaur, R. J. Johnson, B. Carrington, I. Isherwood; Manchester

Purpose: The study was performed to assess the ability of Magnetic Resonance Imaging (MRI) to monitor the response of carcinoma of the uterine cervix to radiotherapy and to demonstrate residual or recurrent disease.

Methods: Pre-treatment MRI was obtained in 100 women with invasive carcinoma of the cervix, repeated routinely 3 and 6 months after external pelvic and/or intracavity radiotherapy, with subsequent scans as clinically appropriate. An imaging protocol utilising conventional spin echo sequences was performed on a $0.26 \mathrm{~T}$ system and MR findings were compared with clinical assessment and biopsy results.

Results: Complete tumour regression was observed on post-treatment MRI in 63 patients who remain well up to 30 months later. Persistent high signal on T2-weighted images was seen 3 months after treatment for bulky stage IIb-IV tumours in 15 patients, six of whom died or developed metastases. In 16 patients, satisfactory regression of cervical primary tumour was associated with progressive lymphadenopathy or pelvic sidewall disease on MRI. Two patients died soon after treatment and one developed endometrial carcinoma. In four patients with clinically suspected recurrence, a reappearance of cervical high signal was observed on T2-weighted images. This proved to be recurrent tumour in two, radiation change in one and both in two.

Conclusions: MRI complements clinical assessment for monitoring treatment response and detecting metastases. Persistent or recurrent cervical high signal demonstrated on MRI after radiotherapy may be due to tumour or radiation change and requires biopsy for further assessment.

\subsection{PM}

639 MR-monitoring of primary irradiated carcinomas of the uterine cervix

F. Flueckiger, F. Ebner, H. Poschauko, K. Arian-Schad; Graz

Purpose: To investigate the reliability of MR-imaging in the evaluation of the effects of primary radiation therapy (RTX) of cervical carcinomas and to monitor the changing appearance of signal intensity (SI) of the tumor.

Methods: We studied 28 patients with carcinomas of the uterine cervix by MRI before and immediately after RTX and further in a six-month-interval (follow up 9-42 months). The results were ascertained by transvaginal biopsy, physical examination and clinical course. 1,5 T; $64 \mathrm{MHz}$; T2: SE TR/TE 2500/30, 90; sagittal, transversal; $1 \mathrm{mg}$ glucagon intravenously.

Results: The majority of tumors exhibited a significant decrease of volume and loss of SI (up to $77 \%$ ) on T2 weighted images after a time interval of 6 months. Residual- and recurrent tumors showed high SI like the primary. Radiation fibrosis showed higher SI in the early stage up to 6 months and low SI in the later stages. The presence of high SI in the irradiated tumor is a usual sequel within the first 3 months after RTX and does not necessarily reflect tumor persistance.

Conclusions: MR is able to proof reliably the effects of RTX. Beneath a decrease of volume significant loss of SI six months after RTX is a characteristic sign for good tumor response. Residual- and non responder tumors do not show this loss of SI in this characteristic amount. Residual- and recurrent tumors can be differentiated well from radiation fibrosis.

\section{$2.50 \mathrm{PM}$}

640 Low field MRI $(0,28 \mathrm{~T})$ of pelvic carcinoma. Advantages of the RARE technique

C. Roy, C. Scheiber, B. Moyses, B. Dumitresco, D. Gounot, J. Chambron; Strasbourg

a) We studied 67 patients with pelvic carcinoma (20 uterine cancer, 15 cervic cancer, 15 bladder cancer, 17 prostatic cancer) to evaluate RARE technique. b) The RARE method (Rapid Acquisition with Relaxation Enhancement) is based on the principle of echo imaging. Echos trains (RARE factor in this case set at 8 ) are generated with each echo being encoded differently for spatial coordinates.

c) With the following acquisition parameters: $\mathrm{TR}=3750, \mathrm{TE}=30, \mathrm{RF}=8$, $\mathrm{NA}=4$, we obtained twelve $256 \times 256$ images in less than $7 \mathrm{mn}$. The contrast was $\mathrm{T} 2$ weighted and intermediate T2 tissues contributed significantly to the image contrast. The image quality was excellent despite the low field strength and the slice thickness of $7 \mathrm{mn}$. The different part of pelvic organs are well 
analysed. The identification of carcinoma is easy. At this field strength, the chemical shift is no significant and the tumor extension is easy to appreciate. Our results was similar to that of the literature with high field strength.

d) The RARE Technique at $0,28 \mathrm{~T}$ is an efficient method to evaluate pelvic carcinoma because of short acquisition time for a $\mathrm{T} 2$ weighted sequence and no chemical shift.

\subsection{PM}

641 MRI evaluation of ovarian masses - comparison with US and CT Pietro Torricelli, Mario De Santis, Piero D'Alimonte, Vincenzo Spina: Modena

1. Purpose: The purpose of this work is to stress the value of MR in diagnostic evaluation of ovarian masses, in particular when compared with US and CT 2. Methods and Materials: 65 patients, ranging in age from 14 to 73 years, affected with ovarian masses, were evaluated by a high field strength MR scanner (General Electric Signa, 1.5 T). All the patients were previously evaluated by US and in 49 cases by CT. In 16 cases a paramagnetic contrast agent (Gadolinium DTPA) was administered intravenously. The results of MR were compared with those of surgery ( 51 cases) and of fine needle biopsy (14 cases).

3. Results: MR assessed better than US and CT the nature of the fluids contained in the cystic masses (serous - hemorragic - proteinaceous); - MR was superior to US and almost equivalent to CT in characterizing dermoid tumors and it was superior to both US and CT in characterizing ovarian fibromas; - in most cases MR did not give, if compared with CT and US, a significant aid in differentiating benign cystoadenomas from their malignant counterparts; - MR was slightly superior to CT in staging the abdominal diffusion of ovarian cancers.

Conclusions: MR can significantly contribute to characterize ovarian masses and to stage ovarian cancer. Since MR is a free-radiation technique it should be preferred, where possible, to CT, especially in evaluating young women in reproductive age.

\subsection{PM}

\section{Benign ovarian lesions: diagnostic accuracy of US and of MRI} A. Vanzulli, S. Sironi, M. Busacca, C. Belloni, A. DelMaschio; Milan

Purpose: Magnetic resonance imaging (MRI) is seldom used in the evaluation of benign ovarian lesions. Purpose of this study is the comparison of diagnostic accuracy of MRI and of ultrasound (US) in adnexal cysts.

Methods: 63 women with adnexal masses have been studied both 0.5 T MRI (SE: $2000 / 40,80 ;$ SE: $500 / 20$ ) and US.

Of these 63 patients 21 resulted to be affected by benign lesions at surgery (12) or at laparoscopy (9)

Diagnostic accuracy of MRI and of US was compared in these 21 patients.

Results: At hystologic examination 6 patients had simple cysts, 9 endometriomas, 3 had ovarian dermoids, 3 flogistic lesions.

MRI diagnosis was correct in 18 patients (MRI sensitivity $=85.7 \%$ ); US in only 10 patients (US sensitivity $=47.6 \%$ ).

In haemorragic cysts MRI had sensitivity $=100 \%$ and specificity $=91.6 \%$. 1 false positive diagnosis resulted in one case of salpingitis.

Results: MRI can be useful in the evaluation of benign adnexal lesions because of the limited diagnostic accuracy of US. MRI demonstration of blood in a cystic lesion can suggest the diagnosis of endometriosis, which can be treated conservatively in early stages.

\subsection{PM}

\section{MRI accuracy in detecting dermoid cysts of the ovary}

S. Tella, B. Beomonte Zobel, C. D'Archivio, C. Catalano, M. Di Girolamo, R. Passariello; L'Aquila

The purpose of our study was to evaluate the capability of MRI in recognizing benign teratomas of the ovary using Spin-Echo (SE) and STIR sequences. We considered MR examination ( 0,5 Tesla) of 40 female patients, previously undergone Ultrasound, with surgically proved dermoid cysts. In 33 cases MRI showed typical findings: a signal intensity pattern similar to that of the fatty tissue with some areas of very low signal intensity due to the presence of calcium and a general appearance of a cystic lesion. In the remaining patients there were an homogeneous pattern, size larger than ten centimeters and signal intensity higher than fat in SE T2 weighted images, one of these was bilateral, so that they all could be confused with ovarian endometrioses. Moreover all these lesions had transonic appearance at ultrasound examination. At surgery the content was liquid and the chemical tests showed that it was made by pure sebum, known to be liquid at body temperature, so the diagnosis of mature teratoma was made in all cases. Our experiences showed that in such a cases the use of STIR sequence was helpful choosing a TI value adequate to nullify the signal of sebum. In conclusion, in about $12,5 \%$ of cases, a correct diagnosis of pelvic teratoma cannot be made by SE T1 and T2 weighted sequences and the use of STIR sequences is mandatory in differentiating teratomas from hemorragic cysts of the ovary.

\section{Room B}

\section{Ss} 2.00 PM

\subsection{Environmental lung disease. HRCT of the lung}

\section{Conventional and high resolution $\mathrm{CT}$ in asbestosis - ROC analysis} H. Schüller, A. Overlack, R. Wickenhöfer, F. Christ; Bonn

Purpose: Changes of the lung interstitium and pleura may be found in patients following exposition to asbestos. Minor pleural thickenings and calcifications as well as small interstitial lesions are not detected reliably when using conventional radiography. In this trial, diagnostic utility of high resolution CT (HRCT) and conventional CT (CCT) were analyzed in a ROC-study.

Methods: 100 patients with asbestos exposure lasting for 1 to 15 years were included. Changes of the lung were evaluated using CCT with a slice thickness of $6 \mathrm{~mm}$ (supine position), and HRCT with a slice thickness of $1.5 \mathrm{~mm}$ and an edge enhancing reconstruction algorithm in the prone and supine position. Results: Pathological changes of the lung interstitium were detected in 68 patients with both CCT and HRCT. However, a significantly higher degree of confidence was calculated for the HRCT examinations. Minor subpleural fibrosis was found in 27 patients. This important diagnostic finding was delineated to better advantage using HRCT. Pleural thickening and calcification, interlobar septa and pseudotumiors were detected equally well with both techniques.

Summary: HRCT was found to be superior to CCT in the detection of minor changes of the interstitium in asbestosis using a ROC-analysis. Pleural thickening and calcifications were verified with equal sensitivity using both modalities.

\subsection{PM}

645 Pleural and non pleural asbestos related diseases: comparison of conventional and high-resolution computed tomography

P. A. Gevenois, S. Dedeire, M. L. Van Sinoy, F. Rypens, P. De Vuyst, J. Struyven; Brussels

Purpose: Comparison of accuracy of conventional and high-resolution CT in detection of asbestos related disease.

Methods: 40 workers with professional exposure to asbestos were investigated Conventional CT and high-resolution CT were obtained and confidence in presence of abnormalities were evaluated: nodules, masses, curvilinear subpleural shadows, septal lines, non septal lines, honeycombing, nondependant opacities, parenchymal band, minimal emphysema, blebs, bulla, round atelectasis, hyaline and calcified pleural plaques.

Results: Conventional CT was more accurate for detection of nodules and hyaline pleural plaques, high-resolution CT for curvilinear subpleural shadows, septal lines, honeycombing, emphysema and blebs. Both methods were equivalent for detection of masses, nondependant opacities, parenchymal bands, round atelectasis and calcified pleural plaques.

Conclusions: For detection of asbestos related disorders, both methods must to be used.

\subsection{PM}

646 The relationship of high resolution computed tomography (HRCT) to the structure and function of the lung in asbestos-related disease (ARD)

M. W. Bourne, G. H. Whitehouse, M. Pearson; Liverpool

High resolution computed tomography (HRCT) is a technique which optimizes the spatial resolution of lung parenchyma, uses thin collimation, reconstruction with a high spatial frequency algorithm, image targeting and sometimes increased $\mathrm{kVp}$ and $\mathrm{mA}$ settings.

In a cohort of 100 asbestos-exposed individuals, the HRCT findings are compared with the type and degree of asbestos exposure, symptoms and clinical signs, pulmonary function testing, plain chest radiograph (using ILO standards) and conventional CT.

HRCT can provide information regarding lung morphology that cannot be obtained using conventional CT or plain radiographs, in asbestos-related disease. 
647 Asbestos related pleural disease: significance of the obliteration of the costophrenic angle

P. A. Gevenois, P.De Vuyst, S. Dedeire, M. L. Van Sinoy, F. Rypens, J. Struyven; Brussels

Purpose: Diffuse pleural thickening corresponds to fibrosis of visceral pleura. It is associated with fibrous strands penetrating into the parenchyma of the lung and with round atelectasis. When associated with parenchymal bands or round atelectasis, visceral pleural thickening can be distinguished from parietal pleural plaques on CT. If in these cases, costophrenic angle is obliterated on conventional chest radiograph, the associated pleural thickening corresponds to fibrosis of the visceral pleura (diffuse pleural thickening).

Methods: 40 workers exposed to asbestosis are imaged by conventional radiograph of the chest and CT. Obliteration of costophrenic angle and pleural thickening, round atelectasis, parenchymal band are respectively evaluated. Results: Round atelectasis or parenchymal band associated with pleural thickening were found in 6 cases. In all of them, obliteration of the costophrenic angle was observed. In 23 cases, isoled pleural plaques, without round atelectasis or parenchymal band were found. Obliteration of the costophrenic angle was never observed in these cases.

Conclusions: When pleural thickening is associated with obliteration of the costophrenic angle, the thickening reflects fibrosis of the visceral pleura.

\subsection{PM}

648 Computed tomography findings in acute and accelerated silicosis: correlation with plain chest films

Serdar Akyar, Cebrail Șimșek, Süreyya Özbek, Cemil Yağci, Sevim Bavbek, Hilmi Gürses, Hüseyin Sumer, Nezihe Enacar; Ankara

1. Purpose: To investigate the role of computed tomography (CT) in the diagnosis and categorization of silica-exposed workers and to correlate CT findings with plain film findings.

2. Methods: CT of thorax was performed in 23 patients wiht heavy silica exposition. Patients were divided into two groups on the basis of chest films: patients with abnormality on chest radiographs $(\mathrm{O} / \mathrm{O})$ (no:11) and patients with definite pathologies on chest radiographs (no:12). CT scans were obtained with $10 \mathrm{~mm}$. contiguous slices; no contrast medium used. Radiographs were taken within a week of CT scans. Readings were made according to the 1980 ILO classification of pneumoconiosis. The chest radiographs and CT scans were read by two independent observers without knowledge of clinical situation. CT scans were scored on the basis of ILO classification as well of the methods defined by Begin and Goddard.

3. Results: CT has been found superior to the chest films in the evaluation of small opacities and increased the scores especially in the first patient group. CT demonstrated small opacities $(\mathrm{p} / \mathrm{q})$ in 8 out of 11 patients where plain radiographs had no sound pathology. Furthermore, CT was more efficient in determining other abnormalities such as adenopathies, emphyseme bullae, pleural changes or cavities in both patient groups. In overall evaluation, CT results higher profusion scores in categorization of the patients. Pulmonay function tests somewhat correlated well with both plain film and CT results.

4. Conclusions: CT of thorax supplied additive informations in the evaluation of silica-exposed workers. CT examination will be useful especially in workers under heavy and short-term exposition with negative chest X-ray.

\subsection{PM}

649 Pulmonary changes in dental technicians: HRCT and pulmonary function

U. Lörcher, H. Kronenberger, J. Peters; Frankfurt/Main

Purpose: Dental technicians are regularly exposed to dust and thus are a group of high risk for pneumoconioses. Our goal was early demonstration of these entities.

Methods: 70 dental technicians were studied clinically, radiologically and functionally (pulmonary function). Besides a chest X-ray a CT was performed ( 1 and $5 \mathrm{~mm}$ technique) using high resolution algorithmus.

Results: In HRCT we found severe fibrotic-emphysematous changes in $30 \%$, moderate changes in $50 \%$ and no changes in $20 \%$ of patients. The CT findings correlate with the values found in pulmonary function test and are more accurate than those found by chest X-ray.

Conclusions: HRCT is very valuable for early diagnosis of pulmonary changes and their distribution in dental technicians.

\subsection{PM}

650 Problems in detecting bronchial carcinoma in silicotic lungs E. Meyer, U. Zimmermann; Freiburg

Purpose: How is it possible to detect and identify a bronchial carcinoma in a silicotic or anthracosilicotic lung? How can we distinguish a bronchial carcinoma from the silicosis-related progressive massive fibrosis (PMF)?

Methods: Analysis of chest X-rays and computed tomographies of 16 patients with simultaneous silicosis and bronchial carcinoma.

Results: PMF and bronchial carcinoma can be distinguished by means of the following radiological criteria: Dynamics, localization, configuration, shape and margin, surrounding reaction.

Conclusions: The combination of PMF and bronchial carcinoma is not rarely encountered in elder men. The consequences make it important to differentiate these diseases. In most cases our criteria allow the differential diagnosis.

\subsection{PM}

651 Airway changes with different aerosols: assessment with high resolution computed tomography

Christian J. Herold, Robert H. Brown, Carol A. Hirshman, Wayne Mitzner, Elias A. Zerhouni; Baltimore, MD

Purpose: Aerosols are frequently used in the diagnosis and treatment of bronchial hyperreactivity, asthma and related disorders. To test the hypothesis that aerosols, independently from their chemical composition, can cause bronchoconstriction by stimulating irritant receptors, we used HRCT to visualize and measure airway responses to different aerosol types.

Methods: Ten anesthetized and ventilated dogs were investigated 1. during a control state, 2. following isotonic saline aerosol application and 3. after histamine aerosol challenge. In each condition, we performed HRCT and determined airway pressure (Paw) as a measure of peripheral airway tone. HRCT (125 kVp, $310 \mathrm{mAs}$, 4-sec scans, ten 2-mm slices through the lower lung lobes, 1-mm table feed) was obtained at functional residual capacity. Scans were digitized and airway lumenal areas were measured using a computer edging process.

Results: Following histamine aerosol challenge, HRCT demonstrated a decrease in airway areas of $43.3 \% \pm 2.4$ (mean \pm SEM) from control. Paw increased $98.7 \% \pm 17.7$. Surprisingly, HRCT demonstrated significant airway constriction $(26.4 \% \pm 2.6)$ also following saline aerosol, whereas Paw, decreasing $4.6 \% \pm 2.4$ ), did not reflect this airway reaction.

Conclusions: Inert aerosols may cause broncho-constriction, probably by stimulating bronchial irritant receptors. HRCT is a new tool to visualize airway reactions and can demonstrate reactions not reflected by standard physiologic measurements.

\subsection{PM}

652 Pulmonary capillary volume changes: evaluation by high resolution CT (HRCT) measurements of parenchymal background density Christian J. Herold, Robert H. Brown, Carol A. Hirshman, Wayne Mitzner, Elias A. Zerhouni; Baltimore, MD

Purpose: Pulmonary capillaries respond to physiologic changes by adjusting blood flow and volume. However, no direct means of measuring changes in the capillary bed existed. We used HRCT as a new method to evaluate changes in capillary blood volume by measuring changes in pulmonary background density during controlled physiologic maneuvers. Changes in HRCT parenchymal background density, at constant lung volume, are proportional to changes in blood volume in capillaries (since the blood volume in the first orders of arteries and veins smaller than 300 microns is minimal).

Methods: Isolated dog lungs were continously perfused at one of two flow rates, while venous outflow was controlled by the height of a reservoir. Airway pressure was maintained at $6 \mathrm{~cm} \mathrm{H}_{2} \mathrm{O}$. Three physiologic states were studied simulating the three Wests zones of the lung. In each condition, we performed HRCT $(125 \mathrm{kVp}, 230 \mathrm{mAs}$, 1-sec scan time, 20 contiguous 2-mm slices, 1-mm table feed). Parenchymal densities were measured with ROI circles of constant size and position.

Results: With pressure and flow changes simulating zone one, zone two and zone three, background density measurements demonstrated changes in pulmonary capillary volume that differed corresponding to the respective zone.

Conclusions: HRCT is a new method to quantify volume changes in the pulmonary capillaries in response to physiologic changes.

\section{Room C}

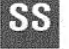

\subsection{Ultrasound}

\section{$2.00 \mathrm{PM}$}

653 Transesophageal endosonography versus CT in the staging of regional esophageal carcinoma

A. Graziano, A. Andriulli, C. De Angelis, T. Gindro, S. Kienle, D. Regge, G. Gandini; Torino

From June 1987 to December 1990 preoperative staging of 70 patients with esophageal neoplasms was performed in our Institution with both CT and 
transesophageal endosonography (EUS). Results of staging were compared with surgical and hystological findings according to the actual TNM classification. EUS was found to be more accurate than CT in the classification of small tumors (T1 - T2) as ist could accurately evaluate the extent of wall infiltration. CT and EUS had similar diagnostic accuracy in large neoplasm (T3T4). Truly esophageal cancer could not be staged accurately with EUS in patients with extensive infiltration of adjacent structures. Furthermore the instrument could not stage patients with severe esophageal strictures. EUS was found to be very accurate in the staging of regional lymphonodes and of vascular involvement. Nodes of $5 \mathrm{~mm}$ in $\varnothing$ adjacent to the esophagus could be clearly appreciated. Concluding we feel that EUS is superior to CT in the staging of both the $\mathrm{T}$ and $\mathrm{N}$ of esophageal neoplasms: CT should be therefore employed only in large neoplasms if extensive infiltration in suspected.

\section{$2.10 \mathrm{PM}$}

\section{The use of intraoperative ultrasound in the management of gastric carcinoma}

M. Bradley, M. Griffin, S. Cheung, A. Li, C. Metreweli; Liverpool

1. The increased sensitivity of intra-operative ultrasound of the liver has been well documented in the evaluation of many abdominal neoplasms, but the aim of this study is to ascertain whether this information affected the surgical management over and above surgical palpation of the liver in patients with gastric carcinoma. In view of the fact that the mortality rate for a radical gastrectomy may approach $5 \%$, it is obviously important to know about liver metastases.

2. 30 patients with gastric carcinoma underwent pre-operative and intraoperative ultrasound of the liver.

3. No malignant pre-operative lesions were seen, but as expected the intraoperative ultrasound was more sensitive ( 20 intra-operative, 10 pre-operative). The surgeon palpated multiple metastases in 2 patients which agreed with the intra-operative ultrasound, but these small lesions $(3-5 \mathrm{~mm})$ were not seen pre-operatively. Thus in these cases the management was not affected by the intra-operative scan. However as regards the non-palpable lesions, 2 solitary metastases were demonstrated by intra-operative ultrasound and positive guided biopsy. Surgical management thus changed in these 2 patients $(6.6 \%)$ due to the intra-operative ultrasound.

4. Therefore we propose that if intra-operative ultrasound demonstrates surgically non-palpable metastases, the surgeon may opt for palliative gastrectomy, but it is of little benefit for multiple metastases as these are likely to be palpable.

\section{$2.20 \mathrm{PM}$}

655 Abdominal ultrasonography in the screening of inflammatory bowel diseases (IBD)

T. Cammarota, S. Barbaro, C. Rigazio, V. Ponti, G. Rossi; Torino

The purpose of this study is to investigate the role of ultrasonography (US) in a group of 52 patients with abdominal pain and diarrhea.

US was performed with $5 \mathrm{MHz}$ linear probe and was positive for bowel wall thickening in 33 patients: in 23 cases the ultrasonographic finding was confirmed by endoscopical and radiological tests.

In conclusion, in our experience, US proved to be a sensible $(81.8 \%)$ and specific ( $71.4 \%)$ test, with a positive predictive value of $87 \%$ in the screening of patients with a clinical suspicion of IBD.

\section{$2.23 \mathrm{PM}$}

656 Ultrasonography in postoperative recurrence of Crohn's disease T. Cammarota, M. Astegiano, A. Sambataro, A. Pera, G. Rossi; Torino

Aim of our study is to evaluate the accuracy of ultrasonography (US) in the diagnosis of postoperative recurrence of Crohn's disease (CD).

Abdominal ultrasonography was performed in 28 patients, who had undergone ileal resection or right hemicolectomy.

A US diagnosis of recurrence was made in 25 patients; the diagnosis was confirmed with clinical-haematological-radiological-endoscopical data in 21 cases $(84 \%)$. In a further 3 patients US diagnosis was negative for recurrence and was confirmed in 2 cases.

US in postoperative recurrence of CD showed a good sensitivity ( $84 \%)$ with a positive predictive value of $95 \%$.

\subsection{PM}

\section{Primary ultrasound of the gut in patients with suspected small bowel Crohn's disease} D. A. Nicholson, D. F. Martin; Manchester

When there is clinical suspicion of small bowel Crohn's disease (CD) then barium examination is the usual initial investigation. Despite attempts to select patients for radiology the majority turn out to have no disease. To assess whether transabdominal intestinal ultrasound (IUS) can detect CD and thus allow more selective use of small bowel barium studies we have undertaken preliminary IUS in all patients referred for barium radiology with the clinical suspicion of small bowel CD. Small bowel wall thickness, presence of peristalsis and intra-abdominal collections were recorded. Barium examination was performed immediately following IUS by a different radiologist who was unaware of the ultrasound findings.

To date 71 patients have been examined, age range 18-84 years (mean 43), 45 female and 26 male. 19 were known to have had CD. In these 19 patients active CD was correctly diagnosed by IUS in 9 of 10 patients and absence of CD was correctly diagnosed in 5 . There were 4 false positive ultrasound diagnoses. In the 52 patients with no history of $\mathrm{CD}$, active disease was correctly diagnosed in 9 of 11 and a normal bowel accurately demonstrated in 40 . There was one false positive. Overall sensitivity was $85 \%$ with a specificity of $90 \%$. The false negative examinations were all encounted early in our experience. We anticipate with increased experience and interpretation, IUS could be used as the primary method of assessment of patients with $\mathrm{CD}$, thus reducing the number of barium studies on patients without disease. Barium radiology and its significant radiation burden could then be reserved for assessment of patients demonstrated to have disease on IUS or those few in whom clinical suspicion remains high despite negative ultrasound.

\subsection{PM}

658 Transrectal endosonography in the evaluation of presumed benign rectal lesions

F.J.H. Hulsmans, E.M.H. Mathus-Vliegen, T.L. Tio, G.N.J. Tytgat; Amsterdam

Transrectal endosonography allows exclusively the visualisation of the separate wall layers and their infiltration or disruption by rectal cancer. Besides for the pre-operative staging of carcinoma, this technique was used in the evaluation of rectal villous adenomas because of their well known highly malignant potential. In 67 patients with an adenomatous lesion in the rectum the previous endoscopy combined with biopsies revealed only tubulo-villous adenoma.

Despite the still not optimal results, endosonography was able to detect eight of the eleven carcinomas. The unfortunate large group of false positives proved to be all previously treated because of their adenoma. In some of the resection specimens distortion of the deeper layers could be detected caused by scarring or inflammation secondary to prior therapy. Beside these histologic features, there are also technical pitfalls which could simulate infiltration or disruption of the deeper layers. Their recognition is essential in the evaluation of rectal lesions.

Although transrectal endosonography is not an infallible technique, because of its inability to differentiate malignant infiltration from inflammatory changes, it would appear to be the most adequate imaging technique for presumed benign rectal lesions. Hallmark is the integrity of the bordering wall layers, without suspicion of infiltration.

\subsection{PM}

659 Contribution of colorectal endosonography in the differential diagnosis between extraparietal and submucosal lesions

P. Fouque, L. Henry, G. Genin, B. Napoleon, B. Pujol, J. C. Souquet, P. J. Valette; Lyon

Endosonography (ENS) is already known as a useful tool for the diagnosis and staging of esophagogastric lesions. This study was designed to evaluate the potential of ENS in the diagnosis of colorectal submucosal lesions.

Methods: During a 3 years period, 27 patients with an edoscopic suspicion of submucosal tumor or extrinsic compression, had colic or rectal ENS. ENS was performed with a rigid endoprobe (Bruel et Kjaer $1850,7 \mathrm{MHz}$ ) in 8 patients until August 88, then with the Olympus echocoloscope (CF EUM3, 7,5 MHz) in 19 patients. The ENS data were correlated with pathological specimen obtained by surgery $(n=12)$, cytology examination $(n=2)$ or endoscopic biopsy $(\mathrm{n}=5)$.

Results: Eight patients presented normal findings using ENS, with clinical normal follow-up from 6 to 36 months. In 9 cases, lesions found by ENS were parietal (carcinoid tumor $\mathrm{n}=5$, leiomyoma, mucosal prolapse, abscess, endometriosis) whereas in 10 cases, lesions were extraparietal (genital cancer $\mathrm{n}=3$, metastatic lymph node $\mathrm{n}=2$, abscess, chondroblastoma, neurofibroma, tailgut cast, pelvic recurrence of an adenocarcinoma). ENS allowed the right location in all the cases. Precise information on the pathological nature of the lesion was not possible.

Conclusions: Based on this still limited series of patients, ENS appears as a powerful imaging modality allowing the differentiation between colorectal submucosal tumor and extrinsic compression. 


\section{US contrast enhancement with $\mathrm{CO} 2$ in hepatic tumours} (ECO-carbography)

F. Garbagnati, A. Marchianò, M. Salvetti, L. Frigerio, G. Piragine, C. Segura, C. Pintaudi, G. Porcelli; Milano

Purpose: To assess whether the selective injection of $\mathrm{CO} 2$ into the hepatic artery can be utilized as contrast agent in the ultrasonographic diagnosis of HCC.

Methods: Twelve patients affected by hepatocellular carcinoma, 1 patient with a regenerating nodule and 1 patient with hepatic haemangioma were submitted to selective $\mathrm{CO} 2$ injection into the hepatic artery, ultrasonographic evaluation of the liver was carried out at the same time.

Results: All cases of hepatocellular carcinoma studied with eco-carbography showed prolonged enhancement by $\mathrm{CO} 2$. Satellite lesions were also demonstrated which could not be identified by conventional ultrasonography.

Imaging of the hepatic haemangioma revealed the same enhancement characteristics after $\mathrm{CO} 2$ injection, while the regenerating nodule did not show any contrast uptake.

Conclusions: Even though the present preliminary study needs further extension, the Authors are positively impressed by the usefulness of this procedure: intra-arterial selective injection of $\mathrm{CO} 2$ can improve the sensitivity of US.

\subsection{PM}

\section{Ultrasound-guided percutaneous laser ablation of liver tissue in a} rabbit model

E.Papini, C.M. Pacella, Z.Rossi, G. Bizzarri, P. Castaldo, F. Garosi, M. Cinti, F. Muzzi, D. S. Paliotta; Rome

Purpose: The aim of our study is to evaluate the efficacy, reproducibility and safety of the liver tissue destruction obtained with laser induced interstitial hyperthermia in a living model.

Methods and Materials: We have placed under ultrasound guidance a spinal needle (18 gauge) into the liver of ten anesthesized New Zealand rabbits. The tip of a $600 \mathrm{~m}$ fiber was passed through the needle and a Nd: Yag laser was used in continous wave mode without coolant flow. Tissue temperature was evaluated at $1 \mathrm{~cm}$ distance from the tip of the fiber. The laser was applied at 1,2 and 3,5 watt for 5 and respectively 10 minutes. Two rabbits were sacrified immediately and the others were followed up for 1-8 weeks.

Results: All animals treated by 1 and 2 watt recovered without complication after the procedure while the 3,5 watt treated had cutaneous and diaphragmatic necrosis. No late complication took place. Liver showed a well defined $2 \mathrm{~cm}$ diameter lesion with a central area of necrosis.

Discussion - The safety and reproducibility of the low wattage technique could be employed in percutaneous treatment of hepatic malignant lesions. This technique could be an alternative procedure to percutaneous ethanol injection (PEI) in treatment of small hepatocellular carcinoma.

\subsection{PM}

\section{Sonographic findings after laproscopic cholecystectomy} W. Pichler, R. Waneck, P. Jiru, K. Lederer, R. Kolb; Vienna

100 patients who underwent laproscopic cholecystectomy have been examined by ultrasound in a prospective study on the first and fourth day after operation. Of these patients 95 remained clinically asymptomatic while 5 patients showed different smaller or even more severe complications such as jaundice, fever, heavy pain and breathing difficulties. There was a good correlation between these clinical symptoms and the ultrasound such as enlargement of the common bile duct, fluid collections in the bed of the removed gallbladder with the differential diagnosis of haematoma or bilioma. In a group of 63 non-symptomatic patients there were however on the first day after operation different sonographic findings such as hyper- or hypoechogenic homogeneous or inhomogeneous structures in the bed of the endoscopical removed gallbladder. They never were larger than $3,5 \mathrm{~cm}$ in diameter and disappeared after 4 days in $31 \%$, they changed their echogenicy from hyper- to hypoechogenic in $35 \%$ and remained in same size $(5 \%)$ or got smaller $(29 \%)$. In 14 patients who showed larger circumscribed fluid collection over $3,5 \mathrm{~cm}$ in diameter in the bed of the removed gallbladder, the major part (8), of these lesions had been resorbed after 4 days or became significantly smaller. 18 patients showed no remarkable findings at all. In $7 \%$ of all patients an enlargement of the common bile duct occured $(6 \mathrm{~mm})$ in 5 patients asymptomatically and in 2 patients with pain and in one of these two with jaundice.

\section{Ultrasound in the follow-up of stage I and II ovarian carcinoma} A. Martínez-Noguera, P. Coscojuela, L. Donoso, M. Izquierdo, B. Ojeda; Barcelona

1. Purpose: In this paper we compare Ultrasound (US) and second-look laparotomy findings and discuss the usefulness of routine US practice in patients with stages I and II ovarian cancer.

2. Methods: Follow-up US studies in 74 patients (mean age: 54) with stage I and II ovarian carcinoma carried out after chemotherapy and prior to second look laparotomy were reviewed.

3. Results: Nine cases of recurrent disease were detected by second-look laparotomy. Five had been detected by US studies and clinical signs had been observed in only three cases. Peritoneal seedlings less than $2 \mathrm{~cm}$ were observed by laparotomy in the four patients with false negative US results. The total number of US studies (378) was very high in relation to the recurrent disease detected by this technique.

4. Conclusions: We conclude that the utility of routine US in the follow-up of stage I and II ovarian carcinoma is questionable in view of the low percentage of recurrent disease detected, the low diagnostic sensitivity, and also the high costs implied. Finally, we propose the determination of tumoral antibodies together with second-look laparotomy and the routine use of US only in stage III and IV ovarian carcinoma.

\section{Room E1}

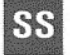

\section{$2.00 \mathrm{PM}$}

\section{Can CT scan still help in the diagnosis of pituitary tumors?} (Keynote Lecture)

J. F. Bonneville; Besançon

Magnetic resonance imaging represents today the method of choice for imaging the hypothalamic-pituitary region, especially for suprasellar tumors. Nevertheless, computed tomography remains an interesting technique for imaging intrasellar pituitary tumors on the condition that high-resolution dynamic CT is used; moreover, dynamic CT allows perfect delineation of cavernous sinus components. Dynamic CT in coronal sections is essential for demonstrating small adenomas. This technique allows visualization of the delay in enhancement of the adenoma by comparison with the healthy pituitary tissue, the controlateral displacement of the secondary pituitary capillary bed, sometimes a very early and transient enhancement of the microadenoma itself corresponding to its direct arterial blood supply, and the intracavernous cranial nerves and veins.

Dynamic scanning requires rapid injection of a bolus of $80 \mathrm{cc}$ contrast medium at $15 \mathrm{cc} / \mathrm{sec}$ pushed by $30 \mathrm{cc}$ saline solution. Six to eight $1.5 \mathrm{-mm}$ sections are carried out beginning with the start of injection. Scan time is $2 \mathrm{sec}$ and the interscan delay time is $2.5 \mathrm{sec}$.

Dynamic CT allows visualization of the intracavernous and supraclinoid internal carotid arteries, central part of the secondary pituitary capillary bed (the pituitary tuft), centrifugal enhancement of the anterior pituitary, posterior pituitary in axial section, and the venous spaces of the cavernous sinus. The intracavernous internal carotid arteries and the branches of their intracranial division are perfectly opacified $10 \mathrm{sec}$ after the end of injection. The high quality of information thus available concerning topography of the carotid siphons today renders carotid angiography superfluous before transphenoidal surgery.

Approximately $10 \mathrm{sec}$ after optimal enhancement of internal carotid arteries, the secondary pituitary capillary bed is visualized as an unpaired median vascular structure situated below the sellar diaphragm, in a prolongation of the direction of the pituitary stalk.

In dynamic axial sections posterior pituitary capillary enhancement occurs very soon after enhancement of the intracavernous carotid arteries due to the direct arterial blood supply. At some time after the bolus injection the posterior pituitary appears less enhanced than the anterior pituitary. Indeed, when contrast media equilibrates between the intravascular and extravascular interstitial spaces, a lesser enhancement of the posterior lobe is seen, correlating with the lesser volume of the interstitial spaces.

The capillary bed is regularly displaced or deformed (tuft sign) in cases of lateralized intrasellar microadenoma.

In general, intrasellar microadenomas are enhanced more slowly and to a lesser extent than the healthy pituitary tissue. In one out of three cases pituitary microadenomas demonstrate very early and transient enhancement due to their direct arterial supply through the artery of the fibrous core. These data only obtained with dynamic CT and ignored with MR imaging, raise many questions:

- Do some pituitary adenomas develop preferentially from some parts of the pituitary gland supplied by the extraportal arterial system?

- Or do some adenomas recruit exportal arteries? 
- These adenomas being situated outside the hypothalamic-hypophyseal portal system are they less dependant of the hypothalamic factors?

- Do these adenomas react differently to medical treatment with dopaminergic drugs?

- What is their specific recurrence rate after surgical removal?

At least, early invasion of the cavernous sinus by a pituitary adenoma can be suspected in the cases where laterosellar veins, particularly the vein of the carotid sulcus are not opacified with dynamic CT.

\subsection{PM}

\section{Pituitary adenoma with intratumoral hemorrhage: MR findings} Kazuhiro Tsuchiya, Kohzoh Makita, Shigeru Furui, Eiichi Takenaka; Saitama

Purpose: Intratumoral hemorrhage (ITH) is known to be frequent within pituitary adenomas. Our purpose was to determine the spectrum of its MR findings.

Methods: We retrospectively evaluated MR (1.5 T) and CT findings of nine cases with surgically proved pituitary ITH.

Results: Clinically, pituitary apoplexy was experienced only in two cases. CT disclosed high density suggesting hemorrhage in five cases. MR findings were classified into three groups. ITH of three cases (group I) showed hyperintensity, on $\mathrm{Tl}$ - and/or T2-weighted images. In four cases (group II), a fluidfluid level was delineated within ITH. The upper part showed hyperintensity, while the lower part demonstrated isointensity or hypointensity on both sequences. In the remaining two cases (group III), ITH showed a mixture of abnormal intensity.

Conclusions: The hyperintensity in groups I and II was considered to reflect the accumulation of free methemoglobin; this was verified in two cases in group II through hemoglobin analysis of surgically obtained hematoma. Hemorrhage within necrotic component was assumed to be the causative mechanism of the fluid-fluid level in group II. In group III, hemorrhages of various stages were probably reflected. Thus, although the MR appearance of the pituitary ITH is somewhat complicated, MR imaging depicts it sensitively and provides detailed information of its contents.

\section{$2.25 \mathrm{PM}$}

\section{MR-imaging of pituitary adenomas: postoperative findings} E. Steiner, E. Knosp, K. Staniszewski, J. Kramer, R. Stiglbauer, H. Imhof; Vienna

MR-Imaging studies of 20 patients with pituitary macroadenomas have been analysed retrospectively in order to determine the typical postoperative changes in the sellar region and to assess the characteristics of residual or recurrent tumors, scar tissue and operatively implanted materials.

Preoperative and postoperative studies were performed on a $1.5 \mathrm{~T}$ unit by means of Tl-weighted sagittal and coronal images before and after administration of Gadolinium-DTPA. 8 patients had more than one postoperative study. All studies were correlated to detailed operative reports and discussed with the neurosurgeon.

The mean size of the adenomas preoperatively was $3.2 \mathrm{~cm} .85 \%$ of the adenomas showed suprasellar extent and $70 \%$ signs of invasion of the cavernous sinus. 17 adenomas were operated transsphenoidally, 4 subfrontally and 1 by both approaches.

$70 \%$ of the patients showed evidence of residual tumors on the first postoperative examination. In all of these patients at least parts of the residual tumors could be found within the cavernous sinus. Therefore, evidence of invasion of the cavernous sinus on a preoperative study is the most important sign that the adenoma might not be totally resectable.

The typical characteristics of residual tumors, scar tissue and implanted materials for sella floor reconstruction (e.g. muscle or fat tissue, bone, fibrine clue and Surgicel) are discussed.

\section{$2.35 \mathrm{PM}$}

\section{Postoperative changes of the sella-evaluation using Gd-DTPA enhanced MRI}

B. Siewert, A. Hochstetter, W. A. Kaiser, K. Bootsveld: Bonn

Purpose: The assessment of postoperative changes of the sella may be difficult due to different surgical techniques (operative access, implantation of various foreign materials). This study was performed to evaluate the differentiation between tumor and scar.

Methods: 21 patients who had been examined from 1985-1990 ( 1 month to 6 years postoperative) were analyzed retrospectively. T1-weighted spin-echo-sequences prior to and following injection of $0.1 \mathrm{mmol} / \mathrm{kg}$ Gadolinium-DTPA were performed in the coronal and sagittal plane.

Results: In 6 cases residual tumor could be assessed following subtotal resection. 6 patients showed a recurrent tumor, and 5 had scars. The differentiation between recurrent tumor and scar was not possible in 4 cases because of signal enhancement after injection of contrast media. In cases of small residual tumors, diagnosis was possible only in combination with an increased hormone level. The radiologist must know whether and which foreign material was used to cover the operative defect at the bottom of the sella or to tamponade a hemorrhage.

Conclusions: With the exception of tiny hormone-active tumor recurrencies MRI is the method of choice for the evaluation of postoperative changes in patients after sella operation.

\subsection{PM}

668 MRI of pituitary adenomas - optimal technique and clinical efficiency

K. Staniszewski, E. Steiner, E. Knosp, H. Imhof; Vienna

Purpose: Determination of the optimal investigative technique for pituitary adenomas with MRI. Testing the influence of contrast agents on evidence and delineation of these tumors and testing if T2-weighted images give conclusions about the tumour consistency (important for determining the optimal operative approach).

Methods: 58 patients wiht pituitary adenomas underwent MRI investigation and were divided into two groups. Group A (consisting of 38 patients) was investigated by means of T1-weighted spin-echo sequence in two planes (coronal and sagittal) before and after application of contrast agent. The influence of contrast agent on the presence and delineation of these adenomas was confirmed.

Group B (consisting of 18 patients with macroadenomas) was additionally subjected to T2-weighted spin-echo sequence in order to assess the tumour consistency.

Results: In group A, 4 cases $(10 \%)$ of tumours were demonstrable only after contrast agent application. The delineation of these tumours from the cavernous sinus after contrast agent application rose from $47 \%$ to $97 \%$.

In group B no significant correlation (Chi-square test) between signal intensity of T2-weighted images and tumor consistency could be demonstrated.

Conclusions: On the basis of our preliminary results, we recommend T1-weighted sagittal and coronal images as well as coronal sections following contrast agent application.

Further we recommend the application of contrast agent in routine investigation of pituitary tumours, as this leads to better demonstration and delineation of adenomas.

T2-weighted spin-echo sequence does not provide any significant additional information (evidency, delineation and tumour consistency). It is also a time intensive sequence. This technique is suitable in cases of suspected haemorrhage into the adenomas or in cases of parasellar tumours.

\subsection{PM}

\section{Panhypopituitarism: MR findings}

\section{R.S. Pozzi-Mucelli, F. Frezza, M. Cova, C. Ricci, L. Dalla-Palma; Trieste}

Purpose: The aim of this paper is to evaluate with MRI the changes of the hypothalamic-pituitary region in patients with global panhypopituitarism in order to see if abnormalities similar to those described in pituitary dwarfism are present.

Methods: Ten patients, 8 males, 2 females, age 26-59, with global panhypopituitarism (predominant gonadotropic hormones insufficiency associated with lower level of other hormones, excluded prolactin hormon), were examined using a Philips Gyroscan 1.5 T magnet. Sagittal and coronal T1 weighted images were obtained using $3 \mathrm{~mm}$ thick slices.

Results: The most important findings are: reduced size of the pituitary gland in $9 / 10$ cases; partially empty sella in 8 and total empty sella in 2 cases; absent hyperintensity of the posterior pituitary in 9 cases; reduced (thin or absent) visibility of the pituitary stalk in 9 cases; ectopic bright spot in the hypothalamic region in 8 cases.

Conclusions: MRI shows the abnormalities of the hypothalamic-pituitary region in patients with panhypopituitarism and may give a contribution to the understanding of the etiopathogenesis of this disease.

\subsection{PM}

670 MR evaluation of suprasellar mass of the brain Shuichi Higano, Shoki Takahashi, Kiyoshi Ishii, Akihiro Sato, Hidetoshi Ikeda, Akira Ogawa, Kiyohiko Sakamoto; Sendai

Purpose: To assess the usefulness of MR imaging for delineation and differentiation of suprasellar mass of the brain.

Methods: The MR images (spin-echo sequence at 1.5 or $0.5 \mathrm{~T}$ ) of 51 patients with a suprasellar mass were reviewed. The various lesions were included: 23 pituitary adenomas; seven craniopharyngiomas; five meningiomas; three each of optic gliomas, germinomas and teratomas/dermoids; two each of aneurysms and cavernous angiomas; one each of chondroma, hamartoma and arachnoid cyst. In most cases, findings of MR were compared with those of CT and of operation and/or pathology.

Results: The lesions containing hemorrhage or hemosiderine deposit (frequently found in cavernous angioma, pituitary adenoma, teratoma), fat 
(teratoma), cyst (craniopharyngioma), flowing blood (aneurysm) were more specifically identified by MR than CT, while the MR intensity and enhancement (Gd-DTPA) pattern of other components was relatively non-specific. The mass extension, which was well detected by MR as correlated to the operative findings, was characteristic in some lesions: other mass than pituitary adenoma seldom extending to the cavernous sinus or sphenoid sinus; craniopharyngioma and teratoma frequently extending to hypothalamus and/or 3 rd ventricle, and compressing optic chiasma downward, etc.

Conclusions: MR allowed more specific diagnosis of suprasellar mass than CT, which was helpful for detection of calcification and fat.

\subsection{PM}

671 Gd-DTPA enhanced MR imaging of prolactin-secreting microadenomas using $0.5 \mathrm{~T}$ field strength

A. Athanassopoulou, A. Gouliamos, A. Kalovidouris, G. R. Kotoulas, A. Souvatzoglou, L. Vlahos, C. Papavassiliou; Athens

A prospective study was performed to assess the role of Gd-DTPA in the detection of prolactinomas.

Examination of the pituitary fossa was performed in twenty-three patients (19 females and 4 males) with prolactin-secreting adenomas using a $0.5 \mathrm{~T}$ system. In twenty-one patients MR scans were taken before and $3 \mathrm{~min}$ after the injection of Gd-DTPA $(0.1 \mathrm{mmol} / \mathrm{kg}$ body weight $)$.

Lesions were identified in 9 of 23 precontrast and in 16 of 21 postcontrast studies. There were 14 microprolactinomas and 3 macroprolactinomas. In 5 patients with normal examination moderate hyperprolactinemia was found. The size of the tumor in all the patients was correlated with the concentration of prolactin levels in the serum. In 14 patients the microadenomas were lateral but within the sella. No suprasellar or inferior extension of the tumor was observed. Invasion of the cavernous sinus was suspected by MR in 4 postcontrast studies.

It is concluded that routine administration of intravenous contrast medium is necessary when investigating hyperprolactinemia up to $100-150 \mathrm{ng} / \mathrm{ml}$ or when there is lateral involvement of the pituitary gland for a better delineation of the adenoma from the cavernous sinus.

\subsection{PM}

672 MR findings in patients with hypothalamic and pituitary low stature: clinical and morphological correlation

F. Cesarani, S. Cirillo, G. Di Rosa, S. Vannelli, L. Benso, T. Avataneo; Torino

Same preliminary studies have proven the presence of morphological changes of the hypothalamic and pituitary axis in patient with hyposomatism but correlation between clinical data and MR findings remains to be established. In our series we have included 23 patients sent to us from the Auxopathology Center of the University of Turin for low stature. The MR exam gave the following results: - normal pituitary gland, - reduced pituitary gland, - interruption of the pituitary stalk with ectopic neurohypophysis, - occuping space process. All patients that had the most severe clinical presentation with multiple pituitary defects and severe stature deficiency at diagnosis show evident morphological alteration of the hypothalamic and pituitary region with interrupted stalk and ectopic neurohypophysis or neoplasm. The good correlation observed between the RM results and clinical data allows us to hypotize the use of MR as a useful diagnostic tool to define the type of alteration behind a growth defect of endocrin origin.

\section{Room E2}

Ss 06.02 Tumours II

2.00 PM

673 MR Imaging in the management of Ewing's sarcoma

D. MacVicar, J. E. Husband, J. Olliff, C. R. Pinkerton, J. Pringle; Surrey

Purpose: To investigate the role of MRI in the management of Ewing's sarcoma.

Methods: In a prospective study CT and MRI have been performed in nineteen patients at the inception of chemotherapy for Ewing's sarcoma, and repeated at three monthly intervals until completion of treatment (a total of 45 scans). Thirteen patients have undergone surgical resection (either amputation or prosthetic replacement) and pathological specimens have been made available for radiologic/histologic correlation. In the remaining patients surveillance with MRI has continued.

Results: Histological correlation showed MR to be comparable to CT in assessing presence or cortical breakthrough of tumour and of soft tissue and marrow abnormality. MR was superior to CT in assessing relationship of disease to joints and epiphyseal plates which is of particular importance in planning prosthetic replacement. MR and CT were unreliable in predicting sites of residual active tumour. However, areas of very high signal on T2 weighted images in bone marrow and soft tissues after chemotherapy correlated histologically to areas of necrosis rather than viable tumour.

Conclusions: MRI is the modality of choice in Ewing's sarcoma. It yields accurate information regarding extent of disease. Signal changes may predict areas of tumour necrosis after chemotherapy, but are unreliable in identifying areas of residual active tumour.

\subsection{PM}

674 MR evaluation of preoperative chemotherapy-induced changes in the osteosarcoma of the extremities

Vincenzo Spina, Pietro Torricelli, Marco Manfrini, Renato Romagnoli; Modena

Purpose: The aim of this work is to assess the accuracy of MR in evaluating the response to preoperative chemotherapy in the osteosarcoma of the extremities. Methods: 46 patients affected with high-grade osteosarcoma were evaluated by a high field strength MR scanner (G.E. Signa 1.5 T), both before and after two cycles of preoperative chemotherapy. 10 patients out of the 46 were also examined after intravenous injection of Gadolinium DTPA. We evaluated the chemotherapy-induced changes in: intra- and extraosseous tumoral mass, soft tissue and joint effusion, tumoral pseudocapsule, intramedullary edema, and signal intensity of the tumoral mass.

All the patients underwent surgery and the MR data were compared with the results of anatomical examination. In particular we related the changes detected by MR with the percentage of the chemotherapy-induced necrosis, histological assessed.

Results: The changes of tumoral size and edges appeared to be well related to the histological changes. In most cases the decrease in signal intensity in T2-weighted images reflected a good response to therapy even if both our results and the literature reports are not homogeneous on this issue. Soft tissue and joint effusion always decreased or disappeared after therapy but this findings are not strictly related to a good histological response.

Conclusions: MR showed to be an accurate, non-invasive method to evaluate the response to preoperative chemotherapy in the osteosarcoma of the extremities.

\subsection{PM}

\section{Neoadjuvant intraarterial chemotherapy of malignant soft tissue} tumours

András Kónya, Zoltán Vigváry; Budapest

Purpose: 51 patients (pts) with soft tissue sarcomas of extremity received neoadjuvant intraarterial (i.a.) chemotherapy via a percutaneously placed catheter. Treatments were aimed at creating prerequisites of limb salvage.

Methods: These were performed as a series of chemotherapy cycles (on average 3 in each patient) and carried out in the form of short term infusions (20-40 min.) of doxorubicin and cisplatin. I.a. infusions were combined with complementary methods (superselective catheterization, tourniquet, occlusion-infusion, chemoembolization).

Results: In 3 pts complete remission and in 21 partial remission were registered, while in 27 pts only minimal response could be achieved, although in this latter group necrosis of 13 tumours proved to be significant value (more than $30 \%$ ). Even these sarcomas demarcated well from its neighbourhood giving adequate surgical margins for limb salvage. 5 additional tumours became operable. In 43 out of 51 pts successful limb sparing surgery could be performed.

Conclusions: Multiple courses of i.a. chemotherapy combined with supplementary methods increasing local cytostatic/cytotoxic effects of drugs seemed to be useful in preparation of soft tissue sarcomas for limb salvage.

\subsection{PM}

\section{FLASH or SE sequences for the investigation of musculoskeletal} neoplasms?

R. Erlemann, G. Reuther, H. Müller-Miny, N. Roos, P. E. Peters; Münster

Purpose: The potential of FLASH sequences for a replacement of spin echo sequences (SES) in staging musculoskeletal neoplasms was evaluated.

Methods: In 121 patients FLASH sequences with flip angles of $10^{\circ}$ (FLASH-10) and $90^{\circ}$ were utilized, the latter with (FLASH-90G) and without Gd-DTPA enhancement (FLASH-90), using a TR of $40 \mathrm{~ms}$ and a TE of $10 \mathrm{~ms}$. Additionally a FLASH-10 with a TR of $320 \mathrm{~ms}$ and a TE of $14 \mathrm{~ms}$ was applied (FLASH-10/320). Image contrasts obtained with these FLASH sequences were compared to that obtained wiht T1-w, T2-w SES and Gd-DTPA enhanced T1-w SES.

Results: Contrast between neoplasms and muscle was highest with FLASH-90G but was $61 \%$ lower than that in the T2-w SES; while all other FLASH sequences were significantly inferior. Contrast between neoplasms and bone marrow or fat was highest in the FLASH-90 and in the FLASH-10 and amounted to $66 \%$ of that in the T1-w images. In sclerotic and fibrotic lesions contrast was much higher in the FLASH-90 than in the FLASH-10, 
while in lytic lesions contrast was higher in FLASH-10. Pertitumorous edema in muscle was visualized by FLASH-90G and FLASH-10/320 and was missed by all others, while edema in fat was mostly missed by all.

Conclusions: FLASH imaging cannot replace SE imaging for staging but it may be recommended for displaying a muculoskeletal neoplasm in additional orientations.

\subsection{PM}

677 Contrast enhanced (Gadolinium-DTPA) MRI of bone and soft tissue tumors

H. De Gryse, F. Ramon, A. De Schepper; Edegem

Signal intensity and morphology of bone and soft tissue tumors on native T1and T2-weighted spin-echo MR images allow a specific diagnosis in a limited spectrum of entities. We retrospectively reviewed the records of 200 patients to determine the value of contrast administration for improvement of tissue characterization and a more accurate staging.

All patients were examined on a $1.0 \mathrm{~T}$ superconductive magnet (Magnetom, Siemens AG) operating at $0.5 \mathrm{~T}$. Native spin-echo $\mathrm{T} 1$ - and $\mathrm{T} 2$ weighted images were compared with contrast enhanced spin-echo T1-weighted images (after $0.1 \mathrm{mmol} / \mathrm{kg}$ Gd-DTPA IV)

Pattern (homogeneous, peripheral, peripheral with papillary internal borders, cocarde-like, pleiomorph) and degree of enhancement were determined. Soft tissue extension and impact on the medullary cavity (edema, infiltration) were analysed. Finally MRI findings were compared with histologic examination. The results indicated no strict correlation between pattern and degree of enhancement and the histologic type of the lesion. Contrast administration neither allowed distinction between benign and malignant lesions. On the other hand, use of Gd-DTPA improved the depiction of intratumoral architecture (eg. periosteal reaction) and the differentiation between viable and non-viable tumor tissue (biopsy guiding). Finally, pattern and degree of enhancement allowed a closer distinction between neoplastic tissue and surrounding edema, hereby better demonstrating the tumor itself and its extension.

\section{$2.50 \mathrm{PM}$}

\section{Magnetic resonance imaging (MRI) of soft tissue tumors} A. De Schepper, H. De Gryse, F. Ramon; Edegem

In order to assess the value of MRI in the histological classification, grading and staging of soft tissue tumors, we reviewed the records of 150 patients examined over a three year period.

All patients were studied with use of a $0.5 \mathrm{~T}$ unit obtaining $\mathrm{Tl}$-weighted spin-echo images in a coronal plane and T2-weighted spin-echo images in an axial plane. In a limited number of cases, T2-weighted gradient-echo images were also obtained. Gadolinium DTPA has been used as a contrast agent in 110 patients $(74 \%)$

Signal intensity (SI) on different pulsing sequences, homogeneity, shape (oval, rounded, lenticular, bifid, stellar, lobular, plexiform, dumbbell, and serpigineous) size, depth, degree and pattern of enhancement after contrast administration of all lesions were evaluated.

Although SI on MRI reflects only gross morphology and not microscopy, it allows a more accurate histological classification compared to all other imaging modalities. Cellularity, pleiomorphism, mitotic rate, necrosis and amount of matrix are histologic parameters which are reflected on the MR images, improving the grading capability of this method. Because size, depth and relationship to the surrounding structures are excellently depicted on MRI, this method is mandatory in the staging of soft tissue tumors.

\subsection{PM}

\section{Muscle texture: a useful pattern in the postoperative MR follow-up of patients with soft tissue sarcomas}

Pietro R. Biondetti, Richard L. Ehman; Padova, Rochester, MN

Purpose: Nonspecific intensity changes in T2w images of extremities following surgery and radiation therapy for tissue sarcomas can make postoperative assessment difficult. This study was designed to determine whether the parenchymal texture of muscle in high-resolution T1w images could be used to differentiate such posttreatment changes from tumor recurrence.

Methods: MR studies of 40 consecutive patients who had surgery and radiation therapy for soft tissue sarcoma of the lower extremities were reviewed. T2w images were evaluated for masses and nonspecific high signal changes; textural characteristics in the corresponding T1w images were classified. All patients had surgical proof and/or a clinical follow-up of at least 16 months.
Results: For patients had obvious masses which were correctly diagnosed as tumor recurrences. Three were fluid collections. In 31 cases $T 2 w$ images showed nonspecific high signal changes. In 23 of these analyses of the T1W images demonstrated textural caracteristics of muscle in the suspicious regions. Although 12 of this group had original interpretations expressing concern about recurrence, none proved to have this.

Conclusions: Recognition of muscle textural caracteristics in high resolution Tlw transverse images of suspicious regions improves the diagnostic specificity of follow-up MR examinations in patients with soft tissue sarcomas.

\section{$3.10 \mathrm{PM}$}

680 Locoregional recurrences of superficial soft tissue sarcomas and melanoma

P. C. Muzzio, A. Tregnaghi, L. Bidoli, C. Lacognata, F. Candiani, R. Rossi; Padua

Soft tissue sarcomas and melanoma present a high incidence of locoregional recurrence after surgery, often a few months from extirpation. At the moment there is not any agreement in the instrumental diagnostic approach. A comparison between Ultrasonography and Computed Tomography in local recurrences of superficial tumors showed a higher capacity of US than CT in detecting the lesion number in each case. Moreover a better delimitation by US is demonstrated. Furthermore US offers a better evaluation of the relationship between the tumor and the contiguous muscular and vascular structures. Only regarding liposarcomatous relapses CT offers a more effective evaluation than US; this is attributed to the high difference in densitometry of this tumor with normal contiguous muscular tissue. In conclusion US is more suitable than CT for a preoperative local staging of patients with recurrence from soft tissue sarcomas and melanoma with the exception of liposarcoma.

\section{$3.20 \mathrm{PM}$}

681 Failures of bone scintigraphy in the diagnosis of intra-capsular osteoid osteoma of the proximal femur - diagnosis by CT

D. Bossard, C. Lapra, T. Tavernier, M. Bochu; Lyon

Purpose: The diagnosis of osteoid osteoma is usually based on clinical findings and positive Radionuclide Bone Scintigraphy (RBS). This study reports 3 cases of surgically proven Osteoid Osteoma (O.O) diagnosticated by computed tomography, with non-suggestive RBS.

Methods and Materials: Over a 2 years period, 3 patients (female $=1$, male $=$ 2 , mean age $=18$ years) have been referred for hip pain evocative of O.O. Plain-films, 99m Tc Bone scan, and high resolution computed tomography were obtained for the 3 patients, and the results correlated with pathological specimens.

Results: Plain-films were negative in the 3 patients. RBS demonstrated diffuse increased activity in the hip of the 3 patients, without focal abnormal finding, suggestings "synovitis" of the hip but not any O.O appearance. High Resolution CT ( $2 \mathrm{~mm}$ continuous thick slices, $256^{2}$ matrix) demonstrated small subperiosteal foci of hypodensity, well delineated, without surrounding osteosclerosis. Joint effusion was also noted in the 3 cases. These findings, very suggestive of intra-capsular O.O, were confirmed after surgery by pathological demonstration of a typical nidus, and non-specific synovitis in the 3 cases.

Conclusions: We suggest that RBS may fail in diagnosing osteoid osteoma, when intra-capsular and associated with synovitis: in these cases, the nidus is likely to be better identified by CT.

\section{Room L}

\section{SS} 2.00 PM

\subsection{Neuro}

682 Pathological white matter development in infants and children - a clinical correlation study

Peter Baierl, Christoph Förster, Monika Popp, Lothar Ziegler, Hannes Schedel; Munich

Purpose: Periventricular white matter lesions, especially in the frontal and occipital subcortical areas, are a common finding in pediatric MRI. In some cases, however, the assignment of the lesion to a particular category (demyelinating/dysmyelinating disorders, anoxic-ischemic damage) is not possible. The purpose of this study was a correlation of the MR patterns with the clinical findings.

Methods: WMR lesions in over 50 infants and children were found in MR images at 1.0 and $1.5 \mathrm{~T}$. Because the discrimination between normal and pathological WMR development is difficult in this age group, 40 normals served as age-matched controls. Three different MR patterns could be differentiated: diffuse confluent/patchy/focal. Clinical evaluation included history, neurological and psychomotoric status, EEG, DSF findings. The degree of retardation was classified as: none/mild/severe. Follow-up evaluations were 
obtained in most patients.

Results: Generally, a positive correlation was found between the degree of retardation and the extent of white matter lesions. However, in several patients with focal lesions the clinical findings were normal. On the other hand, some infants with a severe neurological deficit showed only minor changes.

Conclusions: Discrepancy MRI/clinical findings.

\subsection{PM}

683 MR-neuroimaging in infantile hypopituitarism

M.-H. Smet, F. de Zegher, G. Marchal, G. Wilms, A. L. Baert, M. Vanderschueren-Lodeweyckx; Leuven

Purpose: MRI was performed to document potentially present morpho-structural abnormalities of the hypothalamo-pituitary region in seven infants $(0-14$ months) with hypopituitarism. Four infants had associated congenital cerebrofacial malformations.

Methods: MR imaging was performed with a standard head coil on a 1.5 Tesla Siemens imager. Tl weighted sagittal and coronal sections were obtained with a spin-echo pulse sequence.

Results: The following anatomical abnormalities were identified in variable combinations: ectopic neurohypophysis, absence of the pituitary stalk, extreme elongation of the pituitary stalk, aplasia of the anterior pituitary lobe, hypoplasia of the anterior pituitary lobe and no visualisation of the hypothalamopituitary complex.

All ectopic hyperintense posterior pituitary lobes had a normal function. One infant showed an orthotopic hyperintense posterior pituitary gland in association with diabetes insipidus and holoprosencephaly. Absence of the pituitary stalk was associated with multiple anterior pituitary deficiencies. Visualisation of normal hypothalamo-pituitary anatomy didn't exclude functional impairment: in our series it was associated with less severe hormonal dysfunction.

Conclusions: MRI is very sensitive in the identification of structural abnormalities of the hypothalamo-pituitary region in infants with or without cerebro-facial malformations. However, the MR image does not appear to be a good predictor of endocrine function.

\subsection{PM}

684 Choroid plexus cysts - a marker for trisomy 18: a prospective study and review of the literature

P. Twining, J. Zuccollo, J. Swallow, J. Clewes; Nottingham

Purpose: In order to determine the incidence and significance of choroid plexus cysts, a prospective study was carried out on all fetuses scanned at Queen's Medical Centre, Nottingham.

Methods: Over a 17 month period 6706 singleton pregnancies were scanned at 18-19 weeks gestation and 36 fetuses were identified with choroid plexus cysts. Results: The incidence of cysts is $0.54 \%$. Cysts were unilateral in 17 cases and bilateral in 19 cases. The size of the cysts varied from 3-13 mm in diameter with a mean diameter of $5.6 \mathrm{~mm}$.

Choroid plexus cysts were associated with other abnormalities in 3 cases. Two of these subsequently were found to have a chromosomal abnormality, one case of Trisomy 18 and one Trisomy 21.

A review of the literature reveals 239 cases of choroid plexus cysts associated with 13 cases of Trisomy 18 and 2 cases of Trisomy 21 . This produced an overall incidence of $5.4 \%$ for Trisomy 18 and $0.8 \%$ for Trisomy 21 . Large bilateral cysts were associated with Trisomy disease in 4 cases.

Conclusions: We feel therefore that in fetuses with choroid plexus cysts a carefully detailed scan should be carried out to look for the ultrasound markers of Trisomy 18. If there is an associated abnormality or cysts are large and bilaterial, a karyotyping should be carried out.

\section{$2.30 \mathrm{PM}$}

$685 \mathrm{CT}$ scan findings of spontaneous intracranial hemorrhage due to hemostatic disorders in infants and children

Shin-Lin Shih, Jackson Ching-Tzer Lin, Jon-Kway Huang, Der-Cherng Liang; Taipei

Intracranial hemorrhage remains a serious problem of hemostatic disorders in infants and children. Sometimes intracranial bleeding is more marked than clinically expected. Computed tomography permits accurate and sensitive diagnosis of intracranial hemorrhage.

We retrospectively reviewed the CT scans of brain due to hemostatic disorders for past 6 years 6 months (Jan 1, 1984-June 30, 1990). CT Scans were performed either with Toshiba TCT 80 whole body scanner or Somatom DRH. All the cases were confirmed by laboratory tests except one case due to reactive thrombocytosis probably storage-pool disease. The study group consists of 13 patients. The age incidence is from 6 weeks to 14 years. No neurosurgical procedures were performed except one patient. Three patients died of serious intracranial complication.

Three patients with hypoprothrombinemia showed subarachnoid hermorrhage
$(\mathrm{SAH})$ and subdural hematoma $(\mathrm{SDH})$ in 2 cases. Intracerebral hematoma (ICH) was seen in one case.

Four patients with thrombocytopenia or platelet dysfunction presented SDH in one patient and ICH (including one intracerebellar hematoma) in 3 patients. Six patients with hemophilia A, B or Von-Willebrand's disease revealed ICH in 4 patients, SAH in 5 patients and SDH in 6 patients.

Computed tomography is indicated in all cases of suspected intracranial hemorrhage. The CT scan was used to evaluate the severity and provide a rational basis for therapy.

\subsection{PM}

686 Importance of the detection of "post-therapeutic image" in the prognosis of head and neck rhabdomyosarcoma in the child

R. Gilles, D. Couanet, S. Chevret, L. Shapeero, F. Flamant, M. Meunier, J. Masselot; Villejuif

Purpose: We studied patients with head and neck rhabdomyosarcoma (RMS) to determine whether Computed tomography (CT) findings could be helpful to identify those with high risk of relapse.

Methods and Materials: High dose contrast (CT) was performed in 23 children, aged 2 to 17 years, treated for a histologically proven RMS from 1985 to 1989 .

Results: Retrospective analysis of the CT scans revealed two groups of patients. One group (12 patients) had a "post-therapeutic image" on the CT scan performed at the end of the treatment. "Post-therapeutic image" is defined as soft tissue thickening at the original site with intermuscular fascia enhancement, without or with a little mass effect and which remains stable over a period of three months. Nine of these 12 patients relapsed. The other group (11 patients) demonstrated normal CT scan at the end of the treatment, only three relapsed. Statistical analysis demonstrated that children with a "post-therapeutic image" had a higher rate of relapse (Fisher's test) and a shorter disease-free interval (logrank test).

Conclusions: On our study we found that a "post-therapeutic image" in CT performed after therapy appears to be a prognosis factor of good value.

\section{$2.50 \mathrm{PM}$}

\section{Magnetic resonance imaging of children with suspected spinal dysraphism}

J. M. Hawnaur, J. P. R. Jenkins, I. Isherwood; Manchester

Purpose: Conventional investigation of spinal dysraphism by myelography and computed tomography involves ionising radiation, is invasive, and is often poorly tolerated by children. The aim of this study was to review our experience of spinal Magnetic Resonance Imaging (MRI) in such patients. Methods: MRI findings were reviewed in 46 children aged 3 months to 18 years undergoing spinal imaging for suspected spinal dysraphism. MRI was performed at $0.26 \mathrm{~T}$ using spinal surface coils. Sagittal T1-weighted scans were supplemented as appropriate by T2-weighted, or further T1-weighted sequences in alternative planes. General anaesthesia or sedation was required in 11 patients.

Results: In 21 children, no abnormality was demonstrated. Six patients had scoliosis and/or vertebral segmentation anomalies without associated dysraphism. One patient had a subcutaneous haemangioma with no underlying spinal abnormality. The most frequent manifestation of spinal dysraphism was a low tethered cord, associated in all cases with one or more of the following: (lipo)meningocoele, intradural/subcutaneous tumour (usually lipomatous), syringomyelia or diastematomyelia. In five patients spinal cord involvement by congenital tumour (enterogenous or dermoid cyst) or as part of a meningomyelocoele was the most significant finding.

\section{$3.00 \mathrm{PM}$}

\section{MRI in children with surgically repaired spina bifida aperta} R. Salbeck, H. Hötzinger, M. Göpfrich, H. C. Tödt, H. K. Beyer; Herne

Purpose: To evaluate the accuracy of MRI in children with surgically repaired spina bifida aperta.

Methods and Materials: MRI was performed in 11 children (age ranges, 3 months to 13 years), who underwent surgery for spina bifida aperta shortly after birth. 9 children had progressive neurologic deficits, 2 children had no symptoms. Surgical confirmation of MRI diagnoses was obtained in 7 children.

Results: MRI detected the cause of neurologic symptoms in each case: in 5 children we found dorsal fixation of the neural placode at the operative site ( 3 surgically proven), two children had a dermoid tumor (histologically proven), two other children had a spinal lipoma with fixation of the filum terminale (surgically proven). Additionally hydro-/syringomyelia was found in 4 patients.

Results: MRI is an accurate tool in the diagnostic work-up of children with surgical repair of spina bifida aperta and progressive neurologic deficits. 


\section{Room M}

SS

\subsection{US in urology}

$2.00 \mathrm{PM}$

689 Is screening for neoplasm indicated in acquired cystic disease of the kidney?

J. Hughes, G. V. Thomas, C. Evans; Cardiff

Acquired cystic disease of the kidney is a recognised complication in patients with end stage renal disease on longterm dialysis. Complications of this entity include spontaneous haemorrhage and the development of solid renal tumours, some malignant. The incidence of cystic disease increases with the duration of renal replacement therapy. Investigation of 64 patients in our centre who had been on dialysis for more than five years demonstrated cystic disease in $38 \%$. No tumours were detected.

Review of all patients who had received longterm dialysis in our centre $(1,000$ patients since 1967) did not reveal any deaths from renal carcinoma. We therefore carried out a postal survey of 20 dialysis centres in the UK. The total number of renal tumours identified in these centres and thought to be related to renal cystic disease was 23 over a 21 -year period. The number of patients on longterm dialysis in the UK at present is 6,800 , of whom about one third will have been on dialysis for more than five years. Routine screening of patients for tumours is only carried out in one centre. Despite the recognised increase in renal tumours in patients on longterm dialysis, we suggest that in view of the low yield (one renal tumour per annum approximately in 20 dialysis centres), routine screening is not justified.

\subsection{PM}

690 Ultrasonographic evaluation of the acquired cystic kidney disease (ACKD): a new grading

L. Baldrati, B. Balbi, M. Mughetti, A. Rocchi, R. Rossi, C. Capponcini, C. Feletti, D. Docci; Cesena

ACKD is the result of cyst formation in failing noncystic kidneys; its complication includes hemorrhage, flank pain and neoplasm.

In the ultrasonographic evaluation of the ACKD, several parameters have been considered, such as number, size and extent of cysts. In order to standardize different kind of grading, we set up the following cumulative grading (CG): grade $0,<3$ cysts per kidney; grade $1,3-5$ cysts per kidney, extent (= kidney mass replaced by cysts) $<25 \%$; grade $2,3-5$ cysts, extent $>25 \%$; grade $3,6-10$ cysts, extent $<50 \%$; grade $4,6-10$ cysts, extent $>50 \%$; grade $5,11-15$ cysts, extent $<50 \%$; grade $6,11-15$ cysts, extent $>50 \%$; and grade $7,>15$ cysts per kidney.

In 60 hemodialysis patients, we have found a significant positive correlation between months of dialysis and CG $(R=0.59, p<0.001)$.

\section{$2.13 \mathrm{PM}$}

691 US evolution of acquired cystic kidney disease (ACKD). Our experiences in over 200 patients undergoing hemodialysis

C. Reviglio, M. Dandrone, G. Garofalo, M. T. Sorrentino, T. Cammarota; Torino

US first informed us in the past about the appearance of renal cysts, mostly cortical, and even now our patients are yearly controlled with this method only. Our results demonstrate that the percentage of patients with ACKD increases with the dialytic age; the number and the size of the cysts are related with this age. The intracystic hemorrhage and the rupture usually happen in cysts whose size are at least of $30 \mathrm{~mm}$; the appearance of adenocarcinoma is very rare.

After transplantation, the acquired cysts decrease in number and size and often disappear.

\subsection{PM}

692 The value of sonography in the diagnosis and follow-up of patients with blunt renal trauma

A. Furtschegger, D. Lungenschmid, W. Buchberger, W. Kastlunger, P. Waldenberger; Innsbruck

We have studied the value of sonography in the assessment of renal trauma with particular emphasis on follow-up after both surgical and conservative therapy. Renal lesions were diagnosed by sonography in 145 patients (108 males, 37 females; age range 4-87 years) between April 1982 and October 1990. An additional I.V.P. was performed in 111 patients. 8 patients also underwent CT and 4 underwent angiography. 31 patients with ruptured kidneys and 42 with contused kidneys were followed up sonographically. Equipment used included a PICKER model LSC 7000 or 9500 with either a $3.5 \mathrm{MHz}$ or $5.0 \mathrm{MHz}$ transducer. Rupture of the kidney was diagnosed in 57 patients and contusion of the kidney in 84 . The correlation between operative findings, sonography and urography in 46 patients showed positive sonographic findings to be correct in all patients with rupture of the kidney. In 7 patients with normal sonograms the diagnosis of a contusion was based on the urographic results. An intimal lesion was seen in 2 and a tear of the renal artery in 2.

The results of our investigation suggest that sonography should be applied in the first place in the evaluation of blunt renal trauma. It is the investigation of choice during follow up after surgical as well as conservative therapy.

\subsection{PM}

693 The value of sonography in the diagnosis of ureteral calculi R. Oyen, A. L. Baert; Leuven

Purpose: To evaluate the value of sonography in the diagnosis of ureteral calculi in patients with acute renal colic.

Methods: The files of 80 patients with acute renal colic were reviewed. The diagnosis of ureteral stone was proven by a plain film or by an IVU. In all patients a routine sonogram of the kidneys was performed by different radiologists, most often at the urgency department.

Results: In $90 \%$ the sonogram was abnormal, in $10 \%$ the sonogram was completely normal. In 50 cases $(62,5 \%)$ the ureteral calculus was visualized sonographically: 9 out of 28 calculi in the upper third of the ureter were seen, 2 out of 9 in the middle third and 39 out of 43 in the lower third. In more than half of the patients the calculus was located in the distal part of the ureter which can routinely be visualized on ultrasound. In 4 patients, initially considered to be normal, there was obvious increase in cortical echogenicity with pronounced cortico-medullary differentiation and only minor dilatation of the pelvicalyceal system.

Conclusions: Ultrasound in a patient with renal colic should routinely include the distal ureter. A plain film is essential in a minority of patients in whom sonography is normal or borderline.

\section{$2.36 \mathrm{PM}$}

\section{Endovaginal cystourethrosonography}

M. Bazzocchi, G. B. Bellis, F. Stacul, B. Frea; Trieste

Purpose: To analyze anatomic and physiologic features of female micturition with a new ultrasonographic technique.

Methods: 27 females, age 23-74, with stress incontinence (8), recurrent cystitis (11) and other voiding disorders (8), were examined during micturition with an endovaginal linear probe $(7.5 \mathrm{MHz})$ using an $\mathrm{AU} 590$ Esaote Biomedica equipment. Echographic images during micturition were videorecorded. The urethral flow pattern was evaluated with color doppler as well. A special chair which allows micturition in a physiological position was used. Results were compared with the X-ray cystourethrographic findings.

Results: It was possible to show both morphologic and dynamic features both in normal cases and in several pathologic conditions. Basing upon the direction of the urethra it is possible to establish with a good reliability the change of position of the bladder floor and neck, which are often responsible of stress incontinence.

Conclusions: This new echographic technique turned out to be rather simple and not invasive. The investigation is particularly advisable in fertile-age women because ionizing radiations are not used.

\subsection{PM}

695 Evaluation of female stress incontinence with vaginosonography and MRI: a comparative study H. Hötzinger, E. Herbe, R. Jasper, R. Salbeck; Herne

Purpose: In the evaluation of female stress incontinence besides urodynamic examination MCU has lost acceptance. Vaginosonography (VS) has gained importance. A major disadvantage of VS is the bad visualization of bony reference points in contrast to MRI. In this prospective study VS and MRI are compared for the diagnosis of female stress incontinence.

Methods and Materials: 5 normal women (19-36 years) and 15 patients (41-72 years) with stress incontinence I-III were studied prospectively. VS: Picker 9500, 6,5 MHz, vaginal scanner; MRI: 1,5 T Signa, GE, sagittal sections GRE $50 / 13 / 10$. All examinations were done in relaxation and with maximal pressure. Parameters: $\alpha$ : inclination angle, $\beta$ : vesico-urethral angle, scipp-line, dsu (asu): distance (angle) inferior border symphysis and internal orifice of the urethra.

Results: With VS and MRI the following parameters can be measured:

$$
\text { VS MRI }
$$

$\alpha$

$\beta$

scipp

dsu

asu

$\begin{array}{ll}- & + \\ + & + \\ - & + \\ + & + \\ + & +\end{array}$


VS and MRI results of $\beta$, dsu and asu showed no statistical relevant difference. Conclusions: VS and MRI are suitable for imaging of female stress incontinence. No statistical relevant difference between comparable parameters has been found. MRI in addition to VS allows to define $\alpha$ and the scipp-line which are useful in selective cases.

\section{$2.56 \mathrm{PM}$}

696 The value of sonography and CT in renal trauma due to ESWL - an experimental study with pathologic correlation

Thomas Schaub, Hamada El-Damanhoury, Klaus Pfitzner, Martina Kunisch, Stefan Störkel, Hans Schild, Manfred Thelen; Mainz

Purpose: To test the reliability of sonography and computed tomography in renal trauma after ESWL.

Methods: Domestic pigs were used as animal model. Sonography and CT was done before and after ESWL with second generation lithotripter system. 54 kidneys were treated with shock waves and power setting comparable to patient treatment. All animals were sacrificed and the kidneys examined pathologically.

Results: 39 (72\%) of the 54 kidneys treated showed macroscopic renal bleeding on pathologic examination. Sonography yielded 39/54 (75\%), Computed tomography $45 / 54(83 \%)$ correct findings.

Conclusions: CT is superior in detecting renal bleeding compared to sonography. However macroscopic renal bleeding was not detected in 6 cases by $\mathrm{CT}$ and 9 cases by sonography.

\subsection{PM}

697 Kidney changes after extracorporal shock wave lithotripsy: evaluation using Doppler US

L. E. Derchi, F. Pretolesi, G. Mancini, C. Caviglia, P. Bottino, A. Germinale; Genova

Intraparenchymal hemorrhage and edema from shock waves are well known short-term complications of ESWL for renal stones. We evaluated by US Doppler blood flow changes related to such lesions.

We compared the arterial waveforms from two different sites within the kidney: one near the stone; another at least at $3 \mathrm{~cm}$ from it, obtained before, at $1 \mathrm{~h}$, and $24 \mathrm{~h}$ after ESWL in $17 \mathrm{pts}$. (7 female, 8 male; 35-67 years) with pelvic or calyceal renal stones. Three measurements were obtained at each site, and Resistive Index (RI) was calculated. Patients received 600-2000 shock waves (means 1672) at 18-20 kV. In tissues around stones a highly significant increase of RI $(\mathrm{p}<.0002)$ at $1 \mathrm{~h}$ after ESWL was noted. A significant $(\mathrm{p}<003)$ decrease to values slightly higher than basal followed at $24 \mathrm{~h}$. No changes were noted in RI values far from stones. Reversible flow changes are demonstrated by US Doppler following ESWL for renal stones; they relate well to vascular damage from the intraparenchymal hemorrhage and edema which occur in these situations. Further work is needed to evaluate if non-reversible US Doppler changes occur and if they can be used as prognostic factor for long-term complications of ESWL.

\subsection{PM}

\section{US follow-up of transjugular kidney biopsy}

\section{E. Coderc, G. Rouhana, D. Meseure, N.Sellier, F. Mal, P. Callard; Bondy}

The transjugular kidney biopsy is a new vein approach deriving from the transjugular liver one.

To prove the accuracy of such a procedure, a sonographic follow-up has been performed in 100 patients one hour after biopsy. In case of US abnormality, or secondary clinical diseases (pain, hematuria) the patients have been controlled, twenty hours later.

The most patients present any US finding just after biopsy.

Some present with subcapsular or small parenchymatous hematomas but secondary follow-up were normal.

Few of them (3\%) present with large perirenal hematomas or bladder clots. No surgery was necessary and progressive improvement was observed.

\subsection{PM}

699 Renal colic: which radiological approach?

M. Bazzocchi, F. Stacul, L. Pagnan, G. Festini, D. Marega; Trieste

Introduction: there is no agreement in the current literature on the best radiological approach to the patient with renal colic. The association of the plain film of the abdomen and ultrasonography (U.S.) is suggested by some authors, while others still support the role of IVU as a primary tool. A prospective study in order to set the best approach was begun.

Methods and Materials: The study began on January, 1, 1991 and is expected to end by December, 31, 1991 (preliminary results on the first 8 months will be presented at the congress). All patients with firm diagnosis of renal colic who arrived at the emergency department were enrolled. They underwent plain film of the abdomen and renal and bladder U.S., plus colour doppler evaluation of the ureteral jets. 58 patients were investigated up to March, 15. 44 of them subsequently entered the hospital, while 14 were discharged. Both groups underwent a clinical and radiological follow up (urography was performed in 38 of them). Moreover outpatients were asked by phone on eventual symptoms one month following the colic.

Results: Concerning the 38 patients who underwent IVU as well, 10 had negative plain and U.S. findings and IVU was negative as well (no false negatives). In the remaining 28 patients plain film and/or U.S. showed positive findings (stones and/or hydronephrosis). IVU was positive in 25 of them (3 false positive of the association plain film - U.S.). The evaluation of the ureteral jets by colour doppler did not offer further information.

Discussion: Our preliminary data in this prospective study are consistent with our previous position. The association of the plain film of the abdomen and renal and bladder U.S. seems adequate for the initial management of patients with renal colic. IVU is probably redundant in many cases.

\section{Room N}

\section{SS} $2.00 \mathrm{PM}$

\section{Nuclear cardiology: ventricular function and myocardial perfusion (Keynote Lecture) \\ P. Rigo; Liège}

Accurate evaluation of the repercussion of coronary atherosclerosis requires measurement of regional coronary blood flow reserve and of the impacts of blood flow abnormalities on myocardial function and viability.

Important progresses have been made in the evaluation of myocardial perfusion using nuclear medicine techniques. New generations of PET machines now provide quantitative assessment of regional myocardial perfusion and perfusion reserve. These can be coupled with assessment of myocardial substrates utilization to assess energy requirement and oxygen utilization, and to derive information on viability and potential for recovery after ischemic insults.

The introduction of new perfusion tracers such as $99 \mathrm{mTc}-\mathrm{MIBI}$ and Teboroxime also offers great promise for the evaluation of the extent and repercussions of coronary artery disease.

Acute studies and simultaneous evaluation of perfusion and function are several of the new possibilities offered by these tracers.

Assessment of myocardial viability is one the current challenges in nuclear cardiology. This begins by more precise evaluation of ventricular function impairments both diastolic and systolic. The contribution of nuclear medicine techniques in these perspectives will also be reviewed.

\subsection{PM}

701 Evaluation of coronary bypass graft PTCA by magnetic resonance imaging (MRI) using spin-echo and phase gradient echo sequences H. Mayr, J. Strametz, M. Klicpera, J. Kramer, S. Globits, H. Frank, H. Imhof, D. Glogar; Vienna

It was the purpose of the study to evaluate a potential of MRI in the diagnosis of aortocoronary bypass graft patency. 25 patients ( 19 men, 6 women, mean age 57.6 years) were examined using a 1.5 Tesla Siemens Magnetom. Examinations were done in an axial orientation using $10 \mathrm{~mm}$ slices covering the region from the aortic arch to the diaphragm. Three MR-sequences were compaired: Spin-echo T1, flow rephased FISP and FLASH. All patients had selective bypass angiography within 14 days of the MRI examination. The readouts were done independently by two experienced observers.

Results: Highest sensitivity and accuracy was found with FISP sequences (sens. $93 \%$, acc. $88 \%$ ), followed by FLASH (sens. $88 \%$, acc. $82 \%$ ), and spin-echo $\mathrm{T} 1$ sequences (sens. $79 \%$, acc. $73 \%$ ). In two cases false positive results were found in occluded bypasses because of retrograde flow.

We conclude that MRI has a high sensitivity and accuracy in detecting blood flow in coronary bypass grafts. Fast gradient echo sequences proved to be superior to the spin-echo technique. In respect to bypass function the diagnostic potential of MRI seems to be questionable (organic stenosis, retrograde flow).

However, in patients with chestpain after recent coronary bypass operation, were organic bypass stenoses are unlikely, MRI could be a valuable non-invasive method in the diagnosis of bypass graft patency. 
interobserver variability $(\mathrm{r}=0.95)$. The examination in the short axis took about $18 \mathrm{~min}$, the assessment about $4 \mathrm{~min}$.

Conclusions: Cine-MR is a very reliable method for a semiquantitive evaluation of aortic valve insufficiency. Due to the short time of the examination and the simple assessment our procedure can be used in the routine diagnosis. prediction of immediate outcome possible?

P. Jebavý, R. Feuereisı, V. Ježek, J. Ježková, P. Niederle; Prague

Percutaneous transvenous mitral commissurotomy - Inoue technique (PTMC) was performed in 25 patients. In one patient was procedure completed by tricuspidal balloon commissurotomy. Two large sizes of balloon $(28,30 \mathrm{~mm})$ were used only. Immediate haemodynamic changes after PTMC are given in the table.

\begin{tabular}{llllllll}
\hline & CI & SI & PPA & PLA & MVG & MVF & MVA \\
\hline before PTMC & 2,75 & 40 & 27 & 17 & 10 & 84 & 0.62 \\
after PTMC & 3,11 & 43 & 23 & 11 & 5 & 102 & 1.16 \\
\hline $\mathrm{P}<$ & .01 & n. & .001 & .001 & .001 & .001 & .001 \\
\hline
\end{tabular}

PTMC leads to a significant decrease in pulmonary artery (PPA), left atrial (PLA) pressures and mitral valvular gradient (MVG) due to increased mitral valve area index (MVA) irrespective to a previous surgical commissurotomy (13 pts), or rhythm (SR-14, AF-11 pts), and or echo severity score. Cardiac index (CI) and mitral valvular flow index (MVF) increases, stroke index (SI) remains unchanged. PTMC increased MVA of 96 percent in average, (in 3 patients under 50 percent only). Success rate decreased with increasing age of patients $(r=-0,54)$. Higher body weight or surface area exhibited negative influence on success of procedure marginally significant. No mitral regurgitation was observed after PTMC in all patients. Left-right shunt larger than 20 percent of pulmonary flow (Fick method) after transseptal approach of balloon was detected in 3 patients only. We believe a need for the larger balloon for mideuropean population patients with mitral stenosis is justified. PTMC is useful and save procedure for mitral stenosis treatment irrespective to possible previous surgery.

\subsection{PM}

703 Three-dimensional tomographic imaging of the right ventricle S. Cross, H.S. Lee, M. Norton, S. Walton; Aberdeen

Tomographic imaging of the cardiac blood pool has been shown to be useful for studying the left ventricle. Regional right ventricular function is difficult to examine non-invasively: the right ventricle does not contract uniformly and differs in shape and size between individuals. We describe our findings in examining the right ventricular phase maps in a group of patients undergoing gated blood pool tomography.

After labelling of the red blood cells with technetium, tomographic sections of the cardiac blood pool were obtained in 47 patients admitted for cardiac catheterisation. The images were displayed as a pseudo three-dimensional image on a computer screen. The phase images of the right ventricular blood pool in 14 controls were compared to that obtained in 33 patients with previous myocardial infarction (MI).

From the control group it was decided that abnormal right ventricular phase occurred when an area of right ventricle had a phase angle lying outside the range 150-350 degrees. There were focal areas of abnormal right ventricular phase in $9 / 13$ patients with inferior MI, and in $1 / 20$ patients with anterior MI. Our results show that 3 -dimensional gated blood pool tomography can be used for the study of abnormal right ventricular contraction.

\subsection{PM}

704 Functional diagnosis of aortic valve insufficiency using Cine-MR: a semiquantitive assessment of valvular regurgitation volumes compared with colour-Doppler-echocardiography and cardioangiography

M.-C. Dulce, K. Friese, A. Albrecht, B. Hamm, K.-J. Wolf; Berlin

Introduction: Cine-MR is a non-invasive method demonstrating blood flow phenomena without an application of contrast media. The purpose of our study is to test the reliability of a semiquantitative evaluation of aortic insufficiencies using Cine-MR.

Methods: 40 patients with aortic valve insufficiencies were evaluated with an 1.5 Tesla equipment. We used a gradient-echo-sequence $(\mathrm{TE}=12 \mathrm{~ms} ; \mathrm{FL}=60$; $\mathrm{TR}=\mathrm{RR}$ ). The heart was shown in the anatomical short axis with 12-24 different time frames per cardiac cycle. The aortic insufficiency was quantified with a MR-Score-Index, which takes into consideration the diagonal axis area of the aortic insufficiency-jet as well as its length. It was compared with Colour-Doppler-Echocardiography (CDE) and cardioangiography.

Results: All patients with aortic valve insufficiencies showed a localized signal loss during diastole in Cine-MR. Cine-MR indicated a higher correlation with cardioangiography $(r=0.89 ; p<0.001)$ than $C D E$ with cardioangiography $(r=0.81 ; p<0.001)$. Compared with angiography over- or underestimations occured more rarely in Cine-MR than in CDE. Cine-MR showed a very low

\subsection{PM}

705 The role for ultrafast CT in cardiovascular diagnosis - experience during the first decade

Martin J. Lipton; Chicago, IL

It is almost 10 years now since the first prototype Ultrafast CT scanner (UFCT) became available. An increasing number of departments initially in the United States, later Japan and most recently in Europe have installed this system.

Despite remarkable advances in DSA, MRI and MRS, Ultrafast CT offers unique capabilities for screening patients for coronary artery disease based upon its high sensitivity for detecting calcification in the coronary vessels themselves, and in characterizing and quantitating myocardial function. A large multicenter United States trial of coronary artery bypass grafts showed that Ultrafast CT detected patency with an accuracy approaching that of selective angiography. In addition global and regional myocardial wall motion and thickening dynamics can be measured routinely for both ventricles during a single Ultrafast CT procedure.

Measurements of myocardial tissue perfusion have also been validated using intravenous contrast enhancement. Ultrafast CT requires no ECG gating, unlike radionuclide methods and spin echo and fast MRI imaging, hence, all cardiac levels can be scanned with a total imaging time of only a few seconds. This effectively eliminates respiratory motion artifacts which are a major problem with MRI. This also facilitates good anatomical detail in the heart, great vessels and simultaneously the lungs.

Future improvements in Ultrafast CT image acquisition speed, reconstruction time, and more sophisticated image analysis will occur. Electron beam scanner design may ultimately replace all current mechanical conventional CT units. A very optimistic future role can be expected for Ultrafast CT scanning not only in the heart, but also for whole body diagnosis.

3.05 PM

706 Detection of left atrial thrombi with ultrafast CT in mitral stenosis Tadashi Nakanishi, Seiki Hamada, Hiroaki Naito, Naoaki Yamada, Satoshi Imakita, Yoshiaki Hirose, Tatsurou Kaminaga, Makoto Takamiya; Osaka

Purpose: Usefulness of ultrafast CT (UFCT) for diagnosis of left atrial (LA) thrombi in mitral stenosis was assessed in comparison with echocardiography. Methods and Materials: UFCT and transthoracic echocardiography (TTE) were performed in 90 patients. Transesophageal echocardiography (TEE) was carried out in 41 out of the 90 patients. These 3 examinations were carried out within a month. LA was transversely scanned with $6 \mathrm{~mm}$ slice-thickness and $6 \mathrm{~mm}$ slice-interval. The LA was scanned $30 \mathrm{sec}$ and $5 \mathrm{~min}$ after contrast injection as an early and late phase scan, respectively. LA thrombi were defined as positive, when filling defects were observed at both phases.

Results: Results agreed in $86 \%$ between UFCT and TTE, in $80 \%$ between TTE and TEE, and in $80 \%$ between UFCT and TEE. Analyzing disagreement cases between UFCT and TEE, TEE showed higher detectability in LA appendage (LAA) thrombi. Early defect (ED) with late filling in the LAA was observed in 16 out of 90 cases $(18 \%)$. TEE showed no thrombi in the areas of the filling defect. In the cases of negative TEE and UFCT, those of ED with late filling showed higher incidence of embolic episode (36\%) than the other cases $(19 \%)$. Conclusions: UFCT is a good modality to detect LA thrombi. Combination with early and late phase scan was necessary for LAA thrombi diagnosis. ED with late filling may suggest a jeopardy of embolic episode.

\subsection{PM}

\section{Technetium $99 \mathrm{~m}$ Teboroxime: a new myocardial perfusion agent} R. Carretta, M. Kramer, P. Cronin; Roseville, CA

Technetium $99 \mathrm{~m}$ Teboroxime (TEBO) is a new myocardial perfusion agent that differs significantly from Thallium-201 chloride (T1-201). TEBO is neutral lipophilic compound that is prepared by template synthesis involving boronic acid derivatives. The technetium-based compound is readily available and has significant advantages over T1-201. The technetium energy level is optimized for current gamma camera images and the kit preparation precludes the problems associated with cyclotron-produced radiopharmaceuticals. The high photon flux assures optimal resolution of the myocardial perfusion while the rapid myocardial clearance of TEBO enables a stress/rest study to be performed within 90 minutes. TEBO is rapidly taken up by the myocardium and has a linear relationship to myocardial blood flow. There is no significant redistribution of the agent and washout parameters can be assessed using multidetector camera systems. Both planar and SPECT imaging is possible using TEBO. Total imaging time with single detector systems is less than 15 minutes. Greater patient through-put is possible using TEBO and high quality 
SPECT images can routinely be obtained using current single-headed SPECT systems. TEBO is a welcome addition to myocardial perfusion imaging and provides significant advantages over T1-201.

\subsection{PM}

708 Dynamic quantitative cardiac angiography in patients with MI undergone $\mathrm{CT}$ and delayed PTCA

F. Leisch, A. Savtchenko, A. Smirnov; Moscow

71 pts with myocardial infarction (MI) were studied. All pts were treated by coronary thrombolysis (CT) (within 4 first hrs of the chest plain) and percutaneous transluminal coronary angioplasty (PTCA) of infarct-related artery (IRA) (on 4-10 days of MI). Coronary angiography with left ventriculography were performed twicely in all pts: during PTCA and $6 \mathrm{mo}$. later. Quantification of coronary angiograms was performed by means of computerised semiautomatic system. Quantitative analysis of global and local left ventricular (LV) function was performed with the help of computer equipped by program "AVD" (Siemens). Combined application in MI pts of CT (intravenous administration of 1500000 IU of Streptokinase) and delayed PTCA of IRA produced improvement of IRA patency (decreasing of the main calculated stenosis severity indices). Significant improvement of LV local wall motion in MI zone occurred in these pts by $6 \mathrm{mo}$. of observation. It is considerable that IRA renarrowing after CT + PTCA by 6 mo. of observation didn't influence the LV myocardial function restoration in MI zone.

\subsection{PM}

709 Coronary artery ultrasound thrombolysis in acute myocardial infarction

Cesar Monteverde, Mario Velez, Guillermo Nava, Ricardo Jauregui, J. Bordes; Mexico

Four patients (pts) with acute myocardial infarction were treated with mechanical thrombolysis with ultrasound. On admission a coronary angiogram was performed. Inclusion criteria was: less than 4 hours of the symptoms beginning and a totally occluded coronary artery related with the infarcted zone. We used a prototype device, (Sonic Needle Corp.) Ultrasound frequency was fixed at $22 \mathrm{kHz}$ and transmitted to target areas by a ball-tipped, (titanium alloy) wire probe. The wires were tuned up to work at $22 \mathrm{kHz}$. Ultrasound was delivered in pulses no more than 20 seconds long. Two patients had a total occlusion of the right coronary artery, one of the circumflex artery and one of the proximal left anterior descending artery. Angiographic recanalization was achieved in 3 of the 4 patients in 10 to 15 seconds of continuous sonication. In one patient t-PA was used after ultrasound sonication to achieve distal revascularization of the right coronary artery. No immediate restenoses occurred. Symptomatic improvement was reported immediately after the procedure. Residual stenoses in two patients underwent balloon angioplasty 7-10 days after the procedure. The thrombolytic effect of ultrasound in achieving recanalization in acute myocardial infarction is feasible.

\section{$3.26 \mathrm{PM}$}

710 Early experience with percutaneous cardiopulmonary support in percutaneous transluminal coronary angioplasties and resuscitation U.M. Sivananthan, M. R. Rees, T. Browne, S. Whittaker, G. A. Davies; Leeds

The recent introduction of portable self contained cardiopulmonary bypass systems together with vascular access via percutaneous cannulae has extended the role of extracorporeal circulatory support into coronary angioplasty and resuscitation. The vascular access is via femoral vessels using a modified Seldinger technique with siting of the $17 \mathrm{~F}$ to $19 \mathrm{~F}$ cannulae at the junction of the vena cavae and right atrium, and common iliac artery. The closed circuit system consists of a constrained Vortex pump, oxygenator and heat exchanger. Outputs of up to 6 litres/minute may be obtained.

A total of 19 patients (16M:3F) underwent percutaneous cardiopulmonary support (CPS), eleven of the patients were judged to be high risk (low ejection fraction, target vessels supply greater than $50 \%$ of the viable myocardium, surgically inoperable etc.) for PTCA and were electively supported by CPS. Eight patients underwent emergency CPS as part of resuscitation and 4 of these patients survived to undergo PTCA. One patient was supported during aortic valvuloplasty. Five deaths occurred in total, significantly all in the emergency CPS group.

We have found CPS a useful adjunct in elective PTCA for high risk patients. All 10 out of the 11 elective CPS patients underwent successful PTCA who would ordinarily not have been considered for angioplasty. But this relatively new procedure needs further evaluation before it is widely used.

\section{Room 0}

\section{SS} 2.00 PM

\subsection{New technical developments in CT (II)}

711 New developments in single photon emission computerised tomography (Keynote Lecture)

P. J. Ell; London

Single photon emission computerised tomography (SPET) is a versatile and widely available tomographic imaging technique. It relies on conventional radionuclides which label an increasing range of specific radiopharmaceuticals in order to obtain tomographic maps of organ function/metabolism. In the last few years, the field has been progressing with the arrival of multidetector SPET tomographs, vastly increasing the detection sensitivity of the instrumentation and the development of Technetium-99m labelled and I-123 labelled specific radiopharmaceuticals. The new Technetium-99m labelled brain, cardiac and renal agents and the new I-123 labelled neuroreceptor imaging agents are typical examples of this development.

With the arrival of multi-detector technology, imaging strategies for throughput, information density or reduction in radiation exposure can be chosen in relation to the appropriate clinical request. At least 12 manufacturers offer these new multi-detector SPET tomographs which are optimised for brain or brain and body imaging. At least 3 Technetium- $99 \mathrm{~m}$ cardiac myocardial perfusion agents are now being used and again at least three I-123 labelled neuroreceptor tracers are being applied to the investigation of specific disease states. The subject of this keynote lecture will be a review of the progress achieved in this field over the last 5 years.

\section{$2.15 \mathrm{PM}$}

\section{Densitometric limitations of computed tomography}

\section{S. Tabakov; Plovdiv}

Purpose: The increased role of quantitative computed tomography (QCT) naturally raises the problem of reliability assessment of densitometry and the limits of its application in practice.

Methods: We performed over 3000 density measurements on the image of specially prepared phantoms. The investigations were made at all possible scanning parameters. Densoprofile density definition and averaging the density over a given area were used.

Results: The significant image contour spread in CT (1 to $6 \mathrm{~mm}$ depending on scanning parameters) puts up a lower limit of dimensions of objects, whose density could be precisely defined. Another limitation for precise density measurement is the presence of noises of different nature. This leads to strong decrease both in repeatability of results and in reliability of density measurements in areas below $10 \mathrm{sq} . \mathrm{mm}$. Density measurement is precise and repeatable only in an area above 40 sq.mm. Densitometry for objects below $5 \mathrm{~mm}$ as well as in the periphery of objects is connected with a strongly increasing error. Conclusions: The described densitometric limitations in computed tomography set up the problem of differentiating visualisation abilities in medical imaging (spatial and contrast resolution) from metric abilities of methods and apparatuses. In this connection we put forward a new criterionmetric resolution-reflecting the lower limits of precise quantitative measurements in medical imaging.

\subsection{PM}

713 Three-dimensional volumetric assessment of bone structure by quantitative computed tomography

Claus-C. Glueer, Michelle J. Estilo, Kenneth G. Faulkner, John D. Rosin, Harry K. Genant; San Francisco, CA

Purpose: Quantitative Computed Tomography (QCT) of the spine is an established method for accurate diagnosis of osteopenia. The abilities to predict fracture risk in the individual patient and to assess bone structure, however, are limited. We are developing a 3-dimensional (3-d) volumetric QCT approach to obtain complete 3 -d structural information of bone density both at the spine and the proximal femur.

Methods: Software routines added to our QUIET (Quantitative Image Evaluation Technique) image processing system allow for 3-d assessment of bone structure. These include the generation of object coordinate systems (OCS), 3-d volumes of interest, and subtraction images. The OCS is based on anatomic landmarks such as the vertebral endplates, the center of the femoral head and the femur shaft axis.

Results: Spine and femur imgages on paraplegic patients following a volumetric QCT scanning protocol (GE $9800,80 \mathrm{kVp}, 140 \mathrm{mAs}, 3 \mathrm{~mm}$ contiguous slices) showed residual spatial repositioning errors of about $1 \mathrm{~mm}$. Using their respective OCSs and 3-d image transformation techniques images of follow-up measurements can be aligned to the baseline image.

Conclusions: Volumetric QCT yields 3-d information about the bone mineral 
density of the spine and the proximal femur. The results suggest adequate positioning accuracy for in-vivo QCT studies.

\section{$2.35 \mathrm{PM}$}

714 Determination of iodine concentration in the thyroid tissue: Value of CT

B. Mayr, A. Seebauer, B. Leisner, R. Kantlehner; Munich

Purpose: Opposed to single energy quantitative computed tomography (SEQCT) dual energy quantitative computed tomography (DEQCT) allows to quantify thyroid iodine concentration with the aid of material-selective image calculation.

Methods: 39 thyroid specimens were examined by SEQCT and DEQCT. X-ray fluorescence method was used as gold standard.

Results: DEQCT provided a reliable calculation of iodine concentration in the thyroid tissue $(r=0,922)$, while in SEQCT the correlation was poor $(r=$ $0,53 / 0,64)$. The reproducibility of DEQCT was limited in thyroids with a low iodine concentration.

Conclusions: Only using dual energy CT allows a reliable calculation of the iodine concentration in the thyroid. But this method is limited in the exact measurement of low iodine concentration in the thyroid tissue.

\subsection{PM}

\section{Characterization of DXA measurements in a multi-center clinical} drug trial

Kenneth G. Faulkner, Claus-Christian Glueer, Harry K. Genant; San Francisco, CA

Purpose: The performance of several Lunar DPX and Hologic QDR-1000 series dual energy $\mathrm{x}$-ray bone densitometers is determined as part of a multicenter clinical drug trial.

Methods: A spine phantom designed for DXA quality assurance were sent to ten different bone densitometry centers, seven equipped with Hologic QDR-1000 or QDR-1000/W scanners and three with Lunar DPX systems. Both phantoms were scanned five times, and the short term coefficient of variation $(\mathrm{CV})$ was determined. Longitudinal data were obtained from several patients at each center and compared. In vivo precision was based on the standard error of the estimate from a linear regression for longitudinal studies performed on the same patient.

Results: Precision of spine BMD in vitro was the same for both manufacturers (Hologic $\mathrm{CV}=0.47 \%$, Lunar $\mathrm{CV}=0.45 \%$ ). $\mathrm{CV}$ in vivo of the spinal $\mathrm{BMD}$ measurement varied between 1 and $3 \%$. Phantom data indicate an offset in BMD between the two scanner types of between 10 and $20 \%$.

Conclusions: Longitudinal variations in patient measurements exceed the variations between machines for both systems. Due to differences in calibration, comprehensive quality control programs are needed in order to compare and pool from different systems. We recommend the establishment of an absolute standard for DXA calibration to standardize BMC and BMD measurements.

\subsection{PM}

716 Brain tumour, surrounding edema and regional blood flow, assessed by the stable xenon CT method

Dirk van Roost, László Solymosi, Alexander Hartmann, Christine Bim; Bonn

Purpose: In 15 patients with a brain tumour with marked surrounding edema, we measured the regional cerebral blood flow using stable xenon-enhanced computed tomography.

Methods: Assessing the images, we distinguished the following regions of interest: 1. the vital appearing tumour parts, 2. the necrotic appearing tumour parts, 3. the tumour in its entirety, 4. the surrounding brain edema, 5. the entire tumour-bearing hemisphere, 6 . the contralateral hemisphere, 7. the cortical rim of the contralateral hemisphere.

Results: We found significant differences of blood flow between the necrotic and vital appearing tumour parts, between the tumour in its entirety and the surrounding edema, and also between the tumour-bearing and the contralateral hemisphere. Moreover, the blood flow of both the contralateral $(26,2 \mathrm{ml} / 100 \mathrm{~g} / \mathrm{min})$ and the tumour-bearing hemisphere $(20,9 \mathrm{ml} / 100 \mathrm{mg} / \mathrm{min})$ significantly stayed below the mean value of normal persons of the same age group.

Conclusions: We consider the following possible reasons for the reduced regional blood flow: 1. an autoregulation disturbance, 2. a latent or evident increase of intracranial pressure, 3 . cytotoxic mechanisms, 4 . a diaschisis and 5 . a broader value scattering because of the older mean age of the patients.
3.05 PM

717 Interactive interventional imaging - a new approach to stereotactic CT-guided whole body intervention A. Jacob, G. A. Horstmann, H. F. Reinhardt, W. Steinbrich; Basel

1. Development of an interactive free-hand whole body stereotaxy system.

2. The system consists of 4 components:

2.1 An orthogonal assay of three ultrasound generators.

2.2 An assay of two to four receiving microphones in the interventional instrument.

2.3 An automated computer system, which measures the different sound travelling times from generators to microphones and through matrix transformations calculates the coordinates of the instrument's tip and transfers them to 2.4 the graphical workstation (i386 based). This computer holds a stack of previously recorded CT-slices and picks the appropriate for display. It overlays a crosshairs on this image at the very position of the instrument's tip, providing immediate visual feedback.

3. The system has been successfully used in about 20 cases of open brain surgery. We will report on the first 20 interventional procedures carried out. 4. We believe that this system has the potential of redefining the state of the art in interventional CT through

- visual feedback of needle position during puncture

- working at any off-plane angles,

- accelerating and objectifying the art of puncturing without bringing along much of an overhead.

Right now the system is being transferred to an SUV environment for real-time 3-D-display and inclusion of MR-images.

\subsection{PM}

718 Quantitative Computed Tomography (QCT) in the evaluation of bone mineral content (BMC) of lumbar spine in workers professionally exposed to whole body vibrations: preliminary experiences

G. Guglielmi, N. Zarrelli, G. M. Giannatempo, T. Scarabino, M. Cammisa; San Giovanni Rotondo

QCT can selectively measure the trabecular centrum or spongiosa of vertebral body. The contribution of other mineralized tissues can be excluded using this method. A slice 2-10 mm thick is taken through the centre of the 1st, 2nd, 3rd and 4 th lumbar vertebra parallel to the endplate in the lateral position. An elliptical region of interest in the anterior part of vertebral body is then chosen. Scanning is done with reference solution of known mineral concentration. The Hounsfield units, a measure of attenuation, of the reference solutions are plotted against their known concentrations providing a standard curve. Bone mineral density, mass $(\mathrm{mg})$ per unit volume $\left(\mathrm{cm}^{3}\right)$ is thus obtained. From June 1989 we employed this technique in 15 building workers occupationally exposed to whole-body vibrations. They all were males, aged less than 60 years and working for more than 15 years. In all studied cases the value of BMC was lower than the standard values, related for age and sex, while the standard $\mathrm{x}$-ray was essentially normal, because it can show an osteopenic condition only when almost $30-40 \%$ of bone mass is lost. Therefore we suggest to employ the non-invasive technique QCT for the measurement of $\mathrm{BMC}$ to early evaluate the bone loss and select the risk condition for bone fractures.

\subsection{PM}

\section{Organ doses and effective dose equivalent in computed tomography}

K.A. Jessen, J. Juul Christensen, J.Jørgensen, J.Petersen, E.W. Sørensen; Århus

A nationwide survey has been conducted in Denmark to establish frequency and technical parameters adapted in each type of CT examinations on the facilities in operation primo 1989. Corresponding measurements of doses, free-in.air, at the axis of rotation, for a single slice for the most commonly used sets of scanning parameters by TL dosimeters. Conversion factors given by GSF and by NRPB relating the organ doses to the measured doses are used for calculation of organ doses. The two sets of conversion factors have been compared and found to match for most organs. Differences in the pre-patient filtration give rise to a factor $2-3$ between conversions factors for different scanner models which makes it necessary to know the filtration for each scanner type to achieve a resonable estimate of the organ doses and Effective Dose Equivalent (EDE). The collective EDE from CT in Denmark in 1989 is estimated to 225 man mSv giving $0.045 \mathrm{mSv}$ per caput or an average of $3.1 \mathrm{mSv}$ per examination. The largest contribution from ex. of abdomen. 
720 Possibilities for using artificial intelligence in radiology E. Jugel; Tübingen

In the whole it can be said that computer-controlled pattern recognition in $\mathrm{X}$-ray images is still in its beginnings. This is shown by the small number of published reports, which, moreover, are distributed over the whole world. The goal of computer-controlled pattern recognition in X-ray images is to enhance performance and eliminate errors. The limited ability of the human eye to differentiate between shades of grey is pointed out in the paper. In this field computerized tomography has permitted great advances. Interestingly, however, it is precisely in this sector that no works on pattern recognition have been published as yet. They do exist in the field where pattern recognition is the most difficult methodologically: the X-ray image. This image must first be transformed into a form allowing electronic processing: the digital X-ray image only then can the number of shades of grey to be made visible be essentially increased by appropriate choice in the respective window. Thus, the actual extent of bone resorption in parodontosis can be established. Conceivably, hidden bone metastases might be detected. (orig.)

\section{Room R}

\section{SS}

16.07 Intraoperative external radiotherapy and new modalities of interstitial radiotherapy II

\section{$2.00 \mathrm{PM}$}

\section{Technical controversies in IORT protocols (Keynote Lecture) J.P. Gérard, I. Sentenac; Lyon}

With more than 7000 patients treated in the world IORT is becoming a popular technique for local treatment of cancer. In fact many technical differences exist among the various systems of IORT. The aim of IORT is to deliver a homogeneous single dose to a volume well delineated by an applicator. Usually and at best this dose is delivered with electrons. Depending on the technical facilities quite large variations can be seen in the final outcome of dose delivery.

- The actual dose given in the treated volume on the $90 \%$ isodose can vary by 10 to $25 \%$ (either underdosage or overdosage).

- The volume of this $90 \%$ isodose can vary by 10 to $30 \%$

- The dose given to the normal tissue close to the collimator can vary by 10 to $30 \%$.

The differences can be due to the type of applicator (shape, size, material) the collimating system, the use of a docking or non docking system, the use of a flattening filter, the type of adaptator fixed on the accelerator head. This differences can also influence the safety of the system.

All the differences must be assessed for each individual applicator. A quality control program must be implemented in each institution and international common definition must be used.

\subsection{PM}

722 Surgery and quality control in IORT protocols (Keynote Lecture) H.J. Hoekstra; Groningen

Intraoperative radiotherapy (IORT) involves the delivery of large single doses of electron beam irradiation during surgical procedures to areas of malignant disease. The majority of the patients is treated in phase I or II protocols. Today's clinical experience with IORT is based on these clinical studies as well as experimental canine tissue tolerance studies. A dose of 15-20 Gy IORT is delivered as a radiation boost to tumor masses in combination with wide field external beam irradiation (EBRT) of 40-50 Gy. In IORT single or multiple fields are used.

The surgical approach for IORT procedures requires more extensive incisions and exploration than routinely performed for comparable malignancies. The morbidity of these extensive surgery together with IORT is equivalent to surgery and conventional EBRT.

Phase I and II IORT studies for advanced gastrointestinal malignancies showed an improvement in the local control rate without an increased treatment morbidity, warranting the design of cooperative, EORTC and RTOG, Phase III IORT studies. Quality control of the surgery and radiation therapy is essential in these protocols to establish the final place of this experimental treatment modality in the treatment of cancer.
2.40 PM

723 Photodynamic therapy - a new dimension in radiotherapy (Keynote Lecture)

G. Alth, R. H. Jindra, G. Koderhold, H. Koren, G. Schenk; Vienna

Photodynamic therapy offers a new principle in therapy based on the effect of non-ionising radiation caused by visible light. It is performed in two steps. Firstly tumor tissue is sensibilised by intravenous application of an haematoporphyrin derivative (Photosan III). Secondly, by irradiation of light (either white or monochromatic) the sensibilized cells are destroyed immediately which effect is in contrast to irradiation by electron or gamma therapy. Interstitiell and superficial therapy was carried out in a clinical phase I trial study therefore restricted to worst cases only. From May ' 88 to February ' 9138 persons were treated. Therapy was performed for cancers of the mamma, gynecological organs, throat-nose-ear and of dermatological ones. In 23 cases side effects were observed during sensibilisation and included nausea (2), shivering (11), nausea combined with shivering (8) and cardiovascular effects (2), drop of blood pressure and tachycardy. Complications after therapy occurred in 12 cases, namely oedema (2), oligurie (1), combustio (3), superinfection (12) and sunburning (2). The results include 17 total remissions, 15 partial remissions, 13 recidives. In 4 cases no effect was observed. Summarily this new therapy offers an efficient alternative to common use treatments and demands also for intensive investigation in clinical and theoretical fields.

\subsection{5-3.00 PM: Discussion}

\section{$3.00 \mathrm{PM}$}

724 IR-192, low dose rate endobronchial brachytherapy in the treatment of malignant airway obstruction

P. I. Raju, T. K. Roy, R. D. McDonald, C. Crim, T. M. Hyers, S. J. Marshall, J. A. Ohar, K. S. Naunheim; St. Louis, MS

Endobronchial brachytherapy (EBT) offers significant palliation in patients with malignant airway obstruction. From $9 / 86$ to $4 / 89,39$ patients had 49 catheter placements. An after-loading endobronchial catheter was placed using fiberoptic bronchoscope under fluoroscopy. 38/39 completed the prescribed EBT; one did not (non-compliant). Diagnoses were: recurrent lung cancer in $38 / 39$; squamous cell carcinoma in $28 / 38(74 \%)$; adenocarcinoma in $8 / 38(21 \%)$; and large cell carcinoma in $2 / 38(5 \%)$; and metastatic renal cell carcinoma in $1 / 39.3 / 39$ had laser surgery $2-3$ weeks prior to EBT. 48/49 treatments delivered a prescribed dose of $20 \mathrm{~Gy}$ at $1 \mathrm{~cm} .10 / 39$ had two implants; one patient received $7 \mathrm{~Gy}$ (non-compliant). Follow-up bronchoscopy was performed in $28 / 39$ patients.

Results: $13 / 28(46 \%)$ had complete response (no visible endobronchial tumor); $12 / 28(43 \%)$ had partial response (tumor still present but lumen opened); and $3 / 28(11 \%)$ had minimal response (lumen slightly opened). Clinically, dyspnea improved in $30 / 37(82 \%)$ of patients; hemoptysis in $17 / 19(89 \%)$ of patients; cough in $31 / 39(79 \%)$ of patients; and pneumonia in $21 / 23(92 \%)$ of patients. No patients were cured. No complications were encountered. 2/39 are alive with disease at 120 and 104 weeks and median survival is 20 weeks (range 4-120 weeks).

Conclusions: This technique is simple and well tolerated and offers significant palliation with no major treatment-related complications.

\subsection{PM}

725 Iridium 192 high dose rate (HDR) brachytherapy combined with external beam irradiation (EBR) in unresectable esophageal cancer B. Pakisch, P. Kohek, G. Stuecklschweiger, F. G. Juettner-Smolle, E. Poier, H. Leitner, J. Hammer, A. Hackl; Graz

We investigated the value of EBR combined with $\operatorname{Ir} 192$ HDR intraluminal afterloading concerning both quality and duration of the palliative effect as well as survival in nonresectable cancer of the oesophagus as compared to Ir192 alone.

A group of 68 patients with functionally or both functionally and anatomically nonresectable cancer of the oesophagus and the esophagogastric junction (T1:3, T2:21, T3:43, T3:1, recurrence:5) underwent intraluminal afterloading therapy (5-7 Gy/session, total dose 5-22 Gy, mean $12.4 \mathrm{~Gy}$ ). In 36 cases the general condition allowed additional EBR to be administered (60 Gy total dose: 2 Gy/day).

Durable satisfactory palliation (intake of at least semisolid food) was demonstrable in 61/68 patients and was maintained by radiotherapy alone until the last follow-up investigation or until death in all but 22 patients, who needed additional dilatation and/or laser desobliteration. The median survival time was 6.5 months for the whole collective (4.9 months without, 10.5 months with EBR).

Only in $\mathrm{T} 1$ and $\mathrm{T} 2$ lesions did additional EBR prolong the 1-year survival rate (66\% vs $17 \%$ without EBR: P 0.05$)$. 
726 The role of intraluminal radiation therapy in the multidisciplinary management of extrahepatic bile duct cancer

T. Kamada, H. Saito, G. Irio; Sapporo

Purpose: Intraluminal radiation therapy (ILRT) of the bile duct is an attractive method in the management of extrahepatic bile duct cancer. This is a retrospective analysis of the treatment result of 92 patients with extrahepatic bile duct tumor who referred for ILRT.

Methods: Between 1983 and 1990, 46 cases were referred for the treatment of their unresectable lesions, 36 for post-operative radiotherapy, 8 pre-operatively, and 2 cases were for post-operative recurrence. Sixty-six out of 92 patients were treated with ILRT with or without external beam radiation therapy (ERT) and the remaining 26 were treated with ERT alone.

Results: Sixty-six patients receiving ILRT had better survival compared with 26 patients receiving ERT alone $(14.7 \%$ vs $3.8 \%$ at 5 years $\mathrm{P}<0.001)$. Five year survival rate of $45.5 \%$ was observed in 11 patients who had high risk of microscopic residual disease after complete resection of gross disease, and 3 year survival rate of $13.3 \%$ was observed in 24 unresectable cases who received ILRT (30 Gy or more) and ERT (50 Gy). Incidence of radiation-induced morbidity was relatively high in pre-operatively treated cases (3 early post-operative biliary tract bleeding), whereas other cases remained low.

Conclusions: ILRT in conjunction with ERT may play a significant role in the multidisciplinary management of extrahepatic bile duct tumor.

\section{Room S}

\section{SS}

\subsection{Teaching concepts and techniques in radiological} education

\subsection{PM}

727 Education in radiology: medical school and post-graduate training in various countries of Europe (Keynote Lecture)

I. Isherwood; Manchester

The Diagnostic Radiologist is a clinician concerned with investigative imaging and interventional techniques and should be called a Clinical Radiologist. Investigative imaging embraces the spectrum of diagnostic procedures from conventional radiology to cellular metabolism. Biological events need not be recorded pictorially. The display may be entirely numerical, graphic or spectral. The analog image is frequently derived from digital data. Computation and biochemical literacy are increasingly necessary for the radiologist. The convergence of diagnostic and interventional methods within Clinical Radiology has consequences for radiological and surgical practice. The educational responsibility for change must be addressed nationally and internationally. Sub-specialisation via general radiological training programmes is currently either technique or clinically orientated. Clinical sub-specialisation has the advantage that all modalities can be applied to problem orientated clinical management.

At under-graduate level the opportunities presented by the non-invasive imaging methods to explore structure and function in normal individuals have important implications for teaching anatomy and physiology. All doctors in training need to understand the role of Radiology in clinical management.

The European Association of Radiology (EAR) is the Federation of 27 National Radiological Societies in Europe, each of whom has individual responsibility for education. Professional definitions, training programmes, the relationships between conventional Radiology, Ultrasound, Nuclear Medicine and Radiotherapy vary widely throughout the Continent. Educational requirements and qualifications to practice are not uniform. The EAR is concerned to seek uniformity of standards and opportunities based on the best radiological teaching in Europe. Current activities in the EAR are directed towards an expansion of continuing education programmes and a consideration of continuing assessment systems.

The challenge for European educational programmes of the future is to seek the benefits of the multi-disciplinary multi-national activities of Radiology whilst avoiding fragmentation.

\subsection{PM}

\section{8 "Picturing the Future": electronic imaging and radiology education (Keynote Lecture)} Anne G. Osborn; Salt Lake City, UT

Rapid advances in computer-based interactive training are changing the face of radiology instruction. This lecture will briefly review state-of-the-art technology, present examples of sophisticated interactive educational software, and - perhaps of most interest to practicing radiologists - demonstrate computer-based aids to diagnosis. An elegant program that has been developed for teaching cranial nerve anatomy, imaging, and pathology will be shown along with preliminary results from its use by radiology residents attending the six-week course in radiologic pathology at the Armed Forces Institute of Pathology in Washington, D.C.

\section{$2.30 \mathrm{PM}$}

729 Computerized documentation for clinical radiological research Michael V. Knopp, Monika Martens, Udo Trost, Hans-Ulrich Kauzcor, Stefan Delorme, Alois Knopp, Gerhard van Kaick; Heidelberg

Purpose: To evaluate the requirements of a general data based concept for radiological documentation while supporting clinical research.

Methods and Materials: After an initial system-analysis of two different radiological departments, detailed specifications of requirements were established. An already developed scientificly oriented medical documentation system, H.A.N.D., was chosen as the data base system and modified to include all specifications. The system can be used in a single user or network environment on any MS-DOS or OS/2 operating system personal computer.

Results: Most radiological information systems or data bases are structured to support the administrative data but are frequently insufficient to support clinically oriented research studies. The essential requirements can be summarized as the following: User friendliness, extreme flexibility regarding the number of patients and data per patient, responsiveness to required tasks, direct interface to word and graphic processing as well as statistical analysis.

Conclusions: A computerized documentation system for radiologically oriented research requires a tremendous flexibility regarding the number of patients as well as data per patient. User friendliness, responsiveness of the data base system to requested tasks as well as portability of the data and software to a portable home or PC are absolute necessities for successful use.

\section{$2.40 \mathrm{PM}$}

\section{Hypermedia software: aid in computerized diagnostic imaging} teaching

P. Pavone, C. Catalano, G. De Gasperis, G. Cardone, A. Agnifili, G. Albertini, S. Giuliani, R. Passariello; L'Aquila

Purpose: Hypermedia formats allow access to different kind of information, including text, images, animation, sound. New hypermedia software (Supercard) also allow acquisition and storage of radiological images with 256 gray levels. Aim of this study was to define the possibility of new hypermedia software in radiological teaching.

Methods: The computer used for this evaluation was a Macintosh II (Apple computer, Cupertino, CA), with 8 Mbytes RAM and 80 Mbytes hard disk.

The computer was connected through an ethernet link to the imaging chain of our PACS system, allowing direct retrieval of images or digitization of radiographic films through a CCD digitizer.

Results: Images, text and free sound explanations were included in the program, to generate a homogeneous teaching program of different areas. The first application was on MR applications in evaluation of chest disease. The MR images had to be converted in TIFF files for manipulation by the Macintosh computer.

The final program was prepared by radiological residents, due to the easiness of the software program.

General acceptance of the program as a teaching aid was obtained.

Conclusions: The new hypermedia software provides a further chance to improve the direct access of images during medical teaching in radiology.

\section{$2.50 \mathrm{PM}$}

\section{Computer assisted instruction in radiology}

William J. Hendrick, Jr., Harjit Singh; Albany, NY

We will demonstrate interactive Computer Assisted Instruction (CAI) modules on chest radiology and bone radiology currently in development. These programs show the advantages of new software for the Macintosh computer in authoring of the image/graphic intensive systems required for Radiology CAI. The preliminary teaching modules are currently employed at our Institution for users at multiple levels ranging from third year medical students to thoracic surgery residents.

The software is being developed on a Macintosh II computer with 4 Megabyte RAM, a hard disk drive, and standard RGB monitor with extended 8-bit video card. We are using the new programming environment, SuperCard ${ }^{\circledast}$ (Silicon Beach Software, San Diego, CA), to create the module.

Direct on-screen user interactions with displayed radiographic and microscopic pathologic images as well as hypertext links to appropriate reference materials in anatomy and pathology are incorporated.

Users may approach the subject matter via an interactive lecture or a case oriented teaching file format. A computer-driven self assessment tests the user's ability to envision three dimensional mediastinal anatomy based on axial images.

Techniques of development including goal setting, project design, hardware and software selection, drafting, product refinement and presentation will be included in the discussion. 
732 A comparison of computer assisted instruction versus conventional instructor-based teaching in diagnostic radiology

William J. Hendrick, Jr., Harjit Singh; Albany, NY

We assessed the efficacy of computer assisted instruction in a standard diagnostic radiology curriculum. One group of students received instruction on CT Anatomy of the mediastinum in the traditional light box/instructor format. The second group employed an interactive computer assisted instruction module developed by the authors, based on the same material. The two groups were then compared using an identical multiple choice examination. The second group was also surveyed on their subjective response to using a computer as the primary medium of instruction.

The results of this comparison demonstrate that computer assisted instruction is an effective method of teaching radiology compared to conventional formats, readily accepted by medical students.

\section{$3.00 \mathrm{PM}$}

733 Monitoring radiologist communication style as an index of performance

Arlene Sierra, E. James Potchen, Mark Bisesi; East Lansing, MI

The quality assurance in radiologic procedures has largely been related to measuring image quality and occasionally observer performance. Once the observations are made, however, it is necessary to communicate the information to a referring physician in order to have the procedure benefit the patient. This process of communication has been studied little in diagnostic radiology. We have used computer based report writing and the Right Writer Program software package to monitor the writing style variance of radiologists as they read different types of radiologic examinations. This has provided an opportunity to understand how differing radiologists respond to different radiologic procedures in their style of communication. These stylistic differences may significantly influence the perception of the referring physician as to the certainty and credibility of the radiologist. Our initial studies suggest that the referring physician's perception of the radiologist's certitude is heavily dependent on the writing style and relatively independent of the radiologist's true certitude as indicated by them when they read the film. This paper will demonstrate the differences in radiologists' performance on different studies and between various radiologists as a quality assurance tool in diagnostic radiology.

\section{$3.05 \mathrm{PM}$}

\section{Professor's work station}

S. Balter, D. Trost, T. A. Sos; New York, NY

The Professor's Work Station is a MacIntosh based system which supports the capture of video fluorographic images, electronic pre-press, optical image archiving, and the production of high quality $35 \mathrm{~mm}$ lecture slides. This system produces text and graph slides as well as annotated captioned images. It is constructed entirely from commercially available hardware and software modules. A presentation graphics camera produces the slides. Captured clinical images are electronically cropped, rotated, grey scale corrected, annotated and assembled. Multiple images can be placed on one slide. The quality of the final slides exceeds that of direct photographic reproduction of hard copy images because of the processing capacity of the system. 
dyskinetic cilia syndrome, cystic fibrosis or allergic bronchopulmonary aspergillosis, CT is effective in appreciation of the extent of bronchiectasis and of the quality of bronchial secretion drainage, guiding effectively the fibroscopic suction of mucoid impaction.

In bronchiolitis obliterans, radiogaphic findings include nodular opacities, alveolar opacities and hyperinflation. In diffuse panbronchiolitis, the changes associate disseminated nodular pattern with lower zonal predominance, hyperinflation and cystic ring shadows. On CT scan, micronodules and branching linear opacities can be accompanied by bronchiolectasis.

GE

\section{1}

735 MR-imaging of the liver, pancreas, and spleen E. Rummeny; Münster

Magnetic resonance imaging of the abdomen has undergone rapid technical maturation. Newer imaging techniques and the reduction of motion-related artifacts with the use of signal-averaging, spatial presaturation, and gradient moment nulling have improved image quality considerably.

This course will review the current clinical applications and possible further developments of MR-imaging of liver, pancreas, and spleen. The sensitivity of MR-imaging in the detection of focal liver lesions and its ability to differentiate between malignant and benign liver tumors will be compared to that of contrast-enhanced CT and other imaging modalities. It will be demonstrated how MR signal-intensity characteristics and morphologic patterns can be used to characterize focal liver lesions quantitatively and qualitatively. The role of special pulse sequences, such as chemical shift and STIR imaging as well as the ability to demonstrate hepatic vascular anatomy will be discussed as important applications of MR-imaging for assessing the resectability of liver neoplasms. The efficacy of MR-imaging will be further increased with the use of new techniques, such as high-speed imaging methods (Turbo-FLASH, Echo-Planar Imaging) and the use of new tissue specific MR contrast agents. The improvement in image quality is likely to expand the present role of MR in imaging of the pancreas and spleen. New applications of MR-imaging including dynamic contrast studies and the use of new paramagnetic and superparamagnetic contrast agents for the diagnosis of pancreatic and splenic diseases will also be presented.

\section{MRI of the retroperitoneum}

Joseph K. T. Lee; St. Louis, MS

The retroperitoneum is a difficult area to examine by conventional radiologic methods. Since the advent of computed tomography (CT) and magnetic resonance (MR) imaging, direct visualization of retroperitoneal organs has disease processes involving the aorta (such as aneurysm) and inferior vena cava (including developmental variation and tumor thrombus) can be diagnosed with the use of MR. Isolated or diffuse lymph node enlargement is clearly depicted. Mature fibrosis may be differentiated from active tumor by MR. Disease processes affecting the psoas muscle as well as primary retroperitoneal tumors can likewise be shown.

The advantages and limitations of MR will be stressed. The relationship of MR to other diagnostic methods - including ultrasound, angiography, lymphangiography and CT - will be discussed.

\section{Room B}

\section{GG}

\section{1}

737 Chronic airway diseases: chronic bronchitis, asthma, bronchiectasis, bronchiolitis

Ph. Grenier; Paris

Chronic bronchitis can not be diagnosed radiographically. However, changes can be observed in 20 to $50 \%$ of patients with chronic bronchitis, and include tubular and ring shadows and prominent lung markings. In case of persistent largely irreversible airway obstruction, chest radiography can detect hyperinflation and saber-sheath deformity of trachea.

In asthma, the incidence of abnormality on chest radiography is considerably influenced by the age at onset of the asthma, its severity, and its constancy. In case of acute severe asthma, the most characteristic radiographic findings are pulmonary hyperinflation and expiratory air trapping. In some patients with chronic asthma, the bronchial walls are thickened, sometimes detectable on chest films in both segmental and subsegmental bronchi, then seen as ring or tubular shadows. The chief indication of chest radiography is to exclude other conditions that cause diffuse wheezing. Radiographic abnormalities which affected management of patients were found in $2-9 \%$ of adults with asthma. Consolidation, atelectasis were both the most frequently complications observed, but pneumothorax and pneumomediastinum can also occur.

For bronchiectasis, chest radiography is able to reveal changes highly suggestive of diagnosis in the great majority of patients. CT can be considered the most reliable non invasive imaging method for assessing the presence, severity and distribution of bronchiectasis. However, although bronchography is an invasive investigation that may cause complications, it should be performed on all patients for whom surgical resection is planned. In case of

\section{Pulmonary emphysema}

P. Bernadac, L. Hennequin; Nancy

Emphysema is defined as an "increase beyond the normal of air spaces, distal to the terminal bronchiole, either from dilatation or from destruction of their walls", "without obvious fibrosis".

There are two radiological patterns of emphysema on the chest pa X Ray - emphysema with vascular deficiency (G. SIMON) of high specificity but of poor sensibility

- emphysema with increased markings (W. THULBLECK) of high sensibility but poor specificity.

The chest X Ray is unable to detect pulmonary emphysema with accuracy.

- CT permits anatomic and density evaluation: the practicable resolution of $200 u$, permits to define the centrilobular area, to affirm emphysema and often to precise its histologic type: centrilobular - paraseptal - bullous - panlobular and irregular emphysema.

- CT precises the topography and the profusion of emphysema

- CT precises the hypoattenuation and the air trapping

- CT is able to quantify, emphysema by anatomic study (point counting, planimetry) and by semi-automatic techniques of densitometry (logiciel EVAV Siemens, SAKAI'S method, MERLET's method).

$\mathrm{CT}$ is valuable for therapeutic indication, longitudinal survey of the disease and detection of associated and complicated forms.

After chest X Ray CT provides a greater accuracy in diagnosis and evaluation of $\mathrm{COBP}$.

\section{Room C}

\section{WS}

\section{4}

739 Endoscopic ultrasonography (EUS) of the GI tract tumors P.J. Valette; Lyon

The high frequency ultrasonic beam brought into digestive lumen permits observation with a great resolution of the digestive wall (visible as a complex five-layers structure) and the neighbouring structures. EUS is now routinely used for assessment of tumors of the esophagus, the stomach or the rectum in different indications:

1. Pre-therapeutic staging of digestive cancer

The principles of interpretation are similar for the upper and lower GI tract wall. The diagnosis of tumor extension is done on the basis of the changes of the parietal layers at the tumor site. The lymphnodes are suspected of invasion according to the shape, the size and the echopattern. The rate of accurate diagnosis of the depth of cancer invasion amounts to $90 \%$. The lymphadenopathies detection rate is $60-70 \%$. The prognosis and terapeutic implications of this technique are already effective, specifically in cases of non-surgical treatment.

2. Diagnosis of large fold gastropathies

Diffuse infiltrative process beneath normal mucosa may be difficult to assess due to frequent negative endoscopic biopsies. EUS enables clear visualization of the destruction of the gastric wall and appears to be helpful in the diagnosis of diffuse lymphoma or linitis plastica.

3. Evaluation of sub-mucosal tumors

Submucosal tumors can be readily distinguished from extrinsic compressions by EUS. This technique also allows measurement of the size and may identify the origin layer of the submucosal tumor permitting an attempt of prediction of the histological diagnosis. However malignant and benign tumors may be of same echostructure.

\section{Room E1}

\section{WS}

740 Radiological management of arteriovenous malformations A. Roche; Villejuif

Abstract available at the information desk. 


\section{The radiological management of arterio-venous malformations} J.Weber; Hamburg

Confusion in terminology and classification means one of the most serious problems in congenital vascular malformations (CVM). Syndromes like Klippel-Trenaunay's and F. P. Weber syndrome have been described before angiography had been developed. Above all noninvasive and new imaging modalities (such as ultrasound, computed tomography and magnetic resonance imaging) angiography is the method of choice for classification and decision in finding an adequate therapy. Arteriography above all has to define and localize arteriovenous fistulae and its hemodynamics whereas phlebography must demonstrate the malformative outflow, its extent and degree of pirmay or secondary functional decompensation. Lymphography is only used rarely in detection of veno-lymphatic and lympho-cystic components of the CVM.

Transcatheter embolization in congenital vascular malformations (CVM) is an alternative or additive method in the curative and palliative treatment of hyperdynamic arteriovenous malformations (AVM). Its indications and its mostly interdisciplinary treatment ought to be managed by teamwork. The safe and adequate transcatheter vasoocclusion depends furthermore on an optimal choice of applicating devices (catheters, guide wires etc.) and suitable embolic materials. Semi-fluid Ethibloc is in our eyes actually the most effective embolizing agent for getting a safe and permanent central occlusion of congenital av-fistulae.

\section{Room E2}

\section{RE}

\section{6}

742 Assessment of osteopenia: basic methods

H.E. Meema; Toronto, Ont.

The most basic method of diagnosing osteopenia is visual assessment of bone mineral density, usually from lateral radiographs of the spine. This has, however, been repeatedly shown unreliable in diagnosis of slight to moderate osteopenia, and its main value is to detect and count vertebral compression deformities, i.e. to assess the severity of structural damage. For detection of earlier stages of developing osteopenia, the best method is radiogrammetry, i.e., measuring the Combined Cortical Thickness (CCT) at a standard site in a peripheral bone. It will be shown that when such CCT measurements are made in the second metacarpal and proximal radius, and the values summed, the summed measurement appears to be as sensitive in predicting vertebral fracture risk as measurements in the vertebrae themselves by quantitative computerized tomography, and even more sensitive than dual-photon absorptiometry of the spine. If mass screening programmes are contemplated for prevention of osteoporotic fractures, such CCT measurements are less expensive than the high technology methods referred to, and since the availability of regular X-ray equipment is nearly universal, the smaller centers and economically less advantaged areas would thus also be able to participate in the osteoporosis screening programmes.

\section{Quantitative computer tomography}

J.E. Adams; Manchester

QCT is unique amongst methods of bone mass measurement (BMD) in providing for the separate estimate of cortical and trabecular bone mass in axial (vertebral body) and appendicular (forearm, tibia) skeletal sites. It is more usually applied to measure vertebral trabecular bone and the mineral content is measured as a true density in $\mathrm{mg} / \mathrm{ml}$ of mineral equivalent material, depending on the calibration phantom used (usually $\mathrm{K}_{2} \mathrm{HPO}_{4}$ in fluid phantoms; hydroxyapatite in solid phantoms). A calibration phantom is required to correct for any scanner instability and beam hardening through the patient. QCT is now available in over 2,000 centres worldwide.

The method is quick to perform and includes a lateral scan projection radiograph followed by four $10 \mathrm{~mm}$ sections through the mid-plane of four vertebrae, generally $\mathrm{T} 12$ to $\mathrm{L} 3$ (10 minute scan time, 10 minute operator analysis time). With automation of selection of scan plane and region of interest (ROI), the time required to perform the measurement is even shorter. Thinner sections $(5 \mathrm{~mm})$ may be required in vertebrae that have wedge fractures, to avoid including endplate in the analysis. As with the other quantitative techniques, consistent method and meticulous care are required in performing the measurements, to achieve the high precision (1-3\%) required for BMD measurements to be clinically useful. For single energy QCT a low dose technique should be used $(90 \mathrm{KVp}, 70 \mathrm{MA}, 2 \mathrm{~s})$, to minimize radiation dose to an effective dose equivalent EDE of $30 \mu \mathrm{sv}$, half the dose of a chest radiograph. Although marrow fat can cause some inaccuracies in single energy QCT BMD measurements which can be corrected by a dual energy technique, this is at the expense of precision and doubling of the radiation dose. Dual energy QCT is not essential to obtain clinically relevant BMD results in the vertebrae.

Appropriate reference data must be available so that patients with reduced BMD and increased fracture risk can be identified. The measurement of BMD has an established role in perimenopausal women, equivocal radiographic features of osteopaenia, patients treated with corticosteroids, and primary hyperparathyroidism. QCT would be an appropriate measuring technique to apply to these categories. Since QCT is the only method which allows for the separate measure of trabecular bone, which is some eight times more metabolically active than cortical bone, it is the more sensitive technique for monitoring changes in BMD with time and in predicting fracture risk.

\section{Role of DEXA in the assessment of osteopenia}

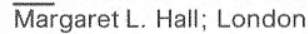

Dual energy X-ray absorptiometry (DEXA) is a relatively new technique for the assessment of osteopenia. The prinicple is similar to that used in dual photon absorptiometry (DPA) only the source of photons is a small X-ray tube. Various machines have been available since 1988 and not only have they been well accepted clinically, they have been financially successful too. Whilst it is often difficult to compare and contrast differing techniques, some of the advantages of DEXA are shown in the table below.

\begin{tabular}{lllll}
\hline & DEXA & DPA & SPA & QCT \\
Precision & $0.9 \%$ & $1-2 \%$ & $1-3 \%$ & $3-5 \%$ \\
Rad. dose & $0.003 \mathrm{mSv}$ & 0.002 & 0.02 (skin) & $2-10$ (marrow) \\
Study time & $<10 \mathrm{~min}$ & $20-30 \mathrm{~min}$ & $10 \mathrm{~min}$ & $20 \mathrm{~min}$
\end{tabular}

With any new method there are initial problems to be overcome and one of these is the non-uniformity of results between systems. This has been partly answered by the development of a European phantom but still requires some work. Future potential and versatility of the technique is likely to outweigh any small initial difficulty. There has been a great advance in the software available and this is likely to benefit all users.

The technique is suitable for all groups of patients requiring bone mineral status assessment. However, the improved precision and speed has opened up new areas such as paediatric and veterinary work with some machines offering rapid scanning or high resolution studies. Screening is made practical by the large possible throughput and ease of use, minimal training is necessary to produce good studies.

DEXA is now an established and efficient method for the assessment of osteopenia. It is to be hoped that the improved clinical interest in osteoporosis will increase the number of patients having accurate bone mineral studies by whatever method possible.

\section{Room L}

RC

\section{Fetal and neonatal hydronephrosis}

$\overline{\text { E. F. Avni, M. Hall, C. C. Schulman; Brussels }}$

Fetal uropathies represent one of the largest group of anomalies detected through obstetrical ultrasound. Their detection has brought many changes in our knowledge of the diagnosis, the natural history, the work-up and the management of these babies after birth. Fetal uropathies can be separated into two groups. Cases of early diagnosis (before 22 weeks gestation) and cases detected later in pregnancy. The first group includes cases with severe anomalies (urethral atresia, Prune-Belly sequence...). An oligohydramnios and pulmonary hypoplasia are usually associated findings. Their outcome is usually rapidly lethal.

The second group, the largest, includes cases in which the diagnosis is made after 30 weeks during routine examinations. The pathology encountered is various: upper or lower urinary tract obstruction, dysplasia, vesico-ureteral reflux.

UPJ obstruction is the most frequent etiology for dilatation of the urinary tract. Diagnosis is based on a renal pelvis dilatation above $10 \mathrm{~mm}$ on a transverse scan.

As for any antenatally diagnosed uropathy, post natal work-up should include an US examination to confirm the anomaly, a VCUG to detect associated reflux and a functional study.

Differential diagnosis includes Multicystic Dysplastic Kidney (MDK) and uretero-vesical junction (UVJ) anomaly.

The diagnosis of MDK is easy in most cases.

Under normal circumstances, a fetal ureter is not demonstrable. Its visualisation in utero implies a U.V. anomaly, (megaureter or reflux).

Another sub-group of uropathies "rediscovered" thanks to antenatal diagnosis is renal duplication.

In the pre-obstetrical US era, very few cases, were discovered in the neonatal period and most cases were ectopic ureteroceles. Nowadays more cases are discovered in the neonatal period equally ectopic ureters or ureteroceles.

It should be stressed that in utero, duplications are usually diagnosed as dilatation without a specific diagnosis. This is of little clinical significance, since all these cases will have a work-up after birth that will clarify the diagnosis. 


\section{Room M}

RE

\section{5}

746 Gynaecological ultrasound - A review

W. R. Lees; London

Institute: Department of Radiology, Middlesex Hospital, London, UK

The benefits and limitations of ultrasound scanning are well understood after 20 years experience. The past few years have dramatically changed the concensus as transvaginal scanning (TVS), Colour Doppler Imaging (DCI) and guided needle biopsy and percutaneous treatment have become routine. Problems of the first trimester of pregnancy are almost exclusively studied transvaginally and are greatly enhanced by DCI to assess viablitiy of intra uterine and ectopic pregnancy. General gynaecological diagnosis involves both conventional and TVS in the majority of cases. Infertility studies are quicker and more accurate with TVS, and CDI is emerging as a useful tool for monitoring ovarian and uterine blood flow through the menstrual cycle.

High speed replay of recorded transvaginal images of the uterus has shown contraction waves of the longitudinal inner myometrial fibres running from cervical canal to cornua - described by Lyons as the "surfing sperm". Variations in amplitude and frequency of contraction are seen and are not only maximal at mid cycle but are suppressed by the oral contraceptive.

Cancer screening, diagnosis and staging are now feasible using all the new methods. Follow up of treated patients and studies of the elderly often require the use of specialised probes, transrectal rather than transvaginal scanning and careful correlation with $\mathrm{CT}$ and MRI. Integrated imaging (including 3D studies) will be the dominant theme of the 1990s.

\section{Diagnostic imaging of the female pelvis: CT and MRI}

\section{B. Hamm; Berlin}

Modern imaging procedures of the female pelvis are endovaginal ultrasound (US), CT and MR imaging. Though these methods are often complementary, each of them can provide specific diagnostic information.

Endosonography has now replaced $\mathrm{CT}$ as the primary imaging technique. However, CT still has its place in screening examinations of the pelvis, such as the search for occult lesions, in the staging of advanced neoplasms, in non-organ-specific imaging, in the evaluation of postoperative complications, e.g. hematoma and abscess, and in the follow-up of pelvic neoplasms after treatment. More recent techniques of pelvic CT include intravenous bolus application of contrast agents and ultrafast scanning.

Completely new dimensions in the evaluation of the female pelvis are opened up by MR imaging. This procedure yields very clear images of both anatomy and pathological processes. A very decisive advantage of MR imaging is the excellent organ specificity. Major indications for MR imaging are uterine malformations, staging of uterine and ovarian neoplasms, and the differential diagnosis of adnexal tumors. The excellent contrast resolution and multiplanar sectioning make MR imaging superior to all other imaging procedures in the evaluation of tumor extension. In the assessment of pelvic and retroperitoneal lymphadenopathy, MR imaging is comparable to CT. Advantages offered by MRI in pelvic examinations include the intravenous application of Gd-DTPA and the use of pelvic surface coils.

\section{Room N}

\section{RC}

\section{7}

748 Imaging of space-occupying lesions of the brain

D. Balériaux; Brussels

Abstract available at the information desk.

\section{Diagnosis and follow-up of intracranial bleeding on MR}

\section{G.Wilms; Leuven}

After extravasation of blood from the vascular bed, a typical sequence of biochemical and physical changes takes place that leads to the typical signal characteristics on MRI. Four stages can be discerned going from hyperacute ( $24 \mathrm{~h}$ ) to acute ( 1 to 3 days), subacute ( 3 to 21 days) and chronic ( $>3$ weeks) hematoma.

In the hyperacute stage no change may be seen. In the acute stage the lesion is isointense on T1 WI and hypointense on T2 WI, due to desaturation of intact red blood cells and formation of deoxyhemoglobin. Deoxyhemoglobin is paramagnetic, due to its 4 unpaired electrons, resulting in T2 shortening. In this stage there is no $\mathrm{T} 1$ effect, since the unpaired electrons are shielded from direct dipole-dipole interactions with water molecules.

In the subacute stage a central hyperintensity appears. This is first visible on T1 WI due to the formation of intracellular methemoglobin, that has $\mathrm{Tl}$ effect due to its 5 unpaired electrons with dipole-dipole interactions with water molecules. After cell lysis the hyperintensity is also visible on T2 WI. Cell lysis eliminates the bulk susceptibility differences, leading to lengthening of T2.
In the chronic stage, a hypointense rim surrounds the hematoma, due to deposition of hemosiderin in the macrophages around the hematoma. In this phase a low intensity centre on T1 WI is present. In the residual stage, only a hemosiderin cleft will be seen on T2 weighted images.

In this workshop these different aspects of hemorrhage in the different stages will be extensively illustrated. These responsible biochemical and physical changes will be explained by presentation of practical diagrams and schemas.

\section{Room 0}

\section{WS}

\section{5}

750 Low field versus high field MR-Imaging. Technical developments in correlation with clinical use: considerations on low field strength MRI Roberto Passariello; L'Aquila

The improvement in magnet design and the use of new permanent magnetic materials has led to increase diffusion of low field MR units. Economical aspects are completely in favour of low field units. We can calculate that even comparing a permanent unit to a $0.5 \mathrm{~T}$ superconductive unit, considering the saving in equipment costs, site preparation, shielding, cryogen consumption, we expect a saving of more than 500,000 US over a 3-year period.

The use of a low field system is also a must for those hospitals or clinics where, for space requirement limitation, installation of a high field unit would be impossible. In fact, the space requirement is $50-60$ sqm for a low field versus $100-150 \mathrm{sqm}$ for a high field, with a fringe field of less than 100 versus $150-250$. Therefore, low field unit can be installed in any floor, including the limited space of a room previously occupied by an X-ray equipment. Downtime of these systems is irrelevant, $5 \%$, versus $14 \%$ of high field unit and this advantage has to be taken into account in the planning.

From the technical point of view, all conventional pulse sequences are available with a low field unit.

Minimum TE is $25 \mathrm{msec}$, because of gradient geometry, and this can account for a reduced T1 weighting of the image. However, T2 weighting can be obtained with shorter TR time, as compared to high field strength. Routine examinations of every organ are possible with high spatial and contrast resolution. Dynamic studies of the heart and joints are also available with cine animation display.

Limitations can be observed during the acquisition of thin slices. However, the use of increased NEX can overcome this problem.

Although ultrafast imaging will present some limits in low field strength systems, the acquisition of MR angiography images has been already achieved with slightly lower resolution, but with clinical relevance. In conclusion, our opinion is that low field MRI systems are satisfactory for routine MRI procedures, such as are needed in clinically oriented facilities.

Research oriented centers, which intend to investigate the feasibility of ultrafast imaging, diffusion imaging or spectroscopy, have to acquire a high field strength unit.

751 Low field versus high field MR-imaging. Technical developments in correlation with clinical use

Georg M. Bongartz; Münster

Beside economical aspects, the spectrum of investigations that can be performed, the differences in image quality, and the different sensitivities to several artifacts are considered in the decision between high or low field strength.

Since the signal to noise ratio is improving with field strength, high resolution imaging enables access to smaller structures like in neurological or orthopedic applications. The optimized signal also supports the performance of gradient echo imaging sequences especially when 3D data sets are acquired. Various clinical trials deal with the benefits of gradient echo sequences as the versatile contrasts, the improved resolution, and the speed of the investigation, compared to standard spin echo imaging.

By improved separation of fat and water peaks with high field strength, frequency-selective saturation enables fat-selective or water-selective imaging, respectively.

The enhanced chemical shift in high field strength imaging require compensation by strong gradients. Motion artifacts dramatically increase with gradient strength and thus limit the MRI results in thoracial or abdominal imaging that require sophisticated compensation regimes like gradient motion rephasing or spatial presaturation.

Ultrafast imaging is able to freeze physiological motion in the chest or abdomen and therefore give access to the heart, the major vessels, and the abdominal organs. The drawback to date is the still inadequate resolution. MR-spectroscopic analysis require a sufficient field strength to separate the metabolic peaks in vivo. Spectroscopy of pathological tissue mainly refers to the evaluation of therapy response in follow up studies than to primary tissue characterization 


\section{Room R}

SS

\subsection{Intraoperative external radiotherapy and new} modalities of interstitial radiotherapy III

4.00 PM

\section{MRI of tumor perfusion under photodynamic therapy}

M. Naegele, F. Gamarra, W. Lumper, A. E. Goetz; Bonn

Purpose: Tumor blood flow reduction after photodynamic therapy (PDT) is considered a relevant mechanism for effective tumor therapy. To study the effects of PDT on the perfusion of solid tumors we measured the signal intensity (SI) behavior of PDT-treated and control tumors at MRI before and after intravenous injection of Gd-DTPA.

Methods: Tumor cells of an amelanotic melanoma were injected subcutaneously into 18 Syrian Golden hamsters at two sites in a dorsal skin fold. At day six of tumor growth (diameter, 5-6 mm), one tumor was treated with PDT-HpD. The second tumor served as an intraindividual control. 24 hours after PDT, MRI of both tumors was performed before and after injection of Gd-DTPA. SI were measured in both tumors and in the dorsal skin.

Results: PDT-treated tumors noted a small increase of SI after Gd-DTPA was given in comparison to the baseline values. Every control tumor showed an increase of SI significantly greater than that of the corresponding PDT-treated tumor.

Conclusions: MRI with Gd-DTPA seems to be a clinically applicable and valuable method to control the reduction of tumor perfusion after PDT.

\subsection{PM}

753 Artificial carriers for radioisotopes "in vivo": tumor selectivity of MAB-labelled liposomes containing 131-lodine

G. Rombi, F. Cossu, G. Melis; Cagliari

Radioisotopes alone or combined with some tumoricidal drugs could be vehicled by using artificial carriers. The purpose of this study is the "in vivo" demonstration of MAB labelled liposomes ability to achieve a selective tumor targeting. Patients affected by colo-rectal, esophageal and pancreatic tumors (CEA and CA 19-9 positives) have been infused with mab-labelled liposomes containing 131-I. The liposome composition was DPC, Cholesterol and 0.6 mol of SPDP modified DPE and monoclonal CEA/CA 19/9 (Fab') 2 $200 \mathrm{gr} / \mathrm{ml}$ with $8: 1 \mathrm{MAB}$-liposomes ratio.

The patients infusion was carried out by using a $100 \mathrm{ml}$ solution containing a final MAB concentration of $15 \mathrm{mg}$ and 131 Iodine with a total activity of 1.5 $\mathrm{mCi}$. The images obtained after scintigraphy have been tested in orderto evaluate 131 Iodine tumor uptake 12-48 and 96 hrs after liposomes infusion. The factor investigated are closely related to the infusion-time. Moreover the calculated enhancement ratio of the Iodine uptake by using MAB-labelled liposomes is 40 times higher than using standard iodinated MAB

The results obtained show that a very selective targeting is possible using this technique to vehicle radioisotopes or drugs

\subsection{PM}

754 Quantitative estimation of therapeutic effects of photodynamic therapy (PDT) during treatment

R. H. Jindra, G. Alth; Wien

Photodynamic therapy is based on the excitation of a photosensitizer which is accumulated in tumor cells by visible light. In contrast to high energy irradiation the effect of photodynamic treatment immediately occurs. Moreover, interaction between light and tissue is more complicated than in the case of ionising radiation because biological tissue is a turbid medium for light. Both prepositions demand for measurement of the progress of photodynamic therapy effects. Since biological tissue provides enlarged absorption and diffraction for light in the visible range sensor mechanism has to be very sensitive, power in the range of picowatt should be detected. Due to inhomogeneities of tissue no curve for constant illumination may be constructed but in observing time dependant changes of power modulation of light information about destruction of tumor cells both qualitatively and quantitatively is presented. Estimation of results of photodynamic therapy in course of treatment are shown. Examples are taken from superficial treatment as well as from interstitial therapy. In addition the results are compared with clinical observations.

\subsection{PM}

755 Optimized stereotactic interstitial neurobrachytherapy for malignant brain tumors: preliminary report Hong W. Chin; Shreveport, LA

Purpose: To improve the survival results and performance status of patients with malignant gliomas, using optimized stereotactic interstitial neurobra- chytherapy (OSINB), external beam radiation treatment (EBRT), with/without debulking surgery (DS).

Methods: Meticulous technique and sophisticated computerized planning programs were developed to optimize implantation dose to adequately cover the entire tumor volume for high dose interstitial Ir-192 boost irradiation $(-40$ Gy). There were two groups in our study; Group I (inoperable patients): OSINB + EBRT (60 Gy), and Group II (operable patients): DS + OSINB + EBRT (60 Gy).

Results: There were no failures (death) as of this report. The treatment response was excellent and good control of the tumors evidenced by PET studies. Three patients in Group I are alive at 24,23 , and 16 months, and the remaining three patients in Group II are at 22,21 , and 11 months.

Conclusions: We are reporting extraordinarily excellent results on the first six malignant brain tumor patients of our study who were treated with optimized implantation technique. Comprehensive analysis of our neurobrachytherapy technique with excellent results is presented.

\subsection{PM}

756 Esophageal and cardial tumors treated by intraluminal Cs-137 irradiation and chemotherapy

Zoltán Vigváry, András Kónya, Ernö Makó; Budapest

168 patient (pts) - mainly with inoperable lumen restricting esophageal and cardial tumors - have been treated since 1984-1989. They obtained Cs-137 intraluminal irradiation with after-loading method. The irradiation was performed with CURIETRON by the help of flexible nylon applicators. The dose delivered was $50 \mathrm{~Gy}$ in 3 or 4 fractions, protracted to $24 \mathrm{~h}$. For setting up correct diagnosis, esophagoscopy, radiography and CT examinations were carried out. In all cases relief of dysphagia and increasing weight was achieved. In 110 cases significant regression could be observed. In cases with extended tumors or distant metastases the treatment was completed by chemotherapy. 16 pts were medicated with a triple-drug regimen (VCR + BLM + MITO-C) and 13 pts with CDDP+BLM+MITO-C. When irradiation was used only the average survival was 10,6 months, in the range of combined treatment 13,0 months.

\subsection{PM}

757 Radiation therapy in combination with intraarterial selective chemotherapy

D. Bilenjki, L. Lincender, B. Bilenjki; Sarajevo

Since 1984, the intraarterial selective applications of cytostatics (IAC) in combination with radiotherapy (EBT) have been performed.

Indications for use of this combined therapy are T3 and T4 tumors of small pelvis, postoperative recidives and as a primary approach in highly riskful radioresistent tumors (radiosensibilization).

Target volume and relation to the surrounding structures are evaluated by CT and angiography. The effects of the therapy are evaluated by radiologic methods.

Seldinger technique is used for catheter insertion for the continuated application of cytostatics. (Platinol, MTX, 5-FU).

The authors have included 120 patients in the study and presented the results of this combined therapy (IAC + EBT). The objective remission is achieved in $46 \%$ of cases. The mean survival is 32 months. The life quality (pain relief) is improved in $90 \%$ of cases. Toxic reactions are neglectable, the method acceptible and applicative. The mean hospitalization 18 days. Cost effect - satisfactory.

\subsection{2-5.30 PM: Round Table Discussion with Keynote Speakers}

\section{Room S}

\section{WS}

\section{4}

Education in radiology

758 Survey of training in radiodiagnosis and medical imaging in Belgium

A. L. Baert; Leuven

1. Undergraduate teaching (medical students)

Courses in diagnostic radiology as well as formal examinations are compulsory at every university.

During the clinical years, a total number of teaching hours varying between 20 hours (min.) and 65 hours (max.) is programmed.

In addition optional tutorials of varying length are available at some universities.

During the preclinical years, radiological anatomy is included in the curriculum at 6 of the 7 universities. The number of teaching hours varies between 6 and 37,5

2. Postgraduate teaching 
Postgraduate training is nearly exclusively organised by the 7 university departments of radiodiagnosis and medical imaging in collaboration with radiological departments in regional hospitals. All radiological departments who train future radiologists need an official accreditation from the Ministry of Health.

2.1. Requirements for entry

A selection procedure, varying from one university to another, is applied. It is mainly based on: the levels and degrees obtained during the preclinical and clinical years of the undergraduate curriculum, optional tutorials as well as on an individual assessment of the general "profile" of the candidate such as personality, motivation, etc.

2.2. Training

5 years compulsory training is required. Besides the practical training "on the job" each future radiologist needs to attend, on a regular basis, a comprehensive theoretical teaching programme of radiology during the duration of his training. This training includes basic radiological training as well as a minimum training in subspecialties such as neuro-, vascular-, and pediatric radiology,

There is no compulsory national board examination at the end of the training period. Each university training center of radiology is responsible for an adequate assessment procedure, the modalities of which vary from one university to another.

There is no official state diploma nor certificate delivered to the radiologist who has successfully finished his training. However, to be accredited for reimbursement by the social security, the radiologist needs the approval of a ministerial committee responsible for evaluation of his or her professional qualification.

2.3. Continuing education

Continuing education is entirely on a voluntary basis. No credit systems are needed for keeping the licence for reimbursement.

\section{Education in radiology}

M. Bellet; Brest

Abstract available at the information desk

\section{Medical school and postgraduate training in Italy}

\section{Carissimo Biagini; Roma}

\section{Undergraduate teaching}

A new teaching model ("Table 18") was provided in 1986 by the Ministry of University, with classified teaching subjects, subdivided in 18 areas for 5500 teaching hours in 6 years. Number of hours per single area compulsory, introduction of the teaching subjects, and hour distribution per subject decided by the faculty. Pre-clinical cycle: radiological anatomy included into area 2 ("human morphology"). Health physics included in area 4 ("integrated biological functions"). In clinical cycle, autonomous area 17, "diagnostic imaging and radiotherapy" includes diagnostic radiology, radiobiology, radiotherapy, nuclear medicine, clinical radiological anatomy. Total number of hours 100. Supplementary teaching hours of radiological interest are neuroradiology in area 10 ("neurosciences"), and radiation oncology in area 12 ("clinical medicine"). Examination for the assessment of radiological knowledge is planned per area.

2. Postgraduate teaching

Recruitment for postgraduate schools of radiology is per public contest in single faculties with a "programmed number" per school. Length of basic training four years. Teaching times and practical applications combine 400 hours per year, 1600 in four years. Assessments of knowledge are provided per year with a final board examination. Different degress in the same school (with first year common) for diagnostic radiology and for radiation oncology. Separate schools for nuclear medicine. No certification for sub-specialties. A new statute is in now in consideration, with the possibility of differentiate schools with official certification for interventional radiology, neuroradiology, and paediatric radiology. Fully separate schools are proposed for diagnostic radiology and radiotherapy,

3. Continuous assessment

A voluntary model of refreshment courses (APC or "Aggiornamento Professionale Continuativo") with a system of credits attributed per year, was organized by Italian Association of Radiology starting from 1980.

\section{Education in radiology: medical school and postgraduate training in Sweden}

Uno Erikson; Uppsala

The medical education is composed by basic medical studies for 24 months, clinical studies for 42 months, rotating internship for 24 months, totally 90 months or 7,5 years.

Then the education for speciality take place and until the last of June 1992 it consists of diagnostic radiology for 4 years, elective e.g. surgery, internal medicine for 0,5 years, totally 4,5 years.

During the period of 4,5 years the radiologists have to attend six one-week long board certifying courses in diagnostic radiology. At the end of each course a written and/or an oral examination is given.

There is no compulsory board certification examination but the Swedish Association of Medical Radiology has since 1987 arranged such an examination twice a year but with a relatively few number of participants.

From the first of July 1992 the medical faculties will have an increased responsibility and courses for the young doctors applying for speciality will be given by the universities. The time for training at hospitals will be 5-7 years. Political and economical considerations threaten the quality of the training.

Each department of diagnostic radiology at the universities will have to take care of the doctors who intend to be specialists and to give them a personal training program with a certain number of courses which, however, are not compulsory.

It is an important task for the universities to take their responsibility for these courses.

\section{Radiological education in Switzerland \\ W.A. Fuchs; Zürich}

Undergraduate teaching of medical students comprises radiology related to anatomy and pathology, basic principles of radiology and radiation protection, fundamental concepts of interpretation in radiodiagnosis of the chest and extremities. The role of imaging including nuclear medicine in the clinical management of patients is explained. Indications and therapeutic results of clinical radiooncology are presented. A final oral examination ensures quality control.

Postgraduate education is divided into the three radiological specialities radiodiagnosis, nuclear medicine, radiooncology.

The curriculum comprises four years of training in radiology and one year of clinical training or pathology.

The accreditation is based upon board examinations organized by the Schweizerische Gesellschaft für Medizinische Radiologie und Schweizerische Ärztegesellschaft and includes the following specialty and subspecialty boards:

- national board examination in general diagnostic radiology. Specialist radiological certification in pediatric radiology and neuroradiology

- national board examination in nuclear medicine

- national board examination in radiooncology

Continuing Assessment

The postgraduate education takes place on a voluntary basis. Credit systems are currently being discussed.

\section{The conditions of education in radiology in the former GDR M. Lüning; Berlin}

The present rules are valid in the new states of the Federal Republic of Germany during an interim period. These rules will continue to apply for different lengths of time in the various states.

1. Undergraduate teaching:

Under the subject medical physics (first and second semesters) the following is covered: basic physics of imaging diagnosis (including ultrasound and magnetic resonance).

75 hours of radiological training (60 lectures, 15 seminars), 32 hours of radiological diagnosis including sonography, radiobiology and radio-protection, 10 hours each of nuclear medicine and radiotherapy, and 8 hours of oncology. Diagnostic radiology in the 7 th semester consisting of 30 hours. Content: radiology (including interventional radiology and neuroradiology).

For stomatology there is a radiology lecture of 14 hours. Practicals and demonstration on diagnostic radiology in the form of seminars with groups of 10 to 12 students (15 hours)

Finally the student undergoes an oral examination on theory of radiology and $\mathrm{X}$-ray film analysis.

2. Postgraduate teaching

Specialization on radiology in the former GDR has been divided since 1986 into the following:

- specialist for radiology-diagnosis

- specialist for radiology-therapy

- specialist for nuclear medicine.

The time for completing specialization is 4 years. The doctor is constantly under the supervision of a specialist. In universities the specialization involves 5 years, during the 5 th year the candidate works in a different clinical department (e.g. internal medicine, surgery, pathology).

Before a doctor is permitted to appear for the radiology examination he has to undergo the following training:

- 24 months radiodiagnosis

-4 months sonography (with certificate)

- 3 months computed tomography

-2 months pediatric radiology

- 4 months angiography, interventional radiology, lymphography

- 1 month mammography

- 1 month neuroradiology

Compulsory courses (each consisting of one or two weeks):

- physics and technology 
- radiobiology, radiotherapy

- nuclear medicine

- course on radio-protection

Further subspecialization (e.g. pediatric radiology) is possible by doing a so-called "additional specialization" (consisting of 3 years).

The additional specialization of radiology-radiotherapy involves at least 3 years of radiotherapy and 1 year of radiodiagnosis, specialization in nuclear medicine involves 30 months, in addition 6 months of clinical internship, 4 months of radiodiagnosis, 3 months of sonography and 1 month of radiotherapy.

Finally the candidate has to pass the examination held by a commission.

3. Continuing assessment of radiologists

Various programs are offered by universities and medical centers, by academies for medical advanced training and the medical-scientific (regional) societies.

\section{Undergraduate medical education in the United States}

Anne G. Osborn; Salt Lake City, UT

This of the program will review the participation of radiologists in undergraduate medical education in the United States including format and content of typical programs. Current status of radiology residency training as well as subspecialty program accreditation and individual certificates will also be presented.

\section{Education in radiology}

\section{P.E. Peters; Münster}

Since it is impossible to cover all aspects of education in radiology I will concentrate my short statement on the final assessment of postgraduate training.

In Germany, at the moment, the final examination consists of an oral test of about 60-90 mins duration. In the light of a united Europe in 1992 there is urgent need for harmonization of the entire process of postgraduate teaching in radiology. The bottom-up solution to that problem would ask for comparable standards in training programs, however, this might take years given the existing disparities.

Thus, the top-down approach is probably better suited to obtain at least mutually acceptable examination results in different European countries.

For a time period it is conceivable that NATIONAL and EUROPEAN examinations coexist, though, mutual reciprocity of final examinations should be the ultimate goal.

Who could possibly set the guidelines for this process of harmonization except the AER? In analogy to the USA and the UK the foundation of an European college of radiology under the roof of AER could provide the organisational base. My questions to the distinguished colleagues at the round table will be: 1. Do you favour an European diploma of radiology (F.E.C.R.) or mutual reciprocity of national examinations?

2. Do you favour European training courses in radiology even if the process of harmonization is slow (as expected)?

3. Do you favour European training courses for teachers in radiology - Teach the teachers how to teach?

4. Can you agree that these courses are to be given in English?

\section{Undergraduate teaching - postgraduate teaching - continuing assessment}

H. Pokieser; Vienna

Statements concerning the three afore mentioned questions:

1. Undergraduate teaching

Basic knowledge of anatomy, physiology, radiophysics, radiobiology and pathology will be conveyed in the course of 30 hours in each preparatory subject.

Before the clinical section there will be a 60-hour compulsory teaching program and 4 hours of practical training. At the end of this there will be a compulsory examination. The examination intends to test theoretical knowledge of radioprotection, radiobiology, basics of imaging diagnosis and radiotherapy (methods, indication, informing the patient, cooperating with the specialist).

Further, the practical ability of the student to recognize acute and frequent diseases on the basis of standard $\mathrm{x}$-ray films is also tested.

During the two-year clinical section the student is free to participate voluntarily in various teaching programs in all subjects.

2. Postgraduate teaching

Requirements for entry into radiology is a one-year clinical internship including a minimum of 3 months of internal medicine.

The four-year basic radiological training includes all aspects of radiological diagnosis with recommended periods of training in each department. Three months of nuclear medicine and 6 months of radiotherapy are compulsory. Each section of the postgraduate training has to be certified by the chiefs of the officially authorized departments. Certification of specialization is issued by the legal professional authorities, without prior examination. Additionally, there is a two-year training program for radiotherapy and nuclear medicine respectively.

In all subspecialities the training guidelines are in the process of being changed, these propose to introduce a clear division in the training regulations for diagnosis, radiotherapy and nuclear medicine.

3. Continuing assessment is voluntary

A credit system does not exist. A large number of teaching programs are offered in areas of dense concentration, e.g. Vienna.

\section{European diplomas}

\section{J.P. Tessier, Ph. Peetrons, L. Jeanmart; Villejuif}

Some questions: Are European diplomas useful? Are they possible? other question: What diplomas?

Actually, an enquiry of 1989 shows there are controls by annual or continuous examinations in 6 countries, final examinations in 10 countries, Theses in 2 countries (see tables). All countries have final certification.

Diploma will not be recognized by medical, academic or political powers. But they can be probably a mark of quality in many countries.

What diplomas:

General radiology diploma has been forsaked but a new formula is possible. The creation of diplomas by organs or specialites is too difficult. Except neuroradiology or radio-pediatric.

On the other hand diploma of technique are easier to imagine $=$ CT, M.R.I., sonography interventional.

One diploma had been created in 1986: the diploma of sonography organised by the education commission (L. Jeanmart, J. P. Tessier and Ph. Peetrons). The results of this diploma are:

\begin{tabular}{|c|c|c|c|c|}
\hline \multirow{6}{*}{1986} & & Inscrits & Recus & \\
\hline & Belgique & 3 & 1 & \\
\hline & Espagne & 136 & 82 & \\
\hline & Italie & 48 & 45 & \\
\hline & France & 11 & 8 & \\
\hline & & $\overline{198}$ & $\overline{136}$ & $=68.7 \%$ \\
\hline \multirow[t]{4}{*}{1987} & Espagne & 103 & 58 & \\
\hline & France & 10 & 9 & \\
\hline & Italie & 27 & 25 & \\
\hline & & $\overline{140}$ & 92 & $=65.7 \%$ \\
\hline \multirow[t]{6}{*}{1989} & Espagne & 41 & 28 & \\
\hline & Italie & 26 & 15 & \\
\hline & France & 12 & 9 & \\
\hline & Belgique & 3 & 3 & \\
\hline & Portugal & 33 & 26 & \\
\hline & & $\overline{115}$ & 81 & $=70.4 \%$ \\
\hline 1990 & $\begin{array}{l}\text { France } \\
\text { Portugal } \\
\text { Italie }\end{array}$ & 13 & 13 & \\
\hline
\end{tabular}

This diploma was justified by the place of sonography in diagnostic imaging. Sonography is complete by part of imaging but is the most shared and the most threatened technique. The ultrasound training is long and complex.

Are other experiences useful? Can sonography diploma be pursued and extended to others countries?

(see tables 1 and 2) 
Table 1

\begin{tabular}{|c|c|c|c|c|c|c|c|c|c|c|c|c|c|}
\hline Pays & $\begin{array}{c}\text { Nbre } \\
\text { d'Années }\end{array}$ & Internat & $\begin{array}{l}\text { Tronc } \\
\text { commun }\end{array}$ & $\begin{array}{l}\text { Année } \\
\text { Clinicat } \\
\text { Préparat. }\end{array}$ & $\begin{array}{c}\text { Durée } \\
\text { Radio Dg } \\
\text { propr.dit }\end{array}$ & $\begin{array}{l}\text { Stage } \\
\text { Tps } \\
\text { Compl } \\
\text { Radio }\end{array}$ & Rémun. & $\begin{array}{c}\text { Formation } \\
\text { Théorique } \\
\text { Radio. } \\
\text { Clin. }\end{array}$ & $\begin{array}{c}\text { Complém } \\
\text { Radio } \\
\text { Thérap }\end{array}$ & $\begin{array}{l}\text { Complém. } \\
\text { Medecine } \\
\text { Nucléaire }\end{array}$ & $\begin{array}{l}\text { Examen } \\
\text { annuel }\end{array}$ & $\begin{array}{c}\text { Examen } \\
\text { final }\end{array}$ & $\begin{array}{c}\text { Mémoire } \\
\text { thèse }\end{array}$ \\
\hline Autriche & 6 & non & non & $\begin{array}{l}\text { oui } \\
1 \text { an }\end{array}$ & $41 / 2$ & 5 & $?$ & 4 & 6 mois & 3 mois & non & non & non \\
\hline Belgique & 4 & non & $\begin{array}{c}\text { non } \\
\text { Créat. } \\
\text { Ens. prob. } \\
\text { Radio } \\
\text { ped. }\end{array}$ & non & 4 & 4 & $?$ & 4 & non & non & non & non & non \\
\hline Danemark & 4 & non & non & non & $2+2$ & 2 & $?$ & 2 & non & non & \multicolumn{3}{|c|}{ en cours d'introduction } \\
\hline D.D.R. & 4 & non & non & non & $31 / 2$ & 4 & $?$ & 4 & 6 mois & non & non & oui oral & non \\
\hline Espagne & 4 & non & 6 mois & non & $31 / 2$ & 4 & oui & 4 & non & non & \multicolumn{2}{|c|}{ contrôle continu } & non \\
\hline Finlande & 4 & non & non & non & 4 & 4 & $?$ & 4 & non & non & non & oui & non \\
\hline France & 4 & oui & oui & non & 4 & $\begin{array}{l}\text { au moins } \\
2 \text { ans }\end{array}$ & oui & 4 & non & non & $\begin{array}{c}\text { oui } \\
\text { modulaire }\end{array}$ & $?$ & oui \\
\hline GB & 4 & non & non & $\begin{array}{l}\text { oui } \\
1 \text { an }\end{array}$ & 3 & 3 & oui & 3 & non & non & oui & oui & non \\
\hline Hongrie & 4 & non & non & non & 4 & 4 & $?$ & 4 & non & non & non & oui & non \\
\hline
\end{tabular}

Table 2

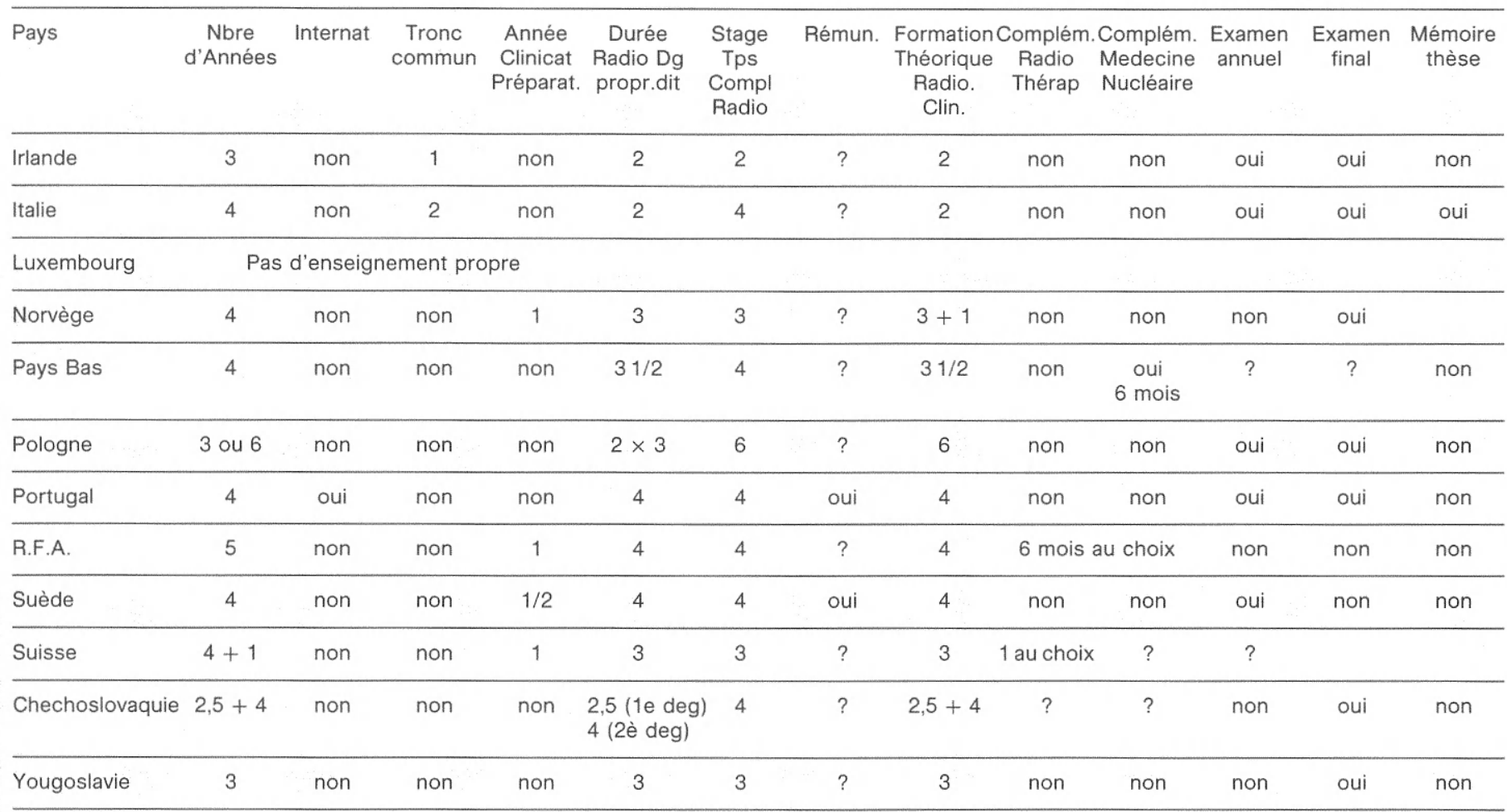

\section{Education in radiology in the Netherlands}

A.E. van Voorthuisen; Leiden

Pre-doctoral teaching in medicine encompasses four years and is usually problem-oriented, built around a core of medical topics. Lecturers from clinical and non-clinical departments participate in each course. The choice of the topics ensures that the whole field of medicine and health care is covered.

Radiology participates in these courses and in the related exams.

The number of hours, spent on radiodiagnosis in the pre-doctoral phase, varies from university to university beween ten and fifty. The teaching of radiation physics, biology and hygiene comprises between seven and fifteen hours.

The post-doctoral phase of two years is composed of clercships. Usually these clerkships are preceded by a course on basical clinical sciences; radiology should have a place in this programme. The time, allocated to radiodiagnosis varies from two days to two weeks.

Electives in research and clinical work are part of the pre-doctoral and post-doctoral training. They can be spent in radiodiagnosis and may last up to three months.

Postgraduate teaching of diagnosis radiology is supervised by a central board; it is concentrated in eight university hospitals and eleven large regional hospitals.

Five years full-time employment is required as well as a solid training program that contains all subspecialties, including nuclear medicine. 
Teaching departments should employ at least as many full-time staff-members as residents. This ensures adequate opportunities for supervision and individual training.

The central board does not require examinations for certification. The Dutch Society for Diagnostic Radiology started in 1981 with written exams and today $70 \%$ of all residents passed these exams before the time of board certification. There is no national registry for subspecialties in diagnostic radiology. 


\section{Room A}

B6 711

\section{MRI of the pelvis}

Hedvig Hricak; San Francisco, CA

MRI is increasingly gaining acceptance in the exploration of diseases involving the male and female pelvis. The superb presentation of soft tissue contrast resolution, allowing the multiplanar cross-sectional display of anatomy and pathology requires detailed knowledge of topographic anatomy. In the male pelvis, the use of MRI has been extended to the study of congenital anomalies when ultrasound is inconclusive. In the staging of carcinoma of the prostate, the role of MRI, although controversial, is being accepted as complementary to transrectal ultrasound and superior to CT. In the evaluation of benign diseases of the female pelvis, MRI is used when ultrasound is inconclusive or technically limited. The use of MRI has been advocated as the primary imaging modality in the staging of carcinoma of the endometrium and cervix. In ovarian cancer, the role of MRI is the subject of ongoing studies. The preliminary results demonstrate that the diagnostic and staging accuracy of MRI is similar to that of CT.

In the short time since the introduction of MRI into clinical practice, this modality has made great strides in its usefulness, and the continuous development of new techniques and application of contrast media are further expanding its horizon.

\section{MRA review}

G. Marchal, H. Bosmans, P. van Hecke; Leuven

MR angiography is a rapidly evolving field in MR imaging, the main goal of which is a non invasive tool for visualizing blood vessels.

The acquisition of continuous images of the blood vessels, comparable to the conventional DSA images, as well as the functional evaluation of blood flow velocities and flow patterns are the subject of continuing developments.

The basic principles of the various techniques and their clinical applications are reviewed and the practical limitations in specific anatomic regions discussed. The review concludes with a survey of future developments. Index Terms: MR angiography, flow patterns, MR inflow effects, MR phase effects Time-of-Flight, phase subtraction, Phase images, velocity quantification

\section{Room B}

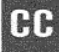

\section{1}

771 Chronic infiltrative lung disease - CT technique - basic CT signs Martine Rémy-Jardin, Jacques Rémy; Lille

Computed tomography (CT) has been demonstrated superior to chest radiography in assessing the presence and extent of parenchymal abnormalities. Advances in CT technology now allow detailed imaging of the lung parenchyma and diseases involving the pulmonary interstitium can be accurately evaluated. If conventional CT can depict many parenchymal lesions, the use of high resolution CT (combination of thin-section CT and the use of a high-frequency resolution algorithm) has been shown superior in assessment of fine parenchymal details. However, the combination of conventional CT and high resolution CT provides the best overall assessment of disease pattern, extent and distribution. Algorithm for the best imaging approach in different groups of patients is developed. Several CT signs indicate the presence of diffuse infiltrative lung disease. These include abnormal interfaces, ground glass opacity, linear pattern with septal and non septal lines, presence of micronodules, nodules and masses and honeycombing. The most common patterns are defined and illustrated. Pathologic-CT correlations are developed to increase our understanding of the pattern and distribution of diffuse infiltrative lung disease.

\section{Chronic infiltrative lung disease: the role of high resolution computed tomography}

\section{G. Gavelli, M. Zompatori, R. Canini; Bologna}

High resolution CT (HRCT) is often used nowadays in subsequent areas: a) detection of diffuse lung disease (DLD), also in patients with negative chest $\mathrm{X}$-ray but with clinical symptoms or signs of pulmonary disease, functional abnormalities and so on.

b) precise localization of the anatomical distribution of DLD, using the lobular anatomical conception.

c) characterization of lung disease (when possible) d) evaluation of disease severity and activity

e) choice of biopsy site and modality

f) follow-up.

DLD most often diagnosed by means of HRCT are: UIP (and related conditions), dip, sarcoidosis, lymphangial carcinomatosis, lam, x-histiocytosis, silicosis, bronchiectases and emphisema.

These topics will be covered in our presentation, with particular emphasis on "interstitial" lung disease.

- Usual interstitial pneumonitis (UIP) in the early stages and during relapses (alveolitis) is characterized by ground glass opacities. Otherwise, HRCT typically shows reticular opacities and honeycombing in peripheral and basilar areas. This presentation is rather specific for UIP, but SSP, rheumatoid lung, asbestosis and mixed connective tissue disease may have similar appearance. - In sarcoidosis, HRCT can detect small nodules clustered in peribroncho-vascular and subpleural regions, septal thickening, adenopathies. Ground glass is visible during activity relapses; crowding and fibrotic distorsion of bronchovascular bundles in end-stage disease.

- Lymphangitis carcinomatosa is characterized by mono- or bilateral, nodular thickening of bronchovascular markings, septal interlobular lines and polygonal opacities, prevailing at the bases. In the clinical context, these lesions are rather diagnostic, but could be produced also by pulmonary edema, sarcoidosis and other, rare conditions.

- HRCT pattern of lymphangioleiomyomatosis consist of diffuse cystic air-space, with thin but perceptible walls, diffusely distributed through the lung parenchyma (cortex and medulla). Lung bases are more involved. Lung volumes are increased.

- X-histiocytosis shows a very peculiar association of nodules, peribronchial cavitating nodules (in the lobular core) and, later, thin walled cysts, prevailing at upper lobes. Many lesions appearing reticular on plain chest film are actually cystic, as shown by HRCT.

\section{Room C}

\section{Imaging of the acute abdomen}

G. R. Schmutz, R. K. Chhem, T. B. Bui, F. R. Plante; Sherbrooke, Quebec

The acute abdomen is a sudden syndrome, affecting the abdominal and pelvic cavities and accompanied by pain and other signs. It is a frequent and potentially life threatening clinical situation in which the radiologist should, only with the usual procedures, decide promptly whether the pathological process requires urgent surgical, medical and/or radiologic interventional therapy. Although clinical findings may lead to a specific diagnosis, a great deal of overlap from extra or intraabdominal disorders exists and radiological studies are often valuable in their classification.

The advent of CT and US has diminished the importance of plain film radiography in the evaluation of acute abdominal diseases, yet, in many conditions the plain radiograph provides definitive or at least highly suggestive diagnostic information.

Obviously, roentgen diagnosis in acute abdominal settings should be assessed in the shortest time with the greatest care and accuracy, for a consequent reduction in the mortality and morbidity rates. It is necessary to choose the appropriate available examinations, reducing the time needed to establish a diagnosis and decreasing the number of examinations required.

The presentation is geared toward the determination and the choice of multiple roentgen procedures rather than toward the specific disorders. I have chosen this approach because many clinical presentations of acute abdominal emergencies overlap.

In this setting, it is useful to categorize the approach as follows: Abdominal plain films; Angiography; Abdominal sonography; CT; Contrast studies.

Conclusions: the acute abdomen is a clinicoradiologic problem that may be resolved by an excellent collaboration between the referring physician and the radiologist. At each step of the diagnostic approach, an interdisciplinary consultation is advised to determine the most appropriate available procedure for an adequate final result.

\section{Room E1}

\section{WS}

\section{2}

774 Techniques, indications and results of renal artery stenting G.Wilms; Leuven

The indications for renal artery stenting are:

- Relapse of stenosis after previous angioplasty or bypass surgery

- Insufficient result after angioplasty, with residual high grade stenosis as frequently seen in ostial lesions of the renal artery

- Complications of angioplasty such as dissections, obstructive intimal flaps and occlusions.

Major requirements for an arterial stent are easy positioning, good and stable 
expansion and poor thrombogenicity, so that thrombosis and intimal hyperplasia do not occur.

For a stent in the renal arteries other proporties of the stent are necessary. The stents must have good radio-opacity in order to allow exact positioning of the stent, with regard to the renal artery ostium. The stent should be flexible, considering the downward course of the renal arteries and their movement with respiration. Finally for exact positioning of the stent, it should shorten as little as possible, during release, in order to prevent misplacement or restenosis. The patency of the stent at late follow-up is influenced by the medical treatment after stenting. Depending on the type of stent anticoagulation can be necessary, while in other cases, antiplatelet aggregation drugs are sufficient.

\section{Renal angioplasty}

J.C. Gaux; Paris

Abstract available at the information desk.

\section{Contraindications for renal angioplasty - renal function before and after angioplasty}

H. Ingrisch; Munich

In case of renovascular hypertension PERCUTANEOUS TRANSLUMINAL RENAL DILATATION (PTRD) is indicated because this therapy results in great benefit (improvement or normalization of blood pressure and improvement of renal function) at little risk for the patient.

PTRD is contraindicated under the following conditions:

- if no improvement is to be expected, e.g. in case of insignificant stenosis

- if applying PTRD seems to prove more dangerous for the patient than the disease itself

- if PTRD is impossible for technical reasons

- as long as treatment of accompanying diseases has not yet been completed

- if personal and/or technical condition are not available

- if any general contraindications exist.

Improvement of renal function by PTRD can be measured by serum-creatinin (global function) and/or by J-Hippuran clearance (function of each kidney). In case of unilateral stenosis measurement of the function of the contralateral kidney allows prognostic predictions about blood pressure after PTRD.

Changes in renal function after PTRD are reported.

\section{Room E2}

\section{RG}

\section{6}

\section{Arthropathies: basic approach to diagnosis}

\section{lain Watt; Bristol}

A simple diagnostic tree will be presented to facilitate the diagnosis of arthritis based upon simple radiological observations. In essence, it depends not upon the joint distribution of disease but upon the distribution of disease within joints. A simplified logical approach is demonstrated below. The essential decision necessary at the outset of diagnosis is which of the three main areas of a joint are involved by the disease processes. Those three areas are, the site of synovial contact with bone - the so-called bare area, the articular surfaces (including hyaline cartilage and subchondrial bone) and the enthesis.

\section{Rheumatoid arthritis of the cervical spine}

\section{W. Dihlmann; Hamburg}

Adult rheumatoid arthritis affects the cervical spine as frequently as if this part of the spine was the 5 th extremity of the human body. In the majority of cases however (about $70 \%$ ) involvement of the cervical spine occurs at least 10 years after onset of the disease.

Granulation tissue in rheumatoid arthritis can affect and destroy joints, bursae, ligaments, disci, and bone structures of the cervical spine. In the feared aftermath of these destructions instability threatening the spinal cord and the medulla oblongata may result. Instabilities of the occipito-cervical junction can occur in all directions. Pathologic motility in the sagittal plain dominates from $\mathrm{C} 3$ on. Conventional radiographs and studies in ante- and retroflexion supply basic information. When pathologic findings are present in the occipito-cervical junction plain film diagnosis is completed by a.-p. tomography and lateral tomography in anteflexion position.

The so-called alarming erosion is pointed out and regarded the most important indication to perform MRI of the occipito-cervical junctions.

\section{Disorders of the temporomandibular joint}

\section{J. L. Termote; Leuven}

This presentation will first demonstrate the functional anatomy of the temporomandibular joint. The current imaging methods: plain film radiography, tomography, computed tomography, arthrography and magnetic resonance imaging of the temporomandibular joint. Bone lesions and lesions of the articular surface as growth problems, posttraumatic disorders, inflammatory disorders, ankylosis, synovial disorders and tumors are discussed. The internal derangements of the temporomandibular joint are also discussed. A review of the current literature concerning the temporomandibular joint will be presented.

\section{Room L}

\section{WS}

\section{8}

780 Modern imaging of the maxillofacial region - normal anatomy Carmen Rodriguez-Guirado; Barcelona

The anatomy of the sinus cannot be interpreted without looking for a dynamic interpretation which explains the frequent variations which are found in this region.

The paranasal sinuses are susceptible to many influences during the approximately ten years of their growth phase. Development of these cavities is particularly affected by infections, malformations, trauma and congenital lesions.

Atypical sinusal growth can also be due to malformations or deformities of the septum, which are of considerable clinical interest because of their great frequency. It is above all the ethmoid which presents major changes in development, especially because of its direct relation with the nasal cavity, forming part of the respiratory area, according to Guillen.

The presence of spurs, adhesions or deviations of the nasal septum should be studied with CT axial or coronal sections, paying special attention to asymmetries of sinus development.

CT is a very suitable method of studying detailed sinusal structures and their anatomical and physiological variants.

\section{3-D imaging of congenital deformities, trauma and neoplasms} Frans W. Zonneveld; Utrecht

Three-dimensional imaging has matured over the past decade from a gimmick to a clinically useful tool. At the same time its application has been increasingly focused on the support of therapy rather than that of diagnostics. Both the quality of 3-D image rendering as well as the quality of the underlying data sets have been steadily improving, while the capability to interact with the data has grown (removal of dental artifacts and anaesthesia tubes, disarticulation of mandible and cervical spine, cut-away view generation, soft tissue segmentation).

3-D imaging is generally applied to complex cases requiring an individual approach. In case of teratogenic deformities it is important to visualize the location and extent of craniofacial dysostosis, synostosis and congenital neoplasms in such a way that the individual pathology is clear to all members of the surgical team and that the treatment approach can be individualized on the basis of the 3-D images. When soft tissues are involved, they are visualized in relation to their bony skeletal context. Segmentation of the soft tissue structures themselves often requires manual interaction when there is insufficient contrast with the surrounding tissues. In trauma, mostly the old cases with delayed or neglected treatment are suitable for 3-D imaging. In oncology, it is important to determine the best surgical approach, how to remove the tumor, and how to reconstruct the defects associated with ablative surgery.

3-D data sets can also be used for multiplanar reformatting, for quantitative analysis (distance, angle, volume, shape), and for the fabrication of physical models (as applied in surgery rehearsal and implant design).

We will illustrate these applications with relevant case material.

\section{Imaging of tumours of the facio-maxillary region} Glyn Lloyd; London

Benign tumours especially those showing bone changes are for the most part adequately demonstrated by CT: the exception is juvenile angiofibroma. Because of the hazard of biopsy with this highly vascular lesion, the radiologist may need to make the initial diagnosis. This can be made by CT, but the extent of the tumour is best demonstrated by MR either with or without the use of GdDTPA.

In malignant tumours of the paranasal sinuses the diagnosis is usually made by biopsy, and the role of imaging is to show the extent and distribution of the neoplasm. This will largely determine the feasibility and mode of treatment. For the preoperative assessment of patients for cranio-facial resection extension of tumour from the ethmoids and sphenoid sinuses into the anterior fossa is best demonstrated by GdMR using inversion recovery sequences. In the orbit often the effect of GdDTPA is to convert tumour tissue to MR signal characteristics close to those of orbital fat, so that in most instances orbital extension of tumour is better demonstrated either without the use of the contrast agent or by the use of subtraction GdMR. For the evaluation of the cervical area, the total coverage of the head and neck by MR is the optimum method of showing both metastases and direct extension of tumour. For recurrent tumours following surgical resection of radiotherapy and for tumours invading the skull base, such as adenoid cystic carcinoma, subtraction GdMR has proved to be the optimum method of demonstrating tumour extent. 
Finally there are two areas in sinus malignancy, which are usefully evaluted by CT rather than MR: early bone erosion of the cribriform plate, which will indicate the need for craniofacial resection; and invasion of the pterygopalatine fossa, which is important for deciding tumour resectability and prognosis.

\section{Room M}

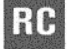

\section{5}

\section{3, 784 Radiology of impotence}

J.L. Struyven and C.P. Delcour; Brussels

This Refresher Course starts with a review regarding the physiology of erection and the pathophysiology of impotence. A description of clinical patient assessment is given and the studies available for evaluating the impotent patient are reviewed. The evaluation of penile blood flow with Doppler techniques is of increasing importance in ultrasonography. Vascular lesions are one of the most frequent courses of organic impotence. The importance of the arterial evaluation of patients with suspected disease of the vessels is emphasized. After the major pelvic vessels are evaluated, pharmacoangiographic techniques are necessary for proper demonstration of the penile arteries, often including selective catheterization of the internal pudendal artery.

The importance of a normal venoocclusive mechanism to produce and maintain a normal erection has become clear and the value of cavernosography and cavernosometry for the diagnosis of abnormal venous leakage will be illustrated.

The technique of the various procedures will be described. It is important that interventional radiologists and uroradiologists understand the interplay between arterial and venous mechanisms of erection. In addition, the use of pharmacologic agents such as papaverine for diagnosis and therapy are basic to the management of these patients.

\section{Room N}

\section{RC}

\section{7}

\section{Cerebral blood flow and neuroreceptor imaging}

I. Podreka; Vienna

Abstract available at the information desk.

\section{Spectroscopy \\ S. Müller; Base}

The rich analytic potential of in vivo magnetic resonance spectroscopy (MRS) for the investigation of organ chemistry and function is now generally recognized. Indeed the noninvasive investigations of human tissue with MRS have produced fascinating results in biology and medicine.

Nowadays combinations of local techniques with spectroscopic imaging have gained wide acceptance: both spatial and spectral information can be measured in a single multidimensional imaging/spectroscopy experiment. Recent applications include $1 \mathrm{H}, 31 \mathrm{P}$ and $13 \mathrm{C}$ investigations, in which the specific physical and biological advantages of each nucleus can be appreciated.

More recently labelling studies especially with $13 \mathrm{C}$ have opened new perspectives for the time resolved studies of metabolism. In combination with spectroscopic imaging new trends in in vivo spectroscopy are therefore in sight which include dynamic metabolite maps of human organs.

In the presentation an overview over recent advances in MR spectroscopy is given including introductions into some important basic spectroscopy methods.

\section{Room 0}

\section{WS}

787 Adverse reactions to contrast media: incidence mechanisms and prevention

Takahiro Kozuka; Osaka

In a large-scale (337,647 cases), nation-wide cooperative clinical study on adverse drug reactions to contrast media (CM) in Japan, the overall incidence of adverse reactions (ADRs) to ionic CM was $12.66 \%$ and to non-ionic CM it was $3.13 \%$. Severe ADRs occurred in $0.22 \%$ of the ionic and $0.04 \%$ of the nonionic contrast media examinations. Use of nonionic contrast media significantly reduced the frequency of severe ADRs to contrast media. Risk factor analysis shows nonionic $\mathrm{CM}$ to be 5.6 times safer than ionic $\mathrm{CM}$. As major risk factors, we verified the following: history of ADR at exposure to $\mathrm{CM}$, history of asthma and underlying cardiac disease. The incidence of severe ADRs in patients with these three risk factors is 120 times higher than in patients without. As a method of predicting severe adverse reactions, pretesting has already been abandoned in most countries. In Japan, however, pretesting is still widely being carried out in many hospitals. In this study, pretesting was also evaluated for its reliability in predicting severe reactions to $\mathrm{CM}$. There was no significant difference in the incidence of severe ADRs between the nonpretested and pretested populations. Severe ADRs occurred in patients with negative pretest. The predictive value of the test was $1.2 \%$ for ionic and $0.0 \%$ for nonionic CM. We conclude that pretesting is not useful in the prediction of severe ADR. In addition, the effect of premedication on patients who had positive results of pretesting was reviewed in the study. Premedication after a positive pretest was not helpful in decreasing the incidence of severe ADR due to $\mathrm{CM}$ injection. The radiologist can, however, significantly reduce the risk of a contrast media examination by choosing a nonionic contrast agent.

Nevertheless, CM should always be injected carefully, with full preparation for an emergency.

\section{Coagulation problems due to the contrast media during angio- graphy}

M. Amiel, R. Eloy, C. Corot; Lyon

1. Coagulation problems and thromboembolic complications (TEC) during angiography are well known since more than 30 years! In many ways the role of contrast medium (CM) has been evocated several years ago. But recently the debate has changed with the various effects shown during coronary angiography and/or angioplasty according to the nature of $\mathrm{CM}$ molecule.

2. Many studies have been made, in vitro and in vivo (with animal models or in clinical conditions), concerning CM interactions with blood cells and proteins involved in the clotting process. They have shown various effects of ionic (I) and non ionic (NI), of high $(\mathrm{H})$ or low $(\mathrm{L})$ osmolaly $\mathrm{CM}$ molecules. For exemple: the fibrinopeptid $\mathrm{A}$ formation is inhibited in an increasing manner by: NI LOCM, ILOCM, IHOCM, respectively.

3. In this particular case, angiography, the good biocompatibility of some $\mathrm{CM}$ molecules may, paradoxally, increase the risk of TEC! Inversely some CM molecules allow, in clinical practice, a protective effect against the clotting activative factors represented by:

- prothrombic patient status,

- catheterism materials (guide wire, catheters, syringe...),

- procedure itself (duration...).

4. More prospective randomized clinical trials are needed to confirm and evaluate precisely the initial data.

\section{Mechanisms of contrast agent toxicity} Peter Dawson; London

Chemotoxicity and osmotoxicity of contrast media (CM) are determined by chemical structure. The lower the chemotoxicity and osmotoxicity of the CM, the less animal toxicity and higher clinical tolerance in humans will be achieved. Nonionic monomers such as iohexol and iopamidol in iodineequivalent concentrations have approximately half the osmolality and therefore half the osmotoxicity of ionic monomers such as diatrizoate, iodamide, iothalamate and metrizoate. Absence of carboxyl groups in nonionic $\mathrm{CM}$, as opposed to the presence of carboxyl groups in ionic $\mathrm{CM}$, results in a lower chemotoxicity in nonionic CM. Similarly, a larger number of hydroxyl groups in nonionic CM than in ionic CM result in a lower chemotoxicity. The lower chemotoxicity of nonionic $\mathrm{CM}$ is reflected as a higher subarachnoid and intravenous tolerance both in animals and in the clinical setting.

When a contrast medium bolus instead of blood flows through a vessel the excess or lack of various plasma electrolytes in the contrast medium solution may contribute to its toxicity. Furthermore a contrast medium bolus does not deliver any oxygen to the tissues and this may also contribute to the adverse effects of the contrast medium solution.

790 Influence of toxicity, osmolarity and viscosity of CM in intravascular injection

Roberto Passariello; L'Aquila

The iodinated uro-angiographic contrast media (CM) cause a range of undesiderable pharmacodynamic effects after their intravascular injection which may or not become evident as adverse reactions.

These undesiderable effects depend on many factors such as molecular toxicity, osmolalty, viscosity, as well as on some components of the formulations as counter-ions and chelating agents.

The review of the very large numbers of papers on these topics does not supply a definite statement on the relative importance of these factors. We intend to discuss mainly about cardio-vascular effects related to intra-arterial injection of the CM.

The molecular toxicity plays a predominant role in compromising the myocardial performance. The normal myocardial electrophysiological processes are troubled both by the molecular toxicity and, only for ionic compounds, by the absence of sodium-ions. A chelating effect of the iodinated 
molecule and the presence of chelating agents as stabilizer may contribute to enhance these effects.

The high osmolalty of the solution causes a decrease of the peripheral vascular resistances with a consequent drop of the blood pressure. The decrease of the peripheral resistance and the rapid expansion of the plasma volume caused by the high osmolality increases the cardiac preload which requires a good myocardial performance.

A high viscosity is not only important in limiting the output of the CM, but it contributes to cause a stasis in the microcirculation with consequent prolongation of an ischemic condition in selective arteriography.

Thus haemodynamic effects caused by a selective arteriography are complex and depend upon several factors such as injection time and rate, diameter of catheter and examined vessels, regional blood flow, osmolalty and viscosity of the CM employed.

\section{Renal tolerance: ionic versus non-ionic contrast media}

Peter Dawson; London

The assumption the iodinated intravascular radiological contrast agents may be associated with clinical nephrotoxicity has been with us for half a century. There is a considerable, but actually rather inconclusive, literature on the subject which only allows certain limited conclusions: Contrast agents may cause physical renal injury resulting in proteinurea but a relationship between this and clinical renal dysfunction has not been demonstrated; many patients exhibit a rise in serum creatinine in association with contrast administration but this is transient and no relationship between this common event and more dramatic manifestations of renal impairment has been demonstrated; angiography in some "risk groups" is associated with clinically apparent impaired renal function in a small number of patients but clinically significant contrast agent nephrotoxicity associated with other procedures, in other risk groups and in the general population remains unproven. Genuine risk factors appear to be dehydration and high dose. Specific contributions to risk of diabetes, old age and pre-existing renal impairment have not been established definitively. Indeed, to echo Katzberg, it may be "that the null-hypothesis represents the truth" - that is to say, contrast agent associated nephrotoxicity in the sense that radiologists have long understood it may not really exist.

Historical and up-to-date evidence, clinical and experimental, concerning this ill-defined entity will be reviewed.

\section{Room R}

\section{RE}

Sarcomas of soft tissue comprise a broad grouping of the various malignant tumors arising from the mesenchymal soft tissue at all sites within the body. In addition, the malignant tumors of peripheral nerves are included. There is a broad spectrum of histologic types; they are usually designated according to the apparent soft tissue of origin. In the U.S. there are approximately 5600 newly diagnosed patients with STS per year.

The role of radiation therapy in the management of a patient with sarcoma of soft tissue will be assessed with reference to radiation alone or radiation in combination with surgery and/or chemotherapy or biological response modifiers. The principal role for radiation in the management of patients with sarcoma of soft tissue is that in combination with surgery. This may be pre-operative, post-operative or intra-operative use of external beam photons, electrons, and protons, brachytherapy and survival and overall survival will be considered for each of the various techniques with respect to size, grade and histologic type. Morbidity associated with these various treatments will then also be considered. 


\title{
Room A
}

\section{SS 11.07 Abdomen II}

\author{
$10.30 \mathrm{AM}$
}

\section{Postoperative assessment of shunts for portal hypertension with $\overline{M R}$ angiography (MRA)}

\section{A. Steudel, F. Träber, W. A. Kaiser, P. van Dijk, M. Reiser; Bonn}

Purpose: Magnetic resonance angiography (MRA) was performed to evaluate patency of 27 shunts. Angiography, Doppler, or MRI were compared with MRA results.

Methods and Materials: 25 patients with biopsy-proven cirrhosis of the liver were studied. MRA was performed on a $1.5 \mathrm{~T}$ system using the $2 \mathrm{D}$-inflow gradient echo sequences (FFE) with 50/15/60 (TR/TE/rf pulse angle). Spatially selective presaturation pulses were acquired and 40-60 slices covered a region of $20-30 \mathrm{~cm}$. All MRA images were assessed for shunt patency and flow directionality in the main portal vein.

Results: Satisfactory MR angiograms were obtained in all but two patients. Findings by MRA did not agree with those by other imaging modalities and by surgery in six cases.

Conclusions: MRA gives reliable and accurate information on portal venous anatomy. It is a safe and noninvasive technique for detecting shunt patency.

\section{$10.40 \mathrm{AM}$}

\section{MR angiography of portal hypertension}

Kouji Morimoto, Yoshiko Aoki, Soom Choi, Yoshiaki Miyata, Kazuo Hara, T. Shirakawa; Osaka

Purpose: The purpose of this study is to visualize various types of portal venous collaterals or anomalies in patients with portal hypertension using non-invasive MR angiographic technique.

Methods: Twenty-eight patients with portal hypertension previously diagnosed by computed tomography, ultrasonography or angiography were analyzed using MR angiography. Studies were performed on a $1.5 \mathrm{~T}$ imaging system (Siemens). Using maximum intensity projection algorithm, projection angiogram was constructed from consecutive coronal images. Each coronal image was obtained during breath hold using the pulse sequence of FLASH-2D. To identify the spatial relationship of the vessels, two images which had different view point (10 degrees apart) were reconstructed and viewed stereoscopically. Also serial reconstructed images of different view point were displayed by cine mode.

Results: In all cases portal vein abnormalities including esophageal varices, spleno-renal shunt, gastric wall collaterals with splenic vein obstruction, umbilical vein, dilated subcutaneous collaterals, dilated azygos vein and/or portal vein aneurysm were demonstrated clearly by this method. Stereoscopic view and cine mode display also demonstrated the precise spatial relationship of the vessels.

Conclusions: MR angiography has an excellent potential in the evaluation of portal vein abnormalities accessible to the quality of direct portography such as percutaneous transhepatic portography.

\section{$10.50 \mathrm{AM}$}

\section{Comparative study of measurement of portal blood flow by MRI and Doppler echo techniques}

Ichizo Suemitsu, Shin Kimoto, Nobuo Sato, Misaki Shiraiwa, Atsushi Ono, Toshio Hiraki; Okayama

Purpose: Portal blood flow volume was determined using the phase contrast method (MRI) and then compared with the color Doppler method (US). Subjects and Methods: Portal blood flow volume was measured during fasting using the phase contrast method and a comparison study was made by simultaneously measuring the same portal blood flow volume using the Color Doppler Echo method on 40 healthy volunteers. A basic experiment was conducted using an electromagnetic flow meter for both MRI and US. The measured values were then corrected according to that data.

Results: The average portal blood flow rate of the 40 healthy volunteers by MRI was $11.0 \mathrm{~cm} / \mathrm{second}$ and the blood flow volume was $11.4 \mathrm{~cm}^{3} / \mathrm{second}$. The blood flow rate using the Color Doppler Echo method was $15.5 \mathrm{~cm} / \mathrm{second}$ and the blood flow volume was $14.0 \mathrm{~cm}^{3} / \mathrm{second}$.

Conclusions: Measurement of portal blood flow by the phase contrast method is believed to be a useful method and should be used in the understanding of the flow of portal blood in cirrhosis and chronic hepatitis.

796 Dynamic contrast enhanced snapshot flash MR imaging in focal nodular hyperplasia of the liver

Didier Mathieu, Alain Rahmouni, Pierre Gheung, Marie C. Anglade, Béatrice Falise, Mondher Golli, Norbert Vasile; Créteil

Purpose: To evaluate contrast-enhanced sub-second Turbo Flash MR imaging in comparison with spin echo (SE) sequences in focal nodular hyperplasia of the liver (FNH).

Methods and Materials: Twenty two patients with 25 pathologically prooven FNH were studied on a $1.5 \mathrm{~T}$ magnet using this protocol: $-\mathrm{T} 2$ weighted images $(\mathrm{TR}=2200 \mathrm{~ms}, \mathrm{TE}=45 \mathrm{~ms}, 90 \mathrm{~ms}),-$ Snapshot Flash $\mathrm{T} 1$ sequence $(\mathrm{TR}=$ $7 \mathrm{~ms}, \mathrm{TE}=3 \mathrm{~ms}, \mathrm{TI}=400 \mathrm{~ms}$, flip angle $=10^{\circ}$, matrix $=128 \times 128$ ) before and after bolus injection of $0.1 \mathrm{mmol}$ Gadolinium-DOTA $/ \mathrm{kg}$. Each image was acquired every $5 \mathrm{sec}$ during 2 minutes $\mathrm{T} 1$ weighted images were then obtained $(\mathrm{TR}=420 \mathrm{~ms}, \mathrm{TE}=15 \mathrm{~ms})$. These sequences were compared with measurements of SD/noise.

Results: 1. Unenhanced Snapshot Flash images demonstrate always the FNHs, as hypointense lesions, 2 . The central scar was depicted by this technique, 3 . Dynamic contrast studies demonstrated always the high arterial enhancement of FNH, 4. In 10 of 25 lesions, an enhancement within the scar was noticed, 5. Delayed postcontrast $\mathrm{Tl}$-weighted images demonstrated always this scar as an hyperintense area, 6. Comparison of unenhanced and enhanced Snapshot Flash sequences with the SE T2-weighted sequences showed significant differences.

Conclusions: Contrast enhanced Snapshot Flash MR imaging allows the hemokinetics study of FNH and the dynamic behaviour of its central scar. It is a powerful adjunct tool to SE sequences for the diagnosis of FNH as well as delayed postcontrast T1-weighted sequences.

\subsection{AM}

797 T1- and T2-weighted turboflash sequences - applications to benign liver tumors

Béatrice Falise, Didier Mathieu, Marie Christine Anglade, Alain Rahmouni, Jean Michel Franconi, Pierre Gheung, Isabelle Attali, Norbert Vasile, Wladimir Kawiecki; Saint Denis

Purpose: To evaluate the benign liver tumors by subsecond T1- and T2-weighted Turbo FLASH (TF) sequences with variations of the parameters of these sequences.

Methods and Materials: Twenty benign liver tumors including cysts, hemangiomas and focal nodular hyperplasias were imaged with this prospective protocol: 1 . T2-weighted images $(2200 / 45,90 \mathrm{msec}), 2$. TF-T1 ( $7 / 4 /$ flip angle: $\left.10^{\circ}\right)$ with TIs varying from 15 to $3000 \mathrm{msec}, 3$. TF-T2 (7/4/flip angle: $\left.10^{\circ}\right)$ with TEs varying from 20 to $300 \mathrm{msec}$. To obtain a T1 contrast, a pulse of inversion recovery is used before the TF sequence. TI is defined as the time between the inversion pulse and the beginning of this sequence. To obtain a $\mathrm{T} 2$ contrast, two preparations have been used: a DEFT sequence: $90^{\circ}$ - TE $/ 2-180^{\circ}$-TE $/ 2-90^{\circ}$-data acquisition and a stimulated echo: $90^{\circ}$-TE $/ 2-90^{\circ}-1 \mathrm{msec}-90^{\circ}$ - TE $/ 2-90^{\circ}$-data acquisition.

Results: 1. All the lesions are depicted on these T1 and T2-TF sequences. 2. By the analysis of the null point of the lesion depending upon TIs, these benign tumors can be differentiated, on T1-TF sequence. 3. By the SI differences/Noise, cyst and hemangioma can be differentiated with a TE of $150 \mathrm{msec}$, on T2-TF sequence. 4. The central scar present in FNH is always depicted as a hyposignal on $\mathrm{T} 1$ and a hypersignal on T2 TF.

Conclusions: By subsecond T1 and T2 Turbo FLASH sequences, these different benign liver lesions can be differentiated.

\section{$11.20 \mathrm{AM}$}

\section{Application of snapshot FLASH for abdominal MRI} J.Tintěra, H. Filipová, J. Lízler; Praha

Quality of abdominal MR spin echo images suffers from breath motion artifacts. A way how to overcome these artifacts is to use a sequence proposed by Haase and coworkers-Snapshot FLASH. In approximately one second we can obtain a motion unaffected image in matrix $128 \times 256$.

We implemented such a type of sequence to Siemens Magnetom 1.5 with $10 \mathrm{mT} / \mathrm{m}$ gradient coils. This sequence is composed from a nonselective inversion pulse followed by a data acquisition part. FLASH with flip angle of $10^{\circ}$, TE of $4.6 \mathrm{~ms}$, TR of $8 \mathrm{~ms}$ is used to acquire all matrix lines. Maximum FOV is $200 \mathrm{~mm}$. We took 4 measurements for every slice to improve $\mathrm{S} / \mathrm{N}$ and each measurement was started by a breath sensor to ensure the defined slice position.

We can achieve very different contrast by varying TI-time distance between the nonselective pulse and the data acquisition. Using this we acquired the selected slice with TI from $100 \mathrm{~ms}$ to $3000 \mathrm{~ms}$.

Possibility to use Snapshot FLASH for liver lesion differentiation in clinical MR examination was investigated. Results of MRI in a group of 25 patients were compared with ultrasound findings and histology. We found Snapshot 
FLASH images having a high diagnostic value mainly due to the absence of motion artifacts, contrast flexibility and short measurement time.

\section{$11.30 \mathrm{AM}$}

\section{Turbo-flash MR-imaging: perfusion patterns of hepatic tumors} E. Rummeny, U.Stöber, Th. Vestring, J., Adolph, H. Daldrup, J. Fervers, P.E. Peters; Münster

Purpose: To evaluate perfusion patterns and contrast-enhancement of hepatic tumors using ultrafast Turbo-FLASH imaging we studied thirty-one patients with proved hepatic tumors ( 9 with hemangiomas; 19 with metastases; 3 with hepatomas) at 1.5 T (Siemens, Magnetom).

Methods: T1-weighted (T1W) Turbo-FLASH (400/7/4 [TI msec/TR msec/TE $\left.\mathrm{msec}, 8^{\circ}\right)$ images were obtained before, during, and after i.v. bolus injection of $0.1 \mathrm{mmol} / \mathrm{kg}$ Gd-DTPA. Conventional TIW FLASH $60(100 / 6$ [TR msec/TE $\mathrm{msec}), 60^{\circ}$ ) images were obtained 4 to 12 minutes after the injection. Twenty to 30 Turbo-FLASH images were obtained in intervals of 5 seconds each. Time-to-peak enhancement (T) of the lesion relative to enhancement of uninvolved liver was calculated [t peak(lesion) - $t$ peak(aorta) $/ \mathrm{t}$ peak(liver) - $\mathrm{t}$ peak(aorta)]

Results: Hemangiomas showed a peripheral fill-in on Turbo-FLASH images, became hyperintense relative to liver $30-40 \mathrm{sec}$ after the aortic peak and were hyperintense on FLASH 60 images. Hypervascular metastases showed central fill-in and appeared hyperintense relative to liver 20-30 seconds after peak aortic enhancement, and were isointense on FLASH 60 images. Hypovascular metastases showed ring enhancement on Turbo-FLASH images, and appeared hypointense on FLASH 60 images.

Conclusions: Subsecond Turbo-FLASH MR imaging can be used to evaluate perfusion patterns of different hepatic tumors and may aid in their differential diagnosis.

\section{$11.40 \mathrm{AM}$}

800 MR imaging features of small HCC treated by percutaneous Ethanol injection (PEI)

S. Sironi, T. Livraghi, A. Vanzulli, A. DelMaschio; Milan

Purpose: We conducted a prospective investigation to evaluate the MR imaging features of small hepatocellular carcinoma (HCC) treated by percutaneous ethanol injection (PEI) under ultrasound guidance.

Methods and Materials: Thirteen patients with 15 histologically proven $\mathrm{HCC}$ lesions (2.3-2.9 $\mathrm{cm}$ in diameter) were studied with MR imaging before and after treatment. T1 weighted (SE 500/20) and T2 weighted (SE 2000/40-90) images were obtained at $0.5 \mathrm{~T}$. In all patients MR imaging findings were correlated with the corresponding CT findings and with the results of the final fine-needle biopsy.

Results: T1 weighted images proved most efficacious for the evaluation of tumor changes after ethanol injection. Before treatment 10 lesions were hypointense and 5 were isointense compared with normal liver on T1 weighted images. After treatment (15-17 days after the last injection) all HCCs (15) became markedly hyperintense relative to liver on T1 weighted images. On posttreatment dynamic CT scans all lesions appeared hypodense; the final fine-needle biopsy confirmed the presence of complete tumor necrosis (coagulative necrosis) in all cases.

Conclusions: In patients with small $\mathrm{HCC}$ treated by PEI, MR imaging can provide a useful means of evaluating the effectiveness of treatment in producing tumor necrosis.

\section{$11.50 \mathrm{AM}$}

\section{$80131 \mathrm{P}$ magnetic resonance spectroscopy of the liver in morbus Wilson}

Hester N. van Wassenaer-van Hall, Jeroen van der Grond, Tjeerd J. L. Hoogenraad, Jan van Hattum, Willem P. Th. M. Malt; Utrecht

$31 \mathrm{P}$ magnetic resonance spectroscopy was performed in the liver of patients with Morbus Wilson $(\mathrm{n}=22)$ and healthy volunteers $(\mathrm{n}=20)$. Image guided localization spectroscopy (ISIS) was used to acquire spectra from a volume of interest within the liver. A significant difference was found in phosphodiesters/ATP ratios between fibrotic/cirrhotic livers as compared to the control group of normals. Furthermore abnormal hepatic iron concentrations could be recognized on the spectra by low signal to noise ratios with broadening of the peak areas. The severity of cirrhosis as well of iron deposition can be semi-quantitative recognized at the spectra. These findings may help to ensure a clinical role for magnetic resonance spectroscopy as a noninvasive alternative for liver biopsy in patients with a diffuse liver disease.

\subsection{PM}

Marie-Curie Lecture (s. page 160)

\section{Room B}

SS

01.06 Chronic diffuse infiltrative lung disease (HRCT) $10.30 \mathrm{AM}$

802 Interstitial lung disease - assessment of HR-CT with pathologic correlation

Stefan Delorme, Michael V. Knopp, Hans-Ulrich Kauczor, Michael Walz, V. Schulz, Gerhard van Kaick; Heidelberg

Purpose: To find patterns visible on HR-CT in patients with chronic interstitial lung disease, regarding topographical and geometrical considerations.

Methods: 60 patients with chronic interstitial lung disease were studied with four slices at a $1 \mathrm{~mm}$ collimation. Image analysis included shape, size and distribution of lesions as well as their relation to anatomical structures of the lung. Diagnosis of inflammatory or fibrosing lung disease was proven by biopsy, suspected airway disease was confirmed by pulmonary function tests, bronchoscopy or bronchography.

Results: The initial lesion in fibrosing alveolitis or interstitial pneumonia consists of diffuse or disseminated ground glass opacities. The findings progress through thickening of the interlobular septa to true honeycombing. In geometric terms the former delines a wide mesh network of lines thinner than $5 \mathrm{~mm}$, the latter a coarse reticular pattern with loss of the enclosed parenchymal structures. Chronic airway disease shows thickening of the bronchovascular bundles, sometimes associated with infiltrates of dependent lobuli. The latter appear as sometimes sharply demarcated polyangular opacifications $2-5 \mathrm{~cm}$ in diameter.

Conclusions: HR-CT allows characterization of the anatomical distribution of a process and thus enables discrimination of airway disease and interstitial disease as well as an assessment of its progression and severity.

$10.40 \mathrm{AM}$

803 High resolution computed tomography in diseased isolated lungs: a radiologic-pathologic correlation

S. Diederich, N. Roos, H. Lenzen, Z. Puskas, A. Fahrenkamp, U. Bick, P. E. Peters; Münster

Purpose: The aim of our study was to establish precise correlations between high-resolution computed tomographic (HRCT) scans and corresponding gross pathological sections.

Methods and Materials: 22 isolated lungs were fixed with a solution containing polyethylene glycol 400 and dried by air inflation applying a constant pressure of $25 \mathrm{~mm} \mathrm{Hg}$. HRCT scans were obtained in standard positions and gross pathological sections were prepared at exactly identical levels. A matrix was projected to CT scans and pathological sections to allow precise correlation between CT, macro- and microscopic findings.

Results: The method applied, particularly with the matrix technique, allowed an exact regional HRCT-pathologic correlation. The patients studied included: $A R D S, n=4$, pneumonia, $\mathrm{n}=3$, tuberculosis, $\mathrm{n}=2$, multiple lung abscesses, $\mathrm{n}=1$, COPD, $\mathrm{n}=1$, pulmonary embolic infarction, $\mathrm{n}=1$, intoxication, $\mathrm{n}=1$, Goodpasture's syndrome, $\mathrm{n}=1$, pulmonary metastasis, $\mathrm{n}=4$, pulmonary lymphoma, $\mathrm{n}=2$. We observed, that even with well established CT-phenomena complex differential diagnoses often had to be considered. Conclusions: Postmortem pathologic correlation of pulmonary HRCT applying the matrix technique allows precise characterization of radiological findings. We therefore conclude that through these observations an optimization of in vivo pulmonary CT imaging can be obtained.

\section{$10.50 \mathrm{AM}$}

804 Pulmonary sarcoidosis: spectrum of CT findings and correlation between $\mathrm{CT}$ extent and pulmonary function tests

F. Laurent, V. Lafarge, B. Houang, M. Joullie, B. Chaulin, J. Drouillard; Bordeaux

The purpose of the study was: 1 . to describe the spectrum of CT findings in sarcoidosis 2. to compare chest film, CT and High Resolution CT (HRCT) in the staging and follow up 3 . to search correlations between pulmonary function tests and pulmonary extent evaluated by CT.

Methods: Sixty patients with a proven thoracic sarcoidosis were explored with chest film, standard CT, High Resolution CT (40 cases) and pulmonary function tests. Frequency of CT findings, staging with Chest film, CT and HRCT were retrospectively assessed. A visual score of pulmonary extent was determined on both plain film and CT and correlated with pulmonary function tests. Follow up over one year was available in 20 patients.

Results: CT modified the staging in $30 \%$ of the cases $(50 \%$ beeing classified stage I) and in $25 \%$ of the patients followed during one year. HRCT improved the visualisation of subpleural lesions but did not modify the staging. Visual scores established with Chest film and CT moderately but significantly correlated with pulmonary function tests $(0,305<\mathrm{r}<0,686)$. Variations of CT visual score during the follow up significantly correlated with forced vital capacity 
and carbon monoxyde diffusing capacity.

Conclusions: CT improves detection and follow up of pulmonary involvement in sarcoidosis. Evaluation of the extent with CT moderately correlates with pulmonary function tests.

\section{$11.00 \mathrm{AM}$}

\section{High resolution computed tomography (HRCT) in sarcoidosis:} usefulness and limits

A. Anglesio, G. C. Cortese, C. A. Cametti, G. P. Vaudano, E. Comino; Torino

Thirty-two patients with sarcoidosis, in most cases histologically proved, were studied with HRCT. The examination consisted of a series of $1.5 \mathrm{~mm}$ thick section, reconstruction with bone algorithm and 256 or 131 field of view. According to the literature, we conclude that HRCT is suitable for an early diagnosis of sarcoidosis even in patients with negative chest X-ray picture and can be very useful in interstitial severe involvement (stage III) when the differential diagnosis is difficult.

A longer time of examination and higher radiation exposure for the patients are the only limits to this method.

\section{$11.03 \mathrm{AM}$}

806 Pulmonary sarcoidosis: evolution of the lesions assessed by CT Michel Brauner, Stéphane Lenoir, Philippe Grenier, Philippe Cluzel, Dominique Valeyre; Bobigny, Paris

Purpose: To describe the evolution of the pulmonary CT findings, spontaneously or under steroid therapy, in order to identify reversible and definite components of pulmonary sarcoidosis.

Methods: Thirty-two patients, studied by $2 \mathrm{CT}$ examinations separated by a 3-27 months delay (mean: 10 months), were classified in 2 groups on clinical, biological and radiographic criteria: 1 . Group $A(n=22)$ included patients who had clear improvement, 2. Group B $(\mathrm{n}=10)$ included evidence of worsening or patients in a stable situation.

Results: CT findings were classified in 3 categories. Category 1 including nodules, confluent nodules, alveolar or pseudoalveolar consolidation, peribronchovascular thickening, decreased or disappeared in all cases of group A but were often stable or increasing in group B. These observations can be considered as active inflammatory lesions. Category 2 including micronodules, irregular interfaces, subpleural thickening, ground-glass opacities, had various evolution in each group that could be explained by various pathologic lesions. Category 3 included linear areas of attenuation, lung distortion, traction bronchiectasis and honeycomb cysts which have never decreased in groups $\mathrm{A}$ and $\mathrm{B}$, and was consistent with fibrosis.

Conclusions: We observed that CT allows better correlations with inflammatory changes detected by means of the evolution than chest radiography. The capacity to predict the likelihood of reversal of lesions is important for the therapeutic indications.

\section{$11.13 \mathrm{AM}$}

\section{$807 \mathrm{CT}$ appearance of pulmonary eosinophilic granuloma}

H.-J. Triebel, G. Kanzow, E. Buecheler, H. Magnussen; Hamburg

Purpose: To characterize the CT-morphology of this disease and to compare it to plain chest radiographs.

Methods and Materials: Standard and HR-CT scans and chest radiographs of 15 patients with histologically proven pulmonary eosinophilic granuloma were compared.

Results: On CT-scans the most striking abnormalities were cysts of variable numbers and sizes ( $\mathrm{n}=12$ patients) partially accompanied by diffuse patchy or reticular densities ( $\mathrm{n}=9$ patients). Nodules were found in four cases. Seven patients showed irregularity of the interfaces and six presented with mediastinal lymph nodes. CT was superior to plain chest films in depicting cystic lesions in nine patients and in detecting nodules in three.

Conclusions: Pulmonary eosinophilic granuloma is suggested by a typical cystic appearance on CT-scans. However similar changes are found in lymphangioleiomyomatosis and congenital air cysts. To our knowledge a differentiation by means of $\mathrm{CT}$ is not yet possible.

\section{$11.23 \mathrm{AM}$}

\section{Differential diagnosis of cystic-bullous changes of the lung} U. Lörcher, H. G. Keul, J. Peters; Frankfurt/Main

Purpose: In pulmonary CT cystic-bullous changes may be found that are difficult to correlate with certain diseases.

Methods: In $50 \mathrm{CTs}$ we studied size and distribution of cystic-bullous pulmonary changes and correlated them with the results of histology.

Results: In idiopathic pulmonary fibrosis we found cysts mainly peripherally and in the lower parts of the lung. Their size was usually below $1 \mathrm{~cm}$. In Histio- cytosis $X$ we found small (below $1 \mathrm{~cm}$ ) and larger cysts $(2-4 \mathrm{~cm}$ ) but no accumulation in the subpleural region. In lymphangioleiomyomatosis cysts are diffusely distributed throughout the lung and may be solitary or multiple. In about $50 \%$ of cases a definite correlation was not possible.

Conclusions: CT very precisely demonstrates cystic changes of the lungs. Correlation with a definite disease is possible in about $50 \%$ of cases.

\section{$11.33 \mathrm{AM}$}

809 Specific diagnosis of chronic diffuse infiltrative lung disease: contribution of high-resolution CT evaluated by Bayes' model Philippe Cluzel, Sylvie Chevret, Dominique Valeyre, Michel Brauner, Claude Chastang, Philippe Grenier; Paris

Purpose: to evaluate the additional accuracy provided by the inclusion of high-resolution computed tomography (HRCT) in generally used strategy for specific diagnosing chronic diffuse infiltrative lung disease (CDILD).

Methods: A model of Bayes theorem was applied to clinical, functional, biological data, chest radiograph (CR) and HRCT findings collected on 118 patients with proved specific diagnosis of CDILD. Bayes' theorem provides the posterior probability of a disease with the knowledge of the prior probability of the disease and the conditional probability of symptoms for each diagnosis. CR and CT scans were read by two observers without knowledge of clinical and pathologic data. They listed findings observed on the films with the aid of a previously established checklist. Prior probabilities were assumed to the prevalence of the diseases in the population. The results were then considered to determine the accuracy in predicting the specific diagnosis before and after taking into account HRCT informations.

Results: the specific diagnosis obtained from the largest Bayesian probability before and after HRCT was correct in 80 and $86 \%$ of cases respectively. A superior to .95 probability provided the correct specific diagnosis before and after HRCT in 49 and $72 \%$ of cases respectively.

Conclusions: HRCT improves confidence level for a specific diagnosis in CDILD, before pulmonary biopsy.

\section{$11.43 \mathrm{AM}$}

810 Impact of (high resolution) CT of the chest on clinical diagnosis and management

F.H. Barneveld Binkhuysen; Amsterdam

The diagnostic and therapeutic effectiveness of CT and High Resolution CT (HRCT) of the chest is evaluated.

The contribution to the diagnostic understanding and therapeutic decisionmaking by (HR)CT of the chest is measured in 100 patients using prospective and retrospective inquiry of referring physicians (before/after analysis). Three questions will be answered namely:

1. What is the diagnostic and therapeutic effectiveness of CT of the chest in our clinical setting?

2. What is the diagnostic and therapeutic effectiveness of routinely HRCT of the chest in our hospital?

3. Are there indications or criteria to point out so CT can be avoided?

$11.48 \mathrm{AM}$

811 Usefulness of HRCT in the evaluation of lymphangitic carcinomatosis

Mario Dragani, Cesario Ciccotosto, Maria Luigia Storto, Lorenzo Bonomo; Chiet

High-resolution CT (HRCT), providing anatomic details and an exact localization of disease processes within the secondary pulmonary lobule, has been advocated in the evaluation of diffuse infiltrative lung diseases.

HRCT can provide findings consistent with lymphangitic carcinomatosis (LC). The authors undertook this study to define the usefulness of HRCT in the evaluation of LC.

Between March and December 1990, 38 female patients (mean age 56 years), who had undergone mastectomy, were included in the study.

All patients undergone high-kV chest radiography and HRCT consisting of a series of $1 \mathrm{~mm}$ thick sections at 6 different levels. The median time interval between surgery and radiological examinations was 36.15 months.

Chest radiographic and HRCT findings agreed in 34 patients and disagreed in 4.

Chest radiography often shows nonspecific findings of infiltrative lung disease and the diagnosis of $\mathrm{LC}$ can be difficult on the basis of plain films; moreover some patients have normal chest radiographs in the early phase of LC. On the other hand HRCT shows some characteristic findings of LC (beaded chain appearance and irregular thickening of the fissures) and it is more sensitive than plain film. 


\section{Room C}

SS

04.08 General interventional radiology, lithotripsy

$10.30 \mathrm{AM}$

812 Interventional procedures in the upper GI-tract (Keynote Lecture) Hans Jantsch; Vienna

The indications, possibilities and complications of interventional procedures of the upper GI tract are discussed, based on the experience of 474 procedures. With the development of balloon catheters with large balloon-diameters it became possible the dilate esophageal obstructions. Eccentric or high grade obstructions with or without bending of the axis are often easier and less risky passed with angiographic guide wires and catheters compared to endoscopic methods. 297 dilatations of benign and 26 malignant obstructions of the esophagus have been performed. The complication rate was low with $0.9 \%$. In $53.3 \%$ the balloon dilatation was performed after failure of endoscopic bouginage. $66.2 \%$ of the benign obstructions were caused by anastomotic scars, and less frequent by peptic stenosis $(16.9 \%)$, achalasia $(7 \%)$, pylorospasm $(5.6 \%)$ and in individual cases with obstructions caused by pemphigus vulgaris, acid burn and one Schatzki-ring. Malignant obstructions which were dilated were cases of recurrent carcinomas of the esophagus or the EG-junction. In 10 patients a combined endoscopic-fluoroscopic guided implantation of an esophageal endoprosthesis was performed after endoscopic failure of tube implantation. In 8 patients with contraindications for implantation of an endoprosthesis such as cervical obstruction and coloninterposition self expanding stents have been implanted. In 10 patients a dislocated endoscopic or operative implanted endoprosthesis was removed or readjusted under fluoroscopic guidance with the use of balloon catheters. 123 percutaneous gastroenterostomies have been performed, which were complicated by 7 cases of tube dislocations and consequent local peritonitis. The indications for gastroenterostomy were mainly end stage carcinomas of the tongue, the hypopharynx, esophagus and the EG-junction.

\section{$10.45 \mathrm{AM}$}

\section{Operator experience versus specimen quality in 15 commonly} used biopsy devises

Kenneth D. Hopper, Dean E. Baird, Steve H., Parker, John F. Cardella; Hershey, PA

Purpose: This study evaluates the role of a radiologist's previous experience in the use of commercially available biopsy needles and biopsy guns versus the quality of specimen obtained for histopathological analysis.

Methods and Materials: Open hepatic and renal biopsies were performed by three radiologists of varying experience in 15 pygmy pigs with each of 15 different biopsy devises. These biopsy devices included the 14 gauge Vim Silverman and Tru-cut needles, five types of 18 gauge aspiration-type needles (Chiba, Turner, Franseen, Cook, and Jamshidi) and five biopsy guns (long-throw and short-throw Bard Biopsy, Cook and 14 and 18 gauge KlearKut). The specimens were evaluated separately in a double-blind fashion by two histopathologists using graded criteria.

Results: The performance of the aspiration-type needles were the most dependent upon the previous experience of the radiologist $(\mathrm{p}<.005)$. The results obtained with the 14 gauge Tru-Cut needle were not experience related. Mixed results were obtained with the 14 gauge Vim Silverman needle and the biopsy guns. However, experience was not a factor in the quality of specimens obtained with the 18 gauge long-throw Bard Biopsy Gun ( $>>0.05)$.

\section{$10.55 \mathrm{AM}$}

\section{Palliative treatment of esophageal self-expanding metal-stents - preliminary results}

obstruction with H. J. Wagner, K. Knyrim, E. Starck; Kasse

Purpose: Self expanding metallic stents may be inserted in a less traumatic technique with fewer side effects and without the need for general anesthesia conventional esophageal prostheses.

Methods: Between $6 / 90$ and $3 / 91$ we attempted to treat dysphagia in seven consecutive inoperable patients (5 esophageal cancer, 1 recurrent gastric carcinoma, 1 radiogenic stricture) with 9 metal stents $(14 \mathrm{~mm}$ diameter, $6-10 \mathrm{~cm}$ implanted length). The stents were placed under fluoroscopic and endoscopic control.

Results: Stent placement was successful in all patients without any procedure related complications. All patients were able to eat solid food initially. The mean follow up period is 10 weeks (4-20 weeks). Three patients died with patent stents. In one patient symptomatic restenosis due to tumor in growth through the meshes occurred after 5 weeks. In another patient stent blockage by granulation tissue was observed after 4 weeks. One patient with a patent stent had a bad functional result. Patients with recurrent dysphagia were treated with percutaneous gastrostomy.

Conclusions: Metal stents can easily and safely be placed at all sites of the esophagus. The initial relief of dysphagia is satisfactory, however long term patency is inferior compared with conventional plastic prostheses. Further studies should define whether a subset of patients may benefit from this procedure.

\section{$11.00 \mathrm{AM}$}

815 Transperineal drainage of pelvic abscesses after amputation of the rectum

F. Karnel, H. Schurawitzki, H. Jantsch, F. Herbst; Vienna

Purpose: to report the operational details and results of a new technique for drainage of pelvic abscesses.

Methods and Materials: In 8 patients, pelvic abscesses were drained using a transperineal approach with $\mathrm{CT}$ guidance. Equipment for the technique included a standard needle, angiographic wire and 8-10F drainage catheters. After the catheter placement patients were treated with gravity drainage and appropriate antibiotics; the follow-up was done with sequential imaging studies.

Results: All abscesses were drained effectively using the transperineal approach; no complications or problems were encountered. Performance of the procedure was quite simple and there was no discomfort to the patient. Compared to the transgluteal approach this method offers advantages related to patient comfort, operator safety, and minimal risk of potential complications such as sciatic nerve injury or abscess tracking.

Conclusions: This transperineal approach is an effective and well-tolerated method for drainage of pelvic abscesses after resection of the rectum.

\subsection{AM}

816 Long-time survival of malignant pelvic tumours after embolization of life-threatening bleedings

Ph. Hendrickx, G. Orth, H. Wagner; Hannover

Percutaneous transluminal embolization is an increasingly used technique for treatment of tumours and hemorrhages.

Based on an experience with 45 patients between 1981 and 1989, long-time survival, using the Kaplan-Meier procedure is discussed. In most of the patients malignant tumours of gynecological (20 pts) or rectosigmoideal (21 pts) origin were embolized; urethral carcinoma (1 pt), Thorotrast-sarcoma (1 pt) or metastases (2 pts) were rare indications. Mostly GAW-coils were used. Up to now 38 patients have died. The median survival time for patients suffering from gynecological tumours was 7.7 months and for those with colorectal carcinomas 9.5 months. Cause of death in most of the patients was tumourcachexia. Recurrent bleeding appeared in 10 patients $(22.2 \%)$, in 3 of them with lethal consequence. In 7 patients a successful re-embolization was performed. Severe complications were seen in only 2 patients (rectal necrosis, occlusion of the external iliac artery).

In severe, life-threatening bleedings resulting from malignancies in the pelvic region percutaneous transluminal embolization showed to be a safe and effective, although palliative measure.

\subsection{AM}

\section{Transcatheter-96\% absolute alcohol-sclerotherapy of lymphoceles}

Okan Akhan, Saruhan Cekirge, Mustafa N. Özmen, Aytekin Besim; Ankara

Purpose: Although percutaneus procedures have been used for treatment of lymphoceles, transcathetersclerotheraphy has not been widely applied previously. We present the results of transcatheter- $96 \%$ absolute alcohol-sclerotherapy of lymphoceles. This is the second study in which single agent used for sclerotherapy of lymphocele after the Gilliland et al's study used providone-iodine.

Methods: We have seven patients with eight lymphoceles. All seven patients had undergone pelvic lymphadenectomy for pelvic malignancy. We performed transcatheter sclerotherapy with $96 \%$ absolute alcohol with sonographic and fluoroscopic guidance.

Results: Seven of eight lymphoceles completeley disappeared. The duration of drainage ranged from 4 to 21 days (mean 9 days). Although one lymphocele did not resolve completely, it did not require surgery since the patient recovered from all complaints.

Conclusions: Being a new agent for lymphocele treatment, absolute alcohol has not been tried in a series of patients until now. We have a high success rate $(87.5 \%)$. If the sclerotherapy of patient, who has got rid of all her complaints although having a fibrotic remnant, is accepted to be successful, the rate will rise to $100 \%$. 


\section{$11.30 \mathrm{AM}$}

818 Extracorporeal shockwave lithotripsy: an effective non-invasive treatment for cholecystolithiasis

C. D. Becker, T. Huber, S. Glättli, C. Seiler; Bern

Purpose: To evaluate prospectively the results and the determinants for success of extracorporeal shock-wave lithotripsy (ESWL) as an outpatient procedure. Methods: In the form of a prospective interdisciplinary trial, 35 patients with symptomatic uncomplicated cholecystolithiasis were to date treated by ESWL using an electromagnetic lithotripter with ultrasound guidance (Lithostar Ultra, special modification). Adjuvant oral bile acid treatment was given in all patients. The stone volume, calculated on the basis of cholecystograms ranged from 0.2 to $8 \mathrm{~cm}^{3}$ (mean volume: $3 \mathrm{~cm}^{3}$ ). The number of calculi ranged from 1-10 (average 2). In the majority of patients, the calculi were radiolucent. ESWL was done as a full outpatient procedure without any analgesia.

Results: An average of 2.3 ambulatory treatment sessions were necessary to obtain fragments of $\leq 4 \mathrm{~mm}$. The total observation period is presently 18 months (average: 7 months). To date, 15 patients are free of calculi and symptoms. The average duration of treatment in these patients was 6.2 months (5-16 months). Two patients underwent cholecystectomy because of treatment failure. One patient had developed a transient acute pancreatitis and one patient acute cholecystitis. No other complications were observed.

Conclusions: ESWL can be performed effectively as an outpatient procedure in well-selected patients. The posttreatment dissolution period is directly proportional to the total stone volume. Expertise in ultrasound imaging is a key determinant for the success of the procedure.

\section{$11.40 \mathrm{AM}$}

\section{In vivo CT of gallstones: prediction of ESWL success} Daniele Regge, Stefano Debernardi, Eliana Carnieri, M. C. Martina, Elisabetta Tola, Giovanni Gandini; Torino

Seventy-four consecutive patients were submitted to CT in order to assess if this exam could aid in patient selection for biliary lithotripsy (BL).

Five $\mathrm{mm}$ sections were performed on the gallbladder region with a General Electric CT Max not more than one week before BL.

Fragments inferior to $10 \mathrm{~mm}$ were obtained in 47 of 74 patients (64\%).

Stones have been subdivided in 6 groups: hypodense, isodense, faint, dense, laminated and rimmed. Rim density and thickness has been related to success rate in the last group. Furthermore success of fragmentation has been related to stone density. Higher success rates were obtained with isodense stones $(71 \%)$ and with stones with rim thickness inferior to $4 \mathrm{~mm}(77 \%)$. Furthermore stones with CT density above $100 \mathrm{HU}$ had fragmentation success of $31 \%$ versus $72 \%$ for stones with densities equal or inferior to $100 \mathrm{HU}$.

Concluding we feel CT does improve selection of patients to be submitted to BL. Systematic use of this exam must be carefully evaluated as this would increase significantly the cost of a form of treatment on whose effectiveness many doubts still remain.

\section{$11.50 \mathrm{AM}$}

\section{Percutaneous extraction of retained bile duct calculi through the} T-tube tract with and without lithotripsy

Christoph D. Becker; Bern

Purpose: To emphasize the usefulness of percutaneous radiologic extraction of retained bile duct stones through the T-tube tract after surgery.

Methods: Over 18 months 14 patients with single $(n=8)$ or multiple $(n=6)$ retained bile duct stones after cholecystectomy were treated by means of percutaneous radiologic stone extraction. Under fluoroscopic control, the T-tube was removed and the calculi engaged with a Dormia basket. The calculi were either extracted in toto or as fragments after mechanical lithotripsy or shock-wave lithotripsy (ESWL).

Results: All calculi were successfully removed in 13 of 14 patients. In one case, an impacted common duct stone could neither be removed radiologically nor endoscopically and repeat surgery became necessary. In 6 patients, stone extraction was feasible without fragmentation in a single session. In eight patients larger calculi required lithotripsy either mechanically $(n=6)$ or by means of ESWL $(n=2)$. In all patients in whom mechanical lithotripsy or ESWL was performed, an additional treatment session was necessary for removal of the fragments. One patient experienced a transient episode of cholangitis. No other complications were observed.

Conclusions: Percutaneous removal of retained bile duct stones through the T-tube tract is an effective and safe interventional radiologic procedure. The present experience as well as the review of larger series from various centers indicate that the risk of serious complications is clearly lower than that of endoscopic retrograde stone removal with sphincterotomy. The percutaneous approach can be done on an outpatient basis. If a T-tube is present in the postoperative period, percutaneous removal of retained calculi via the T-tube tract should be the standard first-line treatment.

\section{Room E1}

SS

\subsection{Imaging and intervention in the abdominal} vasculature

10.30 AM

\section{Visceral arteries: (Keynote Lecture)}

P. Rossi; Rome

Since the advent of new imaging modalities the indications for diagnostic visceral angiography are very limited, mainly to confirm the presence of a vascular pathology (stenosis, obstruction, A.V.M. etc.) or for few neoplastic lesions.

However the indications for interventional procedures still remain as valid as before.

The most important interventional procedures actually are:

- bleeding control, spontaneous or traumatic

- A.V.M. embolizaton

- arterial aneurisms occlusion

- tumor embolization (chemoembolization for active hypersplenism)

- fibrinolythic therapy of occluded arteries.

Techniques, materials and results will be presented and discussed.

$10.45 \mathrm{AM}$

822 The value of a co-axial 3-french tapered catheter for superselective embolization

J. E. Jackson, D. J. Allison; London

Purpose: The embolization of arteriovenous malformations, arteriovenous fistulae, visceral arterial pseudo-aneurysms and some tumours may require super-selective catheterization to preserve surrounding normal tissues and to obtain the best long-term results. The arterial anatomy often precludes successful catheterization with conventional catheters. The introduction of a co-axial 3Fr tapered catheter (Tracker ${ }^{\circledR}$, Target Therapeutics) has made superselective catheterization and embolization of small, and sometimes extremely tortuous, vessels possible.

Methods: Tracker 18 catheters, together with Seeker 16 (Target Therapeutics) guide wires, have been used in 16 patients with a variety of different pathologies, on a total of 20 occasions when conventional catheters have failed to achieve selective catheterization because of small size or tortuosity of the supplying vessels.

Results: Super-selective catheterization has been possible on all occasions and embolization has been successfully performed with a variety of agents including metal coils, polyvinyl alcohol and absolute alcohol. There have been no serious complications.

Conclusions: The Tracker co-axial 3Fr catheter system enables super-selective embolization with resultant preservation of surrounding normal tissues in cases where conventional catheters would be unsuccessful. The method of use of this catheter and wire system will be described.

$10.55 \mathrm{AM}$

823 Percutaneous vascular intervention for hepatic encephalopathy Toshiyuki Matsuoka, Kazuo Kida, Atsuko Morimoto, Sumio Takashima, Haruki Nakatsuka, Kenji Nakamura; Osaka

Purpose: To evaluate the therapeutic effects of embolization of portocaval shunt for the patients with hepatic encephalopathy caused by hyperammonemia.

Methods and Materials: Two patients, aged 45 and 73, with one to two years history of recurrent consciousness disturbance and hyperammonemia in spite of liver dysfunction being mild were found to have collateral pathway; one from IMV to left renal vein and the other from SMV to the inferior vena cava. The tip of a catheter was inserted selectively into the shunt through IVC or portal vein, and then embolized with steel coils.

Results: Angiograms taken immediately after embolization showed that the shunts were completely occluded. Follow-up angiograms taken 4 to 6 weeks later showed that the shunt had been disappeared and new collateral pathways had not appeared. The blood ammonia level gradually decreased to normal and consciousness disturbance did not occur during follow-up, which was done in the first one year after embolization. Side effects and complications did not appear

Conclusions: Transcatheter embolization with steel coils of portocaval shunts is an effective and seemingly safe method to control hepatic encephalopathy in certain cases. It is easier and less invasive than surgical closure. 


\subsection{AM}

824 The value of imaging procedures for the assessment of Budd-Chiari syndrome

M. Bino, F. Antonucci, C. Zollikofer, M. Turina; Zürich

Purpose: Evaluation of radiological methods in diagnosis of Budd-Chiari syndrome for planning a hepato-atrial anastomosis.

Methods: 13 patients with the tentative diagnosis of Budd-Chiari syndrome were investigated by the following radiological methods:

Ultrasonography, CT, MRI, cavography with transcaval hepatic venography, celiacomesentericography, percutaneous transhepatic venography.

Additionally, for planning a hepato-atrial anastomosis, the optimal procedure for the visualization of non-occluded hepatic veins was evaluated.

Results: The diagnostic accuracy was as follows:

Ultrasonography $(\mathrm{n}=4 / 8,50 \%)$, CT $(\mathrm{n}=5 / 6,83 \%)$, MRI $(\mathrm{n}=2 / 2,100 \%)$, cavography with transcaval hepatic venography $(\mathrm{n}=5 / 10,50 \%)$, celiacomesentericography $(n=0 / 8,0 \%)$, percutaneous transhepatic venography $(n$ $=8 / 8,100 \%$.

The best visualization of non-occluded hepatic veins was done by percutaneous transhepatic venography.

Conclusions: Percutaneous transhepatic venography is best suited for the diagnosis of Budd-Chiari syndrome, supplemented by CT and MRI. When preparing the patient for a hepato-atrial anastomosis, the percutaneous transhepatic venography brings most useful information for the surgeon. Additional cavography is necessary to determine the amount of IVC obstruction.

\subsection{AM}

\section{Color Doppler sonography of portosystemic shunt}

C. von Itter, Th. Harder, P. Kolvenbach; Bonn

Purpose: This study was designed to investigate the utility of color Doppler sonography in the evaluation of portosystemic shunts.

Methods: 19 patients were imaged. The types of shunts examined included portocaval, 13 and distal splenorenal (Warren), 6. 17 investigations of portocaval and 7 investigations of Warren shunt were performed. Sonography was performed without knowledge of the status of the shunt. The results were correlated with clinical findings, angiography or MR imaging.

Results: Color Doppler imaging correctly inferred shunt patency in 11 cases and showed the anastomosis in 8 cases of portocaval shunts. In 6 cases the shunt could not be investigated because of ascites, meteorism, adipositas or dyspnoe. Color Doppler sonography successfully inferred shunt patency (3 cases) and thrombosis ( 2 cases) and showed the anastomosis in 1 case of Warren shunt. In 2 cases the Warren shunt could not be investigated.

Conclusions: Color Doppler sonography appears to be a promising new noninvasive method for the evaluation of portocaval and splenorenal shunts.

\section{$11.25 \mathrm{AM}$}

\section{Varicocele is a bilateral malformation}

Slobodan Radojković, D. Ljubisavljević, S. Lavrnić, C. Tulić, S. Mićić; Belgrade

The purpose of this study is to change a well established opinion that varicocele is a left sided malformation. By the method of superselective catheterisation and phlebography of internal spermatic veins we were able to prove that varicocele is a bilateral malformation in over $97 \%$ of randomly, or better to say of all investigated patients. Clinical diagnosis, supported by scrotal scintigraphy, was "bilateral" in less than $20 \%$ of cases. We have modified existing techniques of spermatic veins catheterisation, and this modification enables us to catheterise both sides successfully in such a high percentage of cases. We perform a therapeutic scleroembolisation in the same session in purpose to cure male infertility.

We conclude that our modification of catheterisation technique is very easy to be learned and when applayed permits a precise diagnosis and definitely better results, than up to day data show, in the treatment of varicocele as a cause of male infertility.

\section{$11.35 \mathrm{AM}$}

827 Early vascular thrombotic complications after combined pancreas and kidney transplantation in diabetics: diagnostic accuracy of duplex Doppler sonography

E. Angeli, P. L. Paesano, A. Vanzulli, A. DelMaschio; Milan

Purpose: early vascular complications often represent dramatic events for graft and patient survival. Their timely recognition could allow effective revascularization. We evaluated accuracy of duplex doppler sonography in diabetic patients after combined pancreas and kidney transplantation.

Methods: 22 transplanted patients were examined with duplex doppler sonography in the period Nov. '87-Dec. ' 90 ; 9 patients were clinically suspected of graft vascular thrombosis ( 8 pancreas, 1 kidney), because of sudden worsening of organ function. Clinical course, angiography and surgery were compared with US findings.

Results: at follow-up (clinical course, surgery, angiography) 4 patients had venous thrombosis of the pancreas, one had thrombosis of the renal artery; moreover, one had dehiscence of pancreatic venous anastomosis. US and duplex doppler detected all these vascular complications. In 2 of the other 3 patients with pancreatic malfunction, duplex doppler revealed vascular patency and increased vascular resistance due to rejection. In the last one we were able to visualize the enlarged graft but not its vascular pedicle.

Conclusions: in our experience duplex doppler sonography is a sensitive and reliable technique in the evaluation of graft vascular status, allowing an earlier detection of thrombosis which could lead to successful revascularization.

\subsection{AM}

828 Chemoembolization of hepatocellular carcinomas: personal experience

G. C. Ettorre, A.P. Garribba, G. Francioso, F. Mirizzi, S. Elba, A. Mossa; Bari

Transcatheter selective intra-arterial chemoembolization was established as an effective method for the treatment of unresectable hepatocellular carcinoma We report here our experience in 21 inoperable patients affected by hepatocellular carcinomas (HCC), bigger than $5 \mathrm{~cm}$ in diameter.

Adriamycin and mitomycin, together with lipiodol, were injected; a terminal embolization with gelatin sponge was then performed.

The patients were subjected to CT scans 3 weeks after treatment and every three months in order to control tumor size: that significantly decreased in 10 patients, did not change in 8 patients and increased in three patients only.

7 patients died from six weeks to 22 months after the first embolization.

In 4 patients the death was not due to the cancer, but rather to the complications of the underlying cirrhosis.

We believe that the superselective catheterization is able to save the hepatic parenchyma not yet affected by the tumor.

\section{$11.55 \mathrm{AM}$}

829 Intravascular US (IVUS): evaluation of tumor infiltration into the wall of large vessels

K. Lehner, P. Gerhardt, R. Blasini; Munich

Possibility of radical resection of malignant tumors is often limited by infiltration of major blood vessels. This infiltration of the vessel wall is very often not realized using all modern imaging techniques such as angiography, color doppler sonography, CT and MRI.

IVUS has been shown to be an accurate method of evaluating the structural changes of vessel walls by atherosclerosis and balloon angioplasty. In our first 20 applications of IVUS to patients with mediastinal-, liver- or other tumors of the abdomen a tumor infiltration of the vena cava, aorta and portal vein was proven or excluded with high accuracy. IVUS appears to be a potent adjunct to diagnostic tools in pretherapeutic tumor-staging.

\section{Room E2}

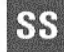

\subsection{Metabolic I}

\section{$10.30 \mathrm{AM}$}

830 Practical applications of musculoskeletal MRI in children (Keynote Lecture) Andrew K.Poznanski; Chicago, IL

MRI plays an important role in the diagnosis of various musculoskeletal disorders in children. Pediatric skeletal disorders often affect the ends of bones which are cartilagineous and are not visualized on radiography. Cartilage is particularly well visualized on gradient echo sequences where it has high signal. Ordinary T1 and T2 weighted spin echo sequences as well as post gadolinium infusions also have some applications. Obtaining quality MRI examinations in small children usually requires sedation and proper monitoring of the patient is essential to monitor potential respiratory depression. A pulse oximeter as well as respiratory and EKG monitors should be used.

In congenital disorders MRI can be very useful in determining the degree of deformity which may not be visible on the radiograph. For example, in infantile foot deformities one can determine the talonavicular relationships before the navicular is ossified. Similarly, MRI can give important information regarding the state of the proximal femur in proximal focal femoral deficiency. The shape of the ends of the bone in the various bone dysplasias can be determined with MRI and this may have important therapeutic ramifications. In acquired disease MRI is the method of choice in evaluating bone and soft tissue tumors and there are also many applications in rheumatic disorders. 
MRI permits the detection of early changes of synovitis, particularly when gadolinium DTPA is used. It is also useful in detecting small erosions, for showing early loss of cartilage, and in outlining synovial outpouching of cysts. In dermatomyositis it permits the localization of muscle involvement which is often spotty thus aiding in localizing the area for biopsy. It can also be used to evaluate the efficacy of therapy. MRI is useful in Calve-Legg Perthes disease both as a diagnostic tool but also in determination of the degree of coverage of the femoral head. Another important use is in determination of possible damage to the growth plate following trauma or infection and in detecting the amount of the growth plate which is closed which has implications regarding surgical management.

\section{$10.45 \mathrm{AM}$}

\section{The value of QCT in assessment of renal dystrophy} K. Wagiel, J. Walecki, J. Imiela, M. Zmijewska; Warsaw

Purpose: The purpose of the study was to determine the utility of QCT in evaluation of various stages of osteodystrophy.

Methods: X-ray and QCT (Picker 1200 Expert, $512 \times 512$ matrix, $140 \mathrm{kV}$, $95 \mathrm{~mA}$, slice thickness $10 \mathrm{~mm}$ ) examinations were carried out in 43 patients with renal failure undergoing dialysis.

Results: X-ray examinations confirmed osteoporosis in 24 cases (54\%), whereas QCT in 32 cases (74\%). The extent of osteodystrophy was varied. No evident correlation between the length of period of time since the first dialysis and the intensity of osteoporosis evaluated by QCT has been observed.

Conclusions: The results obtained show that properly conducted dialysis do not influence osteodystrophy. The correlation between X-ray findings and QCT is weak. QCT being a sensitive and repeateable method can prove itself to be a useful tool to monitor dialysis and assess the efficiency of treatment of osteodystrophy.

\section{$10.55 \mathrm{AM}$}

832 Low-dose measurement of vertebral mineral density with singleenergy quantitative $\mathrm{CT}$ : determination of practical limits and estimate of involved errors

R. Loose, E. Wetzel, T. Frank, F. Herbig, M. Georgi; Mannheim

Single-Energy Quantitative Computertomographie (SEQCT) is a well established method for determination of vertebral bone mineral density (BMD). In comparison with alternate methods (SPA, DPA, DPX), the advantage of SEQCT is a high geometric resolution and an individual selection of scan planes. Regions of interest (ROI) enable selective measurement of trabecular BMD without cortical structures in each vertebral body. A disadvantage of SEQCT with standard scan parameters is the patients total radiation dose of 500-3000 mrem.

Using a phantom of vertebral column and pelvis and a $\mathrm{K}_{2} \mathrm{HPO}_{4}-\mathrm{BMD}$ phantom $\left(0,50,100\right.$ und $\left.200 \mathrm{mg} / \mathrm{cm}^{3}\right)$ we analysed the contribution of systematic and statistical errors (Scanner General Electric CT/T 9800). Scans were performed with $5-200 \mathrm{~mA}$ anode current of the X-ray tube and a scan time of $4 \mathrm{sec}(20-800 \mathrm{mAs})$. Statistical errors were calculated by mean and standard deviation of 20 measurements for each set of parameters. Furthermore we determined the contribution of the size of ROI to the total error.

Our results demonstrate that measurements with a dose of less than $100 \mathrm{mAs}$ $(25 \mathrm{~mA})$ imply a significant statistical error. Also the size of the ROI determines the accuracy of the BMD-measurement. With a simple scan protocol the recommended minimal dose for any scanner/phantom combination can be determined. Thus the recommended dose for imaging (up to $400 \mathrm{mAs}$ ) can be reduced by $50 \%-200 \%$ without loss of accuracy.

\section{$11.05 \mathrm{AM}$}

\section{Determination of different rates of bone loss in postmenopausal} women by single-energy QCT

G. Reuther, M. Dören, R. Erlemann, P. E. Peters; Münster

Purpose: A follow-up study was performed to evaluate single-energy QCT in identifying increased rates of bone loss ("fast bone looser").

Methods and Materials: 54 untreated postmenopausel women without osseous symptoms were yearly examined by spinal QCT (L2-L4) for up to 4 years.

Results: Variation of vertebral cancellous bone density ranged from increases of $12 \mathrm{mg} \mathrm{K}_{2} \mathrm{HPO}_{4} / \mathrm{cm}^{3}$ to decreases of $18 \mathrm{mg} \mathrm{K}_{2} \mathrm{HPO}_{4} / \mathrm{cm}^{3}$ per year and exceed the statistical error of the measuring technique. Losses of more than $11 \mathrm{mg}$ $\mathrm{K}_{2} \mathrm{HPO}_{4} / \mathrm{cm}^{3}$ were not compensated for in following intervals by significant increases.

Conclusions: Single-energy QCT allows a differentiation between physiologic variations in vertebral bone density and uncompensated decreases. Within intervals of 2-3 years individuals with increased bone loss may be identified.
$11.15 \mathrm{AM}$

834 Improved assessment of osteoporosis and vertebral stability by texture analysis of vertebral bodies based on HR-CT

A. Mundinger, E. Dinkel, A. Helwig, A. Beck, B. Wiesmeier; Freiburg

Purpose: Purpose of our study was to assess morphometrical criteria of vertebral body texture in patients with normal and reduced bone density, as well as in osteoporotic patients with and without fractures.

Methods: Seventy-one women (64 postmenopausal) and 16 men were examined by one midvertebral high resolution CT scans through a non-fractured lumbar vertebra, and by osteodensitometry (SEQCT). Variations in the architecture of trabeculae and intertrabecular spaces were studied by a computer assisted imaging analysis system, and statistically analyzed.

Results: Percentual trabecular area as well as mean and variance of the thickness of trabecular plates and intertrabecular spaces were the most relevant texture parameters and showed characteristic age and sex dependent changes $(\mathrm{p}<0.01)$. Vertebral mineral content of patients with silent osteoporosis (without fractures) compared with patients suffering from manifest osteoporosis (with fractures) was similar. Both groups, however, showed a discriminating pattern of spongiosa texture changes $(p<0.05)$.

Conclusions: Vertebral texture analysis can assess remodelling changes of the spongiosa architecture and, in combination with osteodensitometry, promises further understanding of the osteoporotic process and individual risk for fractures.

\subsection{AM}

835 Agreements in the evaluation of bone density and mass assessments by means of computed tomography

A. Høiseth; Oslo

Purpose: Agreements, and not the error or measurements determine the usefulness of measurements. This paper focuses on the agreements and draws attention to problems of expressing agreements, with attention to traditional use of regression and correlation.

Results: Bone density and mass are assessed in femur specimens by means of $\mathrm{CT}$ and related to the Ca content measured chemically and to the mechanical strength. Data are analyzed, for the whole material and for subgroups, by regression and correlation and by paired differences for the actual measured values and for standardized values.

Results: For the subgroups and the whole material inconsistent results were obtained when data was analyzed by regression and correlation. Consistent results were obtained by calculation of paired differences of the standardized values.

Conclusions: The use of correlation coefficients and regression analyses are to a substantial extent influenced by the material. For this reason these methods do not give adequate descriptions of agreements. Paired differences between standardized values do not have these limitations, and are recommended.

\section{$11.35 \mathrm{AM}$}

\section{Diagnostic approach to osteopenia/osteoporosis - comparison} of two systems

H. R. Kistenich; Krefeld

For Diagnosis of Osteopenia/Osteoporosis in $1200 \mathrm{CT}$ scans (CIRS) women and men were performed 140 patients with diagnosed Osteoporosis are compared with values of BMC in HOLOGIC-QDR-1000 System.

- In 78\% different values were found - in QDR-1000 normal values versus pathologic results in CT scanning;

- the various values are discussed:

1. The different Reference Groups in all systems.

2. The various measuring systems and ways to the results

a) in volume versus square to find BONE MINERAL CONTENTS

b) in various phantoms and - only in CIRS-System - well defined BMC-Phantoms.

The most important and accurate Diagnosis to find in ROIs taking of changed trabecular structures in CT scanning.

3. The difficulties of interpretating dates taken by QDR-1000 of changed vertebra caused in broken trabecular structures and degeneratic alternations. - The Diagnosis by CT scanning shows highest accuracy in Diagnostic approach to Osteopenia/Osteoporosis.

\section{$11.45 \mathrm{AM}$}

837 Bone mineral assessments by computed tomography: is simultaneous calibration really necessary?

A. Høiseth, K. Tangen; Oslo

Purpose: A simultaneous calibration is required to correct a lack of from-scan-to-scan reproducibility. A valid simultaneous calibration requires 
that the object and the calibration phantom is subject to identical errors. The purpose of this paper is to evaluate: a) The need for simultaneous calibration in quantitative CT. b) To assess the prerequisite for a valid simultaneous calibration.

Methods: Short term inter and intra scanner variability was assessed on two different CT scanners. Using body phantoms of different densities, the densities were assessed in objects (located inside body phantoms) and in the calibration phantoms (placed in the usual location outside the body phantom). Results: The inter and intra scanner variability was small. By different body densities there was a substantial inconsistency between the measured density in the object and in the calibration phantom, showing a substantial variation in the measuring error in the two locations.

Conclusions: Any long term scanner variation or any inter scanner variation can be coped with by calibrations at regular intervals. There is however, hardly a need for making a calibration for each scan and the validity of such calibrations must be questioned.

\section{$11.55 \mathrm{AM}$}

838 Results of osteodensitometry in pre- and perimenopausal women R. Hauck-Schröder, R. Hauck, R. Busley; Munich

322 women up to the age of 55 underwent osteodensitometry (DPA/DPX) in the radiologic office.

In the premenopausal group (age under $42, n=17$ ) $41 \%$ showed asymptomatic but significantly decreased bone mineral density (BMD), caused in $46 \%$ by early ovarial insufficiency.

In the perimenopausal group (age $42-55, \mathrm{n}=305$ ) $25 \%$ showed oligosymptomatic pathologically decreased BMD, $67 \%$ of these pathologic results caused by early ovarial insufficiency or ovarectomy. The important role of the sufficient estrogen substitution controlled by osteodensitometry especially in the group with anamnestic early ovarial dysfunction is discussed.

\section{Room L \\ SS \\ 08.04 Facial skeleton}

$10.30 \mathrm{AM}$

839 Precision of three-dimensional CT-assisted model production in the maxillofacial area

Mario Traxler, Peter Solar, Christian UIm, Herwig Imhof, Georg Watzek; Vienna

Purpose: In the mid-80s, CT-assisted three-dimensional images and plastic models of skull structures were introduced into preoperative diagnostics in maxillofacial surgery. The aim of the present study was to collect information on the reproducibility of a skull model milled from hardened polyurethane foam.

Methods: The model was based on the CT data of a real skull. 21 comparative studies were carried out both, on the model and on the original skull.

Results: The model showed an average inaccuracy of $1,5 \mathrm{~mm}$. The deviations ranged between 0,1 and $3 \mathrm{~mm}$ - the general trend did, however, favour enlargements. As far as the total of all measurings is concerned, the deviation of the model as compared to the original skull was $2 \%$.

Conclusions: A convincing aspect of the model, which cannot be obtained by any other method, is its plasticity and the possibility of planning operations on a model in 1:1 scale. The model's inaccuracy of $1,5 \mathrm{~mm}$ average has no influence on the result of reconstructive surgery after trauma or tumor resection but makes the method not enough reliable for planning enosseous implantation in the lower jaw, where the inferior alveolar nerve could be damaged.

\section{$10.40 \mathrm{AM}$}

840 Tumors of the facial area: evaluation with 3D CT Robert Sigal, Guy Michel, Gérard Mamelle; Villejuif

Purpose: This study is aimed at evaluating the respective advantages and drawbacks of 3D CT in patients presenting with malignant tumors adjacent to the skull base.

Methods and Materials: 35 patients with malignant tumors of the facial area, nasopharynx and parapharyngeal spaces were prospectively studied. $2.5 \mathrm{~mm}$ thick, overlapping slices were obtained with iodine infusion. 3D CT (Excel 2400 Elscint) was used for bony and soft tissues volumetric display. Trackball driven software allows for rotation and pealing of the facial structures at various angles. Console manipulations were done both by radiologists and surgeons. MR imaging was available in most cases. Correlation with intraoperative findings was obtained in 18 cases.

Results: 3D CT is less accurate than MR in the delineation of the meningeal and cerebral extension as well as cavernous sinus and perineural involvement. Differentiation between tumor and inflammation is not optimal. 3D CT clearly visualizes the skull base orifice, but overestimates the osseous extension (false holes). In fact, the major advantage of $3 \mathrm{D} \mathrm{CT}$ is to offer to surgeons a volumetric display of the tumor with clear depiction of its relationship to adjacent structures. This is particularly useful for tumors located in the infratemporal fossa.

Conclusions: 3D CT does not constitute a valuable tool in the depiction of facial tumors, since it is less sensitive than MR. However it is a valuable tool for pre-operative planning, since it allows the surgical team to perform assisted computed simulated intervention.

\section{$10.50 \mathrm{AM}$}

841 3D-MRI of head and neck tumors: clinical studies and direct operative correlation

Th. Vogl, C. Wilimzig, J. Assal, G. Laub, J. Lissner; Munich

Purpose: Several attempts have been made to develop a new technique that offers the possibility to visualize the third dimension. Particularily the possibility of preoperative planning and radiotherapy planning by this technique could be a new dimension of the diagnostic value of the MR imaging-technique. In our studies 3D-MRI was performed by implementing topographical structures in a 3D-reconstruction of the whole head.

Methods and Materials: Twenty-one healthy volunteers and fourty-two patients with head and neck lesions were so far primarily examined with standard 2D MR imaging and additional 3D-Flash Imaging on a $1.0 \mathrm{~T}$ and a 1.5 T MAGNETOM. The patients were measured before and after application of Gd-DTPA. The 3D-reconstruction of the data cube for visualization of inner parts of the 3D-reconstructed head performed at a separate processing unit. All 3D-reconstructions were compared by surgical findings.

Results: The diagnosis included benign lesions as well as malignant lesions of the head and neck. The application of the contrast medium Gadolinium-DTPA proved to be helpful in $82 \%$ of the cases. 3D-reconstruction revealed clear topographic details of the lesions, including the spread of infiltration and destruction of soft tissue. Especially the demonstration of the reconstructed images by preoperative movie-technique enabled us to simulate the surgery showing the important topographic structures.

Conclusions: The important aspects of the lesions are much easier to evaluate by $3 \mathrm{D}$-reconstructed images than by mentally reconstructing the morphology from individual slices. Methods for the direct display of the 3D-reconstructions during surgery have to be further investigated as well as the value of this method in radiotherapy planning.

\section{$11.00 \mathrm{AM}$}

\section{High resolution CT: pre-operative assessment of chronic rhino-} sinusitis

J. C. Ferrie, P. Vandermarcq, O. Azais, J.M. Klossek, J. Drouineau, D. Barret, C. Gasquet; Poitiers

The better understanding of chronic rhinosinusitis and the advance of endoscopic endonasal surgery have modified the radiologic pre-operative investigation.

The ethmoid labyrinth not accessible by the endoscopic way can be explored by axial and coronal high-resolution thin section CT $(2 \mathrm{~mm})$. One hundred CT scans with endoscopic correlation have allowed to assess the accuracy of this pre-operative approach.

This anatomical study shows up the presence and the extent of paranasal sinus diseases. The beginning of these diseases wich is usually focused in the areas of the ostiomeatal unit and anterior ethmoid, is demonstrated. The visualisation of anatomical variations, that have been reported to predispose sinusitis, is always possible. They are located in the nasal septum, the middle turbinate, the unciform process or the cells of unciform process and the ethmoidal bulla. The endoscopic endonasal surgery landmarks, the individual morphological variations and the relationships of the ethmoid are well shown by CT.

This precise radiologic pre-operative assessment is necessary for a safe surgery.

\section{$11.10 \mathrm{AM}$}

843 Two year's experience with a multimodality equipment for maxillofacial radiology

P. Paukku, D. Hallikainen, T. lizuka, J. Edgren, C. Lindqvist; Helsinki

Purpose: To evaluate clinical usefulness and application areas of a piece of equipment performing narrow beam radiography and spiral tomography of the maxillofacial area.

Methods: The material consists of 2095 patients referred to radiological examination by the department of maxillofacial surgery during 4.2. 1988-4.2. 90. The radiological procedures and diagnoses were classified. The effect of the radiological statement on clinical treatment as well as clinical confirmation of radiological findings were recorded.

Results: Classification of diagnoses: Trauma 18\%, tumours $15 \%$, cysts $8 \%$, TMJ disorders $11 \%$, diseases of dental origin $30 \%$. Radiological procedures: Panoramic examinations covered $79 \%$ and $21 \%$ consisted of tomography in 
one or two directions and detailed images. Tomography was especially useful in cases of osteosynthesis, where metal artefacts impaired the CT images. Conclusions: A multimodality equipment is effective in maxillofacial radiology. Close co-work between radiologists and clinicians improves the results.

\section{$11.20 \mathrm{AM}$}

844 Performances of a storage phosphors digital radiography system applied to orthopantomography

P. Burci, C. Malagola, R. Stamati, M. Miceli, S. Sartoni Galloni, A. Pastremoli; Bologna

Purpose: A comparative study between conventional radiography (CR) and digital radiography (DR) was performed, in order to evaluate the performances of a photostimulable phosphors DR system (Philips - PCR Graphics 1) in orthopantomography.

Methods: Orthoralix Philips was used (12 sec. rotation time, $60-80 \mathrm{kV}, 4-14$ $\mathrm{mA}$ ) utilizing, at the same time, plain film cassette $(15 \times 30 \mathrm{~cm})$ and $D R$ imaging plate $(15 \times 30 \mathrm{~cm}, 1576 \times 1976 \times 12$ bits matrix, $0.15 \mathrm{~mm}$ pixel size, $3.3 \mathrm{lp} / \mathrm{mm}$ ). Four Radiologists tested the better detectability of: temporomandibular joint (condilons, glenoid, zygoma); paranasal sinuses; apex of upper and lower molars, bicuspids, canines, incisors; mandibular nerve, on 50 examinations performed with both CR and DR.

The statistical analysis used a binary alternative test.

Results: DR system generally was shown to be superior in $30 \%$ of cases and the same of CR in $60 \%$ of cases. Nevertheless in particular anatomical structures DR demonstrated a much better diagnostic accuracy (incisors area, gum soft tissue).

Conclusions: Photostimulable phosphors DR system, already routinary applied in our Department to thoracic and skeletal examinations, can provide very high performances in orthopantomography.

Moreover dose absorption data examined after exposition of a human phantom show that exposition more than 50\% reduced on DR allows to obtain images as good as CR ones.

\section{$11.30 \mathrm{AM}$}

\section{High resolution $\mathrm{CT}$ in the diagnosis of periodontal disease}

\section{H. Schüller, M. Frentzen; Bonn}

Purpose: Traditionally periodontal diseases are documented with orthopantomograms (OPG) and introral films (IOF). With these techniques it is difficult to analyze the amount and location of bone loss on all surfaces of the teeth roots especially buccal, palatal, lingual and in the bifurcation.

Methods: In this prospective study 10 patients with extensively documented periodontal disease were examined using OPG, IOF and high resolution CT (HRCT). HRCT slices of $1.5 \mathrm{~mm}$ thickness parallel to the occlusal plane were performed.

Results: All bony lesions shown in OPG and IOF were detected in HRCT Bone loss at the bifurcation was visualized with superior sensitivity on HRCT. The amount of bone loss could be measured without geometric distortion. Summary: Using HRCT the status of periodontal disease can be assessed with minimal patient discomfort. HRCT is superior to OPG and IOF in the diagnosis of periodontal disease of the maxilla. The amount of bony destruction around the roots of the teeth can be measured quantitatively without probing the desmodontal space.

\section{$11.40 \mathrm{AM}$}

846 Clinical and radiologic follow-up on mandibular condyle fractures S. D. Bianchi, F. Tarello, G. C. Cortese, G. P. Vaudano, G. Ramieri; Torino

A group of 86 patients treated for mandibular condyle fractures have been considered in this study in order to investigate the radiographic morphology of condylar remodelling and of its correlation with functional scores.

The patients were examined with regard to sex, age, other mandibular or maxillary fractures, the condylar head dislocation, both medio-laterally and antero-posteriorly, the mandibular ramus shortage and the mandibular laterodislocation. Patients were then clinically reevaluated and the following parameters were recorded: laterodeviation, opening, protrusion, laterotrusion, protrusive and opening deviation, TMJ sounds, pain and malocclusion. By means of orthopantomogram and PA radiography at follow up the condylar position was compared with the controlateral side and with the post-trauma location. CT scans has been used for morphological investigation of condylar remodelling and articular space, arthrosis and ankylosis evaluation. The different radiographic appearances of fracture sites and dislocation were used for dividing the cases in different classes. In these classes, both clinical and radiographic data were measured by means of the ANOVA and multiple correlation investigation statistic.

The results are very interesting and similar to the ones reported by literature: a larger and proper use of CT is stressed.

\section{Evaluation of orofacial bone destructions with 3 D-CT} C. Zwicker, M. Langer, B. Erdtmann; Berlin

Purpose: To evaluate in a prospective study the quality and the impact on therapy planning of $3 \mathrm{D}$-CT for tumorous osseous destructions and fractures of facial and oral bones.

Methods and Materials: 52 patients were investigated. The examinations were performed with the Somatom Plus, Siemens and $2 \mathrm{~mm}$ overlapping slices $(0,7 \mathrm{~s}$ scanning time, $120 \mathrm{kV}, 60 \mathrm{mAs} / \mathrm{scan}$ ) were used. The time for investigation and 3 D surface reconstruction was $30-45 \mathrm{~min}$, so that this procedure could be performed routinely.

Results: For 24 patients very good image quality was obtained. Metal artifacts reduced image quality in 25 patients, but image quality was still diagnostically sufficient. Only in 3 uncooperative patients the $3 \mathrm{D}$ images had no adequate quality.

Conclusions: 3 D-CT reconstruction proved to be of useful in therapy planning and control for patients with orofacial bone destructions.

\section{$11.53 \mathrm{AM}$}

\section{Computed tomography and alveolar pockets - first results} R. Kaczmarek, P. Meriot, J. Abjean, A. Renault, M. Bellet; Quimper

Computed tomography is performed in adults undergoing endodontic treatment for deep periodontal lesions (alveolar pockets), to evaluate this technique versus routine dental radiographs.

All scans were performed on an Elscint 2400 scanner. After scoutviews were performed, axial, contiguous $1.5 \mathrm{~mm}$ scans, parallel to the hard palate, were obtained. In some cases, coronal scans were done. In addition, reformatted images were post-processed first results suggest computed tomography to be useful in evaluating patient's denture status, alveolar crest height, extent of the alveolar bone defect. It allows recognition of endo-antral syndrome (E.A.S.) and also the course of the inferior alveolar nerve canal.

So, a new radiograph algorithm including computed tomography is able, to reduce the number of routinely taken radiographs in periodontal disease (alveolar pockets) and modify the approach of endodontic treatment.

\section{Room M}

\section{SS 05.08 Penis and urethra}

$10.30 \mathrm{AM}$

849 Velocity-time diagrams for the evaluation of penile blood flow in erectile dysfunction

P. Hübsch, N. Nürnberger, S. Trattnig, D. Pölzleitner, F. M. Kainberger; Vienna

Purpose: Although pulsed Doppler measurements of blood flow in the penile arteries are extensively used to assess vasculogenic disorders causing erectile dysfunction, there is until now no standard examination technique. In this paper, we suggest a new examination modality on the basis of color coded Doppler sonography.

Methods: The patient is examined in seating position. At the beginning, blood flow in the cavernosal arteries is assessed in the flaccid state. Then, Prostaglandine $\mathrm{E}_{1}$ is injected in a dosage, which has been ascertained before (dosage needed for maximum erection achievable without discomfort). Afterwards, systolic and diastolic blood flow velocity in both cavernosal arteries is measured by turns during 20 minutes (every minute at least one measurement / vessel). Additionally, the tumescence and rigidity of the penis is graded $\left(\mathrm{E}_{0}-\mathrm{E}_{5}\right)$. The results are displayed in a diagram ("Velocity-Time-Diagram"). Results/Conclusions: With the method explained above, the disadvantages of single measurements are compensated by their multiplicity. The diagram better reflects the dynamic process of erection; possible errors of the "single measurement technique" are eliminated. Color coded Doppler sonography is mandatory for the technique described above.

$10.40 \mathrm{AM}$

850 (CDS)

K. Brandstetter, K. Mühlbauer, J. U. Schwarzer, J. Dörrler, J. Gmeinwieser; Munich

25 cases of erectile dysfunction were studied with $\operatorname{CDS}(7.5 \mathrm{MHz}$, Acuson) before and after intracaverosal injection of papaverine. Penile vascular anatomy was mapped and measurements of maximum systolic velocity were performed. In $15 / 25$ patients internal pudendal angiography was performed as gold standard.

CDS was $100 \%$ sensitive in detecting penile arteries. In 14/15 patients CDS 
and angiography led to identical evaluation of penile blood supply. We conclude that CDS is a sensitive non-invasive method for evaluation of suspected arteriogenic erectile dysfunction.

\section{$10.43 \mathrm{AM}$}

\section{Pharmacodynamic high-resolution MR imaging in erectile dysfunction \\ Th. Vestring, J. Sulke, G. Bongartz, J.Adolph, C. Drews, P.E. Peters; Münster}

Purpose: Evaluation of MRI in the assessment of erectile dysfunction.

Methods and Materials: High-resolution MRI (surface coil, FOV: 80-100 mm, slice thickness: $3 \mathrm{~mm}, \mathrm{~T} 1$ - and T2-weighted spin-echo pulse sequences, 1.5 Tesla) of the penis was performed in 15 patients with erectile dysfunction (psychogenic: $\mathrm{n}=5$, organic: $\mathrm{n}=10$ ) before and after intracavernosal injection of 5-15 micrograms prostaglandine El.

Results: After prostaglandine application the cavernous corpora demonstrated higher T2-values in all cases. The diameter of the cavernous arteries generally increased from $1 \mathrm{~mm}$ to $2 \mathrm{~mm}$ in psychogenic $(\mathrm{n}=5)$ and neurogenic $(\mathrm{n}=5)$ erectile dysfunction, but failed to enlarge in arterial $(n=5)$ erectile dysfunction.

Conclusions: MRI can differentiate between the arterial form and other causes of erectile dysfunction.

\section{$10.46 \mathrm{AM}$}

852 Gd-enhanced sequential MR-imaging of the penis in patients with erectile dysfunctions

B. Wein, M. Sohn, K. Bohndorf, K. Ch. Klose; Aachen

Purpose: A new functional approach was studied by observing penile arteries' flow signal and the inflow of Gd-DTPA into the corpora cavernosa in $10 \mathrm{sec}$ intervals using MRI-FLASH-2D sequences.

Methods and Materials: The MRI-investigations were performed with a superconducted 1.5 T Magnetom and a surface-coil $(12 \mathrm{~cm} \varnothing)$. FLASH-2D echo gradient sequences $\left(\mathrm{TR}=35 \mathrm{~ms}, \mathrm{TE}=12 \mathrm{~ms}\right.$, Flip angle $=60^{\circ}$ ) were applied in the coronal plane through the very anterior part of the symphysis. 12 to 15 minutes after injection of $20 \mu \mathrm{g}$ Prostaglandin E1 or $25 \mathrm{mg}$ Papaverin into the corpora cavernosa inflow of Gd-DTPA (i.v. administration, $0.2 \mathrm{mmol} / \mathrm{kg}_{\mathrm{BW}}$ ) was observed every $10 \mathrm{sec}$ for 3.5 minutes. One late image was obtained $10 \mathrm{~min}$. post i.v. injection. 65 investigations were performed in 50 patients with proven organic dysfunction.

Results: In more than $75 \%$ of the examinations flow signal of penile arteries corresponded to findings in angiography and intraoperative dopplersonography. Calculated Gd-DTPA-uptake and intracavernosal Gd-distribution depended closely on etiology (e.g. fibrosis, angiopathy and intracorporal microangiopathy).

Conclusions: Gd-enhanced MRI of penis may give additional and more specific informations about causes of erectile dysfunction due to anatomical and/or vascular abnormalities.

\section{$10.56 \mathrm{AM}$}

\section{Colour and pulsed Doppler in male impotence}

G. Corsi, C. Del Boca, C. Ferrari, A. C. Giuberti, G. Raieli; Lodi

The vascular mechanism of tumescence has been exhaustively explained by angiography/cavernosography combination. Colour (CD) and pulsed Doppler (PWD) combination represent a valuable alternative in term of accuracy and non invasivity. We have studied 48 patients with vascular male impotence and 12 normal subjects using CD to map the anatomy of the penile vasculature combined with PWD measurement of the systolic peak velocity (Vmax), the end diastolic velocity (Vmin) and the pulsatility index (PI), before and after the intracavernous administration of $30 \mathrm{mg}$ of papaverine sulphate. According to the literature we graded the rectile response in 5 steps $(0-4)$. In normal subjects we found a Vmax range to be $0.50 \mathrm{~cm} / \mathrm{sec}$, mean $+-\mathrm{SD}=$ $13.5+-9.8$ and $V \min : 0-11 \mathrm{zcm} / \mathrm{sec}$, mean $+-\mathrm{SD}=0.36+-1.2$ before papaverine injection and we found a Vmax range to be $26 \cdot 108 \mathrm{~cm} / \mathrm{sec}$, mean $+-\mathrm{SD}$ $=37.2+-9$ and Vmin: $0 \cdot-6.4$, mean $+-S D=-3.2+-2.5$ after pharmacological stimulation (grade 4)

In 21 patients with known generalized arterial disease only 2 bad Vmax of greater than $30 \mathrm{~cm} / \mathrm{sec}$ (range: $5 \cdot 31$, mean $+-\mathrm{SD}=7+-9$ ) and only 1 had $\mathrm{Vmin}$ greater than $5 \mathrm{~cm} / \mathrm{sec}$ (range: $-5 \cdot 6 \mathrm{~cm} / \mathrm{sec}$, mean $+-\mathrm{SD}=-2.8+-2.4$ ) In 27 patients with venous leaks, confirmed by cavernosography, only 1 had $V \max$ less than $30 \mathrm{~cm} / \mathrm{sec}$ (range $28 \cdot 60 \mathrm{~cm} / \mathrm{sec}$, mean $+-\mathrm{SD}=45+-13$ ) and two had $V \mathrm{~min}$ less than $8 \mathrm{~cm} / \mathrm{sec}$ (range: $6 \cdot 24,5 \mathrm{~cm} / \mathrm{sec}$, mean $+-\mathrm{SD}=$ $12+-10.7)$.

CD and DWP combination, executed before and after papaverine stimulation, is an accurate way to evaluate male impotence, because of its ability to differentiate the three main causes of male impotence: arteriopathic, venous and psychogenic, without the invasivity of both angiography and cavernosography.

\section{$11.06 \mathrm{AM}$}

\section{Ultrasound and MRI in the diagnosis of penile induration} (Peyronie's disease)

G. Helweg, K. Wicke, W. Judmaier, D. zur Nedden, O. Ennemoser; Innsbruck

Purpose: Penile induration, a disease of connective tissue, requires accurate delineation and differentiation of inflammatory changes (plaques) for an accurate Ontosein ${ }^{R}$-therapy. In order to evaluate the diagnostic impact of MRI a prospective study was initiated.

Methods and Materials: 58 patients with IPP (Kelamy classification) underwent ultrasound (Siemens 7,5 MHz), 25 of them MRI (1,5 T, Magnetom Siemens). For MRI a $15 \mathrm{~cm}$ surface coil was used and $\mathrm{T} 1$ and $\mathrm{T} 2$ weighted images had been acquired in all patients, in 9 cases additional $\mathrm{Tl}$ images were performed after application of Gd DTPA $(0,1 \mathrm{mmol} / \mathrm{kg})$ within 4 weeks.

Results: MRI was more sensitive in detection of inflammatory changes (T2 prolongation and moderate GD DTPA enhancement) $63 \%$ MRI versus US $47 \%$. Plaques were identified by US $100 \%$ versus MRI $71 \%$.

Conclusions: US is the modality of choice in diagnosis of IPP consisting of the overall sensitivity to plaques, MRI on the other hand may offer valuable additional information in case of inflammatory changes, especially by use of Gd DTPA.

Therefore MRI has the potential to improve therapeutic planning and should be used in monitoring Ontosein ${ }^{\mathrm{R}}$ effects.

\subsection{AM}

\section{Endovaginal sonography for urethral diverticula}

R. Oyen, I. Reymen, P. Willemen, L. Baert, A. L. Baert; Leuven

Purpose: To compare the diagnostic accuracy of micturation urethrography, high-pressure double balloon urethrography and vaginal sonography in patients with recurrent lower urinary tract infections.

Methods and Materials: The 3 exams were performed in 47 patients. For the retrograde high pressure urethrography a Davis type catheter was used. Micturation urethrography was performed in an upright, slightly oblique projection, the endovaginal sonography in the supine position using a $7 \mathrm{MHz}$ transducer.

Results: Diverticula of the urethra were seen in 6 patients. In 2 patients the diverticulum was not seen at micturation urethrography. In 1 patient the diverticulum was not filled at the high pressure urethrography. All diverticula were recognized sonographically. The content and the wall of the diverticula were evaluated. 2 diverticula were filled up with pus, therefore not seen on the retrograde urethrogram. 1 patient had several urethral diverticula and an invasive adenocarcinoma, probably originating in a diverticulum. 2 Diverticula were punctured under sonographic guidance for bacteriologic examination, contrast studies, or injection of dye blue or gel to facilitate surgery. In 2 patients the diagnosis of bullous trigonitis was made sonographically, in 4 urethritis was diagnosed. Additionally in 1 patient a retention cyst of Bartholin's gland was correctly made sonographically.

Conclusions: Endovaginal sonography is recommended when urethral pathology is suspected.

\subsection{AM}

856 Stenosis of penile and bulbar urethra: diagnosis by ultrasound V. García-Medina, J. D. Berna, J. Llerena, J. García-Medina, J. L. Rico, N. Tomás, J. Guirao, J. L. Genovés; Murcia

Purpose: We evaluated the role of urethral sonography as an alternative technique top conventional retrograde and or voiding urethrography in the diagnosis of penile and bulbar urethral stenosis.

Methods and Materials: Sonography examination of the urethra was performed using a $7.5 \mathrm{MHz}$ and $10 \mathrm{MHz}$ transducer that is applied over the penis. The lumen of the urethra was well seen after the instillation of 6 of $7 \mathrm{cc}$ of sterile saline within the distal portion of the urethra. Dynamic sonographic examination of the urethra was also performed while the patient was voiding. In both instances the anechoic fluid within the urethral lumen gave us an excellent view of the penile and bulbar portions of the urethra.

Results: Fourteen patients with ages ranging between 35 and 68 years underwent urethral sonography for suspected stenosis. All the patients had routine retrograde and voiding urethrography after urethral sonography. Complete correlation between the results of urethral sonography and conventional uretrography was found among the 10 patients with stenosis in the penile urethra. Three of four (75\%) of the bulbar stenosis were correctly diagnosed by urethral sonography. There were no complications related to the procedure. 
11.29 AM

857 Echographic evaluation of anterior urethra

D. Mancino, A. M. Giambersio, V. Barile, M. Mancino; Potenza

Echomorphologic features of penile and bulbous urethra of 30 patients with congenital or acquired urethral stenoses (Group A) and of 10 patients without urethral obstructive pathology (Group B) were prospectively analyzed by means of high-resolution realtime ultrasonography (US). All patients underwent conventional cystourethrography (CU). Urethral US was performed in longitudinal and transverse planes with a $7.5 \mathrm{MHz}$ linear transducer during retrograde urethral infusion of physiologic solution. The characteristics of normal anterior urethra were identified in the patients of Group A. All the strictures observed at CU were clearly demonstrated at US; moreover US allowed a more accurate evaluation of the strictures such as urethral gauge, urethral wall and length of the stenoses. We conclude that US gives more informations in the study of stenoses of antherior urethra than conventional CU; the method is particularly helpful in the evaluations of therapies (e.g. dilatations) and in the identifications of recurrences.

\section{$11.32 \mathrm{AM}$}

858 A new treatment for recurring urethral strictures: temporarily implanted long-term urethral stent-Urocoil ${ }^{\mathrm{P}}$ Daniel Yachia, Mordechay Beyar; Hadera

A new urethral stent developed for use in non-surgical treatment of recurring urethral strictures is presented. This stent which is made of medical grade stainless steel alloy is self-expanding and self-retaining when released from its introducer reaching its maximal caliber of $26 / 32 \mathrm{~F}$ by radial expansion to its wavy form.

This stent was used in 55 patients who had recurrent strictures necessitating frequent dilatations or urethrotomies. The stent is introduced under fluoroscopy after adequate dilatation of the stricture either using high pressure urethral balloons or visual urethrotomy to accomodate the stent. Strictures of $0.5-8.0 \mathrm{~cm}$ long were treated successfully with this device. All strictures were distal to the external sphincter. No serious complications which could be attributed to the device were recorded. When necessary repositioning could be done either under fluoroscopy or endoscopically. The large internal caliber of this stent allows passage of instruments up to $17 \mathrm{~F}$ into the bladder. It can be removed anytime either by threading a small caliber Foley catheter (12-14F) through the stent and inflating the balloon with $3-4 \mathrm{ml}$ of saline and pulling the catheter outwards together with the stent; or endoscopically. Either the insertion or the removal is done under topical anesthesy of the urethra using $2 \%$ Lidocaine jelly for 10 minutes.

During our mean followup period of 14 months (range 3-24 months) no incrustations were observed in the stents and no stent became incorporated into the urethral wall. They were removed $6-8$ months after insertion. $88 \%$ of the patients remained free of stricture 6-16 months (mean 11 months) after the removal of the stents. The video presents the stent and the technique for its insertion and removal.

\section{$11.42 \mathrm{AM}$}

\section{A new, self-expanding and self-retaining temporary, long-term} intraprostatic stent: Prostacoil ${ }^{\mathrm{R}}$

Daniel Yachia, Mordechay Beyar; Hadera

Currently 3 different intraluminal stents are available for stenting the obstructed prostatic urethra which can be used instead of indwelling catheters: The metallic spiral stents with fixed caliber (20F) based on the spiral developed by Fabian; an intraurethral catheter with an external caliber of $16 \mathrm{~F}$ developed by Nissenkorn and the permanent, expandable woven metallic stent with large caliber $(32 \mathrm{~F})$ developed by Wallsten (originally developed for vascular use). The first 2 stents have an external configuration which do not fit well to the configuration of the prostatic urethra and in many cases it may migrate either into the bladder or into the anterior urethra. Their small caliber do not allow passage to instruments through their lumen when the need arises for bladder evacuation or endoscopic examinations. The third device allows passage to instruments through its lumen but becomes completely imbedded into the urethral epithelium within 4-6 months becoming permanent implants. Their removal necessitates traumatic surgical manipulations. This limitations led us to develop a new, temporary, self-expanding and self-retaining large-caliber $(26 / 32 \mathrm{~F})$ stent which is inserted under fluoroscopy in reduced caliber (20F) When released from its introducer it expands radially to its maximal caliber in a wavy form.

During the last 2 years this stent was inserted to 35 surgically poor risk patients who needed indwelling catheterization. Most of these patients $(90 \%)$ could pass urine spontaneously with good sphincteric control within $24-48$ hours after insertion of the stent.

The video presents the new stent and the technique of insertion and removal.
$11.52 \mathrm{AM}$

860 Urodynamic U.S. study of anterior urethral stenosis before and after surgery: comparison with micturional cystourethrography P. Corpino, G. Carro, G. F. Chabert, F. Floris; Cagliari

The authors tried to give a correct evaluation of the urethra during micturional phase in patients with anterior urethra stenosis before and after surgery. 22 patients were studied both with micturional cystourethrography and with U.S. so as to visualize the various portions of the urethra; the transrectal probe can demonstrate the prostatic portion and the 7.5 probe with oil bath the anterior urethra. This method allowed U.S. to identify correctly both the length and the calibre of the stenosis in the anterior urethra and in the post bulbar tract, this last one often due to prostatic problems.

Comparison between echography and traditional radiology showed higher precision with the former, this, together with the absence of radiation makes it the first choice for urodynamic study in anterior urethral obstructions.

Post surgical controls have confirmed the value of the method with the clear sonographical evidence of the removal of the obstruction.

$11.57 \mathrm{AM}$

861 Use of a self-expandable stent in the therapy of the recidivous stricture of the urethra

D. Liermann, W. W. Meyer, W. Boeckmann, J. Kollath, D. Jonas; Frankfurt/Main

Purpose: The recidivous stricture of the urethra of the man is a common problem. Recurrences often appear independent from the genesis of the primary stricture. In some cases it was necessary to underwent 6 and more recidivous strictures surgical treatment in a short interval. The use of the stent technique allows an easier to manage and cheaper alternative.

Methods: In case of recidivous stenosis in the proximal urethra except the pars pendulans we dilate the urethra up to 30 Charière and deliver afterwards the wallstent which expends itself to a maximal diameter of $10 \mathrm{~mm}$. Up to now only patients will be treated which are elder than 20 years and developed the third recurrence of urethrastricture.

Results: All patients which have been treated with this method remain without any recurrence over a period longer than 12 months.

Conclusions: In case of multiple recidivous stenosis in the urethra of the man transurethral stent implantation will be more effective than several time surgical treatment and the result after stent will be better than any other treatment before.

\subsection{AM}

862 The Nissenkorn prosthesis as a nonsurgical alternative in the treatment of urinary retention

V. Garcia-Medina, J. D. Berna, M. M. Tomas, J. Garcia-Medina, J. L. Genoves, J. Llerena, W. R. Castañeda-Zuñiga; Murcia, Minneapolis, MN

We evaluated the role of the Nissenkorn endourethral prosthesis as a new alternative treatment of urinary retention in high-risk surgical patients.

40 patients (mean age: 68 years) with symptoms of urinary retention secondary to $\mathrm{BPH}$ (36) or prostate cancer (4) underwent endourethral placement of a IGH polyurethane double Malecot prosthesis at the prostatic urethra with the aid of an introducer sheath.

In all cases the prosthesis was placed in adequate position without immediate complications. Follow-up evaluations with retrograde and voiding cystourethrograms, urodynamics studies, urethroscopy and urine cultures were performed 24 hours and seven days post-procedure and then monthly. At this time we have followed-up these patients between two and twelve months. All of them have shown radiographic and urethroscopic evidence of adequate patency of the prostatic urethra with no evidence of residual urine within the bladder. No cases of infection have been found.

\subsection{AM}

\section{Retrograde urethrography with controlled pressure} Sandor Lovasz, György Nádas; Budapest

A well known disadvantage of the usual method of retrograde urethrography is that the intraurethral pressure is unknown, perhaps sometimes unnecessarily high, which may de dangerous. Iatrogenic infections and injuries may occur. Our new method of retrograde urethrography allows a continuous control of filling pressure of the contrast medium. This method offers the possibility to produce X-ray picture series made with stepwise rising pressure. These visualize most clearly the elastic end rigid parts of the urethra. Pressures higher than $50 \mathrm{H} 20 \mathrm{~cm}$ do not improve the diagnostic value of the procedure and may cause extravasates and represent a higher risk of iatrogenic infections. The method of controlled pressure helps to follow up the patients with urethral lesions or after internal urethrotomy because only the X-ray pictures made by the prescribed way are really comparable to each other. With this method the 
doctor may be absent from the direct beam of X-rays thus decreasing the radiation dose of the examining doctor. Preventing the extreme high pressures the subjective complain of the patients may be eligible.

\section{Room N}

\section{SS 07.06 Vascular II}

$10.30 \mathrm{AM}$

\section{MR angiography in cerebral lesions (Keynote Lecture) K.Voigt, D. Petersen; Tübingen}

MR-angiography with 3D- and 2D-inflow techniques and maximumintensity-projecions displays vascular anatomy not only of arteriovenous malformations but also of vascular anomalies, aneurysms, stenoses and occlusions. The acquisition is noninvasive and postprocessing allows projections in additional directions, which cannot be achieved by conventional DSA. To prevent misinterpretation of MR angiography it is essential not to confuse it with DSA, even if the images in some instances may look similar. Knowledge of MR flow phenomena and especially of saturation effects are the key to clinical understanding of inflow MRA. Examplary investigation results of volunteers and patients demonstrate the influence of sequence parameters and volume selection on depiction and contrast of cerebral vessels, leading to different indications for 3D- und 2D-techniques. Furthermore, for high resolution imaging of slow-flow-vessels such as the cerebral veins the advantages of postcontrast 3D-MRA are demonstrated.

\section{$10.45 \mathrm{AM}$}

\section{MR-angiography of intracranial aneurysms: a prospective comparison to X-ray angiography}

S. Felber, W. Judmaier, F. Aichner, G. Birbamer, H. Lehner; Innsbruck

MR-Angiography (MRA) has recently matured to a routine modality and first results on intracranial aneurysms have been reported. Our purpose was to evaluate sensitivity and specificity prospectively in patients with subarachnoid hemorrhage $(\mathrm{SAH})$

38 patients suffering from SAH underwent MRA supplementary to routine MRI. All were consciousness and cooperated into the examination. Transfemoral X-Ray angiography served as "gold standard". Another 11 patients with evidence of incidental aneurysm on MRI/MRA underwent confirmatory X-Ray AG. MRA was performed on a $1.5 \mathrm{~T}$ system (Siemens, FRG) using a circular polarized head coil and flow compensated 3D-FFT gradient-echo sequences (FISP, TR: $40 \mathrm{~ms}$, TE: 7-9 ms, : $15^{\circ}$, ptt $=64$, $\mathrm{s} 1=0.8-1.2 \mathrm{~mm}$ ). 19 (38) patients had no evidence of aneurysms on MRA and repeated X-Ray AG thus $\mathrm{SAH}$ was proven by $\mathrm{CT}$ and lumbar puncture. 21 aneurysms were present in the other 19 patients with SAH. MRA missed 2 small $(3-4 \mathrm{~mm})$ aneurysms of the cavernous carotid artery and a post. comm. artery aneurysm in a local hemorrhage. All MRA suspected non-ruptured (incidentical) aneurysms were proven by X-Ray AG. MRA again missed 2 small aneurysms. In conclusion, MRA was specific for aneurysms but sensitivity (5 false negatives) presently is not sufficient to replace X-Ray-AG prior to surgery. However, MRA may represent a valuable noninvasive screening modality in patients at risk for aneurysms (family history, collagen deficiencies, polycystic kidneys).

\section{$10.55 \mathrm{AM}$}

\section{Comparison of MR-angiography with i.a. DSA in intracranial aneurysms}

G. Schuierer, G. Bongartz, B. Kleemeyer, W. J. Huk, P. E. Peters; Münster

In 31 patients with 36 intracranial aneurysms MRA was performed with a flow-compensated 3-D-FISP sequence at 1.5 or $1.0 \mathrm{~T}$. The in-plane resolution was about $0.8 \mathrm{~mm}$ with a $256 \times 256$ matrix, the single slice thickness $1.0-1.25 \mathrm{~mm}$. The data sets were postprocessed using a ray tracing algorithm including a MIP-algorithm to generate MR angiograms in multiple views. A complete cranial i.a.-DSA study in at least 2 projections served as the reference investigation in all patients.

- MRA showed 30 of the 36 aneurysms (83.3\%) correctly, however in 7 of these the demonstration on MRA was incomplete. In 5 cases MRA was equivocal and one aneurysm was missed.

- The single slices of the 3-D-data set were superior to the reconstructed MR-angiograms in detecting small aneurysms and allowed the differentiation of thrombosis like T1-weighted MRI-scans

- The main restriction of MRA is the limited resolution due to limitations of the available hard- and software. Slow and/or turbulent flow are further problems reducing the reliability of MRA. MRA requires more cooperative patients than i.a. DSA.

At present MRA is clearly inferior to i.a.-DSA in the diagnosis of intracranial aneurysms. However if high resolution sequences become available MRA may provide a noninvasive alternative to angiography.

\section{$11.05 \mathrm{AM}$}

867 MRA in the evaluation of the anatomy and functionality of the circle of Willis' Technique, results and pitfalls

G. Marchal, P. Vanhoenacker, P. Aerts, H. Bosmans, E. Kersschot, G. Wilms, S. Verellen, R. Suy, A. Nevelsteen, A. L. Baert; Leuven

Purpose: Functional anatomic connections between the different vascular territories of the brain are of vital importance in patients with severe stenosis or obstruction of the carotid and/or vertebral arteries. The major collaterals, the anterior and posterior communicating arteries in Willis' circle, are easily visualized by 3-D MRA. In patients with flow impairment, the functionality of these vessels can be assessed by saturating the flow from either the right or left carotid artery.

Methods and Materials: We evaluated this technique in 12 normals and in 20 patients. Selectivity was optimized using oblique rather than orthogenal saturation pulses.

Results: Both volunteers and patients showed a similar \% of anatomical variants of Willis' circle. These include aplasia, hypoplasia and fetal branching of the posterior cerebral arteries. Selective MRA shows the flow direction over the communicating arteries. For an exact interpretation of selective MRA additional information about the flow in the cervical arteries is needed. Saturation pulses set over the carotids can also affect the vertebrals, leading to misleading images.

Conclusions: Selective MRA provides interesting additional information in the selection of patients for surgical treatment.

$11.15 \mathrm{AM}$

868 Hemorrhage in cerebral arteriovenous malformations: detection in $\overline{\mathrm{MRI}}$ and comparison with clinical history

L. Prayer, D. Wimberger, R. Stiglbauer, J. Kramer, B. Richling, G. Bavinski, Th. Czech, H. Imhof; Vienna

Purpose: To evaluate the utility of MRI in the detection of blood breakdown products in patients with cerebral AVMs in comparison with clinical history of hemorrhage.

Methods and Materials: 63 patients with 71 angiographically proved cerebral AVMs were examined by high-field MRI performing T1- and T2-weighted SE sequences in at least two imaging planes.

Results: Evidence of previous bleeding was seen in $85.8 \%$ of patients with verified cerebral hemorrhage, in $45.5 \%$ of patients with symptoms suggestive of an episode of hemorrhage and in $18.6 \%$ of patients with completely negative record.

Conclusions: MRI can make important contributions to further management of patients with cerebral AVMs by demonstrating the presence of blood breakdown products in patients with and without adequate clinical history.

\section{$11.25 \mathrm{AM}$}

869 MR-angiography of the vertebro-basilar system: methods and clinical application

Klaus U. Wentz, J. Röther, F. Gückel, W. Jaschke, C. Hitzigrath, G. Laub, W. Rauthenberg, R. Loose, M. Hennerici, G. Georgi; Mannheim

The diagnostic value of MR-angiography of the vertebro-basilar system was examined in patients with clinical signs for ischemic lesions of the brain-stem and cerebellum. 20 symptomatic patients who had been examined with Doppler-ultrasound (DUS) and selective digital subtraction angiography (DSA) were selected for MR-arteriography. Two axial sets of flow sensitive 3D gradient-echo images were acquired in each patient (3D-FISP: $\mathrm{TR} / \mathrm{TE} / \mathrm{flip} / \mathrm{slab} / \mathrm{NEX}=32 \mathrm{~ms} / 7 \mathrm{~ms} / 15^{\circ} / 70 \mathrm{~mm} / 1$ ). Signal from venous inflow was eliminated by a presaturation slice parallel and above the imaging volumes. Using a targeted maximum-intensity-projection algorithm (MIP), the data were post-processed to obtain angiograms. In order to discriminate between vascular occlusion and low flow rates an additional sequential 2D-gradient-echo-sequence was used $($ FLASH $2 \mathrm{D}: \mathrm{TR} / \mathrm{TE} / \mathrm{flip} / \mathrm{gap} / \mathrm{Acq}=$ $\left.32 / 10 / 30^{\circ} / 0,5 / 1\right)$.

There was an overall close correlation between DSA, DUS and MRA where the later tends to overestimate stenoses but well demonstrates hemodynamics. While AICAs are rarely visualized SCAs and PICAs are seen in a high pecentage of patients. MRA might become a powerful non-invasive screening method and could help to reduce indication for invasive methods in these patients. 
11.35 AM

870 Clinical assessment of new biocompatible embolization microspheres

R. Beaujeux, A. Laurent, M. Wassef, A. Aymard, P. Gobin, C. Shenker, J. J. Merland; Paris

The authors describe the use of a new type of acrylic microspheres. They have been tested with 80 patients: 17 tumors, 8 facial AVM, 30 spinal cord AVM, 20 cerebral AVM, 5 miscellaneous.

For all of them we have noted the feasibility and easiness of the embolization. For 19 patients ( 13 tumors and 6 facial AVM) we have compared the use of 2 types of microspheres: narrow peak distribution (of different calibers $200 \pm 50 \mu$, and $400,600,900 \pm 100 \mu)$ and a wide distribution of $500 \pm 300 \mu$. The efficiency of these microspheres, for these 19 patients, has been determined by following criterias: angiographic devascularisation, surgical benefits and histological consequences.

For the 80 patients, embolization was possible and easy, without any aggregation problem either in the catheters or in the vascular network.

For the 19 patients: the use of narrow peak allowed the targeting of the embolization (in this case the microspheres are very numerous in the tumors and necrosis is widely spread), on the opposite, the use of widely distributed microspheres allowed only a proximal embolization without necrosis.

The use of these particles embolization enable us to accurately target the embolization.

\section{$11.45 \mathrm{AM}$}

\section{Hemodynamics of the circle of Willis: MR-angiography using} arterial presaturation

F. Gückel, K. U. Wentz, J. Röther, W. Rauthenberg, P. Busch, W. Jaschke, R. Loose, H. Mattle, G. Laub, M. Hennerici, M. Georgi; Mannheim

This study was designed to provide functional information about collateral circulation in the circle of Willis using MR angiography. In 25 patients with occlusions or stenosis of carotids or vertebro-basilar arteries MR angiography was performed together with transcranial Doppler sonography and conventional angiography. First one axial flow sensitive 3D gradient-echo data set was acquired at the level of the circle of Willis (3D-FISP: $\mathrm{TR} / \mathrm{TE} / \mathrm{flip} / \mathrm{slab} / \mathrm{NEX}=35 \mathrm{~ms} / 7 \mathrm{~ms} / 25^{\circ} / 32 / 1$ ). Using a maximumintensity-projection algorithm (MIP), the data were post-processed to obtain a projection angiogram. Further $3 \mathrm{D}$ angiograms were produced, using presaturation pulses. They consist of a slice selective $90^{\circ}$-pulse followed by a spoiler gradient $(5 \mathrm{mT} / \mathrm{M})$. These 1 to $2 \mathrm{~cm}$ thick slices are positioned vertically or oblique to the imaging volume in order to selectively eliminate flow signal from selected arteries (ICAs, basilar artery and/or ophthalmic arteries).

The technique described allowes a precise analysis of flow-directions and a semi-quantitative estimation of flow-dynamics in the circle of Willis. The results show a good correlation with transcranial Doppler sonography and conventional angiography.

\section{$11.55 \mathrm{AM}$}

\section{Carotid and vertebral artery dissection - angiographic and MRI correlations}

D. Tampieri, S. Grahovac, D. Melanson, R. Ethier; Montreal, CDN

Carotid and vertebral arteries dissections are dramatic presentation in young adult without any previous history of trauma.

The radiological files of 20 patients presenting with an acute neurological deficit and having carotid or vertebral artery dissection have been revised. All the patients underwent a complete angiographic evaluation. We observed 13 cases of carotid and 7 cases of vertebral dissections. Among the vertebral artery dissection, two patients had bilateral lesion. In 18 patients CT scan was performed and in eight patients an MRI examination was also available. Finally, two patients underwent also MRI angiography.

The angiographical characteristics of the carotid and vertebral dissections have been fully reviewed as well as the extension of the stenosis, the presence of pseudo-aneurysm or double-lumen.

The complete angiographic evaluation of the vessels of the neck is the examination of choice for diagnosis of dissection. Magnetic resonance allows the visualization of clot within the wall of the vessel. Finally, MRI angiography will permit in the near future the accurate investigation of these patients.

\section{Room 0}

Ss

\subsection{Contrast media}

$10.30 \mathrm{AM}$

873 Optimum utilisation of intravascular radiological contrast media Ronald G. Grainger; Sheffield

a) To optimise the use of the three types of intravascular contrast media 1 . Conventional high osmolar ionic, 2. Low osmolar ionic, 3. Low osmolar non-ionic in order to provide the best patient benefit.

b) \& c) During the last decade, we have greatly increased our knowledge of the potential advantages, disadvantages and specific indications of these three types of contrast agents. The major contrast medium problem for the 1990's is how best to use this information to provide optimum patient care within our resources, which are unfortunately not infinitely expandable.

There is still inappropriate use of excessively large volumes and concentrations of contrast agents, of unnecessary contrast examinations, inappropriate application of Digital Imaging and careless wastage of the media.

Recent surveys and analyses provide new helpful data for the optimum use of these three types of agent consistent with the best available cost-benefit return. In any resource-limited organisation, it may be inappropriate or even irresponsible to inject the more expensive low osmolar media in every patient, for this may deprive our patients of other facilities which may well provide greater benefit.

\section{$10.40 \mathrm{AM}$}

874 Suitability of Isotonic iotrolan versus lowosmolal lopromid in femoral and penile arteriography

J. Gmeinwieser, K. Mühlbauer, St. Feuerbach; Munich

Purpose: The aim of the study was, to evaluate the suitability of iotrolan (Schering), an isotonic nonionic dimer, in penile and in global and selective femoral arteriography.

Methods and Materials: In a randomized double-blinded trial the sensations of pain and heat, the systemic side-effects and the contrast quality after the injection of iotrolan $(280 \mathrm{mgI} / \mathrm{ml})$ were compared versus lowosmolal iopromid $(300 \mathrm{mgI} / \mathrm{ml})($ Schering $)$ in penile angiography $(\mathrm{n}=20)$ and in global $(\mathrm{n}=20)$ and selective $(n=20)$ femoral arteriography.

Results: There were no systemic side-effects. Especially in penile angiography and in selective femoral arteriography iopromid caused strong sensations of heat and pain in the majority of the patients. The local tolerance of iotrolan was statistically highly significant better. Inspite of its lower iodine content there were no differences in contrast quality. Iotrolan's higher viscosity did not impair its injectability.

Conclusions: Iotrolan proved to be well suited as a contrast agent for penile and femoral arteriography. In angiographies, were even the use of lowosmolal contrast agents is frequently associated with severe discomfort, heat and pain sensations could be minimized by using iotrolan.

\section{$10.50 \mathrm{AM}$}

875 Experimental studies on mechanisms of Lipiodol hepatic artery infusion for diagnosis and treatment of $\mathrm{HCC}$

Y. Ni, J. Du, G. Marchal; Leuven

Purpose: Study of the biodistribution and resorption of intraarterial lipiodol in rats with normal, cirrhotic and cancerous liver.

Methods and Materials: A surgical technique was developed to selectively infuse the hepatic artery with preservation of the patency of the hepatic artery after the procedure.

This infusion technique was applied in 84 rats. 42 had diethylnitrozamine induced cirrhosis with superimposed HCC. The rest was used as controls.

$0.04 \mathrm{ml}$ of lipiodol per $100 \mathrm{gr}$ body weight was injected. Rats were sacrificed in groups of 6 rats at $1 / 2 \mathrm{hr}, 24 \mathrm{hr}, 24 \mathrm{hr}, 3,7,14$ and 21 days.

The rats were analysed with: radiography, macroscopy, microscopy with HE and oil red 0 lipid staining, emzymatic histochemical assays for non-specific esterase, eletronmicroscopy and autoradiography after infusion of radiolabelled lipiodol (125 I-LPD).

Results: The biodistribution differs in normal and cirrhotic animals. In normals the main deposition of the intraarterial lipiodol is in the liver whereas in cirrhotic rats a large amount is also drained to the lungs.

30 minutes after injection the lipiodol is in the intrahepatic and intratumoral vessels. Later on lipiodol is progressively cleared from the vessels probably by endocytosis of the hepatocytes. Once intracellular the lipiodol is progressively metabolised. This phenomenon was not observed in the tumor. Here the lipiodol is either more slowly cleared probably by progressive washout into the surrounding parenchyma, or retained in necrotic areas.

Discussion

These findings clearly differ from current literature. 
11.00 AM

876 Adverse reactions to nonionic contrast media with special regard to high-risk patients

U. Fink, B. K. Fink, D. Jung; Munich

Nonionic contrast media $(\mathrm{CM})$ proved to be significantly safer than ionic $\mathrm{CM}$. Nevertheless deaths were reported after application of nonionic agents. Aim of the study was to investigate the rate of adverse reactions to nonionic CM in non-risk patients in comparison to high-risk patients after premedication. In a prospective study over about two years 12.995 examinations with intravenous or intraarterial application of nonionic $\mathrm{CM}$ were evaluated. A premedication with $\mathrm{H} 1$ - and $\mathrm{H} 2$-antagonists was used in high-risk patients with known adverse reaction to $\mathrm{CM}$, history of allergy or severe cardiac or pulmonary disease.

For all examinations there were $143(1.10 \%)$ adverse reactions (mild in $0.58 \%$, moderate in $0.41 \%$ and severe in $0.05 \%$ ). In high-risk patients there were adverse reactions in $4.37 \%$ without and in $1.57 \%$ with premedication. There was no severe adverse reaction in the high-risk patients after premedication. Age of the patient, CM-dosage and CM-concentration proved to be no risk-factors in the present study.

In conclusion, the additional premedication with $\mathrm{H1}$ - and $\mathrm{H} 2$-antagonists could be an effective agent to reduce the risk of mild and moderate adverse reactions and to avoid severe adverse reactions in high-risk patients.

11.10 AM

877 Investigation of the systemic reactions of loxitalamate $(5.000$ $\overline{\mathrm{CT}} /$ scans-examinations)

J. M. Caillé, A. M. Bidabé, J. F. Dartigues, M. Allard; Bordeaux

The purpose of the present work is to study the adverse reactions of sodium Ioxitalamate and Meglumine ( $380 \mathrm{mg}$ of iodine per $\mathrm{ml}$, Telebrix $38^{\mathrm{TM}}$ ) in 5.000 cephalic CT/scans.

The medical records analysed by the computer department included $63 \%$ males and $37 \%$ females, with a mean age of 52.6 years $( \pm 18.5)$.

There were no exclusion criteria. Among the patients, there were $1.5 \%$ of severe cardiac failures and $2.1 \%$ of severe respiratory failures.

Premedication was administered to $8.77 \%$ of patients in the two days preceding the examination, because of the presence of a risk factor, the most frequently being a history of a reaction to intravascular contrast media during previous examinations or a known history of allergy.

We observed adverse reactions in $2.9 \%$ of cases, $98 \%$ of which were minor or moderate. The remaining $2 \%$ were classified as severe because they needed the assistance of an anesthesiologist. In no case were there any sequelae of deaths. The present work will establish the correlation between the incidence of adverse reactions to Ioxitalamate and premedication received by high risk patients.

The analysis of the 5.000 medical records will allow the authors to present and discuss the results.

\subsection{AM}

\section{Blood pool X-ray contrast media: evaluation of a new iodinated polymer}

C. Chambon, S. Le Greneur, X. Violas, D. Revel; Aulnay sous Bois, Lyon

Purpose: Water soluble contrast media are distributed throughout the vascular space and diffuse through the interstitial space across the capillary wall, rapidly leading to low vascular iodine concentrations.

As a consequence of these pharmacokinetics, vascular imaging involves selective intraarterial or multiple peripheric contrast media injections.

The difficulty could be overcome by using high molecular weight contrast media.

Methods: An iodinated carboxymethyl dextran was obtained by a 2 step reaction:

- Dextrane activation

- CMD reaction with an aromatic triiodinated amine.

Its plasma pharmacokinetics and urinary excretion were studied in anesthesised rabbits $(n=5)$ and compared to Telebrix ${ }^{\oplus}(n=5)$ and Iopamiron ${ }^{\oplus}(\mathrm{n}=5)$ at the dosage of $340 \mathrm{mg}$ Iodine $/ \mathrm{kg} \mathrm{B.W}$.

Results: Iodinated polymer injection results in plasma iodine concentration three times higher than Iopamiron ${ }^{\circledast}$ or Telebrix ${ }^{\oplus}$ do.

As a result from the low distribution volume $(130 \mathrm{ml} / \mathrm{kg})$ and the relatively low clearance $(3 \mathrm{ml} / \mathrm{min})$ the plasma half-life of the iodinated polymer is three times longer than the elimination half-life of the conventional X-ray contrast media.

The urinary elimination remains satisfactory as $61 \%$ of the injected dose is excreted within the $4 \mathrm{~h}$ following IV injection.

Conclusions: In conclusion, this iodinated polymer is a potential contrast media for X-ray vascular and perfusion imaging. Animal imaging studies in normal, ischemic and tumor-bearing models are therefore in progress.
11.30 AM

879 A clinical trial of Optiray in intravenous urography F. Joffre, F. Blain, C. Azau; Toulouse

OPTIRAY is a new low osmolar non-ionic contrast medium with original physicochemical properties (low viscosity and high hydrophilicity).

A randomized, double blind, controlled trial was performed comparing OPTIRAY $300(60 \mathrm{ml})$ and IOPAMIRON $370(50 \mathrm{ml})$ on 80 patients requiring intravenous urography. The diagnostic efficacy was assessed by the same radiologist by means of opacification score noted after each injection and for each level of the urinary tract. Patient tolerance was determined by side effects which were described according to their nature, intensity and outcome over a 30 minutes period. The results are presented and compared to the following european trials: 7 comparative studies (253 patients) and 2 open studies (65 patients).

In this study, for a similar amount of iodine, we found no difference in efficacy between products. In the other european studies, ioversol-enhanced pelvicalyceal films and ureterograms were of higher quality than those using contrast media with an identical iodine concentration and identical volumes.

These data indicate that OPTIRAY provides a good opacification of the urinary tract when compared to other non ionics and that it has an excellent safety profile.

\section{$11.40 \mathrm{AM}$}

\section{Radiological surveys with intravenous contrast medium in dialysis} patients

M. Bertoli, A. Meneghello, M. Urso, L. Benedetti; Padova

The need of radiological surveys with i.v. contrast media (ivem) in dialysis patients is increasing due to the large number of patients and the increasing life during maintenance dialysis. The use of ivcm in renal failure is dangerous because of the reduced renal elimination. There is the risk of volume overload and of the enhanced systemic toxicity.

We reported two cases of vicarious elimination of ivcm by liver with gallbladder's opacification, and by small bowel, and consequent paralytic ileum. All the symptoms disappeared after following dialysis.

Therefore, we studied with chromatographic assay the plasma iopamidol's levels during hemodialysis in 6 patients. All the patients performed radiological examinations with i.v. iopamidol (mean dosage $65.2 \pm \mathrm{SD} 25.2 \mathrm{~g}$.) only for the basic survival of the patient. A dialytic treatment was performed within 2 hours after i.v. infusion and the plasma samples were taken at the beginning and at the end of treatment and after 60 and 90 minutes. The dialysis reduced significantly $(\mathrm{p}<0.01)$ the plasma level of iopamidol (half life $2.08 \pm$ SD 0.46 hours).

Our results suggest that the use of contrast media, with rapidly following dialytic treatment is safe, and can be used in larger indications than the basic survival of the patient.

\subsection{AM}

\section{Air embolism after contrast enhanced thoracic CT} S. Cantoni, C. Frola; Genova

The occurrence of air embolism after contrast enhanced CT has been already emphasized by some authors who refer a percentage of $23 \%$ of air embolism without any side effect.

As we observed one case of moderate air embolism complicated with cardiorespiratory distress after contrast enhanced thoracic $\mathrm{CT}$, we reviewed the latter 120 thoracic CT performed in our center.

We found air embolism in $33 \%$ of cases, minimal in 19 cases and moderate in 5 ; three of these complained acute respiratory and cardiac symptoms that were referred to an allergic reaction.

We were not able to explain if a moderate air embolism could cause those symptoms, neither why we found a so high percentage of air embolism.

\section{$11.53 \mathrm{AM}$}

882 MR imaging of normal and ischemic rat myocardium with Gd-BOPTA/Dimeg

F. Cavagna, F. Maggioni, E. Vicinanza, E. Felder, D. de Haën, C. B. Higgins; Milan

Doses of $250 \mu \mathrm{mol} / \mathrm{kg}$ of either Gd-BOPTA/Dimeg ${ }^{+}$or Gd-DTPA/Dimeg were administered i.v. to two groups of five rats each, respectively. Two groups consisted of normal animals, while the other two were formed of animals which had been subjected to occlusion of the lower anterior descending coronary artery. The contrast agent was given $60 \mathrm{~min}$ after occlusion. MRI was performed prior and up to $60 \mathrm{~min}$ after contrast agent administration. Normal myocardium signal to noise ratio (SNR) enhancement was measured in normal animals, and the normal area-injured area contrast to noise ratio (CNR) was determined in the animals with coronary artery occlusion. 
Gd-BOPTA/Dimeg produced a SNR enhancement (96\%) in normal myocardium and a normal area-injured area CNR enhancement (17 fold) which both were by far stronger and more persistent than those produced by Gd-DTPA/Dimeg. Infarcts were still clearly delineated $45 \mathrm{~min}$ after administration of Gd-BOPTA/Dimeg, while they were barely visible $30 \mathrm{~min}$ after administration of Gd-DTPA/Dimeg.

Thus beyond being a general extracellular and hepatobiliary contrast agent, Gd-BOPTA/Dimeg promises utility in myocardial imaging.

${ }^{+}$Gd-BOPTA/Dimeg is Gadolinate (2-), (4R,S)-[4-carboxy-5,8,11-tris(carboxymethyl)-1-phenyl-2-oxa-5,8,11-triazatridecan-13-oato $\left(5^{-}\right)$] dihydrogen compound with 1-deoxy-1-(methylamino)-D-glucitol (1:2)

\section{$11.56 \mathrm{AM}$}

\section{Polylysine-(Gd-DTPA): a new macromolecular MR contrast agent} for imaging of tumors

P. Landwehr, A. Benkert, D. Höhmann, W. Ebert, H. Schmitt-Willich, Th. Krahe, K. Lackner; Würzburg

Purpose: Until now, Polylysine-(Gd-DTPA) has been used experimentally as an intravascular MR contrast agent due to its high molecular weight. We report on the use of Polylysin-(Gd-DTPA) for imaging of animal tumors compared with conventional Gd-DTPA.

Methods: Tumors of 3 different histologies (melanoma, melanoblastoma, lung carcinoma) were produced by subcutaneous implantation in 18 athymic mice. Dynamic MRI (SE 200/30, $1.5 \mathrm{~T}$ ) was performed every minute $(0-20 \mathrm{~min})$, every 10 minutes (20-120 min), every hour (2-6h) and 1, 2, 3, 5, 7, 9 days after injection of Polylysine-(Gd-DTPA) [mean MW $50000 \mathrm{D}, \mathrm{R} 1=11.6 \mathrm{~L} / \mathrm{mmol}$ $\times \mathrm{s} ; \mathrm{LD} 50$ (mouse) $=15 \mathrm{mmol} / \mathrm{kg}](\mathrm{n}=11)$ and conventional Gd-DTPA ( $=7)(1 \mathrm{mmol} \mathrm{Gd} / \mathrm{kg}$, respectively). Standard-related percent signal intensity was calculated by: $\% \mathrm{SI}=\left(\mathrm{SI}_{\text {after }} / \mathrm{SI}_{\text {before }}\right) \times 100$.

Results: In tumors \%SI ${ }_{\max }$ after Polylysine-(Gd-DTPA) was higher than after Gd-DTPA $(241.1 \pm 68.4$ vs $176.1 \pm 15.2)(\mathrm{p}<0.01)$, whereas in skeletal muscle $\% \mathrm{SI}_{\max }$ was not significantly different $(140 \pm 28.7$ vs $150.7 \pm 20.7) . \% \mathrm{SI}_{\max }$ in tumors was reached 3-4 minutes after injection of Gd-DTPA with a marked reduction of \%SI after 2 hours. Using Polylysine-(Gd-DTPA), \%SI ${ }_{\max }$ could be measured 2-3 hours after injection with a long lasting plateau up to several days.

Conclusions: Polylysine-(Gd-DTPA) accumulates significantly in malignant tumors with a strongly prolonged enhancement compared with Gd-DTPA. The high "tumor-to-muscle"-contrast after Polylysine-(Gd-DTPA) characterizes this substance as a potential tumor-selective MR contrast agent.

\section{Room R}

\section{SS} $10.30 \mathrm{AM}$

\section{Quality control in radiotherapy (Keynote Lecture) F. Eschwege; Villejuif}

Improvement of results on survival and quality of life after radiotherapy may be obtained by the improvement of the local control.

One of the ways to improve the results is an amelioration of the quality of radiotherapy treatments. The chance of tumor cure, the incidence of complications are critically dependant on many technical factors.

A long chain of events appears during radiotherapy treatment, from the simulation of the patients to the long series of the sessions. During this period, a large number of human errors deviations, technical and mechanical failures are possible.

The quality control (Q.C.) tries to analyse and eliminate the vaste majority of the problems. One of the best way to improve the quality of treatment is to incorporate such a QC in the trials in radiotherapy (RT). A large number of European and American phase II and III RT trials, are now connected with Q.C. program.

Numerous analysis of QC reports have been published and may explain controversial results obtained in clinical R.T. trials.

Recommendations have been proposed for the amelioration of the treatment including number of medical and non medical radiotherapy staff, specific and individual data and physicists tasks.

\section{$10.45 \mathrm{AM}$}

885 European quality assurance protocols. Actions in concert or solo performances? (Keynote Lecture)

John S. Clifton; London

The European Federation of Organisations for Medical Physics (EFOMP) has member organisations in 24 countries representing some 3000 medical physicists and is in a unique position to encourage the harmonisation and use of Quality Assurance procedures in Radiation Therapy throughout Europe.
The role and responsibility of the medical physicist in promoting quality assurance procedures in the context of total quality management of the treatment of the patient will be discussed. Protocols published by member organisations and other interested bodies will be compared, the extent of their use reviewed and proposals for harmonisation and concerted action to promote quality assurance presented.

\subsection{AM}

886 Clinical feasibility of megavoltage CT scanning in the set-up and verification of radiation therapy

K. Nakagawa, Y. Aoki, A. Akanuma, Y. Onogi, K. Karasawa, N. Muta, A. Terahara, K. Hasezawa, Y. Sasaki; Tokyo

Purpose: A megavoltage CT scanning system using 4 and $6 \mathrm{MeV}$ treatment beam of a linear accelerator has been developed to improve accuracy in patient set-up and treatment verification of radiation therapy.

Methods: The detector array is composed of 120 sensor units arranged in an arch at the distance of $160 \mathrm{~cm}$ from the source. Each sensor unit consists of a CdWO4 crystal and a photo-diode. Heavy metal collimator is attached to exclude scattered dose, which results in poor spatial resolution, that is pixel size of $3 \mathrm{~mm}$.

Results: Clinical application of this system is as follows. For a treatment requiring accurate set-up, multi-slice megavoltage CT images are taken, followed by a quickly performed radiation treatment planning. After the planning set-up is performed automatically according to the treatment parameters. As for treatment verification, the same detector receives exit dose during an actual irradiation to superimpose high dose area on the megavoltage CT image. Linear attenuation co-efficient distribution is used to calculate absorbed dose. A measurement with Mix-Dp phantom revealed that absorbed dose for a scan with 4 and $6 \mathrm{MeV}$ X-ray was approximately 1.4 and $2.8 \mathrm{rad}$ respectively. Preliminary image quality is enough to be utilized in radiation therapy treatment planning.

Conclusions: Megavoltage CT scanning is useful when an accurate patient's set-up is highly required.

\section{$11.15 \mathrm{AM}$}

887 Digital portal radiography in radiation therapy - pros and cons Roland J.Scheck, Rainer Rienmüller, Norman Willich, Manfred Schätzl; Munich

Purpose: To evaluate the potential of digital luminescence radiography to improve image quality, esp. contrasts resolution of portal films in radiotherapy.

Methods: Both conventional and digital verification films of an antropomorphic phantom and various irradiation ports in patients were obtained with high energy photon beams (Co $60,6 / 8 / 15 \mathrm{MeV}$ ). For both techniques conventional films (Structurix D2, Agfa) and storage phosphor screens (SPS) were placed into a cassette with steel intensifier screens (front $1 \mathrm{~mm} \varnothing$, back $0.5 \mathrm{~mm}$ $\varnothing$ ). Exposed SPS were processed by a semiautomatic mode, an optimized contrast curve and moderate edge enhancement and printed as hardcopies (FCR-7000, Digiscan, Siemens).

Results: Digital radiographs were easier to read (no need of visual adaptation or spotlight) than conventional portal films and needed a shorter exposure time ( $5 \%$ of conventional films), thus lowering motion artefacts. In both techniques the delineation of anatomical structures was comparable.

Special treatment settings (e.g. small ports) and several types of artefacts revealed some problems of the digital technique.

Conclusions: Digital luminescence radiography yields portal radiographs, which are easier to read and comparable in diagnostic quality to conventional films in most treatment settings.

\subsection{AM}

888 Quality of life study in lung cancer patients treated with once a week radiation therapy

M. Bindi, F. Velicogna, E. Paci, E. Tucci, F. Pepi, L. Pirtoli; Siena

Seventy patients with III and IV stage lung cancer were treated with once a week radiation therapy. Treatments were carried out with six fractions of 880 cGy (14 pts.) or eight fractions of $550 \mathrm{cGy}$. In both cases the total dose was 4400 cGy. All patients had symptoms of locally advanced cancer, were out-patients and presented a performance status ranging 50-80 using the scale of Karnofsky. The survival of this group of patiens does not differ from the results obtained by conventional radiation therapy technique reported in literature. Quality of life was recorded using the Functional Living Index-Cancer (FLIC). The 22 -item questionnaire evaluated the psychological state, the sociability and the somatic sensations. The FLIC factor 1 analysis (physical well being) indicated a good satisfaction correlated to the relief of symptoms. The factor 2 analysis (psychologic state) showed low levels of anxiety and depression. The factor 3 and 4 (family interactions and sociability) indicated pleasure of the patient to remain most of time at home, during the treatment course. 
In conclusion, the FLIC is a simple instrument, specific for cancer patients, allowing to compare groups of patients vis-a-vis their overall functional response both to cancer and to different treatment modalities.

11.35 AM

889 Dosimetry and clinical results of total skin electron beam therapy of cutaneous T-cell lymphoma

C. Rübe, N. Willich, Th. Wendt, H. Feist, F. HeB, N. Sepp, P. Kaudewitz; Munich

We report on 19 consecutive patients with mostly advanced stage cutaneous T-cell lymphoma (Mycosis fungoides), who were treated by total skin electron beam therapy with total doses between 8 and 36 Gy in fractions of 200 to $400 \mathrm{cGy}$ one to four times a week. All patients had progressive disease under all other kinds of treatment. The treatment regimen consisted of a irradiation from six directions in $300 \mathrm{~cm}$ distance. Before the treatment of the patients was started, we carried out numerous dose measurements with LiF TL-rods (TLD 100 ) on the Alderson phantom. Moreover, dose measurements have been carried out at least once on each patient. Low dose spots and tumorous skin-areas were boosted individually. 5 patients achieved complete remission with a mean disease free survival of 11.4 months, 4 patients had a partiell remission, which changed into a complete remission after several months, 10 patients in most advanced stages had partiell remission with a mean duration of only 3.9 months with a good palliative effect on the skin tumors. Data on dosimetry and the clinical results are presented.

\section{Room S}

\section{SS} $10.30 \mathrm{AM}$

890 Imaging methods in the diagnosis of osteomyelitis: bone scan, leukocyte scan, and MRI

Ch. Ehrenheim, G. Ulferts, N. Südkamp, H. Hundeshagen; Hannover

Purpose: Different diagnostic imaging methods were to be evaluated in patients with clinical signs of acute or chronic osteomyelitis (OM).

Patients and methods: 24 patients with acute $(9$, hematogeneous 6$)$ or chronic OM (15, posttraumatic 11) were examined. The preferred localization of the inflammatory processes was the lower extremity. X-ray and dynamic bone scan was performed in all cases. Adults also underwent a leukocyte scan using $111 \mathrm{In}$-oxin labelled leukocytes. MR images were obtained using T1- and T2-weighted sequences. 10 patients received the paramagnetic contrast agent Gd-DTPA.

Results: Both, acute and chronic OM showed hyperperfusion and subsequently an increased uptake in the blood pool and late phase images of the bone scan. Leukocyte scan, however, usually allowed the differentiation between acute and chronic stage of osteomyelitis, as chronic processes did not show leukocyte migration in this study. Characteristic morphological patterns were also found in MR images: in acute OM cortical bone was mostly intact $(6 / 9)$, bone marrow edema was revealed $(6 / 9)$ as well as periostal reaction and soft tissue edema in all cases. Cortical bone was thickened or destroyed $(11 / 15)$ in chronic OM, spotted lesions were found in the marrow space $(14 / 15)$ with high signal intensity pointing to necrosis $(9 / 15)$. This finding was supported by the contrast enhanced images.

Conclusions: The high resolution and excellent soft tissue contrast facilitates the differentiation between acute and chronic OM. MRI provides high sensitivity, and improves the specificity of imaging techniques in the diagnosis of osteomyelitis.

\section{$10.40 \mathrm{AM}$}

891 Diagnosis of osteomyelitis: is anti-granulocyte antibody imaging superior to three phase bone scan?

J. Scheidler, G. Leinsinger, M. Pfahler, C.-M. Kirsch, J. Lissner; Munich

The aim of this study was to define if there is a diagnostic superiority of anti-granulocyte antibody (MoAGAb)-imaging to conventional three phase bone scan (BS) in osteomyelitis (OM).

BS and MoAGAb-imaging was performed in 37 patients (pts). 24 h p.i. of 180 $\mathrm{MBq}^{123} \mathrm{~J}-\mathrm{MoAGAb}$ (Granuloscint ${ }^{\circledast}$ ) static and, if appropriate, SPECT images were acquired. MoAGAb-imaging was done at least $48 \mathrm{~h}$ after BS to avoid superimposition. BS was considered positive for OM when increased radionuclide activity was seen in all phases. Diagnosis was verified by surgery/biopsy or clinical follow-up.

OM was diagnosed in 17 pts. BS was considered being positive in all pts with OM (sensitivity 100\%). MoAGAb-imaging was able to detect all infectious lesions (sensitivity 100\%). 20 pts had no OM or non-infectious bone diseases. 15 out of these pts revealed no MoAGAb accumulation (specificity $75 \%$ ). $10 / 20$ pts without infection were negative for OM in BS. In contrast, 10
OM-negative pts showed questionable BS results. 6 out of these pts were additionally found to be negative in MoAGAb-imaging. False positive (with respect to OM) MoAGAb-results were obtained in aseptic bone necrosis (2), loosening of prostheses (1), gout (1), and trauma (1).

In conclusion MoAGAb-imaging is a highly sensitive diagnostic modality and provides acceptable specificity. For cost effectiveness BS should be performed prior to MoAGAb-imaging. No additional gain can be obtained by MoAGAbimaging when three phase BS is positive. However, MoAGAb are helpful for the exclusion of infection in pts with questionable BS results.

10.50 AM

892 Infectious sacro-iliitis. The use of CT and MRI scanning C. Le Breton, I. Frey, M. F. Carette, J. Richaud, A. Kujas, J. Korzec, J. M. Bigot; Paris

Purpose: to examine the use of CT and MRI in the diagnosis of septic sacroiliitis.

Methods: 14 cases of septic sacro-iliitis, all explored by CT scan of whom 2 had been investigated by MRI.

Results: the cases comprised 6 females and 8 males of whom 9 were African. 8 had tuberculous sacroiliitis and 6 pyogenic sacro-iliitis. All cases showed a pre sacroiliac soft tissue swelling with ring-like enhancement after intravenous contrast caused by to a psoas abscess in 5 cases and an iliac abscess in 6 cases. Joint narrowing was found in 4 cases and widening in 8 cases with local osteoporosis. The MRI showed low signal T1 weighted images and high signal T2 in the subchondral opaces, with soft tissue abscesses.

Conclusions: the CT appearance of septic sacro-iliitis is very different from inflammatory sacro-iliitis and useful in diagnosis. The MRI appearance can be misleading and resembles that of a tumour.

$11.00 \mathrm{AM}$

893 Infectious sacroiliitis imaging P. Chevalley, J. Garcia; Riaz

Purpose: About 11 cases of various infectious sacroiliitis, we will discuss the clinical, biological and especially the radiological findings.

Acute and subacute infectious sacroiliitis can be associated with another arthritis and are often accompagnied with soft tissue lesions.

Methods: Radiological investigations are very useful for the diagnosis, because of the unspecific clinical and biological findings.

Review of all the radiological techniques useful for the diagnosis (Radiography, tomography, CT, MRI and bone scintigraphy): their advantages and inconvenients.

Results: Radiography shows advanced osteoarticular remodelling but the subtle lesions are often unappearent.

Bone scintigraphy shows a focal hypercaptation but is unspecific.

Tomography and CT point out the erosions. With CT, soft tissue lesions are well demonstrated and a diagnostic punction with a fine needle is easely guided.

MRI can indicate a bone marrow oedema and her quality for soft tissue lesions is well known.

Conclusions: MRI could become a technique of choice especially in acute infectious sacroiliitis.

\subsection{AM}

\section{MRI of skeletal infections}

R. S. Pozzi-Mucelli, M. Cova, C. Ricci, L. Dalla-Palma; Trieste

Purpose: To determine MRI capabilities and limitations in the evaluation of skeletal infections.

Methods: 38 patients - 16 with clinical diagnosis of osteomyelitis of the upper or lower limbs and 22 with spondylodiscites were imaged using a Philips Gyroscan 1.5 T magnet. SE T1 and T2 weighted images were obtained. Signal intensity and morphological features were then evaluated. In 15 patients T1 weighted images were obtained also following intravenous injection of Gd-DTPA $0.2 \mathrm{mmol} / \mathrm{kg}$; T1 and T2 weighted images were compared with enhanced T1 weighted images. A limited group of patients was examined using also a SPIR sequence. Nine patients underwent MRI follow-up.

Results: MRI identified the lesions in $100 \%$ of cases and the nature in $92 \%$ of cases. MRI was highly sensitive in identifying lesions in the early stage and in demonstrating lesion regression, as well as in identifying complications. Compared to $\mathrm{T} 1$ - and $\mathrm{T} 2$-weighted images, $\mathrm{T} 1$-weighted enhanced images allowed a better evaluation of the extent of the disease. The specificity of MRI was improved by Gd-DTPA.

Conclusions: These results suggest that MRI provides significant informations in skeletal infections. Gd-DTPA may be of value in better evaluating the extent of the disease and improving the specificity of the technique. 
11.20 AM

895 Selective arteriography of the hip in femoral neck fractures about 250 cases

A. Tournade: Colmar

Selective arteriography is carried out in emergency conditions for predicting and results concerning arterial data of the femoral neck.

Methods and Materials: - 250 examinations are carried out using either contro-lateral either ipsilateral route.

- 2 arteriographies are performed in each case, before and after the fracture reduction, studying the medial circonflexe artery which surrounds the femoral neck posteriorly. Then an arterial data and a classification are established.

Results: - 4 groups of vascularisation are described after the fracture reduction.

- In $75 \%$ of the cases concerning the most frequent of the fractures the selective injection of the medial circonflex artery shows no change in the caliber and the course of the artery.

Conclusions: It is of very great interest to perform this sort of examination to conserve the femoral head with good conditions of arterial supply.

\subsection{AM}

\section{DSA in patients with femoral head necrosis and pedicled pelvic} bone graft

R. Langer, A. Scholz, M. Langer, G. Schwetlick; Berlin

Purpose: By this prospective study the femoral head perfusion pre and post pedicled pelvic bone graft in patients with femoral head necrosis was evaluated.

Patients and methods: 37 patients with femoral head necrosis were evaluated preoperatively by superselective intraarterial (i.a.) DSA of the medial circumflex femoral artery - which mainly supplies the femoral head in adults -, the deep circumflex iliac artery, and the deep branch of the superior gluteal artery. 19 patients underwent posttransplant angiography. DSA was performed as transfemoral crossover angiography, using a 4 or $5 \mathrm{~F}$ Cobra catheter for superselective catheterization.

Results: In femoral head necrosis the typical angiographic findings are a rarefication or an occlusion of the nutricial branches of the medial circumflex femoral artery, which could be demonstrated in $97 \%$.

Postoperative DSA revealed an adequate arterial supply and venous drainage of the pedicled pelvic bone graft in 17 out of 19 cases. In 2 patients the supplying artery was occluded.

Conclusions: I.a. DSA is the method of choice for pretransplant evaluation of the arterial perfusion of the femoral head and for therapeutic control postoperatively in patients with femoral head necrosis.

\section{$11.40 \mathrm{AM}$}

\section{MR in the follow-up of the healing process in bone-grafts-} Initial-experiences

M. Schratter, J. Kramer, D. Kropej, P. Ritschl, H. Imhof; Wien

We present our first experience with MR in bone grafts in 23 patients. Graft implantation followed curretage of benign bone lesions. The locations were, rather exclusively, the long tubular bones. T1- and T2-weighted spin echo-sequences including intravenous application of Gadolinium-DTPA were performed (Magnetom 63, 1,5 T).

A total of 29 examinations ( 1 follow-up control in 6 pat.) was devided into 5 groups, with regard to different stages after surgery, which varied from 1 week to more than 3 years postoperatively. The MR results were related to the respective groups.

The results show typical stages of bone graft incorporation, but also greater individual variations in the duration of incorporation. Our first results, however, represent only "snapshots" of different patients at different stages after bone graft surgery. Therefore some important questions are still unanswered but may perhaps be explained with the help of subsequent prospective studies.

$11.50 \mathrm{AM}$

898 Detection of osteomyelitis with sonography

A. Furtschegger, W. Buchberger, W. Kastlunger, D. Lungenschmid, T. Penz; Innsbruck

The methods for diagnosis of osteomyelitis include conventional radiography, bone scintigraphy, gallium-67 scintigraphy, MR-imaging and in the recent time sonography.

12 patients (nine males, three females; age range $12-55$ years) with clinically suspected osteomyelitis were studied sonographically between September 1987 and November 1990. Sonography was performed with a PICKER model LSC 7000 or 9500 using either a $5.0 \mathrm{MHz}$ or $7.5 \mathrm{MHz}$ transducer.

Seven patients had abnormalities detectable on sonograms. Five of these seven had an abnormal fluid collection adjacent to and contiguous with the bone and an elevated periosteum. Findings from subsequent radiographic and scintigraphic studies confirmed the diagnosis of osteomyelitis. In the other two patients a disruption in the anterior cortical surface of the bone and a sequestrum were demonstrated sonographically.

The findings of our study suggest that fluid around the bone seen on sonograms indicate acute osteomyelitis.

\subsection{AM}

899 Differential diagnosis in acute tumorous and inflammatory spinal lesions

H. ABmann, G. Krzok; Erfurt

$5 \%$ of the spinal CT-examinations are performed as non-traumatic spinal emergencies ("acute spinal cord"). In regard of the differential diagnosis of spondylitis or spinal tumour, there was obvious that also in the acute phase, the combination of myelography followed by Myelo-CT has stood its test.

(MR-Imaging is not available in case of spinal emergency.) 12 patients with acute spondylitis, and 52 patients with acute spinal cord owing to tumour destruction have retrospectively been analysed for more than 4 years.

Experiments are running on elaborating differential diagnostic criterions that determine further diagnostic and therapeutic proceedings.

Typical case demonstrations and post-operative results (stabilizing and fusion operations) illustrate the roentgen morphological spectrum of the Myelo-CT.

\section{$11.56 \mathrm{AM}$}

\section{Rhabdomyolysis by CT}

R. Bassaganyas, J.Ferrer, J.Pont, X. Serres, J. Barceló, J. C. Vilanova; Girona

The Rhabdomyolysis is a muscular lesion which produces an alteration of the muscular membrane allowing the release of intracellular substances towards the plasma.

We present four cases where the predisposition to cause Rhabdomyolysis, in two cases, was alcohol associated with pharmaceutical products and the other two cases were induced by hard drugs (cocaine and heroine). Although the diagnosis is based mainly on the analytical data, we think that muscular CT should be carried out in those cases which present symptoms of focal muscular lesion, in order to:

- establish morphological criteria of the extension and intensity

- plan the treatment

- later check-ups. 


\section{Room A}

\section{Marie Curie-Lecture}

901 Prospects in radiation oncology - its relationships with medical imaging

M. Tubiana; Villejuif

From the outset there has been close relationships between oncology and medical imaging.

The diagnosis of a tumor and its precise delineation require the help of medical imaging. For example without mammography, screening of breast cancer would be impossible. The discovery of CT-Scan has greatly contributed to the progress in radiotherapy because it has allowed a much more precise study of dose distribution in the tumor and normal tissues. It has been both possible in several instances to reduce the size of the irradiated volume and in others to enlarge it when deemed useful. The protection of critical normal tissues became more effective, reducing the severity of side effects. Magnetic resonance imaging can contribute in some types of patients to this endeavour. In the future the advances in 3-D dosimetry will be linked with the progress in 3-D imaging and display. Moreover magnetic resonance and nuclear medicine (in particular PET and SPET) will help the oncologists to study the biological characteristics of a tumor. It is already possible to follow hormones (such as estrogens) or growth factors and to assay their uptake in the tumor, evidencing the presence of specific receptors. For some tumors monoclonal antibodies are already able to detect small amounts of neoplastic tissues and open the way to metabolic radiotherapy. The experience gained during the past four decades in the use of radioactive iodine for the treatment of thyroid cancer shows that its contribution has been duofold: early detection of metastases not detected on conventional X-ray or CT scan and identification of patients with a sufficient uptake who can benefit from radioactive iodine therapy. Thyroid cancer can be a model for the development of the cooperation between medical imaging and radiation oncology. 
the outer half of the myometrium (Stage I C). In evaluating the exact degree of tumor invasion EV-US had sensitivity $=64.7 \%(22 / 34)$ and MRI had sensitivity $=70.6 \%(24 / 34)$. With EV-US 8 patients were overstaged and 4 patients understaged. With MRI 6 patients were overstaged and 4 understaged.

Conclusions: There is no statistically significant difference (chi square test) in the sensitivity of EV-US and MRI in evaluating the degree of myometrial invasion of endometrial carcinoma (Stage I).

\section{Room A}

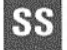

\subsection{Pelvic organs II - Bones}

$2.00 \mathrm{PM}$

902 Liver lesions - comparison of CT and T2-weighted fat-suppression, breath-hold FLASH and dynamic Gd-DTPA-enhanced FLASH at $1.5 \mathrm{~T}$ MR

R. C. Semelka, M. A. Kroeker, J. P. Shoenut, C. S. Yaffe, H. M. Greenberg, F. C. Simm, R. Kroeker, A. B. Micflikier; Winnipeg, CDN

Purpose: To compare high field MR sequences: T2-weighted suppression (T2FS), breath-hold FLASH, and dynamic Gd-DTPA enhanced FLASH, with dynamic contrast $\mathrm{CT}$.

Methods: 60 patients scheduled for CT studies were selected for MRI studies on the basis of a strong history for liver disease. CT studies were performed on a GE 9800 scanner. MR studies were performed on a $1.5 \mathrm{~T}$ cryogenic magnet (Helicon, 63SP, Siemens, Erlangen). CT and MR images were independently interpreted by experienced investigators using ROC analysis. Confirmation of disease was made by surgery $(n=22)$, biopsy $(n=14)$, or clinical/imaging follow up $(\mathrm{n}=24)$. Lesion detection and characterization were determined. Qualitative and quantitative $\mathrm{SD} / \mathrm{N}$ values were made on MR images.

Results: T2FS detected the most lesions (229) followed by Gd-DTPA enhanced FLASH (200), CT (193) and FLASH (185). Lesion characterization was highest for Gd-DTPA enhanced FLASH (194), followed by CT (176), FLASH (159), and T2FS (139). Liver lesion - liver SD/N was highest for T2FS (14.5 \pm 3.5$)$. Conclusions: T2FS has good lesion detection and Gd-enhanced FLASH good lesion characterization. In combination they may provide more information than $\mathrm{CT}$.

\subsection{PM}

\section{MRI evaluation of pathologic condition during pregnancy} B. Beomonte Zobel, S. Tella, M. Innacoli, C. Catalano, C. D'Archivio, R. Passariello; L'Aquila

Some authors suggested that MRI could play an effective diagnostic alternative in the study of pathologic conditions of mother and foetus during pregnancy. To verify the role of MRI in these conditions, we examined 26 patients in the 3rd trimester of pregnancy after an ultrasonographic (US) preliminary examination: 22 patients presented fetal or placental pathologies; 3 patients underwent pathological condition during pregnancy; in 1 case the MR examination resulted normal. Regarding to placental pathology we studied a case of placental cyst, three hematomas between the placenta and the uterine wall, two cases of partial placenta previa; regarding to foetal malformations we evaluated: an omphalocele, a Prune-Belly syndrome, femoral asymmetry, a tanatoforic dwarfism, a case of thoracophagus twins with cardiovascular abnormalities, fetal idrocephaly and abdominal cysts. Regarding to maternal pathology during pregnancy, we observed a case of subserous uterine fibromyoma, a right hydronephrosis, and a large ovarian cyst. In our experience, the high contrast sensitivity and the large field of view produced by MRI, was useful in the investigation of different pathologies occurred during pregnancy. In the evaluation of abnormality of fetus and placenta, especially during the 3rd trimester, MRI gives important informations so that it can be considered a complementary technique compared to US to investigate fetal malformations and intrauterine growth retardations.

\subsection{PM}

904 Transvaginal US examination and MR imaging in staging endometrial carcinoma (FIGO stage I)

A. Vanzulli, S. Sironi, D. Spagnolo, C. Belloni, G. L. Taccagni, A. DelMaschio; Milan

Purpose: Recently ultrasound examination (US) with endovaginal high frequency probes (EVUS) has been shown to be able to detect the invasion of myometrium of endometrial carcinoma. Aim of the present study was to compare the accuracy of MRI and EV-US in staging endometrial carcinoma (FIGO stage I).

Methods: We evaluated with 0.5 T MRI (SE: 2000/40-80) and EV-US 34 women with endometrial carcinoma (Figo stage I). Hysterectomy was performed in all the patients and the pathologic specimen was evaluated according to the revised FIGO criteria.

Results: At the histologic examination 4 patients had no invasion of the myometrium (Stage I A); 12 patients had involvement of the inner half of the myometrium (Stage I B) and 18 patients had endometrial carcinoma involving

\subsection{PM}

905 Is T1-relaxation time a diagnostic parameter for malignant lymph nodes of squamous cell carcinoma?

C. Wagner-Manslau, B. Clasen, U. Gebhardt, R. Dorn, P. Lukas, H.W. Pabst; München

Purpose: MRI and maybe also relaxometry should be settled for a better TNM-classification. Relaxometry is to be proven as a reliable tissue change parameter.

Methods and Materials: We investigated 24 patients having different malignant head and neck tumours using IE-mode (TR1400/TE30/TI120 ms). These inoperable tumours ( $\mathrm{T} 3$ or T 4 tumour), were investigated before therapy, after the first and second course of combined RCT (Mitomycin C and 5 Fluoruracil during the first four days of both courses of radiotherapy with each $30 \mathrm{~Gy}$ for the head and neck region and each 10-15 Gy on supraclavicular lymph nodes). $\mathrm{T} 1$ times were calculated with additionally IE-sequences $(\mathrm{TI}=$ $300,400,500 \mathrm{~ms}$ with reduced resolution and acquision time) determining $\tau_{0}$. Results: In correlation to literature (DEWES, DOOMS) malignancy shows an elevated relaxation time, T1 values of lymph nodes were prolonged to $830-1200 \mathrm{~ms}$. Radiochemotherapy reduces the T1 values, detectable lymph nodes have a T1 time from 500 to $800 \mathrm{~ms}$.

Conclusions: Relaxometry seems to be a specific parameter for better determination between malignant and hyperplastic lymph nodes and a reliable tissue change parameter for better TNM-classification and therapy monitoring.

\subsection{PM}

906 Evaluation of abdominal extent of malignant lymphatic disease: gradient-echo MR imaging versus $\mathrm{CT}$

G.P. Krestin, B. Marincek, W. Gross-Fengels; Zürich, Köln

Purpose: Evaluation of fast gradient echo MR imaging during suspended respiration for the assessment of the exact extent and for treatment planning in malignant abdominal lymphatic disease.

Methods: 35 patients with proven malignant lymphoma were included in the study. MRI was performed at $1.5 \mathrm{~T}$ with gradient echo sequences in transversal and coronal plane. In 27 cases additional iv. administration of Gd-DTPA was used for better delineation of anatomical structures. All patients had state of the art CT examinations with iv. contrast administration.

Results: All lesions could be assessed with both methods. MRI had clear advantages in delineation of retroperitoneal encased or displaced vessels ( $\mathrm{n}=$ 7), of renal infiltration or displacement $(\mathrm{n}=5)$ and splenic involvement $(\mathrm{n}=$ 3). Evaluation of the cranio-caudal extent of the disease was more accurate ( $\mathrm{n}$ $=4$ ) on coronal MR sections.

Conclusions: MRI with fast gradient echo sequences during suspended respiration is a true alternative to $\mathrm{CT}$ for evaluation of abdominal involvement in patients with malignant lymphoma.

\subsection{PM}

907 Magnetic resonance imaging (MRI) for the diagnosis of primary lymphoma of bone (NHL/S)

R. Stiglbauer, I. Augustin, J. Kramer, H. Schurawitzki, H. Imhof, Th. Radaszkiewicz; Vienna

Purpose: Aim of the study was to determine whether NHL/S exhibit a "typical appearance" on MR-images allowing this entity to be differentiated from other malignant bone tumors.

Methods: Eight patients (mean-age: $34 a$ ) with histologically and clinically verified NHL/S (distal femur, proximal tibia, distal tibia, os ilium: 4-2-2-1) were examined using a $1.5 \mathrm{~T}$ unit (T1- and T2-W SE sequences, 6 cases: 0.1 mmol Gd-DTPA i.v.). All MR-examinations were performed prior to therapy, none of the patients had received blood transfusions. Another 7 patients (mean-age: $14 a)$ with malignant bone tumours $($ PNET $=7$ ) were examined using comparable parameters.

Results: In most cases NHL/S appeared hypointense on T2-W images, corresponding to a high content of fibrous tissue visualized by silver staining in histologic specimens, whereas all tumors of the $2^{\text {nd }}$ group exhibited hyperintensity on T2-W images (only minimal content of fibres). Contrast enhancement was moderate to marked. NHL/S were predominantly localized epimetaphyseally in long bones; in 5 cases invasion into adjacent joints could be demonstrated, characterized by intraarticular formations showing signal characteristics equal to the lesion itself.

Conclusions: We conclude that hypointense signals due to a high content of 
fibrous tissue within lesions located epimetaphyseally in long bones along with a tendency to invade adjacent joints is probably characteristic of $\mathrm{NHL} / \mathrm{S}$.

$2.55 \mathrm{PM}$

908 MRI or scintigraphy for the assessment of response to chemotherapy in malignant bone tumors?

R. Erlemann, A. Sciuk, A. Roessner, P. Wuisman, C. Kusnierz-Glaz, J. Ritter; Münster

Purpose: MRI including Gd-DTPA enhancement and dynamic FLASH (TR $=40 \mathrm{~ms}, \mathrm{TE}=10 \mathrm{~ms}$, flip angle $=90^{\circ}, \mathrm{NSA}=2$ ) studies were utilized to assess response to chemotherapy.

Methods: In 18 patients suffering from osteosarcomas and 6 from Ewing's sarcomas MRI was performed before and after chemotherapy and the results were compared with quantitative 3-phase skeletal scintigraphy.

Results: Static MRI including Gd-DTPA enhanced imaging proved not valuable in differentiation viable from non-viable tumor tissue. Decrease of tumor size was not correlated with response. In responders $(n=13)$, after chemotherapy the slopes of the curves obtained with dynamic MR studies decreased significantly as compared to the values before chemotherapy, while in non-responders $(\mathrm{n}=11)$ slope values remained mostly constant $(\mathrm{p}<0.01)$. The accuracy of assessment of response amounted to $83 \%$. In skeletal scintigraphy differences between responders and non-responders concerning changes of values of perfusion and soft tissue phase were not significant $(p>0.05)$; while values of osseous phase decreased in responders significantly $(\mathrm{p}<0.05)$. However, accuracy of assessment of response amounted to only $73 \%$.

Conclusions: Dynamic MRI is a new non-invasive technique, which allows a reliable assessment of the efficacy of preoperative chemotherapy and seems to be superior to skeletal scintigraphy.

\subsection{PM}

909 MRI of Ewing's sarcomas: the potential of dynamicGd-DTPA-scanning for quantifying the chemotherapy-response R. Maas, M. Reuter, U. Heise, G. Delling; Hamburg

Introduction: Chemotherapy of Ewing's sarcomas has developed tremendously during recent years. After pre-operative chemotherapy the tumor may be resected locally. It remains a diagnostic challenge to answer the question as early as possible during preoperative chemotherapy whether the tumor is responding or not, so that the treatment modalities can be adapted. We are able to present our experience with dynamic Gadolinium-DTPA scanning in the follow-up of six Ewing's sarcomas.

Methods: During the last 5 years we examined 22 Ewing's sarcomas with MRI. Six had a follow-up-study for a 2 to 5 months period. They received a dynamic-GD-scanning, which consists of one slice which is scanned repetitively in rapid successions using a T1-weighted spin-echo sequence. GD-DTPA is given as a bolus immediately after the first scan. Quantification is done by means of an "irregular region of interest" (ROI). A phantom was introduced as a standard. The time-intensity-diagram reflects the significant increase and high signal intensity level, indicating a hyperperfusion of a malignant tumor. During chemotherapy perfusion decreases and correspondingly the signal intensity declines, indicating a responder. The resected specimen is then mapped by the bone-pathologist for estimation of remaining vital tumor cells. Results: Dynamic Gadolinium-DTPA scanning seems to be an excellent tool for quantifying tumor response during chemotherapy. There were 4 responders and 2 non-responders, whose correlation with the histopathologic mapping were excellent.

\subsection{PM}

\section{Enchondroma: effective diagnosis using gradientecho sequences and Gd-DTPA}

T. Yousry, U. Fink, B. Fink, S. Breitner, J. Lissner; Munich

Enchondroma: Effective Diagnosis using gradientecho sequences and Gd-DTPA

- Enchondromas are often difficult to differentiate from bone infarcts. The correct diagnosis is important as only Enchondromas have to be operated.

- We therefore examined 18 patients with equivocal radiographic patterns. T1 and T2 as well as gradientecho sequences with long TR were used. After Gd-DTPA application the T1 and the gradientecho sequences were repeated. Histologic confirmation was obtained in 15 patients.

- The diagnosis was established by MR in all the cases. Hyaline cartilage has a relatively hyperintense signal compared to bone infarcts which remain hypointense. Uncalcified tumor areas, not suspected before, were easily delineated. Gradientecho sequences improved the anatomic discrimination, had a similar signal behaviour as T2 weighted sequences and were less time consuming. In case of dense calcifications the uptake of Gd-DTPA by the tumor matrix helped establishing the diagnosis.

- In summary only one sequence the gradientecho sequence with long TR is necessary, with Gd-DTPA to be applied in only selective cases.

MR is therefore a fast and very reliable method in differentiating enchondromas from bone infarcts.

\section{$3.20 \mathrm{PM}$}

\section{MR findings in usual and unusual variants of osteosarcoma} B. Stöver, G. Sigmund, C.P. Adler; Freiburg

Based on histological classification an attempt was made to define variants of osteosarcoma by MR findings.

Consecutive investigations of 11 patients with osteosarcoma aged 13-50 years were reviewed. 3 were classified as osteogenic, 2 patients had teleangiectatic, 2 fibrogenic, 2 chondrogenic and 2 parosteal variants.

Intramedullar and extraosseous tumor components as well as joint and/or neurovascular involvement was clearly depicted in all patients.

Parosteal and teleangiectatic variants showed moderate signal intensity on $\mathrm{T} 1$ weighted images - whereas SI in all but the fibroblastic type was low in T1 sequence.

Fat-tumor contrast and evidence of a pseudocapsule however allowed no differentiation.

\subsection{PM}

912 High-resolution MRI of carpal and tarsal bones: imaging of pathologic changes

Th. Heuchemer, M. Wüstner, G. Hofmann, W. Weidenmaier; Ulm

Using surface coils and high resolution matrix, magnetic resonance imaging of carpal and tarsal bones and their pathologic changes as tumors, fractures, necrosis, cysts, intraosseous ganglions, pseudarthrosis and bone infection is sufficient. We introduce an examination technique which considers the limited examination time and guarantees excellent images of the small bones, joints and the connected soft tissues. We use T1 (TR $600 \mathrm{~ms} / \mathrm{TE} 15 \mathrm{~ms}$ ), double-echo T2 (TR $2500 \mathrm{~ms} /$ TE 20 and $90 \mathrm{~ms}$ ) and FISP (TR $400 \mathrm{~ms} /$ TR $12 \mathrm{~ms}$; flip-angle $70^{\circ}$ ) sequences. By the first 30 patients we demonstrate the possibilities and limitations of this examination technique.

\subsection{PM}

913 Magnetic resonance imaging in the evaluation of prognostic grade in patients with non-Hodgkin lymphoma

Rickard S. Nyman, Suzanne M. Rehn, Bengt L. G. Glimelius, Hans E. Hagberg, Anders L. Hemmingsson, Göran O. Sperber; Uppsala

Purpose: To find out if the degree of inhomogeneous signal intensity pattern in lymph nodes with non-Hodgkin lymphoma (NHL) might be related to the grade of malignancy as determined by histopathology.

Methods: Seventy patients with NHL were investigated with MRI $(0.5 \mathrm{~T})$. The signal intensity in the lymph nodes were evaluated in T2-weighted and pre- and post Gd-DTPA enhanced T1-weighted images and determined to be either inhomogeneous or homogeneous. A method to measure the degree of inhomogeneity was developed and compared with a subjective evaluation

Results: A large proportion of the high grade NHL (about $70 \%$ ) had an inhomogeneous signal intensity and almost all low grade NHL (about 90\%) had a homogeneous pattern. The degree of inhomogeneity was not related to lymph node size. The discrimination between high and low grade NHL nodes was further improved with Gd-DTPA enhancement. The inhomogeneity was to some extent related to necrosis at histopathology. The survival in patients with homogeneous lymph nodes was significantly better than in those with inhomogeneous nodes.

Conclusions: The degree of inhomogeneous signal intensity pattern is possible to measure and seems to be useful in the prediction of prognostic grade in patients with NHL.

\section{Room B}

SS 2.00 PM

\section{Diagnosis and therapy of spinal AV-malformations (Keynote Lecture)}

J. J. Merland; Paris

Abstract available at the information desk. 


\section{$2.15 \mathrm{PM}$}

\section{Benefits and pitfalls of MRI in the detection of spinal vascular pathology}

D. Wimberger, A. Neuhold, P. Samec, K. Heimberger, H. Imhof; Vienna

Purpose: To define characteristical MRI patterns of spinal vascular pathology. Methods and Materials: MR images of 43 patients with suspected spinal vascular pathology were evaluated retrospectively and compared with superselective spinal angiographies $(17 \times)$ and/or myelographies $(21 \times)$ and/or spinal CTS $(12 \times)$ and/or operation reports. MRI was done using $0,5 \mathrm{~T}$ or $1,5 \mathrm{~T}$ superconducting systems surface coils, T1, T2 w. SE- and GE sequences in 2 planes.

Results: 24 MRI diagnoses of spinal vascular pathology were proved. $2 \times$ MRI was the only method to identify operatively proved vascular pathology. In 2 cases with pathological vessels in MRI operative findings were negative, $3 \times$ MRI did not show vascular pathology which was found angiographically or intraoperatively. 19 MRI diagnoses could not be verified up to now.

Conclusions: Characteristical MRI patterns for spinal vascular pathology are: 1. intramedullary vessels with or without hemorrhages. 2. extramedullary serpigineous vessels 3 . medullary lesions in presence of widenened vessels. Identification of an increased number of vessels only, has to be interpreted rather as sign of the presence of another than a vascular pathology.

\subsection{PM}

\section{Automated percutaneous lumbar discectomy (APLD) method and one year follow up}

Christian Luft, Jörg Weber, Werner Horvath, Paul Pürgyi; Linz

Purpose: The APLD is described as a very safe interventional radiological technique for the treatment of lumbar protrusions and covered disc herniations. The one year follow up of our own material will be presented.

Methods: The Nucleotome probe is a $2 \mathrm{~mm}$ rounded blunt tipped needle with a single side port. Movable disc material will be aspirated by a vacuum and cutted by a little blade guillotin-like. After CT-planning the probe is located in prone position by a sheath under rotating fluoroscopy using a posterolateral approach. The selection criteria are a positive CT scan in correlation to radicular leg pain and failure of at least 6 weeks of conservative therapy, Absolute contraindications are signs of free fragment (sequester). From July 89 to December 9096 discs on 93 patients were treated. $63 \times$ level $\mathrm{L} 4 / 5$, $31 \times \mathrm{L} 5 / \mathrm{S} 1,1 \times \mathrm{L} 3 / 4$ and $1 \times \mathrm{L} 5 / 6$. Average age was 41,7 years, 64 female and 32 male.

Results: Ca. $80 \%$ of the patients have good to excellent results in a follow-up period of one year $(n=52)$. No major complications appeared. 2 of the unimproved had laminectomy.

Conclusions: Our experience indicates that APLD has to be an alternative to laminectomy and chemonucleolysis for uncomplicated herniated lumbar discs.

\subsection{PM}

917 A new deal with far lateral lumbar disk herniations (FLDH) automated percutaneous discectomy

F. Vanneroy, F. Courtheoux, H. Huet, J. Theron; Caen

AIM: The authors put forward that percutaneous discectomy may be proposed for treatment of FLDH with the same successful results obtained with intraspinal herniations.

Methods: After informed consent, percutaneous discectomy was carried out in 17 patients presenting with such FLDH among 273 patients treated by the method for lumbar disk herniation between February 1988 and July 1990.

Results: Very good and good results were obtained in 12 patients (success rate $=70 \%$ ) with a follow-up of at least 5 months. 3 cases among the 5 failures were later treated by injection of Chymopapain with success in 2 cases. Only 2 patients were operated in the end.

Conclusions: The authors emphazise the value of percutaneous discectomy in the treatment of FLDH.

\subsection{PM}

918 Postoperative lumbar spine: value of contrast-enhanced MR imaging

B. Hamm, B. Häring, M. Mayer, H. Traupe; Berlin

Purpose: Prospective study to assess the value of contrast-enhanced MRI in differentiating scar and recurrent disk herniation.

Methods: 112 patients with suspected recurrent disk herniation in a total of 133 segments were examined by MRI [1.5 T; T1-weighted spin echo sequence before and after intravenous bolus application of Gd-DTPA $(0.1 \mathrm{mmol} / \mathrm{kg})]$. 52 segments in 47 patients had been examined by CT. The results were evaluated separately by two independent radiologists.

Results: Contrast-enhanced MRI improved the differential diagnosis of scar and recurrent disk hernation significantly for both examiners in comparison to plain imaging and CT. 31 patients underwent renewed surgery, and in 29 cases $(94 \%)$ the MRI findings were confirmed $(13 \times$ recurrent disk herniation, $9 \times$ postoperative scar with inclusion of small sequesters, $7 \times$ postoperative scar). In 2 cases the intraoperative findings contradicted the results of MRI $(1 \times$ postoperative scar, $1 \times$ postoperative scar with inclusion of small sequesters).

Conclusions: Contrast-enhanced MRI is the most accurate method at present for differentiating recurrent disk herniation and scar in the postoperative lumbar spine.

\subsection{PM}

\section{Digital luminescence radiography (DLR) in myelography}

R. Erlemann, W. Wiesmann, G. Reuther, T. Vestring, J. Adolph, J. Fervers, P. E. Peters; Münster

Purpose: The value of DLR ( $2.5 \mathrm{lp} / \mathrm{mm}$, gray-scale: $10 \mathrm{bit})$ was evaluated in myelography (decision making depends more on image contrast than on spatial resolution). DLR was compared with conventional myelography using ROC methodology.

Methods and Materials: 95 conventional (CR), digital (DR) and edge enhanced digital (EDR) myelographic images (49 disk herniations, 46 normal) were read independently by 5 board certified radiologists with no clinical information. Findings were scored using a 5 point confidence-scale and a ROC analysis was performed based on CT-myelography as gold standard.

Results: Higher confidence levels were used in making both correct and incorrect diagnosis with EDR than with CR. However, no statistically significant differences between AUC (area under the curve) values obtained with $\mathrm{CR}(0.784 \pm 0.037), \mathrm{DR}(0.776 \pm 0.0132)$ and $\mathrm{EDR}(0.768 \pm 0.042)$ were noted (all $\mathrm{p}>0.05$ ). With EDR, sensitivity at a $10 \%$ false positive level was slightly lower in the lumbar spine $(50 \%)$, and due to smoothing contrast differences within the cervical-thoracic junction sensitivity was clearly lower in the cervical spine ( $24 \%$ ) than with CR ( $62 \%$ and $37 \%$, respectively).

Conclusions: In myelography, no increase in diagnostic accuracy but some decrease in sensitivity was obtained particularly with contrast postprocessing in DLR compared with conventionial radiographs.

\subsection{PM}

920 Three-dimensional imaging in the trauma of the dorsolumbar spine R.Tran, A. Bouamama, C. Louail, D. Gene de Beaufort, J. M. Vital, J. M. Caillé; Bordeaux

The authors evaluated the contributions of three dimensional reconstructions with CT-scan in the traumatic pathology of the dorsolumbar region.

Methods: $3 \mathrm{D}$ imaging was performed routinely in the pretreatment evaluation, following a standard CT-scan examination, using a Somatom DRH Siemens. In 25 cases, the results of $3 \mathrm{D}$ imaging performed before treatment were compared to the surgical findings.

Results: 3D reconstruction can determine the mechanism of fracture whether it is by compression, flexion-distraction and translation of pure distraction, allowing a better surgical approach.

However, the native CT-scan slice remains indispensable to visualize the small details of the fracture, which are not observable with the $3 \mathrm{D}$ reconstruction because of smoothing of the image.

In three cases of rotational fracture-luxation with facet-locking not well visualized by a simple X-ray, and in a case of severe burst, 3D allowed the modification of the therapeutic conduct and the performance of a well adapted surgical procedure.

The authors emphasize the particular interest of this technique in the exploration of posterior arch lesions and complex displacements. It must be noted that there is probably an increase of back-waid movement of the posterior vertebral wall, in certain bursts without neurologic complications.

Conclusions: The scanner with $3 \mathrm{D}$ reconstruction appears as an useful examination in the pretreatment evaluation of traumatisms of the dorsolumbar spine, because it determines the mechanism of the fracture. Thanks to this technique, the utilisation of emergency conventional tomography will probably be avoided in the future.

3.15 PM

\section{Percutaneous discectomy on an ambulatory basis}

Alvaro Cebrian de Almeida Magalhães, Fernando Pedrosa; São Paulo

25 patients with lumbar disk herniation were treated by percutaneous discectomy on an ambulatory basis. All patients were selected and evaluated following Onik's criteria. The procedure was performed under local anesthesia and took approximately 90 minutes. The nucleus pulposus was suctioned by a $2 \mathrm{~mm}$ aspiration probe, placed into the disk under fluoroscopic guidance. No complications were observed.

$80 \%$ achieved good to excellent results. Follow up from 3 to 30 months is presented. The preliminary results indicate that percutaneous discectomy is a low morbidity procedure that can be done on an outpatient basis. 


\subsection{PM}

922 Spontaneous intraspinal epidural hematoma - report of two cases J.Barcelo, J.Teruel, V. Calatayud, J. C. Vilanova, J. Ferrer, F. Castaner, I. Llado; Girona

The spontaneous intraspinal epidural hematoma (SIEH) is an uncommon pathology, arising from venous bleeding according to several authors. We present two cases of SIEH examined with magnetic resonance (MR), myelography, and angiography; with the surgical and histological correlation. These examinations did not identified any underlying pathological process. In both cases a blood clot of the thoraco-lumbar epidural space was extracted. The prognosis of SIEH is usually poor unless surgical decompression is performed. From that, it follows the importance of an accurate diagnosis when an acute spinal or radicular pain with neurological signs of spinal cord compression is presented.

\subsection{PM}

923 Symptomatic intervertebral disc destruction or collapse: a diagnostic challenge

K. York Chynn, Ethimios Spyropoulos, Meyer Alpert, R. D. Singson; New York, NY

Neck pain and back pain with destructive process of the intervertebral disc and vertebral body endplates present a diagnostic challenge to general radiologists and neuroradiologists alike.

The clinical data, conventional radiographs and when available, CT scans, myelogram, and MRI in 73 patients with pathologically proven lesions involving the intervertebral discs were reviewed. (Fifty-nine patients with percutaneous needle biopsy and 14 with open surgery.) The study extends from 1979 through 1990.

The purpose of the study is to illustrate the important radiological features and their limitations in the diagnosis of pyogenic, tuberculous spinal osteomyelitis and other conditions. The percutaneous needle biopsy has been proved to be a safe procedure in this series, provided meticuolous technique and strict precautions are used.

\subsection{PM}

924 Functional MR imaging of the cervical spine after distortion injury W. Weidenmaier, B. Wallner, K. A. Schumacher; Ulm

Distortion injuries of the cervical spine are a diagnostic problem for the clinician as well as for the radiologist. In order to find pathomorphological correlates for neurological deficits, invasive procedures like myelography and post-myelography $\mathrm{CT}$ are required.

In 31 patients who suffered from distortion injury of the cervical spine functional MR imaging was performed. The patients were placed in a device, that allowed four to seven defined positions of the cervical spine from maximum reclination to inclination. Images were acquired in the different positions using a $\mathrm{T} 2$-weighted gradient echo sequence. Instability of the dorsal longitudinal ligament and disc protrusion, that was only visible in certain positions of the cervical spine, could be visualized in $80 \%$ of these patients. We conclude, that functional MR imaging of the cervical spine may be a useful tool in evaluation of patients with distortion injury of the cervical spine. It provides information about instability of the discs and ligaments, otherwise not available with MR imaging.

\section{Room C}

\section{Ss} $2.00 \mathrm{PM}$

\subsection{Biliary interventional radiology}

925 Percutaneous transhepatic cholangioscopy and biopsy using a small cholangioscope $(9,3 \mathrm{~F})$

K. H. Hauenstein, R. Salm, Ph. Vinieé, J. Sontheimer; Freiburg

Purpose: Optical and histological determination of the tumor borders in patients with obstructive jaundice.

Results: Due to the flexibility and an external control mechanism of our thin calibrated cholangioscope $(9.3 \mathrm{~F})$ an inspection of the bile ducts via a percutaneous transhepatic access is possible without too much discomfort for the patient. A 3.6 F working canal enables target specific biopsies under optical control. It does not only allow the histological diagnosis of the tumor itself but also the exact definition of proximal and distal tumor borders. Percutaneous stone extraction by contact lithotripsy or with a Dormia-basket is possible via the working canal under endoscopic view. An inspection of the peripheral branches of the same and the other liver lobe from only one access has been made possible by the easy manoeuverability and flexibility of the endoscope. Results: Up to now we successfully used this method in 49 patients with obstructive jaundice (in 4 cases before and after intracavity radiation therapy). In 2 cases we extracted stones under optical control.

Conclusions: Due to the thin calibrated cholangioscope $(9.3 \mathrm{~F})$ an inspection of the bile ducts via a percutaneous transhepatic access is possible without any discomfort for the patient.

\subsection{PM}

926 Percutaneous transhepatic endoscopy as adjunct to diagnosis and treatment of biliary strictures

Elvira V. Lang, Gerhard R. Wittich, Denise L. Johnson, Jeffrey H. Reese, Clyde A. Stevick; Palo Alto, CA

Purpose: When radiographically guided biopsies or bile cytology in patients with biliary strictures do not reveal malignant cells, the diagnosis of benignity is uncertain. During internal biliary stenting sparing of the ampulla and ductal branch points is important to prevent reflux or stent induced obstruction. Distortion of the anatomy by the stricture, drainage catheters or safety wires may preclude exact radiographic localization of the end points of the lesion and hence introduce error in deployment of biliary stents. We therefore investigated the value of percutaneous endoscopy in these situations.

Methods: 15 patients with biliary strictures underwent percutaneous biliary endoscopy with $2.8-5 \mathrm{~mm}$ endoscopes. After balloon plasty, temporary external/internal stents were placed across benign strictures and self-expanding internal biliary wall stents across malignant strictures.

Results: In 7 patients endoscopic inspection of strictures with or without visually guided biopsy allowed a confident diagnosis of benignity which was confirmed during 6-18 months follow-up. In 8 patients with malignancies, percutaneous endoscopy allowed evaluation of extent and type of lesions and custom-tailored deployment of the wall stents in all cases. It was crucial in sparing of the ampulla.

Conclusions: Percutaneous biliary endoscopy is a useful adjunct to diagnosis and custom-tailored treatment of biliary strictures.

\subsection{PM}

927 Long-term follow up in benign biliary strictures treated with metallic stents

P. Rossi, P. Ricci, M. Bezzi, F. Maccioni, F. M. Salvatori; Roma

Purpose: Since 1983 we have treated 79 patients with benign biliary strictures (BBS). In the group considered for statistical evaluation the success rate was $70 \%$ after balloon dilatation. Eighteen patients who underwent repeated dilatations and long-term stenting, without resolution, were considered for metallic stents implantation (MSI).

Methods: Initially all patients with recurring BBS underwent MSI. In the last year no more stents were positioned, not because of unsatisfactory results, but in order to observe the clinical evolution and the efficency of these new devices in the biliary tree. The first patient was treated in November ' 88 , while the last patient in November ' 89 . The stents placed were self-expandable and made according a Gianturco-Rosch. We had no complications in stent positioning. Results: Our follow-up is now 16-26 months (average 18). Actually we have no recurrence in $12 / 18$ patients $(67 \%)$ and symptomatic recurrence in $3 / 18$ patients (17\%). Two patients died for liver failure with multiple stenoses and intrahepatic cholangitis $(2 / 18-11 \%)$ and one with cholangiocarcinoma $(1 / 18$ $-5 \%$ ).

Conclusions: While the first treatment of a newly diagnosed BBS should be surgical, the recurrence after surgery do benefit of percutaneous balloon dilatation. The use of metallic stent, in recurrent cases, may increase the rate of success of percutaneous maneuvres.

\section{$2.30 \mathrm{PM}$}

928 Percutaneous use of the Wallstent in advanced malignant hilar lesions

J. S. Laméris, J. Stoker, H. G. T. Nijs, M. van Blankenstein, O. T. Terpstra; Rotterdam

Purpose: The value of placement of Wallstents in palliating patients with malignant hilar lesions, causing biliary obstruction, was studied.

Methods: Between 04-89 and 12-90 50 Wallstents were placed in 36 patients with inoperable malignant biliary obstruction, located in the hilum of the liver. Six patients had a type I lesion, 10 a type II lesion and 19 a type III or IV lesion. In 11 of 17 patients with incomplete drainage, who had a normal common bile duct outflow tract, had prepapillary positioned stents.

Results: In all but 6 patients (type III or IV) bilirubine levels turned to normal values. The median survival of 15 patients who died was 4.3 months $(0.7-8 \mathrm{~m})$. Twenty-one patients are alive after a median period after stent placement of 8 months, 5 of them are surviving longer than one year. Recurrence or worsening of the jaundice was seen in 10 patients, 1-6 months (median 4 months) after stent placement. Eight of these patients had signs of cholangitis. Tumor overgrowth at the proximal end of the stent(s) was the cause in 7, at the distal end in 2 and tumor ingrowth was seen in 1 patient. Six patients underwent a 
re-intervention, 4 died without further interventions.

Conclusions: From our experience sofar we conclude that the use of the Wallstent in advanced malignant hilar lesions offers several advantages. The open mesh of the stent allows drainage of second order ducts. The prepapillary position of the stents seems to reduce the chance on septic complications. The rate of re-interventions is low. Tumor overgrowth remains the most frequent cause for recurrent jaundice.

\subsection{PM}

929 Metallic biliary endoprosthesis occlusion - the problem and the solution

A. Adam, J. Jackson, M. E. Roddie, N. Chetty, I. S. Benjamin; London

Purpose: To establish the cause and rate of occlusion of self-expandable metallic biliary endoprostheses (Wallstent, Medinvent SA) and to develop a satisfactory method of overcoming this problem.

Methods: Over 27 months biliary endoprostheses were inserted in 54 patients with malignant obstructive jaundice. The diagnosis of stent occlusion was established by ultrasonography and cholangiography. A second endoprosthesis was advanced into the occluded stent in a position where it overlapped the proximal or distal end of the original endoprosthesis and extended beyond the occluding mass. In one case a plastic endoprosthesis was inserted through a metallic stent.

Results: None of the stents have become occluded due to ingrowth of tumour through the stainless steel mesh. Occlusion due to encrustation of bile has not been observed. However, during the follow-up period 4 patients were readmitted to hospital with stent occlusion due to growth of tumour above or below the endoprosthesis, one of the patients presenting twice with this complication. The procedures were successful in relieving the obstruction in all cases. Conclusions: Occlusion is less common with metallic than with conventional plastic stents and the usual cause is tumour overgrowth. This can be managed more simply than plastic stent replacement.

\subsection{PM}

\section{Preliminary experience with two different metallic stents for the} treatment of biliary stenoses

W. Jaschke, H. P. Busch, E. Wetzel, M. Georgi; Mannheim

Purpose: Metallic stents were recently introduced for the treatment of benign and malignant biliary stenoses. Currently, 2 different types of stents (balloon expandable and self expanding) are available for applications in the biliary system. We report our experience with both types in 30 patients.

Methods: All stents were implanted following transhepatic catheter drainage for 3 days. A $23 \mathrm{~cm}$ long $8 \mathrm{~F}$ sheat was used for introduction of the stent into the biliary system. Clinical follow-up included physical exams and laboratory tests. The longest follow-up period was 18 months.

The results may be summarized as follows. Approximately $25 \%$ of all biliary stenoses could not be dilated adequately. Thus, implantation of a balloon expandable stent was impossible. However, a self expanding metallic stent could be successfully used in all these cases. The mean survival time was 4 months. Symptoms of obstructive jaundice were relieved in more than $90 \%$ of all patients. Reocclusion was encountered in 3 patients. This was due to tumor invasion of the stent ( 1 patient), tumor overgrowth ( 1 patient) and bile incrustation ( 1 patient).

\subsection{PM}

\section{Biliary wallstent endoprostheses: two years results}

Günter E. Klein, Klaus A. Hausegger, Johannes Lammer, Florian Waltner, Fritz Flückiger; Manuela Aschauer; Graz

Purpose: The longterm results of transhepatic treatment of obstructive jaundice $(\mathrm{OJ})$ by metallic biliary prostheses (bP) are evaluated. Secondly we compare the results of metallic bP and plastic bP according to a randomized protocol.

Methods: Step I: 82 patients were treated with metallic bP (71 malignant, 11 benign obstructions). Control interval: $6-30 \mathrm{mth}$. Step II: 22 patients, 13 treated with plastic, 9 with metallic bP. All obstructions were malignant. Control interval: $3 \mathrm{mth}$.

Results: Step I: Successful primary implantation in $100 \%$. The 30 days mortality was $8.2 \%$. No death related to the procedure was observed. Stent occlusion occurred in 13 patients. Of those in 4 cases tumor ingrowth could be histologically proven. Step II (preliminary results): Successful implantation in $100 \%$. About the same 30 days mortality (12.8\% plastic vs. 10.8 metallic bP). One dislocation of a plastic bP, no stent occlusion.

Conclusions: Metal bP offer the advantage of a primary implantation procedure. Due to a smaller transhepatic working channel (7F compared to 12 F) the implantation of metal bP is less traumatic. Although we could not observe a significant difference in the complication rate and 30 days mortality yet, metallic bP seem to be superior to plastic bP in treatment of OJ.
932 Wide-gauge $\mathrm{Y}$-shaped endoprosthesis stent (F16) with a subcutaneous port system - a new device for biliary drainage in Klatskin tumors K. H. Hauenstein, R. Salm, Ph. Vineé, J. Sontheimer; Freiburg

Purpose: Surgical therapy or implantation of stents in cases of central tumor biliary obstruction including both bile ducts are in most cases impossible. The development of a $16 \mathrm{~F}$ Y-shaped-polyurethane endoprosthesis stent enables to drain the left and right biliary system simultaneously. The implantation of a subcutaneous port system connected to the prosthesis allows not only X-ray examination of the function, but also lavage of the prosthesis and the biliary ducts.

Methods: An extern-intern percutaneous transhepatic biliary drainage (F7 or F8) is the basis of the implantation of the endoprosthesis. The endoprosthesis is placed via two long guide wires $(0,0035 \mathrm{inch})$ by a combination of external transhepatic and endoscopic approach, the so called "Rendezvouz-Technique" by push from the oral end and pull from transhepatic ends with two catheters.

Results: Since 1988 we have been able to implant this wide gauge Y-shaped endoprosthesis without any complications in 46 cases. In the last 10 months we used the implantation of a subcutaneous port system connected to the prosthesis in 4 cases. In this way the patency of the prosthesis can be maintained substantially longer.

Conclusions: The implantation of a wide gauge Y-shaped endoprosthesis stent facilitates a successful therapy in inoperable cases of patients suffering from obstructive jaundice by Klatskin tumors.

\subsection{PM}

\section{Biliary wallstent endoprostheses: histologic findings in men}

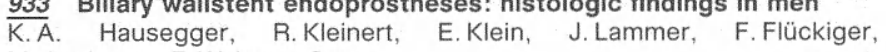
M. Aschauer, F. Waltner; Graz

Purpose: To evaluate the histologic changes adjacent to wallstent biliary endoprostheses in patients treated for malignant obstructive jaundice.

Methods: Necropsy specimens were available from 7 patients, surgical resection material from 1.6 patients suffered from pancreatic, 2 from gallbladder carcinoma. The stent was in place 5 days to 12 months.

Results: All stents were patent and free from intraluminal tumorous masses. The stents were easy to remove from the specimen in 4 cases (stent in place 5, 7 , 14 days and 6 months respectively). In 4 cases the stent was well embedded and macroscopically covered by a thin tissue layer (stent in place 6-12 months). Within the first 2 weeks stromal compression and unspecific inflammatory changes, after 2 weeks minimal foreign body reactions with multinucleated cells were observed. In the tumorfree segments fibroblastic cells covered the stent-pseudoendothelialisation. In the tumor-segments tumorcells invaded the stent without any stromal component. Intraluminal proliferation was not observed.

Conclusions: The wallstent biliary endoprosthesis does not induce major granulation tissue formation. Tumorcells tend to invade the stent without stromal components. Thus early exfoliation prevents reocclusion of the endoprostheses by tumorous masses.

\section{Room E1}

\section{SS}

\subsection{New technique in PTA (II)}

\subsection{PM}

934 Results of a randomised trial of conventional versus laser thermal PTA of total peripheral artery occlusions

Anna-Maria Belli, D. C. Cumberland, A. E. Procter, C. L. Welsh; London

The aim of the trial was (1) to assess whether there is any difference in primary success rate in treating total peripheral artery occlusions between the two methods, and (2) to assess any difference in the rate of recurrence.

81 patients with 84 peripheral artery occlusions suitable for PTA by the ipsilateral femoral approach were randomised to conventional PTA or laser thermal PTA using a $2.5 \mathrm{~mm}$ hybrid laser probe. There were 68 femoropopliteal and 16 iliac occlusions. Patients were followed up clinically and by ankle/brachial doppler indices for up to 1 year.

The results showed no statistical difference in primary success rate between the two methods in crossing femoro-popliteal occlusions, but the laser was unsuccessful in crossing the iliac occlusions in this trial. The primary success rate for femoro-popliteal occlusions was further increased by combining the two methods and using the mechanical properties of the laser probe.

Follow-up shows no statistical difference in the patency rates between the two groups at 1 month or one year. 


\subsection{PM}

935 Experimental and clinical results with a pulsed dye laser for angioplasty

Stephan H. Duda, Peter E. Huppert, Volker Arndt, Manfred Wehrmann, Claus D. Claussen; Tübingen

We investigated a pulsed $504 \mathrm{~nm}$ dye laser (Candela Corp.) for its angioplasty capabilities.

The system is operated at a pulse duration of $1.25 \mu$ s and a pulse rate of $10 \mathrm{~Hz}$. Laser energy was delivered via $7 \mathrm{~F}+9 \mathrm{~F}$ multi-fibre ring catheter $(200 \mu \mathrm{m}$ fibres, $.035^{\prime \prime}$ wire).

Our experimental data showed an effective ablation of atheroma and disruption of calcified plaque at an energy transmission of $60-80 \mathrm{~mJ} / \mathrm{pulse}$ under blood. Histologically there was only minimal thermal injury to the adjacent tissue $(10-20 \mu \mathrm{m})$. There was no charring. Irregular tissue borders after energy exposure under blood provide strong evidence for an ablation mechanism via shock or pressure waves. We performed laser-assisted balloon angioplasty in 25 pts. with total occlusions of the iliac and femoropopliteal arteries. Lesion length had a mean of $5.5 \mathrm{~cm}$. Total success after primary guide-wire passage was $24 / 25(96 \%)$. Stand-alone laser angioplasty with flexible multi-fiber laser catheters $(7+9 \mathrm{~F})$ was successful in 5 instances $(21 \%)$. 19 patients had subsequent conventional balloon angioplasty. There were no complications.

We conclude that the over-the-wire approach for delivery of pulsed dye laser light is a safe and efficient tool in laser angioplasty.

\subsection{PM}

936 In vitro experiments and initial clinical experiences in angioplasty of vascular occlusive disease with a pulsed dye-laser system

M. Zwaan, H.-D. Weiss, H. Kagel, M.Scheu, J.H. Göthlin, E. Gmelin, D. Kummer; Lübeck

Purpose: The ability of selective plaque ablation with laser radiation at $450-500 \mathrm{~nm}$ has already been demonstrated. We investigate then a new pulsed dye-laser device in vitro and in vivo for the ablation of atheromas, calcified lesions, and intimal hyperplasia.

Methods: In the in vitro study, laser induced fluorescence signals (LIF) of 376 different vessel wall specimens were detected at $495 \mathrm{~nm}$ wavelength and $2 \mu \mathrm{s}$ pulse duration. The particle sizes of different lesions during shock wave generation at $30,50,60 \mathrm{~mJ}$ were examined. The in vivo study included the treatment of 3 patients with shunt-fistula stenoses, 5 with altogether 8 lesions in the SFA, and 3 patients with iliac artery lesions.

Results: A discrimination between calcified and other lesions is with LIF made possible. The created particles are normally beneath the capillar diameter. In vivo the technical success of recanalization was $3 / 3$ in the iliac artery, $4 / 5$ in the SFA, and $3 / 3$ in the shunt-fistulas. We got 2 early reocclusions caused by a bad run-off, and 1 perforation in the SFA. Additional balloon angioplasty was necessary in 8 of 14 lesions. The AAI increased on average of 0.68 to 0.97 . The shunt patients were reintegrated in the dialysis programme.

Conclusions: Pulsed dye-laser angioplasty promises to be an effective and fast method for plaque ablation. The first clinical experiences confirmed the in vitro results, specially in the treatment of rigid shunt stenoses.

\subsection{PM}

\section{Experimental vascular tissue ablation using a pulsed infrared} holmium laser

D. Vorwerk, G. Zolotas, S. Hessel, F. Wondrazek, G. Adam, R. W. Günther; Aachen

The ablative potentials of a pulsed $2100 \mathrm{~nm}$ holmium laser on normal and diseased vascular tissue were tested in vitro. It was transmitted by a $600 \mu \mathrm{m}$ fiber and applied on post-mortem aortic specimens $(n=72)$ of normal tissue $(n=24)$, lipomatous $(n=24)$ and calcified plaques $(n=24)$. Energy per pulse varied from 120 to $890 \mathrm{~mJ}$. The specimens were analyzed histologically. In normal tissue and lipomatous plaques, an effective ablation was obtained even with low energy of $120 \mathrm{~mJ}$. Perforation and minor thermal changes occurred frequently. Mean ablation rate per pulse increased significantly from $67 \pm 30 \mu \mathrm{m}(120 \mathrm{~mJ})$ to $553 \pm 235 \mu \mathrm{m}(580 \mathrm{~mJ})$ in normal tissue and lipomatous plaques; no significant difference in ablation depth was found between both types of tissue. In calcified plaques, ablation was significantly reduced to $35 \pm 33(205 \mathrm{~mJ})$ and $126 \pm 96 \mu \mathrm{m}(315 \mathrm{~mJ})$; ablation was first obtained with $205 \mathrm{~mJ} /$ pulse. Damage to the fiber did not occur.

All types of atherosclerotic tissue were effectively ablated by the fiber-transmitted holmium laser. Distinct difference in ablative thresholds for lipomatous and calcified material compromises its clinical applicability and requires further safety considerations.

\subsection{PM}

938 A critical report on first clinical experiences with an excimer long-pulse laser

G. Küffer, F. A. Spengel, D. Hahn, W. Nathrath; Munich

Purpose: To report our first experience with the new excimer long-pulse generation for recanalization of femoropopliteal occlusions.

Methods and Materials: Device: Technolas excimer laser MAX 10LP. XeCl with $308 \mathrm{~nm}$, pulse length $115 \mathrm{~ns}$, pulse rate $20-40 \mathrm{~Hz}$, energy $240 \mathrm{~mJ}$. Use of $9 \mathrm{~F}$ ring catheters with $63 \mathrm{~mJ} / \mathrm{mm}^{2}$ mean energy density. Patients: 10 patients with 10 femoropopliteal occlusions. AVK stage: $6 \times$ II b, $1 \times$ III, $3 \times$ IV. Mean occlusion length $5.7 \mathrm{~cm}(2-15)$. Calcified 5/10. Two angioiscopic checks. Results: Technical success $8 / 10$. Clinical success $6 / 10$. Recanalization without guiding aid $4 / 10$ ( $3 / 4$ no Terumo probing possible). Stand-alone operation 3/9; with PTA 2/9, with PTA/Atherectomy 4/9. Complications: Contrast medium extravasation: $4 / 9$. One impending perforation. Embolism: $3 / 8$ (twice requiring urokinase). Early occlusion within $24 \mathrm{~h}: 2 / 8$ (once provoked by large inguinal hematoma). Technical problems: Fibre defects due to plaque calcifications (4/5) resulting in visible thermal effects on excised material.

Conclusions: In narrow, diffusely stenosed or occluded fem.pop. vessels a stand-alone technique is possible. Calcified plaques can be ablated. Improvement is required in the durability of the fibres, the effective crosssectional area and the steerability of catheters.

\section{$2.45 \mathrm{PM}$}

939 Development and use of a special sapphire tip for laser recanalization as sole therapy without PTA

D. Liermann, R. Goebel, J. Kollath; Frankfurt/Main

Purpose: The most results published about laser longterm results are in reality results of the PTA following the laser treatment and insofar not different from the results after PTA. The single use of a laser beam was not sufficient to create a greater diameter during the recanalization. The development of a special laser saphire tip allows to treat occlusions and stenosis only with laser but without balloon angioplasty.

Methods: Concerning our stent application and the problems with intimahyperplasia in the stented area in the adductorial channel, we searched for laser system, which will be able to create a channel which is big enough for sufficient bloodflow in the stent (approx. $6 \mathrm{~mm}$ ) but prevent from a necessary mechanical force of a PTA to the intima after recanalization. The development of a frosten saphire tip allows using a tip with a diameter of $3 \mathrm{~mm}$, insertable through a $9-\mathrm{F}$ sheat creating of a channel diameter $6 \mathrm{~mm}$ in pulsed wave and continuous wave technique of a Neodyn Yag Laser.

Results: It was possible to recanalize narrowed or occluded stent areas only by laser treatment to a diameter of $6 \mathrm{~mm}$ which allows a sufficient bloodflow without the use of mechanical forces of a balloon.

Conclusions: If longterm results after single use of laser will be better than PTA results, this method could be an alternative.

\subsection{PM}

940 Pulsed holmium: YAG lasers: large lumen and sole laser therapy early experience

P. Gaines, D. C. Cumberland; Sheffield

Laser devices used in the legs traditionally provide only narrow lumen that subsequently require balloon dilatation.

We have early clinical experience using pulsed Holmium: YAG laser energy of wavelength $2.1 \mathrm{um}$ : pulse duration $250 \mathrm{usec}$, at a frequency of $5 \mathrm{~Hz}$ (Trimedyne Inc., Tustin, California). The laser energy has been delivered by three different catheters in 13 patients with 21 femoropopliteal or tibial lesions ( 17 stenoses, 4 occlusions).

14 of 16 limbs were successfully treated (primary success $87.5 \%$ ). The average ABI rose from 0.7 pre-treatment to 0.84 following therapy.

In 7 lesions the lumen produced prior to balloon dilatation was larger than the tip of the catheter probe.

In 6 limbs only sole laser therapy was used without adjuvant balloon dilatation. In these patients the average pre-treatment $\mathrm{ABI}$ equals 0.66 . Six weeks following sole laser therapy $80 \%$ remain improved with a mean $\mathrm{ABI}$ of 0.73 . These early results suggest sole laser therapy is possible with implications for re-stenosis.

\subsection{PM}

941 Ultrasonic angioplasty: experimental in vitro penetration of fibrous and calcified atherosclerotic plaques

C. Müller-Leisse, T. Schmitz-Rode, U.Böhm, S. Biesterfeld, B. Klosterhalfen, R. W. Günther; Aachen

Purpose: To study the effect of an ultrasonic angioplasty device under precisely defined conditions to investigate their penetration depth on healthy 
human intima, fibrous and calcified plaques.

Methods and Materials: An ultrasonic angioplasty device consisting of a $1.2 \mathrm{~mm}$ diameter flexible wire with a $2 \mathrm{~mm}$ ball tip which was ensheathed in a $7 \mathrm{~F}$ guided catheter and guided vertically through a glass tube and attached to a power source generating ultrasonic energy of $26 \mathrm{kHz}$ was used. The ultrasound energy was applied to 137 segments of human cadaver atherosclerotic arteries, 90 with calcified, 47 with fibrous plaques and to 100 segments of healthy swine aorta. The bearing pressure and transmission time were varied.

Results: The average depth of penetration is dependent on the bearing pressure, the transmission time and the degree of atherosclerosis. There is significantly better penetration through fibrous than through calcified plaques. There was one perforation of a fibrous plaque using a bearing pressure of 200 p. Healthy intima was never injured.

Conclusions: Catheter-delivered ultrasound energy has a significant potential for recanalizing both fibrous and calcified atherosclerotic arterial obstructions. The risk of perforation and the injury of healthy intima is minimal even under extreme conditions.

\subsection{PM}

942 Angiosonoplasty: percutaneous intravascular plaque ablation with ultrasound - twenty-one months follow up

Mario Velez, Cesar Monteverde, Ricardo Jauregui, Arturo Abundes, Julian Borges; Vista del Valle

Peripheral arterial obstructive disease has been treated with ultrasound ablation. Thirty-two patients (pts), mean age $67 \pm 10$ years, underwent angiosonoplasty with an ultrasound prototype generator, (Sonic Needle Corp.), due to severe claudication $(31 \%)$, rest pain $(44 \%)$, rest pain and ulceration $(25 \%)$ Angiosonoplasty was successful in 26/32 (81\%) stenoses, (1/2 iliac, $9 / 12$ femorals, $11 / 12$ popliteals, $5 / 6$ tibials). Angiography before and after angiosonoplasty confirmed that the lesions were successfully opened, $(81 \%, \mathrm{p}-0.05)$. A mean of $3 \pm 2$ passes were required to adequately remove sufficient plaque. Balloon angioplasty was used in combination with angiosonoplasty in $11 / 32$ (34\%) occluded vessels. Complications included groin hematoma in 4 pts $(12 \%)$ and vascular spasm in two patients $(6 \%)$. Within 24 hours, $27 / 32(94 \%)$ pts were discharged clinically improved.

Reocclusion occured in 4 pts $(12 \%)$ immediately after the procedure. Anklebrachial indices significantly increased from $0.52 \pm 0.10$ before to $0.70 \pm 0.12$ after $(\mathrm{p}-0.05)$. In conclusion, the use of ultrasound energy to ablate atherosclerotic plaque exhibits its predictability in safely removing peripheral arterial stenosis with a low incidence of complications and late restenoses.

\subsection{PM}

943 New percutaneously implantable catheter port system for loco-regional chemotherapy of advanced malignant neoplasm within the pelvis

E. P. Strecker, W. Michaelis, S. Vetter, T. Claus, P. Tsikuras; Karlsruhe

Purpose: The commonly used catheter port systems for chemotherapy require the surgical insertion of a catheter leading to the tumor feeding artery, and a subcutaneous implantation of the connected port. This invasive method for endstage tumor disease patients has a questionable value because there are accompanying complications and the prognosis is unpredictable. Therefore a nonoperative and better treatment is mandatory.

Patients and methods: In the tumor-feeding internal iliac artery a 4 French polyurethane catheter is introduced by common angiographic techniques and connected to a newly developed cylindrically shaped port which is implanted subcutaneously through an introducer sheath. So far 25 patients with advanced carcinomas of the urinary bladder, prostate, cervic and uterus have been treated by the infusion of mitomycin and carboplatin.

Results: Follow-up up to 3 years including computertomography and cystoscopy revealed disappearance ( 10 cases) and improvement $(5$ cases) of the neoplasmas within the pelvis. There was one local tumor recurrency. Major complications related to the new system did not occur.

Conclusions: The advantages of the new port systems are:

less invasiveness than surgery, more exact catheter placement under angiographic conditions for selective chemotherapy, optional accompanying embolization of normal arteries for better blood flow distribution.

\section{Room E2}

SS $2.00 \mathrm{PM}$

\section{Osteoarthritis - a major radiological challenge? (Keynote Lecture) lain Watt; Bristol}

Traditionally $\mathrm{OA}$ has been seen as a wear and tear disorder of joints, with a relentless progression to joint failure. However this view has been overturned by fresh reappraisal in several major centres. Many of the time honoured concepts are shown to be unsound.

At least some of the classical radiological signs are known to be simply age-related rather than indicative of disease. Crystal deposition is seen to be a marker of bone and joint response or lack of it, not the cause of arthropathy itself. The predilection of joints which are involved may be at least partly governed by evolutionary underdesign.

Physiologically OA can be shown to have episodic crises which are detectable by radionuclide scintigraphy and possibly MR. Evidence of healing is commonplace, particularly when the conservative approach to therapy is practiced or enforced.

$\mathrm{OA}$ is shown not be a single disease but many, a changing spectrum even within an individual patient. Currently almost nothing is known of the pathogenesis of these disorders, although some understanding of the mechanisms of tissue response to disease is now possible by studying the features demonstrated by modern imaging.

$\mathrm{OA}$ is a major problem in an ageing European population. Urgent research is necessary if joint failure, with its consequent economic burden, is to be avoided.

\subsection{PM}

945 MRI of rheumatoid arthritis: contrast enhanced studies; perfusion dynamics

M. Naegele, V. Kunze, B. Woell, M. Reiser; Bonn

Purpose: Purpose of the study was to determine the value of contrast enhanced MRI and perfusion dynamics in rheumatoid arthritis for early detection of synovial proliferation and assessment of inflammatory activity of disease.

Methods: The hands of 43 patients with RA were examined by MRI. Sequences: SE T1 pre and post Gd-DTPA, SE T2, FFE dynamic scans (first image pre Gd-DTPA and 7 images post Gd-DTPA over a time span of $160 \mathrm{sec}$ ). Contrast material Gd-DTPA $.1 \mathrm{mmol} / \mathrm{kgbw}$, surface coil.

Results: T1W pre Gd-DTPA: No distinction between synovial hypertrophy and effusion possible. TIW post Gd-DTPA: Synovial hypertrophy reveals high signal intensity (SI) increase, high contrast between joint effusion and proliferations. T2W: Inhomogeneous high SI pattern of proliferations, poor differentiation from joint effusion. FFE dynamic scans: assessment of perfusion of synovial proliferation possible; high inflammatory reaction-strong and rapid signal intensity increase; inactive proliferation-low-medium SI increase. Conclusions: Contrast enhanced MRI of RA supports diagnostic workup of severity and exact extent of synovial proliferations and joint destruction. Dynamic studies might assist clinical evaluation of therapy protocols.

\section{$2.25 \mathrm{PM}$}

946 MR imaging of the cervical spine in rheumatoid arthritis: interest of Gadolinium-DOTA IV administration

J. Assoun, C. Poey, M. Pages, A. Roux, T. Billey, B. Fournie, J. J. Railhac; Toulouse

Purpose: Cervical spine involvement is frequent in rheumatoid arthritis (RA), but it is difficult to appreciate joint lesions except with roetgenograms. It is known that Gadolinium-DOTA (Gd-DOTA) IV administration is taken up by inflamed synovium and we report the study with MRI and Gd-DOTA of 12 patients with cervical spine arthritis in RA.

Methods: The 12 patients had classical of definite RA according to the ACR criteria, examined at our center in the years 1989 and 1990. All patients had clinical symptoms of cervical spine involvement (pain, stiffness, medullary or other neurological compressions). Plain radiographs are always performed (normal findings, erosions, subluxation, diastasis of $\mathrm{C} 1-\mathrm{C} 2$, inflammatory spondylodiscitis). MRI is realized on 0,5 Tesla Magniscan with a cervical coil in all patients: $\mathrm{Tl}$-weighted sequences before and after Gd-DOTA; T2-weighted sequences in sagittal and axial planes.

Results: MRI with Gd-DOTA IV administration seems very helpful in cervical spine of RA and allows better findings than T2-weighted sequences. Lesions are always most important in comparison with standard X-rays.

Conclusions: In our opinion, these methods can be helpful in therapeutic approach and neurosurgery in cervical spine of RA.

\section{$2.35 \mathrm{PM}$}

$\frac{947}{4 p}$

A. Giovagnoni, A. Lorenzoni, A. Corvetta, P. Ercolani, P. Busilacchi; Ancona

The purpose of this work is to define the potential role of US and MRI in definition of rheumatoid arthritis (RA) lesions of the hand in patients in early phase of disease and in follow up during medical treatment.

21 patients $(17 \mathrm{~F}-4 \mathrm{M})$ with clinical-laboratory diagnosis of RA (ARA criteria) underwent US and MRI of the hands. US investigation were performed by US scanner Ansaldo AU 450 with $7,5 \mathrm{MHz}$ transducer. MRI 
investigations were performed by a subconductive $1.0 \mathrm{~T}$ (Magnetom) scanner using a round surface coil. Coronal and axial SE T1 (500/17) and GE (FLASH$\left.200 / 10-70^{\circ}-15^{\circ}\right)$ images were obtained. In 15 patients i.v. Gd-DTPA was also administrated. US and MRI findings were blinded evaluated and correlated with clinical-laboratory data.

US appears to be a useful method for minimae soft tissue's lesions evaluation of the hand in patients with RA. MRI permits the best stage assessment of disease in relations to its optimal soft tissues and bone lesions identifications. Besides the use of contrast agent (Gd-DTPA i.v.) allow to evaluate the inflammatory activities of the pannus, already demonstrated by means US examinations.

Our experience suggests that US could be used as screening method in patients with early clinical-laboratory diagnosis of RA or as follow-up during medical therapy. MRI should be represent the gold standard image modality at beginning and at final point of treatment.

2.45 PM

$\frac{948}{\mathrm{MR}}$

G. Reuther, U. Cordes, T. Eger, R. Erlemann, P. E. Peters; Münster

Purpose: To assess the value of dynamic imaging in postoperative ligamentous instability of the wrist in lunatomalacia.

Methods and Materials: 20 patients with surgically treated lunatomalacia were examined with cine fluoroscopy and video loops of static MR images in different radial and ulnar deviations.

Results: Pathologic motion patterns of the proximal carpals could be detected with either technique. However, projection fluoroscopy better delineates the three-dimensional carpal shifts $(n=16)$, while MRI visualizes pathologic compressions of the triangular fibrocartilage (TFC) between the triquetrum and the ulnar styloid $(\mathrm{n}=18)$.

Conclusions: Dynamic MRI of the wrist is the method of choice for the evaluation of dynamic pathology of the TFC, while it does not add information about carpal instabilities and ligamentous lesions which cannot be derived from cine fluoroscopy. Fluoroscopy remains the most efficient imaging technique in carpal instabilities.

\section{$2.55 \mathrm{PM}$}

\section{Digital subtraction arthrography in the diagnosis of loosening hip prosthesis}

B. K. Fink, U. Fink, M. Hansen, M. Pfahler; Munich

Purpose: The most common cause of failure of total hip replacement is loosening of one or both components of the prosthesis. Beside standard radiographs and radionuclide bone scans arthrography can provide important evidence of looseness. A manual subtraction technique was proposed to demonstrate the extension of contrast media throughout the bone/cement and the prosthesis. This study presents the results of digital subtraction arthrography (DSAr) in detection of loosening.

Methods and Materials: DSAr was performed in 99 patients with painful hip arthroplasties. $10 \mathrm{ml}$ of contrast medium were injected into the hip in bolus technique. During injection digital subtracted images were acquired. The arthrographic findings were compared with plain radiographs, radionuclide bone scans and a detailed clinical examination. Surgical findings were the golden standard in 47 cases.

Results: By DSAr even minimal gaps at the interface of the prosthesis, the cement and the bone can be seen. Additional advantages were real time visualization and the possibility of postprocessing. Aspiration of the joint fluid excludes sepsis. The accuracy of DSAr was $90 \%$ for the acetabular and $79 \%$ for the femoral component.

Conclusions: DSAr proved to be indispensable in the presurgical examination of painful arthroplasties.

\subsection{PM}

\section{Ultrasonography of the ankle}

R. K. Chhem, G. Schmutz, B. Bui, S. Groleau, F. R. Plante, A. Lussier; Sherbrooke, CDN

Purpose: To evaluate the role of ultrasonography (US) as a non invasive modality for assessing non traumatic ankle lesions.

Methods: US of the ankle was performed in 34 extremities in 17 patients. The patient population included 17 men and 10 women who ranged in age from 12 to 76 years. All the cases were specifically referred for evaluation of painful and/or swollen ankle. The control population included 12 healthy volunteers. US was performed in each patient using state of the art real time equipment with a $7.5 \mathrm{MHz}$ transducer. Standard study included longitudinal and transversal cross sectional orientations. In many cases the uninvolved ankle was used as a control. The US exams were correlated with clinical, biologic, radiologic, pathologic findings and follow-up.

Results: We found 7 tenosynovitis. Among them six were related to rheumatoid arthritis and one was associated with septic arthritis. Three bursitis were found, two were related to rheumatoid arthritis and one was an isolated finding. There were also two cases of ankle joint effusion. One case was associated with juvenile rheumatoid arthritis and the other was infectious. There was one abscess of the soft tissue secondary to subacute osteomyelitis. One case of osteochondroma pressing against the Achille's tendon without any sign of tendinitis.

Conclusions: In this small serie, US proved to be a useful, non invasive, simply performed technique for assessing non traumatic painful and/or swollen ankle.

\subsection{PM}

951 Dynamic imaging of the joints by ultrafast MRI techniques F. G. Shellock, T. Foo, J. H. Mink; Los Angeles, CA

Many types of joint abnormalities are not appreciated by static MRI views because the pathology may be related to a particular position within the range of motion of the joint. Recently, ultrafast MRI techniques have evolved that allow imaging of joints during active movement.

Purpose: To assess the joints during movement by ultrafast MRI.

Methods: A 1.5 T MR scanner (General Electric Co.) was used along with several different ultrafast MR imaging strategies to acquire images during active movement of the joints for "true" dynamic studies. Patellofemoral joints, TMJs, wrists, and ankles of normal subjects and patients were evaluated by these techniques. Examination times ranged between 7 and 12 seconds for each slice location obtained through the joints. Fields of view and slice thicknesses were adjusted to optimize the assessment of the joints and ranged from 8 to $32 \mathrm{~cm}$ for FOV and 3 to $7 \mathrm{~mm}$ for slice thickness.

Results: Joint abnormalities related to motion (i.e., patellar maltracking, TMJ disk displacements with and without recapture, carpal instabilities of the wrist, and bony and soft tissue impingements of the ankle) were observed during active joint movement using ultrafast MRI techniques.

Conclusions: Examination of the joints during movement by ultrafast MRI techniques is a useful adjunct to standard static MRI views.

\subsection{PM}

952 MRI for evaluation of therapeutic effects on patients with Heberden's and Bouchard's osteoarthritis treated with the non-steroidal antirheumatic substance Ademetionin

M. Kevenoglu, H. König, H. Stahl, K.-J. Wolf; Berlin

Purpose: The therapeutic effect of the non-steroidal antirheumatic Ademetionin in patients with finger osteoarthritis was evaluated by MRI with regard to the qualitative morphological alterations and the cartilage signal intensity in DIP and PIP joints.

Patients and methods: 23 patients with clinically diagnosed finger osteoarthritis were examined clinically, radiologically and by MRI over a period of 6 months. In 15 patients an MRI of the hand was carried out before treatment and 6 months after oral application of Ademetionin. 8 untreated patients served as a control group. A double-spin echo sequence (TR 1200, TE 22, $90 \mathrm{msec}$ ) and a 3D-FLASH sequence (TR 30, TE $6 \mathrm{msec} .30$ grade) were applied. In addition to the evaluation of morphological alterations we measured the signal intensity of the articular cartilage of the DIP and PIP joints.

Results: Among the 15 patients treated, the findings were better/ unchanged/worse in $6 / 6 / 3$ (control group $1 / 5 / 2$ ) by clinical criteria, by MRI in $7 / 6 / 2$ (control group $2 / 4 / 2$ ) for cartilage signal intensity and in $3 / 10 / 2$ (control group 1/6/1) by morphological MRI pattern.

Conclusions: MRI permits a morphological evaluation of the effect of drug therapy in patients with finger osteoarthritis especially with regard to alterations of the joint cartilage.

\subsection{PM}

953 White blood cell scintigraphy (WBC) with monoclonal antibodies (MAB) in septic loosening of endoprostheses

J. Sciuk, C. Puskás, B. Greitemann; Münster

The septic loosening of an endoprosthesis is a severe complication, which requires special operative management. X-ray does not allow for differentiation between septic and aseptic loosening; MRI and CT are unsuitable due to artefacts caused by the prosthesis.

We evaluated the WBC-scintigraphy (Tc-99m-, J-123-labelled monoclonal antibodies) in 28 patients with clinically suspected septic loosening of an endoprosthesis (hip $\mathrm{n}=26$, knee $\mathrm{n}=2$ ). In 17 cases the comparison with a bone scan was possible. The final diagnosis was established by open surgery, histology and bacteriology. 10 patients had septic, 18 patients had aseptic loosening.

WBC-scintigraphy revealed the following results: true positive $\mathrm{n}=10$, true negative $\mathrm{n}=13$, false negative $\mathrm{n}=0$, false positive $\mathrm{n}=5$ (Sensitivity $100 \%$, Specificity $72 \%$, Accuracy $88 \%$ ). The 5 false positive scans correlated with 
damage of the polyethylene surface of the prosthesis $(n=2)$, metallosis $(n=2)$ and periprosthetic granuloma $(\mathrm{n}=1)$.

In defining the periprosthetic uptake of $\mathrm{MAB}$ as infectious lesion or as bone marrow island, comparison with skeletal scintigraphy was helpful. In the first case an increased and in the second case a normal bone turnover was observed. In conclusion, WBC with monoclonal antibodies is a safe, easy and simple in vivo method with high accuracy for diagnosis of septic loosening of endoprostheses.

\section{Room L}

\section{SS}

$2.00 \mathrm{PM}$

\subsection{Skeleton, general}

\section{Radiological investigations in children presenting with growth failure (Keynote Lecture)}

G. Kalifa, C. Adamsbaum; Paris

In most instances, conditions associated with growth failure can be differentiated on the bases of history, physical examination or by laboratory tests. However, radiological examination is mandatory to estimate the skeletal development, and to evaluate grossly the bone mineralisation. This is done mainly through a hand and wrist radiography (Greulich and Pyle's method). In addition, these films may display intrinsic bone abnomalities. No other $\mathrm{X}$-ray and U.S. examination should be routinely performed in these patients. But further radiological investigations will be proposed only according to the initial clinical and biological findings.

For instance a complete neuroradiological work-up is justified in case of pituitary dwarfism; skeletal survey may be useful in case of intra-uterine growth retardation, or clinical evidence of intrinsic bone disease.

A practical algorithm will be proposed for these various causes of short stature.

\subsection{PM}

\section{The Q-score and metacarpophalangeal pattern profile analysis} P.F. Dijkstra, H.W. Venema; Amsterdam

The Q-score is a new approach to standardization of bonelength measurements, to quantify congenital skeletal dysplasia of the hands, in the form of metacarpophalangeal pattern profile analysis (MCPP).

Purpose: The method is devised because of inaccuracies in the Z-score of the classic MCPP: the prominent scatter in the S.D. of the reference length, the tabulated reference data available only at one year intervals and the age of appearance of the epiphyses, which may vary considerably.

Methods: The Q-score simply expresses the length of each bone relative to the reference. For a better reference we calculated the sum of the length of the bones for each age and fitted a polynomial through these points. The percental fraction which each bone contributes to the added length, can be described by an exponential which approaches an asymptotic level. Reference length bone $=\operatorname{sum}(\mathrm{y})^{*}$ fraction $(\mathrm{y})$. We use a standard without epiphysis for ages below 7 years.

Results: The advantages of the Q-score are that no S.D. is needed and no rounding errors occur because the exact age of the patient can be used. The deviation of the reference length for both girls and boys is at most $2 \%$ for any age, eliminating the rounding error for age. Especially at low age the rounding errors can be substantial.

Conclusions: The Q-score is an easy and accurate way to standardize bonelength and lacks many of the drawbacks inherent to the Z-score, which will be demonstrated on a diversity of skeletal dysplasias.

\subsection{PM}

\section{MR imaging of epiphyseal dysplasia of the femoral head} G. Ranner, F. Ebner, W. Linhart, R. Wildling; Graz

Purpose: To assess the value of MRI for diagnosis of dysplasia epiphysealis capitis femoris (Morbus Meyer).

Methods and Materials: In 83 children with suspected LCPD and/or transient coxitis plain radiography, radioisotope bone scan and MRI were done.

Results: In 6/83 patients the course and the radiologic findings led to the diagnosis of epiphyseal dysplasia. Radiography showed mostly a slight flattening of the epiphysis and little alteration of the osseous contour. In infants the ossification center was narrowed or granulated. One case of bilateral involvement showed symmetrically radiolucent areas within the epiphysis. Scintigraphically no abnormality, a slight increase of tracer accumulation or a transient activity defect could be determined. MRI revealed in $5 / 6$ cases a sharply confined area of decreased signal intensity within the epiphysis. Conclusions: A single X-ray or MR study do not allow to diagnose epiphyseal dysplasia of the femoral head. If the bone scan is normal or shows a slight increased uptake dysplasia epiphysealis is strongly suggested. In cases of decreased uptake however follow ups are necessary to differentiate abortive LCPD from necrosis complicating epiphyseal dysplasia.

\subsection{PM}

957 Low dose computed radiography of scoliosis in children: review of $\overline{1600}$ examinations

John A. Kalmar, C. B. Merritt; New Orleans, LA

Over 1600 spine examinations in children ranging in age from 4 to 18 years have been performed using low dose techniques with computed radiography over a 5 year period. Records were reviewed to calculate the total savings in radiation burden resulting from the use of low dose techniques and the adequacy of diagnostic image quality in the serial evaluation of scoliosis. Our studies indicate that clinically useful studies can be routinely achieved at average exposure levels of less than $5 \%$ of those required for normal radiography, with significant reduction in cumulative dose to bone marrow and breast tissue in patients receiving serial examinations.

\subsection{PM}

958 Staging of synovial hypertrophy and cartilaginous destruction in hemophilic arthropathy of children and adolescents R. Erlemann, H. Pollmann, P. E. Peters; Münster

Purpose: Degree of synovial hypertrophy (SH) and of cartilaginous destruction (CD) in young hemophilics receiving substitution therapy were assessed and diagnostic value of MRI was compared to that of radiographs. Methods: 60 ankle and knee joints with bleeding history were investigated radiographically and with MRI using a 3-D FLASH sequence (40/12/flip angle $\left.15^{\circ}\right)$, and degrees of $\mathrm{SH}$ and $\mathrm{CD}$ were scored.

Results: Due to susceptibility, hemosiderinladen synovia showed very low signal intensity on FLASH images, while normal cartilage was bright. Stages of SH: I: broad dark membranes attached to the capsule (15\%), II: membranes extending between the articulating cartilages without $(20 \%)$, III: and with cartilaginous destruction ( $25 \%$ ). Stages of CD: I: circumscribed signal decrease $(20 \%)$, II: marginal destructions $(20 \%)$, III: decrease in cartilage thickness $(20 \%)$, IV: cartilage loss $(3 \%)$. Degree of SH correlated with recent bleeding frequency $(r=0.79)$. On radiographs, osteoporosis was exclusively, and osseous erosions or epiphyseal overgrowth were better demonstrated. In stage I of $\mathrm{SH}$ or $\mathrm{CD}$, no osseous abnormalities except epiphyseal overgrowth were found on radiographs. In 5 cases of epiphyseal overgrowth, no SH or CD were seen with MRI.

Conclusions: 3-D FLASH imaging is valuable for staging synovial hypertrophy and cartilaginous destruction, however, detection of some osseous abnormalities is more reliable with radiographs.

\subsection{PM}

959 Diagnostic advantage of ultrasound imaging in the detection of progressive muscular dystrophy

J. Kocsis, I. Horváth, A. László; Szeged

During a period of 8 years, observations were made on 42 myopathic children: 32 had recessively inherited progressive muscular dystrophy (PMD), 3 Becker's myopathy, 1 mitochondrial myopathy, and 6 neurogenic myopathy. The examinations revealed that ultrasonography is suitable for the demonstration of myopathies. The progression of the process can be followed in PMD patients. The changes detected by ultrasound correlated well with the histological changes. The ultrasound data are of assistance in the differentiation of PMD and neurogenic myopathies.

\section{$2.51 \mathrm{PM}$}

\section{Ultrasound markers in chromosomal disease: a retrospective study}

P. Twining, J. Zuccollo; Nottingham

Purpose: In order to assess the antenatal ultrasound markers of chromosomal disease a retrospective study was carried out.

Methods: Over an 18 month period 31 pregnancies were complicated by a chromosomal abnormality. Antenatal and postnatal scans were reviewed and post mortem specimens were also assessed for potential ultrasound markers. Results: In the two cases of Trisomy 13, holoprosencephaly with midline facial clefting, polydactyly and growth retardation were seen. In the nine cases of Trisomy 18, abnormalities of the extremities, face and heart were common findings. Growth retardation, diaphragmatic hernia and choroid plexus cysts were also demonstrated. In the fourteen cases of Trisomy 21, the main abnormalities were cardiac. There were four atrioventricular canal defects, four ventriculo-septal defects and one tricuspid regurgitation. Two cases of duodenal atresia were seen, one associated with choroid plexus cysts. In the two cases of Triploidy $69 \mathrm{xxx}$, a large hydropic placenta was seen in one case and holoprosencephaly in the other. All four cases of Turner's Syndrome 
demonstrated a cystic hygroma and two showed hydrops.

Conclusions: From the antenatal scans only nine cases of chromosomal disease were suspected. However, the routine assessment of cardiac structures at 18 weeks gestation should detect more cases of Down's Syndrome. The presence of choroid plexus cysts, cardiac anomalies, diaphragmatic hernia and severe growth retardation should prompt assessment of extremities for markers of Trisomy disease. The ultrasound markers of chromosomal disease are demonstrated with pathological correlations.

\section{$3.01 \mathrm{PM}$}

961 Abdominal ultrasound in Noonan syndrome: a study of 45 patients E. J. Adam, C. George, M. Sharland, M. Patton; London

Purpose: To establish the abdominal ultrasound findings in Noonan syndrome; in particular to assess the frequency of renal abnormalities which are well described in Turner's syndrome, a condition with a similar phenotype. Methods: 45 patients with Noonan syndrome were scanned. The age range was 7 months to 37 years, there were 17 males and 24 females.

Results: 17 scans were normal and 28 abnormal. 5 patients had renal abnormalities consisting of two unilaterally absent kidneys, one duplex kidney, bilateral cysts with scarring and one dilated renal pelvis. 23 patients had splenomegaly, and 6 of these had associated hepatomegaly. In all cases the echo texture of the liver and spleen was normal. One patient was found to have a choledochal cyst in addition to a unilaterally absent kidney.

Conclusions: The frequency of renal abnormalities in Noonan syndrome is low but splenomegaly, with or without hepatomegaly is a common finding. In view of the normal morphological appearance of both liver and spleen, the aetiology of the enlargement is obscure.

\section{$3.11 \mathrm{PM}$}

\section{Color coded Doppler sonography - new perspectives in pediatric ultrasound diagnosis}

Klara A. Vergesslich, Walter Ponhold; Vienna

Color-coded Doppler sonography allows on-line assessment of blood flow during real-time exams, providing both qualitative and quantitative information.

The clinical application comprises the depiction of cerebral hemodynamics in infants, where characteristic blood flow patterns can be found in patent ductus arteriosus, hypoxic-ischemic encephalopathy, cerebral hemorrhage, hydrocephalus, a.-v. malformations or in brain death. Moreover, color-coded Doppler sonography plays an important role in the evaluation of renal hemodynamics, especially in kidney transplants. Finally, the examination of the portal venous system can demonstrate thrombosis of the portal vein, porto-systemic collaterals or hepatofugal blood flow in severe portal hypertension. Thus, invasive investigative procedures may become unnecessary in certain cases.

The future potential of this technique may lie in the possibility of tissue characterisation (tumor flow!) and the development of quantitative measurements of volume flow.

\section{Room M}

\section{SS}

\subsection{Scrotum}

\subsection{PM}

\section{Scrotal imaging (Keynote Lecture)}

\section{A. Haubek; Aarhus}

Before the advent of the new imaging methods - ultrasound, CT, and MR only few clinical problems related to the scrotum could be elucidated by radiological or scintigraphic procedures (e.g. angiography in search for non-descended testis, scintigraphy in suspected torsion). On the contrary, the value of especially ultrasound including colour Doppler as a tool in the evaluation of a broad spectrum of scrotal pathology is now well established, and a possible role for $\mathrm{MR}$ is the subject for ongoing research.

The clinical entities could be summarized under the following headings "acute scrotum" (torsion, acute epididymitis, trauma), testicular mass (neoplasia, orchitis, neglected torsion), paratesticular mass (epididymitis, spermatocele, hydrocele), varicocele, and non-descended testis (NDT). Ultrasound is the method that has been most extensively evaluated with a number of established indications. Torsion can be diagnosed with the use of colour Doppler with a high accuracy according to recent reports, and the norma testicular flow is displayed as well. Scintigraphy remains a less specific alternative. Since urgent surgical intervention is mandatory, treatment should not be delayed by imaging. Testicular cancer exhibits a substantial rise in incidence in most European countries, and an important progress in prognosis has been experienced as well. High frequency ultrasound can reveal testicular tumours, including non-palpable lesions. The main objective is to separate intratesticular from epididymicial masses. According to echopattern discrimination between malignant and benign lesions is not very accurate. Benign lesions are most often related to inflammatory conditions and will include signs of epididymitis. Furthermore, ultrasound can characterize paratesticular pathology according to topography, solid or cystic nature, and vascularization.

A number of different conditions are known to carry an increased risk of developing germinal cell tumours, and a premalignant pathological entity is also defined - carcinoma in situ. Both ultrasound and MR have recently been tested in identifying diagnostic criteria according to echopattern or relaxation time measurements in high risk groups. While the importance of MR in scrotal imaging is still a subject for investigation, a basic difference between ultrasound and MR, the former carrying a better spatial resolution and the latter a better contrast resolution, may be an indicator for their future role and relative importance.

For reasons of spatial and contrast resolution and because of radiation dose CT has little to offer in scrotal imaging. Non-descended testis is one exception, but also with this indication ultrasound and MR are prevailing. In inguinal retention ultrasound has in more series been accurate in identifying the non-descended testis, and in a few reports also in an exact topographic localization. Controverses exist according to the ability of the methods to identify abdominally retained testes, and consequently to the reliability of diagnosing the abscence of a testis.

In conclusion scrotal imaging is characterized by the dominating role of ultrasound and Doppler, and by an interesting and comprehensive research in both ultrasound, Doppler, and MR.

\subsection{PM}

\section{Ultrasound evaluation of blunt scrotal trauma} W. Buchberger, A. Furtschegger, W. Kastlunger, T. Penz; Innsbruck

a) Blunt scrotal trauma poses a diagnostic challenge to the radiologist. Most extratesticular lesions can be treated conservatively, whereas testicular rupture needs early surgical exploration and repair. Because physical examination is often difficult, ultrasound has emerged as the diagnostic imaging modality of choice in the evaluation of patients with scrotal trauma.

b) 80 patients with blunt scrotal trauma underwent scrotal sonography with a real-time unit and $5 \mathrm{mHz}$ or $7 . \mathrm{mHz}$ transducers. Sonographic features of testicular and extratesticular lesions were reviewed retrospectively.

c) 14 testicular ruptures and 8 testicular hematomas without disruption of the tunica albuginea were correctly diagnosed. Diagnoses could be confirmed at operation in 14 cases and by clinical and sonographic follow-up in 8 cases. Other ultrasound diagnoses included 28 hematoceles and 17 lesions of the epididymis (hematoma or contusion).

d) Sonography is able not only to differentiate testicular from extratesticular lesions, but also testicular rupture from testicular hematoma without disruption of the tunica albuginea. A heterogeneous parenchymal pattern, contour abnormalities, poorly defined margins and obvious fragmentation of the testicle strongly suggest testicular rupture.

\subsection{PM}

\section{Diagnostic value of MRT in diseases of the testis and scrotum} B. M. Cramer, E. Schlegel, J. Thüroff; Wuppertal

Purpose: To determine the value of MRI in diseases of testis and scrotum. Methods: 300 patients with diseases of the testis and scrotum underwent examination on a Siemens Magnetom 1.5 using surface coils. Surgical and bioptic comparison was possible in more than 100 patients.

Results: Malignant tumors of the testis were diagnosed without exception. There were no false-positive or false-negative readings. Embryonal cancers and seminomas showed different morphologies. With some limitations differentiation of Beta-HCG-positive seminomas appears possible. In benign diseases differentiation of inflammatory and other lesions as delayed torsions seems possible. With testicular trauma rupture of the tunica albuginea can be visualized.

Conclusions: MRI is an important tool for diagnosis of diseases of the scrotum and testis, whenever indication for surgery was not clear, MRI greatly helped in decision-making. Also, mm-size tumors were detected and differentiated from other type lesions.

\subsection{PM}

\section{MR imaging of the scrotum - a review of 20 cases}

R. Oyen, S. Verellen, G. Marchal, L. Baert, A. L. Baert; Leuven

Purpose: To evaluate the potential role of MRI in the diagnosis of scrotal pathology.

Methods: 20 patients had high resolution magnetic resonance imaging $(1.5 \mathrm{~T})$ with a surface coil. The results were compared with those at high resolution ultrasound $(7 \mathrm{MHz})$. An in vitro MRI was performed on 3 specimens.

Results: The distinction between testicular and extratesticular pathology had been correctly made in all patients. Seminomatous tumors appeared to be well circumscribed low intensity lesions on T2-weighted images. Non-semino- 
matous tumors were ill-defined with inhomogeneous signal characteristics on T2-weighted images. However these lesions could not be differentiated from a burned-out tumor, a necrotic seminoma, a Leydig cell tumor nor from a malignant lymphoma. In 2 patients with macrogonadism MRI showed normal, homogeneous signal intensities in both testes, as was proven by an open testicular biopsy. In 2 patients the extent of epididymo-orchitis, 1 with abscedation was clearly shown. In 1 patient the diagnosis of an old paratesticular hematoma had been correctly made by MRI. In 2 others a simple hydrocele was found.

Conclusions: It remains impossible to make a histologic diagnosis. US remains the primary imaging modality for scrotal disease. MRI is indicated when the findings at physical examination and US are discrepant and when infiltrative disease is suspected.

\subsection{PM}

967 Assessment of testicular function by in vivo phosphorus magnetic resonance spectroscopy

J. van der Grond, J. S. E. Laven, T. M. T. W. Lock, W. P. Th. M. Mali; Utrecht

Purpose: Information about testicular function is mainly based on the results of semen analysis and determination of serum FSH, LH and testosterone levels. Since it is known that in vivo ${ }^{31} \mathrm{P}$ MR spectroscopy can provide, in a non invasive fashion, metabolic information about several organs, this technique could also provide useful information about testicular function, in case of idopathic oligozoospermia and azoospermia.

Methods: In vivo ${ }^{31} \mathrm{P}$ MR spectroscopy of the human testis was performed on healthy volunteers $(n=17)$, oligozoospermic $(n=42)$ (both groups approved by semen analysis and analysis of serum hormone levels), azoospermic (Sertoli cell only, approved by testicular biopsy) $(n=6)$ and azoospermic patients of which serum hormone levels were within normal limits and who were suspected from epididymal obstructions $(n=7)$.

Results: The PM/ATP ratio was $1.72 \pm 0.03$ for healthy volunteers and dropped to $1.22 \pm 0.07$ for azoospermic patients $(\mathrm{P} \leq 0.001)$. The PD/ATP ratio did show an increase from $0.49 \pm 0.05$ (healthy volunteers) to $0.85 \pm 0.15$ (Sertoli cell only) ( $\mathrm{P} \leq 0.05$ ). The $\mathrm{PM} / \mathrm{ATP}$ and $\mathrm{PD} / \mathrm{ATP}$ ratio of the oligozoospermic patients amounted to $1.34 \pm 0.06$ and $0.74 \pm 0.05$ respectively. The MR spectra of five azoospermic patients who were suspected to suffer from epididymal obstructions did not show significant differences to MR spectra of healthy volunteers, whereas for 2 patients the MR spectra showed that also spermatogenesis was affected. For the patients who were suffering from epididymal obstructions in five cases showed a normal functioning spermatogenesis epididymo-vasography might be helpful to repair male fertility. For the two patients in which also spermatogenesis was affected, unnecessary epididymovasography might be avoided.

\section{$2.55 \mathrm{PM}$}

968 Clinical diagnostic value of radionuclide scrotal blood pool scintigraphy in male infertility with a varicocele

Rongfu Wang; Suresnes

This study demonstrates the eluate of technetium-99m scrotal blood pool scintigraphy in male infertility with a varicocele. Twenty normal volunteers and two hundred and thirty patients who were clinically, biochemically and surgically determined were performed in this study. The 20 normal scintigrams indicated a bilaterally symmetric distribution of radioactivity, which was considered as negative (normal). 199 of 210 patients with a varicocele markedly showed an unilaterally, particularly in left abnormal important activity (positive), of them including 27 of 30 without clinical evidence. 20 cases had bilateral accumulation. There were 11 cases of false negative due to slightly bilateral varicocele and 5 cases of false positive caused by testicular tumors, torsion other disorders. This technique, a higher probability of $94 \%$, therefore, not only has the avantage that is simple, noninvasive, economic and widely used as compared with varico-veinography, Doppler ultrasound scans, ect., but also really help the clinical surgeon confirm the diagnosis, greatly suitable for examining the subclinical varicocele, and determine surgical protocol. In the appropriate clinical setting, we can easily exclude the false positive and negative so as to improve considerably the diagnostic accuracy. This makes it very useful clinically.

\subsection{PM}

969 Treatment of male infertility by occlusion of varicocele with Vilanol V. Janik, J. Zvěřina, I. Kawaciuk; Prague

The authors describe algorithm of diagnostic procedure and method of treatment of male infertility in 56 men, aged 19-45 years, with varicocele. After spermiogram, ultrasound and doppler examination and percutaneous transfemoral catheterization, occlusion of the spermatic vein with the Czechoslovak preparation Vilanol (hydrolyzed copolymer of polyvinyl acetate and polyvinyl alcohol in $96 \%$ ethylalcohol) was performed by coaxial catheter system. Ten patients were treated on account of marked subjective complaints and 46 because of reduced infertility. The spermiogram improved after occlusion in 41 of them. The authors describe the diagnostic procedure, the technique of occlusion, its complications and results.

\subsection{PM}

\section{Percutaneous sclerotherapy in idiopathic varicoceles} M. Lenz, M. Strotzer, B. Kersting-Sommerhoff, N. Hof; München, Tübingen

Purpose: To demonstrate the role of sclerotherapy of the internal spermatic vein in the ambulatory treatment of idiopathic varicoceles - analysis of 1369 patients.

Methods: A specially designed catheter is inserted percutaneously into the femoral vein and - via caval and renal vein - placed in the internal spermatic vein, which is then obliterated with Varicocid ${ }^{\mathrm{R}}$.

Results: $1158(84.6 \%)$ of 1369 patients were treated successfully. In the last 380 patients the success rate reached $93,7 \%$ depending on anatomic variations of the vein. Recurrent varicoceles were found in $5,8 \%$ of patients (415 DopplerUltrasound controls). Acute complications were a) venous trauma $(2,4 \%)$ and b) plexus pompiniformis phlebitis $(3,6 \%)$. No complications with permanent damage were observed.

Conclusions: Sclerotherapy of the internal spermatic vein is an effective, low cost and low risk method for treatment of idiopathic varicocele. Sclerotherapy has taken the place of surgery as the method of choice.

\subsection{PM}

\section{Clinical evaluation of spermatic vein embolization for varicocele} using Ethanol

Sumio Takashima, Masami Toyoshima, Toshiyuki Matsuoka, Kenji Nakamura, Haruki Nakatsuka, Noriaki Usuki, Kazuo Minakuchi, Yasuto Onoyama; Osaka

Purpose: To evaluate the efficacy of ethanol as an embolic agent in the treatment of varicocele.

Subjects and methods: Twenty patients aged 13-46 years were treated for varicocele by embolization with ethanol. The chief complaint was sterility in 12 patients, scrotal swelling or pain in five, and hematuria in three. Ethanol was injected into the internal spermatic vein involved in the varicocele by spermatic venography. Usually $1-3 \mathrm{ml}$ of ethanol was injected at a peripheral portion of the vein, this was immediately repeated, usually for a total of two or three times, more proximally when necessary. The total volume used was $0.5-15 \mathrm{ml}$. In four patients, ethanol diluted with saline was used.

Results: Embolization was useful in all patients. During follow-up, the varicocele became smaller or disappeared in 19 of the 20 patients. In one patient it appeared again 5 months after embolization. In this patient, embolization of a collateral vein was unsuccessful. The sperm count or motility (or both) improved in five of the six patients tested before and after treatment. Side effects were not serious.

Conclusions: Embolization with ethanol was easily performed and seemed to be safe, effective, and inexpensive.

\section{Room N}

\section{SS} $2.00 \mathrm{PM}$

\section{New development in MR of the thorax (Keynote Lecture)} Gordon Gamsu; San Francisco, CA

Developments in MR of the thorax have been hampered by the physicochemical properties of the lung, which reduce imaging of structures within the lung parenchyma. Three areas that are receiving attention and for which information is available are:

1. Mediastinal staging of lung cancer. Several large and detailed studies have shown equivalency for MR and CT in detecting mediastinal adenopathy, although both techniques are less accurate than previously shown. MR is probably better than CT for detecting direct mediastinal and chest wall invasion.

2. Demonstration of central pulmonary and mediastinal vessels and quantification of flow in the central pulmonary arteries. Using the ability of gradient-recalled MR imaging to show and quantify intravascular signal, several studies have

a) measured differential lung blood flow and

b) shown the causes of central vascular obstruction.

3. Ultrafast imaging techniques using sub-millisecond echo-time can image the lung parenchyma. These images lack contrast but are a promising avenue for thoracic imaging. 
973 First clinical applications of MR-angiography in thoracic surgery H.-U. Kauczor, G. Layer, M. Müller-Schimpfle, S. Tuengerthal, W. Semmler, G. van Kaick; Heidelberg

The diagnostic contribution of MR-Angiography in combination with MRT in patients with intrathoracic lesions undergoing surgical intervention is assessed. Sequential scan acquisition on a 1.5 Tesla Siemens Magnetom SP was done using Flash-2D gradient echo sequences (TR $30 \mathrm{msec}$, TE $10 \mathrm{msec}, \mathrm{FA} 30^{\circ}, 1$ Ac, slice thickness $5 \mathrm{~mm}$, overlap $1 \mathrm{~mm}$, matrix $256 \times 256$, FOV $400 \mathrm{~mm}$.) in a 10 second breath-hold period.

$2 \mathrm{D}$-scans were postprocessed by maximum intensity projection. The resulting 3D-angiograms should be displayed by cine-loop for better spatial impression. The angiograms display mediastinal and hilar as well as peripheral intrapulmonary and intercostal vessels. For confident anatomical identification and complete diagnostic evaluation 2D- and 3D-images are necessary. In intrathoracic masses MRA reveals vessel displacement and stenosis due to tumor compression or infiltration. Additionally, poststenotic perfusion deficit or lumen patency is shown. In vena cava superior syndrome residual lumen could not be detected because of slow or turbulent flow. Although the sensitivity can not be compared to conventional angiography, MRA is superior to contrast-enhanced CT in vessel visualization, thus determining surgical procedure. Display of perfusion deficits makes MRA extremely useful in planning pneumenectomy or sleeve resection.

MRA illustrates morphologic and functional features of intrathoracic lesions making it a valuable completion of MRI, probably leading to a new position among staging examinations.

\subsection{PM}

\section{Vascular involvement by mediastinal neoplastic lesions:} diagnosis with conventional and refocused MR images

Paolo Pavone, Paolo Di Renzi, Ernesto Di Cesare, Silvia Giuliani, Giampiero Cardone, Luca Marsili, Roberto Passariello; L'Aquila

Purpose: To verify the ability of MRI to detect the vascular infiltration by neoplastic mediastinal lesions and to determine the improvement obtained by the use of refocussed sequences.

Methods: 14 patients with mediastinal tumors were studied. A 0.5 T superconductive equipment and a $0.2 \mathrm{~T}$ permanent unit were employed. The conventional spin echo sequences were acquired with a TR of $520 \mathrm{msec}$. and a TE of 20 sec. on axial planes. The refocussed sequences were acquired both without and with cardiac gating. The latter sequences allowed cine display of the images. TR values varied according to the R-R period. TE was $20 \mathrm{msec}$.

Results: In 6 cases involvement of the major mediastinal vessels was present (pulmonary artery in 3, superior vena cava in 2 and aorta in 1).

With the use of refocussed sequences correct evaluation of the status of the lumen of the major mediastinal vessels was obtained in every case. Bright signal intensity was displayed by the lumen of patent vessels also when the flow was slow and orizzontal (parallel to the imaging plane).

Conclusions: The use of refocussed sequences improves the diagnostic yield in the evaluation of the patency or involvement of major vessels during neoplastic mediastinal spread of different lesions.

\section{$2.35 \mathrm{PM}$}

975 Opacification of the esophagus during magnetic resonance imaging of the chest with use of a new paramagnetic preparation P. Pavone, G. Cardone, S. Giuliani, N. De Stefano, G. Albertini P., R. Passariello; L'Aquila

Purpose: To improve the anatomical evaluation of the esophagus during MRI of the chest, using a specific contrast agent.

Methods: 16 patients undergoing MRI of the chest for different reasons (excluding esophageal carcinoma) were included in the study. The examinations were performed with a $0.5 \mathrm{~T}$ superconductive unit, employing non gated Spin Echo sequences (TR $260-520 \mathrm{msec}$, TE $20 \mathrm{msec}$, NEX 8). Axial and sagittal or coronal images were acquired with $10 \mathrm{~mm}$ slice thickness. Paramagnetic esophageal contrast agent was administered prior to the examination, with the patient already lying on the MRI table. The new contrast agent was prepared by using a specific esophageal CT agent, presenting high viscosity (TAC Bario, distributed by Bracco I.C. in Italy and produced by EZ.E.M, U.S.A.). A paramagnetic MR contrast agent (Gd-DTPA, commercial name Magnevist, Schering, Germany) was emulsified to the specific esophageal agent.

Results: In all but two cases good opacification of the esophageal lumen was obtained in the MR images acquired. In two cases of lung cancer contiguous to the esophagus the opacification proved useful for the identification of the organ.

Conclusions: The preparation described here in provides a valid opacification of the esophageal lumen, and can be employed for routine chest MRI studies.

\subsection{PM}

976 Progress of nuclear medicine in imaging of the chest (Keynote Lecture)

J. Peter Lavender; London

This presentation will attempt to cover recent advances in lung imaging using radionuclides.

The major application of lung scanning is in the diagnosis of pulmonary embolic disease and the wider use of aerosols and dispersed particles has made high resolution ventilation imaging now more readily available. However, an alternative approach is the direct imaging of thrombus to screen patients at risk of embolic disease by tracers with high affinity for thrombus. Two approaches are available; fibrin directed tracers such as antifibrin monoclonal antibodies, fragment E1, or alternatively tracers with affinity for platelets such as platelet associated monoclonal antibodies and various peptides now under development.

Ventilation perfusion imaging can also be applied to non-embolic disease, particularly where unexplained hypoxia can be shown caused by non-ventilated perfused lung in patients with relatively normal chest radiographs. Of particular importance is the loss of ventilation in the left lower lobe in patients with cardiomegally, a phenomenon which may explain the high incidence of postoperative collapse of this lobe in patients following cardiac surgery.

Either Indium or Technetium labelled autologous white blood cells can be used to show migration of cells into patients with lung sepsis. Diffuse lung hold up is shown in patients with adult respiratory distress syndrome, but also in certain patients with vasculitis:

Lastly, agents designed to locate receptors have been widely used in the brain. In the lung sympathetic innovation can be shown with MIBG; beta receptors in the lung may also be imaged and give us a new approach to the treatment of asthma.

\subsection{PM}

\section{Positron emission tomography (PET) of the thorax: applications in} the preoperative staging

Michael V. Knopp, Ludwig G.Strauss, Uwe Haberkorn, Helge Bischoff, Antonia Dimitrakopoulou, Josef K. Doll, Detlef Branscheid, Ingo Vogt-Moykopf, Gerhard van Kaick; Heidelberg

Purpose: To evaluate the use of positron emission tomography (PET) with F-18-labelled deoxglucose (FDG) in the preoperative staging of indeterminate thoracic lesions.

Methods and Materials: FDG-PET studies of 100 patients with thoracic lesions were performed using a whole-body PET scanner. Each patient was furthermore studied by CT. Additional procedures including MRI were obtained when resonable. All histologies were confirmed by mediastinoscopy, surgery or bronchoscopy.

Results: Histology revealed 83 malignant tumors and 17 benign lesions. 82 of the malignant tumors showed intense FDG uptake with values above 1.8 SUV at 60 minutes whereas 16 of the benign lesions showed uptake values below 1.8 SUV. PET confirmed the previous T-Staging in $63 \%$ of the cases and changed T in $37 \%$. The T-Staging by PET agreed in $96 \%$ with surgical staging. PET enabled the differentiation of atelectatic areas from tumor thus imaging the real tumor mass.

Conclusions: PET of the thorax shows to be of great benefit in the preoperative staging of thoracic lesions if they are to be considered indeterminate by the more conventional techniques. FDG-PET enables a metabolic oriented approach to image lesions with good image quality and thus introduces new approaches in the staging of thoracic tumors.

\subsection{PM}

\section{PET imaging of malignant pleural mesothelioma}

Michael V. Knopp, Ludwig G. Strauss, Helge Bischoff, Uwe Haberkorn, Detlef Branscheid, Franz Oberdorfer, Wolfgang Maier-Borst; Heidelberg

Purpose: The non invasive evaluation of pleural mesothelioma presents a major problem in staging and therapeutic management even with the use of CT and MRI. The study is designed to evaluate if FDG PET can be used to detect mesothelioma as well as to monitor a response in follow up studies.

Methods: A whole-body PET scanner (WB 2048/7) manufactured by GE-Scanditronix with a rotating point source for transmission scans were used. Endpoint measurements 50 min. post iv. injection of $440 \mathrm{MBq}$ FDG with emission scans of $15 \mathrm{~min}$. followed by $5 \mathrm{~min}$. transmission scans were used. Each patient was studied by CT prior to the PET exam and in some cases by MRI.

Results: All malignant pleural mesotheliomas showed significant FDG accumulation and were consistently detected by PET. Ten patients are included in this ongoing study and histology was confirmed in all cases. Five patients were consecutively surgically resected.

Conclusions: FDG PET imaging can be used to detect a delineate malignant pleural mesothelioma especially in patients where CT and MRI offer equivocal 
findings. The changes in FDG uptake characteristics can be used to monitor therapy response or tumor progression.

3.15 PM

979 Cyst ventilation behaviour in fibrosing alveolitis compared with emphysematous bullae

N. H. Strickland, A. N. Adam, J. M.B. Hughes, J.P. Lavender; London

Purpose: To compare the ventilation characteristics of cysts in cryptogenic fibrosing alveolitis (CFA) with those of emphysematous bullae.

Methods: Patients with CFA were examined using inspiratory and exspiratory CT, with planar and SPECT krypton-81 ventilation and technetium- $99 \mathrm{~m}$ albumin perfusion isotope imaging to demonstrate the ventilation and perfusion characteristics of their lung cysts. The results were compared with the ventilation and perfusion characteristics of emphysematous bullae.

Results: We have correlated three imaging techniques and have shown both anatomically and physiocologically that cysts in CFA appear to behave differently from emphysematous bullae. CFA cysts are non-perfused but are ventilated, giving mismatched ventilation-perfusion defects.

This contrasts with emphysematous bullae which are non-perfused and are usually unventilated, giving matched ventilation-perfusion defects.

Conclusions: The ventilation characteristics of lung cysts in fibrosing alveolitis appear to differ from those of emphysematous bullae. CFA cysts largely ventilate unlike emphysematous bullae which usually do not. This leads to mismatched V/Q defects in CFA in contrast to matched defects in emphysematous bullae.

\section{Room 0}

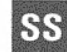

\subsection{Metabolic II}

\section{$2.00 \mathrm{PM}$}

980 Femoral density or mass in cancellous, cortical bone or in the two combined. Which is most relevant?

A. Hoiseth, A. Alho; Oslo

Purpose: Bone mineral can be expressed as density and as mass in the cancellous or cortical bone or in both combined. Which of these parameters is most relevant in the femur?

Methods: Femoral density and mass in cortical, cancellous and in combined cancellous/cortical bone were assessed by means of computed tomography, in specimens, in patients with femoral neck fractures and in control subjects. The measures were related to age, bone strength and to the risk of femoral neck fracture. A discrimination between cancellous and cortical bone was made only in locations with substantial amounts of both.

Results: Mechanical strength had a better agreement with mass in combined cancellous/cortical bone than with any density measure. There was a substantial age related loss of cortical bone mass (the cancellous loss may contribute less to the total loss). Mass, but not density, discriminates between patients with femoral neck fractures and controls.

Conclusions: Density in isolated cancellous bone is for several purposes not very informative. Bone mass and even cortical bone may be very relevant.

\section{$2.10 \mathrm{PM}$}

981 DXA (dual-energy X-ray absorptiometry): measurements of total body mineral content (BMC) in patients with renal osteodystrophy F. Kainberger, G. Seidl, A. Sterk, P. Pietschmann, P. Hübsch, B. Ludvik, O. Traindl; Vienna

Purpose: To determine the influence of renal transplantation on the calcium content of the bone as measured with dual-energy projection methods.

Methods: BMC-measurements of the total body were obtained in 21 patients (14 male, 7 female; $48 \pm 11.7$ years) who underwent DXA 2-4 weeks after transplantation with a follow-up study $3-8$ months later. BMC-values were compared with standard magnification radiographs of the hands.

Results: The follow-up study showed a significant increase of BMC values after renal transplantation $(2501 \pm 437 \mathrm{~g}$ versus $2582 \pm 411 \mathrm{~g}, \mathrm{p}=0,105)$. Results of magnification radiograms showed no significant differences in these patients.

Conclusions: Despite sophisticated dialysis techniques, renal transplantation may lead to significantly increased calcium uptake of the bone which may not be observed with magnification radiography.

\subsection{PM}

982 Hypoestrogenism as a risk factor of reduced bone density in endurance sportswomen and anorexia nervosa

R. Weiske, K. Wurster; Stuttgart

Endurance sportswomen (especially long distance runners) often have disturbances of menstrual cycle extending to amenorrhea as a s sign of great psychological and physical stress. The generative hormone system is considerably or fully suppressed to post-menopausal levels.

We studied the effect of hypoestrogenic metabolism on bone density of 34 sportswomen (including 4 women formerly with anorexia nervosa) which was determined in trabecular and cortical bone of the lumbar spine by QCT.

A particular low bone density was measured in those athletes with long existing cyclic disturbances, who started endurance training in their prepuberal period and with previous or existing eating disorders extending to anorexia.

Our results indicate a higher risk of stress fractures in female long distance runners with cycle irregularities. For their future life problems regarding the menopause are to be expected.

A reduction of training intensity, changing alimentary habits, increase in body weight and compensation of the hormone deficit (as most of 11 re-examined women did) are therapeutical possibilities.

\subsection{PM}

983 Effectiveness of transdermal estrogen substitution for osteoporosis prophylaxis in oophorectomized women

D. Felsenberg, H. Lübbert; Berlin

Purpose: In a prospective study, 80 women received transdermal estrogen substitution therapy after having undergone a bilateral oophorectomy. The effectiveness of this treatment as a prophylaxis against the development of osteoporosis was examined by determining bone mineral density (BMD).

Patients and methods: 110 patients, aged between 30 and 56, who had been submitted to a bilateral oophorectomy were followed up postoperatively. The estrogen substitution was done transdermal via a plaster (Estraderm TTS 50) with $50 \mu \mathrm{g} 17-\beta$-Estradiol/day. The change in BMD over a period of 2 years was determined by QCT of the lumbar spine (lumbar vertebrae 1-3) with the single- and dual-energy method and compared with an age-correlated control group. Calcaneus and radius examinations were performed with SPA. The examinations took place preoperatively, 3, 6, 12, 18 and 24 months postoperatively.

Results: In a preliminary analysis 75 patients were evaluated, 40 of them had completely finished the study. In the lumbar spine a bone mineral loss of $4.2 \mathrm{mg} / \mathrm{cm}^{3}$ on average was calculated in the group of oophorectomized patients. In the reference collective a decrease of bone mineral of $7,23 \mathrm{mg} / \mathrm{cm}^{3}$ was measured.

Conclusions: The transdermal application of $50 \mu \mathrm{g}$ 17- $\beta$-Estradiol/day prevented an unphysiological decrease of bone mineral in oophorectomized women.

\subsection{PM}

984 Changes of vertebral bone density under hormone substitution G. Reuther, M. Dören, H. P. G. Schneider, P. E. Peters; Münster

Purpose: The effect of hormone substitution on vertebral bone density in postmenopausal women was to be evaluated.

Methods and Materials: Single-energy QCT was performed in 2 groups of postmenopausal women with estrogen and either continuous $(n=70)$ or intermittent $(n=50)$ progestagen substitution for up to 4 years.

Results: Both forms of hormone substitution lead to significant increases in bone density, but continuous progestagen substitution proved to be more effective. The effect was strongest during the 1st year and in individuals with low bone density.

Conclusions: In contrast to previous assumptions remineralization takes place in osteopenic postmenopausal women under combined hormone substitution. QCT provides effective monitoring already after 1 year of therapy.

\subsection{PM}

985 Bone metabolism in acromegaly and bone mineral density measured by DEXA

P. Hübsch, H.Kotzmann, F.M. Kainberger, A. Luger, P. Pietschmann, E. Knosp; Vienna

AIM: The lumbar spine and the femoral neck are the main sites for routine measurements of bone mineral density (BMD) by using dual energy X-ray absorptiometry (DEXA). This study was undertaken to evaluate, how those regions reflect the alterations of bone metabolism in patients with active acromegaly.

Methods: 14 patients ( $4 \mathrm{~m}, 7 \mathrm{f}$, mean age $46.9 \mathrm{a}$ ) were studied. In 4 patients with hypogonadotropic hypogonadism sex steroids were replaced. Blood concen- 
trations of hormones including $\mathrm{GH}$ and osteocalcin were determined and the concentration of hydroxyproline in the urine was measured. BMD of the lumbar spine and the femoral neck was measured by DEXA (Norland XR-26). 14 controls were also studied (matched for age and sex).

Results: There was significant correlation of the plasma osteocalcin level with BMD of the femoral neck in the acromegaly group $(r=0.7)$, whereas no correlation with BMD in the lumbar spine was observed. BMD was significantly elevated only in the femoral neck $(p=0.01)$ in the acromegaly group compared to normal controls.

Conclusions: Although both bone formation and bone resorption are increased in acromegaly, the BMD of the femoral neck seems to be a good indicator mainly for bone formation. BMD of the spine is normal.

\section{$3.00 \mathrm{PM}$}

986 Reflex sympathetic dystrophy syndrome - use of MRI as a new diagnostic method

S. Schimmerl, H. Schurawitzki, H. Imhof, G. Canigiani, J. Kramer, V. Fialka; Vienna

Purpose: Establishment of diagnostic criterias in magnetic resonance imaging (MRI) for the study of reflex sympathetic dystrophy syndrome (RSDS or morbus Sudeck)

Methods: 20 patients with the clinical diagnose of Sudeck's disease in different stages, who had positive results in radiograph and three-phase radionuclide bone scanning were examined by MRI using T1- and T2-weighed spin-echo-sequences before and after injection of contrast medium.

Results: All patients in acute stage $(n=9)$ showed inhomogeneousness in bony pattern with diffuse hypointense spots on T1-weighted images, thickening of periarticulare soft tissue structures and enhancement of the reactive-dystrophic areas in bone, muscle and subcutaneus fat. In healing stages we saw lesser fatty-marrow changes, atrophy of soft tissue and little or no more contrast enhancement.

Conclusions: MRI proved to be a reliable diagnostic tool to differentiate between acute and healing stages, which is of great importance for therapeutic management. MRI features can be explained by histopathological findings.

\subsection{PM}

987 Radiological findings of amyloid arthropathy (beta 2 microglobulin-related amyloidosis) in long term haemodialysis

A. Talegon Melendez, C. Rios Camacho, L. Gil, A. Valenzuela, M. Gonzalez Guirao, S. Gomez Benitez; Sevilla

1. Radiological features of cystic lesions due to beta 2 microglobulin-related amyloidosis have been studied in 88 patients receiving haemodialysis for more than 4 years.

2. Amyloid arthropathy was confirmed by demonstration of amyloid-beta 2 microglobulin deposition on different substrates.

3. Plain radiographs of shoulder, spine, pelvis, hands and knees revealed cystic lesions in 31 patients (35.2\%), being its distribution: $71 \%$ shoulder, $56.7 \%$ carpal bones, $25.8 \%$ pelvis, $16.1 \%$ femur, and $13.3 \%$ knees. Discitis was found in 34 patients $(38.6 \%)$, pathological fracture of femur in 1 patient $(1.1 \%)$, and erosive arthropathy of hands in $65(77.4 \%)$. The occurrence of lytic lesions was frequent in patients suffering from amyloid arthropathy $(63 \%$ vs. $23 \%, p=$ $0.001)$. On the other hand no significant differences were found in frequency of discitis or erosive arthropathy of hands among both groups. The former was related to age and the latter to secondary hyperparathyroidism.

4. We stress the typical radiographic appearance and the high incidence of these lesions in patients on long-term haemodialysis.

\section{$3.20 \mathrm{PM}$}

\section{Poems syndrome; skeletal radiologic features}

X.Tomás, J.A. Clavero, T. Pujol, J.Berenguer, C. Nicolau, N. Bargalló, A. Salvador; Barcelona

We describe skeletal radiologic findings observed with $\mathrm{X}$-ray films and $\mathrm{CT}$ in a 31-year-old man with POEMS syndrome, a rare variety of plasma cell dyscrasia with polyneuropathy, organomegaly, endocrinopathy, monoclonal M-protein and skin changes.

A skeletal survey detected several abnormalities. There were a lytic lesion with a mild sclerotic margin in both ilium. New bone was noted arising from the posterior elements of lumbar vertebrae, discovertebral junctions and tibial and fibular margins.

All those radiologic features may allow the radiologist to suggest the diagnosis first.

\subsection{PM}

989 Reference BMD for British women using SPA, QCT and DEXA J.E. Adams, R.W. Whitehouse, A.Silman, P.H. Adams, C. Kay; Manchester

Ethnic and national differences in bone mass are well recognised. Methods for bone mineral density (BMD) measurement (SPA, QCT and DEXA) are widely available, and generally relevant reference data are provided with the equipment by the manufacturer. This study was undertaken to ascertain if these reference data were generally applicable to the local community.

Methods: Over 6 months, 200 women (aged 45-75 years), drawn at random from a local medical practice, were invited to attend for BMD measurement. To date 142 women of 200 invited (response rate $71 \%$ ) have attended. SPA (Nuclear Data) were performed in 2 sites in the forearm (proximal and ultradistal). Single and dual energy QCT (GE9800) was performed in the spine (T12-L3). DEXA (Lunar DPX) measurements were made in the spine and both femoral necks. The data collected were compared with the reference data provided.

Results: SPA (distal site) compared with reference data gave a significantly higher mean value $(+0.52 \mathrm{SD} ; \mathrm{p}<0.001)$. The proximal forearm measure was not significantly different from those of a European population (Brunner study). DEXA measurements in the femoral neck were significantly higher $(+1.179 \mathrm{SD} ; \mathrm{p}<0.001)$ than American reference data provided. Spinal BMD was also significantly higher $(+0.294 ; \mathrm{p}<0.016)$ than this reference data. QCT results were not significantly different from published data (Block et al [1989] J Bone \& Mineral Research 4, 249-257).

Conclusions: The results confirm that national differences exist between reference data for forearm, hip and lumbar spine. Institutions providing BMD measurements should ideally obtain their own local reference data and where possible not rely on data provided from different populations and ethnic groups.

\section{Room R}

\section{Ss} $2.00 \mathrm{PM}$

\subsection{Quality control in radiotherapy II}

990 The importance of good practice and quality assurance programmes for the treatment outcome in radiotherapy (Keynote Lecture)

G. E. Hanks; Philadelphia, PA

Abstract available at the information desk.

2.20 PM

991 The IAEA programme for quality assurance in radiotherapy (Keynote Lecture)

K. Zsdánszky, H. Svensson; Vienna

An international Network of Secondary Standard Laboratories has been organized by the IAEA and WHO in 1976 in order to improve accuracy in applied radiation dosimetry throughout the world. Periodic comparisons are organized for reference dosimeters of the SSDLs to ensure adequate consistency with the international system of measurements. The IAEA Dosimetry Laboratory performs also calibrations for SSDLs using its secondary standards calibrated at the "Bureau International des Poids et Mesures" (BIPM). The SSDLs are then calibrating dosimeters used by radiotherapy centres. An "instrument traceability" is thus provided from the BIPM through the IAEA and SSDLs to the radiotherapy centres.

The IAEA also directly performs dosimetry calibration checks for radiotherapy centres. In 1966 a postal dosimetry service for ${ }^{60} \mathrm{Co}$ radiation was initiated by the IAEA for radiotherapy centres using lithium fluoride thermoluminescent dosimeters (TLD). The WHO joined the programme in 1968 and organized a service in co-operation with its Regional Offices to cover all parts of the world. The participants should irradiate the TLDs to a specified dose (2 Gy) to water. The IAEA Dosimetry Laboratory compares the dose reported by the participant (radiotherapy centre) with the dose measured by IAEA. In addition to the accuracy of the dosimeters also the proper use is checked by this service, i.e. a "measurement traceability" is provided.

In 1989 a Co-ordinated Research Programme (CRP) was started by IAEA. The programme is planned as follows:

a) machine dose calibration including a check of beam quality,

b) simple patient dosimetry using a geometric phantom with multiple field irradiation, and

c) complex dosimetry including tissue inhomogeneities using simulated anatomical phantoms. 
992 Former experiences of quality assurance programmes for international radiotherapy clinical trials in Europe (Keynote Lecture)

J. Chavaudra, J. C. Horiot, K. A. Johannsson; Villejuif, Dijon, Gothenburg

It is now well established that randomized clinical trials can improve the practice of radiotherapy, provided that suitable Quality Assurance Programmes are applied to ensure the compliance of the treatments with the treatment regimes. Such Quality Assurance Programmes have existed for a long time at local or national levels in different countries. The requirement of larger and larger numbers of patients have made international trials unavoidable, especially in Europe, where the different levels of development of the Radiotherapy Practice and the existence of strong medical and radiotherapy tradition can introduce limitations in efficiency or biases without Quality Assurance.

EORTC initiated such programmes especially devoted to clinical trials in Europe at the beginning of the eighties, after previous dosimetric International Intercomparisons performed by the IAEA and the Nordic Therapy Centres. The experience gained from the EORTC, and the IGR in a clinical trial including about 30 European centers, enable us to present an overall prospective of typical european centers involved in clinical trials.

Such data are mainly based on (1) enquiry on human environment and equipment (2) mail dosimetric intercomparisons (3) on-site visits for the review of the reliability of clinical data and basic dosimetry of treatment units $(4$ review of the treatment records for individual patients. The later has proven to be a very important part of the Quality Assurance Programme, and it provides information which cannot be found through the classical dosimetric investigations and on-site visits.

\section{$3.00 \mathrm{PM}$}

993 Prognostic factors for patients with osseous metastasis: a multiinstitutional prospective study

Teruki Teshima, Masashi Chatani, Toshihiko Inoue, Yutaka Hirokawa Kouzou Kasiwado, Masayuki Kagemoto, Shizutomo Katsuta, Yoshifumi Honke, Tadashi Koyama, Youko Makidono, Hiroshi Katayama, Tetuji Kiso; Osaka

A multiinstitutional prospective study for the analysis of prognostic factors for patients with osseous metastasis was performed. From February 1986 through June 1988, a total of 216 patients were included in this study. Cox's regression model made it clear that the most significant overall prognostic factor was primary site $(\mathrm{p}=0.0002)$. In the lung cancer group, performance status ( $\mathrm{p}=$ $0.0036)$ and metastasis of organs than bone $(\mathrm{p}=0.0105)$ were also significant prognostic factors. In the breast cancer group, no significant factors were obtained. In the hepatoma group, the values for alkaline phosphatase (ALP) (p $=0.0021)$, lactate dehydrogenase $(\mathrm{LDH})(\mathrm{p}=0.0195)$, and $\operatorname{sex}(\mathrm{p}=0.0264)$ proved significant. In the group of other cases, the most significant prognostic factor was the value for urinary hydroxyproline/creatinine ratio $(p=0.0001)$, followed by the pain score of RTOG $(\mathrm{p}=0.0018)$. These factors and actual survival periods obtained in this study will be useful for the future stratification of patients for individualized optimal radiation schedules.

\subsection{PM}

994 Six decades of radiation therapy for meningiomas at the University of Illinois College of Medicine at Chicago

Eduard Dvořák, Edwin J. Liebner, Richard E. Haas; Chicago, IL

Two distinct periods can be observed: in the essentially orthovoltage era (1931-1969), 10 evaluable patients were irradiated (median follow-up: 70.5 months; $5 / 10$ tumors were malignant). Of these 10 patients, 7 had history of subtotal tumor resection(s), 2 had exploration with biopsy only, and one patient is without surgical data. The survival of these patients, using KaplanMeier method, was $77 \%$ and $51.5 \%$ at 5 and 10 years, respectively. From 1970-1977, there were no referrals; starting in 1978 and, by December 1989.2 patients could have been analyzed (median follow-up: 35 months; $7 / 21$ tumors were malignant). Of these, 17 had subtotal resection(s) and 3 gross total resection preceding radiation therapy; one was diagnosed by clinical and radiographic features only. The average target volume dose of 19 patients who completed their course was 55.8 Gy. A survival of 21 patients using KaplanMeier method is $89.5 \%$ at 3 and 5 years. Radiation therapy remains an established modality for achieving long-term tumor control both in incompletely resected meningiomas and as the initial treatment in unresectable tumors. Prognostic factors will be briefly discussed.
995 HDR brachytherapy of cervix carcinoma applied to 272 younger and 119 older than 60 years patients: comparison of 5 -year results and complications

S. Čikarić; Belgrade

In 1984 we treated 272 younger and 119 older than 60 years patients with cervix carcinoma of all stages using Cathetron (HDR Co-60) for brachytherapy, and Linear accelerator $(10 \mathrm{MeV})$ for external beam therapy. The treatment regimes were equal for both groups Cathetron: radical irradiation $-4 \times 1000 \mathrm{cGy} / \mathrm{A}, 1$ fraction/week, irradiation after surgery (Wertheim-Meigs) - $4 \times 750$ cGy $/ 0,5 \mathrm{~cm}, 1$ fraction/week. Linear accelerator: $4600 \mathrm{cGy}, 22$ fractions, 2 opposit fields with central lead shields, after surgery $-3600 \mathrm{cGy}, 18$ fractions, 2 opposit fields without central lead shields. The 5 year survival of patients was: the younger group: the older group - st. I - 79/94 $(84,0 \%): 10 / 14(71,4 \%)$, st. II $-74 / 98(75,5 \%): 30 / 46(65,2 \%)$. st. III - 35/78 $(44,9 \%): 20 / 58(34,5 \%)$, st. IV $0 / 2: 0 / 1$, all stages - 188/272 (69,1\%):60/119 (50,4\%).

Late postirradiation sequelae were: the younger group - $27 / 272(9,9 \%)$ and the older group - 14/119 (11,8\%). Local recurrences: the younger group - 24/272 $(8,8 \%)$, the older group - $11 / 119(9,2 \%)$. Distant metastases: $5,9 \%$ and $7,6 \%$.

\section{Room S}

\section{SS}

\subsection{Contrast media in MR}

\section{$2.00 \mathrm{PM}$}

996 Macromolecular Gd-DTPA compounds as contrast agent for MR-lymphography: initial experimental results in rabbits G. Branding, B. Hamm, S. Wagner, M. Taupitz, K.-J. Wolf; Berlin

Purpose: To investigate the suitability of macromolecular (Gd-DTPA)-compounds as signal-enhancing lymphographic agents in MR imaging.

Methods and Materials: Two (Gd-DTPA)-polylysine compounds and (Gd-DTPA)-albumine, with molecular weights of $48.000,139.000$ and 87.000 daltons, respectively, were tested in rabbits at Gd doses of 5 and $15 \mu \mathrm{mol}$ per animal. 18 animals were examined (3 animals/dosage/compound) with T1-weighted sequences. The iliac lymph nodes were imaged prior to and during unilateral endolymphatic infusion into a femoral lymph vessel, as well as over a period of 2 hours thereafter.

Results: All contrast agents showed a significant signal enhancement during infusion. For each compound the optimal dose was found to be $5 \mu \mathrm{mol}$ Gd per animal, whereas overdosage effects occurred at $15 \mu \mathrm{mol}$. For the lower molecular weight (Gd-DTPA)-polylysine the signal enhancing effect decreased 1 hour after stop of infusion and the enhancement pattern of the lymph nodes became inhomogeneous. For the high molecular (Gd-DTPA)-polylysine and the (Gd-DTPA)-albumine the homogeneous signal remained unchanged during the entire period of 2 hours. (Gd-DTPA)-albumine revealed the best results.

Conclusions: High molecular weight (Gd-DTPA)-polylysine compound and (Gd-DTPA)-albumine appear promising for MR-lymphography.

\subsection{PM}

997 Fast high resolution Gd-DTPA Polylysine enhanced MR angiography: technique and animal studies

G. Marchal, H. Bosmans, P. Van Hecke, Y. Jiang, P. Aerts, A. L. Baert, H. Bauer; Leuven, Berlin

Purpose: Evaluation of fast high resolution CE projection MRA using Gd-DTPA Polylysine.

Methods: Contrast enhanced MRA is based on the selective shortening of the $\mathrm{T} 1$ of the blood by so called "blood pooling agents". In present study in the rabbit, extreme shortening of the $\mathrm{T} 1 \mathrm{of}$ the blood (about $20 \mathrm{msec}$ ) was obtained by the IV injectioin of $\pm 0.5 \mathrm{mmol} / \mathrm{kg}$ Gd-DTPA Polylysine. Projection MRA was acquired in the absence of a slice selection gradient.

The signals of soft tissues and fat, with longer T1 than the CE blood, were suppressed by presaturation and inversion recovery pulses. The imaging part of the sequence was based on flow compensated FLASH or SE pulses.

Materials: CE MRAs of the vessels of the lower limbs and the abdomen were evaluated in 15 rabbits. Results were compared to conventional DSA, and both 2-D and 3-D inflow MRA.

Results and conclusions: The main assets of presented technique are: short acquisition times ( $0.5-2$ minutes); improved vascular detail; lower sensitivity to flow; no projection reconstruction needed.

The major drawback is the simultaneous visualization of both arteries and veins. 
998 Superparamagnetic iron oxide-detection of liver tumors at different concentrations - results of MRI at $1.5 \mathrm{~T}$

Pierre Gheung, Alain Rahmouni, Jean Luc Montazel, Didier Mathieu, Serge Derhy, Anne Arthaud, Alain Roche; Créteil

Purpose: This study was to assess the value of MR imaging of hepatic malignant tumors after injection of different concentrations of superparamagnetic iron oxyde (AMI-25) at $1.5 \mathrm{~T}$.

Methods and Materials: Fourteen patients with pathologically proved hepatic malignant tumors were imaged on a $1.5 \mathrm{~T}$ magnet. $\mathrm{T} 1$ ( $\mathrm{TR}=400 \mathrm{~ms}, \mathrm{TE}=20$ $\mathrm{ms})$ and $\mathrm{T} 2(\mathrm{TR}=2000 \mathrm{~ms}, \mathrm{TE}=40,90 \mathrm{~ms})$ sequences were obtained before and one hour after intravenous injection of varying concentrations of AMI-25 (10-20 micromol $/ \mathrm{kg}$ body weight). Signal intensity measurements were obtained from tumor, liver and background noise. The contrast-to-noise ratio was calculated as the lesion-to-liver signal intensity difference scaled to image noise. Statistical tests were applied to compare the lesion-to-liver contrast before and after injection.

Results: 1. After injection, the contrast-to-noise ratio increased significantly on all the sequences and for the three different concentrations, 2. The increase of the contrast was maximal on the 2000/40 SE sequence associated with the injection of $20 \mathrm{micromol} / \mathrm{kg}$ and reached $420 \%, 3$. Additional lesions were depicted after AMI-25.

Conclusions: In opposition with previous reports at $1.5 \mathrm{~T}$, we have observed an improvement in contrast and a depiction of numerous additional lesions on T2 weighted images, whatever the AMI-25 injected concentration.

\subsection{PM}

999 Dynamic MRI during fast infusion of Gd-DTPA: results in hepatic and pancreatic enhancement

Paolo Pavone, Silvia Giuliani, Giampiero Cardone, Rossella Occhiato, Giorgio Albertini P., Engin Aytan, Roberto Passariello; L'Aquila

Purpose: To evaluate the effect of continuous flow of Gd-DTPA at vascular level during MR imaging of the upper abdomen.

Methods: 13 patients studied for hepatic lesions (n.8) and pancreatic pathologies (n.5) were submitted to this evaluation. The examinations were performed with a 0.5 Tesla superconductive unit. Breath holding, rapid Acquisition Spin Echo (RASE) sequences were employed. TR was $230 \mathrm{msec}$, TE $16 \mathrm{msec}$., NEX 1 and matrix $128 \times 256$, for a total acquisition time of $30 \mathrm{sec}$. Gd-DTPA was injected at a dose of $0.2 \mathrm{mmol} / \mathrm{kg}$. In order to have a continuous flow of contrast agent during the acquisition of the sequence, a particular injection technique was employed. First, the contrast agent was diluted in $40 \mathrm{ml}$. of water. Second, the injection was started $15 \mathrm{~seconds}$ prior to the beginning of the pulse sequence. Third, the injection lasted for a period equal to that of the acquisition time of the sequence $(30 \mathrm{sec}$.).

Results: The images obtained with the technique described presented a strong enhancement of the pancreatic parenchyma, stronger than that obtained in images acquired immediately after, related to the arterio-capillary nature of pancreatic enhancement. No improvement in the hepatic enhancement was obtained.

Conclusions: This technique could be used mostly for the evaluation of pancreatic tumors by MRI.

\subsection{PM}

1000 Manganese-DPDP as a MR contrast agent for enhanced

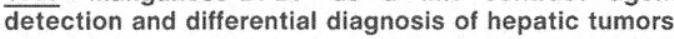

E. Rummeny, J.Adolph, U.Stöber, S. Menker, H. Raber, P.E. Peters; Münster

Purpose: We evaluated the use of Manganese-DPDP (Mn-DPDP), a new paramagnetic hepatobiliary contrast agent, in the detection and differential diagnosis of local liver lesions.

Methods: Twenty patients with proved hepatic tumors [14 with metastases; 3 with hepatomas (HCC), 2 with focal nodular hyperplasia (FNH), 1 with hepatic adenoma] were studied at $1.5 \mathrm{~T}$ (Siemens). T1-weighted (T1W) images were obtained before and 0.5 to $1 \mathrm{hr}$ after i.v. injection of $0.01 \mathrm{mmol} / \mathrm{kg}$ and $0.005 \mathrm{mmol} / \mathrm{kg}$ of Mn-DPDP using gradient-echo Flash 60 (100/5-6 [TR $\mathrm{msec} / \mathrm{TE} \mathrm{msec}], 60^{\circ}$ ) and spin-echo (SE) 500/15/4 (TR/TE/NEX) pulse sequences. Late enhanced T1 W images were obtained 24 to 48 hours after the injection of Mn-DPDP in 11/20 patients. Unenhanced T2-weighted images were obtained with the SE $2500 / 90 / 2$ pulse sequence.

Results: After injection of Mn-DPDP tumor-liver contrast-to-noise (CNR) increased by $165 \%(-8.6 \pm 4.1$ vs. $-22.4 \pm 10.5)$ on Flash 60 images and by $154 \%$ on SE $500 / 15$ images. On enhanced T1-weighted images CNR was significantly $(\mathrm{p}<.001)$ greater as compared to unenhanced T2-weighted images

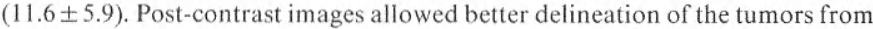
uninvolved liver tissue, and increased the detection rate of small metastases $(<1.5 \mathrm{~cm})$. HCC showed peripheral enhancement in $2 / 3$ cases and FNH exhibit inhomogeneous intratumoral enhancement in all patients. No major side effects were reported.

Conclusions: Mn-DPDP is a promising new contrast agent for improved detection and differential diagnosis of hepatic tumors.

\subsection{PM}

1001 MR imaging of liver metastases enhanced with manganese-DPDP

Klaus P. Aicher, Andreas F. Kopp, Eckart Grönewäller, Ernst Rummeny, Michael Laniado; Tübingen

Manganese-DPDP (Mn-DPDP) as a new paramagnetic contrast agent was evaluated in a phase II clinical trial in 21 patients with liver metastases. Tolerance and lesion conspicuity were specially sought.

Imaging was performed at $1.5 \mathrm{~T}$ employing axial T1-weighted and balanced/T2-weighted SE sequences (SE 500/10;15 and SE 2.500/15;90, respectively). T1-weighted sequences were repeated 30 minutes post contrast. Mn-DPDP (Byk, Germany) was administered intravenously $0.005 \mathrm{mmol} / \mathrm{kg}$ body weight $(2.0 \mathrm{ml} / \mathrm{min})$. Pre and up to 24 hour post Mn-DPDP serum chemistry tests, hematological tests and urin analysis were performed. Subjective and objective events were recorded. Contrast-to-noise ratios were calculated on the basis of signal intensity measurements in lesion, liver and noise.

Eight patients reported a total of ten side effects (metallic taste, feeling of warmth, flush). All events were short-lived, no therapy was required. No change in ECG, blood pressure or labor parameters was observed. Mean values of CNR were as follows: precontrast $\mathrm{T} 1=11.4$; precontrast balanced $\mathrm{SE}=3.7$; precontrast $\mathrm{T} 2=12.6$; postcontrast $\mathrm{T} 1=19.2$. In the vast majority of the cases Mn-DPDP caused a superior lesion conspicuity compared to plain images.

Mn-DPDP is a promising contrast agent for MRI of liver metastases.

\section{$3.00 \mathrm{PM}$}

1002 Mn-DPDP in MR imaging of the liver: tumor detection with a hepatobiliary contrast agent

A. Steudel, M. Reiser, W. A. Kaiser, B. Siewert, K. Bootsveld; Bonn

Purpose: A new paramagnetic hepatobiliary contrast agent (Manganese-DPDP) was evaluated for contrast enhanced MR imaging of liver cancer.

Methods and Materials: Twenty patients with proved malignant liver tumors (16 with metastases, three with hepatomas, one with lymphoma) were studied at $1.5 \mathrm{~T}$ (Philips). T1-weighted SE 500/17 (TR/TE) and FFE 40/20/45 (TR/TE/rf pulse angle) were obtained before and 0.5-1 hour after intravenous injection of 0.01 or $0.005 \mathrm{mmol} / \mathrm{kg}$ of Mn-DPDP. Unenhanced T2-weighted images were obtained with SE 2000/90 (TR/TE).

Results: Tumor-liver contrast-to-noise (CNR) of enhanced SE 500/17 increased by $144 \%$, on T1-weighted FFE by $152 \%$. CNR of both T1-weighted $\mathrm{SE}$ and FFE sequences were significantly $(\mathrm{p}<0.005)$ greater than on unenhanced T2-weighted SE sequences. Postcontrast images allowed better tumor delineation from uninvolved liver tissue.

\section{$3.10 \mathrm{PM}$}

1003 Comparison of the contrast media Gadolinium, Ytterbium and iodine: CT studies in vitro and in vivo

C. Zwicker, M. Langer, V. Ulrich, R. Felix; Berlin

Purpose: To evaluate the enhancement of different concentrations of gadolinium (gd), ytterbium (yb) and iodine in vitro and in vivo.

Methods and Materials: Comparison of the enhancement (HU) of gd, $\mathrm{yb}$ and iodine in concentrations ranging from $0,1-30 \mathrm{mg} / \mathrm{ml}$ using a Somatom Plus (120 and $137 \mathrm{kv}$ ). Measurement of the sensitiveness to gd, $\mathrm{yb}$ and iodine (specific $\mathrm{cm}$ enhancement). In vivo dynamic CT studies without table incrementation were performed in 10 dogs with $1 \mathrm{ml} 0,5$ molar gd, yb and iodine $/ \mathrm{kg}$. Time-density curves were achieved in aorta and liver.

Results: The in vitro studies confirmed a decrease of the enhancement in this order: gd $(120 \mathrm{kv})>\operatorname{gd}(137 \mathrm{kv})>\mathrm{yb}(120)>\mathrm{yb}(137)>$ iodine $(120)$ $>$ iodine (137). The specific enhancement of gd was $40,8(120 \mathrm{kv})$, of yb 34,2 and of iodine $29,6 \mathrm{HU} / \mathrm{mg} / \mathrm{ml}$. As shown in vitro the enhancement of the liver decreases from $21 \mathrm{HU}(\mathrm{gd})$ to $19 \mathrm{HU}(\mathrm{yb})$ and $12 \mathrm{HU}$ (iodine). Gd and yb achieved a higher aortal enhancement than iodine (190 vs $157 \mathrm{HU})$. All contrast media were well tolerated.

Conclusions: Equimolar concentrations of gadolinium and ytterbium are superior to iodine in the in vitro contrast and the in vivo enhancement of the aorta and liver. 
$1004 \mathrm{CT}$ enhancement of the liver, liver abscess, spleen and vessels $\overline{\text { after }}$ iv bolus injection and slow infusion of Perfluorooctylbromide Gerhard Adam, Harald Görtz, Clemens Schiffer, Rolf W. Günther, Jörg Limmer; Aachen

We investigated the enhancement of the liver, liver abscess, spleen and major vessels after i.v. bolus application and slow infusion of PFOB in an animal model. After percutaneous CT controlled abscess induction, 21 rabbits received an i.v. bolus injection of $3 \mathrm{~g} / \mathrm{kg} / \mathrm{bw}$ PFOB and were examined on a Somatom Plus CT scanner in intervals ranging from 2 to 48 hours after contrast medium application. In the second group 21 animals received the same dosage $\mathrm{PFOB}$ as infusion over half an hour and were controlled at the same time intervals. After bolus injection peak enhancement of the liver, the liver abscess membrane and the spleen occurred after 48 hours, while it was found in the infusion group after 24 hours. Maximum enhancement of the aorta and the IVC was found immediately after bolus injection and after 2 hours, while it was significantly lower after infusion of PFOB. The maximum density increase was $50 \mathrm{HU}$ for the liver, $45 \mathrm{HU}$ for the abscess membrane, $305 \mathrm{HU}$ for the spleen and $100 \mathrm{HU}$ for the vessels in comparison to the precontrast scans. We conclude, that $\mathrm{PFOB}$ permits best vascular imaging after bolus injection within the first two hours, while liver and spleen CT imaging should be performed between 24 and 48 hours, depending on the application mode.

\subsection{PM}

1005 A comparison of lopamidol and loxaglate in CT enhancement C. Del Favero, G. Rossini, N. Trenti; Como

AIM: to compare the tolerability of intravenous administration of iopamidol $300 \mathrm{mgl} / \mathrm{ml}(616 \mathrm{mOsm} / \mathrm{kg})$ and ioxaglate $320 \mathrm{mgI} / \mathrm{ml}(600 \mathrm{mOsm} / \mathrm{kg})$ in CT enhancement at doses of $0.8 \mathrm{gI} / \mathrm{kg}$. The relatively high doses of the 2 low-osmolal contrast media (CM) will allow to compare the incidence of adverse reactions (AR) due to the intrinsic chemotoxicity of the molecule. Study design: double-blind comparison on 2 parallel groups of 300 patients (pts.) each. Evaluation criteria: subjective AR, laboratory parameters before and after contrast administration, radiographic quality, evaluation of $\mathrm{HU}$ on 3 reference points.

Results: 330 pts. have been recruited so far. Preliminary analysis on 276 pts. without breaking the randomization code revealed the occurrence of $46 \mathrm{AR}$ in 26 pts. $(9.5 \%)$. All the AR were of mild to moderate intensity, only 6 reactions required pharmacological treatment. No severe reactions occurred. Since the reaction rate was significantly higher than the ones reported by us and other authors with nonionic $\mathrm{CM}$ (range $1.2 \%-3.13 \%$ ) we decided to break the randomization code. Two pts. in the iopamidol group (1.45\%) and 24 in the ioxaglate group (17.4\%) reported AR. The study so far was therefore able to distinguish the two $\mathrm{CM}$ as far as mild and moderate $\mathrm{AR}$ is concerned. The rate of reported severe AR ranges from $0.01 \%$ to $0.06 \%$ with nonionic $\mathrm{CM}$, and is $0.22 \%$ with ionic high osmolality CM in the study by Katayama et al., it is therefore unlikely that the completion of this study will be able to detect a severe reaction in both groups. We have therefore decided to terminate this study that indicates that the intrinsic chemotoxicity of the molecule is more important than the osmolality of the solution in inducing AR.

\subsection{PM}

1006 lodixanol, an isotonic dimeric X-ray contrast medium. Results from six phase II trials

B. H. Grynne, K.W. Borch, J. Ø. Nossen, B. Lundby, B. Bolstad, D. T. Kristoffersen; Oslo

Iodixanol is a new dimeric, non-ionic, hexaiodinated $\mathrm{x}$-ray contrast medium, now under clinical development.

The phase II program comprised 100 patients referred to cardioangiography ( 3 trials), peripheral arteriography ( 2 trials) and cerebral arteriography (1 trial). The trials were open, and patients received iodixanol 320,270 or $150 \mathrm{mg} \mathrm{I} / \mathrm{ml}$. Patients were asked about discomfort (i.e. sensation of warmth, coldness or pain during injection). Adverse events which occurred up to $24 \mathrm{~h}$ after the examination and radiograpic efficacy were assessed. Further, vital signs (heart rate, ECG and blood pressure) were monitored (4 trials) and clinical chemical parameters in blood ( 2 trials) and special renal function parameters ( 3 trials) were performed.

Generally iodixanol was well tolerated and no serious reaction occurred. Sensation of warmth was experienced by 85 patients, usually of mild intensity. Adverse events were reported by 25 patients. No clinical important effects on vital signs or blood parameters were observed. Iodixanol promoted a transient change in renal function parameters, but the effect was similar to or less than what is generally observed after injections of monomeric, non-ionic contrast media.

Based on the phase II results iodixanol is forwarded to comparative phase III trials. 
mation is certainly invaluable but the ICU chest film also contains a great deal of physiologic information that may be crucial to hour-by-hour treatment. Unfortunately, ICU patients not only encompass the entire spectrum of diseases found elsewhere but also manifest multiple superimposed iatrogenic disorders, creating a radiograph which is very complex. The abstraction of the essential quantitative physiologic data requires therefore that the interpreter be radiologically quite sophisticated, have a thorough grounding in physiology and a complete grasp of the special radiologic findings which allow him or her to follow abnormalities of blood volume, flow and distribution, and of extra and intravascular water, and to differentiate between all forms of hydrostatic versus injury lung edema. The accuracy and objectivity of the data from a correctly interpreted ICU chest radiograph equals and may exceed that of complex invasive techniques. It is probable that "severely ill" "fragile" patients should be x-rayed even more frequently than at present for more precise fine-tuning of their treatment.

Causes cartilage, ligaments, tendons, bone marrow, synovial fluid etc.

Of all joints the knee joint is probably the most frequently examined one. Meniscal tears are visualized excellently, reaching a sensitivity of $95 \%$. Because of the high negative predictive value, exploratory arthroscopy may be obviated. Ligament lesions can be exactly evaluated and graded, this being important for therapy.

Hyaline cartilage is best visualized on GE-sequences. Nevertheless in many cases hyaline cartilage and synovial effusion cannot be exactly differentiated. Depending on the stage of disease, different kinds of therapy are performed. MR-Arthrography seems to solve this problem. Avascular necrosis (AVN) of the bone, particularly in the hip joint, can be visualized and staged best with MRI. In early cases of AVN it is already a standard diagnostic imaging modality. Transient bone marrow edema is described as a selflimiting disease itself. There is some evidence that this disease may be only a special type of AVN. Rotator cuff diseases in the shoulder joint are staged and visualized with MRI excellently. It may become a routine method in a short time.

Triangular fibrocartilage abnormalities in the wrist and degeneration of the disk in the temporomandibular joint are also becoming standard indications for MRI, as other joints of the body.

\section{Soft tissue tumors}

\section{Georg M. Bongartz; Münster}

The wellknown capability of magnetic resonance imaging to depict soft tissues ensures its leading role in the evaluation of soft tissue tumors. In comparison to computed tomography or sonography, MRI reveals its superiority by the variety of contrasts. Multiplanar capabilities directly demonstrate the extention of a tumorous mass along all geometrical axes. The results of a MR investigation are readily demonstrable and transferable.

Identification of a tumor is directly connected to its contrast to peritumorous tissues which again directly relies on its signal behaviour in different sequences (T1-, T2-weighted) or on the tumor affinity to contrast media. Improved resolution enables the assessment of tumorous margins as well as tumor invasion into adjacent structures. Therefore presurgical staging of soft tissue tumors today is a domain of MRI. Imaging should precede and guide surgical biopsy in order to ensure optimal results and facilitate treatment. Follow up studies after irradiation or surgery demonstrate recurrence or postoperative residues. Local edema after treatment may hamper the evaluation of remission in early post-therapeutic stages. The role of spectroscopy in staging and follow up studies still has to be discussed.

\section{$1009 \mathrm{MRI}$ of primary bone tumors}

\section{Vanel, L. G. Shapeero; Villejuif, San Francisco, CA}

Because of its excellent soft tissue contrast, and multiplanar capabilities, MRI is the best technique for local evaluation of primary bone tumors: their extent and involvement of bone marrow, soft tissues, joint, and neurovascular structures.

MRI is rarely helpful for specific tumor diagnosis. Relaxation time measurements are non-specific, and small calcifications may be missed on MRI. Furthermore, the size of the tumor is sometimes overestimated on T2-weighted images.

In addition, MRI allows an accurate evaluation of effectiveness of preoperative chemotherapy and of local recurrences when the patient has a non-paramagnetic prosthesis (titanium or vitalium).

\section{Room B}

\section{RG 801}

1010 Sophisticated critical care unit radiology - non-invasive monitoring of cardiopulmonary pathophysiology

Eric N. C. Milne; Irvine, CA

ICU films have traditionally been read to determine whether tubes and catheters are correctly positioned and to detect relatively gross anatomic abnormalities such as consolidation, atelectasis and pneumothorax. This infor-

\section{Cardiogenic and non-cardiogenic edema}

\section{Miniati, M. Pistolesi, E. N. C. Milne, C. Giuntini; Pisa, Irvine, CA}

Left heart failure, overhydration (caused by excess saline infusion or renal failure), and injury to the lung microcirculation are the three major causes of lung edema occurring in clinical practice. Although the radiographic aspects of cardiac, overhydration, and injury lung edema have been described in the literature, it has long been alleged that cardiogenic and noncardiogenic edema cannot be differentiated from each other on the basis of their radiographic appearance. To solve this controversy, a retrospective study was carried out on 216 chest radiographs obtained from patients with cardiac disease, renal failure, and lung microvascular injury (Milne et al. AJR 1985; 144: 879). Two independent observers, unaware of the patients' clinical diagnosis, analyzed the chest radiographs and were asked to state, on the basis of their previous experience, what type of edema was present in any given case. In the evaluation of chest films, the following radiographic signs were considered: heart size and shape, vascular pedicle width, hilar abnormalities, pulmonary blood flow distribution, pulmonary blood volume, lung density increase (qualitative features and regional distribution), septal lines, peribronchovascular cuffs, air bronchogram, and pleural effusion. The prevalence of the radiographic findings was compared, among the three groups, by the chi square test. The two observers assigned the chest radiographs to the correct group with an accuracy of $86 \%$ and $90 \%$, respectively. Patients with injury lung edema were characterized by the highest prevalence of normal heart shape, the fewest hilar abnormalities, normal pulmonary blood flow distribution, absence of septal lines, rare peribronchovascular cuffs, rare pleural effusion, and frequent air bronchograms. In cardiac and renal patients, haziness of lung fields was observed. For patients with injury lung edema, the increased lung density often had a parchy configuration and a peripheral distribution. A base-to-apex blood flow inversion and an even distribution of the increased lung density were more frequently observed in cardiac patients than in renal and injury cases. The distinctive features of renal patients were an enlarged vascular pedicle, a balanced pulmonary blood flow distribution, an increased pulmonary blood volume, and a central distribution of the increased lung density. To further assess the value of the chest radiograph in differentiating various types of edema, an independent two-observer study was performed on 119 chest films from patients with cardiac disease, renal disease, and lung injury (Miniati et al. Invest Radiol. $1988 ; 23: 433$ ). In this study, the radiographic findings used by the readers in identifying the etiology of lung edema served as input variables for discriminant analysis. Computer-generated numerical functions identified the etiology of edema with an overall accuracy of $88 \%$. When the groups were compared as pairs, percentages of correct classification were $91 \%$ (cardiac vs. renal), $93 \%$ (cardiac vs. injury), and $100 \%$ (renal vs. injury). Thus, a standardized reading of the chest radiograph may be considered a reliable clinical method for identifying the etiology of lung edema.

\section{Room C}

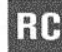

\section{4}

1012 Abdominal interventional procedures guided by CT and US R. Ch. Otto; Baden

Though new imaging modalities like CT, Ultrasound and MRI are capable to detect pathological alterations in the body with a high degree of certainty, they cannot always differentiate between malignant and benign lesions. The more invasive procedure, the percutaneous biopsy under permanent visual control, allows for more precise evaluation of focal lesion and provides minute tissue probes for cytological, histological and/or bacteriological examination at a virtually low risk. In more than $90 \%$ it is possible to get tissue probes of the region of interest in different parts and organs of the body. Prerequisites, equipment and precautions will be discussed as well as the difference of fine needle puncture and larger biopsy. Moreover, several curative or palliative and pseudocysts, nephrostomy and even tumor treatment. The special interventions may be performed without operation like drainage of abscesses 
technique using different imaging modalities for biopsy and therapy will be discussed as well as the results.

\section{Interventional radiology of the biliary system}

\section{Hans Jantsch; Vienna}

Biliary drainage procedures provides an effective nonsurgical intervention for advanced nonresectable malignant biliary obstruction. Furthermore preoperative biliary drainage procedures are still recommendable to optimize patients in poor general condition (e.g. sepsis with cholangitic abscesses) and in patients with difficult postoperative anatomic conditions (e.g. after liver transplantation or liver resection). But biliary interventions are also indicated in benign deseases as removal of intraoperative overlooked or operative non removable common and intrahepatic bile duct stones, balloon dilatation of benign strictures of the common bile duct but also of intrahepatic bile ducts. The technique of biliary drainage procedures has changed in the last years since the catheters, guide wires and endoprostheses have become more sophisticated. At the present time we strive for the single procedure insertion of an endoprosthesis. This is only recommendable in case of no or minor hemobilia which is more frequent to achieve by using only a thin needle ( 22 gauge) for diagnostic and therapeutic puncture and the use of 0.18 inch guide wires with gold or platinium tip and coaxial dilatation of the tract. For stenting we prefer in our institution the wall stent, which is an self expanding metal stent, which is easy to insert through a small tract ( 7 French) but is difficult to position.

\section{Room E1}

\section{RC 805}

1014, 1015 Anatomy and pathology of the retroperitoneum: an integrated approach

M. A. M. Feldberg (1014) and M. S van Leeuwen (1015); Utrecht

Modern cross-sectional imaging techniques provide detailed anatomical information in virtually all patients under study. The correct interpretation of these studies depends on a thorough working knowledge of the regional anatomy and the patterns of spread of different disease processes. In this course the anatomy and pathology of a variety of diseases will be discussed emphasising the relationship between cross-sectional and surgical anatomy.

The anterior pararenal space; harbouring pancreas, duodenum and the larger parts of descending and ascending colon is a transition zone between the true retroperitoneum and the subperitoneal space; i.e. the ligaments and mesenteries. Therefore it is anatomically, embryologically and (patho)physiologically intimately related to the alimentary tract.

The perirenal space contains kidneys and adrenals and is contained between a conspicuous anterior and posterior layer of renal fascia. The majority of disease within this compartment originates from the kidney or the urinary tract and may affect the psoas compartment. Midline communication however is a matter of discussion.

The posterior pararenal space does not contain organs, but its continuity with the great vessels and psoas muscles medially and the properitoneal flank fat laterally provides a pathway for fluid and solid pathological processes.

Ultrasound, Computed Tomography and Magnetic Resonance Imaging will be used to illustrate the anatomical pathways between, and within, the different compartments. An effort will be made to emphasize a practical, problem solving approach toward imaging the anatomy and pathology of the retroperitoneum.

\section{Room E2}

\section{WS}

1016 Pediatric hip: ultrasonography in infancy Marleen Smet; Leuven

Real time ultrasonography provides valuable morphological and functional information of the hip joint in infancy. The dynamic relationship of the cartilaginous head to the acetabulum is clearly defined and instability, subluxation and dislocation can be demonstrated. The validity of the clinical and sonographic examination relies heavily on the experience of the examiner. In addition to a brief description and explanation of the examination technique, this workshop will focus some of the benefits, limitations and potential therapeutic implications of this imaging method. The clinical, sonographic, radiological and therapeutic correlations will be illustrated and discussed on the basis of several interesting and challenging cases.

\section{Pediatric hip - the child with a limp}

\section{Herbert J. Kaufmann; Berlin}

In the diagnostic approach to this problem the value of the different imaging modalities and their respective indications will be emphasized.

\section{Ultrasonography:}

Even after infancy US is being more and more applied as the primary examination to determine the presence of fluid/blood in the joint. In addition it is possible to evaluate the integrity of the femoral head comparing the two sides. Thereby it is possible to avoid examinations with ionising radiation in the presence of "fleeting coxitis", "hemophiliac joints" and other conditions.

2. Conventional X-rays:

Conventional roentgenograms are required if US is negative or equivocal and particularly if a congenital or acquired bony abnormality of leg length difference is present or suspected. Tomograms may be of value in individual instances.

3. Skeletal Scintigraphy:

Performed early, radionuclide studies are highly informative for the recognition of the initial phase of aseptic necrosis of the femoral head (M. Perthes) if a "cold lesion" is demonstrated. Infectious lesions and tumors can be readily demonstrated as "hot spots". Especially if multiple such lesions are suspected scintigraphy is the preferred examination.

4. Magnetic Resonance Imaging:

It is amazing how rapidly MRI has been introduced for the diagnosis and follow-up of pathology of the hip in infancy and childhood. Destructive lesions of the femoral head or the immediately adjacent bony structures of the pelvic ring and pathology of soft tissue structures can now be diagnostically approached.

5. Computertomography:

CT-examination is indicated only if bony pathology in the area of the femoral neck or head is strongly suspected and results with the other imaging modalities are inconclusive. Due to the inclusion of the gonads in the region investigated only clear indications are acceptable for this examination.

The emphasis will be less on the multitude of reasons for a limp, but rather on the respective value of the different imaging modalities, especially those "not using ionising radiation".

\section{Aseptic necrosis of the child's hip} Helen Carty; Liverpool

The femoral head is the most frequent site of aseptic necrosis of bone in both children and adults. The causes vary in different age groups and in different populations. While the sequence of histological and radiological changes are well known, and many aetiological factors are recognised, the precise cause of the osteonecrosis is not known. There is clinical and experimental evidence that the induction of necrosis may be multifactorial and include alterations in lipids, fatty embolisation to bone and nitrogen embolisation. It is postulated that these raise intra osseous pressure, and reduce bone perfusion leading to necrosis. The effects may be increased by inflammatory reaction to the response. Raised joint pressure may also have a role in producing vascular tamponade of the femoral head, and this is shown scintigraphically on occasion in an acutely septic hip. Alteration in cellular lipid metabolism is probably the mechanism in steroid induced avascular necrosis. Whatever the cause, there is resultant osteocyte death with repair by osteoclast resorption and bone repair in cortical and subchondral bone. The subchondral bone becomes weakened by the resorptive process, and may fracture at sites of high mechanical load, leading to further compromise and collapse of the femoral head.

The radiological features of aseptic necrosis have been well documented. The diagnostic features seen in the adult population are also seen in children's hips. These features will be reviewed and illustrated. The role of scintigraphy, C.T. and M.R. in the diagnosis of aseptic necrosis will be discussed. The results of a survey of 60 consecutive cases of avascular necrosis of the femoral head, presenting in a children's hospital will be presented to demonstrate the causes in children.

\section{Room L}

\section{WS}

\section{9}

1019 Management of children in imaging procedures: how to do body $\mathrm{CT}$ in children

Javier Lucaya, Joaquim Piqueras; Barcelona

When we do Pediatric CT good technique should be our primary concern. With good technique diagnosis will be quite easy. On the other hand to make good diagnosis with poor exams is extremely difficult. Therefore we would like to start this talk by emphasizing the importance of good technique.

All studies should be tailored to the specific clinical situation, therefore prior to planning the CT we should go through all the available information on the patient such as the clinical history, previous X-rays, ultrasound and so forth and then we will decide not only the preparation required but also the technical factors of the exam such as the use of contrast by mouth by enema or intravenously, the thickness and number of slices to be taken and so on. As sedation and/or intravenous contrast material will be used in a high percentage of cases and infants and younger children are often sedated we make our patients NPO in the following manner: 
- Newborn NPO as necessary in consultation with neonatologist

- Less than 1 year NPO for 3 hours

- Over 1 year NPO for 4 hours

- Adolescents NPO after midnight for morning exams

During this time both food and liquid are withheld except for oral contrast material given to opacify the gastrointestinal tract. For oral contrast material we use a 3 per cent concentration of gastrografin diluted either in the formula or in a flavored vehicle such as Kool Aid or Tang which helps to mask the unpleasant taste of the contrast.

The contrast is given orally or through a nasogastric tube 20 to 30 minutes before actual scanning begins. If we have used a nasogastric tube it should be withdrawn prior to scanning so as not to cause high density artefacts on scans. When examining the pelvis we give two equal volume doses of oral contrast: one 3 hours prior to the exam and a second dose 30 minutes before scanning begins. When it is very important to fill the entire colon we either give an additional dose of contrast 12 hours prior to the examination or use a 3 per cent gastrografin enema which will be administered just prior to starting the exam. When all we want to see of the large bowel is the location of the rectum we place a small tube in the rectum and inject a small amount of a 3 per cent concentration of gastrografin. We usually inject no more than 20 to $30 \mathrm{cc}$ and in most instances leave the tube in the rectum while scanning the patient. If the child is to be sedated and/or if the child is young, it is best to have an intravenous line in place before the child is called to the CT room. This can then be used for IV contrast administration without disturbing a child who is either sedated or frightened.

In order to achieve good imaging it is very important that the patient remains still. Non-cooperative patients will require sedation. In infants less than 3 months of age we usually use Chloral Hydrate which is a harmless and safe drug at doses ranging between 50 and $100 \mathrm{mg}$ per kilogram. We administer the chloral hydrate mixed either with the infant formula or with the oral contrast material about 30 minutes prior to scanning. The onset of sedation in most infants is 20 to 45 minutes.

After the age of 3 months we prefer to use IV nembutal at a dose of $6 \mathrm{mg}$ per kilo with a maximum total dose of $100 \mathrm{mg}$. The onset of action is rapid, toxicity is minimal and duration of action is not prolonged. In patients older than 3 months we will decide whether they need sedation depending on their behavior once they have been placed in the gantry and are ready to be examined. If we decide to sedate the patient the first thing we do is dilute his total dose of nembutal, that is $6 \mathrm{mg}$ per kilogram up to a maximum dose of $100 \mathrm{mg}$ in $100 \mathrm{c.c}$. of saline.

In order to avoid mistakes the syringe containing nembutal is always marked with a big letter "N". We then inject about half of the total dose and wait for approximately 5 minutes. If the patient falls asleep we start scanning. If he remains awake we then inject very slowly the other half of the dose. Occasionally the patient will require an additional dose of $2 \mathrm{mg}$ per kilogram which will only be administered if the patient remains awake 20 to 30 minutes following the administration of the initial dose. Oxygen should be administered to all patients receiving sedative medication with the possible exception of neonates at risk of retinopathy of prematurity. Administration of supplemental oxygen markedly increases polmonary oxygen reserves and permits prolonged apnea or airway obstruction without hypoxia. We administer the oxygen through simple devices such as a mask or a tube taped to the patients forehead.

In our experience this sedation regime has been successful in about 95 per cent of cases. Once the patient has been sedated he has to be immobilized usually with sand bags or velcro tapes. All children are monitored using visual inspection and a pulse oximeter. Oximetry is rapidly becoming a standard for anesthetic procedures. It can be used reliably in CT without affecting image quality. The sensor can be placed on an extremity outside the gantry and the monitor can be placed at some distance from the scanner.

In very small infants when visual inspection is somewhat difficult we place a paper cup on top of their abdomens. This facilitates control of their respiratory movements from the remote console.

The next step is to take a scout view of the region of interest and decide the technical factors we are going to use for the exam. We usually select the lowest m.A. possible, usually around $100 \mathrm{mAs}$, we choose the fastest scan time possible, usually 0.5 or 1.2 seconds, and elect slice thickness and spacing, usually contiguous $10 \mathrm{~mm}$ thick slices.

After reviewing the available non contrast slices we will decide whether the patient needs either additional non contrast slices or if the exam should be repeated following the administration of intravenous contrast material.

When we need IV contrast we use non ionic low osmolar contrast agents which in our experience have proved to be less painful at the injection site and cause fewer reactions, particularly nausea and vomiting, than hyperosmolar contrast agents.

Whenever we decide the patient needs an exam using intravenous contrast we use dynamic scanning with bolus injection technique. The paucity of body fat in the pediatric patient makes delineation of anatomic structures very difficult. This is precisely why the use of dynamic scanning with bolus technique is so important in children.

For dynamic scanning and bolus technique we prefer to use the largest bore butterfly needle possible, in most cases we will be using either a 19-gauge or a 21 -gauge needle. Non ionic low-osmolar contrast material is administered intravenously as a bolus injection at a dosage of 2 to 3 c.c. per kilogram, up to a maximum of 100 c.c. Approximately one-half of the total contrast dose is injected as rapidly as possible, and then the technician is signalled to begin the preprogrammed scanning. The second half of the intravenous contrast dose is then injected at a slower rate so as to last throughout the first 6 to 8 slices. It is extremely important to test the IV line before starting the bolus injection in order to avoid extravasation. When injecting very small infants is not unusual to notice some signs of discomfort, such as agitation or withdrawal of the injected limb. In such cases we modify our method and do not begin scanning until 80 per cent of the total dose of contrast material has been, very slowly, injected trying to avoid any discomfort which may cause the patient to move or cry. By so doing we have reduced the incidence of images degraded by motion artefacts.

When examining the chest we prefer to inject the intravenous contrast in a left upper extremity vein. This allows good opacification of the innominate vein which is an excellent anatomical landmark.

When examining the abdomen any upper extremity vein will do. We do not like to use lower extremity veins since the high opacification of the inferior vena cava will cause artefacts.

\section{How to do MR in children}

\section{Graeme M. Bydder, Jacqueline M. Pennock; London}

There are several important differences of technique in MRI of children. Immobilisation requires sedation and while general anesthesia is acceptable for major illness other methods are necessary for less serious cases. Oral chloral hydrate administered by nasogastric tube is effective and predictable. For neonates, we rely on natural sleep and place the infants on their side.

Spherical receiver coils provide improved signal to noise for imaging the brain. They need to be available in a range of sizes.

The infant brain contains a higher proportion of water than adults and has a much longer T1 and T2. In order to maintain similar contrast it is useful to lengthen TE, TR and TI for neonates and progressively shorten them during the first two years of life.

During this period myelination is occurring and data from a normal set of controls is necessary in order to identify delays or deficits in this process.

Unmyelinated white matter in the frontal and occipital lobes has a particularly long T1. This decreases to the value of gray matter at three to six months. Subarachnoid hemorrhage is a very common finding in neonates requiring intensive care and mild degrees probably have benign implications. Otherwise patterns of hemorrhage are similar to those seen in adults.

Many pathological processes increase $\mathrm{T} 1$ and $\mathrm{T} 2$ but the proportional changes in neonates are much less than in adults, resulting in lower contrast between normal and abnormal areas of the brain.

\section{Room M}

\section{WS}

\section{Inferior vena cava filter}

$\overline{\text { R.W. Wünther; Aachen }}$

Pulmonary embolism is still a significant cause of morbidity and mortality in hospitalized patients. Standard therapy consists of anticoagulation. If this fails or is contraindicated, a mechanical barrier in the inferior vena cava is the last resort to prevent potentially life-threatening pulmonary embolism.

Ligation of the common femoral vein or the inferior vena cava was an early form of treatment. Later on, caval clipping simplified the procedure, but still required a major operative approach. Operative insertion of vena cava filters using the femoral approach via a venous cut-down led to a further simplification of the procedure. The final step in simplification was an exclusively percutaneous approach.

A variety of filter types have been developed for percutaneous insertion, such as Birds nest filter, LGM filter, basket filter, Kimray-Greenfield filter (titanium), DIL filter and nitinol filter.

Regardless of which filter type is used, it should be borne in mind that filters are foreign bodies in the inferior vena cava which may have side-effects such as filter breakage, filter migration, and thrombosis of the inferior vena cava. For this reason, the indications for filter placement should be strictly observed and restricted to:

1. Recurrent pulmonary embolism despite anticoagulation,

2. Pulmonary embolism when anticoagulation is contraindicated,

3. Free-floating emboli in the iliac and femoral veins, or in the inferior vena cava,

4. Prophylactic filter placement. This is only indicated in high-risk patients in iliac or femoral vein thrombosis.

The filter is usually placed in an infrarenal position, suprarenal filter placement may be used when thrombi extend up to or above the renal veins, or when renal vein thrombosis is a source of recurrent pulmonary embolism. 


\section{Venous stents}

C. L. Zollikofer; Winterthur

Percutaneous angioplasty (PTA) of venous stenoses generally shows a high recurrence rate. Often the tumorous or fibrotic nature of the underlying disease is primarily resistant to PTA. Therefore endovascular stents have been used quite successfully to overcome these problems.

To date there are five main groups of diseases where stents may be used successfully in the venous system.

1. Palliative treatment of tumor compression of the vena cava and large central veins.

2. Non tumorous external compression of the vena cava such as in Budd-Chiari syndrome or mediastinitis.

3. Postoperative strictures in large native veins.

4. Postthrombotic stenoses of large veins, venous spur (May-Thurner syndrome), congenital webs.

5. Stenoses of the venous outflow tract including brachiocephalic veins in chronic hemodialysis fistulas.

The technique of stent insertion, the instrumentation as well as specific indications will be discussed in this workshop. Results, problems and pitfalls will be shown by means of cases performed during the last five years. The main aim of this workshop is to mediate practical guidelines as to the use and indications of successful stenting in the venous system.

\section{Room N}

\section{RC}

\section{Investigation of congenital spinal malformations}

$\frac{1023}{\text { D.P.E. Kingsley; London }}$

A knowledge of the embryology of the spine provides an understanding of the types of congenital lesions that are encountered.

Clinical manifestations of congenital spinal malformations are very varied bu physical examination often reveals surface dimples, hairy patches, swellings etc., indicating the likely level of the lesion.

Investigation of such lesions depends on access to MRI, which should be the first investigation since it provides much useful information and is non-invasive. A combination of sagittal, oblique and axial T1 weighted sequences provides most of the basic anatomical information such as the position of the conus, the relation of the cord to bony spurs, lipomas etc. and the integrity of the cord itself, but localised volume imaging, such as across a gibbus, can provide greater anatomical detail.

More detailed anatomy may require CT myelography to resolve the nerve roots particularly when the cord merges with a thickened film terminal or when there is possibility of useful neural tissue at the level of the placode whose function must be preserved during surgery. MRI at present is not a reliable procedure for this purpose.

In the very young, sonography can provide useful adjunct information regarding elements of the anatomy not revealed by CT myelography or MRI. There is evidence that it may provide useful functional information in the assessment of cord tethering both before surgery and in the follow-up period.

\section{Imaging of space-occupying lesions of the spine}

\section{F. E. Zanella; Hamburg}

Abstract available at the information desk.

\section{Room 0}

\section{WS}

\section{DSA, myth and reality}

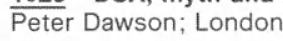

The future of medical imaging is undoubtedly a digital future. Digital angiographic systems are already in widespread use throughout the world and enough experience has been gained to allow conclusions to be reached about the place of digital angiography in modern imaging. As usual with new technology some exaggerated expectations have been disappointed, but in other areas there has been agreeable surprise in unpredicted usefulness.

There will be discussion of the state of the technological art in digital angiographic systems; of the proper role of DSA in modern angiography; of intravenous vs intraarterial studies; and of applications of digital systems in interventional radiology.

\section{DSA: myth or reality}

E. Zeitler; Nürnberg

In the last decade, the equipment for digital subtraction angiography (DSA) has undergone extreme changes and developments. Nevertheless, the great hope for a non-invasive angiography with peripheral and intravenous application of contrast media could not be fulfilled, except in a few special cases. But the hopes which were placed too high (the myth!) have launched an evolution which led to the result that the conventional cut-film-angiography with AOT and Puck-Changer was more and more frequently replaced by digital systems. Even though the spatial resolution of image intensifier units with a matrix of $1024 \times 1024$ has improved extremely, it still is higher with classical analogous techniques. - Also, the quantity of contrast medium (CM) necessary for leg angiography is still higher today with digital systems as it is with conventional angiography when using automatic table shifting or bit cut films. But there is no way backwards. DSA has made angiography less invasive! DSA has opened new indications and gave the possibility of outpatient studies in many instances. Intravenous studies with preartial catheter placement as well as intraaterial studies after the use of small size catheters (4 or 5 French) on the transfemoral or transbrachial route procure an in- and outpatient reality which has helped to reduce the cost and increase early diagnosis. The need of higher quantities of CM is due to new and more extreme questions to the radiologist and not only to the change from an analogous to a digital technique. Furthermore, many of the vascular interventional radiological (IR) procedures are more successful and less risky with DSA. Thus, in 1991 , there still remain a small number of indications for the classical angiography due to the higher spatial resolution, but in most cases, the increased contrast resolution of DSA will help to forget this unsolved residual problem in practice.

\section{Room R}

\section{Ss} 4.00 PM

\subsection{Quality control in radiotherapy III}

\section{Management of patients with early stage carcinoma of the floor} of the mouth with the aim of mandible preservation

H.Ikeda, T.Inoue, S. Murayama, T. Teshima, H. Fuchihata, K. Kubo, K. Shimizutani; Osaka

Purpose: To search the most suitable treatment combination relating to the tumor extension for the patients (pts.) with early stage carcinoma of the floor of the mouth with the aim of mandible preservation.

Methods: A retrospective analysis was made of 103 pts. with ca. of the floor of the mouth (16 pts. with Stage I, 33 St. II, 40 St. III and 14 St. IV after UICC-TNM '87) treated with radiotherapy, solely or in combination, from 1980 through 1987 . In most pts. with St. I, permanent implant of ${ }^{198} \mathrm{Au}$ grains was applied, to a median permanent dose of $89 \mathrm{~Gy}$ (range 65-140 Gy). In most St. II, a concomitant combination of external radiotherapy (ERT) of a dose of $30 \mathrm{~Gy} / 3$ weeks and intravenous infusion of either Bleomycin (BLM), $90 \mathrm{mg} / 3 \mathrm{w}$, or Peplomycin (PEP), a derivative of BLM, $60 \mathrm{mg} / 3 \mathrm{w}$ before applying boost brachytherapy or radical surgery.

Results: In pts. with St. I, perfect local control (13/13) was achieved when applied with ${ }^{198} \mathrm{Au}$ grains. In all pts. with St. II, ultimate local control rate of $73 \%(23 / 33)$ was obtained. In 26 pts. treated with combination of ERT + $\mathrm{BLM} / \mathrm{PEP}$, clinical complete remission (CR) before brachytherapy or surgery was obtained in 10 , and led to definite local control in 9 . In contrast, in pts. who did not achieve CR, better local control was obtained with further treatment by surgery $(3 / 4)$ than by brachytherapy $(6 / 11)$. Mandible was preserved in all with St. I $(16 / 16)$ and in some St. II who were treated with ERT + BLM/PEP with brachytherapy $(14 / 14)$, whereas it was not in those treated with surgery $(0 / 5)$. Overall 5 -year survival by Stage was, $93 \%$ for St. I, $69 \%$ St. II, $45 \%$ St. III and $43 \%$ St. IV, respectively.

Conclusions: 1. Permanent brachytherapy with ${ }^{198} \mathrm{Au}$ grains is the treatment of choice in superficial tumor as Stage I. 2. Assessment of response at the end of $\mathrm{ERT}+\mathrm{BLM} / \mathrm{PEP}$ is useful in selecting the further modality in Stage II. 3. When treated with radiotherapy, mandible was preserved in all patients with Stage I and II. 
1028 Patient individual automatic set up using the Beamview portal imaging system

F. Van den Heuvel, W. De Neve, M. Coghe, F. Krispel, G. Storme; Brussels

A method for patient individual set up for the Siemens Beamview ${ }^{(B)}$ system is presented. The portal imaging device consists of a fluorescent screen placed behind the patient. The image is recorded with a SIT video camera with adjustable gain, offset and black level. The inspiration and experience for system set up was acquired during a clinical survey using the Beamview ${ }^{\circledR}$ system on 800 patient fields on a dual energy (6 MV and 18 MV) KDS2 Siemens linear accelerator. Fields where taken in the pelvic, thorax and Head \& Neck regions. Observations in the different settings led to physical experiments yielding favorable results. Black Level only had to be changed in an initial set up. The offset is found to be directly dependent on the outgoing dose rate (i.e. dose rate on the fluorescent screen) and of the beam energy. Camera gain is only affected by the kind of contrast wanted. An automatic set up using these parameters is proposed making this system usable for routine purposes. A clinical survey of pelvic fields will act as a test of the system where scores will be attributed to every image.

\subsection{PM}

1029 Continuous treatment verification by an ionisation chamber-

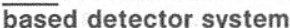

A. Habegger, A. Meister, N. Lombriser, U. Dechantsreiter, U. M. Lütolf; Zurich

New diagnostic methods in radiation therapy such as CT and MRI are contributing to a more precise determination of tumour volume.

Accurate repeated positioning of a patient during therapy remains a major problem. Reproducibility is especially important when applying a therapeutic dose near critical organs such as the brain or spinal cord.

Recently, an ionisation chamber-based detector has been developed which allows $x$-ray data to be electronically acquired during treatment. The resulting images, stored in a computer, are available for further processing.

The continuous monitoring of the patient's position will allow us to minimize the discrepancy between the simulated and irradiated volume. This results in better quality assurance during treatment and should finally enable us to increase the tumour dose.

At the University Hospital in Zurich such a system is currently being tested in clinical practice. We are conducting research into image processing that facilitates the recognition of anatomical structures and landmarks.

This work is supported by grant No. 1841.1 of the KWF (Commission for Scientific Research, Berne, Switzerland).

\subsection{PM Roundtable Discussion with Keynote Speakers}

\section{Room S}

\section{WS 810}

1030 Future of contrast media

Gerald L. Wolf; Charlestown, MA

Abstract: Early investigators believed that MR imaging would always be slow and would never require contrast agents. However, several excellent proton imaging schemes now provide subsecond scanning, and paramagnetic extracellular fluid indicators are now used in more than $30 \%$ of brain MRI exams. The detector and special MR indicators are now locked in a fascinating and intimate pas de deux. Not only is the MR detector a major advance in imaging biologic tissue, but the MR contrast agents are detected with sufficient sensitivity to allow chemical diversity not achievable for $\mathrm{x}$-ray or ultrasonic inquiry. The near future is dominated by development of blood pool, lymphographic, monoclonal, intracellular, specific pathology, and receptor agents. With this expanded range of indicators, the combination of fast imaging and adequately safe agents allow for in vivo measurements of such tissue functions as perfusion, receptor density, and increased local vessel or cell permeability. MRI and MR contrast agents allow sophisticated tissue characterization in vivo with excellent spatial resolution. Spatially resolved tissue characterization minimizes averaging errors associated with systemic clearance determinations and has non-invasive, non-destructive advantages over biopsy methods. While scientific focus is on the details and the opportunities, society is entreating scientists to determine the effect of new technology upon outcomes and to measure its cost-effectiveness. Soon this plea will also come from our colleagues in clinical practice who have been denied reimbursement for their examinations.
1031 Particulate contrast agents for CT

David D. Stark; Boston, MA

Particulate CT contrast agents are designed to increase the accuracy, efficiency, and safety of hepatosplenic imaging. During the last two decades, several different approaches have been tried. Iodinated oils, liposomes, and solid particles made from derivatized contrast media have all shown promise, but none of these compounds have been developed for routine use, principally due to toxicity considerations.

A new carbonate ester of metrizoic acid has been developed (Nycomed, Inc.). This material shows favorable pharmacokinetics and biodistribution, with $60 \%$ of the injected dose accumulating in the rat liver, and clearance from this organ with a half-life of $11 \mathrm{hr}$. At the $100 \mathrm{mg} \mathrm{l} / \mathrm{kg}$, liver attenuation increased from 63 to $106 \mathrm{HU}$, without any tumor uptake. As a result, tumor-liver image contrast enhanced dramatically. Biodegradability and urinary excretion of the iodinated metabolite metrozoic acid may be responsible for the low acute toxicity $(2000 \mathrm{mg} \mathrm{I} / \mathrm{kg}$ in mice). This class of hydrophilic biodegradable particulates shows promise for further development.

\section{Ultrasound contrast agents}

Thomas Fritzsch; Berlin

Agents which enhance backscattered ultrasound signals are employed as ultrasound contrast agents (USCA). Several approaches have been used to develop agents with high reproducibility of the contrast effect and ease of use, which are effective and safe. Solid particles or solutions which alter the speed of sound were used as well as emulsions or bubble-containing solutions. However, only the latter have proven to be useful. Typical examples of USCA of this type are sonicated X-ray contrast media, agitated polygeline solution and suspensions of galactose particles with microbubbles. After i.v. injection, these preparations lead to opacification of the right heart. The number and size of the bubbles are important for contrast effect and tolerance. The principle of the galactose associated microbubbles has advantages due to standardization, i.e. physical binding of preformed gas bubbles to microparticles. Arterial contrast effects need a catheter injection, since the bubbles do not cross the lung capillaries. USCA with stabilized gas bubbles which can provide left heart contrast after i.v. injection were recently described. Animal experiments and first investigations in man show promise. USCA have shown an additional diagnostic potential in combination with Doppler-techniques by increasing the signal-to-noise ratio. Provided the bubbles are small and their size spectrum is narrow, the velocity in the blood is not different from red blood cells. Thus the Doppler-shift, and thereby the basis of quantification, is not changed.

In conclusion, USCA have demonstrated a useful diagnostic potential. Moreover, number and nature of side-effects seem to be minimal. USCA may contribute significantly to diagnosis, can probably complement most of the current methods and will certainly replace some of them.

\section{Blood pool agents}

Catherine Chambon; Aulnay sous Bois

Several research groups are trying to develop blood pool contrast media (BPCM) in order to avoid the rapid extravascular diffusion of the currently available iodinated contrast media or MR imaging agents. The theoretical applications of these new contrast media will be perfusion imaging studies, angiography and the detection of abnormal vessel permeability.

According to the literature, BPCM can be classified as macromolecular compounds and particulate agents.

The distribution of macromolecular agents (albumin and dextran derivatives) which is not strictly intravascular, is a potential disadvantage. Their elimination is either by glomerular filtration or metabolisation.

Particulate agents are taken up by the Macrophage Phagocytic System, metabolised and eliminated either by the renal or hepatic route.

In perfusion imaging studies, the great challenge of these BPCM in terms of efficacy is that they are expected to enhance the contrast of a whole organ while being limited to the vascular space.

\section{Liposomes in contrast enhancement} Christoph de Haën; Milan

Liposomes are in preclinical development as imaging agents applicable in X-ray examinations, MRI, echography and scintigraphy, depending on whether they are filled or associated with iodinated compounds, paramagnetic material, gas or radionuclides. The chemical composition and the method of preparation jointly determine the highly varied structures and physicochemical imaging properties of liposomes. The following structural parameters determine the stability and fate of liposomes in the body: size, net electrical charge, hydrocarbon and polar head group composition of amphipathic lipids and the content in lipophilic molecules such as cholesterol. Size determines the extent to which liposomes may leave the vasculature through endothelial fenestrae or defects. The other parameters are responsible for membrane fluidity and permeability (e.g. to contrast agent, water) as well as for affinity for serum opsonins and dysopsonins. All properties mentioned together determine the differential efficacy with which liposomes are trapped by small capillaries 
(e.g. in lung) or captured by macrophages in various organs. Variations in structural parameters can thus yield blood pool or pulmonary contrast agents or agents with tissue selectivity for hepatic or splenic macrophages as well as sites of defective endothelium (tumors, inflammation sites). Additional tissue targeting may be obtained by binding of address molecules, such as antibodies or hormones, to the surface of liposomes. Liposomes made from natural phospholipids and lipids are in general well tolerated. In certain cases reversible splenomegaly has been observed. However, it is important to remember that liposomes deliver contrast agents that are typically confined to the extracellular compartment into target cells possibly with novel toxic consequences. The potential problem is at least partially mitigated by the fact that in any case all cells absorb limited amounts of extracellular agents by bulk phase pinocytosis. Preclinical imaging studies with all four imaging modalities i.e. X-rays, MRI, echography and scintigraphy bode well for the clinical future of liposomes as contrast agent vehicles. 


\section{Room B}

\section{Room A}

\section{C6. 911}

\section{Spinal cord}

William G. Bradley; Long Beach, CA

The most common presenting complaint for a spinal cord neoplasm in an adult is pain; in a child, it is difficulty walking. Approximately $1 / 5$ of all tumors of the central nervous system involve the spinal cord; approximately $1 / 5$ of these are gliomas, i.e., astrocytomas or ependymomas. The most common appearance of an ependymoma is that of a cystic, enhancing neoplasm most often involving the lower cord or conus. Ependymomas account for $65 \%$ of all cord gliomas and are usually fairly easily resected surgically. They find to respond to radiotherapy. Astrocytomas tend to occur in the cervical region and, in fact, may involve the entire cord. These tend to be less surgically resectable due to their infiltrative nature and they are also less responsive to radiation therapy. Either type of glioma tends to enhance with Gadolinium-DTPA and may be associated with cord cysts. These cysts may be subdivided into tumoral cysts (which show little CSF flow on CSF velocity imaging studies) or with cysts of the cord (e.g., syringes) which are glia-lined cystic cavities above or below the tumor.

When syringohydromyelia is identified within the cord, a Chiari I malformation, i.e., cerebellar tonsillar ectopia, should be sought. If the cerebellar tonsils are $3 \mathrm{~mm}$ or more below the foramen magnum, then the syrinx is presumed to represent congenital hydromyelia, i.e., dilatation of the central canal which is lined by ependyma. If a Chiari I malformation is not identified, Gadolinium is administered to exclude a syrinx associated with a tumor. Inflammatory conditions of the spinal cord can simulate tumors and produce myelopathy. Transverse myelitis is a monophasic illness, probably autoimmune in etiology, which generally recovers over a period of months. Multiple sclerosis of the cord is generally associated with little mass effect and with similar intracranial lesions involving the periventricular white matter. The clinical course is characterized by fluctuating lesions in space and time. Acute tumefactive MS, on the other hand, may enhance with Gadolinium-DTPA and may perfectly simulate a cord neoplasm. Occasionally, such lesions may not be associated with additional intracranial white matter abnormalities in which case biopsy may be necessary to distinguish it from tumor. If biopsy can be put off for a period of 6-12 weeks, acute tumefactive MS will generally resolve while the tumor will remain unchanged or grow.

\section{Degenerative discs}

M. Heller; Hamburg

Contribution available at the information desk.

\section{Bone lesions of the spine}

\section{K. Bohndorf; Aachen}

This course will provide an overview of the role MRI in the evaluation of spinal changes due to bone marrow disease, primary and secondary bone tumours, ischemic necrosis, infection and trauma. The signal pattern and information that MRI provides in each of these disease processes will be described.

The indication for the use of MRI, in comparison with CT, plain radiography and radionuclide studies will be discussed. Attention will be focused on the use of Gd-DTPA and technique of investigation. The value of contrast media and gradient-echo sequences, STIR and conventional SE-techniques will be demonstrated.

Case examples will be used to illustrate that MRI has become the imaging modality of choice for the evaluation of diffuse bone marrow disease of the spine, spondylitis and of those tumours of the spinal column encroaching on the spinal canal. However, the ability to predict the histologic nature of tumors and tumor-like lesions from MRI studies is only possible in certain cases, for example, in vertebral haemangioma. The possible distinction between neoplastic and inflammatory disease will be examined.

The normal conversion patterns of red (haematopoietic) to yellow (fatty) marrow in the axial skeleton from adolescence to adulthood will be discussed and, having established the appearance of healthy marrow on MRI, the appearance of marrow diseases will be considered. These will include infiltrative disorders of marrow, such of leukemia and multiple myeloma, and reconversion patterns of yellow to red marrow, as seen in anaemia and malignancies.

Trauma of the chest resulting from motor vehicle and work accidents are responsible for 150,000 deaths annually in the USA. Since Corean and Vietnamese wars, one knows that the outcome of the patient is directly related to the severity of the chest trauma, the delay time to admit the patient and the identification of the injuries.

New diagnostic modalities such as digital angiography, ultrasonography and computed tomography, dramatically changed the diagnostic approach and algorithms of the radiologist in the emergency room. Our purpose is to review the respective place and role of conventional and modern diagnostic procedures for the diagnosis of the traumatized chest wall, pleura, lungs, heart, diaphragm, tracheo-bronchial tree and the large thoracic vessels.

\section{Room C}

RC

1039 Diagnostic strategies in pancreatic disease Claus D. Claussen; Tübingen

Methods now available for evaluating the pancreas include endoscopic retrograde cholangiopancreatography (ERCP), angiography, ultrasound, computed tomography $(\mathrm{CT})$, and percutaneous fine-needle aspiration. The exact capability and usefulness of magnetic resonance imaging has yet to be defined. Clinicians are confronted with the problem of selecting the single most efficacious procedure or best sequence of imaging techniques for evaluating a patient with suspected pancreatic disease. None of the methods will provide all necessary information for every clinical situation. It is important to decide which particular problem is to be addressed before a rational choice of examination can be made.

Developmental disorders, i.e. pancreas divisum and annular pancreas, can be proofed only by ERCP.

Acute pancreatitis is primary a combined clinical and laboratory diagnosis. Imaging is necessary to define the extent of the inflammatory process, to detect complications, and to find the cause of the disease. When compared to CT, sonography is less precise in defining extrapancreatic fluid collections and parenchymal necrosis. ERCP is more and more accepted in early stage of the disease to ascertain a biliary origin. Fine-needle aspiration of pancreatic tissue or peripancreatic fluid collection can be used to detect the presence of infection.

Radiological diagnosis of chronic pancreatitis is based on changes in the pancreatic duct and parenchymal morphology and on the presence of ductal calculi. In advanced stages a significant correlation is found between clinical and morphologic disease severity in most cases, whereas the correlation is poor in patients with mild-to-moderate disease. The findings and complications of chronic pancreatitis can be detected by sonography, CT, ERCP, and angiography. ERCP is the most accurate method for diagnosing chronic pancreatitis. Subtile changes of the pancreatic duct and side branches are specific for the disease.

Significant advances have been made in the radiological diagnosis and staging of pancreatic carcinoma. Sonography and CT are the initial imaging modalities for evaluation of patients with suspected carcinoma. An important aspect of CT is determing tumor resectability. In nonspecific or equivocal findings adjunctive use of ERCP, angiography and percutaneous fine-needle biopsy may be valuable.

Cystic neoplasm of the pancreas are more frequently detected and surgically treated. CT can often differentiate microcystic adenoma from mucinous cystic tumors.

The most accurate combination for the preoperative detection of pancreatic islet-cell tumors is CT and angiography.

\section{Room E1}

\section{RG 907}

1040 Spinal trauma imaging

J. C. Dosch; Strasbourg

The three main spinal trauma imagings are: conventional plain film, CT and MRI.

- First of all the plain film examination. A minimum of two views in perpendicular plan is essential. Additional views and tomography can be used for going into more details. This first step permits to classify different situations: an evident lesion - luxation, fracture, dislocation - immediately needs a chirurgical therapeutic to avoid further spinal cord damage. An unstable lesion - 
severe sprain, tear drop fracture - needs a temporary immobilisation before further exploration. The lesion is unknown but one or more specific signs of injury, can be found.

- CT evaluation can be done in different manner: with and without contrast medium, after intravenous injection (in case of radicular pain), after intrathecal injection (in case of cord compression). CT reveals especially compression lesion, as well as pericanalar and intracanalar fragments. Hyperflexion lesions can be suspected.

- MRI exploration is particulary helpful in neurological spinal trauma without bone lesions. Cord compression, intramedullary lesions, ligamentous injuries are well documented by $\mathrm{T} 1$ and $\mathrm{T} 2$ weighted sequences.

We do not need all this explorations but each lesion has it own specific imaging procedure.

\section{Diagnosis of lumbar disc prolapse}

A. Chevrot, C. Vallee, F. L'Huillier, B. Rousselin, O. Richard, D. Godefroy; Paris

Lumbar disc prolapse is suspected when characteristic clinical signs appear: radiculalgias or sciaticas during or after raising effort.

Plain films datas remain uncertain: obvious reduction of the intervertebral height CT scan shows the bulging disc more or less far from this level. It allows to determine the direction of the hernia: posterior, postero-lateral, foraminal or extra foraminal. It can sometimes help to see if there is or not disruption of the posterior longitudinal ligament and if the herniation is extruded.

MRI gives comparable datas and moreover shows the subarachnoidian spaces and the conus medullaris.

Lumbar myelography remains a procedure of choice when intermittent herniation which could only appear in upright position.

Discography and CT discography can be used in case of uncertain diagnosis of recurrence after surgery.

\section{Room E2}

\section{RC}

1042 Initial imaging strategies in spinal trauma patients

Erkki M. Laasonen; Helsinki

Patients with spinal trauma may come to a local hospital or health center at any time. Let us follow such a patient during his first 24 hours and work out how the often insufficient radiological resources should be optimally used.

The facilities at a local hospital are very limited; a physician on duty, conventional $\mathrm{x}$-ray room, and at best, a general radiologist. The more severely injured patients cannot be treated optimally and have to be sent on to a specialised Trauma Center. Two problems arise; how to select the right patient to send and how to avoid further neurological deterioration? The medical team in the local hospital has to answer following:

- did the patient arrive in walking, sitting, or lying on a stretcher?

- are they alert/unconscious, cooperative/uncooperative?

- do they have neurological symptoms referrable to the spine?

- do they have localising pain in the spine?

On this basis a simplified algorithm can be built up suggesting which plain films should be taken when. No tomography, CT, or MRIs are recommended here. The capacity of the algorithm to cope with the above mentioned problems is also analysed.

Even with prompt transfer to a Trauma Center, some six hours will already have passed. Now the fate of the poor patient will depend tremendously on the policies of the Trauma Center, as well as the radiological facilities available around the clock. We know that cortico-steroid therapy started within eight hours of spinal trauma improves the chances of recovery of neurological deficit, but that does not require any radiology, just a skillful neurological assessment. Stabilising and/or decompressive operations may be done as an emergency but opinions differ as to whether these facilitate the patient's recovery. Do we get MRI immediately and if so, is the prognostic information obtained necessary now? Amidst these controversial issues, definite algorithms cannot be given. Nevertheless, opinions on the use of radiological procedures within the first 24 hours in a Trauma Center are presented, bearing in mind the eventual neurological deterioration. Only relevant radiological information that significantly affects treatment or prognosis of our poor patient should be sought. Perfectionism should be reserved for scientific projects.

\section{3,1044 Imaging in the management of patients with spinal injuries} H. Imhof and R. N. Bodley; Vienna, Stoke Mandeville

Despite the recent dramatic revolution in imaging procedures, in the vast majority of cases, initial evaluation is by plain radiography. Modern equipment allows images to be taken in all possible directions without moving the patient. The first and most important radiograph is still the lateral view. This should be reviewed before further evaluation as it has been reported to be positive in $70-90 \%$ of cases. Additional views (cervical spine: AP, open mouth and obliques; thoracic and lumbar spine: AP) are obtained if no other life threatening disorders demand urgent treatment. Flexion and extension views of the cervical spine are reserved for patients with abnormal alignment and should be done only under the guidance of an experienced physician.

Depending on these first imaging steps and the clinical (neurological) status of the patient, either further conventional radiography, CT or MRI can be performed. Conventional tomography supplements CT in the diagnosis of axially oriented fractures, facet injuries and multilevel trauma but has the disadvantage that the patient must change position for lateral examinations. The CT examination, which is vital for assessing the spinal canal patency and encroachment by osseous fragments, must be adequate for surgical planning and for evaluation of associated soft tissue injuries, both local and distant. MRI is an exciting new development as intervertebral discs, ligamentous injuries, haematomas and spinal cord damage are all excellently visualised and may influence acute management. With the advent of MRI the use of myelography (in combination with CT) is now limited to cases which cannot be evaluated by MRI, such as nerve root avulsions or suspected dural tears.

Patients with spinal injuries need regular monitoring to pre-empt complications. As well as the complications common to all severely injured patients (infection, haemorrhage etc) early problems may occur with spinal stability (for which repeat $\mathrm{CT}$ and MR evaluation may be required), ectopic ossification (generally in the thighs and which required differentiation from deep venous thrombosis) and renal tract complications related to long term catheterisation and the loss of normal bladder control.

Later complications requiring investigation relate to spinal instability, muscle spasms and pressure sores but particularly to spinal cord changes (MRI or CT myelography is needed to evaluate atrophy or syringomyelia) and to the renal tract. Ideally, annual review with ultrasound and KUB or intravenous urography is instituted so complications such as reflux, stones or obstruction may be diagnosed before irreparable damage has occurred.

\section{Room L}

\section{WS}

\section{8}

$1045,1046,1047$ Modern imaging of the orbit - respective roles of ultrasonography $\mathrm{CT}$ scanner and magnetic resonance imaging O. Berges, J. Vignaud, G. Wilms; Paris, Leuven

Ultrasonography (US), CT Scanner (CT) and Magnetic Resonance Imaging (MRI) are the three imaging modalities to explore ocular and orbital disorders. Ultrasonography is non invasive, efficient, reliable and inexpensive and gives dynamic, real time information. However US is examiner dependent. Recently, Colour Doppler Flow Imaging (CDFI) has added useful information with regards to the vascularization of ocular and orbital lesions. CT is an excellent method to analyze orbital space occupying lesions (S.O.L.) due to its excellent spatial resolution and since it gives the best analysis of the walls of the orbit. New programs, reformated images and $3 \mathrm{D}$ reconstructions are of great help in some circumstances. MRI of the orbit is interesting since it does not require iodine contrast medium, it does not deliver irradiation and allows to obtain sagittal oblique sections along the great axis of the orbit. The superficial situation of the orbit allows the use of surface coils that give a good spatial resolution.

OCULAR LESIONS: The main role of US of the globe is to measure the axial length of the eyeball to allow the calculation of the intra ocular lens in case of cataract surgery and to evaluate the fundus in case of opaque media preventing ophthalmoscopy. US, with the help of CDFI, also is the method of choice for the pretherapeutic evaluation of intra ocular tumors (mainly retinoblastoma in children and melanoma in the adult) and for the follow-up of these lesions in case of conservative treatment. CT is mainly indicated to detect an extra ocular extension in case of retinoblastoma and in the study of microphthalmos. MRI is useful only to study the posterior segment of the eyeball after vitreous retinal surgery when the eyeball is filled with gaz or silicone oil.

ORBITAL LESIONS: One should always prefer to start with US. For the anterior (palpable) lesions, inflammatory pseudotumors, Graves' disease, varices, optic nerve sheath meningioma and for the lesions with intra- and extra-orbital compartments, CT, after US, is the examination of choice. In case of typical clinical findings in favor of Graves' disease or varices, CT may be performed directly without the ultrasonic step. At present, the only indication of orbital phlebography are the anteriorly located venous malformations that have to be surgically removed since recurrence of the retrobulbar ones is constant. For pure intraconal lesions, optic nerve glioma and for the lesions situated near the orbital floor and the orbital roof, MRI is usefully associated to US for it gives a good tissular characterization approach, it better analyzes the relationship of a SOL with the normal orbital structures and is able to perform oblique sagittal sections. For lesions located at the orbital apex and the ones with intra- and extra-orbital compartments, MRI is useful after CT, especially with the use of Gadolinium. At the present time, arteriography is only indicated in case of high flow carotid cavernous fistulae to allow embolization. 


\section{Room M}

\section{RC 905}

\section{Interventional uroradiology update}

M. J. Kellett; London

This update will cover interventional procedures in the urinary tract involving percutaneous puncture of the kidney. The method of percutaneous needle nephrostomy and its applications including percutaneous nephrolithotomy (PCNL) and percutaneous pyelolysis will be discussed. In addition the results of percutaneous resection of low grade transitional cell tumours will be covered.

Extracorporeal shock wave lithotripsy (ESWL) is now the treatment of choice for small intrarenal calculi. The place of percutaneous nephrolithotomy will be discussed and in particular cases where extracorporeal shock wave lithotripsy is not indicated will be covered. New generation lithotriptor can treat renal calculi without any anaesthesia or analgesia and their role in the current management of calculus disease will be discussed. Large staghorn calculi are best treated by debulking PCNL to remove the central mass of the stone and any calyceal fragments that are easily accessible. A double $\mathrm{J}$ stent is usually inserted in order to treat the remaining calculi by ESWL. The role of ureteric stenting will be discussed in the treatment of urinary calculi.

An acute stricture in the ureter may be successfully dilated via a percutaneous track. The results with dilating chronic strictures more than three months standing are less good but it may be worth attempting these before reverting to open surgery. The method of stricture dilatation and insertion of antegrade double $\mathrm{J}$ stents will be discussed both for benign and malignant obstructions. Conclusions: The role of the radiologist and that of the urologist are rapidly changing. Interventional radiological techniques using percutaneous tracks are at the moment under external $x$-rays or ultrasound guidance but real-time intraluminal ultrasound may lead us closer to the actual target of interest. Urological endoscopy has lead to the development of smaller more versatile endoscopes which can carry energy through smaller channels. The two disciplines of interventional radiology and endourology are beginning to overlap in the speciality of minimally invasive therapy.

\section{Room N}

\section{WS}

\section{2}

\section{Arterial stents}

J. Triller, F. Mahler, D. Do; Bern

G. Nöldge; Freiburg, E.P. Strecker; Karlsruhe

The aim of this project is to evaluate the efficiency of the vascular endoprosthesis in arterial occlusion disease in comparison to the percutaneous transluminal angioplasty (PTA).

Between July 1987 and April 1991 we implanted 78 vascular endoprostheses in 53 patients: femoro-popliteal artery 64 , iliac artery 7 , abdominal aorta 3 , renal artery 3 , subclavian artery 1 . In 74 cases a Wallstent was used, in 4 cases a Strecker stent was implanted.

In 26 patients with extensive femoro-popliteal occlusions an endoprosthesis was primarily implanted in 45 cases. The long-term results of this study were compared with another group of patients which were treated exclusively with PTA. - In a further 27 patients a stent was secondarily implanted in 30 cases in the femoro-popliteal artery, the iliac artery, the subclavian artery and the abdominal aorta. The indication was an unsuccessful result after PTA (dissection) or a recurrence with a balloon resistent stenosis.

The results of the femoro-popliteal stent implantation study showed, that the vascular endoprosthesis is not more efficient in the treatment of extensive femoro-popliteal vascular occlusions in comparison with normal treatment using PTA alone. The patients treated with endoprostheses showed a higher rate of early recurrency, which required an additional catheter thrombolysis or an aspiration thrombembolectomy. Due to neointimal hyperplasia a stenosis occurred relatively early, which required a further recanalisation with PTA The patency-rate after 18 months was approximately $50 \%$ and comparable with PTA.

The indication for a stent is basically to discuss, if after an initial PTA an unsatisfactory result due to dissection exists and an occlusion of the artery should be prevented. With the presence of a recurrence a vascular endoprosthesis is indicated if after a following PTA a balloon resistent stenosis still exists.

\section{Room O}

RE

\section{5}

1050 Color Doppler: abdomen

Michel Lafortune; Montreal, Quebec

The colored display of doppler shifts arising from vessels (and other moving structures) of the abdomen allows the creation of a non invasive "angiogram". Physiological information about flow characteristics can then be added by examining the spectral display of the doppler shifts at varying points of interest.

Increased sensitivity to slow flow and improvements in spatial resolution of the image are recent technical improvements. Color doppler has now become an invaluable adjunct to the sonographic examination of the abdomen.

I shall focus on the investigation of the patient with liver or kidney disease. The diagnosis of portal hypertension is treatly facilitated, as portosystemic collateral routes are readily outlined, and flow direction and changes in flow velocity monitored as the disease progresses. This is done in an entirely physiological way. Flow in the intrahepatic vessels is readily outlined: Thrombosis tumor invasion or arterio-venous fistulae can be identified. Surgical portosystemic shunts and their repercussions on intrahepatic flow are readily assessed, as are vascular complications (chiefly thrombosis of the hepatic artery or portal vein) following hepatic transplantation. The assessment of liver transplant rejection with doppler has been disappointing to date.

Vascularisation of the kidney, especially following trauma, shock, surgery or transplantation is readily evaluated with color doppler. The color image is an excellent guide to placing sample volumes for analysis of the doppler shifts arising from the segmental arteries. These can then be used to assess vascular rejection or other causes of high vascular resistance such as vasculitis, edema or compression of the renal parenchyma. Serial examinations are possible and especially valuable for monitoring the progress of the disease.

Percutaneous intervention in the abdomen is facilitated by the use of color doppler; vascular aneurysm are distinguished from other fluid collections. Multiloculated abscesses are more easily drained (by showing movement of PUS). Biopsy is facilitated by easier visualisation of needle tip and identification of nearby vascular structures.

\section{Peripheral vessels}

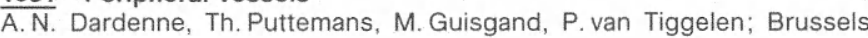

Unlike arteriography, which yields only anatomic information, and the indirect noninvasive vascular procedures, which reflect quantitative hemodynamic measurement, duplex scanning is unique in combining both anatomic and physiologic data.

Extracranial arterial disease: Duplex scanning was first applied to the study of the carotid artery in the neck. This unusual bifurcation, with the dilatation a the level of the proximal internal carotid artery, is a very common site for the development of atherosclerosis. Ultrasonic techniques are well adapted for monitoring patients with high vascular risk.

Duplex Doppler precisely shows stenoses and plaques and demonstrates their hemodynamic changes. Furthermore it is advantageous from a cost-benefit point of view, as it spares many angiographic procedures.

Moreover, intracranial arteries are now accessible by low frequency pulsed Doppler.

The problem of operator dependence will be lessened by the availability of color-modulated imaging.

Peripheral arterial system: There are important differences between disease in the carotid system and arterial disease in the lower extremity in which, due to the diffuse availability of collateral circulation, severe disease is frequently tolerated well with relatively few symptoms.

The indications for duplex scanning are essentially the same as for arteriography.

As in the carotid area the quality of information obtained from duplex ultrasonography of the lower extremity arterial system is highly operator dependent but is also obtained easier with a color modulated imaging.

Peripheral veins: Undiagnosed deep venous thrombosis (DTV) is thought to be responsible for the majority of pulmonary embolism.

Newly developed real-time Doppler color flow imaging (DCFI) permits visual identification of nonocluding intraluminal thrombus. The major benefits of DCFI, is the ease and rapidity in which the lower extremity veins can be evaluated, including not only the femoral and popliteal system, but also the major calf veins.

\section{Color-coded Duplex sonography of the breast, thyroid and genitals \\ Franz Fobbe; Berlin}

Color-coded duplex sonography (CCDS) allows the simultaneous display of soft tissue and blood movement over the entire scan field. Blood movement is visualized even in vessels too small to be seen in the gray scale image. CCDS thus permits for the first time the detection of the blood supply in small parenchymal organs both non-invasively and in real-time. 
The clinical application of this new method for examining the breast, thyroid and the genitals will be discussed. In patients with goiter, the vascularization allows the differentiation between a simple and a nodular goiter on the one hand and a goiter with an autonomous adenoma or a carcinoma on the other. CCDS makes it possible to differentiate reliably between a torsion of the testis and an acute inflammation in patients with an "acute scrotum" thus avoiding diagnostic surgery. Varicocels can be diagnosed in a matter of minutes by demonstrating the retrograde blood movement in the pampiniform plexus during a Valsalva's maneuver. The available data indicate that demonstration of the vascularization of breast tumors does not allow a differentiation between malignant and benign ones.

CCDS is the method of choice in patients with an "acute scrotum" and for the diagnosis of varicoceles. Demonstration of the blood movement permits the exclusion of an autonomous adenoma or a carcinoma in goiter patients. Further differentiation of breast tumors on the basis of the vascularization does not seem to be possible.

\section{Room R}

RG

916

1053 Combined modality in the treatment of rectal cancer L. Cionini; Florence

Local recurrence (LR) rate after apparently radical resection in carcinoma of the rectum ranges from $15 \%$ to $40 \%$; the major factor affecting probability of LR is the extent of tumor infiltration at the time of surgery: when the muscolaris propria is not penetrated the risk of LR is negligible, but when tumor infiltrates the muscolar layer or extends into the perirectal tissues, the probability of LR raises to $30 \%-50 \%$. A large number of patients developing a LR also have concomitant or subsequent distant metastases; the presence of involved nodes in the operative specimen carries the highest risk to develop both local and distant failures. LRs are rarely curable when they became clinically evident and their occurrence always produces heavy subjective symptoms affecting quality of life; therapeutic efforts must therefore be addressed to prevent their occurrence trying to destroy viable cells left in the surgical bed. Radiotherapy (RT) has been largely used to this purpose both pre, post and intraoperatively; in the more recent years radiations have also been used in combination with 5-Fluoruracil (5FU). The comparison of the large number of studies reported in the literature is very difficult: different criteria were in fact used in the selection of patients and in the evaluation of the LR rate; radiation doses and fractionation regimens varied to a large extent particularly in the preoperative options; the follow time and the follow up procedures were very different. A definitive agreement on the value of adjuvant RT in cancer of the rectum and on its optimum modality is therefore not yet achieved. Based on the most recent randomized trials some conclusions can however be drawn. Both pre and post operative RT demonstrated to significantly improve the LR rate but not the survival. Preoperative RT to be effective requires a dose not below $40 \mathrm{~Gy}$ in 20 fractions in 4 weeks (or a biologically equivalent regimen); its main advantage is the excellent tolerance; the difficulty of selecting the patient at high risk of LR and the interference of downstaging in the evaluation of results are the main disadvantages. In postoperative RT the radiation dose is usually $45-50$ Gy in 25 fractions in 5 weeks; the proper selection of patients at higher risk of LR based on the pathological data represents the main advantage; the complication rate is however not negligible due to the larger amount of small bowel included in the treated volume after the surgical removal of rectum. The combination of radiations with 5FU given concomitantly proved in some recent randomized trials to improve both LR rate and overall survival; this treatment modality is therefore becoming more and more popular as the standard adjuvant treatment modality in cancer of the rectum. The results of the reported trials need however further observation to reconfirm the advantage and to clarify its mechanism; it is in fact unclear yet whether $5 \mathrm{FU}$ works because of its radiosensitising effect carrying a further reduction of LR rate or as a systemic agent decreasing the distant metastases rate. 


\section{Room A}

\section{SS 11.02 Joints I}

$10.30 \mathrm{AM}$

\section{Articular cartilage defect detectability in human knees with MR-arthrography}

A. Engel, J. Kramer, R. Stiglbauer, P. C. Hajek, H. Imhof; Vienna

Purpose: To demonstrate the cartilage defects, their extent and location. Methods: 113 knees of 112 patients were examined by MR arthrography (MRA). MRI was performed on a $1.5 \mathrm{~T}$ unit using surface coils. Standard T1-weighted spinecho sequences (SE) and T2-weighted gradient echo sequences (GE) were obtained in sagittal and axial slice orientations. $40 \mathrm{ml}$ of a $2 \mathrm{mmol}$ Gd-DTPA solution were injected intraarticularly. The MR description of the surface of articular cartilage defects was classified according to Outerbridge (I: normal cartilage surface and thickness; II: surface normal or slightly irregular [focal/diffuse]; III: severe surface irregularities and cartilage defects [focal diffuse]; IV: extensive cartilage defects, scar formation [fibrous cartilage]). MR-findings were correlated to arthroscopy and arthrotomy ( $\mathrm{n}=$ 28).

Results: Out of 113 knee joints 48 showed cartilage defects (medial/lateral femoral condyle: 19/4; patella: 34). Findings were distributed as follows: grade I-II $: \mathrm{n}=3 ; \mathrm{II}: \mathrm{n}=20 ; \mathrm{II}-\mathrm{HII}: \mathrm{n}=2 ; \mathrm{III}: \mathrm{n}=15 ; \mathrm{III}-\mathrm{IV}: \mathrm{n}=10 ;$ IV $: \mathrm{n}$ $=6$. In $98 \%$ MR-imaging findings correlated exactly with arthroscopy/arthrotomy.

Conclusions: MRA appears to be the method of choice for the evaluation of unspecific complaints of the knee joint. An exact assessment of the hyaline cartilage is possible and is therefore important for therapeutic decisions.

\subsection{AM}

\section{Contrast-enhanced dynamic MR imaging in rheumatoid arthritis: identification and follow-up of hypervascular pannus after cortisol therapy}

H. König, J. Sieper, K.-J.Wolf; Berlin

Purpose: To identify and localise pannus tissue and to quantify the effects of cortisol drug therapy by MRI.

Patients and methods: 18 patients with rheumatoid arthritis (RA) of the knee joints were examined with MRI primary 2 and 8 weeks after intraarticular cortisol drug therapy ( $25 \mathrm{mg}$ prednisolone). $0.1 \mathrm{mmol} / \mathrm{kg}$ of Gd-DTPA given as a bolus injection was followed by a fast low-angle shot (FLASH) sequence (TR 30 , TE $10 \mathrm{msec}$, excitation angle $70^{\circ}$ ) repeated 15 times within 120 seconds. Primary to contrast a double echo sequence (TR 1.600 , TE $22+110 \mathrm{msec}$ ) was applied. Enhancement of pannus and pannus-like structures has been measured as signal-intensity time curves.

Results: Hypervascular pannus showed a fast and significant enhancement compared to other soft tissues and compared to synovial fluid. 2 weeks after therapy a reduction of the initial enhancement could be noted in 12 patients but no reduction of pannus masses. 8 weeks after therapy a further reduction of the initial enhancement could be seen in 5 patients combined with a reduction of pannus masses.

Conclusions: Using postcontrast dynamic MRI hypervascular pannus can be identified and cortisol drug therapy effects on pannus vascularity can be monitored.

\subsection{AM}

\section{MR evaluation of bony bridges after growth plate injuries} J. Lizler, P. Havranek, J. Tintera; Praha

Bony bridge may develop as a complication of an injury interrupting the line of the epiphyseal growth plate. Several surgical methods of its treatment have been developed, all of them requiring an exact delineation of the extent and shape of the bridge. Plain radiograms and tomograms often do not allow to determine this information exactly and CT is also limited in this indication. MR images parallel to the long axis of the bone reveal well the site of the plate disruption together with the confirmation of the bone bridge presence using T1 weighted and FLASH sequences, the latter displaying clearly the cartilage itself together with its lesion.

A group of seven children aged between nine and twelve with the clinical and radiological signs of bony bridge occurrence was examined. Four of them had surgery, with MR findings being crucial for the operation planning. In the post-operative evaluation of one of the patients we obtained exact information about the position of the acrylic interpositum replacing the resected bony

bridge.

MRI is a method providing confirmation, exact quantification and delineation of a bony bridge occurring after epiphyseal growth plate injury and may help both operation planning and post-operative evaluation.

11.00 AM

\section{MR arthrography of the knee}

J. Kramer, A. Engel, R. Stiglbauer, P. C. Hajek, H. Imhof; Vienna

Purpose: To demonstrate the diagnostic capabilities of MR arthrography in the first 100 routine clinical cases.

Methods and Materials: 100 patients with intraarticular knee problems (chondromalacia, osteochondritis dissecans (OD), meniscus lesions, synovitis, cruciate ligament tears) were included in this study and diagnosis was proved by arthroscopy or arthrotomy $(\mathrm{n}=68)$. MR imaging was performed on a $1.5 \mathrm{~T}$ system using surface coils. 2D-spinecho (SE) and $3 \mathrm{D}$-gradient echo sequences (GE) following intraarticular injection of $40 \mathrm{ccm}$ of $2 \mathrm{mmol}$ gadolinium-DTPA were obtained. The first 75 cases were also imaged without intraarticular contrast agent.

Results: MR arthrography allowed for a superior imaging quality compared to plain MR. This resulted in a far better delineation of intraarticular structures, especially of cartilage defects (condyl and patella preinjection T1:24, GE: 36 , post injection T1:67, GE: 79), synovitis (pre T1:2, GE:6; post T1:27, GE: 27), plica syndrome (pre T1/GE: 0 , post T1/GE: 16) and exact staging of OD (improvement due to contrast injection in 43\%). 3D gradient echo imaging was found to be the most efficient sequence technique. Sensitivity and specificity as well as overall accuracy were $100 \%$ for cartilage defects (grade II-IV according to Outerbridge).

Conclusions: MR arthrography is superior to conventional MR imaging:

1. Sensitivity and specificity are equal to arthroscopy.

2. Good assessment of details is relevant for adequate therapy planning.

3. The method is excellently suited for cartilage imaging.

\section{$11.10 \mathrm{AM}$}

1058 Improved MR imaging evaluation of chondromalacia patellae using a device for cartilage compression

H. König, F. Dinkelaker, K.-J. Wolf; Berlin

Purpose: More precise evaluation of chondromalacia patellae by means of MR imaging performed with a specially constructed device for compression of the retropatellar cartilage.

Patients and methods: Two volunteers and 18 patients were examined 1-4 weeks before arthroscopy and cartilage biopsy. Imaging parameters included spin-echo (SE) $(1.600 / 22+110 \mathrm{msec})$ and fast low-angle shot (FLASH) $\left(50 / 10 \mathrm{msec}, 10^{\circ}\right.$ excitation angle) as well as fast imaging with steady precession (FISP) $\left(50 / 10 \mathrm{msec}, 90^{\circ}\right.$ excitation angle) sequences, $4 \mathrm{~mm}$ section thickness, and sagittal and axial views. FLASH and FISP measurements were done without and with compression. Cartilage thickness and signal intensities were measured at 3 different points of the retropatellar facet.

Results: Cartilage thickness showed no significant reduction in stage 0 , III and IV, whereas in stage I and II reductions of up to $25 \%$ could be measured. Signal intensities showed no significant changes in stage 0 and III, in stage I and II an increase of signal intensity could be seen whereas in stage IV a reduction of signal intensities could be noted.

Conclusions: The compression method seems to be useful in stages I and II of chondromalacia patellae.

\subsection{AM}

\section{MR imaging scaphoid pseudarthrosis using Gd-DTPA}

H. Schedel, Th. Vogl, K. Wilhelm, L. Ziegler, D. Hahn, J. Lissner; Munich

Purpose: to detect vitality of bony fragments in patients with scaphoid pseudarthrosis especially using Gd-DTPA.

Methods: 45 patients with scaphoid pseudarthrosis were examined with a $1.5 \mathrm{~T}$ Siemens Magnetom using coronal T2- and T1-weighted images before and after application of Gd-DTPA.

Results: 31 patients showed signal loss on plain images mostly in the proximal part of the scaphoid. In 10 patients signal intensity raised after application of Gd-DTPA meaning vital bony structures. In 4 cases interpretation was limited by artifacts caused by metallic implantation material.

Conclusions: MR imaging using Gd-DTPA is the most accurate method to answer the question of vitality in scaphoid pseudarthrosis thus influencing the therapeutic strategy. 
1060 MR imaging of sacroiliitis using Gd-DTPA

L. Ziegler, S. Spaethling, H. Kellner, H. Schedel, Th. Vogl, D. Hahn; Munich

Purpose: Evaluation of Gd-DTPA enhanced MR imaging of sacroiliitis caused by spondylitis ankylosans, Lyme disease, Reiter syndrome and arthritis psoriatica.

Methods: 25 patients with suspected sacroiliitis were examined with $\mathrm{T} 1$ and $\mathrm{T} 2$ weighted SE- and GRE sequences (Flash $40^{\circ}$ flip angle) and T1 weighted examinations after intravenous application of Gd-DTPA. Images were obtained with a 1.5-T Siemens Magnetom in transverse and coronal planes. The MR findings were compared with plain film examinations and correlated with the clinical outcome.

Results: We found normal sacroiliac joints in 11 patients, in 6 patients we diagnosed an acute sacroiliitis, in 7 patients changes of the joint space, fatty degeneration of spongiosa and sclerosis and in 1 patient a complete ancylosis. Changes of the joint space were best delineated by gradient echo, sclerosis and fatty degeneration by native TI weighted sequences. Diagnosis of an acute inflammation was easily possible by visual analysis of a marked increase of signal intensity after contrast media application. Measurable abnormalities, not clearly seen on plain radiographs, were demonstrated by MR imaging.

Conclusions: Gd-DTPA enhanced MR imaging is the method of choice in the assessment of an acute or old inflammatory process and improves diagnosis and subsequent treatment of sacroiliitis

\section{$11.40 \mathrm{AM}$}

1061 MRI guided aspiration fine needle biopsy: feasibility with a high field $(1.5 \mathrm{~T})$ magnet

J.D. Tesoro-Tess, L. Balzarini, E. Ceglia, R. Petrillo, Y. Reyner,

R. Musumeci; Milano

1. CT guided aspiration cytology is a valuable tool for oncological diagnosis. The standard needles used for CT-guided biopsy are unsuitable for MR-guided biopsy because ferromagnetic artifacts obscure the underlying anatomy.

2. The availability of a new needle specifically designed for use with MRI permitted to performe MR-guided aspiration biopsies in a group of 17 patients with different neoplastic diseases. To localize the site and the depth of the lesion a glass pipette containing a diluted solution of paramagnetic contrast medium (GD-DTPA) was placed on the skin surface.

3. In 13 out of 17 patients ( $76.5 \%)$ biopsy was successful and histological and/or cytological diagnosis was achieved. In spite of a longer measurement time T1-weighted SE images demonstrated the best quality image/artifact dimension ratio. On the contrary FAST-scan images (FLASH $15^{\circ}$ to $90^{\circ}$ ) with or without breath holding were very short time consuming $(7 \mathrm{sec}$.) but poorly useful mainly in small lesions frequently obscured by artifacts.

\section{$11.50 \mathrm{AM}$}

1062 Precontrast and postcontrast (Gd-DTPA) MR imaging of hand joints in patients with rheumatoid arthritis

V. Jevtić, B. Rozman, M. Kos-Golja, O. Jarh, F. Demšar; Ljubljana

In patients with rheumatoid arthritis the estimation of activity is difficult on the basis of conventional radiologic procedures. MRI, owing to its high contrast resolution, enables clear noninvasive presentation of inflammed para and intra articular soft tissue structures. Standard spin-echo sequence, especially $\mathrm{T} 2$ weighted images are sensitive enough to present accumulation of fluid (edema, effusion), which usually corresponds with clinically more active disease. However, the basis for the beginning and evaluation of therapy is prove of acute synovial inflammation. The signal intensity from actively inflammed sinovium and from synovial fiuid in joint effusion or in an inflammatory pseudocyst is similar or the same on $\mathrm{T} 1$ and $\mathrm{T} 2$ weighted images. On the other side actively inflammed sinovium is hypervascular and pathologically permeable enabling accumulation of intravenousely administrated gadopentetic acid (Gd-DTPA).

In our prospective study 50 patients with clinically estimated active initial or advanced excerbated rheumatoid arthritis are investigated by means of conventional radiography and MRI. In all the patients standard a-p hand and wrist radiographs are taken. The next day MRI is performed on a 2.35 T Bruker scanner. A spin echo sequence with T1W precontrast images (TR $603 \mathrm{msec}$, TE $30 \mathrm{msec}$ ), TIW postcontrast images (iv. administration of Gd-DTPA, $0,1 \mathrm{mmol} / \mathrm{kg}$ body weight), and T2W images (TR 2045, TE $90 \mathrm{msec}$ ) is used. A field of view is $12 \mathrm{~cm}$ with data acquisition matrix of $256 \times 256$. The images are performed in coronal plane ( $3 \mathrm{~mm}$ thick consecutive slices). The purpose of our study is to evaluate the diagnostic efficacy of TIW precontrast, TIW postcontrast and T2W MRI in proving active synovial inflammation in early and excerbated rheumatoid arthritis.
1063 MRI of the operated anterior cruciate ligament M. Sens, G. Bachmann, W. Rau; Giessen

We investigated patients with a tear of the anterior cruciate ligament of the knee which were treated with an implantation of the tendon of the semitendinosus muscle. We performed a first examination right after the operation and follow-up examinations at one, three, six and twelve months after surgery. We looked especially for the time course of the signal intensity of the operated ligament. Immediately after the operation we saw the normal dark signal which increased in the following and was nearly isointense with the surrounding tissues at 6 months. During this stage it was very difficult to estimate whether the ligament was intact or not. In the following 6 months the signal intensity of the ligament nearly returned to normal and had a dark appearance at one year after surgery. The increase in signal intensity of the operated anterior ligament was seen in every patient and seems to reflect the normal healing process, so that one can judge a delay of this process if a different as the expected signal intensity is encountered in a special patient.

\section{Room B}

\section{SS}

$10.30 \mathrm{AM}$

1064 Eosinophilic lung disease (Keynote Lecture) A. G. Wilson; London

The term eosinophilic lung disease includes those disorders associated with blood and/or tissue eosinophilia in which the major airways and/or lung are affected. It is a more comprehensive term than pulmonary eosinophilia or pulmonary infiltration with eosinophilia (P.I.E. syndrome).

Eosinophilic lung disease can be usefully subdivided into three subgroups: (i) pulmonary conditions in which tissue and/or blood eosinophilia is pathogenetically important; (ii) pulmonary conditions in which blood and/or tissue eosinophilia is minor or occasional and (iii) pulmonary conditions with coincidental blood eosinophilia.

This review is mainly concerned with conditions in the first group and a classification scheme is presented based on their pathogenesis and pathology. The main divisions of such a classification are those of eosinophilic bronchitis, eosinophilic pneumonia and vasculitic/granulomatous disorders. It is possible using such a scheme to understand and predict the important imaging findings in the major forms of eosinophilic lung disease.

\section{$10.45 \mathrm{AM}$}

1065 Comparison between conventional radiography and storage phosphors digital radiography in interstitial pulmonary diseases S. Sartoni Galloni, M. Burzi, P. Burci, R. Stamati, M. Miceli; Bologna

Purpose: A comparative study between conventional radiology (CR) and digital radiology (DR) was performed in order to evaluate the diagnostic possibilities of a storage phosphors DR system (Philips PCR Graphics 1) in interstitial pulmonary diseases.

Methods: 80 patients with AIDS underwent at the same time CR examination (Kodak T-Mat G film) and DR examination (Fuji II St. imaging plate) with the same technical data ( $140 \mathrm{kV}$ with automatic exposure)

Imaging plates (IP) were read by high intensity scanning laser beam; digital images obtained were processed using "standard chest" software and recorded on hard copy (Fuji CR 633 film).

Presence or absence of interstitial pathology was evaluated performing as gold standard mainly HRCT examination or Ga 67 scintigraphy, BAL and eventually biopsy, shortly after plain CR and DR.

Four radiologists observed at random $\mathrm{CR}$ and DR examinations, ignoring clinical data about patients, and they indicated their level of confidence (1-4) about presence or absence of interstitial disease.

Results: No statistically significant difference was observed between CR and $\mathrm{DR}$ in this experience using a ROC analysis $(\mathrm{CR} \mathrm{Az}=0.84, \mathrm{DR} \mathrm{Az}=0.82 ; \mathrm{p}$ $=0.4$ ).

Conclusions: The results suggest that the diagnostic accuracy of CR and DR are substantially the same in the detection of interstitial pulmonary pathology. At present lower spatial resolution of this storage phosphors DR system $(2.5 \mathrm{lp} / \mathrm{mm}$ for $35 \times 43 \mathrm{~cm}$ IP) can be reasonably counterbalanced by its higher contrast resolution (10 bits) and by its dynamic range and spatial filtering postprocessing possibilities on display. 
1066 The lung in the SLE: analysis of 104 cases with special attention to the pulmonary hemorrhage induced lupus and its pathogenesis J.Fernandez Cruz, S. Gomez, P. Escobar, A. Glez, M. A. Glez Guirao, A. Martinez, J. M. Varela, J. Sanchez Roman; Sevilla

Pulmonary disease is a recognized complication of Systemic Lupus Erythematosus (SLE) and occurs in as many as $50-70 \%$ of patients during the course of their illnes. The most commun pulmonary complications are pleuritis and interstitial pneumonitis. Other complications are nonspecific - and include infection, thrombosis and hyaline membrane formation.

Pulmonary Hemorrhage (PH) in SLE has been recognized since 1904 when Osler described a 24-year-old-woman with nephritis, hemoptysis, and bilateral pulmonary infiltrates.

We have studied 104 patients with SLE, studying their lung alterations, specially the $\mathrm{PH}$, the pathogenesis, clinical, evolution, radiological manifestations and treatment.

Our patients had a mean age of 38.1 years and the rate $\mathrm{M} / \mathrm{F}$ was 6.8 years. Forty-one patients had pulmonary disease: Unilateral effusion pleural 16, Bilatera E.P. 11, Lupus pneumonitis 4, pleuritis 3, Alveolar Hemorrhage 4, and pulmonary thromboembolism 1 .

The coagulation profile was normal in 2 and anormal 2 cases.

The PH is a rare, but often fatal complication of SLE. Clinical findings and radiological manifestations of $\mathrm{PH}$ make difficult the differential diagnosis with other pulmonary complications and invasive diagnostic procedure frequently are required. The fast response of the steroids supports the diagnosis of this rare complication.

\section{$11.05 \mathrm{AM}$}

1067 Pulmonary lymphoproliferative disorders with affinity to lymphoma: a clinic-phato-radiologic study of 16 cases J. Fernandez Cruz, A. Glez. Garcia, S. Gomez Benitez, M. A. Glez. Guirao, P. Escobar Casas, F. Borderas Naranjo; Sevilla

Pulmonary Lymphoproliferative Disorders (PLD) include plasma-cells granuloma, Castleman's disease, Pseudolymphoma, Lymphocytic interstitial pneumonitis, Angioimmunoblastic Lymphadenopathy and Lymphomatoid Granulomatosis. They have morphologic features of an immunologic response and are thought to represent a hyperplasia of the pulmonary lymphoid system in response to chronic antigenic stimulation. The point in pathogenesis of malignant lymphoma from some of these "benign" lymphoid disorders, is often unclear.

We study 16 cases distributed as they follow: 8 LIP, 5 LYG, 2 Plasma cells granuloma (Lung's Histiocytoma) and 1 Angioimmunoblastic Lymphadenopathy. These cases have been survived in the past 10 years from which, 1 LIP and 3 LYG degenerated into Non-Hodgkin's Lymphoma and the Angioimmunoblastic lymphadenopathy had a malignant evolution a short period of time. We analyze the symptomatology, clinical findings, evolution in the course of time and the response to the treatment, emphasizing the phato-Radiologic manifestations. We described the CT appearance of the 3 LYG, 2 LIP and 2 Plasma cells granuloma.

We explain the aspects known nowadays about these entities, discussing about their ethiopathogenic, their association to autoimmunity diseases and their occasional malignant degeneration.

Finally we discuss if the plasma-cells granuloma should be included in this group of disease.

\subsection{AM}

\section{Radiological assessment of pulmonary edema - a new principle} Göran Wegenius, Carl-Johan Wickerts, Göran Hedenstierna; Uppsala

Purpose: Histologic and physiologic evidence suggests that in pulmonary edema the sequence of fluid accumulation in the lung is quantal (all-or-none). We have proposed that this is reflected in the presentation at chest radiography of patients with pulmonary edema treated with PEEP (positive and expiratory pressure). Also, increased variation of radiographic density with respiration in patients with pulmonary edema has been attributed to quantal alveolar behavior.

Methods: To test this hypothesis pulmonary edema was induced in pigs by i.v. infusion of oleic acid. Ventilation with a constant tidal volume was instituted and pulmonary extravascular lung water (EVLW), was monitored with the double indicator dilution method. CT-scans of the chest in inspiratory and expiratory breath-holding were performed to match these observations. Also, effect of PEEP was analyzed.

Results: An increase in variation of radiographic density with respiration was always seen in pulmonary edema. However it did not always match EVLW. We propose that this variation mainly reflects alveolar edema. Institution of PEEP often resulted in elimination of alveolar edema.

Conclusions: Applying the principle of quantal alveolar behavior is a useful model for analysis of pulmonary edema and its relation to ventilation.
1069 High resolution computed tomography (HR-CT) for preoperative patient assessment for lung transplantation

H. Schurawitzki, R. Stiglbauer, O. Ch. Burghuber, W. Klepetko; Vienna

Purpose: Lung transplantation (LTX) has become a widely accepted procedure for patients with endstage lung disease. Careful patient evaluation is necessary to select optimal candidates for the procedure.

Methods: We evaluated 29 pts. with endstage lung disease by using HR-CT. Sixteen pts. had fibroses (group A) and 13 suffered from emphysema (group B). HR-CT was performed with $2 \mathrm{~mm}$ collimation at deep inspiration. HR-CT scans were analyzed according to the following questions: evidence of marked pleural thickening, subpleural bullae, difference of parenchymal alterations between both lungs.

Results: Group A: Marked pleural thickening was demonstrated in 7 pts.; subpleural bullae larger than $5 \mathrm{~cm}$ in 6 pts. A significant difference in lung involvement between both lungs could be shown in 10 pts. (62\%). Group B: HR-CT revealed large bullae in 7 pts, and a significant difference in structural damage between both lungs in 10 pts. (76\%). Marked pleural thickening was evident in one patient. The final decision for acceptance of patients and choice of the procedure (SLTX, DLTX) was highly influenced by HR-CT findings in $35 \%$.

Conclusions: Marked pleural thickening as well as bullae can cause problems during surgery (e.g. bleeding, contralateral pneumothorax). HR-CT is an excellent method to demonstrate the extent and severity of parenchymal and pleural alterations thus enabling preoperative planning of the particular transplant procedure.

\section{$11.35 \mathrm{AM}$}

1070 Pulmonary changes in patients with Kaposi-sarcoma

V. Jacobi, I. Harth, B. Mulert-Ernst, H. Fuchs, Chr. Friebe, S. Staszewski, S. Rehmet; Frankfurt/Main

Kaposi-sarcoma was found in 116 out of 300 patients who had died of AIDS. $20 \%$ of these patients had pulmonary manifestations which had been evident in preceding chest radiographies. Pulmonary complications in Kaposi-sarcoma can be seen radiologically before clinical symptoms are stated.

There are two types of patterns: the more common linear type and the nodular type. There are also mixed patterns. Other guide signs are Kerley-B-lines, pleural effusions of different intensity and pericardial effusions in a few cases. In general a chest radiography is sufficient for the diagnosis of Kaposisarcoma. A computed tomograph scan might be useful for the diagnosis of an additional pneumonia.

\section{$11.38 \mathrm{AM}$}

1071 Pulmonary alveolar microlithiasis: CT findings in five cases Philippe Cluzel, Philippe Grenier, Pierre Bernadac, Francọis Laurent, Jean-Daniel Picard; Paris

We report the results of conventional and high-resolution CT scans of the lung performed in five patients with confirmed diagnosis of pulmonary alveolar microlithiasis. This disease is characterized by widespread intraalveolar calcification of both lungs. Conventional CT confirmed the inferior and posterior predominance of the lesions in four of our cases and showed an exceptionally high concentration of microliths in the subpleural parenchyma and along the bronchovascular bundles. High-resolution CT revealed a perilobular and bronchovascular distribution of the disease process at the level of the secondary pulmonary lobule. This pattern correlated closely with the pathologic findings.

\section{$11.41 \mathrm{AM}$}

1072 Lymphography and biopsy in the nails syndrome

B.P. Villacastín. A. G. Robledo, C. Verdejo, M. Górgolas and G. Marín: Madrid

The "yellow nails" syndrome is characterised by yellow nails, Lymphoedema and pleural effusion. Bronchiectasis, chronic sinusitis and pulmonary infections can be other components as well. Around a hundred of cases with the mentioned triad and some variants - (Chest-1990) has been reported.

Four cases of the "yellow nails" syndrome seen at the F.J.D. are reviewed; three of them had pleuropulmonary alterations and two patients had Lymphoedema. A bilateral pedia Lymphography was performed showing the typical Lymphographic images that are the main interest of this communication. We also obtain tissue for histological study. Lymphography has been performed in only 17 "yellow nails" syndrome reported cases in the literature, and we have no references that any biopsy has been taken for histopathological studies. 


\subsection{AM}

1073 High resolution CT appearances in fibrosing alveolitis as a predictor of natural history

D. M. Hansell, A. U. Wells, C. M. Black, D. Davidson, R. M. Dubois; London

Lung disease in systemic sclerosis is histologically identical to lone cryptogenic fibrosing alveolitis; open lung biopsy appearances reveal a mixture of fibrosis and inflammation. It has been established that appearances on high resolution computed tomography (HRCT) are a useful guide to histological findings. A reticular pattern correlates with a fibrotic biopsy whereas amorphous parenchymal opacification is associated with increased cellularity. To evaluate the predictive value of HRCT appearances the initial scan was categorised as predominant reticulation (group A), equal proportions of reticulation and parenchymal opacification (group B) or predominant parenchymal opacification (group $\mathrm{C}$ ); changes in pulmonary function parameters over the next year were tabulated and changes in HRCT appearance and extent of disease were assessed $24 \pm 10$ months later.

Alteration in pulmonary function was seen less frequently in group $\mathrm{A}(5 / 23$, $22 \%)$ than in group B $(7 / 15,47 \%)$ or group $\mathrm{C}(5 / 7,71 \%), \mathrm{p}<0.05$. A change in extent or appearance on HRCT of disease was also less frequent in group A $(3 / 20,15 \%)$ than in group $\mathrm{B}(8 / 14,57 \%)$ or group $\mathrm{C}(4 / 7,57 \%), \mathrm{p}<0.05$. These findings suggest that HRCT appearances indicative of predominant lung fibrosis are usually associated with stable disease in the context of systemic sclerosis, whereas HRCT appearances suggestive of significant inflammation are associated with an increased likelihood of change in lung function and CT appearances during follow-up.

\subsection{AM}

$107499 \mathrm{mTCDTPA}$ and high resolution $\mathrm{CT}$ : an anatomical and functional correlation of disease activity in fibrosing alveolitis

A. U. Wells, D. M. Hansell, R. Lawrence, C. M. Black, R. M. Dubois; London

The fibrosing alveolitis of systemic sclerosis (SSc) consists histologically of a mixture of fibrosis and inflammatory cells, in variable proportions. Increased lung clearance of inhaled $99 \mathrm{mTCDTPA}$ has been employed as a marker of alveolar inflammation in many pulmonary diseases including SSc. High resolution CT (HRCT) has been successfully used to categorise the extent of lung disease and predict the likely histological findings at open lung biopsy. To explore the relationship between lung epithelial permeability and the presence, extent and activity of lung disease, the HRCT appearances of 78 patients with SSc were compared with DTPA clearance.

DTPA clearance was abnormally fast in $5 / 16(31 \%)$ patients with normal HRCT appearances and in $42 / 62(68 \%)$ with disease on HRCT. DTPA clearance was abnormal in $29 / 30(97 \%)$ patients with more than $20 \%$ involvement on HRCT, but only $18 / 48(37 \%)$ with less than $20 \%$ involvement $(\mathrm{p}<0.0001)$. There was no significant difference in clearance abnormalities between patients with predominant reticulation $(16 / 24,67 \%)$ and predominant parenchymal opacification $(4 / 6,67 \%)$ on HRCT. We conclude that DTPA clearance is a sensitive indicator of alveolar inflammation. Alveolar epithelial abnormalities are almost invariably present if lung disease is extensive $(>20 \%)$ in systemic sclerosis whether HRCT appearances suggest fibrotic disease or increased cellularity.

\section{Scientific Exhibition Awards}

The awards will be presented to the winners by Prof. Dr. J. Lissner, President of ECR ' 91 and Prof. Dr. H. Imhof, Chairman of the Scientific Exhibition Committee.

\section{Room C}

\section{Ss}

\subsection{Pancreas}

\subsection{AM}

\section{Diagnosis of the pancreas with spiral CT}

\section{W. Bautz, G. Luttke, W. Riffeser, M., Lenz; Munich}

Purpose: CT scanners with rotating measurement systems enable continuous scanning of a section of the body when combined with continuous patient transport (Spiral CT), for the pancreas with its relatively small volume the complete organ can be scanned in a single breathhold.

Methods and Materials: For pancreatic examinations continuous data acquisition of $24 \mathrm{~s}$ with a table feed of $10 \mathrm{~mm} / \mathrm{s}$ was used on a Somatom Plus. A power injection of $50 \mathrm{ml}$ contrast media (CM) was administered immediately before measurement starts. CT images with a nominal slice thickness were reconstructed from the volume data set at $2 \mathrm{~mm}$ intervals. 96 patients with pancreatitis, carcinoma or metastases of the pancreas, endocrine active tumors and echinococcus were examined both with conventional and Spiral CT.

Results: Problems experienced with poorly reproducible inspiration levels in conventional CT are completely eliminated. CM studies of the pancreas with spiral CT are showing an improved enhancement of the parenchyma. The result is an increased detail recognition not only in the pancreas (e.g. pancreatic duct) but also in the parapancreatic space (e.g. lymphomas and vessels). Compared to conventional CT of the pancreas, the $\mathrm{CM}$ volume can be reduced tue the short examination time, even in endocrine active tumors.

Conclusions: Spiral CT opens new perspectives for CM examinations of the pancreas. Compared to conventional CT, detail diagnosis is improved, measurement time and CM volume are reduced significantly.

\section{$10.40 \mathrm{AM}$}

\section{Improved contrast enhancement of the pancreas by spiral scanning}

G. Marchal, H. Rigauts, A. L. Baert; Leuven

Purpose: The influence of the type of scanning on the contrast enhancement of the pancreas is evaluated.

Methods: Sixty patients with suspicion of pancreatic disease are studied either with dynamic sequential scanning (cycle times of 5 seconds), or with spiral scanning (cycle times of 1 second).

For both scanning techniques, mean attenuation values of the pancreas are measured before and after a single bolus injection of $72 \mathrm{ml}$ of a $38 \%$ diluted iodined contrast agent. The differences in attenuation values of the pancreas before and after contrast injection reflect the level of contrast enhancement in both methods.

Results: After a single bolus injection, the level of contrast enhancement of the pancreas is statistically significantly higher in spiral scanning than in dynamic sequential scanning. This is due to the lower total acquisition time of spiral scanning that allows the entire pancreas to be scanned during the short arterial peak of contrast enhancement, whereas in dynamic scanning only two or three levels of the pancreas can be scanned during the same period.

Conclusions: Compared to the conventional dynamic scanning, spiral scanning allows to reduce the total amount of contrast medium to be injected without compromising the level of contrast enhancement.

\section{$10.50 \mathrm{AM}$}

\section{Pancreatic imaging using ultra-fast magnetic resonance} P. Reimer, S. Saini, M. S. Cohen, P. F. Hahn, T. J. Brady; Charlestown, MA

Purpose: Pancreatic imaging with conventional MR is of limited value due to motion artifacts and lack of a bowel contrast agent. We undertook the present study to evaluate the potential of ultrafast, motion-artifact free MR to image the pancreas.

Methods: MRI was performed on a $1.5 \mathrm{~T}(\mathrm{GE})$ system, with ultrafast imaging capability (Instascan, ANMR). High resolution $10 \mathrm{~mm}$ thick slices ( $5 \mathrm{~mm}$ gap) were obtained with fat suppressed spin-echo pulse sequences (TR $6 \mathrm{sec}$, TE $26 / 50 / 100 / 150 \mathrm{msec})$ in 10 subjects using single-shot $(128 \times 128)$ and mosaic $(256 \times 128)$ techniques (FOV $40 \times 20 \mathrm{~cm}$, pixel size $1.5 \times 3.0 / 1.5 \times 1.5 \mathrm{~mm})$. With this technique, 21 slices were sufficient to cover the liver and pancreas in a single $6 \mathrm{sec}$ breath hold (single shot) or $12 \mathrm{sec}$ (mosaic). In 4 subjects $200 \mathrm{ml}$ of water were administered orally prior to imaging.

Results: The entire pancreas (head, body, and tail) was visible in the ultrafast MR images in all subjects. Oral water intake improved differentiation of the pancreas from adjoining bowel. The common bile duct was visible in the head of the pancreas in all subjects. T2 related signal changes were clearly visible at the multiple TE's. When 12 second breath holds were practical, visible improvements were apparent in the mosaic images.

Conclusions: The capability of ultra-fast methods to reliably visualize moving structures of the abdomen offers important advantages for pancreatic imaging. Water might serve as a potential bowel contrast agent on heavily $\mathrm{T} 2$ weighted images.

\section{$11.00 \mathrm{AM}$}

1078 Ultrasound guided fine needle biopsy of the pancreas: smear cytology versus microhistology

R. Lencioni, P. Bagnolesi, A. Cilotti, D. Caramella, C. Bartolozzi; Rome

Purpose: of the work are: 1. compare the results of smear cytology (SC) and microhistology $(\mathrm{MH})$ in the microscopic diagnosis of pancreatic masses obtained through us-guided FNB. 2. verify whether the use of a cutting needle increases the risk of the procedure.

Methods: within a 5 -years period 73 patients with pancreatic mass underwent us-guided FNB. SC (Chiba needle $22 \mathrm{G}$ ) was performed in 70 cases, while $\mathrm{MH}$ (Surecut cutting needle 19-22G) in 29. In 26/73 patients SC and MH were both carried out. Surgery, autopsy or a minimum 6 months clinical/instrumental follow-up were employed to check all cases.

Results: retrieval rate was $95.7 \%$ for SC and $86.2 \%$ for MH. Sensitivity was $80.9 \%$ for SC and $85.0 \%$ for $\mathrm{MH}$. The combined use of SC and $\mathrm{MH}$ allowed $90.9 \%$ to be reached. No false positives were found. In no case we observed significant post-bioptic complications. 
Conclusions: SC and MH play a complementary role: SC is less sensitive than $\mathrm{MH}$ when diagnosing well differentiated carcinoma, but it furnishes a higher retrieval rate when dealing with colliquated or necrotic lesions. Both methods are safe and can be used in combination thus improving diagnostic accuracy.

\section{$11.10 \mathrm{AM}$}

1079 Interest of endosonography in duodeno-pancreatic tumors S. Chiche, V. Séné, M. C. Iseni, O. Marty, J. L. Bouillot, C. Guettier, M. C. Plainfosse; J.P. Petite; Paris

Purpose: Echography, CT and cholangiography are commonly used for the diagnosis and the assessement of local invasion in duodeno-pancreatic tumors. Their accuracies rapidly decrease when the tumor's diameter is less than $3 \mathrm{~cm}$. We hope that Intestinal Endosonography will be able to detect T1 tumors of Duodenum or Pancreas.

Methods and Materials: The Echoendoscope OLYMPUS EUM3 was used for our studies. The US frequency was 7,5 or $12 \mathrm{MHz}$. It was possible to switch the frequency from 7,5 to $12 \mathrm{MHz}$ during the US examination. The penetration depth of ultrasound was limited to $10 \mathrm{~cm}$.

Endosonography has been done in 13 patients suspected of duodenal, biliary or pancreatic lesion.

Results: Endosonography was not contributive in three patients and revealed the injuries in the others. In 7 patients ES depicted the tumor and was able to distinguish the localization between the duodenal wall, the papilla vater, the main bile duct and the head of the pancreas. The accuracy of ES was high when the tumor diameter was less than $2 \mathrm{~cm}$. But it was lower in the assessment of local invasion of $\mathrm{T} 2-\mathrm{T} 3$ tumors.

Conclusions: Intestinal endosonography would become an important technique for the assessment of small duodeno-pancreatic tumors.

\subsection{AM}

1080 Pancreatic insulinoma identification: sensitivity of US, CT, angiography and MRI (1980-90: 17 proved cases)

E. Angeli, A. Vanzulli, P. L. Paesano, S. Sironi, M. Castrucci, G. M. Crespi, A. DelMaschio; Milan

Purpose: to evaluate the sensitivity of US, CT, Angiography, MRI in the last ten years.

Results: in the last ten years 17 surgically confirmed pancreatic insulinomas (range: $1-4.3 \mathrm{~cm}$; mean: $1.84 \mathrm{~cm}$ ) were examined: 15 US, $16 \mathrm{CT}, 15$ Angiographic, 8 MRI studies were performed. Sensitivity of each test was calculated. To evaluate the diagnostic assessment of the 4 modalities we also separately considered studies performed before and after January 1987 (introduction of $0.5 \mathrm{~T}$ MRI and convex US probes in our department): 7 patients were examined before 1987, ten after 1987 . The last eight patients were submitted to all procedures.

Results: sensitivities were: $53.3 \%(8 / 15)$ for US; $31.3 \%(5 / 16)$ for CT; $53.3 \%$ $(8 / 15)$ for Angiography; $62.5 \%$ (5/8) for MRI. No statistical difference was found for CT and Angiography in these two separate periods; US sensitivity was $16.6 \%(1 / 6)$ before 1987 and $78 \%(7 / 9)$ in last four years. Association of CT and Angiography failed diagnosis in 5/17 patients (29.4\%); association of non-invasive techniques (US + MRI) localized insulinoma in each patient studied with both modalities $(8 / 8=100 \%)$.

Conclusions: in our experience only US improved sensitivity in the last four years. The combination of US and MRI is sufficient for preoperative localization of insulinoma.

\subsection{AM}

1081 MRI of small pancreatic islet cell tumors: a screening procedure? P. Pavone, S. Giuliani, G. Cardone, G. Albertini P., R. Occhiato, N. de Stefano, R. Passariello; L'Aquila

Purpose: To evaluate the feasibility of screening patients with suspected pancreatic islet cells tumors by MRI, after initial US workout.

Methods: 12 patients with suspected pancreatic islet cell tumors were included in the study. Large lesions identified by initial US workout were excluded from the study.

MRI was performed with a $0.5 \mathrm{~T}$ superconductive system, using a short TR (260-320 msec.), short TE (16-20 msec.), high NEX (8-16). Matrix was $256 \times 256$. T2 weighted sequences were also acquired with a TR of $2000 \mathrm{msec}$., a TE of $30-70 \mathrm{msec}$. and a matrix of $128 \times 256$.

Results: In 8 patients a lesion was identified, with size ranging from 0.7 to $2.5 \mathrm{~cm}$. The lesions were also evident at CT in only 5 cases, and were evident at angiography in 6 cases.

In only one patient angiography was positive when MRI did not detect a lesion.

In the remaining 3 patients no technique identified the lesion. However, none of these patients was submitted to surgery, for the mild symptoms. Clinical follow up is under way.

Conclusions: The high sensitivity of MRI and the lack of false positives in this series allow to state that screening use in the detection of pancreatic islet cell tumors is justified.

\section{$11.40 \mathrm{AM}$}

1082 Radiologic appearance of intra-pancreatic metastatic disease F. Boudghène, P. Deslandes, J. D. Grange, M. F. Carette, J. M. Bigot; Paris

These lesions are rarely diagnosed, in comparison with autopsy reports $(5 \%$ cancer). But they are often small, not symptomatic, and may occur late (12 of 21 cases), particularly in renal carcinoma (5 cases). They are also often observed in broncho-pulmonary neoplasms ( 10 cases), and sometimes in breast carcinoma and melanomas.

In the 21 intra-pancreatic metastases of this study, explorations were performed with US, CT, and sometimes angiography. The lesions were diffuse in 7 cases, and solitary in 9 cases. They appear hypoechoic on US in 12 cases, and hypodense on CT in 9 cases; sometimes they may be hypervascularised (3 cases), or pseudocystic (3 cases). Percutaneous biopsy was diagnostic for 7 cases, and guided the treatment, with surgical resection, or specific chemotherapy, regarding on the histologic type of the primitive malignancy, Results observed in the 21 cases of this study, were compared and appeared similar, for primitive neoplasms, location and aspect of these lesions on US and $\mathrm{CT}$, with the 82 cases described in literature reports.

\section{$11.50 \mathrm{AM}$}

1083 Enlargement of the pancreas in non alcoholic liver cirrhosis - a radiological and pathological analysis

Toru Muranaka, Akira Kato, Yasuo Matsuura, Takahisa Yoshida, Takeji Toda, Yoshihiko Oshiumi; Fukuoka

In order to evaluate the effect of hepatic dysfunction on pancreas morphology, anteroposterior width of the pancreas was measured by $\mathrm{CT}$ in 50 non alcoholic liver cirrhotic patients without pancreatic disease (group I) and 221 age and sex-matched normal subjects (group II). In addition, we reviewed the histological findings of the pancreas in 15 autopsy cases in group I and 14 cases without hepatic or pancreatic disease. The mean width of the pancreatic head in group I was $29.4 \pm 3.4 \mathrm{~mm}$ (mean $\pm \mathrm{SD}$ ), which was significantly larger than that in group II $(21.7 \pm 3.2 \mathrm{~mm})(\mathrm{p}<0.001)$. The width of the pancreatic body in group I was also significantly larger than that in group II $(19.5 \pm 2.4 \mathrm{~mm}$ vs $15.0 \pm 3.0 \mathrm{~mm})(\mathrm{p}<0.001)$. Histological investigation revealed that lipomatosis and intralobular fibrosis increased with age in the control group, however, they were seldom observed in autopsy cases of group I, and hypertrophy of islet cells was evident in 6 cases out of $15(40 \%)$.

The present study suggested that advanced liver dysfunction might provoke hypertrophy of the pancreas.

\section{Room E1}

\section{Ss}

\subsection{Diagnostic and interventional procedure in the head and neck}

$10.30 \mathrm{AM}$

1084 Carotid and vertebral vessels: diagnostic and interventional procedures (Keynote Lecture)

J. J. Merland; Paris

Abstract available at the information desk.

$10.45 \mathrm{AM}$

1085 PTA of carotid and vertebral arteries

R. Kachel, S. Basche, I. Kassel, K. Grossmann; Erfurt

We present our experience with more than 122 patients in whom PTA was performed in 129 stenosed or occluded supra-aortic arteries.

PTA was successful in 41 stenoses of the carotid artery, 18 stenoses of the vertebral artery and 59 obliterations of the subclavian or innominate artery. There were 2 minor-complications (1 TIA, 1 small thrombus of the internal carotid artery which was detected by 111 -indium platelet scintigraphy and treated by thrombendarterectomy before the appearance of neurological symptoms) and 1 major-complication (1 hemiparesis).

121 of 122 patients were symptomfree after angioplasty. During observations period of 3 to 114 months (average 61 months) there were only two cases with restenosis after subclavian-angioplasty.

The results of more than 700 own and international published PTA's of supraaortic arteries are presented. The results suggest that PTA of carotid and vertebral artery is an effective method. On strict definition of the indication the complication rate for angioplasty of the vertebral and carotid artery is not likely to be higher than for operative treatment. 
1086 Local intra-arterial fibrinolytic therapy in central retinal artery occlusion

M. Schumacher, A. K. Wakhloo, D. Schmidt; Freiburg

Central retinal artery occlusion can be treated with fibrinolytic agents improving visual function. However systemic application of fibrinolytic agents may lead to cerebral haemorrhage. To avoid life-threatening complications, local intra-arterial fibrinolytic therapy was performed in 6 patients over a period of 70 to 150 minutes. A total amount of 500.000 to 900.000 I.U. Urokinase diluted in saline solution was administered via a transfemoral introduced coaxial cathetersystem. The tip of the microcatheter was placed superselective at the proximal portion of ophthalmic artery. Subsequently 20.000 I.U./d Heparin was administered for the following 3 days. Besides blood pressure control, hemodilution was performed in two patients with elevated hematocrit additionally. The cause of the central retinal artery occlusion were traumatic dissection of carotid artery, aortic stenosis, severe high blood pressure, mitral insufficiency, carotid artery occlusion and polyglobuly. In the latter case the microcatheter was placed at the distal part of maxillary artery and the fibrinolytic therapy was performed via cross flow between maxillary artery and ophthalmic artery. In 5 patients fluorescence angiography was carried out before and after fibrinolytic therapy confirming the significant improvement of retinal and choroidal perfusion as seen on the carotid angiogram. 4 patients showed only a slight improvement of visual function due to delayed fibrinolytic therapy of 7 to $10 \mathrm{~h}$ after onset of central retinal occlusion. However two patients showed a complete recovery of visual acuity. No complications occurred during the whole investigation.

\subsection{AM}

\section{Endovascular treatment of basilar bifurcation aneurysm - strategy and pitfalls \\ M. Schumacher, A. K.Wakhloo, W. Radü; Freiburg}

The surgical and endovascular treatment of basilar bifurcation aneurysms continues to remain problematic and is afflicted with severe complications. Advantages of temporary balloon occlusion with fully conscious patient prior to definitive balloon detachment performed in 8 patients are described. Different aspects of aneurysm, i.e. size, shape, neck configuration and location concerning the posterior cerebral arteries and thalamoperforating arteries are discussed for the planning of endovascular treatment. Handling of double lumen catheter and advantages of balloon occlusion combined with microcoils are described. The possibility of correction of definitive detached balloon if not tolerated by patient, with a balloon-push technique is shown.

\section{$11.5 \mathrm{AM}$}

\section{Magnetic resonance angiography: evaluation of clinical value for} detection of atherosclerotic diseases

\section{J. L. Struyven, B. Poncelet, T. Metens, D. Wery, C. Delcour; Brussels}

Purpose: Comparison of accuracy of conventional angiography and magnetic resonance angiography (MRA) in detection of atherosclerotic diseases. Methods: MR two-dimensional angiography is performed exploiting inflowgenerated contrast and amplitude. The data are acquired by repeated singlesection imaging with a flow compensated gradiant echo-sequence. Presaturation follows selective imaging of the arteries or veins. Processing consists of reconstruction of slices to produce $2 \mathrm{D}$ projective images. Conventional angiography is performed by arterial DSA. We did compare the images of both methods in 34 patients, 29 males and 6 females. Mean age was 65 years ranging from 42 to 79.249 arterial segments were investigated and compared.

Results: Mostly, image quality was from good to acceptable in both methods. For detection of carotid lesions MRA was inferior compared to DSA. For aortic and peripheral arteries lesions MRA and DSA showed globally similar results but in some cases MRA was more performant.

Conclusions: MRA is a promising new imaging modality providing valuable clinical informations. Informations are from different value compared to DSA since rules of contrast media do not apply.

\subsection{AM}

1089 Thromboembolic phenomena in clinical angiography - Role of materials and technique

Peter Dawson; London

Thromboembolism is a well recognised and much feared complication of clinical angiography. When it occurs in the cerebral or coronary circulation it may be disastrous and it is not surprising, therefore, that most of the data concerning its incidence and contributory factors have been collected in the context of these studies. There has been intense interest and heated controversy, particularly in the United States, during the last three years since claims were made that newer non-ionic contrast agents might actually positively contribute to the danger of thromboembolism. Far more heat than light has been generated in the United States and now, with the Committee on the Safety of Medicines (CSM) in the UK poised to follow the lead of the US Food and Drugs Administration (FDA), it seems likely that the debate will soon concern British radiologists and cardiologists.

An objective review will be given of all the known factors, both materials and technique related, which may contribute to thromboembolic phenomena in clinical practice.

\subsection{AM}

\section{Carotid Doppler velocimetry in patients with brain tumors} Noriaki Tomura, Toshio Kato, Susumu Fushimi, Tetsuya Sakamoto; Akita

Purpose: To study the influences of brain tumor on the carotid arterial flow by pulsed Doppler velocimetry.

Methods: Subjects were twenty-five patients with various brain tumors (astrocytomas, glioblastomas, oligodendrogliomas, metastases, meningiomas, etc.). We used a RT-3600 scanner (U-sonic, YMS) with $5 \mathrm{MHz}$ probe, and measured systolic velocity (SV), diastolic velocity (DV), mean velocity (MV) and pulsatility index (PI) of the common carotid artery. Computed tomography (CT) and conventional angiography were performed in every patient. We studied the relationships among Doppler velocity, PI, CT appearances (maximum diameter of tumor and the degree of the midline structure) and angiographic findings (tumor vascularity).

Results: In 6 of 7 patients with large tumor more than $7 \mathrm{~cm}$ in maximum diameter, SV on affected side was lower than those on its contralateral side. In 8 of 9 patients with shift of miline structure, SV and MV on the affected side were lower than those of its contralateral side. And, in 8 of 10 patients with hypervascularity and/or early venous filling on angiograms, SV on the affected side was higher than that of contralateral side.

Conclusions: This study confirmed that Doppler flow of the common carotid artery reflected the degree of tumor vascularity and the mass effect on the main intracranial arterial branches in patients with brain tumors.

\section{$11.45 \mathrm{AM}$}

1091 Three dimensional (3D) MR angiography at high resolution: a new approach in the evaluation of vascular diseases

Luigia Rota, Andrea Villa, G. Carlo Barazzoni, Paolo Poggi, Rizzardo Anguissola, Riccardo Rodriguez, Adelaide Martelli; Pavia

Purpose: We evaluated the accuracy of MR Angiography in depicting the vessel lumen both in cerebral and in the neck vessels.

Methods: 260 patients were studied at our Institution using a $1.5 \mathrm{~T}$ system (Magnetom-Siemens); 52 patients were volunteers without any known vascular disease; the other 208 had vascular diseases (aneurysms, MAV, stenosis, tortuosity) preliminarily investigated: $\mathrm{Ct}$ with contrast medium and/ or angiography were performed in patients with cerebral vascular diseases; US doppler and/or angiography were performed in suspected peripheral diseases. We used Three-dimensional gradient-echo pulse sequence at high resolution of $0.7 \times 0.9$ voxel size.

Results: We obtained good quality images in $93 \%$ of our patients; 3D-MR Angiography provided accurate evaluation of the major bronches of the carotid, vertebral and basilar arteries and the circle of Willis; the tortuosity and the bifurcation of the common and internal carotid arteries were also well documented. However we observed that the smaller distal cerebral vessels were better documented on angiography and we estimated like stenosis at the carotid bifurcation 10 normal vessels, due to the signal loss.

Conclusions: MR Angiography is a new approach in the evaluation of cerebral and peripheral vessels. Actually, the major problems are the limited spatial resolution, the fast flow and motion artifacts. The 3D technique at high resolution is important to improve the diagnostic accuracy.

\section{$1155 \mathrm{AM}$}

1092 Large systemic arteriovenous malformations - is there an angiogenesis factor?

Peter Dawson, Ann Kennedy, Richard Petty; London

Large systemic arteriovenous malformations represent an uncommon but important vascular pathology. There is little understanding of the factors which drive their development and treatment options are limited. Transcatheter embolization remains the best therapy for most patients and excellent results have been achieved in good centres. However, such treatment is usually only palliative and it is a common place observation in centres where such procedures are performed that, following successful embolization of these lesions and their important arterial feeders, new feeders quite rapidly appear and previously noted but unimportant feeders become larger. A fundamental biological question is, is there an angiogenesis factor influencing these lesions as is frequently the case in malignant tumours? Results of some recent studies on this important question will be presented 


\section{Room E2}

SS

$10.30 \mathrm{AM}$

\subsection{D Imaging}

1093 Introduction to the technique of three-dimensional imaging (Keynote Lecture)

Frans W. Zonneveld; Utrecht

Medical 3-D imaging was introduced in the late seventies. It used the tools of computer grapics on the basis of volumetric medical imaging data sets (e.g. CT, MRI, US). The tissue structure of interest is usually isolated from the data set (segmentation) and then an image of this structure is synthesized (rendering). The most common segmentation technique is thresholding. The earliest rendering technique assigned a shade of gray to the distance between viewer and imaged structure and was called "depth-encoding". Today more sophisticated techniques are applied using virtual light sources (surface rendering) or using different degrees of transparency for different tissues in combination with a projection technique (volume rendering). Each technique has its own virtues and, within techniques, different degrees of sophistication are may result in different levels of image quality. Generation of two or more images with slightly differing viewing angles allow for stereo-pair display and dynamic display respectively thus producing more realistic images.

Segmentation can be restricted to a part of a given type of tissue. In this way, a mandible or a skull base can be imaged separately (disarticulation). Different segmentations can be combined in one image. This can apply to bony and soft tissues or even to tissue structures from different modalities such as CT and MR (multi-modality imaging). Segmentations can also be used for volume calculations (voxel addition) and for the fabrication of physical models (surgery rehearsal and implant design). The normal anatomy can be simulated by mirror imaging of the non-affected side. We will illustrate the techniques mentioned above with relevant case material.

\section{$10.45 \mathrm{AM}$}

\section{Marker-guided multimodality matching of the brain} Petra A. van den Elsen, Max A. Viergever; Utrecht

Purpose: The aim of this study is to combine CT, MRI, SPECT, and EEG or MEG derived dipole information, so as to improve diagnosis in neurological studies.

Methods: Use is made of external markers, which are easy to apply to the skin. Known markers have distinct disadvantages, e.g. low accuracy in standard image protocols employing thick slices and/or large interslice gaps, or special positioning requirements. A marker design is presented which achieves subslice accuracy in indicating points, and can even pinpoint reference points outside the scanned volume. The same markers are used in MRI and CT, which allows leaving them in place if both examinations are done successively. However, if necessary, the marker design allows precise repositioning. Results: The approach has been applied successfully to EEG/CT and EEG/MRI fusion in studies of romandic epilepsy. The markers locate the reference points of the dipole calculations in the CT and MR images with great precision. For neurosurgical studies, EEG/MRI/CT/SPECT fusion is currently investigated. Results of the method are presented by means of $2 \mathrm{D}$ as well as $3 \mathrm{D}$ visualizations.

Conclusions: Marker-guided matching is a useful tool to accurately register electromagnetic dipole data with tomographic brain images, acquired from multiple modalities.

\section{$10.55 \mathrm{AM}$}

\section{Determination of intracranial EEG electrode-position on three} dimensional surface MR-images of the brain

K. Bootsveld, B. Siewert, F. Träber, W. A. Kaiser, M. Reiser, C. E. Elger; Bonn

Purpose: The precise determination of the position of intracranial EEG electrodes is important for the appropriate interpretation of EEG-curves in epileptology.

Methods: 15 patients were examined using T1-weighted 2D-FFE-images with a slice thickness of $3 \mathrm{~mm}$ and less. These $30-60$ slices were reconstructed to 3D-rendered images of the brain.

Results: The EEG electrodes can be precisely depicted on the brain surface due to their specific signal void and allow for reliable monitoring of their position in relation to relevant gyri and sulci. The visualization of small sulci, however, is often difficult using this method.

Conclusions: Three-dimensional surface MRI is superior to other imaging modalities for localization of intracranial EEG-electrodes. For preoperative evaluation of patients with epilepsy it allows for accurate interpretation of EEG measurements.
11.05 AM

1096 A computer environment for stereotactic neurosurgery R. Verbeeck, D. Vandermeulen, P. Suetens, G. Marchal, J. Gybels; Leuven

Purpose: A system for planning and verification of stereotactic neurosurgery based on a flexible integration, processing and display of multimodality image data.

Methods: The software package was developed on a general purpose workstation equipped with a 3-D interaction and stereoscopic viewing system for easy access and manipulation of CT, MR and DSA data.

Results: All images, acquired using a patient-fixed stereotactic frame, are stored on a PACS archiving system and can be directly accessed by the workstation. A user-friendly display module allows the easy identification of target and entry points on any image. Stereoscopic DSA images are used for the determination of avascular electrode trajectories.

MR Angiography (MRA) can also be used for stereotactic planning. MRA is safe and non-invasive and has some potential advantages with respect to cerebrovascular imaging, more particularly the simultaneous acquisition of brain tissue and vascular morphology. Some methods were developed to visualize these important structures in a single view.

Conclusions: This workstation is used for the planning of stereotactic neurosurgical procedures including biopsies and implantation of recording or stimulating electrodes.

\subsection{AM}

1097 Knee MRI: Three dimensional rendering using a combination of surface and volume reconstruction

Martin Vahlensieck, Philipp Lang, Wing Chan, Harry K. Genant; San Francisco, CA

Purpose: The purpose of this study was to improve image quality of 3-D MR reconstruction of the knee joint anatomy and pathology by combining surface and volume rendering techniques.

Methods: 3-D displays of 2-D MR images were generated in 15 patients with knee disorders (MaxiView Workstation Dimensional Medicine, Inc.). In a first step, bone, menisci, anterior ( $\mathrm{ACL}$ ) and posterior ( $\mathrm{PCL}$ ) cruciate ligaments and pathologic processes were reconstructed with surface and volume rendering, respectively. Then both techniques were combined in one display. Results: The combination of volume and surface reconstruction yielded superior results compared to both techniques applied separately. Appearance of artifacts in the combined technique ( 7 cases) was comparable to volume reconstruction alone ( 6 cases) but more often seen in surface reconstruction ( 15 cases). Delineation of the crucial ligaments in the combined method was comparable to surface reconstruction alone but superior to volume reconstruction: only with the volume reconstruction we saw insufficient delineation of ACL origin ( 2 cases), ACL insertion (3 cases), PCL origin ( 3 cases) and PCL insertion ( 1 case). Delineation of pathology was comparable in all three techniques.

Conclusions: 3-D reconstruction of MR images of the knee by a technique combining volume and surface rendering is characterized by a reduction of artifacts and improved anatomical delineation and illustration of pathology.

\subsection{AM}

\section{Image enhancement and soft segmentation of MR angiography} for an improved visualization of blood vessels

D. Vandermeulen, D. Delaere, P. Suetens, H. Bosmans, G. Marchal; Leuven

Purpose: Visualization of three-dimensional images requires a preliminary classification of structures to be visualized. The type of classification is dependent on the type of rendering: a binary, "hard" classification for Surface Rendering and a continuous, "soft" classification for Volume Rendering. The simplest binary and continuous classification methods are based on photometric properties (grey values, gradients) only. Such a classification works well for high-contrast structures such as bone on CT. However, most tissues visible in MRI are not unambiguously characterized by local photometry. Consequently, additional knowledge (geometry, semantics, context) about the objects has to be applied in order to obtain a better classification. Results: Two techniques have been investigated for improved analysis of 3-D-MR angiography.

- A 3-D non-linear filter that enhances the contrast of bloodvessel-like structures. A subsequent Maximum Intensity Projection results in an enhanced visualization of small low-contrast blood vessels.

- A soft classification technique assigns continuous values to voxels, reflecting their probability of belonging to a blood vessel. These values can be converted into a opacity value needed for volume rendering. The probabilities are estimated by a stochastic sampling process of the a posteriori probability density function. This function incorporates the relationship between blood vessels and their signal in the image as well as a continuity and shape constraint. 
1099 Use of hypermedia software for diagnostic images display

Paolo Pavone, Carlo Catalano, Giovanni de Gasperis, Antonio Agnifili, Giorgio Albertini P., Giampiero Cardone, Roberto Passariello; L'Aquila

Purpose: New hypermedia softwares allow access to different information, including text, sound and images, with 256 gray leves. Our study was undertaken to evaluate the possibility to include diagnostic images in the program, for storage and teaching purposes.

Methods: A Macintosh computer (Apple Computer, Cupertino, CA) was used, with 8 Mbytes RAM and 80 Mbytes Hard Disk.

The computer was connected with ethernet link to our PACS system, allowing retrieval of images directly, or through a CCD digitizer. The images were then transformed into a TIFF format, allowing displaying and evaluation on the monitor.

A "Supercard" hypermedia software was used for image management and manipulation.

Results: The image quality on the Macintosh computer was considered very good for teaching purpose, and sub-optimal for diagnostic purposes. The first option was therefore stressed. Images can be easily accompanied by explaining texts or voice, allowing an easy interface with the user, with mouse control. Conclusions: The new softwares show promises as aid in diagnostic imaging manipulation and teaching.

\subsection{AM}

\section{Towards 3D computed angiography}

\section{Saint-Felix, C. Picard, A. Rougee, Y. Trousset; Buc}

The objective is to provide a three-dimensional (3D) image of a vasculature using a set of 2D DSA images taken around the patient. Such 3D quantitative information can be useful for accurate stenosis sizing, surgery planning or guidance of interventional radiology procedures.

The underlying reconstruction problem is considered as a reconstruction problem with missing date because of the low number of available projections, their truncature and their restricted distribution in the space around the patient. An original regularizing method has been developed which consists of two successive steps. First, a volume of interest (VOI) containing the vessels to be reconstructed is detected given the DSA images. Second, the value of the voxels belonging to the VOI is estimated using a computationally efficient multi-resolution approach.

This technique has been tested on several anatomical phantoms, among which cadaver hearts and heads. High resolution $\left(512^{3}\right)$ reconstruction of the opacified vasculature of these phantoms have been obtained. Results are fully satisfactory for the above mentioned applications, even when using a low number of projections (10 to 30 ) located on a semi-circular source-detector trajectory.

\section{Room L}

\section{SS}

\subsection{AM}

1101 Magnetic Resonance Imaging of the head and neck: examination technique, clinical usefulness, limitations - A retrospective analysis of six year's experience with progressive technical and methodological improvements

M. Lemort, Ph. Haesendonck, H. Maisonnier, G. Andry, G. Chantrain, D. Larsimont, P. Dor, D. Balériaux; Bruxelles

The intensive head and neck surgical activity of the Institute J. Bordet, a comprehensive cancer centre, permitted the development of a long-standing experience in imaging head and neck tumors or tumor-like conditions with CT and, since 1984, with MRI ad 0.5 and 1.5 Tesla, in selected cases. Improvement in the imaging equipment and the increasing information provided by the literature as well as the know-how acquired through our experience, has lead to the progressive modification of the examination methodology and some aspects of our conception on the present and future role of MRI in the initial evaluation and follow-up of head and neck patients. On the basis of imaging and clinical files, retrospective analysis was carried out with the intend to define the true impact of MRI on our practice. We point out the difficulties and pitfalls of the method and suggest an optimal examination technique, the actual specific indications and the expectations for the near future. Among the aspects considered in this study, are: the relative disappointment with $\mathrm{T} 2$ or fast imaging, the growing importance of the contrast media, the complementarity of MR and CT, the importance of an appropriate selection of patients. Our assessment is that MRI was not a complete revolution in the evaluation of head and neck tumors, but was able to compensate for certain insufficiencies in other methods, particularly CT. MRI gained an important complementary place, that will grow in the future.
10.40 AM

1102 High resolution ultrasonography (US) in benign and malignant superficial lymphadenopathy: a sonographic-pathologic correlation Pierre Vassallo, Georg Edel, Nikolas Roos, Peter E. Peters; Münster

Purpose: In recent years, changes in internal architecture of superficial lymph nodes (LNs) on high resolution US have been described, which may assist in differentiating malignant from reactive disease. By directly comparing sonographic and pathologic sections, we are assessing the significance of these US criteria.

Methods and Materials: A total of 50 superficial LNs (cervical, axillary and inguinal) from patients with known or suspected malignant disease were examined with $7.5 \mathrm{MHz}$ ultrasonic probes. All LNs were documented on in vivo and/or in vitro sonograms in coronal, sagittal and transverse planes. The completely excised LN was then microsectioned in one identical plane to allow direct correlation with sonographic findings. US features (including long-to-short diametric ratio, presence and width of central hilus and concentric or eccentric parenchymal widening) were graded on a 6 level scoring scale (0-5). Maximal nodal diameter was also measured.

Results: All LNs scored 4 or 5 at sonography were malignant, whereas all scored 0 were benign. LNs scored 1 were benign in $83 \%$ of cases whereas those scored 2 and 3 were malignant in 57 and $63 \%$ of cases respectively. $79 \%$ of nodes were correctly classified when scores of $0-1$ were considered benign and 2-5 malignant. Strong criteria for malignancy included an $\mathrm{L} / \mathrm{S}$ ratio less than 2 $(100 \%)$, absence of a central hilum (100\%) and eccentric parenchymal widening $(77 \%) .72 \%$ of nodes with an $\mathrm{L} / \mathrm{S}$ ratio over 2 were benign irrespective of nodal size.

Conclusions: Direct pathologic correlation allows characterisation of high resolution US findings in LN disease. The objective evaluation of these features using a scoring scale may improve the specificity of US in the analysis of benign and malignant nodal disease.

\subsection{AM}

1103 High resolution $\mathrm{CT}$ for the detection of cervical lymph node metastases

Stefan Delorme, Michael V. Knopp, Hans-Ulrich Kauczor, Friedhelm Raue, Heinz Buhr, Gerhard van Kaick; Heidelberg

Purpose: To develop a technique with improved detail resolution in CT imaging of the cervical region.

Methods: 30 patients who had undergone thyreoidectomy and modified neck dissection for medullary thyreoid carcinoma were studied in continuous slices with $4 \mathrm{~mm}$ thickness from the mandibula to the carina enrolled in a clinical follow-up-study. The patients received intravenous contrast medium at a controlled infusion rate. Images were reconstructed with a high resolution algorithm at an average $16 \mathrm{~cm}$ field of view. Attenuation values of vessels, lymph nodes, muscles and sonographically suspected metastases were measured and compared to former studies without contrast medium.

Results: The presented technique enabled a significant improvement of contrast between muscular and vascular structures. The improved resolution as well as the optimised contrast enhancement by a controlled infusion technique enables excellent anatomical identification of all structures; lesions as small as $5 \mathrm{~mm}$ were reliably identified as adenopathy which could be confirmed by surgical resection.

Conclusions: The use of a high resolution reconstruction technique combined with a standardised contrast infusion procedure enabled a significant improvement of topographic orientation, thereby permitting the reliable evaluation of adenopathy as small as $5 \mathrm{~mm}$. It seems superior to ultrasound regarding a standardised objective and reliable evaluation especially in patients with slowly growing head and neck tumors.

\section{$11.00 \mathrm{AM}$}

1104 Value of contrast enhanced $\mathrm{CT}$ in recurrent malignancy of the head and neck region

M. Lenz, B. Kersting-Sommerhoff, W. Riffeser, H. Bongers; München, Tübingen

Purpose: 544 CT examinations in 231 patients (115 recurrent tumors, 76 recurrent lymph node metastases) were analyzed retrospectively to assess the role of contrast enhanced CT in the posttherapeutic monitoring of patents with head and neck neoplasms in comparison and as a supplement to clinical examination.

Methods: CT scans were obtained with a scan time of 1-3 sec and 4-5 mm slice thickness in contiguous scanning. Contrast media were applied as a $50 \mathrm{ml}$ bolus injection followed by $100 \mathrm{ml}$ fast infusion $(300 \mathrm{mg}$ Iodine $/ \mathrm{ml})$. Recurrent malignancies were identified using the criteria mass lesion, hyperdensity, enhancement and structural characteristics (inhomogeneity ect.).

Results: CT $(80 \%)$ was inferior to clinical examination $(87 \%)$ in the detection of recurrent malignancy, but superior in depicting the extent of larger lesions. CT $(95 \%)$ was superior to clinical examination $(80 \%)$ in the detection of 
recurrent lymph node metastases.

Conclusions: Contrast enhanced CT is especially suitable for detection of recurrent lymph node metastases.

CT supplements the clinical examination in the detection of recurrent malignancy by depicting the extent of the lesion. A base line CT examination is of great importance.

\subsection{AM}

1105 Imaging of extra-nodal, lymphatic \& extralymphatic, Non-Hodgkin-Lymphoma (NHL) in the head and neck region

M. Horvath, J. L. Termote, S. F. Lemahieu; Leuven

Purpose: To evaluate the distribution, the different patterns, and the differential diagnostic problems in various extranodal locations.

Methods and Materials: Retrospective study of (50) patients examined mainly by $\mathrm{CT}$ scan. Because of the very different pattern according to location, six subgroups are considered. The location in the Waldeyer's ring is the best known and this lymphatic type represents more than $50 \%$ of the extranodal NHL. Between the nonlymphatic type, the orbital location takes an important place. The soft-tissue location affects either the superficial facial structures (cheek, nose, paranasal sinuses) or the deep retrofacial tissues. The location in the salivary gland, and the osseous lesions of NHL are peculiar problems, as are the rare locations in the thyroid gland.

Results: CT imaging allows a precise detection and localization of extranodal NHL, useful for the planning of the treatment. The differential diagnostic problems in these usually aspecific lesions are stressed.

Conclusions: An attempt is made to a more precise diagnostic approach in the polymorphic appearance of extranodal NHL of the head and neck.

\section{$11.20 \mathrm{AM}$}

1106 Value of sonography as preoperative localization method in hyperparathyroidism

A. Talegon Melendez, P. Escobar Casas, A. Gonzalez Garcia, M. Iribarren Marin, M. Calero Garcia, I. Galan Parra; Sevilla

1. The value of sonography as initial method of preoperative localization has been studied in 85 patients with hyperparathyroidism (HPT)

2. 61 patients (group A) had HPT $1^{\circ}, 47$ without cervical surgery (group A-1), and 14 with previous cervical surgery (group A-2). 24 patients (group B) had HPT $2^{\circ}$. All the patients were examined with real-time equipment and transducers of $7.5 \mathrm{MHz}$, and underwent surgery.

3. Out of 135 glands removed, sonography identified 107, 28 passed unnoticed, and 4 were false positive (sensitivity $79.2 \%$; specificity $98 \%$ ). Sensitivity was higher in group A $(83.3 \%)$ vs group B (76\%), as well in group A-1 $(85.4 \%)$ vs group A-2 (71.4\%). In uremic patients, sonography was useful to control the glandular growth.

The capacity of detection depended on the glandular size and weight. The most frequent errors were the coexistence of thyroid nodules, and glands of low size and weight. The majority of glands were straight, oval or spherical shaped, solid homogeneous and low echogenicity.

4. Sonography have high sensitivity $(79.2 \%)$ and specificity $(98 \%)$ for the preoperative detection of parathyroid disease. Because of its low cost, noninvasivity, results, and ample availability, we propose sonography as the first preoperative method in patients with HPT.

\section{$11.30 \mathrm{AM}$}

1107 Cervical ultrasound combined with parathyroid hormone assay

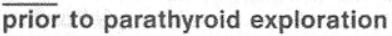

H. Mäkäräinen, R. Takalo; Oulu

The purpose was to analyze ultrasound (US) and fine needle aspirations (FNA) for parathyroidhormone (PTH) in the preoperative evaluation before parathyroid exploration and compare the results to FNAs for cytology.

Methods: During Nov. 1987-Dec. 1990, 20 patients with hyperparathyroidism (HPT) had cervical US combined with FNAs for PTH and cytology. US examinations were done with a real-time scanner using $7.5 \mathrm{MHz}$ transducer and direct contact method. The aspiration method was modified according to Doppman et al. The main differences were that we used US-guidance instead of CT-guidance and an immunoradiometric assay for the direct quantitative determination of intact PTH instead of a mid-region specific radioimmunoassay. Free-hand technique and 23 or 24 gauge needle were used and the aspirated specimen was soluted to a volume of $1 \mathrm{ml}$ saline. The ice was needed to freeze the sample immediately.

Results: The normal serum-PTH varies from 10 to $55 \mathrm{ng} / \mathrm{ml}$ and s-PTH of these patients aspirated varied from 85 to 175 . The values for PTH content from suspected parathyroid varied from 200 to 78400 . The greater values were achieved after immediately freezing the samples. FNAs for cytology were too unsensitive to be useful in routine practise.

Conclusions: US combined with FNA for PTH assay is the primary imaging method of choice prior to parathyroid re-exploration, more invasive imaging methods, sclerotherapy and in selected cases before primary exploration.

\section{$11.40 \mathrm{AM}$}

\section{Color Doppler in thyroidal pathology}

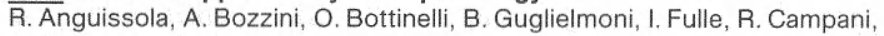
E. Genovese; Pavia

This work is meant to show how useful color doppler may be in the study of the thyroidal pathology, particularly in cases of a change in the functionality of the gland, in thyroiditis and in the focal pathology.

50 patients (aged $20-78$, suffering from thyroidal pathology) were taken into consideration: 76 multinodular goitres, 20 diffused goitres, 18 cases of thyroiditis, 36 single nodules, of which 10 carcinoma. Two linear probes $(5$ and $7.5 \mathrm{MHz}$ ) with color doppler were used.

The study of the thyroid by means of color doppler allows to check the functionality of the gland with respect to its vascularization. This is very useful in cases of subclinical hyperthyroidism, in cases of associated pathology (chronic thyroiditis associated with functioning adenoma). It may, moreover, help to characterize the single nodule, to show the presence of tumour in diffused goitres and precociously point out relapses in operated patients.

Sonography used with color doppler is, therefore, systematic, not invading. It gives important informations about the focal or diffused pathology of thyroid. It shows, better than scintigraphy and cytology, the presence of tumour particularly in multinodular goitres.

\section{$11.50 \mathrm{AM}$}

1109 Parathyroid colour Doppler sonography in primary and secondary hyperparathyroidism: correlation with biochemical parameters and blood flow velocities

F. Calliada, M. Bergonzi, R. Campani, C. Passamonti, G. Sala, O. Bottinelli; Lodi, Pavia

Colour Doppler provides a simple, non invasive and low cost method in parathyroid examination. It is very helpful in identifying parathyroid glands, characterized by a typical blood flow pattern.

The combined use of B mode and Colour Doppler US allows the vascular features of suspicious parathyroid nodules to be satisfactorily demonstrated, with easy differentiation between parathyroid adenomas, featuring diffuse internal vascularization, lymph nodes, thyroid adenomas and cysts that shows different kind of vascular pattern.

We studied with a linear $7 \mathrm{Mhz}$ probe coupled with a Colour Doppler device 103 patients ( 48 males and 55 females) with clinical and biochemical signs of hyperparathyroidism ( 18 primary and 6 secondary, surgically proven and 79 secondary, in emodialized and kidney transplanted patients). Every patient was scanned both with B mode and Colour Doppler sonography. Fine needle aspiration biopsy was performed in non surgical patients.

For simplicity we considered the expected glands in primary HPT to be one for each patient ( $85 \%$ with one adenomatous gland and $15 \%$ with more than one hyperplastic gland) and 4 for each patient in secondary HPT.

At surgery we found 18 adenomas in primary HPT patients vs 16 localized with US/CD association: 2 false negative, no false positive, with a sensitivity rate of $88 \%$ and specificity rate of $100 \%$. In 6 secondary HPT affected patients we found at surgery 23 hyperplastic glands vs 21 localized with US/CD association: 2 false negative, no false positive, with a sensitivity rate of $87.5 \%(91.3 \%$ vs surgery) and specificity rate of $100 \%$.

Peak velocities recorded both with Colour and pulsed Doppler showed a velocity range between 4 and $44 \mathrm{~cm} / \mathrm{s}$. No significant correlation was found between detected peak velocities $\rightarrow$ range: $4-44 \mathrm{~cm} / \mathrm{sec}$, mean $\pm \mathrm{SD}$ : $14.6 \pm 11.7 \mathrm{~cm} / \mathrm{sec}$, parathormone $\rightarrow$ normal values: $10-65 \mathrm{pg} / \mathrm{ml}$ (intact molecule Nichols Institute), found range between 122 and $2920 \mathrm{pg} / \mathrm{ml}$, mean 土SD: $794 \pm 773$ and other biochemical parameters: calcium: $9.0 / 12.3 \mathrm{mg} / \mathrm{dl}$, mean \pm SD: $10.4 \pm 1.4$, phosphate: $2.4 / 7.5 \mathrm{mg} / \mathrm{dl}$, mean $\pm \mathrm{SD}: 4.1 \pm 1.6$, alkaline phosphatase 290/3760 IU/1, mean \pm SD: $579 \pm 232$.

Colour Doppler is very useful in the diagnostic approach to differentiate hyperplastic parathyroid glands from other solid masses in the neck. However peak velocities recorded using Colour and pulsed Doppler did not correlate with the activity of the pathological parathyroid glands. 
Room M

Ss

\subsection{Interventional procedures}

$10.30 \mathrm{AM}$

1110 Selective salpingography and tubaric catheterization in diagnosis and treatment of Fallopian tube obstruction

Eugenio Zanon, Giovanni Gandini, Dorico Righi, Paolo Fonio, Ugo Maisano; Torino

In the last two years we have performed hysterosalpingography in 203 women with infertility to diagnose and localize obstructive diseases of Fallopian tubes and to correct proximal tubaric occlusion.

We have used a coaxial system of 8 French and 4.5 French catheters with appropriate guide wires.

We have obtained the following results:

141 patients with patent tubes

32 patients with one occluded tube

23 patients with bilateral occlusion

7 patients with hydrosalpinx

Successful tubaric catheterization and selective salpingography was obtained in 72 of 78 tubes with an overall success rate of $92.3 \%$.

Maneuvers failed in 6 cases.

The total number of pregnancies will be evaluated in June '91, six months after the last examination.

There were no complications due to the procedure.

We believe that the good diagnostic and therapeutic results, the low cost of the procedure and the absence of complications justify the use of this technique to investigate female infertility and to correct proximal tubaric occlusions.

\section{$10.40 \mathrm{AM}$}

\section{Fallopian tube catheterization and recanalization}

Simon P. Strijk, Peter E. J. M. Sallevelt, Wim N. P. Willemsen, Ton Franssen; Nijmegen

Purpose: Fallopian tube (FT) obstruction is a relatively common finding in women with disturbed fertility. Surgical reconstruction or in-vitro-fertilization are the usual therapeutic options to induce pregnancy in selected cases. Preliminary results of FT catheterization are promising and may prove to be competitive. A survey of the literature and our own results will be presented. Methods: With the use of a commercially available coaxial catheter-set selective catheterization of the tube(s) is performed via a transvaginal, transcervical access. Hospitalization is not necessary. Apart from an antibiotic prophylaxis, no medication is needed.

Results: Twenty-two women with a total of 32 occluded tubes were treated. Recanalization was successful in $78 \%$ of the tubes, or in $72 \%$ of patients. Clinical significant complications did not occur. Four patients conceived between 2 and 14 months (median 2 months) after the procedure, including one tubal pregnancy. Persistence of tubal patency was checked after 6 months in 6 non-pregnant women and was demonstrated in $87 \%$ of the tubes. Updated results will be presented.

Conclusions: FT-catheterization is a valuable diagnostic and therapeutic technique in women with infertility. In selected cases it possibly improves the conception rate. In clinical and economical aspects, it compares favorably with other treatment modalities.

\section{$10.50 \mathrm{AM}$}

1112 Is there a need for transcervical Fallopian tube recanalization? K. Wadin, C. Rasmussen, M. Lönnemark, A. Magnusson; Uppsala

Purpose: Since a few years several reports, mainly from the United States, have been published on a new technique of transcervical fallopian tube recanalization as a treatment of infertility. According to these reports proximal obstruction of the fallopian tubes occur in up to $25 \%$ of hysterosalpingograms (HSG). A relatively large number of the obstructions are caused by intraluminal debris and can be treated with recanalization. We wanted to offer our patients this treatment, but we had the impression that proximal obstructions were not frequently found. Therefore a retrospective review of HSG was undertaken in order to find the frequency of proximal obstructions in our patient population.

Methods: 527 HSG examinations performed 1986-1988 were retrospectively reviewed.

Results: In 205 cases the HSG was normal. 60 patients had bilateral obstruction of the fallopian tubes, but only in 12 cases the obstructions were proximal. 85 women delivered babies within 18 months after the HSG, and in at least 51 cases no treatment had been given after HSG. In the latter group the average duration of infertility was 2,5 years.

Conclusions: Only a very limited number of patients with proxima obstruction of the fallopian tubes were found. There is no reason to believe that the patient population in Sweden in this matter differ from that in the United States. Is the explanation to be found in the technique of performing the HSG?

\section{$11.00 \mathrm{AM}$}

1113 Malignant ureteral obstruction: treatment by percutaneous placement of self-expandable metal stents (wallstents)

F. Flueckiger, J. Lammer, G. E. Klein, F. Waltner, K. Hausegger, M. Aschauer; Graz

Purpose: Long-term treatment of ureteral obstruction is usually done by placement of double-J-stents (DJS) which tend to occlude within few months by incrustation. If a highly resistant stenosis is present, only small DJS can be placed, which occlude still earlier. In such cases we implanted Wallstents to get sufficient ureteral dilatation.

Methods: After percutaneous nephrostomy the stenosis was dilated with a $5 \mathrm{~F}-7 \mathrm{~mm}$-balloon-catheter and then a Wallstent of $7 \mathrm{~mm}$ diameter was placed and dilated additionally. Follow up was done by antegrade pyelography or fluoroscopically supervised IVP (to watch ureteral peristalsis). Up to now we treated 6 pat. with pelvic ureteral stenoses

Results: In 5 cases the procedure was successful and we observed during follow up ( 3 to 12 months) continuous spasm of the ureter adjacent to the stent, but no obstruction. In 1 case the Wallstent was compressed totally by a hard tumor so that we had to place an extern-internal-drainage.

Conclusions: Wallstent placement is an alternative treatment of malignant ureteral obstruction. It generates an adynamic segment with continuous adjacent ureteral spasm. Therefore this procedure should be reserved to the pelvic ureter to avoid iatrogenic hydronephrosis due to disturbed peristalsis.

\subsection{AM}

1114 Percutaneous nephrostomy in the management of acute and chronic pelveoureteral obstruction

P. Lukes, G. Tidebrant, A. Wihed, L. Norlén; Göteborg

107 patients with acute or chronic pelveoureteral obstruction primarily treated with percutaneous nephrostomy were studied to evaluate the effects of nephrostomies prior to the definite treatment as well as procedure related complications and technical difficulties. The obstructions were of malignant origin in $65 / 107(61 \%)$ and benign in $42(39 \%)$. The latter usually being impacted ureteral stones.

- The nephrostomies were performed under local anaesthesia using ultrasonographic and fluoroscopic guidance.

- In $52 / 65(80 \%)$ of the patients with malignant ureteral obstruction causing renal failure with elevated serum creatinine a substantial beneficial effect was noted. The mean survival rate being 7.6 months (4-21). In 22/27 (81\%) patients with obstructing ureteral stones these could be removed via the nephrostomy channel. Renal pelvic bleedings occurred in $8 / 107(7.5 \%)$ patients. Dislodgment of the nephrostomy catheters occurred in 31/107 (28\%). Significant urinary leakage along the catheter took place in $20 / 107(18 \%)$ and $12 / 107(11 \%)$ of the catheters had to be replaced because of partial breakage. Conclusions: Percutaneous nephrostomy is usually easily performed with good effect in patients with pelveoureteral or ureteral obstructions

Restoring the renal function by decompression is mandatory prior to definite treatment especially with malignant obstructions.

Malfunctions and displacements of the catheters are frequent problems.

Further improvements of technique and catheters seem desirable.

\subsection{AM}

1115 Percutaneous dilatation of benign ureteral strictures following radical hysterectomy

J.W. M. Hoe, E. C. Tan, K. H. Tung, A. Ilancheran, P. Singh; Singapore

Following radical hysterectomy in 42 patients with early invasive carcinoma of the cervix, ureteral strictures developed in 7 patients. These were long smooth strictures situated in the distal ureter and thought to be of benign origin and related to devascularization of the ureter. All except one were diagnosed within 3 months of surgery and all were treated by a percutaneous approach using a combination of dilating catheters, balloon catheters and ureteral stenting. In 5 patients $(83 \%)$ the strictures were successfully treated. In one patient there was an equivocal result as she developed tumour recurrence at 8 months while in another the procedure failed, most likely because the stricture was not detected for 12 months and she had also had radiotherapy. The follow up period ranged from 6 months to 26 months (mean 15 months).

Although previous authors have indicated that ureteral strictures following radical hysterectomy would not be expected to respond to transluminal dilatation in view of the devascularisation of the ureter, our experience shows that the majority of these partially devascularised ureters are amenable to percutaneous techniques if detected within $2-3$ months following surgery. 


\section{$11.30 \mathrm{AM}$}

1116 Self expanding metallic stents in malignant extraluminal ureteral stenoses

H. Lugmayr, W. Pauer; Wels

As described in the literature, $45 \%$ of double-J-endoprostheses in the ureter are compressed by the extraluminal tumor and are therefore unable to drain anymore. During the second half of 1990 we implanted selfexpanding wallstents in 12 ureters in 10 patients. 3 stents were localized from above via a nephrostomytract, 9 stents from below via cystoscopy. In all cases good urinary drainage could be observed immediately after insertion of the stents. Three weeks after the intervention the stents were almost completely covered by urothelium as endoscopy showed. In three ureters 2 to 3 months later an additional double-J-pigtail catheter had to be inserted to overcome obstruction by extensive hyperplasia of the urothelium in the stent area. Thus good drainage could be obtained over a observation period of another three months. The double-J did not obstruct again because the wallstent with-stood tumorcompression.

Late results cannot be obtained up to now. From our point of view malignant extraluminal strictures of the ureter are another indication for implantation of metallic wallstents, which allow patients to live in better conditions than with a percutaneous nephrostomy.

\section{$11.40 \mathrm{AM}$}

1117 Clinical evaluation of intermittent arterial infusion with implanted

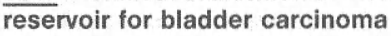

Kenji Nakamura, Sumio Takashima, Haruki Nakatsuka, Toshio Kaminou, Kazuo Minakuchi, Yasuto Onoyama; Osaka

Purpose: Chemotherapy with intermittent arterial infusion from an implanted reservoir was developed to treat advanced bladder carcinoma. Eighteen patients were treated, and the clinical value of this method was evaluated. Patients and methods: The tip of an infusion tube was inserted selectively into an internal iliac artery. The other internal iliac artery was then embolized with steel coils, and the opposite end of the infusion tube was connected to a reservoir implanted intradermally. Treatment consisted of intermittent injection of doxobubicin ( $10 \mathrm{mg} /$ body), cisplatin ( $10 \mathrm{mg} /$ body) and/or Mitomycin C ( $4 \mathrm{mg} /$ body) in a 10 -minutes period once weekly.

Results: Among 13 evaluable patients who have received at least four injections so far, nine patients had more than a $50 \%$ reduction in tumor size, as calculated from US and CT scans. No serious side effects, such as myelosuppression or renal and/or liver dysfunction, occurred.

Conclusions: The results suggested that intermittent arterial chemotherapy with an implanted reservoir was useful clinically. It seems safe and was easily done in outpatient clinic for the treatment of advanced bladder carcinoma.

\section{$11.50 \mathrm{AM}$}

\section{CT guided percutaneous nephrostomy placement}

\section{Siddhi J. Shah, Jaime Tisnado; Richmond, VA}

Percutaneous nephrostomy is a safe and effective technique for establishing access to the upper urinary tract.

The collecting system can be imaged under sonographic, fluoroscopic and/or combined sonographic/fluoroscopic guidance.

To our knowledge, nephrostomy placement under CT guidance has not been described.

We have developed a technique where the collecting system is approached under CT guidance.

There are many advantages for this approach. Some serious complications, such as perforation of bowel, pneumothorax, and failure to place the tube, are avoided. One major advantage is no radiation to the operator.

To date, we have used this method successfully in 10 patients. Average procedure time has been $60-70$ minutes.

In this presentation technical aspect and our experience in selected cases will be discussed.

\section{$11.55 \mathrm{AM}$}

\section{Combined percutaneous and endoscopic approach in the} treatment of ureteral fistula

M. Šurlan, J. Knific, D. Pavčnik, M. Mihelič, J. Klančar; Ljubljana

Ureteral fistulas and leaks are common complications following surgery and radiation, as well as a consequence of penetrating trauma, neoplasms, and regional inflammation. Surgical repair of fistulas and leaks is notoriously difficult, and has a high rate of complications and morbidity.

Percutaneous nephrostomy and stent placement offer effective treatment and provide better results than primary surgery. This technique has been well known for years.

In cases where fistula cannot be crossed with a guide wire by percutaneous or transureteral endoscopic approach, we suggest our combined new technique. Our idea was to capture the guide wire in fistula with endoscope when the whole ureter is affected by fistula, and thus all antegrade and retrograde attempts at wire passage fail. In such case it may be possible to place the guide wire into the fistula percutaneously and capture it with transureterally introduced endoscope. Once the wire is passed through the area of the fistula a stent can be placed over the wire.

The new technique was succesful in use of 7 patients with 9 fistulas complicating Wertheim operation. In two patients the fistulas were bilateral.

Primary technical and clinical success judged by fistulas sanation was $100 \%$. Complete heading of fistulas was observed in 6 patients $(85,5 \%)$. In one patient, unilateral hydronephrosis followed healing of the fistula. The stenosis was removed surgically. No recurrence of fistula or stenosis was noted in 6 months follow up of treated patients.

\section{$12.00 \mathrm{AM}$}

1120 Treatment of varicocele by coaxial coil embolization of the spermatic vein

Ine-Marie Obdeyn, Jelle O. Barentsz; Nijmegen

Purpose: The purpose of this study was to evaluate the results of spermatic vein occlusion by coil embolization as a first choice treatment for varicocele.

Methods and Materials: In an outpatient procedure 40 men with clinical or subclinical varicocele were referred for venography and embolization.

Venography was obtained via transfemoral catheterization by placing a 6.7 French Marsman catheter near the left renal hilus. For embolization the tip of the Marsman catheter was positioned in the spermatic vein ostium and multiple coils were introduced through a coaxial 3 French catheter into the spermatic vein.

Results: Embolization by mini-coils was a simple outpatient procedure which caused no discomfort for the patient. The embolization was technically successful in 34 of the 39 cases $(87 \%)$. In 1 patient there was no varicocele and in 5 cases it was not possible to place a coil. Seventy-five percent of the patients showed improvement of the semen quality. We saw a recurrent varicocele in 3 patients ( $8 \%)$. Except for a minor extravasation in 2 patients, which did not influence the embolization, no complications were seen.

Conclusions: Coil embolization of the spermatic vein is a safe and effective outpatient treatment of a clinical or subclinical varicocele. Surgical ligation is indicated only when embolization is not possible.

\section{Room N}

\section{SS} $10.30 \mathrm{AM}$

\subsection{Neuro - head, spine, spectro}

\section{Computer tomography and MR-tomography in tuberosis} sclerosis

H. Hadidi, P. Benz, U. Hau, H.-J. Richter, A. Seeliger, G. Brill, W. Dewes, U. Piepgras; Homburg/Saar

9 children (6 months- 7 year old) with tuberosis sclerosis were examined in magnetic resonance (MR) and computer tomography (CT) since 1987.

4 characteristic types of lesions were observed in MR

All children were epileptic, 4 children had tumors of the heart, 3 children had tumors of the retina, only in 3 children changes of the skin were visible, 7 children were mentally or physically retarded, 2 children had vessel or skeletal changes.

MR-tomographie is able to detect even early changes, which has therapeutic and humangenetic consequences.

$10.40 \mathrm{AM}$

\section{MRI of intracavernous meningiomas}

G. Dooms, P. Mathurin, G. Cornélis; Brussels

Purpose: A retrospective study was performed to assess the value of MRI for demonstrating intracavernous meningiomas and their full extent.

Methods: Fifteen patients ( 6 males and 9 females, mean age: 54 years) were included in the study; final diagnosis was confirmed by biopsy or partial surgical resection for every patient. MR was performed with a superconducting magnet Philips Gyroscan S15 operating at 1.5 Tesla. T1 weighted images $(\mathrm{TR}=0.415 \mathrm{msec}$ and $\mathrm{TE}=20 \mathrm{msec}$ ) were performed in the three orthogonal planes, before and after contrast medium (Gd-DTPA) IV injection in every patient. T2 weighted images $(\mathrm{TR}=2.18 \mathrm{sec}$ and $\mathrm{TE}=50$ and 1000 msec) were performed in 5 patients.

Results: Intracavernous meningiomas were identified as cavernous sinuses enlarging masses presenting with signal intensity similar to that of normal brain tissue on both $\mathrm{T} 1$ and $\mathrm{T} 2$ weighted images ( 13 cases), and enhancing after Gd-DTPA IV injection (15 cases). In 13 patients, they induced a concentric stenosis of the lumen of the intracavernous portion of the carotid artery and 
extended outside the lateral wall of the cavernous sinus. In 11 patients, the gasserian ganglion was unidentified on the homolateral site of the intracavernous meningioma and tumor extended along the free edge of the tentorium cerebelli.

Conclusions: In conclusion, MRI is the first imaging modality for studying parasellar masses, including intracavernous meningiomas and for showing their full extent.

\section{$10.50 \mathrm{AM}$}

1123 The use of image analysis in monitoring the progression of the cerebral atrophy changes in children infected with the HIV virus N. Roberts, M.W. Bourne, G. H. Whitehouse; Liverpool

Children infected with HIV develop a progressive encephalopathy in 30-50\% of cases. This manifests as loss of developmental milestones, impaired brain growth and progressive motor dysfunction. On MRI this is seen as marked diffuse cerebral atrophy with sulcal and ventricular dilatation. A reduction in these changes has been demonstrated after treatment with anti-viral agents. The progression of cortical and deep atrophy changes as shown by MRI correlates well with the progression of neurological dysfunction. Using maximum likelihood image classification techniques, training areas comprising of fewer than $1 \%$ of the field of view have been applied to multiple echo (i.e. TE 30 and TE 80 msecs.) images of a $5 \mathrm{~mm}$ thick axial slice at the level of maximum ventricular cross section. This technique divides the pixels in the field of view into two classes; i.e. brain tissue and CSF. Additional image analysis techniques, using a line detection algorithm and binary morphological operations are used to determine the inner boundary of the skull, and pixels outside of this boundary are excluded from the analysis which follows. The ratio of internal to external CSF space, and the ratio of CSF space to brain substance at the level of the image are determined and changes in these ratios, between examinations at 6 monthly intervals over a period of two years have been monitored. The degree of atrophy measured by this method is correlated with the neurological complications and antiviral treatment.

\section{$11.00 \mathrm{AM}$}

1124 Optimization of MR sequences: when fast scans should be used?

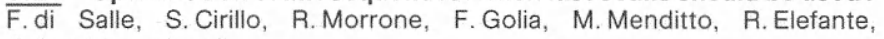
F. Smaltino; Napoli

The Authors examine limits and advantages of Fast imaging MR sequences, in comparison with Spin-Echo images, and they try to point out when a fast sequence cold be useful to the diagnostic evaluation of the main brain pathologies.

A careful "in vitro" experience has been carried on test objects with well-known relaxometric characteristics, utilizing both Fast sequences and Spin-Echo sequences. Spin-Echo signal intensity had a good correlation with $\mathrm{T} 1$ and T2 values of test tubes; data obtained by echo-gradient did not show a similar linear correlation with relaxation parameters.

This non-linear correlation between signal intensity and relaxation parameters in fast imaging could limit the clinical usefulness of echo-gradient sequences. On the other hand the high sensibility to magnetic field dishomogeneity of echo-gradient scans can be useful when it is expected to find out any pathology (i.e. angiomas, haemorrhage) stirring up a local magnetic dishomogeneity. Spin-Echo and Gradient-Echo diagnostic effectiveness is thus compared in the main brain pathologies to assess the role the latter can have in the diagnostic algorithm.

Further useful application of fast sequences are considered such as the study of vascular pathology by flow selective fast images.

\subsection{AM}

1125 A comparison of single voxel versus CSI techniques for localized ${ }^{1} \mathrm{H}$ MRS of the human brain

R. Sauter, M. Schneider, K. Wicklow, H. Kolem; Erlangen

Single voxel techniques based on the stimulated echo (STEAM) or second spin echo (SE) provide a high degree of localization and spectral resolution. Chemical shift imaging (CSI) techniques offer the possibility of spatial mapping of metabolites. It has been the purpose of this study to evaluate the performance of CSI and single voxel techniques with respect to localization, sensitivity and spectral resolution.

Experiments on phantoms and volunteers have been carried out on a $1.5 \mathrm{~T}$ whole-body MR scanner (SIEMENS Magnetom) using the standard circularily polarized head coil. The STEAM-, SE- and CSI-localization techniques have been implemented and compared for the echo times TE $=270 \mathrm{~ms}, 135 \mathrm{~ms}$ and $40 \mathrm{~ms}$. The typical spatial resolution was $8 \mathrm{ml}$. For the CSI experiments a large VOI (typically $80 \times 80 \times 20 \mathrm{~mm}^{3}$ ) was preselected with the SE-technique to avoid contamination from subcutaneous fat.

Results of phantom and volunteer measurements show similar sensitivity for CSI and single voxel techniques when echo times of $270 \mathrm{~ms}$ or $135 \mathrm{~ms}$ are used. The SE-technique shows the expected twofold improvement in SNR when compared to the STEAM-technique. For TE $=40 \mathrm{~ms}$, single voxel techniques are superior in sensitivity and spectral resolution, while the CSI spectra are affected by residual eddy currents. Furthermore, CSI spectra show decreased spectral resolution in locations near strong susceptibility changes (frontal sinus, auditory canal). Single voxel techniques show a clear superiority with respect to the delineation of the VOI.

We conclude that CSI techniques are especially useful for follow up studies of cerebral infarction and therapy, where the spatial distribution of lactate, cholines, creatine and NAA is of primary interest. Providing optimal delineation of the VOI and access to a larger number of cerebral metabolites, short TE single voxel techniques will be preferred for biochemical studies.

\section{$11.20 \mathrm{AM}$}

\section{Localized 1H MR-spectroscopy in acute stroke} S. Felber, F. Aichner, G. Birbamer, F. Gerstenbrand; Innsbruck

Initial applications of $1 \mathrm{H}$ MR-Spectroscopy (MRS) have shown potential to observe ischemic metabolism in-vivo. This study was initiated to assess the impact of 1 H MRS into the routine diagnosis of acute stroke.

MRS was performed not later than 8 hours after ischemic stroke in 10 patients. Spectra of 8 volunteers and 8 chronic infarctions served as base-line. All exams were performed on a $1.5 \mathrm{~T}$ system (Siemens, FRG). The protocol consisted of $\mathrm{T} 1$ and $\mathrm{T} 2$ weighted sequences followed by shimming on the volume of interest (VOI) and a 3-12 min acquisition of localized spectra using a stimulated echo sequence (STEAM, TR: $1500 \mathrm{~ms}$, TE: $270 \mathrm{~ms}$, NEX: 128-512).

All spectra had sufficient signal/noise and resolution for evaluation. T2 weighted images were mandatory to target MRS in acute ischemia. Lactate was present in acute infarctions, reflecting anaerobic glycolysis, up to 2 weeks in follow-up. $\mathrm{N}$-acetyl-aspartate (NAA) as indicator of viable neurons, decreased according to size and duration of ischemia. Cholines and Creatine/phosphocreatine maintained volunteer levels in the acute phase.

$1 \mathrm{H}$ MRS can routinely extent diagnostic information from morphology to biochemistry and holds promise to monitor pharmacologic effects for an improved management of stroke in future.

\subsection{AM}

1127 Localized brain proton MR spectroscopy of chronic MS patients $\bar{P}$. Van Hecke, K. Johannik, C. Van Ongeval, S. Verellén, P. Demaerel, G. Marchal, G. Wilms, H. Carton, A. L. Baert; Leuven

Purpose: To investigate the origin of the altered $\mathrm{N}$-acetyl-aspartate (NAA), phosphocreatine + creatine $(\mathrm{PCr}+\mathrm{Cr})$ and choline $(\mathrm{Cho})$ peak intensities in brain localized proton spectroscopy of chronic MS patients.

Methods: $\mathrm{N}$-acetyl-aspartate, creatine and choline peak intensities were measured at $1.5 \mathrm{~T}$ in 22 chronic MS patients and 17 healthy volunteers, using the STEAM localized proton spectroscopy method. Possible changes in metabolite $\mathrm{T} 1$ and $\mathrm{T} 2$ relaxation times were investigated using different values of TR and TE.

Results: The ratios NAA/Cho and NAA/Cr were significantly smaller (factor $0.79)$ in the MS than in the normals $(\mathrm{p}<0.001)$; the ratio of $\mathrm{Cho} / \mathrm{Cr}$ was not significantly altered $(p>0.5)$. Spectra did not reveal meaningful lactate or lipid peaks. No significant difference was found between the relaxation times T] and T2 of the MS $(n=5)$ and of the control group $(n=6)(p>0.5)$.

Conclusions: The decrease in the metabolite ratios NAA/Cho and NAA/Cr is not a relaxation effect but is the result of metabolite concentration changes. Since the ratios NAA/Cho and NAA/Cr are reduced by the same factor and the ratio $\mathrm{Cr} / \mathrm{Cho}$ is unchanged, the reduced ratio is attributed to a decrease of NAA concentration in the plaque containing tissue of chronic MS patients.

\section{$11.40 \mathrm{AM}$}

$\frac{1128}{\text { brain }}$

P. R. Luyten, J. A. den Hollander, J. Bunke; Hamburg

Purpose: To observe noninvasively the spatial distribution of metabolite levels in the human brain.

Methods: All measurements were performed on a regular $1.5 \mathrm{~T}$ whole body MR imager. Water suppression was achieved by a selective adiabatic inversion pulse at the water frequency. For spectroscopic imaging a combination of spatially selective excitation and phase encoding in two orthogonal directions was implemented. Threedimensional volume selection was used to obtain both slice selection and suppression of the very intense lipid signals originating from subcutaneous fat and bone marrow. Whole Hahn echoes were acquired using a $90^{\circ}-180^{\circ}-180^{\circ}$ sequence. Slice widths of 1.5 to $2.5 \mathrm{~cm}$ and $32 \times 32$ phase encoding steps over a field of view of $22.5 \mathrm{~cm}$ yielded nominal voxel sizes of about 1 cc. Metabolite images were reconstructed by calculating signal integrals for the resonances of interest and representing these values on a grey-scale.

Results: The implemented technique allowed the acquisition of 1024 spectra over a large region of the human brain in a single measurement. Metabolite 
maps of choline, creatine, $\mathrm{N}$-acetyl aspartate and lactate were reconstructed with an in plane resolution of $7 \mathrm{~mm}$. This opened the possibility to observe regional differences in metabolite levels for different parts of the brain. In clinical studies metabolic heterogeneity could be demonstrated for several kinds of lesions.

Conclusions: The capability of $1 \mathrm{H}$ MR spectroscopic imaging to detect the spatial distribution of metabolite levels in the human brain makes it a valuable tool in the examination of patients.

\section{$11.50 \mathrm{AM}$}

\section{Quantitative 31P MR spectroscopy of the normal brain} R. Longo, C. Ricci, R. Vidimari, A. Giorgini, C. M. Segebarth, L. Dalla Palma; Trieste

Purpose: To determine the spectral characteristics of the 31P MR brain spectra obtained from a control population composed of adult healthy volunteers. Introduction: 31P MR spectroscopy has been applied extensively to assess energy metabolism of tissues in humans. 31P MR studies of infant brain, in particular, have shown that the cerebral $31 \mathrm{P}$ MR spectra vary considerably during brain maturation. Considering that the establishment of the spectral characteristics of a normal population is a key step towards eventual clinical applications of cerebral 31P MR examination, we have assessed whether any variations with age of the spectral patterns could also be observed on the $31 \mathrm{P}$ MR spectra obtained from the adult brain.

Methods: $1 \mathrm{H}$ image-guided 31P MR spectra were obtained from the brain of 41 healthy volunteers. Age ranged between 25 and 85 years. The spectral parameters were determined by means of a time domain fitting routine. Standard deviations of the parameters were obtained by the Cramer-Rao theory.

Results: There is a significant albeit small age dependency of the 31P MR spectral patterns of adult brain. A significant increase with age is found in the phosphocreatine line area, $\mathrm{pH}$ exibits no age-dependency. The accuracy of the spectral parameters is such that significant differences can be detected between individuals from a same age-group.

Conclusions: 31P MR metabolites ratios have been determined for a population of normal volunteers, showing a slight increase with age in the normalized phosphocreatine line area.

\subsection{AM}

\section{Magnetic resonance imaging in eclampsia}

G. Dooms, P. Mahieu, O. Vandenplas, P. Mathurin, G. Cornélis; Brussels

A retrospective study was performed to assess the initial and follow up MRI findings in patients with eclampsia. Three patients (age 24-40) were included in the study. They presented with particulary severe neurological manifestations, including marked brainstem dysfunction. In all three patients, initial MRI studies revealed multiple hyperintense areas on the T2 weighted images throughout the brain, basal ganglia and brainstem (suggestive for edema and/or ischemia). Focal intraparenchymatous hematomas were also identified in 2 patients. Despite these diffuse lesions, under adequate medical treatment, all three patients made a progressive neurological recovery and follow up MRI examinations demonstrated complete resolution of the hyperintense foci.

\section{Room 0}

\section{SS} $10.30 \mathrm{AM}$

1131 Transbrachial catheter angiography in supraaortic branches: advantages and limitations for out-patients A. Schwartz, M. Mull, A. Link, W. Rautenberg, A. Aulich; Düsseldorf

Purpose: Concerning the approach via the groin the transfemoral selective catheterization of the brain supplying vessels means a bleeding risk and immobilization for the patient. Experienced in retrograde brachial angiography we examined the advantages and limitations of transbrachial selective catheterization by small sized catheters in a follow-up study.

Methods: During one year transbrachial catheterization was carried out in 228 patients by a new 4 French catheter designed by Mallinckrodt under fluoroscopic control. The follow-up 3 to 6 months later (now 70 patients) inclosed neurological, angiological, and ultrasound (colour-coded Duplex sonography).

Results: We found in 5 cases a mild adverse reaction due to contrast media, in two patients with pior ischemic attacks TIA occured during examination. The selective approach to the supraaortic branches was possible without problems in all patients, even in those two cases with a left side common subclavian trunc. Major bleeding or median nerve lesions were not seen.

Conclusions: Transbrachial catheter angiography is an efficient and safe technique for selective imaging of supraaortic vessels in stroke-risk out-patients.

\subsection{AM}

1132 Intraarterial dynamic CT for treatment planning before regional chemotherapy or arterial tumor-embolization

A. Rieber, H.-J. Brambs; Tübingen

a) The effect of locoregional tumor-therapy depends on the complete perfusion or embolization of the tumor and the sparing of normal tissue. For the improvement of this concept the intraarterial dynamic CT was performed as a pretherapeutic monitoring.

b) The method was performed in 8 patients with inoperable non small lung cancer and in 8 patients with breast cancer before a planned regional chemotherapy and in 2 patients with liver tumors before tumor-embolization. The angiographic catheter was placed superselective in coaxial technique. c) The perfusion of normal tissue could be considerably reduced and a complete perfusion or embolization of tumor could be guaranteed. A partial deficiency of contrast enhancement suggested in 2 patients with lung cancer a second tumor-feeding artery, which could be perfused in a second angiography.

d) The intraarterial dynamic CT improves the treatment planning before a regional chemotherapy or tumor-embolization and visualizes, whether it is necessary to change the position of the angiographic catheter. The damage of normal tissue can be reduced and this of the tumor can be improved.

\section{$10.50 \mathrm{AM}$}

1133 Hepatic artery embolisation of non resectable hepatocellular carcinoma

A. Scholz, R. Langer, M. Langer, R. Felix, P. Neuhaus; Berlin

Purpose: In a prospective study 35 hepatic artery chemoembolizations were performed in 22 patients with non resectable hepatocellular carcinoma.

Methods and Materials: $70-120 \mathrm{mg}$ doxorubicin and cisplatin was mixed with 7-12 ml ionized oil to prepare a water-in-oil emulsion that was injected into the hepatic artery, after application of Spherex-particles.

Results: After chemoembolization CT-scans, including dynamic CT, were performed. Changes in blood chemistry were controlled. Patients complained about vomiting $(n=9)$, diarrhea $(n=5)$, and hair loss $(n=6)$. Leukocyte depression was registered 12-14 days after chemoembolization in 4 patients. Four weeks after therapy 13 patients felt better than before. The CT follow up revealed tumor regression in 15 , and ascites reduction in 7 patients.

Conclusions: We think, that even in multilocolar hepatocellular carcinoma and advanced tumor stage, intraarterial chemoembolization should be performed for tumor reduction. A subjective amelioration of patients complaints can be achieved.

\subsection{AM}

1134 Localization and radiological signs of deep venous thrombosis after elective total hip replacement - Phlebographic results of a multicenter trial

W. Krings, D. Gill, P. E. Peters; Münster

In a double-blind randomised multicenter trial efficacy and safety of two regimens for the prevention of postoperative venous thromboembolism - low molecular weight heparin (LMWH) CY 216 and unfractionated heparin (UH) - were compared in 341 patients undergoing elective total hip replacement. Deep vein thrombosis (DVT) was assessed by bilateral phlebography on day $14 \pm 1$ after surgery. Phlebography was successfully performed in 273 patients (136 patients of the LMWH group, 137 of the UH group). DVT occurred in 45 of 137 patients $(33,1 \%)$ treated with LMWH and in 47 of 136 patients $(34,3 \%)$ receiving $\mathrm{UH}$. The incidence of proximal DVT (popliteal and iliofemoral veins) was $10,3 \%$ in the LMWH group and $19 \%$ in the UH group. Isolated muscle vein thrombosis in the lower leg occurred in 10 of 136 patients treated with LMWH and 4 of 137 patients on the UH group. A (statistical) analysis of phlebographic pattern and extent of DVT is given for the two prophylactic regimens.

\section{$11.10 \mathrm{AM}$}

1135 Diagnostic application of $99 \mathrm{~m}$ Tc-HMPAO thrombocytes in patients (Pts) with pulmonary thromboembolism

I. Kostadinova, Chr. Hadgicostova; Sofia

The localisation of the source of the active thromboembolism is of a great importance for a surgical or conservative treatment. The aim of the work was, applying radionuclide labelled thrombocytes (Thr), to evaluate their diagnostic application in searching of an active thrombosis in Pts with proved pulmonary thromboembolism. In the all investigated $18 \mathrm{Pts}-$ at first - a lung scintigraphy with ${ }^{99 m}$ Tc-MAA was carried out for a localising of the area with 
an absent perfusion and subsequently - a whole body scintigraphy with ${ }^{99 \mathrm{~m}} \mathrm{Tc}$-HMPAO-autologous labelled Thr. In 14 of the Pts - an increased uptake of the label led cells in the blood vessels of the lower extremities or in cardiac chambers was registered and only in 3 Pts there was a slightly increased uptake in the big vessels of the lung. We have considered, that the relatively long period between the onset of the desease (from 2 weeks to 2 months) from one side, and the impossibility of a cessation of the treatment with oral anticoagulants in some of the Pts at that period, are the main factors, depressing active uptake of the cells in the lung. It was concluded, that this sequence of radionuclide methods - lung perfusion scintigraphy and whole body scintigraphy with labelled Thr, was a helpful strategy for a localization of active thromboembolism and choosing of an appropriate treatment.

\section{$11.20 \mathrm{AM}$}

1136 Adrenal venous sampling analysis of primary aldosteronism: value of ACTH stimulation in the differentiation of adenoma and hyperplasia

Koh Tokunaga, Tsutomu Hashimoto, Norio Hirabuki, Hironobu Nakamura, Chikazumi Kuroda, Takahiro Kozuka; Osaka

Purpose: To determine the value of ACTH stimulation in the differentation of adenoma and bilateral hyperplasia.

Methods and Materials: Selective vadrenal venous blood sampling before and after ACTH stimulation was performed to evaluate aldosterone assay in patients consisting of 25 patients with unilateral adenomas and five with bilateral hyperplasia. Our sampling techniques were; 1 . to obtain blood from both adrenal veins selectively, 2 . to infuse intravenously in five minutes a half amount of $0.25 \mathrm{mg}$ of $\mathrm{ACTH}$, and 3. to repeat the sampling from both adrenal veins during a continuous infusion of the remaining ACTH solution.

Results: Before ACTH stimulation, ratios of aldosterone level in the blood in adenoma side to that in the normal side ranged from 1.52 to 115.9 (mean 35.3). Ratios of the aldosterone level in the higher side to the lower side in bilateral hyperplasia ranged from 1.04 to 6.73 (mean 3.16). After ACTH stimulation, ratios in the adenoma ranged from 2.05 to 1098 (mean 98.8), and those in the hyperplasia ranged from 1.27 to 2.3 (mean 1.75). These results indicate that by $\mathrm{ACTH}$ stimulation, the ratios increase in a patient with adenoma and decrease in a patient with hyperplasia.

Conclusions: ACTH stimulation is useful in the differentiation of adenoma from bilateral hyperplasia.

\subsection{AM}

\section{Fallopian tube recanalization}

D. Kelekis, I. Fezoulidis, Th. Petsas, A. Tsapalira, N. Kelekis; Patras

Purpose: To evaluate the efficacy and safety of transcervical recanalization of obstructed fallopian tubes.

Methods: Transcervical recanalization of obstructed fallopian tubes was performed in 20 patients. A coaxial catheter system was used and the procedures were carried out on a digital subtraction apparatus.

Results: Recanalization of one or both obstructed fallopian tubes was achieved in $18(90 \%)$ out of 20 patients. In a mean follow up period of 12 months $9(50 \%)$ pregnancies were recorded.

Conclusions: Selective transcervical tube recanalization, compared to laparoscopy and laparotomy, is a quick, safe, less invasive and less expensive treatment of fallopian tube obstruction. It eliminates the need of reconstructive tubal surgery in selected patients. The use of the road map facility of the digital subtraction apparatus is very helpful.

\section{$11.40 \mathrm{AM}$}

\section{The radiology of Behcet's disease}

Tariq Linjawi, M. Banna, A. Dalaan; Riyadh

Behcet's Disease, originally described as a triad of iridiocyclitis, oral and genital ulcerations, is presently considered a rare multisystem immune related vasculitis. The disease is more prevalent in the Mediterranean, Middle East and Japan. This paper presents our radiological findings in 46 patients. The age range is between 15-58 years and the male to female ratio was $4.1: 1$.

Pulmonary findings were present in $25 \%$ and consisted of pulmonary artery aneurysms with or without infarction in four patients, occlusion of the lower lobe vessels in one patient and high probability VQ mismatch in four patients. Neuroradiological findings were present in $44 \%$ and in these, CT was non contributory except in one patient with dural sinus thrombosis. MRI in 9 patients showed foci of high signal intensity on T2 weighted images within the brainstem, basal ganglia and cerebral hemispheres, with no predilection to involve the periventricular area. Cardiovascular involvement was present in $18 \%$. Of these, there were two cases with MRI documentation of right ventricle and right atrium thrombosis, two cases with thoracic aortic aneurysms and four cases with IVC thrombosis.

\section{$11.50 \mathrm{AM}$}

1139 Analysis of aspiration after cerebral injury Chr. Hannig, A. Wuttge-Hannig, M. Hörmann; München

a) Considering the fact, that $6 \%$ of all patients suffering from a cerebrovascular injury die from aspiration pneumonia within the very first year, the analysis of the pathomechanism of chronic aspiration becomes of crucial importance also for the further therapeutic

b) work-up. The high temporal resolution of High-Speed-Cineradiography (HFK) using 50 frames/sec. provides the possibility to recognise the various motoric events during the

c) short period of $0.7 \mathrm{sec}$., in which pharyngeal swallow is accomplished. Five case-examples are presented (out of the neurological impaired patient's group, $\mathrm{n}=143$ ) illustrating the possibility to differentiate between three types of aspiration by means of cineradiography. These types are the so-called "pre-, intra- and postdeglutitive aspiration". This differentiation turned out to be of crucial therapeutic importance. Frame-by-frame analysis of the disturbed deglutition allows to distinguish between episodes of aspiration occurring before, during or after the triggering of the swallowing reflex.

d) Moreover it is possible to analyse the disturbances of the pharyngo-laryngeal motility and the temporal coordination which helps to elaborate for each patient an individual surgical and/or conservative program for rehabilitation.

\section{$12.00 \mathrm{AM}$}

\section{Collateral lymphatic circulation in the region of pelvis} J. Bruna, O. Ishida; Prague, Osaka

Collateral lymphatic circulation is not only one of the most interesting fields of the lymphographic diagnosis but also clinically important finding. Collateral lymphatic circulation depends on the topographic localization of the lymphatic obstruction, on the anatomical variation of the lymphatic and venous systems. We studied the collateral lymphatic circulation on diseases of protein-loosing enteropathy (20 patients), filariasis (41 patients) and on malignancy of lymph nodes (more than 1000 patients) using direct oil lymphography. The lymphatic collaterals were classified as typical and non-typical. The typical lymphatic collaterals are the perivisceral, the parietal, and the subcutaneous ones. The filariasis and malignancy (metastatic involvement of lymph nodes) produce in addition to these the non-typical collaterals as lymphovenous communications and internal lymphatic fistulas. The different types of the collaterals are visibly demonstrated only during the filling phase of the direct oil lymphography. We shall demonstrate the different types of the lymphatic collaterals.

\section{$12.05 \mathrm{AM}$}

\section{Clinical utility of to-and-fro dynamic CT}

Vassilios Raptopoulos, Michael Caputo, Barbara Biber; Boston, Worcester, MA

Purpose: We evaluated the utility of a CT technique which combines dynamic scanning without and dynamic scanning with table incrementation.

Methods: Technique: CT images are obtained in rapid sequence over several consecutive levels, scanned repeatedly back and forth during administration of intravenous contrast material. Up to 30 images can be obtained. Usually areas of $3-5 \mathrm{~cm}$ in length are scanned 4 to 6 times over a period of $2-4 \mathrm{~min}$.

Results: The technique provided useful information of intravenous contrast material dynamics over large regions (e.g. large or multiple lesions) or when exact pre-contrast localization of an abnormality is difficult (e.g. small or isodense lesions, uncooperative patients). It was used in over 250 patients and provided useful information in a variety of abnormalities, including vascular anomalies $(n=32)$, differentiation of vessels from masses $(n=26)$ and distinction of vascular malformations from solid masses $(\mathrm{n}=21)$.

Conclusions: To-and-fro dynamic CT is a valuable and simple technique which in selected cases may provide information otherwise unobtainable or requiring notable expertise, critical planning and considerable effort.

\section{Room R}

\section{SS}

16.11 Importance of fractionation and dosage in radiotherapy with a view on results and sequelae I

10.30 AM

1142 The importance of fractionation in radiation therapy with a view to sequelae and results (Keynote Lecture)

Luther W. Brady; Philadelphia, PA

Recent clinical trials using radiation therapy alone have been directed toward increasing the effectiveness of the treatment regimens. This concept has 
emerged as a consequence of the difficulties in controlling more advanced and larger volume disease.

Altered fractionation techniques represent a major area of investigation to improve the potential for local regional control. These may include split course treatment, hyperfractionation, accelerated hyperfractionation and concomitant boost to radiation therapy.

The preliminary results from these clinical trials clearly indicate the advantages that accrue to these techniques. These data will be presented along with the sequelae for complications.

\section{$10.50 \mathrm{AM}$}

1143 The importance of the time factor in the response of normal and malignant human tissues to dose protraction (Keynote Lecture)

J. H. Hendry; Manchester

Current estimates of the increases in isoeffective dose with increasing overall time of treatment for normal tissues and tumours will be reviewed. Examples will include: a) the Royal College of Radiologists survey among 172 U.K. radiotherapists regarding variations in tolerable dosage prescriptions for 6 case types. For radical treatments of breast and vocal cord, the analysis of the prescriptions showed a high $(\alpha / \beta)$ ratio $(\sim 40 \mathrm{~Gy})$ with a time factor $(\gamma / \alpha)$ of about $0.6 \mathrm{~Gy} /$ day.

b) a series of head and neck results from the Christie Hospital, showing also a very high $(\alpha / \beta)$ ratio for tumour control and a significant time factor of about $0.8 \mathrm{~Gy} /$ day from 9 to 42 days overall time.

\subsection{AM: Discussion}

\subsection{AM}

1144 How many equivalent factors are necessary changing a LDR-into an HDR-brachytherapy?

R. Schulz-Wendtland, M. Bauer, F. Richard; Freiburg

The steep decrease of dose and dose rate in brachytherapy implies very different radiobiological considerations of the biological effectivity. Therefore, in imitation of the clinical procedure, we compared the LDR-and HDR-brachytherapy. We carried out experiments on epidermoidal cervix carcinoma cells (Ca Ski-cells) and human primary keratinocytes obtained after transfection with human papillomavirus type 16 DNA varying the dose rate (27 $\mathrm{cGy} / \mathrm{h}-8.000 \mathrm{cGy} / \mathrm{h}$ ), the dose $(1 \mathrm{~Gy}-100 \mathrm{G}$ ) and fractioning (protracted, 3,6 , 12 and 18 fractions).

Our conclusions are:

- Changing a LDR-into an HDR-brachytherapy the equivalent factors close to the source have to be selected low and with increasing distances from the source high respectively higher.

- The reduction of the dose in HDR-radiation-therapy is a compromise in order to limit side effects caused by a radiation. The trade-off is a small therapeutic range and reduced therapeutic effectivity at the tumour.

- Each increase in the dose rate requires higher fractionation but perhaps it is not possible to replace an LDR-radiation by an HDR-radiation by a high fractionation because tumour cells and healthy cells show different reactions. - The percutanous dose at the pelvis wall has to be reduced if at the same time an HDR-brachytherapy will be carried out - to avoid side effects.

\section{$11.30 \mathrm{AM}$}

1145 Late effects of radiotherapy: a comparison between brachytherapy and superficial X-irradiation in the treatment of skin cancers

D. A. L. Morgan; Nottingham

Radiation damage to late-reacting tissues from low-dose-rate continuous irradiation would be expected theoretically to be less marked than from an equivalent treatment, in terms of tumoricidal effect, given as a small number of large fractions of x-rays. To assess whether this is borne out in practice, we conducted a retrospective study.

Twenty-nine patients were traced who had been treated for skin cancer at least eight years previously (range $8-32$, mean 18 years) and had been continuously disease-free. Of these, 14 had been treated by brachytherapy, using moulds, 15 by $116 \mathrm{kV}$ x-rays. Both modalities are known to be highly effective, and comparably so, in terms of cure. Late skin changes were scored by two observers independently, using EORTC criteria; means were taken when the two observers differed (in three cases, none by more than one grade).

The mean late damage score of the brachytherapy group was 1.53 , and none scored higher than 2 . The mean score of the $\mathrm{x}$-irradiation group was 2.43 , significantly worse $(p<0.001, t$ test).

This study bears out, in clinical practice, the predicted superiority of brachytherapy in terms of late damage.
$11.40 \mathrm{AM}$

1146 Accelerated radiotherapy (RT) and concomitant chemotherapy $\overline{(C T)}$ in head and neck cancer (H\&NC): seven years Munich experience T. G. Wendt, T.P. U. Wustrow, A. Schalhorn; Munich

Altered fractionation of RT and simultaneous CT are employed to overcome the resistance of locally advanced H\&NC. In a prospective phase II study patients (pts.) were treated by three cycles of cis-platinum $60 \mathrm{mg} / \mathrm{sqm}$ day 1 , 5-FU $350 \mathrm{mg} / \mathrm{sqm}$ day 1 i.v. bolus, leucovorin (LV) $50 \mathrm{mg} / \mathrm{sqm}$ day 1 i.v. bolus, 5 -FU $350 \mathrm{mg} / \mathrm{sqm} / 24 \mathrm{~h}$ and $\mathrm{LV} 100 \mathrm{mg} / \mathrm{sqm} / 24 \mathrm{~h}$ days $1-5$. RT is given with two fractions (fr.) per day, $13 \mathrm{fr}$. of 1.8 Gy each per cycle are administered from day 2 to day 10 . This regimen is repeated on day 22 and 44 . Total dose of RT is $70.2 \mathrm{~Gy} / 39 \mathrm{fr} / 8$ weeks. Acute bone marrow and mucosal toxicity was acceptable (J. Clin. Oncol. 7, 471, 1989).

From 1984 to 1989109 pts. were treated, 4 pts. died during therapy, 105 (male : female $=94: 11)$ evaluable pts. had primaries (T2: 4, T3:34, T4:67) of the hypopharynx (26), oropharynx (44), oral cavity/floor of mouth (16) and supraglottic larynx (19) and were followed up from 12 to 67 mos. 45 pts. died from uncontrolled cancer (median survival: 5.5 mos.). 32 failed locally or regionally or at both sites (median latency: 6.5 mos.), 12 pts. experienced distant spread. At 3 yrs. corrected survival was $50 \%$, local control $70 \%$ and node control $77 \%$ (Kaplan Meier). N3 $(n=15)$ was significantly less frequently controlled than N1-N2C $(n=64)[p<0.01]$. Site of primary, age and gender were not found prognostic variables. Disease free survival will be analysed with respect to the drug doses administered and unscheduled treatment breaks. A randomized protocol to evaluate the value of chemotherapy will be outlined.

\section{$11.50 \mathrm{AM}$}

1147 Post treatment acute toxicity after different curative strategies for Hodgkin's disease

K. Dieckmann, S. Sehlen, D. Matthaei, H. Kirschner, M. Busch, V. Diehl, E. Dühmke; Göttingen

Acute toxicity following treatment of lymphoma arises as a cumulative effect of different therapeutic models in use. Its importance is only scarcely understood. Thus acute toxicities can either be interpreted as negative side effects or as a measure of the curative late results that can be expected.

The german Hodgkin's disease study comprises an extended field radiotherapy for lower stages (HD4), whereas higher stages of the disease are treated in a combined manner in two different studies (HD5, HD6). Here first observations are reported, that for 260 patients show the amount of anemia, leucopenia, pharyngitis, and nausea after radiotherapy alone and after radiotherapy following two (HD5) or four (HD6) courses of chemotherapy. The amount of acute toxicity as expected was higher in combined therapy (leucopenia $46 \%$ (HD5) and 23\% (HD6)) than in radiotherapy alone (leucopenia 12\% (HD4)). In combined strategies the toxicity seems to depend from the size of the volume of radiotherapy used ((HD5) extended $=45 \%$ vs. (HD6) involved field $=$ $23 \%$ ). Comparable effects could be seen for the other above parameters. It should be mentioned, that these results do not represent parameters of the effectiveness of treatment. However, if these study results are arising the knowledge of acute toxicities can probably influence the therapeutic strategies with respect of patient comfort. An interpretation of the results is given with respect to acute toxicities reported in other Hodgkin's disease studies.

\section{Room S}

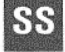

\subsection{Miscellaneous}

$10.30 \mathrm{AM}$

1148 Dialysis associated destructive spondylarthropathy of the atlanto-axial region: demonstration of $\beta_{2}$-Microglobulin related amyloid deposits by MRI

A. Stäbler, G. Kröner, M. Seiderer, W. Samtleben, J. Lissner; Munich

Purpose: Patients receiving chronic hemodialysis can develop severe bone and joint diseases due to amyloid deposits, which are of $\beta_{2}$-microglobulin origin. The dialysis associated spondylarthropathy mostly affects the cervical spine, but no case of manifestation in the atlanto-axial region has been reported.

Methods: We investigated the atlanto-axial region of 6 patients, 17,8 years (mean) on hemodialysis by MRI. Sagittal and transverse Tl-w $(\mathrm{TR} / \mathrm{TE}=$ $500 \mathrm{~ms} / 17 \mathrm{~ms})$ and opposed long TR GE images $(\mathrm{TR} / \mathrm{TE} / \alpha=$ $\left.500 \mathrm{~ms} / 17 \mathrm{~ms} / 90^{\circ}\right)$ were obtained $(\mathrm{sl}=4 \mathrm{~mm}, 1$. OT, Magnetom, Siemens $A G)$.

Results: In 5 of 6 patients we found soft tissue around the dens axis with high signal on GE-images. In 3 of these patients the amyloid deposits caused destruction of the dens, the patients had severe neurological symptoms (tertraspastic) due to cord compression. $\beta 2 \mathrm{~m}$-related amyloid could be confirmed in one case by operation, in another case by autopsy.

Conclusions: MRI is highly sensitive to show amyloid deposits in the 
atlanto-axial region, so patients on chronic hemodialysis with signs of cervical myelopathy should be investigated by MRI. Opposed long TR GE-sequences are of great value. Instabilities can be shown by MRI in ventral flexion.

$10.40 \mathrm{AM}$

1149 Quality assurance in radiology: concepts for large multicenter studies

Claus-C. Glueer, Michelle J. Estilo, Kenneth G. Faulkner, John D. Rosin, Harry K. Genant; San Francisco, CA

Purpose: Large scale clinical trials often require sample sizes that exceed the capacity of individual hospitals or research centers. If carried out in a multicenter setting, quality assurance (QA) programs need to be implemented to ensure cost-effectiveness and optimum inter-center compatibility.

Methods: We have developed QA concepts for radiologic studies (e.g. for osteoporosis research). These include radiologic QA tests for qualitative assessments (e.g. interpretaton of radiographs), semi-quantitative evaluations (e.g. scores based on visual interpretation of radiographs), and quantitative image evaluation techniques (e.g. Quantitative Computed Tomography, Dual-Energy X-Ray Absorptiometry).

Results: Currently, the ORG is monitoring the quality of radiologic examinations of multiple clinical trials in more than 110 centers. QA measures have revealed differences ranging from $25 \%$ to more than $200 \%$ in the reliability of bone mineral density measurements of the spine in centers using identical radiologic equipment. Implications for sample size estimations will be given. Conclusions: Based on the observed inter-center differences, a need for rigorous QA measures can be deduced. Implementation of QA measures may reduce sample size requirements. Optimum results are obtained if QA measures are supervised by an independent QA center with an excellent scientific reputation, managerial capacities, and computerized data management facilities.

\section{$10.50 \mathrm{AM}$}

\section{Phosphor plate computed radiography system in musculos-} keletal imaging

Mark D. Murphey, James L. Quale, Norman L. Martin, Larry T. Cook, Samuel J. Dwyer III; Kansas, KS

Purpose: Phosphor plate computed radiographic (CR) systems have numerous advantages compared to conventional screen-film methods. These include increased film latitude and improved image storage, retrieval, transfer, and processing. Our purpose is to evaluate the application of this technology to musculoskeletal examinations.

Methods: From January 1989 to the present over 200 musculoskeletal examinations have been collected on our CR system. Comparison to an equivalent number of screen-film technique examinations using ROC analysis was performed. Examinations included lateral thoracic spine (40), lateral cervical spine (60), Swimmers views of cervical spine (40), scoliosis (40), and lateral "shoot through" hips (20). Exposure dose variations were available for cervical spine and scoliosis techniques.

Results: The results of the ROC analyses reveal that no significant difference in diagnostic quality existed between screen-film and phosphor plate examinations. This was achieved at 30 to $50 \%$ reduction in exposure dose compared to screen-film techniques. The interactive image workstation component of the CR system provided an effective method to modify display parameters and can be used to salvage improperly obtained images.

Conclusions: In conclusion, the phosphor plate CR system provides high quality musculoskeletal images comparable to screen-film technology with $30-50 \%$ reduction of exposure dose.

\section{$11.00 \mathrm{AM}$}

1151 Photostimulable phosphor computed radiography in the study of the hand: comparison with high resolution technique

G. M. Giannatempo, M. G. Bonetti, M. A. Dicembrino, M. Cammisa; San Giovanni Rotondo

Computed Radiography (CR) is a newly developed technique that employs a phosphor photostimulable imaging plate instead of conventional film/screen combination. The Authors applied direct magnification CR to hand study; a Toshiba TCR 201 system was used together with high resolution Imaging Plates (matrix: $1770 \times 2370 \times 10$ bits; pixel size: $0.1 \times 0.1 \mathrm{~mm}$ ) and $2 \times$ direct magnification. Results were compared with conventional radiography (high MTF industrial type film, without screens, $5 \times$ optic enlargement reading). In both cases, a mammographic unit was employed for film-taking.

40 pts. (bearing renal osteodistrophy, psoriatic arthropathy or rheumatoid arthritis) and 20 normal subjects underwent the study. Digital images were processed by a particular characteristic curve and spatial filtering with unlinear unsharp masking.

$\mathrm{CR}$ hand images allowed good visualization of small lesions (sub-periosteal resorption, cortical bone tunnelling, minute periosteal neostosis), not inferior to conventional technique. CR has, moreover, adjunctive advantages over conventional technique: low biological costs, wide exposure and registration range, image post-processing capabilities, easy archiving and networking.

$11.10 \mathrm{AM}$

1152 Comparative imaging of tendons in the horse distal forelimb J.-M. Denoix, N. Crevier; Maisons-Alfort

Purpose: The purpose of the study was to document tendon injuries with ultrasonography (US), radiology, and magnetic resonance imaging (MRI) and to compare the semiological datas offered by each imaging technique.

Methods and Materials: 15 isolated forelimbs were used. 7 of them were taken on horses examined for locomotor diseases and euthanasied. The other 8 limbs were collected in a slaughter-house and selected because of the evidence of lesions identified by inspection and palpation. Each lesion was documented by radiology, US and MRI. US scans were performed with a 7,5 MHz linear scan head and MR Images were made using T1 weighted sequence. After completion of the imaging procedures, anatomopathological studies were made on tendon injuries.

Results: Tendon injuries appeared to be more precisely documented with MRI than with US, especially at the metacarpophalangeal level. Chronic tendinitis with fibroplasia were characterized by hypoechoic areas on US scans and a high signal intensity on MR Images. Insertion tendinopathies gave abnormal findings on X-rays and US-scans but were more thoroughly documented on MRI scans.

Conclusions: Tendon lesions are very frequent in horses and appear to be a very good model in comparative pathology.

11.20 AM

\section{Three-dimensional $\mathrm{CT}$ in bone diseases} M. Ernsting, H.W. Stedtfeld, E. Zeitler; Nürnberg

Three-dimensional surface reconstructions were generated in more of 100 patients with various disorders. The comprehension of complex bony structures was easier than with ligaments or tendons. 3D-CT was particularly useful in preoperative planning of hip and shoulder surgery while it also simplified the intraoperative orientation and give an good overview. 3D-CT proved to be a useful diagnostic method supplementing axial Computed tomography.

\section{$11.30 \mathrm{AM}$}

1154 Reasonable clinical applications of 3D-GE sequences in MRI

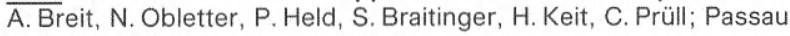

AIM: In the field of neuroradiology and orthopedics there are many situations where slice thickness and consequently spacial resolution of conventional magnetic resonance examinations are still not optimal. For this reason, the examination of relatively large volumina with high resolution has not yet been possible in classic MRI with spin-echo sequences.

Number of patients:

353 examinations of the spine

227 examinations of the region of the sella

613 examinations of joints

Technique:

Spin-echo sequences, T1, T2-weighted,

in comparison with 3-D-FLASH-40,

before and after Gadolinium-DTPA.

All patients were examined with T1- and T2-weighted spin-echo sequences, the patients with lesions in the sella with optimized spin-echo sequences with a matrix of $512 \times 512$ or $394 \times 512$. In the same course, the patients were examined with 3D-FLASH-40 sequences, before and after administration of Gadolinium-DTPA. For examinations of the spine we used 3D-FLASH and 3D-FISP sequences in combination.

Results: Advantages of examinations with 3D-gradient-echo sequences:

- Far (remarkably) better solution in MRI

- Extremely good imaging of cartilage

- Reconstruction of irregular and curved slices in relatively short examination time.

\section{$11.40 \mathrm{AM}$}

1155 Detectability of hyaline cartilage defects in osteoarthritis: 2D spin-echo versus $3 \mathrm{D}$ gradient-echo MR imaging in an animal model G. Adam, C. Nolte-Ernsting, M. Bühne, A. Prescher, K. Bruchmüller, W. Küpper, K. Bohndorf, R. W. Günther; Aachen

We evaluated the diagnostic accuracy of MR imaging for the assessment of hyaline cartilage defects caused by osteoarthritis in an animal model in 10 dogs.

After transsection of the anterior cruciate ligament of the knee joint, resulting in rapid development of osteoarthritis, animals were followed up by MR 
imaging using T1- and T2-weighted spin-echo and gradient-echo sequences. They were sacrificed at different times after surgery ( 1 month-12 months) and final MR measurements were compared with the pathohistologic findings.

Grade 1 and 2 hyaline cartilage lesions were not detectable by MR imaging, neither by spin-echo nor by 3D gradient-echo imaging. Grade 3 an 4 lesions were all visible on 3D MR images, showing signal loss on T1- and T2 weighted gradient-echo scans and loss of cartilage thickness. 2D spin-echo imaging detected accurately only $56 \%$ of grade 3 lesions and $85 \%$ of the grade 4 lesions, while the remaining were underestimated or were not detectable.

We conclude, that 3D gradient-echo imaging permits exact delineation of grade 3 and 4 hyaline cartilage lesions, but fails in the detection of grade 1 and 2 defects. 2D spin-echo imaging seems to be an inadequate tool for the assessment of hyaline cartilage lesions in vivo.

\subsection{AM}

1156 Calcifying tendinitis of the rotator cuff: does it exclude rupture? F. Sáez, J. Negueruela, J. C. Martín, I. Azkuna, E. Elizagaray, A. Aguinaga; Bilbao

We have studied 187 patients by means of plain films, ultrasound and CT-arthrography to evaluate if the presence of calcifying tendinitis excludes the possibility of cuff rupture.

Results: 31 cases of calcifying tendinitis of the rotator cuff, in the appearance of the formative phase, have been found. Only two cases were associated with rotator cuff tear. 76 cuff tears (partial and complete) were demonstrated, accounting for $2.6 \%$ the association between cuff tears and calcifying tendinitis. Significant statistical correlation was found between calcifying tendinitis and absence of rotator cuff tear.

Conclusions: Rotator cuff tears are very unlikely when calcifying tendinitis (formative phase) is seen.

\section{$11.53 \mathrm{AM}$}

1157 Renal osteodystrophy: radiological findings versus plasma BGP (osteocalcin) levels

G. Guglielmi, S. Modoni, F. Aucella, G. Ritrovato, C. C. Guida, A. Simeone, G. Valle, V. Frusciante; San Giovanni Rotondo

The Authors report their experience in the diagnosis of renal uremic osteopathy.

A study was carried out to evaluate the relationships between radiological findings vs. plasma Bone Gla Protein and other biochemical parameters in patients in routinary dialysis treatment. 73 haemodialyzed patients (mean age $55 \pm 14$ yrs.; dialytic age $56 \pm 36 \mathrm{mths}$ ) were studied by means of conventional radiology and hand microradioscopy (Meema method); in selected cases other imaging methods were used (CT, computed radiography, bone scintigraphy): CT densitometry was performed in some typical cases.

All the patients were allowed to group A (hyperparathyroidism) or group B (osteomalacia).

Student $\mathrm{t}$ test and linear regression were used for statistical analysis.

High BGP levels were found in 68 pts. and correlated with PTH $(\mathrm{p}<0.00001)$, $\operatorname{ALP}(\mathrm{p}<0.0005)$ and dialytic age $(\mathrm{p}<0.005)$.

In group A mean BGP level was $51.2 \pm 15.4 \mathrm{ng} / \mathrm{ml}$, while in group B was always below $30 \mathrm{ng} / \mathrm{ml}(\mathrm{p}<0.0001)$. Between these groups statistically different were PTH $(\mathrm{p}<0.0001)$ and $\operatorname{ALP}(\mathrm{p}<0.0006)$ too.

Our studies confirm that BGP levels strongly correlates with other biochemical parameters and may be useful with skeletal $\mathrm{x}$-rays as a non invasive tool for bone hystopathology assessment. 


\section{Room A}

\section{SS}

\subsection{Joints II - Soft tissue}

$2.00 \mathrm{PM}$

1158 Correlation of magnetic resonance imaging and histomorphology in bone marrow edema of the hip

S. Hofmann, A. Neuhold, A. Engel, J. Kramer, K. Leder, H. Plenk jr.; Vienna

Purpose: The nonspecific MRI pattern of bone marrow edema (BME) of the femoral head is interpreted clinically either as "transient bone marrow edema syndrome" or "transient osteoporosis", but also as early form of "avascular necrosis (AVN)". In this study histomorphological evidence of BME and early AVN is presented for the first time in such cases of MRI-detected BME. The histomorphological verification of this BME-syndrome is of clinical importance.

Methods: 16 bone cores from femoral heads of 15 patients exhibiting MRI signs of BME were examined histologically using undecalcified sections. Radiographs were inconspicuous or nonspecific with only signs of "focal osteoporosis". Trauma, osteomyelitis, acute sickle cell crisis, and neoplasms could be excluded clinically.

Results: All patients showed the typical MRI pattern of BME and no focal abnormalities characteristic for AVN. Histologically increased tissue fluids were found in the marrow spaces. All bone trabeculae showed a normal volume density, but were covered with wide osteoid seams and active osteoblasts. Extended fatty marrow degeneration alternated with fibrovascular regeneration of the marrow.

Conclusions: Our histological findings confirm the existence of BME in the femoral head, as demonstrated by MRI. The bone and marrow changes correspond well with early AVN. However, the term "transient osteoporosis" seems no longer appropriate. Whether BME of the femoral head constitutes a distinct transient syndrome or represents the initial stage of AVN, as supported by our findings, remains a controversial issue.

\subsection{PM}

1159 Osteonecrosis of the hip - primary diagnosis and follow up with MR imaging using Gd-DTPA and 3D-sequences

H. Schedel, Th. Vogl, L. Ziegler, D. Hahn, J. Lissner; Munich

Purpose: to evaluate the sensitivity of MR imaging in osteonecrosis of the hip and the effectiveness of therapeutic strategy especially using Gd-DTPA and 3D-sequences.

Methods: 40 patients with suspected osteonecrosis of the hip were examined with a 1.5 T Siemens Magnetom using coronal and paraxial T2- and T1-weighted sequences before and after application of Gd-DTPA. Facultatively FISP 3D-sequences with a flip angel of 70 degree were performed. The results were compared to conventional radiography and in a few cases to bone scanning.

Results: 37 cases of osteonecrosis ( 3 Ficat I, 22 Ficat II-IV, 15 postoperative), 1 transient osteoporosis and two coxitis were diagnosed. In early stages and in postoperative cases Gd-DTPA detected vitality of osteonecrosis or of bony implantation material. 3D sequences were equal to plain SE-sequences.

Conclusions: MR imaging especially using Gd-DTPA is the method of choice diagnosing osteonecrosis of the hip and vitality in early and postoperative stages. 3D imaging could replace SE-sequences in future.

\subsection{PM}

\section{Shoulder instability: CT-arthrography vs 0.5-T MR}

F. Sáez, E. Elizagaray, J. Negueruela, J. C. Martín, A. Ferrero, J. C. Ruiz; Bilbao

Purpose: To compare the diagnostic performance of CT-arthrography and 0.5-T MR imaging for shoulder instability.

Methods and Materials: 153 patients have been studied by means of CT-arthrography and in 46 of them 0.5-T MR imaging was also performed. Results: Sensitivities were as follows:

Hill-Sachs lesion

Bankart (bony)

Bankart (fibrous)

Loose bodies

\begin{tabular}{|c|c|}
\hline CT-Arthro & MRI \\
\hline $30 / 30(100 \%)$ & $8 / 10(80 \%)$ \\
\hline $12 / 12(100 \%)$ & $3 / 6 \quad(50 \%)$ \\
\hline $52 / 53(98 \%)$ & $12 / 19(63 \%)$ \\
\hline $5 / 6(83 \%)$ & $1 / 3 \quad(33 \%)$ \\
\hline
\end{tabular}

The evaluation of capsular volume and insertion could only be done routinely by CT-arthrography. MRI was helpful only in a few cases with glenohumeral effusion.

Conclusions: CT-arthrography is clearly superior to 0.5-T MRI for shoulder lesions related to instability.

\subsection{PM}

1161 Instability of the shoulder joint: a comparison between double contrast $\mathrm{CT}$ and double contrast MRI

W. Judmaier, K. Wicke, A. Oberhauser, G. Helweg, D.zur Nedden, H. Resch; Innsbruck

A: Double contrast CT (DC-CT) is a well established method for evaluation of the glenohumeral joint in patients with recurrent shoulder dislocation. We examined 15 patients by both DC-CT and double contrast MRI (DC-MR) to rank the value of each method.

B: After administration of both iodine and gadolinium containing contrast agent and air into the shoulder joint, each patient had a standard CT scan immediately followed by a multiplanar MR exam. The studies were evaluated independently by two radiologists.

C: In DC-MR a sufficient layer of contrast agent outlined the cartilaginous structures of the joint in all cases, making the exam comparable to DC-CT Fine anatomical details, especially of the underlying bony structures were better demonstrated by DC-CT. The superior soft tissue contrast and the multiplanar capability of MR facilitated the assessment of coexistent muscle and ligamentous injuries.

D: Under present technical conditions DC-MR does not allow a better evaluation of the cartilaginous and bony structures of the shoulder joint compared with DC-CT. MR however, allows for a better visualization of the stabilizing soft tissues.

\section{$2.40 \mathrm{PM}$}

1162 MR imaging of the rotator cuff: evaluation of criteria for diagnosis of full thickness tear

T. E. Farley, C. H. Neumann, L. S. Steinbach, F. W. Morgan, R. G. Holt, A. H. Jahnke, S. S. Petersen; San Francisco, CA

Purpose: Distinction of a full thickness tear from other rotator cuff pathology has important clinical and surgical implications. Criteria for MR diagnosis of a full thickness tear of the rotator cuff are statistically evaluated. MR features found in patients with surgically confirmed diagnoses of inflammation, degeneration, and partial tears are described.

Methods: MRI was performed on shoulders of two hundred patients using a $1.5 \mathrm{~T}$ superconductive system. The rotator cuff was evaluated by a radiologist experienced in musculoskeletal MR imaging. Seventy-two of these patients had subsequent surgical correlation.

Results: The following criteria predicted (all with $\mathrm{P}<0.0001$ ) a full thickness tear of the rotator cuff: presence of subacromial fluid-sensitivity $(93 \%)$, specificity $(72 \%)$; presence of tendinous gap on the T2 weighted image-sensitivity $(67 \%)$, specificity $(96 \%)$ and musculotendinous retraction-sensitivity $(80 \%)$, specificity $(91 \%)$. Obliteration of the deltoid fat stripe had a positive predictive value of $.25(\mathrm{P}<0.19)$.

Conclusions: MR imaging is useful for diagnosis of full thickness rotator cuff tears. The most sensitive sign for complete tear is presence of subacromial fluid; however, this sign lacks specificity with a positive predictive value of 0.48 . Criteria highly specific for complete tear with high positive predictive value are presence of a tendinous gap, and musculotendinous retraction. Obliteration of the subdeltoid/subacromial fat stripe is not a useful criteria for diagnosis of full thickness tear.

\section{$2.50 \mathrm{PM}$}

1163 Soft tissue tumors; substantial improvement using gradientecho sequences with long TR

T. Yousry, U. Fink, S. Backmund, C. Gärtner, S. Breitner, J. Lissner; Munich

Soft tissue tumors are often difficult to differentiate from surrounding structures. We wanted to establish the most effective imaging strategy, yielding in the least time the highest diagnostic information.

We examined 54 patients with soft tissue tumors, 11 up to 8 times. Radiographs, CT scans and MR images were compared. T1 and T2 weighted as well as gradientecho sequences were applied. After the application of Gd-DTPA the T1 weighted and the gradient-echo sequences repeated.

MR is the superior method in delineating the tumor margin, detecting involvement of the neuromuscular bundle and determining the tumor rise. MR and CT are equal in detecting cortical destruction. With respect to soft tissue tumors gradientecho sequences revealed a similar signal behaviour as T2 weighted sequences with an essential improvement in the anatomic discrimination and only requiring half the measurement time. By the application of Gd-DTPA the delineation of the tumor margin and demarcation of its structure is further improved. The difference is significant with respect to $\mathrm{Tl}$ 
and $\mathrm{T} 2$ weighted sequences not to gradientecho sequences.

For the diagnosis and follow up of soft tissue tumors, MR is the superior method. Gradientecho sequences replace T2 weighted sequences. Gd-DTPA is only to be applied in selective cases. Therefore MR is the only method required after radiography with only $\mathrm{Tl}$ weighted and gradientecho sequences to be applied.

\subsection{PM}

1164 MR findings in skeletal muscles and biochemical changes after

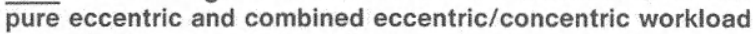
K. Wicke, A. Koller, Ch. Haid, W. Judmaier; Innsbruck

a) In comparison to our previous work on pure eccentric workload (e.g. see European Congress of NMR or World Congress of Sports Medicine, 1990) we wanted to find out if additional concentric loads leed to an increase of the MR and/or biochemical changes. This would be of high importance in the understanding of training effects of the muscle.

b) One group of 6 volunteers underwent pure eccentric workload on a specially designed machine, another group of 6 volunteers had to do additional concentric exercise on a Cybex II +. Creatinkinase and Myoglobin were assessed before and immediately after exercise and on day 1,3,6,9 and 12. MR imaging was performed on a 1.5 Tesla Unit in axial T2 weighted $8 \mathrm{~mm}$ thick slices through the muscles of the thigh on day 3,6 and 9 .

c) Creatinkinase-levels showed a high increase in the second group, while changes in MR signalintensities where not significantly different between the two groups.

d) The discrepancy between CT levels and MR findings give evidence that CK levels are not only a marker for musclefibre damage as assumed so far.

\subsection{PM}

$1165{ }^{31}$ P-MR spectroscopy of the calf muscle in peripheral arterial occlusive disease: results before and after angioplasty

P. Landwehr, R. Schindler, Th. Krahe, K. Lackner; Würzburg

Purpose: To evaluate ${ }^{31} \mathrm{P}-\mathrm{MRS}$ of the calf muscle in patients with peripheral arterial occlusive disease (PAOD) before and after PTA.

Methods: 13 normals, 14 PAOD-patients. Protocol: clinical examination; standardized pain-free walking distance; Doppler pressure index. ${ }^{31} \mathrm{P}-\mathrm{MRS}$ of the calf muscle (1.5 T, 4 spectra/min) at rest, during and up to 10 minutes after ergometry (plantar flexion $80 / \mathrm{min}$; individual exercise time up to claudication or fatigue). Calculation of $\mathrm{PCr} / \mathrm{Pi}$ by fitting the FID. Complete protocol before $/ 2$ weeks/ 3 months after PTA.

Results: Mean work during MRS was $1386 \mathrm{~J}$ (normals) and $314 \mathrm{~J}$ (patients before PTA) $(\mathrm{p}<0.01)$. In patients, mean recovery time of $\mathrm{PCr} / \mathrm{Pi}$ was longer than in normals $(97.1$ s vs. $34.8 \mathrm{~s})(\mathrm{p}<0.01)$. In the sub-group with clinical successful PTA an improvement of the mean walking distance $(77 \mathrm{~m}$ before, $496 \mathrm{~m}$ after) and the Doppler index ( 0.76 before, 1.02 after) could be observed $(\mathrm{p}<0.01)$; however, the mean recovery of $\mathrm{PCr} / \mathrm{Pi} 2$ weeks after PTA didn't improve significantly $(77.5 \mathrm{~s}$ before, $60.7 \mathrm{~s}$ after). The data of additional patients and follow-up studies 3 months after PTA will be presented.

Conclusions: ${ }^{31}$ P-MRS allows identification of patients with PAOD. However, preliminary short-term results after PTA demonstrate insignificant changes in phosphorus metabolism. Follow-up studies are on the way to clear up the discrepancies between clinical and spectroscopic findings.

\section{$3.20 \mathrm{PM}$}

\section{Localized in-vivo ${ }^{1} \mathrm{H}$ spectroscopy of human bone and soft tissue tumors}

H. Bongers, F. Schick, M. Skalej, W. I. Jung; Tübingen

Purpose: To assess the value of 'H MRS to characterize soft tissue tumors and to detect biochemical changes during treatment.

Methods: We report the results of $21{ }^{\prime} \mathrm{H}$-MRS studies in 14 patients with malignant soft tissue and bone tumors including cases of osteosarcoma, ewing sarcoma, malignant histiocytoma and plasmocytoma. We used a double spin echo localisation method implemented at a 1.5 Tesla whole body unit TR was chosen $2 \mathrm{sec}$; the shortest TE was $50 \mathrm{msec}$. Localisation voxels of $(13 \mathrm{~mm})^{3}$ or $(20 \mathrm{~mm})^{3}$ were available. A head and Helmholtz coil were preferred. A suppression of the water resonance was necessary in all cases.

Results: The most common resonances detected in these tumors were those of choline at $3.2 \mathrm{ppm}$ and phosphocreatine at $3.1 \mathrm{ppm}$. In contrast to spectra of skeletal muscles the choline resonance dominated in all cases. Lipid resonances (methyl at $1.2 \mathrm{ppm}$ and methylene at $1.5 \mathrm{ppm}$ ) were recorded in most cases. In addition resonances between 1.8 and $2.5 \mathrm{ppm}$ were seen and might refer to aminoacids. The T2-relaxation behaviour of these metabolites could be studied varying the echo time TE. In 2 cases the spectra were very poor and contained only a small signal in the lipid range besides the water resonance. Possible reasons (short T2-relaxation) are discussed. In 3 patients follow up studies were performed during and after chemo- or radiotherapy. The choline-, phosphocreatine- and aminoacid-resonances were diminished or undetectable after some weeks. In these cases the MRI studies and bone scans gave evidence of tumor response to therapy.

Conclusions: Our results indicate that 'H MRS might be useful to look into tumor biochemistry noninvasively and give new data for treatment response monitoring.

\section{Room B}

\section{SS}

\subsection{Diagnostic and interventional procedures in pulmonary and venous systems}

\section{$2.00 \mathrm{PM}$}

\section{Interventional radiology in the pulmonary circulation (Keynote Lecture)}

D. J. Allison; London

The presentation will focus on the radiological management of pulmonary arteriovenous malformations (PAVMs). These malformations, producing shunting from pulmonary arteries to veins, are an important curable cause of morbidity producing hypoxaemia and cerebral complications if untreated. Surgical management of such lesions is invasive, sometimes impossible and almost always unnecessary. PAVMs are ideally treated by therepeutic embolization with coils or balloons. The success rate of such procedures is very high and the complication rate low.

The indications for PAVM embolization will be reviewed and details of the investigation, treatment, success rates and complication rates for the procedure presented with illustrative case histories.

\subsection{PM}

1168 Vascular cerebral complications associated with pulmonary arteriovenous malformations (PAVM)

R.I. White Jr., P. B. Fayad, J. S. Pollak, L. Charnas, C. Davenport, L. M. Brass; New Haven, CT

PAVMs are variably associated with hereditary hemorrhagic telangiectasias (HHT), and represent a source of hypoxemia, as well as infectious and paradoxical embolism. Although stroke has been reported with both entities, its incidence and etiology are less clear. Since 1978, we studied both prospectively and retrospectively 99 patients with PAVMs, to determine: incidence, risk factors, and type of vascular cerebral complications. All our patients were treated with balloon embolotherapy.

Vascular cerebral complications occurred in 28/99 patients (28\%); 20 women and $8 \mathrm{men}$. This was the presenting manifestation in 10/28 (35.7\%). Mean age was 40.7 (range 17-76). Events consisted of: transient ischemic attacks (TIA) (7), and infarctions (21) [symptomatic (11) and silent (10)]. Recurrences occurred in 9 patients mainly before treatment ( 5 TiAs, 4 infarcts, and 1 hemorrhage). A family history of stroke was present in 16/28 (57\%). HHT was present in $26 / 28(92.8 \%)$ patients.

Since screening is simple and treatment is available, a diagnosis of PAVM or HHT, either sporadic or as part of the HHT spectra, should be entertained in young patients with stroke. If HHT diagnosed, their families need to be screened. Patients with PAVMs should be considered for balloon embolization, an effective therapy with small risks.

\section{$2.25 \mathrm{PM}$}

1169 Superior vena cava syndrome stenting: for whom and when D. Vavasseur, M. Sapoval, S. Indushekar, M. Gad, M. F. Cosset, A. Roche; Villejuif

AIM: to study the clinical and superior phlebocavographic (SPC) features in patients referred for possible and/or useful Superior Vena Cava (SVC) stenting.

Methods and Materials: 31 patients underwent SPC during a 9 months period between April 90 and December 90 . Of these, 12 patients had clinically obvious SVC syndrome, and were good candidates for SVC stenting. Among these 12 patients, 7 underwent percutaneous stenting of SVC. In all patients, clinical (duration and severity of symptoms, etiology) and venographic features (level, type and length of obstruction, anatomy of collateral veins) were studied. Particular emphasis was placed on 5 patients in whom stenting was not possible.

Results: in $18(58 \%)$ patients the obstruction was due to tumor or post radiotherapy narrowing, among them 12 had obvious SVC syndrome. Stenting was not performed in 3 cases as the lesion was extensive. In these 3 patients the mean duration of SVC syndrome was 9 months as opposed to 7 weeks in patients who underwent stenting. We found no correlation between severity of SVC syndrome and extension, level of obstruction or collateral pathways. Conclusions: The feasibility of stenting is more dependent on duration and extent of obstruction rather than severity of clinical symptoms. The onset of 
SVC syndrome should drive one promptly to perform SPC to consider stenting.

\subsection{PM}

1170 Treatment of superior vena cava (SVC) and inferior vena cava (IVC) occlusions with balloon dilatation and rotational angioplasty and wallstents

Turhan Cumhur, Tulay Olcer, Serdar Karakose, Ahmet Mavis, Erkin Aribal, Oguz Tasdemir, Murat Beyazit, Cumhur Ceken; Ankara

Purpose: To treat different types of SVC and IVC occlusions with balloon dilatation and rotational angioplasty and wallstents.

Methods and Materials: Five patients with a mean age of 51 years were treated. One patient had SVC occlusion due to thrombosis resulting from cardiac surgery. The occlusion is treated with rotational and balloon dilatation angioplasty and wallstent is placed after reestablishment of the passage through the occlusion. Two patients had congenital occlusions in the IVC, one of which had a complete membraneous obstruction and the other had segmental constriction. The former is treated with rotational and balloon dilatation angioplasty and the later is treated with balloon dilatation angioplasty only. Two patients had IVC strictures due to liver cyst hydatid compression. Both of the patients are treated with balloon dilatation angioplasty and wallstent placement.

Results: In all of the cases occlusions are treated and pressure gradients returned to normal. In one patient dislodgement of the wallstent in the IVC is observed. In the rest 4 patients the SVC and IVC were patent during the follow-up.

Conclusions: SVC and IVC occlusions, due to different causes, can be treated with balloon and rotational angioplasty and wallstents, selected cases, as an alternative to surgery.

\subsection{PM}

1171 MR-Venography with 2D-inflow technique

B. Siewert, W. A. Kaiser, F. Träber, K. Bootsveld; Bonn

Purpose: Aim of this study was to compare the results of conventional phlebography with MR-TOF-Venography.

Methods: 28 patients (20 venous systems of the legs, 4 mediastinal and cervical veins, 4 sinus veins) were examined. MR-measurement was performed with 2D-TOF-technique in contiguous transversal slices with arterial presaturation and secondary $3 \mathrm{D}$-reconstruction.

Results: Using MR-Venography diagnosis of deep venous thrombosis could be verified in 16 cases. No false positive or negative results were obtained in this series. When flow signal is present on MR-Venography, complete thrombosis could be reliably excluded. The assessment of pathological changes in calf veins, however, was possible in only $50 \%$ of cases, probably due to a very slow flow. Postthrombotic changes in the iliac and femoral veins were seen on MRI in 3 of 4 cases.

Conclusions: MR-Venography showed good agreement with the results of phlebography concerning deep venous thrombosis in the popliteal, femoral and iliac veins and is well suited as a noninvasive diagnostic tool of vein diseases.

\section{$2.55 \mathrm{PM}$}

1172 Recanalization in acute massive pulmonary embolism by balloon-catheter and local thrombolysis

St. Basche, G. Oltmanns, W. Hahn, R. Kachel; Erfurt

The mortality rate in patients with acute massive pulmonary embolism (PE) is high. A very important factor for the ultimate prognosis is a rapid recanalization. By balloon-catheter-dilatation the time for recanalization can be reduced markedly. In 7 patients with acute massive $\mathrm{PE}$ a mechanical alteration by balloon-catheter-dilatation-technique (diameter $8-20 \mathrm{~mm}$ ) were performed and subsequently streptokinase or urokinase were administered via the pulmonary artery as close as possible to the thrombus.

After balloon-dilatation in 6 of 7 patients ( 5 patients with shock) systolic pulmonary artery pressure, patient situation and the angiographic findings improved impressively.

The 7 th patient died in shock (autopsy: massive tumour embolism). This combined form of balloon-recanalization and local thrombolysis of acute massive PE described above represents thus a highly successful form of treatment.

The interventions were without complications.
Evaluation of the vascular anatomy of the iliocaval junction was formerly almost exclusively done by ascending contrast venography. We evaluated the iliocaval junction in 22 patients by both ascending and descending venography to compare their efficacy in revealing pathology in the iliocaval junction and to correlate the findings with the hemodynamic profile obtained concurrently. 22 patients consecutively referred for evaluation of refractory pain and swelling of the lower extremity(s) were selected. Standard contrast venography was performed by the ascending route. We then performed selective percutaneous transbrachial catheterization. Utilizing a commercial pressure injector we performed rapid sequence filing of the opacification of the iliocaval junction with the patient in $70^{\circ}$ upright position. Following radiographic evaluation hemodynamic pressure measurements were obtain both at rest and after the raising exercise both in the IVC as well as both common iliac veins. Of the 22 patients, 16 showed suggestion for abnormality at the iliocaval junction on ascending venography. Following descending venography 20 of 22 patients were shown to have radiographic pathology at the iliocaval junction. 6 of the 22 patients had increased transiliocaval ambulatory venous pressure. 19 patients in this group went to surgery for autogeneous vein angioplasty. Web formation at the site of the radiographic abnormality was found in 15 of 19 patients. Normalization of transiliocaval pressures occurred in 2 of the 6 patients.

Compression of the left iliocaval junction by the right common iliac artery is a not uncommon cause for left lower extremity venous insufficiency. Ascending venography will frequently fail to reveal the extent of pathology that the descending technique demonstrates. We conclude that although ascending venography can suggest the presence of any abnormality, the descending technique is far superior in demonstrating the specific abnormality.

\section{$3.15 \mathrm{PM}$}

1174 Time dependent defects and complications of vena caval filters long-term follow-up studies

Gerhard Miersch, Wolfgang Münster, Wolfdietrich Schöpke, Gisela Stobbe; Berlin

The long-term follow-up five years after implantation of vena caval basket filters in 60 patients demonstrates time dependent complications of those filters: Fractures of the anchoring struts $(53,2 \%)$, broken baskets $(21,6 \%)$, distal migration, penetration into the aortic wall, the small intestine, the iliopsoas muscle, and the diaphragm. Embolized pieces of the filters were seen in the pulmonary arteries and the hepatic veins $(6,6 \%)$. Inspite of these multiple complications no clinical signs or symptoms were apparent. Further implantation of these otherwise optimal filters is with the present quality and stability of the material not recommended. Long-term follow-up studies of all types of vena caval filters are necessary.

\subsection{PM}

\section{Percutaneous placement of Hickman catheters}

M. Maynar, R. Reyes, J.M. Pulido-Duque, E. Gorriz, C. Garcia-Morales, J. Garcia-Medina; Las Palmas

One hundred Hickman catheters were placed in 95 patients. There were 48 females and 47 males, with age range from 9-74 years with a mean of 38 . The principle indication for catheter placement was chemotherapy infusion. Thirty percent of the patients had less than 50,000 platelets at the time of percutaneous Hickman line placement. The internal jugular vein was used in all patients and the procedure was performed under local anesthesia in 98 instances, except in two pediatric patients.

Results: Length of procedure varied from 15-30. The technical success was $100 \%$. In ninety-three percent of the patients, the catheter remains patent for the length of catheter stay or until the patients demise. The mean duration of catheter implantation was 138 days with a range of 5-330. Early complications included 5 hematomas in the subcutaneous tract which were treated conservatively. Late complications included accidental dislodgement in 2 cases, hemorrhage in the subcutaneous tract in 1 case, and catheter obstruction in 3 cases.

\subsection{PM}

1176 Insertion of chronic indwelling central venous catheters under radiological control

O. Chan, D. Hughes, P. Gishen; London

A prospective study was undertaken over a 2 year period to assess the feasibility of insertion of chronic indwelling central venous catheters (Hickman lines) under radiological control. Over 100 patients, mainly with haematological disorders (malignancy) had Hickman lines inserted for long-term venous access. The lines were inserted in the Radiology department under 
aseptic conditions using a subclavicular approach. Prior digital subtraction angiography (DSA) was performed to confirm patency and for initial mapping of the venous anatomy. The technique will be described in detail, with emphasis on antibiotic regime and modifications/refinement of equipment. The results will be discussed with special emphasis on complications, in particular infection and thrombosis.

\section{$3.24 \mathrm{PM}$}

1177 A new retrievable percutaneous vena cava filter: in vivo- and in vitro-studies

Jörg Neuerburg, Rolf W. Günther, Dierk Vorwerk, Stefan Handt, Kirsten Tonn; Aachen

A new retrievable percuteneous caval filter was tested in vivo for its biocompatability and in vitro for its hydrodynamic features and its efficiency.

In 8 dogs, 15 new retrievable stainless steel 1/2-basket filters (Cook, Europe), were inserted percutaneously through a $10 \mathrm{~F}$-catheter into the lumbar and the hepatic segment of the vena cava. Follow-up studies (cavography, external and intravascular ultrasound, CT), post-mortem examinations ( 3 or 6 months following filter insertion) and histologic specimens were obtained. Retrievability of 13 filters was tested in 7 additional dogs 2 to 3 weeks after filter insertion. The flow characteristics and the clot trapping efficiency were tested in a flow phantom.

There was no occlusion or filter migration; a slight decentration of the filter post implantation occurred in 2 of 28 filters. Ten of 11 filters were retrieved 14 days post implantation without complications. Three weeks post implantation the filters were firmly fixed by a fibrotic wall reaction. Post-mortem studies showed a normal width of the caval lumen in all cases. Histology revealed no foreign-body reaction. In vitro, only slightest turbulences were detectable behind the filter. The device was very effective in trapping medium and large sized thrombi.

\section{Room C}

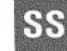

\subsection{Liver MRI}

\section{$2.00 \mathrm{PM}$}

1178 Contrast media in MRI of the liver (Keynote Lecture) J.M. Bigot; Paris

Abstract available at the information desk.

$2.15 \mathrm{PM}$

1179 MRI of the liver - value and diagnostic statement of Mn-DPDP Th. Vogl, B.Schnell, S. Steiner, A. Eibl-Eibesfeld, C. Wilimzig, J. Lissner; Munich

Introduction: In an open-label, prospective, multicenter study on 20 patients with suspected liver tumors, tolerance and diagnostic value of the new hepatobiliary contrast agent Mn-DPDP was examined.

Methods: All studies were performed using a 1.5 T-Magnetom with a body coi and an abdominal belt for reducing motion artifacts. Images were obtained before and after application of $5 \mu \mathrm{mol}$ or $10 \mu \mathrm{mol} \mathrm{Mn}-\mathrm{DPDP} / \mathrm{kg}$ b.w., Routinely T1-and T2-weighted SE-sequences and two GE-sequences (breathhold-Flash and Turboflash) were applied.

Additionally hematologic and circulation parameters were evaluated before and after Mn-DPDP-injection.

Results: None of the studied patients reported subjective complaints and no pathological changes of the laboratory parameters were seen after Mn-DPDP application. Mn-DPDP significantly increased the signal/noise-ratios of liver parenchyma in all T1 weighted sequences. In the Flash sequences (T1 weighted) the percentage of enhancement reached $120 \%$ at the higher dose of Mn-DPDP. The technical and diagnostic quality of MRI studies was significantly improved after application of Mn-DPDP. Histology revealed a malignant involvement of liver parenchyma in 15 cases, in 5 patients benign lesions were identified. The MR-findings correlated with Histology at $88 \%$ of the patients.

Conclusions: Our series prove the intravenous contrast agent Mn-DPDP to be a safe, specific drug for hepatobiliary imaging. It proves valuable extending the imaging window in MRI. The diagnostic results of liver-MRI are improved by a higher accuracy in the judgement of tumor size and tumor detection.
1180 Physiological changes of portal flow - a study using double oblique through plane MR imaging

C. Thomsen, F.Ståhlberg, O. Henriksen, Scandinavian Flow Group; Hvidovre

Purpose: The aim of the present study was to find a normal range for portal flow during fasting, and to quantify the increase in portal flow after eating using a non-invasive MR imaging flow measurement method.

Methods: Measurements were performed during a single breath hold. The flow was calculated from the phase difference in two rapid gradient echo sequences, the first one compensated for flow and the second one flow-sensitive in the slice selective direction. Both sequences were fully flow compensated in the readout direction.

Five healthy volunteers were studied after seven hours of fasting. A standard nutrition meal was served within the magnet and portal flow was measured immediately before and 5, 15 and 30 minutes after intake of the meal.

Results: During fasting the volume flow in the five volunteers was $0.85 \mathrm{l} / \mathrm{min}$ (range $0.40-1.531 / \mathrm{min}$ ). Following the standard meal the flow increased with a factor of $1.41,1.60$ and 2.52 after 5,15 and 30 minutes, respectively. The increase was seen to depend upon an increased velocity as well as upon an increase in size of the portal vein.

Conclusions: The study shows that non-invasive MR flow studies can be made in the portal vein and that physiological changes in flow can be quantified. If a time schedule for fasting and intake of meal is established, the method could prove useful for accurate determination of liver malfunction.

\section{$2.35 \mathrm{PM}$}

\section{Magnetic resonance arterial portography (MRAP) with paramag-} netic agents: definition of the technique

P. Pavone, S. Giuliani, G. Cardone, P. Di Renzi, C. Simonetti, N. De Stefano, R. Passariello; L'Aquila

Purpose: To assess the optimal parameters to obtain selective liver enhancement with intraarterial injection of Gd-DTPA in the superior mesenteric artery.

Methods: 12 patients were included in the study who had to undergo angiography for different reasons (intraarterial chemotherapy, pre operative vascular mapping etc.). The patients were divided in three groups. One group (n.2) was studied with conventional, non breath holding, T1 weighted spin echo sequences (TR 260, TE 16, NEX 8, acq. time $4.5 \mathrm{~min}$.). The second group (n.4) was studied with Gradient echo breath holding sequences (TR 100, TE 13, NEX 2, acq. time 28 sec.). The third group (n.6) was studied with a Rapid Acquisition Spin Echo (RASE) sequence (TR 230, TE 16, NEX 1, acq. time 30 sec.). $4 \mathrm{ml}$. of $0.5 \mathrm{mmol} / 1 \mathrm{Gd}$-DTPA solution were injected into SMA diluted with $20 \mathrm{ml}$. of water, starting $15 \mathrm{sec}$. prior to the sequence and lasting $30 \mathrm{sec}$. Results: The first group did not provide good results, with contemporary enhancement of the liver and liver lesions. In the second group a good improvement of contrast to noise ratio was obtained, with selective liver enhancement (SE/N 8.4, vs. 0.6 of liver lesions). Better results were obtained in the third group, with SE/N of 9.5 in the liver and 0.5 in the lesions.

Conclusions: This experience shows the superiority of RASE sequences in the dynamic post-contrast evaluation of the liver after MRAP.

\section{$2.45 \mathrm{PM}$}

1182 Comparison between turboflash and spin echo sequences in detection and characterization of liver tumors

F. Laurent, V. Lafarge, B. Houang, B. Chaulin, J. Drouillard; Bordeaux

Purpose: To compare plain T1 weighted (T1W), T2 weighted (T2W), and contrast enhanced Turboflash with conventional spin echo (SE) sequences in the detection and characterization of liver tumors.

Methods: Twenty-two patients with 30 liver tumors (hepatomas: 10, metastasis: 8 , hemangiomas: 6 , focal nodular hyperplasia: 3 , adenoma: 1 , biliary cysts: 2 ) were prospectively studied on a Magnetom SP $1.5 \mathrm{~T}$ with the following protocol performed with 8 to $10 \mathrm{~mm}$ slices on the whole liver (maximum gap between slices was $2 \mathrm{~mm}$ ):

- SE T1W $(400,15,4$ Acq, $160 \times 256)$

- SE T2W $(2000,45 / 90,2$ Acq, $160 \times 256)$

- Turboflash T1W $\left(7,3,1\right.$ Acq, $\left.10^{\circ}, 128 \times 128\right)$

- Turboflash T2W $\left(6,40,1\right.$ Acq, $\left.8^{\circ}, 128 \times 128\right)$

- Dynamic incremental TIW Turboflash (DOTAREM $0,1 \mathrm{mMol} / \mathrm{kg}$ ).

Comparison included the number of lesion detected, their characterization and the measurement of contrast to noise ratio (CNR).

Results: Contrast enhanced Turboflash showed the highest CNR. The number of lesion detected by contrast enhanced, T1W Turboflash and T2W SE sequences were not statistically different. T2 SE was more reliable than T2 W turboflash in the characterization of hemangiomas and cysts.

Conclusions: Although T1 W Turboflash can replace T1 W SE in liver 
imaging, T2 SE remains the most reliable sequence both for detection and characterization of liver tumors at $1.5 \mathrm{~T}$.

\section{$2.55 \mathrm{PM}$}

1183 MR differentiation of small hepatocellular carcinoma from small cavernous hemangioma

Kuni Ohtomo, Yuji Itai, Yasuhito Sasaki, Yumiko Ohtomo, Tsutomu Araki; Tokyo

Purpose: to evaluate the efficacy of transverse relaxation time (T2) in differential diagnosis between small hepatocellular carcinomas (HCCs) and cavernous hemangiomas.

Methods: Fifty-two HCCs and 106 cavernous hemangiomas less than $2 \mathrm{~cm}$ in diameter were studied with magnetic resonance (MR) imaging at $1.5 \mathrm{~T}$. Protondensity and T2-weighted spin echo images were obtained. The time parameters used were 2000/28, 75 (TR msec/first-echo TE, second echo TE msec) or $2000 / 22,90$. T2 values were calculated by two point method from a pair of images with different TEs.

Results: T2 values were shorter than $60 \mathrm{msec}$ in the all HCCs and longer than $60 \mathrm{msec}$ in 91 hemangiomas. When the T2 borderline between HCCs and hemangiomas was set at $60 \mathrm{msec}, 143$ of 158 lesions $(90.6 \%)$ were correctly classified. The sensitivity of T2 differentiation for HCCs was $100 \%$.

Conclusions: MR imaging with T2 measurement was very useful for differentiation between HCCs and hemangiomas less than $2 \mathrm{~cm}$ and should be performed after screening ultrasound to avoid unnecessary invasive examinations in patients with hemangiomas.

\subsection{PM}

\section{$1184 \mathrm{MRI}$ of focal nodular hyperplasia}

V.Vilgrain, J.F. Fléjou, J.Belghiti, L. Arrivé, D. Najmark, Y. Menu, J.P. Benhamou, H. Nahum; Clichy

Purpose: to study the diagnostic value of magnetic resonance imaging (MRI) in histologically proven focal nodular hyperplasia (FNH).

Methods and Materials: twenty-three patients ( 22 female, one man) with one or more FNH were prospectively studied. All patients had MRI examination. Five patients had T1 weighted sequence after Gadolinium-DOTA intravenous injection.

Surgery was performed in all cases. Radioanatomic correlations were performed. Signal analysis on MRI was compared to histological findings. Results: surgery disclosed 29 tumors in 23 patients. Typical features of FNH: homogeneous mass, intensity slightly different from the liver intensity on all sequences, hyperintensity of the central scar on T2 weighted sequences. In six patients the scar was hypointense on T2 weighted sequences, and in six other patients the scar was not seen. In two cases the mass was not visible on MRI. T1 weighted sequences performed after Gadolinium injection showed better visualization of the central scar in all cases. Presence and size of the central scar on MRI were well correlated with macroscopic findings in all cases. In nine out of the 12 hyperintense scars on T2 weighted sequences: histological analysis found predominant edema. In three out of the four hypointense scars on T2 weighted sequences, histologic analysis discovered marked fibrosis.

Conclusions: in this surgical study, MRI allows specific diagnostis of FNH in $40 \%$. There is an excellent radioanatomic correlation about the presence and size of the scar. There is a relationship between the signal intensity of the central scar and the histological composition of the scar.

\subsection{PM}

1185 Dynamic contrast-enhanced MR imaging of liver lesions: improved diagnosis of focal-nodular hyperplasia?

B. Hamm, M. Lüning, M. Taupitz, Ch. Dewey, G. Branding, M. Mühler, A. Speidel, K.-J. Wolf; Berlin

Purpose: Assessment of the diagnostic value of dynamic MRI (fast imaging in combination with intravenous bolus injection of Gd-DTPA) in suspected focal-nodular hyperplasia (FNH).

Methods: Standardised dynamic MR examination of liver lesions in 96 patients $(1.5 \mathrm{~T}, 0.1 \mathrm{mmol} \mathrm{Gd}-\mathrm{DTPA} / \mathrm{kg}$, imaging up to $10 \mathrm{~min}$. after application of contrast agent by rapid, T1-weighted pulse sequences; two-center study carried out at Klinikum Steglitz and Charité).

Qualitative and quantitative evaluation of 29 patients with confirmed FNH and of 67 patients with malignant liver tumors.

Results: Only $53 \%$ of FNH showed the known isointensity to the surrounding liver tissue in T1- and T2-weighted plain imaging. However, using dynamic MRI, all FNHs showed a strong contrast agent enhancement with a maximum already $15 \mathrm{sec}$. after application and subsequent continuous decrease of signal intensity. In contrast, the central scar (confirmed in $49 \%$ of cases) showed late enhancement. In comparison with 67 dynamic MR examinations of malignant liver tumors, similar contrast agent dynamics were observed only in two cases. Conclusions: Dynamic contrast-enhanced MRI improves the diagnostic accuracy in FNH compared to plain imaging and facilitates differential diagnosis of FNH and malignant liver tumors.

\section{Room E1}

\section{SS}

02.09 Imaging in aortic and peripheral vascular disease $2.00 \mathrm{PM}$

1186 Growth rate of aortic aneurysm as measured by computed tomography

Yoshiaki Hirose, Seiki Hamada, Satoshi Imakita, Hiroaki Naito, Tadashi Nakanishi, Tatsurou Kaminaga, Makoto Takamiya; Osaka

Purpose: Computed tomography (CT) is superior to angiography and ultrasonography in several points, but there have been reported few papers related to the growth rate of aortic aneurysm measured by CT. We analysed the growth rates of thoracic and abdominal aortic aneurysms with CT examinations to evaluate the natural history of aortic aneurysm.

Methods and Materials: From May 1980 to August 1990, sequential CT examinations were performed on 160 patients ( 126 males and 34 females) aged 32-85 years with at least 6 -month intervals (mean 32 months). The number of measured diameters of aneurysms was 195 (ascending: 10, arch: 33, descending: 31, suprarenal: 18, infrarenal: 103).

Results: The mean growth rates of the thoracic and abdominal aortic aneurysmal diameters were $0.42 \pm 0.41$ and $0.28 \pm 0.24 \mathrm{~cm} /$ year, respectively. The difference between these two groups has stastical significance $(p<0.005)$. Abdominal aortic aneurysms with an initial diameter exceeding $4 \mathrm{~cm}$ showed a significantly faster increase in size compared with smaller aneurysms $(\mathrm{p}<0.01)$.

Conclusions: Our study showed quantitatively not only the growth rates but their differences in initial diameters with stastical analysis.

\subsection{PM}

1187 Inflammatory aneurysms of the abdominal aorta: role of ultrasound

A. Cilotti, P. Bagnolesi, R. Lencioni, D. Caramella, C. Bartolozzi; Pisa

Purpose: Establish the diagnostic accuracy of US in the diagnosis of the inflammatory aneurysms of the abdominal aorta as far as the nature and the complications are concerned.

Methods: Thirty-three inflammatory aneurysms were found within a patient-population of 200 abdominal aortic aneurysms, all submitted to US and CT (16,5\% incidence).

Results: The inflammatory nature was demonstrated on US in 27 out of 33 cases with diagnostic accuracy of $78 \%$; as to the complications (ureteral narrowing, caval narrowing and enteric involvement) US did not reveal themselves as highly accurate. In all cases CT correctly pointed out the inflammatory nature and the complications (with the exception of the enteric involvement). Surgery was performed in 23 patients.

Conclusions: US can demonstrate the inflammatory nature up to $78 \%$ of the cases (against the referred-in-the-literature 33\%); this high percentage significantly drops when complication are concerned.

The inflammatory nature often goes unnoticed either because of knowledge of this pathology or because of unproper technique of examination (higher frequences probes and proper gain settings are required); the inflammatory aneurysms may have higher incidence than the commonly referred 5-15\%; likewise, periaortic fibrosis may also accompany mild aortic dilatation.

\subsection{PM}

1188 The value of emergency CT in cases of acute symptomatic inflammatory aneurysms of the abdominal aorta

E. Rinast, E. Gmelin, I. Grande-Nagel; Lübeck

a) Abdominal aortic aneurysms associated with retroperitoneal fibrosis are called inflammatory aortic aneurysms. Surgical measures are much more difficult in those cases than in usual atherosclerotic aneurysms. Therefore pre-operative diagnosis of that special type of aneurysm is particularly valuable for adequate planning of surgical intervention.

b) In a retrospective study 346 consecutive emergency computed tomographic examinations were compared with intraoperative findings.

c) In 35 cases ( $10.1 \%$ ) the typical peri-aortic retroperitoneal fibrosis could be demonstrated preoperatively by computed tomography. CT sensitivity to demonstrate involvement of periaortic organs was as follows: duodenum $89 \%$, left renal vein $100 \%$, renal arteries $74 \%$, and ureter $94 \%$.

d) Even in cases of clinical and/or sonographic unequivocal acute symptomatic abdominal aortic aneurysms preoperative CT should be performed to realize the potential inflammatory character of the aneurysm. 
2.30 PM

1189 The role of MRI in the preoperative evaluation of abdominal aortic aneurysms

Peter E. J. M. Sallevelt, Jelle O. Barentsz, Olette C. Hartog, Willem P. T. M. Mali, Sjef H. J. Ruys, Frans M. J. Heystraten; Nijmegen

Purpose: Assessment of the preoperative value of MRI compared to angiography and ultrasound in patients with abdominal aortic aneurysm.

Methods: A prospective comparative study was performed on 21 patients having an aneurysm of the abdominal aorta. All patients underwent angiography and ultrasound as well as MRI. The results were interpreted by 3 observers independently and finally compared with findings at operation. We used $\mathrm{Tl}$-weighted spin echo sequences $(1,5 \mathrm{~T})$, in at least 3 planes.

Results: MRI provided correct information about the involvement of coeliac, mesenteric, and renal arteries. In 2 out of 5 patients double renal arteries were not noted. These could be recognised retrospectively however. The presence of renal or iliac artery stenosis was false-negative in one patient, and in another false-positive. MRI provided additional information such as a thrombosed suprarenal saccular aneurysm, periaortitis, and quality of the thoracic aorta. Ultrasound proved to be not reliable, while angiography was overstated in one patient.

Conclusions: We conclude that MRI is a reliable alternative in the preoperative management of aneurysms of the abdominal aorta. Only in the detection of double renal arteries angiography scores slightly better. This drawback however will be overcome through the implementation of angiographic software in MRI.

\subsection{PM}

\section{CT of acute rupture of aortic abdominal aneurysm (A.A.A.)}

J. A. Jimenez Lasanta, J. M. Rius Chornet, A. Alvarez Castells, A. Segarra, C. Torrens, S. Pedraza; Barcelona

All the CT performed in Emergency Department of our Hospital with clinical suspicion of rupture are revised.

In 21 cases, $\mathrm{CT}$ confirmed the AAA diagnostic with intraabdominal bleeding. In another case $\mathrm{CT}$ showed that the bleeding came from a splenic artery giant aneurysm.

CT was able to determinate the size and real extension of the aneurysm as well as the rupture point of direct form in a few of cases or indirectly according to bleeding location.

In most of cases the bleeding was located at left posterior pararenal space but almost always with extension to others compartments.

Occupation of pararenal anterior space was confirmed in almost two thirds of patients. Other places with a lower location frequency are: right pararenal posterior space, abdominal wall, mesentery, the hepatorenal space and gutter.

\subsection{PM}

1191 Cine MRI of aortic dissection - Comparative study with spin-echo static MRI, contrast enhanced CT and DSA -

Toshiaki Nitatori, Hajime Takaki, Junichi Hachiya, Tateo Korenaga, Minoru Okada, Yoshiro Furuya; Tokyo

Purpose: Investigation of applicability of cine MRI in the evaluation of aortic dissection.

Methods: Cine MRI was performed in 25 cases of aortic dissection with double barreled structure and comparative study among cine MRI, spin echo static MRI, contrast enhanced CT and digital subtraction angiography was made. Results: Cine MRI accurately detected aortic dissection. It was found to be most accurate among various diagnostic methods in demonstration of entry site of dissection (Cine MRI; $90.5 \%$, static MRI; $52.4 \%$, enhanced CT; $27.8 \%$, IVDSA; $56.3 \%$ )

Take-off of renal artery and its relation to true and false channels was also accurately demonstrated by cine MRI.

Conclusions: The above results suggest that cine MRI can be an important diagnostic modality in the examination of aortic dissection.

\section{$2.53 \mathrm{PM}$}

\section{Diagnostic utility of ultrafast CT for dissection of the ascending aorta}

Makoto Takamiya, Seiki Hamada, Hiroaki Naito, Satoshi Imakita, Naoaki Yamada, Mitsushige Ohta, Koji Kimura; Osaka

Purpose: Evaluation of detectability of the intimal flap and the tear in the ascending aorta with ultrafast CT (UFCT).

Methods and Materials: Seventeen cases of dissection of ascending aorta ( 7,8 and 2 cases of DeBakey-I, DeBakey-II and thrombosed dissection, respectively) were studied using an UFCT scanner with a scan time of 100 or $200 \mathrm{~ms}$, a slice-thickness of $6 \mathrm{~mm}$, a slice-interval of $10 \mathrm{~mm}$ and a bolus injection of non-ionic contrast media. The patients did not hold the breath during scanning. Intraarterial DSA was concurrently carried out in 13 out of 17 cases. Results: Respiratory movement did not degrade image quality of the aorta in $200 \mathrm{~ms}$ scan. Intimal flaps and tears were clearly depicted in all except one case of Marfan syndrome, which had to be studied with scan time of $100 \mathrm{~ms}$. One out of 15 cases had multiple entries, which could be depicted with UFCT. Ulcer-like projections in thrombosed dissection were also clearly depicted. Detectability of the intimal flaps with UFCT was superior to that of DSA. Conclusions: Detectability of the intimal flap with UFCT and the tear with UFCT was feasible to eliminate preoperative angiographic study in almost all of emergency cases. Scanning without breath hold and short total scanning time were especially beneficial for severe patients.

\section{$3.03 \mathrm{PM}$}

\section{Single-Breathhold volumetric CT (SVCT) in suspected aortic} dissection

M. Soucek, P. Vock, W. Kalender; Bern

Purpose: Although dynamic CT (DCT) is a reliable method in aortic dissection, artifacts within the ascending aorta may still be difficult to differentiate from an intimal flap. We hypothesized that single-breathhold volumetric CT (SVCT), a method for 3D data acquisition based on continuous tube rotation with table incrementation, might reduce these artifacts.

Methods: Twenty patients (ages $25-76$ years) with suspected thoracic aortic dissection, 19 of which had streak artifacts on DCT scans (contrast bolus $100 \mathrm{ml}, 10$ contiguous $1 \mathrm{~s}$-scans in $35 \mathrm{~s}$, collimation $10 \mathrm{~mm}$ ), were studied by SVCT $(60 \mathrm{ml}$, continuous $12-24 \mathrm{~s}$-scans, feed $5-8 \mathrm{~mm} / \mathrm{s}$, collimation $5-8 \mathrm{~mm}$ ). Helical raw data were interpolated to generate planar data and images every $4 \mathrm{~mm}$. Image quality of SVCT was compared to DCT.

Results: SVCT findings were correct in all cases. Image quality of SVCT was identical to DCT in 11 , superior in 8 cases and inferior in 1 case. Dissection was excluded or diagnosed when DCT findings were non-diagnostic $(n=4)$. Closely spaced SVCT images allowed for excellent reformations.

Conclusions: In suspected aortic dissection the accuracy of CT can be improved by adding volumetric scanning during apnea. The authors suggest to use SVCT alone in order to reduce the contrast dose and examination time needed.

\subsection{PM}

\section{Massive hematemesis due to an aberrant right subclavian artery- esophageal fistula}

Manuela Weissgerber, Georg Küffer, Dietbert Hahn; Munich

Introduction: This communication describes the erosion of a lusoric artery into the esophagus caused by mechanical pressure of a nasogastric tube.

Case Report: A patient with an isolated head trauma developed fulminant gastrointestinal bleeding. No bleeding source could be detected gastroscopically or at laparotomy. Upper esophageal bleeding was suspected and aortic arch angiography performed, demonstrating the erosion of a lusoric artery into the esophagus.

Comment: A lusoric artery-esophageal fistula should be considered in the differential diagnosis of massive hematemesis in presence of a nasogastric tube.

\section{$3.16 \mathrm{PM}$}

\section{Translumbar catheter angiography with a new 5-F needle-sheath} system

\section{E. Rinast, E. Gmelin, M. Zwaan; Lübeck}

We present a newly developed 5-F needle-sheath system, which allows translumbar introduction and interchange of 4-F-catheters with various lengths and shapes.

The system was used in 236 patients. In 227 cases a pigtail catheter was introduced for outflow studies of the lower extremities or visualization of the aortic arch, respectively. In 9 cases a selective catheter (sidewinder, headhunter, cobra) for angiography of a total of 19 vessels (carotid arteries, visceral arteries, renal arteries) was used. No major complications related to aortic puncture or catheter manipulation occurred, no relevant retroperitoneal hematomas were observed.

A new 5-F needle-sheath catheter delivery system allows translumbar selective and non-selective angiography. It is a safe and simple alternative to the more precarious transbrachial technique.

\subsection{PM}

1196 Initial observation on penetrating atherosclerotic ulceration of the descending thoracic aorta with transesophageal echocardiography Toshio Yamaguchi, Kazunori Kuroki, Tohru Ishikawa; Kawasaki

Penetrating Atherosclerotic Ulceration of the descending thoracic aorta is a poorly understood entity. We describe our initial observation on this entity by 
transesophageal echocardiography (TEE) in 3 patients with typical CT features. A thick crescent-shaped echogenic aortic wall was present in all 3 , suggesting intramural hematoma. Multiple low echoic area with arterial flow was noted in this hematoma. Flow through the intima was seen in 2, indicating penetrating portion of atherosclerotic ulceration. TEE may be useful for detecting the penetrating site and residual flow within dissecting hematoma of descending thoracic aorta.

\section{$3.22 \mathrm{PM}$}

\section{Obstructive arterial disease in patients with pathology glucose test (PGT)}

H.-J. Maurer, Marta Marin-Grez; Kuala Lumpur

Due to WHO PGT belongs to the Diabetics Group. Therefore 148/170 angiographies: 78 non-diabetics, 58 diabetics (11 type I, 47 type II), and 12 pat. with PGT, are retrolectively evaluated. About $25 \%$ of non-diabetics and PGT pat. are women, in diabetics there is no sex difference. Due to risk factors no difference could be found in PGT pat. compared with non-diabetics, but both groups show different high risk factors compared with diabetics. Angiographic findings are separately evaluated for right and left limb, the results had been statistically controlled. Generally no difference was shown between non-diabetics and PGT pat., but as known lower limb arteries are more often involved in diabetics. Severity of obstructive arterial disease was equal in all groups. In our collective no differences are found due to arterial obstructive disease regarding distribution and risk factors between non-diabetics and PGT pat., but both groups differ statistically significant to diabetic group.

\section{Room E2}

\section{SS} $2.00 \mathrm{PM}$

1198 Postoperative conditions and fracture healing (Keynote Lecture) Niels Egund; Odense

Radiology play an integral part in the monitoring of musculo-skeletal injuries and disorders, and in the assessment of their treatment being conservative or surgery. Plain radiography is still the most important modality following bone and joint surgery and there is a need for further development of precise methods of measurements obtained from radiographs due to the high accuracy of modern orthopedic surgery. The role of CT, US and scintigraphy is limited in the first postoperative period, but increase at later follow-up of surgery, in particular in the assessment of complications. The role of MR imaging has rapidly expanded in the follow-up of non-prosthetic joint surgery by its noninvasive ability to visualize postoperative healing e.g. recovery of subchondral lesions and regeneration of cartilage in osteoarthrosis and remodelling of the cartilaginous epiphysis after varus osteotomy in Legg-Calvé-Perthes disease. Recent experience indicates that MR imaging is particular valuable in evaluating the surgical result of repair of soft tissue lesions, tendons and ligaments as well as complications to treatment. In these aspects the interpretation of the MR images may, however, interfere with lack in our knowledge about the appearance of the "normal" postoperative process of recovery.

\subsection{PM}

1199 MR and discography of intervertebral disk trauma due to vertebral fractures

B. Allgayer, St. v. Gumppenberg, B. Kersting-Sommerhoff; Munich

Purpose: Evaluation of MR findings of intervertebral disks of fractured vertebrae and comparison with discographic findings.

Methods and Materials: 54 disks in 27 pat. with fractured vertebrae were studied with MR imaging and discography.

MR measurements were obtained using a $0,5 \mathrm{~T}$ and a $1,5 \mathrm{~T}$ superconductive unit. The protocol included $5 \mathrm{~mm}$ thick sagittal images (T1-, protondensityand T2-weighted sequences). Discography was performed intraoperatively with fluoroscopic monitoring.

MR and discographic appearance were categorized in 3 types: no damage (type I), minor damage (type II) and major damage (type III).

Results: The sensitivity of MR compared with discography was $96 \%$, the specificity $75 \%$ and the accuracy $88 \%$.

Conclusions: MR is an appropriate method for detecting and categorizing traumatized intervertebral disks.

\section{MRI evaluation of TMJ abnormalities related to cervical} extension-flexion injuries (WHIPLASH)

F. G. Shellock, B. D. Pressman, J. Schames, M. Schames; Los Angeles, CA

Purpose: Patients with cervical extension-flexion injuries (whiplash) from motor vehicle accidents often have TMJ-related symptoms. Therefore, MRI was used to evaluate TMJ abnormalities in this patient group.

Methods: 33 symptomatic patients were evaluated by MRI; 13 patients had no direct trauma to the jaw, mouth, head, or face during the automobile accident and none of the patients had a prior history of TMJ symptoms. A 1.5 T MR scanner was used along with dual $3^{\prime \prime}$ surface coils to obtain Tl-weighted, closed and opened-mouth views in the sagittal plane and closed-mouth views in the coronal plane. T2-weighted, closed-mouth views in the sagittal plane were also obtained to optimize identification of edema/fluid.

Results: $50 \%$ of the TMJs had ane of the following abnormalities: 19 - anterior disk displacement with reduction, 9 - anterior disk displacement without reduction, 4 -medial or lateral disk displacement, and 1 - posterior disk displacement. On T2-weighted images, $36(55 \%)$ TMJs had joint fluid localized to the joint space and/or lateral pterygoid muscles.

Conclusions: These data demonstrated that a high percentage of patients had abnormalities of the TMJ related to whiplash injury. The predominant finding was associated fluid/edema, indicating that T2-weighted images are especially useful for assessment of patients that present with whiplash injury.

\section{$2.35 \mathrm{PM}$}

\section{Three-dimensional computed tomography of fractures} M. Ernsting, H.W. Stedtfeld, E. Zeitler; Nürnberg

Computer programs that produce $3 \mathrm{D}$-surface reformations from sets of axial CT's were used in evaluating a variety of fractures. The 3D-Images were useful in complex displaced fractures and in some cases also to show the extent of fine fracturelines. 3d-Reconstructions are in many cases helpful to show an overview of the extent of fractures and the displacement.

$3 \mathrm{D}$ brings a good orientation for a reconstruction surgery; it is helpful in surgery of fractures from shoulder, scapula, hips and hipjoint, calcaneus and middle-face-injuries.

\subsection{PM}

\section{Osseous 3D-CT in traumatology}

P. Uhrmeister, F. Astinet, M. Langer; C. Zwicker, B. Erdtmann, R. Felix; Berlin

Purpose: Transversal CT slices do not always provide the optimal spatial information of fractures. This study was performed to evaluate the feasibility of the threedimensional CT (3D-CT) in skeletal traumatology.

Methods and Materials: 32 patients with fractures in different locations were examined by $\mathrm{CT}$ with following threedimensional reconstruction. The thickness of the slices was $2 \mathrm{~mm}$. The time for scanning and 3D-reconstruction was $30-45$ minutes.

Results: The image quality was excellent without any artefacts in 15 cases. A slight reduction of image quality was due to motion and metal artifacts in further 13 patients. In all these cases the threedimensional images were classified as helpful for demonstration of the fracture extension but not as decisive for diagnosis. In 4 uncooperative patients sufficient threedimensional images could not be achieved.

Conclusions: The threedimensional CT facilitates the delineation of complex fractures and has therefore a widespread acceptance.

\subsection{PM}

1203 Acetabular fractures: value of three-dimensional CT imaging J.J. Railhac, M. Brunet, P. Maquin, P.Samaran, J.Assoun, E. Bloom; Toulouse

Purpose: Evaluation of $3 \mathrm{D}$ imaging in the pre-operative analysis of acetabular fractures.

Methods: Twenty-five patients with a recent acetabular fracture were submitted to plain radiographs and conventional CT scans along with $3 \mathrm{D}$ imaging. All patients were evaluated for the type of the fracture, degree of communition and displacement, intra-articular fragments. Each of the techniques was compared in respect to its diagnostic accuracy.

Results: $-3 \mathrm{D}$ imaging did not bring any complemental information than conventional explorations. Plain radiographs and axial CT scans were more sensitive than the $3 \mathrm{D}$ images in detecting undisplaced fractures.

- However, $3 \mathrm{D}$ technique allows a perfect spatial representation of the lesion. The multidirectional view is most helpful in the evaluation of the importance, the disruption and the orientation of the bone fragments. It has an overwhelming interest in the study of the fractures involving posterior acetabular wall and comminuted fractures which analysis is known to be most 
difficult. It provides the surgeon with an anatomical representation of the lesion, much alike its surgical appearance.

Conclusions: The $3 \mathrm{D}$ images are useful in complex displaced fractures.

3.05 PM

1204 High frequency sonography for evaluation of traumatic lesions of skeletal muscles

W. Dock, W. Happak, F. Grabenwöger, V. Metz, K. Eibenberger, W. Gruber; Vienna

Purpose: Evaluation of the value of high frequency sonography for diagnosis and classification of traumatic lesions of skeletal muscles.

Methods: In order to evaluate the normal sonomorphology of skeletal muscles 8 musculi graciles of human corpses have been examined sonographically with high frequency ultrasound probes $(7,5$ or $10 \mathrm{MHz})$. Sonographic-histologic correlations revealed that the smallest structures of skeletal muscles which may be differentiated sonographically are the primary bundles and the internal perimysial septa. This knowledge has been applied for the evaluation of 145 patients with clinically suspected muscular trauma. The mean age of the 94 male and 51 female patients was 43 years ( $15-72$ years)

Results: In 68/145 patients muscle tears could be detected, in $77 / 145$ cases a muscle tear could be excluded. In 19 cases a complete tear was diagnosed. In 49 cases the rupture was classified as incomplete. The knowledge about normal sonomorphology of skeletal muscles provided new signs for detection and classification of muscle tears.

Conclusions: High frequency sonography is an excellent method for evaluation of muscle trauma and should be performed routinely if a muscle tear is suspected clinically.

\subsection{PM}

1205 Digital luminescent radiography for diagnosis of skeleton injuries B. Buddenbrock, R.-D. Müller, V.John, H.-J. Kock, M. VoB, G. Schmidt, E. Bosch, E. Löhr, K.-P. Schmit-Neuerburg; Essen

The image quality of digital luminescent radiographs is proved for patients with traumatic skeleton and joint lesions.

Methods: Screen film radiographs were done with Agfa Curix ortho STG, combined with Curix ortho medium/fine. The post processing of the digital data shows images with different characteristics such as similar to conventional screen film radiographs, edge enhanced radiographs and images processed by the use of optimized weighting factors. The evaluation was performed by three radiologists and two specialists for accident surgery. Criteria were the image quality concerning compacta, spongiosa, soft tissue, fractures and osteosynthesis.

Results: The demonstration of important skeleton details is often significantly better on conventional radiographs $(p<0.05)$ except the recognition of soft tissue on the edge enhanced digital image $(\mathrm{p}<0.05)$. The detection of compacta and spongiosa details is improved by appropriate digital post processing $(\mathrm{p}<0.05)$ (high-frequency filtering, gray scale inversion, contrast variation). No advantages in the recognition of fractures are demonstrable on digital images, because loosening signs around osteosynthetic material are greatly diminished due to special artifacts.

Conclusions: Improvement of the available standard digital imaging is necessary, since the required image quality can up to now only be reached by interactive image processing. High resolution imaging plates in all sizes should be available to improve detail recognition.

\section{$3.25 \mathrm{PM}$}

\section{New morphological MRI findings on articular-cartilage changes:} in vitro measurements using a specially compression device R. Andresen, M. Bollow, H. König, K.-J. Wolf; Berlin

Purpose: Establishment of a clinically applicable standard curve for quantification of the compressibility and signal characteristics of healthy and pathologically altered retropatellar articular cartilage.

Methods and Materials: The examination was performed in ten knees removed immediately post mortem, six with arthrotic changes of varying degrees and four without degenerative alterations.

Examination methods included exploratory axial measurement (SE $100 / 15 \mathrm{~ms}$ ), sagittal double-echo measurement (SE 1600/22-90 ms, $4 \mathrm{~mm}$, $1 \mathrm{Ac}$ ) and gradient echo sequences in axial and sagittal sectioning (FLASH $50 / 10 \mathrm{~ms}, 4 \mathrm{~mm}, 4 \mathrm{Ac}$ each with $10^{\circ}$ - and $30^{\circ}$-flip angles) at a defined increasing compression $(0,10,15,20,25,30 \mathrm{kp}$ and maximal $\mathrm{kp})$ using a specially constructed compression device. Decreases in cartilage thickness and changes in signal intensity were measured in three different reproducible areas. Results: Degeneratively altered cartilage showed a significantly lower decrease in thickness and a less marked loss of signal intensity under compression than histologically normal cartilage.

Conclusions: This recently developed MR-specification can add important information to the known criteria for cartilage diagnostics.

\section{Room L}

SS

\subsection{Gastro-Intestinal}

$2.00 \mathrm{PM}$

1207 Presentation and diagnosis of esophageal perforation in children J. E. Benson, G. A. Taylor, J. P. Dorst, A. Jabra, T. D. Trinh; Baltimore, MD

Six patients ( 4 females, 2 males, age 2.5-17 years) had varied clinical and radiographic presentations of esophageal perforation. Five patients had history of recent anti-reflux surgery or instrumentation; the sixth had dermatomyositis. All esophagrams were performed with water-soluble contrast medium. Chest film findings just prior to the diagnosis of perforation included localized infiltrate, localized hydropneumothorax, pleural effusion, and pneumomediastinum. Residual soft tissue air and edema from recent surgery often complicated and delayed diagnosis of perforation which, in one case, was remote from the site of surgery. In another, diagnosis was made after successive normal examinations.

Conclusions: 1. The appearance of mediastinitis on chest film often preceeds esophageal perforation. Since the esophagus has no serosa, it may be hypothesized that the inflammation may in fact cause perforation where there was no previous tissue trauma. 2. Water-soluble contrast is useful for serial studies of the esophagus because it is completely resorbed with no residue to complicate further interpretation. In iso-osmolar dilution, non-ionic contrast is still visible radiographically while diminishing risk in case of aspiration.

\subsection{PM}

1208 Hypertrophic pyloric stenosis: use of ultrasonography in diagnosis and demonstration of pyloric changes after pyloromyotomy T. Sindel, E. Lüleci, S. Yilmaz, M. Melikoğlu; Antalya

23 infants referred with suspected diagnosis of hypertrophic pyloric stenosis (HPS) were examined by real-time ultrasonography (US) in order to evaluate the accuracy of US in this disorder. For each patient, measurements of pyloric muscle-wall thickness (MT), diameter (PD) and maximum pyloric length (PL) were made while gastric peristaltic waves and emptying were observed. In 20, diagnosis of HPS was established by US and confirmed by operation.

In a comparable group which comprises 20 infants hospitalized for conditions unrelated to HPS, US examinations were performed and the results were compared with those of 15 patients with HPS. No overlap was found between MT, PD and PL values of HPS patients and those of control group.

After pyloromyotomy, ultrasonographic pyloric measurements were made on 15 patients with HPS in the 1st, 4th and 8th post-operative weeks and at the end of the 4 th month. Changes in pyloric tumor were noted. It was observed that all parameters returned to normal by the end of the 8th week and didn't change significantly thereafter.

This study suggests that, taking all the diagnostic criteria into consideration, ultrasonographic diagnosis can be accurately made in the great majority of HPS cases. Furthermore, post-operative changes in pyloric dimensions can be followed easily and reliably.

\subsection{PM}

1209 Umbilical artery Doppler in high risk pregnancy: relationship to outcome

P. Twining, J. Swallow, E. Lewin; Nottingham

Purpose: In order to assess the role of umbilical artery Doppler in high risk pregnancy a retrospective study was carried out.

Methods: Over a 12-month period systolic/diastolic ratios of umbilical artery blood flow were obtained in 119 high-risk pregnancies. The main indications for a Doppler study were: suspected or documented growth retardation, pregnancy induced hypertension and reduced fetal movements. Systolic/diastolic ratios were classified into four groups: I. Normal; II. A raised ratio which subsequently returned to normal; III. Raised systolic/diastolic ratio but positive diastolic flow; IV. Absent end diastolic flow.

Results: Patients with absent end diastolic flow had significantly higher Caesarean section rates, and Caesarian section for fetal distress was five times higher. Fetuses with absent and diastolic flow were delivered at an earlier gestational age (33.6 weeks), had lower birth weight $(1.68 \mathrm{~kg})$ and spent more time on the Special Care Baby Unit (15.4 days) than fetuses with normal ratios ( 38.6 weeks, $2.7 \mathrm{~kg}$. and 1.3 days, respectively). Patients with raised ratios but positive diastolic flow were delivered of babies with intermediate values for gestational age at delivery (37.6 weeks), birth weight $(2.2 \mathrm{~kg})$ and Special Care Baby Unit stays (6.2 days).

Conclusions: We feel that umbilical artery Doppler is a useful parameter in monitoring high-risk pregnancies. Abnormal Doppler ratios are associated with an adverse outcome. 
1210 Duplex-Doppler in differential diagnosis of pediatric masses M. Torres, B. Asenjo, Mad. Martinez-Piazza, R. Vicente, L. Ceres, I. Alonso; Malaga

Purpose: We present our experience in the use of Duplex-Doppler in the differential diagnosis of benign masses (both inflammatory and non-inflammatory) and malignant masses in pediatric patients, based on the flow patterns found in intralesionary vessels.

There is at present no agreement in this matter.

Methods: We studied 48 patients aged between 1 day and 12 years: 14 infectious-inflammatory, 25 benign non-inflammatory and 9 malignant lesions. We carried out Doppler study of intra- and peri-lesional vessels in all patients and colour Doppler in 15. We measured quantitative (systolic and diastolic peaks and resistance index) and qualitative (spectral curve patterns) parameters. We considered velocities greater than $70 \mathrm{~cm} / \mathrm{s}$ or $3 \mathrm{KHz}$ and flow patterns of both shunts and neo-formed vessels to be representative of malignancy.

Results: In the benign masses, we found criteria of malignancy in $25 \%$ of inflammatory and $16.6 \%$ of non-inflammatory benign lesions. Normal or absent flows were present in $37.5 \%$ of malignant masses.

Conclusions: Comparing our results with the literature we conclude that Doppler flow patterns should be interpreted more as an expression of vascular architecture than as demonstrative of a benign or malignant lesion.

\subsection{PM}

\section{MR study of the most frequent solid tumors of childhood} Stefano Brusori, Francesco Gigli; Bologna

25 examinations using Magnetic Resonance were carried out on 11 patients suffering from neuroblastoma and Wilms tumours. The results were compared with those obtained using alternative diagnostic methods such as CT and US in order to verify the reliability of the examination in the location and staging of lesions. In the study of the signal behaviour registered in the images $\mathrm{T} 1$ and $\mathrm{T} 2$-weighted using various repetition times and echo times, it was attempted to identify the presence of pathognomonic findings and to differentiate tumour from posttherapy fibrosis. All the evidence was confirmed by bioptic examination or, in the case of patients who were subjected to surgical operation, by the operating report. In order to carry out all the examination it was necessary to sedate the patients or apply a general anaesthesia. One of the main advantages of $\mathrm{MR}$ is its ability to image in any plane which generally enables one to ascertain the extent of the lesion and the organ it belongs to. The additional advantages of MR over CT should not be underestimated: it allows, in fact, one to obtain images of the abdomen devoid of artifacts resulting from respiration and two provide a sharp contrast between pathological and healthy tissue without the aid of contrast medium. In all test cases, MR proved itself to be superior to CT in the evaluation of the intraspinal extension of the paraspinal lesions. MR also enabled regular examination to be carried out on the children, without the risk of subsequent proteximetric problems, allowing masses to be distinguished from fibrous tissue in all patients.

\subsection{PM}

\section{$1212 \mathrm{MRI}$ of pelvic masses in children}

\section{Hugosson, B. Jacobsson, H. Jorulf, K. Sackey; Riyadh}

The value of MRI was studied in 24 children between 9 months and 14 years of age with pelvic masses.

Spinecho technique with T1 coronal and sagittal and T2 axial images were performed in all patients and additional images if necessary. In 6 patients Gadolinium-DTPA (Gd-DTPA) was administrated.

The findings at MRI were compared with the findings at US and CT. MRI demonstrated superior delineation towards surrounding structures. The origin of the mass could be predicted in 17 out of the 24 patients with MRI but only in 10 patients with CT. Change in size after treatment was seen equal by US, CT and MRI. Change in signal intensity at MRI during treatment was noticed in 11 patients. Enhancement of tumour with Gd-DTPA was demonstrated in 2 out of 6 patients.

MRI offers high soft tissue contrast resolution and capacity of multidirectional imaging and appears superior to US and CT for assessment of pelvic tumours in children.

\subsection{PM}

1213 Pediatric intraperitoneal cystic masses: ten years follow-up and literature review

C. Lopez, M. Miralles, S. Borruel, L. Peral, G. Pozo, A. Muñoz, M. Martinez, A. Ramos; Madrid

Abdominal cystic masses non solid organ dependant are named mesenteric or omental because of their location or gross appearance. It is a descriptive and not histopathologic definition. These unusual tumors are benign. The present
True cysts, with endothelial inner lining (lin-phangiuoma), epithelial (duplication and neuroentheric cysts) or mesothelial; b) Pseudocysts, which have a fibrous wall without inner lining: non pancreatic pseudocysts.

We report a series of 18 cases of intraperitoneal cystic masses collected during the last 10 years in our institution. Radiologic and sonographic findings are described, and histopathological correlation documented. There were 18 cases: $43 \%$ lymphangiomas, $35 \%$ duplication and enteric cysts, $14 \%$ nonpancreatic pseudocysts, and in the remaining $8 \%$ we found 3 mesenteric hydatidosis and one case of peritoneal tuberculosis. Duplication cysts and lymphangiomas have specific ultrasonographic characteristics, useful in the preoperative diagnosis and differentiation from other intraperitoneal cysts.

\subsection{PM}

121411 new cases of pediatric-focal nodular hyperplasia of the liver value of imaging

E. Dion-Voirin, D. Pariente, F. Brunelle, M. Hadchouel, F. Gauthier, O. Bernard, P. Chaumont; Paris

Purpose: We present a retrospective clinical and radiological study over 20 years of 11 pediatric cases of focal nodular hyperplasia.

Methods and Materials: 11 cases of FNH have been observed in 10 girls and 1 boy, aged from $11 / 2$ to 11 years. US was performed in 10 cases, scintigraphy in 3, CT in 9, MRI in 3 and angiography in 11.

Results: The tumor was discovered fortuitously in 7 cases. Biological findings were always negative. The tumor was large, $(5$ to $10 \mathrm{~cm})$, unique and polylobulated. It appears isoechoic in 8, with dense contrast enhancement on CT in all cases, and on T2-weighted images iso intense in 2 and hyperintense in 1 . Central fibrous scar was evident in 7. Angiography demonstrates constant hypervascularity with peripheral venous network. 9 children were operated on with complete resection in 8 . Evolution was favorable in all patients with a follow-up of 9 and 11 years for 2 non operated tumors.

Conclusions: Diagnosis of FNH may be suggested in front of an asymptomatic large tumor with negative alphafetoprotein level, which appears homogeneous, hypervascular and centered by a fibrous scar, even in very young children.

The treatment of this benign tumor of unknown etiology is still debatable.

\subsection{PM}

1215 Jaundice as the first symptom of hydatidic liver disease in children: sonographic evaluation

C. Serrano, I. Cano, M. Miralles, A.Ramos, G. De Orbe, D. Tejedor, F. Olcoz; Madrid

We describe two cases of hydatidic liver disease in childhood with clinical manifestation of cholostasis as the first symptom, caused by rupture of the cyst content into the biliary tract. Involvement of the biliary tract depends on the stage of evolution of the cyst and its location, Jaundice is more commonly caused by cysts arising in segments 1 and 8 . Although communication between the cyst and biliary tract occurs in $60 \%$ of the cases, it is very uncommon to find biliary tract obstruction.

Hydatidic liver disease in childhood is evaluated by ultrasound and usually no other imaging modalities are required. In our two cases sonographic features included intra and extrahepatic bile ducts dilatation and solid, mixed echogenic hepatic masses representing the echinococcal cysts. Moreover in one of these patients solid material inside the coledocal lumen was found, representing ruptured membranes.

Jaundice as a result of the rupture hydatidic cyst in the biliary tract is an uncommon manifestation of echinococcus infection. The purpose of this work is to show our experience and to assess the value of ultrasound in evaluating this rare complication of hydatidic disease.

\section{Room M}

\section{SS} $2.00 \mathrm{PM}$

\subsection{Ultrasound - Doppler}

\section{Echo-Doppler in portal hypertension (Keynote Lecture)} Marco Zoli; Bologna

In the last years pulsed echo-doppler and color doppler have been extensively used in the non-invasive evaluation of portal haemodynamics, to obtain qualitative and quantitative data on the portal flow. Qualitative data (doppler spectrum and flow direction) are easy to obtain and are reproducible, while quantitative (flow velocity and volume) may be biased by the measurements of the $\theta$ angle and of the cross sectional area of the vessel.

In liver cirrhosis with portal hypertension, echo-doppler shows that the portal blood is generally directed towards the liver, but at a velocity lower than in control subjects $(10.5 \pm 4.2 \mathrm{~cm} / \mathrm{sec}$ vs $16.0 \pm 3.5)$. A reversed flow is present in a 
minority of patients: $2-8 \%$ in the splenic and superior mesenteric veins, $1 \%$ in the main portal vein. On the contrary, an hepatofugal flow may be frequently observed in the gastric and in the umbilical veins.

The effects of physiological conditions (exercise, meal) and of drugs was extensively studied. In particular echo-doppler proved that propranolol (a $\beta$-blocker useful in the prevention of bleeding from esophageal varices) reduces the portal flow of about $35-40 \%$ in cirrhotic patients.

More recently this non-invasive technique has obtained an important role in the pre- and postoperative assessment of patients undergoing porto-systemic shunts of liver transplantation.

\subsection{PM}

1217 Complex colour Doppler study in viral hepatitis and liver cirrhosis M. Elwertowski, S. Leszczynski, J. Cianciara, A. Sadownik, H., Oziemska, B. Zielecka; Zalesie Gorne

Measurements of Doppler signals arising from hepatic veins and portal vein compromising both velocity and signal spectrum evaluation appear to reflect intensity of inflammatory processes affecting liver parenchyma.

Doppler study was performed in the group of 360 patients with histologically proved liver function impairment. Abnormalities of venous flow seem to be related with the status of sinusoidal vessels, which are easily affected by liver edema and congestion (liver venous flow may remain normal in patients with portal vein thrombosis and end to side porto-caval shunts). Portal flow alterations reflect better irreversible changes associated with regeneratory processes leading to liver cirrhosis and portal hypertension. In our study portal flow changes were observed in 152 patients, while venous flow was altered in 182 of them. Biggest discrepancies were demonstrated in cases of acute hepatitis (53 patients) with portal flow altered in $28 \%$ of cases versus $72 \%$ abnormal venous flow and aggressive hepatitis (46 patients) with $46 \%$ versus $65 \%$. Colour Doppler (Thoshiba 270a) helped in elimination of errors associated with signal measurements, specially in patients with abundant parenchymal changes and narrow vessels.

In most cases when healing occurred venous flow returned to normal values. In aggressive and acute hepatitis Doppler evaluation of liver venous system is a better indicator of liver impairment degree than portal flow estimation. It can also serve as an excellent prognostic factor in follow up studies.

\subsection{PM}

1218 Liver tumors - comparison between echo Doppler - color Doppler flow imaging and ultra fast CT flow mode

M. C. Plainfosse, A. Hernigou, C. Mainard, C. Delattre, M. Marotel, D. Chossiere; Paris

Methods and Materials: CT densities of aorta-tumors and normal liver were studied with the Flow mode ( 20 images during 60 secondes after contrast injection $45 \mathrm{cc}-6 \mathrm{cc} / \mathrm{sec}$ ) - pulsed doppler and colored doppler were realised with a Radius machine.

Results: 1. in 8 hepatocarcinomas: curves were evocative with a peak 5-10 seconds after the aortic peak, arising to the same values that the adjacent liver but 20 seconds earlier. Then the tumor curve decreased and crossed the liver curve.

- The systolic doppler signal was between 1,5 and $6 \mathrm{KHz}$ and resistance index lower than $65 \%$ in 6 . Doppler was negative in one and unrealisable in the last. - The high systolic peaks were correlated with the visualisation of abnormal vessels inside the tumor

2. in metastases: A density peak appeared early at 10 seconds in 4 but values never reached the liver values and curves never crossed.

- Doppler signal was absent or lower than in hepatocarcinomas with index higher than $65 \%$

Conclusions: Doppler signals and CT density curves are well correlated with few overlapping between hepatocarcinomas and metastases.

Both techniques look contributive especially for detection of hepatocarcinomas.

\subsection{PM}

1219 How reliable are Doppler measurements of superior mesenteric artery blood flow?

Reinhold Mallek, Gerhard Mostbeck, Dimiter Tscholakoff, Adolf Mostbeck; Vienna

Purpose: To evaluate reproducibility and inter- and intraobserver variability of quantitative Doppler measurements of superior mesenteric artery (SMA) blood flow.

Methods and Materials: Doppler blood flow measurements were performed in 20 consecutive volunteers ( 11 male, 9 female, 23 to 60 years) by two independent, equally experienced investigators (RM, GHM) on the same ultrasound unit. 3 measurements of SMA diameter (DIA), resistive index (RI), time average velocity (TAV), peak systolic velocity (PSV) and blood flow (BF) were performed in the SMA $2 \mathrm{~cm}$ distant from the origin within 30 minutes of total investigation time.

Results: Intraobserver variability for the two investigators was excellent for measurements of DIA $(5.3 \%, 4.1 \%)$ and RI $(4.1 \%, 2.9 \%)$. However, variability for TAV $(24.5 \%, 22 \%)$, PSV $(19.2 \%, 14.7 \%)$ and BF $(28.5 \%, 26.6 \%)$ was much higher. For interobserver variability, similar results were obtained. Linear correlation of DIA and RI between the two observers was excellent $(\mathrm{n}=60$, $r=0.83$ and $r=0.87, p<0,001$ respectively), but $r$ was between 0.5 and 0.63 for TAV, PSV and BF. Additionally, the Wilcoxon-test revealed statistically significant differences between the two investigators in measurement of TAV $(24.5$ ( \pm 13$)$ vs. $18.1( \pm 7.4) \mathrm{cm} / \mathrm{sec}, \mathrm{p}<0.001), \operatorname{PSV}(2.4( \pm 0.9)$ vs $1.6( \pm 0.5)$ $\mathrm{m} / \mathrm{sec}, \mathrm{p}<0.001)$ and BF $(505( \pm 270)$ vs $383( \pm 178) \mathrm{ml} / \mathrm{min}, \mathrm{p}<0.001)$.

Conclusions: Measurement of the RI in SMA is a reliable hemodynamic parameter with small intra- and interobserver variability. TAV, PSV and BF reveal a high variability probably due to Doppler angle assessment and ultrasound gain setting.

\section{$2.45 \mathrm{PM}$}

1220 Color-Doppler and angio-MRI of portal vein thrombosis C. Radier, M.F. Bellin, S. Lenoir, Ph. Curet, J.C. Bousquet, D. Valla, J. Grellet; Paris

Purpose: The goal of this study is to compare color Doppler and angio MRI is the assessment of portal vein patency.

Methods and Materials: 15 patients with a portal vein thrombosis proved by $\mathrm{CT}$ and/or angiography were studied.

Results: Color Doppler examination was performed with an Acuson 128 unit equipped with a $3,5 \mathrm{MHz}$ transducer. A lack of color within the lumen indicated absence of blood flow.

MRI was performed on a Signa GE 1,5 T, in a body coil, using fast gradient echo imaging (GRASS). The absence of bright signal within the lumen of the vessel was diagnosis for thrombosis.

For color Doppler, 2 patients had a technically unadequate examination. On MRI, intense artefacts were seen on all patients with ascitis. Thrombosis of the portal vein was well defined in all technical adequate examinations in color Doppler and MRI.

Both color Doppler and MRI could not differentiate tumor from cruoric thrombus. Collateral pathway secondary to thrombosis was better imaged by MRI.

Conclusions: Both color Doppler and MRI can demonstrate portal vein thrombosis. Color Doppler offers the advantages of ease of examination and lower cost. MRI should be performed when color Doppler is technically inadequate.

\subsection{PM}

1221 Quantitative Doppler flowmetry of the portal vein in hepatic allografts

Gregory Foster, Myra Rapaport, Howard Sankary, Ester Brown; Chicago, IL

Purpose: Early detection of hepatic allograft injury would allow more rapid alterations in treatment of permit early retransplantation to improve recipient survival. The hepatic microvasculature is affected in ischemic preservation injury and chronic rejection, resulting in diminished sinusoidal blood flow. We investigated whether portal vein volume flow as determined by doppler ultrasound could provide an index of graft function.

Methods: Using duplex doppler ultrasound, we determined the cross-sectional area of the main portal vein (A) and the angle-corrected mean portal venous velocity $(\mathrm{V})$. Using the even insonation method, we computed portal vein volume flow $(\mathrm{Q})$ as $\mathrm{Q}=\mathrm{VA}$.

Results: In an animal model for ischemic preservation injury we found significantly reduced portal flow in animals with allograft injury. No significant difference in flow volume was demonstrated between normally functioning allografts and non-operated controls. We have evaluated 14 human patients with hepatic allografts. In patients with normally functioning livers $(\mathrm{N}=11)$, mean $Q=1458 \pm 415 \mathrm{ml} / \mathrm{min}$. Patients with allograft failure $(\mathrm{N}=3)$ demonstrated markedly reduced flow $(\mathrm{Q}=542 \pm 96 \mathrm{ml} / \mathrm{min} ; \mathrm{p}<0.001)$. This reduced flow volume is similar to that seen in cirrhotic patients undergoing preoperative evaluation for liver transplantation $(\mathrm{Q}=668 \pm 129 \mathrm{ml} / \mathrm{min}$ ). Conclusions: Decreased portal vein volume flow is associated with hepatic allograft dysfunction. Quantitative doppler flowmetry of the portal vein is a promising new non-invasive technique which may provide a unique physiologic indicator of graft viability.

\section{$3.00 \mathrm{PM}$}

\section{Duplex Doppler and color Doppler imaging in Budd-Chiari} syndrome

M. F. Bellin, C. Radier, J.C. Bousquet, S. Attias, D. Valla, P. Opolon, J. Grellet; Paris

Purpose: The aim of this study is to compare the effectiveness of conventional ultrasonography, pulsed Doppler and color Doppler in the diagnosis of 
Budd-Chiari syndrome (BCS).

Methods and Materials: Twelve patients with a surgically proved BCS were prospectively examined on an Acuson 128 unit, using real time ultrasonography, followed by pulsed Doppler and color imaging. CT was performed in 8 cases, MRI in 9 cases and angiography in 10 cases.

Results: In all cases, the hepatic veins appeared abnormal on ultrasound. Color Doppler was found useful to detect a non obstructive thrombus and to assess the direction of flow within collateral accessory veins. The velocity of blood flow in venous patent segments was assessed by pulsed Doppler. A caval thrombosis was identified in 2 cases. Pulsed and color Doppler correctly assessed the direction of portal flow, the presence of portal vein thrombosis and the direction of flow in portosystemic collateral veins. Patency of portocaval shunts $(\mathrm{n}=5)$ was clearly demonstrated by color Doppler.

Conclusions: We conclude that pulsed Doppler and color Doppler provide additional information that is similar to information obtained by invasive or more expensive imaging modalities, such as angiography.

\subsection{PM}

1223 Preoperative diagnosis of portal venous confluence involvement in pancreatic carcinoma - Duplex Doppler ultrasonography in comparison with angiography

Nico J.Smits, Jacques W.A.J. Reeders, Ad C.Wevers, Dirk J.van Leeuwen; Amsterdam

Purpose: To compare Doppler Ultrasonography (Doppler-US) and angiography for assessment of Portal Venous Confluence (PVC) involvement in patients with pancreatic head carcinoma.

Methods and Materials: 32 patients underwent both Doppler-US and angiography. Imaging results were compared with surgical and histopathological findings. Doppler-US was considered as abnormal correlating with PVC involvement only if pulsed-Doppler showed an abnormal flow pattern.

Results: PVC involvement was seen in $47 \%$ of the patients at surgery. In predicting of PVC involvement Doppler-US had a sensitivity of $40 \%$ and a specificity of $100 \%$ in comparison with angiography $66 \%$ and $100 \%$ respectively. In predicting of irresectability the results for both procedures were similar: sensitivity $60 \%$ and specificity $100 \%$.

Conclusions:- A normal Doppler-US may suggest: either no PVC involvement at all, or minor PVC involvement due to contiguity of tumor or slight compression and the tumor may be still resectable.

- An abnormal Doppler-US always concludes major PVC involvement and the tumor may be irresectable, or only resectable after venous vascular reconstruction.

- Doppler-US seems as accurate as angiography in diagnosing major PVC involvement and seems the examination of choice for PVC evaluation in carcinoma of the pancreatic head.

\section{Room N}

\section{SS}

$2.00 \mathrm{PM}$

1224 Oral Magnetic Particles (OMP) as an oral contrast agent for abdominal and pelvic MRI - Results from clinical trials

T.F. Jacobsen, T. Bach-Gansmo, A. Børseth, I. Haugen, B. Lundby, M. Svaland, A. Øksendal; Oslo

Purpose: To determine the safety and efficacy of Oral Magnetic Particles (OMP) used in MR examinations of patients with abdominal and pelvic pathologies.

Methods: OMP consists of crystalline magnetic iron oxides supported on a carrier matrix and suspended in a viscous solution. The contrast agent reduces the signal intensity from the contrast filled bowel. Approximately 450 adult male and female patients were included in a clinical trial program (phase II and III) in Europe. An MR examination after ingestion of OMP was compared to a non-enhanced MR examination. The OMP concentration and the volume given was in most cases $0.5 \mathrm{~g} / \mathrm{l}$ and $800 \mathrm{ml}$, respectively. During the phase II trials, various concentrations, volumes and viscosities were evaluated. Spin Echo and fast and ultrafast Gradient Echo sequences were applied at different field strengths from $0.02 \mathrm{~T}$ to $1.5 \mathrm{~T}$.

Results: OMP gives good contrast enhancement and shows diagnostic value in both upper and lower abdomen. The signal void is apparent on all applied sequences. The acceptance is good, and no serious adverse events have been reported.

Conclusions: The results from this clinical trial program implies that OMP satisfies a need of an oral contrast agent for MRI.
1225 Superparamagnetic particles as oral gastrointestinal contrast agent in MRI of abdomen

F. Boudghène, J. M. Faintuch, C. Wallays, T. B. Gansmo, M. Lambrechts, J. M. Bigot; Paris

A superparamagnetic iron oxide preparation for oral administration was tested in 35 patients referred to abdominal MRI examination.

On a $1.5 \mathrm{~T}$ superconducting magnet, $\mathrm{T} 1$ and $\mathrm{T} 2$ spin-echo sequences $(500 / 20$, $2000 / 50,100)$, were applied. In addition, these sequences were followed by gradient-echo ultrafast low angle sequences $\left(30 / 14 / 20^{\circ}\right)$, obtained with apnea $(10 \mathrm{~s})$, in the same transversal plane. MRI was performed in all patients before and just after oral ingestion of about $800 \mathrm{ml}$ of contrast agent, taken in portions during a two hours period.

On enhanced images, we obtained a continuous and homogenous distribution of this iron oxide suspension, with a marked loss of signal on all T2 sequences, and a good extinction of proximal and distal bowel lumen. A better delineation of the pancreas, pararenal anterior space and hollow viscera was obtained, for the post-contrast scan particularly on ultra-fast imaging sequences with good distinction of the vessels. No serious adverse reactions were noted, but 7 of the first 17 patients didn't drink the all volume, finding it too important. No contrast medium related artifacts were observed, and even less peristaltim related artifacts were noted on T2 spin-echo sequences ( 10 before contrast and 6 after contrast in the first 17 patients). More informations were obtained in 7 cases for the 17 initial patients.

The use of this oral contrast agent for abdominal MR imaging, will allow a better evaluation of intra-abdominal viscera and lesions, and T2 gradient-echo sequences appeared promising, on the preliminary results of this study.

\subsection{PM}

\section{Gd-DTPA as an agent for the gastrointestinal tract}

L. Vlahos, A. Gouliamos, V. Clauss, A. Kalovidouris, S. Trakadas, A. Athanassopoulou, A. Hadjioannou, C. Papavassiliou; Athens

The purpose of this phase III clinical trial was to determine the effect of oral or rectal administration of Gd-DTPA on the MR appearance of the abdomen and pelvis.

MR examination of the abdomen was performed in 40 patients using a special formulation with $1 \mathrm{mmol}$ Gd-DTPA and $15 \mathrm{~g}$ Mannitol/litre. T1 and T2 weighted SE-images were obtained in each patient before and after the administration of $600-1000 \mathrm{ml}$ of the Gd-DTPA solution orally or of $200-300 \mathrm{ml}$ rectally. 25 patients received $1 \mathrm{mg}$ Glucagon intravenously, in order to reduce the peristalsis and to suppress the motion artifacts.

Both examinations were graded by three separate investigators with respect to: a) Filling of the gastrointestinal tract on oral/rectal administration, b) Gastrointestinal contrast in the region of interest, and c) Diagnostic yield compared to CT with oral and/or oral and intravenous contrast medium, or plain MR.

Gd-DTPA achieved adequate filling and visualization of the gastrointestinal tract allowing satisfactory recognition of the adjacent structures and pathological processes. The diagnostic yield of MR of the abdomen after administration of Gd-DTPA was comparable to CT with oral contrast medium, but superior to plain MR.

The administration of glucagon resulted in increased clarity of bowel visualization. Finally, Gd-DTPA produced no side effects, like nausea, diarrhoea, or allergic reactions. The taste of the new MR contrast medium was accepted by all patients without any complains.

\section{$2.30 \mathrm{PM}$}

\section{Oral Gd-DOTA: use in MR imaging of the abdomen}

Y. Miaux, J. Frija, C. Dubourdieux, F. Cyna-Gorse, P. Ollier, M. LavalJeantet; Paris

Purpose: To test Gd-DOTA as an oral MR imaging contrast agent to improve contrast between the gastrointestinal tract and other abdominal organs in MR imaging of the abdomen.

Methods: 29 patients (age range, 20 to 76 years) presenting with varied abdominal pathologies were studied two times the same day with a 0.5 Tesla MR imaging system. The second time they ingested $1000 \mathrm{~mL}$ of a solution $(10 \mathrm{~g} / \mathrm{L}$ Glucose, $2 \mathrm{mmol} / \mathrm{L}$ Gd-DOTA, GUERBET), one hour before undergoing MR imaging. T1- and T2-weighted axial images were obtained in the two studies. Side effects of this ingestion were noted. These $29 \mathrm{MR}$ studies with and without oral Gd-DOTA were reviewed retrospectively and blindly by two independent radiologists. Delineation and visibility of the gastrointestinal tract, intraabdominal organs and abnormalities were evaluated and statistical analysis of these results was made.

Results: two cases of vomiting could be imputed to ingestion of Gd-DOTA.TIand T2-weighted images with oral Gd-DOTA showed better delineation of the entire pancreas and spleen and better visibility of the stomach and duodenum. The diagnosis was made easier in five cases. 
Conclusions: we suggest that oral Gd-DOTA is useful as a contrast agent improving MR imaging of the stomach, duodenum, spleen and above all pancreas.

\subsection{PM}

1228 A new paramagnetic preparation for specific esophageal opacification

Paolo Pavone, Rossella Occhiato, Nicola de Stefano, Silvia Giuliani, Giampiero Cardone, Giorgio Albertini P., Roberto Passariello; L'Aquila

Purpose: To obtain a preparation for specific esophageal opacification in MRI.

Methods: A paramagnetic agent and a viscous paste support were thought to be necessary for the preparation of this agent. After different trials, we concluded that the use of already existing esophageal paste would be advisable. We therefore used a CT esophageal barium paste with high viscosity (TAC Esofago, distributed in Italy by Bracco I.C., and produced by EZ.E.M., U.S.A.). Gd-DTPA was added as paramagnetic agent (Magnevist, Schering, Germany). The two contrast agents were emulsified for homogeneous density. Results: The in vitro evaluation showed a high signal intensity of the preparation in $\mathrm{T} 1$ weighted sequences, contrasting with the low signal intensity of the water phantom employed.

In vivo studies also showed a specific, long lasting opacification of the esophageal lumen, with good delineation of the esophageal wall in normal and in cancer patients.

Conclusions: This preparation shows promises for selective opacification of the esophagus. Its use in routine MRI of the chest could be suggested for the identification of the normal esophagus and for the evaluation of esophageal cancer.

\subsection{PM}

1229 MR-Lymphography using superparamagnetic iron oxide particles: first results of animal studies

B. Hamm, M. Taupitz, S. Wagner, D. Pfefferer, R. Lawaczeck, K.-J. Wolf: Berlin

Purpose: The suitability of superparamagnetic iron oxide particles (SIP) for MR-lymphography was tested in animal studies.

Methods: a) During the initial study phase, the endolymphatic application of 5 different dosages $(0.01-1 \mu \mathrm{mol} \mathrm{Fe} / \mathrm{kg})$ of SIP was tested in 15 rabbits $(1.5 \mathrm{~T}, 12$ different pulse sequences); b) in the second study phase, the contrast agent effect was studied in 18 animals after interstitial application of 6 different dosages $(2-50 \mu \mathrm{mol} \mathrm{Fe} / \mathrm{kg}) ; \mathrm{c})$ in the third study phase, the diagnostic effect of the contrast agent was investigated in 16 tumor-bearing rabbits.

Results: a) After endolymphatic injection of SIP, maximal contrast enhancement of the lymph nodes is achieved with a dosis of $0.5 \mathrm{umol} \mathrm{Fe} / \mathrm{kg}$. The contrast agent effect is used best with a proton density-weighted pulse sequence (SE 2000/15). b) After interstitial injection of SIP, good signal enhancement of the lymph nodes is obtained at a dose of $20 \mu \mathrm{mol} \mathrm{Fe} \mathrm{per}$ extremity. c) Thirteen of 16 tumor-bearing animals had histologically confirmed lymph-node metastases (tumor diameters $2-12 \mathrm{~mm}$ ). Whereas the metastatic lesions could not be recognized during plain imaging, this was possible in $89 \%$ of the examined lymph nodes after application of SIP.

Conclusions: MR-lymphography using superparamagnetic iron oxide particles is a new and promising method for the detection of lymph-node metastases.

\section{$3.00 \mathrm{PM}$}

1230 Evaluation of the renal tolerance of Gd-DOTA in patients with chronic renal failure

G. Deray, M. F. Bellin, E. Auberton, C. Jacobs, J. Grellet; Paris

Gd-DOTA is an intravenous contrast medium used in MRI. The clinical and biological tolerance of Gd-DOTA was studied in patients with chronic renal failure (eg. having a glomerular filtration rate less than $60 \mathrm{ml} / \mathrm{mn}$ ). Twenty patients were randomized into two groups. In the control group, spin-echo (SE) T1 and T2-weighted images of the kidneys were obtained without injection of Gd-DOTA. In the DOTA-group, patients received $0,2 \mathrm{ml} / \mathrm{kg}$ of Gd-DOTA. SE T1-weighted were obtained before and after injection; a T2-weighted sequence was performed before injection. Clinical data, serum creatinine and laboratory parameters were estimated before, 24 and 48 hours after MRI. No adverse reaction was reported after injection of Gd-DOTA. Mean serum creatinine and glomerular filtration rate remained unchanged in both groups. Five patients in the control group and 3 patients in the DOTA-group increased their serum creatinine (SCR) of more than $10 \%$ and less than $25 \%$.

\begin{tabular}{lll}
\hline & DOTA & $\begin{array}{l}\text { Control } \\
\text { group }\end{array}$ \\
SCR $\mu$ mol $/ 1($ mean $\pm \mathrm{SD})$ & group & $330 \pm 180$ \\
Basal & $331 \pm 141$ & $340 \pm 192$ \\
$24 \mathrm{~h}$ & $327 \pm 152$ & $345 \pm 216$ \\
$48 \mathrm{~h}$ & $306 \pm 149$ &
\end{tabular}

In conclusion, we have shown in a randomized trial that Gd-DOTA is not nephrotoxic in patients with chronic renal failure.

\subsection{PM}

\section{Pharmacokinetics and tolerance of Gd-DOTA in healthy volun- teers and in patients with chronic renal failure}

B. Bonnemain, A. Arthaud, P. Molinier, J. L. Gayet; Villepinte

Purpose: The aim of this study is to assess pharmacokinetics and tolerance of Gd-DOTA (DOTAREM) in not dialysed patient with a chronic renal failure. Methods: 8 patients with chronic renal failure (clearance $=10$ to $60 \mathrm{ml} / \mathrm{mn}$ ) and healthy volunteers have been administrated with $0,1 \mathrm{mmol} / \mathrm{kg}$ Gd-DOTA by intravenous route. Blood samples and urinary samples were collected during 24 to 72 hours according to the importance of the renal failure in order to assess the pharmacokinetics parameters. Meanwhile Gd-DOTA tolerance has been studied (vital signs, biological parameters, adverse reactions).

Results: In healthy volunteer, Gd-DOTA is rapidly distributed in the extracellular space and almost totally eliminated by urinary route (glomerular filtration) ( $>80 \%$ in 6 hours).

For the first patients included with chronic renal failure, a delayed and almost total elimination via urinary route can be observed with a correlation between Gd-DOTA clearance and plasma creatinin clearance: tolerance is good (particularly with no significant modification of the renal function). These results need to be confirmed on this whole studied population.

Conclusions: The injection of Gd-DOTA in patients with chronic renal failure does not affect the renal function. Therefore, this product can be used safely for these patients.

\subsection{PM}

1232 Discrepancy between MRI and light microscopy in the pharmacodynamics of iron oxide in the rabbit liver P. Van Hecke, G. Marchal, B. Van Damme, A. L. Baert; Leuven

During our pharmacodynamic study of iron oxide (AMI-25, Guerbet, France) in the rabbit liver, the decrease in MRI signal was compared with the number of iron carrying Kupffer cells.

Liver specimens obtained 3 hours and 7 days after injection of doses between 0,5 and $4 \mathrm{mg} \mathrm{Fe} / \mathrm{kg}$, were processed for histology, stained with Perl's blue and studied with light microscopy.

The MRI intensities decreased for increased iron dose, and recovered to their original value as a function of time, as expected. Light microscopy however was unable to detect iron carrying cells at doses below $2 \mathrm{mg} \mathrm{Fe} / \mathrm{kg}$ and surprisingly, detected more cells after 7 days, in apparent contradiction with the MRI results. Two hypotheses based on iron oxide metabolisation and/or clustering will be proposed to reconcile both results.

It is concluded that light microscopy is not appropriate under these conditions for quantification of iron oxide in the liver.

\subsection{PM}

\section{Ozone, contrast media in digital angiography}

J.C. Uqarte, J. Alfonzo, J.Banasco, J. García, E. Moreno, R. Wong, M. Gómez, S. Menéndez, D. Meńdez, E. Barber, H. Gómez, E. Fermín; Havana

Digital Substraction Angiography (DSA) offers a possibility for obtaining high quality vascular images by intra-arterial administration of iodine contrast media; but adverse reactions were seen in patients with low tolerability. A lot of experiences using intra-arterial ozone as therapeutic agent in patients with vascular disease, have been reported. We introduced the ozone as a negative contrast media in DSA of the kidney and lower limbs.

Studies of images quality, physicochemical properties, side effects and genotoxicity were performed in experimental animals with good results. Later on DSA examinations were performed in 97 patients who had low tolerability to the iodine contrast media. Good images quality with no damage or complications were obtained. Some advantages of intra-arterial DSA with ozone as a contrast media, are evident:

1. Reduced risk in patients with iodine allergy, renal failure and heart and liver failure.

2. Decrease of the side effects.

3. Lower cost.

4. Therapeutic effects. 


\subsection{PM}

1234 Gd-BOPTA/Dimeg: relationship between pharmacokinetics and MRI liver signal enhancement

Vito Lorusso, Italo Poggesi, Friedrich Cavagna, Christoph de Haën, Piero Tirone; Milano

Gd-BOPTA/Dimeg is a newly developed octadentate chelate of $\mathrm{Gd}^{3+}$, currently under evaluation as a polyvalent MRI contrast agent. The excretion of the compound was studied in the rabbit after i.v. administration at infusion rates ranging from 3.75 to $22.5 \mu \mathrm{mol} \times \mathrm{min}^{-1}$. Both urinary and saturable biliary excretion were observed. The latter was characterized by a maximum excretion rate of $0.87 \mu \mathrm{mol} \times \mathrm{min}^{-1} \times \mathrm{kg}^{-1}$. Half-maximal biliary transport was seen a plasma level of $0.53 \mu \mathrm{mol} \times \mathrm{mL}^{-1}$. Biliary excretion was further characterized by a Hill coefficient of 1.6. After a bolus dose of $250 \mu \mathrm{mol} \times \mathrm{kg}^{-1}$, biliary excretion amounted to $25 \%$. Previous studies in rats showed $52 \%$ biliary excretion after the same i.v. dose.

Maximum signal intensity enhancement in liver MRI was obtained at plasma levels that were below those which gave half-saturation of biliary transport. This is consistent with the finding that while bromosulfophthalein was able to inhibit the biliary excretion of Gd-BOPTA almost completely, the effects on the MRI signal were maintained.

We concluded that MRI signal enhancement by Gd-BOPTA/Dimeg in the liver is not proportional to tissue concentrations.

\section{Room 0}

\section{SS $2.00 \mathrm{PM}$}

1235 Duplex sonography for the assessment of tumor vascularisation W. Dock; F. Grabenwöger, V. Metz, K. Eibenberger, M. T. Farres; Vienna

Purpose: Determination of the value of duplex sonography for the differentiation of malignant from benign lesions by evaluation of peak systolic flow values $($ meter $/$ second $=\mathrm{m} / \mathrm{s}$ ).

Methods: In 120 patients with 42 tumors of the breast, 42 mass lesions of the liver, and 36 primary or secondary tumors in other regions tumor duplex sonography was performed. All breast lesions and 59/78 other lesions were excised and examined histologically. The remaining 19 diagnoses were verified with long term follow up examinations (at least 1 year).

Results: A peak flow of $0.4 \mathrm{~m} / \mathrm{s}$ turned out to be the best value to separate benign from malignant lesions (ROC curves): sensitivity $=0,63$, specificity $=$ 0,83 , predictive value of a negative test $($ PVNT) $=0,31$, predictive value of a positive test (PVPT) $=0,95$. Isolated evaluation of tumors of the breast provided the following results (value of separation $=0,4 \mathrm{~m} / \mathrm{s}$ ): sensitivity $=$ 0,65, specificity $=0,85$, PVNT $=0,5$, PVPT $=0,91$. Further detailed statistical analyses will be presented.

Conclusions: Negative results of Doppler examinations are meaningless. High systolic peak flow values indicate the presence of a malignant process. Thus, if high Doppler flow values are found in a mass lesion further examinations should be performed even if the lesion provides a benign aspect.

\section{$2.10 \mathrm{PM}$}

1236 Colour Doppler contribution to US diagnosis of breast masses: three years experience

M. Bergonzi, F. Calliada, C. Passamonti, G. Corsi, G. Sala, G. B. Raimondi; Lodi

The authors report on their experience with Colour Doppler sonography in the diagnosis of solid breast masses.

122 patients were examined; breast lesions were studied with B-mode US first, to evaluate their patterns (size, margins, echotexture, tumoral calcifications and acoustic shadows) and then with CD US to depict eventual tumour vascularization. The examinations were performed with linear-array $7 \mathrm{MHz}$ transducer (Acuson 128).

Final diagnoses were obtained by means of surgical excision (in 67 lesions) and fine-needle aspiration (in 55 cases) and were correlated with US results. Colour Doppler US demonstrated only one vascular pole, or no Doppler signals, in histologically confirmed benign masses. On the contrary, in $92,8 \%$ of histologically confirmed malignant masses, Colour Doppler easily depicted two or more groups of nutritional arteries.

Colour Doppler makes the diagnosis of malignant masses easier, thus allowing, in the author's opinion, a reduction in the number of biopsies of solid breast masses clinically mammographically detected. The technique is non invasive, fast and easy and its widespread use would translate into advantages for both the patient and the physician.

In conclusion the use of CD is suggested in those cases where mammography is not conclusive, because CD allows accurate assessment of neovascularity, associated with breast malignancies. In addition, CD offers more rapid evaluation of blood flow distribution compared to pulsed Doppler analysis.

\subsection{PM}

1237 Pulsatility Index in foetal vessels: normal values C. Passamonti, R. Campani, D. Garzia, G. Raieli, C. Sabbia; Lodi

Colour Doppler visualization of the blood flow jets in foetal vessels allows the sample volume to be correctly positioned and the vessel/Ultrasound beam angle to be adequately adjusted. Consequentially, semiquantitative velocimetric measures should be regarded as reliable.

We examined 85 foetuses at 2 th and 3 th trimester of gestational age and we recorded Pulsatility Index (PI) in the lumen of umbilical artery and middle cerebral artery. Every measure was repeated at least three times and the mean was taken as recorded value.

All of the examined gestation had a regular course and a normal delivery. The newborns weight was between 2500 and 3500 gr. and the Apgar index was 10 . In the table we present the recorded values as mean \pm SD for every gestational age.

$\begin{array}{llc} & 2^{\text {th }} \text { trimester } & 3^{\text {th }} \text { trimester } \\ \text { umbilical artery } & 106 \pm 20 & 96 \pm 18 \\ \text { middle cerebral artery } & 191 \pm 34 & 178 \pm 34\end{array}$

Those values should be considered as normal values in a regular pregnancy for an European country.

\subsection{PM}

1238 Duplex sonography of the carotid arteries in newly diagnosed type 2 diabetics

Jana Brunová, Josef Bruna; Prague

The duplex sonography of carotid arteries were made in the group of newly diagnosed Type 2 diabetic men, aged 45-64 years. The atherosclerotic changes of arteria carotis were found in $44 \%$ of patients. However arterial stenoses were mostly non-significant. Diabetics with atherosclerotic changes of the investigated arteries had significantly increased ischemic heart disease compared with diabetics without atherosclerosis $(\mathrm{p}<0,01)$. Diabetics with the macrovascular disease of the carotid arteries had significantly higher levels of triglycerides, total cholesterol/HDL-coholesterol ratio and ischemia compared with diabetics without ultrasonographically diagnosed atherosclerotic lesions.

\section{$2.33 \mathrm{PM}$}

\section{Is aspiration necessary in fine needle biopsy?} J. Kreula, P. Virkkunen; Helsinki

Purpose: Fine needle aspiration biopsies (FNAB) are increasingly performed by radiologists. Despite of widespread use of FNAB, views on the mechanism of cell detachment differ considerably.

Methods: The effect of suction on specimen weight and cell count was studied in vitro using an automatic FNAB sampler. Bovine liver and testis and porcine kidney were used as target tissues. The behaviour of tissue in a needle during FNAB was visualized using a transparent glass needle.

Results: Specimen weight and cell count increased linearly $(\mathrm{p}<0.001)$ and rapidly with suction force, when suction was combined with standard needle movement. However, in the absence of needle movement, the suction force, duration of suction and rate of increase of suction had no effect on the scanty specimen size. Needle movement alone, without suction, produced meager specimens.

Conclusions: Suction itself does not seem to be the factor leading to cell detachment. The cells are not aspirated from the biopsy sites. However, suction causes tissue to bulge into the needle lumen. When needle moves forward, this bulge is sliced of by cutting edge of the needle. Simultaneous aspiration and needle movement are essential.

\section{$2.43 \mathrm{PM}$}

1240 How to avoid unsatisfactory specimens in fine needle aspiration biopsy?

P. Virkkunen; Helsinki

Purpose: Fine needle aspiration biopsy is a safe method of diagnosing lesions detected by imaging methods. Wide variation in incidences of unsatisfactory specimens containing too few representative cells have been reported. Methods: An automatic sampler was constructed to study in detail the dependence between sampling technique and specimen size in vitro. Bovine liver and porcine kidney were used as target tissues.

Results: Specimen size increased linearly $(\mathrm{p}<0.001)$ and markedly with needle diameter when a standardized suction and needle movement was used. 
Number of needle strokes used had also a marked and linear $(\mathrm{p}<0.001)$ effect, when standardized suction was used, and when the needle was angled between the strokes into different directions in a fan-like pattern. However, when the needle was stroking repeatedly in the same channel, less and less tissue was detached per stroke. The effect of the amplitude of needle strokes on specimen size was linear $(p<0.001)$ and marked, after an initial plateau: the smallest amplitudes $(<3-4 \mathrm{~mm})$ were ineffective. Specimen weights were significantly larger when a few needle strokes with long amplitudes were used than when a large number of strokes with small amplitudes were employed. Needle movement alone, in the absence of suction, produced only small specimens. Conclusions: Poor biopsy techniques may be responsible for high incidences of unsatisfactory specimens in FNAB.

\section{$2.53 \mathrm{PM}$}

1241 Percutaneous fine needle biopsy in the management of patients with a history of lymphoproliferative disease

R. M. Walter, G. R. Wittich, K. Nowles, D. E. Lucas, K. Terasaki, D. Swanson; Vienna

Purpose: Tissue diagnosis has to be established for patients with a history of lymphoproliferative disease presenting with a new or growing lesion. The role of fine needle biopsy (FNB) for establishing a definitive diagnosis of lymphoma has been controversial. We reviewed the results of FNB in 85 patients with a history of lymphoma and 2 patients with a de novo diagnosis. The purpose was to determine the sensitivity of FNB in diagnosing Hodgkin's disease (HD) or Non-Hodgkin's Lymphoma (NHL), incidental carcinoma or infection.

Methods: Included were 87 pts. who underwent CT-guided, fluoroscopically guided or US-guided biopsies and had available clinical or histological follow-ups. Adequacy of specimen was assessed by immediate cytological evaluation. Processing cytological specimen included tumor marker studies. Results: The sensitivity for diagnosing NHL was $88 \%$, for HD $75 \%$. Most of the pts. with HD had nodular sclerosing HD. Incidental carcinoma was diagnosed in 7 pts.; in 3 pts. with biopsies negative for Ly or CA a definitive diagnosis of infection was established. In one pt. wich cyclosporin A (Cs A) medication largely necrotic material tested positive for $\mathrm{EB}$-virus and $\mathrm{Cs} \mathrm{A} / \mathrm{EBV}$-related lymphoma was diagnosed. Re-biopsies had the same sensitivity as initial biopsies.

Conclusions: FNB including tumor marker studies has a high sensitivity in detecting and defining NHL and CA. Results for HD may be enhanced by core biopsies with large needles. Re-biopsies after negative results of initial biopsies should be a standard option.

\section{$3.03 \mathrm{PM}$}

\section{Role of percutaneous fine needle cytology in suspected recurrent lymphoma \\ Th. Dao, J.Fleury, D. Mathieu, C. Haioun, F. Reyes, N. Vasile, J.F. Bernaudin; Paris}

Purpose: To evaluate the accuracy of percutaneous fine needle cytology to assess the status of residual masses or suspected new locations of lymphoma after treatment.

Methods and Materials: Percutaneous fine needle cytology was performed under computed tomography (CT) guidance over a period of 4 years in 15 patients with residual masses $(n=8)$ or suspected new location after treatment. Percutaneous biopsy was performed in only 4 patients when safe approach was possible. These results were also compared to clinical and radiological follow-up over 18 months.

Results: The cytology of residual masses demonstrated 4 active diseases, 3 cases of blood or necrosis, and one case of fibrosis. In 5 other cases, cytology provided unexpected diagnosis of non-related neoplasms: lung carcinoma (3), renal adenocarcinoma (1), metastatic lymph nodes (1). In the 2 remaining cases, cytology concluded to fibrosis and nocardiosis. In all the cases, cytologic samples were in total agreement with biopsy, clinical and radiological follow-up.

Conclusions: Our results suggest that percutaneous fine needle cytology under CT guidance is a reliable method for the evaluation of either suspected residual masses or unexpected new location of lymphoma.

\section{$3.13 \mathrm{PM}$}

\section{Value of percutaneous fine needle cytology in the diagnosis and} classification of lymphoma

T. H. Dao, J. Fleury, D. Mathieu, C. Haioun, F. Reyes, N. Vasile, J.F Bernaudin; Paris

Purpose: To evaluate the accuracy of percutaneous fine needle cytology to assess the diagnosis and the classification of lymphoma.

Methods and Materials: Thoracic and abdominal percutaneous fine needle cytology were performed under computed tomography $(\mathrm{CT})$ guidance over a period of 4 years in 32 patients with a suspected lymphoma. Any peripheral lymphadenopathy or masses were palpable. Cytology was performed in all patients and biopsy in only 12 patients, when safe approach was possible. The cytologic samples were compared to biopsy, clinical and radiological follow-up over 18 months.

Results: The cytology demonstrated a newly diagnosed lymphoma in 29 cases $(90,6 \%)$ and allowed a classification of lymphoma into non-Hodgkin's lymphoma $(\mathrm{NHL})(\mathrm{n}=25)$ and Hodgkin's disease $(\mathrm{n}=4)$, with a subclassification of NHL according to the International Working Formulation. In 3 patients, no sufficient tissue was obtained because of hemorrhagic sample $(\mathrm{n}=$ 1) or necrosis $(n=2)$. In 12 patients, immunochemical studies could be performed for immunologic subclassification of lymphoma on the basis of cystology $(\mathrm{n}=6)$ and biopsy $(\mathrm{n}=6)$.

Conclusions: Our results suggest that percutaneous fine needle aspiration cytology is a reliable method for the diagnosis and classification of lymphoma. Especially immunohistologic studies can be made on cytology alone if biopsy is not safe.

\subsection{PM}

1244 Palmar hyperhydrosis - Treatment by percutaneous transthoracic chemical sympathicolysis - Follow-up three and two years Olga B. Adler, Ahuva Engel; Haifa

Palmar hyperhydrosis is a disabling entity effecting emotional, social and professional life. The accepted treatment consisted of surgical ablation of the third thoracic sympathic ganglion, for sweat glands are innervated by the sympathic chain of the autonomous nervous system. For the past three years we are performing percutaneous thoracic chemical sympathicolysis; during careful computed tomographic monitoring a special phenol solution is injected into the paravertebral space at the height of the third thoracic vertebra. Positive response - i.e. dryness of the hand is obtained during the procedure which is done for both extremities in the same session.

Our patient material comprises 60 patients. Results and complications will be presented in three groups of patients: one year, two year and three year follow-up.

\subsection{PM}

1245 Ultrasound and thermography in the evaluation of the state of tissues in the area of late radiation fibrosis A. F. Tsyb, I. Kh. Mukhamedzhanov; Obninsk

90 patients with late radiation fibrosis of the skin and underlying tissues following radiation therapy of tumors of organs of the small pelvis and the mamma were examined. The skin was found to be thickened in $76,1 \%$, echostructure of the subcutaneous fat became more homogeneous, echogeneity increased (mean value was 17,06 grey scale units, normally 9,68 units). In $16,7 \%$ of cases, focal changes such as hypoechogeneous areas, cavities and echodense areas occurred. Morphologically, destructive changes, cavities, necrosis and calcifications were observed. Thermography revealed in $97,6 \%$ of patients a distinct hyperthermia in the fibrotic area. Thermoasymmetry made up $2,33^{\circ}$. Echography provided objective data concerning changes of the skin, subcutaneous fat, fascias and muscles in the area of radiation fibrosis.

\section{Room R}

SS

\subsection{Importance of fractionation and dosage in radiotherapy with a view on results and sequelae II}

$2.00 \mathrm{PM}$

\section{Progress in radiotherapy is impossible without radiomodification} (Keynote Lecture)

\section{S. P. Yarmonenko; Moscow}

The development of methods of combined use of hyperthermia and other radiomodifying agents (polyradiomodification) is a promising approach to the enhancement of radiotherapy efficacy. The clinical use of hyperthermia with irradiation (thermoradiotherapy) gave the possibility to rise 1,5-2 times the probability of local control of several types of tumors as well as the survival of patients during 3-5 years period. It follows from experiments on rodents with transplantable tumors that the outcome of thermoradiotherapy could be made substantially better by simultaneous use of some modalities which decrease blood circulation and $\mathrm{pH}$ in tumors. The most effective schedules so far used rise the therapeutic gain factor threefold in comparison with irradiation alone and more than twofold in comparison with thermoradiotherapy.

The clinical use of polyradiomodification both with traditional and new types of fractionation seems to be one of the fruitful approaches to radiation therapy improvement. 
2.20 PM

1247 Dosimetry of CT-based radiotherapy of breast cancer, treated by $\overline{c o n s e r v a t i v e ~ s u r g e r y ~(K e y n o t e ~ L e c t u r e) ~}$

G. Gyenes, L. Pap; Budapest

The postoperative irradiation after conservative breast surgery poses some questions and problems concerning dose distribution of the breast lymph areas and healthy tissues.

From 1985-1990 nearly 500 patients were treated by radiotherapy after conservative breast cancer surgery. The breast and lymph node areas were treated by telecobalt resp. linear accelerator to 45-50 Gy. Boost to the tumor site was performed by $\mathrm{T} 2$ tumors by electron beam $(6 \mathrm{MeV})$. The goal of radiotherapy is to eradicate the tumor, without damage of healthy tissues, that's why all patients underwent CT-topometry and CT-dosimetry, while the isodose curves in the lung tissue, heart muscle and spinal cord have been studied. The conclusion was made, that CT-topometry and densitometry can influence the choice of the radiation beam ( 60 Co or megavoltage X-Ray), the irradiated volume and total dose as well. The dose absorbed by lung tissue, hert and spinal cord, give important data for short term complications, long term survival, and late consequences.

\subsection{PM: Discussion}

\subsection{PM}

1248 Radioiodine dose-response in differentiated thyroid carcinoma M. E. A. O'Connell, M. A. Flower, C. L. Harmer, V. R. McCready; Sutton

Purpose: Dose-response curves have been constructed to determine the tumoricidal dose for differentiated thyroid carcinoma and thus enable precise activities of radioiodine to be prescribed to maximise tumour killing and minimise morbidity.

Methods and Materials: Tumour and normal residual thyroid doses from I-131 have been determined with improved precision using special high-resolution dual-head rectilinear scanning and positron emission tomography.

Results: Dose-response data have been obtained for 41 patients. Following total thyroidectomy and $3 \mathrm{GBq}$ I-131, a mean absorbed dose of 384 Gy achieved complete ablation of thyroid and remnants in $72 \%$ of patients. Patients who had persistent uptake in the thyroid region on subsequent radioiodine scanning received a mean dose of only $69 \mathrm{~Gy}$. Cumulative doses in excess of 150 Gy were found to eradicate cervical nodal metastases following neck dissection. Patients with bone metastases (who generally have a very poor prognosis) received doses of the order of 20 Gy to the lesions.

Conclusions: The dose response curves explain the varying response to fixed activities of radioiodine. In future they will enable precise prescription of radioiodine to achieve tumoricidal doses avoiding the morbidity of excessive therapy.

\subsection{PM}

1249 Comparative evaluation of modalities of $\mathrm{X}$-ray treatment and hyperthermia

C. Sawas-Dimopoulou, I. Iordanou, N. Ouzounoglou, V. Dardufas, V. Kouloulias, T. Maris, K. Papavassiliou; Athens

A controversy remains as to the treatment schedule permitting the better synergism between X-ray therapy and hyperthermia. We report our experience concerning a combined therapy of the solid tumor Walker carcinoma. Male Wistar rats were submitted to treatment the 9 th day after transplantation of the tumor. Two groups of rats received either a single X-ray dose of 800 Rads by a $6 \mathrm{MeV}$ linear accelerator (Mevatron-Siemens), or treatment by $434 \mathrm{MHz}$ microwaves with a continuous control of tumor tissue temperature to $44 \pm 1^{\circ} \mathrm{C}$ for $45 \mathrm{~min}$. Another group of rats was submitted to a combined treatment: the $\mathrm{X}$-ray therapy preceding hyperthermia by $24 \mathrm{hrs}$. The last group of animals contained control rats. The greatest reduction in tumor size and the longest survival time were obtained by the above combined treatment, with a gain factor of 1.93 for tumor size, compared to 1.46 after $\mathrm{x}$-ray therapy and 1.12 after hyperthermia, and 1.85 for survival time, compared to 1.30 after $x$-ray therapy and 1.05 after hyperthermia.

In conclusion, the results suggest that in the above schedule of combined treatment, hyperthermia applied to a solid tumor 24 hrs after a single dose of $\mathrm{X}$-ray, enhances the beneficial effect of therapy.

\subsection{PM}

\section{Radiotherapy of the NSCLC. Results of a standardized treatment schedule}

D. Ch. Vogel, B. Kimmig, H. Bülzebruck, M. Wannenmacher; Heidelberg

Between 1982 and 1989537 patients with non small cell lung cancer were irradiated at the primary tumor side at the department for radiooncology university of Heidelberg; 381 primary and 96 after surgery. Surgical treatment and clinical follow up were performed at the hospital for lung diseases Heidelberg-Rohrbach.

Primary radiotherapy was used in fact of internistic or technical inoperability and for patients, who had declined a surgical treatment. Indications for postoperative radiotherapy were stage III of disease or incomplete tumor resection.

The five years survival of the primary treated patients is $6 \%$, for the postoperative irradiated patients $21 \%$.

The stage depending survival rates for the primary radiotherapy are $16 \%$ for stage I, $8 \%$ for stage II and $4 \%$ for stage III and IV. The postoperative irradiated patients with stage III have significant $(\mathrm{p}<0,03)$ better five years survival rates than only surgical treated patients; although there is no significant difference in the local relapse free survival. Beside the well known effects on survival for primary radiotherapy our results demonstrate the improvement in treatment of advanced lung cancer by postoperative radiotherapy.

\subsection{PM}

1251 Mixed gamma-neutron irradiation of advanced laryngeal carcinoma

Y. S. Mardynsky, A. S. Sysoev, V. G. Andreev, I. A. Gulidov; Obninsk

The purpose of the study was to evaluate a new technique of radiation therapy using fast neutrons produced by the research reactor. A total of 46 patients with advanced laryngeal cancer underwent mixed gamma-neutron irradiation. Neutrons (E-1MeV) were produced on BR-10 research reactor. Neutron dose was $20-40 \%$ of total dose. RBE was considered to be 3,5-5. Photon irradiation was delivered by Co-60 units. Total dose was isoeffective $60 \mathrm{~Gy}$ of conventional photon radiation. We used a split-course technique. Neutrons were delivered as a priming treatment. 30 patients with partial response after priming treatment continued to receive gamma-irradiation. The rest 16 patients with no response underwent radical operations. Complete response was in 19/30 (63\%). Partial response was in 11/30 (37\%) patients. Most severe acute skin and mucosa reactions were found to be moist desquamation in $5 / 46$ (11\%) patients and spotted mucositis in $7 / 46(15 \%)$ patients, respectively. Thus, mixed gamma-neutron irradiation was quite well tolerated by surrounding normal tissues and provided satisfactory tumor response in advanced laryngeal carcinomas.

\section{Room S}

\section{SS} $2.00 \mathrm{PM}$

15.01 New technical developments in CT (I)

1252 X-ray computed tomography: state of the art and new developments (Keynote Lecture)

Willi Kalender; Erlangen

To assess the state of the art in computed tomography, a review of image quality and performance parameters of today's CT scanners will be given. Further improvements in some of the basic parameters, like spatial and low-contrast resolution, will be limited for physical reasons; in contrast, dynamic capabilities will be extended due to technological advances. Continuously rotating scanners are well established by now and will dominate the high-performance end in the future. CT has also established itself in a number of special applications, e.g. bone mineral or cerebral blood flow measurements. This will be demonstrated by clinical examples, in particular including dynamic CT studies.

The future of CT can be seen in the direction of rapid 3D volume data acquisition. This will build upon the technology provided by continuously rotating systems using Spiral CT scanning. A practical implementation has recently been presented for the Siemens Somatom Plus in a special scanning mode where continuous patient transport is synchronized with the continuously rotating X-ray tube and detector system. Presently, it is possible to scan volumes of up to $30 \mathrm{~cm}$ in $30 \mathrm{~s}$. Future demands include improved X-ray power and computer and storage capacities. Physical performance characteristics of volume scanning modes and clinical examples will be presented. The latter will include $3 \mathrm{D}$ and cine displays by video tape.

\subsection{PM}

1253 Spiral scanning and the influence of the reconstruction algorithm on image quality

H. Rigauts, G. Marchal, A. L. Baert; Leuven

Purpose: In the present clinical study with spiral scanning artifacts induced by reconstruction algorithms are evaluated.

Methods: Spiral scanning is a new method of computed tomography. Data acquisition is obtained by continuous table incrementation during continuous scanning.

Out of the same acquisition data, image is reconstructed with two different algorithms. The first one using the 0.7 second reconstruction algorithm (this 
algorithm uses projections over $240^{\circ}\left(180^{\circ}\right.$ rotation and $60^{\circ}$ detector array)). The second algorithm is especially designed for spiral scanning and uses weighted interpolations over $360^{\circ}$ rotation with previous and following slices. The results of both reconstruction algorithms were evaluated in 20 thoracic and 150 abdominal studies.

Results: Images reconstructed with the 0.7 reconstruction algorithm are of good diagnostic value but show streak artifacts in 30\%. Images reconstructed with the specially designed interpolation algorithm are free of streak artifacts, have better signal to noise but show volume averaging artifacts.

Conclusions: Although both reconstruction algorithms have their advantages and drawbacks, both produce images of sufficient quality for routine clinical use.

\section{$2.25 \mathrm{PM}$}

\section{Improved spatial and contrast resolution in spiral CT scanning by image deconvolution}

G. Marchal, D. Ooms, P. Suetens, H. Rigauts; Leuven

Purpose: Which factors affect the image quality in spiral scanning? Can image deconvolution improve the image quality in spiral scanning? Methods: Spatial scanning combines continuous tube rotation with continuous table incrementation. On the Siemens' Somatom plus both slice thickness and table speed can be selected. Volumes up to $24 \mathrm{~cm}$ can be acquired during one single breathhold. Image reconstruction uses raw data interpolation for optimal results.

In spiral scanning true-plane resolution not only depends on slice thickness selected but also on the table incrementation speed and interpolation technique. The total smoothing is characterized by the pointspread function which can be calculated with a simulator or measured on phantom.

Conclusions: A spiral-scan-simulator was developed to calculate the optimal combinations of slice thickness, table incrementation speed and raw data interpolation.

If the pointspread function is well defined, invers filtering in the frequency domain definitely improves the true-plane resolution.

The technique was tested on clinical images of both abdominal and thoracic spiral scans. Results will be presented.

\section{$2.35 \mathrm{PM}$}

1255 Cine mode display of images reconstructed out of spiral scans H. Rigauts, G. Marchal, A. L. Baert; Leuven

Purpose: Spiral scanning combines continuous tube rotation with continuous table incrementation. This technique has the advantage of scanning an entire volume without any gaps. This cannot be obtained with incremential scanning because of inconsistency in breath-holding during the consecutive scans.

The raw data of a spiral scan, can be reconstructed with different reconstruction intervals. If a sufficiently small reconstruction interval is chosen and images are assembled in image stacks, they can easily be displayed in a cine mode. The possible advantages of this approach were clinically evaluated. Methods: Fifty patients were examined on the Somatom plus (Siemens Erlangen, W. Germany). Spiral scans were performed with a slice thickness of 3 to $5 \mathrm{~mm}$., a table incrementation speed of 5 to $10 \mathrm{~mm}$. pro second. Reconstructions were made with a reconstruction interval of 0.1 second. The scan volume varied between 12 to $24 \mathrm{~cm}$.

Results: The combination of spiral scanning and cine display simplifies the three dimensional interpretation of the C.T. data. Particularly the exact anatomy of the structures with complex topography or shape is better appreciated.

Conclusions: We feel that cine mode display is the method of choice to take optimal advantage of the actual possibilities of spiral scanning.

\subsection{PM}

\section{Clinical use of a localization device in CT-guided biopsies}

\section{G. Reuther, R. Erlemann, P. E. Peters; Münster}

Purpose: A new localization device for CT-guided punctures that avoids calculations of entry point and angulation by external landmarks was tested in clinical routine.

Methods and Materials: The device was used in more than 80 biopsies of difficult lesions and the results compared to those of freehand guidance in 40 biopsies.

Results: Without time-consuming preparations the easy-to-handle device proved valuable in all thoracic and abdominal lesions. The number of localization scans and correction manipulations were reduced by half compared to freehand guidance and no serious complications were noted. The non-invasive anticipation of the needle path was particularly helpful to keep an access or to determine changes in angulation for further passes.

Conclusions: The new localization technique facilitates the approach to difficult lesions and is recommended for routine use in all CT-guided extracranial biopsies.
1257 Application of and indications for low-dose CT in the diagnosis of the paranasal sinus

Anne Sparenberg, Dieter Felsenberg, Willi Kalender, Michael Pohly, K.-J. Wolf; Berlin

Purpose: Computerized tomography (CT) is characterized by a number of systematic and standardized examination strategies. However, the problem of minimizing radiation exposure has so far played only a minor role in these considerations. It is the aim of the present study to investigate otorhinological indications for CT examinations with a reduced radiation dose.

Methods and Materials: The examinations were done using a Somatom DRH (Siemens AG, Erlangen, FRG) at $85 \mathrm{KV}$ and 1.7-3.0 mGy (instead of $125 \mathrm{KV}$ and 20-50 mGy), and the results were compared with representative scans obtained in the normal mode. The low-dose mode was used in a prospective study including 50 patients with diseases of the paranasal sinus.

Results: 1 . The radiation dose was reduced by a factor of 6-10.2. Good visualization of inflammatory processes of the paranasal sinus in the low-dose mode. 3. Good visualization of fractures. 4. Tumorous alterations require clarification in the normal mode.

Conclusions: The low-dose mode yields satisfactory results for the planning of surgery in cases of inflammatory processes and traumatological disorders of the paranasal sinuses.

\subsection{PM}

\section{Digital luminescence radiography of intensive care chest}

\section{T.E. Mayer; Frankfurt}

Because of the worse signal to noise ratio of DLR compared to a conventional film-screen combination, higher dosages are necessary to obtain adequate presentation of feeble lines, especially in light (high density) regions, of catheter material, tubes and pneumothorax in our experience of 20.000 DLR chest images.

This could be proved with a special low contrast high spatial frequency phantom.

\subsection{PM}

1259 Functional imaging of neuronal brain activities: overlay of distributed neuromagnetic current density images and morphological MR images

Walter H. Kullmann, Manfred Fuchs, Olaf Dössel; Hamburg

Neuromagnetic imaging is a relatively new diagnostic tool in order to image functional electrical activities in the nervous system. It is based on the noninvasive detection of extremely weak magnetic fields around the human body with SQUID (Superconducting Quantum Interference Device) detectors. Conventionally the 'equivalent current dipole' model is used to describe the location of the total electrical activity. New current reconstruction methods enable the imaging of the spatial extension and internal structure of neuronal activities.

For practical use in medical diagnosis a combination of the abstract neuromagnetic images with MR- or CT-images is required in order to match the functional activity with anatomy and morphology. The neuromagnetic images can be overlayed onto three-dimensional morphological images with spatially arbitrarily selectable slices. The matching of both imaging modalities is discussed.

Based on the detection of auditory evoked magnetic fields neuromagnetic images are reconstructed with linear estimation theory algorithms. The MR images are used as a-priori information of the volume conductor geometry and they allow an attachment of functional and morphological properties.

\subsection{PM}

1260 A post-processing algorithm to reduce metallic clip artifacts in CT images

H. K. Tuy, R. A. Cecil; Highland Heights, $\mathrm{OH}$

The CT images of human organs with foreign objects such as metallic prosthetic devices, dark and white bands of streaks emanating from the area of the high density metallic objects hide the anatomical structure in the region covered by the streaks. An algorithm to reduce the artifacts caused by metallic clips in CT images will be presented. The algorithm requires the estimation of the CT data of the clips alone using the forward projection of their image. Original data along rays going through the high density objects are modified based on the data along rays near the clips and on the difference of the above estimated data from the original CT data. This process reduces a significant amount of streaks from the original CT images. Further improvements of the image quality can be achieved by estimating the errors introduced by the above process. The estimation of these errors is done by first create a high contrast object model from the previous corrected image, and then apply the process to 
the model. The evaluations of this algorithm on clinical data will be presented. With fast hardware forward-projectors producing high quality data, the proposed method can be made practical clinically. 


\section{Friday}

4.00-5.30 PM

\section{Room A}

\section{GC}

\section{1}

1261 Considerations of acquiring MR equipment: low versus high field, site planning and economics

Stuart W. Young; Palo Alto, CA

Acquiring the appropriate MRI system has become more complex during the last five years. In 1987, with an installed base of 1,304 MR scanners. $58 \%$ were high field (HF) units. Although the installed base will have increased to over 3,700 MRI scanners, in 1991, only $23 \%$ of MRI unit sales (approximately 800 scanners) will be high field systems with $45 \%$ operating at mid field (MF) and $32 \%$ at ultra low field (ULF). The MRI acquisition question has become more complex because of the increasing number of MR scanners available, rapid developments in instrumentation, applications of instrumentation, cost containment efforts, and competitive factors.

The fact that the signal to noise ratio (SNR) increases approximately linearly with field strength is generally accepted at this point. High field systems are thus the clear winners when signal strength is the main objective of the clinical imaging center. Patient throughput can also potentially be traded off against the higher signal to noise of high field systems in that fewer excitations are necessary to achieve images with a diagnostic quality. Patient throughput is recognized by nearly everyone as a critical success factor, however, the types of patients being scanned, system reliability and availability of replacement parts, size of the acquisition buffer automated protocols and the systems input buffer and the ability to perform multiple tasks (filming, scanning, archiving, etc.) simultaneously, in fact, impact patient throughput to a greater extent than SNR

In an ever more competitive imaging environment, the interrelationship between instrumentation, MRI application, and cost factors has become increasingly important. The acquisition price is, of course, important but, product cycle costs are also important and mid and low field systems are generally estimated to cost only $30 \%$ to $40 \%$ of high field systems over a five-year time frame. Siting, shielding, manufacturer's reputation and stability, local referral patterns and the types of systems operated by competitors, pulsing sequence and RF coil flexibility, system reliability and local service availability, upgrade ability, patient comfort, and architecture of the system represent a complex fabric of interrelated factors which vary in importance from practice to practice.

\section{MR - economical strategies}

R. Felix, W. Schörner; Berlin

Magnetic Resonance Imaging (MRI) is now established as an extremely useful tool for the imaging diagnosis of the central nervous system and is gaining increasing acceptance in other diagnostic fields. Because MRI is expensive, fast imaging strategies (multi-slice, gradient echo) are being developed to increase patient throughput; on the other hand, the improvement of imaging standards often implies higher investments (high-field systems) and sophisticated time-consuming imaging strategies. In the course, individual factors influencing the cost per MR-examination will be discussed on the basis of the German system (investment, daily operating time, personel, archiving, additional diagnostic measures such as contrast agents, ECG-gating, anesthesia and coil change). Also, different models of reimbursement will be evaluated and a break-even-point analysis will be presented for systems with different field strengths ( 0.5 and $1.5 \mathrm{~T})$.

\section{Room B}

\section{CC}

1263 Interventional radiology of the chest: percutaneous procedures R.F. Dondelinger; Luxembourg

Percutaneous tissue sampling, percutaneous drainage of fluid collections, percutaneous drug instillation and percutaneous neurolysis of the sympathetic chain have become standard radiologically guided interventional procedures in the chest. Uniplanar or biplanar fluoroscopy, ultrasound and computed tomography are used as guidance imaging techniques: technical imperatives, advantages and disadvantages are stressed. Technique of percutaneous pulmonary and mediastinal biopsy, choice of biopsy needle, handling of cytological and histological specimens are revised. Emphasis is put on prevention and management of pneumothorax on an outpatient basis. Technique, indications and results of percutaneous drainage of pleural empyemas, pulmonary abscesses and mediastinal collections are presented.
Technique, results and complications of percutaneous blockade of the upper thoracic sympathetic chain are detailed in vasomotor syndromes and hyperhidrosis of the upper limbs.

\section{Interventional radiology of the chest: vascular procedures} J. Rémy, M. Rémy-Jardin; Lille

The thoracic vascular interventional radiologist must be considered as a consultant with a lot of experience in both diagnostic imaging and endovascular procedures.

1. Pulmonary embolism

Mechanical actions in facilitating emboli resolution such as endovenous aspirations and fragmentations may be helpful for cardio-pulmonary hemodynamics and thrombolytic therapy.

2. Vaso-occlusion of endo and transthoracic shunts, of congenital and acquired origin, between systemic and pulmonary circulations have been developed in order to eliminate the need for surgery or to significantly simplify surgical procedures.

3. Vaso-occlusion of pulmonary arteriovenous malformations (PAVM) must be considered as the treatment of choice, replacing surgery, except when the PAVM is not easily catheterizable. A long term follow-up of the obstructed pedicles is needed.

4. Embolization of pulmonary or systemic supply of the lung for treatment of hemoptysis become progressively a less dangerous procedure with anatomic knowledges and monitoring of somato-sensory evoked potentials.

5. Angioplasties and stents placements in vascular stenoses

6. Intra arterial chemotherapy

7. Angioscopy of pulmonary artery

- Repositioning or percutaneous extraction of central venous catheters

9. Endovascular cytologies and biopsies are other well accepted or work in progress procedures.

\section{Room C}

\section{WS}

\section{Imaging of liver tumors} H. Schild; Mainz

The diagnosis of primary and secondary liver tumors has dramatically improved over the last 15 years, however it is still far from being perfect. This is documented by the number of diagnostic modalities that are used either solitary or in combination with others. In the first part of the session common diagnostic procedures are discussed, including their basic principles, diagnostic and differential diagnostic capabilities, and drawbacks. Special emphasize is on ultrasound, CT, MRI, and angiography.

As exact lesion localization becomes increasingly important for planning of surgical and interventional procedures, the second part of the work shop gives an introduction and practical guide to lesion localization.

The work shop is addressed to the practising general radiologist, not to the subspecialist.

\section{Room E1}

\section{WS}

\section{2}

1266 Non invasive vascular ultrasound imaging from peripheral to abdominal vessels, technical and practical aspects: carotid duplex sonography

J. C. Kurdziel; Luxembourg

Stroke secondary to atherosclerotic disease is still among the leading causes of death in developed countries and accounts for significant neurologic impairment among survivors. Atherosclerotic disease develops preferentially at the level of the carotid bifurcation. Intraarterial DSA (IA DSA) is considered the golden standard for morphological examination of the carotid vessels and has supplanted conventional angiography. It remains an invasive and expensive test. Among the various non invasive tests developed for examination of the carotid circulation, "duplex ultrasound" remains the most popular, since it gives a high quality gray-scale image of the carotid vessels that can be combined with physiologic blood flow measurements given by pulsed doppler. The technique has been enhanced by the introduction of color doppler US which shortens examination times and gives more confident and reproducible results. Carotid duplex sonography allows recognition of high grade stenoses and differenciates those low grade stenoses which do not require any further examination from those which may be considered as surgical indications (hemorrhagic plaque, ulceration) because they may be a source for emboli, even if not flow limiting. Carotid duplex sonography can be considered more specific than IA DSA which is limited to the evaluation of the degree of stenosis, without giving information about the precise morphology and nature of the plaque. The course will focus on technical aspects, results and 
difficulties of carotid sonography and underline the remaining indications for IA DSA, in a combined approach.

1267 Non invasive vascular ultrasound imaging - from peripheral to abdominal vessels - technical and practical aspects

F. Weill; Besançon

Pulsed and Duplex Doppler (DD) enable one to display as a graph the blood velocity. The pattern of the velocity curve characterizes the flow pattern: normal arterial or venous pattern: particular pattern in some particular vessels, such as the hepatic veins; abnormal patterns of vascular stenosis or thrombosis. Recently, color doppler (CD) has brought about a new mode of dynamic bidimensional flow representation.

We want to help clarifying what kind of additional diagnostic information CD is able to yield. With that purpose, we shall start in peripheral vascular networks and end up within the liver, the basic data of arterial and venous velocity curves will be described and compared to $\mathrm{CD}$,

- regarding arteries, $C D$ enables one to obtain a color-mapping of the arterial flow, and to pinpoint abnormal zones where DD will be carried out as a complement.

In peripheral veins, $\mathrm{CD}$ also yields a color mapping of the blood flow. In case of sonotransparent partial venous thrombosis, conventional ultrasound is delusive, particularly if $\mathrm{DD}$, centered on the persisting incomplete lumen shows a normal velocity spectrum. In such cases, $\mathrm{CD}$ is able to display directly the narrowed lumen, the colored lumen outlining the thrombi. Color mapping helps also in the evaluation of the extension of the thrombosis. However, the quality of $\mathrm{CD}$ can be impaired, as is DD, by non adequate beam angulation. What has just been described for peripheral veins applies to the vena cava, the hepatic veins and the portal network. Examples of Budd Chiari and of thrombosed portal veins will be given.

We found CD particularly efficient in two fields of application:- the follow-up of the transplanted liver (arterial thromboses, pseudoaneurysms, venous thrombosis).

- the preoperative assessment and the follow-up of multilocular ecchinococcosis.

The study of the vascularization of liver tumors is another field of application. $\mathrm{DD}$ and $\mathrm{CD}$ are complementary. Morphologic data yielded by $\mathrm{CD}$ are decisive in many circumstances.

\section{Room E2}

\section{RC 1006}

\section{Postoperative orthopedic imaging: the painful arthroplasty} Kristian Herrlin; Lund

In the radiologic evaluation of arthroplasties, a thorough understanding of the surgical procedure, of the biomechanical demands on the prosthesis and of the pathophysiology of subsequent complications is mandatory.

In this lecture with an emphasis on cemented total hip and knee replacements, technical aspects of the surgical procedure relevant to complications, and the role of the prosthetic orientation in the development of subsequent failure will be discussed.

Prosthetic complications, such as infection, loosening, foreign body reaction, metal fatique, ileopectineal bursitis, dislocation and prosthetic wear are presented and the merit of different imaging modalities including bone scan, arthrography, ultrasonography and CT is illustrated.

\section{Fibrosis in the spinal canal}

\section{McCall; Oswestry}

Modern surgical techniques have significantly reduced the soft tissue trauma of disc prolapse removal. Nevertheless, haemorrhage within the spinal canal continues to occur and this can be demonstrated by CT scans in the immediate post-operative period which shows heterogeneous material of lower attenuation than disc and with blurred margins in a high percentage of cases. Fibrosis around the nerve roots and dural sac develop over the subsequent weeks in a proportion of patients and appear as a mass of tissue attenuation with 30-50 $\mathrm{HU}$, and varying in position and area. The dural sac may be distorted by attraction to the operated side and extensive fibrosis may obliterate the dural sac completely. Epidural fibrosis is also clearly seen on magnetic resonance as hypointense to the annulus on T1 SE and hyperintense on T2. Differentiation of fibrosis from disc may be enhanced on both CT and MR by the use of IV contrast agents which highlight the fibrosis at the expense of disc material. Recent studies on MR suggest accuracy levels of between $89 \%$ and $96 \%$ in this regard. The clinical significance of fibrosis has been questioned however as recent studies found it present in extent and severity in both asymptomatic and symptomatic post-operative patients.
1270 Healing and non-union of fractures J.Freyschmidt, A. Kasperczyk; Bremen

The healing of fractures can be differentiated patho-anatomically and radiologically into a primary and a secondary form. Disturbing factors such as inadequate immobilisation, vascular damage and infection can lead to a delayed healing or non-union of the fragments. One talks about "delayed healing" if a fracture - depending on patient age, localisation and severity does not heal within 16 to 18 weeks. If there is no consolidation within 8 months, one speaks about "non-union". Here one can differentiate between a hypertrophic (reactive), atrophic (areactive) and infected form.

The course of the healing process is not influenced negatively by posttraumatic cyst formation, which can develop after subperiosteal or intratrabecular hemorrhage or after interposition of soft tissue. In the daily routine one reason for a delayed healing process or a non-union is often overlooked: a primary or secondary bone tumor, on the ground of which a spontaneous fracture has happened.

The different forms of normal and disturbed healing of fractures with or without osteosynthetic intervention can be sufficiently imaged and therapy can be adequately planned with the help of today's imaging modalities (plain-film, scintigraphy, computed tomography and magnetic resonance imaging). We will discuss the adequate use of the different imaging techniques.

\section{Room L}

\section{WS 1009}

1271 Management of children in imaging procedures: how to do conventional gastrointestinal procedures in children?

F. Diard, J.F. Chateil; Bordeaux

Digestive endoscopy is a more severe procedure in children than in adults, so the indications of conventional radiology are widely extended in pediatrics, owing to its easier use. A few indications have been done away by U.S. which is the ideal additional technique.

Conventional gastrointestinal procedures will be described according to their use on emergency or in daily practice.

ON EMERGENCY a frontal chest X-Ray and plain films of the abdomen, upright and supine A.P. views, and a prone lateral view, are first performed. They allow the diagnosis of obstruction or perforation.

Opacification is performed depending upon the entity suspected:

- In case of incomplete high obstruction, with green vomiting upper gastrointestinal tract (U.G.I.T.) opacification is the best tool of duodenal stenosis diagnosis.

- In case of neo-natal distal intestinal obstruction iso-osmolar aqueous contrast enema is necessary for diagnosis. Hyperosmolar contrast may be used for meconium ileus therapy.

- In case of intussusception plain films of the abdomen and U.S. are reliable enough for the diagnosis, but reduction is basically carried out by aqueous contrast or barium enema.

- Necrotizing enterocolitis is best explored by plain films.

IN DAILY PRACTICE:

- Conventional radiology remains the best technique to study the swallowing disorders.

- Opacification of the oesophagus is the major tool for the diagnosis of intrinsic (peptic, caustic, congenital) or extrinsic (vascular signs) stenosis. Conventional radiology is easier to use than P.H. study for gastroesophageal reflux, but its sensibility is far less. The availability of U.S. is on evaluation. - When hypertrophic pyloric stenosis is suspected, an A.P. upright plain film of the abdomen, without eating since six hours and U.S. allow the right diagnosis in most of the cases. U.G.I.T. opacification is only performed when U.S. is negative.

- The small bowel pathology is the privilege of barium opacification except for intestinal malabsorptions which are diagnosed by biopsy, and Non-Hodgkin lymphomas where U.S. is most of the time sufficient.

- The colon is well imaged by single or double contrast enema which allow the diagnosis for polyps and polyposis, ulcerative colitis and Crohn disease. During the nineteenth all these applications will persist but digital radiology will progressively take the place of conventional radiology.

\section{Management of children in imaging procedures: how to do conventional GU-studies in children?} Ulrich V. Willi; Zürich

Conventional voiding-cystourethrography (VCU) and excretion urography (EU) should be kept in professional training and routine practice. They remain relatively simple and easily available procedures yielding, in good hands, a most complete overall information on the anatomy and function of the kidneys, ureters, bladder and urethra in a majority of patients.

Circumstances: Care for proper surroundings, time, assistance and equipment (including fluoroscopy) to do the studies. A friendly room, calm athmosphere and dim light are essential. Explain briefly why the test is done. With the 
mother convinced that things in process are necessary and well done, the examination is usually successful.

VCU: Usually done in normally hydrated outpatients without medical sedation (a sweetened pacifyer often helps in babies) in supine position. For girls, frog leg position and a bright light source are indispensable. Gently spread the labia. Identify the urethral orifice. After local desinfection, introduce a French 8 (F5 in babies) catheter by a gentle but determined forward move. If the orifice is not visible, place catheter in exact midline above hymen; as the catheter enters the orifice, it often becomes visible. Fix catheter next to labium. - In uncircumcised boys, try to free the glans for desinfection. In physiological phimosis, desinfect and aim with catheter to center of glans through phimosis, pulling the foreskin gently backwards. Stay with mild constant pressure by the catheter against closed sphincter, avoiding back-and-forth movements. The catheter will eventually glide in as the child is crying, taking a deep breath or attempting to void. Fix catheter along shaft of penis. Fill bladder continuously under hydrostatic pressure $(60-90 \mathrm{~cm} \mathrm{H} 20)$ to capacity, i.e. initiation of voiding. Check briefly catheter position as contrast begins to enter the bladder. Have mother help to calm the child if needed. Encourage voiding while trying to relax the child. Encouragement to exert pressure is usually counterproductive. Appreciate the child's cooperation. Do oblique spotfilms of each ureter insertion site, straight spotfilms of occurring renal reflux, of urethra (oblique in boys), of bladder and kidney regions at end of voiding. Leave catheter during voiding.

EU: In moderate to severe reflux, leave the catheter under drainage to prevent reflux. Wait for proper drainage of upper tracts and do abdominal film before injecting contrast intravenously. Do 3-minute (after beginning of injection) film of renal area and 15-minute abdominal film. In obstructive uropathy, follow-up abdominal films at 45 or 60 (120) minutes may be necessary, always with the bladder empty either by catheter drainage or by active voiding prior to exposure.

After the study, explain findings to mother (parents). Drawings may help.

\section{Room M}

\section{RE}

1005 Diagnosi precoce dei tumori urologici: rene, vescica, prostata, testicoli (This Refresher Course will be translated simultaneously from Italian to English and vice versa)

\section{Early detection of urological tumors: kidney R.S. Pozzi-Mucelli; Trieste}

The new diagnostic imaging modalities have increased the detection of renal tumors and, among these, of small renal tumors (less than $3 \mathrm{~cm}$ in diameter). This is due to two major factors:

1. improved visualization of the kidneys;

2. increased number of kidneys visualized in the general population.

The imaging modalities available for the early diagnosis of kidney tumors are intravenous urography (IVU), ultrasonography (US), computed tomography (CT), magnetic resonance imaging and arteriography. However only US and CT have had a real impact on the diagnosis of small renal tumors. Among the two techniques CT has shown a greater sensitivity (94-100\%) than US (nearly $80 \%$ ). Both techniques are superior than IVU which shows only $67 \%$ of small renal tumors.

Although US and CT are both sensitive in detecting small renal tumors, they raise problems of differential diagnosis with benign (adenoma, oncocytoma), malignant lesions (metastases, lymphoma) and with non tumorous conditions (pseudotumors, etc.).

Furthermore, the early detection of kidney tumors has opened some controversies about the surgical treatment.

\section{Early detection of urological tumors: bladder P. Pavlica; Bologna}

Bladder tumors are the most common tumors of the urinary tract and represent about $3 \%$ of all neoplasms. They include a heterogeneous group of lesions that vary considerably in their histologic appearance and malignancy. The treatment, the frequency of recurrences and prognosis of urothelial carcinomas are influenced by stage and grade of disease at the time of the first examination. The over-all 3 years survival rate of high stage tumors (T3-T4) is $15-27 \%$, while that of low stage (Ta to T2) is 53-90\%. The early diagnosis of small superficial bladder cancer is therefore the main factor influencing the survival rate. Excretory urography is the most common radiographic method for the identification of bladder cancer but has a low sensitivity in detecting small neoplasms: the accuracy rate is $30-65 \%$ depending on the size of the tumor.

Transabdominal ultrasonography has been reported to be more effective in the identification of small carcinomas with a detection rate of $75-80 \%$ for lesions from 2 to $5 \mathrm{~mm}$ in size. When used in conjunction with urinary cytology, US is a valuable procedure in the early diagnosis or follow up of patients surgically treated; the diagnostic accuracy is $85-95 \%$. This association is indicated in the diagnostic phase and also to monitor the previously treated patients to reduce the frequency of cystoscopy in the follow-up. Furthermore U.S. is useful in the diagnosis of tumor located in a diverticulum which are difficult to see with cystoscopy.

Urography is still indicated to detect synchronous and metachronous carcinomas of the upper urinary tract.

\section{Early detection of urological tumors: prostata \\ G. Di Candio; Pisa}

The early diagnosis of prostatic tumors introduces the following problems: - biopsy of all the hypoechoic lesions, all the palpable nodules or only the hypoechoic and palpable lesions;

- biopsy of peripheral and lateral areas of the gland or biopsy of the anterior and central areas too.

We think that the common sense has to drive the decision, especially when we use a technique which, with the increase of the frequency, easily detects even minimal differences in the US structure of the tissue. Infact not always these modifications correspond to a neoplastic area. It is important then to integrate the clinical-bioumoral criteria and the echographic findings.

Some cancers are palpable and US detectable whereas others are not palpable and not US detectable; others are non palpable and US detectable, because of these difficulties some rules have to be followed.

A) Biopsy of all the palpable areas without definite characteristics of benignancy (asymmetric, hypertrophic nodule, area of dense fibrosis, calcifications or cysts). The existence of isoechoic tumors justifies, in our opinion, the above policy.

It is for sure less dangerous the underestimation of a not palpable tumor, then probably small and in an early stage, than the underestimation of a cancer already clinically manifest.

B) When the prostate is clinically normal (no palpable nodules), the hypoechoic areas, especially if located at the periphery of the gland, are the target of the US-guided biopsy. Also in this case it is very important to determine the size of the lesion and the presence of a pathological PSA/Yang dosed level. If the area is less than $5 \mathrm{~mm}$ in diameter and the PSA is normal, it is possible to avoid the biopsy, whereas if the area is between 5 and $10 \mathrm{~mm}$ the biopsy is necessary only with an elevated PSA. Lee points out a PPV of $0 \%$ for lesions of the above diameter when PSA and DRE (digital rectal examination) are normal. Nevertheless every case does need a strict evaluation, because it has been demonstrated that US tends to underestimate the size of the lesion. When the lesion is greater than $1 \mathrm{~cm}$ in diameter, the biopsy is mandatory, even if the DRE and PSA are normal.

\section{Early detection of urological tumors: testis G. Rizzatto; Gorizia}

Advances in early diagnosis and innovations in therapy have made cure the expected outcome for most patients with testicular tumours.

Sensitivity values higher than $98 \%$ and the lack of adverse biological effects make high resolution U.S. the best tool for screening patients at risk. The population to be screened will include family members of men who had testicular tumour, patients with posttraumatic atrophy or infections and patient with maldescent testis.

U.S. screening should also be applied to the palpably normal testicle in patients with controlateral tumour to detect either simultaneous or metachronous bilateral tumours.

Tumours larger than $3 \mathrm{~mm}$ are easily recognized; most of them are hypoechoic. Unfortunately many small benign processes may mimic these neoplastic changes: Doppler and color Doppler analysis are not reliable. For small hypoechoic lesions specificity is less than $75 \%$. Fine needle aspiration biopsy under ultrasonic guidance is required to avoid false positive results; $23 \mathrm{G}$ needles are used for this $94 \%$ accurate and safe procedure.

\section{Room N}

\section{RG 1007}

1277 Chemonucleolysis in the treatment of lumbar herniae J.P. Braun; Colmar

Chymopapainnucleolysis was introduced by SMITH in 1964 as a percutaneous method of treatment of lumbar disk herniation through enzymatic hydrolysis of the condromucoprotein part of the nucleus pulposus. It appears to reduce the pressure exerted by the herniated disk material on adjacent spinal nerves. The technique of chemonucleolysis must be applied under strict asepsis conditions and after neuroleptanalgesis preparation of the patient. The disk is punctured from the left side and a good nucleography is always practised to directly reveal the state of the disk to be treated.

In certain cases, we extend our examination by a CT discography in order to appreciate the topography and volume of the hernia as well as the rupture of the posterior ligament. 
We treat all typical or atypical sciatic pain of hernial origin after a meticulous clinical anamnesis and CT location of the hernia.

We observed over $80 \%$ good results in a series of 1400 patients over 8 years. We have not observed any anaphylactic shock, only a few minor allergic complications without sequellae.

Chymopapainnucleolysis, in the properly selected patient, is an effective alternative to surgical diskectomy for the treatment of lumbar disk herniation. Thus it has no post-surgical fibrotic recurrence and permits future surgical treatment if necessary.

1278 Automated percutaneous lumbar discectomy (percutaneous nucleotomy)

H. Weigand; Wiesbaden

The percutaneous nucleotomy or the Onik-procedure is described as a new interventional percutaneous radiological technique for lumbar disc removal. The own results in 350 patients since October 1987 are perfectly correlated with other surgical and microsurgical techniques with and without endoscopic control. The percutaneous nucleotomy seems to be very valuable in every level of the lumbar spine.

Precise indications and technique under fluoroscopic control and documentation may ensure 70 to $80 \%$ good and excellent results. In comparison with other interventional treatments the percutaneous nucleotomy is safe, relatively simple and without major complications. In the near future the percutaneous nucleotomy will have a certain place in the treatment program of radicular back pain.

\section{Room 0}

\section{WS}

1279 Breast cancer screening

N. Bjurstam; Gothenburg

Abstract available at the information desk

1280 Frequency and predictivity of recalls and calls for biopsy in the Florence screening programme

Stefano Ciatto, Marco Rosselli Del Turco; Florence

Recall rates in the Florence program decreased progressively, according to changes in recall criteria, from $20.3 \%$ in $1979-81$ to $1.4 \%$ in $1987-89$ when the presence of radiologic abnormalities suspicious for cancer were the most common criterium. Subjective symptoms other than pain were also criteria for recall as they were unfrequent $(0.2 \%)$ but had a high predictivity $(22.7 \%)$ and were the only reason for recall in 2 of 69 cancers detected. Recall rates decreased and predictivity increased with age. Recall rates were double (2.2 vs. $1.2 \%)$ and predictivity was half ( 11.7 vs. $21.7 \%)$ at first compared to repeat screening when comparison with a previous test was possible. Diagnostic assessment was increasingly complex and in 1987-1989 was based on physical examination, detail mammography, direct magnification, sonography, and cytology of palpable or nonpalpable (stereotaxic or US-guided) lesions. This allowed a reduction in the frequency (from 0.9 in $1979-81$ to $0.3 \%$ in 1987-89) and an increase in the predictivity (from 0.33 in 1979-81 to 0.92 in 1987-89) of biopsy calls. Recalls for diagnostic assessment and calls for biopsy account for a relevant proportion of screening costs and should be optimized according to a reference standard.

1281 Breast cancer screening in the Netherlands is a cost-effective programme: what does this mean, and might it be true for all countries? H. J. de Koning; Rotterdam

Breast cancer screening is generally accepted as an effective means of reducing breast cancer mortality in post-menopausal women. The impact on quality of life of such programmes and the attainable mortality reduction in a real population, however, remain to be discussed. Seemingly contradictory, there is much pressure to expand screening programmes for women younger than 50 or older than 70 years of age, and more and more (European) countries are discussing the possibilities of breast cancer screening.

In this lecture, the main favourable effects of the Dutch screening policy will be presented (e.g. breast cancer mortality reduction, reduction in treatment for advanced disease, shift from mastectomy to breast conserving therapy), opposed to inevitable unfavourable effects (e.g. additional number of primary treatments, increase in follow up procedures). It will be shown that mammographic screening for women aged 50 and over with a 2 or 3 year interval is cost-effective, compared to other health care programmes. The Dutch organization structure will shortly be presented. A large number of factors influence both the favourable and unfavourable effects of screening and its cost-effectiveness. In this lecture, I will focus on those factors where the radiologist might play an important role: capacity of screening units, quality of mammographic reading or criteria for referral (all in relation to the screening situation), and attitude of radiologists or general practitioners in respect to requests of women (outside a screening programme) or especially for non invited women.

At present, screening women under 50 is not to be recommended. Even when assuming a small mortality reduction for this age group, an extremely unfavourable balance between effects and costs will evolve. The cost-effectiveness of breast cancer screening in different European countries will be discussed.

1282 Breast cancer screening programme in Germany H.-J. Frischbier, W. Hoeffken; Hamburg

The cancer screening programme of the statutory health insurance system in Germany includes a physical examination of the breast and instructions in breast self examination for all women aged over 30 years. Mammography is reserved for high risk women (positive family history, previous abnormality), and of course for diagnosis of breast lesions in symptomatic women.

Approximately 1700 units examine 2,5 million women each year. Screening mammography for all symptomless women is not yet included in the health insurance items. From 1971 to 1986 a controlled screening study was performed in Hamburg at the university hospital. Based on the results of the different European screening studies with reduction of the mortality rate in breast cancery the German Society of Senology constituted a committee of experts to find out how to organize a screening programme in Germany. This committee developed a programme in cooperation with the Ministry of Health and the legal health insurances. In 1989 the "Deutsche MammographieStudie" was founded. This study has been given the assignment to develop training of physicians and quality control measures in order to prepare introduction of screening mammography in Germany. The health system in Germany does not allow specialized screening centers. Screening mammography would be performed by radiologists and gynaecologists in their own office practices. Understandable questions can be rised whether the results of the randomized studies can also be transferred to our health system. Up to now two service regions with about 40 mammographic physicians are involved. Women over 40 years of age are invited. The first three mammography rounds including clinical examination will take place with an annual interval, then a two year interval will be recommended.

On the basis of the results of this German study the introduction of a screening mammography in the early detection of breast cancer will be considered by our government and the health insurances.

\section{Overview on the Belgian breast cancer mass screening projects} W. Gordenne; Liege

Several cancer screening centers have existed in Belgium for many years, under the responsibility of oncologists and gynecologists; breast cancer screening is essentially based on physical examination.

The index of frequentation of these centers is low.

Since 1985 some radiologists are sensitized by the results of foreign trials based on systematic mammography but have had many difficulties in convincing most of the radiologists of the difference between diagnostic and screening mammography. Moreover, they had difficulties in situating the role of screening mammography beside BSE and physical examination and in convincing general practitioners of the role they must play in this public health problem.

Another important fact is that social security has up to now decided not to play a responsible role in preventive medicine.

+ ince the beginning of 1990, a national group getting together representatives of universities, public and private medicine, has worked to set up a scientific "white book" on breast mass screening playing the role of official reference in this field.

Different local groups are undertaking pilot studies based on the specialized and the non specialized models. They are advised by the national group in order to present their first results before 1994, year selected by "Europe against Cancer" to support some projects in our country.

\section{Room R}

\section{SS}

16.12 Importance of fractionation and dosage in radiotherapy with a view on results and sequelae II

\subsection{PM}

1284 Reactor fast neutrons in the combined and radiation treatment of some human tumors

I. A. Gulidov; Obninsk

From October 1985 till now at the Institute of Medical Radiology (Obninsk, USSR) 150 patients with laryngeal, breast carcinoma and osteogenic sarcoma have received mixed gamma-neutron radiation therapy or combined treatment (preoperative neutron or gamma-neutron therapy and surgery) using reactor neutron beam. In all cases acute local reactions allowed realization of the treatment plan. Reactor fast neutrons in the preoperative radiation therapy did 
not deteriorate of wound healing.

Described techniques were shown to be promising treatment modality allowing to increase efficacy of radiation and combined treatment of some human tumors.

\subsection{PM}

1285 Local-regional relapses in early breast cancer treated by conservative surgery and radiotherapy

H. Bašić, M. Mušanović, A. Drljević, A. Tatarević, S. Strinić; Sarajevo

At our Institution between 1980 and 1984, 56 breast cancer patients with clinical Stage I or II (with tumour less than $3 \mathrm{~cm}$ in pathological diameter) were treated by conservative surgery and radiotherapy. Radiotherapy was administered with Co-60, $5500 \mathrm{cGy}$ in 5 and half weeks to the breast, and $5000 \mathrm{cGy}$ to the axilla and supra-clavicular region in patients with pathologically positive axillar lymphnodes. Sixteen out of 56 patients had a boost 1000 to $2000 \mathrm{cGy}$ to the tumour bed. Seven out of $56(12,5 \%)$ patients developed local-regional failure, three of them in breast, three in axillar nodes, and one patient had both breast and axillar failure. Only one out of 4 patients who developed breast failure had got boost irradiation. All patients with axillar recurrence had palpable lymphnodes before the surgical treatment.

4.06 PM

12864 years experience with radio-chemotherapy of advanced head and neck carcinomas with carboplatin

Th. Schnabel, N. Zamboglou, C. Kolotas, M. Daniel, G. Schmitt; Düsseldorf

From 1987 to 1991101 patients with advanced head and neck carcinoma (T2-4, N0-3) were treated with radio-chemotherapy with Carboplatin. Tumours were located in the oral cavity in 33 patients, oropharynx in 9 patients and hypopharynx in 7 patients. Two patients had a tumour of the epipharynx, two patients a tumour of the larynx and 48 patients an involvement of two or more compartments. Radiotherapy was performed with 60 Co-rays or $8 \mathrm{MeV}$ photons in a fractionation of $5 \times 2 \mathrm{~Gy} /$ week up to a dose of $50 \mathrm{~Gy}$. Carboplatin was administered in a dose of 60 or $70 \mathrm{mg} / \mathrm{m}^{2}$ from day $1-5$ and from 29-33. After a two week interval the tumour involution was evaluated and decision was made in the patients operability. In case of inoperability radiotherapy was continued up to a target absorbed dose of 70-74 Gy. Up to now 101 patients have entered the study. 93 of them have completed treatment up to now and three of them died during the treatment period. 30 patients were operated after $50 \mathrm{~Gy} .7$ patients showed a histologically complete remission, 10 microscopic tumour residual and 13 had macroscopic tumour left behind. 63 patients were only irradiated. 35 of them achieved a CR and 27 a PR. In eight of these 26 patients the residual tumour could be operated. At the end of treatment 70 patients showed a complete remission.

4.09 PM: Roundtable Discussion with Keynote speakers

\section{Room S}

WS

1015

1287 Fast CT of the cardiovascular system

P. F. Sheedy II; Rochester, MN

Development utilization of a Fast CT scanner that uses an electron beam rather than a rotating $\mathrm{x}$-ray tube has enhanced the ability of radiologists and cardiologists to examine the heart and vascular system with $\mathrm{x}$-ray computed tomography.

The beating heart can be examined with simultaneous contiguous multilevel scans that can be sequentially displayed to depict cardiac dynamics. Highly accurate ventricular volumes can be determined in various physiologic and pathologic states. Also flow sequence of opacification studies for determination of vessel patency and organ perfusion can be conducted using volumetric sequential scanning. Both clinical and research applications are being developed.

The Fast CT scanner, originally fashioned for cardiac scanning, was modified so it might also be used for high resolution conventional applications in all areas of the body. This change allowed it to be used, to great advantage, in evaluation of the vascular system, especially the aorta.

The short scan time ( $100 \mathrm{msec})$, the short scan interscan time and lack of $\mathrm{x}$-ray tube heat limits permits scanning to occur at a rate as fast as 40 scans (with table incrementation) in less than 70 seconds. Rapid examination of both chest and abdominal vascular structures can be conducted in a short time with maximum opacification of vessels using standard (or less) doses of contrast material. Protocols for examination of all blood vessels and of vascular structures within organs are being developed and modified in an effort to improve the quality of imaging of the heart and the vascular system. 
